\title{
EAPC Abstracts
}

\section{EAPC President's Welcome}

Dear Colleagues, Dear Friends,

On behalf of the Board of EAPC, and as President, I am delighted to welcome you to our $16^{\text {th }}$ World Congress in Palliative Care. As ever, this Congress is two years in the planning and it has been my honour to work with a dedicated group of colleagues, who have worked tirelessly to bring this programme to fruition.

Berlin is an amazing choice of venue for our Congress for many reasons. Everyone can find something to capture the imagination in this beautiful city. It resonates with history, diversity and vibrancy but most of all, with life. And that, of course, is a key message for palliative care. We who work in this field are as much about life as death, supporting patients and families to live and live well until the end. So, as the people of Berlin, we should embrace life and savour every moment that this Congress and this city will offer us over the next few days.

The $16^{\text {th }}$ World Congress focus on global health is a strong message that EAPC is a world player in the development and practice of palliative care. We have a voice which echoes the interface between clinical, research and policy engagement. We represent a critical perspective in national and international understanding of palliative care and showcase our work through this Congress and that of the EAPC Research Network (don't forget Palermo 2020 !). Please take time to sit and listen, debate and reflect on the important issues to be discussed here at Congress. Your views are important and help us to shape our EAPC thinking on the way forward. I am also delighted that for the first time, we will have a parallel day on children's palliative care, ably organised by our CEO, Dr. Julie Ling. We expect over 700 delegates will attend this event, a remarkable achievement and one which heralds a new strand in our Congress material which I hope will continue in future years.

There are many people to thank for this event. Our Co-Chairs, Dr. Sébastien Moine and Dr. Anne Letsch, who was also leading the local organising committee, our debt of thanks for the hard work and long hours. Thanks also to the members of the Scientific Committee and Local Organising Committee who have provided the ideas and proposals which shape the 2019 Congress. Your support is, as always, much appreciated. We cannot forget our Conference Partner, Interplan and Elke Jaskiola in particular for organising us and keeping us to task. Of course, my personal thanks to EAPC Head Office, Julie, Eleanor, Cathy and Avril for the logistics, organization and managing all the other EAPC demands at the same time as bringing a world congress together. I would also like to give sincerely thanks to our colleague Claudia Sütfeld our congress administrator who has worked tirelessly to ensure that everything runs smoothly.

Finally, I wish to thank our German friends and colleagues for welcoming us to Berlin and for agreeing to host this Congress.

Liebe Freunde,

die Wahl des Landes und des Tagungsorts für einen EAPC-Kongress ist immer eine Herausforderung. Diesmal allerdings fiel es uns wirklich leicht, denn Sie haben uns überaus herzlich willkommen geheißen. Im Namen des EAPC-Vorstands danken wir der Deutschen Gesellschaft für Palliativmedizin für alles, was sie in den letzten zwei Jahren geleistet hat.

So, I wish you a wonderful Congress, time to meet old friends and make new ones and opportunities for creativity and relaxation when you can. Welcome to Berlin 2019.

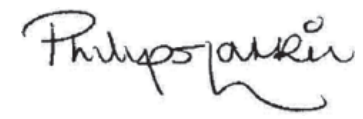

Professor Philip J Larkin

President, European Association for Palliative Care 


\section{Welcome from the Chairs of the Scientific and Local Organising Committee}

Dear Congress Participant,

It is a pleasure to welcome you to the $16^{\text {th }}$ World Congress of the EAPC in Berlin. As questions are asked related to equitable access to palliative care in Europe and around the world, the congress "Global Palliative Care - Shaping the future" will explore how palliative care fits with the current global health agenda. You will be at the core of the discussions and events which aim to address the emerging challenges and opportunities our discipline will face in policy, research and clinical practice.Five years on from the adoption of the World Health Assembly Resolution on Palliative Care, the EAPC World Congress is the perfect time to reflect on our progress in the development of palliative care globally and to identify areas where more needs to be done. Our Congress takes place shortly after the 2018 adoption of the new Declaration of Astana which highlights palliative care as one of the key components of primary health care, after the United Nations High Level Meeting on non-communicable diseases and before the United Nations High Level Meeting on universal health coverage which will take place in New York in September 2019. The successes of palliative care now transcend its usual boundaries and the work of building bridges toward new landscapes is now deeply rooted in its DNA. Berlin provides you with the opportunity to examine the extent to which palliative care has been included in universal health coverage (UHC) and within our various health systems.

The increasing recognition of the importance of palliative care in society is reflected in the growing number of EAPC congress delegates who represent a range of disciplines from many countries throughout Europe and beyond. The EAPC World Congress provides a unique opportunity to network and meet with colleagues, clinicians, caregivers, researchers, volunteers, patients advocates and educators in palliative care from across the globe. You will exchange knowledge with champions and enthusiasts to celebrate diversity and richness, make new links, new collaborations and develop projects during the time spent together. We are expecting more than 3000 participants to attend the congress.

Berlin is the capital of Germany and the country's largest city. It is the major centre for politics, science, culture and art. Like Palliative Care, Berlin is dynamic, colourful and diverse and honoured and delighted to host the EAPC World Congress.

On behalf of all involved in EAPC 2019, we welcome you to Berlin and the 16th World Congress of the European Association for Palliative Care.

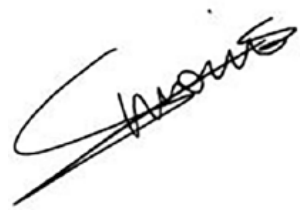

Dr Sebastien Moine

Chair of the Scientific Committee

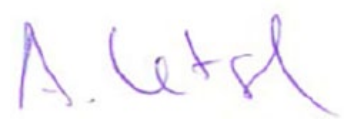

PD Dr Anne Letsch Co-Chair of the Scientific Committee Chair of the Local Organising Committee 


\section{Committees}

\section{Scientific Committee}

Sebastien Moine (France) - Chair

Anne Letsch (Germany) - Co-Chair

Phil Larkin (Ireland)

Christoph Ostgathe (Germany)

Irene Murphy (Ireland)

Libby Sallnow (United Kingdom)

Malgozata Krajnik (Poland)

Saskia Teunissen (The Netherlands)

Lieve Van den Block (Belgium)

Lukas Morin (France)

Local Organising Committee

Anne Letsch - Chair

Simona Blankenburg

Michael Ewers

Heiner Melching

Christof Müller-Busch

Urs Münch

Wiebke Nehls

Angelika Odening

Martina Preisler

Lukas Radbruch

Beate Rothermel

EAPC Head Office

Julie Ling, Chief Executive Officer

Eleanor Blake, Administrator

Cathy Payne, Project Officer

Avril Jackson, Information Officer \&

Social Media Editor

Claudia Sütfeld, Congress Secretary

\section{Reviewers}

Aass, Nina (Norway)

Agar, Meera (Australia)

Alonso Babarro, Alberto (Spain)

Alt-Epping, Bernd (Germany)

Amblas, Jordi (Spain)

Antunes, Barbara (Portugal)

Baracos, Vickie (Canada)

Barnard, Alan (South Africa)

Bates, Ursula (Ireland)

Bausewein, Claudia (Germany)

Beaussant, Yvan (United States)

Becker, Gerhild (Germany)

Benitez-Rosario, Miguel Ángel

(Spain)

Benkel, Inger (Sweden)

Bernard, Mathieu (Switzerland)

Beyer, Sigrid (Austria)

Bhatnagar, Sushma (India)

Bitschnau, Karl W (Austria)

Bluebond-Langner, Myra (United

Kingdom)

Boland, Jason (United Kingdom)

Bollig, Georg (Denmark)

Bolognesi, Deborah (Italy)

Bolt, Eva (Netherlands)

Bone, Anna (United Kingdom)

Borasio, Gian Domenico

(Switzerland)

Boyd, Kirsty (United Kingdom)

Boyd, Michal (New Zealand)

Brazil, Kevin (United Kingdom)
Cohen, Joachim (Belgium)

Connor, Stephen (United States)

Costantini, Massimo (Italy)

Craig, Finella (United Kingdom)

Csikos, Agnes (Hungary)

Cuervo Pinna, Miguel Ángel (Spain)

Curiale, Vito (Monaco)

Currow, David (Australia)

De Conno, Franco (Italy)

De Simone, Gustavo (Argentina)

de Visser, Marianne (Netherlands)

Downing, Julia (United Kingdom)

Droney, Joanne (United Kingdom)

Dziegielewska-Gesiak, Sylwia

(Poland)

Eisenchlas,Jorge Hugo (Argentina)

Engels, Yvonne (Netherlands)

Ewers, Michael (Germany)

Ewing, Gail (United Kingdom)

Eychmueller, Steffen (Switzerland)

Fainsinger, Robin (Canada)

Fallon, Marie (United Kingdom)

Filbet, Marilène (France)

Fineberg, Iris Cohen (United States)

Finucane, Anne (United Kingdom)

Firth, Pam (United Kingdom)

Flores Pérez, Luis Alberto (Spain)

Foley, Kathleen (United States)

Forbes, Karen (United Kingdom)

Frantzen, Tone-Lise (Norway)

Froggatt, Katherine (United

Kingdom)

Fürst, Carl Johan (Sweden)

Goncalves, Antonio (Portugal)

Gonçalves, Edna (Portugal)

Gootjes, Jaap (Netherlands)

Gove, Dianne (Germany)

Grande, Gunn (United Kingdom)

Gretton, Sophy (United Kingdom)

Griffo, Yvona (United States)

Groenvold, Mogens (Denmark)

Guldin, Mai-Britt (Denmark)

Gwyther, Liz (South Africa)

Harding, Richard (United Kingdom)

Hasselaar, Jeroen (Netherlands)

Hegedus, Katalin (Hungary)

Heimerl, Katharina (Austria)

Hockley, Jo (United Kingdom)

Hodiamont, Farina (Germany)

Hoenger, Catherine (Switzerland)

Hopkinson, Jane (United Kingdom)

Hoskin, Peter J. (United Kingdom)

Houska, Adam (Czech Republic)

Hudson, Peter (Australia)

Hughes, Sean (United Kingdom)

Jakubikova, Hedviga (Slovakia)

Johnston, Bridget (Ireland)

Johnstone, Ros (United Kingdom)

Jones, Laura (Switzerland)

Jox, Ralf J. (Switzerland)

Junghanß, Christian (Germany)

Kilic Öztürk, Yasemin (Turkey)
Letsch, Anne (Germany)

Leysen, Bert (Belgium)

Likar, Rudolf (Austria)

Limon, Esther (Spain)

Ling, Julie (Ireland)

Lloyd-Williams, Mari (United

Kingdom)

Lorenzl, Stefan (Germany)

Lunder, Urska (Slovenia)

Lundström, Staffan (Sweden)

Mackova, Marie (Czech Republic)

Maddocks, Matthew (United

Kingdom)

Maia e Moura, Claudia Maria

(Portugal)

Martino, Ricardo (Spain)

Martins Pereira, Sandra (Portugal)

Mattos-Pimenta, Cibele (Brazil)

McCaffrey, Nikki (Australia)

McEnhill, Linda Susan (United

Kingdom)

Mcllfatrick, Sonja (United Kingdom)

Miccinesi, Guido (Italy)

Minton, Ollie (United Kingdom)

Mitrea, Nicoleta (Romania)

Moine, Sébastien (France)

Morgan, Deidre (Australia)

Morin, Lucas (Sweden)

Morita, Tatsuya (Japan)

Mosoiu, Daniela (Romania)

Müller-Busch, Christof (Germany)

Murphy, Irene (Ireland)

Murtagh, Fliss (United Kingdom)

Mystakidou, Kyriaki (Greece)

Nauck, Friedemann (Germany)

Neergaard, Mette Asbjoern

(Denmark)

Nicholson, Caroline (United

Kingdom)

Nijs, Ellen (Netherlands)

Noble, Simon (United Kingdom)

Noguera Tejedor, Antonio (Spain)

Nolan, Steve (United Kingdom)

Normand, Charles (Ireland)

O'Brien, Tony (Ireland)

O'Connor, Margaret (Australia)

Oechsle, Karin (Germany)

Olagunju, Andrew (Nigeria)

Oliveira, José Eduardo (Portugal)

Oliver, David (United Kingdom)

Onwuteaka-Philipsen, Bregje

(Netherlands)

O'Reilly, Maeve (Ireland)

Ostgathe, Christoph (Germany)

Paal, Piret (Germany)

Panagiotou, Irene (Greece)

Papadatou, Danai (Greece)

Parker, Deborah (Australia)

Pascual, Antonio (Spain)

Pasman, Roeline (Netherlands)

Pautex, Sophie (Switzerland)
Reyners, An (Netherlands)

Rhee, Joel (Australia)

Rietjens, Judith (Netherlands)

Riley, Julia (United Kingdom)

Robinson, Jackie (New Zealand)

Rocafort Gil, Javier (Spain)

Rodio, Gustavo Francisco

(Argentina)

Rolke, Roman (Germany)

Rombouts, Walter (Belgium)

Rukhadze, Tamari (Germany)

Ryan, Karen (Ireland)

Salazar, Helena (Portugal)

Sallnow, Libby (United Kingdom)

Sampson, Elizabeth (United

Kingdom)

Sapeta, Paula (Portugal)

Schell, Matthias (France)

Schildmann, Eva (Germany)

Schildmann, Jan (Germany)

Schmidlin, Esther (Switzerland)

Schneider, Nils (Germany)

Scott, Ros (United Kingdom)

Seymour, Jane (United Kingdom)

Simões, Angela (Portugal)

Simões, Catarina (Portugal)

Simon, Steffen (Germany)

Sinclair, Shane (Canada)

Sjøgren, Per (Denmark)

Slama, Ondrej (Czech Republic)

Sleeman, Katherine (United

Kingdom)

Speck, Peter (United Kingdom)

Sterie, Anca Cristina (Switzerland)

Stiel, Stephanie (Germany)

Stone, Carol (United Kingdom)

Stone, Paddy (United Kingdom)

Strasser, Florian (Switzerland)

Tarquini, Daniela (Italy)

Taubert, Mark (United Kingdom)

Tavares, Miguel (Portugal)

Taylor, Ros (United Kingdom)

Teike Lüthi, Fabienne (Switzerland)

Teno, Joan (United States)

Teunissen, Saskia (Netherlands)

Thomas, Keri (United Kingdom)

Timm, Helle (Denmark)

Tishelman, Carol (Sweden)

Toma, Stefan (Romania)

Tuffrey-Wijne, Irene (United

Kingdom)

Turner, Mary (United Kingdom)

van de Geer, Joep (Netherlands)

Van den Block, Lieve (Belgium)

Van Den Noortgate, Nele (Belgium)

van der Heide, Agnes (Netherlands)

van der Steen, Jenny (Netherlands)

Van Nes, Marie-Claire (Belgium)

van Nus, José (Netherlands) 
Broeckaert, Bert (Belgium) Bruera, Eduardo (United States) Brunelli, Cinzia (Italy) Burucoa, Benoit (France) Busch, Christian (Denmark) Callaway, Mary (United States) Capelas, ManuelLuís (Portugal) Carneiro, Rui (Portugal) Centeno, Carlos (Spain) Chambaere, Kenneth (Belgium) Chambers, Lizzie (United Kingdom) Chan, Kin-Sang (Hong Kong) Chaumier, François (France) Ciurlionis, Marius (Lithuania) Cleary, James (United States) Coast, Joanna (United Kingdom)
Kabelka, Ladislav (Czech Republic) Keegan, Orla (Ireland)

Kiyange, Fatia (Uganda)

Klepstad, Pål (Norway)

Koffman, Jonathan (United Kingdom)

Krakowiak, Piotr (Poland)

Kreicbergs, Ulrika (Sweden)

Krizanova, Kristina (Slovakia)

Kurita, Geana (Denmark)

Larkin, Philip (Switzerland)

Leget, Carlo (Netherlands)

Lehto, Juho (Finland)

Leppert, Wojciech (Poland)
Payne, Sheila (United Kingdom)

Pelttari, Leena (Austria)

Peruselli, Carlo (Italy)

Petruta, Anania (Romania)

Pigni, Alessandra (Italy)

Pivodic, Lara (Belgium)

Pleschberger, Sabine (Austria)

Porta-Sales, Josep (Spain)

Portenoy, Russell (United States)

Powell, Richard (Kenya)

Preston, Nancy (United Kingdom)

Pype, Peter (Belgium)

Radbruch, Lukas (Germany)

Rasmussen, Birgit Holritz (Sweden)

Read, Susan (United Kingdom)

Reigada, Carla (Spain)

Reitinger, Elisabeth (Austria)

Rémi, Constanze (Germany) 


\section{Contents}

- Foreword

- Professor Philip Larkin

- Dr. Sébastien Moine, PD Dr. Anne Letsch

- Committees

- Plenary Sessions

- PL01 Floriani Lecture: Access to Palliative Care and Pain Relief - an Imperative of Universal Health Coverage

- PLO2 Early Palliative Care - When and How for Generalists?

PL03 The Language of Dying

PL04 Is Palliative Care Biased?

PL05 Increasing the Value of Research in Palliative Care

PL06 From Crisis to Sustainability - Building Palliative Care with Limited Resources

Best Abstract: Matters of Life and Death - An Experimental Study Investigating Interventions to Encourage End-of-Life Discussions

- Best Abstract: Coping Strategies of Patients with Advanced Lung or Colorectal Cancer in Six European Countries: Insights from the ACTION Study

- Best Abstract: The Liminal Space Palliative Care Volunteers Occupy and the Roles They Perform within it: A Qualitative Study

- Winner of the Post-Doctoral Award: Building a Palliative Care Research Career across Borders

- Winner of the Clinical Impact Award: Bringing palliative Care to the Public: the Impact of Social Media

- Winner of the Palliative Medicine Early Researcher Award: People, Palliative Care and Multiple Perspectives: my Contributions and Future Directions

- Parallel Sessions

- PS01 From Ideas to Action:Achieving Universal Access to Palliative Care

PSO2 Understanding Complexity in Palliative Care

PS03 Shaping the Future for Bereavement Care in Palliative Care

PS04 Separating the Wheat from the Chaff: Deprescribing in Advanced Illness

PS05 Palliative Care for Patients with Neurological Conditions - Collaboration and Interaction

PS06 What Happens to People with Intellectual Disabilities at the End of Life? Findings from Across Europe

PS07 Delivering Palliative Care in Ageing Populations: Comprehensive Interventions and Clinical Models

PS08 Increasing the Value of Randomized Controlled Trials (RCTs) for Complex Interventions in Palliative Care

PS09 Global Prospects in Palliative Care: Measuring the Burden, Responding to Crises

PS10 End of Life and Prison: Inequalities and Vulnerabilities

PS11 Medication Safety in Palliative Care

PS12 Implementing ACP across the Healthcare System in Europe and the US: the Results of Three ACP Trials.

PS13 Providing Palliative Care for Refugees and Migrants

PS14 Understanding Health Promotion in End of Life Care: from Policy Change to Community Action

PS15 The Challenge of Integrating Palliative Care for People with Chronic Obstructive Pulmonary Disease (COPD)

- PS16 Enabling Person-Centred Care for Palliative Care Patients and Their Informal Carers: Definitions, Models of Practice and Implementation

- PS17 Developments in Global Palliative Care Policy and Strategy: Opportunities and Challenges

PS18 What is New about Palliative Management of Breathlessness in People with Heart Failure

PS19 What is the Impact of Assisted Dying on Health Care Professionals Working in Palliative Care?

- PS20 Using Big Data to Improve End-of-Life Care: Opportunities and Challenges

- Meet the Expert Sessions

- ME01 Time to Change?: Current Practices in Delirium Care

ME02 Palliative Care and the Homeless Population: Challenges, Solutions and Lessons from Others

ME03 Maximising Global Impact in Palliative Care: Achieving Effective Knowledge Transfer and Exchange

ME04 How to Develop an Academic Palliative Care Network

ME05 How to Integrate Palliative Care and Pain Relief in Primary Health Care?

ME06 Enhancing Transparency in Systematic Reviews of Qualitative Literature: a Practical Approach

ME07 Role of Social Work in Supporting the LGBT Community

- ME08 Delievering Bereavement Care in Palliative Care - Can This Be Provided by Volunteers or Is It the Remit of Professionals? Evidence or Opinion?

- ME10 Managing Substance Use Disorder Treatment at the End of Life

- ME11 Enabling the Voice of Patients, Carers and Family Members to Improve Advocacy on Access to Palliative Care

- ME12 The Significance of a New Model of Social Capital in Redressing Inequalities in End of Life Care.

- ME13 New Findings on the Status of Palliative Care Globally from the 2nd Edition of the WHPCA/WHO Global Atlas of Palliative Care (2018)

- ME14 National Actions to Facilitate International Visibility and Networking in Palliative Care

- ME15 What Do the Arts Bring to Palliative Care?

- ME16 Practical Aspects of Spirituality

- $\quad$ Free Communication Sessions

- FC01 Older People: Dementia and Frailty Near the End of Life

FC02 Patient-Reported Outcome Measures (PROMs) and Routinely Collected Data

FC03 Living with Non-Malignant Conditions

- FC04 Pain, Breathlessness and Other Symptoms 


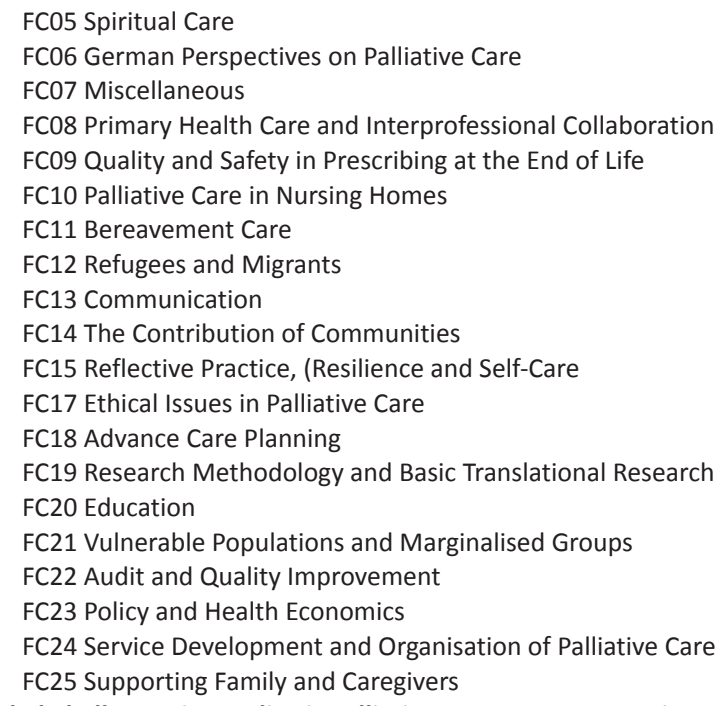

- Global Challenges in Paediatric Palliative Care: An EAPC Seminar

- PPC1 Prevalence of Life-Limiting Conditions in Paediatric Palliative Care

- PPC2 Information and Planning in Children's Palliative Care

PPC3 Symptom Managemant in Paediatric Palliative Care

PPC4 Contemporary Issues in Children's Palliative Care

PPC5 Challenges in Paediatric Palliative Care

- Integration of Palliative Care and Oncology: An EAPC-ESMO-Seminar

- ME9 The Rapidly Moving Field of Cancer Immunotherapy - Indications, Response Prediction and Management of Side Effects

EE1 Global Developments in Integration of Oncology and Palliative Care

EE2 Integration of Oncology and Palliative Care

FC16 Palliative Care for People with Cancer

- EE3 Podium Discussion. How Does Integration of Oncology and Palliative Care Change in the Light of a New Era in Oncology? A

Multiprofessional Challenge

- Digital Legacy Conference 2019 


\section{Plenary Sessions}

PL01

Access to Palliative Care and Pain Relief - An Imperative of Universal Health Coverage

Rajagopal M.

R. Pallium India, Trivandrum, India

WHO defined health in 1948 as physical, mental and social well-being and not merely absence of disease or infirmity. The Astana Declaration in 2018 reiterates that health for all has to be health with all.

But healthcare systems, while continuing to improve health in some by curing diseases, also destroy health all over the world in many ways including causing financial destruction, a globally growing phenomenon. As reported in India in 2008, 55 million people are driven below poverty line in a year by out of pocket health expenditure.

Globally, palliative care has been addressing health in its true sense. The report of the Lancet Commission on Global Access to Palliative Care and Pain Relief of 2017 expands the scope of palliative care to include true healthcare in all serious health related suffering. Its potential to relieve suffering and to restore true health can be realised only if we take on board two messages from the Astana Declaration - "include the public in designing and controlling health systems." and "achieve integration of multiple sectors like education, agriculture, transport, commerce, religion, housing,trade and health".

In one way these are easy to achieve, in terms of costs or mechanics. In another way this is difficult because palliative care experts don't find it easy to engage the community in planning healthcare systems, leave alone giving control to them as advised by the Astana Declaration.

In Kerala, palliative care delivery systems have achieved to some extent what the Astana Declaration asked us to do. They engage the community,make use of social capital, welcoming volunteers to be partners in delivery of healthcare.

This would mean that the current healthcare governance,management and experts have to give up some of their authority and engage the common man. Such a change will need some considerable facilitation by experts who believe in the power of the community. But it has the potential to transform healthcare to true healthcare.

\section{PL02}

\section{Early Palliative Care: When and How for Generalists?}

MurrayS.

University of Edinburgh, Primary Palliative Care Research Group, Edinburgh, United Kingdom Presenting author email address: scott. murray@ed.ac.uk

It is increasingly advocated that Palliative care should be started sooner than later for patients with life-threatening illness, when there is still time to respond to patient's wishes and prevent suffering. However many patients in every country still fail to receive a palliative care approach, or receive it only in the last days or weeks of life.

A rationale will be presented as to when early palliative care should start to be integrated in the care that generalists - both working in hospital and in primary care can provide for people will different illnesses. Various tools and training materials to promote systematic early identification of people who are likely to benefit from palliative care will be suggested and illustrated.

A framework of how integrated palliative care might be delivered in practice by hospital specialists and in primary care and by communities will then be considered, examining outcomes and benefits for patients, professionals and health services. The new EAPC Toolkit for integrating palliative care in primary health care systems throughout Europe will be launched.
PL03

\section{The Language of Dying}

Renz M.

Kantonsspital St. Gallen, Psychoonkologie, St. Gallen, Switzerland

End-of-life care entails more than understanding patients' wishes. We have to explore the dyings inner perspective and their experience of transcending our everyday consciousness.

Approaching death, patients' identity, spirituality, but also their inner world, fear and dignity change. Their hearing sensitivity increases. Their fundamental sense of time, space and body shifts as perception changes. I speak of a transformation of perception: patients seem to pass an inner invisible threshold of consciousness. Crossing the threshold, patients pass into a mystery beyond words. Yet immediately before that sphere, there appears to be an anteroom. Here the dying no longer think or experience in rational-logical terms. Their communication is analogical and metaphorical. They often see or talk about symbols (e.g. the coming of a ship, a holy seat, a light in darkness, a staircase resembling a stairway to heaven). Symbols are never coincidental; they represent energy, a particular theme, the pressure to change, the particular situation of exactly this person. What patients experience in symbols is comparable to mythical and fairy tale metaphors. As caregivers, we need sensitivity and knowledge to sense and decipher these symbols. To illustrate my point, I will talk about the fear when crossing the threshold of consciousness. Patients may consciously cope with fear, or avoid and ignore it. Yet, crossing the threshold of consciousness during the dying process fear grips many patients. One patient cried, "I'm falling", another "darkness, help me", a third just stared into the void. Not they manage symptoms and metaphors but are psychologically determined by them. After crossing the threshold of consciousness, they felt relief, instead of talking about falling the patient said: "I'm flying, I'm afloat", another uttered "beautiful" (the darkness was gone), or the gaze became light and steady again after the fearful staring. All these were hints that patients had found into peace.

\section{PL04}

\section{Is Palliative Care Biased?}

\section{Gott M.}

Te Ārai Palliative Care and End of Life Research Group University of Auckland, Auckland, New Zealand Presenting author email address: m.gott@auckland.ac.nz

All clinical and academic disciplines are a product of the society that created them. Palliative care is no exception. Our research, practice and policy attentions are therefore inevitably influenced by both the conscious and the unconscious biases we individually, and collectively, hold. In this presentation I will argue that we urgently need to critically examine our discipline to identify those biases and make them visible. I will provide examples of how they influence who receives palliative care services, as well as the extent to which those services meet peoples' needs and aspirations. Promoting diversity of thought about the purpose and delivery of palliative care requires diverse voices to be heard and power for decision-making (of all types) to be reconsidered. The challenge for researchers in supporting an agenda that promotes equity of end of life experience is to critically consider what evidence is needed to support change, as well as, more fundamentally, what counts as 'evidence'.

\section{PL05}

\section{Increasing the Value of Research in Palliative Care}

Sleeman $K$

E. King's College London, Cicely Saunders Institute of Palliative Care, Policy and Rehabilitation, London, United Kingdom Presenting author email address: katherine.sleeman@kcl.ac.uk

Biomedical research ultimately aims to improve clinical care. An important question is: how much of the research that we do achieves this aim? 
In 2009, Glasziou and Chalmers claimed in The Lancet that as much as $85 \%$ of global investment in biomedical research may be wasted. That is, it does not impact on clinical care. This waste accumulates over five stages of the research process: 1 ) when the wrong research question is asked; 2) because an inappropriate study design/method is used; 3) through inefficient and overly burdensome research regulation; 4) through biased under-reporting of data; and 5) because findings are reported in such a way that they are unusable.

This plenary will unpick the concept of research waste, drawing on evidence from across basic and applied research, and exploring the relevance to palliative care. It will consider the questions: how much research carried out in palliative care makes a difference for patients, carers and society? How much might, according to Glasziou and Chalmers' criteria, be considered wasted? And how can the value of research in palliative care be increased?

\section{PL06}

From Crisis to Sustainability - Building Palliative Care with Limited Resources

Ntizimira $C^{1,2}$

${ }^{1}$ Harvard Medical School, Global Health and Social Medicine, Framingham, MA, United States, ${ }^{2}$ City Cancer Challenge Foundation, City Manager, Kigali, Rwanda Presenting author email address: ntiheritier@gmail.com

Multi-disciplinary palliative care for patients with any disease is rarely integrated into the public healthcare system at all levels in Africa. Rwanda rose up from ashes after the Genocide against the Tutsi in 1994 which resulted in one million deaths in 100 days. Humanity itself seemed lost that time. In Rwanda, the Ministry of health has developed palliative care services in district hospitals and linked these services to home care. In 2011, the country issued a national palliative care policy and guidelines that enabled development of palliative care services within the public healthcare system. Available services include inpatient and home care provided by physicians, nurses, social workers, and pharmacists with training in palliative care and home hospice care provided by a private home hospice organization with which the Ministry signed an MOU. In addition, the Ministry of Health approved a palliative care training curriculum for home-based care practitioners employed by the government who will expand and make sustainable palliative home care. Patients in need of palliative care are referred to Hospitals from throughout the district and the country. Anecdotal data indicates a high level of satisfaction by patients and family members with palliative care services provided and a reduced tendency of patients with end-stage diseases to pursue costly treatment abroad. Is it the palliative care approach that will contribute to rebuilding the sense of humanity lost during the Genocide?

\section{Best Abstract}

Matters of Life and Death - An Experimental Study Investigating Interventions to Encourage End-of-Life Discussions von Blanckenburg P., Nagelschmidt K., Leppin N., Rief W. Clinical Psychology and Psychotherapy, Philipps University of Marburg, Marburg, Germany

Background: End-of-life discussions can alleviate distress and uncertainties and are associated with higher satisfaction with palliative care in both old and young adults. Advance care planning is a possibility to realize a structured discussion about relevant end-of-life topics. Nevertheless, the majority of the western population do not use those programs. Although talking about death is often seen as alleviative and worthwhile, it has become a taboo in our society, both in healthy and ill populations.

Aims: To investigate short interventions encouraging end-of-life discussions in healthy adults.
Methods: 184 participants (group 1: young adults: $18-35$ years, group 2: old adults: $65-88$ years) were randomized to four different interventions (EG1: value-based intervention, EG2: communication-based intervention, EG3: combination of EG1 and EG2, CG: control group). The primary outcome was the change in readiness to engage in end-of-life topics and Advance Care Planning. Secondary outcomes were changes in distress and sense of coherence. Assessments took place before, directly after the intervention and at two weeks follow up.

Results: $\mathrm{EG} 2$ and $\mathrm{EG} 3$ reported significant more changes in the readiness to engage in end-of-life discussions than the $C G\left(F(3,160)=7.21, p<.001, \eta^{2}=.12\right)$ directly after the intervention. Young adults reported significant more changes in their readiness to engage in end-of-life discussions than older adults $\left(F(1,160)=18.3, p<.001, \eta^{2}=.10\right)$. Moreover, there were significant effects of the intervention on changes in sense of coherence. Thus, EG1 and EG3 showed significant changes to a more positive sense of coherence than the $C G$ after the intervention. All results stayed stable at follow up.

Discussion: Short interventions can be useful to encourage end-of-life discussions and could be integrated in health care programs. The efficacy and effectiveness of those interventions in palliative patients are currently examined by our research group.

\section{Best Abstract}

Coping Strategies of Patients with Advanced Lung or Colorectal Cancer in Six European Countries: Insights from the ACTION Study

Jabbarian L. ${ }^{1}$, Korfage I. ${ }^{1}$, van der Heide A. ${ }^{1}$, Wilcock A. ${ }^{2}$, Deliens $L .{ }^{3,4}$, van Delden H. ${ }^{5}$, Cerv B. ${ }^{6}$, Miccinesi G. ${ }^{7}$, Thit Johnsen A. ${ }^{8,9}$, , Payne S. ${ }^{10}$, Rietjens J. ${ }^{1}$, on behalf of the ACTION Consortium

${ }^{1}$ Public Health, Erasmus MC, Rotterdam, Netherlands, ${ }^{2}$ Medicine \& Health Sciences, University of Nottingham, Nottingham, United Kingdom, ${ }^{3}$ End-of-Life Care Research Group, Vrije Universiteit Brussel, Brussels, Belgium, ${ }^{4}$ End-of-Life Care Research Group, Ghent University, Ghent, Belgium, ${ }^{5}$ University Medical Center Utrecht, Utrecht, Netherlands, ${ }^{\circ}$ Ljubljana University Medical Centre, Ljubljana, Slovenia, 7Istituto per lo Studio, la Prevenzione e la Rete Oncologica - ISPRO, Florence, Italy, ${ }^{8}$ University of Southern Denmark, Odense, Denmark, ${ }^{9}$ Bispebjerg and Frediksberg University Hospitals, Copenhagen, Denmark, ${ }^{10}$ Lancaster University, Lancaster, United Kingdom

Background: Patients with advanced cancer often experience multiple physical symptoms and psychological distress. Even when medical treatment options are limited, supporting patients' adaptive coping strategies has the potential to improve quality of life. Better understanding of coping and its associated variables is necessary to develop high quality coping support.

Aims: To characterize the prevalence of coping strategies and their associated sociodemographic and clinical characteristics.

Methods: This study used sociodemographic information and baseline data from the ACTION trial, comprising measures of Denial, Acceptance, and Problem-focused coping from the COPE and Brief COPE inventory in patients with advanced cancer from Belgium, Denmark, Italy, the Netherlands, Slovenia, and the United Kingdom. Health professionals provided clinical background characteristics. Multilevel analyses were performed.

Results: Data from 675 patients were used; mean age 66 (10 SD) years, $60 \%$ male, 342 (51\%) stage III/IV lung cancer and 333 (49\%) stage IV colorectal cancer. Overall, patients scored low on Denial (i.e. using it a little bit) and scored high on the coping strategies Acceptance and Problem-focused coping (i.e. using them a lot). The use of coping strategies differed between subpopulations. Older patients and patients living in Italy and Denmark scored high on Denial and patients with more years of education scored high on Acceptance.

Conclusion: Different patterns in the use of coping strategies are seen among an international sub-population of patients with advanced cancer. 
Our findings may help inform the design and implementation of tailored interventions to enhance patients' coping strategies.

\section{Best Abstract}

The Liminal Space Palliative Care Volunteers Occupy and the Roles They Perform within it: A Qualitative Study Vanderstichelen S. ${ }^{1}$, Cohen J. ${ }^{1}$, Van Wesemael Y. ${ }^{2}$, Deliens L. ${ }^{1,3}$, Chambaere . $^{1}$

${ }^{1}$ Faculty of Medicine and Pharmacy, End-of-Life Care Research Group at the Vrije Universiteit Brussel and Ghent University, Brussel, Belgium, ${ }^{2}$ Palliabru, Brussels, Belgium, ${ }^{3}$ Department of Public Health and Primary Care, Ghent University, Ghent, Belgium

Objectives: Volunteers have an important place in palliative care, positively influencing quality of care for seriously ill people and those close to them and providing a link to the community. However, it is not well understood where volunteers fit into palliative care provision in relation to other caregivers, nor how to adequately support them.

Aim: To describe volunteer roles across palliative care settings through the perspective of those closely involved in the care of terminally ill people.

Methods: A qualitative study was conducted using semi-structured focus groups with volunteers, nurses, psychologists and family physicians and interviews with patients and family caregivers. Participants were recruited from hospital, home, day care and live-in services.

Results: 79 people participated in the study. Two volunteer roles were identified. The first was 'being there' for the dying person. Volunteers represent a more approachable face of care, focused on psychological, social and existential care and building relationships. The second was the 'liaison' role. Volunteers occupy a liminal space between the professional and the family domain, through which they notice and communicate patient needs missed by other caregivers. Patient-volunteer matching was a facilitator for role performance; barriers were lack of communication opportunities with professional caregivers and lack of volunteer coordination.

Conclusion: Volunteers complement professional caregivers by 1 ) occupying a unique space between professionals, family and patients and fulfilling a liaison function, and 2) being a unique face of care for patients. Healthcare services and policy can enhance volunteer role performance by ensuring the accessibility of nurses and psychologists within health services and by appointing nurses as volunteer coordinators.

Funding was provided by the Agency for Innovation through Science and Technology (I.W.T.) as part of the INTEGRATE-project. [IWT-SBO 140009]

\section{EAPC Researcher Award Plenary: Winner of the Post-Doctoral} AwardBuilding a Palliative Care Research Career across Borders Kurita G.P.

Rigshospitalet Copenhagen University Hospital, Palliative Research Group, Department of Oncology and the Multidisciplinary Pain Centre, Department of Neuroanaesthesiology, Copenhagen, Denmark Presenting author email address: geana.kurita@regionh.dk

After my second year in the nursing undergraduate program at the University of Sao Paulo in Brazil, I applied for a volunteer student position in an academic association that treated patients with chronic pain (Pain League), where I had the opportunity to develop clinical and research activities related to patients with cancer pain. This experience sparked in me an interest that I definitely knew I wanted to pursue.

I consider myself a privileged researcher, because I have had remarkable mentoring, built strong links to national and international research groups (European Association of Palliative Care, European Palliative
Research Centre, and University of Sao Paulo), and have had the opportunity to develop a research collaboration between my native (BR) and adopted (DK) countries. Such collaborations have enabled me to appreciate both fundamental similarities as well as differences in palliative care across different countries.

For the last decade, my main scientific contributions have been within the field of chronic pain and opioids with a focus on cognitive functioning in cancer and non-cancer patients, which has provided me the opportunity to transfer/test/develop knowledge in both populations. Over the years my portfolio has evolved and includes epidemiologic investigations on opioid use, experimental studies of opioid effects on the central nervous system, validation of neuropsychological tests in patients with advanced cancer, palliative interventions for patients with cancer, and genetic and renal aspects associated with opioid effects in cancer. Hopefully, these studies are informing health professionals, researchers and health authorities to take measures to improve palliative care.

I am honored to have won the Post-Doctoral Award. Building this research career has been a very exciting journey, which would have not been possible without the support and kindness of everyone I have met along the way.

\section{EAPC Researcher Award Plenary: Winner of the Clinical Impact Award}

\section{Bringing Palliative Care to the Public \& the Impact of New Media} Taubert M. ${ }^{1,2,3}$

${ }^{1}$ Cardiff University, Diploma and MSc Palliative Care, Velindre NHS Trust Palliative Medicine Department, Cardiff, United Kingdom, ${ }^{2}$ Byw Nawr Live Now Coalition Wales, National Lead for Advance Care Planning, Welsh End Of Life Care Board, Cardiff, United Kingdom, ${ }^{3}$ Bevan Commission Wales, Swansea, United Kingdom

How do we bring complex matters that come up in our daily practice to wider attention? How do we share some of the most challenging moral conundrums in palliative care and ascertain public attitude towards them? In this plenary talk, Dr Mark Taubert will outline his experience of making sensitive use of sound and vision via new media in order to ask pertinent questions that have been with our specialty for decades.

How do we teach an old dog new media tricks? Initially strongly advised not to go ahead and create social media content videos for the public to discuss 'Do Not Attempt Cardiopulmonary Resuscitation' (DNACPR) decisions online, an app and a website called \#TalkCPR http://talkcpr.com were nevertheless created with extensive patient support.The Welsh website and Youtube Byw Nawr TalkCPR videos has been accessed over a million times from all over the world. TalkCPR video media pads can now be found in all general practice surgeries in Wales and in hospitals and nursing homes, but they have been used as far away as Lesotho. This is real shared patient and public engagement.

But there is a raw and dark side to new media. The sometimes emotive topics of palliative \& end of life care can attract elements to this forum that wish to hurt and disrupt. Without expert input, the quality of the debate can suffer and build on fallacies. This plenary talk will outline how Quality Improvement methodology was used to evaluate sense and sensibility of advance care planning resources, and how expertise from colleagues who work in IT, television, social media and government was vital in stepwise national project outcomes.

Dr Taubert is national strategic lead for Advance and Future Care Planning in Wales, a role that requires sound knowledge of Twitter, Facebook and Youtube. He will outline some of the conundrums he has faced, and perhaps convince new media phobics at EAPC World Congress to join the often passionate online debate. His Twitter account is @ DrMarkTaubert 


\section{EAPC Researcher Award Plenary: Winner of the Palliative Medicine Early Researcher Award}

Older People, Palliative Care and Multiple Perspectives: My Contributions and Future Directions

Heckel M.

Universitätsklinikum Erlangen, Friedrich-Alexander-Universität ErlangenNürnberg (FAU), Palliativmedizinische Abteilung, Erlangen, Germany

At all ages and in all settings, palliative care services should provide support for patients and their families following the holistic palliative care approach to meet complex needs. With my background in social work and nursing science, I am active in palliative care research. I am truly honoured to have been selected for the early researcher award. My presentation will focus on the benefits of exploring end-of-life care issues from multiple perspectives. People may die at any age, but becoming older the probability increases by nature, health status often deteriorates, symptoms occur, the social mesh becomes smaller and may lose some of its supportive value whereas support needs individually increase. High quality palliative care may become necessary. However, measuring quality of end-of-life care is challenging. Our group approached informal caregivers and health professionals as surrogates for quality of dying and death (QoDD) and care of the dying (CODE). The German validation studies of both questionnaires provide their psychometric properties and allow for international comparisons of care outcomes. In a project on multidrug-resistant bacterial microorganisms (MDRO) a mixed-methods approach was used. We succeeded in developing national recommendations based on patients', families' and team members' as well as institutional representatives' perspectives. The recommendations allow for the development of new strategies to enhance the quality of end-of-life care for (older) patients with palliative care needs and MDRO diagnosis. In the aforementioned project, geriatric patients were included besides palliative care patients. The heterogeneous and complex needs of older people at the end of life will have to receive more attention in palliative care due to ageing societies. With my social gerontology perspective I would like to share my ideas on age and ageing that seem relevant in end-of-life care for both palliative care services and research.

\section{Parallel Sessions}

PS01 From Ideas to Action: Achieving Universal Access to Palliative Care

No Abstracts available

PS02 Understanding Complexity in Palliative Care Understanding Complexity - A System Approach to Palliative Care Situations

Hodiamont F., Schildmann E., Bausewein C.

Ludwig-Maximilians-Universität München, Department of Palliative Care, München, Germany

Background: As a complex problem a palliative care (PC) situation is characterized by reciprocal, non-linear relations and uncertainties. Dealing with complex problems necessitates problem-solving methods tailored to specific situations. The theory of complex adaptive systems (CAS) provides a framework for locating problems and solutions. A CAS consists of relatively autonomously acting agents, which interact. It is part of a superordinated system and consists of interacting subsystems itself.

Aim: To describe criteria contributing to complexity of PC situations and develop a conceptual framework to promote the understanding of complexity of a PC situation by locating the complex problem in a CAS.

Method: Qualitative interview study with semi-structured expert (clini$\mathrm{cal} /$ economical/political) interviews. Audio-recorded interviews were transcribed verbatim. Data was analysed applying framework method. Thematic framework developed inductively. Categories were reviewed, subsumed and connected considering CAS theory.
Results: 42 interviews from 6-10/2015. The CAS of a PC situation consists of 3 subsystems: system patient, social system, system team. Agents in the system patient are allocated to further subsystems on patient level: physical, psycho-spiritual, and socio-cultural. Social system and system team are composed of social agents, who affect the CAS as carriers of characteristics (e.g. carer burden), roles (e.g. minor children) and relationships (e.g. cooperation with external service providers). Agents within subsystems and subsystems themselves interact on all hierarchical system levels and shape the system behaviour of a PC situation. Discussion: All complexity factors described by experts could be translated into a CAS. System elements and their relations give insight into possible behaviour of the PC situation. It is shown that elements cannot be understood independently from the overall PC situation. This independent research is funded by a private charity.

PS02 Understanding Complexity in Palliative Care Understanding Patients' Needs: A National Qualitative Study with Patients, Family Carers and Professionals

Pask S. ${ }^{1}$, Pinto C. ${ }^{2}$, Bristowe K. ${ }^{2}$, van Vliet L. ${ }^{2}$, Nicholson C. ${ }^{3,4}$, Evans C.J. ${ }^{2,5}$, George R. ${ }^{4}$, Bailey K. ${ }^{2}$, Davies J.M. ${ }^{2}$, Guo P. ${ }^{2}$, Daveson B.A. ${ }^{2}$, Higginson I.J. ${ }^{2}$, Murtagh F.E.M. ${ }^{1,2}$

${ }^{1}$ Wolfson Palliative Care Research Centre, Hull York Medical School, University of Hull, Hull, United Kingdom, ${ }^{2}$ Cicely Saunders Institute of Palliative Care, Policy \& Rehabilitation, Florence Nightingale Faculty of Nursing, Midwifery \& Palliative Care King's College London, London, United Kingdom, ${ }^{3}$ Florence Nightingale Faculty of Nursing, Midwifery \& Palliative Care, King's College London, London, United Kingdom, ${ }^{4} \mathrm{St}$ Christopher's Hospice, London, United Kingdom, ${ }^{5}$ Sussex Community NHS Foundation Trust, Brighton, United Kingdom

Background: The comprehensive holistic assessment is intended to identify patients' needs, but it is challenging to classify needs in a meaningful way to represent complexity.

Aim: To explore palliative care stakeholders' views on patient needs and how complexity can be effectively represented.

Methods: Face-to-face, semi-structured interviews across the UK with stakeholders in palliative care. Participants were sampled purposively by location, background, experience and setting. The COREQ guidelines were used to enhance methodological rigour. Framework analysis was used to analyse data.

Results: 65 participants recruited (10 patients and family carers, 38 healthcare professionals, and 17 managers and senior leads). The person's needs are directly influential and central to how complex their care needs are. Participants often described needs using the common holistic assessment domains (i.e. physical, psychological, social and spiritual), but other aspects were considered important to delivering effective care. These included: communication, social needs, and demographic and clinical characteristics. A patient's circumstance before they receive palliative care and the number of needs were thought to influence patient complexity and how effective care may be in addressing their needs. Participants described other aspects that should be captured by clinical assessment beyond the four holistic domains; including 'pre-existing', 'cumulative', and 'invisible' aspects of needs. Conclusions: In palliative care, it essential to go beyond physical, psychological, social and spiritual needs. A person's characteristics, how complex a patient is before they receive palliative care and the number of problems a person has are also important. The holistic common assessment should consider incorporating these additional factors to ensure a comprehensive assessment.

PS02 Understanding Complexity in Palliative Care Optimising Teamwork Using Complexity Science Principles

Pype P.

Ghent University, Gent, Belgium Presenting author email address: peter.pype@ugent.be 
Background: Complexity science (CS) has been introduced in healthcare as a theoretical framework to better understand complex situations. Within the complex palliative care situation, an interprofessional healthcare team can be viewed as a Complex Adaptive System (CAS). CS focuses more on the interactions between individuals than on the individuals' characteristics. These interactions allow a system to adapt to changes by developing new emergent behaviour. The aim of this study is to explore the functioning of a healthcare team as a CAS and to evaluate the usefulness of CS to optimise the team functioning.

Methods: Results from two studies will be presented: a qualitative interview study and a quantitative questionnaire study, both in primary palliative care. The qualitative study focused on team members' interactions. A two-step analysis consisted of a deductive approach, which uses the CAS principles as coding framework for interview transcripts, followed by an inductive approach, which identifies patterns in the codes for each CAS principle. The quantitative study used the Complex Adaptive Leadership questionnaire to evaluate the team as self-organising team. Descriptive statistics were used to analyse the data.

Results: All CAS characteristics can be identified in the team members interactions. Study results show how patterns of interactions between individuals define team behaviour. Workplace learning as emergent behaviour indicates system self-organisation towards a higher level. Reviewing the team members' interactions using the Complex Adaptive Leadership questionnaire confirms that palliative care teams function as a CAS in complex situations and identifies areas for quality improvement. Conclusion: Looking at interprofessional healthcare teams through the lens of CS provides us with insights on how to optimise team functioning.

PS03 Shaping the Future for Bereavement Care in Palliative Care Carers' Grief when a Family Member Has Dementia

Moore K.J. ${ }^{1}$, Crawley S. ${ }^{1}$, Cooper C. ${ }^{2}$, Relf M. ${ }^{3}$, Vickerstaff V. ${ }^{1}$, Davies N. ${ }^{1}$, Harrison Dening K. ${ }^{4}$, King M. ${ }^{2}$, Sampson E.L. ${ }^{1,5}$

${ }^{1}$ University College London, Marie Curie Palliative Care Department, Division of Psychiatry, London, United Kingdom, 2University College London, Division of Psychiatry, London, United Kingdom, ${ }^{3} \mathrm{Oxford}$ University Hospitals NHS Foundation Trust, Bereavement Service, Sir Michael Sobell House, Oxford, United Kingdom, ${ }^{4}$ Dementia UK, London, United Kingdom, ${ }^{5}$ Barnet, Enfield and Haringey Mental Health Trust, North Middlesex University Hospital Mental Health Liaison Team, London, United Kingdom Presenting author email address: kirsten. moore@ucl.ac.uk

Background: Family/friend carers of people living with dementia experience a number of losses as dementia progresses, called grief before death. One of the factors associated with complex grief is not being prepared for a death, yet many carers do not consider dementia a disease you can die from.

Hypothesis: This study tests the hypothesis that modifiable factors indicating preparation for end of life are associated with lower grief before the death in carers of people with dementia. It will also explore more broadly carers' recognition of grief and strategies for coping with loss and grief.

Method: We used a cross-sectional design interviewing 150 family carers of a person with dementia. We used the Marwit-Meuser Caregiver Grief Inventory Short Form (MMCGI) to assess severity of grief before death. We assessed dementia severity, preparation for end of life, advance care planning, depression, anxiety, relationship quality, coping strategies, dementia knowledge and relationships with healthcare professionals and social network.

Results: Analysis of the first 50 participants indicated that $38 \%$ were spouse carers, $52 \%$ adult children and $70 \%$ female with a mean age of 61.7 (Standard Deviation 12.6). There was a higher representation of people caring for someone with young onset dementia (diagnosed younger than 65 ) representing $16 \%$ of the sample (compared with $5 \%$ in population). Participants were representative in terms of rurality and deprivation status. Forty percent cared for someone with severe dementia and $44 \%$ moderate. Thirty percent cared for someone in a care home. Grief scores were consistent with previous research in the US indicating experiencing a moderately high intensity of grief before death. We will present regression analysis findings to test the study hypothesis.

Conclusion: Identifying modifiable factors that impact on grief severity can inform service delivery to optimise support for carers experiencing grief whilst caring for a relative with dementia.

PS03 Shaping the Future for Bereavement Care in Palliative Care The Three Year Bereavement Trajectory for Carers and Prevention of Adverse Bereavement Outcomes

Guldin M-B. ${ }^{1}$, Nielsen M.K. ${ }^{2}$, Bidstrup P.E. ${ }^{3}$

${ }^{1}$ Aarhus University Hospital, Palliative Care Team, Århus C, Denmark, ${ }^{2}$ Aarhus University, Research Unit for General Practice, Aarhus C, Denmark, ${ }^{3}$ Danish Cancer Society, Copenhagen, Denmark Presenting author email address: m.guldin@ph.au.dk

Aim: In our studies on bereavement, we have focused on describing grief responses and bereavement trajectories based on national population-based cohorts and register-based data to increase knowledge on caregiver's grief. In our latest studies, we aimed to identify common grief trajectories from before the loss to three years post-loss based on grief symptoms and to identify risk factors for adverse bereavement outcomes

Method: In order to perform a comprehensive description of grief responses and bereavement trajectories, data from a prospective questionnaire study from before the loss and up to three years after loss and national health register-based studies were used. Trajectories were described based on caregiver questionnaires for prolonged grief (Prolonged Grief-13 scale) in 1735 caregivers using group-based trajectory models. Prospective register-based studies using data from the national health registries were conducted within a nationwide cohort of 1.4 million bereaved persons to identify risk factors for adverse outcomes (i.e. suicide and psychiatric diagnoses).

Results: We identified five specific grief trajectories. The majority of caregivers had a low grief or moderate-decreasing grief trajectory, a smaller group had a high-decreasing or late grief trajectory and a minority of caregivers suffered from persistent high grief. A range of risk factors for adverse outcomes were identified and confirmed in additional registerbased studies.

Discussion: Knowledge on grief responses and bereavement trajectories can inform carers for patients at the end-of-life on possible developments and vulnerabilities. Information on risk factors is crucial for identifying persons in need of support and for the development of an optimal support strategy starting before the death of the patient. Thereby, predeath bereavement studies may even aid in the prevention of adverse grief responses in caregivers.

\section{PS03 Shaping the Future for Bereavement Care in Palliative Care} Reaching an Agreement to Establish the Framework to Guide the Development of Bereavement Care in Palliative Care in Europe- a Delphi Study

Keegan $0 .{ }^{1}$, Murphy I. ${ }^{2}$, Guldin M. ${ }^{3}$, Relf M. ${ }^{4}$, Benkel I. ${ }^{5}$, Limonero J.T. ${ }^{6}$

${ }^{1}$ Head of Education, Research \& Bereavement, Irish Hospice Foundation, Dublin, Ireland, '2Director of Bereavement \& Family Support Services, Marymount University Hospice, Cork, Ireland, 3Palliative Care Team, Aarhus University Hospital, Aarhus, Denmark, ${ }^{4}$ Sobell House, Oxford, United Kingdom, ${ }^{5}$ Gothenburg University Hospital, Palliative Department, Gothenburg, Sweden, ${ }^{6}$ School of Psychology, Universitat Autònoma de Barcelona, Barcelona, Spain

Background: The WHO definition of palliative care includes bereavement care. Our survey of bereavement care in palliative care found a lack 
of bereavement guidance and policy across European countries (Phase 1, EAPC taskforce, Guldin et al, 2015). Therefore, development of a framework is required.

Aims: Phase 2 of the EAPC bereavement taskforce:

1. Formulate recommendations for bereavement care principles, structures, processes and delivery based on current practice and evidence.

2. Establish consensus on these recommendations.

A Delphi process was deemed appropriate to formulate recommendations and to establish consensus and core shared priorities. Routine clinical practices and expert knowledge can be integrated through a Delphi process.

Methodology: A 4-round Delphi process was completed (R1 to R4). R1: Based on literature review and consultations with expert advisory group (EAG) and stakeholders, a Delphi survey of 54 Likert-scale statements (strongly disagree to strongly agree with a 1 - 5 score) under six dimensions was designed. Consensus criteria were agreed as $>80 \%$ strongly agree/agree + median score of $5=$ 'Very Strong Consensus'; 75 - 80\% strongly agree/agree + median score of $4=$ 'Strong consensus'. Statements $<75 \%$ agreement were to be referred to EAG for review. R 2: Following a pilot trial, the survey was emailed to a panel of phase 1 survey respondents and nominated bereavement experts ( $n=376)$. R3: Items with low consensus/ requiring adaptation based on qualitative comments were re-sent to the panel to test agreement. Round 4: Final recommendations were discussed and confirmed by EAG.

Results/Interpretation: Response rate: R2, 23\% (87/376); R3, 79\% (69/87). After R2, 24 statements had 'very strong' consensus; 24 had 'strong' consensus and eight statements did not reach thresholds /needed clarification based on open-ended comments. Out of six dimensions, full consensus was reached on three: definition of bereavement; definition of bereavement care; and integrating bereavement care in the community. The eight non-consensus statements concerned defining bereavement care, volunteer competence, professional coordination roles, point of referral assessment and practical bereavement support. All eight nonconsensus statements were returned to R3 and consensus achieved. R4 classified 'Essential' and 'Desirable' features of bereavement care.

Conclusion: A final EAPC bereavement care guidance in palliative care has been developed. This guidance include 78 statements within six dimensions. Of them, 26 recommendations were qualified as essential (exceeded $80 \%$ agreement with a median of 5 ) and 52 recommendations were qualified as desirable (exceeded $80 \%$ agreement with a median of 4 ). The implications for Bereavement Services will be discussed.

PS 4 Separating the Wheat from the Chaff: Deprescribing in Advanced IIIness

Models Used in Deprescribing

Holmes $\mathrm{H}$.

McGovern Medical School, Division of Geriatric and Palliative Medicine, Houston, Texas, United States

This session will highlight different frameworks that can be useful to facilitate deprescribing in patients with advanced illness. Deprescribing is a systematic process by which all medications are reviewed and considered for their eligibility for discontinuation in the context of an individual patient's functional status and goals of care. Patients with advanced illness may require different levels of deprescribing intensity, depending on the potential benefit of reducing medicines and the potential harms of polypharmacy. Frameworks that specifically consider advanced illness can be helpful to further prioritize medications that are most likely to be of benefit and to be aligned with a patients preferences for care. Ultimately, while deprescribing is based on common foundational principles regarding medication safety, deprescribing in advanced illness requires a highly individualized approach.

PS 4 Separating the Wheat from the Chaff: Deprescribing in Advanced Illness

Utility and Futility of Medications for Neurodegenerative Diseases McPherson M.L.

University of Maryland Baltimore, Pharmacy Practice and Science, Baltimore, MD, United States Presenting author email address: mmcphers@rx.umaryland.edu

Patients with neurodegenerative disease such as Amyotrophic Lateral Sclerosis (ALS), Alzheimer's disease, multiple sclerosis and Parkinson's disease routinely receive medications that aim to slow disease progression or treat the complications of the disease. At some point when the patient's disease or comorbid conditions indicate the patient is near the end of life, decisions must be made about the medications used for their neurodegenerative disease. For example, practitioners must weigh the benefits and burdens of continued therapy. Is there still benefit to the drug therapy? Is the patient experiencing adverse effects that cause greater burden than any real or perceived benefit? What will the impact be on discontinuing drug therapy - no impact, disease state worsening, or potentially even enhancing the patient's status? This presentation will address these issues, as well as how to have the conversation with patients, families and caregivers on this sensitive topic - how and when to stop medications that the prescriber advised "you'll be taking this until you die."

\section{PS 4 Separating the Wheat from the Chaff: Deprescribing in Advanced Illness}

Examples and Conversations in Deprescribing

Todd A.

Newcastle University, School of Pharmacy, Newcastle upon Tyne, United Kingdom

Interest in the area of "deprescribing" is rapidly growing with researchers and clinicians alike seeking ways to reduce inappropriate polypharmacy and improve health outcomes for older people. While our understanding of deprescribing as a process has greatly improved, with several recommendations specifically published for addressing deprescribing in people with diminished life expectancy [1], there is limited literature exploring the individual patient context in deprescribing decision-making. For any deprescribing episode to be successful, it is critical that the approach is centred on patients. A recent framework - termed the deprescribing rainbow[2] - has captured the complexity of deprescribing decision-making, and outlines five different deprescribing determinants. These determinants consists of clinical, psychological, social, financial and physical factors. A rainbow analogy has been used to highlight that deprescribing should be considered as a positive process, and that the relationship between the different determinants is fluent, and may change over time. If deprescribing processes are to be ethical, respectful and successful - and truly patient-centred - it is important that deprescribing considers the individual needs of the patient, and what factors are important to them. We apply this framework to a complex patient case to highlight the importance of individual patient context, and how the clinical, psychological, social, financial and physical deprescribing determinants should be addressed in any conversations relating to deprescribing.

\section{References}

1. Todd A, Holmes HM. Recommendations to support deprescribing medications late in life. Int J Clin Pharm. 2015;37(5):678-81

2. Todd A, Jansen J, Colvin J, McLachlan AJ. The deprescribing rainbow: a conceptual framework highlighting the importance of patient context when stopping medication in older people. BMC Geriatr. 2018;18(1):295. 
PS 5 Palliative Care for Patients with Neurological

Conditions - Collaboration and Interaction

Evaluation of Short-term Integrated Palliative Care (SIPC) to OPTimise CARE for People Severely Affected by Long-term Neurological Conditions (LTNCS)

Gao W. ${ }^{1}$, Wilson R. ${ }^{1}$, Hepgul N. ${ }^{1}$, Yi D. ${ }^{1}$, Evans C. ${ }^{1}$, Bajwah S. ${ }^{1}$, Crosby V. ${ }^{2}$, Higginson I. ${ }^{1}$, OPTCARE Neuro Trial

${ }^{1}$ King's College London / Cicely Saunders Institute, London, United Kingdom, ${ }^{2}$ University of Nottingham, Nottingham, United Kingdom

Background: Early palliative care has shown some benefits in people with LTNCs. However, the best evidence to date is mainly from smaller studies focusing on a single condition.

Aim: To determine the effectiveness and cost-effectiveness of SIPC in improving symptoms and palliative care outcomes and caregiver burden for people severely affected by LTNCs.

Study population and settings: Multiple Sclerosis, Parkinsonism \& related disorders and Motor Neuron Disease patients experiencing a) an unresolved symptom and b) another symptom, cognitive problems, complex psychological or social need; and their family caregiver. This trial was conducted at 7 centres in the UK.

Study design and methods: A pragmatic Phase III, randomised controlled, multicentre, fast-track trial of a complex intervention, with an embedded qualitative component. Patients referred by neurologists were randomised to receive SIPC immediately or after 12-weeks. Patient and caregiver outcomes were assessed 6-weekly up to 24 weeks. Primary outcome at 12 weeks was 8 key symptoms. Recorded service use was used to determine cost-effectiveness. A qualitative component explored the value, impact of SIPC and linkages between the mechanisms and key outcomes.

Data analysis: Primary analysis was on an intention-to-treat basis using generalised linear mixed model, adjusting for baseline scores and any variables found imbalanced at baseline. Economic evaluation was conducted from health service and societal perspectives. Qualitative data were analysed thematically.

Results: We recruited 350 patients and 229 carers, making it the world's largest palliative care trial in a non-cancer condition. We will introduce more details about the trial, present the study findings and discuss the implications. This definitive trial will determine whether SIPC is effective across neurological diseases and can influence future directions of care. Acknowledgement: NIHR HS\&DR(12/130/47), CLAHRC South London.

PS 5 Palliative Care for Patients with Neurological Conditions Collaboration and Interaction Neuro-Pall Guidelines in Progress Across Europe Voltz $R$.

University of Cologne, Palliative Medicine, Köln, Germany

Recently, The Lancet Neurology highlighted the topic of palliative care in neurology in an Editorial. This was prompted by the European Association for Neuro-Oncology guideline for palliative care in adults with glioma. An EAN Task Force is currently working on a European Guideline on Palliative Care and Multiple Sclerosis. Earlier, a consensus review on the general principles in palliative care for patients with chronic and progressive neurological disease was published, jointly developed by the EAN and the EAPC. With this growing interest and number of guidelines, the question arises how general and disease-specific guidelines can complement each other, and how separately or jointly from "general" palliative care these should be developed. A solution could be based on the palliative care framework of the German Guideline Program in Oncology (www.leitlinienprogramm-onkologie.de/english-language) which encompasses general palliative care principles that are independent from the underlying diagnoses (ie, a horizontal guideline), together with guidelines on disease-specific aspects. Specific guidelines should refer to the principles outlined in the horizontal guideline and provide only guidance on disease-specific aspects.
To achieve the high-quality evidence for such guidelines and to guide patient care, increased funding for research in this area is clearly needed. We hope that funders and reviewers of proposals in the future acknowledge the clinical relevance of this new and important topic in neurology, and that at the same time the connection to "general" palliative care is not lost.

PS 5 Palliative Care for Patients with Neurological Conditions -

Collaboration and Interaction

Palliative Care and Decision Making - How Neurologists and Palliative Care Specialists Can Help Each Other

Borasio G.D.

CHUV, University of Lausanne, Service de Soins Palliatifs et de Support, Lausanne, Switzerland

Difficult end-of-life decisions are frequent in neurology. They include but are not limited to - the following topics:

- Discontinuation of disease-oriented treatment

- Do-not-resuscitate order

- Discontinuation of ventilation

- Discontinuation of nutrition/hydration

- Palliative sedation

- Voluntary stopping of eating and drinking (VSED)

- Assisted suicide / euthanasia

The inherent difficulty of end-of-life decision-making is often compounded for neurological patients by prognostic uncertainty, long disease courses, early loss of competence, and behavioral disturbances. The prominent role of family caregivers also requires proper acknowledgement and implication in the decision-making process.

There is a generalized need to focus on the crucial role of medical indication in decision-making, as it is often overlooked. If a treatment is not medically indicated, it must not be administered, regardless of the patient's wish. This is easier said than done, however.

Palliative care specialists can help neurologists with their experience in family conferences, transcultural sensitivity, interprofessional communication and knowledge on ethico-legal aspects at the end of life (incl. e.g. palliative sedation, withdrawal of life support, VSED). On the other hand, neurologists can be of invaluable help for palliative care specialists due to their specific competence on symptoms and foreseeable courses in advanced neurological disease, allowing to establish best-case prognostic scenarios that can form the basis for effective shared decision-making (examples will be given).

Both disciplines should start to cooperate early on in the disease course, one goal being to establish a joint process of advance care planning. This can greatly enhance the probability of the patient's wishes actually being followed, and can reduce stress, anxiety and depression in family caregivers. The earlier the cooperation begins, the better for the patients and families.

PS 6 What Happens to People with Intellectual Disabilities at the End of Life? Findings from Across Europe Death and End of Life Care Outcomes of People with Intellectual Disabilities in Care Home Settings - Findings from Two UK-Wide Surveys

Todd, $S$.

University of South Wales, Pontypridd, United Kingdom Presenting author email address: stuart.todd@southwales.ac.uk

EOLC for people with intellectual disabilities ID may experience disadvantage at the end of life. However, the evidence base for this limited. This presentation will present data from 2 studies. The first examined death and EOLC outcomes for people with ID within a cohort of 13,000 adults with ID living in ID care homes. Data were obtained on 222 deaths using a modified version of VOICES-SF. The second examined deaths of 
people with ID in non ID care homes given that as people with ID age there is an increasing risk that they experience transitions in care from small ID care settings to larger more generic care settings. Dara were obtained on 132 people with ID in those settings.

The data from ID care settings confirmed the risk of premature mortality in people with ID. However the relative risk of death within this population was lower than expected. There were about 11 deaths per 1000 people supported per year. This is attributable to the lower numbers of people with ID living within those settings. Only $7 \%$ of the living cohort was aged 70 or older. The data also suggest that many people with ID were supported to die in their place of care, and the extent to which this happened increased if the death had been anticipated for 3 months of more. However, only one in three deaths of people with ID had been anticipated for 3 months or more. Setting characteristics also shaped EOLC outcomes even when death had been anticipated. ID settings are not homogenous, and poorer outcomes were reported for expected deaths in smaller settings.

The data suggest that a major disadvantaged here is that deaths infrequently expected. ID services are dealing with complex deaths and there is more research required on how death is anticipated within this population. There is also more research required to examine how setting characteristics influence EOLC for this population, including people with ID living in non ID care and nursing homes.

PS 6 What Happens to People with Intellectual Disabilities at the End of Life? Findings from Across Europe Health Status, Use of Medical Services and End-Of-Life Decisions of People with Disabilities - a Longitudinal Study (Switzerland) Wicki M.T. University of Applied Sciences of Special Needs Education, Institut of Professionalization and System Development, Zurich, Switzerland

Aims: International research shows significant differences in the health and health care of people with and without disabilities. But in Switzerland it is not known how good the health services are in residential homes for adults with disabilities. The aim of this study is to capture the health and health services as well as end-of-life decisions for persons who are older than age 50 and who have had lifelong disabilities in Switzerland.

Methods: To capture context factors, state of health, access to health services, and end-of-life decisions for persons with disabilities, the research project conduct a longitudinal study as a census, with two measurement time points ( $t 1$ and $t 2$ ) in six cantons. The time between t1 and $t 2$ is 350 to 370 days.

Results: Results of the first wave are available. A total of 383 people were interviewed. 134 live in their own apartment or with relatives, 241 in a residential facility. Furthermore the survey delivered detailed information on 161 people (101 male, 60 female) who had died between 2015 and 2017 in 77 residential homes for people with disabilities.

Results produce a picture of health status, use of health services and end-of-life decisions in residential homes for adults with disabilities. A total of $56.1 \%$ of all deaths in the study population were nonsudden and non-unexpected and therefore eligible for end-of-life decisions. In more than 4 of 5 non-sudden deaths (46.5\% of all sampled deaths, 74 persons), at least one end-of-life decision was made. It becomes apparent that people with a disability pension who live independently have the worst assessment of their health condition and also feel the restriction in everyday life most strongly due to a health problem.

Conclusion: Detailed results will be presented and discussed to contribute towards overall improvement of health services for people with disabilities in Switzerland.

PS 6 What Happens to People with Intellectual Disabilities at the End of Life? Findings from Across Europe The Experiences of People with Intellectual Disabilities at the End of Life: Lessons Learnt from over a Decade of Research
Tuffrey-Wijne I.

Kingston University \& St George's University of London, Faculty of Health, Social Care and Education, London, United Kingdom

Background: The end-of-life and palliative care needs of people with ID can be difficult to assess, as this is an often hidden population with needs that can be difficult for them to communicate.

Aims: To give an overview of experiences and needs of people with ID from their own perspective.

Methods: The author has conducted inclusive research into dying, death, bereavement and ID for over a decade. Methods have included observation, interviews and focus groups with people with ID. Creativity in data collection methods was often needed, including the use of pictures and story-telling, for example. She has also worked with coresearchers with ID, who have added their own perspectives to data analysis.

Results: Overall, the palliative care needs of people with ID are no different from those of the general population, but they face particular challenges that make it more difficult to meet those needs. We found that people with ID are well able to express their wishes, but need help to do so. Those with severe and profound ID, in particular, depend strongly on carers who know them well. Those who were able to tell us, placed a strong emphasis on the importance of relationships, feeling safe, and good symptom control. Involvement in decision-making, and being helped to understand what was happening, was also valued.

Conclusion: It is important to understand the palliative care journey of people with ID in the context of their life story. What matters most to them, and what makes for a good quality of life, may not be immediately obvious to those who do not know the person well. It is important, therefore, to make sure the person with ID themselves, and their family/ carers, are listened to carefully. Palliative care for people with ID needs to be highly personalised. If professionals are able to do this well, it will benefit all their patients, not just those with ID.

PS 7 Delivering Palliative Care in Ageing Populations: Comprehensive Interventions and Clinical Models Recommendations on Implementation of Palliative Care Interventions in Long Term Care Facilities (EAPC White Paper)

Froggatt K. ${ }^{1}$, Collingridge Moore D. ${ }^{2}$, Payne S. ${ }^{2}$, Van den Block L. ${ }^{3}$ ${ }^{1}$ Lancaster University, International Observatory on End of Life Care, Lancaster, United Kingdom, ${ }^{2}$ Lancaster University, Lancaster, United Kingdom, ${ }^{3} \mathrm{VUB}$, Brussels, Belgium Presenting author email address: k.froggatt@lancaster.ac.uk

Background: The number of older people dying in long term care facilities (LTCFs) is increasing. Interventions to improve palliative care delivery in LTCFs are shown to be effective. Less is known about the nature of successful implementation processes.

Aim: To identify palliative care implementation practices from current evidence to inform policymakers and practitioners

Design: Two stage development process: 1 . Scoping review with a thematic synthesis, using ENTREQ guidelines 2. Transparent Expert Consultation event and online prioritization process.

Methods 1. Scoping review: Published literature identified from electronic databases (including MEDLINE, EMBASE, PsycINFO, CINAHL, Proquest, Cochrane Library, Web of Science). Controlled, non-controlled and qualitative studies and evaluations were included. Studies that met the inclusion criteria were sourced and data extracted on the study characteristics, the process of, and facilitators and barriers to implementation. 2. Transparent Expert Consultation event. International experts in palliative care, gerontology and care homes attended a workshop to identify recommendations. These were prioritized using an on-line survey with a wider group of stakeholders. 
Results: The review identified 8,902 abstracts; 62 studies were included. Preliminary findings were shared with 19 experts from 13 countries. The online survey was conducted with 28 experts from 16 countries; response rate was $71 \%$.

A matrix of implementation was developed with 4 implementation dimensions (facilitation, change recipients, joint working and education/training); and 3 implementation stages (conditions to introduce the intervention, embedding the intervention within practice and sustaining ongoing change). From this matrix 30 recommendations were developed.

Conclusion: Identifying implementation processes can be difficult as they are often underreported. A matrix model of implementation provides a clear structure for recommendations.

PS 7 Delivering Palliative Care in Ageing Populations: Comprehensive Interventions and Clinical Models End of Life Directions in Aged Care in Australia Parker D. ${ }^{1}$, Yates P. ${ }^{2}$, Tieman J. ${ }^{3}$

${ }^{1}$ University Technology Sydney, Faculty of Health, Sydney, Australia, ${ }^{2}$ Queensland University of Technology, School of Nursing, Brisbane, Australia, ${ }^{3}$ Flinders University, Nursing and Health Sciences, Adelaide, Australia Presenting author email address: deborah.parker@uts.edu.au

Aims: End of Life Directions in Aged Care (ELDAC) is a nationally funded project to improve palliative care and advance care planning for older Australians. Specifically the aims of the program are to:

$<$ ol style="list-style-type: decimal; direction: Itr;" $>$

- Provide specialist palliative care and advance care planning advice to aged care providers and GPs providing health care for recipients of aged care services:

- Improve linkages between aged care services and palliative care services;

- Improve the palliative care skills and advance care planning expertise of aged care service staff and GPs providing health care for recipients of aged care services; and,

- Improve the quality of care for aged care recipients, prevent unnecessary hospital admissions, and shorten hospital stays.

This session will explore how to use and apply the innovative ELDAC solutions that are designed to improve end of life care for older people in Australia living in long term care.

Methods:

The ELDAC project commenced in June 2017 and includes four interrelated streams of work:

1. Capacity building resources and advisory services

2. Technology solutions

3. Policy enablers

4. Service and sector development and advisory services

Results:

This presentation will provide an overview of the first two years of the project and specifically showcase online knowledge translation and education products to improve advance care planning and palliative care for older Australians in long term care.

PS 7 Delivering Palliative Care in Ageing Populations: Comprehensive Interventions and Clinical Models What are the Components of an Effective Model of Palliative Care for Dementia?

Fox S. ${ }^{1}$, Drennan J. ${ }^{2}$, Guerin S. ${ }^{3}$, Kernohan G. ${ }^{4}$, Murphy A. ${ }^{5}$, Timmons S. ${ }^{6}$ ${ }^{1}$ University College Cork, Centre for Gerontology and Rehabilitation, School of Medicine, University College Cork, Cork city, Ireland, 2University College Cork, School of Nursing and Midwifery, Cork,
Ireland, ${ }^{3}$ University College Dublin, School of Psychology, Dublin Ireland, ${ }^{4}$ Ulster Univerisiity, School of Nursing and Health Research, Antrim, United Kingdom, ${ }^{5}$ University College Cork, Department of Economics, Cork, Ireland, ${ }^{6}$ University College Cork, Centre for Gerontology and Rehabilitation, Cork, Ireland Presenting author email address: s.fox@ucc.ie

Aims: Palliative care has been identified as a priority in dementia care, and research into "effective models" of palliative care for people with dementia has been recommended by several European agencies. The Model for Dementia Palliative Care Project is currently underway in Ireland, aiming to develop an acceptable, evidence- and practicebased model for palliative care for people with dementia in the community.

Methods: Project Phases I-II are complete. Phase I involved a scoping review of the evidence for various components of a dementia palliative care model. In Phase II, a web-based survey was designed to i) explore key stakeholders' preferences for a model, and ii) identify existing services in the Republic of Ireland, Northern Ireland, England, Wales, and Scotland. Preliminary results of Phase III, evaluations of existing services, are available.

Results: There is limited published research supporting components of dementia palliative care models in the community. However, the scoping review suggests that the core areas of an effective community model include: advance care planning, education on the disease process, 24-hour nurse phone support, improved care co-ordination and family support. Survey respondents rated the three most essential aspects of a model as: "Care for People at all Stages of Illness" (77\% considered 'essential'), "Information Continuity" (75\% 'essential') and "Defined Pathways for Specialist Service Input" (58\% 'essential'). In addition, good communication between palliative care and dementia care teams was perceived to be core to a successful model, with a key worker needed to facilitate this communication, and to support families e.g. around care transitions. Conclusion: Consistent aspects of an effective model were identified. This work builds on existing theory and research to ensure that future models of care in Ireland will be effective, evidence-based, feasible to adopt and acceptable to healthcare workers and potential users.

PS 8 Increasing the Value of Randomized Controlled Trials (RCTs) for Complex Interventions in Palliative Care MORECare Methodological Guidance on Increasing the Value of RCTs for Complex Palliative Care Interventions

Evans C. ${ }^{1,2}$, Higginson I. ${ }^{1}$, on behalf of MORECare and OPTCare Elderly

${ }^{1}$ King's College London, Cicely Saunders Institute of Palliative Care, Policy and Rehabilitation, London, United Kingdom, ${ }^{2}$ Sussex Community NHS Foundation Trust, Brighton, United Kingdom Presenting author email address: catherine.evans6@nhs.net

Aim: To identify, appraise and apply 'best practice' methods to address common challenges in experimental trials of complex interventions in palliative care.

Methods: Methods of Researching End of life Care (MORECare) built on Medical Research Council guidance on the development and evaluation of complex interventions. We used systematic review and consensus methods (nominal group and online) to develop the MORECare statement on best practice in EoLC research, including recruitment, ethics, attrition, mixed methods, complex outcomes and economic evaluation. We critically consider the guidance by applying in a mixed method feasibility trial called OPTCare Elderly - optimising palliative care for people in the community. Trial eligibility aged $75+$ years, $2+$ unresolved symptoms or concerns, non-cancer, Clinical Frailty Score $>4$ (range 4-7). Intervention short-term integrated palliative and supportive care (SIPS) and usual care or usual care, main outcome 5 key symptoms measured Integrated Palliative care Outcome Scale (baseline, 6 weeks and 12 weeks), and exploration economic evaluation, including formal and 
informal care costs (CSRI) and effectiveness (EQ5D, ICECAP-O and main outcome)

Results: Integration of data from 3 systematic reviews, 5 consensus groups and 133 online responses to form the MOREcare statement comprising 36 best practice solutions. Application in trial, recruited to target 50 patients and 26 carers, randomised to SIPS $(n=24)$ or usual care $(n=26)$. Findings that feasible to identify and recruit, key intervention ingredients (comprehensive assessment, case management and accessibility), effectiveness on main outcome at 6 weeks, and 'best approach' for cost-effectiveness formal service costs and main outcome.

Conclusions: Application of the MORECare statement can improve trial design by anticipating and planning for common challenges to enable recruitment, examine effectiveness and cost-effectiveness, and linkage between the problem and outcomes.

PS 8 Increasing the Value of Randomized Controlled Trials (RCTs) for Complex Interventions in Palliative Care Mixed-Method Process Evaluations as Necessary Requirements Alongside RCTs of Multicomponent Interventions: Examples of Guidelines Implementation Studies and Nonpharmacological Interventions

Agar M. .,2,3 $^{2}$

${ }^{1}$ University of Technology Sydney, IMPACCT, Sydney, Australia, ${ }^{2}$ UNSW, South West Sydney Clinical School, Sydney, Australia, ${ }^{3}$ Liverpool Hospital, Palliative Medicine, Sydney, Australia Presenting author email address: meera.agar@uts.edu.au

The goals of palliative care delivery are multidimensional, and to achieve these aims interventions require multicomponent strategies. Understanding processes and factors which influence implementation and the subsequent adherence and 'dose' of components delivered, interpretation of the primary and secondary outcome findings, and insights into sustainability are critical components of intervention evaluation. This session will utilise the examples of three Australian clinical trials - a cluster randomised controlled trial of facilitated family case conferencing in advanced dementia (the IDEAL Study), ${ }^{1}$ a stepped wedge cluster trial evaluating cancer pain guideline implementation, ${ }^{2}$ and a non-pharmacological multicomponent intervention to prevent delirium in hospitalised older people (PRESERVE study) ${ }^{3}$ to illustrate the value add of mixed method evaluation.

1. Agar M, Luckett T et al. Effects of facilitated family case conferencing for advanced dementia: A cluster randomised clinical trial. PLoS ONE 2017; 12(8): e0181020

2. Luckett T, Phillips J, Agar M et al. Protocol for a phase III pragmatic stepped wedge cluster randomised controlled trial comparing the effectiveness and cost-effectiveness of screening and guidelines with, versus without, implementation strategies for improving pain in adults with cancer attending outpatient oncology and palliative care services: the Stop Cancer PAIN Trial. BMC Health Services Research. 2018, 18;558.

3. Hosie, A., Phillips, J., Kochovska, S., Brassil, M., Noble, B., Kurrle, S., Cumming, A., Caplan, G.A., Chye, R., Le, B., Ely, E.W., Lawlor, P.G., Bush, S.H., Davis, J.M, Lovell, M., Brown, L., Fazekas, B., Cheah, S.L. Edwards, L. and Agar, M. Multicomponent non-pharmacological intervention to prevent delirium for hospitalised people with advanced cancer: study protocol for a phase II cluster randomised controlled trial. BMJ Open. 2019. 9(1);e02617728

PS 8 Increasing the Value of Randomized Controlled Trials (RCTs) for Complex Interventions in Palliative Care Theory-based Methods to Understand Whether, How and When Complex Palliative Care Interventions Work: Examples of Palliative Care and Advance Care Planning
Van den Block L.

Vrije Universiteit Brussel \& End-of-life care Research Group, Brussel, Belgium

Aims: To illustrate how the Theory of Change methodology, developed by the Aspen Institute, can be integrated into the UK MRC Framework for Complex Interventions, to improve the development and evaluations of complex palliative care interventions.

Methods: A 'Theory of Change' (ToC) is 'a theory of how and why an initiative works which can be empirically tested by measuring indicators for every expected step on a hypothesized causal pathway to impact'. It is visually represented in a ToC map. As part of two randomized controlled trials (on implementing advance care planning in nursing homes and integrating an early palliative care intervention for frail older people), we have developed a ToC during the first development phase of the UK MRC Framework, using literature review and intensive and systematic stakeholder consultation and backwards outcome mapping. Intervention components were identified as part of the ToC and defined as activities needed to achieve the different steps towards achieving the desirable impact.

Results: Using the ToC methodology, the intervention programmes that were developed, differentiate between several micro (e.g. conversations with patients and family), meso (e.g. education of professionals) and macro level (e.g. sensibilization and information to the wider system) components of the intervention, which all need to be addressed to achieve meaningful change in real practice. The ToC map also guided outcome and process evaluations in subsequent pilot RCTs.

Conclusion: Developing a Theory of Change helps in clearly identifying and describing all intervention activities and components of complex interventions and whether and how to achieve change. This is necessary to improve comparability of interventions, replicability and implementation in practice.

PS 9 Global Prospects in Palliative Care: Measuring the Burden, Responding to Crises

Who Needs Palliative Care?

Krakauer E.

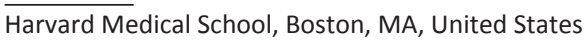

According to the World Health Organization (WHO), palliative care is first and foremost a response to suffering of any kind, physical, psychological, social or spiritual. Among the serious health problems that most often produce suffering requiring palliation, the most common are life-threatening conditions such as malignancies and major organ failure. However, moderate or severe physical, psychological, social, or spiritual suffering also occurs among patients who can recover from their health problem or whose health problem can be cured or controlled for many years. Curable conditions that may generate a need for palliative care include drug-resistant tuberculosis, some hemorrhagic fevers such as Ebola, and some malignancies. Conditions from which patients can recover but which may generate a need for palliative care include serious injuries and burns. Conditions that may be controlled for many years but which may generate a need for palliative care include HIV/AIDS and some musculoskeletal disorders. The specific types and severity of suffering vary by geopolitical situation, socioeconomic conditions and culture. People in low and middle income countries often endure less healthy social conditions. They also typically have less access to disease prevention, diagnosis and treatment, to social support, and to specialists and specialized services of many kinds than people in high-income countries. Thus, in low-resource settings, a wider variety of people with a wider variety of physical and psycho-social problems need palliative care than in high-resource settings.

PS 10 End of Life and Prison: Inequalities and Vulnerability Ageing and Dying in Prison: Exploring the Vulnerability of Older Prisoners and the Challenges in Providing Equitable Palliative Care 
Turner $M$

University of Huddersfield, Department of Nursing \& Midwifery, Huddersfield, United Kingdom Presenting author email address: m.turner@hud.ac.uk

This paper draws on findings from a UK study of palliative care in prison to explore the vulnerabilities of older prisoners facing the end of life in custody. England \& Wales has the highest prison population rate in Western Europe, and the numbers of older prisoners and deaths in custody are rising rapidly. Many older prisoners are classed as 'vulnerable' because of their age, frailty or the nature of their offence, which puts them at risk of bullying from other prisoners. The study used participatory action research to investigate and improve the provision of palliative care to this vulnerable population.

The national End of Life Care Strategy for England \& Wales (2008) advocates high quality and equitable palliative and end of life care for all who need it, regardless of care setting. The paper discusses some of the challenges in providing palliative care in a prison setting, and highlights issues such as prison design and layout, access to opioid medication, lack of equipment and other resources, and the constraints inherent in the prison regime. Implications for staff are also considered, and some initiatives to improve practice that emerged from the study are presented.

PS 10 End of Life and Prison: Inequalities and Vulnerability How to Communicate with End of Life Patient in Prison? A Prospective National Survey of the Palliative Care of Inmates in France Chassagne A.

University Hospital, CIC-Inserm 1431, Besancon, France

The French prison population is aging and particularly ill. Every year, about 100 inmates die in prison from illness. In France, there is no specific system for palliative care for inmates unlike some other countries (USA and UK) which have been developing hospice care programs. What are inmates experiencing at the end of their life? How to communicate with those patients/inmates about death and dying ?

The National Survey of the Palliative Situation among Inmates in French Prisons Project is a mixed-method study that combining quantitative and qualitative approaches (2012-2015). We conducted a census in all dedicated health care facilities $(n=190)$ in each French prison during 3 months and identified 50 inmates requiring palliative care. Then, we conducted interviews to identify the difficulties in developing appropriate global palliative care, especially the barriers to communicate with the end of life patients/inmates in prison.

70 Interviews were conducted individually with the inmates themselves, physicians, nurses, social and probation workers and sometimes families. Three factors appear to be some major barriers to communicate about death and dying: 1 / The tension between healthcare professionals and prison staff; 2 /The lack of palliative care training for health care professionals ; 3 / The uncertain temporality of death juxtaposed with the hope of being released for inmates.

Prison seems not to be a place to communicate about death and dying. Can and should the system which has been developing for "free end of life patients" be implemented for inmates?

\section{PS 11 Medication Safety in Palliative Care} Neither Shaken Nor Stirred - Drug Incompatibility in Palliative Care Dickman A.

Royal Liverpool and Broadgreen University NHS Hospitals Trust, Academic Palliative and End of Life Care Centre, Liverpool, United Kingdom

Mixing of incompatible parenteral drugs is seen as an important medication error. Avoidance of incompatibilities and instabilities of drug combinations represents prevention of medication error.
The characteristic physicochemical properties of a drug molecule are governed by functional groups. When two or more drugs are mixed together for parenteral administration, different functional groups may interact giving rise to the long-recognised problem of physicochemical interactions

Incompatibility refers to a theoretically reversible physicochemical change that may result in precipitation or insolubility, which may not always be visible. Physical incompatibility is a term that is occasionally used to describe visual changes that may occur when drugs are combined, such as particulate formation, haze, turbidity, crystallisation, colour change, or gas evolution. Instability refers to irreversible chemical degradation of the active compound, resulting in loss of potency. Incompatibility of drug admixtures can be as a result of a number of factors including change of $\mathrm{pH}$ of the solution, dilution of co-solvent, salting out and cation-anion interactions. Since most drugs are small organic electrolytes which, in aqueous solution, behave as weak acids, weak bases or both, a rudimentary understanding of acid-base chemistry can be used to explain, identify and predict many drug-drug incompatibilities that result in precipitation. Drugs in solution can be susceptible to instability due to irreversible chemical degradation. The most common chemical reactions implicated in parenteral drug degradation involve hydrolysis and/or oxidation

The best confirmation of stability and compatibility will always be the performance of an appropriate laboratory analysis to examine the range of concentrations commonly used, prepared in the preferred diluent(s) and stored over a range of temperatures and light exposure, reflecting use in the clinical situation.

\section{PS 11 Medication Safety in Palliative Care Off-Label-Use in Palliative Care} Hagemann $V$.

LMU Munich, Department of Palliative Medicine, University Hospital, LMU Munich, Munich, Germany

Aims: To identify the status quo of prevalence of off-label drug use in palliative care and to describe the clinical practice with regard to the awareness and management strategies of healthcare professionals regarding off-label-use.

Methods: In total we performed three studies. First a systematic review of international literature to determine the status quo, second a survey among German palliative doctors regarding existing guidelines for and views on off-label drug use in palliative care and third a cross-sectional analysis of prescribing data from a palliative care unit in Germany. Results: In the international literature the frequency of off-label-use in palliative care ranges between 14.5 and $35 \% .85 \%(n=95)$ of the doctors participating in the survey reported that no guidance on off-label-use or documentation was available. $75 \%$ of the participants regularly used offlabel therapies with little or no evidence base. $55 \%$ of all drug applications on the palliative care unit were off-label.

Conclusion: The prevalence of off-label drug use in palliative care is high. Available data is heterogeneous making comparisons difficult. In summary little is known on off-label-use in clinical practice. There is a lack of evident information and guidance and a potential discrepancy between the suspected frequency and the actual need for off-label-use in palliative care.

PS 11 Medication Safety in Palliative Care Drug Information - Ensuring Medication Safety in Palliative Care Remi C.

Ludwig-Maximilians-University, Munich University Hospital, Department of Palliative Medicine, München, Germany

Drug therapy is a fundamental part of symptom control in palliative care. As well as its potential benefits, it also carries risks and a major aim 
should always be not to harm the patient. Examples for risky areas in palliative care drug therapy are off-label drug use, polypharmacy and the mixing of drugs in infusion devices. Access to structured drug information may enable the prescriber to use all the possibilities of medication while limiting side effects. This lecture therefore aims to provide an overview on medication safety and to sensitize professionals to risk factors in the palliative care medication process. Strategies for every day clinical practice to manage these challenges will be presented. The goal is to encourage participants to continue to learn and practise ways to improve the safety of medication use in order to avoid harm to the patient and to optimize drug therapy and subsequently symptom control.

PS 12 Implementing ACP across the Healthcare System in Europe and the US: the Results of Three ACP Trials Improving the Initiation of Advance Care Planning (ACP) in General Practice for People with a Chronic Life-Limiting Illness: An Exploratory Phase II Cluster Randomised Controlled Trial

Dierckx S. ${ }^{1}$, Pardon K. ${ }^{1}$, Pype P. ${ }^{2}$, Deliens L. ${ }^{1}$, De Vleminck A. ${ }^{1}$

${ }^{1}$ Vrije Universiteit Brussel (VUB) \& Ghent University, End-of-life Care Research Group, Brussels, Belgium, ${ }^{2}$ Ghent University, Departement of Public Health and Primary Care, Ghent, Belgium

Background: Evidence on the form and effectiveness of ACP for general practice is lacking.To address this gap, we developed an intervention to improve the initiation of ACP in general practice for people with a chronic life-limiting illness.

Aims: to understand the feasibility and acceptability of this complex intervention and to evaluate the potential effectiveness of this intervention on patients' quality of life and the quality of GP-patient communication.

Methods: We conducted an exploratory cluster-RCT; individual GPs served as unit of randomization. Semi-structured interviews with patients and GPs, questionnaires and detailed documentation of the study procedures were used to evaluate feasibility, acceptability and preliminary effectiveness of the intervention.

Results: A total of 32 GPs agreed to participate in the study of which 18 were randomized to the intervention group. In total, 45 patients were considered eligible by the GPs and participated in the study. Most GPs and patients valued the ACP discussions that took place: GPs were pleased that, in response to discussions, patients were prompted to think more about end-of-life issues or discuss their preferences with relatives, and that patients were reassured that some fears or uncertainties for the future were addressed. We identified barriers at both the GP- and patient-level to discuss ACP, as well as difficulties with some study procedures that affected feasibility and acceptability. No differences in preliminary effectiveness on the quality of life of patients and quality of physician-patient communication between intervention and control group were found.

Conclusion: We found a complex multi-component intervention to improve the initiation of ACP in general practice to be feasible and acceptable to both GPs and patients. This study also highlighted some important challenges regarding the study procedures and outcomes measures that will need to be addressed in a future large-scale trial.

PS 12 Implementing ACP across the Healthcare System in Europe and the US: the Results of Three ACP Trials Improving Advance Care Planning with Easy-to-Use, Patient-Facing, Evidence-Based Tools: The PREPARE Program

Sudore R.L., Schillinger D., Katen M.T., Shi Y., Boscardin J., Osua S., Barnes D.E.

UCSF, San Francisco, CA, United States

Aims: To overcome literacy and language barriers to advance care planning (ACP), we created easy-to-read advance directives (ADs) and an online advance care planning program (PrepareForYourCare.org) in
English and Spanish. In 2 randomized trials we compared the efficacy of PREPARE plus an easy-to-read AD (PREPARE) with an AD alone to increase ACP documentation and patient-reported engagement.

Methods: Two comparative efficacy randomized trials among English and Spanish-speaking patients, age $\geqslant 55$ years, with $\geqslant 2$ chronic or serious illnesses from (1) a Veterans Hospital and (2) 4 safety-net, primarycare clinics in San Francisco, California USA. Participants were randomized to PREPARE plus an easy-to-read AD (PREPARE) or the AD alone. There were no clinician/system-level interventions. Staff were blinded. The primary outcome was new ACP documentation in the medical record at 15 months. Patient-reported outcomes included ACP engagement at baseline, 1 week, and 3, 6, and 12-months. We used intention-to-treat, mixed-effects logistic and linear regression, controlling for time, health literacy and baseline ACP, clustering by physician, and stratifying by language.

Results: 1400 patients enrolled in the 2 trials. The mean (SD) age was 63.3 years ( \pm 6.4 ), $40 \%$ had limited health literacy, and $45 \%$ were Spanishspeaking. No participant characteristic differed between arms; retention was $86 \%$. Compared to the AD alone, PREPARE resulted in higher ACP documentation (adjusted $43 \%$ vs. $32 \% ; p<0.001$ ) and higher self-reported increased ACP engagement scores (98.1\% vs. 89.5\%; $p<0.001)$. Results remained significant among English and Spanish-speakers.

Conclusions: PREPARE and an easy-to-read AD, without clinician/systemlevel interventions, increased ACP documentation and engagement, with higher gains for PREPARE. These tools may allow patients to begin ACP on their own and improve the process for diverse populations.

PS 12 Implementing ACP across the Healthcare System in Europe and the US: the Results of Three ACP Trials

Advance Care Planning in Patients with Advanced Cancer: Main Results from a Cluster Randomized Trial in Six European Countries; the ACTION Trial

Korfage I. ${ }^{1}$, Miccinesi G. ${ }^{2}$, van Delden J.J.M. ${ }^{3}$, Deliens L. ${ }^{4}$, Groenvold M. ${ }^{5}$, Lunder U. ${ }^{6}$, Payne S. ${ }^{7}$, Pollock K. ${ }^{8}$, van der Heide ${ }^{*}$ A. ${ }^{1}$, Rietjens $* J .{ }^{1}$, ACTION consortium

${ }^{1}$ Erasmus MC, Public Health, Rotterdam, Netherlands, ${ }^{2}$ ISPO Cancer Prevention and Research Institute, Clinical and Descriptive Epidemiology Unit, Florence, Italy, ${ }^{3}$ Medical School of Utrecht University, Julius Center for Health Sciences and Primary Care, Utrecht, Netherlands, ${ }^{4}$ Vrije Universiteit Brussel (VUB) \& Ghent University, End-of-life Care Research Group, Brussels, Belgium, ${ }^{5}$ University of Copenhagen, Department of Public Health, Kobenhavn, Denmark, ${ }^{6}$ University Clinic for Respiratory and Allergic Diseases Golnik, Golnik, Slovenia, ${ }^{7}$ Lancaster University, International Observatory on the End-of-Life Care, Lancaster, United Kingdom, ${ }^{8}$ University of Nottingham, School of Health Sciences, Sue Ryder Centre for the Study of Supportive, Palliative and End of Life Care, Nottingham, United Kingdom

Background: Advance care planning (ACP) encourages people to define, discuss and record their goals and preferences for future care. Despite being internationally recommended, robust studies on ACP in European patients with advanced cancer are scarce.

Methods: We performed a cluster-randomized trial in patients with advanced lung or colorectal cancer in 23 hospitals across six European countries. In intervention hospitals, patients were offered the ACTION Respecting Choices ACP intervention, that includes scripted ACP conversations with trained facilitators, alongside usual care. Questionnaires at baseline and at 10-12 weeks follow-up assessed emotional functioning (EORTC) and symptoms (EORTC-QLQ-C15) as primary outcomes, along with coping, patient satisfaction, and shared decision-making. Use of care was derived from medical files. Multilevel regression analyses were performed taking into account potential clustering at hospital level.

Findings: Between 2015 and 2018, 1117 patients (442 intervention; 675 control) were included, of whom 810 (73\%) completed the follow-up 
questionnaire. In the intervention group, 396 (90\%) patients participated in the intervention. Facilitators' fidelity to the intervention was very good with $86 \%$ of key topics consistently addressed. The average change in patients' emotional functioning at follow-up was comparable in intervention vs. control group: -0.07 vs. $-0.23, p=0.35$. We did not find differences in change scores for symptoms, coping, patient satisfaction, and shared decision-making either. In the intervention group, medical files more often included advance directives ( $7 \%$ versus $3 \%, p=0.003$ ) and more patients used specialist palliative care ( $36 \%$ versus $27 \%, p=0.003$ ).

Conclusion: In this large trial in patients with advanced cancer, the ACTION Respecting Choices ACP intervention had no effects on emotional functioning and symptoms and limited effects on secondary outcomes, including use of care.

Funding: EU FP7.

PS 13 Providing Palliative Care for Refugees and Migrants Need for Guidance for a White Paper on Palliative Care for Refugees and Migrants

Nauck F. ${ }^{1}$, Marston J. ${ }^{2}$, Leng M. ${ }^{3}$, Radbruch L. ${ }^{4}$

${ }^{1}$ University Göttingen, Department of Palliative Medicine, Göttingen, Germany, ${ }^{2}$ ICPCN Global Ambassador for Children's Palliative Care, Clinical Manager Hospice, Bloemfontein, South Africa, ${ }^{3} \mathrm{Global}$ Health Academy, University of Edinburgh, Department of Palliative Medicine, Edinburgh, United Kingdom, ${ }^{4}$ University Hospital of Bonn, Department of Palliative Medicine, Bonn, Germany Presenting author email address: friedemann.nauck@med.uni-goettingen.de

Background: Most European countries are hosting refugees, with some hosting large numbers in relation to their country population. Over half of them are under 18 years of age. Countries with high numbers of refugees and migrants such as Turkey report that their health systems are struggling to cope with the increased need for health services; while in refugee camps and settlements health care is often provided by humanitarian non-government organizations such as Doctors without Borders (MSF), that have no formal palliative care programs. A number of ongoing studies highlights the palliative care needs in migrant and refugee populations in Palestine, Uganda and Bangladesh.

Aim: The EAPC has set up a task force to develop a White Paper on palliative care for refugees and migrants in Europe - undertaking a literature review, assessing the palliative care needs of refugees and making recommendations for palliative care professionals as well as for professionals working in humanitarian organizations. The white paper is to describe structural and organizational aspects as well as methods for screening, assessment and treatment. The paper will be used to formulate the agenda for the Task Force's later activities and producing an Action plan for the Task Force and the EAPC.

Conclusion: There is a need for guidance on palliative care for refugees and migrants both for palliative care professionals and humanitarian organizations. The EAPC task force will collaborate with PALCHASE, which has been established as a collaborative of palliative care and humanitarian aid practitioners, ethicists, educators, researchers and advocates promoting palliative care integration in all humanitarian aid situations.

PS 13 Providing Palliative Care for Refugees and Migrants The Loss and the Making-of-Home: Support for Refugee Children Who Live Betwixt and Between Worlds

Papadatou D.

National and Kapodistrian Univesrity of Athens, Nursing, Athens, Greece

As of January 2019, there are 71.200 refugee and migrants in Greece, among them 27.000 children. The latter include 3.400 unaccompanied children, most of whom are adolescent boys, older than 14 years of age. They live "betwixt and between" worlds, with an undefined social status and future, hoping to reunite and/or settle in northern or central Europe, despite the growing opposition of European countries to host refugees. While living in limbo, they often maintain multiple representations of home: their home of origin (past), the home of their aspired destination (future), and a home they inhabit during a period of protracted liminality (present). These home representations are associated to their identity that is challenged and re-shaped in liminality. Recounting stories of life back home, is more than an act of remembrance, since it reflects a need for self-affirmation, while stories of resettlement in a desired home allow children to project self into the future. By engaging in 'home-making practices' children introduce a sense of predictability, order and belonging, which enhances their ability to tolerate ambiguity and make the most of living in the present. This presentation aims: (a) to describe the challenges that unaccompanied refugee children experience while living "between and betwixt worlds" for a prolonged time; (b) to highlight their need to maintain alternative representations of "home" and re-create a sense of identity; and (c) to present a model that illuminates how lossrelated, traumatic and enriching experiences affect the developmental and their psychosocial well-being in times of ambiguity and liminality.

PS 13 Providing Palliative Care for Refugees and Migrants A Rapid Appraisal of Palliative Care Needs in a Humanitarian Crisis: Experiences from the Rohingya Refugee Crisis Doherty M. ${ }^{1}$, Khan F. ${ }^{2}$

${ }^{1}$ Children's Hospital of Eastern Ontario, Pediatrics, Ottawa, ON, Canada, ${ }^{2}$ Fasiuddin Khan Research Foundation, Dhaka, Bangladesh Presenting author email address: megan.elizabeth.doherty@gmail.com

Aims: Palliative care has been neglected during humanitarian crises as the imperative to save lives has often meant that the suffering of those who cannot be cured is forgotten. To date, very few programs provide palliative care in humanitarian crisis situations. Our aim was to assess the palliative care needs of those with life-limiting illnesses, and their caregivers during an evolving humanitarian crisis situation.

Methods: Structured interviews were conducted with 156 individuals living with life-limiting illnesses and 157 caregivers about their palliative care and support needs, in the context of their broader needs and experiences as refugees in four settlement areas in Cox's Bazar, Bangladesh during the Rohingya refugee crisis in November 2017.

Results: The most common illnesses which were reported by patients were severe physical disabilities $(98,63 \%)$, treatment-resistant tuberculosis $(33,21 \%)$, cancer $(16,10 \%)$, and HIV/AIDS $(3,2 \%)$. Pain was a significant problem which was reported 114 (73\%) of patients surveyed. Only one health facility had strong opioids available, and no patients had received opioids. None of the health facilities surveyed were providing any palliative care and were instead focused on acute medical issues. The majority of patients surveyed $(87,56 \%)$ reported significant financial problems which prevent them from getting medical care, other common barriers to medical care included a lack of suitable treatment at local facilities $(28,18 \%)$ and difficulties with transportation $(20,13 \%)$.

Conclusions: The findings from this rapid appraisal of palliative care needs in the Rohingya refugee crisis in Bangladesh illustrates the specific and concrete needs in this particular context and has implications for the development of palliative care for humanitarian interventions in a global context. The methodology employed in the current study may prove useful in assessing palliative care needs of specific disadvantaged populations globally.

PS 14 Understanding Health Promotion in End of Life Care: from Policy Change to Community Action The Role of National Policy Change in Improving Access to Pain Relief: an Indian Example

\section{Rajagopal M.R.}

Pallium India, Trivandrum, India Presenting author email address: chairman@palliumindia.org 
To advance palliative care anywhere, we need to have facilitatory policy by governments and health care delivery agencies, access to essential medicines and concurrent education of professionals and common man. Though palliative care took birth in India in 1985, even today less than $2 \%$ of the population has access to it. Earlier efforts at education increased awareness but had no major impact in access to care. A collaborative of Indian palliative care activists with Pain and Policy Studies Group at Madison-Wisconsin from 1995, analyzed opioid policy and worked systematically. Though a faciliatory opioid regulations resulted in 1998 , it had very little impact on access to palliative care nationally till a multi-pronged approach was started in 2005.

The initiative aimed at the following:

1. Legislation for reform of law governing opioids. Though the efforts took 19 years, the Indian Parliament enacted a legislative reform in 2014.

2. Influencing health policy of central and state governments. Government of Kerala state created a palliative care policy in 2008. Government of India announced a national program for palliative care in 2012. The new health policy of India in 2016 included palliative care.

3. Quality assurance. A national task force created by Pallium India created a minimum standards document in 2006.

4. Inclusion of palliative care as part of medical and nursing education. In 2010, medical council of India accepted palliative medicine as a specialty. Post-graduate degree courses were started in Palliative Medicine in 2012. In 2016, health university in Kerala included Palliative Care as part of medical curriculum.

Above reforms in the last one decade have together made the current environment conducive to growth. But improvement in access to palliative care continues to be slow. The missing link at national level appears to be adequate public awareness. It appears that with the buildup of the background work, what is needed is adequate public advocacy.

\section{PS 14 Understanding Health Promotion in End of Life Care: from Policy Change to Community Action}

The Development of Individual and Community Capacity in End of Life Care: A UK Example

Sallnow $L$.

University College London, St Christopher's Hospice, London, London, United Kingdom Presenting author email address: libby.sallnow@nhs. net

The Ottawa Charter for Health Promotion presents five pillars through which healthcare services, communities and individuals can create health and improve wellbeing. These principles have been applied to all clinical specialties in addition to schools, workplaces and urban environments. The application of health promotion principles to end of life care involves taking a broad perspective about the factors that impact on experiences and outcomes at the end of life, looking beyond healthcare services. Using strategies to prevent or reduce the effect of factors such as loneliness, inequity of access to services, poor health and death literacy and poor bereavement outcomes, it is hoped that broader experiences of end-of-life care will be improved across a community.

This talk will illustrate how the principles of the Ottawa Charter can be applied to end-of-life care through the presentation of the results of a large mixed methods study which evaluated 'Compassionate Neighbours', a health promoting intervention developed by a hospice and the local community in the UK. This intervention built links with members of the local community as they became Compassionate Neighbours and local advocates for end of life care.

Twenty-one interviews, two focus groups and 19 episodes of participant observation were conducted,11 documents and service data on 180 Compassionate Neighbours and 173 Community Members was included.
Impacts from the work included a reduction in loneliness, improvements in wellbeing and changes to hospice practice. Further to this, three underlying drivers emerged that underpinned the work as a whole. They translated the observed actions of the project into the impacts and included: altered power dynamics,expression of reciprocity in relationships and the development of agency. This study provides a reflexive and critical account of the processes and impacts of compassionate communities in practice and poses new research questions for this important field.

PS 15 The Challenge of Integrating Palliative Care for people with Chronic Obstructive Pulmonary Disease (COPD) How Physicians Discuss End of Life Issues with Patients with COPD: A Systematic Review and Experiences of Respiratory Physicians from Slovenia Lunder U. ${ }^{1}$, Froggatt $K^{2}{ }^{2}$, Preston $N^{2}$

${ }^{1}$ University Clinic for Respiratory and Allergic Diseases Golnik, Slovenia, Research Department, Golnik, Slovenia, 'Lancaster University, Lancaster, United Kingdom Presenting author email address: urska. lunder@klinika-golnik.si

Background: Patients with COPD rarely receive timely end-of-life discussions, and deterioration of their disease is often perceived as unexpected. The reluctance of physicians to initiate and conduct end-of-life discussions is one of the main reasons that patients do not usually receive palliative care in advanced COPD.

Methods:

1. We conducted an integrative literature review and searched four databases (PubMed, CINAHL, PsycINFO and Web Of Science). We identified 11 studies.

2. Semi-structured interviews with 14 physicians caring for patients with COPD on the respiratory wards in two hospitals in Slovenia were conducted and thematically analysed.

Results: Findings from the integrative review and qualitative study about the end-of-life conversations and experiences of physicians from Slovenia showed some common features. These include uncertainty in prognostication, a focus on 'living/saving life' and feelings of feeling conflicted in complex active management of COPD and a palliative care approach. The initiation of end of life conversations in the earlier stages raised concerns about preservation of hope. Physicians perceive that it is difficult to invite patients in EOL decision-making discussions, especially about the possibility of introduction of invasive ventilation and intubation. This may lead to avoiding difficult discussions or physicians reframe the information. Conclusion: Physicians experience prognostic uncertainty and lack of common consensus about when and how to introduce end-of-life discussions in advanced COPD as the main barriers in initiating and conducting such conversations. Deciding between active management of the disease and a palliative care approach presents a tension, which can lead to less open communication.

PS 15 The Challenge of Integrating Palliative Care for people with Chronic Obstructive Pulmonary Disease (COPD)

Palliative Care Integration in Advanced COPD: Learn from the Past, Live the Present and Plan the Future

Fusi-Schmidhauser $T_{.}{ }^{1,2}$, Riglietti A. ${ }^{3}$, Froggatt $K^{4}{ }^{4}$, Preston $N^{4}$

1Palliative and Supportive Care Clinic, Institute of Oncology of Southern Switzerland and Ente Ospedaliero Cantonale, Lugano, Switzerland, 2Internal Medicine Department, Ospedale Regionale di Lugano, Ente Ospedaliero Cantonale, Lugano, Switzerland, ${ }^{3}$ Respiratory Care Department, Ospedale Regionale di Lugano, Ente Ospedaliero Cantonale, Lugano, Switzerland, ${ }^{4}$ International Observatory on End of Life Care, Lancaster University, Lancaster, United Kingdom 
Background: Chronic obstructive pulmonary disease is recognized as being a life-limiting condition with palliative care needs. Nonetheless, palliative care delivery in this population is sporadic. The unpredictability of the disease trajectory and misconceptions in both patients and healthcare professionals about palliative care being exclusively relevant in cancer and in the last days of life limit palliative care provision in advanced COPD. This systematic review aimed to explore how palliative care is provided in advanced COPD and to identify elements defining integrated palliative care.

Methods: Eight databases, including MEDLINE, EMBASE and CINAHL, were searched using a comprehensive search strategy to identify studies on palliative care provision in advanced COPD, published from January 1 , 1960 to November 30, 2017. Citation tracking and evaluation of trial registers were also performed. Study quality was assessed with a critical appraisal tool for both qualitative and quantitative data.

Results: Of the 458 titles, 24 were eligible for inclusion. Experiences about living with advanced COPD, palliative care timing, service delivery and palliative care integration emerged as main themes, defining a developing taxonomy for palliative care provision in advanced COPD. This taxonomy involves different levels of care provision and integrated care is the last step of this dynamic process. Furthermore, palliative care involvement, holistic needs' assessment and management and advance care planning have been identified as elements of integrated care.

Conclusions: This literature review identified elements that could be used to develop a taxonomy of palliative care delivery in advanced COPD. This taxonomy will be linked to an action learning study of a joint respiratory and palliative care initiative in Southern Switzerland, aimed at developing new ways to integrate palliative care provision for patients with advanced COPD

PS 15 The Challenge of Integrating Palliative Care for people with Chronic Obstructive Pulmonary Disease (COPD) Development of an Intervention to Promote Integration of Palliative Care in COPD Management for a Multicentre Study Preston N.J. ${ }^{1}$, Payne S. ${ }^{1}$, Walshe C. ${ }^{1}$, Marsden P. ${ }^{2}$ ${ }^{1}$ Lancaster Univeristy, International Observatory on End of Life Care, Lancaster, United Kingdom, ${ }^{2}$ Wythenshawe Hospital, Respiratory Medicine, Manchester, United Kingdom Presenting author email address: n.j.preston@lancaster.ac.uk

Chronic obstructive pulmonary disease (COPD) accounts for $5 \%$ of deaths globally (WHO statistics 2015). Most people with COPD do not receive any sort of specialist palliative care involvement. Recognition that it is a terminal condition can be quite late in the disease trajectory with limited time to discuss future care plans. This is due in part to the difficulty in prognostication for this group but also reluctance by health professionals to initiate such conversations. This results in poor identification of palliative care needs of patients with COPD and they then receive less than optimal care.

A critique of tools used to identify end of life care needs of people with COPD will be presented. Based on this critique a novel hospital based approach, the CLEAR Framework has been developed to identify people with palliative care needs who have a COPD diagnosis and deliver improved care, which recognises end of life care needs of patients with advanced COPD. The aim is to identify people with COPD who have end of life needs, communicate effectively with them, their family and all members of the healthcare team, to decide upon the level of escalation in treatment patients require if experiencing an exacerbation of their condition. In addition, advance care planning is introduced and these plans reviewed regularly. The framework brings together evidence on identification tools, integrated care guidelines and previously tested models of care delivery for this group of patients. The CLEAR Framework [C: communication LE: Level of escalation A: Advance care planning R: Review] is initiated in the hospital during an acute hospital admission by the respiratory team with palliative care support. This framework could be refined and adopted internationally.
PS 16 Enabling Person-Centred Care for Palliative Care Patients and Their Informal Carers: Definitions, Models of Practice and Implementation

Person-Centred Care: Principles and Practice Barriers Diffin J. ${ }^{1}$, Öhlén J. ${ }^{2}$

${ }^{1}$ University of Manchester, Manchester, United Kingdom, ${ }^{2}$ University of Gothenburg, Gothenburg, Sweden Presenting author email address: janet.diffin@manchester.ac.uk

Aim: Barriers to the implementation of person-centred care within palliative care practice have been identified for patients and family carers. A shared understanding of (i) the principles of person-centred care, and (ii) how to successfully implement person-centred interventions is therefore required. This paper aims to to define the key principles of personcentred care and identify barriers and enablers to implementation success within palliative care practice.

Methods: This paper brings together the international evidence on person-centred care, and reviews the enablers and barriers to implementation within palliative care practice. It will draw on the experiences of the development of person-centred care practice and the implementation of the Carer Support Needs Assessment Tool intervention, a person-centred process of assessment and support for family carers.

Results: Person-centred care is characterised by practitioners initiating, working through and safeguarding a partnership 'with' patients and/or family carers. As such, a change in practice from a practitioner-led approach, to one which is led by a patient/family carer is often required. Implementation barriers at the individual level include viewing a person-centred approach as an 'add-on' to existing practice, and at the organisational level include a lack of managerial support and protected time. Training is therefore required for the individual practitioner to help them integrate this approach into their everyday practice, and for the organisation to ensure adequate preparation, and the establishment of a strategy to support implementation. A team of practitioners within the organisation is needed to drive this implementation planning and ensure organisational readiness for change.

Conclusions: The evidence presented provides vital lessons learned for implementation of person-centred interventions for patients or family carers within palliative care practice.

PS 16 Enabling Person-Centred Care for Palliative Care Patients and Their Informal Carers: Definitions, Models of Practice and Implementation

KOMMA: Person-Centred Assessment and Support for Carers in the German Language

Pleschberger S. ${ }^{1}$, Kreyer C. ${ }^{2}$

${ }^{1}$ Gesundheit Österreich / The Austrian Public Health Institute, Wien, Austria, ${ }^{2}$ UMIT - Private Universitiy for Health Sciences, Medical Informatics and Technology, Dep. for Nursing Science \& Gerontology, Hall i. T., Austria Presenting author email address: sabine. pleschberger@goeg.at

Background: The Carer Support Needs Assessment Tool (CSNAT) intervention, developed in the UK, is effective in assessing and addressing support needs of family carers of palliative patients. KOMMA (Kommunikation mit Angehörigen) is the translated and culturally adapted German version of CSNAT which uses a person-centred process of assessment and support for carers.

The aim of this presentation is to reflect on adapting the CSNAT and implementing its person centred approach in the context of different national service frameworks.

Methods: The KOMMA Approach was implemented in four hospice and palliative home care services in Germany. This was accomplished in three stages: (1) translation of CSNAT following ISPOR criteria and validation; 
(2) adaption of the UK-CSNAT-training and piloting (3) implementation of the approach facilitated by regional champions. A mixed-methods evaluation was conducted.

Results: A multi-step process of translation and piloting led to a culturally well acceptable German tool (KOMMA). Carers and champions reported that the KOMMA was easy to understand and to complete, and that the domains adequately addressed carers' support needs. Using KOMMA afforded sensitivity in person centred support and balanced the focus on family carers with patients. The training was modified to include a trial phase of using KOMMA, which was later evaluated as beneficial by professionals. A key issue raised by teams in the implementation project was who has responsibility to do systematically assess and address family carers' needs in palliative home care.

Discussion \& Conclusion: The innovative training method was effective in preparing practitioners in Germany to use the KOMMA Approach in palliative home care. However, any process of implementation in hospice and palliative care needs to be culturally and regionally sensitive and take account of differences in ways of approaching family carers in services as well as different professional backgrounds.

PS 16 Enabling Person-Centred Care for Palliative Care Patients and Their Informal Carers: Definitions, Models of Practice and Implementation

Enabling Person-Centred Care for Patients: The Support Needs Approach for Patients (SNAP)

Gardener A.C. ${ }^{1}$, Ewing G. $^{2}$, Farquhar M. ${ }^{3}$

${ }^{1}$ Univeristy of Cambridge, Public and Primary Care, Cambridge, United Kingdom, ${ }^{2}$ University of Cambridge, Centre for Family Research, Cambridge, United Kingdom, ${ }^{3}$ University of East Anglia, School of Health Sciences, Norwich, United Kingdom

Aims: The Support Needs Approach for Patients (SNAP) is an intervention to enable delivery of person-centred care, originally developed for patients with advanced Chronic Obstructive Pulmonary Disease (COPD). Modelled on the Carer Support Needs Assessment Tool (CSNAT) Approach, SNAP comprises an evidence-based set of questions to help patients consider where they need more support (the SNAP tool) and a needs-led conversation between the patient and healthcare professional (HCP). This presentation will outline the development and clinical utility of SNAP.

Methods: SNAP has been developed within a four-stage mixed-methods programme using data from patients, informal carers and HCPs. The SNAP programme comprised: 1) SNAP tool development; 2) SNAP tool validation; 3 ) development of SNAP Training to enable HCPs to deliver the SNAP intervention and 4) a pilot study to assess the feasibility and acceptability of SNAP in primary, secondary and community care settings (currently on-going, completes February 2019).

Results: 1) The developed SNAP tool asks patients to consider whether they need more support across 15 broad areas of support need e.g. "knowing what to expect in the future" and "getting out and about." 2) The SNAP tool is valid for use with patients with advanced COPD. 3) Face to face and online SNAP Training have been developed. 4) Early results from the pilot suggest SNAP is feasible and acceptable to HCPs and patients with COPD across the range of settings: patients report increased legitimacy and visibility when considering and expressing need, and HCPs report working with patients to respond to identified needs including facilitation of future care conversations. Data analysis is on-going.

Conclusion: The SNAP intervention comprises the validated SNAP tool and needs-led conversation between the patient and HCP. It appears feasible and acceptable across a range of settings, enabling care to be more person-centred. Website: theSNAP.org.uk
PS 17 Developments in Global Palliative Care Policy and Strategy: Opportunities and Challenges

Development of Palliative and Home Care Strategic Framework in Jordan - A Demonstration Project

Shamieh O. ${ }^{1}$, Harding R. ${ }^{2}$, Mansour A. ${ }^{3}$, Abdelrazeq H. ${ }^{4}$, Al-Tarawneh M. ${ }^{5}$, Al Tabba' A. ${ }^{4}$, Payne S. ${ }^{6}$

${ }^{1}$ King Hussein Cancer Center, Palliative Care, Amman, Jordan, ${ }^{2}$ Kings College London, London, United Kingdom, ${ }^{3}$ King Hussein Cancer

Center, Director General, Amman, Jordan, ${ }^{4}$ King Hussein Cancer Center, Amman, Jordan, ${ }^{5} \mathrm{High}$ Health Council, Secretary General, Amman, Jordan, ${ }^{6}$ Lancaster Univeristy, Lancaster, United Kingdom Presenting author email address: oshamieh@khcc.jo

Introduction: Palliative care in Jordan is better developed compared to most of Middle Eastern countries but still lagging behind western countries. Currently, palliative care is only accessible to a small portion of cancer patients, mainly at King Hussein Cancer Centre, in Amman, the capital city of Jordan. It is estimated that more than $70 \%$ of cancer patients who died in Jordan in 2016 did not have access to palliative care. A national palliative care strategic framework is needed to advance palliative care development.

Objectives: To describe the development of the national palliative and home care strategic framework in Jordan.

Methods: A systematic content analysis of palliative care needs in Jordan, SWOT analysis and multistage consensus procedure and national and international expert consultations were used to develop and revise the national strategic framework in Jordan

Results: A detailed content analysis of needs was completed. The development of the strategic framework involved multiple steps, the first draft was developed using the WHO framework by the national Palliative care committee, the draft was then revised by international experts. To reach a consensus, the second draft was revised in a national strategic multidisciplinary workshop of multi stakeholder experts. The final version was approved by the national and the international experts. The final framework contains 6 domains; policy, finance, capacity building/ workforce development, service delivery, Opioids/ Essential Medications, and information/research/monitoring and evaluation

Conclusions: The multistage process of development and the involvement of key stakeholders led to significant revision of the framework and was successful in reaching a national consensus. The strategic framework was endorsed by the Minister of Health of health in late 2017 and was officially launched on April 2018.

\section{PS 17 Developments in Global Palliative Care Policy and} Strategy: Opportunities and Challenges

Using Palliative Care Providers to Support National Health Policy: South African Hospices Providing Essential Government Services Gwyther L. ${ }^{1,2}$

${ }^{1}$ University of Cape Town, Family Medicine, Cape Town, South Africa, ${ }^{2}$ Hospice Palliative Care Association of South Africa, Cape Town, South Africa Presenting author email address: liz@hpca.co.za

The development of the South African National Policy Framework and Strategy for Palliative Care has been a long journey with strong support from hospices and from the Hospice Palliative Care Association(HPCA). As in many countries, palliative care was first provided to patients and families requiring this care in the non-governmental sector. The work of hospices, providing compassionate care to patients and families with life-threatening and life-limiting illnesses received recognition by the communities they served, the government and funders. Hospices have been providing this essential health service for over 30 years. SA Department of Health now recognise care as part of Universal health Coverage and include palliative care in the planned National health Insurance. HPCA have an active advocacy role in increasing the reach of palliative care working closely with departments of health. Thus, South 
Africa was primed to comment on and to co-sponsor the World health Assembly resolution 67.19 on palliative care. Recognising the responsibility to enact the recommendations of the WHA resolution,Tte Minister of Health appointed a steering committee for palliative care - chaired by a high-level official the MEC for Health in Kwa-Zulu Natal. The national steering committee has members from the National Department of Health, from HPCA, from professional councils and academic palliative care groups. There are 7 task teams each allocated to address a specific WHA recommendation and the first success has been the development of the National Policy Framework and Strategy for Palliative Care which was approved by the National Health Council in April 2017. However, there is no budget; so the Funding Task Team led by the department of health has failed in its task! This presentation will explore the steps taken to implement the policy and the importance of developing champions to drive the process both at poly level and on the ground.

\section{PS 17 Developments in Global Palliative Care Policy and Strategy: Opportunities and Challenges Generating Relevant Evidence to Inform Local Policy \& Strategy: Examples from Global Health Research and Capacity Building Harding $R$. \\ King's College London / Cicely Saunders Institute, London, United Kingdom}

Aim: Research to improve access to palliative care in low and middle income countries (LMIC) requires strategic planning and sustained partnerships. We present achievements and challenges from global health palliative care research in building capacity and impact.

Methods: The design and conduct of timely and robust primary research in LMIC is driving both capacity building (apprenticeships in research methods and study delivery, academic fellowships, postgraduate degrees, local mentorship cascades) and driving impact (dissemination activities, advocacy partnerships, implementation and quality improvement). Results: Research partnership guidelines underpin sustained, productive programmes. Outcome measurement research (as an exemplar) recruited the first African palliative care research nurses, generated publications with co-authorship, informed locally-led quality improvement strategies, embedded measurement within standards and guidance, and enabled both cohort and RCT studies. This led to post-graduate degrees, and mentored studies led by LMIC PI's, and to mhealth technology pilot studies. Capacity-building grants with post-docs are adapting measurement to conflict settings, while advances in paediatric measurement led from Africa are informing studies in high income settings. A number of challenges persist. While the increasing number of PhDs from LMIC is encouraging, post-doc career pathways are few. Grant funds from within LMIC are rare, and more co-PIs from LMIC must lead applications to high income funders. Paediatric research remains relatively poorly funded, and RCTs are rare. The body of evidence is small compared to the increasing need for palliative care and to competing health fields in LMIC.

Conclusion: Growth of individual researchers, and engagement with NGOs and advocacy bodies who lobby for evidence into policy, are crucial. Research partnerships are mutually beneficial, bringing methodological innovation and two-way learning.

\section{PS 18 What is New about Palliative Management of} Breathlessness in People with Heart Failure Different Types of Breathlessness in People with Heart Failure Sobanski P.

EAPC Task Force on Palliative Care for People with Heart Disease Spital Schwyz, Department of Internal Disease, Palliative Care Ward and Competence Centre, Schwyz, Switzerland

Breathlessness is a universal complaint among people living with heart failure (HF). In less advanced stages of HF, it is only precipitated by exercise, which exceeds ordinary levels. As the severity of HF progress, the capacity for exercise decreases and the threshold of exercise evoking breathlessness drops down. In the end stages of HF, those affected experience breathlessness after slight exercise or even at rest. Up to $90 \%$ of people living with advanced HF report experiencing breathlessness, which limits their basic activities of daily living, sometimes reducing them to the very minimum. Breathlessness evoked by heavy exercise and that which is present at rest are different entities, in respect of different initiating stimuli, signal transmission and processing, as well as their perception. As a consequence, various pathways, neurotransmitters and receptors might be involved. When interpreting the results of research investigating therapeutic interventions and tailoring their management, the potential differences should be acknowledged.

Breathlessness, which persists despite optimal causative management, should be considered as refractory and result in the initiation of effective symptomatic, palliative treatment, optimally together with the adjustment of disease specific medication. The participating factors or disease aggravating or evoking breathlessness should be actively sought, as in as many as $50 \%$ of people with an obvious, well-known disease usually causing breathlessness (such as advanced HF) additional conditions (both extracardiac and cardiac) can be identified. They should be specifically managed if possible and if appropriate. If this is not the case, or if this causative treatment is not effective enough to alleviate the suffering, palliative treatment must be employed. Different types of breathlessness related to $\mathrm{HF}$ or commonly coexisting with $\mathrm{HF}$ and the tools to assess breathlessness will be addressed.

\section{PS 18 What is New about Palliative Management of} Breathlessness in People with Heart Failure Non-medical Management of Breathlessness in Heart Failure Fettes L.

Cicely Saunders Institute of Palliative Care, Policy \& Rehabilitation, Florence Nightingale Faculty of Nursing, Midwifery \& Palliative Care, King's College London, London, United Kingdom

People living with heart failure have a high symptom burden, with breathlessness being consistently rated as one of the most distressing symptoms. People with heart failure frequently experience both episodic and continuous breathlessness, with episodes triggered by activity, position changes or anxiety, as well as episodes with no obvious cause. This can be a difficult symptom to manage pharmacologically, with limited options available to clinicians.

Increasingly, research is demonstrating the benefits of non-pharmacological management of breathlessness in those with a range of advanced diseases, including heart failure. This has been found to be particularly beneficial when delivered as a complex, multi-disciplinary intervention and has been shown to improve self-awareness of the symptom and selfefficacy, thereby improving quality of life.

This lecture will discuss the latest research on non-pharmacological breathlessness management and outline current best practice in this field. It will provide information directly applicable to clinical practice including practical ideas for managing symptoms.

PS 18 What is New about Palliative Management of Breathlessness in People with Heart Failure Medical Management of Breathlessness in Heart Failure Currow D.

University of Technology Sydney, Sydney, Australia

In 2017, an international Delphi process defined a clinical syndrome of chronic breathlessness. This is an important step forward for people where the underlying causes optimally treated and, despite this, the person continues to experience disability. In terms of the modified Medical Research Council (mMRC) breathlessness scale, these people will be 
short of breath when walking along the flat at their own pace requiring them to stop every 100 metres or breathlessness enough to be housebound or breathless getting dressed or undressed. These are significant levels of disability that effect people with chronic heart failure numbers across our communities.

Chronic breathlessness is systematically under-recognised and, when recognised, under-treated. The recognition of chronic breathlessness is a responsibility of every health professional. Better recognition and clinical response would help to relieve avoidable suffering in our communities.

Whatever the underlying aetiology for the cardiac failure, a prevalent end point is chronic breathlessness. When the underlying cause(s) is optimally treated, the residual breathlessness needs to be treated symptomatically.

The clinical expectation is to reduce the impact of that breathlessness on a person's day to day life. It appears that worst breathlessness is the target that will most benefit patients. Non-pharmacological interventions such as oxygen have not been shown to significantly reduce chronic breathlessness. Regular, low dose, systemic opioids have a role, with most evidence in studies of morphine. Having said this, there is a questions as to whether a) there is a class effect of opioids; and b) fluctuations in renal function are more limiting for use of morphine in heart failure.

\section{PS 19 What is the Impact of Assisted Dying on Health Care Professionals Working in Palliative Care? What Is the Experience of Assisted Dying for Dutch Healthcare Professionals Working in a Hospice or Chronic Disease Care Centre? \\ Lewis D.A. \\ Post Graduate Lancaster University, Faculty of Health and Medicine, Lancaster, United Kingdom Presenting author email address: deborahann.lewis@btinternet.com}

Background: Assisted dying is a contemporary issue with worldwide interest with debate often focused on the right-to-die. Thus far comprehensive consideration of the experience of healthcare professionals has been lacking.

Aim: The aim of this study was to explore and gain a deeper understanding of the assisted dying experience for Dutch healthcare professionals who have the longest experience of permissive legislation.

Methods: This research utilised a constructivist qualitative approach, with thematic analysis, to analyse the care experience of 21 physicians, nurses and therapists working in a Dutch hospice or chronic disease care centre.

Findings: All professional groups received requests for assisted death. Large numbers of requests were heard at the hospice where half of admitted patients wanted to discuss the option. This could be onerous for professionals with a conscientious objection. All requests required an assessment of seriousness, exploration of patient fears, observation of psychological status and possible coercion. Opening up dialogue, optimising palliative care and resolving practical care issues were priorities. Applying such measures resolved the majority of requests and, in chronic disease, also delayed assisted deaths.

Care continuity was valued, but such deaths were challenging and perceived as not normal. The administration of lethal drugs and post-death case scrutiny were stressful for physicians. Post-death debriefing and psychological support was valued, but adjustment to such deaths took time. Some physicians required a recovery period before considering new requests rising equality and equity issues.

The involvement of nurses and therapists including previously unreported activities safeguarded patients. Assisted dying was perceived as significantly increasing workload, but this was not resented. Support for the provision of assisted dying was overwhelming, but this was unqualified in only a third of the participants.
PS 19 What is the Impact of Assisted Dying on Health Care

Professionals Working in Palliative Care?

Hospice Professionals' Experiences of the Death with Dignity Act in Washington State

Gerson S.M. ${ }^{1}$, Grinyer A. ${ }^{2}$, Bingley A. ${ }^{2}$

${ }^{1}$ University of Glasgow, School of Interdisciplinary Studies, Dumfries, United Kingdom, ${ }^{2}$ Lancaster University, Division of Health Research, Lancaster, United Kingdom Presenting author email address: sheri. gerson@glasgow.ac.uk

Background: Several jurisdictions around the world have passed laws that allow a physician, or, in some areas, a nurse practitioner to legally prescribe life- ending medications to individuals faced with a serious illness. There are some individuals receiving hospice services that also die by suicide without legal lethal means.

Aim: The aim of this study is to understand hospice professionals' experiences with home hospice patients who have died by suicide or hastened death utilising the Death with Dignity Act.

Methods: This qualitative study undertaken in Washington State, where the Death with Dignity Act was implemented in 2009, investigates home hospice professionals' experiences with patients who died by suicide or utilised the Death with Dignity Act. Data were collected in 2015 through in-depth, semi-structured interviews with seven nurses, seven social workers, four physicians, and three chaplains.

Results: Analysis of these data indicates participants' interpretation of suicide is blurry but there are some patients receiving home hospice services who die by suicide because they are not eligible for, have no knowledge of, or lack access to the option of medical aid in dying. Participants in this study work with patients who utilise medical aid in dying despite dilemmas related to personal values, or organisational policies that conflict with the principle of non-abandonment. Many deaths by suicide may be interpreted as rational but a perceived taboo about suicide limits open conversations among professionals and within organisations.

Conclusion: Culture, location, socioeconomic disparities, and organisational policy impact how patients perceive, accept, or afford medical aid in dying in Washington State. Palliative care professionals working in the home setting need open communication within teams and training about assessing patients at risk of suicide and those who seek to utilise medical aid in dying.

PS 19 What is the Impact of Assisted Dying on Health Care Professionals Working in Palliative Care?

Palliative Care Physicians' Experiences of the Swiss Model of Assisted Suicide

Gamondi C. ${ }^{1,2}$, Borasio G.D. ${ }^{3}$, Oliver P. ${ }^{4}$, Preston N..$^{5}$, Payne S. ${ }^{5}$ ${ }^{1}$ Oncology Institute of Southern Switzerland, Palliative Care, Bellinzona, Switzerland, ${ }^{2}$ Palliative and Supportive Care Service, Lausanne University Hospital, Lausanne, Switzerland, ${ }^{3}$ Palliative and Supportive Care Service, Lausanne University Hospital, Service de Soins Palliatifs, Lausanne, Switzerland, ${ }^{4}$ Pam Oliver Ltd, Research and Evaluation, Waiheke Island, New Zealand, 5 International Observatory on End of Life Care, Lancaster University, Faculty of Health and Medicine, Lancaster, United Kingdom

Background: In Switzerland euthanasia is not permitted, but assisted suicide is permitted. If the assistance has no selfish motivations, those providing it are not prosecuted. There is no federal law regulating assisted suicide provision in Switzerland, although two cantons have enacted legislation regulating assisted suicide in public institutions. Switzerland constitutes a unique "civil model". Assisted suicide mainly occurs at the patient's home with the assistance of right to die associations. The rightto-die associations assess the patient's medical documentation for eligibility, determine their mental capacity, can refer to a physician to obtain a prescription for the lethal drug, and offer the support of a volunteer to 
assist the patient during this process. The assigned volunteer evaluates the patient's metal capacity prior to ingestion.

Aims: To investigate their perspectives and involvement in assisted suicide practice.

Methods: Twenty-three palliative care physicians were interviewed; thematic analysis was used.

Results: It emerged that they receive assisted suicide requests regularly and tended to follow a common pattern in responding to them. They tend to set and communicate early on boundaries on their level of willingness to support a patient's assisted suicide request. They also play an active role in the decision-making process by exploring patients' reasons, offering alternatives, and discussing assisted suicide risks. In conclusion. Participants had received little training on responding to assisted suicide requests.

Conclusion: The Swiss civil model of assisted suicide permits palliative care physicians to develop individualised roles which reflect each practitioner's personal, ethical and professional belief systems and their responses seemed to be largely shaped by personal values and experiences. Responding to assisted suicide requests remains a difficult task for Swiss palliative care physicians

\section{PS 20 Using Big Data to Improve End-of-Life Care: Opportunities and Challenges \\ Using Big Data to Study End-Of-Life Care: Opportunities and Challenges \\ Onwuteaka-Philipsen B.}

Amsterdam UMC, Location VUmc, Dept. of Public and Occupational Health, Amsterdam, Netherlands

Aim: Big data ('big' with regard to the four Vs: volume, variety, velocity and veracity) provide opportunities for researchers, clinicians and policy makers for measuring and comparing actual health care practice during the last years, months, weeks and days of life. Aim of this first presentation of the session on the use of 'big data' in palliative care research is to give an overview of the possibilities of this type of research with a focus on discussing the opportunities these studies can bring and the challenges that need consideration when using or interpreting this type of research.

Opportunities: Several opportunities will be discussed. That it concerns routinely collected data is related to several opportunities, for instance that no costly data collection is needed, that patient's and caregivers are not burdened by the data collection. In addition it means that sampling errors, selection bias and recall bias are avoided. Other opportunities stem from the possibility to use innovative methodologies or analyses, such as the use of machine learning or algorithms for prediction

Challenges: That the data used are routinely collected also brings challenges. For instance with regard to the suitability of the data for research. Another challenge is that palliative care is recorded and, hence, can be recognized in databases can differ. This is especially challenging in international comparative research. 'Hindsight knowledge' and controlling for confounding are methodological challenges, especially in - the until now most frequently used - mortality follow back designs. Finally there are also ethical challenges e.g. related to consent for secondary data analyses or the use of algorithms.

In conclusion: It will be argued that the opportunities outweigh the challenges if the latter are considered and solved or taken into account in interpretation. The EAPC task force 'Big Data in end-of-life care research' aims at supporting this.

PS 20 Using Big Data to Improve End-of-Life Care: Opportunities and Challenges

Evaluating the Impact of Palliative Home Care on Outcomes and Costs of Care Using Routinely Collected Data

Cohen J.
Vrije Universiteit Brussel, End-of-life Care Research Group, Brussel, Belgium

Background: Big data can provide evidence about effectiveness of interventions at the end of life that is difficult to provide via clinical studies. For instance, because palliative home care is -in many countries- a patient right, it is impossible to conduct a randomized controlled trial (RCT) allocating a group to receiving palliative home care and withholding such care to a control group.

Aim: This presentation evaluates the impact of palliative home care on the quality of care and costs at the end of life using a propensity score matching technique.

Methods: A matched retrospective cohort study was conducted using individual health claims data from all people who died in Belgium in $2012(N=107,847)$, linked to information from several other populationlevel databases. 8837 people who received palliative home care support (exposed group) were matched to 8837 people who received usual care (unexposed) based on the propensity to receive palliative home care as estimated by a number of pre-exposure characteristics (e.g. diagnosis, comorbidities, care dependency, prior health care use intensity, sociodemographics). Outcomes concern previously validated indicators of appropriateness and inappropriateness of end-of-life care and total inpatient and outpatient health care costs in the last 14 days of life (postexposure period).

Results: Palliative home care (exposed vs control group) resulted in a larger likelihood of a home death (56\% vs. $14 \%$ ) and a higher average of family physician contacts ( 3.1 vs. 0.8 ), a lower risk of hospital admission ( $27 \%$ vs $61 \%$ ), intensive care unit admission ( $18 \%$ vs $40 \%$ ), emergency department admission (15\% vs $28 \%$ ) and lower total average costs of care in the last 14 days of life (€3081 vs. €4698).

Conclusion: This presentation illustrates how big data that simulate the controlled circumstances of an RCT as much as possible are a good opportunity to provide the best possible evidence about the impact of palliative home care on quality and costs of care.

\section{PS 20 Using Big Data to Improve End-of-Life Care: Opportunities} and Challenges

Healthcare Utilization Near the End of Life: What Can We Learn from Big Data?

Morin L.

Karolinska Institutet, Stockholm, Sweden

Routinely collected clinical and administrative data are increasing in volume and improving in quality. This opens many opportunities for palliative care research, especially for describing and evaluating the effectiveness of healthcare services provided to people near the end of life.

During this presentation, we will discuss some of the opportunities and challenges related to the availability of 'big data' for studying healthcare utilization at the end of life.

We will take advantage of high-quality registers with national coverage in Sweden to illustrate with concrete examples how this type of data can be used to answer different research questions, by applying different study designs to investigate different outcomes in different populations.

The presentation will be highly interactive. After a short introduction, the audience will be asked to make decisions regarding the research question, the study design, the definition of the study population, and the statistical models. Data will be analysed in real-time based on the choices made by the participants, and the results will serve as a springboard for discussing the methodological caveats of this type of studies.

In conclusion, we will highlight the current knowledge gaps that could be filled by using 'big data' and we will make simple recommendations for ensuring the validity and the transparency of future studies. 


\section{Meet the Expert Sessions}

ME01 Time to Change? Current Practices in Delirium Care Time to Change? Current Practices in Delirium Care Agar M. ${ }^{1}$, Bush S. ${ }^{2}$

${ }^{1}$ University of Technology Sydney, IMPACCT, Sydney, Australia, ${ }^{2}$ University of Ottawa, Department of Medicine, Ottawa, ON, Canada Presenting author email address: meera.agar@uts.edu.au

Delirium is a highly prevalent and distressing clinical syndrome in palliative care. Delirium is universally under-detected and poorly managed, in a wide range of health care settings including palliative care. Improvements in delirium care require attention to prevention, assessment, management, and communication with patients and their families. Quality initiatives to enhance delirium care require an interprofessional approach, underpinned by policy, evidence-based guidelines and education. Approaches, evidence and resources developed in other health care settings also need to be translated to suit palliative care practice. In this session, delegates will have the opportunity to discuss the individual, service, system and policy level challenges in delirium practice change, and have the opportunity to learn in more detail about frameworks, guideline development and international initiatives that enable quality delirium care in the palliative care setting, and consider how these can be applied in their practice context. This session is led by two experienced delirium researchers and advocates for improved delirium care in the palliative care setting; through their leadership of the 2018 Delirium in Adult Cancer Patients: ESMO Clinical Practice Guidelines and European Delirium Association Board Membership respectively. The session will facilitate an interactive discussion around the key clinical challenges faced in palliative care practice and debate what is best practice in relation to screening for delirium, prevention, non-pharmacological care, pharmacological approaches and support for carers. Delirium care is an interdisciplinary enterprise; the session will consider how we can best engage our teams in delirium care, improve communication about delirium and feel supported when witnessing delirium in our patients distresses us.

ME 2 Palliative Care and the Homeless Population: Challenges, Solutions and Lessons from Others

Palliative Care and the Homeless Population: Challenges, Solutions and Lessons from Others

Conneely A. ${ }^{1,2}$, Shulman C. $3,4,5$

${ }^{1}$ Academic Department of Palliative Medicine, Our Lady's Hospice \& Care Services, Dublin, Ireland, ${ }^{2}$ St Francis Hospice, Dublin, Ireland, ${ }_{3}^{3}$ Marie Curie Research Department, University College London, London, United Kingdom, ${ }^{4}$ Pathway Charity, London, United Kingdom, ${ }^{5}$ King's Health Partnership, London, United Kingdom Presenting author email address: aoibheann.conneely@gmail.com

People experiencing homelessness often have very poor health and a higher risk of death than the housed population. Average age of death is in their 40's and standardised mortality rates 8 and 12 times higher than housed populations. They often present with a combination of severe physical and mental health difficulties, complicated by substance misuse (trimorbidity). Trimorbidity, lack of stable accommodation and lack of family to provide informal care make delivery of palliative care challenging. Delegates will be encouraged to think about the homeless population as not just the visible homeless, but as a broader vulnerable population including the hidden homeless.

In this session two speakers from different professional backgrounds of palliative medicine and general practice will present. Both work with people experiencing homelessness and have conducted research on the delivery of palliative care to this population. The speakers will discuss the challenges identified from their research and work. Recommendations, along with tools and resources, will be presented. The presentation will also include reference to international research and will draw on knowledge and experience from other specialties such as public health, and the emerging field of inclusion medicine.

The health service utilisation by people experiencing homelessness, and how interactions with staff and services can differ from the housed population, will be discussed. Speakers will challenge current models of care, and encourage the delegates to think about service delivery from a different perspective.

This session aims to encourage delegates to think about their own local palliative care services and what initiatives could be undertaken to address the palliative care needs of this marginalised population.

\section{ME 3 Maximising Global Impact in Palliative Care: Achieving}

Effective Knowledge Transfer and Exchange

Maximising Global Impact in Palliative Care: Achieving Effective

Knowledge Transfer and Exchange

Guerin S. ${ }^{1,2}$, McKeon M. ${ }^{3}$, Kernohan G. ${ }^{4}$

${ }^{1}$ University College Dublin, Dublin, Ireland, ${ }^{2}$ AllHPC Palliative Care Research Network, Dublin, Ireland, ${ }^{3}$ All Ireland Institute of Hospice and Palliative Care, Dublin, Ireland, ${ }^{4}$ Ulster Univerisiity, Newtownabbey, United Kingdom

Knowledge transfer and exchange refers to the process by which researchers work to engage with policy and practice settings to maximise the likelihood that evidence-based knowledge may be positioned to inform practice. This has been identified as a challenge in many areas of health services research, including palliative care. In a global research context this challenge can be increased due to inequalities in access to traditional dissemination products, such as peer-reviewed journals and professional conferences. There is a need to consider how to promote effective knowledge transfer and exchange in a global community. The aim of this session is to support health care professionals, researchers and those interested in ensuring that evidence-based palliative care knowledge reaches its beneficiaries. Central to the session is a peer reviewed evidence-based knowledge transfer and exchange model "EMTReK". EMTReK - the Evidence-based Model for the Transfer and exchange of Research Knowledge was developed based on a systematic review of models relating to knowledge transfer in health services research. EMTReK encourages researchers to consider the multiple messages from research that may be relevant to different audiences at different stages of the research process. By the end of the session it is hoped that delegates will be able to (i) identify challenges around achieving effective sharing of palliative care knowledge to ensure access for all; (ii) apply the key components of EMTReK to their dissemination activity; (iii) develop an effective dissemination plan for palliative care knowledge; (iv) recognise and use traditional and non-traditional dissemination tools and communication channels; and (v) understand the importance of continuous evaluation during the dissemination process and develop an evaluation plan alongside these activities.

ME 4 How to Develop an Academic Palliative Care Network How to Develop an Academic Palliative Care Network - a FrenchGerman-Swiss Experience

Borasio G.D. ${ }^{1}$, Aubry R. ${ }^{2}$

${ }^{1}$ CHUV, University of Lausanne, Service de Soins Palliatifs et de Support, Lausanne, Switzerland, ${ }^{2} \mathrm{CHU}$ Besançon, Département Douleur et Soins Palliatifs, Besançon, France

Looking at historical precedents, the establishment of a strong academic presence of palliative care (including but not limited to physicians) will most likely be a decisive factor in the development, and even survival, of the discipline. The presenters have contributed to the creation of networks of multidisciplinary palliative care professorships, with both private and public funding, in France (R.A., 10 professorships), as well as Germany and Switzerland (G.D.B., 4 and 3 professorships, respectively). 
We would like to share our experiences, including opportunities and pitfalls, strategies that worked and those that didn't, in order to support colleagues around the world who share the same goal, in the face of sometimes challenging academic environments. We hope that our experiences may motivate and help the delegates in developing strategies aimed at improving the academic standing of palliative care in their respective countries through the creation of university positions and the establishment of academic networks.

\section{ME 5 How to Integrate Palliative Care and Pain Relief in Primary} Health Care?

How Should Palliative Care Be Integrated into Primary Health Care? Krakauer E.

Harvard Medical School, Boston, MA, United States

WHO recommends a stepwise approach to integrating palliative care into health services in general, and into primary care in particular, in a cost-effective manner, to reach all those in need. This strategy includes:

- updating existing policies on palliative care and creating new ones where none exist;

- assuring safe accessibility of essential palliative medicines, including oral fast-acting morphine;

- initiating required training in palliative care for all clinicians who treat people with palliative care needs, including training in basic palliative care for all primary care workers;

- implementing palliative care services that are integrated into all health care systems at all levels, including palliative home care as part of primary care.

In settings where primary care is underdeveloped, primary care and palliative care can be developed and expanded at the same time. The same infrastructure and human resources that provide primary care both can and should provide basic palliative care; similarly, efforts to implement community-based palliative care both can and should strengthen primary care.

WHO recommends an essential package of palliative care for primary health care (EP PHC) that is designed to assist health care and primary care policy-makers, planners, implementers and managers to integrate palliative care into community-oriented primary care. The EP PHC is the minimum package that should be accessible in primary care clinics, and it is designed to address the palliative care needs of both adults and children. The package comprises:

- safe, effective, inexpensive, off-patent and widely available medicines;

- $\quad$ simple and inexpensive equipment;

- basic intersectoral social supports; and

- human resources needed to apply each element of the EP PHC effectively and safely.

ME 6 Enhancing Transparency in Systematic Reviews of Qualitative Literature: a Practical Approach

Enhancing Transparency in Systematic Reviews of Qualitative Literature: A Practical Approach

Preston $\mathrm{N}^{1}{ }^{1}$, Kars $\mathrm{M}^{2}$

${ }^{1}$ Lancaster University, International Observatory on End of Life Care, Lancaster, United Kingdom, ${ }^{2}$ University Medical Center Utrecht, Medical Humanities/Nursing Science, Utrecht, Netherlands Presenting author email address: n.j.preston@lancaster.ac.uk

The benefits of conducting systematic reviews of research about interventions are well known. There are clear methods to conduct systematic reviews of research findings related to effects of interventions. A great deal of research, particularly in palliative care, is qualitative where experiences are explored. How do you synthesis findings from this research, which may cover a range of qualitative methods and where authors present their research findings in varying levels of detail? While systematic review methods to synthesise qualitative data are developing, there is less consensus on how to synthesise findings in these reviews. Even when applying these methods, challenges are encountered due to the heterogeneity of palliative care in practice, the often explorative character of review questions and poorly defined keywords and concepts in the databases.

This session aims to highlight the range of methods to conduct systematic reviews of qualitative research studies including but not exclusively narrative synthesis, integrative reviews, thematic synthesis and realist reviews. These will be used as exemplars to consider how these systematic review methods can be applied and will help you to decide which review method to use. In part, these choices will be based upon the design of the included research studies but also your underlying epistemology. We draw attention to a newly developed approach that focuses your search to ensure relevant studies are captured. We will cover whether the different review methods need a comprehensive search, or are you looking for data saturation? We question which tools incorporate theory and which critical appraisal tools are available. We conclude with an overview of reporting guidelines.

ME 7 The Role of Social Work in Supporting the LGBT

Community

Meet the Expert

Firth P. ${ }^{1}$, Marshall S. ${ }^{2}$

${ }^{1}$ Independent Consultant, St. Albans, United Kingdom, ${ }^{2}$ Kings College Hospital NHS Foundation Trust, London, United Kingdom Presenting author email address: p.firth97@btinternet.com

Aims: Palliative Care Social Workers make an important contribution working with LGBT people, but more knowledge is needed.

Whilst focussing upon the needs of the lesbian, gay, bisexual and trans (LGBT) community at the end-of-life and in bereavement, this session will also explore the contribution of social workers within multi-disciplinary palliative care teams. The Chairs and facilitators, two experienced palliative care social workers, will be joined by an experienced researcher katherine Bristowe whose work focuses on LGBT palliative care and bereavement.

Method: The session will begin with brief presentations around the unique palliative care needs of the LGBT community and the role social workers can play when working with this cohort. Recent research findings will be shared. Small group discussion will also focus on some case studies and how LGBT needs in palliative care can be taken forward on an international level

Conclusion: It is hoped that the session will attract clinicians from all disciplines with an interest in this area and we encourage newly appointed and attending palliative care social workers to join us to meet task force members. The facilitators are active researchers who hope to encourage other social workers to undertake research exploring psychosocial issues in palliative care, as well as research focussed upon the needs of marginalised groups.

Group work will be facilitated by EAPC Palliative Care Social Work Task Force.lt is an opportunity to extend our knowledge and work in this area.

ME 8 Delivering Bereavement Care in Palliative Care: Can this Be Provided by Volunteers or is it the Remit of Professionals? Evidence or Opinion?

Delivering Bereavement Care in Palliative Care: Can This Be Provided by Volunteers or Is It the Remit of Professionals? Evidence or Opinion? Relf $M .^{1}$, Scott $R^{2}$ 
${ }^{1}$ Sir Michael Sobell House, Bereavement Service, Oxford, United Kingdom, ${ }^{2}$ University of Dundee, Dundee, United Kingdom Presenting author email address: smsh.bereavement@ouh.nhs.uk

Through discussion participants from different settings and countries will be challenged to consider different ways that bereavement support may be offered and to examine policy and service implications. In some countries there is a strong tradition of volunteering and volunteers play a major role in delivering bereavement care both in hospices and in the wider field. Without volunteers few people would have access to support. In other countries the involvement of volunteers is controversial and bereavement care is delivered by professionals. Dr Marilyn Relf is a pioneer in the development of palliative care bereavement services. She has been involved in research, service management, education and supervision as well as clinical work. Dr Ros Scott has researched the involvement of volunteers in hospices and community services and has a wealth of experience in the field. Together they will lead a discussion about the advantages and limitations of involving volunteers in bereavement care and the structures that need to be in place to ensure quality services are delivered to meet a wide range of needs.

\section{ME 10 Managing Substance Use Disorder Treatment at the End of Life}

Managing Substance Use Disorder Treatment at the End of Life
McPherson M.L. ${ }^{1}$, Gourlay D. ${ }^{2}$

${ }^{1}$ University of Maryland Baltimore, Pharmacy Practice and Science, Baltimore, MD, United States, ${ }^{2}$ Private Practice, Toronto, Ontario, ON, Canada

Substance use disorder and the opioid epidemic has added a new layer of complexity in patient care today. To be certain, it has made the management of acute and chronic pain far more complicated, and patients routinely do not get effective pain relief. What is the responsibility of the hospice or palliative care team when a patient has substance use disorder? This brings up a variety of ethical, psychosocial, and clinical management issues such as "Should we treat substance use disorder in a patient close to the end of life?" "How should we handle opioid agonist therapy in a recovering substance use disorder patient when they are terminally ill?" "If the patient cannot get to the opioid agonist therapy provider, how does the hospice/palliative care team handle this clinically, particularly if the patient also has concurrent pain?" Drs. Gourlay and McPherson are prepared to handle all these questions and more as we wrestle with "dealing with substance use disorder in advanced illness."

ME 12 The Significance of a New Model of Social Capital in Redressing Inequalities in End of Life Care Collective Social Capital: A New Model of Social Capital in End-Of-Life Care

Sallnow L. ${ }^{1}$, Kellehear A. ${ }^{2}$

1St Chistopher's Hospice, University College London, Palliative Care, London, United Kingdom, 2University of Bradford, Digital Health Enterprise Zone, Bradford, United Kingdom Presenting author email address: libby.sallnow@nhs.net

This session will explore and critique the new concept of 'collective social capital'. This concept extends current understanding of social capital to include reciprocal relationships between healthcare organizations and local communities. How this relates to and challenges our existing understanding of the relationship between communities and institutions will be explored and the novel perspectives this affords for the new public health approach and end of life care in general will be evaluated.
ME 13 New Findings on the Status of Palliative Care Globally from the 2nd Edition of the WHPCA/WHO Global Atlas of Palliative Care (2018)

Preliminary Findings on the Status of Palliative Care Globally from the 2nd Edition of the WHPCA/WHO Global Atlas of Palliative Care (2019) Connor S. ${ }^{1}$, Varghese C. $^{2}$

${ }^{1}$ Worldwide Hospice Palliative Care Alliance, London, United Kingdom, ${ }^{2}$ World Health Organization, Geneva, Switzerland Presenting author email address: sconnor@thewhpca.org

The second edition of the Global Atlas of Palliative Care, a joint WHPCA/WHO publication, will be released later in 2019. The original edition published in 2014 was the first Atlas to describe the status of palliative care worldwide and offered a number of benchmarks for advocacy purposes. These included a first estimate of the number of adults and children needing palliative care worldwide, a description of the level of palliative care for all countries using a six level schema, and a number of measure of barriers including opioid use, numbers of providers to population, and an estimate of the number of those receiving palliative care. There was also a description of model programs globally and the resources available for palliative care development. The second edition will compare progress in all these areas over the past 5 years and will feature some new information. The recent Lancet Commission estimate of the need for palliative care will be utilized, data from WHO's country capacity surveys will be presented, and some workforce analysis will be included. Preliminary findings from the New Global Atlas will be shared noting areas of progress and areas needing improvement.

ME 14 National Actions to Facilitate International Visibility and Networking in Palliative Care National Actions to Facilitate International Visibility and Networking in Palliative Care

Silove L., Renard C.

Société Française d'Accompagnement et de Soins Palliatifs, Paris, France

Networking and taking action at an international level is not always easy, especially in a foreign language, demanding time and energy. Coordinating, implementing and communicating these actions on a national level can be complex. In 2015, the French national palliative care association, the SFAP, decided to form a national multidisciplinary working group to address some these challenges collectively.

We will share our experience of creating our International Relations Working Group (IRG) and setting it in motion. We will talk about how, though this joint national venture, we move forward together in a dynamic and powerful way, how we are inspired from abroad, and the impact of our work. We will also discuss the barriers and unexpected opportunities we have encountered and what adjustments we have made along the way. Collaborating in this way is exciting and stimulating, and it is a pleasure to work with a group of motivated colleagues. Our model is an evolving one, we are learning on the way and readjust as required. It is one that can be replicated, but also adapted to different cultures. On a practical level we will be offering support and working with participants who are interested in setting up a similar initiative in their own countries. We hope that delegates will leave with an understanding of why and how we formed the IRG, and how we work. We hope they will be inspired by our initiative and part with ideas, and be motivated, to make changes in their own countries.

ME 15 What Do the Arts Bring to Palliative Care? A Creative Response - What Do the Arts Bring to End of Life Care? Hartley N. ${ }^{1}$, Filbert M. ${ }^{2}$

${ }^{1}$ Mountbatten, Newport, United Kingdom, ${ }^{2}$ Institut de Cancérologie des HC, Lyon, France Presenting author email address: nigel.hartley@ mountbatten.org.uk 
An overview of the literature and research re the development of the arts as used in end of Life Care. Also there will be an emphasis on the benefits that artists bring to senior clinical and management roles in palliative care, focusing on the benefits of when artists become leaders and how the professional discipline of a leader in End of Life Care can bring something unique and powerful to strategic thinking and development. Goodhead A \& Hartley N 2018 Spirituality in Hospice Care Publ. Jessica Kingsely publications

Hartley N. 2013 'End of life Care - A guide for Artists, Therapists and Arts Therapists Publ. Jessica Kingsley Publications, London

Hartley N \& Payne M 2008 'the Creative Arts in Palliative Care Publ. jessica Kingsely publications, London

\section{ME 16 Practical Aspects of Spirituality} Recognising and Exploring Spiritual Issues into Daily Palliative Care Practice

Engels $Y_{.}{ }^{1}$, Boyd $K^{2}{ }^{2}$, van de Geer $\mathrm{J}^{3}$

${ }^{1}$ Radboud University Medical Center, Anesthesiology, Pain, Palliative Medicine, Nijmegen, Netherlands, 'University of Edinburgh, Anesthesiology, Pain and Palliative Medicine, Edinburgh, Netherlands, ${ }^{3}$ Agora, Bunnik, Netherlands Presenting author email address: yvonne. engels@radboudumc.nl

Background: People living with a progressive, life limiting illness, their family members and close friends are all confronted with spiritual issues as they try to find meaning and retain hope. The WHO definition of palliative care says that spiritual care should be proactively integrated into daily palliative care. However, many clinicians (GPs, hospital specialists, nurses, and other health and care professionals have had little training in how to recognise or explore existential issues.

During this meet the expert session, we will invite two members of the EAPC taskforce on spiritual care to give a short presentation where they share the way they integrate active exploration of spiritual issues into daily palliative care. The first, Dr Joep van de Geer (the Netherlands) is currently leading a nationwide initiative of the Ministry of Health to increase spiritual care in primary care. He worked as a chaplain in a hospital and did his PhD on "Learning spiritual care in Dutch hospitals". He was also member of the Dutch committee on spiritual care, which recently released updated Guidelines aimed at health care professionals.

After both presentations, we will have a discussion with the audience.

\section{Free Communications}

FC 1 Older People: Dementia and Frailty Near the End of Life Frequent Emergency Department Attendance among Older People in the Last Three Months of Life and Associated Factors Bone A.E. ${ }^{1}$, Evans C.J. ${ }^{1,2}$, Henson L.A. ${ }^{1}$, Gao W. ${ }^{1}$, Higginson I.J. ${ }^{1}$ ${ }^{1}$ Cicely Saunders Institute of Palliative Care, Policy and Rehabilitation, King's College London, London, United Kingdom, ${ }^{2}$ Sussex Community NHS Foundation Trust, Brighton, United Kingdom

Background: Frequent visits to the emergency department (ED) towards the end of life disrupt care continuity and are contrary to most peoples' preferences for home-based care. We aimed to determine factors associated with frequent $(3+)$ ED attendances for older people in the last three months of life.

Methods: Mortality follow-back survey investigating healthcare at the end of life using pooled data from two studies in rural and urban areas of England. Respondents were the next of kin of people aged $65+$ who died four to ten months previously. We used multivariable modified Poisson regression to examine factors associated with $3+$ ED visits in the last three months of life.

Results: Of the 688 respondents, most were sons/daughters ( $n=416$, $60.5 \%$ ) or spouses $(n=150,21.8 \%)$. Mean age at death was 85 years,
$36.5 \%$ had a primary diagnosis of cancer $(n=251), 26.2 \%$ cardiovascular $(n=180)$ and $16.3 \%$ respiratory disease $(n=112) .12 \%$ of decedents $(n=80)$ experienced $3+$ ED visits in the last three months of life, accounting for $43 \%$ of all ED visits. In an adjusted model, respiratory disease and $2+$ comorbidities were associated with frequent ED attendance (prevalence ratio (PR) 2.12, 95\% Cl 1.21-3.71 (reference cancer); PR 1.81, 95\% Cl 1.07-3.06, (ref 0 comorbidities), respectively). Those with $7+$ community nursing contacts were more likely to have $3+$ ED visits (PR 2.65, 95\% $\mathrm{Cl}$ 1.49-4.72, ref 0 contacts). Risk of frequent ED visits was reduced among those who identified a key health professional (PR 0.58, 95\% Cl 0.37-0.88)

Conclusion: Assignment of a key health professional to older people at increased risk of frequent end of life ED visits, e.g. those with respiratory disease and/or multiple comorbidities, may reduce repeat ED visits and improve care quality. Our unexpected finding that greater number of community nursing contacts is associated with increased risk of frequent ED visits warrants further investigation.

Funding: Cicely Saunders International;The Atlantic Philanthropies

\section{FC01 Older People: Dementia and Frailty Near the End of Life} Making Decisions on Behalf of Someone with Dementia at the End of Life

Davies N. ${ }^{1,2}$, Rait G. ${ }^{1}$, Sampson E.L. ${ }^{2,3}$

${ }^{1}$ Department of Primary Care and Population Health, University College London, London, United Kingdom, ${ }^{2}$ Marie Curie Palliative Care Research Department, University College London, Lodnon, United Kingdom, ${ }^{3}$ Barnet Enfield and Haringey Mental Health Trust Liaison Team, North Middlesex University Hospital, London, United Kingdom

Background: There are a range of symptoms and challenges at the end of life which someone with dementia and their family may face. Despite attempts to increase advance care planning, many people with dementia reach the end of life without a plan. This means decisions are often left to family caregivers.

Aim: The aims of this study were

1) Explore what significant decisions family caregivers need to make when caring for someone with dementia at the end of life;

2) Understand how family caregivers make these decisions. This will inform the development of a decision aid to support family caregivers at the end of life.

Methods: Semi-structured interviews were conducted with family caregivers $(n=16)$ of someone with dementia at the end of life. Participants were recruited through general practice, memory services and an online research register. Interviews were analysed using thematic analysis.

Results: Six main decisions were discussed by caregivers:

1) the best place of care and when was the right time to move;

2) managing agitation;

3) managing nutrition and hydration;

4) how to ensure the person is washed and bathed;

5) how do I know if something is wrong;

6) when to stop treatment or interventions.

These decisions were broken down into a series of stages, with considerations at each stage. Each decision will be presented as a diagram. Caregivers discussed balancing the impact on their life with the effect on the person with dementia. Caregivers were often managing difficult family dynamics with conflicting views, or a lack of input from some members which impacted their decision making processes.

Conclusion: The findings from this study are informing the development of a decision aid to support family caregivers of people with dementia at 
the end of life. Caregivers often make a series of decisions which they are unaware of. This study has highlighted the unconscious decision processes and considerations caregivers make when making these decisions.

FC01 Older People: Dementia and Frailty Near the End of Life Medical Cannabinoids in Patients with Severe Dementia and Neuropsychiatric Symptoms: A Feasability Study

Pautex S. ${ }^{1}$, De Saussure C. ${ }^{2}$, Langlois A. ${ }^{2}$, Wampfler J. ${ }^{2}$, Revol A. ${ }^{2,3}$, $\overline{\text { Broers } B .{ }^{4}}$

${ }^{1}$ Rehabilitation and Geriatrics, Division of Palliative Medicine, Collonge-Bellerive, Switzerland, ${ }^{2} E M S$ les Tilleuls, Geneva, Switzerland, ${ }^{3}$ University of Geneva, Geneva, Switzerland, ${ }^{4}$ Division of Primary Care, Geneva, Switzerland

Most patients with severe dementia will experience neuropsychiatric symptoms over the course of their disease. Pharmacologic treatment is challenging, as currently available medications have important drawbacks concerning the benefit-to-risk ratio. $d$ might therefore serve as an alternative pharmacologic treatment.

Method: This study was a feasibility study. Patients with severe dementia and neuropsychiatric symptoms refractory to available medications were recruited in one in long term care facility after informed consent signed by a proxy. Tincture of Cannabis sativa prepared by a pharmacist containing $5 \%$ THC and CBD was introduced in all patients

Results: 10 patients $(79.5 \pm 41.1$ years) with severe dementia were included. Initial dosage was 8 drops 3 times/day (approximately $7 \mathrm{mg}$ of $\mathrm{THC} /$ day. Doses were increased in all patients according to the positive effect, with a maximal dosage of $15 \mathrm{mg}$ of THC / day. Because of mouth irritation tincture was replaced by oil in all patients. No other side were noted. Effect of the introduction of treatment at 2 weeks and one month are summarized at inclusion / 2 weeks/ 1 month

- $\quad$ Cohen-Mansfield Agitation index mean (SD) 74.5 (30.1)/62.2 (17.1)/ 62.3 (22.7)

- $\quad$ Neuropsychaitric Index mean (SD) 71.1 (32.5)/ 53.1 (21.4)/ 43.1 (21.2)

- $\quad$ Barthel score mean (SD): 13 (19.9)/14.5 (19)/ 9.5 (11.1)

- $\quad$ Rigidity unified Parkinson disease rating scale mean (SD) 3.4 (0.9)/ $2.7(0.8) / 2.2(0.9)$

Conclusion: We could demonstrate that it's feasible to introduce medical cannabinoids in patients with severe dementia and neuropsychiatric symptoms with improvement of the patients and no significant side effects. The next step s to conduct a randomised study to demonstrate the efficacy.

FC01 Older People: Dementia and Frailty Near the End of Life An Innovative Intervention Using the $\mathrm{PARO}^{\circledR}$ Robot for the Management of Acute Pain in Advanced Dementia

Demange M., Pino M., Charlieux B., Kerhervé H., Rigaud A.-S., Cantegreil-Kallen I.

Broca Hospital, Assistance Publique-Hôpitaux de Paris, Paris, France

Background: Pharmacological intervention is considered a first-line treatment for pain in persons with advanced dementia (PwAD), in spite of the known side effects. Research on older adults supports the hypothesis that non-pharmacological interventions, using the distraction method, have a positive impact on pain modulation (eg music therapy, audiovisual stimuli). However, to date, little is known on the effect of this method on acute pain in PwAD.

Aims: This study examined the effect of the use of a social robot (PARO ${ }^{\circ}$ ), as a distracting and pleasant stimulus, during painful care situations in PWAD, as well as its impact on analgesic medication and anxiety related to care.
Method: A crossover randomized clinical trial was conducted among 53 PWAD allocated to two groups. One group received a 3-weeks PARO mediated intervention during painful care situations, followed by a 3-weeks control period not involving the robot. The second group received the same intervention conditions in the opposite order. A 1-week washout period was applied between the two intervention phases of the study. The use of pain medication was evaluated at baseline and at the end of each intervention phases. Pain and anxiety were assessed during painful care with the ALGOPLUS ${ }^{\infty}$ and the COVY ${ }^{\star}$ scales respectively.

Results: Behavioral manifestations of pain were significantly lower in the PARO -mediated intervention period compared to the control period $(p<.0001)$. Results also showed a significant positive correlation between acute pain and anxiety in patients during painful care situations. Finally, there were no significant differences regarding analgesic consummation between the robotic intervention and the control periods.

Conclusion: $\mathrm{PARO}^{\circ}$ robot seems to provide an effective therapeutic approach, as a complement to the medication, to the needs of pain management in PwAD.

Source of funding: This research was supported by the Paul Bennetot Foundation and by the "Fondation de France"

FC01 Older People: Dementia and Frailty Near the End of Life What Is Valued Most in End-of-Life Care for People with Dementia? A Qualitative Exploration of their Loved Ones' Experiences Bolt S.R. ${ }^{1}$, van der Steen J.T. ${ }^{2,3}$, Schols J.M.G.A. ${ }^{1}$, Zwakhalen S.M.G. ${ }^{1}$, Meijers J.M.M. ${ }^{1}$

${ }^{1}$ Department of Health Services Research, Maastricht University, Maastricht, Netherlands, ${ }^{2}$ Department of Public Health and Primary Care, Leiden University Medical Center, Leiden, Netherlands, ${ }^{3}$ Department of Primary and Community Care, Radboud University Medical Center, Nijmegen, Netherlands

Background: Palliative care for older people with dementia is often suboptimal. Understanding the experiences with care of loved ones of people with dementia at the end of life may help improve palliative care. Moreover, loved ones' experiences may relate to grief. Needs and experiences may differ between nursing home and home settings and thus require different approaches.

Aims: To investigate loved ones' experiences with end-of-life care for people with dementia, and compare experiences between the nursing home and home setting.

Methods: This qualitative study adopted a critical realist approach. We conducted semi-structured, in-depth interviews with a purposive, criterion-based sample. We performed thematic analysis using Braun \& Clarke's paradigm. We interviewed 32 individuals bereaved (in the past year) of someone with dementia who received nursing home or home care from one of three collaborating long-term care organizations in the province of South Limburg in the Netherlands.

Results: Experiences with end-of-life care translated into four themes: acknowledging human dignity; being recognized as an important caregiver; (not) talking about death and dying; making decisions -together. Overall, a personal approach was valued and positive experiences prevailed. Person-centeredness was sometimes missed in the nursing home. Loved ones at home took on a more prominent position within the care team and were more appreciative of their caregiving role compared to the nursing home. End-of-life communication and decisionmaking brought forth similar challenges in both settings.

Conclusion/ discussion: Nursing home and home care professionals should inform and support loved ones in decision-making. Loved ones of people with dementia at the end of life primarily valued authentic and compassionate attention from professional caregivers. We suggest that nursing homes may learn from home care practice to endorse personcenteredness and establish care relationships. 
FC01 Older People: Dementia and Frailty Near the End of Life What Are the Palliative Care Needs of Older People with Frailty near to the End of Life, and Can Primary Care Identify when to Help? Stow D., Matthews F., Hanratty B.

Institute of Health and Society, Newcastle University, Newcastle upon Tyne, United Kingdom

Background: Frailty increases a person's vulnerability to poor health outcomes and death. An increasing number of people now live with frailty but they are among the least likely to access palliative care services. Knowing what services to provide and when, are two key challenges to improving access. In the UK, GPs must now identify patients with severe frailty. In most cases this will be done using the electronic frailty index (eFI), a 36-item cumulative deficit frailty score, derived from electronic health records (EHRs). The use of the eFI to predict mortality in individuals is unclear.

Aims: Synthesise the evidence on palliative care needs of older people with frailty and investigate if the eFI could support clinical judgements about when someone is nearing end of life and may require palliative care.

Methods:

1) Systematic review of literature on people with frailty nearing the end of life, to identify the potential needs of this group.

2) Longitudinal analysis of 26,298 EHRs in England using monthly eFI scores calculated over one year. Frailty trajectories were examined, and discrimination methods were used to identify eFl items best suited for mortality prediction.

Results: 20 articles were identified, reporting on 18,698 people with frailty. A range of physical and psychosocial needs were identified, including evidence that people with frailty are likely to experience pain, be functionally impaired and require support with activities of daily living. Analysis of EHRs identified three distinct trajectories of frailty. Rapidly rising frailty was associated with a $180 \%$ increase in mortality over a stable trajectory. We report our findings on the efl items with the best discrimination for mortality.

Discussion: Observing rapid change in frailty could be used to support clinical judgement about where someone is at a higher risk of dying. Primary care services are well placed to identify and meet many of the needs of people with frailty.

Funding: NIHR SPCR

FC 2 Patient-Reported Outcome Measures (PROMs) and Routinely Collected Data

Palliative Care Outcome Measures and Patient Choice. How Do they Influence Place of Death?

Edwards J. ${ }^{1}$, Moody J. ${ }^{2}$, Hooper D. ${ }^{1}$, Kneen K. ${ }^{1}$, Draper K. ${ }^{2}$, Connolly J. ${ }^{3}$, Eagar K. ${ }^{3}$, Saurman E. ${ }^{4}$, Blackburn P. ${ }^{3}$, Allongham S. ${ }^{3}$

${ }^{1}$ Palliative Care Service, Sydney Adventist Hopsital, Wahroonga, Australia, ${ }^{2}$ Eastern Palliative Care Association Inc, Mitcham, Australia, ${ }^{3}$ Palliative Care Outcomes Collaboration, University of Wollongong, Wollongong, Australia, ${ }^{4}$ Broken Hill University Department of Rural Health, University of Sydney, Broken Hill, Australia

Background: Evidence suggests that preference for home death may not be as high as thought. Patient choice and preference for place of care/ death are integral to patient-centered palliative care. However, patient choice is dynamic, may change over time, and affects the experience of dying.

Aims: This study examines the place of death for palliative care patients, the proportion who die in their place of choice and any preference changes over time. Relationship between preferred place, routine clinical assessments, and patient reported symptoms is examined too.

Methods: This is a retrospective, longitudinal study linking preferred place of care/death with routinely collected patient outcome data.
Cohort is all patients ( $n=2735$ ) from two Australian community palliative care services from 01-08-2016-31-08-2018.

Descriptive statistics will report proportions of patients' place of death, preferred place, and change in choice. Logistic regression investigates the relationship between patient reported pain and symptom distress, socioeconomic status, level of functional dependence, preferred place, and preference changes as they near death.

Results: Preliminary data from one site shows that $86 \%$ of patients died in their place of choice. When asked for the last time, $44 \%$ reported a choice to die in hospital, $28 \%$ at home, and $27 \%$ at a residential aged care facility. When asked more than once about their preferred place, 1 in $3 \mathrm{had}$ a preference change while receiving palliative care. Findings on the relationship between preferences and outcome measures will be presented at EAPC 2019.

Conclusion: It is possible to support patients to die in their preferred place. Patient-centred care demands regular conversation about preferences because choices change with time. Results of outcome measures and preferences will be presented. Study results have implications for clinical practice, service planning, policy, and national outcomes programs.

\section{FC 2 Patient-Reported Outcome Measures (PROMs) and} Routinely Collected Data

Electronic Palliative Care Coordination Systems (EPaCCS): A Systematic Review of the State of the Science

Leniz J. ${ }^{1}$, Weil A. ${ }^{1,2}$, Higginson I.J. ${ }^{1}$, Sleeman K.E. ${ }^{1}$

${ }^{1}$ Cicely Saunders Institute of Palliative Care, Polity and Rehabilitation, King's College London, London, United Kingdom, ${ }^{2}$ North London Hospice, London, United Kingdom

Introduction: Electronic Palliative Care Coordination Systems (EPaCCS) are web-based electronic registers that facilitate documentation and sharing of information about patients' preferences and plans for care, and have been promoted by the UK Department of Health. Concerns have been raised around the evidence base for EPaCCS. Our aim was to systematically search, evaluate and report the state of the science of EPaCCS.

Methods: We searched on CINAHL, MEDLINE, Embase and The Cochrane Library for articles evaluating or discussing UK-based electronic systems to facilitate sharing of information about advance care plans. Two independent review authors screened full-text articles for inclusion, assessed quality and extracted data. Articles were evaluated according to their position in a hierarchy of evidence.

Results: In total, 26 articles were included. 11(42.3\%) were 'expert opinion' articles (editorials or commentaries), 11(42.3\%) were quantitative studies, $3(11.5 \%)$ were qualitative studies and one was a $(3.8 \%)$ mixedmethod study. All quantitative studies had an observational design, and two included control groups. Quantitative studies reported associations between use of EPaCCS with hospital utilisation and advance care planning documentation. Qualitative studies were mainly focused on challenges of EPaCCS design and implementation.

Discussion: Much of the current scientific literature on EPaCCS comprises expert opinion, and there is an absence of any experimental studies evaluating the impact of EPaCCS on end-of-life outcomes. Given the current drive for national roll out of EPaCCS by 2020, it is essential that rigorous evaluation of EPaCCS is prioritised.

\section{FC 2 Patient-Reported Outcome Measures (PROMs) and} Routinely Collected Data

Assessing Quality of Life in People Receiving Palliative Care: Empirical Comparison of Four Questionnaires (EORTC QLQ-C15-PAL, FACT-G7, FACIT-Pal, FACIT-Pal-14)

McCaffrey N. 1,2, Agar M.3, 3,5, Currow D. 2,3,6, Hardy J. ${ }^{7}$, Fazekas B. ${ }^{2}$, King M. ${ }^{8}$

${ }^{1}$ Deakin Health Economics, Deakin University, Burwood, Australia, ${ }^{2}$ Palliative \& Supportive Care, Flinders University, Adelaide, Australia, 
IIMPACCT, Faculty of Health, University of Technology Sydney, Sydney, Australia, ${ }^{4}$ SW Sydney Clinical School, University of NSW, Sydney, Australia, ${ }^{5}$ Ingham Insitute for Applied Medical Research, Sydney, Australia, ${ }^{6}$ Wolfson Palliative Care Research Centre, Hull York Medical School, University of Hull, Hull, United Kingdom, ${ }^{7}$ Dept Palliative \& Supportive Care, Mater Health Services, Mater Research, University of Queensland, Brisbane, Australia, ${ }^{8}$ School of Psychology \& Medical School, University of Sydney, Sydney, Australia

Background: Health-related quality of life (HrQOL), typically assessed with a self-reported questionnaire, or 'patient-reported outcome measure' (PROM), is a key outcome in palliative care research. Despite recommendations for head-to-head PROM comparisons, there are no published international data to guide choice.

Aims: The aim of this study was to empirically compare the reliability, validity and responsiveness of four PROMS: EORTC QLQ-C15-PAL, FACT-G7, FACIT-Pal and FACIT-Pal-14.

Methods: Secondary analysis of data from two Phase III randomised trials was conducted. Sub-groups were defined by Australia-modified Karnofsky Performance Status (AKPS) and global impression of change (GIC). Reliability, construct validity and responsiveness were evaluated using: internal consistency (Cronbach alpha, $\alpha$ ) and test-retest reliability (intra-class correlation coefficient, ICC) in participants with stable AKPS and 'no change' on GIC; a priori hypotheses about sensitivity of PROM scores to AKPS groups; and comparison of PROM change scores with GIC groups using analysis of variance.

Results: FACIT-Pal had better internal consistency ( $\alpha$ ranged 0.59-0.80, $15 / 18 \geqslant 0.70)$ than QLQ-C15-PAL $(0.51-0.85,4 / 8 \geqslant 0.70)$ and FACT-G7 $(0.54-0.64,0 / 2 \geqslant 0.70)$. FACIT scales had better test-retest reliability (FACIT-Pal $11 / 27$ ICCs $\geqslant 0.70$, FACT-G7 $2 / 3$ ICCs $\geqslant 0.70$ ) than QLQ-C15-PAL $(2 / 30$ ICCs $\geqslant 0.70,18 / 30 \leqslant 0.5)$. Four scales demonstrated sensitivity to AKPS: QLQ-PAL-15 Physical Functioning and Global QOL, FACT-G Functional Well-being and FACIT-Pal Trial Outcome Index (TOI). Nine scales demonstrated responsiveness: QLQ-C15-PAL Pain and Fatigue; FACIT-Pal Palcare, TOI and Total; FACT-G Physical Well-being and Total; FACIT-Pal-14; and FACT-G7.

Conclusions: No PROM was clearly superior, confirming that choosing the best PROM requires careful consideration of the research goals, patient population and the domains of HrQOL targeted by the research.

\section{FC 2 Patient-Reported Outcome Measures (PROMs) and Routinely Collected Data}

The Internal Structure of the Integrated Palliative Care Outcome Scale (IPOS): Evidence for a General Palliative Care Factor in Addition to Symptoms, Emotional Well-being and Quality of Care as Domains of Palliative Care

Ramsenthaler C. 1,2, Davies J.M.2, Higginson I.J.2, Murtagh F.M.2,3, Bausewein $\mathrm{C}^{1}$

${ }^{1}$ Department of Palliative Medicine, University Hospital Munich, Munich, Germany, ${ }^{2}$ Department of Palliative care, Policy and Rehabilitation, King's College London / Cicely Saunders Institute, London, United Kingdom, ${ }^{3}$ Wolfson Palliative Care Research Centre, University of Hull, Hull, United Kingdom

Background: The Integrated Palliative care Outcome Scale (IPOS) is one of the main outcome measures in palliative care (PC) with good psychometric properties.

Aim: To further its use for clinical and research purposes, the aim of this study is to validate a summary score and to determine which domains are most central to PC assessment.

Methods: The IPOS was used in two routine samples comprising inpatient PC, hospital PC teams and outpatient services

(a) at a German university hospital ( $n=1,881$ patient and staff self-reports), and (b) at two hospital trusts and a hospice in the United Kingdom ( $\mathrm{n}=$ 6,137) from January 2016 to August 2018.

In confirmatory factor analyses six pre-specified conceptual models of subscales were tested. The best fitting model was used to investigate the reliability of the sum score using omega hierarchical coefficients. Multiple-indicator multiple-causes (MIMIC) models were used to explore construct validity of the sum score with clinical assessments (Australiamodified Karnofsky Performance Status, phase of illness and subsequent mortality status).

Results: Both the bi-factor and second-order models showed acceptable fit. The fit indices were consistent in cancer and non-cancer samples. After exploring local areas of poor fit of the models in the German sample, the model with the single higher order factor $\left(X^{2}=1095.7, \mathrm{df}=\right.$ 102, RMSEA $=0.048[90 \% \mathrm{Cl}: 0.046-0.051], \mathrm{CFI}=0.974, \mathrm{TLI}=0.965$ ) was used for deriving the sum score. Reliability for this sum score was excellent $(\omega>0.8)$. Based on weightings and MIMIC models, the IPOS sum score and the domain scores showed a differential pattern of relationships to performance status and phase of illness in cancer and noncancer patients.

Conclusions: Our results empirically support a measurement model of the IPOS producing a summary score in addition to the subscale scores. Its responsiveness and minimal important difference as a primary outcome should be investigated next.

\section{FC 2 Patient-Reported Outcome Measures (PROMs) and Routinely Collected Data}

How Many Patient Deaths Has our Team to Cope with - 7 Years Analysis of Routine Data from a Palliative Care Unit in a Tertiary Centre

Ostgathe C. ${ }^{1}$, Müller M. ${ }^{2}$, Herget I. ${ }^{3}$, Oderbein S. ${ }^{1}$, Bieberstein $\mathrm{H}^{3}{ }^{3}$, Klein $\mathrm{C}^{3}$

${ }^{1}$ Palliative Medicine, Friedrich-Alexander-Universität Erlangen-

Nürnberg, Erlangen, Germany, ${ }^{2}$ Universität in Bern, Bern, Switzerland, ${ }^{3}$ Universitätsklinikum Erlangen, Erlangen, Germany

Background: Palliative care focusses on patients at their end of life. Hence, death and dying are rather common in specialized services. In a Germany-wide survey in 2008, respondents stated that more than 4 deaths a week are perceived as critical for a team to cope with. Consecutive patient deaths were rated as being significantly more stressful than evenly spread deaths over the week. Detailed information on deaths, death rate, chronological sequence and number of deaths per employee on palliative care units are rather scarce.

Methods: Routine data from 2011 - 2017 including occupancy, time of death, and number of deaths per day were merged with structural data on shifts of nurses and physicians and analysed descriptively using SPSS 21.

Results: In 2557 days under review 2432 patients (pts) were admitted; of these 1504 (61.8\%) died. Mean annual death rate range between 57\%$67.5 \%$. Per week a mean of 4.1 pts died (median 4, range 0-12 per week). In $39 \%$ of the weeks more than 4 pts died. Mean number of deaths per fulltime nurse increased from 15.2 to 18.6 per year. The nightshift (as the longest shift) had the highest number of deaths $(n=614)$. As for physicians, the number of deaths during their presence increased from 57 to 80 pts per year. The day with the highest numbers of deaths is Friday $(n=247)$, lowest is Sundays $(n=185)$. The longest phase with at least one deceased per day was 10 , the longest without 11 days.

Conclusions: Death is an expected outcome on a palliative care unit. Nevertheless, in addition to the case complexity which was not surveyed here, frequency and chronological sequence can have an influence on the strain on employees. The data from our 12-bed palliative care ward show that the number of weeks in which the number of deaths and the sequence was described as acceptable by employees is often exceeded. Above that the death burden per employee increased over time. Extra support for the employees and adequate staffing level could be helpful. 


\section{FC 2 Patient-Reported Outcome Measures (PROMs) and}

Routinely Collected Data

Evaluating the Appropriateness of End-of-Life Care in People with COPD at a Population Level Using Quality Indicators

De Schreye R. ${ }^{1}$, Smets T. ${ }^{1}$, Deliens L. ${ }^{1,2}$, Annemans L. ${ }^{3}$, Gielen B. ${ }^{4}$, Cohen J. ${ }^{1}$

${ }^{1}$ End of Life Care Research Group, Vrije Universiteit Brussel \& Ghent University, Brussels, Belgium, 2Department of Medical Oncology, Ghent University, Ghent, Belgium, ${ }^{3}$ Public Health, Ghent University, Ghent, Belgium, ${ }^{4}$ InterMutualistic Agency, Brussels, Belgium

Introduction: People dying from COPD are best served by end-of-life care focused on symptom treatment and comfort, forgoing aggressive treatment. To evaluate the quality of care they receive, full-population evaluations of the quality of end-of-life COPD care are needed. Using a previously validated set of population-level, COPD-specific quality indicators (QIs) this study aims to:

1) assess appropriateness of end-of-life care in all people dying from COPD in Belgium in 2012 and 2) establish relative standards for appropriate end-of-life care in this population.

Methods: We conducted a retrospective observational study of all deaths from COPD (ICD10-codes J41-J44) in 2012 in Belgium. Seven routinely collected national administrative databases were linked, to calculate a previously validated set of 26 Qls. QI scores were risk-adjusted for comparison between 14 care regions. Based on this comparison, we established the best scoring quartile for each QI as a relative (and realistic) performance standard.

Results: 4,231 people died from COPD in Belgium, 2012. During the last 30 days of life, 60 percent was admitted to hospital, 65.9 percent underwent diagnostic testing, while 11.8 percent received specialized palliative care. A large regional variation was observed and based on the best scoring quartile we could set relative standards, e.g. specialized palliative care use at at least 23.2 percent and diagnostic testing in the last 30 days of life below 54.9 percent.

Conclusion: Our study suggests that end-of-life care in people with COPD could be improved. Particularly the prevalence of diagnostic testing and hospital admissions in the final month of life seem relatively high and the proportions receiving specialized palliative care low. Moreover, the high risk-adjusted variation between regions suggests that quality improvement of end-of-life care in COPD is realistic and achievable. The identified relative standards can be set as goals for endof-life COPD care.

FC03 Living with Non-Malignant Conditions Frailty Scoring in Patients with End Stage Renal Failure: Identifier of Declining Health?

Prentice J. ${ }^{1,2,3}$, Hetherington L. 1,2, Findlay M. ${ }^{2}$, Collidge T..$^{2,4}$

${ }^{1}$ The Beatson West of Scotland Cancer Centre, NHS Greater Glasgow and Clyde, Glasgow, United Kingdom, ${ }^{2}$ Scottish Renal Palliative Collaborative Group, Glasgow, United Kingdom, ${ }^{3}$ University of Glasgow, Glasgow, United Kingdom, ${ }^{4}$ The Glasgow Renal \& Transplant Unit, NHS Greater Glasgow and Clyde, Glasgow, United Kingdom

Background: In the end stage renal failure (ESRF) population frailty is associated with early mortality, increased hospitalisations, and significant symptom burden. After identifying a high degree of frailty in patients withdrawing from dialysis in our population we examined the use of formal frailty scoring and its use in identifying deteriorating patients on renal replacement therapy (RRT).

Methods: The Rockwood Clinical Frailty Scale (CFS) is a 9-point scale which enables measurement of frailty it has high inter-rater reliability and correlates well with objective measures of frailty and has been validated in the ESRF population. We introduced routine recording of the
CFS for all ESRF in our region for patients at three monthly intervals and for low clearance patients at the time of RRT education.

Results: A total of 1663 scores (range 1-9) have been recorded in 798 patients. Mean age 63.9 years. The median CFS score was 4 . Of those patients currently undergoing haemodialysis the median CFS score was $4(\mathrm{~N}=533)$. The median score for transplant patients was $3(\mathrm{~N}=39)$; for conservative care, $6(\mathrm{~N}=23)$; and low clearance patients, $4(\mathrm{n}=106)$. Evidence of deterioration in CFS score (last score greater than the first) was present in $50 \%(14 / 28)$ of those who were deceased at follow-up, whereas a deteriorating score was only present in $22.3 \%$ (97/435) of those who remained alive, $p=0.001$. A documented score of 6 or greater was present in $51.4 \%(38 / 74)$ of those who subsequently died vs. $21.7 \%$ $(158 / 727)$ of those who remained alive, $p<0.001$.

Conclusion: In our unit, deterioration in frailty score is associated with death at follow-up. Furthermore, a score 6 or greater at any point is predictive of death at follow-up. Routine monitoring of frailty using the CFS provides a simple tool to identify patients who are deteriorating and at risk of death. High or deteriorating CFS score should trigger clinical review and anticipatory care planning where appropriate.

FC03 Living with Non-Malignant Conditions Referral Practices of Cardiologists to Specialized Palliative Care Bonares M., Mah K., Mclver J., Ross H., Zimmermann C., Wentlandt K. University Health Network, Toronto, Canada

To describe current referral practices of cardiologists to specialized palliative care (SPC) in Canada and define demographic characteristics, practice and opinions associated with referral.

Cardiologists were invited to participate in an anonymous survey assessing referral practices to SPC. Associations between likelihood of referral to SPC and perceptions of these services were analyzed using multiple linear regression.

The response rate was $51 \%$ (551/1082 physicians); $69 \%$ primarily practiced in an academic centre, $21 \%$ at a community hospital, and $10 \%$ in private practice. $84 \%$ reported SPC was available to their inpatients but only $57 \%$ of these had access to services for outpatients. Only $37 \%$ referred terminally ill inpatients always/usually and generally for uncontrolled symptoms or discharge planning late in the disease course. Almost $50 \%$ would refer to SPC earlier if it was named supportive care and only $1 / 3$ felt SPC services had adequate knowledge in titration of cardiac-specific treatments.Predictors of higher referral frequency included comprehensiveness of available services $(p=0.002)$, satisfaction with SPC services $(p<0.0001)$, SPC acceptance of patients on continuous infusions $(p<0.001)$ and believe patients wait longer for PCU placement then cancer patients $(p=0.008)$. Lower predictors of referral frequency included male gender $(p<0.0001)$, practicing at a community hospital $(p=0.026)$, feel patients without symptoms do not need palliative care until the last few weeks of life $(p<0.0001)$, wait on SPC referral due to uncertainty in predicting prognosis $(p=0.004)$, and feel that SPC services prioritize patients with cancer $(p=0.003)$.

Cardiologists referred patients infrequently to SPC; often later in the disease course for patients with uncontrolled symptoms. Availability of comprehensive SPC services, especially those for patients requiring cardiac-specific treatments and outdated understanding of SPC appear to be the main barriers preventing SPC referral.

\section{FC03 Living with Non-Malignant Conditions} Does Use and Timing of Palliative Home Care Have an Effect on Resource Use in the Last Month of Life in COPD? A Full-population Retrospective Study

Scheerens C. ${ }^{1}$, Faes $K^{1}{ }^{1}$, Pype P. ${ }^{1}$, Beernaert $K^{1}{ }^{1}$, Joos G. ${ }^{2,3}$, Derom E. ${ }^{2,3}$, Cohen J.4, Deliens L. ${ }^{1,4}$, Chambaere K. ${ }^{1}$

${ }^{1}$ Department of Public Health and Primary Care, End-of-Life Care Research Group, Ghent University, Ghent, Belgium, 2Internal Diseases, Ghent University, Ghent, Belgium, ${ }^{3}$ Respiratory Diseases, Ghent 
University Hospital, Ghent, Belgium, ${ }^{4}$ Family Medicine and Chronic Care, Vrije Universiteit Brussel \& End-of-Life Care Research Group, Brussel, Belgium

Background: COPD patients often receive extensive resource use, e.g. frequent hospitalisations, close before death. Early palliative home care (PHC) can lower extensive resource use. However, population studies studying this possible association for COPD are lacking.

Aim: To study the effect of use and timing of $\mathrm{PHC}$ on resource use in the last 30 days before death (DBD) for COPD.

Methods: We conducted a population retrospective study of Belgian decedents in 2010-2015 who died of COPD or of cardiovascular diseases with COPD. We calculated odds ratios (OR) for resource use between using $\mathrm{PHC}$ versus not using, controlled for age, education, comorbidities, income, year of death, home care with a PHC character given by a community nurse.

Results: 1751 patients (2.3\% of all COPD cases) used PHC, of which 638 (36.4\%) earlier than 30 DBD. Using PHC (versus not using) decreased the OR for hospital (0.38) and intensive care unit (0.16) admission, specialist contacts (0.62), invasive ventilation (IV) (0.13), medical imaging (0.39), RX thorax (0.39) and sedatives (0.50) and increased the OR for home care (3.47), general practitioner contacts(4.66), palliative care unit admission(2.83), non-IV (3.00), gastric tube (2.33), oxygen(2.48), opioids (4.27)and morphine (5.56) $(p<0.001)$. Using PHC 90-31 DBD (versus not using) decreased the OR for hospital and emergency room admission (both 0.31), RX-thorax and medical imaging (both 0.21) and increased the OR for non-IV(4.29), morphine (5.28) and opioids (3.98) $(p<0.001)$. Conclusion: $\mathrm{PHC}$ use was associated with less extensive resource use in the last 30 DBD for COPD. No clear timing effect was found, although less extensive resource use was mostly seen when using PHC 'late', namely 90-31 DBD. The results suggest that health policy and services should focus on increasing $\mathrm{PHC}$ access for COPD, while research should further explore timing effects of early PHC for COPD.

Funding: (SBO IWT nr. 140009)

FC03 Living with Non-Malignant Conditions

Use of the 'Surprise Question' in Predicting Adverse Outcomes among Frail Older Patients after Hospital Admission

Gaffney L. ${ }^{1,2}$, Judge C. ${ }^{2}$, Morrison L. ${ }^{2}$, Costello M. ${ }^{2}$, Small C. ${ }^{2}$, Robinson S. ${ }^{2}, O^{\prime}$ Caoimh R. ${ }^{1,2}$

${ }^{1}$ Geriatrics, NUI Galway, Galway, Ireland, ${ }^{2}$ Geriatrics, University Hospital Galway, Galway, Ireland

Introduction: The 'surprise question'(SQ)(Would you be surprised if this patient died in the next 12 months?") is considered useful in predicting mortality and the need for palliative care, though recent meta-analysis suggests it has only modest accuracy in predicting death. It is unknown if it has predictive validity for adverse outcomes or if it correlates with frailty on admission.

Methods: Consecutive patients aged $>70$ attending a large Irish university hospital Emergency Department(ED)had the SQ scored by a trained clinician after completing a detailed comprehensive geriatric assessment.Additional measures of frailty including the Clinical Frailty Scale (CFS) and Risk Instrument for Screening in the Community (RISC) mortality sub-score,along with the caregiver burden score (CBS) and Euroqol EQ-5D quality of life (QOL)measure were also recorded.

Results: The SQ was available for 191 patients, median age $79+/-10$, $55 \%$ female.In all, 56/191(29\%)screened SQ positive (i.e. not surprised). There was no statistically significant difference in age (80 versus 79 years, $p=0.06$ ) or gender( $55 \%$ versus $46 \%, p=0.07)$ between SQ positive and negative patients.SQ positive patients were frailer, median CFS 6/9 versus 4/9 $(p<0.001)$ and had lower EQ-5D scores $(p<0.001)$. Correlations between the SQ and CBS ( $r=-0.35)$ and RISC ( $r=-0.68)$ were moderate-strong but poor for measures of frailty and the EQ-5D(all < $0.1)$.While SQ positive patients had significantly reduced survival times
(LogRank $X^{2}=14.5, p<0.001$ ), accuracy in predicting one-year mortality, admission, prolonged LOS and readmission were also poor(area under the curve $<0.65$ )

Conclusion: A large proportion aged $>70$ attending ED,approximately one-third,screened positive on the SQ.These were significantly frailer with lower QOL.The SQ correlated with caregiver burden but not with measures of frailty or QOL.As with mortality,the predictive accuracy of the SQ for healthcare system-important outcomes appears poor.

FC03 Living with Non-Malignant Conditions Patient-Empowerment Programme' Improved Depression, Anxiety and 6minutes Walking Test in Advanced Heart Failure Patients - a Single Centre Randomised Control Trial Zainal Abidin M.Z., Katiman D., Zainal Abidin H.A., Isa R., Ahmad Ainuddin $\mathrm{H}$. Internal Medicine, Faculty of Medicine, Universiti Teknologi MARA, Sungai Buloh, Malaysia

Introduction: Progressive Muscle Relaxation Therapy (PMRT) and Energy conservation technique (ECT) has been used among chronic illness patients to reduce symptoms burden and improve quality of life. However, data regarding these therapies among advanced heart failure patients remain limited. This study aimed to evaluate the effects of PMRT and ECT on functional capacity, symptoms assessment scores and quality of life amongst advanced heart failure patients.

Methods: This was a 12-week, two-arm, parallel, non-blinded, randomized control trial in a single centre. The study compared 42 patients receiving standard care (SC) against 48 patients receiving "PatientEmpowerment Programme (PEP)" (SC+PMRT+ECT). 90 advanced heart failure patients were enrolled. The intervention group received two PEP reinforcement sessions four weeks apart. Patients were asked to implement the programme at least three times a week at home, using an audiovisual aid. Assessment tools comprised of questionnaires on demographics, 6-minute walking test (6MWT), New York Heart Association (NYHA) classifications, Revised version of the Edmonton Symptoms Assessment System (ESAS-r), Brief Fatigue Inventory (BFI), New York Heart Association (NYHA) Classification and McGill Quality-of-Life Questionnaire (MQOL-R) administered on recruitment and upon study completion.

Result: 76 (84.4\%) were male. Mean age was 59.93 years $(+11.16)$ and majority was from the lower income group. There was a $11.99 \mathrm{~m}(95 \% \mathrm{Cl}$ : $5.99,15.98)(p<0.05)$ improvement in the 6MWT between intervention and standard group. All symptoms showed improvement after intervention, however, only the depression and anxiety items were significant. There was improvement in the fatigue scale, fuctional status and quality of life in the intervention group however it is not statistically significant. Conclusion: ECT and PMRT are useful in reducing depression and anxiety scores, and improve the 6MWT distance.

FC03 Living with Non-Malignant Conditions Hypnosis: An Effective Complementary Tool in the Palliative Management of Severe COPD Patients Delignières A. ${ }^{1}$, Anllo H. ${ }^{2,3}$, Bocahu Y. ${ }^{1}$, Brefort S. ${ }^{1}$, Haniez $F^{1}{ }^{1}$, Herer B. ${ }^{1}$, Jaillet H. ${ }^{1}$, Mach V. ${ }^{1}$, Pytlak C. ${ }^{1}$, Saada M. ${ }^{1}$, Secundo I. ${ }^{1}$, Larue F. ${ }^{1}$ ${ }^{1}$ Centre Hospitalier de Bligny, Briis sous Forges, France, ${ }^{2}$ Bligny Hospital, Briis sous Forges, France, ${ }^{3}$ Waseda University, Watanabe Lab, Tokyo, Japan

Purpose and context: Dyspnea in patients with severe COPD is an anxiety-provoking symptom difficult to control. Use of hypnotic complementary therapy in our hospital has consistently improved our patients'. The present work constitutes an attempt at validating this therapeutic benefit in a systematic fashion through a controlled study

Method: 12 male and 7 female, committed COPD patients, were tested in an intra-group design that consisted of two conditions: 
1) hypnosis session $(\mathrm{H})$,

2) an attention exercise mimicking a hypnotic interaction (A).

Both procedures were of identical duration and conducted randomly at 2 different days, within a 24 to 72 hour window. Both conditions were controlled for patient expectation. Data was collected before and after each session. Data related to respiratory quality included Respiratory Rate (RR), Oxygen Saturation in blood (SaO2) and Borg score (self-assessment of dyspnea). The anxiety component of dyspnea was also assessed with the 6-item State Trait Anxiety Inventory (STAI-6)

Results and discussion: Patient characteristics indicated that they all suffered of severe COPD. The following changes (mean \% of change) were observed in groups $\mathrm{H}$ and $\mathrm{A}$, respectively: $\mathrm{RR}=-10.4$ vs +3.0 (effect of $\mathrm{H}>\mathrm{A}$ treatment, $\mathrm{p}<0.05$ ); Borg score $=-37.5$ vs -26.7 (comparable variation of the 2 groups between the beginning and the end of the session, $\mathrm{p}<0.001$ ); $\mathrm{SaO} 2=+2.1$ vs +1.4 (comparable variation of the 2 groups between the beginning and the end of the session, $p<0.001$ ); STAI-6 $=-26.0$ vs -9.0 (effect of $\mathrm{H}>$ A treatment, $\mathrm{p}<0.05$ ). Results, hence, confirm our hypothesis: hypnosis can reduce anxiety and respiratory rates above control

Conclusion: We conclude that hypnosis has a favorable effect on dyspnea for severe COPD patients. This study further underscores the interest of using non-drug complementary therapies in palliative respiratory care and opens the door to other questions, such as what are hypnosis' long-term effects on COPD symptoms.

\section{FC04 Pain, Breathlessness and Other Symptoms} Hospital Care - A Risk Factor for Dying with Unrelieved Pain Klint Å. ${ }^{1}$, Rasmussen B.H. ${ }^{1}$, Bondesson E. ${ }^{2}$, Fürst C.J. ${ }^{1}$, Schelin M.E. ${ }^{1}$ ${ }^{1}$ Institute for Palliative Medicine, Lund University, Lund, Sweden, ${ }^{2}$ Department of Pain Rehabilitation, Lund University/Skåne University Hospital, Lund, Sweden

Background: Fear of dying in pain resonates in most people but despite an increased global focus on palliative care, dying with unrelieved pain is still paramount, also in high-resource countries like Sweden.

Aim: To investigate risk factors for unrelieved pain in a nationwide observational cohort study.

Methods: The unique, population-based Swedish Register for Palliative Care was used, and data from the 161762 expected deaths during 20112015 were analysed. The investigated risk factors include cause of death, place of death, end-of-life conversation and contact with a pain management team. Cause-of-death information was obtained from the Swedish Cause of Death Register. Adjusted log-binomial regression models were fitted to estimate risk ratios (RR) with corresponding $95 \%$ confidence intervals $(\mathrm{Cl})$ for unrelieved pain.

Results: $68 \%$ of the total population reported pain during the last week of life, for $25 \%$ of these the pain remained unrelieved, despite prescription of opioids "as needed" in $97 \%$ of the cases. Significant risk factors for unrelieved pain included hospital death $(R R=1.82$, $95 \%, \mathrm{Cl} 1.77-1.86)$, as compared to dying in specialized palliative care or in a community setting, not having had an end-of-life conversation ( $R R=1.39,95 \%, \mathrm{Cl} 1.36-1.43)$ and cancer/metastases in bones $(R R=1.28,95 \%, \mathrm{Cl} 1.22-1.33)$ and lung $(R R=1.23,95 \%, C l$ 1.19-1.26), as compared to dying from non-cancer causes. Cancer in the brain or liver did not confer any increase risk of dying with unrelieved pain.

Conclusion: Prescription of opioids "as needed" is made to virtually all dying patients, yet unrelieved pain is still prevalent. Health care providers in general, and hospitals in particular need to assure professional competency in order to earlier identify palliative care needs and intervene through adequate pharmacological and non-pharmacological therapy in order to relieve pain at the end of life.

Funding: No external funding
FC04 Pain, Breathlessness and Other Symptoms

Virtual Reality for Symptom Control in Palliative Care - A Pilot Trial Guenther M. ${ }^{1}$, Krueger J. ${ }^{2}$, Hofmeister U. ${ }^{2}$, Lenz . $^{3}$

${ }^{1}$ Department of Palliative Care, University Medicine Muenster, Muenster, Germany, 2Palliativnetz Muenster, Muenster, Germany, ${ }^{3}$ Department of Palliative Care, University Medicine Muenster, Münster, Germany

Background: Life-limiting diseases are often associated with a loss of mobility due to physical decline, isolation by infectiousness or immune deficiency. These limitations of the individual radius lead to an aggravation of various symptoms. Virtual Reality (VR) allows to temporary escape from this isolation and depressing atmospheres. The aim of the study is to evaluate VR for symptom control such as pain in palliative care (PC). Methods: During this on-going observational prospective pilot study patients are offered a VR experience. Patients' and procedural characteristics are collected and pain was evaluated before, during and after the procedure by using a visual analogue scale. Health-related quality of life (HRQOL) is measured by EQ-5D-5L questionnaire (ClinicalTrials.gov Identifier: NCT03698526).

Results: Up to now, 11 patients were enrolled in this study, one patient was excluded because of acute loss of visual acuity. 4 male and 6 female patients participated at an average age of $52 \pm 11$ years (33 - 70). The Karnofsky of $45 \pm 14 \%$ and the self-reported HRQOL of $45 \pm 24$ shows the bad overall condition of the study population. $9 / 10$ patients watched three or more different video experiences with a duration of $32 \pm 17$ min. 8/10 patient suffered from malignant diseases, 2 were isolated and one patient was on an intensive care unit. During the intervention pain was significantly lower compared to the situation before $(18 \pm 25 \mathrm{~mm}$ vs. $34 \pm 35, P=0.039)$. The pain control tends to be effective directly after VR ( $25 \pm 25 \mathrm{~mm}, P=0.098)$, but no significant effect was seen $1 \mathrm{~h}$ after the VR intervention. 8/10 patients saw VR as a valuable experience and all of them would like to use the device again.

Discussion: VR seems to be a modern possibility to reduce pain and other symptoms in PC. The usage of VR in PC has a broad acceptance and might be a valuable tool for the future.

\section{FC04 Pain, Breathlessness and Other Symptoms}

A Novel Integrated Model of Palliative Rehabilitation Significantly Reduces Healthcare Utility and Improves Functional Outcomes in Patients with Chronic Non-malignant Causes of Dyspnea Neo H.Y. ${ }^{1}$, Choo A. ${ }^{2}$, Cheong S.K. ${ }^{2}$, Lee L.T. ${ }^{2}$

1Palliative Medicine, Tan Tock Seng Hospital, Singapore, Singapore, ${ }^{2}$ Ren Ci Hospital, Singapore, Singapore

Background: The Integrated $\underline{\text { Care for }}$ Advanced REspiratory disorders (ICARE) is a novel service that integrates key skillsets from Palliative Care and Rehabilitative Medicine to deliver stay-in, goal-directed palliative rehabilitation for patients with chronic, non-malignant lung diseases. Patients are stepped-down from the tertiary hospital to a community hospital for ICARE, as early as 4 days into their admission. Through weekly multi-disciplinary meetings, ICARE proactively screens and treat 10 selected physical and psychological comorbidities. It also directs evidence-based pharmacologic and non-pharmacologic interventions towards enhancing self-mastery of total dyspnea.

Aim and method: This study prospectively evaluated program efficacy. The primary objective is to compare the Total Length of Stay (TLOS) in tertiary hospitals, 6 months prior to enrolment versus 6 months ensuing discharge from ICARE. Secondary outcomes include increment in 6-minute walk distance (6MWD) and Modified Barthel Index (MBI) at the end of ICARE. Patients who died or had repeated ICARE admissions during the 6 month follow-up and those with unplanned discharges within 7 days of ICARE enrolment were excluded.

Results: 51 admissions fulfilled selection criteria. $>80 \%$ of subjects have COPD. Overall, ICARE reduces median TLOS from 17 to 7 days ( $p<$ 
0.001). Subjects with $\geqslant 3$ admissions in the 6 months prior to ICARE showed the greatest reduction in TLOS (from 27 to 8 days; $p<0.01$ ). Subjects also demonstrated increment in both median 6MWD $(30 \mathrm{~m})$ and MBI (14.5 points). (Both $p<0.001)$. Subjects with poorer 6MWD $(<$ $100 \mathrm{~m})$ and lower $\mathrm{MBI}(<80)$ at point of enrolment exhibited greatest improvement. (Both $\mathrm{p}<0.01$ ).

Conclusion: ICARE significantly reduces tertiary hospital TLOS. It also improves patients' exercise tolerance and their ability to self-care. The frailest cohort exhibited greatest improvement, suggesting significant reversibility of multi-faceted umet needs. Cost efficacy analysis is underway.

\section{FC04 Pain, Breathlessness and Other Symptoms}

Association between Quality of Life (QOL) and Survival Time in 28,264 Danish Cancer Patients.

Data from the Danish Palliative Database

Hansen M.B. ${ }^{1,2}$, Petersen M.A. ${ }^{1}$, Ross L. ${ }^{1}$, Adsersen M. ${ }^{1}$, Rojas-Concha L. ${ }^{1,2}$, Groenvold M.1,2

${ }^{1}$ The Research Unit, Department of Palliative Medicine, Bispebjerg / Frederiksberg Hospital, University of Copenhagen, Copenhagen, Denmark, ${ }^{2}$ Department of Public Health, University of Copenhagen, Copenhagen, Denmark

Background: Large, nationally representative studies of the association between $\mathrm{QOL}$ and survival time in cancer patients in specialized palliative care (SPC) are missing.

Aims: To investigate whether QOL at the time of admission to SPC, may help predict survival, in addition to basic variables (gender, age and cancer diagnosis).

Methods: Data from the Danish Palliative Care Database was used. Cancer patients who died in 2010-2017 and who completed EORTC QLQC15-PAL at admission to SPC were included. Cox regression analyses were performed to test if the basic variables and QOL domains (pain, dyspnea, sleeplessness, appetite loss, constipation, fatigue, nausea/ vomiting, emotional function, physical function and $\mathrm{QOL}$ ) were associated with survival time. Since survival was expected to differ for inpatients at hospice and outpatients in palliative care units (PCUs), the analysis was stratified by type of SPC.

Results: 28,264 patients ( $84 \%$ from PCUs) were included in the study. The gender distribution and average age differed between hospice (56.4\% women, 71.8 years) and PCUs ( $47.2 \%$ women, 68.9 years). For both settings, lung cancer was the most common diagnosis. The regression analyses found shorter survival in men compared to women in hospice (Hazard Ratio= 1.16, $\mathrm{p}<0.0001$ ) and PCUs (HR=1.21, $\mathrm{p}<0.0001)$. Diagnosis was significantly associated with survival time in hospice and PCUs $(p<0.0001)$. Except for constipation and nausea in hospice and overall QOL in both settings, all QOL domains were significantly associated with survival time (all $p<0.05$ ). However, except for physical function in hospice ( $H R=1.13$, for a ten-point decrease in physical function), the effect of the QOL domains on survival time was small.

Conclusion: Gender and cancer diagnosis were associated with survival time. For most QOL domains, a significant, but small, association with survival time were found. Reduced physical function was associated with shorter survival time in hospice patients.

FC04 Pain, Breathlessness and Other Symptoms

Factors Affecting the Wish to Die in Patients with Advanced Disease: A Navarra Study in Two Different Centers

Belar A. ${ }^{1}$, Arantzamendi M. ${ }^{1}$, Santesteban Y. ${ }^{2}$, Martinez M. ${ }^{3}$, López-

Fidalgo J. ${ }^{1}$, Lama M. ${ }^{2}$, Rullán M. ${ }^{1}$, Centeno C. ${ }^{1,4}$

${ }^{1}$ Universidad de Navarra. Instituto Cultura y Sociedad, Pamplona, Spain, ${ }^{2}$ Hospital San Juan de Dios Pamplona, Pamplona, Spain, ${ }^{3}$ Clínica Universidad de Navarra, Pamplona, Spain, ${ }^{4}$ Instituto de Investigación Sanitaria de Navarra (IdiSNA), Pamplona, Spain
Background: Wanting to die may occur with or without a desire to shorten or hasten death. In patients with advanced disease, the increase in life expectancy, changes in value systems and the meaning of the disease, facilitate the expression of wish to die (Wtd). In the Spanish context, the phenomenon of the wish to die has scarcely been studied and we do not even know which factors are associated with a more intense expression.

Aims: We aim

a) to know the prevalence of Wtd in patients with advanced disease

b) to determinate associated factors of $\mathrm{Wtd}$,

c) to study how exploring Wtd affect the expression.

Methods: Cross-sectional study with consecutive patients admitted in two hospitals in Pamplona (Navarra). Data about symptom burden (ESAS-r), anxiety and depression (HADS), demoralization (DS-II-es), perceived self-dignity (PDI) and wish to die (AFFID interview) were collected through two face to face interviews (within 48-72h).

Results: Of 409 patients admitted to the hospital, 213 met the inclusion criteria (52\%), 180 (84\%) accepted participate and 148 (82\%) completed both interviews. Prevalence of Wtd was $20 \%$ in the first interview and $18 \%$ in the second one (no significant difference, $p=0.51$ ). We find significative $(p<0.05)$ moderate correlation of Wtd with demoralization (Spearman rho $=0.43$ ) and significative low correlation with current depression ( $r h o=0.34)$, symptom burden $(r h o=0.33)$, emotional distress (rho $=0.31$ ), less informed patients $(r h o=0.30)$, perception of loss of dignity ( $r$ o $=0.27)$, previous depression $(r h o=0.22$ ).

Conclusion: One of five patient studied express Wtd. Exploring Wtd doesn't increase that feeling in our study. Existential and mood aspects of the patient have been found to be associated with the Wtd.

Founding: Project funded by the Spanish Ministerio de Sanidad y Consumo (Fondo de Investigación Sanitaria), project PI18/01703.

\section{FC04 Pain, Breathlessness and Other Symptoms} The PRESERVE Pilot Study: A Phase 2 Cluster Randomised Waitlist Controlled Trial of a Multi-component Non-pharmacological Intervention to Prevent Delirium in Patients with Advanced Cancer Hosie A. ${ }^{1}$, Phillips J. ${ }^{1}$, Lam L. ${ }^{1}$, Kochovska S. ${ }^{1}$, Brassil M. ${ }^{1}$, Noble B. ${ }^{1}$, Kurrle S. ${ }^{2}$, Cumming A. ${ }^{3}$, Caplan G. ${ }^{4,5}$, Chye R. ${ }^{6}$, Le B. ${ }^{7}$, Ely E.W. ${ }^{8}$, Lawlor P. ${ }^{9}$, Bush S. ${ }^{9}$, Davis J.M. ${ }^{10}$, Lovell M. ${ }^{1,2,11}$, Brown L. ${ }^{1}$, Fazekas B. ${ }^{1}$, Cheah S.L. ${ }^{1}$, Edwards L. ${ }^{1}$, Green A. ${ }^{1}$, Agar M. ${ }^{12}$

${ }^{1}$ IMPACCT (Improving Palliative, Aged and Chronic Care through Clinical Research and Translation), University of Technology Sydney, Sydney, Australia, ${ }^{2}$ Northern Clinical School, University of Sydney, Sydney, Australia, ${ }^{3}$ Australian Commission on Safety and Quality in Health Care, Sydney, Australia, ${ }^{4}$ Geriatric Medicine, Prince of Wales Hospital, Sydney, Australia, 5 University of New South Wales, Sydney, Australia, ${ }^{6}$ Sacred Heart Palliative Care Service, St Vincent's Hospital Sydney, Sydney, Australia, ${ }^{7}$ Royal Melbourne Hospital, Melbourne, Australia, ${ }^{8}$ Center for Critical Illness, Brain Dysfunction \& Survivorship (CIBS), Nashville, United States, ${ }^{9}$ Department of Medicine, University of Ottawa, Ottawa, Canada, ${ }^{10}$ Palliative Care Service, Calvary Health Care Kogarah, Sydney, Australia, ${ }^{11} \mathrm{HammondCare,} \mathrm{Greenwich}$ Hospital, Sydney, Australia, ${ }^{12}$ IMPACCT (Improving Palliative, Aged and Chronic Care through Clinical Research and Translation), University of Technology Sydney, Ultimo, Australia

Aim: To determine if a multicomponent non-pharmacological delirium prevention intervention, adapted from effective interventions in older hospitalised patients, is feasible and acceptable for inpatients with advanced cancer.

Methods: A phase 2 cluster randomised waitlist controlled trial conducted in four Australian palliative care units. All units implemented delirium screening and diagnostic assessment, plus prevention strategies addressing sleep, vision and hearing, eating and drinking, 
orientation, mobility, and family partnership in the intervention phase. The primary outcome was adherence to the intervention; secondary outcomes were rates of interdisciplinary involvement and completion of study measures, delirium incidence and severity, and adverse events. Patient, family, staff and volunteer perspectives were obtained via brief interviews.

Results: Data were collected for 65 adult patients with advanced cancer (20 intervention, 25 control, 20 waitlist intervention). Highest adherence was for eating and drinking, orientation, and sleep strategies; the lowest for family partnership. Overall adherence was higher at waitlist intervention sites, where $28 \%$ of patients received all prevention strategies (and $50 \%$ received any strategy) within at least four domains for at least five of the first seven days of admission. Nursing (67\%) and medical staff (16\%) were most involved, family and volunteers less so $(7 \%$ and $1 \%$, respectively). There was $98 \%$ completion of delirium screening, $77 \%$ completion of diagnostic assessment. Delirium incidence was lower in the intervention (20\%) and waitlist intervention sites (19\%) compared to control (35\%). No adverse events were related to the intervention. Qualitative findings were that the intervention was feasible and acceptable for interview participants $(n=39)$, who also gave insights into refining the intervention. Conclusion: A phase 3 trial of the intervention in inpatients with advanced cancer is feasible.

\section{FC05 Spiritual Care}

Effectiveness of Spiritual Care Using Spiritual Pain Assessment Sheet for Advanced Cancer Patients: A Pilot Non-randomized Controlled Trial

Ichihara K. ${ }^{1}$, Ouchi S. ${ }^{2}$, Okayama S. ${ }^{3}$, Kinoshita F. ${ }^{4}$, Miyashita M. ${ }^{5}$, Morita T.6, Tamura K. ${ }^{1}$

${ }^{1}$ Department of Human Health Sciences, Graduate School of Medicine Kyoto University, Kyoto, Japan, ${ }^{2}$ Department of Nursing, Kyoto University Hospital, Kyoto, Japan, ${ }^{3}$ Department of Nursing, Takarazuka Municipal Hospital, Takarazuka, Japan, ${ }^{4}$ Department of Nursing, Gratia Hospital, Mino, Japan, ${ }^{5}$ Division of Palliative Nursing, Tohoku University Graduate School of Medicine, Health Sciences, Sendai, Japan, ${ }^{6}$ Department of Palliative and Supportive Care and Seirei Hospice, Seirei Mikatahara General Hospital, Hamamatsu, Japan

Background: Spiritual Pain Assessment Sheet (SpiPas) was developed an intervention which can be available in daily practice. The clinical trial has not been performed to clarify whether intervention using the SpiPas was beneficial for patients themselves.

Aims: To obtain preliminary knowledge to design a randomized controlled trial to clarify the effects of spiritual care using SpiPas.

Method: The study was designed as a non-randomized controlled trial. The study took place between January 2015 and July 2015 in a hematology and oncology ward and 2 palliative care units in Japan. Among 54 eligible patients with advanced cancer, 46 patients were recruited ( 24 in the control group vs. 22 in the intervention group). The intervention group received spiritual care using SpiPas and usual care, and the control group received usual care. The primary outcome was the Functional Assessment of Chronic Illness Therapy-Spiritual (FACIT-Sp). The secondary outcomes were the Hospital Anxiety and Depression Scale (HADS), Comprehensive Quality of Life Outcome (CoQoLo) and evaluation items of spiritual care.

Results: A total of $33(72 \%)$ and $23(50 \%)$ patients completed 2-week and 3-week follow-up evaluations, respectively. The intervention group maintained or improved in total scores of the FACIT-Sp, HADS, and CoQoLo 3-week, whereas the control group's scores worsened during. The evaluation items showed tendency to increase opportunities to tell their own spirituality and relief spiritual pain. The differences in the changes during 2 weeks in total scores of FACIT-Sp and HADS were significant ( $95 \%$ confidence intervals, 3.65 to $14.4, p<0.01 ;-11.2$ to -1.09 , $\mathrm{p}=0.02$, respectively). No significant changes were observed in the total score of the CoQoLo.
Conclusion: Spiritual care using the SpiPas might be useful for improving patient spiritual well-being. This controlled clinical trial could be performed and a future clinical trial is promising if outcomes are obtained within 2 weeks.

\section{FC05 Spiritual Care}

Finding the Spiritual Dimension of Palliative Care in Everyday Care Practices: A Study into Ritualized Practices in a Dutch Hospice Setting van der Weegen K. ${ }^{1}$, Timmermann M. ${ }^{2}$, Hoondert M. ${ }^{3}$, van der Heide A. ${ }^{1}$ ${ }^{1}$ Public Health, Erasmus MC, Rotterdam, Netherlands, ${ }^{2}$ Stichting Groenhuysen, Roosendaal, Netherlands, ${ }^{3}$ Culture Studies, Tilburg University, Tilburg, Netherlands

Background: Despite acknowledging the importance of the spiritual dimension of palliative care, many health care professionals find it difficult to deal with spirituality. This study introduces an approach to the spiritual dimension of care that involves a focus on everyday care practices. Beyond their functionality, care practices have the ability to create and express meanings and values through processes of ritualization.

Aims: The aim of this study is to explore how ritualization take place during everyday care activities in an inpatient hospice facility.

Methods: Data were collected during two months of participant observation in a Dutch inpatient hospice facility. In addition, semi-structured interviews were conducted with hospice staff, patients and next of kin. The constant comparative method was used to analyze data.

Results: Ritualized practices were common during everyday care practices at the hospice. Ritualization occurs during activities surrounding meals, bathing, night time and medical interventions. Ritualized practices in this context relate to human connection, trust, ambiance and security. When asked about the true meaning of hospice care, professionals, patients and next of kin indeed mentioned particular moments of significance that we identify as ritualized practices.

Conclusion / Discussion: Through ritualization everyday care practices can become sources of meaning and create opportunities to address the spiritual dimension of palliative care. Creating more awareness among health care professionals that their practices may have a meaning beyond their direct functionality might help health care professionals to further incorporate the spiritual dimension in palliative care practice. Funding for this study was provided by The Netherlands Organisation for Health Research and Development (ZonMw).

\section{FC05 Spiritual Care}

What Do We Mean, when We Address Spiritual Care at the End of Life? An Action Research Study on Spiritual Care from the Perspective of Patients and Personnel at Hospices in Denmark Viftrup D.T. ${ }^{1}$, Madsen L.D. ${ }^{2}$, Horstmann M. ${ }^{3}$, Hvidt N.C. ${ }^{1}$

${ }^{1}$ Research Unit for General Practice, University of Southern Denmark, Odense, Denmark, ${ }^{2}$ Arres $\varnothing$ dal Hospice, Frederiksværk, Denmark, ${ }^{3}$ Hospice Sydfyn, Svendborg, Denmark

Background: Research suggests Danes practice their spirituality and religiosity in private, and therefore tend to have fewer spiritual and religious resources and existential concepts compared to more religious people. However, international and Danish studies have shown correlations between life-threatening disease and intensification of existential and/or spiritual needs and beliefs. A Danish study substantiates how patients at hospices are less secularized and individualized but more open to traditions and religion than the general Danish population. Furthermore, international studies show that spiritual care may increase quality of life for dying patients and patients with life-threatening diseases. Therefore, spiritual care is highly prioritized at hospices in Denmark. However, studies in Denmark have found that due to lack of existential concepts, insecurity marks spiritual care practices and understandings in hospices in Denmark. 
Aim: Of the study is to explore how spiritual care is practiced and understood in hospices in Denmark from the perspective of both patients and personnel.

Method: Applied for studying spiritual care in hospices in this study is action research. Data collection consists of observations as well as semistructured interviews with individuals and groups.

Results: Personnel emphasize how spiritual care should be understood as an individual, relational, embodied, and verbal concept. Patients, on the other hand, emphasize spiritual care as a lived phenomenon centred on needs for: 1) Being meet with attempts of understanding, 2) being treated as a whole person, and 3) Being allowed to contribute.

Funding: The study has received funding from: Jascha fonden, AgeCare - Academy of Geriatric Cancer Research, M. L. Jørgensen og Gunnar Hansens fond, Arresødal Hospice and Hospice Sydfyn.

\section{FC05 Spiritual Care}

Health Care Workers, Patient Spiritual Counselor's Communication Interface to Improve the Quality of Care in Palliative Care: Case Study from Tanzania

Kayange $A_{.}{ }^{1,2}$, Kiwanga $\mathrm{F}^{3}$, Manase $\mathrm{F}^{4}$

${ }^{1}$ Uhuru Medical Centre (UMC), Dar es Salaam, Tanzania, United Republic of, ${ }^{2}$ Christian Leadership University (CLU)-PhD in Theology Candidate, Broadway St. Cheektowaga, United States, ${ }^{3}$ Ocean Road Cancer Institute, Dar es Salaam, Tanzania, United Republic of, ${ }^{4}$ The Community Centre for Preventive Medicine [CCPmedicine Medical Center], Dar es Salaam, Tanzania, United Republic of

Introduction: Communication is an exchange of information done by speaking, writing, or using any other medium such as television, etc. Lack of an effective communication between health care workers, patient and spiritual counselors may deter any efforts implemented to improve quality of care to people living with chronic illness.

Patient description: Patient ND 47 year's male diagnosed with prostate cancer and not started on treatment. He had a history of generalized body malaise, wasted and seemed to be in severe pain. Pre-treatment investigations conducted and while waiting for the results, the patient sought the permission from the health care worker to go and meet the spiritual counselor. Upon meeting the spiritual counselor (Prophet), the patient was asked not to seek further treatment as there was no physical illness to him but rather spiritual. The patient decided not to go back to the hospital. The condition got deteriorated and other family members decided another course of patient management.

Intervention and outcome: Family members met the spiritual counselor whom this time agreed the patient to seek treatment from the hospital. The patient was convinced and admitted; pre-treatment investigations were re-done. The patient started treatment, including pain management. He was again linked to another Spiritual counselor upon request from the patient. The patient's hope regained, health condition improved and pain was controlled.

Conclusion: To ensure the quality of care is maintained, both Spiritual counselors and health care workers need to speak the same language lest the patient get confused and abandon the planned treatment.

\section{FC05 Spiritual Care}

Recognising Spirituality: The Impact of Training on Healthcare Professionals Supporting Patients' Spiritual Needs at End of Life - A Qualitative Study

Groves $K^{1}{ }^{1}$, Jack B. ${ }^{2}, O^{\prime}$ Brien $M .^{2}$, Kinloch $K^{2}{ }^{2}$

1Palliative Medicine, Supportive \& Specialist Palliative Care Services, Southport, Merseyside, United Kingdom, ${ }^{2}$ Evidence Based Practice Research Centre, Edge Hill University, Ormskirk, United Kingdom

Background: Supporting patients spiritually as they approach the endof-life is vital. If spiritual needs are unmet, patients are at increased risk of poorer psychological outcomes, quality of life is diminished, and a reduced sense of spiritual peace ensues. Yet many healthcare professionals feel unprepared to appreciate, assess and tackle patients' spiritual issues.

Cheshire \& Merseyside Palliative \& End of Life Care Network has run the 'Opening the Spiritual Gate' course, across the UK for a number of years, to address this training need. This aims to increase awareness of spiritual and religious needs and facilitate recognition of spiritual distress.

Aim: To explore participant perceptions of spiritual care and the impact of the training on their clinical roles after completing the course.

Methods: A qualitative methodology was adopted, using digitally recorded semi-structured interviews. Purposive sampling of healthcare professionals who had undertaken the course, in either the North West or South West of England between 2015-2017, resulted in 21 participants. Data were subject to thematic analysis.

Results: All participants reported on the value of the course and the impact on their clinical roles, including being better able to recognise when spiritual distress may be evident. Two main themes were identified; recognising spirituality, containing sub-themes of what spirituality means and what matters, and supporting spiritual needs with subthemes of recognition of spiritual distress, communication skills, not having the answers and going beyond the physical.

Conclusions: The course is clearly effective in preparing staff to recognise opportunities to provide individualistic spiritual care. Acknowledging that spiritual care is as important as physical care, and having the skills to address it, is vital for delivery of best holistic care.

Funding: Cheshire and Merseyside Palliative \& End of Life Network Education Strategy Group

\section{FC05 Spiritual Care}

Palliative Caregivers' Spirituality, Views about Spiritual Care and Associations with Spiritual Well-being: A Mixed Methods Study

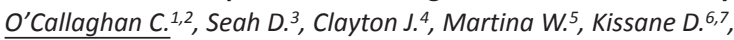
Georgousopoulou E. ${ }^{7}$, Michael N.1,7,8

${ }^{1}$ Palliative and Supportive Care Research Department, Cabrini Health, Melbourne, Australia, ${ }^{2}$ Institute for Ethics and Society, University of Notre Dame, Sydney, Australia, ${ }^{3}$ Sacred Heart Hospice, St Vincent's Hospital Sydney, Sydney, Australia, ${ }^{4}$ Hammond Care, Sydney, Australia, ${ }^{5}$ Cabrini Medical Centre, Melbourne, Australia, ${ }^{6}$ Szalmuk Family PsychoOncology Research Unit, Cabrini Health, Melbourne, Australia, ${ }^{7} \mathrm{School}$ of Medicine, University of Notre Dame, Sydney, Australia, ${ }^{8}$ Faculty of Medicine, Nursing and Health Sciences, Monash University, Melbourne, Australia

Background: Provision of spiritual care is a focus of palliative care as spirituality allows for purpose, meaning and connectedness to the sacred or important. Palliative caregiver spirituality may assist with coping, warranting exploratory inquiries to improve support.

Aims: To examine palliative caregivers' spirituality, religiosity, views on spiritual care and associations with spiritual wellbeing.

Methods: Convergent mixed methods design. Convenience sampling recruited caregivers of patients with $\leqslant 12$ month prognosis from 3 Australian hospitals. A semi-structured anonymous survey examined views about spiritual care, support received and spiritual well-being (FACIT-Sp-12). Data analysis used descriptive statistics, association tests and qualitative content analysis. Significance level adjusted for multiple comparisons ( $n=50$ ) was 0.001 .

Results: 109 surveys were returned (47\% responded). 69\% reported being spiritual, $64 \%$ religious and $23 \%$ neither. Median spiritual wellbeing of caregivers was $30.5\left(1^{\text {st }}, 3^{\text {rd }}\right.$ quartiles: 25.0, 38.0). Following patients' diagnoses, caregivers reported increased meditation $(p=0.002)$, which brought peace and strength. $83 \%$ were comforted by pastoral care visits if received $(n=35)$, with no difference in spiritual wellbeing between recipients and non-recipients $(p=0.53)$. Caregivers with spiritual needs supported to any extent by the hospitals had greater spiritual wellbeing 
than those reporting no support ( $p<0.001) .79 \%$ wrote that hospitals should support caregivers' spiritual needs due to their stress, vulnerability and existential uncertainty. Spiritual care appreciated by caregivers included both staff characteristics and organizational tone.

Conclusion: Vigilance toward caregivers' spiritual distress is needed. Spiritual care, including support, ritual and access to contemplative spaces, needs to be concomitantly extended to caregivers alongside palliative care patients.

Main Funding: Cabrini Foundation

\section{FC06 German Perspectives on Palliative Care}

Extended Evidenced-based National Guideline on Palliative Care for Adult Patients with Incurable Cancer in Germany

Simon S.T. ${ }^{1}$, Voltz R. ${ }^{1}$, Pralong A. ${ }^{1}$, König S. ${ }^{1}$, Radbruch L. ${ }^{2}$, Bausewein

C. ${ }^{3}$, on behalf of the German Guideline Group Palliative Care

${ }^{1}$ Zentrum für Palliativmedizin, Uniklinik Köln, Köln, Germany, ${ }^{2}$ Palliative Care, University Hospital Bonn, Bonn, Germany, ${ }^{3}$ Palliative Care, University Clinic Munich, Munich, Germany

Background: Palliative care aims to maintain the best quality of life for patients with life limiting disease and their carers.

Aim: To improve palliative care for patients with incurable cancer by provision of evidence-based and expert consented recommendations for a brought range of clinical and organizational topics.

Methods: PubMed, Embase, and Cochrane Library databases were systematically searched for relevant publications (guidelines, systematic reviews, primary studies), and assessed as recommended by the Scottish Intercollegiate Guidelines Network. Representatives of 61 professional societies and relevant groups were included to achieve a brought consensus in a structured and defined national process. This guideline had been funded by the German Cancer Aid and supported by the German Guideline Program in Oncology.

Results: The expert panel agreed on 442 recommendations and statements of palliative care principles focused on 15 topics based on best available evidence: Breathlessness, Cancer Pain, Anxiety, Depression, Constipation, Nausea and Vomiting, Sleep Disorders, Fatigue, Malignant Intestinal Obstruction, Malignant Wounds, Communication, Therapeutic Goal and Criteria of Decision-Making, Wish to Hasten Death, Dying Phase, and Organization of Palliative Care. For some topics (e.g. Breathlessness, Pain or Depression) the evidence base is good - however for some topics (e.g. Wish to Hasten Death or Malignant Wounds) more studies are needed.

Conclusion: Patients with incurable cancer need to be offer best and high quality care, including palliative care. There is growing evidence from well-designed studies for further enhancement of palliative care provision. This National Guideline provides high quality recommendations for the best palliative care based on available evidence and a broad national expert consensus.

\section{FC06 German Perspectives on Palliative Care} Last Year of Life in Cologne: What Do Transitions Mean to Patients and Relatives?

Schippel N. ${ }^{1,2}$, Hanke G. ${ }^{1}$, Strupp J. ${ }^{1}$, Rietz C. ${ }^{3}$, Voltz R. ${ }^{1,4,5}$, Cologne

Research and Development Network (CoRe-Net)

${ }^{1}$ Department of Palliative Medicine, University Hospital of Cologne, Cologne, Germany, ${ }^{2}$ Institute for Medical Sociology, Health Services Research and Rehabilitation Science (IMVR), University of Cologne, Cologne, Germany, ${ }^{3}$ Faculty of Social and Educational Sciences, University of Education Heidelberg, Heidelberg, Germany, ${ }^{4}$ Center for Integrated Oncology (CIO), Cologne, Germany, ${ }^{5}$ Medical Faculty, University of Cologne, Center for Health Services Research, Cologne, Germany

Background: The end of life is often characterized by an increase in patient transitions between care settings, which might not improve symptom control or quality of life for patients. There is a lack of valid data about the amount, types, reasons and effects of transitions at the end of life in urban areas in Germany.

Objective: To provide an in-depth insight into reasons for transitions and perceived effects on patients' well-being.

Methods: In a sequential explanatory design, a retrospective survey with 359 bereaved relatives was followed by in-depth interviews ( $N=40)$, which were sampled purposefully by stratifying with pre-defined variables from the questionnaire (length of time of communication of foreseeable death, perceived quality of care).

Results: The most frequent five transitions identified in the survey were hospital to home $(42,6 \%)$, vice versa $(28,8 \%)$, hospital to nursing home $(5,6 \%)$, vice versa $(5,5 \%)$ and hospital to hospital $(5,2 \%)$. For those transitions, the interviews show common reasons, processes and effects. For instance, in positively experienced transitions the cooperation between health services and transparency in communication was highlighted. Major problems in transitions were reported in cases where social service in hospitals was not involved and information flow was therefore poor. When further care planning including next care settings or coordination of services was not provided in advance, patient burden was experienced as particularly high. Perceived burden could be reduced in cases where flexibility in the date of transitions was given. A successful transition from hospital to home was facilitated by systematic inclusion of all necessary out-patient providers at an early stage.

Discussion: By using a mixed-methods approach including the views of bereaved relatives, we were able to show that high burden in transitions for patients and relatives is preventable in many cases.

Funded by the Federal Ministry of Education and Research (BMBF\#01GY1606)

FC06 German Perspectives on Palliative Care Advance Directives and Living Wills in Nursing Home Residents in Germany - A Nursing Staff Prospect

Dempwolff C. ${ }^{1}$, Stanze H.1,2, Jansky M. ${ }^{1,2}$, Hummers E..$^{1,2}$, Nauck $F_{.}^{1,2}$ ${ }^{1}$ Clinic for Palliative Medicine, University Medical Center Goettingen, Goettingen, Germany, ${ }^{2}$ Institute for General Practice, University Medical Center Goettingen, Goettingen, Germany

Background: Since 2009 the German Civil law Code \$1901a includes advance directives (AD). Hereby any person is enabled to compile their specific desire for medical treatment in case one's capacity to decide on their medical treatment is limited. Although nursing staff often reports first to the scene in case of emergency, there is still little understanding of nursing staff's knowledge about ADs and their application.

Aim: The goal of this survey was to obtain a better comprehension of how each nursing employee evaluates his own knowledge background on ADs.

Methods: Anonymous questionnaires containing 15 items derived from literature were distributed among 55 regional nursing homes in a region in Lower Saxony, Germany. Data was analyzed descriptively using SPSS. Results: Analysis of 620 data sets shows that the majority of the nursing staff rated their knowledge about ADs as moderate, despite regarding them as relevant for daily work life. More than 70 percent addressed ADs when new residents moved into the facility. Two thirds considered it very important for them to know their residents' treatment preferences. Furthermore the majority of nursing staff pointed out ADs to emergency physicians. More than half of the nursing staff disagreed with the statement that handling ADs is only limited to physicians. However, employees stated there were scarce educational offerings by their employer addressing ADs specifically.

Conclusion: Many participants are willing to comprehend current issues of their residents regarding ADs. Nursing home staff regards the process of decision making at the end of life as relevant for their daily work. Therefore nursing home administrations should offer educational elements for their employees in the future. Further education may help to 
improve the nursing staff's relevant background knowledge and courses of action for ADs.

FC06 German Perspectives on Palliative Care Palliative Care at the End-of-Life: Who Receives what Type of Care in Germany? - A Claims Data Analysis

Ditscheid B. ${ }^{1}$, Krause M. ${ }^{1}$, Jansky M. ${ }^{2}$, Lehmann T. ${ }^{3}$, L'hoest H. ${ }^{4}$, Maddela S. ${ }^{3}$, Mboulla M. ${ }^{4}$, Meißner W. ${ }^{5}$, Schulz S. ${ }^{1}$, Stichling K. ${ }^{1}$, Vollmar H.C. ${ }^{1,6}$, Wedding U. ${ }^{5}$, Freytag A. ${ }^{1}$, for the SAVOIR Study Group

${ }^{1}$ Institute of General Practice and Family Medicine, Jena University Hospital, Jena, Germany, ${ }^{2}$ Clinic for Palliative Medicine, University Medical Center Göttingen, Göttingen, Germany, ${ }^{3}$ Center for Clinical Studies, Jena University Hospital, Jena, Germany, ${ }^{4}$ Department of Medicine and Health Services Research, BARMER Statutory Health Insurance Fund, Wuppertal, Germany, ${ }^{5}$ Department of Palliative Care, Jena University Hospital, Jena, Germany, ${ }^{6}$ Institute of General Practice and Family Medicine, Ruhr University Bochum, Faculty of Medicine, Bochum, Germany

Background: A growing part of the population needs palliative care (PC) at the end of life. Although most patients with PC receive general PC, some patients need specialized PC. In Germany, little is known about the utilization of the different types of PC.

Aims: How many deceased received PC, particularly, general and/or specialized outpatient PC? What were the characteristics of patients receiving the different types of PC?

Methods: We conducted a German-wide retrospective observational cohort study using claims data from adult deceased in 2016 and insured with the second largest health insurance fund BARMER. PC was assumed if billing codes for general or specialized outpatient PC or inpatient PC were documented within the last six months before death. Descriptive analyses of patient cohorts were performed using SAS EG 7.1.

Results: The study population consists of 95,962 deceased. Preliminary results showed that $26,243(27,3 \%)$ received PC at all: $22,171(23,1 \%)$ received exclusively outpatient $\mathrm{PC}, 4,072(4,2 \%)$ exclusively inpatient PC and $3,611(3,8 \%)$ received both outpatient and inpatient PC. Among outpatient PC, $15,454(16,1 \%)$ received exclusively general and $3,243(3,4 \%)$ exclusively specialized PC, whereas $3,474(3,6 \%)$ received both. At the time of submission, data preparation and analysis were not finished yet. At the congress we will provide data for socioeconomic and medical care related characteristics of the different patient cohorts.

Conclusion: The results on characteristics of patients receiving general versus specialized outpatient PC may deliver new insights for the discussion of need and supply in palliative care.

Funding: Our study is part of the SAVOIR project which is funded by Federal Joint Committee German Innovations Fund to evaluate SAPV (Specialized Outpatient Palliative Care) in Germany.

\section{FC06 German Perspectives on Palliative Care} Feasibility of Early Palliative Care for Outpatients with Incurable Advanced Cancer in Real-world Settings - Analysis from a Prospective, Multicenter, Mixed-methods, Sequential Control Group Study (EVI Project)

Orellana-Rios C.L. ${ }^{1}$, Siemens W. ${ }^{1}$, Seibel K. ${ }^{1}$, Schoensteiner S. ${ }^{2}$, Schaekel U. ${ }^{3}$, Kessler J. ${ }^{4}$, Eschbach C. ${ }^{5}$, Viehrig M. ${ }^{6}$, Gärtner J.7, Becker G. ${ }^{1}$ ${ }^{1}$ Clinic for Palliative Care, Medical Center, University of Freiburg, Faculty of Medicine, University of Freiburg, Freiburg, Germany, ${ }^{2}$ Department of Internal Medicine III, University Hospital UIm, UIm, Germany, ${ }^{3}$ Department of Internal Medicine V, University Clinic Heidelberg, Heidelberg, Germany, ${ }^{4}$ Center of Pain Therapy and Palliative Care Medicine, Department of Anesthesiology, University Hospital Heidelberg, Heidelberg, Germany, ${ }^{5}$ Department of Thoracic Oncology, Member of the German Center for Lung Research (DZL), University Hospital Heidelberg and Translational Lung Research
Center Heidelberg (TLRC-H), Heidelberg, Germany, ${ }^{6}$ Department of Radiooncology, Palliative Care Unit, University Hospital of Tübingen, Tübingen, Germany, ${ }^{7}$ Center for Palliative Care Hildegard, Basel, Switzerland

Background: Evidence and recommendation of early palliative care (EPC) are based on randomized controlled trials which often lack generalisability. Investigations in real-world clinical scenarios are urgently needed.

Objectives: Primary aim: to assess whether EPC can be implemented into comprehensive cancer centers (CCCs) under real-world conditions. Methods: We conducted a multicenter, mixed-methods, prospective cohort study with a sequential control group design (DRKS00006162) in 23 oncology departments of 5 CCCs in Southern Germany. We included outpatients with metastatic cancer diagnosed within the last eight weeks. They were treated with standard oncology care or standard oncology care plus "as needed" PC by a PC physician or PC nurse. EPC was considered as feasible if $\geqslant 75 \%$ of eligible patients would be referred to a PC physician and agreed to receive EPC. Interviews with PC physicians, oncologists, patients and relatives were conducted after both study phases. Acceptability, demand and practicality of EPC were analyzed using content analysis.

Results: For the intervention group, we screened 1716 patients, 293 patients were eligible and 254 of them $(87 \%>75 \%)$ were referred to the PC physician and agreed to receive EPC.

A total of 174 interviews were analyzed (44 patients, 40 relatives, 67 oncologists and 23 PC specialists). EPC was well accepted among oncologists and patients. Patients and relatives emphasized a benefit in the areas of symptom treatment and communication quality. Oncologists appreciated the reinforcement of high-quality care. Most oncologists supported a consolidation of the intervention. The interviews with PC specialists revealed existing tensions between both professions.

Conclusion: EPC is feasible in German CCCs under real-world clinical conditions, but clarification of roles and tasks is needed in order to foster a successful professional collaboration.

Funding: Robert Bosch Stiftung: 11.5.1364.0055.0.

\section{FC06 German Perspectives on Palliative Care} Quality of End-of-Life Care - Comparing Claims Data Based Quality Indicators between Different Types of Palliative Care in Germany Krause M. ${ }^{1}$, Ditscheid B. ${ }^{1}$, Hennig B. ${ }^{2}$, Jansky M. ${ }^{3}$, Lehmann $T^{4}{ }^{4}$, Maddela S. ${ }^{4}$, Meißner W. ${ }^{5}$, Schulz S. ${ }^{1}$, Stichling K. ${ }^{1}$, Vollmar H.C. ${ }^{1,6}$, Wedding U. ${ }^{5}$, Freytag A. ${ }^{1}$, for the SAVOIR Study Group

${ }^{1}$ Institute of General Practice and Family Medicine, Jena University Hospital, Jena, Germany, ${ }^{2}$ Department of Medicine and Health Services Research, BARMER Statutory Health Insurance Fund, Wuppertal, Germany, ${ }^{3}$ Clinic for Palliative Medicine, University Medical Center Göttingen, Göttingen, Germany, ${ }^{4}$ Center for Clinical Studies, Jena University Hospital, Jena, Germany, ${ }^{5}$ Department of Palliative Care, Jena University Hospital, Jena, Germany, ${ }^{6}$ nstitute of General Practice and Family Medicine, Ruhr University Bochum, Faculty of Medicine, Bochum, Germany

Background: There are numerous approaches to describe the quality of End-of-Life-(EoL-)care based on quality indicators. Knowing their limitations, health insurance claims data can describe EoL-care in large populations. The study is part of a German-wide claims data study on palliative care (PC)

Aims: To investigate quality in EoL-care focussing on potentially aggressive treatments and compare these between patients receiving general versus specialized outpatient PC.

Methods: A retrospective observational cohort study has been conducted using claims data from adults insured with the second largest health insurance fund BARMER and deceased in 2016. PC was assumed if billing codes for general or specialized outpatient PC were documented 
within the last six months before death. The quality indicators we apply are internationally established and basically refer to the last three months before death. Where necessary and possible, we apply shorter time spans.

Results: The study population consists of 95,962 deceased. Preliminary results showed that 22,171 of these received outpatient PC: 15,454 received general and 6,717 received specialized outpatient palliative care. At the time of submission, data preparation and analysis had just started. At the congress we will provide the results for the following indicators: rates of patients with intensive care treatment, rates of chemotherapy in cancer patients, rates of PEG tube applications and parental nutrition. Conclusion: The given overview on the use of potentially aggressive therapies at the EoL in general versus specialized outpatient PC may support the ongoing discussion on how much of this might be avoidable.

Funding: Our study is part of the SAVOIR project which is funded by Federal Joint Committee German Innovations Fund to evaluate SAPV (Specialized Outpatient Palliative Care) in Germany.

\section{FC07 Miscellaneous}

Integrated Palliative Care Is Missionary Work; The Admission of Palliative Care in Five European Health Care Settings

Van Gurp J. ${ }^{1}$, van Wijngaarden J. ${ }^{2}$, Hasselaar J.3, on behalf of the InSup-C Consortium (Pecs University Medical Center, Lancaster University, Universitats Klinikum Bonn, UZ Leuven, Radboudumc Nijmegen

${ }^{1}$ Q Healthcare, Radboud University Medical Center, Nijmegen, Netherlands, 2Erasmus University Rotterdam, Rotterdam, Netherlands, ${ }^{3}$ Radboud University Medical Center, Nijmegen, Netherlands

Background: Integration of palliative care with curative care is expected to lead to better results for quality of life, costs, and perhaps even survival. Realization of such integration, however, remains limited; it is available for only a small group of people. Throughout Europe, integration of palliative care meets several obstacles among which are the recognition of palliative care values and limited trust between health care professionals.

Aims: This paper aims to explain whether and how normative integration - the integration of goals, implicit values, and practical norms - of palliative care with the currently dominant health care structures takes shape in five European countries.

Methods: As part of the EU-funded research project InSup-C (20122016), 19 semi-structured group interviews in 5 European countries (GE, $\mathrm{UK}, \mathrm{BE}, \mathrm{NL}, \mathrm{HU}$ ) were conducted. The analysis of the English translated data followed a grounded theory approach. Extensive international peer review was applied to guarantee the validity of the analysis conducted by the Dutch research team.

Results: Normative integration is about missionary work. The latter involves a) to find an entrance (for telling a normative story about palliative care), and b) to maintain and deepen relationships (in order to build trust with other caregivers). Palliative care providers require the virtue of diplomacy to realize this missionary work. Towards patients (e.g., by using the appropriate words and sending a univocal team message) and towards other health care professionals (e.g., by being grateful, modest, and aiming for a quiet revolution).

Conclusion/discussion: Diplomacy appears, for now, to be an essential virtue necessary for normative integration. It requires hard work from palliative care professionals, practical wisdom about the culture and goals of regular curative care, and finding a middle road between assimilating with regular curative care and standing up for the basic palliative care values.

\section{FC07 Miscellaneous}

Prevalence of Uncertainty in Illness in Family Caregivers of People in Palliative Care at a Colombian Health Institution Arias M. ${ }^{1}$, Carreño S. ${ }^{2}$
${ }^{1}$ Faculty of Nursing, Universidad de Antioquia, Medellin, Colombia, ${ }^{2}$ Faculty of Nursing, Universidad Nacional de Colombia, Bogota, Colombia

Background: Family caregivers of patients in palliative care assume the responsibilities of caring for the patient in many cases without any knowledge about aspects related to the disease. Assuming the care of the patient without knowledge leads to experiencing high levels of uncertainty in illness in the caregiver.

Aims: To describe the level of uncertainty in illness of family caregivers of patients in palliative care and to explore what factors of the characteristics of the participants may be associated with the development of uncertainty.

Methods: Correlational descriptive study with non-probabilistic sampling, carried out with 300 family caregivers of patients in palliative care hospitalized in a healthcare institution in Bogotá-Colombia. The sociodemographic characterization questionnaire of the patient-caregiver family dyad and the Uncertainty in illness scale of the family caregiver's were used. Descriptive statistics were performed for sociodemographic variables and Spearman's Rho correlation test was applied.

Results: The participants had an average age of 50.17 and a level of uncertainty in illness was in average 91.7 points. The analyzes performed revealed significant direct correlations between the level of uncertainty and the degree of dependence of the patient $(r=0.187, p=0.001)$, the evaluation of symptoms $(r=0.312, p<0.001)$ and time as caregiver $(r$ $=0.131, p=0.023$ ). On the other hand, a negative correlation was found between the level of uncertainty and the perception of support that the caregiver had with the healthcare professionals $(r=-0.16, p=$ $0.048)$, family $(r=-0.145, p=0.012)$. ) and religious support $(r=$ $-0.131, p=0.050)$.

Conclusion: The results suggest that the control of symptoms in the patient, the degree of functionality, professional, family and religious support are factors that can be taken into account to address the uncertainty in illness presented by the family caregiver.

\section{FC07 Miscellaneous}

Experiences of Oldest-old Carers whose Spouse is Reaching End-oflife: A Systematic Review and Narrative Synthesis

Morgan T., Bharmal A., Duschinsky R., Barclay S.

Public Health and Primary Care, University of Cambridge, Cambridge, United Kingdom

Background: Globally ageing populations have rapidly increased the number of people requiring palliative care. In resource-limited healthcare systems, spouses are increasingly providing end-of-life care to enable their partners to remain at home. Little attention, however, has been paid to oldest-old spousal carers who are often managing their own comorbidities whilst caring.

Aim: To review the literature concerning experiences of carers aged 75 and over whose partner is approaching end-of-life.

Methods: Systematic review and narrative synthesis of the empirical literature published between 1985-April 2018 across six databases. Gough's weight of evidence and Morgan's feminist appraisal tool used to determine quality.

Results: From the initial 6950 titles, eight qualitative studies and six quantitative studies were included. We identified three themes.

1) "The embodied impact of care". Many oldest-old carers experienced a 'double jeopardy' while providing emotionally and physically taxing care and managing their own diminishing health. They often subordinated their own health needs to continue caring.

2) "Marital and gendered conceptualisations of caring". Oldestold carers strove for normality in their self and marriage identities by providing care for their spouse with little additional 
support and by incorporating their caregiving within their established gender roles.

3) "Learning to care". Oldest-old carers had to learn new skills and ways of coping to keep their spouses at home. They became the expert of their partners' condition as well as having to take over all household tasks.

Conclusion: This review highlights the importance of oldest-old carers contributions to their spouses' end-of-life care. Oldest-old carers see it as their duty to care, but the caregiving itself involves learning new practical and emotional skills. Future research needs to consider how to best support these carers to do so without grave impact to their own health.

\section{FC07 Miscellaneous}

Four Profiles of Informal Caregivers who Provide Care to Patients at Home at the End of Life

Bijnsdorp F.M. ${ }^{1}$, Pasman H.R. ${ }^{1}$, Boot C.R.L. ${ }^{2}$, van Hooft S.M. ${ }^{3}$, van Staa A. ${ }^{3,4}$, Francke A.L. ${ }^{1,5}$

${ }^{1}$ Department of Public and Occupational Health, Amsterdam UMC, Vrije Universiteit, Public Health Research Institute, Expertise Center for Palliative Care, Amsterdam, Netherlands, ${ }^{2}$ Department of Public and Occupational Health, Amsterdam UMC, Vrije Universiteit, Public Health Research Institute, Amsterdam, Netherlands, ${ }^{3}$ Research Centre Innovations in Care, Rotterdam University, Rotterdam, Netherlands, ${ }^{4}$ Erasmus School of Health Policy \& Management, Erasmus University Rotterdam, Rotterdam, Netherlands, ${ }^{5}$ Nivel, Netherlands Institute for Health Services Research, Utrecht, Netherlands

Background: To prevent overload of informal caregivers of patients at the end of life and enhance their capacity to provide care it is important that support is deployed in time. However, it is unclear which types of informal caregivers provide care to patients at the end of life and which type of support they prefer.

Aim: To identify profiles of informal caregivers who care for patients at the end of life at home.

Design: A Q-methodological study in which informal caregivers ranked 40 statements on support needs and attitudes towards the care situation followed by interviews about their ranking. By-person factor analysis was conducted to identify corresponding ways in which informal caregivers ranked statements. Those with the same views shared the same factor. Factors were interpreted as informal caregiver profiles.

Participants: 41 current and former informal caregivers with a variety on background characteristics who recently provided care for someone at the end of life (e.g., person diagnosed with incurable cancer, dementia, organ failure, stroke) at home were included.

Results: Four distinct profiles of informal caregivers were identified; those who want appreciation and an appointed contact person (1), those who are positive about sharing care $(2+)$ or wish to share care but are not able to or unsatisfied (2-), those who want information and practical support (3) and those who need off-time (4). These profiles reflect different needs and experiences with support.

Conclusions: Informal caregivers of patients at home at the end of life have varying support needs and one size does not fit all. The profiles are relevant for healthcare professionals and volunteers in palliative care as they provide an overview of the main characteristics and support needs among informal caregivers of patients near the end of life and can be helpful in proving more targeted support.

Funding: The Netherlands organisation for health Research and Development

FC07 Miscellaneous

Guided Internet Cognitive Behavioral Therapy for Insomnia After the Loss of a Child: Randomized Controlled Trial
Sveen J. ${ }^{1}$, Jernelöv S. ${ }^{2}$, Pohlkamp L. ${ }^{1}$, Kreicbergs U. ${ }^{3}$, Kaldo V. ${ }^{2}$

${ }^{1}$ Palliative Research Centre, Ersta Sköndal Bräcke University College, Stockholm, Sweden, ${ }^{2}$ Karolinska Institutet, Stockholm, Sweden, ${ }^{3}$ Ersta Sköndal Bräcke University College, Stockholm, Sweden

Background: Disturbed sleep is a common problem in bereaved individuals (Buckley et al., 2012).

Aims: The aim was to evaluate satisfaction with and effects of internetdelivered cognitive behavior therapy (ICBT) treatment for insomnia in parents up to 5 years after the loss of a child to cancer.

Methods: Participants were 21 bereaved parents with insomnia. The parents were randomized to an intervention or a control group. The intervention was a 9-week ICBT for insomnia. The control group received a short booklet with psychoeducation on sleep via the internet. Primary outcome was symptom of insomnia and secondary outcomes were prolonged grief, depression, posttraumatic stress, grief rumination, assessed pre- and post-intervention with follow-up after 9 and 18 months. Satisfaction with treatment was assessed at postintervention $(n=7)$ and by telephone one month after $(n=6)$ the intervention.

Results: The intervention group improved from pre- to post-intervention with regard to symptoms of insomnia (Cohens $d=1.56$ ), however there was no significant difference between the two groups at postmeasure $(d=0.11)$ due to a large reduction also in the control group ( $d=1.12$ ). At the 9 months follow-up there was a significant difference between the two groups in symptoms of insomnia $(\mathrm{d}=1.54)$ as well as the secondary measures $(d=0.62-1.54)$. Of six individuals who responded by telephone, no one had experienced any negative consequences of the treatment. Some parents thought it was time-consuming. One person reported being quite dissatisfied with the treatment, although the majority of participants reported being very satisfied.

Conclusion: The preliminary results, due to small sample size, indicate that the internet-delivered treatment with CBT had a positive effect on reducing insomnia as well as psychological distress in bereaved parents, short-term and long-term.

\section{FC07 Miscellaneous}

The Effect of Music Therapy on the Quality of Life of Hospice Patients: A Critical Realist Trial

Porter S. ${ }^{1}$, McConnell T. ${ }^{2}$, Graham-Wisener L. ${ }^{3}$, Kirkwood J. ${ }^{4}$, Clarke M. ${ }^{5}$, Regan J. ${ }^{6}$, Reid J. ${ }^{7}$

${ }^{1}$ Social Sciences and Social Work, Bournemouth University, Bournemouth, United Kingdom, ${ }^{2}$ School of Social Sciences, Education \& Social Work, Queen's University Belfast, Belfast, United Kingdom, ${ }^{3}$ School of Psychology, Queen's University Belfast, Belfast, United Kingdom, ${ }^{4}$ Every Day Harmony Music Therapy, Belfast, United Kingdom, ${ }^{5}$ School of Medicine, Dentistry and Biomedical Sciences, Queen's University Belfast, Belfast, United Kingdom, ${ }^{6}$ Marie Curie Hospice Belfast, Belfast, United Kingdom, ${ }^{7}$ School of Nursing and Midwifery, Queen's University Belfast, Belfast, United Kingdom

Background: There is positive but weak evidence that music therapy (MT) can have a beneficial effect upon the quality of life of palliative care patients.

Aims: To provide evidence about MT's effect on the quality of life of hospice patients.

To establish the feasibility of carrying out a critical realist trial in a hospice setting.

Methods/Design: A critical realist pilot trial which supplemented outcome measurements with causal analysis. The outcomes of interest were measured by means of a single-centre, two-armed randomised trial. Outcomes were explained using qualitative data that identified the influence of contextual mechanisms, intervention mechanisms and agency. 
Data collection: Outcome data: McGill Quality of Life (MQoL) questionnaire was administered to 51 participants.

Process data: Open text answers from participants receiving MT and focus groups with hospice professionals.

Analysis: Effectiveness was assessed by determining the change between baseline score and one week after baseline. During this period the intervention group received two MT sessions.

Qualitative data were thematically analysed using a theoretical framework developed from a realist review of the literature.

Results: The overall difference in MQoL between the groups was nonsignificantly in favour of MT. There was a statistically significant improvement in existential well-being for the MT group. Results for physical sub-measures were contradictory.

MT contains multiple mechanisms that can provide physical, psychological, emotional, expressive, existential and social support.

The hospice context, animated by a holistic approach to healthcare, is a significant facilitator of the effects of MT.

Patients' responses were influenced by their biographical specificities such as their religiosity.

Discussion: The results provide further evidence of the benefits of MT for palliative patients, but indicate the need for a fully powered trial.

\section{FC07 Miscellaneous}

The Berlin Trilogy: David Bowie, Palliative Care and Berlin - A Public Reading

Taubert M. ${ }^{1}$, Minton $0^{2}{ }^{2}$, Keeley $P^{3}$

${ }^{1}$ Palliative Care, Cardiff University School of Medicine, Cardiff, United Kingdom, ${ }^{2}$ Brighton and Sussex Medical School, Brighton, United Kingdom, ${ }^{3}$ Glasgow Royal Infirmary, Glasgow, United Kingdom

Background: The Berlin Trilogy consists of three consecutively released studio albums by singer David Bowie: Low (1977), Heroes (1977) and Lodger (1979). The albums were recorded after Bowie took up residence in West Berlin in late 1976. The 'EAPC Berlin Trilogy 2019' will consist of public readings and displays of relevant Bowie texts that resonate with contemporary end of life care issues and public understanding.

Aim: In three readings, three palliative care professionals will read texts and letters relating to Bowie and to end-of-life care. Mark Taubert will read his 2016 letter to Bowie. Taubert wrote a posthumous letter to the Starman after the singer's death. A letter that went viral over social and print media after Bowie's son shared it. A letter that has inspired a musical composition premiered on BBC Radio 3. A letter that has been read out aloud by Jarvis Cocker and Benedict Cumberbatch in public readings. Why so much fuss over a letter? Probably best to hear it here. Ollie Minton and Paul Keeley will read texts from the Blackstar album and from one the Berlin trilogy albums.

Conclusion: After the readings, an open discussion forum will be held. Whilst in palliative care we often talk about improving global understanding about end of life care and advance care planning, we don't always manage to get our key messages through as effectively as we'd wish. Culture, music and shared memories, anywhere in the world, can be a powerful way to encapsulate good palliative care, similarly to what happened in the patient encounter that is described or in the words of a song. In the end, what binds us all together is our shared enjoyment of the simple things in life, like music, poetry and Bowie.

Additonal Information: After these readings, the presenters will invite delegates to join them on a trip to nearby 'Das Neue Ufer Café Bar', one of Bowie's favourite Berlin locations, followed by an optional visit to Hansa Studios, were the Trilogy albums were recorded.

FC08 Primary Health Care and Interprofessional Collaboration How Do Primary Care Teams Make Sense of their Role at End of Life Care at Home?
Meléndez A. ${ }^{1}$, Reigada C. ${ }^{2,3}$, Sáenz de Ormijana A. ${ }^{4}$, Almaraz M.J. ${ }^{1}$, Delgado A. ${ }^{5}$, García de Albéniz $A .{ }^{6}$

${ }^{1}$ Palliative Care Team, Araba Integrated Healthcare Organization, Basque Health Service, Vitoria-Gasteiz, Spain, ${ }^{2}$ Institute for Culture and Society, ATLANTES, University of Navarra, Pamplona, Spain, ${ }^{3}$ Instituto de Investigación Sanitaria de Navarra (IdiSNA), Pamplona, Spain, ${ }^{4}$ Integrated Care Development Department, Araba Integrated Healthcare Organization, Basque Health Service, Vitoria-Gasteiz, Spain, ${ }^{5}$ Zaramaga Primary Care Unit, Araba Integrated Healthcare Organization, Basque Health Service, Vitoria-Gasteiz, Spain, ${ }^{6}$ Norte-Zuia Primary Care Unit, Araba Integrated Healthcare Organization, Basque Health Service, Vitoria-Gasteiz, Spain

Background: Caring for people at their end-of-life (EOL) at home is part of the role of Primary Care teams (PCT). Collaboration between PCT and Palliative Care Community Support Teams (PCCST) is crucial, particularly in the management of complex cases. Even so, the so-called "team collaboration" faces difficulties mainly related to professional EOL care practices in the home setting. These difficulties may even restrain the continuity and quality of care.

This study aims to understand the way PCTs make sense and describe their role and responsibility when caring for patients at their EOL in the homecare setting.

Methods: Qualitative study, based on the dialogical method using indepth interviews and focus groups (FG). A theoretical sampling of PCT health professionals were selected trying to include a high range of realities regarding the phenomenon of caring for people at the EOL at home. A thematic analysis was used.

Results: Eight interviews (Physicians= 5; Nurses=4) and three FG ( $N=19)$ were conducted. The understanding of PCT's role and practices at EOL care varies amongst professionals. The study identifies three main themes supporting those different ways of understanding role and practices: 1) the professional-self; 2) the clinical practice; 3 ) and the context of practice. Each area itself results from a complex integration of multiple components such as the PC approach, the self-awareness (responsibility) of EOL practices, the personal and professional growth through previous EOL experiences. PCTs holding larger knowledge and further self-awareness of their role at EOL care tend to more easily accept PCCST support. Conclusion: Understanding the way different PCT professionals make sense of their own EOL homecare role and practice, as well as the elements shaping those realities, may help PCCST tune and adjust their support role, as to make themselves more accessible. Efficient collaboration between PCT and PCCST is an essential element of good EOL care at home.

FC08 Primary Health Care and Interprofessional Collaboration Advance Care Planning at the Interface of Home Care Professionals and Inpatient Facilities

Lehmann N. ${ }^{1}$, Fliedner M. ${ }^{1}$, Eychmüller S. ${ }^{1}$, Linhart M. ${ }^{2}$

${ }^{1}$ Universitäres Zentrum für Palliative Care, Bern, Switzerland, ${ }^{2}$ Department of Health Professions, Bern University of Applied Sciences, Bern, Switzerland

Background: General practitioners (GP) and home care nurses play an important role in promoting advance care planning (ACP) activities due to their close relationship with their patients. However, roles and responsibilities within this process remain frequently unclear, especially when patients move between in- and outpatient care during the last months and weeks of life.

Aims: To understand the home care nurses' and GPs (home care professional's) roles and responsibilities in the process of performing ACP, particularly regarding the intersection between the hospital and home care setting.

Method: Three focus groups and three expert interviews were conducted with 12 GPs and 10 home care nurses in a mixed rural and urban 
region in Switzerland. Qualitative content analysis was utilized for analysis.

Findings: A major barrier for GPs and home care nurses to implement ACP is the deficiency in financial reimbursement and the therefore occuring time constraint. Important tasks of home care professionals such as initiating ACP and coordination of care are regarded as being essential but are not fully financially reimbursed. Furthermore, ACP documentation (written and spoken word) between various care settings is not structured in terms of content and responsibilities. Despite being in favor of ACP, both GPs and home care nurses displayed restraint in addressing the topic of ACP because they were afraid to lose the patients' trust.

Conclusion / Discussion: In sum GPs and home care nurses in Switzerland feel uneasy in the way, ACP is currently offered and implemented. Unclear role definitions, fear of losing trust and gaps in financial reimbursement seem to be major reasons for this and counteract the policy to strengthen palliative care on a national level. Therefore, in order to guarantee all patients' access to ACP, ACP activities should become a routine topic in primary care and financial reimbursement has to be clarified by the responsible department.

FC08 Primary Health Care and Interprofessional Collaboration Primary Palliative Care Team Perspectives on Coordinating and Managing People with Advanced Cancer in the Community: A Qualitative Study

Hackett J. ${ }^{1}$, Ziegler L. ${ }^{2}$, Godfrey M. ${ }^{2}$, Foy R. ${ }^{2}$, Bennett M. ${ }^{2}$

${ }^{1}$ University of York, York, United Kingdom, ${ }^{2}$ University of Leeds, Leeds Institute of Health Sciences, School of Medicine, Leeds, United Kingdom

Background: Primary health care teams are key to the delivery of care for patients with advanced cancer during the last year of life. The Gold Standards Framework is proposed as a mechanism for coordinating and guiding identification, assessment, and support. There are still considerable variations in practice despite its introduction. The aim of this qualitative study is to improve understanding of variations in practice through exploring the perspectives and experiences of members of primary health care teams involved in the care of patients with advanced cancer. Methods: Qualitative, semi-structured interviews, focus groups, and nonparticipatory observations involving 67 members of primary health care teams providing palliative care. Data were analysed using a grounded theory approach.

Results: We identified distinct differences in the drivers and barriers of community advanced cancer care coordination, which relate to identification and management, and access to effective pain management, and go some way to understanding variations in practice. These include proactive identification processes, time and resource pressures, unclear roles and responsibilities, poor multidisciplinary working, and inflexible models for referral and prescribing. These provide valuable insight into how professionals work together and independently within an infrastructure that can both support and hinder the provision of effective community palliative care.

Conclusions: Whilst the GSF is a guide for good practice, alone it is not a mechanism for change. Rather it provides a framework for describing quality of practice that was already occurring. Consequently, there will continue to be variations in practice.

FC08 Primary Health Care and Interprofessional Collaboration Strategy for Inserting Palliative Care in Primary Health Care in Brazil: Looking at Nursing

Barrioso P.D.C. ${ }^{1,2,3}$, Zoboli E.L.C.P. ${ }^{1}$

${ }^{1}$ Departamento de Saúde Coletiva, Escola de Enfermagem da Universidade de São Paulo, São Paulo, Brazil, 2'Serviço de Cuidados Paliativos, HSPE- São Paulo, São Paulo, Brazil, Instituto Paliar, São Paulo, Brazil
Aim: To propose nursing actions in Palliative Care (PC) for primary health care nurses in the context of public health in Brazil.

Method: Considering the lack of consensus for the classification of the levels of complexity of nursing actions in PC in Brazil, the study used the Delphi Technique with a panel of nurses specialized in PC to classify forty-eight nursing actions in PC, from a level of basic or specialized action. This study considers basic level actions to be actions in PC that all the professional nurses should be able to perform whereas actions at the specialized level are those that only professionals with specialized training or training in PC would be prepared to perform.

Results: Twenty-four professionals who formed the panel of experts participated in this study. Most of the participating nurses (92\%) have PostGraduation (masters and doctorate). Regarding the time of training (67\%) have more than 10 years of professional experience. As for the Palliative Care experience, $50 \%$ have 5 years of professional practice in the specialty, highlighting that one of the criteria for exclusion was less than 03 years of experience in the PC are. Twenty-three of the fortyeight nursing actions in $\mathrm{PC}$ were classified as basic level actions and were subdivided into four categories: symptom control in PC; Broad view in PC: culture, spiritual dimension and values; Continuity of care and integral care; the home as a place of care.

Conclusion: Nursing is considered a strategic profession to disseminate the PC approach, since it is present in all levels of health care. We obtained a list of twenty-three nursing actions which have been proposed for insert to Palliative Care in Primary Health Care from this study wich can be referenced for trainings and public policies in this field.This study serves as a starting point for professionals working at the community level to deal with the increasing demand of PC in Brazil. This study was funded by author's resources.

FC08 Primary Health Care and Interprofessional Collaboration Advance Care Planning for Patients with Cancer in the Palliative Phase in Dutch General Practices

Ermers D. ${ }^{1}$, van Bussel K. ${ }^{2}$, Perry M. ${ }^{3}$, Engels . $^{1}$, Schers $H_{.}{ }^{2}$

${ }^{1}$ Anaesthesiology, Pain and Palliative Medicine, Radboudumc, Nijmegen, Netherlands, ${ }^{2}$ Primary and Community Care, Radboudumc, Nijmegen, Netherlands, ${ }^{3}$ Geriatrics, Radboudumc, Nijmegen, Netherlands

Background: Advance care planning (ACP) is a crucial element of palliative care. It improves the quality of end-of-life care and reduces aggressive and needless life-prolonging medical interventions. However, little is known about its application in daily practice.

Aims: This study aims to provide better insights into the application and documentation of ACP in general practice.

Methods: We performed a retrospective cohort study in eleven general practices in the Netherlands. Electronic patient records (EPRs) of deceased patients with colorectal or lung cancer were analysed.

Data on ACP documentation, correspondence between medical specialist and general practitioner (GP), and healthcare use in the last year of life were extracted.

Results: Records of 163 deceased patients were analysed. In $74 \%$ of the records, one or more ACP items were registered. Three or more ACP items were found in $36 \%$ of the records. GPs especially documented patients' preferences for euthanasia (58\%), palliative sedation (46\%) and preferred place of death (26\%). Most information was registered in the last three months of life. Per patient, GPs received on average six letters from medical specialists. These letters mainly contained information regarding medical treatment and only rarely ACP items. In the last year of life, patients contacted the GP over 30 times on average, and 51\% visited the emergency department at least once, of whom $54 \%$ in the last month. The number of contacts with the GP was not related to ACP documentation.

Conclusion: Registration of ACP items in GPs' EPRs appeared to be limited. ACP elements were rarely subject of communication between 
primary and secondary care, which may impact the continuity of patient care during the last year of life. More emphasis on registration of ACP items and better exchange of information regarding patients' preferences is needed.

Funding: National Health Care Institute Netherlands (funding 2016/969/ $\mathrm{OOL})$.

FC08 Primary Health Care and Interprofessional Collaboration A Cluster Randomised Feasibility Trial (cRCT) to Test the Routine Use of the Needs Assessment Tool: Cancer (NAT:C) in Primary Care to Reduce Unmet Patient and Carer Needs and Determine the Feasibility of a Definitive Trial

Clark J. ${ }^{1}$, Amoakwa E. ${ }^{2}$, Allgar V. ${ }^{3}$, Blenkinsopp J. ${ }^{4}$, David C. ${ }^{1}$, Amanda F. ${ }^{5}$, Robbie F. ${ }^{6}$, MacLeod U. ${ }^{1}$, Meads D. ${ }^{6}$, Wright-Hughes A. ${ }^{5}$, Johnson M. ${ }^{1}$ ${ }^{1}$ Wolfson Palliative Care Research Centre, Hull York Medical School, Hull, United Kingdom, ${ }^{2}$ UCEN Manchester, Manchester, United Kingdom, ${ }^{3}$ University of York, Hull York Medical School, York, United Kingdom, ${ }^{4}$ Newcastle Business School, Northumbria University, Newcastle, United Kingdom, ${ }^{5}$ University of Leeds, Leeds Institute of Clinical Trials Research, Leeds, United Kingdom, ${ }^{6}$ University of Leeds, Leeds Institute of Health Sciences, Leeds, United Kingdom

Background: People with cancer commonly have distressing symptoms and unidentified palliative care needs. The Needs Assessment Tool:Cancer (NAT:-C) is a validated tool to improve needs-based access to palliative care.

Aims: To assess the feasibility and acceptability of conducting a definitive CRCT of the NAT:-C in primary care in terms of

1) recruiting 4 GP practices and $40-60$ patients (plus carers),

2) Uptake of NAT:PD-C,

3) Questionnaire completion rates

4) acceptability of study measures

Methods: A non-blinded cluster randomised trial with parallel process evaluation. Patients with active cancer were identified through cancer registries or in clinic and invited to participate and nominate a carer if they wanted to. Participants completed study questionnaires at baseline and 1, 3 and 6 months. Patients sought a 20 minute appointment postbaseline. Patients, carers and GP practice staff were invited to participate in interviews/focus groups.

Results: Five GP practices were approached and 4 recruited and trained in the use of the NAT:-C. Practices were cluster randomised (1:1). Fortyseven patients and 17 carers were recruited. Early recruitment problems were addressed by including a pre-paid expression of interest form with the study invitation. Recruitment rate was appropriate for a definitive multicentre trial. $44(94 \%)$ of patients received a study appointment, 42 (95\%) attended and 32 (76\%) received a NAT-guided consultation. Item completion rates of the proposed primary outcome measure the Supportive Care Needs Survey at baseline were 96.4\%. 15 patient interviews and 4 focus groups with GP practices were conducted. Participants supported the need for a definitive study and found all measures acceptable. Process evaluation informed effective refinement of study invitations.

Conclusion: The feasibility trial indicated that the recruitment rate, intervention uptake and data collection were appropriate, with refinements, for a definitive CRCT is feasible.

FC09 Quality and Safety in Prescribing at the End of Life Exploring Opioid Errors in Australian, Inpatient Palliative Care Services: A Mixed Methods Study

Heneka N. ${ }^{1}$, Shaw T. ${ }^{2}$, Rowett D. ${ }^{3}$, Lapkin S. ${ }^{4}$, Phillips J.L. ${ }^{1,5}$ ${ }^{1}$ University of Notre Dame Australia, Broadway, Australia, ${ }^{2}$ University of Sydney, Sydney, Australia, 3University of South Australia, Adelaide,
Australia, ${ }^{4}$ University of Wollongong, Wollongong, Australia, ${ }^{5}$ University of Technology Sydney, Ultimo, Australia

Background: Opioids are a high-risk medicine routinely used to manage palliative patients' pain and other symptoms. Despite the high volume of opioid use in palliative care services, little is known about opioid errors in this clinical setting.

Aims: To identify

i) opioid error incidence, patient impact and error contributing factors; and

ii) barriers and facilitators to safe opioid delivery processes in inpatient palliative care services.

Methods: A two-phase, explanatory sequential mixed methods study, guided by a multi-incident analysis framework. Three Australian palliative care inpatient services participated in this study.

Results: Opioid errors accounted for $32 \%$ of all reported medication errors, equating to a mean 0.9 ( \pm 1.5 ) opioid errors per 1000 occupied bed days. Opioid administration errors comprised $76 \%$ of reported opioid errors, $33 \%$ of which were omitted dose errors. Opioid prescribing errors (15\%) were less frequently reported overall, but were proportionally higher in services that did not employ an on-site clinical pharmacist. Over half $(53 \%)$ of opioid errors required a clinical intervention to preclude or manage patient harm. For $57 \%$ of patients, opioid errors resulted in opioid under-dose, with subsequent adverse impact on pain management. Suboptimal skill mix, poor clinical communication, and absence of support from central functions (e.g. pharmacy) were perceived to contribute to opioid errors. A strong opioid safety culture was evident in all participating services, and was seen as critical to supporting safe opioid delivery.

Conclusion: Opioid errors in palliative care inpatient services contribute to iatrogenic patient harm, and occur as a result of differing combinations of individual, team, environmental and organisational factors. A commitment to creating and sustaining a strong opioid safety culture, and enabling an adequately resourced workplace, is fundamental to reducing opioid errors in inpatient palliative care.

FC09 Quality and Safety in Prescribing at the End of Life Antibiotic Use in Advanced Dementia Patients Near End of Life: Comfort or Conflict Francisco E.P. ${ }^{1}$, Tay R.Y. ${ }^{1}$, Hum A.Y.M. ${ }^{1,2}$

${ }_{1}^{1}$ Programme Dignity, Dover Park Hospice, Singapore, Singapore, ${ }^{2}$ Pallaitive Medicine, Tan Tock Seng Hospital, Singapore, Singapore

Background: Advanced Dementia (AD) is a terminal illness. Many $A D$ patients die from infections, the commonest of which is pneumonia. Evidence about the use of antibiotics for symptomatic relief in $A D$ patients suffering from infection is conflicting. Erring on the side of caution, physicians frequently escalate antibiotics even when patients are at the end of life (EOL).

Aim: To determine if the use of antibiotics affects comfort of AD patients at the EOL and to ascertain if enteral feeding affects the prevalence of infection with antibiotic usage.

Methods: Patients with FASTC (Functional Assessment Staging Test) Stage 7 and beyond with albumin level $<35 \mathrm{~g} / \mathrm{L}$, a feeding tube, or pneumonia were recruited. The Comfort Assessment in Dying with Dementia (CAD-EOLD) Scale was used to assess patients' comfort when prognosis was short. The presence of enteral feeding, type and frequency of infections, and use of antibiotics in the last 2 weeks of life were reviewed. Mann-Whitney $U$ test and Chi-square test were used for between group comparisons of continuous and categorical variables respectively. Results: Of 343 patients, $78.1 \%(n=268)$ died of sepsis, with pneumonia being the commonest cause (83\%). 69\% ( $n=186$ ) of patients with sepsis were given antibiotics in the last 2 weeks of life. Median CAD-EOLD score 
of patients who used antibiotics ( $M d=37, n=130$ ) was not significantly different from patients who did not use antibiotics ( $M d=38, n=104)$, $p=0.216$. Patients on enteral feeding were 2.8 times more likely to suffer from pneumonia than patients without, $\chi^{2}(1, n=337)=17.8, p<0.0001$, phi $=0.24$, hence increasing the likelihood of antibiotics being given by a factor of $1.9\left[\chi^{2}(1, n=337)=8.2, p=0.004\right.$, phi=0.16].

Conclusion: Infection is prevalent in AD patients at the EOL especially for those with enteral feeding. Antibiotics are frequently given for symptomatic relief but ironically did not increase AD patients' comfort at the EOL. Antibiotic therapy should hence be offered judiciously.

\section{FC09 Quality and Safety in Prescribing at the End of Life} Regular Oxycodone for Chronic Breathlessness - Answers from a Randomised Controlled Trial

Currow D. ${ }^{1,2}$, Ferreira D. ${ }^{1}$, Agar M. ${ }^{2}$, Louw S. ${ }^{3}$, McCloud P. ${ }^{3}$, Fazekas B. ${ }^{1}$, McDonald C.., 5 , Clark K. 6,7, McCaffrey N. ${ }^{8}$, Ekström M. ${ }^{9}$

${ }^{1}$ Flinders University, Palliative Care Clinical Studies Collaborative (PaCCSC), Adelaide, Australia, 2University of Technology Sydney, ImPACCT, Sydney, Australia, ${ }^{3} \mathrm{McCl}$ loud Consulting Group, Belrose, Australia, ${ }^{4}$ Austin Health, Melbourne, Australia, ${ }^{5}$ University of Melbourne, Melbourne, Australia, ${ }^{6}$ Northern Sydney Local Health District, Sydney, Australia, 7 University of Sydney, Sydney, Australia, ${ }^{8}$ Deakin University, Melbourne, Australia, ${ }^{9}$ Lund University, Faculty of Medicine, Department of Clinical Sciences Lund, Respiratory Medicine and Allergology, Lund, Sweden

Background: Morphine can relieve breathlessness when the underlying disease is optimally treated - chronic breathlessness. It is unclear whether oxycodone has a similar effect.

Aim: This randomized, placebo-controlled, double-blind, parallel-arm study aimed to evaluate the effects of regular, low-dose, controlledrelease (CR) oxycodone for chronic breathlessness.

Methods: Participants were randomised to $15 \mathrm{mg}$ of oral $\mathrm{CR}$ oxycodone ( $5 \mathrm{mg}$ every 8 hours) or placebo for seven days. Up to 6 doses/day of 'as needed' immediate release morphine $(2.5 \mathrm{mg} /$ dose $)$ were available for both arms as required by one of the Ethics Committee overseeing this trial. The primary endpoint was change from baseline in the intensity of breathlessness now (0-100mm visual analogue scale [VAS]) between arms. Secondary endpoints included other measures of breathlessness; quality of life; function; and adverse events.

Results: From February 2010 to July 2015, 215 participants were randomized and 213 participants were included in this study that did not reach target recruitment (oxycodone $n=74$; placebo $n=139$ ). Primary outcomes of breathlessness now were similar between arms. The placebo arm used more 'as needed' morphine solution (mean 9.0 versus 4.6 daily doses; $p \leqslant 0.001$ ). The oxycodone arm reported more nausea as a treatment emergent adverse event and more constipation, and appetite loss in the EORTC-QLQ-15.

Conclusions: This randomized controlled trial demonstrated no benefit of oxycodone over placebo for treatment of chronic breathlessness. An adequately powered study should be conducted without "as needed" opioids. Future research should explore a different primary outcome (worst breathlessness) in a specific sub-population (modified Medical Research Council scale 3 or 4).

FC09 Quality and Safety in Prescribing at the End of Life Methadone Rotation in a Palliative Care Cohort: A Multicentred Retrospective Review

Tuffin P., Corfias E., DOrazio M., Guelfi K., Listina I., Popowicz N. Faculty of Health and Medical Sciences, Centre for Optimisation of Medicines, University of Western Australia, Perth, Australia

Background: Different guidelines for rotation of opioids to methadone are used internationally without consensus on the most efficacious.
Guidelines were developed based on literature and expert opinion of palliative care clinicians and implemented across all palliative care centres in our community to provide a consistent rotation procedure.

Aims: To evaluate the efficacy and safety of opioid rotation to methadone and adherence to guidelines in a multicentre series.

Methods: Patients from 8 palliative care centres converted to methadone between Jan and Sept 2018 were included. Measured outcomes included efficacy (assessed by pain score and oral morphine equivalent (MEQ) dose changes and time to stable dose) and safety (by reported adverse effects). Patient demographics, pain history, symptom monitoring, pain scores and opioid dosing were collected retrospectively from medical records. Loading, regular, breakthrough doses and dose adjustments were assessed for adherence to guidelines.

Results: Of 43 patients, rotation to methadone appears effective, with a decrease in average pain scores from pre-rotation to final assessment (3.2 to 1.6, $p=0.002$ ), and safe, with no serious adverse events reported. Participants were prescribed a range of opioids prior to rotation with an average daily dose of $376( \pm 282)$ mg MEQ which reduced to $272( \pm 161)$ $\mathrm{mg}$ with methadone. The most common reasons for rotation were inadequate analgesia and adverse effects with current opioid. Although complete adherence to guidelines was low $(19 \%)$, most conversions (53\%) met a minimum of 2 criteria and modifications were commonly use of a lower dose than recommended. A trend to fewer days required until dose stability was observed in patients who received a loading dose compared to those who did not (median[IQR], 6[6-10] vs 8.5[6-10], $\mathrm{p}=0.16)$.

Discussion / Conclusion: Although complete adherence to the guidelines is low, methadone conversions are safe and effective with decreased pain scores and MEQ doses.

FC09 Quality and Safety in Prescribing at the End of Life Breakthrough Medications in Unresponsive Palliative Care Patients: Indications, Practice and Efficacy

Barbato M. ${ }^{1}$, Barclay G. ${ }^{1,2}$, Yeo W.2,3,4, Potter J.2,4,5

${ }^{1}$ Palliative Care Services, Illawarra Shoalhaven Local Health District, Warrawong, Australia, ${ }^{2}$ Graduate School of Medicine, University of Wollongong, Wollongong, Australia, ${ }^{3}$ Division of Medicine, Illawarra Shoalhaven Local Health District, Wollongong, Australia, 4 Illawarra Health and Medical Research Institute, Wollongong, Australia, ${ }^{5}$ Division of Aged Care, Rehabilitation and Palliative Care, Illawarra Shoalhaven Local Health District, Wollongong, Australia

Background: An unresponsive patient's distress and their response to breakthrough medication are determined by clinical assessment and/or observational measures. How closely these methods match the patient's experience is unknown.

Aims: To determine the efficacy and effectiveness of breakthrough medication in unresponsive patients and the perception of patient comfort made by nurses and family.

Methods: A prospective study of breakthrough medication in 40 unresponsive patients. The Richmond Agitation-Sedation Scale (RASS) and Patient Comfort Score (PCS) were compared with time-matched Bispectral Index (BIS) scores. The effects of opioid versus opioid plus benzodiazepine breakthroughs and the relation between synchronous nurse and family measurements of the PCS were evaluated. ANOVA and paired t-tests were used for BIS analyses and non-parametric Mann Whitney tests for RASS and PCS.

Results: There were 112 breakthrough medication doses recorded. Significant score reductions at 30 and 60 minutes following breakthrough medication were noted for BIS $(p<0.0004)$, RASS ( $p=0.043$ and 0.004 respectively) and PCS $(p<0.0004)$. The most common indication for breakthrough medication was respiratory distress. A direct comparison of the effect of opioid breakthrough medication(12.5\% of doses) versus opioid plus benzodiazepine ( $45 \%$ of doses) revealed no significant difference (BIS, $p=0.512$; RASS, $p=0.195 ; P C S, p=0.119$ ). Of the 157 
synchronous nurse and family measures of patient comfort, families rated patient discomfort significantly higher than nurses $(p<0.0004)$. Conclusion: This study provides additional evidence for the efficacy and effectiveness of breakthrough medication and the merit of observational measures in determining a patient's response. The onset of action is evident at 30 minutes following injection. Family assessment of patient comfort may be more nuanced than that of nurses and they not uncommonly rate patient discomfort higher than nurses.

\section{FC09 Quality and Safety in Prescribing at the End of Life Use of Tramadol in Palliative Care and Pain Treatment: Potential Consequences of Placing it under International Control De Lima L. ${ }^{1}$, Radbruch L. ${ }^{1}$, Pettus K. ${ }^{1}$, Pastrana T. ${ }^{2}$ \\ ${ }^{1}$ IAHPC, Houston, United States, ${ }^{2}$ RWTH Aachen University, Aachen, Germany}

Background: Tramadol is a synthetic opioid analogue of codeine, recommended by the World Health Organization (WHO) as a step-2 analgesic in the guidelines for cancer pain relief. Currently tramadol is not subject to international control ("scheduling"). The WHO Expert Committee on Drug Dependence (ECDD) will be reviewing tramadol and evaluating whether it should be placed under international control.

Aims: To explore the clinical use of tramadol and the impact that the proposed scheduling may have on access for legitimate medical needs in pain treatment and palliative care (PC).

Method: Cross over study using an online survey conducted in October 2018 among members of the International Association for Hospice and Palliative Care.

Results: The survey was sent to 672 prescribers worldwide and 470 participants responded (RR 70\%). Of these, 415 participants prescribe tramadol, working most of the time in PC $(60 \%)$, or half time in PC $(27 \%) .41 \%$ prescribe it daily or at least once a week (32\%) Most use it for the treatment of mild to moderate pain $(53 \%)$ and moderate to severe pain (43.4\%). However, $4 \%$ of participants from 9 countries use it for all levels of pain, having no other analgesic available. According to the participants, placing tramadol under international control would have a negative impact (mean 72.0; SD=32.46) (100 being "Extremely negative impact on access to pain treatment"). Participants from Low Income and Low Middle Income countries rate a higher negative effect on access to pain treatment if tramadol is placed under international control, compared to participants from High Income Countries $(p \leqslant 0.000)$, where other analgesics are available.

Conclusions: Tramadol is widely used by PC and pain professionals. The negative consequences of placing it under international control outweigh any benefits related to curbing misuse, especially for patients living in poor resource settings in all WHO regions, deepening inequity for vulnerable populations.

\section{FC10 Palliative Care in Nursing Homes}

Use of Sedatives at the End of Life in a German Nursing Home: A Pilot Retrospective Cohort Study

Schildmann E., Bolzani A., Meesters S., Marheineke A., Bausewein C. Department of Palliative Medicine, University Hospital, LudwigMaximilians-Universität (LMU) München, Munich, Germany

Background: Sedatives are known to be frequently used at the end of life in specialist palliative care (SPC). There is scarce information about their use in nursing homes.

Aim: To assess

1. the use of sedatives in general and

2. the use of "sedatives with continuous pharmacological effects", based on objective operational criteria, within the last 7 days of life in a nursing home.
Methods: Pilot retrospective cohort study as part of a multi-centre mixed-methods study, using medical records of residents who died in a German nursing home from $1 / 2016$ to $12 / 2017$. Sedatives recorded were benzodiazepines, levomepromazine, haloperidol $\geqslant 5 \mathrm{mg} /$ day and propofol. Explorative statistical analysis (R version 3.4.3).

Results: 30/112 (27\%) deceased residents received any sedative at least once during the last 7 days of life, none labeled "sedation". The term "palliative" was significantly more often documented for residents who received sedatives $(p=0.004)$. Oral lorazepam was used most frequently $(n=28$; midazolam $n=2)$, mainly for anxiety $(n=12)$ and dyspnea $(n=8)$, but also due to "palliative status" $(n=2)$ and "patient's wish" $(n=1)$. The median total daily dose of lorazepam increased from $1 \mathrm{mg}$ (range 0.5 - 3) six days before death to $2 \mathrm{mg}$ (range 0.5 - 5) on the day of death. 17 residents had "sedatives with continuous effects" on at least one day, median 2 days (range 1-7). Two of the three residents under the care of a SPC team received this treatment.

Conclusion: For the first time, this pilot study provides data on sedation practice at the end of life in a German nursing home. Sedatives are frequently used, mostly orally. Median doses are low, probably with a mainly anxiolytic and mild to moderate sedating effect. This stands in contrast to international data on continuous deep sedation. Multi-centre mixed-methods research is needed to describe sedation practices in German nursing homes and inform respective guidance. Funding: Federal Ministry of Education \& Research (SedEol)

FC10 Palliative Care in Nursing Homes Predicting Mortality in People with Dementia and Pneumonia: Time to Update the Risk Score

van der Steen J.T. ${ }^{1,2}$, Heymans M.W. ${ }^{3}$, van der Maaden T. ${ }^{4}$, Mehr D.R. ${ }^{5}$, Kruse R.L. ${ }^{5}$, de Vet H.C.W. ${ }^{3}$, Rauh S.P. ${ }^{3}$

${ }^{1}$ Public Health and Primary Care, Leiden University Medical Center, Leiden, Netherlands, ${ }^{2}$ Primary and Community Care, Radboud University Medical Center, Nijmegen, Netherlands, ${ }^{3}$ Epidemiology and Biostatistics, Amsterdam Public Health Research Institute, Amsterdam UMC, Location VU University, Amsterdam, Netherlands, ${ }^{4}$ National Institute for Public Health and the Environment (RIVM), Bilthoven, Netherlands, ${ }^{5}$ Family and Community Medicine, School of Medicine, University of Missouri, Columbia, United States

Background: People with dementia often die from pneumonia. According to the EAPC dementia white paper, optimal prognostication combines clinical judgement with evidence-based prediction models. However, models may need revision when applied in different (e.g., sicker) populations or when care changes, such as altered management of cardiovascular risk or awareness of sufficient food and fluid intake.

Aims: To evaluate, in a recent cohort, the performance of a model developed in the 1990s for predicting 14-day mortality risk in antibiotictreated nursing home residents with dementia and pneumonia.

Methods: The original prediction model included gender, respiratory rate, respiratory difficulty, pulse rate, decreased alertness, fluid intake, eating dependency and pressure sores. We applied the model to recent pneumonia episodes (380 in the Dutch PneuMonitor study, 2012-2015). Conforming to current standards, we considered additional variables and model revision. Model performance was evaluated by: HosmerLemeshow statistics ( $\mathrm{HL})$, calibration graphs, and area under the receiver operating characteristic curve (AUC) to assess discrimination. We internally validated using bootstrapping.

Results: The newer cohort had lower 14-day mortality and was less often dehydrated or malnourished. Median AUC of the original model over imputed datasets was 0.76 (IQR: $0.76-0.77$ ), compared to 0.80 in the old cohort. Augmenting the model with dehydration, bowel incontinence, increase in eating dependency, and cardiovascular history, while removing pressure sores, improved AUC: 0.80 (IQR: 0.80-0.81) after internal validation. Calibration remained adequate $(\mathrm{HL}: \mathrm{p}=0.67)$. We also revised the risk score for use in practice. 
Conclusion: Despite beliefs that nursing home residents are sicker now, the recent cohort of residents with pneumonia was less severely ill, with lower mortality. Extending the model retained performance to distinguish between those at low and high mortality risk.

\section{FC10 Palliative Care in Nursing Homes}

Does the Namaste Care Family Program Improve Quality of Life of People with Advanced Dementia?

Smaling $H_{.}{ }^{1,2}$, Joling $K^{3}{ }^{3}$, Achterberg $W^{2}{ }^{2}$, Francke A. ${ }^{1,4,5}$, van der Steen J. ${ }^{2,6}$

${ }^{1}$ Department of Public and Occupational Health, Amsterdam UMC, VU University Medical Center, Amsterdam, Netherlands, ²Department of Public Health and Primary Care, Leiden University Medical Center, Leiden, Netherlands, ${ }^{3}$ Department of General Practice and Elderly Care Medicine, Amsterdam UMC, VU University Medical Center, Amsterdam, Netherlands, ${ }^{4}$ Netherlands Institute for Health Services Research (NIVEL), Utrecht, Netherlands, ${ }^{5}$ Expertise Center Palliative Care, VU University Medical Center, Amsterdam, Netherlands, ${ }^{6}$ Department of Primary and Community Care, Radboud University Medical Center, Nijmegen, Netherlands

Background: Many people with advanced dementia die in nursing homes. Family caregivers often find it difficult to communicate meaningfully with them, and judge quality of life and end of life as poor. The daily Namaste Care Family program - derived from the US Namaste Care program - involves family caregivers and integrates personalized and palliative care with meaningful activities for people with advanced dementia. There is some evidence that Namaste Care is beneficial, but this needs confirmation with robust research designs.

Aims: To examine if the Namaste Care Family program improves quality of life of people with advanced dementia in nursing homes.

Methods: Randomized controlled trial in 19 nursing homes, of which ten implemented Namaste Care Family and nine provided usual care. Data collection started May 2016 and will conclude December 2018. Quantitative data about the quality of life of 231 persons with advanced dementia are collected during the baseline assessment and at 1, 3, 6, and 12 months using a validated questionnaire (Quality of Life in LateStage Dementia, referring to last week) and direct observations (Discomfort Scale-Dementia of Alzheimer Type). Data are analyzed using mixed model analysis.

Results: With data collection still ongoing, data analysis for this abstract focused on the first three months. Preliminary results indicate that observed discomfort decreased over time, with the Namaste group showing a significantly lower level of discomfort compared to the control group after 3 months. No group difference was found on the quality of life measure.

Conclusion / discussion: Preliminary findings indicate that Namaste Care Family can decrease observed discomfort of people with advanced dementia. The results for the total research period will be added at the time of the conference.

\section{FC10 Palliative Care in Nursing Homes}

The Complexities of the Use of Palliative Sedation in Nursing Homes: A Qualitative Exploration of Physicians' and Nurses' Perspectives Robijn L. ${ }^{1,2}$, Deliens L. ${ }^{1,2}$, Rietjens J. ${ }^{3}$, Pype P. ${ }^{1,2}$, Chambaere $K^{1,2}$ ${ }_{1}^{1}$ Department of Family Medicine and Chronic Care, End-of-Life Care Research Group at the Vrije Universiteit Brussel and Ghent University, Brussels, Belgium, 2Department of Public Health \& Primary Care, Ghent University, Ghent, Belgium, ${ }^{3}$ Department of Public Health, Erasmus University Medical Center Rotterdam, Rotterdam, Netherlands

Background: While deciding on and performing palliative sedation is replete with challenges, research suggests that the challenges are particularly pervasive in low-care settings such as nursing homes with limited relevant infrastructure and where individual and organisational factors may further complicate good practice.

Aims: This study identifies barriers for the decision-making, communication, and performance of continuous sedation until death in nursing homes as experienced by professionals involved.

Methods: Ten focus groups were held with 71 healthcare professionals including 16 palliative care physicians, 42 general practitioners and 13 nursing home staff (nurses and care assistants), representing a broad range of views and experiences regarding the use of continuous sedation until death in Flemish nursing homes. Grounded theory principles were applied.

Results: Barriers were classified according to three levels.

(1) Personal barriers related to knowledge and skills including the lack of clarity of what sedation should be used for (linguistic ambiguity) and how it should be used (practical ambiguity);

(2) Relational barriers concerning communication and collaboration both between professionals and with the family. Respondents in our discussions indicated e.g. that they do not know how to best approach the family, or that there is multiple contact points for the family as a result of which the family often receive contradictory messages;

(3) Organizational barriers related to the organization of care in nursing homes where e.g. there is no on-site physician responsible for all residents or who is not necessarily a nursing home specialist.

Conclusion: Whilst the findings uncover the complexities in deciding on and performing continuous sedation, they suggest considerable challenges for sound decision making, communication and performance of palliative sedation - particularly communication with the family deserves attention.

\section{FC10 Palliative Care in Nursing Homes}

Physical Limb or Trunk Restraint Use in Nursing Home Residents' Last Week of Life: An Epidemiological Study in 6 European Countries Pivodic L. ${ }^{1}$, Smets T. ${ }^{1}$, Gambassi G. ${ }^{2}$, Kylanen M. ${ }^{3}$, Pasman H.R. ${ }^{4}$, Payne S. ${ }^{5}$, Szczerbińska K. ${ }^{6}$, Deliens L. ${ }^{1}$, Van den Block L. ${ }^{1}$, on behalf of PACE ${ }^{1}$ End-of-Life Care Research Group, Vrije Universiteit Brussel \& Ghent University, Brussels, Belgium, ${ }^{2}$ Universita Cattolica del Sacro Cuore, Rome, Italy, ${ }^{3}$ National Institute for Health and Welfare, Helsinki, Finland, ${ }^{4}$ VU University Medical Center, Amsterdam, Netherlands, ${ }^{5}$ Lancaster University, Lancaster, United Kingdom, 6 Jagiellonian University Medical College, Cracow, Poland

Background: Challenges regarding end-of-life care in nursing homes may foster use of physical restraints, despite evidence that they are neither safe nor effective. We studied the prevalence of physical restraint use in the last week of life of nursing home residents in six European countries and its association with resident and facility characteristics.

Methods: Cross-sectional survey; proportionally stratified random sample of nursing homes in Belgium (BE), England (ENG), Finland (FI), Italy (IT), Netherlands (NL), Poland (PL). We identified all deaths of residents of the preceding three months. Staff (nurse or care assistant) most involved in the resident's care indicated in a structured questionnaire whether trunk or limb restraints were used during the last week of life 'daily', 'less frequently than daily' or 'not used'. Analysis included generalized linear mixed models.

Results: In 322 nursing homes, staff returned questionnaires regarding 1384 deceased residents (response rate $81 \%$ ). Limb and/or trunk restraints were used daily in the last week of life in $8 \%$ (BE), 1\% (ENG), $4 \%(\mathrm{FI}), 12 \%(\mathrm{IT}), 0 \%(\mathrm{NL}), 0.4 \%$ (PL) of deceased residents; and 'less frequently than daily' in $4 \%$ (BE), $0 \%$ (ENG), $0.4 \%$ (FI), 6\% (IT), $0 \%$ (NL), 3.5\% $(P L)$. Older residents $(p=0.006)$ had higher odds of being physically restrained. Restraint use was not significantly associated with resident's 
gender, dementia, staffing level, care intensity within facility, or facility status (public/private, for-/not-for-profit).

Conclusion: A concerning proportion of dying nursing home residents are physically restrained, especially older residents and those in IT and $B E$. Lower restraint use in other countries suggests that they can be prevented. Absence of restraint use in NL indicates that policy strongly advising against it may be effective. These data indicate a need for tailored country-level strategies, such as staff education, to limit restraint use in at-risk countries, facilities and residents.

\section{FC10 Palliative Care in Nursing Homes}

Implementing a Palliative Program in Long Term Care Homes Reduces Hospital Use at End of Life

Kaasalainen S. ${ }^{1}$, Sussman T.S. ${ }^{2}$, Thompson G.T. ${ }^{3}$, Sinclair S. ${ }^{4}$, Brazil K. ${ }^{5}$, Parker D. ${ }^{6}$, SPA-LTC

${ }^{1}$ McMaster University, Hamilton, Canada, ${ }^{2}$ McGill University, Montreal, Canada, ${ }^{3}$ University of Manitoba, Winnipeg, Canada, ${ }^{4}$ University of Calgary, Calgary, Canada, ${ }^{5}$ Queen's University Belfast, Belfast, United Kingdom, ${ }^{6}$ University of Technology Sydney, Sydney, Australia

Background: Palliative care in long term care (LTC) homes is a concern worldwide.

Aim: The aim of this pilot study was to evaluate the Strengthening a Palliative Approach in Long Term Care (SPA-LTC) program in Canada. Methods: A mixed method design was used in four Canadian LTC homes. Pre- and post-intervention surveys were completed by staff and bereaved family members to assess their perceptions about care and resident comfort at end-of-life. Facility-level data was collected on hospital use over a one-year period both pre- and post- intervention. Residents who scored $<40 \%$ on the Palliative Performance Scale and their families were invited to attend a Family Care Conference (FCC). Qualitative interviews were conducted to explore family perceptions about attending a FCC.

Findings: In total, 40 residents/families agreed to participate in the study. Descriptive statistics showed residents who had an FCC tended to have dementia, higher Charlson Comorbidity Index, and were hospitalized more often during the last year. The most commonly discussed domains in FCCs were physical issues, end-of-life and social care; loss and grief were the least common. Family felt well supported at the FCC and that it was helpful for them; the majority of families reported that their concerns and the kind of care and services wished for at end-of-life were addressed at the meeting. There were statistically significant reductions $(p<.05)$ in ER visits and hospital deaths for residents who participated in the intervention. However, there were no significant prepost differences in either: staff comfort in providing palliative care, or bereaved families' perceptions of residents' end of life comfort and families' overall perceptions of care.

Conclusions: The SPA-LTC program shows promise to improve quality of living and dying for LTC residents and their family.

\section{FC11 Bereavement Care}

Does Informal, Community or Professional Bereavement Support Help? An Irish Population Study

Roberts A. ${ }^{1}$, Keegan $0 .{ }^{1}$, Aoun S. ${ }^{2,3}$, Weafer J. ${ }^{4}$

${ }^{1}$ Irish Hospice Foundation, Bereavement Development, Dublin, Ireland, ${ }^{2}$ La Trobe University, Palliative Care Unit, School of Psychology and Public Health, Melbourne, Australia, ${ }^{3}$ Perron Institute, Nedlands, Australia, ${ }^{4}$ Weafer and Associates, Dublin, Ireland

Background: Information about how contemporary grieving is experienced and supported through time in Ireland, as elsewhere, is scarce. This study set out to explore the bereavement experiences and support profiles of bereaved Irish adults.

Aims: The study sought to establish proportions of self-reported bereaved people in Ireland; describe the impact of the bereavement on wellbeing; and investigate experiences of support (formal and informal).

Methods: This study is a population-based cross-sectional study and entailed a telephone survey of 1000 adults in Ireland (response: $\mathrm{n}=908$, $90 \%)$. The survey tool was adapted with permission from an Australian study. For comparability this paper outlines a sub-analysis of those bereaved within the previous two years at the time of data collection $(n=281)$.

Results: Participants accessed informal support (e.g. family friends) most frequently and most found this type of support helpful. Community and professional supports (i.e. formal support) were accessed less frequently and more likely to be found less helpful.

Participants' who reported deterioration in one aspect of wellbeing (i.e. physical health, 75/27\%; mental health, 96/35\%; and financial situation, $52 / 19 \%)$ were more likely to report deterioration in another aspect. In addition, a vulnerable group who did not receive sufficient support were identified ( $n=90,36 \%)$. They were more likely to report a deterioration in wellbeing, rate support from family/friends negatively and more likely to access community or professional supports.

Conclusion: In common with the Australian study, a public health approach to bereavement care is endorsed. Developing guidance for bereavement care may be a first step to address some of the issues identified with formal support. A framework providing guidance on need, corresponding level of service provision and associated staff competencies/training needs is in development.

\section{FC11 Bereavement Care}

The Empty Chair Project: A Qualitative Study Exploring the Impact of Death in the Haemodialysis Community

Durley K.E. ${ }^{1}$, Sutherland S. ${ }^{1}$, Lowney A.C. ${ }^{1}$, Gillies K. ${ }^{1}$, Glogowska M. ${ }^{2}$, Lasserson D. ${ }^{3}$, Pugh C.W..$^{1,4}$

${ }^{1}$ Oxford University Hospitals NHS FT, Oxford, United Kingdom, ${ }^{2}$ Nuffield Department of Primary Care Health Sciences, University of Oxford, Oxford, United Kingdom, ${ }^{3}$ University of Birmingham, Birmingham, United Kingdom, ${ }^{4}$ Nuffied Department of Medicine, University of Oxford, Oxford, United Kingdom

Background: Patients attending haemodialysis units form a community of people who see each other three times a week often for many years. Mortality rates are high and sudden cardiac death is the single most common cause of death amongst these patients. This high risk of sudden death means that the dialysis community commonly experiences unexpected bereavements.

Aims: To explore the experience of the death of a patient on haemodialysis amongst fellow patients on a unit and determine the impact a death has on the surviving patient's understanding of their own mortality.

Methods: This is a qualitative study using semi-structured interviews and was conducted in a tertiary dialysis centre. Patients were purposively sampled and a topic guide was used to inform the interviews. After interviews were transcribed, they were thematically analysed.

Results: 10 participants completed the interviews and data saturation was reached ( 6 female and 4 male with an age range of $42-88$ years). Main findings from the interviews included:

i) Patients described a haemodialysis community which includes haemodialysis nurses as core members

ii) The death of a fellow patient in the haemodialysis unit focused patients' minds on their own mortality and advance care planning

iii) Patients noticed avoidance behaviour by staff in relation to discussing death in the unit and would prefer a culture of open acknowledgment.

Conclusion / Discussion: Acknowledgment of death is of central importance to haemodialysis patients who feel that the staff are part of their 
community. If death is not acknowledged, grief may become disenfranchised. Ethical frameworks in which professionals work may contribute to the avoidance behaviour perceived by the patients.

Funding: Oxfordshire Health Services Research Committee Grant.

\section{FC11 Bereavement Care}

Effects of Receiving a Therapeutic Conversation Intervention Before and in the Bereavement Phase on Bereaved Family Cancer Caregivers Outcomes: A Quasi-experimental Study

Petursdottir A.B. ${ }^{1,2}$, Sigurdardottir V. ${ }^{3}$, Rayens M.K. ${ }^{4}$, Svavarsdottir E.K. ${ }^{5}$ ${ }^{1}$ University of Iceland, Reykjavik, Iceland, ${ }^{2}$ Palliative Home Care Unit, Landspitali University Hospital, Kopavogur, Iceland, ${ }^{3}$ Palliative Care Unit, Landspitali University Hospital, Kopavogur, Iceland, ${ }^{4}$ College of Nursing, University of Kentucy, Lexington, United States, ${ }^{5}$ Faculty of Nursing, University of Iceland, Reykjavik, Iceland

Background: The effect of cancer caregiving may have profund impact on the psychosocial health of family caregivers (FCGs) and become evident post-loss. Providing a family-oriented intervention when preparing for a death of a family member due to cancer, may positively impact bereavement outcomes.

Aim: To evaluate the impact of a Family Systems Nursing strength-oriented therapeutic conversation intervention (FAM-SOTC) on bereaved FCGs outcomes when offered before and after a death of a close relative.

Methods: A quasi-experimental design; 51 bereaved FCGs who had received advanced cancer care from a specialized palliative home-care unit were recruited; 26 FCGs in an experimental group and 25 in a control group. The intervention was delivered at the home of 26 bereaved FCGs (prior participated in a trial intervention) over a 2-year period versus usual care. Two hypotheses regarding positive outcomes of psychological distress and grief reactions were tested.The Depression, Anxiety and Stress, and the Adult Attituded to Grief scales were used to assess the psychological distress levels and grief reactions at 3 time points. Results: The Anxiety (4.53 vs 7.88; $p=0.04 ; 3$ months post-loss) and Stress ( 4.96 vs $9.66 ; p=0.02 ; 6$ months post-loss) subscale scores were significantly lower in the intervention group than in the usual care group. The study also identified lower intensity of vulnerability in grief ( 3 and 5 months post-loss) among bereaved FCGs who received the intervention compared to usual care. However, the depression subscale scores were not statistically significant.

Conclusion: The results support that the FAM-SOTC intervention offered before and after a loss, is of benefit to bereaved family cancer caregivers when compared to usual care.

Funding source: The study was funded by grants from the Landspitali University Hospital Scientific Fund, the Scientific Fund of the Nurses Association in Iceland and the Research Fund of Ingibjorg Magnusdottir.

\section{FC11 Bereavement Care}

Effects of Financial Status on Depression and Grief among Bereaved Family Members of Cancer Patients

Aoyama M. ${ }^{1}$, Igarashi ${ }^{1}{ }^{1}$, Sakaguchi ${ }^{2}{ }^{2}$, Morita T. ${ }^{3}$, Shima $Y^{4}$, Miyashita $M$. $^{1}$

${ }^{1}$ Tohoku University Graduate School of Medicine, Sendai, Japan, ${ }^{2}$ Kwansei Gakuin University, Osaka, Japan, ${ }^{3}$ Seirei Mikatahara General Hospital, Hamamatsu, Japan, ${ }^{4} T$ sukuba Medical Center Hospital, Tsukuba, Japan

Background: Cancer treatment can impact not only the patient's but also their family members' financial status. However, little is known about the level of impact and how this may affect their mental health. Aim: To examine the bereaved family member's current financial status, change before and after bereavement, and its effect on depression and grief.
Methods: We conducted a nationwide cross-sectional questionnaire survey involving 787 bereaved family members of cancer patients in 71 institutions in Japan from May to July, 2016. We asked about their perceived level of concern regarding their current financial status and whether it changed after bereavement. We also collected information on demographic factors, and included the Patient Health Questionnaire-9 and Brief Grief Questionnaire to assess participants' grief and depression, respectively. We conducted bivariate and multivariate analysis to examine the relationship between financial status and these factors.

Results: A total of 491 (62\%) questionnaires were returned. The majority ( $n=382,78 \%$ ) of the participants reported having none or mild concerns about their financial status, while $19 \%(n=95)$ had moderate to severe concerns. Regarding the changes in financial status after bereavement, $7 \%(n=35)$ reported an improvement, $28 \%(n=131)$ reported worsening, and $65 \%(n=308)$ reported no change. The prevalence of possible depression and complicated grief were $22 \%(n=108)$ and $9 \%(n=41)$ respectively, and were significantly lower among participants with less concern regarding their financial status, and if it had not changed after bereavement (both $\mathrm{p}<0.05$ ).

Conclusion/discussion: Almost one-fifth of the bereaved family members reported financial difficulties to some extent which were significantly associated with depression and grief. Our findings provided evidence of the necessity for psychosocial support to family members of cancer.

This work was supported by JSPS KAKENHI Grant Number JP $16 \mathrm{H} 06645$

\section{FC11 Bereavement Care}

A Qualitative Study of Perceptions of Clinical and Non-clinical Supportive Care Interventions among Bereaved Family Members after In-hospital Patient Death

Kelemen $A .{ }^{1}$, Johnson $\mathrm{S}^{2}$, Grimes $\mathrm{C}^{2}$, Groninger $\mathrm{H}^{3}{ }^{3}$

${ }^{1}$ Palliative Care, MedStar Washington Hospital Center, Washington, United States, ${ }^{2}$ Catholic University School of Social Work, Washington, United States, ${ }^{3}$ Georgetown University Medical Center, Washington, United States

Background: Studies show that while most adults prefer to die at home, nearly $60 \%$ die in the hospital. The prevalence of in-hospital death signals a need to study the quality of in-hospital care provided at end-of-life to patients and their families. To date, few studies have focused on families' perceptions of the clinical/non-clinical care (e.g. room setting, visiting hours, lighting in room) they receive in the hospital and its impact on the end-of-life care (EOLC) experience.

Aims: We wished to deepen understanding of in-hospital EOLC experiences of bereaved family members, by identifying quality improvement opportunities regarding non-clinical supportive interventions to family members experiencing in-hospital death of a loved one.

Methods: Between December 2017 and May 2018, we conducted semistructured individual interviews with 18 family members whose loved ones had died at a 926-bed urban tertiary care hospital at least one year prior to the interview. Family members wesre capacitated, Englishspeaking, and over age 18. Interviews were audio-recorded and transcribed. Two independent researchers performed content analysis using QDA Miner.

Results: Participants' in-hospital death experiences organized into themes that encompass both positive (e.g. acceptable symptom management and EOLC communication) and negative (e.g. perception of hastening death) care experiences. Findings highlight aspects of participants' in-hospital care experiences that were helpful and should be continued, along with potential ways of improving hospital-based EOLC, both clinical (e.g. earlier prognostic discussions) and non-clinical (e.g. noise, post-death care, flexible visitation hours).

Conclusion: A year or more after the in-hospital death of a loved one, bereaved family members continue to hold specific memories and 
details of final days of care. These experiences should inform end-of-life care quality improvement processes in the hospital setting.

\section{FC12 Refugees and Migrants}

The Colours and Contours Of Compassion: The Findings from a Systematic Review on the Perspectives of Compassion among Ethnically Diverse Patients and Healthcare Providers

Singh P., King-Shier K., Sinclair S.

Faculty of Nursing, University of Calgary, Calgary, Canada

Background: Compassion is considered a key characteristic of quality healthcare. However, there is a lack of understanding of how different ethnic groups perceive and experience compassion in healthcare.

Aims: To identify and describe the perspectives, experiences, importance, and impact of compassionate care among ethnically diverse population groups.

Methods: A systematic review of peer-reviewed literature published between 1947 to 2017, utilizing a narrative synthesis approach, focused on compassionate care in ethnically diverse populations.

Results: A total of 2296 abstracts were retrieved and screened to include a total of 23 articles. Synthesis of the literature identified the perspectives, facilitators and barriers of compassion in healthcare within ethnic groups. Compassion was described as being comprised of healthcare provider (HCP) virtues (honesty, kindness, helpful, non-judgment) and actions (smile, touch, care, support, flexibility) aimed at relieving the suffering of patients. The importance and impact of providing compassion to ethnically diverse patients was also identified. This review also identified the need for more contextual studies directly exploring the topic of compassion from the perspectives of individuals within diverse ethnic groups, rather than superimposing a pre-defined, enculturated and researcher-based definition of compassion.

Conclusion: This review synthesizes the current evidence related to perceptions of compassion in healthcare among diverse ethnic groups and the role that compassion can play in bridging ethno-cultural differences and associated challenges, along with identifying gaps in literature related to compassionate care within diverse ethnic groups. Establishing an evidence base grounded in the direct accounts of members of diverse ethnic communities can enhance culturally sensitive compassionate care and improve compassion related health outcomes among diverse ethnic groups.

\section{FC12 Refugees and Migrants}

Significant Reductions in Hospital Deaths and Increases in Home Deaths for Cancer Patients over a Decade across All Ethnic Groups in England but Significant Differences in Place of Death Remain Verne J., Pring A., Bowtell N.

Public Health England, Bristol, United Kingdom

Background: Previous studies, based on numbers of patients, suggested that black and minority ethnic groups (BAME) are under-represented in hospice and palliative care provision in England.

Aims: To use national datasets to describe current variation and recent trends in place of death by ethnic group for people who died from cancer.

Methods: The analysis used national mortality data with standardised ethnicity status determined from linked hospital admissions records for patients treated in England and who died between 2008 and 2017 with an underlying cause of cancer. Ethnicity groups: White (W), Black (B), Asian (A) Chinese (C). Age at death, gender, place of death and cause were derived from mortality data. 3-year rolling averages were used because of small numbers of cancer BAME decedents (W 356,189, A 6,885, B 5,317, C 690, 2015-17).

Results: $2008-17$, the proportion of cancer deaths in hospital in all ethnic groups reduced (W 43.0 - 35.5\%, A 54.5 - 47.5\%, B 53.1 - 47.8\%, C 51.0
-42.2) and increased at home (W $26.4-30.5 \%$, A $28.1-34.0 \%$, B 19.2 $24.9 \%$, C $17.7-25.0 \%)$. Significant historic and current differences persist. In 2015-17 significant differences also remain in care home deaths (W 14.7\%, A 4.3\%, B 8.6\%, C 10.7). Asians have fewer deaths in hospice (W 17.8\%, A 12.5\%, B 16.8\%, C 21.0\%). The \% home deaths increase with age for Asian (30.6\% 0-74, 40.3\% 85+) \& Black patients (20.6\%, 31.5\%), but decreased for White patients $(32.2 \%, 25.6 \%)$.

Conclusions: All ethnic groups have benefited from National Palliative Care Policy in England since 2008 which supports patient choice in place of care and death, with more cancer patients choosing not to die in hospital. Persistent higher hospital deaths for BAME may reflect lower referral to Hospital Palliative Care teams who could plan community care. The continuing ethnic differences may represent cultural preferences in place of care as well as inequalities. Further analysis suggests type/time of migration relevant.

FC12 Refugees and Migrants Attitudes, Experiences, Challenges and Wishes from People with Migration Background Concerning End-of-Life Care in Germany Froning F. ${ }^{1}$, Rolke R. ${ }^{2}$, Pastrana T. ${ }^{2}$

${ }^{1}$ Gynecology and Obstetrics, Klinikum Hochsauerland, Aachen, Germany, ${ }^{2}$ Palliative Care, Uniklinikum Aachen, Aachen, Germany

Background: Demographic changes such as an aging society and increasing migration demand increased empirical research activity concerning perspectives of people with migration background regarding health care at the end of life. Compared to international researcher results Germany lacks involvement in the issue and needs to catch up.

Objective: The objective of this research project was to identify attitudes, experiences, challenges and wishes regarding end-of-life care from the perspective of affected people in palliative situations with migratory background.

Methods: Explorative, open and three-phasic interviews according to the concept of Honer were conducted after carrying out a systematical literature review. The interviews were analyzed by grounded theory approach using the software of MaxQDA 11.

Results: Attitudes, challenges and wishes of patients with migration background and palliative needs regarding end-of-life care are highly individual and unforeseeable. However, relevant topics could be identified. Namely, the importance of the family as support as well as source of conflict, personal coping-strategies, the bargaining about the preferred place of dying and burial, the obstacles to good palliative care such as the lack of knowledge, language barriers, experiences of discrimination and disregard in health care systems as well as the desire for quality of life, normality, analgesia, empathic care and support in organisational affairs. Conclusion: Based on the results the consequent involvement of language mediators as well as the clinical Ethics Committee for unsolvable conflicts is urgently needed. Furthermore target-oriented information delivery on palliative care as well as advanced care planning by health care institutions and health care workers concerning individual and migration specific context factors for an adequate health care at the end of life without stereotyping, generalizations and (positive) racism is vital.

\section{FC12 Refugees and Migrants} Integration of Palliative Care into Healthcare Provision for South Sudanese Refugees in Adjumani District, Uganda: A Rapid Systems Appraisal Evaluation

Leng M..$^{1,2,3}$, Opia V. ${ }^{4}$, Purewal G. ${ }^{2}$, Venkateswaran $C^{5}{ }^{5}$ Namukwaya E. $^{1}$, Bagasha P. ${ }^{1}$, Grant L. ${ }^{3}$, Downing J. ${ }^{1}$

${ }^{1}$ Internal Medicine, Makerere and Mulago Palliative Care Unit, Kampala, Uganda, ${ }^{2}$ Cairdeas International Palliative Care Trust, Aberdeen, United Kingdom, ${ }^{3}$ Global Health Academy, Edinburgh University, Edinburgh, United Kingdom, ${ }^{4}$ Peace Hospice, Adjumani, Uganda, ${ }^{5}$ Mehac, New Delhi, India 
Background: In humanitarian settings palliative care $(\mathrm{PC})$ is rarely prioritised. New WHO \& SPHERE guidelines support integration. Evidence is needed from field settings to inform service development \& enable integration within a health system strengthening approach. Uganda hosts one of the largest refugee populations and in Adjumani District the 240,000 population hosts 260,000 refugees.

Aim: To evaluate the setting \& systems affecting chronic disease \& health related suffering for refugee and host populations in Adjumani District.

Methods: Using a Rapid Systems Appraisal all documentation were reviewed and roles of key mapped. Interviews were conducted with 4 groups: leaders in the refugee and host community, Ministry of Health, Peace Hospice and humanitarian stakeholders; those living with chronic disease including mental health issues; PC providers \& those who refer or interact with $\mathrm{PC}$, alongside field observations.

Results: From 69 qualitative interviews in five refugee communities the following themes emerged: PC is missing in almost all communities and recognised by stakeholders 'It has been a key lacking point'; challenges in coordination, and integrating resources within the fragile health systems 'integration is the way forward'; cultural barriers to care; significant stigma and effects of trauma, limited PC education, need and impact of PC from patients and families. PC 'is like losing hope - then hope appears suddenly like the sun'.

Conclusion: At all levels PC service integration and training are needed in this excluded population. Refugee communities must have empowerment and ownership to ensure issues of culture and stigma are understood and host communities included to avoid a 2 tier system. Further evidence on the palliative care need will be collected alongside a comprehensive education package for health care workers, village health teams and family caregivers. $\mathrm{MOH}$ and humanitarian stakeholders are being included and to allow wider scale up.

\section{FC12 Refugees and Migrants}

Do Patients with a Migrant Background Present a Challenge for Palliative Medicine? The Limitations of Palliative Care from the Perspective of Clinical Personnel

Schade F., Banse C., Owusu-Boakye S., Jansky M., Nauck F.

Clinic of Palliative Medicine, University Medical Center Goettingen, Goettingen, Germany

Background: Studies show that access to palliative care is difficult for patients with a migrant background. To date, little attention has been paid to the challenges faced by clinical personnel when providing care to such a group of patients that is perceived as being underrepresented, and how these personnel explain the conflicts with patients from a migrant background.

Aims: The objective of this study, which was conducted in Germany, was to find out how clinical personnel experience problems and conflicts when providing palliative care to people with a migrant background, and which causes and conditions they identify.

Method: Qualitative research design, narrative interviews with health care professionals $(n=20)$ working in palliative care. Interpretative analysis using Grounded Theory.

Results: A key result of the study shows that clinical personnel regard a lack of routine in dealing with patients from a migrant background as a problem in providing care. Furthermore, they perceive high expectations of patients who are unfamiliar with the palliative concept. The health care professionals interviewed sometimes feel that they are in rigid (political, legal or everyday) structures and experience a standardisation of the healthcare system that gives little consideration to the migrant background. This makes it difficult to fulfil patients' wishes. The result is insecurity in dealing with patients, which is expressed in various conflicts.

Conclusion / Discussion: With the help of an explanatory model the research project was able to work out the causes and conditions that make it difficult to care for people with a migrant background. The conflict between expectation of care and the reality faced by the patient is experienced as difficult to manage. The standardisation of the healthcare system should be questioned, and an intercultural opening of palliative medicine is desirable.

FC12 Refugees and Migrants

High Admittance to Palliative Care Team and Low Admittance to Hospice for Immigrants from Non-Western Countries. A Nation-wide Register-based Study of Patients with Cancer Adsersen M. ${ }^{1}$, Thygesen L.C. ${ }^{2}$, Kristiansen M. ${ }^{3,4}$, Hansen M. B. ${ }^{1,3}$, Neergaard M.A. ${ }^{5}$, Petersen M.A. ${ }^{1}$, Grønvold M. ${ }^{1,3}$

${ }^{1}$ Department of Palliative Medicine, Bispebjerg Hospital, The Research Unit, Copenhagen, Denmark, ${ }^{2}$ University of Southern Denmark, Copenhagen, Denmark, ${ }^{3}$ Department of Public Health, University of Copenhagen, Copenhagen, Denmark, ${ }^{4}$ Center for Healthy Aging, University of Copenhagen, Copenhagen, Denmark, ${ }^{5}$ The Palliative Team, Department of Oncology, Aarhus University Hospital, Aarhus, Denmark

Background: The population of immigrants in Europe is aging. Accordingly, the number of immigrants with chronic and life-threatening diseases and a need of specialised palliative care (SPC) will increase. Admittance to SPC for immigrants has mainly been studied in US and in relation to hospice, while it is rarely investigated in a European context. Aim: To investigate whether country of birth was associated with admittance to SPC, overall and separately for hospital-based palliative care team and hospice ('type specific').

Methods: The data sources were the nation-wide clinical database, Danish Palliative Care Database and several other nation-wide registers. The associations between country of birth and admittance to SPC, overall and type specific, were investigated in logistic regression analyses adjusted for sex, age, cancer diagnosis, residential geographic region in Denmark, cohabitation status, income and education.

Results: In 2010-16, 104,611 patients died from cancer in Denmark. Of these, $96 \%$ were born in Denmark, $2 \%$ in other Western and $2 \%$ in nonWestern countries. Overall admittance to SPC was higher for immigrants born in other Western $(\mathrm{OR}=1.2 ; 95 \% \mathrm{Cl}: 1.1-1.3)$ and non-Western countries $(\mathrm{OR}=1.4 ; 95 \% \mathrm{Cl}: 1.3-1.5)$ than for Danish born. Similar results were found for admittance to hospital-based palliative care team. No difference in admittance to hospice was found for immigrants born in other Western countries $(\mathrm{OR}=1.1 ; 95 \% \mathrm{Cl}: 1.0-1.2)$ compared to Danish born, while lower admittance was found for non-Western immigrants (OR= 0.7; 95\% Cl: 0.6-0.8).

Conclusion: Immigrants born in other Western and non-Western countries had higher admittance to hospital-based palliative care team, and immigrants from non-Western countries had lower admittance to hospice, compared to Danish born. To ensure that admittance to SPC is based on the patient's wish, and not country of birth, health care professionals should have the knowledge and skills to handle cultural differences.

\section{FC13 Communication}

"Massage the Heart", "Break Some Ribs", "Give You an Electric Shock": Explanations of CPR During Hospital Admission Interviews with Geriatric Patients

Sterie A.-C., Jones L., Jox R., Rubli Truchard E.

CHUV, Chair of Geriatric Palliative Medicine, Lausanne, Switzerland

Background: International standards stipulate that patient wishes regarding cardiopulmonary Resuscitation (CPR) have to be elicited and documented in the case of a hospitalization. Although it is known that patient preferences are influenced by the way physicians provide information, there is little research into how CPR is explained by physicians at patient's hospital admission. 
Aim: This project used natural data to explore the ways in which CPR is explained in physician-patient discussions in a Swiss geriatric rehabilitation hospital.

Methods: Fifty-one patients and 17 physicians consented to their hospital admission interviews being audio-recorded. Discussions about CPR were transcribed verbatim. Qualitative discursive thematic analysis was combined with a quantitative content analysis to analyse how CPR was explained to the patients.

Results: Resuscitation is poorly explained by physicians. Explanations about CPR mostly concern the steps involved (essentially cardiac massage, intubation, and defibrillation) and the unpredictability of the outcome. Physicians warn that risks and complications are possible, especially when old age is involved, but rarely provide details of what they might entail. Chances of survival after CPR are only mentioned in one conversation. On the patients' side, CPR is frequently referred to as "abusive life extension" or "futile care", but their comprehension of the procedure is rarely unpacked.

We discuss two roles that CPR explanations may play: before asking for the patient's wish, as means to enable the patient to make an informed decision, or after the patient's decision, as a clarification and confirmation of it.

Discussion: Explaining CPR is essential for equipping patients with the information that they need to make autonomous decisions about their future care. The lack of such explanations highlights the needs and opportunities for training as means of improving physician-patient communication on sensitive topics.

\section{FC13 Communication}

Is Information-provision about Benefits and Risks of Treatment Options Associated with Receiving Person-centered Care? A Survey among Incurable III Cancer Patients

\section{van Vliet $L{ }^{1,2}$, de Veer $A .{ }^{1}$, Raijmakers $N .^{3}$, Francke A. ${ }^{1,4}$}

${ }^{1}$ Nivel (Netherlands Institute for Health Services Reserach), Utrecht, Netherlands, ${ }^{2}$ Dept of Health, Medical, and Neuropsychology, Leiden University, Leiden, Netherlands, ${ }^{3}$ Netherlands Comprehensive Cancer Organization (IKNL), Utrecht, Netherlands, ${ }^{4}$ Department of Public and Occupational Health, Amsterdam Public Health Research Institute, Amsterdam UMC, Vrije Universiteit Amsterdam, Amsterdam, Netherlands

Background: In an increasingly complex medical world, incurable ill cancer patients are confronted with various life-prolonging or symptomrelieving treatment options. Being informed of treatment benefits and risks is important, but information-provision might be suboptimal. We assessed the extent to which patients with incurable cancer feel informed about benefits and risks of possible treatments, and whether this relates to their self-perceived receipt of person-centered care.

Methods: Patients with incurable cancer ( $N=212$, nation-wide sample) reported the degree to which they felt informed about treatment benefits and risks. Person-centered care was operationalized as 'feeling involved in care' and 'feeling that preferences were taken into account'. (Logistic) regression analyses assessed the relation between feeling informed and receiving person-centered care, exploring moderating influences of background characteristics (age, education, gender).

Results: Two-third (66\%) of patients felt incompletely informed about treatment benefits and risks. Two-third (65\%) of patients felt always involved in their care and $60 \%$ felt that their preferences were taken into account by all providers. If patients felt completely informed, they also felt they received more person-centered care $(p=<0.01)$. $76 \%$ and $81 \%$ of completely informed versus $58 \%$ and $50 \%$ of incompletely informed patients felt they were, respectively, always involved and preferences were taken into account by all providers. Background characteristics did not moderate these relationships, but influenced perceptions of received information and person-centered care.
Conclusion: Complete information provision about treatment benefits and risks is, according to cancer patients, not commonplace. Yet such information is related to receiving person-centered care. More research into what specific information is preferred, provided and remembered is warranted, to achieve optimal information provision and person-centered care.

\section{FC13 Communication}

"Optimistic or Realistic? He's a Dying Man to Me.": Incorporating Patient and Relative Views in the Design of a Complex Intervention for Managing Clinical Uncertainty Yorganci E. ${ }^{1}$, Evans C. ${ }^{1,2}$, Yi D. ${ }^{1}$, Gao W. ${ }^{1}$, Barclay S. ${ }^{3}$, Pickles A. ${ }^{4}$, Murtagh F.E.M. ${ }^{1,5}$, Johnson H. ${ }^{1}$, Wilson R. ${ }^{1}$, Higginson I.J. ${ }^{1}$, Koffman J. ${ }^{1}$ 1Palliative Care, Policy and Rehabilitation, Cicely Saunders Institute, London, United Kingdom, ${ }^{2}$ Sussex Community NHS Foundation Trust, Brighton General Hospital, Brighton, United Kingdom, ${ }^{3}$ Primary Care Unit, Department of Public Health and Primary Care, University of Cambridge, Cambrige, United Kingdom, ${ }^{4}$ Clinical Trials Unit, King's College London, London, United Kingdom, ${ }^{5}$ Wolfson Palliative Care Research Centre, Hull York Medical School, University of Hull, Hull, United Kingdom

Background: Interventions designed to manage clinical uncertainty are often based on clinicians' views. The AMBER care bundle, a complex intervention, places emphasis on training healthcare professionals to discuss and manage uncertain recovery. But patient and relative views must be incorporated into interventions before implementation and evaluation, to ensure good acceptability.

Aim: Explore hospital patient and relative views about care for uncertain recovery as part of feasibility cluster RCT of AMBER care bundle.

Methods: Semi-structured qualitative interviews. Purposively sampled (by trial arm, disease group, age, gender) patients and relatives receiving care in the cluster RCT. Findings analysed thematically using Framework approach.

Results: 12 intervention, 12 control interviews with 8 patients and 17 relatives at 4 hospitals in England. Overall, participants in both trial arms had insight into their uncertain clinical situation; discussions about uncertain recovery, treatment escalation decisions, and resuscitation status were acceptable to them. Three themes to refine intervention delivery emerged:

(i) honest discussions about uncertainty were sought - participants spoke of euphemistic reference to their situation;

(ii) brief, frequent updates from nursing staff - often discussions included clinicians who were not always on the wards;

(iii) relatives as experts - their prior knowledge was often overlooked, causing distress.

Conclusions: Whilst participants accepted the situation, healthcare professionals require further training to increase honesty and avoid euphemism. Access to up to date information from ward staff other than clinicians is essential, as is acknowledgement that relatives are part of the 'unit of care'. Co-production of interventions with patient and relative input could lead to refinement of interventions at earlier stages.

\section{FC13 Communication}

Being the Child of a Parent with a Life-threatening Illness: Minor Children's Self-reports on Illness-related Information and Family Communication

Eklund . $^{1}$, Kreicbergs U. ${ }^{1,2}$, Alvariza A. ${ }^{1,3}$, Lövgren M.1,2

${ }^{1}$ Department of Health Care Science/Palliative Research Center, Ersta Sköndal Bräcke University College, Stockholm, Sweden, ${ }^{2}$ The Department of Women's and Children's Health, Paediatric Oncology and Haematology, Karolinska Institutet, Karolinska University Hospital, 
Astrid Lindgren Children's Hospital, Childhood Cancer Research Unit, Stockholm, Sweden, ${ }^{3}$ Capio Palliative Care, Dalen Hospital, Stockholm, Sweden

Background: Open and honest communication between parents and children when a parent has a life-threatening illness seems to be important in helping the children in everyday life. Previous research on bereaved children shows that inadequate information and poor communication between family and professionals during the illness trajectory increase the risk for long-term psychological distress years after the loss. Aim: To explore minor children's self-report of illness-related information and family communication when living with a parent with a lifethreatening illness who received specialized palliative home care.

Methods: This study used baseline questionnaire data from an intervention that aimed to open up for communication about the parent's illness and support the family in their situation. A total of 48 minor children (aged 7-19 years) from 30 families were recruited from four specialized palliative home care units in Stockholm, Sweden. The questionnaire data were analysed with descriptive statistics.

Results: All but one of the 48 children reported that someone had told them about the parent's illness; however, two-thirds (32/48) wanted more illness-related information. When asked whether they could talk about how they felt or show their feelings to someone in the family, nearly half of the 20 children aged 8-12 years reported themselves partially or completely unable to do so. Half of the children in the same age group wanted to be able to talk and/or show more about how they felt. A quarter of the teenagers reported that they had questions about the illness that they did not dare to ask.

Conclusion: Minor children of parents with a life-threatening illness want and request more communication about illness-related issues and their own feelings. To support these children, interventions should be developed that foster family communication and improve communication between family/children and healthcare professionals.

\section{FC13 Communication}

Do our Estimates Correlate with What We Disclose to Advanced Disease Patients?

\section{Tavares $F^{1,2}$}

${ }^{1}$ Centro Hospitalar Universitário Lisboa Norte,EPE, Medicina Paliativa, Lisboa, Portugal, ${ }^{2}$ Nucleo de Cuidados Paliativos, Centro de Bioética, Faculdade de Medicina de Lisboa, Lisboa, Portugal

Three discrete types of prognostic conversations (PgC) have been described in Palliative Care (PC), based on 8 communication elements. However, it is unclear to what extent predictions of survival are included and influence the style, content and framing of prognostic disclosure.

Aim: To investigate whether clinical survival estimates (CPS) PaP score and modified Glasgow Prognostic Score (mGPS) correlate with specific patterns of $\mathrm{PgC}$ to patients with advanced diseases.

Methods: Prospective, single-centre, descriptive study; for a month, immediately after every hospital-based PC program consultation, a "communication checkbox" (initiator, timing of discussion, topic, quantity, conditionality, elicited emotions, optimistic and pessimistic cues about prognosis) was completed by the physician. Demographic and clinical data (including a CPS estimate in weeks), PaP score, and mGPS were collected. Survival at one month after the last inclusion was determined. Descriptive analysis and nonparametric tests for differences and correlations between groups were used.

Results: One hundred and fifty-six PgC, from 103 patients (58\% female, median age $76,12 \%$ non-cancer, $18 \%$ inpatient, $48 \%$ no disease-modifying-treatment, $32 \%$ terminal or unstable phase) were approached. A PgC was impossible in 6 cases. The patient participated in $78 \%$ of conversations; $14 \%$ included only family members. Sixty four percent of patients were alive at study end. Significant correlations were found between both CPS and PaP and the pattern of PgC: "navigating options and goals" median CPS 13 weeks / PaP 4.0; "facilitating quality of life goals" median CPS 6 weeks / PaP 7.0 and "preparing for end-of-life" median CPS 2 weeks / PaP $13.0(p<0.001)$. The mGPs risk groups were associated with distinct PgC - for a mGPS $=2$ doctors prepared $78 \%$ for end of life vs $7 \%$ of those with a $\mathrm{mGPS}=0(p<0.001)$.

Our results support that $\mathrm{PgC}$ styles may reflect clinicians' subjective but also more objective estimates.

\section{FC13 Communication}

Factors Associated with Accurate Prognostic Awareness in Patients with Cancer: A Systematic Review

Vlčková K., Tučková A., Poláková K., Loučka M.

Center for Palliative Care, Prague, Czech Republic

Background: Prognostic awareness (PA) relates to patients' capacity to understand their prognosis and the likely future illness trajectory. There is mixed evidence about which factors have positive or negative effect on PA in patients with cancer. The aim of this study is to synthesize the available literature on factors associated with accurate prognostic awareness.

Methods: Four databases (MEDLINE, CINAHL, Embase, Psyclnfo) were searched within the data range from 1990 to December 2017 including studies reporting on adult patients with cancer. The initial search terms had three components and all of them were combined using operator AND: 1 . cancer; cancer staging; 2 . awareness; health knowledge; attitudes; understanding; 3. prognosis; diagnosis; perception. Standard procedures for systematic reviews were used for data extraction. Methodological quality of identified studies was assessed by Joanna Briggs appraisal tools. Results: Out of 26597 articles 61 met the inclusion criteria. In these studies, we found 87 of factors to be related to PA. Included studies differ in a way of measuring prognostic awareness, in methodology and study design. Identified factors were divided into 4 thematic groups: personal factors, disease factors, psychological factors and factors related to illness trajectory.

Conclusion: Prognostic awareness is influenced by number of factors, reflecting patients' health status as well as psychosocial context of their situation. This complexity must be taken into account during communication about prognosis with patients and their families. More research is needed on the role of some specific factors such depresivity, quality of life, fatigue and anxiety.

FC14 The Contribution of Communities

A Therapeutic Community? Informal Interactions in Cancer Treatment Settings - An Ethnographic Study

Grant M..$^{1,2}$, Philip J. ${ }^{1}$, Deliens L. ${ }^{3}$, Komesaroff P. ${ }^{2}$

${ }^{1}$ University of Melbourne, VCCC Palliative Medicine Research Group, Melbourne, Australia, ${ }^{2}$ Department of Primary Health, Monash University, Melbourne, Australia, ${ }^{3}$ End-of-Life Care Research Group at the Vrije Universiteit Brussel and Ghent University, Brussels, Belgium

Background: The role of relationships and exchanges outside the clinical encounter has attracted relatively limited study. In hospital settings patients, visitors and staff engage in numerous informal interactions. While many of these may be inconsequential, other encounters may be of significant importance as a forum to share information, experiences, support and companionship.

Aim: This study proposes the novel idea that "informal interactions" play an important role in cancer treatment settings, and it seeks to explore their nature and content.

Methods: The researchers conducted an ethnographic study in cancer treatment settings in an Australian hospital from April 2017 to June 2018. Data was collected through passive observation and recorded as field-notes. Analysis was conducted through a hermeneutic framework structured on vignettes, a novel technique informed by I-poem analysis. 
Results: Informal interactions are widespread in the hospital space, exist in a diverse array of forms and purpose. Frequently these sporadic encounters addressed profound issues, such as mortality, emotional distress, significant gaps in information, and social stressors. The interactions may serve multiple purposes, including therapeutic, supportive, educational, social, and sharing of illness discourses. The language used in informal encounters was narrative, in contrast to the logico-scientific language of formal encounters. These interactions existed within a network of relationships in the hospital and pre-existing social ties, in which other individuals would enter into these encounters.

Conclusions: These interactions represent a wide network of informal communities that remain unrecognised within the hospital environment. They frequently focus on needs which are identified as being inadequately met through formal health provision. These interactions represent forums where patients and carers may engage in shared discourse regarding health concerns and narratives of illness.

FC14 The Contribution of Communities

Compassionate Communities.

Hospice from Childrens Point of View - Talking with Children about

Life and Death

Mejdal S., Thomsen I.H.

Hospice Limfjord, Skive, Denmark

Every year 1400 Danish school children lose a parent while 12.000 school children are living with the loss of a parent. That is an average of 6-7 children in every school in DK. $10 \%$ of these children have a prolonged and complicated grief process. Although death is more openly talked about now compared to just a few years ago, it still remains a taboo in DK and this fact complicates the grief process for children who lose a parent.

This project aims to make the subject of death less taboo. It also seeks to communicate to pupils that death is part of life and that it is important to support a grieving friend.

Design: This project is a action research project, which in collaboration with the Municipality seeks to develop an educational programme that focuses on death and the act of talking about death. Students, teachers, and local priests are participants alongside the project supervisors. The educational programme is developed from the principles of 'cooperative learning'.

Results: A three hour educational programme has been developed, and is hosted by Hospice with the following themes: Palliative care, grief and euthanasia. The content has been developed on an on-going basis in collaboration with the participants. 22 classes and a total of 489 students and their teachers have participated in the programme. During sessions, the students were very inquisitive and actively engaged. There are written evaluations from each session. They show that the students have gained greater insight about death and grief reactions - insights that the students believe can help them the next time a friend is grieving. Votes after sessions show that students have become more nuanced in their opinions.

Conclusion: The educational sessions have contributed to making death less of a taboo subject and have strengthened the students' willingness to support a grieving friend. The concept could with benefit be extended to the rest of the country's schools in collaboration with a local hospice.

\section{FC14 The Contribution of Communities}

Developing the Death Literacy Index: The Progress so far Noonan K. ${ }^{1,2,3}$, Leonard R. ${ }^{1}$, Horsfall D. ${ }^{1}$, Rosenberg J. ${ }^{4}$, Rumbold B. ${ }^{5}$, Grindrod A. ${ }^{5}$, Kelly M. ${ }^{1}$

${ }^{1}$ Social Sciences and Psychology, Western Sydney University, Penrith (Kingswood), Australia, ${ }^{2}$ The GroundSwell Project, Woodford, Australia, ${ }^{3}$ Palliative Care, Liverpool Hospital, Liverpool, Australia, ${ }^{4}$ NHMRC Centre for Research Excellence in End of Life Care | End of Life Directions for Aged Care [ELDAC], Queensland University of Technology, Kelvin Grove, Australia, ${ }^{5}$ Palliative Care, La Trobe University, Melbourne, Australia

Background: Despite growth in public health approaches to palliative care, there is limited research demonstrating its impact. In particular, how community development programs may contribute to transforming current service dominant approaches to end of life care. The death literacy index (DLI) is being developed to address this gap. We define Death Literacy as knowledge about, and understanding of the death system to strengthen community capacity to take action on caring at end of life. Aims:

1. To develop a rigorous, comprehensive, useful and generalizable DLI;

2. As a tool to measure the impact of interventions; and

3. Determine the state of death literacy in Australia

\section{Methods:}

1) Extensive qualitative research had been conducted with carers, communities, and service providers across Australia

2) Focus groups and interviews with national and international experts were conducted to develop DLI items.

3) A representative sample of Australian adults completed an online survey.

Exploratory and confirmatory factor analyses, structural equation modelling and regression analyses were used to identify the DLI and its relationship to demographic variables and people's previous experience with death, dying and bereavement.

Results: The analysis showed that death literacy has conceptual validity. That is, it can be reasonably measured. The snapshot of Australians death literacy challenged some assumptions regarding how people learn about death and dying and how many people are able and willing to contribute to caring for people at end of life. These have implications on the development of social interventions and programs we will further discuss these in this paper.

Conclusion: The death literacy index is a promising development in our understanding of community knowledge and know-how related to dying, death and loss. The DLI can provide baseline measurements for assessing change and cross cultural understandings of death literacy.

\section{FC14 The Contribution of Communities}

The International Transfer and Translation of 'Death Café': A New Social Movement?

Richards N. ${ }^{1}$, Koksvik G. ${ }^{2}$, Gerson S.M. ${ }^{2}$, Clark D. ${ }^{2}$

${ }^{1}$ Social and Political Sciences, University of Glasgow, Dumfries, United Kingdom, ${ }^{2}$ University of Glasgow, Dumfries, United Kingdom

Background: Death cafes are 'pop up' events where people, usually strangers, meet over food and drink to discuss issues related to death and dying. The aim is to encourage people to discuss more openly their thoughts about their own mortality. Death cafes are often positioned alongside other 'compassionate community' actions but to date there has been very little research into them.

Aims:

1. To identify how the death cafe initiative has spread around the world and what form death cafés take in different cultural contexts

2. To assess to what extent the motivations of death café organisers reflect the original ethos of the death café/'café mortel' initiative 
3. To determine how far death cafes can be considered a new social movement.

Methods: This was an inductive, qualitative design, involving semi-structured interviews of death café facilitators from as many countries as possible. Interviews were conducted via Skype. Thematic analysis was undertaken following close reading of interview transcripts and team discussions of emerging themes.

Results: Thirty-eight interviews were conducted with death café facilitators in 31 countries. Most death cafes take place in high income countries in which death is professionalised and medicalised, with resulting social attitudes. Death cafes in the 'global south' are often run by expatriots. While there is evidence of widespread respect/reverence for the original vision behind death cafe, we found wide variation in how death cafés are run, and specifically whether organisers comply with the ethos that death cafes are non-facilitated, non-didactic events.

Conclusion / Discussion: This is the first study to show that death cafés have spread to a significant number of countries globally. The death café concept has significant and growing public recognition and is being used by activists in the death positivity movement as a tool for reconfiguring people's relationship with death and dying.

\section{FC14 The Contribution of Communities}

Hospices Harnessing Communities: Challenges and Benefits of Community Volunteering Programmes

Scott R. ${ }^{1}$, Hindmarch J. ${ }^{2}$

${ }^{1}$ School of Education and Social Work, University of Dundee, Dundee, United Kingdom, ${ }^{2}$ Hospice UK, London, United Kingdom

Background: Hospice volunteers in the UK are now much more involved in directly supporting patients and carers in the community. In order to inform future development, Hospice UK undertook a study to explore the number of programmes, structures, management, and impact of such volunteering. This abstract reports on the extent, challenges and benefits of UK adult hospice community volunteering projects.

Aims: The aims were to:

- assess the extent of hospice community volunteering programmes

- $\quad$ understand the different structures, management and costs

- identify challenges and benefits

Methods: The study sample comprised all member organiations of Hospice UK. An online questionnaire was sent to all 225 hospices of which 115 were adult hospices. This was followed by a number of semistructured interviews to explore emerging themes in more depth. A framework approach was used for the analysis of qualitative data and quantitative data used descriptively.

Findings from adult hospices: In total 80 adult hospices had community volunteering programmes (CVP) (70\%). Of the 35 hospices without CVP, 21 had plans to develop this. Only 4 hospices considered their CVP to be volunteer led $(7 \%)$.

Challenges included:

- funding and resources

- recruitment of suitable volunteers in the right location

- managing flexibility and availability of volunteers

- balancing the number of referrals with the number of volunteers and managing boundaries

Benefits included improved:

- wellbeing, independence, and reduced isolation (patients and carers)
- health and mobility (patients)

- management of illness, stress and anxiety (patients)

- access to care and other services (patients and carers)

- respite for carers and reduction in carer burnout

- $\quad$ caring relationships

Conclusions: Hospice community volunteering models in the UK are growing along with the desire to move towards informal community led models. As a result of the study, an online resource hub is being compiled to support hospices with such development.

FC15 Reflective Practice, (Resilience) and Self-Care Strong Emotional Reactions Experienced by Doctors in Palliative Care: The Causes, Management and Implications

Hubik D. ${ }^{1}$, Dwyer J. ${ }^{2}$, O'Callaghan C. $^{2}$

${ }^{1}$ Psychosocial Cancer Care, St Vincent's Hospital Melbourne, Fitzroy, Australia, ${ }^{2}$ Psychosocial Cancer Care, St Vincent's Hospital Melbourne, Melbourne, Australia

Background: To date, research on doctors' strong emotional reactions (SER) to clinical work and their impact has not focused on those directly working in specialist palliative care. Understanding how doctors emotionally manage working in palliative care has implications for patient care as well as doctor wellbeing.

Aim: We sought to explore what experiences led to SER when working as a doctor in specialist palliative care and to identify the implications (if any) for practice.

Design: A qualitative descriptive design was used which included grounded theory techniques. Semi-structured, audio-recorded interviews explored doctors' memories of strong emotional reactions (SER) and challenging aspects in palliative care work, how emotions were managed and views about support strategies.

Setting: The study setting was a specialist palliative care service within a public health network in Melbourne, Australia. The palliative care service comprised two inpatient units, a consult service and outpatient clinic.

Results: Twenty doctors participated in interviews with mean length 38 $\min (S D=11 \mathrm{~min})$. Three themes and 13 category findings became apparent as data analysis proceeded. Experiences that were found to elicit SER included patient, family and staff distress as well as system issues. SER were found to impact clinical behaviours and patient care as well as a doctor's personal life. Strategies developed for managing SER included debrief and self-reflection as well as non-work strategies. Doctors not skilled in debrief were found to struggle.

Conclusion: This study provides an important insight into what experiences lead palliative care doctors to experience strong emotional reactions and how they are subsequently managed. Whilst challenging experiences are unavoidable and necessary to help a doctor develop strategies to deal with subsequent experiences, doctors need to be supported through this to avoid adversely impacting patient care or doctor wellbeing.

FC15 Reflective Practice, (Resilience) and Self-Care The Internal World of the Schwartz Round in an Acute Trust Meystre C. ${ }^{1}$, Chaplin D. ${ }^{2}$

${ }^{1}$ Haematology Oncology, University Hospitals Birmingham, Solihull, United Kingdom, ${ }^{2}$ Corporate Nursing, Patient Experience and Bereavement Service, University Hospitals Birmingham, Birmingham, United Kingdom

Background: Schwartz Center Rounds (SCR) originated in the US to foster compassionate care, coming to UK Hospices and Trusts in 2009. Uptake rose after a mention in the 2013 Francis Report. SCR are a venue for discussion of the experience of care delivery and facilitators ensure 
process discussion does not supervene. Benefit has been demonstrated in repeated attendance which reduces isolation, increases teamwork, communication, empathy, and compassion towards colleagues and patients. This is important for staff and patient well-being and may help recruitment and retention, stress, burnout and clinician suicide. Attendee feedback, focus groups and staff surveys have been investigated, but not SCR content, which is relevant to the investigation of effectiveness.

Aims: To undertake a mixed methods evaluation of the Schwartz Rounds within an Acute Trust.

Methods: Quantitative analysis of feedback from 20 rounds using inferential statistics and Interpretative Phenomenological Analysis of 23 rounds facilitator notes. Consent to share the learning but not attribute content is agreed at each SCR.

Results: 55\% attendees were doctors; $8 \%$ nurses; $8 \%$ PAM; $4 \%$ other $25 \%$ undeclared. $71 \%$ gave feedback. $80 \%$ rated rounds excellent or exceptional, with no difference between doctors and other staff. 'Developing insight into how others think and feel in delivering care', scored higher than 'knowledge to deliver patient care'. 7 superordinate themes were: Alone and Fearful; Chaos and Tumult; Psychological Defences; Failure and Loss; Recognising Humanity; Responsibility and Courage; and Encouragement. Stress from death and dying was a common coding item.

Conclusion: SCR rate highly but nurses rarely attend. SCR successfully address clinician experience rather than process. SCR content showed staff shared trauma, challenge, and coping, telling of courage, advising and encouraging others in teamwork. Coding notes about death and dying suggest end of life care include staff health.

FC15 Reflective Practice, (Resilience) and Self-Care Concerns of Cancer Patients with Young Children

Kojima R., Takada H., Ishiki H., Shimizu M., Kiuchi D., Satomi E. Department of Palliative Medicine, National Cancer Center Hospital, Tokyo, Japan

Background: The number of cancer patients has been increasing in Japan. It is estimated that 56,143 parents with 87,017 young children (under 18 years old) are diagnosed with cancer per year in Japan. The aim of this study is to investigate the concerns of cancer patients who have young children.

Method: The survey was conducted at the National Cancer Center Hospital between February and April 2018. We distributed to the questionnaires among the cancer patients who have young children (ages 0?17). It was consisted of items about parenting concerns evaluated with the 4-point Likert scale. The items included "cancer notification to their children", "parental anxieties", and others. The items were analyzed separately. Results: The respondents were 152 (143 cancer patients (94回) and 9

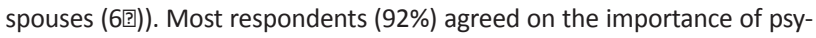
chosocial support for the children and parents in hospital. The average age of their young children $(n=237)$ was 9.5(ages 0?17). The result showed that respondents (93.2\%) did not want to give burdens to children. Most parents (84.5\%) also felt guilty to their children because they had not done sufficient parents' role. This study also revealed that parents were struggling with the conflict over telling their children the truth. One hundred and forty eight (62] ] children were told true parents' illness.

Conclusion: This study describes how cancer has impacts on parents for child rearing. Almost all of the patients with minor children had been found psychologically distressed. It is necessary to improve an effective psychosocial support system in hospitals for cancer patients with young children based on their concerns.

FC15 Reflective Practice, (Resilience) and Self-Care Evaluation of a Mindfulness and Compassion-course for the Palliative Care Team
Lautwein F. ${ }^{1}$, Schallenburger M. ${ }^{1}$, Scherg A. ${ }^{1}$, Schlieper D. ${ }^{1}$, Regel Y.U. ${ }^{2}$, Schwartz J. ${ }^{1}$, Neukirchen M. ${ }^{1}$

${ }^{1}$ Interdisciplinary Center for Palliative Medicine, University Hospital of Düsseldorf, Düsseldorf, Germany, ${ }^{2}$ Paramita, Practice for Mindfulness, Meditation and Stress Management, Bonn, Germany

Background: Palliative care teams work under challenging conditions that can lead to tensions within and between the employees. At the same time the team itself represents a highly relevant protective factor as it helps coping with the burdens of dying and bereavement. To strengthen the resilience of the individual and the team while fostering empathy, mindfulness and compassion-based practices are promising tools. Correspondingly, an 8 week-course was conducted, partly integrated in the daily routine of an interdisciplinary palliative care center. Aims: To investigate feasibility, satisfaction and impact of a mindfulness and compassion-course in the palliative context. We focused on shortterm possibilities and limitations regarding knowledge conveyance and attitude change.

Methods: A retrospective course evaluation has been carried out. It comprised questions concerning demographics, course satisfaction and comparative self-assessment gain (CSA-Gain) of operationalized learning objectives. Free text data about personal experiences/emotions were evaluated using content-structuring analysis.

Results: 25 staff members ( $93 \%$ of $n=27$ ) took part in the evaluation after the course. $58 \%$ attended at least 4 of 7 "mindfulness-days". $91 \%$ were satisfied with the course and would recommend it for palliative work settings. CSA-Gain was high (28-59\%) concerning familiarity with the practices and their application at the workplace. It was rather low (9-26\%) regarding enhanced self-reflection. In between ranged learning objectives concerning the team relationship (20-27\%). From the qualitative content analysis, four main categories emerged: the course, empathy, professional- and personal-life. The opportunity of experiencing self-care was highlighted.

Conclusion: The conducted course is a feasible instrument to introduce palliative care teams to mindfulness-based practices. Yet for (self-)compassion to lastingly support the caregivers, consolidation and refreshing seem indispensable.

FC15 Reflective Practice, (Resilience) and Self-Care The Effects of Mindfulness-based Interventions on Nurses in Palliative and End-of-Life Care (PEoLC): A Systematic Review with a Focus on Health and Burnout

Cho M. ${ }^{1}$, Yi D.H. ${ }^{2}$

${ }^{1}$ Sam International Hospital, Anyang, Korea, Republic of, ${ }^{2 K i n g s}$ College London, London, United Kingdom

Aim and Objectives: The aim of this study is to evaluate the effects of MBIs on nurses' health and burnout in PEoLC. There are four objectives in this review; 1) to investigate what kinds of MBls have been applied and their feasibility in the population of nurses, 2) to evaluate whether MBls affect the mental and physical health of nurses, 3) to evaluate whether MBIs can improve nurses' burnout, and 4) to examine doserelated effects of MBIs on nurses' health and resilience.

Design: A systematic literature review followed the PRISMA guideline. This review adapted a narrative synthesis.

Data Sources: Six databases (MEDLINE, CINAHL, PsycINFO, Web of Science, and EMBASE, Cochrane Library) were searched from the inception to October 2017. For grey literature search, the two databases, ProQuest Dissertation and Theses and Bielefeld Academic Search Engine were explored.

Results: A total of eight randomized controlled trials (RCTs) were included ( $n=277)$. All of the studies in this review have low quality with high risk of bias and half of the studies (4/8) are small sample sized pilot trials. Results suggests that the abbreviated MBIs (5/8) are feasible and acceptable for nurses in PEoLC. Regarding the effectiveness of MBIs on nurses' health and burnout, this review found insufficient evidence to 
draw a clear conclusion. This review found that there was no dosedependent effectiveness of MBls on burnout, albeit this analysis was significantly limited due to the underreporting of compliance.

Conclusion: Although the significant methodological defects were hindered in drawing a robust conclusion regarding $\mathrm{MBI}$ effects on nurses in PEoLC, the included studies contributed as the groundwork for future research building. For further expansion of this research area, consensus for standardisation of MBIs, applying active control group(s) and longterm follow-up are warranted.

\section{FC15 Reflective Practice, (Resilience) and Self-Care}

Professional Boundaries of Counseling in Palliative Care

Stinghe $A$.

Fundatia Hospice, Social, Brasov, Romania Presenting author email address: alinastinghe@hospice.ro

Introduction: Counseling is a support services for those who are in crises that requires changing habits and routines. The role of a counsellor is complex. It requires knowledge and abilities that will lead to problem identification and solving in order to obtain improvement in the quality of life of Palliative Care (PC) beneficiaries. In the interdisciplinary PC team, all professionals have to master competencies needed for counseling patients: empathy, non-verbal communication, active listening, dealing with dilemmas, and guidance in decision making.

Goal: Identifying professional boundaries of PC professionals used in counseling patients in a home care service setting.

Method: Prospective quantitative observational cross-sectional study using a checklist with 16 questions with predefined answers. $25 \%$ of questions were related to counseling in own specialty and $75 \%$ were looking at psycho-emotional, social, spiritual counseling.

Results: All 17 professionals in our home PC service completed the survey: 4 physicians, 7 nurses, 2 psychologists, 3 social workers, 1 spiritual counselor. $80 \%$ of professionals have experience over 7 years in PC. Counselling is performed by all members of the home PC team at horizontal level of professional competency. Clinical experience is a major factor contributing at developing and improving the counselling competency. It remains a field of dilemma when it comes to determine its limits/ boundaries for each PC professional. Multiple factors determine this situation; patients' and families' preferred professional being one of the major determinants.

Conclusions: It is acknowledged and accepted by the members of the PC interdisciplinary team that counselling is a complex professional competency, both common and specific. It's up to the team dynamic, individual open mindedness and adopted conflict resolution processes for this competency to be efficiently used in the process of caring for patients and families.

\section{FC17 Ethical Issues in Palliative Care}

Challenges in Applying the Legal due Care Criteria for Euthanasia and Assisted Suicide (EAS) for People with Intellectual Disabilities (ID) and/or Autism Spectrum Disorder (ASD) in the Netherlands Tuffrey-Wijne I. ${ }^{1}$, Curfs L. ${ }^{2}$, Finlay I. ${ }^{3}$, Hollins S. ${ }^{4}$

${ }^{1}$ Faculty of Health, Social Care and Education, Kingston University \& St George's University of London, London, United Kingdom, ${ }^{2}$ Maastricht University, Maastricht, Netherlands, ${ }^{3}$ Cardiff University, Cardiff, United Kingdom, ${ }^{4}$ St George's University, London, United Kingdom

Background: EAS is legally possible in the Netherlands, provided that statutory due care criteria are met, including 'unbearable suffering without prospect of improvement' and 'voluntary and well-considered request'. There is post-euthanasia scrutiny of all cases, and annual reporting, by the Euthanasia Review Committee (RTE).

Aims: We investigated whether any particular difficulties or challenges arise when the EAS due care criteria are applied to patients with ID and/ or ASD.
Method: The 416 case summaries available on the RTE website (20122016) were searched for ID (6 cases) and ASD (3 cases). Direct content analysis was used on the case summaries.

Results: Assessment of decisional capacity was mentioned in eight cases, but few details given. There was an emphasis on assessing the consistency of the patient's request. In three cases, there was physician disagreement or uncertainty about capacity. For most patients, suffering was due to an inability to cope with changing circumstances or increasing dependency; in several cases, suffering was described in terms of characteristics of living with ASD, rather than an acquired medical condition. Treatment refusal was a common theme, leading physicians to conclude that euthanasia was the only remaining option.

Discussion: Whilst impairment of decision-making capacity lies on a continuum, the judgment of decisional capacity is an all-or-nothing concept. The bar should be set high for a decision as serious as EAS, but it appears that capacity tests were not sufficiently stringent. Difficulties for physicians to understand suffering from the patient's perspective were particularly striking for patients with ASD. We conclude that the Dutch euthanasia due care criteria are not easily applied to people with ID and/ or ASD, and do not appear to act as adequate safeguards.

FC17 Ethical Issues in Palliative Care

Day-to-Day Ethics in Palliative Care: A Systematic Review of the Ethical Challenges Identified by Specialist Palliative Care Practitioners in their Clinical Practice

Schofield G. ${ }^{1}$, Dittborn M. ${ }^{2}$, Huxtable R. ${ }^{1}$, Selman L. ${ }^{3}$, Brangan E. ${ }^{4}$ ${ }^{1}$ Centre for Ethics in Medicine, Medical School, University of Bristol, Bristol, United Kingdom, 'Florence Nightingale Faculty of Nursing, Midwifery \& Palliative Care, King's College London, London, United Kingdom, ${ }^{3}$ Population Health Sciences, Medical School, University of Bristol, Bristol, United Kingdom, ${ }^{4}$ National Institute for Health Research Collaboration for Leadership in Applied Health Research and Care West (NIHR CLAHRC West), University Hospitals Bristol NHS Foundation Trust, Bristol, United Kingdom

Background: Ethical issues arise daily in the delivery of palliative care. Despite much (largely theoretical) literature, evidence from specialist palliative care practitioners (SPCPS) about day-to-day ethical challenges has not previously been synthesised.

This evidence is crucial to inform education and training and adequately support staff.

Aim: To synthesise the evidence regarding the ethical challenges which SPCPs encounter during clinical practice.

Methods: A prospectively registered systematic literature review using narrative synthesis methodology (Popay et al 2006) was conducted. Key words and subject headings of 8 databases (MEDLINE, Philosopher's Index, EMBASE, PsycINFO, LILACS, WHOLIS, Web of Science and CINAHL) were searched without time limits. Eligible papers reported original research using inductive methods to describe SPCP-reported ethical challenges, in any language. Quality was dual assessed using the MixedMethods Appraisal Tool. Tabulation, textural description, concept mapping and thematic analysis were used to develop and present the narrative.

Results: 7023 records were screened. 12 studies from 9 countries were included. A broad range of challenges were identified in 5 themes: patient, patient-family, clinical, organisational, and wider system. Examples within these themes include: autonomy, truth-telling; boundaries of confidentiality; palliative sedation, artificial nutrition; value differences between professions and care settings; euthanasia and organ donation respectively.

Conclusion: SPCPs encounter a broad range of ethical challenges, albeit not all of which are recognised in the ethics literature or form part of training curricula. Findings of the review are relevant to SPCP education, training and support.

Funding: GS is supported by the Wellcome Trust Fellowship Grant No. 208129/Z/17/Z. 


\section{FC17 Ethical Issues in Palliative Care}

Differences in the Perception of Quality of Life, Dignity and Control among Patients with Different Degrees of Wish to Hasten Death Crespo I. ${ }^{1}$, Rodriguez-Prat A. ${ }^{2}$, Guerrero-Torrelles M. ${ }^{3}$, Porta-Sales J., ${ }^{1,4}$, Balaguer A. ${ }^{1}$, Monforte-Royo C. ${ }^{3}$

${ }^{1}$ Faculty of Medicine and Health Sciences, Universitat Internacional de Catalunya, Sant Cugat del Vallès, Spain, ${ }^{2}$ Faculty of Humanities, Universitat Internacional de Catalunya, Sant Cugat del Vallès, Spain, ${ }^{3}$ Department of Nursing, Universitat Internacional de Catalunya, Sant Cugat del Vallès, Spain, ${ }^{4}$ Palliative Care Service, Institut Català de Oncologia, Barcelona, Spain

Background: There is evidence that the wish to hasten death (WTHD) is related to poor health-related quality of life (QoL). E.g. Patients with the WTHD perceive a loss of dignity and lack of control and autonomy due to the disease; this is related to poor QoL.

Aim: To compare perceptions of QoL, dignity and control between groups of advanced patients with

1) clinically relevant WTHD

2) minimal/mild WTHD and

3) no WTHD.

Methods and design: Comparative cross-sectional study.

Data collection: 153 adult advanced cancer patients were assessed for WTHD with the Desire for Death Rating Scale. Scores 1-2 indicate mini$\mathrm{mal} / \mathrm{mild}$ WTHD $(\mathrm{n}=37)$, scores $\geqslant 3$ indicate clinically relevant WTHD $(n=14)$ and scores of 0 imply no WTHD ( $n=102)$. QoL was assessed using the European Organization for Research and Treatment of Cancer Quality of Life Core 15 items Palliative Questionnaire (EORTC QLQ-C15PAL), the perceived loss of dignity was assessed with the Patient Dignity Inventory and control was assessed using the General Self-Efficacy Scale. Analysis: The ANOVA and the Kruskal-Wallis test depending on whether the variable had a Gaussian distribution.

Results: Patients with clinically relevant and minimal/mild WTHD perceived worse emotional functioning $(p<.001)$ and greater appetite loss $(p=.044)$ than those without WTHD. Patients with clinically relevant and minimal/mild WTHD perceived a greater loss of dignity than those without WTHD $(p<.001)$. Both groups with the WTHD showed greater psychosocial and existential distress and a greater sensation of dependence than the group without the WTHD $(p<.001$ and $p=.009)$.

The group with clinically relevant WTHD had the lowest sense of control. Those with minimal/mild WTHD had a lower sense of control than those without WTHD $(p=.002)$.

Conclusion: The greater the WTHD, the worse the patients' perception of their dignity, control and emotional QoL. However, patients with the WTHD do not perceive worse physical symptoms or physical function.

\section{FC17 Ethical Issues in Palliative Care} Meaning of Caring for Cancer Patients from Time of Incurable Diagnosis to Last Days of Life: A Qualitative Study in Family Caregivers Wikert J. ${ }^{1}$, Treutlein M. ${ }^{1}$, Oechsle K. ${ }^{1}$, Bergelt C. ${ }^{2}$, Marx G. ${ }^{3}$, Bokemeyer C. ${ }^{1}$, Ullrich A. ${ }^{1,2}$

${ }^{1}$ Palliative Care Unit, Center of Oncology, University Medical Center Eppendorf, Hamburg, Germany, ${ }^{2}$ Department of Medical Psychology, University Medical Center Eppendorf, Hamburg, Germany, ${ }^{3}$ Department of Palliative Medicine, University Medical Center Goettingen, Goettingen, Germany

Background: Family caregivers' (FC) situation may differ across the trajectory of patients' (PT) disease and may escalate when PT are nearing death.

Aims: To explore what it means for FC to care for PT with advanced cancer at time of incurable diagnosis, during palliative care and at end-of-life.
Methods: Qualitative semi-structured interviews using open-ended questions with 17 bereaved FC (15 spouses). Data analysis using grounded theory and abductive reasoning.

Results: We found a core phenomenon in each of the three phases. At diagnosis, the incurable disease becomes center of FC's life (phenomenon). Threat to life, uncertainty and unpreparedness contribute to a feeling of overstraining when FC face their responsibility. Strategies include denial of own needs and setting aside painful thoughts. Experiences are contextualized by ambivalence of hope/fear, PT's autonomy/caring needs and increasing awareness of life's finiteness. Admittance to a palliative care ward serves as a cut, allowing FC to view PT's death as inevitable and eliciting strong moral obligation. FC elevate PT's will to be their ultimate compass (phenomenon). Consequently, FC show no or little self-care, and if yes, often induced by others. Strategies are various, including extensive communication. Experiences are contextualized by PT's appreciation for caregiving, FC's powerlessness and sense of loosening ties with PT. At end-of-life, a positive parting is most meaningful for FC (phenomenon). Enabling PT and themselves to say goodbye leads FC to escalate into a state of mere functioning. Strategies include self-abandonment, preparing for things to come and settling conflicts. Experiences are contextualized by appropriateness of place of death, feeling endowed to care for a dying person, but also alienation from the loved one.

Conclusion: Some aspects gained or lost meaning over time, thus indicating that preference-sensitive support requires iterate evaluation of FC's situation and needs.

FC17 Ethical Issues in Palliative Care Why Do we Not Understand Each Other? Differences in End of Life Priorities between Patients and their Caregivers

Tučková A., Loučka M., Houska A., Poláková K., Vlčková K.

Center for Palliative Care, Prague, Czech Republic

Background: For successful planning of care at advanced stages of disease is crucial to explore patients' values and when possible to reach consensus on future priorities between patients, their relatives and clinicians.

Aim: To analyze differences between patients and caregivers views on aspects considered important at advanced stage of chronic disease.

Methods: The study was a cross-sectional survey of seriously ill patients $(n=170)$, their relatives $(n=108)$ and physicians $(n=113)$. Participants were asked to rank 40 factors, previously identified in interviews with seriously ill patients as being important at the end of life, on 5 point Likert scale considering their importance $(1=$ least important, $5=$ extremely important). Statistical analysis was used to examine distribution of responses for all 40 items. Significance of differences and agreement was explored by ANOVA and Bonferonni test.

Results: Most factors were considered to be important (only 4 items have mean $<3.5$ ) in all three group. ANOVA showed that responses in groups did not differ in 13 items out of $40(p>.05)$ which are connected mostly to physical symptoms and place of death. There was significant difference between patients and caregivers in factors connected to informational needs, feeling of energy and sense of usefulness, with patients putting more importance on these factors compared to relatives and physicians $(p<.001)$.

Conclusions: This study showed that there are still aspects of care, which might be underestimated by caregivers compared to views of patients with advanced disease. Supporting patients in addressing these needs must be incorporated in treatment plans. More research is also needed on understanding what are the reasons behind the differences among patients and their caregivers.

Research was supported by Czech Science Foundation grant No. $17-26722 Y$ 
FC17 Ethical Issues in Palliative Care

The Minutes Until the Emergency Doctor Arrives on Scene - Ethical Dilemma in Paramedic Emergency Personnel when Encountering Comorbid and Palliative Stage Patients

Mühe K. ${ }^{1}$, Nauck F. ${ }^{2}$, Alt-Epping B. ${ }^{2}$

${ }^{1}$ Medical Faculty, University of Goettingen, Goettingen, Germany, ${ }^{2}$ Dept. of Palliative Medicine, University Medical Center Goettingen, Goettingen, Germany

Background: In Germany, prehospital emergency medicine implies a "rendez-vous" system where paramedics and an emergency physician approach separately. Mostly, paramedics arrive first, and are legally obliged to perform all possible action to maintain the patient's (pt) life. Emergencies with 'palliative' or old, comorbid pts may lead to severe distress and ethical dilemma in paramedic personnel when comfort care might be more appropriate than aggressive procedures.

Methods: We explored paramedic personnel by using a pre-piloted questionnaire. Participants were asked to comment on the given legal restraints. Quantitative data were analyzed by descriptive statistics. Free text answers were analyzed by using qualitative methods.

Results: From all over Germany, 283 paramedics participated. On average, every paramedic recalled 5.7 palliative stage pts within 6 months; 3.5 of those encountered without a physician on scene. In $38 \%$ of those first-in-place situations, paramedics decided not to intervene, e.g. by refusing to call a doctor, leaving the pt at home or to refrain from ventilator support. $62 \%$ of participants had acted against legal allowance by setting limits. Similar results were stated for CPR scenarios in old and co-morbid pts, where CPR was omitted or terminated in one third, each. In total, almost $80 \%$ of the paramedics perceived distress in performing CPR in comorbid pts or in caring for palliative stage pts. $71 \%$ of the participants felt competent enough to decide against CPR by themselves, but only $51 \%$ felt competent enough to decide to refrain from further interventional therapy in palliative stage pts.

Discussion: Our findings support that emergencies in 'palliative' pts lead to feeling of dilemma and moral burden. Legal constraints were deliberately broken, and a high degree of self-competence in deciding by oneself whether to begin or stop interventions was stated, associated with claims for independent responsibility and unequivocal regulations.

\section{FC18 Advance Care Planning}

VSD Vorsorgedialog ${ }^{\circledR}$ - A Nationwide Instrument for Advance Care

Planning in Nursing Homes in Austria

Beyer S., Dzaka N., Pelttari L.

Hospice Austria, Vienna, Austria

Introduction: "VSD Vorsorgedialog" (VSD) is a new nationwide instrument for advance care planning and end-of-life decisions in nursing homes in Austria. Over a process of two years, the instrument was developed and discussed and ultimately gained acceptance of all stakeholders (e.g. Ministry of Health and Social Affairs, Medical Association, Federation of Social Insurance Institutions). It is now enshrined in Austrian law.

VSD is an ongoing communication process between residents, their families, nurses and physicians. They talk about the resident's needs and expectations for a good life as well as for a dignified end of life. The results are documented and regularly re-evaluated. If a resident is not able to communicate her or his will, a VSD-conversation offers the opportunity to ascertain the resident's presumed will.

Method: A quantitative and qualitative survey is conducted in participating nursing homes during the nationwide pilot phase (1.1.201731.12.2021). In September 2018, an interim analysis of the quantitative data of four nursing homes, which had been offering VSD-conversations for 6.7 months on average, was carried out.

Results: During the survey period, 95 VSD-conversations were conducted. In 27 of those, the residents expressed their needs and expectations personally. 42 investigated the resident's presumed will and in 14
VSD-conversations, the resident's ability to make judgements couldn't be determined (missing data: $\mathrm{x}=12$ ). On average, a VSD-conversation lasted 46 minutes, nurses' preparation and follow-up took 60 minutes. 78 emergency information sheets were filled out. In 20 emergency situations the staff acted in accordance with the resident's will, in one emergency situation they didn't act in accordance with it.

Conclusion: The VSD is a suitable instrument not only to ascertain the resident's will, but also the resident's presumed will. The staff is able to act in accordance with the residents' needs and expectations in emergency situations.

\section{FC18 Advance Care Planning}

Effectiveness of Advance Care Planning in Improving End of Life Care for Patients with Advanced Heart Failure

Malhotra C. ${ }^{1}$, Sim D.K.L. ${ }^{2}$, Jaufeerally F. ${ }^{3}$, Vikas N.N. ${ }^{1}$, Hu M. ${ }^{1}$, Finkelstein

E. ${ }^{1}$, ACP Trial Team

${ }^{1}$ Duke-NUS Medical School, Singapore, Singapore, ${ }^{2}$ National Heart Center Singapore, Singapore, Singapore, ${ }^{3}$ Singapore General Hospital, Singapore, Singapore

Background: Advance care planning (ACP) is a widely discussed intervention to help people receive end-of-life (EOL) care consistent with their wishes but evidence on its effectiveness remains inconclusive.

Aims: Primary aim was to assess, among advanced heart failure patients, effectiveness of ACP in ensuring EOL care consistent with patient wishes. Secondary aims were to assess its impact on patients' decisional conflict, discussion of care preferences with surrogates, illness understanding, anxiety, depression and quality of life.

Methods: We conducted a randomized controlled trial of ACP (based on Respecting Choices Model) versus usual care in Singapore. 282 patients hospitalized with heart failure and New York Heart Association Classification III and IV symptoms were randomized to ACP (93) or control (189) arm. They answered up to 6 follow-up surveys conducted every 4 months. Primary outcome was assessed in deceased sample (89; 23 in ACP, 66 in control) using chi-square/fisher's exact test. Secondary outcomes were assessed through mixed-effects models. Both intentionto-treat and per-protocol analyses were done.

Results: $63 \%$ of ACP arm received intervention. Deceased patients in ACP arm were no more likely to have their wishes followed for EOL treatments (full/limited additional/comfort treatment; $35 \%$ in ACP vs $44 \%$ in control; $p=0.47$ ) but were more likely to have their wishes followed for cardiopulmonary resuscitation ( $83 \%$ in ACP vs $62 \%$ in control, $p=0.12$ ) though the difference was not statistically significant. At first follow-up, ACP patients had lower decisional conflict $(\beta=-10.8, p<0.01)$ and were more likely to discuss preferences with their surrogate decision maker $(\beta=1.3, p=0.04)$. Both arms did not differ on other patient outcomes. Per-protocol analyses showed similar results.

Conclusion: ACP has benefits in improving decision making for patients. Findings raise questions on its effectiveness in ensuring EOL treatment consistent with patient wishes.

\section{FC18 Advance Care Planning}

Patient-Surrogate Agreement in Advance Care Planning: Who are the Surrogates and are They Making the Right Decisions?

Sim L.K., Low J., Tan L., Khemlani M.

Yishun Health, Singapore, Singapore

Objectives: The aim of the study is to examine the extent of agreement between the end-of-life patients and their surrogates on the patient's preferences in advance care planning.

Methods: In this cross-sectional study, participants consisted of pairs of end-of-life patient and their surrogate recruited from the advance care planning department of an acute hospital in Singapore. The survey included surrogates' socio-demographic status, a rating of how well the 
surrogate know the patient, and the patient's wishes on end-of-life care based on their understanding of the patient.

Results: A total of 30 patient-surrogate pairs participated in the study between January 2015 to January 2018 . Only $40 \%$ of the patient-surrogate pairs agreed that the patient would not want to be attempted cardiopulmonary resuscitation during a medical crisis. Moderate agreement was seen the patient's and surrogates' reported choices on patient's place of medical intervention ( $k=0.40,95 \% \mathrm{Cl} 0.17-0.63)$. Fifty percent of the pairs agreed on the option of place of death and moderate agreement was observed $(\mathrm{k}=0.62,95 \% \mathrm{Cl} 0.45-0.94)$.

Discussion: Surrogates tended to overestimate the patient's preference on CPR status. Surrogates' knowledge of CPR was found to be poor. This advocates a need to continue improving substitute decision-making so that patients can be more confident that surrogates can make decisions consistent to their wishes.

Conclusion: Surrogates' perceptions of patient preferences are often inaccurate, particularly for those patients who do not want to be resuscitated. ACP provides a platform for surrogates to better understand patients' preferences and make end-of-life decisions for the patient's care consistent with the patients' preferences.

\section{FC18 Advance Care Planning}

\section{Advance Care Planning Documentation in Patients Known to the} Specialist Palliative Care Team in an Acute Hospital Setting Mulqueen L.

Our Lady of Lourdes Hospital, Palliative, Drogheda, Ireland Presenting author email address: laramul101@hotmail.com

Background: The acute hospital setting treats critically ill patients facing uncertainty with respect to outcome. Advance Care Planning (ACP) should be discussed early and documented clearly as this supports the patient and family in making informed decisions and ensures appropriate ceilings of care are adhered to. The Specialist Palliative Care Team (SPCT) in a hospital setting work alongside medical and surgical teams, therefore a consistent approach from all health care professionals is key. Aims: To review documentation in a patient population known to the SPCT, and assess communication and transparency with respect to ACP. Methods: The medical records for inpatient deaths over one month were reviewed retrospectively. An independent reviewer assessed documentation of prognosis, ceiling of care, resuscitation status and communication with the patient/family. Additionally, who initiated these conversations and timing of referral to the SPCT was reviewed.

Results: 21 records were identified. Resuscitation status and ceilings of care were well documented however not easily found in the notes. Prognosis was often omitted. In many cases there was a delay between identifying a life limiting illness and specialist palliative care needs and referral to the SPCT. Consequently ACP with patients and family was delayed as the SPCT carried out the majority of these conversations.

Conclusion: There is an apparent ambiguity around goals of care and a reluctance to initiate $A C P$, which could potentially negatively impact a patients care. Though ceilings of care and resuscitation status are being documented and discussed with patients and family members, they are not easily identifiable and referenced in the medical records.

Given these findings, consideration could be given to a standardised ceiling of care document to be held in healthcare records for specific patients under the care of the SPCT, which would be reviewed and updated as appropriate.

\section{FC18 Advance Care Planning}

Palliative Care Support Needs of Cancer Patients Identified in Regular Multiprofessional Team Meetings - Analyses of Advance Directives, Distress and Performance Status

Missala I., Alex Pellegrin M., Preisler M., Letsch A.

Medical Department, Division of Hematology and Oncology, Campus
Benjamin Franklin, Charité - Universitätsmedizin Berlin, Corporate Member of Freie Universität Berlin, Humboldt-Universität zu Berlin, and Berlin Institute of Health, Berlin, Germany

Background: There is accumulating evidence that outcomes of cancer patients are improved when palliative care and oncology work synergistically. More difficult to define is how integrated palliative cancer care (IPCC) can be achieved and implemented. Regular multi-professional team meetings (MTM) comprising all various health care professionals as well as oncologists and palliative care specialists could function as an effective mean of integration.

Methods: Detailed analyses of cancer patients ( $n=107)$, who were rated to qualify for complex palliative treatment during a regular weekly oncopalliative MTM of the Department for Hematology and Oncology of a German University Clinic during December 2017 and February 2018.

Results: During multiprofessional team discussions, 107 patients were identified to require complex palliative treatment. $47 \%$ were female, $53 \%$ male with a median age of 65.2 years and $55 \%$ of patients with advanced oncological and $45 \%$ with advanced hematological malignant diseases. The median ECOG performance status was 2.9. Using the distress thermometer (DT) the median psychological distress level was 6.9 with high levels of DT $\geqslant 5$ in $97 \%$ of patients. Despite the high prevalence of psychological distress and low performance status, advance directives (AD) at baseline were only available in $19 \%$ of these patients. Patients with $A D$ did not differ with regard to age (median 66.5 ), gender ( $45 \%$ female, $55 \%$ male), performance status (median 2.85), psychological distress (median 7.0) or kind of malignant disease ( $60 \%$ oncological and $40 \%$ hematological).

Discussion: Only a minority of this group of patients with advanced hematological and oncological malignancies and a high prevalence of psychological distress and low performance status had an advance directive at baseline. Factors, which may influence the completion of ADs in cancer patients, should be explored and the potential of IPCC to impact the completion of ADs should be fully exploited.

\section{FC18 Advance Care Planning}

Advance Care Planning in Underserved Populations Using Lay Patient Navigators: Have You had the Conversation?

Fink R. ${ }^{1}$, Kline D. ${ }^{1}$, Bailey F.A. ${ }^{1}$, Handel $D .{ }^{2}$, Lum H. ${ }^{2}$, Fischer $S .{ }^{1}$

${ }^{1}$ General Internal Medicine, University of Colorado Anschutz Medical Campus, Aurora, United States, ${ }^{2}$ School of Medicine, University of Colorado Anschutz Medical Campus, Aurora, United States

Aims: To develop a model for community-based advance care planning (ACP) in rural populations with low English language fluency and healthcare access. To implement the ACP program throughout rural communities and conduct a program evaluation.

Methods: Two bilingual patient navigators (PNs) facilitated ACP conversations in schools, businesses, churches, libraries, nursing homes, clinics, and local government districts. Trained in a group session framework, PNs facilitated one-hour English and Spanish sessions in a community setting. Participants received information by poster or PowerPoint format including: Frequently Asked Questions, a low literacy Advance Directive (www.prepareforyourcare.org), and goal setting form in English or Spanish. Participants were asked to share concerns about choosing a medical decision-maker and finalizing an ACP. They completed the ACP Engagement survey (ACP-4) post session.

Results: Over 18 months (2017-2018), we led 74 ACP sessions engaging 1,034 participants; $38 \%$ were ethnically diverse and $69 \%$ female. A nurse or physician co-facilitated $49 \%$ of the sessions with the PN. Forty seven percent of participants completed an ACP-4. $29 \%$ planned on naming a decision-maker in the next 6 months and 21\% in the next 30 days; $26 \%$ ready to talk about future healthcare decisions with decision-maker in the next 6 months and $22 \%$ in the next 30 days; $31 \%$ ready to talk to their provider about healthcare preferences in the next 6 months and $14 \%$ in the next 30 days; $31 \%$ ready to complete an $A D$ in the next 6 months and 
$22 \%$ in the next 30 days. Evaluations showed 57\% extremely satisfied, $41 \%$ satisfied, with $98 \%$ reporting the session was the right length of time. Conclusions: Bilingual, bicultural PNs effectively engaged underserved, diverse populations in ACP in rural community settings. Our model can be readily adapted by other providers to improve ACP in underserved populations.

Funding: Colorado Health Foundation grant

FC19 Research Methodology and Basic Translational Research A Systematic Review of International Palliative Care Research Priorities and a Thematic Synthesis of Findings

Hasson F. ${ }^{1}$, Nicholson E. ${ }^{1,2}$, Muldrew D. ${ }^{1}$, Payne S. ${ }^{3}$, Mcllfatrick S. ${ }^{1}$ ${ }^{1}$ School of Nursing, Ulster University, Co. Antrim, United Kingdom, ${ }^{2}$ UCD School of Nursing, Midwifery \& Health Systems, University College Dublin, Dublin, Ireland, ${ }^{3}$ Lancaster University, Lancaster, United Kingdom

Background: Increasing evidence contributing to the global research agenda for palliative care has been published on research priorities, however, to date there has been a lack of synthesis of this evidence featuring commonalities, differences and gaps.

Aim: To identify and synthesize global palliative care research priorities. Design: A systematic review of research priorities in palliative care was undertaken and guided by the PRISMA statement for reporting systematic reviews. Studies that elicited palliative care research priorities were eligible for inclusion.

Data Sources: Six databases were searched between 2008-2017 including CINAHL, EMBASE, PubMed, SCOPUS, Web of Science, and PsycINFO, as well as grey literature. Eligibility criteria were applied and the Joanna Briggs Institute Appraisal Tools were used to assess quality.

Results: The search strategy yielded 2288 unique citations, 51 full text articles were reviewed, and seven met the inclusion criteria. Seven priority areas were identified: Service Models, Continuity of Care; Training and Education; Inequality; Communication; Living well and independently; and Recognising family/carer needs and the importance of families. Methodological approaches and process of reporting varied. There was little representation of patient and caregiver driven agendas.

Conclusion: Research priority setting activities in palliative care elicited a broad range of topics, however approaches and samples varied questioning the credibility of findings. The findings of this study may serve as a template to understand the commonalities of research and enhance dialogue in palliative care research. A more standardised approach for priority setting will allow for increased validity and comparability of findings from across palliative care settings.

FC19 Research Methodology and Basic Translational Research Application of van Manen's Approach of Data Analysis Utilising Lifeworld Existentials in a Hermeneutic Phenomenologic Study to Understand Mutuality in a Palliative Approach for Advanced Parkinson's Disease

Arshinoff R. ${ }^{1}$, Preston N. ${ }^{2}$, Grinyer A. ${ }^{2}$

${ }^{1}$ Religious and Spiritual Care, Baycrest, Toronto, Canada, ${ }^{2}$ Faculty of Heath and Medicine, Lancaster University, Lancaster, United Kingdom

Background: Hermeneutic phenomenology focuses on analysis of the lifeworld or lived experience to understand meaning. van Manen elucidates everyday life as the lifeworld, and names four lifeworld themes or existentials that are interdependent and form a unity for each person: spatiality (lived space), temporality (lived time), corporeality (lived body), and relationality (lived other). The four existentials are both interdependent and at times intertwined. They offer a holistic framework in understanding the phenomenon under study for the researcher to explore layers of meaning in the four unique contexts. They help the researcher to promote the uniqueness of each individual as he/she experiences their lifeworld.
Aims: To apply the four existentials to the analysis of data obtained from 12 participants in a study about mutuality in advanced Parkinson's Disease (PD) with the ultimate goal of creating a holistic palliative approach for chronic illnesses.

Methods: Twelve caregiving spouses of PD patients participated in a single face to face interview. Data were analysed using each existential and upon completion, cross-cutting and common themes across all transcripts were identified for each existential. The hermeneutic circle was utilised for writing and analysing the data by moving between the parts and the whole.

Results: Relationality emerged as influencing mutuality the most and strongly overlapping with spatiality as couples spend much time together, often in stressful environments. Temporality overlappped less and corporeality the least. This is not to be assumed that the three existentials of spatiality, temporality, and corporeality are less important, but that relationality is the one that contributes most to the mutuality experience.

Conclusion: The framework of the four existentials provides a structure offering a holistic approach to data analysis. It is well suited to explore complex phenomena in palliative care.

FC19 Research Methodology and Basic Translational Research Are Palliative Care Inpatients Willing to Be Recruited to a Study Exploring the Use of a Technical Device to Assess Level of Consciousness? An Observational Study for I-CAN-CARE Krooupa A.-M. ${ }^{1}$, Stone P. ${ }^{1}$, McKeever S. ${ }^{1,2}$, Vivat B. ${ }^{1}$

${ }^{1}$ Marie Curie Palliative Care Research Department, UCL, London, United Kingdom, ${ }^{2}$ Department of Children's Nursing, London South Bank University, London, United Kingdom

Background: Previous I-CAN-CARE research found that clinicians considered that palliative care patients would be unwilling to participate in research using a technical device (the Bispectral index (BIS)) for monitoring consciousness, owing to the visual impact and apparent invasiveness of the device. Patients and relatives, however, were less concerned. We therefore began a prospective observational study to investigate using BIS in practice.

Objective: To systematically explore recruitment to a study using BIS with hospice inpatients.

Methods: We documented recruitment of all current inpatients and new admissions to the hospice electronically, logging patients' eligibility for the study and whether they had: i) been approached by clinicians, ii) agreed to see the researcher, and iii) consented to take part. Reasons for non-approach/non-participation were recorded at each stage.

Results: A total of 313 patients were screened for inclusion; 142/313 $(45 \%)$ were ineligible, mainly due to lack of capacity $(59 / 142 ; 42 \%)$ or being too unwell (59/142; 42\%). Clinicians approached almost all eligible patients $(156 / 171 ; 91 \%)$; most of those were then seen by the researcher (137/156; 88\%). For BIS monitor-related reasons (either restriction of movement or possible discomfort of sensor strip), one patient refused to see the researcher, and five of those who did $(5 / 137 ; 4 \%)$ did not consent. Overall, $38 / 137(28 \%)$ of those approached by the researcher participated (38/313 (12\%) of all screened patients), while 75/137 (55\%) refused, due mostly to experiencing distressing pain and/or fatigue (41/137; 30\%).

Conclusions: Potential participants in our study were mostly undeterred by the BIS device itself. The main recruitment challenges, similarly to other palliative care studies, were related to the nature of the patient population, but not to "gatekeeping" by clinical staff.

Funding: Marie Curie I-CAN-CARE programme grant (ref: MCCC-FPO-16-U).

FC19 Research Methodology and Basic Translational Research Ensuring Meaningful Patient and Public Involvement in Palliative Care and Rehabilitation Research: A Focus Group Study

Johnson H. ${ }^{1}$, Brighton L.J. ${ }^{1}$, Etkind S. N. ${ }^{1}$, Yu P. ${ }^{1}$, Oluyase A. ${ }^{1}$, Chukwusa E. ${ }^{1}$, Ogden M. ${ }^{2}$, Bailey S. ${ }^{2}$, Smith P. ${ }^{2}$, de Wolf-Linder S. ${ }^{3}$, Koffman J. ${ }^{1}$, Evans C.J. ${ }^{1}$ 
${ }^{1}$ Cicely Saunders Institute of Palliative Care, Policy and Rehabilitation, King's College London, London, United Kingdom, ${ }^{2}$ Patient/Carer Representative, Cicely Saunders Institute of Palliative Care, Policy and Rehabilitation, King's College London, London, United Kingdom, ${ }^{3}$ School of Health Professions, Institute of Nursing, Zurich University of Applied Sciences, Winterthur, Switzerland

Background: Patient and public involvement (PPI) can improve the quality, relevance and impact of research, but is rarely evaluated.

Aim: To evaluate the process of PPI and emergent outcomes at a palliative care and rehabilitation institute in London, UK.

Methods: PPI was evaluated against existing strategies (local and national) which prioritise early, flexible involvement throughout the research cycle. We surveyed researchers to describe PPI activity, then undertook separate focus groups with PPI members and researchers to explore experiences. Participants were purposively sampled. Topic guides were co-designed by researchers and PPI members. Transcripts were double coded and analysed thematically together with PPI members, noting divergent views.

Results: $25 / 28$ research projects at the institute included PPI. Four focus groups and two interviews were conducted with PPI members and researchers ( $n=26 ; 77 \%$ female; $58 \%$ with $3+$ years of PPI experience). PPI members felt involvement was 'educational' and 'collaborative', and researchers felt it increased research relevance and offered a 'grounding' perspective. All agreed that role negotiation to establish clear relationships early on enabled meaningful involvement, particularly to share sensitive experiences. Many pursued early involvement and integration throughout the research cycle, however some raised concerns that attention was too fixed on breadth of involvement, at the expense of meaningful depth. A standardised approach did not suit the diversity of research projects, with all suggesting that increased flexibility was needed.

Conclusions: Enabling meaningful collaboration required tailoring PPI methods to individual project aims and designs and ensuring strong relationships through clarity of role. PPI was included in most projects, with increasing integration. PPI is essential to ensure relevance of palliative and rehabilitation research for clinical-effectiveness.

Funders: NIHR CLAHRC SL \& NIHR RCF

FC19 Research Methodology and Basic Translational Research Volatile Organic Compounds Predict the Last Week of Life in Lung Cancer Patients

Coyle S. ${ }^{1,2}$, Chapman E. ${ }^{2}$, Hughes ${ }^{2}{ }^{2}$, Mason S. ${ }^{2}$, Boyd M. ${ }^{2}$, Probert C. ${ }^{2,3}$, Ellershaw $J_{.2,3}$

${ }^{1}$ St Helens and Knowsley Hospitals Trust, St Helens, United Kingdom, ${ }^{2}$ University of Liverpool, Liverpool, United Kingdom, ${ }^{3}$ Royal Liverpool and Broadgreen University NHS Hospitals Trust, Liverpool, United Kingdom

Background: Recognising dying is difficult and is an ongoing difficulty for doctors. We believe there is a process to dying and animal studies support this theory. We previously demonstrated that a number of volatile organic compounds in urine, change in the last weeks and days of life of patients in a small mixed cancer group.

Aim: To verify this finding in a suitably powered follow-up study.

Method: We prospectively collected urine samples from people with lung cancer. We aimed to compare samples from 25 people in each of the last 3 weeks of life to a control group, 50 people with lung cancer who lived 3 or more months from the time of sampling. The urine samples were analysed for volatile organic compounds by gas chromatography mass spectrometry (GC-MS).

Least Absolute Shrinkage and Selection Operator (LASSO) logistic regression was used to analyse the GC-MS data and create a statistical model. Results: We recruited 162 people in total; 29 in the last week; 28 in the second last week; 30 in the third last week of life; 74 controls i.e. samples taken $>3$ months from death; 424 urine samples. This demonstrates studies of this size and type are feasible.
A model was created to predict whether a patient would die within 1 week. It has an optimism corrected AUC of $0.851(95 \% \mathrm{Cl}$ : 0.767, 0.911);

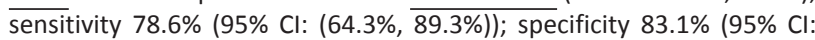
$\overline{(69.9 \%, 92.3 \%))}$. The model identified a selection of compounds that contributed to the identification of patients who were close to death. Discussion:

- The results confirm that volatile organic compounds can predict when people with lung cancer are in the last week of life.

- Our model can predict when a person with lung cancer is in the last week of life with approximately $80 \%$ accuracy.

FC19 Research Methodology and Basic Translational Research Touchless and Burdenfree Monitoring of Heart Rate and Breathing as Surrogate Parameters for Symptom Burden and its Amelioration - A Pilot Study

Steigleder T. ${ }^{1,2}$, Malessa A. ${ }^{1}$, Neumann N. ${ }^{1}$, Shi K. ${ }^{3}$, Michler F. ${ }^{3}$, Schellenberger S. ${ }^{4}$, Heckel M. ${ }^{1}$, Kölpin A. ${ }^{4}$, Ostgathe C. ${ }^{1}$

${ }^{1}$ Department of Palliative Medicine, University Hospital Erlangen, Friedrich-Alexander University Erlangen-Nürnberg (FAU), Erlangen, Germany, ${ }^{2}$ Department of Neurology, University Hospital Erlangen, Friedrich-Alexander University Erlangen-Nürnberg (FAU), Erlangen, Germany, ${ }^{3}$ Department of Technical Electronics, Technical Faculty, Friedrich-Alexander University Erlangen-Nürnberg (FAU), Erlangen, Germany, ${ }^{4}$ Department for General and Electrical Measurement Technology, Brandenburgische Technische Universität, CottbusSenftenberg, Germany

Background: Palliative Care (PC) is focussed on personal encounters and individualized care. To reinforce this, $\mathrm{PC}$ as a rule dispenses with technological diagnostics. Nevertheless, biomarkers (BM, e.g. heart rate (HR) and breathing frequency (BF)) could provide important additional evidence of health status and symptom burden and help to adapt treatment individually. Radar technology (RT) is a highly innovative approach of capturing BM touchless and burden-free. RT detects changes in distance of micrometers from several feet afar permeating materials like clothing or blankets. Learning algorithms (LA) evaluate and interpret the data automatically to extract information on heartbeat and breathing. Methods: $\mathrm{N}=30$ healthy subjects were examined synchronously by non-contact RT from 2 feet distance and gold standard (GS) methods (6-channel ECG, impedance measurement) under controlled conditions (tilt table, Valsalva, command breathing). Endpoints:

(a) recall and precision as reported by F1 scores of radar-based compared to GS assessment methods of $\mathrm{HR}$ and $\mathrm{BF}$;

(b) positive and negative predictive properties of algorithm to detect simulated pathological patterns in participants and death in a model of a corpse.

For comparison of GS methods and RT F1-scores were computed. Results:

(a) Heartbeats were correctly detected in $95 \%$ with a tolerance of $<50 \mathrm{~ms}$ (compared to ECG) and $89 \%$ with a tolerance of $<$ $30 \mathrm{~ms}$ for data set analysis;

(b) F1 scores for heartbeat detection are $97.6 \%$ for data set and 95.8\% for real-time analyses

(c) F1 score for respiration is $98 \%$

(d) LA detected changes in breathing patterns and death (simulated model) with a reliability of $100 \%$.

Conclusion: Touchless and burden-free recording of BM by means of RT is possible. In the context of palliative medicine, RT can facilitate diagnostics for individual therapy adaptation without restricting social participation and personal encounters.

Funding: This study is funded by the BMBF (16SV7695). 


\section{FC20 Education}

Virtual Reality in Palliative Care Teaching - Results of a Longitudinal Survey Study

Taubert M. ${ }^{1}$, Webber L. ${ }^{2}$, Hamilton T. ${ }^{3}$, Carrr M. ${ }^{3}$, Hapgood G. ${ }^{4}$, Harvey M. ${ }^{3}$, Patel M. ${ }^{5}$, Daisley-Devoy T. ${ }^{3}$

${ }^{1}$ Palliative Care Department, Cardiff University School of Medicine, Cardiff, United Kingdom, ${ }^{2}$ Cardiff University School of Medicine, Cardiff, United Kingdom, ${ }^{3}$ Velindre NHS Trust, Cardiff, United Kingdom, ${ }^{4}$ Orchard 360 Media and VR Doctor, Cardiff, United Kingdom, ${ }^{5}$ Cardiff University, Cardiff, United Kingdom

Background: Virtual reality immersive environments have been shown to be effective in medical teaching. Our university hospital received funding from our deanery to film teaching videos with a 360 degree camera.

Aims: Evaluate whether Virtual Reality is an effective teaching environment. Virtual Reality headsets were set up for medical students who rotated through Velindre Cancer Hospital's Palliative Care department.

Methods: Students watched a 27 minute video on nausea \& vomiting in palliative care settings in a virtual tutorial space. They subsequently viewed a radiotherapy treatment experience.

Results: Of the 72 medical students who participated, 70 found the experience comfortable, with 2 students stating they found it uncomfortable (tight headset, blurry visuals). Numerical scoring on ability to concentrate in VR from 0-10 ( $0=$ worst, $10=$ best) scored an average of 8.44 (range 7-10). Asked whether this format suited their learning style, average score was 8.31 (range $6-10) .97 .2 \%(n=70)$ students stated that they would recommend this form of learning to a colleague, with 1 student saying he/she would not recommend and 1 student stating he/she was unsure. Students left positive \& negative free text comments which helped frame future needs in this emerging area and will be presented. Discussion: This study indicates that there is room for exploring new ways of delivering teaching and expanding it more widely in palliative care, but also provides feedback on areas that need further careful attention. Comments from students included: "Might have been the novelty factor but I learned more from this 20 minute VR thing than I have from many lectures"

Summary: The project has proved so popular in medical student feedback that the VR experience is now available on Youtube \& has been expanded to routine teaching. It has been viewed worldwide incl Africa, so this format of teaching could prove valuable and promising in its potential for global reach.

Funding: Wales Deanery

\section{FC20 Education}

Palliative Care Undergraduate Nursing Education in Central and Northern Italy

Ramon Codina M. ${ }^{1}$, D'Angelo D. ${ }^{2}$, Stefanelli M.C. ${ }^{3}$, Pairona G. ${ }^{4}$, Malinverni E. ${ }^{5}$, Guarda M. ${ }^{6}$, Prandi C. ${ }^{7}$, Mastroianni C. ${ }^{3}$

${ }^{1}$ Universidad Nacional de Educación a Distancia (UNED), Rome, Italy, ${ }^{2}$ Istituto Superiore di Sanità, Center CNEC (Centre for Clinical Excellence and Quality of Care), Rome, Italy, ${ }^{3}$ Palliative Care Center Antea, Rome, Italy, ${ }^{4}$ ASST Grande Ospedale Metropolitano Niguarda, Dipartimento di Ematologia ed Oncologia, S. C. Dipartimentale Cure Palliative - Hospice Milano, Milano, Italy, ${ }^{5}$ Luce per la Vita Onlus, Rivoli, Torino, Italy, ${ }^{6}$ Unità di Cure Palliative - Hospice S. Marco, Latina, Italy, ${ }^{7}$ Italian Society of Palliative Care (SICP) SUPSI, Dipartimento Economia Aziendale Scienze Sanitarie e Sociali, Manno, Switzerland

Background: Undergraduate nursing education should include palliative care (PC) as the European consensus suggest. The European Society of Palliative Care (EAPC) issued a guide for the development of palliative nurse education in Europe. The Nursing Commission of the Italian Society of PC care (SICP) decided to carry out a process of mapping the undergraduate nursing education in Italian Universities.
Objective: To describe the characteristics of Italian PC undergraduate nursing education and the extent to which the contents taught in Italian nursing undergraduate courses matches with the EAPC guide on nursing education.

Methods: A questionnaire of 27 items was developed by the Italian Nursing Commission. From may 2016 to October 2016, directors and/or coordinators of all Italian nursing undergraduate courses $(n=221)$ were invited to complete it online. They were invited to participate by phone and by e-mail.

Results: A total of 98 (59\%) nursing undergraduate courses (entirely located in central and northern Italy) answered to the survey. The results show that PC education is included in $100 \%$ of nursing undergraduate courses. Only $34 \%$ of the Italian curricula had compulsory PC courses formally declared, of whom, $84 \%$ included within the nursing discipline. The most frequently addressed topics in the nursing curricula, included in the patient and family dimension of the EAPC guide and were related to pain, symptom management $(61,5 \%)$, and care of family $(50 \%)$.

Discussion: The survey results highlight the growing attention that $\mathrm{PC}$ is deserving in undergraduate nursing education in Italy. Nevertheless, the heterogeneity of the teachings let us to suppose that PC contents are not adequately addressed and important dimensions (recommended by the EAPC nursing education guide) such as Team, Society and Health care system are still neglected.

\section{FC20 Education}

Measuring the Effectiveness of an Online Decision Training Resource for UK Medical Students to Help Recognise Whether a Person Is Dying: A Randomised Controlled Trial (the ORaClES Study) White N. ${ }^{1}$, Oostendorp L. ${ }^{1}$, Harries P. ${ }^{2}$, Yardley S. ${ }^{1,3}$, Tomlinson C. ${ }^{1,4}$,

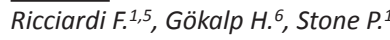

${ }^{1}$ Division of Psychiatry, University College London, London, United Kingdom, ${ }^{2}$ Centre for Applied Health and Social Care Research (CAHSCR), Kingston University \& St George's, University of London, London, United Kingdom, ${ }^{3}$ Central and North West London NHS Foundation Trust, London, United Kingdom, ${ }^{4}$ Department of Surgery \& Cancer, Imperial College London, London, United Kingdom, ${ }^{5}$ Department of Statistical Science, University College London, London, United Kingdom, ${ }^{6}$ Department of Electrical and Electronic Engineering, Ondokuz Mayis University, Samsun, Turkey

Background: All doctors need to be able to recognise when patients are imminently dying. However, few training resources have been developed to teach this clinical skill.

Aim: To assess the effectiveness of an online training resource for medical students to recognise whether a person is imminently dying.

Methods: An online double-blind randomised controlled trial at 5 UK medical schools. Senior students were randomised to either the intervention group (training) or the control group (no training). The intervention detailed how experts in a previous study had weighted various clinical factors when reviewing patient summaries ("vignettes"). Students reviewed the same vignettes as the experts before, after, and 2 weeks after the intervention and provided survival estimates $(0 \%$ certain survival to $100 \%$ certain death). Primary outcome:

(1) The percentage estimate of survival post intervention to calculate the Mean Absolute Difference (MAD) between the students and the experts. Secondary outcomes: The percentage estimates were used to calculate

(2) the judgement weight attributed to each clinical factor;

(3) a maintenance effect; and

(4) a measure of expertise, using the Cochran-Weiss-Shanteau (CWS) index.

Results: 168 eligible students enrolled, 135 completed the trial (82\%) of whom 66 received the intervention (49\%). The intervention group showed 
(1) significantly more agreement with the experts than the control group $\left(\delta_{\text {MAD }}=-3.43,95 \% \mathrm{Cl}-5.3\right.$ to $\left.-1.56, p<0.001\right)$;

(2) weighting of information became more like the experts; and

(3) better consistency and discrimination in their decision making (intervention CWS = 146.04 [sd 140.21], control CWS= 110.75 [sd 104.05]).

(4) There was no maintenance effect $\left(\delta_{\mathrm{MAD}}=1.50,95 \% \mathrm{Cl}-0.87\right.$ to $3.86, p=0.21$ ).

Conclusion: The online training resource proved effective in improving the decision making of medical students. Further testing using realworld prognostic tasks is warranted.

Funding: Marie Curie (MCCC-FPO-16-U)

\section{FC20 Education}

A New Competency-Based Instrument to Assess Resident Physician Knowledge and Self-efficacy in Primary Palliative Care

Moyer K. ${ }^{1}$, Morrison L. ${ }^{2}$, Encadela J. ${ }^{2}$, Kennedy C. ${ }^{2}$, Ellman M. ${ }^{3}$ ${ }^{1}$ Nationwide Children's Hospital, Columbus, United States, ${ }^{2}$ Yale School of Medicine, New Haven, United States, ${ }^{3}$ Internal Medicine, Yale University School of Medicine, New Haven, United States

Background: There is a need to improve both primary palliative care (PC) education and its assessment in graduate medical education. We developed a new instrument based on published PC competencies to assess resident physician (RP) competency and educational interventions.

Aims: To describe the development and psychometric properties of a novel, competency-based instrument to measure RP knowledge and self-efficacy in primary PC.

Methods: We created a two-part instrument comprised of a knowledge test (KT) and a self-efficacy inventory (SEI) addressing 18 core resident PC competencies across five domains: Pain and Symptom Management; Communication; Psychosocial, Spiritual, \& Cultural Aspects of Care; Terminal Care \& Bereavement; and Palliative Care Principles \& Practice. The instrument was distributed to all internal medicine RPs at our institution during the first quarter of academic years 2015-2016 and 20162017. A standard item analysis was performed on the KT. Internal consistency (Cronbach's Alpha) and variable relationships (Factor Analysis) were measured for the SEl. A power analysis was conducted to determine adequate sample size.

Results: One hundred forty-four RPs completed the survey. For $15 \mathrm{KT}$ items, difficulty ranged from $0.17-0.98$, with seven items ranging 0.20 0.80 (typical optimum difficulty); discrimination ranged from 0.03-0.60 with 10 items $\geqslant 0.27$ (good to very good discrimination). Cronbach's Alpha was 0.954 for 35 SEl items. Factor analysis of combined 2015-2016 items yielded four factors explaining the majority of variance for the entire set of variables.

Conclusions / Discussion: Our novel instrument demonstrates promising psychometric properties and reliability in probing the constructs of PC and can be further utilized in PC graduate medical education research to assess learners and evaluate new and existing PC educational interventions.

\section{FC20 Education}

Program of Experience in the Palliative Approach (PEPA) - Improving Palliative Care for Australians Since 2003

Mander $T^{1}{ }^{1}$, He W. ${ }^{2}$, Klug $D .{ }^{2}$, Cosgrove S. ${ }^{2}$, Yates P. ${ }^{2}$

${ }^{1}$ Centre for Palliative Care, St Vincent's Hospital Melbourne,

Melbourne, Australia, ${ }^{2}$ Institute of Health and Biomedical Innovation, Queensland University of Technology, Brisbane, Australia

Background: The palliative approach (PA) aims to improve quality of life for people with a life-limiting illness. It may commence when curative treatments are no longer working, or if someone chooses to stop curative treatment. The focus is on comfort, symptom control and psychosocial support to enable people to live well until death.

The purpose of the Program of Experience in the Palliative Approach (PEPA) is to increase capacity of non-palliative care health professionals to deliver the PA. Elements to achieve this are:

- Workshops

- Placements with palliative care services

- Post-placement support to allow assimilation of new skills

- Provision of opportunities to enhance linkages among palliative and non-palliative providers

Aim:

1. Demonstrate the impact of PEPA on non-palliative care health professionals' ability to provide the PA.

2. Describe the impact of PEPA from the perspective of:

- Placement participants

- Workshop participants

- Employers of placement participants

- Host sites for placement participants

$\bullet$

Method: PEPA invites all stakeholders (participants, employers, host sites) to submit evaluation surveys. Surveys include fixed response items using Likert scales and open-ended questions. Surveys are analysed using the RE-AIM framework, which assesses sustainability of a program against concepts of: Reach, Adoption, Effectiveness, Implementation and Maintenance.

Results: Data demonstrate:

- Increase in number of placement and workshop participants every year

- Participants are from diverse disciplines, location and ethnicity

- Positive impact on palliative care practice

- Positive impact on links between palliative care and other health sectors

Conclusion: PEPA has been delivered in Australia since 2003. PEPA provides experiential education that supports integration of the PA into practice. Participants describe gaining knowledge, skills and confidence in caring for people with palliative care needs. Continued demand shows it is relevant and sustainable.

\section{FC20 Education}

Facing Future Social Challenges: Leadership Skills for Advancing Palliative Care in Eastern and Western Europe

Frerich G. ${ }^{1}$, Koffman J. ${ }^{2}$, Mosoiu D. ${ }^{3}$, Krakowiak P. ${ }^{4}$, Evans C. ${ }^{2}$, Dumitrescu M. ${ }^{3}$, Janowicz A. ${ }^{4}$, Baker-Schuster L. ${ }^{5}$, Voltz R. ${ }^{1,6,7}$ ${ }^{1}$ Department of Palliative Medicine, University Hospital of Cologne, Cologne, Germany, ${ }^{2}$ Cicely Saunders Institute of Palliative Care, Kings College London, London, United Kingdom, ${ }^{3}$ Hospice Casa Sperantei, Brasov, Romania, ${ }^{4}$ Department of Educational Sciences, Nicolaus Copernicus University, Torun, Poland, ${ }^{5}$ Robert Bosch Stiftung, Stuttgart, Germany, ${ }^{6}$ Clinical Trials Center Cologne, Cologne, Germany, ${ }^{7}$ Center for Integrated Oncology Köln Bonn, University Hospital of Cologne, Cologne, Germany

Aims: The development of Palliative Care (PC) across Europe is heterogeneous but countries are recognising the need for PC, especially in the face of current epidemiological developments. The European PC leadership programme is a joint project of four prestigious academic institutions, two in Eastern and two in Western Europe. Its mission is to pioneer tomorrow's leaders in the field across Europe to address anticipated future challenges in our societies. 
Methods: Based on findings of an online needs assessment, the curriculum was developed by a steering committee and an advisory board of international PC experts. Evaluations have been conducted. The programme is to be completed part-time over 1.5 years. It is currently in the third course run comprising up to 20 participants, each of which have been carried out a personal project.

Results: The modules comprise Personal Development, Project Management, Teamwork, Research, Advocacy and Local Best Practice of each host country. Modules are arranged in line with project rationale and to develop leadership habitus. 59 Alumni and participants ( $\mathrm{m}: 20$; f: 39; average age: 41) of 22 Eastern and Western countries (50\% each) have taken part. Various projects have been conducted (16 concerning PC service development, 14 manuals, 12 research, 9 education, 5 others). Quantitative methods were used in 21 projects, in 18 qualitative. Master-level certification was awarded in 2016 (27 ECTS), highlighting coherence, internationality and integration of theory.

Conclusion: Leadership skills are crucial for advancing PC across Europe. Six years of learning from each other in Eastern and Western Europe have been successful. Pioneers have been enabled to face social challenges. This unique program is excellently positioned in the education and job market of European PC. An impact assessment is planned. Fundraising activities aim to secure long-term sustainability and accreditation as European Master with 60 ECTS is strived for.

FC21 Vulnerable Populations and Marginalised Groups The Palliative and Long Term Care and Support Needs of People who Were Formerly Street Homeless

Shulman C. ${ }^{1}$, Hudson B..$^{1}{ }^{1}$, Rice B. ${ }^{2}$, Cornes M. ${ }^{3}$

${ }^{1}$ Marie Curie Palliative Care Research Department, Pathway and UCL, London, United Kingdom, ${ }^{2}$ BR-Research, London, United Kingdom, ${ }^{3}$ Social Care Workforce Unit, Kings College London, London, United Kingdom

Background: Homelessness is associated with severe ill health, exclusion and early death. Research suggests people living in homeless hostels have poor access to palliative care. Little is known about challenges for formerly street homeless people who now live in socially rented accommodation (Clearing House accommodation) and receive floating support from tenancy sustainment teams (staff).

Aims: Explore

1) circumstances surrounding deaths of a cohort of Clearing House residents (clients),

2) insights of current clients and staff around support currently received and

3) what may be needed to improve health and wellbeing.

Methods: Analysis of Clearing House records, semistructured interviews with key workers of 11 deceased clients and focus groups with clients and staff. Qualitative data were analysed using thematic analysis.

Results: Average age of death was 56 years. Heavy substance and alcohol misuse was common. Clients $(n=8)$ described how health and wellbeing was severely impacted while living on the streets. Stigmatization and negative help-seeking experiences made engagement with healthcare services difficult. Loneliness was commonly described. Some felt these were triggers for relapse. Staff $(n=14)$ described working in isolation to manage challenging and distressing cases, difficulties accessing medical information, service inflexibility and lack of multiagency working.

Conclusions: People who access independent tenancies with support through Clearing House have a lower average age of death than the general population and face particular health issues. Continuous and reliable multiagency support is needed to improve and maintain wellbeing in this context. High caseloads and barriers to health and social care services barriers impact on the support of people with high support needs. Access to appropriate level of support and more flexible and sensitive access to services, peer support and meaningful activities were suggested to combat social isolation.

FC21 Vulnerable Populations and Marginalised Groups Defining the Need for Palliative Care Amongst Prisoners in England and Wales

Burtonwood J.1,2, Forbes $K^{2}{ }^{2}$

${ }^{1}$ School of Medicine, Cardiff University, Cardiff, United Kingdom, ${ }^{2}$ Palliative Medicine, University Hospitals Bristol NHS Foundation Trust, Bristol, United Kingdom

Background: In 2017, 184 prisoners died of natural causes in prisons in England and Wales (henceforth 'prisons'). This number has doubled over the last decade and is expected to increase. There is thought to be a significant unmet symptom burden amongst prisoners approaching the end of life (EoL); this suggests a significant need for palliative care (PC) within prisons. Confirming this need has been challenging, however, due to a lack of relevant data collection in prisons. Key to providing effective PC for this population is to first define the underlying demographics and demand for care.

Aims: To define and assess the underlying need for PC in prisons.

Methods: In the UK, all deaths in custody are reported on by an Ombudsman. These reports were used as a data source for descriptive statistical analysis. Data including age, co-morbidity and cause of death were analysed.

Results: The study analysed the reports of 88 deaths in prison in 2016. Average age at death of prisoners was younger than that of the general population (median 68 years). $69 \%$ of the population were multi-morbid and $10 \%$ had a known terminal illness at sentencing. $61 \%$ of all deaths were due to cancer. Respiratory pathology was the predominant cause of death $(43.1 \%)$ and co-morbidity $(44.3 \%)$. This was significantly higher than reported in non-incarcerated populations.

Conclusions: To our knowledge, this study is the first to provide an overview of the demographics of prisoners across England and Wales at the end of life. The study was also a proof of concept of a novel method which could be used for future research. The causes of illness identified in the study population are known to be associated with significant symptom burdens. Many prisoners were frail, and some terminally ill, at the time of incarceration. Thus, the study supports the assertion that demand for $\mathrm{PC}$ care in prisons is high.

Part-funded by a bursary from Health Education England

FC21 Vulnerable Populations and Marginalised Groups Rapid Evidence Assessment of End of Life Care for People with Alcohol and Drug Problems Witham G. ${ }^{1}$, Galvani S. ${ }^{2}$, Peacock M. ${ }^{3}$

${ }^{1}$ Nursing, Manchester Metropolitan University, Manchester, United Kingdom, ${ }^{2}$ Social Care, Manchester Metropolitan University, Manchester, United Kingdom, ${ }^{3} \mathrm{Health}$ and Social Care, Edge Hill University, Omskirk, United Kingdom

Aim: The aim of this rapid evidence assessment, conducted August 2016- August 2017, was to explore the peer-reviewed evidence base in relation to end-of-life care and problematic substance use.

Design: A Rapid Evidence Assessment (REA).

Methods: The following databases were searched using date parameters of $1^{\text {st }}$ January 2004-1 $1^{\text {st }}$ August 2016: Amed, Psycharticles, Ovid, Ageinfo, Medline, Ebscohost, ASSIA, Social Care Online, Web of Knowledge, Web of Science, SSCI, Samsha, NIAAA. Data were extracted using a pre-defined protocol incorporating inclusion and exclusion criteria. Given the dearth of evidence emerging on interventions and practice responses, the inclusion criteria were broadened to include any peerreviewed literature focussing on substance use specifically and end of life care. 
Results: There were 60 papers that met the inclusion criteria. Metaanalysis was neither possible nor appropriate and therefore a systematic mapping approach to the literature was adopted. Using a thematic analysis to categorise findings, papers fell into three broad groups

i) pain and symptom management,

ii) homeless and marginalised groups, and

iii) alcohol-related papers.

Conclusion: There was only a small and diverse literature that lacked depth and quality. There are clear challenges for health and social care professionals in meeting the end-of-life needs of this heterogeneous population since people with substance problems often present with multiple co-morbidities and complex health needs. Addressing issues like safe prescribing for pain management becomes more challenging in the presence of alcohol and illicit drug use and requires flexible service provision from both drug and alcohol services and end of life care providers. More research is needed, particularly to identify models of good practice in working with co-existing substance use and end of life conditions as well as prevalence studies to provide a wider context for policy and practice development.

FC21 Vulnerable Populations and Marginalised Groups The Application of SDM Regarding People with Intellectual Disabilities in the Palliative Phase: A Scoping Review

Noorlandt H.W. ${ }^{1}$, Korfage I.J. ${ }^{1}$, Echteld M.A. ${ }^{2}$, van der Heide A. ${ }^{1}$

${ }^{1}$ Department of Public Health, Erasmus MC, University Medical Center Rotterdam, Rotterdam, Netherlands, ${ }^{2}$ Avans University of Applied Sciences, Breda, Netherlands

Background: Shared decision making (SDM) is the process in which the professional caregiver and patient (or the representative) jointly discuss and decide which treatment policy will be followed. The importance of shared decision making is increasingly being endorsed, also in palliative care. Little is known about SDM in people with intellectual disabilities (ID). Aims: To provide an overview of the application of SDM in people with ID in the palliative care phase.

Methods: In this scoping review, we systematically searched in the Embase, Medline and PsychINFO databases for studies that evaluated the SDM process in people with ID in the palliative phase.

Results: Of 402 titles and abstracts 21 full studies were included. Some papers fall under multiple categories. Twelve were empirical studies, 11 were opinion papers and 7 were legal reports. Ten papers focused on medical decisions in general, 10 papers specifically on medical end-oflife decisions (e.g. foregoing life-sustaining treatment) and 9 papers emphasized end-of-life decisions without a specific medical context. Even though many authors stressed the importance of involving patients in the decision-making process, none of the papers described SDM in the palliative care phase, and no best practices, guidelines or definitions were shown for SDM in the palliative care phase. Papers show an increasing focus on the involvement of people with ID themselves, or at least their loved ones, in making difficult medical decisions around the end of their lives.

Conclusion: This study shows that there is no univocal meaning about what SDM regarding people with an ID should look like. General recommendations indicate that we should involve people with an ID more in the decision-making process by providing them an appropriate environment full of support. More knowledge about the preferences and values, quality of life and life history of people with ID could improve the decision-making process.

FC21 Vulnerable Populations and Marginalised Groups 'We Are Who We Are, Not What We've Done': End of Life Care for People with Alcohol/Other Drug Problems
Ashby J. ${ }^{1}$, Wright S. ${ }^{2}$, Galvani S. ${ }^{2}$

${ }^{1}$ Psychology, Manchester Metropolitan University, Manchester, United Kingdom, ${ }^{2}$ Social Care and Social Work, Manchester Metropolitan University, Manchester, United Kingdom

Aims: As part of an interdisciplinary, multi-method scoping study, this qualitative research aimed to explore:

1. How substance use and hospice services support people with alcohol/drug problems who are approaching the end of their life.

2. The good practice and challenges that people with alcohol/ drug problems face in accessing support and their suggestions about how care may be improved.

Design: Accessed through hospices, substance use services and peer communities, eleven people with life-shortening conditions (who had used substances problematically) participated in semi-structured interviews.

Analysis: Interview transcripts were subject to inductive thematic analysis and detailed case studies were developed to contrast between interviewees accessing hospice care and those not.

Results: Interviewees described experiencing multiple complex health problems, including mental health and social care support needs. Yet many were minimising contact with services or trying to manage how healthcare professionals perceived them in order to avoid stigmatising attitudes and discrimination. Fragmented healthcare delivery and poor communication about their approaching end of life left them little scope to come to terms with dying. The often unpredictable and rapidly deteriorating nature of their health conditions, combined with poor mobility and memory problems, made it extremely challenging for many to access the support they desperately needed - although good care from hospices was commonly described.

Conclusion: Palliative care has much to teach substance use services about explicitly addressing life-shortening conditions with service users. Moreover, cross-fertilization of palliative and substance use approaches, would allow behavioural cues (eg: substance use relapse, self-isolation and deteriorating mental health) to receive greater attention and tailor palliative care better to individual needs.

Funding: Big Lottery Fund (UK)

FC21 Vulnerable Populations and Marginalised Groups Do Adult Persons with Chronic Psychiatric Conditions Receive Fair Access to Specialist Palliative Care? Results from a Systematic Review Martins Pereira S. ${ }^{1,2,3}$, Antunes B. ${ }^{4,5,6}$, Azenha S. ${ }^{7}$, Hernández-Marrero $P^{1,2,3}$

${ }^{1}$ Instituto de Bioética, Universidade Católica Portuguesa, Porto, Portugal, ${ }^{2}$ UNESCO Chair in Bioethics, Institute of Bioethics, Universidade Católica Portuguesa, Porto, Portugal, ${ }^{3}$ CEGE, Centro de Estudos em Gestão e Economia, Porto Católica Business School, Universidade Católica Portuguesa, Porto, Portugal, ${ }^{4}$ Faculdade de Medicina (FMUP), Universidade do Porto, Porto, Portugal, ${ }^{5}$ CEISUC, Centro de Estudos e Investigação em Saúde, Universidade de Coimbra, Coimbra, Portugal, ' Cicely Saunders Institute, King's College London, London, United Kingdom, ${ }^{7}$ Hospital de Apoio de Braga, Braga, Portugal

Background: The linkages between palliative care (PC) and psychiatry are widely described in the literature. Little is known on whether persons with chronic psychiatric conditions have access to specialist PC.

Aims: To review the existing evidence about PC for persons with chronic psychiatric conditions with respect to the access to specialist PC and its specificities.

Methods: Systematic review, following PRISMA 2009. Data sources: PubMed, Web of Science, PsycINFO, EBSCOhost, CINAHL, MEDLINE, Nursing \& Allied Health Collection: Comprehensive, Database of Abstracts of Reviews of Effects, Cochrane Central Register of Controlled Trials, 
Cochrane Database of Systematic Reviews, Cochrane Methodology Register, Library, Information Science \& Technology Abstracts, MedicLatina, Health Technology Assessments, NHS Economic Evaluation Database, PsycARTICLES, Psychology and Behavioral Sciences Collection. Search terms: 'palliative care' OR 'end of life care' AND 'psychiatry' OR 'mental illness' OR 'mental health' OR 'mental disorder' OR 'chronically mentally ill' OR 'serious mental illness' OR 'psychiatric disorders' OR 'severe mental Illness'. Inclusion criteria: studies focusing on PC provision for adults with chronic psychiatric conditions, in English, German, Spanish and Portuguese. Articles independently reviewed by two researchers. Quality appraisal was performed.

Results: 293 articles retrieved, 4 met inclusion criteria. Three articles focused on professionals' perspectives about palliative and end of life care provision, suggesting the need for further education and collaboration between psychiatry and PC. The other article revealed that patients with psychiatric conditions were less likely to receive specialist PC when compared to other patient groups.

Conclusions: This study shows the lack of research on the access and provision of PC for persons with chronic psychiatric conditions. It emphasizes the ethical issue of justice in the access to PC at the end of life.

\section{FC22 Audit and Quality Improvement}

Half a Million Patient Registrations Later - Where Do We Stand and What Are We Aiming for?

Lundström S. ${ }^{1,2}$, Melin-Johansson C. ${ }^{3}$, Fransson G. ${ }^{4}$, Lundorff L. ${ }^{5}$, Martinsson L. ${ }^{6}$

${ }^{1}$ Palliative Medicine, Stockholms Sjukhem Foundation, Stockholm, Sweden, ${ }^{2}$ Oncology-Pathology, Karolinska Institutet, Stockholm, Sweden, ${ }^{3}$ Health Care Sciences/Palliative Research Centre, Ersta Sköndal Bräcke University College, Stockholm, Sweden, ${ }^{4}$ Palliative Medicine, Kalmar County Hospital, Kalmar, Sweden, ${ }^{5}$ Palliative Medicine, Uddevalla Hospital, Uddevalla, Sweden, ${ }^{6}$ Radiation Sciences, Umeå University, Umeå, Sweden

Background: The Swedish Register of Palliative Care (SRPC) is a national quality register which through a web-based end-of-life questionnaire (ELQ) gathers data on quality of care for dying patients, regardless of care setting, age or diagnosis. The ELQ, with 30 questions, is based on the principles of a good death proposed by the British Geriatrics Society and is completed by healthcare staff after the death of a patient. The SRPC started in 2005 and today collects data from approximately two thirds of all deaths in Sweden; $90 \%$ of all cancer deaths are reported.

Aims: To give an update on the current status of the register, achievements so far and plans for the future.

Methods: Data collected in the ELQ is matched with the central population register for validation purposes. Validity is also examined via visits to a selection of units. Add-on questions to the ELQ on specific research questions are now in use through separate modules. Aggregated results are accessible online at the register's website.

Results: Data from over 530000 patients has been registered since 2005 and over 4000 units are reporting data each year. Quality indicators with corresponding goals defined by the Swedish National Board of Health and Welfare shows continuous national improvement. Systematic improvement is now being organized by the government with support from the SRPC. Since 2011, 24 scientific papers based on register data have been published. The focus has mainly been on comparing quality of care between different diagnoses and care settings.

Discussion: The SRPC forms the basis for measurement of quality of endof-life care in Sweden and the working method has proven to be both feasible and valid. Management of more than 4000 reporting units and decreasing financial support to national quality registers from the government are challenges met. Data collection from electronic medical records, bypassing the ELQ, and further use of add-on modules are opportunities in the near future.
FC22 Audit and Quality Improvement

"Did a Member of the Healthcare Team Talk to You about what to Expect when Your Relative was Dying?” - “No." Quality of Care for Cancer Patients Dying in Hospitals: First Results from the International CODE (Care of the Dying Evaluation) Survey

Gerlach C. ${ }^{1}$, Mayland C. ${ }^{2,3}$, Hufthammer K.O. ${ }^{4}$, Tripodoro V. ${ }^{5,6}$, Goldraij

G.7, Leppert W. ${ }^{8,9}$, Wolszczak K. ${ }^{10}$, Garcia Yanneo E. ${ }^{11}$, Nalin Passarini J.12, Bredda Saad I.12, Tuen Hansen M.I. ${ }^{13}$, Faksvåg Haugen D. ${ }^{13,14}$, ERANet-LAC CODE Consortium (www.icode7.org)

${ }^{1}$ Interdisciplinary Palliative Care Unit, University Medical Center of the Johannes Gutenberg University, Mainz, Germany, ${ }^{2}$ Department of Oncology and Metabolism, University of Sheffield, Sheffield, United Kingdom, ${ }^{3}$ Palliative Care Institute, University of Liverpool, Liverpool, United Kingdom, ${ }^{4}$ Centre for Clinical Research, Haukeland University Hospital, Bergen, Norway, ${ }^{5}$ Institute of Medical Research, A. Lanari University, Buenos Aires, Argentina, 6 Instituto Pallium Latinoamérica (NGO), Buenos Aires, Argentina, ${ }^{7}$ Hospital Privado Universitario de Córdoba, Cordoba, Argentina, ${ }^{8}$ University of Medical Sciences, Poznan, Poland, ${ }^{9}$ Department of Quality of Life Research, Medical University of Gdansk, Gdansk, Poland, ${ }^{10}$ Sue Ryder House, Pallmed, Bydgoszcz, Poland, ${ }^{11}$ Palliative Care Unit, Mutualista Asociación Hospital Evangélico, Montevideo, Uruguay, ${ }^{12}$ Sumaré State Hospital, Campinas, Brazil, ${ }^{13}$ University of Bergen, Bergen, Norway, ${ }^{14}$ Regional Centre of Excellence for Palliative Care, Haukeland University Hospital, Bergen, Norway

Background: Quality of care for dying cancer patients has global relevance. Although many cancer patients wish to die at home, a substantial group will need hospital care in their final days. The aims of the ERANetLAC CODE project (2017-2020) were to conduct an international postbereavement survey of hospital cancer deaths and subsequently use the results for direct quality improvement work.

Aims: To assess quality of care, as perceived by bereaved relatives, for adult cancer patients dying an 'expected' death in hospitals across seven countries.

Methods: Post-bereavement survey (August 2017-September 2018) by post (Germany, Norway, UK) or interview (Poland, Argentina, Brazil, Uruguay) using the international version of the CODE questionnaire (i-CODE). Analysis of responses according to the $\mathrm{i}-\mathrm{CODE}$ user guide. Results from near complete dataset.

Results: 1485 eligible deaths identified; 866 relatives responded, minimum 100 per country. Response rates $33-95 \%$, depending on recruitment strategy. $57 \%$ of the deceased were male; $67 \%$ of the respondents female. $49 \%$ died on a medical or surgical ward, $25 \%$ on a palliative care unit. Respondent cases did not differ from non-respondents with respect to patients' gender, age, type of cancer, and type of ward. Overall score for the primary outcome respectful and dignified care of the dying was 3.7 (SD $\pm 0.8,95 \% \mathrm{Cl}$ 3.6-3.8, $0=$ "never", 4="always"), and adequate support for the family $89 \%(95 \% \mathrm{Cl}$ 87-91\%; variation between countries $82-97 \%$ ). $87 \%$ (variation $79-96 \%$ ) were informed about impending death, but $36 \%$ (variation $11-52 \%$ ) lacked information about the dying process. Several other areas for improvement were identified in individual countries.

Conclusion / Discussion: The overall quality of care and support was rated well by most participants. The perceived poor communication about what to expect in the dying phase is a complex discovery and will be further explored. Areas needing improvement will be targeted in the last project year.

FC22 Audit and Quality Improvement Healthcare Professionals' Views on Implementation of Advance Care Planning for Patients with Advanced Pulmonary Disease - A Pilot Study

Hjorth N.E. ${ }^{1,2}$, Schaufel M.A. ${ }^{3,4}$, Sigurdardottir K.R. ${ }^{4,5}$, Haugen D.F. ${ }^{2,4}$ ${ }^{1}$ Palliative Care Team, Centre for Pain Management and Palliative Care, Haukeland University Hospital, Bergen, Norway, ${ }^{2}$ Department 
of Clinical Medicine K1, University of Bergen, Bergen, Norway, ${ }^{3}$ Department of Thoracic Medicine, Haukeland University Hospital, Bergen, Norway, ${ }^{4}$ Regional Centre of Excellence for Palliative Care, Western Norway, Haukeland University Hospital, Bergen, Norway, ${ }^{5}$ Sunniva Centre for Palliative Care, Haraldsplass Deaconess Hospital, Bergen, Norway

Background and aim: Advance care planning (ACP) is a communication process about preferences for end-of-life care. ACP is not routinely used in any hospital in Norway. During the period 2014-2017, we conducted a pilot introducing ACP on a thoracic medicine ward in a Norwegian teaching hospital. The attending physician or a study nurse performed ACP conversations with 51 patients suffering from life threatening pulmonary disease, and documented a summary in the patient's medical record.

The aim of this study was to explore healthcare professionals' experiences and opinions about ACP, based on this pilot.

Methods: The first and second author observed and took field notes. Two focus groups were conducted, with a purposeful sample of 1 physician and 7 nurses; age 25-58 years, all Norwegians, 7 females. Additional comments were purposely collected by e-mail from 4 male physicians. Analysis was by systematic text condensation.

Results: Healthcare professionals reported inclusion barriers, fearing they would deprive patients of hope. Suboptimal information and understanding about the project led to disagreements between the study nurse and healthcare professionals about referrals. Reading the ACP summaries, they found new and valuable information that sometimes was passed on to the primary care services to influence care decisions. ACP was by some suggested as an optional part of discharge planning, still respecting the patient's limits for open communication and wish for confidentiality. Several participants saw ACP as a means to improve communication with patients and relatives, and they called for time and skills to integrate ACP into their daily clinical practice.

Conclusion: Healthcare professionals called for training, feasible routines, as well as allocated time for sustainable implementation of ACP. The training should include skills to identify patients in need of ACP and to recognize turning points in the disease trajectory as triggers for these conversations.

FC22 Audit and Quality Improvement

Global Population Level Cancer Palliative Care Indicators Currently in Use: A Mixed Method Scoping Review to Inform Quality Measurement from Population Data in Australia

Phillips J. ${ }^{1}$, Virdun C. ${ }^{1}$, Luckett T. ${ }^{1}$, Heneka N. ${ }^{1}$, Currow D. ${ }^{1}$, Agar M. ${ }^{1,2,3}$ ${ }^{1}$ Improving Palliative, Aged and Chronic Care through Clinical Research and Translation (IMPACCT), University of Technology Sydney, Ultimo, Australia, ${ }^{2}$ Liverpool Hospital, Liverpool, Australia, ${ }^{3}$ University of NSW, South West Sydney Clinical School, Sydney, Australia

Background: In order to improve palliative care for people with cancer, and their families/carers, we need to measure practice in a feasible and sustainable way.

Aims: To identify global cancer-palliative care indicators that could measure the effectiveness of cancer-palliative care provision in Australia using population level data.

Methods: A review of existing cancer-palliative care policy frameworks and/or indicators for countries ranked as leaders in cancer and palliative care provision ( $n=24)$. Our methodology included: a systematic review of policy frameworks and/or indicators; semi-structured interviews with cancer palliative care stakeholders; and a modified Delphi consensus process to identify the most relevant and feasible indicators for measuring cancer-palliative care quality at population level in Australia. Indicators were reviewed against pre-defined inclusion criteria, themed, classified in line with the Donabedian model, and analysed within the context of Australian policy and data sources.
Results: 321 indicators were identified, of which 64 met criteria for final analysis. The Delphi process selected four indicators:

1. proportion of patients with painful bone metastases receiving single versus multiple fraction, external beam radiotherapy dose with palliative treatment intent;

2. proportion of patients who died from advanced cancer admitted to the intensive care unit in the last 30 days of life;

3. proportion of patients receiving cytotoxic therapy within 2 weeks of death;

4. proportion of last 6 months of life spent at home/community setting.

Conclusion: Globally, numerous approaches have been taken to measuring the quality of cancer-palliative care at the population-level. A combination of indicators may be optimal, that incorporate both service and consumer perspectives, and adhere to a principle of most benefit to most people most of the time, accepting that no indicator will have perfect sensitivity and specificity.

\section{FC22 Audit and Quality Improvement}

Improving Efficiency and Responsiveness in a Community Palliative Care Service: A Quality Improvement Project

Coop H., Magee C.

Coventry and Warwickshire Partnership Trust, Coventry, United Kingdom

Background: Within our community palliative care team in the UK we have seen increasing numbers of patient referrals, with a perceived increase in levels of complexity. The current triage system was leaving patients deemed to be non-urgent waiting a long time before their first contact.

Aim: To improve the efficiency and responsiveness of a community palliative care service within the constraints of existing resources.

Methods: A baseline review of all Clinical Nurse Specialist (CNS) referrals was conducted in December 2017. Using Plan, Do, Study, Act (PDSA) methodology a telephone triage tool, CNS triage rota and telephone triage caseload was instituted. Within this new system the CNS's contacted patients directly to determine the appropriateness and urgency of the referral. The impact of these interventions was reviewed in March 2018. Results: Changes implemented improved efficiency of the triage process. Previously the triage process had taken up to 9 days with up to 5 contacts, mainly with community nurses. After implementation $84 \%$ of triage was performed in one contact, with patients and relatives contacted in $62 \%$ of cases. After triage only $47 \%$ patients referred needed a face to face assessment. For non-urgent patients requiring face to face assessment the waiting time was halved from up to 20 working days to less than 10. There was no impact on urgent referrals who continued to be seen within 2 working days. The triage process ensured the most appropriate multidisciplinary team member reviewed the patient.

Conclusions: This quality improvement project has enabled the community palliative care team to be more responsive to patients referred into the service. The triage process has been streamlined with the majority of assessments now made in one contact. The CNS team were involved throughout, acknowledging change was needed and embracing it, which led to the success of the project.

\section{FC22 Audit and Quality Improvement}

Medical End-of-Life Advanced Directives Knowledge in a Tertiary Hospital

Barros N. ${ }^{1}$, Furini A.F. ${ }^{2}$, Rocha $T^{3}{ }^{3}$, Freitas T. ${ }^{3}$, Couto E. ${ }^{4}$, Campos L. $^{1}$ ${ }^{1}$ Serviço de Dor e Cuidados Paliativos, Hospital Nossa Senhora da Conceição, Porto Alegre, Brazil, ${ }^{2}$ Serviço de Emergência, Hospital Nossa Senhora da Conceição, Porto Alegre, Brazil, ${ }^{3}$ Unidade de Pronto Atendimento, Hospital Nossa Senhora da Conceição, Porto Alegre, Brazil, ${ }^{4}$ Hospital Centenário, São Leopoldo, Brazil 
Background: Previous studies have shown that knowledge on end-of-life advanced directives is low among health care professionals.

Objective: To quantify the knowledge of the end-of-life advanced directives among health care professionals of a public tertiary hospital. Method: Application of questionnaires regarding the knowledge of the directives (8 questions) and 4 questions about end-of-life concepts. Results: Participants: 233 , age $37.28 \pm 8,34$ years old: 68 physicians (29.2\%), 53 nurses (22.3\%), 98 nursing technicians $(42.1 \%), 15$ other professionals (6.4\%). Two hundred and three participants (87, $1 \%$ ) scored 5 or more questions about directives. There was a statistically significant difference between the percentage of correct physicians $(68,100 \%)$ and nursing technicians $(75,74.5 \%)$ answers. (Pearson's chi-square $>0.001)$. One hundred and seventy-eight (76.4\%) individuals stated that they understood the definitions of "comfort measures" and "no ressusitation order", among them only $27(15.2 \%)$ answered at least 3 questions correctly. Conclusion: The discrepancy between the knowledge of the end of life advanced directives and the understanding of the usual terms in end-oflife care alerts to the difficulty of implementing the directives. Strategies aimed at improving communication between teams are necessary to address the desires and values of patients.

\section{FC23 Policy and Health Economics}

Transformation of Palliative Care and End of Life Care through a Whole System Approach: System not Silos

Grimes $C$.

Public Health Agency, Nursing and Allied Health Professions, Belfast, United Kingdom Presenting author email address: corrina.grimes@ hscni.net

Background: A health system consists of all organisations, people and actions whose primary intent is to promote, restore or maintain health. Historically palliative and end of life policy implementation to practice has been in silos, silos of conditions, professions and sectors. The WHO health system framework outlines system building blocks which are needed to improve outcomes.

Aim: To develop a whole system palliative care approach, from policy to practice, for a population of circa 1.8 million, within an integrated health and social care system.

Methods: January-March 2016; review of related policy and strategic documents. Public, user and clinical engagement exercises. March - October 2016; development and implementation of programme mandate and whole system work plan. October 2016 - June 2018; implementation of work plan. June 2018 - March 2020; development of phase 3 work plan and impact evaluation.

Results: A whole system approach to palliative care has been achieved, with membership from all sectors across the system, with clinical leadership, and co- production with service users/ carers. The members work together to deliver a shared vision; that all people who could benefit from a palliative care approach, regardless of their condition are identified, allocated a key worker, have the opportunity to discuss and record their advance care planning decisions, and be supported with appropriate generalist and specialist palliative care services. In June 2018 the programme secured circa $f 700 \mathrm{~K}$ for $2018 / 19$ and potentially a significantly higher allocation for 2019/20.

Conclusion: A whole system approach has increased policy influence, enabled the delivery of the shared vision, improved partnership working and service re-design with the ultimate goal of improving the quality of life for those with palliative and end of life care needs and those important to them.

FC23 Policy and Health Economics

Decreased Hospitalizations due to Proactive Palliative Care in Long-term Care Facilities: A Cost-effectiveness Analysis alongside a Cluster Randomized Trial (PACE)
Wichmann A.B. ${ }^{1}$, Adang E.M.M. ${ }^{1}$, Vissers K.C.P. ${ }^{1}$, Szczerbińska K. ${ }^{2}$, Kylänen M. ${ }^{3}$, Payne S. ${ }^{4}$, Gambassi G. ${ }^{5}$, Onwuteaka-Philipsen B.D. ${ }^{6}$, Smets ${ }^{T}{ }^{7}$, Van den Block L. ${ }^{7}$, Deliens L. ${ }^{7}$, Vernooij-Dassen M.J.F.J. ${ }^{1}$, Engels $Y^{1}{ }^{1}$, PACE

${ }^{1}$ Radboudumc, Nijmegen, Netherlands, 2Jagiellonian University Medical College, Kraków, Poland, ${ }^{3}$ National Institute for Health and Welfare, Helsinki, Finland, ${ }^{4}$ Lancaster University, Lancaster, United Kingdom, 5Università Cattolica del Sacro Cuore, Rome, Italy, ${ }^{6} \mathrm{VUmc}$, Amsterdam, Netherlands, ${ }^{7}$ Vrije Universiteit Brussel \& Ghent University, Brussels, Belgium

Background: In the EU, numbers of residents in long-term care facilities (LTCFs) in need of palliative care are growing. This will significantly increase percentages of budgets spent on palliative care. However, robust evidence on the cost-effectiveness of palliative care interventions in this setting is lacking.

Aim: Assessing the cost-effectiveness of the 'PACE Steps to Success' intervention, aiming at integrating general palliative care into day-to-day routines in LTCFs by means of a train-the-trainer approach, in LTCFs throughout seven EU countries (BE, FI, IT, NL, PL, EN, SW).

Methods: A cluster RCT was conducted in 78 LTCFs within the PACE project. LTCFs retrospectively reported deaths of residents. For each case, a questionnaire about the last month of life was filled in by a staff member most involved. Direct medical costs (related to hospital admission, health care professional visits and medical treatments), QALYs based on the EQ5D-5L and costs per quality increase as measured with the QODLTC and CAD-EOLD were outcome measures. Intervention related costs were calculated. Differences in both costs and effects were analyzed using a mixed model approach.

Results: Data on 1593 residents from 73 LTCFs were collected. Significant cost-savings were achieved in the intervention group (€983,28, p-value $0.020)$, which mainly resulted from decreased hospitalization related costs (€919,51, p-value 0.018). Also, an increase on the QOD-LTC was found (3.40, p-value 0.00). Since no significant differences were found on EQ5D-5L and CAD-EOLD outcomes, QALYs were not calculated and cost-minimization was pursued.

Conclusion: The 'PACE Steps to Success intervention' showed to be costeffective. Significant cost savings and improved quality of EoL were realized. In line with earlier findings, we conclude that investing in palliative care training in LTCFs can increase quality of EoL and reduce medical costs in the last month of life, mainly due to decreased hospitalizations.

\section{FC23 Policy and Health Economics} Improving Access to Palliative Care Medicines in South Africa Sithole Z. ${ }^{1}$, Gray A. ${ }^{2}$

${ }^{1}$ Adcocacy, Hospice Palliative Care Association of South Africa, Cape Town, South Africa, ${ }^{2}$ Division of Pharmacology, Disciple of Pharmaceutical Sciences, University of KwaZulu-Natal, Durban, South Africa, Durban, South Africa

Aims: South Africa's National Policy Framework and Strategy on Palliative Care 2017 - 2022 was developed by a Steering Committee supported by Task Teams (TTs). The Drug Availability TT was tasked to recommend palliative care medicines for inclusion on the Essential Medicines List, develop a national morphine monitoring system and recommend regulations to allow nurses to prescribe, dispense and administer necessary analgesics.

Approach:A TT member who served on the Essential Medicines List Committee tabled a position paper which called for "a carefully considered approach to the level of evidence required for listing medicines for palliative care, taking into account the specific exigencies of palliative care, the degree to which off-label use has to be resorted to in this field, and the existing evidence base". The design of the proposed morphine monitoring system was based on data obtained from the KwaZulu-Natal Provincial Medicines Procurement Unit, combined with denominator 
data obtained from the District Health Information System. A draft set of regulations was proposed to the South African Nursing Council (SANCA). Results: Separate chapters on palliative care and pain have been included in the Paediatric Hospital and Primary Health Care Standard Treatment Guidelines published in 2017 and 2018, and will be included in the next Adult Hospital edition. The proposed morphine monitoring system is being implemented at the national level, using data from the RSA Pharma database.The indicator will be proposed for inclusion in the National Indicator Dataset. The SANC has progressed the draft regulations and they are about to be submitted to the Minister of Health.

Lessons learned: Ensuring that a new policy document is not developed in isolation, but is clearly linked with and co-ordinated with existing structures and processes is critical to its success. The development of a simple, yet sensitive indicator of access will allow for regular monitoring of implementation.

\section{FC23 Policy and Health Economics}

Widening of the Palliative Care Access Abyss: Projections of Serious Health-related Suffering up to $\mathbf{2 0 3 0}$ by Age Groups and World Regions Sleeman K.E., De Brito M., Etkind S.N., Nkhoma K., Guo P., Higginson I.J., Gomes B., Harding R.

Cicely Saunders Institute of Palliative Care, Polity and Rehabilitation, King's College London, London, United Kingdom

Background: The global unmet need for palliative care has been described as an "access abyss". Worldwide, ageing populations and a rise in non-communicable diseases will increase pressure on struggling health systems. Understanding the impact of these changes on the need for palliative care is an ethical and economic imperative. Our aim was to project the global and regional burden of serious health-related suffering up to 2030, to inform policies that strengthen health systems.

Methods: The burden of serious health-related suffering was estimated using WHO mortality projections for 2015 and 2030, incorporating estimates of physical and psychological symptom prevalence in 20 conditions, carrying forward methods developed by the Lancet Commission on Palliative Care and Pain Relief (2018).

Findings: The global burden of serious health-related suffering is projected to increase in both absolute and relative terms. By 2030 there will be an estimated 33 million people dying each year with serious healthrelated suffering. This is a $27 \%$ increase compared to 2015 , mainly driven by rises in expected deaths from malignant neoplasms ( $45 \%$ increase), respiratory disease ( $42 \%$ increase) and stroke (28\% increase). Globally, this burden is projected to fall among the youngest age groups ( $18 \%$ decrease in 0-4 years old), and to increase most rapidly in people aged over 70 (50\% increase). Low-income regions will experience the largest proportional increase in serious health-related suffering ( $35 \%$ increase) compared to $30 \%$ in low-middle, $24 \%$ in upper-middle, and $21 \%$ in high-income regions. Interpretation: By 2030, the burden of serious health-related suffering will grow by $50 \%$ among the oldest old, and by more than a third in lowincome regions, populations which have least access to palliative care. Moving towards Universal Health Coverage as one of the Sustainable Development Goals requires immediate action by governments to integrate palliative care into health systems.

\section{FC23 Policy and Health Economics}

Research Funding for End of Life Care Past and Present - Are We Doing Enough?

Crumpler C. ${ }^{1}$, Best S. ${ }^{1}$, Mutabbir A. ${ }^{2}$, Davies L. ${ }^{3}$, Thakrar S. ${ }^{1}$

${ }^{1}$ Research Management, Marie Curie, London, United Kingdom,

${ }^{2}$ National Cancer Research Insitute, London, United Kingdom,

${ }^{3}$ International Cancer Research Partnership, London, United Kingdom

Background: The National Cancer Research Institute (NCRI) maintains a record of funded research grants, the Cancer Research Database (CaRD) that includes grant data from the 19 largest cancer research funders in the UK. The International Cancer Research Partnership (ICRP) is an alliance of cancer research organisations from 7 countries. The ICRP maintains the only public source worldwide of cancer research grants, totalling over $\$ 50$ billion in cancer research since 2000 . It has previously been reported that in 2012 , only $0.24 \%$ was allocated to cancer-related end of life care (EoLC) research in the UK [Sleeman, Gomez and Higginson, 2012].

Aim: To understand whether investment levels in cancer-related EoLC research have changed since 2012.

Methods: NCRI and ICRP code grant data according to the Common Scientific Outline (CSO). An analysis of the NCRI CaRD was undertaken to identify awards which had a CSO code 6.6 (End of life care) as well as an analysis of the dataset of the ICRP which represents about $60 \%$ of international cancer research funding [Davies L, personal communication]. Results: Between 2002-2017 NCRI partners spent $€ 7.4$ billion on cancer research, but only $0.18 \%$ (between $0.09 \%$ and $0.32 \%$ annually) of this total was spent on EoLC. The percentage that Marie Curie contributed to EoLC research funding has increased from $7 \%$ in $2002 / 03$, to $55 \%$ in 2016/17. Most of Marie Curie's EoLC research spend (87\%) is not cancer site specific. The ICRP analysis showed that between 2005-2015 the USA was the top funder internationally, ahead of Canada and the UK. Marie Curie was the second largest funder internationally in 2015.

Conclusion: Analysis of the NCRI CaRD has demonstrated that despite there being an increase in EoLC cancer research spend, investment remains low and EoLC research is still underfunded. Analysis of the ICRP database highlights the importance of Marie Curie's charitable contribution to EoLC research funding internationally.

FC24 Service Development and Organisation of Palliative Care Integrated Oncology and Palliative Care: Analysis of a New "Triggers" Service for Lung Cancer Patients

Droney J. ${ }^{1}$, Nevin J. ${ }^{1}$, Kano Y. ${ }^{1}$, Kamal L. ${ }^{1}$, Wiseman T. ${ }^{2}$, Popat S. ${ }^{2}$, Perkins M. ${ }^{2}$, Oloko R. ${ }^{1}$, Mohammed K. ${ }^{2}$, Kennett A. ${ }^{1}$, Wood J. ${ }^{1}$, Stevens A.-M. ${ }^{1}$ ${ }^{1}$ Symptom Control and Palliative Care, Royal Marsden Hospital, London, United Kingdom, ${ }^{2}$ Royal Marsden Hospital, London, United Kingdom

Background: Global leading cancer organisations recommend that palliative care should be available to all patients, regardless of stage of disease.

We established a novel Integrated Palliative Care service ("Triggers" Service) for lung cancer outpatients. New patients are screened by oncologists using a brief palliative care referral "Triggers" tool to identify patients who should be undergo full assessment. Assessment of palliative care need is completed using validated tools (IPOS Integrated Palliative Outcome Scale, Performance status).

Aims: Evaluation of the feasibility and effectiveness of the "Triggers" service using data collected from the initial 12 months of the service.

Methods: Prospective observational service evaluation, using descriptive statistical analyses. Approved by the local Committee for Clinical Research.

Results: 496 eligible patients were identified, of which 433 (87\%) were reviewed through the "Triggers" service within 8 weeks of initial oncology outpatient clinic consultation.

$62 \%$ (272) of patients were positive for at least one Palliative Care referral "Trigger" tool item. IPOS and performance status data were available for 269 of these. $79 \%$ (212/269) patients had at least 1 "severe" or "overwhelming" palliative care need. 76\% (205/269) patients were performance status 0-1.

At time of analysis, 120 patients had died. The median (range) time between earliest palliative care review and death/censor date was 197 days (3-485 days), compared to 79 (10-1315) days at baseline before establishment of "Triggers" service.

As a result of this service, the number of new patients reviewed annually by the hospital Palliative Care team increased by $30 \%$. 
Conclusion/ discussion: The "Triggers service appears feasible and effective in establishing early palliative care for patients with lung cancer. Further evaluation and economic modelling is required to facilitate roll out to other tumour groups and services.

FC24 Service Development and Organisation of Palliative Care Complexity of Patients with Palliative Care Needs in a Department of Internal Medicine, Emergency ant ICU of a Private Hospital Carneiro R. ${ }^{1}$, Simões C. ${ }^{1,2}$, Carneiro A. ${ }^{3}$

${ }_{1}^{1}$ Palliative Care Team, Hospital da Luz Arrábida, Vila Nova de Gaia, Portugal, 'Escola Superior de Saúde de Santa Maria, Porto, Portugal, ${ }^{3}$ Hospital da Luz Arrábida, Vila Nova de Gaia, Portugal

Background: The integrated palliative care model can be an excellent solution so that even small hospitals can have the right resources to meet complex palliative needs.

Aims: To identify patients with complex palliative care needs in an Internal Medicine Department (IMD) of a private portuguese hospital and to analyse the integrated intervention of the IMD palliative care team. Methods: Analytical cross sectional syudy conducted in the IMD (Inpatient, ICU and emergency) of a private Portuguese hospital between January and June of 2018. Sample: 400 patients; Average age 76 (35101). A data collection tool was used: Goldstandard Framework (GSF), Prognostic Indicator Guidance ( $2^{\text {nd }}$ edition) and Diagnostic Instrument of Complexity in Palliative Care (ICD-Pal)

Results: $40 \%$ of the patients were in an end-of-life scenario, according to GSF. More than one third of the patients had complex or highly complex needs. Nearly three thirds of all complex or highly complex patients were in an end of life scenario when the GSF was applied. More than two thirds of patients with highly complex palliative care needs were supported by the Palliative Care Team (Equipa de Acompanhamento, Suporte e Paliação).

Conclusion: The results show that half of the patients admitted to the Internal Medicine Department (inpatient clinic, ICU and internal consultation) had, at the time of data collection, a limited prognosis according to the GSF. Most of these patients had complex or highly complex palliative care needs. Patients with palliative care needs can be easily identified and specific resources must be available to answer to the complexity of patient and family situation. Palliative care teams must adapt their structure in order to meet the dynamics of the institutions and the patients they are following up.

FC24 Service Development and Organisation of Palliative Care Recognising Palliative Care Needs in a General Medical Unit: A Nurse-led Initiative?

Whelan T. ${ }^{1}$, Marco D. ${ }^{2,3}$, Bryan T. ${ }^{1}$, Weil J..$^{1,3,4}$

1Palliative Care Consultative Service, St Vincent's Hospital, Melbourne, Australia, ${ }^{2}$ Centre for Palliative Care, St Vincent's Hospital Melbourne, Melbourne, Australia, ${ }^{3}$ Department of Medicine, University of Melbourne, Melbourne, Australia, ${ }^{4}$ Centre for Palliative Care, St Vincent's Hospital, Melbourne, Australia

Aims: Hospital-based palliative care consult services (PCCS) generally receive high referral rates from medical units. Many of these patients, however, are referred too late to benefit from symptom management and psychosocial support that palliative care can provide when integrated with appropriate treatment for their underlying illness. Instead patients are often in their final admission, or indeed days of life, limiting the benefit of palliative care interventions.

The Supportive and Palliative Care Indicators Tool (SPICT) is a validated screening tool that can be used in routine care to identify patients with as yet unmet palliative care needs, and who may be at risk of dying. This project aims to test the feasibility and utility of nurse-led implementation of the SPICT tool to identifying patients who may benefit from palliative care assessment.
Methods: A point prevalence audit was conducted on two medical wards at a large public Melbourne metropolitan hospital. The SPICT tool was used by a nurse practitioner to gather information from paper based hospital records and from the hospital patient administration system. Only patients under the care of general medical teams were audited in this 'snapshot'.

Results: In this sample, all patients had at least one advanced disease documented. 40 of $43(93 \%)$ of patients were identified as being SPICT positive (ie having at least 2 indicators of general deterioration). $90 \%$ of the SPICT positive patients had three or more indicators of deterioration, and $75 \%$ had poor or deteriorating function explicitly documented by clinicians. However, less than $10 \%$ of SPICT positive patients had an active PCCS referral in place.

Conclusions: This point prevalence study demonstrates the feasibility of a nurse-led SPICT tool initiative, and demonstrates the high degree of unmet palliative care needs of the general medical inpatient population.

FC24 Service Development and Organisation of Palliative Care Palliative Care in the Finnish Hospitals in the Home

Pöyhiä R ${ }^{1,2}$, Ohvanainen A. ${ }^{3}$, Niemi-murola L. ${ }^{4}$

${ }^{1}$ Kauniala Hospital, Kauniainen, Finland, ${ }^{2}$ Oncology/Palliative Medicine, University of Turku, Turku, Finland, ${ }^{3}$ Home Based Care, Doctagon, Helsinki, Finland, ${ }^{4}$ Anaesthesia and Intensive Care, University of Helsinki, Helsinki, Finland

Background: Although advanced home care is available in many western countries, detailed information of hospitals-in-the-home (HIH), hospitallike care at home, is lacking.

Aims: This study was performed to find out how the hospitals in the home $(\mathrm{HIH})$ function particularly in the end-of-life $(\mathrm{EOL})$ care in Finland. Methods: $56 \mathrm{HIHs}$ where identified using an internet search and a list list of participants of annual meeting of Finnish HIH staff. Two web-based questionnaires were emailed to the nurses-in-charge of the hospitals. One questionnaire asked information about the structure and the other one about EOL care. The performance during 2015 was analyzed. The sizes of the catchment areas for the HIHs were checked in the National Statistics of Finland.

Results: 34 (61 \%) hospitals answered to the first questionnaire and 18 (21\%) to the second one. $20,6 \%$ of the hospitals had been opened in the 1990 's, 35,3 \% in 2000 's and $44,1 \%$ in the 2010 's. 62 \% of HIHs functioned as a part of municipal hospital or home care. A median population of the catchment area for one HIH was 40970 (6988-635181). A typical staff included nurses, physicians, clerks and physiotherapists. Only few HIHs had volunteers. 544 (31-5614) patients were seen in average in a HIH during $2015 ; 24,9 \%$ of these for palliative/EOL care. Palliative care was based on nursing. Physicians met $44,1 \%$ of patients in EOL care. Syringe drivers were used in $94 \%$, ascites or pleural fluid drainage were performed in $94 \%$ or $33 \%$, resp, and spinal analgesia managed in $56 \%$ of units. $66,7 \%$ of HIHs had a dedicated hospital ward if in-ward care was needed. Median number of deaths at home was 12 (0-316) in 2015. ESAS was used only in $32,4 \%$.

Discussion: An increasing number of $\mathrm{HIHs}$ has been established in Finland as part of the public health care. The daily care is based on nursing. Many invasive palliative procedures are safely done at home. More effort is needed for quality control. HIHs suffer from lack of personnel.

FC24 Service Development and Organisation of Palliative Care The Birth of a Discipline: Development of Academic Professionalisation in German Palliative Medicine from a Linguistic Viewpoint Peters J. ${ }^{1}$, Dykes N.M. ${ }^{2}$, Heckel M. ${ }^{3}$, Ostgathe C. ${ }^{3}$, Habermann M. ${ }^{1}$ ${ }^{1}$ German Linguistics, Friedrich-Alexander-Universität ErlangenNürnberg, Erlangen, Germany, ${ }^{2}$ Computational Corpus Linguistics, Friedrich-Alexander-Universität Erlangen-Nürnberg, Erlangen, Germany, ${ }^{3}$ Department of Palliative Care, Universitätsklinikum Erlangen, Erlangen, Germany 
Background: Modern palliative medicine as a discipline has - compared to other fields in medicine - relatively recently developed an independent academic paradigm with specific guiding concepts, goals and values. Academic publications allow to trace the historical development of the discipline and the emergence of palliative vocabulary.

Aims: The study aims to illustrate the emergence of institutionalised palliative care in the last 20 years on the basis of a large data sample of scientific texts.

Methods: A corpus of articles from the leading German medical journal Deutsches Ärzteblatt between 1973 and 2018 (c. 400.000 words) was analysed with qualitative linguistic analysis. The dataset allows the exploration of attitudes towards palliative medicine from the perspective of general medicine. A second corpus from the German journal Zeitschrift für Palliativmedizin (2000-2017, c. 4.5 million words) was used to determine keywords in modern-day palliative care during three different time periods.

Results: The analysis of 1492 articles from $D \ddot{A}$ showed that palliative medicine was increasingly mentioned since the mid-1990s. Certain term pairs like palliative-curative develop a specific function referring to palliative settings and become established as fixed idioms. The word palliative is enriched with positive concepts like relief, autonomy, dignity, support, inclusion or spirituality. The 'creation' of the discipline is accomplished through the display of independent medical professionality and a strong identity as specialists for terminal illnesses. Compared to other disciplines, the vocabulary of palliative medicine turned out to be particularly patient-oriented and holistic, improving the quality of life of patients and their families facing the problems associated with lifethreatening illness.

Conclusions: The analysis of large amounts of academic texts allows an empirical observation of the development of palliative medicine based on its central concepts.

\section{FC25 Supporting Family and Caregivers}

Nursing Interventions to Support Family Caregivers in Palliative Home Care: A Systematic Review

Becqué Y. ${ }^{1}$, Rietjens J. ${ }^{2}$, van Driel A.G. ${ }^{1}$, van der Heide A. ${ }^{2}$, Witkamp E. ${ }^{1}$ ${ }^{1}$ Research Centre Innovations in Care, Rotterdam University of Applied Sciences, Rotterdam, Netherlands, ${ }^{2}$ Department of Public Health, Erasmus University Medical Center Rotterdam, Rotterdam, Netherlands

Background: Family caregivers are crucial in end of life care. However, family caregiving may involve a significant burden with negative health consequences such as distress and anxiety. Nurses are in a unique position to support family caregivers at home. Little is known about which components of nursing interventions in this context are effective. We performed a literature review to provide an overview of available supportive nursing interventions for family caregivers in palliative home care.

Methods: A systematic literature review was conducted in the electronic databases: Embase, Medline Ovid, Web of Science, Cochrane Central and Google Scholar. We included quantitative studies that report about nursing interventions to support family caregivers in palliative home care. We extracted data on intervention components and family caregivers' outcomes. Methodological quality was assessed with the Cochrane Risk of Bias Tool.

Results: Out of 1004 titles and abstracts, eight articles were included, reporting about seven studies and seven interventions. Three studies concerned randomized controlled trials, one a non-randomized trial and three a single group prospective study. The interventions studied incorporated one or more components (needs-assessment, psychoeducation, practical support and peer support). Psychoeducation was the most common component. Nursing interventions positively affected the preparedness, competence, rewards and burden of the family caregivers. Multicomponent interventions seemed to be most effective, with needsassessment and psychoeducation as potentially effective components.
Conclusion: Few interventions to support family caregivers in palliative home care for nursing practice have been published. The interventions that were found positively affected family caregivers' outcomes. This review shows that nurses can play an important role in supporting family caregivers in end of life care.

\section{FC25 Supporting Family and Caregivers}

Caring for Carers in the UK: Findings from the Hospice UK Organisational Survey of Carer Assessment and Support (HUK-OSCAS) Higgerson J. ${ }^{1}$, Ewing G. $^{2}$, Rowland $\mathrm{C}^{3}{ }^{3}$, Grande $\mathrm{G} .{ }^{3}$

${ }^{1}$ Nursing, Midwifery \& Social Work, University of Manchester, Manchester, United Kingdom, ${ }^{2}$ Centre for Family Research, University of Cambridge, Cambridge, United Kingdom, ${ }^{3}$ Nursing, Midwifery and Social Work, University of Manchester, Manchester, United Kingdom

Background: In the UK, end-of-life care services are increasingly delivered to patients in the home setting. Accordingly, the role of unpaid, informal carers becomes more pivotal, as does the need to ensure carers are appropriately supported. Previous work by the authors established 10 recommendations for key factors required to implement comprehensive, person-centred carer assessment and support within palliative care services.

Aims: To identify the current provision of assessment and support services for carers of patients receiving end-of-life care through hospice organisations in the UK, in relation to the 10 recommendations.

Methods: All hospices providing adult services within the membership of Hospice UK $(n=200)$ were surveyed in a cross-sectional study between March and June 2018. Descriptive statistics were used to examine responses.

Results: 115 (57.5\%) hospices responded to the survey. 12.5\% reported a specific action plan for carers and $37.4 \%$ using a formal carer assessment process. $94.8 \%$ of hospices reported recording demographic data, $87.0 \%$ reported consistent identification and recording of data around carers, $77.9 \%$ receiving senior management support, and $71.1 \%$ regularly communicating around carers. Approximately half provisioned training for carer support (48.7\%), carer support as part of work description $(54.9 \%)$ and use of carer champions $(56.0 \%)$. The least frequently met recommendations were monitoring and auditing of carer support $(30.6 \%)$, and the storage of carer data separate to patient (17.6\% doing this all the time).

Discussion: Most organisations provided services for carers, although comprehensiveness of provision varied. While the foundations for carer assessment and support are largely in place, there is a need for improvements in how services are implemented. Particular areas for improvement include the use of person-centred processes specific to carers: formal assessment, action plans, recording processes and monitoring systems.

\section{FC25 Supporting Family and Caregivers}

Family Meetings for Hospitalized Palliative Care Patients: Do They Improve Outcomes for Family Caregivers?

Hudson P. ${ }^{1,2,3}$, Thomas K. ${ }^{1}$, Girgis A. ${ }^{4}$, Mitchell G. ${ }^{5}$, Philip J. ${ }^{1,2,6}$, Parker

D. ${ }^{7}$, Currow D. ${ }^{7}$, Liew D. ${ }^{8}$, Le B. ${ }^{9}$, Moran J. ${ }^{10}$, Brand C. ${ }^{8}$

${ }^{1}$ Centre for Palliative Care, St Vincent's Hospital Melbourne,

Melbourne, Australia, ${ }^{2}$ The University of Melbourne, Melbourne, Australia, ${ }^{3}$ Vrije University Brussels, Brussels, Belgium, ${ }^{4}$ The University of New South Wales, Sydney, Australia, ${ }^{5}$ University of Queensland, Brisbane, Australia, ${ }^{6}$ Victorian Comprehensive Cancer Centre, Melbourne, Australia, 7University of Technology Sydney, Sydney, Australia, ${ }^{8}$ Monash University, Melbourne, Australia, ${ }^{9}$ Melbourne Health, Melbourne, Australia, ${ }^{10}$ Austin Health, Melbourne, Australia

One of the primary goals of palliative care is to improve the quality of life of family caregivers. However, approximately $40 \%$ of family caregivers may experience psychological distress which is typically under-recognised 
and consequently not addressed. Family meetings are commonly recommended as a means whereby health professionals, patients and family caregivers discuss issues and plan care. However, there has been limited empirical research to determine the effect of these meetings in palliative care.

Aims: To determine if family caregivers of hospitalised patients referred to palliative care who receive a family meeting report significantly: lower psychological distress (primary outcome), fewer unmet needs, improved quality of life and feel more prepared for the caregiving role.

Methods: 297 family carers were enrolled into a cluster type randomised trial in Australia. Participants in the control group $(n=153)$ received usual care from the palliative care team and participants in the intervention group $(n=144)$ received usual care plus a structured family meeting (conducted in accordance with published guidelines). Participants completed outcome measures upon consent (Time 1); 10 days later (Time 2) and two months after the patient died (Time 3). Regression analyses were undertaken to make adjustment for differences between the two groups at baseline.

Results: At Time 2, the intervention group had significantly lower distress scores (-1.61, 95\% confidence interval $[\mathrm{Cl}]-2.86$ to -0.35$)$ and significantly higher preparedness scores $(2.02,95 \% \mathrm{Cl} 0.64$ to 3.39$)$ compared to the control group. There were no significant changes at Time 3.

Discussion/ conclusion: Family meetings conducted in a hospital setting for patients with advanced disease receiving palliative care may be helpful in reducing family caregiver distress and enhancing their sense of preparation for the caregiving role.

\section{FC25 Supporting Family and Caregivers}

Variations in Grief, Anxiety, Depression and Health among Family Caregivers before and after the Death of a Close Person in Palliative Home Care

Holm M. ${ }^{1}$, Årestedt K.2,3, Öhlen J.4,5, Alvariza A.6,7

${ }^{1}$ Department of Nursing Sciences, Sophiahemmet University, Stockholm, Sweden, ${ }^{2}$ Faculty of Health and Life Sciences, Linnaeus University, Kalmar, Sweden, ${ }^{3}$ Department of Research, Kalmar County Hospital, Kalmar, Sweden, ${ }^{4}$ Institute of Health and Care Sciences, University of Gothenburg, Gothenburg, Sweden, ${ }^{5}$ Centre for PersonCentred Care, Sahlgrenska Academy, University of Gothenburg, Gothenburg, Sweden, ${ }^{6}$ Department of Health Care Sciences, Ersta Sköndal Bräcke University College, Stockholm, Sweden, ${ }^{7}$ Dalen Hospital, Stockholm, Sweden

Background: Feelings of grief, symptoms of anxiety and depression, and poor health are often observed in family caregivers both before and after an expected death. However, the variations over time needs to be explored further and it needs to be examined if support during caregiving could influence these emotional reactions.

Aims: The aim was to investigate longitudinal variations in grief, symptoms of anxiety and depression and self-rated health among family caregivers in palliative home care who participated in a randomised psycho-educational intervention study.

Methods: Data derived from a randomised intervention study with two arms (intervention and control). Data were collected through selfreported questionnaires at four time points: baseline, after the intervention, two months later and six months after the patient's death. Analyses were performed using a mixed model ANOVA for pre-death grief ( 3 measurements) and anxiety, depression and health (4 measurements). Post-death grief was measured at one occasion, after the patient's death. Unpaired T-tests were used to examine differences between interventions and controls after death.

Results: In total, 117 family caregivers completed all measurements. Grief was stable across the three pre-death measurements with no significant variations, while anxiety, depression and health varied significantly across the four time points. After the patients' death, the level of anxiety and depression decreased, and health increased. No significant differences were found between interventions and controls for any of the variables neither pre- or post-death.

Conclusion: This study showed different patterns in grief compared to anxiety and depression and health. This adds to a body of research showing that grief is distinct from symptoms of anxiety and depression. Further, it also demonstrates the complex emotional reactions of being a family caregiver in palliative care.

FC25 Supporting Family and Caregivers

Using a Comprehensive Evidence Based Tool (KOMMA) to Identify Support Needs of Family Carers in a German Palliative Home Care Setting

Kreyer C. ${ }^{1}$, Pleschberger $S{ }^{2}$

${ }^{1}$ Department of Nursing Science and Gerontology, UMIT - Private Universitiy for Health Sciences, Medical Informatics and Technology, Hall in Tyrol, Austria, ${ }^{2}$ GÖG - The Austrian Public Health Institute, Vienna, Austria

Background: KOMMA (Kommunikation mit Angehörigen) is the validated German version of the Carer Support Needs Assessment Tool (CSNAT). It is an evidence-based tool to identify carer support needs in palliative home care. The KOMMA-tool comprises 14 domains, enabling carers to indicate the need for more support. As a practice intervention, it is integrated into a person-centred approach of assessment and support (the KOMMA Approach). As part of an implementation project, the KOMMA Approach was used in palliative home care in Germany.

Aim: To identify family carer support needs indicated by carers using the KOMMA-tool

Methods: The KOMMA Approach was implemented in four regional hospice and palliative home care services as a service development. Practitioners were trained by champions in use of the KOMMA-tool and approach. Over a 10 month period (7/2017 to 4/2018) the intervention was delivered to family carers of cancer and chronically ill patients. Selfreported support needs indicated on the KOMMA-tool were analysed using statistical methods.

Results: In total 202 KOMMA-tools were completed (70\% female; mean age 61.5 years). Most carers wanted more support with 'knowing what to expect in the future' (70\%) and 'having time for yourself in the day' (65\%). There were significant differences in support needs identified between female and male caregivers, e.g. men needed more 'practical help in the home' ( $p=0,017$ ), whereas women expressed more frequently the need of 'having time for yourself in the day' $(p=0,043)$ and 'dealing with your feelings and worries' $(p=0,039)$.

Discussion: This is the first study to report family carer support needs in end-of-life home care in Germany. Compared to similar studies from the UK and Australia the top priority is the same, however, 'Dealing with your feelings and worries' which was number two priority in UK and AUS ranked lower in Germany (rank 5), which might refer to cultural differences in dealing with death and dying.

\section{Global challenges in Paediatric Palliative Care: An EAPC Seminar}

PPC1 Prevalence of Life-Limiting Conditions in Paediatric Palliative Care

The Number of Children Requiring Palliative Care; Key Challenges and Using Data Appropriately

Fraser L. ${ }^{1}$, Quinn C. ${ }^{2}$

${ }^{1}$ University of York, York, United Kingdom, ${ }^{2}$ National University of Galway, School of Nursing and Midwifery, Galway, Ireland Presenting author email address: lorna.fraser@york.ac.uk 
This interactive session will have a short presentation on the prevalence data on the numbers of children and young people from the UK and Ireland with Life-limiting conditions and then a discussion session with the audience on how to interpret these data and use these for service delivery and planning.

Key challenges will also be discussed.

PPC2 Information and Planning in Children's Palliative Care End of Life Decision Making in the Perinatal Period: A PopulationBased Study in Fetuses, Neonates and Infants

Beernaert K. ${ }^{1}$, Dombrecht L. ${ }^{1}$, Chambaere $K^{1}{ }^{1}$, Cools F. ${ }^{2}$, Roets $E^{3}{ }^{3}$, Goossens L. ${ }^{3}$, Naulaers G. ${ }^{4}$, Deliens L. ${ }^{1}$, Cohen J. ${ }^{1}$

${ }^{1}$ End-Of-Life Care Research Group, Ghent University \& Vrije Universiteit Brussel (VUB), Gent, Belgium, ${ }^{2}$ Universitair Ziekenhuis Brussel, Brussels, Belgium, ${ }^{3}$ Ghent University Hospital, Gent, Belgium, ${ }^{4}$ Leuven University Hospital, Leuven, Belgium

Despite improvements in prenatal diagnostic techniques and medical and technical interventions for critically ill neonates, about 8.7 per thousand children still die during the perinatal period in Flanders. This death is frequently preceded by difficult decisions such as termination of pregnancy, withholding or withdrawal of life-sustaining treatment or administering drugs or medical interventions; with or without the intention to hasten death. Population-based studies of incidence and characteristics of such end of life decisions (ELDs) in the perinatal period are rare. Therefore, we conducted a population mortality follow-back survey in Flanders for all stillbirths from 22 weeks of gestation onwards occurring between 1 January and 31 December 2017 and for all deaths of infants under the age of 1 year between 1 October 2016 and 31 December 31 2017. For each death case a postal questionnaire, including questions on ELDs was sent to the attending physician. Anonymity of the child, parents and physician is ensured by a rigorous mailing procedure involving a lawyer as trusted third party. We had a high response rate of $71 \%$ $(194 / 275)$ and $82 \%(245 / 297)$ for neonates and infants. We will present the incidence of termination of pregnancies and the incidence of withholding or withdrawal of life-sustaining treatment with the explicit, with co-intention and without intention of hastening death and the administering of drugs with the explicit, with co-intention and without intention of hastening death. We will also give more information and discussion about the ELD process and the relation with clinical and patients' characteristics.

PPC2 Information and Planning in Children's Palliative Care Information and Decisions Regarding Medical Treatment for Severely III Children with Spinal Muscular Atrophy

Kreicbergs U. ${ }^{1,2}$, Beernaert K. ${ }^{3}$, Sejersen $T^{4}$, Werlauff U.5, Rakbek J.5, Jeppesen J. ${ }^{5}$, Hjorth E. ${ }^{1}$, Lövgren M. ${ }^{1,6}$

${ }^{1}$ Ersta Sköndal Bräcke University College, Stockholm, Sweden, ${ }^{2}$ Karolinska Institutet, Karolinska University Hospital, Astrid Lindgren Children's Hospital, Childhood Cancer Research Unit, Stockholm, Sweden, ${ }^{3}$ Ghent University, Ghent, Belgium, ${ }^{4}$ Karolinska Institutet, Stockholm, Sweden, ${ }^{5}$ RehabiliteringsCenter for Muskelsvind, Copenhagen, Denmark, ${ }^{6}$ Karolinska Institutet, Karolinska University Hospital, Astrid Lindgren Children's Hospital, Childhood Cancer Research Unit, Stockholm, Sweden

Parents receiving information that their child has a life limiting illness is devastating. Communication in pediatrics has been studied extensively, predominantly in oncology. This has been found to be crucial for families not only in understanding the seriousness of the illness but also for compliance to treatment and coping with bereavement. Notably, there is a difference between families of a child with cancer compared to a child with severe SMA. Thus, in this genetic neuromuscular condition, which occurs in 1 of 10000 live births, there is no curative treatment. Children with SMA are commonly subgrouped in three categories based on the severity of dysfunction. Children with SMA 1 have a short life expectancy, which, however, may be extended by respiratory support. The decision about this treatment option rests upon the care givers conferring with the parents. Recently, a new therapeutic drug has become available, which may mitigate the progression of the disease and, hence, improve the prognosis, even for children with SMA 1. Lifelong treatment poses a difficult decision for the parents, which should be grounded on solid information conveyed prudently.

We explored communication, knowledge and decisions in the care of children with severe SMA. Interviews with parents and health care staff were conducted to develop a questionnaire for parents used in two nation-wide studies, Sweden and Denmark. A total of 64 children were identified, 59 were found eligible and 57 were represented by at least one parent. The participation rate was $(n=95,84 \%)$, with an almost equal distribution of mothers and fathers. No parents reported that healthcare professionals made treatment decisions without informing them beforehand. Yet, not all parents received information to fully understand the condition, the treatment options and consequences for the life of their child. Thus, there is a need of improved strategy in supporting and communicating with the families.

PPC2 Information and Planning in Children's Palliative Care Advance Care Planning in Pediatric Palliative Care - The View of Parents and Health Care Professionals

Führer M., Knochel K., Zaimovic V., Reimann D., Lotz J., Borasio G.D., Hein $K$.

University Children's Hospital Munich, Center for Pediatric Palliative Care, Munich, Germany Presenting author email address: monika. fuehrer@med.uni-muenchen.de

"We knew what will happen at the end - that we will not kill him with the medication, but ease his way. And the discussions have reassured me", Despite compelling evidence for the effectiveness of advance care planning in adults, there is still a debate whether this concept can be adapted to meet the needs of children with a life-limiting disease and their parents as well as the necessities of health care professionals (HCPs).

We conducted a series of studies aiming to explore the needs and perceptions of parents and HCPs about pediatric advance care planning (pACP), to identify the main challenges during pACP discussions, and to describe core elements for a successful pACP process. Our final goal is to develop a comprehensive pACP guideline. To this end, we used a participatory approach involving bereaved parents and HCPs from all professions concerned.

Main results were:

1) Parents prefer that physicians lead the pACP conversations. They should be familiar with the child's medical condition in order to provide them with the necessary medical information. HCPs involved in PACP should know the family and guide them during the decision-making process.

2) Parents want to prepare for crisis situations, however, advance directives (ADs) are of minor importance to them. HCPs would prefer clear directions for future medical treatment and receiving ADs signed by the parents.

3) HCPs perceive a need for guidelines and professional education in pACP and advocate for a systematic implementation of pACP in health care systems.

4) Both parents and HCPs prefer an early beginning of pACP in the course of the disease, but they also mention that parents must be ready for it and that the correct timing of discussions is crucial for a successful pACP.

"These conversations are also very difficult. But in the end, I have realized that this has been effective. Not to avoid this. [...] But to repeatedly work a little in this direction. But then, you need really sensitive people." 


\section{PPC3 Symptom Management in Palliative Care}

Evidence-Based Prescribing in Paediatric Palliative Medicine

Jassal S.

Rainbows Childrens Hospice, Loughborough, United Kingdom

Presenting author email address: sat.jassal@gmail.com

Aims: To consider why evidence based prescribing is so important in PPM.

Methods: We will look at the history of prescribing in PPM and the pitfalls of the past particular with drugs and routes such as morphine, midazolam and methadone. We will look at the structure used by the APPM formulary to review evidence for drugs in the APPM National Formulary and how the work continue to evolve. Finally we will consider new drugs and pitfalls in prescribing in PPC.

Results: Prescribing in the past was extrapolation from adult medication and guess work and this has led to many errors. We now use very robust systems to review evidence. Future research is limited by small numbers and the special groups we work with.

Conclusion: We have finally understood the importance of evidence based medicine and the future requires collaborative working nationally and internationally.

\section{PPC3 Symptom Management in Palliative Care}

We Need To Talk About Cannabis

McCulloch $R$.

Great Ormond Street Hospital Institute of Child Health, The Louis

Dundas Centre for Paediatric Palliative Care, London, United Kingdom

We need to talk about the cannabis conundrum in current paediatric practice. There is considerable confusion amongst the public, health care professionals and policy makers regarding the various forms of cannabis oils, their use and legality, in addition to the related risks and safeguarding issues associated with administration in children.

Current international discussion focuses on 2 chemicals (cannabinoids) found within cannabis, delta-9-tetrahydrocannabinoid (THC) and Cannabidiol (CBD). Whilst the debate on the use and effectiveness of cannabis rages on, a blurring of lines has occurred between unregulated 'botanoceutical' cannabis oils and 'pharmaceutical' grade cannabinoid use.

Clinical evidence supporting the use of cannabis derived products in children for medicinal use is very limited, there are still no large scale robust well designed randomised controlled studies to determine efficacy. However, a wealth of anecdotal reports claiming that cannabis is able to treat numerous conditions as well as help reduce or prevent a variety of adverse effects, abound on media platforms, presenting enough of a compelling argument for parents (patients) to warrant a trial of unregulated 'botanoceutical' cannabis products for their children. This is occurring with increasing frequency in children with life limiting and life threatening conditions.

This presentation will open up the discussion, provide an overview of the status quo regarding current research and evidence and suggest an approach to management in paediatric palliative care patients.

\section{PPC3 Symptom Management in Palliative Care}

Assessment and Management of Neuroirritability in Pediatric Palliative Care Patients with Severe Psychomotor Impairment (SPMI) Zernikow B.

Witten/Herdecke University, Faculty of Health, School of Medicine, Chair Children's Pain Therapy and Paediatric Palliative Care, Datteln, Germany

Aims: Delegates will learn about children with SPMI in pediatric palliative care. The most frequent and important symptoms, neuroirritability and sleep disorders, will be the focus.
Methods: Based on real case reports and considering a bio-psychosocial-spiritual approach, the existing scientific evidence will be presented

Results: Delegates will gain an understanding of diagnosis and assessment of neuroirritability, including use of validated tools for sleep assessment, and a structured approach for treatment of children with life limiting conditions and SPMI.

Conclusion: Although often a matter of trial-and-error, assessment and treatment of children with SPMI suffering neuroirritability can follow a rational approach.

PPC4 Contemporary Issues in Children's Palliative Care Aprepitant: Our New Friend for an Old Adversary? Patel B. ${ }^{1}$, Bayliss J. ${ }^{2}$, Stephenson A. ${ }^{2}$, Bluebond-Langner M. ${ }^{2,3}$ ${ }^{1}$ Louis Dundas Centre for Paediatric Palliative Care / Pharmacy, Great Ormond Street Hospital NHS Foundation Trust, London, United Kingdom, ${ }^{2}$ Louis Dundas Centre for Paediatric Palliative Care, Great Ormond Street Hospital NHS Foundation Trust, London, United Kingdom, ${ }^{3}$ Faculty of Population Health Sciences, Louis Dundas Centre for Paediatric Palliative Care,University College London, Institute of Child Health, London, United Kingdom

Background: Nausea and vomiting is a common symptom in children through their end of life journey. Uncontrolled nausea and vomiting places a huge psychological and emotional burden on families and children as well as the physical burden, reducing quality of life for the child and family. Aprepitant, a NK-1 antagonist, has become a potent weapon in the fight against chemo-induced nausea and vomiting. Aprepitant's use in palliative care, and refractory nausea and vomiting, has been limited, due to limited experience or evidence of long-term use. However, emerging evidence suggests that long-term (LT) use is not only safe, but also effective in patients with nausea and vomiting refractory to multiple lines of anti-emetic therapy.

Aim: Evaluate the safety and efficacy of LT aprepitant administration for nausea and vomiting, unresponsive to at least 2 previous lines of standard anti-emetic therapy in children.

Method: We conducted a single centre retrospective chart review of children, given daily aprepitant for nausea and vomiting, unresponsive to at least 2 prior lines of anti-emetic therapy. Objective responses from parents, impact of nausea on mobility and on feeding, were used as proxy efficacy markers. Time to effect, duration of effect and toxicity was also evaluated.

Results: 7 children received LT aprepitant and showed resolution of nausea and vomiting, increased ability to mobilise and feed tolerance, within 24 hours of starting aprepitant. Emetic control was maintained with oral medication until end of life in 6/7 children, maintaining home as place of care and death in $85 \%$ of children. No adverse events were noted.

Conclusion: This review is the first report of aprepitant use in this manner in the paediatric palliative care setting and represents the largest case review published within a paediatric cohort. Our review suggests a role for aprepitant in management of refractory nausea and vomiting, prior to use of parenteral therapy, demonstrating safety and efficacy.

PPC4 Contemporary Issues in Children's Palliative Care Exploration of the Term 'Quality of Life' in Consultations of Children with a High Risk Brain Tumour over the Course of the Illness Beecham E..$^{1,2}$, Langner R. ${ }^{2}$, Hargrave D. ${ }^{3}$, Bluebond-Langner M. ${ }^{2,4}$ ${ }_{1}$ Marie Curie Palliative Care Research Department, Department of Psychiatry, University College London, London, United Kingdom, ${ }^{2}$ Louis Dundas Centre for Children's Palliative Care, Institute of Child Health, University College London, London, United Kingdom, ${ }^{3}$ Paediatric Oncology Unit, Great Ormond Street Hospital, London, United Kingdom, ${ }^{4}$ The State University of New Jersey, Rutgers University, New Brunswick, United States 
Background: Discussions of quality of life (QoL) are being increasingly advocated between healthcare professionals (HCPs), parents, and children and young people (CYP) with serious illness, even when chance of cure is good. There is no current consensus on an accepted concept of QoL yet it is used in consultations about a patient's care and treatment. It is therefore important to understand how each stakeholder understands the term QoL.

Aims:

1) To explore if QoL emerges as a topic in consultations between CYPs with high risk brain tumours (HRBTs), parents and HCPs.

2) To explore how QoL is conceptualised during the interaction in these consultations.

Methods: Data is taken from a 20 month longitudinal, prospective, ethnographic, participant-observation study following 24 families from diagnosis to death in all interactions among CYP with HRBTs, parents and HCPs. We present results from the first phase of analysis focusing on consultations with Neuro-Oncology. Data was analysed using both inductive thematic analysis and deductive coding.

Results: The term QoL arose 32 times in 14/24 cases (58\%). Occurrences of the term QoL were overwhelmingly raised by HCPs compared to parents and CYP. When exploring emerging conceptions of QoL the three stakeholders had different perspectives on what quality looked like to them and their priorities. For parents the focus in the consultation was on length of life and time left, HCPs focused on physical status of the patient and avoiding side effects and the CYP's QoL related concerns were relational.

Conclusion / Discussion: A lack of alignment of views and priorities was found among the CYP, parent and HCP views of QoL. Given that their conceptions of QoL may be different, it is important to elicit what QoL means to each of them at each turning point in the illness trajectory. Funding: Marie Curie Research Grants Scheme, grant MCCC-FCO-16-U and Health Foundation.

PPC4 Contemporary Issues in Children's Palliative Care Adolescent Palliative Care in Uganda: Assessing and Addressing Needs

Merriman $R^{1}{ }^{1}$, Doherty $D .^{2}$, Mwebesa $E .^{3}$

${ }^{1}$ King's College London, London, United Kingdom, ${ }^{2}$ University College Dublin, Dublin, Ireland, ${ }^{3}$ Hospice Africa Uganda, Kampala, Uganda

Background: It is recognised that adolescent palliative care patients, facing multiple periods of transition, have specific psychological and clinical needs. For patients in developing countries, further challenges arise. Due to differences in demographics, culture and healthcare provision, better understanding is required to ensure age-appropriate, holistic care.

Aims: To identify and explore the needs of adolescent palliative care patients in a resource limited setting. To highlight the barriers to care, and consider practical solutions.

Methods: With ethical approval, a qualitative study was conducted in three community-based Hospice sites across Uganda. Data was collected using semi-structured interviews with current patients aged 13-19 $(n=21)$, and multidisciplinary team focus-groups $(n=2)$.

Results: Patients highlighted three main areas of concern:

1. Uncertainty regarding diagnosis, prognosis and treatment side effects.

2. The impact of illness on education.

3. Self-image, being 'different', and the effect this has on relationships.

Multiple barriers were identified. Many patients had high, sometimes unrealistic, expectations of Hospice services, with most suggesting financial support as a way to meet their needs. Information sharing was often limited. Families, wanting to 'protect' their child, would conceal their diagnosis. Patients, too, were often reluctant to talk openly. Additionally, a lack of monitoring, integrated care and communication between health services meant that key information was not readily available when required. In general, staff felt unequipped to deal with sensitive issues specific to their teenage patients. This was largely due to gaps in training and service provision. As a result, these important aspects were often overlooked.

Conclusion: Palliative care needs of adolescent patients in resource limited settings are complex and multifaceted. A proactive, sensitive, and multidisciplinary approach to care is essential.

This study received no funding.

PPC4 Contemporary Issues in Children's Palliative Care Clonidine for Intractable Irritability in Children with Severe Neurological Impairment - A Case Series

NíLaoire Á. ${ }^{1}$, Twomey M. ${ }^{1}, O^{\prime}$ Reilly M. ${ }^{1}$, Colleran M. ${ }^{2}$, Devins M. ${ }^{3}$

${ }^{1}$ St. Luke's Radiation Oncology Centre, Dublin, Ireland, ${ }^{2}$ St. Brigid's Hospice, Kildare, Ireland, 'Our Lady's Children's Hospital, Dublin, Ireland

Background: Irritability in children with severe neurological impairment (SNI) is a complex and distressing symptom which is challenging to treat. Clonidine is a centrally acting $\alpha 2$ adrenergic receptor agonist with a potential unique role to reduce irritability by treating dystonia, dysautonomia and nociceptive pain. Despite this, minimal research exists on its use in this setting.

Aim: To explore the use of clonidine by a specialist palliative care (SPC) team for intractable irritability in children with SNI.

Methods: A retrospective case series was conducted by chart review in a tertiary referral centre on all children with SNI who were prescribed clonidine by SPC for intractable irritability over a 3-year period. Due to lack of a validated outcome measure for irritability in SNI, parental subjective assessment, opioid requirements, frequency of contact to SPC and side effect profile were used to assess response.

Results: Seven children (5 male, age range 3-11 years) with SNI were prescribed clonidine for intractable irritability during the study period (mean dose $3.5 \mathrm{mcg} / \mathrm{kg}$ QDS). Contributory diagnoses included dystonia, visceral hyperalgesia, cyclical vomiting and dysautonomia. All patients suffered irritability despite the use of Gabapentinoids. Parental reports of improvement in irritability and a reduction in phone calls to SPC followed the introduction of clonidine in all children. Six patients had reduced opioid requirements (increased in one child due to a dislocated hip). Asymptomatic hypotension occurred in one patient without need for dose adjustment. Intravenous, subcutaneous, transdermal and enteral routes of administration were used.

Conclusion: This case series suggests that Clonidine is well tolerated, opioid sparing and easy to administer in children with SNI and intractable irritability. In these complex cases, Clonidine may be a very useful addition to the multimodal approach necessary to provide optimal symptom control.

PPC4 Contemporary Issues in Children's Palliative Care Tapentadol for Pain Therapy in Children - An Promising Alternative to Combining Coanalgetics with Classical Opioids - An Retrospectve Data-analysis

Gronwald B., Keller C., Welsch K., Gottschling S.

Centre for Palliative Care and Paediatric Pain, Saarland University Hospital, Homburg/Saar, Germany

Acute pain in children is frequent, likewise chronical pain occurs during childhood. Still there are only a few analgetic drugs licensed for children. Tapentadol displays a relatively new pharmaceutical class ( $\mu$-opiodreceptor agonist and noradrenalin re-uptake inhibitor). To date studies 
on the analgetic potency with children are missing, just as pediatric dosage recommendations.

Medical charts of 29 children at the age from 6 to 19 years were evaluated for retrospective data-analysis. All of them had been treated with tapentadol for chronic pain. The analysis was performed on basis of the noted therapeutic course and results rated by the attending physician, the patient himself and/ or the parents.

Neuropatic pain or symptoms for mixed pain were reported by the majority of patients $(69 \%)$. Before treatment with tapentadol the pain level on the numeric rating scale (NRS) was recorded $8.10 \pm 1.37$. The pain level while dosage was finally titrated scored only $4.52 \pm 1.48$., showing a significant reduction $(t(28)=9.97 ; \mathrm{p}<.001)$. Average duration of intake was 58 weeks (SD: 73.25; 4-312 weeks). The average startingdose was $82.41 \mathrm{mg}$ (SD: 48.75), average end-dose $248.33 \mathrm{mg}$ (SD: 168.73). Calculated on mg per kg body weight we found the starting-dose 1.69 (SD: 0.95$)$ with end-dose 5.72 (SD: 4,12). Overall 13 patients complained for side-effects (nausea: 6, constipation: 4, pruritus: 1, tiredness: 6, sleep disturbance: 2 , depressive mood: 1 ). Only in 2 cases tapentadol had to be discontinued.

The analgetic effect on a wide spectrum of diseases was demonstrated, with particular effectiveness on neuropatic pain elements. Side-effects were reported in $45 \%$, these were clearely less distinct then reported from Borys et al (2015). They are comparable to other established opioids. We found tapentadol could be a promising alternative for the treatment of neuropatic pain in children and adolescent, instead of combining opioids with coanalgetic drugs, a „single-pill-regime” faciliates.

PPC4 Contemporary Issues in Children's Palliative Care The Experience of Having a Second Child with Palliative Care Needs: The Case of Jordanian Mothers

Atout M. ${ }^{1}$, Hemingway P. ${ }^{2}$, Seymour $\mathrm{J}^{3}$

${ }^{1}$ Faculty of Nuring, Philadelphia University, amman, Jordan, ${ }^{2}$ University of Nottingham, Nottingham, United Kingdom, ${ }^{3}$ Sheffield University, Sheffield, United Kingdom

Background: The purpose of this study was to explore the experience of communication in the care of children with palliative care needs, from the perspective of physicians, nurses, and mothers in Jordan.

Methods: This study employed a collective qualitative case study approach. Data were collected in three paediatric units in a Jordanian hospital. Two data collection methods were employed: participant observation and semi-structured interviews.

Findings: Twenty five interviews have been collected with fifteen mothers.The findings of the interviews indicate that some mothers who had a second child with the same disease were stronger because the experience increased their ability to adapt themselves to the second situation. However, these mothers reported enormously painful experiences if they were admitted to the same room in which their previous child had been cared for or treated by the same health professionals who had treated the deceased child.The mothers who had more than one child with the same illness had a tendency to dredge up unhappy memories, and some of them refused to talk with students who they expected to ask them to review the family history. The mothers avoided these kinds of discussions because of these painful memories, and also as a means of maintaining their strength in the face of the current situation.

Conclusion: The clinical practice could benefit from these finding by avoiding stimulating the painful memories of parents who had deceased children.This could help the parents to adapt to their children's illness. However, it is important to assess parental preferences before such intervention to account for individual differences among parents. Therefore, a clear discussion with parents who have a second child with the same illness, in order to understand their preferences regarding the medical environment and the medical teams they would like to treat their second child could significantly improve parental satisfaction with hospitalization.
PPC5 Challenges in Paediatric Palliative Care

Assessing Attitudes of Irish Consultant Physicians in Palliative Medicine towards Providing Palliative Care for Paediatric Patients Hennelly C. ${ }^{1}$, Devins M. ${ }^{2}$, Dunne M. ${ }^{3}$, Twomey M. ${ }^{4}$, O'Reilly M. ${ }^{4}$ 1Palliative Medicine, Our Lady's Hospice \& Care Services, Dublin, Ireland, 2Palliative Medicine, Our Lady's Children's Hospital, Crumlin., Dublin, Ireland, ${ }^{3}$ Radiation Oncology, St. Luke's Radiation Oncology Centre, Dublin, Ireland, 4Palliative Medicine, St. Luke's Radiation Oncology Centre Rathgar, Dublin, Ireland

Background: The Report of the National Advisory Committee on Palliative Care in Ireland recommends that palliative care (PC) for children is best provided at home, except in extraordinary circumstances, with the family closely supported by the General Practitioner and the Public Health Nurse, and also a specialist PC team when required.

Aims and Objectives: To assess Consultant Physicians in Palliative Medicine's attitudes towards providing PC for children in the community. Methods: Ethical approval was sought and granted for this cross-sectional study. Our postal survey was based on a survey piloted and used by a study group at the Department of Family and Community Medicine at the University of Toronto. Descriptive statistical analyses were performed using SPSS.

Findings and Results: 31 consultants of the $44(70 \%)$ surveyed responded. Consultants had been practicing PC for a median of 16 years (range: 6-31). Consultants provided PC for a median of 5 children; an average of one child every 3 years (range: 1.2-14.5 years). Although $57 \%$ of respondents perceived the amount of clinical training they received in paediatric palliative care (PPC) to be insufficient based on their current practice, and $29 \%$ had had no clinical training (patient exposure) in PPC, $97 \%$, would be willing to assess and manage a paediatric patient without further training. The importance of access to specialist PPC advice was consistently reported in open ended questions. There was a statistically significant association between respondents' reported familiarity with medications to treat pain in dying children and whether respondents had had any clinical training (patient exposure) in PPC ( $p=.019)$.

Conclusion(s): There is evident willingness on behalf of consultants to continue to support the provision of PPC services but concerns about current levels of training and patient exposure need to be explored.

PPC5 Challenges in Paediatric Palliative Care Inequalities in Children's Place of Death: A Nationwide Registry Study

Wolff S.L. ${ }^{1,2}$, Christiansen C.F. ${ }^{2}$, Johnsen S.P. ${ }^{3}$, Schroeder H. ${ }^{4}$, Darlington

A.-S. ${ }^{5}$, Jespersen B.A. ${ }^{1}$, Olsen M. ${ }^{6}$, Neergaard M.A. ${ }^{1}$

${ }^{1}$ Department of Oncology, Aarhus University Hospital, Aarhus, Denmark, ${ }^{2}$ Department of Clinical Epidemiology, Aarhus University, Aarhus, Denmark, ${ }^{3}$ Department of Clinical Epidemiology, Aalborg University Hospital, Aalborg, Denmark, ${ }^{4}$ Department of Pediatrics, Aarhus University Hospital, Aarhus, Denmark, ${ }^{5}$ University of South Hampton, South Hampton, United Kingdom, 'Department of Pediatrics, Rigshospitalet Copenhagen University Hospital, København, Denmark

Background: Place of death is often used as an indicator of quality when evaluating end-of-life care. However, place of death among children is likely to vary according to etiology of the illness along with demographic and socio-economic characteristics of the child and family. Knowledge on factors influencing where children die is very limited.

We aimed to determine predictors of home death among children.

Methods: We conducted a population-based cross-sectional registry study including children age 1-18 years, who died from natural causes in Denmark between 2006-2017, excluding infants ( $<1$ year). Frequencies of home versus hospital death were explored in relation to the child's gender, age $(</ \geqslant 10$ years) and diagnosis (cancer/non-cancer) as well as household income. 
Results: The preliminary results show that the study population comprised 932 children. The frequency of home death was $16 \%$ and $67 \%$ died at hospital throughout the study period (for $17 \%$ place of death was not registered). There was no difference in place of death according to the gender of the child (RR 1.0; 95\% Cl 0.9-1.1) or age under 10 years (RR $1.1 ; 95 \% \mathrm{Cl} 0.9-1.2$ ), additional analysis using multiple age cut-offs and test for trend did not alter this conclusion.

Cancer diagnoses accounted for $26 \%$ of deaths. Compared to non-cancer, having a cancer diagnosis was strongly related to home death (RR 1.8; $95 \% \mathrm{Cl} 1.4-2.2$ ). When comparing the highest and lowest quartiles of household income, children from the more affluent families were more likely to die at home, (RR 1.3; $95 \% \mathrm{Cl} 1.1-1.5)$.

Conclusion: Neither gender nor age appeared to influence the likelihood of home death among children above one year of age. We found disparities in children's place of death, however, with children who died from cancer and children from families with higher household income being more likely to die at home. Future research should aim to determine the mechanisms behind these inequalities.

PPC5 Challenges in Paediatric Palliative Care

Challenges of Decision-Making in Pediatric Palliative Care

Verhagen $E$.

University Medical Center Groningen, Pediatrics, Groningen,

Netherlands Presenting author email address: a.a.e.verhagen@umcg.n

Parents and of children with advanced life-threatening conditions confront daunting medical decisions. One of the major decisions is whether to initiate a limit to life-saving interventions for their child. The intention of such decisions is to prevent suffering and to promote comfort, quality of life, and dignity. This is in line with the presumption in pediatric bioethics that decisions should be taken in the child's best interest. Yet, despite the importance and frequency of these decisions, it remains difficult for clinicians and parents to know how these decisions should be made at the bedside.

The challenge of optimizing pediatric end-of-life decision-making in the Netherlands has resulted in the publication of the first national evidence-based guidelines for pediatric palliative care in 2013. This guideline has sparked the development of a series of palliative care initiatives. Among them are the establishment of local palliative care teams in all pediatric hospitals, building care networks for children with complex diseases and the start of a national knowledge center for pediatric palliative care connecting practice to knowledge development and implementation. Multidisciplinary experts and parents linked-up to form consultation spots for other parents and professionals facing decision-making dilemmas. We also developed a functional individualized advanced care plan to promote timely decision-making and high-quality palliative care in daily practice.

In our experience, these initiatives have made complex decision-making in pediatric palliative care somewhat easier because knowledge, experience and functional tools are now better (and digitally) available for all stakeholders

Eduard (A.A.E.) Verhagen, MD, JD, PhD

Professor of Pediatrics, Head of Department

University Medical Center Groningen

Beatrix Children's Hospital

P.O. Box 30.001

9700 RB Groningen, The Netherlands

telephone: +31503612470

PPC5 Challenges in Paediatric Palliative Care

Accompanying the Child at the End of Life

Papadatou $D$.

National and Kapodistrian Univesrity of Athens, Nursing, Athens, Greece Presenting author email address: dpap@nurs.uoa.gr
Our role as companions of children whose life come to an end, is quite similar to the role of midwives. Midwives accompany a woman through labor, and help the newborn to 'pave' its way into life. We create conditions for the child to 'pave' a dignified way out of life, and support parents and siblings to 'carve' a pathway from a life with to a life without the child. The goal of this presentation is threefold: (a) to describe some of the challenges that children and adolescents experience when death is ambiguous, and when death is impending; (b) to explore how children and adolescents communicate their awareness, concerns, and needs at the end of life, and how professionals may facilitate enriching encounters; (c) to discuss the prerequisites for providing a "safe haven" and a "secure base" that enable patients and families to attribute meaning to their experiences and to a life worth living. Such a life may be filled with ordinary activities and ordinary relationships which are experienced as extra-ordinary and lived with an acute vitality and a renewed sense of existence into this world. Finally, personal reflections will be shared about our professional capacity to remain 'vulnerable enough' in our encounters with dying children and their families.

\section{Joint symposium of the EAPC and ESMO "Integration of Oncology and Palliative Care"}

ME09 The Rapidly Moving Field of Cancer Immunotherapy - Indications, Response Prediction and Management of Side Effects

No abstracts available

EE1 Global Developments in Integration of Oncology and

Palliative Care

WHO's Global Policies and Partnerships to Bridge the Gaps in Access to Palliative Care

Ullrich A.

Charité Universitätsmedizin Berlin, Gynaecology, Conches, Switzerland Presenting author email address: ullricha@outlook.com

According to WHO, each year, an estimated 40 million people need palliative care $(\mathrm{PC})$ including most of the annual 9.4 million people who die from cancer. However, only about $14 \%$ of people who need PC currently receive it. Reason for these gaps are overly restrictive regulations for morphine and lack of training and awareness of PC among health professionals. The global need for palliative care will continue to grow as a result of the rising burden of noncommunicable diseases and ageing populations. Access to adequate PC services will much depend on national health policies and systems which include PC, increasing capacity and skills among health professionals and population access to opioid pain relief in accordance with international conventions on access to essential medicines. However, there is potential for a game change. In 2014, the first ever global resolution on PC (WHA 67.19) called upon WHO and Member States to improve access to palliative care as a core component of health systems. WHO's work to strengthen PC is focused on: integrating palliative care into all relevant global disease control and health system plans, developing guidelines on integrated palliative care across disease groups, supporting Member States in improving access to palliative care medicines through improved national regulations and delivery systems, promoting increased access to palliative care for children, monitoring global PC access and evaluating progress made in palliative care programmes, encouraging adequate resource allocation for national PC programmes especially in resource-limited countries and building evidence of models of PC that are effective in low- and middle-income settings. Professional organisations such as ESMO and EAPC together with other international PC organisations have a crucial role in catalysing changes through advocacy and capacity building among health care providers for cancer management. 
EE1 Global Developments in Integration of Oncology and Palliative Care

Global Reach of ESMO Designated Centres of Integrated Oncology and Palliative Cancer Care Gudrun Kreye, Nicola Latino, Stein Kaasa

Kreye G. ${ }^{1}$, Latino N. ${ }^{2}$, Kaasa S. ${ }^{3}$

${ }^{1}$ UK Krems, Krems, Austria, ${ }^{2}$ European Society for Medical Oncology Head Office, Lugano, Switzerland, ${ }^{3}$ Oslo University Hospital and Institute of Clinical Medicine, Department of Oncology, Oslo, Norway

Introduction: The European Society for Medical Oncology (ESMO) has developed multiple programmes to promote palliative integration in oncology. One of these programmes was established in 2003 by ESMO to accredit centres who provide a good level of integration of Palliative Care in oncology structures.

By undergoing a rigorous anonymous review process by members of the ESMO Designated Centres Working Group, centres can apply to receive the 'ESMO Designated Centres of Integrated Oncology and Palliative Care (ESMO-DCs)'status. ESMO-DCs are considered as centres that deliver highly integrated care through different care models. To achieve recognition as ESMO-DC-centre, 13 criteria have to be fulfilled and a structured description of the centre has to be provided.

Results: To date, there are 213. ESMO-DCs all over the world. Most centres are located in Europe 158 followed by 38 centres in the Middle East and Asia, 4 in Australia, 13 in South and Northern America including Canada.

Conclusion: Early integration of palliative care in oncology is a hot topic in current research projects. A research collaboration of ESMO-DCs all over the world may be able to develop new networks and insights in ongoing developments not only nationwide, but also in global aspects. Collaboration between ESMO-DCs, coordinated by the ESMO-Designated Centre Working Group could help to support interesting research projects in the future.

\section{EE1 Global Developments in Integration of Oncology and Palliative Care}

The New WHO Guideline for the Management of Cancer Pain in Adults and Adolescents and Findings of the WHO Country Capacity Survey

Bruera E.

MD Anderson Cancer Center, Palliative Care, Rehabilitation \&

Integrative Medicine, Houston, TX, United States

The first WHO Cancer Pain Guideline was produced in 1986 and it was one of the most successful documents published by the WHO. This initial guideline included the concept of the analgesic ladder used for education on the use of non-opioid, weak opioid, and strong opioid analgesics for increasing severities of pain. The second edition was published in 1996 and it included a guide to opioid availability. In 1998 the WHO published a guideline on cancer pain relief and palliative care in children. Unfortunately, as of 2006 approximately 5.5 billion people ( $83 \%$ of the world's population) lived in countries with low or non-existing access to adequate pain management. While significant progress has occurred in the assessment and management of pain in many countries, the majority of cancer patients will die without having ever received a single dose of an opioid analgesic.

On the other hand, in the last 15 years in developed countries there has been a growing concern about the effects of larger availability of opioids in the community as a source of nonmedical opioid use, opioid use disorders, and opioid related mortality. Within this context it became appropriate to update the WHO guidelines for the pharmacological and radiotherapeutic management of cancer pain in adults and adolescents.

The current guidelines published in 2018 have followed updated methodology recommendations and they provide a useful update on the current role of pain evaluation and management.
This presentation will discuss the main changes in the new guidelines and also the palliative care related findings in the 2017 WHO global survey on capacity for non communicable diseases.

There will be some recommendations for implementation of these recommendations in different countries.

EE2 Integration of Oncology and Palliative Care Introduction to the Lancet Oncology Commission Paper - How to Organize and Integrate Palliative Care

Kaasa S., Loge J.H., Lundeby T.

Oslo University Hospital, Department of Oncology, Oslo, Norway

There is a high level of evidence showing that integration of oncology and palliative care will improve several patient outcomes: survival, symptom control, overall quality of life and patient satisfaction.

Full integration will rely on specific knowledge and skills of the two modes of care: the tumor directed approach and the host directed approach, the latter may also be called patient centered care or palliative care.

Systematic assessment and use of patient reported outcomes and active patient involvement in the decisions of cancer care is central. The absence of international agreements on the content and standards of the organization, education and research on palliative care in oncology are major barriers for a successful integration.

Use of standardized care pathways and multidisciplinary teams are proposed as methods to achieve full integration between oncology and palliative care.

\section{EE2 Integration of Oncology and Palliative Care} The Need for Training and Education

Sjøgren $P$.

Cpenhagen University, Section of Palliative Medicine, Cpoenhagen, Denmark Presenting author email address: per.sjoegren@regionh.dk

Education in palliative care has been identified as a key factor for the promotion of integration of oncology and palliative care. Medical education from undergraduate to postgraduate levels is the primary focus of this talk, with the awareness that palliative care shall be delivered by multidisciplinary staff. At present, the need for basic and specialist competence in palliative care is unmet at all levels of health care, which calls for massive increased educational efforts. The skills and the capacity of tertiary palliative care specialists located in comprehensive cancer centers are essential to support primary and secondary providers of palliative care. These demands expand the responsibilities of tertiary palliative care specialists beyond the cancer center setting through education and mentorship programs aiming to upskill primary and secondary health-care providers. Education in palliative medicine is recommended by several societies to be included in undergraduate and postgraduate curricula, updated by continuing education, lectures, courses, and conferences. No overall data on the amount and content of palliative care teaching in postgraduate oncological accreditation programs exist as well as different recommendations exist for oncology teaching in postgraduate palliative care specialization programs. In addition, clinical rotation of trainees and fellows can be implemented to promote integration. Rotations may nurture mutual common understanding, strengthen partnerships, develop new clinical pathways and enhance research collaboration. The certification in palliative medicine falls into three categories, depending on the postgraduate educational system of the different countries: specialty, subspecialty, and general competence in the field. Palliative medicine is increasingly achieving specialty or subspecialty status in many countries world-wide. The integration of palliative care and oncology might be highly dependent on the level of accreditation. 
EE2 Integration of Oncology and Palliative Care Barriers of "Integration of Palliative Care into Oncology": Are We Talking About Patients' Needs or Conflicting Concepts? Gaertner J.

Palliative Care Center Hildegard, Basel, Switzerland Presenting author email address: jan.gaertner@pzhi.ch

The ASCO guideline for "the integration of Palliative Care (PC) into oncology" recommends that patients "should be referred to interdisciplinary PC teams $(\ldots)^{\prime \prime}(1)$. This exemplifies one obstacle towards the improvement of PC in oncology. PC is often used synonymously with specialist PC (SPC), though it has always been a basic part of oncology (i.e. initiating end-of-life discussions). Therefore, the term "integration" may increase misperceptions, because we are not talking about different concepts. Yet, it is about effective interaction of general- and SPC. In addition to such terminological obstacles further barriers for the improved provision of PC been identified.

Training: Cancer specialists should receive PC expertise and -attitude in the course of their training, but PC specialists must also hold sufficient knowledge of cancer care.

Attitude: PC specialist must acknowledge oncologists' dedication to serve their patients as universally as possible. They should foster the pre-existing trustful patient-oncologist relationship and avoid therapeutic chaos. Oncologists must not reduce the role of SPC as mere end-oflife care and recognize the value of concurrent SPC. Thus, cooperations require trust, clearly defined assignments and close communication.

Models of care: These must be tailored individually according to the context of each programme, the available resources and the needs of specific patient groups.

Evidence base: Meta-analyses have identified challenges in the interpretation of trial findings (2). This is necessary to avoid "black and white" interpretations and recommendations.

1. Ferrel B et al JCO 2017

2. Geartner J et al BMJ 2017

EE2 Integration of Oncology and Palliative Care

The Effect of Early and Systematic Integration of Palliative Care in Oncology on Quality of Life and Health Care Use at the End-of-Life: A Randomized Controlled Trial

Vanbutsele G. ${ }^{1,2}$, Van Belle S. ${ }^{3}$, Surmont V. ${ }^{4}$, De Laat M. ${ }^{5}$, Colman R. ${ }^{6}$, Eecloo K. ${ }^{1,2}$, Naert E. ${ }^{3}$, De Man M. ${ }^{7}$, Geboes K. ${ }^{7}$, Deliens L. ${ }^{1,2}$, Pardon K. ${ }^{1}$ ${ }^{1}$ End-of-Life Care Research Group, Ghent University \& Vrije Universiteit Brussel (VUB), Ghent, Belgium, ${ }^{2}$ Department of Public Health and Primary Care, Ghent University, Ghent, Belgium, ${ }^{3}$ Department of Medical Oncology, Ghent University Hospital, Ghent, Belgium, ${ }^{4}$ Department of Respiratory Medicine/Thoracic Oncology, Ghent University Hospital, Ghent, Belgium, ${ }^{5}$ Palliative Care Team, Ghent University Hospital, Ghent, Belgium, ${ }^{6}$ Biostatistics Unit, Department of Public Health and Primary Care, Ghent University, Ghent, Belgium, ${ }^{7}$ Department of Gastroenterology, Division of Digestive Oncology, Ghent University Hospital, Ghent, Belgium

Purpose: Many studies have examined the effect of early palliative care on quality of life (QOL) soon after diagnosis. This study evaluated if early integrated palliative care (PC) in oncology resulted in higher QOL near the end-of-life of advanced cancer patients.

Method: Patients with advanced cancer and a life-expectancy of one year were randomly assigned to either early and systematic integration of PC into oncological care (intervention) or standard oncological care alone (control). In a secondary analysis, QOL was assessed with the EORTC QLQ C30 global health status/ QOL scale and McGill Quality of Life (MQOL) Single Item Scale and Summary Scale at six, three and one month prior to death. Use of health care resources was collected from chart review in patients' electronic medical file of participants who died.
Results: Of the 186 randomized patients, 147 had died by November 2017. Patients in the intervention group scored significantly higher on global health status/quality of life in the EORTC QLQ C30 at six months (difference: 5.9 [0.06; 11.1], $\mathrm{p}=0.03$ ), three (difference: 6.8 [1.0; 12.6], $\mathrm{p}=0.02$ ) and one month (difference: $7.6[0.7 ; 14.5], p=0.03$ ) prior to the patient's death compared to the control group. Similar results were found for the Single Item Scale and Summary Score of the MQOL. We did not observe differences in use of health care resources between groups. Discussion: Early integrated palliative care does not only benefit QOL of cancer patients soon after diagnosis, but benefits are sustained until the last month of life. Future research should examine which components of early palliative care lead to patient benefits.

FC16 Palliative Care for People with Cancer

Efficacy and Safety of Parental Nutrition on Quality of Life Deterioration-free Survival among Palliative Cancer Patients: A Prospective Multicenter Randomized Control Trial (ALIM-K)

Cornet C. ${ }^{1}$, Anota A. ${ }^{2,3}$, Bouleuc C. ${ }^{4}$, Grodard G. ${ }^{1}$, Mathieu-Nicot F. ${ }^{1}$, Cretin E. ${ }^{1}$, Pazart L. ${ }^{1}$, Aubry R. ${ }^{1,5}$, AlimK study Group

${ }^{1}$ Clinical Investigation Center, Inserm CIC1431, University Hospital of Besançon, Besancon, France, ${ }^{2}$ Methodology and Quality of Life in Oncology Unit (INSERM UMR 1098), University Hospital of Besançon, Besancon, France, ${ }^{3}$ French National Platform Quality of Life and Cancer, Besancon, France, ${ }^{4}$ Department of Supportive Care, Institut Curie, Paris 05, France, ${ }^{5}$ Department of Palliative Care, University Hospital of Besançon, Besancon, France

Background: Malnutrition is a common complication in patients at the palliative stage of cancer. Although parenteral nutrition is associated with discomfort and adverse effects, it may improve health-related quality of life (HRQoL). However, there is lack of high-level evidence to evaluate the efficacy and safety of parenteral nutrition among palliative cancer patients.

Aims: To evaluate the impact of parenteral nutrition on HRQoL deterioration-free survival (DFS).

Methods: Randomized controlled trial following Zelen's single-consent design in 13 French cancer centers. Malnourished cancer patients at the palliative stage with functional digestive tract were randomly assigned (1:1) to receive parenteral nutrition (PN) or oral feeding (OF). HRQoL deterioration-free survival was defined as primary end point. HRQoL was measured with the QLQ-C15-Pal questionnaire targeting global health, physical functioning and fatigue. DFS was defined as a definitive deterioration $\geqslant 10$ points compared to baseline or death with $1.66 \%$ level of significance. Secondary end points were overall survival (OS), adverse effects and nutritional parameters.

Results: Between June 2012 and March 2017, 111 patients were included in analysis (PN Arm $n=48$ /OF arm $n=63$ ). $98 \%$ were metastatic with estimated life expectancy less than one year. There was a trend favoring HRQoL DFS in the oral feeding group with a median of 2 months for OF arm vs. 1 month for PN arm (HR=1.31(0.88-1.94) for global health, 1.58 (1.06-2.35) for physical functioning, 1.19 (0.80-1.77) for fatigue). In as treated analyses, adverse effects are numerous in $\mathrm{PN}$ arm vs OF arm ( $p$-value $=0,0122$ ).

Conclusion / Discussion: Parenteral nutrition did not improve healthrelated quality of life deterioration-free survival among palliative cancer patients. Our findings do not support the use of parenteral nutrition for these patients.

This project has been funding by the French National Cancer Institute (INCa) through PHRC 2011.

FC16 Palliative Care for People with Cancer

Where Do Oncological Patients Die?

Gómez Bravo R. ${ }^{1}$, Sánchez González J.C. ${ }^{1}$, Núñez García B. ${ }^{1}$, Morito

Aguilar A.M. ${ }^{1}$, Ruano Raviña A. ${ }^{2}$, Tojar Grisolia T. ${ }^{3}$, Provencio Pulla M. ${ }^{1}$ 
${ }^{1}$ Medical Oncology, Hospital Puerta de Hierro, Majadahonda, Spain, ${ }^{2}$ Preventive Medicine and Public Health, Universidad De Santiago de Compostela, Santiago de Compostela, Spain, ${ }^{3}$ Palliative Care, Hospital Puerta de Hierro, Majadahonda, Spain

Cancer is one of the main causes of death and morbidity. Apart from giving active treatments, it is also very important an appropriate management of symptoms at the end of life and caring how and where patients die. Being able to determinate the place of death is considered as a quality of life (QoL) criteria, both for patients and caregivers. Some studies have shown that patients usually prefer dying at home, instead of at hospital, which often includes a more aggressive processing.

We conducted a retrospective observational study selecting all patients with a first consultation in our hospital during 2014 and 2015, which died before December 31st, 2017. We describe where patients died: acute hospital, hospice or home. We aim to assess if there is any association between the place of death and some variables such as time from last chemotherapy (CT) to death or assessment by palliative care unit (PCU): hospital palliative care unit (HPCU), hospice care home unit ( $\mathrm{HCHU}$ ) or none.

We have analysed information about 662 patients. Patients who were not assessed by PCU, $19.5 \%$ died at home and $80.5 \%$ at an acute hospital and those assessed by $\mathrm{HCHU}, 41 \%$ at home $30 \%$ at an acute hospital and $29 \%$ at a hospice. From those with a follow-up by a PCU less than 30 days before dying, $19 \%$ died at home, $45 \%$ at an acute hospital and $36 \%$ at a hospice; and those with more than 30 days follow-up, $36 \%, 27 \%$ and $37 \%$ respectively. Patients which received last $\mathrm{CT}$ in the last month of life, $13 \%$ died at home, $76 \%$ at an acute hospital and $11 \%$ at a hospice; by contrast, among those who didn't received CT in the last 6 month of life, $33 \%, 36 \%$ and $30 \%$ respectively.

Where patients die depends on several factors. In our study, an early assessment by PCU and $\mathrm{HCHU}$, increases the number of patients who die at home. Supporting PCU could improve QoL at the end of life and a reduction in hospital costs.

FC16 Palliative Care for People with Cancer Effects of Corticosteroids on Quality of Life in Cancer Patients with Advanced Disease and Short Life Expectancy Paulsen $\varnothing .{ }^{1,2}$, Klepstad P. ${ }^{3}$, Kaasa S. ${ }^{4}$, Aass N. ${ }^{4}$

${ }^{1}$ Palliative Care Unit, Telemark Hospital Trust, Skien, Norway, ${ }^{2}$ European Palliative Care Research Centre (PRC), Norwegian University of Science and Technology (NTNU), Trondheim, Norway, ${ }^{3}$ Department of Circulation and Medical Imaging, Norwegian University of Science and Technology (NTNU), Trondheim, Norway, ${ }^{4}$ European Palliative Care Research Centre (PRC), Department of Oncology, Oslo University Hospital, Oslo, Norway

Background: Corticosteroids (CS) are frequently utilized for symptom control in cancer patients. Side effects from CS's remains a concern, including cognitive impairment and upper gastrointestinal discomfort. Aims: The aim of this study was to examine quality of life (QoL), cognitive function (CF) and gastrointestinal (GI) symptoms in data from a randomized placebo-controlled trial (RCT) assessing the analgesic properties of CS. Methods: The primary RCT compared the analgesic effects of methylprednisolone $16 \mathrm{mg}$ twice daily or placebo for seven days in advanced cancer patients. Inclusion criteria were $>18$ years, taking opioids for cancer pain, and pain $\geqslant 4$ (numerical rating scale $0-10$ ). QoL was reported as change from baseline and assessed using the European Organisation for Research and Treatment of Cancer Quality of Life Questionnaire-C30 (EORTC QLQ-C30) (0-100). Assessments also included the Mini Mental Status Examination (MMSE) (0-30) for CF, and items 1-5 of the Gastrointestinal Symptom Rating Scale (GSRS) (0-30) for upper GI symptoms.

Results: 49 patients were included in the RCT with a mean Karnofsky Performance score of 67 . The EORTC QLQ-C30 symptom items appetite* and fatigue* improved significantly by $25.5(p=0.003)$ and $20.0(p=0.003)$ at evaluation Day 7 in the CS group compared to the placebo group, respectively. Pain improved by $14.7(p=0.05)$. The EORTC QLQ-C30 function items global health was improved by $18.7(p=0.01)$, and role function by $17.4(p=0.03)$. There was no difference in CF as measured by MMSE. Gl symptoms (GSRS) were improved by 2.35 (SD 3.7, $p=0.03$ ) in the CS group compared to placebo.

Conclusion: CS administration for seven days improved global health and role function in addition to appetite and fatigue in cancer patients with metastatic disease. Upper gastrointestinal symptoms, originally included to assess side effects, were significantly improved by CS. CS did not alter cognitive function as measured by MMSE.

*Previously reported

FC16 Palliative Care for People with Cancer

Assessment of Symptom Burden and Palliative Care Needs of Cancer Patients at Diagnosis of Incurability and during the Course of Cancer Treatment - Final Results of a Prospective Longitudinal Multicenter Cohort Study of the Arbeitsgemeinschaft Palliativmedizin (APM)

Vogt J. ${ }^{1}$, Beyer F. ${ }^{1}$, Sistermanns J. ${ }^{2}$, Kuon J. ${ }^{3}$, Kahl C. ${ }^{4}$, Alt-Epping B. ${ }^{5}$, Stevens S. ${ }^{6}$, Ahlborn M. ${ }^{7}$, Dieterich M. ${ }^{8}$, Heider A. ${ }^{9}$, Tienken M. ${ }^{1}$, Loquai C. ${ }^{10}$, Stahlhut K. ${ }^{11}$, Ruellan A. ${ }^{12}$, Kubin T. ${ }^{13}$, Dietz A. ${ }^{14}$, Oechsle K. ${ }^{15}$, Mehnert-Theuerkauf A. ${ }^{16}$, van Oorschot B. ${ }^{17}$, Thomas M. ${ }^{3}$, Ortmann $O{ }^{18}$, Engel $C .{ }^{19}$, Lordick $F^{1}{ }^{1}$, on behalf of the Arbeitsgemeinschaft Paliativmedizin (APM) of the German Cancer Society (DKG) ${ }^{1}$ Universitäres Krebszentrum Leipzig, Universitätsklinikum Leipzig, Leipzig, Germany, ${ }^{2}$ Klinik für Strahlentherapie, Kliniken Maria Hilf, Mönchengladbach, Germany, ${ }^{3}$ Abteilung Onkologie und Innere Medizin, Thoraxklinik Heidelberg, Lungenkrebszentrum am Nationalen Centrum für Tumorerkrankungen, Heidelberg, Germany, ${ }^{4}$ Klinik für Hämatologie, Onkologie und Palliativmedizin, Klinikum Magdeburg, Magdeburg, Germany, ${ }^{5}$ Klinik für Palliativmedizin, Universitätsmedizin Göttingen, Göttingen, Germany, ${ }^{6} \mathrm{Klinik}$ für Internistische Onkologie/Hämatologie, Kliniken Essen Mitte, Essen, Germany, ${ }^{7}$ Klinik für Hämatologie und Onkologie, Städtisches Klinikum Braunschweig, Braunschweig, Germany, ${ }^{8}$ Universitäts-Frauenklinik und Poliklinik, Klinikum Südstadt Rostock, Rostock, Germany, ${ }^{9} \mathrm{Klinik}$ für Hämatologie/Onkologie/Palliativmedizin/Schmerztherapie, Klinikum Leverkusen, Leverkusen, Germany, ${ }^{10}$ Hautkrebszentrum Rhein-Main, Universitätsmedizin Mainz, Mainz, Germany, ${ }^{11}$ Praxis für Hämatologie/Onkologie/Palliativmedizin, Immanuel Klinik und Poliklinik Rüdersdorf, Rüdersdorf, Germany, ${ }^{12}$ Tumorzentrum, Städtisches Klinikum Karlsruhe, Karlsruhe, Germany, ${ }^{13}$ Abteilung für Hämatologie/Onkologie/ Palliativmedizin, Klinikum Traunstein, Traunstein, Germany, ${ }^{14} \mathrm{Klinik}$ und Poloklinik für Hals-, Nasen-, Ohrenheilkunde, Universitätsmedizin Leipzig, Leipzig, Germany, ${ }^{15} \mathrm{Klinik}$ und Poliklinik für Onkologie und Hämatologie, Universitätsklinikum Hamburg-Eppendorf, Hamburg, Germany, ${ }^{16}$ Abteilung für Medizinische Psychologie und Medizinische Soziologie, Universitätsmedizin Leipzig, Leipzig, Germany, ${ }^{17} \mathrm{Klinik}$ und Poliklinik für Strahlentherapie, Comprehensive Cancer Centre Main-Franken, Würzburg, Germany, ${ }^{18}$ Klinik für Frauenheilkunde und Geburtshilfe, Lehrstuhl der Universität Regensburg, Caritas-Krankenhaus St. Josef, Regensburg, Germany, ${ }^{19}$ Institut für Medizinische Informatik, Statistik und Epidemiologie, Universität Leipzig, Leipzig, Germany

Background: Symptom burden and care needs of patients (pts) at the time of diagnosis of incurable cancer and in the disease trajectory are poorly studied. However, this information is required for optimizing the integration of palliative care early in the course of incurable cancer treatment.

Aims: The study aimed to assess symptom burden and palliative care needs of patients at the time of diagnosis of incurable cancer and during 12 months follow-up.

Methods: A prospective non-interventional longitudinal multicenter study was performed in 20 German centers, assessing pts with newly 
diagnosed incurable cancer. Pts received validated questionnaires (FACT-G, SEIQoL-Q, PHQ-4, modified SCNS-SF 34, Distress Thermometer [DT]) at the time of diagnosis (T0), and after three (T1), six (T2) and twelve months (T3). Pts' medical information was provided by their treating physicians.

Results: Within two years (Oct 2014 to Oct 2016), 502 pts participated in the study (281 male, 219 female, 2 unknown; mean age 64.2 years; median ECOG PS 1 (TO)). Underlying diagnoses are: lung (219 pts), gastrointestinal (156 pts), head and neck (55 pts), gynecological cancer (57 pts) and malignant melanoma (15 pts). Pts reported significant distress (DT scale $\geqslant 5$ ): $67.1 \%$ (T0), $51.7 \%$ (T1), $47.9 \%$ (T2) and $48.7 \%$ (T3). Pts described a broad spectrum of physical symptoms and care needs with strong variations in intensity both between and also within the different tumor entities. Most common symptoms reported over the course of disease were asthenia, pain, weight loss, side effects and nausea. Most described care needs fall within the domains physical functioning and psychological support.

Conclusions: A broad spectrum of symptoms and care needs were identified at diagnosis and over the trajectory of incurable cancer disease. This emphasizes the importance of an early individualized support by multidisciplinary palliative and supportive care services to provide an adequate treatment for these patients.

\section{FC16 Palliative Care for People with Cancer}

Emotion and Symptom-focused Engagement (EASE): A Randomized Phase II Trial of an Integrated Psychological and Palliative Care Intervention for Patients with Acute Leukemia

Rodin G. ${ }^{1,2,3}$, Malfitano C. ${ }^{1}$, Rydall A. ${ }^{1}$, Schimmer A. ${ }^{4,5}$, Marmar C. ${ }^{6}$, Mah K. ${ }^{1}$, Lo C. ${ }^{1,2,7}$, Nissim R. ${ }^{1,2}$, Zimmermann C. ${ }^{1,2,8}$

1Princess Margaret Cancer Centre, University Health Network, Supportive Care, Toronto, ON, Canada, ${ }^{2}$ University of Toronto, Department of Psychiatry, Toronto, ON, Canada, ${ }^{3}$ University of Toronto, Global Institute of Psychosocial, Palliative and End-of-Life Care (GIPPEC), Toronto, ON, Canada, ${ }^{4}$ Princess Margaret Cancer Centre, University Health Network, Department of Medical Oncology and Hematology, Toronto, ON, Canada, 5 University of Toronto, Department of Medical Biophysics, Toronto, ON, Canada, ${ }^{6} \mathrm{New}$ York University Langone Medical Center, Department of Psychiatry, New York, NY, United States, ${ }^{7}$ University of Geuelph-Humber, Department of Psychology, Toronto, ON, Canada, 8 University of Toronto, Department of Medicine, Toronto, ON, Canada

Background: Patients with acute leukemia (AL) commonly experience significant traumatic stress symptoms as well as severe pain and other symptoms from the illness and its treatment. However, they typically do not receive psychotherapeutic or early palliative care support to address these issues. We designed a novel integrated psychosocial and early palliative care intervention called Emotion And Symptom-focused Engagement (EASE) for this clinical group. EASE includes:

i) 8-12 psychotherapeutic sessions over 8 weeks (EASE-psy); and

ii) weekly screening of physical symptoms with triggered referral to palliative care (EASE-phys).

Aims: To test the feasibility and preliminary efficacy of EASE over usual care (UC) in a phase II randomized controlled trial (RCT) in patients newly diagnosed with AL.

Methods: Patients were recruited within 1 month of hospital admission and randomized to EASE + UC or UC alone. We assessed traumatic stress symptoms, physical symptom burden, and quality of life at baseline, 4, 8, and 12 weeks. Between-group differences were evaluated using multilevel modeling.

Results: Forty-two patients were randomized to EASE $(n=22)$ or UC $(n=20)$, with $76 \%$ retention at 12 weeks. Predefined feasibility outcomes were met: $86 \%$ of EASE participants completed $\geqslant 50 \%$ of EASE-psy sessions (goal $\geqslant 64 \%$ ); $100 \%$ received Edmonton Symptom Assessment System (ESAS, modified for AL) screenings, $64 \%$ of whom completed $\geqslant 50 \%$ of planned screenings (goal $\geqslant 50 \%$ ); and $100 \%$ who scored $\geqslant 4 / 10$ on any physical ESAS-AL item had $\geqslant 1$ meeting with the EASE-phys team (goal 100\%). EASE patients showed significant improvement compared to UC patients in traumatic stress symptoms at 4 and 12 weeks and in pain intensity and interference at 12 weeks ( $p$ 's $<.05$ ).

Conclusions: EASE is feasible in patients newly diagnosed with AL and shows promising efficacy. These results warrant a larger RCT to provide rigorous evidence for its effectiveness with AL patients

FC16 Palliative Care for People with Cancer Quality of Life, Symptom Burden and Needs of Newly Diagnosed Incurable Advanced Cancer Outpatients of Comprehensive Cancer Centers (EVI Project) - Baseline Data from a Prospective, Multicenter, Mixed-methods, Sequential Control Group Study Siemens W. ${ }^{1}$, Schoensteiner S. ${ }^{2}$, Schaekel U. ${ }^{3}$, Bardenheuer H.J. ${ }^{4}$, Eschbach C. ${ }^{5}$, Viehrig M. ${ }^{6}$, Becker G. ${ }^{1}$, Gaertner J. ${ }^{7}$ ${ }^{1}$ Clinic for Palliative Care, Medical Center, University of Freiburg, Faculty of Medicine, University of Freiburg, Freiburg, Germany, ${ }^{2}$ Department of Internal Medicine III, University Hospital UIm, Ulm, Germany, ${ }^{3}$ Department of Internal Medicine V, University Clinic Heidelberg, Heidelberg, Germany, ${ }^{4}$ Center of Pain Therapy and Palliative Care Medicine, Department of Anesthesiology, University Hospital Heidelberg, Heidelberg, Germany, ${ }^{5}$ Department of Thoracic Oncology, Member of the German Center for Lung Research (DZL), University Hospital Heidelberg and Translational Lung Research Center Heidelberg (TLRC-H), Heidelberg, Germany, ${ }^{6}$ Department of Radiooncology, Palliative Care Unit, University Hospital of Tübingen, Tuebingen, Germany, ${ }^{7}$ Center for Palliative Care Hildegard, Basel, Switzerland

Background: Early palliative care (EPC) in oncology is increasingly recognized but information from large cohort studies about the PC needs are lacking. Aims: To describe quality of life (QoL), symptoms and needs of outpatients in German comprehensive cancer centers.

Methods: This cross-sectional analysis of baseline data is part of a prospective, multicenter study (DRKS00006162, primary aim: feasibility). We included adult outpatients with incurable advanced cancer diagnosed within the last eight weeks. Patients' QoL, symptoms and needs were assessed with the Palliative Outcome Scale (POS, 0-40; 40=maximal burden), Hospital Anxiety and Depression Scale (HADS, 0-20; $>10=$ moderate or severe) and European Organization for Research and Treatment of Cancer QoL Questionnaire-C30 (0-100; QoL and function: $0=$ maximal burden; symptoms: $100=$ maximal burden).

Quantitative data analysis was performed using $\mathrm{R}$.

Results: Data from 481 patients (mean age: 62.4 , female: $50.9 \%$ ) were analyzed. Descriptive statistics showed that patients' overall burden was low to moderate.

Yet, a closer look revealed that 41 (8.5\%) patients were in the upper half of the POS Score $(>20)$ and the QoL of 89 patients $(18.5 \%)$ was in the lower quarter $(<26)$ of the scale. Anxiety was moderate or severe in 85 $(17.7 \%)$ and depression in $98(20.4 \%)$ patients. Role functioning was in the lower quarter $(<26)$ of the scale in $180(37.4 \%)$ patients. A number of patients were in the upper quarter $(>75)$ of the symptom scales: fatigue ( $n=162,33.7 \%)$, pain $(n=88,18.3 \%)$ and insomnia $(n=101,21.0 \%)$. Physical symptoms were the most relevant problem reported in the POS free text item ( $n=93,26.8 \%)$.

Conclusion: A relevant number of patients had a low QoL, considerable symptom burden and an impaired role functioning. This data highlights the necessity of EPC and indicates that PC needs may be addressed by different professions and as general or specialist PC.

Funding: Robert Bosch Stiftung: 11.5.1364.0055.0. 
EE3 Podium Discussion. How Does Integration of Oncology and Palliative Care Change in the Light of a New Era in Oncology? A Multiprofessional Challenge

No abstracts available

\section{Digital Legacy Conference}

SPS6 Digital Legacy conference

Do We Prepare Patients for their Digital Legacy? A Survey of Palliative Care Professionals

Coop H., Marlow C. West Midlands Collaborative Actioning Research in End of life and Supportive Care (WM CARES)

The Royal Wolverhampton NHS Trust, Wolverhampton, United Kingdom

Background: Social media is becoming a more prominent part of our everyday lives. As a consequence we will be leaving a digital legacy after death. Digital legacy is the digital information that is available about someone following their death, which may include website or blog listings, social media profiles, photos, videos and gaming profiles. Many will find it a comfort that their legacy remains on social media but there can also be negative consequences in relation to death, dying and bereavement. This survey was carried out to establish whether we prepare patients for their digital legacy within the West Midlands.

Methods: An online survey was sent to 11 hospices within the West Midlands, UK, over a 6 week period in 2017. The survey was circulated by the hospices to the doctors, nurses and social workers.

Results: 210 responses were collected (35\% response rate). $96 \%$ had never discussed digital legacy with patients. Reasons for not having such discussions largely related to it not being considered, lack of knowledge about digital legacy or it not being felt to be appropriate or important.

Discussion: This survey of health and social care professionals shows that we do not discuss digital legacy with patients. There is a potential unmet need that we are not addressing for patients. There is an education need across the region to raise awareness and confidence of digital legacy.

\section{SPS6 Digital Legacy conference}

Usque Twitterati ad Mortem

Minton O. ${ }^{1}$, Norris J. ${ }^{2}$, Taubert M. ${ }^{3}$

${ }^{1}$ Brighton and Sussex University hospitals, Brighton, United Kingdom, 2Dead Social, London, United Kingdom, ${ }^{3}$ Velindre NHS trust, Cardiff, United Kingdom

Aim: We wanted to share our experience to encourage more sharing of data, opinions and joining a growing palliative MDT community online. Methods: One of us (MT) has had global recognition for his letter to David Bowie while others have had blogs in the BMJ supportive care (OM). All of us use twitter and its interactive nature to promote a spirit of unity and encouragement for end of life care more broadly. Given twitter is a public forum this is not without some controversy but the opportunity to discuss funding/ advance care planning / organ donation and research updates, blogs.

Results: We all feel a regular and interactive presence online help our professional lives - many opportunities have arisen from this - radio \& TV work aside - we have all been able to attend meetings where the prior introduction has been made virtually. There is of course no absolute scientific way of measuring "impact" but this is not that different from a research paper but we do all the dopaminergic likes and retweets to keep us motivated. We want to encourage others to be part of our gang and to dilute the in-jokes and UK centric perspective although we do have a southern hemisphere contingent the time difference does limit us.

Conclusion: If one person has made a will or had a serious illness conversation as a result of our presence then this has made everything worthwhile. The untold actions can't be counted but as public health England like to believe - nudging goes a long way and with over 10,000 followers between us there will be impressions being made and while we know it is all a dark art we do hope that some of those have led to someone instigating a more concrete plan.

\section{SPS6 Digital Legacy conference}

Digital Legacy and Future Care Planning - Stories from a Cancer

Hospital

Taubert M.1,2

${ }^{1}$ Cardiff University School of Medicine, Cardiff, United Kingdom, ${ }^{2}$ Digital Legacy Association/ My Wishes, London, United Kingdom

The use of internet enabled devices by patients at the hospital;/hospice bedside appears to be increasing. Digital connections are everywhere, and can bring new opportunities when we are seriously ill. But what makes a good digital death? And how can digital media bring meaning during serious life-limiting illness?

In this talk, Dr Mark Taubert will outline his personal and professional experience with patients, carers, researchers and fellow healthcare professionals working in palliative care, and how since 2013 he has tried to make good use of new media. The taboo around death and dying is seemingly starting to crumble on Youtube, Instagram, Facebook and Twitter with many more opportunities for us in palliative care to influence debate. How celebrities die, for instance, is increasingly commented on, but Mark will also tell stories from the wards and clinics, where people have asked him questions about what they should do with digital content when faced with the end of their lives.

Mark is a palliative care doctor at Velindre NHS Trust cancer hospital in Cardiff (UK) and a senior lecturer at Cardiff University. He is national strategic lead for advance and future care planning in Wales and holds roles with the Bevan Commission, Byw Nawr and the End of Life Care board in Wales. Mark is a regular speaker and editor in areas relating to palliative care, digital media, resuscitation and end of life care. He also contributed to a BBC Radio 4 programme in the UK called "My Digital Legacy" in 2017 with the presenter Joan Bakewell.

Mark will illustrate a story of a patient he worked with who created digital legacy content in the form of videos and messages, to be viewed at significant future dates in his family's life. The journey to achieve this was in many ways harder than achieving good symptom control and took a significant emotional toll, but was what the patient truly wanted. The story was reported on by BBC World in 2019, as part of a report about digital legacy.

\section{SPS6 Digital Legacy conference}

Kids These Days: Caring for Parents Who Are Patients in the Internet Age.

Strand J.

Mayo Clinic, Rochester, MN, United States

Discussion of the complexities in caring for parents who are patients in the age of online patient support platforms, virtual grief support groups and Dr. Google. This session will engage participants in a casebased discussion of working with this patient population surrounding topics such as

- Finding information about death and dying online

- Terminal illness support online

- Grieving online

Additional attention will be given to strategies for engaging interdisciplinary resources in attending to these needs. 


\section{SPS6 Digital Legacy conference}

Digital Legacy, Digital Assets and Advance Care Planning

Norris J.

Digital Legacy Association, Leighton Buzzard, United Kingdom

Presenting author email address: james@digitallegacyassociation.org

This abstract focuses on how the role of the healthcare and social care professional is changing following recent changes in attitudes and behaviours around end of life planning, death and the internet.

It will examine how professionals are embracing recent technological and communicative developments to better support patients with their digital assets and their digital legacy. It will question how technology is changing the ways in which the general public plan for death and how this relates to both advance care planning and documenting other important areas within a Last Will \& Testament. Case studies will lead to a critical analysis around the impact of grieving online and the influence and importance of digital dead.

Digital assets and digital legacy resources currently being used by clinicians and the general public will be reviewed and discussed. Online planning tools that assist patients and the general public to plan for their digital death, and tools that enable the deceased to interact with their loved ones 'into perpetuity', will evoke ethical, moral and sociological questions. The session will conclude with the question; "should conversations around someone's digital wishes form part of a holistic approach to advance care planning or should this area be addressed somewhere else?"

\section{Poster Exhibition}

\section{(Poster Set 1)}

Palliative Care in Childrens and Adolescents

Basic and Translational Research

Communication

Family \& Care Givers

Health Services Research

Medical Sociology

Policy

Primary Health Care \& Primary Care

Public Health \& Epidemiology

Research Methodology

Spirituality

Social Care and Social Work

Psychology and Psychiatry

Volunteering

Organ Failure (Heart, Respiratory, Kidney, and Liver Diseases)

Neurological Conditions

Frailty

Pharmacology \& Polypharmacy

Palliative Care for Older People

Homelesness

Palliative Care for Refugees and Migrants

Other Patient Groups

Abstract number: P01-001

Abstract type: Poster

The Labour and Birth of a Perinatal Palliative Care Framework Bengree L. ${ }^{1,2}$, Cochran C. ${ }^{3}$

${ }^{1}$ Child Health, Southampton Children's Hospital, Southampton, United Kingdom, ${ }^{2}$ Naomi House Children's Hospice, Winchester, United Kingdom, 3Paediatric Cardiology, University Hospital Southampton, Southampton, United Kingdom

Background: Around $5 \%$ of pregnancies are complicated by congenital structural abnormalities, $15 \%$ of which are life-limiting or potentially life-limiting. Over recent years it has become apparent that there is a need for clearer palliative care pathways for this group of infants that can begin prior to delivery.

Method: To establish seamless links between the multidisciplinary teams within the tertiary centre and the district general hospitals (DGH) through the design of the Perinatal Palliative Care (PPC) framework. This supports facilitation of PPC and delivery closer to home, provision of specialist input, advice and training to support teams within the region. Results: With the implementation of the pathway, six families have been supported with PPC in January to July 2018 (Table 1.0); this is a vast increase on previous referral rates. Within the six cases the PPC focus was the physiological symptom management of the infant, the psychological, spiritual, social and emotional support of the family both antenatally and postnatally. Bereavement and hospice support has been instrumental in the facilitation and delivery of PPC.

Conclusion: The delivery of PPC framework has highlighted the benefits of a more formal pathway to ensure that PPC is delivered effectively and is used within PPC cases, where appropriate. The purpose of the PPC framework is to enable clinicians to deliver consistent, high quality care for families electing to continue pregnancy with a fetus with a life-limiting condition, the development of advance care plans and to help ensure uniform standards of care regardless of where the baby is cared for.

Table 1.0 PPC outcomes.

\begin{tabular}{lll}
\hline Case & Diagnosis & Outcome \\
\hline B1 & $\begin{array}{l}\text { Congenital Heart } \\
\text { Disease } \\
\text { B2 }\end{array}$ & $\begin{array}{l}\text { Died 7 days following } \\
\text { delivery in DGH }\end{array}$ \\
B3 & $\begin{array}{l}\text { Renal dysplasia, } \\
\text { oligohydramnios \& } \\
\text { pulmonary hypoplasia }\end{array}$ & $\begin{array}{l}\text { Died } \text { in tertiary centre } \\
\text { delivery in DGH }\end{array}$ \\
B4 & $\begin{array}{l}\text { Congenital Heart } \\
\text { Disease }\end{array}$ & $\begin{array}{l}\text { Died 2hrs following deliver } \\
\text { in tertiary centre }\end{array}$ \\
B5 & $\begin{array}{l}\text { Congenital Heart } \\
\text { Disease }\end{array}$ & $\begin{array}{l}\text { Remains alive 21 days later } \\
\text { at DGH, discharged home } \\
\text { planned. }\end{array}$ \\
B6 & Trisomy 18 & Awaiting delivery \\
\hline
\end{tabular}

Abstract number: P01-002

Abstract type: Poster

A New Last Aid Course for Kids from 8-14 Years - A Pilot-test Bollig G..$^{1,2,3}$, Mainzer K. ${ }^{4}$, Fiedler $H_{.}{ }^{5}$, Pothmann R. ${ }^{4}$, Barra F. ${ }^{6}$

1Palliative Care Team, Medical Department, South Jutland Hospital, Sønderborg, Denmark, ²Palliative Care Research Group, University of Southern Denmark / Institute of Regional Health Research, Sønderborg, Denmark, ${ }^{3}$ Last Aid International, Schleswig, Germany, ${ }^{4}$ Pediatric Palliative Care Team (Kinder-PACCT), Hamburg, Germany, ${ }^{5}$ Pediatric Palliative Care Team, (Kinder Palliativteam), Frankfurt, Germany, ${ }^{6}$ Childrens Hospice Sternenbrücke, Hamburg, Germany

Background/aims: Last aid courses for adults have been introduced to educate the public about Palliative care and to enhance the public discussion about death and dying $(1,2)$. The aim of the present study was to investigate the feasibility and acceptance of a similar approach adapted to the needs of children.

Methods: Based on the Last Aid course for adults $(1,2)$ a multi-professional working-group adapted the curriculum for children between 8 and 14 years. The first pilot-course was evaluated by oral feedback of the 
children after the course and a questionnaire filled out by the participants some weeks after taking part in the Last Aid course.

Results: 10 children between 9 and 14 years participated in the Last Aid course that was held within 3 hours on one afternoon. 9 out of 10 participants had experience with the death of family members. All children stated that they would recommend the course to other children and that also adults should participate in Last Aid courses. The children appreciated the course and most felt somehow more prepared for a potential death in their social network.

Conclusions: The results of the pilot-test are very encouraging. More Last Aid courses for children will be taught within the next year. A scientific evaluation with a mixed-methods approach will be part of the pilot-testing-phase.

\section{Literature:}

1. Bollig G, Kuklau N. Der Letzte Hilfe Kurs - ein Angebot zur Verbesserung der allgemeinen ambulanten Palliativversorgung durch Information und Befähigung von Bürgerinnen und Bürgern. Z Palliativmed 2015; 16:210-216.

2. Bollig $G$, Heller A. The last aid course - a simple and effective concept to teach the public about palliative care and to enhance the public discussion about death and dying. Austin Palliat Care 1(2): id1010 (2016)

3. www.lastaid.info

\section{Abstract number: $\mathrm{P} 01-004$ \\ Abstract type: Poster}

Looking Back to Move Forward: Cannabis Use in Children with Life-threatening Conditions

Vadeboncoeur C. ${ }^{1,2,3}$, Doherty M.1,2,3, Attala M. ${ }^{1}$

${ }^{1}$ Medicine, UOttawa, Ottawa, Canada, ${ }^{2}$ Pediatrics, CHEO, Ottawa, Canada, ${ }^{3}$ Pediatric Palliative Care, Roger Neilson House, Ottawa, Canada

Background: Used for centuries to treat insomnia, nausea, vomiting, loss of appetite, epilepsy, pain and depression, benefits of cannabis extracts are being studied in children. A systematic review of 11 studies suggest benefit for treatment-refractory epilepsy. Synthetic cannabinoids are effective antiemetics in children undergoing chemotherapy. Studies in chronic neuropathic pain have shown minimal analgesic effect. One study of children with life-limiting conditions with spasticity shows promise.

Aims: Describe the use of medical cannabis in children with life-threatening or life-limiting conditions including benefits and adverse effects.

Methods: Review records of children followed by the palliative care team and receiving medically prescribed cannabis products between Jan 2010-June 2018. Children using non-prescribed cannabis before or during the study period were excluded. Effects were recorded using patient, parent and physician comments.

Results: Cannabis was prescribed in 11 children ( 6 female) aged 3.2 to 18.9 years (mean 9.9) between 2014 and 2018. As an antiepileptic in 8 children, $62.5 \%$ experienced a $75 \%$ reduction in seizure frequency. In some cases this effect faded after 4-6 months. Insomnia and sedation were reported in 1 child each. Treatment of nausea and vomiting was successful in 1 child. Two children had positive results stimulating appetite and helping treat their weight loss. One of these reported mild insomnia. In treating pain, one child with neuropathic leg pain and another with abdominal pain reported effectiveness with no adverse effects.

Discussion: Medical cannabis led to reduction (transiently) in seizure frequency in children using cannabis for anti-epileptic effects. It had a positive effect on nausea/vomiting, pain and appetite in this small cohort of children. Reported adverse effects included insomnia and sedation. In future we will prospectively enrol children to determine clinical and adverse effects in real time.

Abstract number: P01-005

Abstract type: Poster
Shaping the Future for Paediatric Palliative Care Patients with Multidrug Resistant Bacteria: A New Inpatient Approach Schmidt P., Hartenstein-Pinter A., Hasan C., Wager J., Zernikow B. Paediatric Palliative Care Centre and German Paediatric Pain Centre, Children's and Adolescents' Hospital Datteln, Department of Children's Pain Therapy and Paediatric Palliative Care, Witten/Herdecke University, Faculty of Health, School of Medicine, Datteln, Germany

Background: Multidrug resistant (MDR) bacteria are an important healthcare issue throughout Europe. Because children and adolescents with life-limiting conditions (LLC) are particularly vulnerable to MDR bacteria and nosocomial infections (NI), much effort must be undertaken to avoid new colonisations in these patients. An important concurrent aim in the treatment of children with LLC is to maximize quality-of-life by enabling social participation. In children with both MDR bacteria and LLC, these two treatment goals are important considerations in the ongoing challenge to prevent NIs without minimizing social participation. A new inpatient hygiene concept for pediatric palliative care (PPC) patients with MDR bacteria, called PALLINI, has been developed. PALLINI follows risk-adapted hygienic guidelines and at the same time enables the patients to participate in the daily routine of the unit.

Aim: The current study aims to evaluate the safety and acceptability of PALLINI.

Method: Over a period of two years (02/2018 - 01/2020), a surveillance will be conducted to detect NIs due to MDR bacteria. All patients on the PPC unit will be screened for MDR bacteria at admission and discharge. To investigate the acceptability semi-structured interviews with families and staff members will be conducted.

Expected results: Results of the first 12 months of data collection will be presented. We expect that the rate of NIs due to MDR bacteria will be close to zero and that PALLINI is accepted by families and staff members as a safe and patient-friendly hygiene concept for patients who are colonised with MDR bacteria.

Conclusion: Outcome of this study will be a safe hygiene concept with regard to the prevention of NIs and at the same time a patient-friendly approach that allows social participation. It will be transferable to other settings, e.g. hospice, respite care or nursing homes.

The study is funded by the German Federal Ministry of Education and Research (01GY1713)

Abstract number: P01-006

Abstract type: Poster

Hide and Seek: Discovering what's Helpful for the Evidence Base in Irish Children's Palliative Care

Quinn C., Devins M., Twomey M., Mc Carthy S., Ling J., O'Reilly M.

NUI Galway, School of Nursing and Midwifery, Galway, Ireland

Presenting author email address: claire.quinn@nuigalway.ie

Research aims: Irish children's palliative care (CPC) is an evolving speciality. There is a need to develop an evidence-based approach and in order to do this in a systematic way, identification of the research priorities in CPC in Ireland is required. Although Delphi studies have been undertaken in several countries, findings are not transferable between countries due to a variety of factors including: different healthcare delivery systems, varying policy initiatives, differences in geography and variation in access to services.

Study design: The Delphi method is a consensus building approach to data collection. Experts in CPC were identified by a steering committee and were invited to participate to ascertain their opinion on the research priorities nationally. This iterative process of data collection utilised 'rounds' to achieve consensus. Classic Delphi studies use four rounds, although three is often viewed as sufficient to achieve consensus and avoid participant fatigue. The first 'round' aims to establish a list of topics or questions which are then put forward in subsequent rounds through the use of questionnaires to seek group consensus relating to the relative importance of 
each topic. In general, surveys are used during the first round to establish the key areas to be put forward for review in later rounds.

Results: Following a robust 4 round Delphi study, consensus was reached between a group of key health care professionals and stakeholders in CPC in Ireland ( $n=54$ ) on the future research priorities. Findings from this Delphi fit broadly into five themes: Needs Assessment (including a database of children with life-limiting conditions, accurate prevalence); Service Development; Policy; Education/ Training and Care Supports.

Conclusion: The wide range of topics emerging from the Delphi process reflects the current lack of documented findings to support service development and confirms the need for greater evidence within this emerging specialty.

Abstract number: P01-007

Abstract type: Poster

Prospective Survey of Wellbeing and Quality of Life in Parents and Siblings of Children and Adolescents with Life-limiting Conditions in Palliative Home Care

Kuhlen M. ${ }^{1,2}$, Dinkelbach L. ${ }^{1}$, Danneberg M. ${ }^{1}$, Gagnon G. ${ }^{1}$, Trocan L. ${ }^{1}$, Dechert O. ${ }^{1}$, Hoell J. ${ }^{1,3}$, Janßen G. ${ }^{1}$

${ }^{1}$ Heinrich Heine University Duesseldorf, Dep Ped Oncology, Hematology and Clin. Immunology, Düsseldorf, Germany, ${ }^{2}$ Klinikum Augsburg, Children's Hospital Augsburg, Swabian Childrens' Cancer Center, Augsburg, Germany, ${ }^{3}$ Martin Luther University Halle-Wittenberg, Department of Pediatric Hematology and Oncology, Halle, Germany

Background: Pediatric palliative care aims to achieve best possible quality of life (QoL) for children and adolescents (CAs) with life-limiting conditions (LLCS) and their entire family. However, data on the needs of parents and siblings in the course of palliative care in the home setting (SPPHC) are still lacking. Indeed, from a Public Health perspective, it is important to realize needs, excessive stress and non-existing coping resources of affected families to prevent secondary diseases.

Aim: To survey the needs, psychosocial burden and health-related QoL in parents and siblings of CAs with LLC

Design and methods: Development and validation of surveys for parents and siblings (for the latter based on existing instruments (KINDL-R, LARES, SDQ-Deu; separated by age groups 8-11, $12-13$ and $14-18$ years); assessment at three time points during SPPHC.

Here, we present preliminary findings of this ongoing, prospective longitudinal survey.

Results: To date, 17 families participated in the parents' survey at the first two time points, of these 15 were mothers (88\%). Median age was $40 \pm 8.8$ years. The need for psychosocial support was highest at referral (Wilcoxon's $Z=-2.2, p=.028$ ), while the QoL increased and nursing burden descriptively decreased over time.

In the siblings' survey, 30 CAs participated (20 females). Median age was $13.3 \pm 4.7$ years. Disease knowledge correlated significantly with psychosocial wellbeing $(p=.038) ; 27.6 \%$ of the siblings felt uninformed and wanted to learn more about the LLC of the affected child. In comparison to normative cohorts, siblings showed specific impairments in healthrelated QoL but increased prosocial behavior (each $p<.05$ ).

Conclusion: As part of SPPHC, knowledge about the needs of the entire family of CAs with LLCs is inevitable. First findings of a prospective survey point to an impairment of the psychosocial wellbeing and health-related QoL of affected parents and siblings, which needs to be confirmed in larger samples.

Abstract number: P01-008

Abstract type: Poster

\section{An Assessment of the Learning Needs in Paediatric Palliative}

Care

Whitla L. ${ }^{1}$, Devins M. ${ }^{2}$, Molloy E. ${ }^{1,3}$, Twomey M. ${ }^{2}$, O'Reilly M. ${ }^{2}$, Balfe J.1,4

${ }^{1}$ Tallaght University Hospital, Dublin, Ireland, ${ }^{2}$ Our Lady's Children's
Hospital, Crumlin, Dublin, Ireland, ${ }^{3}$ Trinity College Dublin, Dublin, Ireland, ‘LauraLynn- Ireland's Children's Hospice, Dublin, Ireland

Aim: To determine baseline learning needs of Paediatricians in Ireland when caring for children with palliative care needs.

Methods: A survey of Paediatricians working in Ireland was conducted. The Quality of Care Collaborative for Australia in Paediatric Palliative Care Project Research Study Group Learning Needs Assessment template was used with kind permission. Ethical approval was granted.

Results: One hundred and eleven people responded to the survey, $69.4 \%$ female. The majority were specialist registrars but almost half were consultant paediatricians (45.3\%). Almost all worked in a tertiary $(56.8 \%)$ or regional hospitals $(36 \%)$. The median number of children cared for with a life limiting condition per year was 10 (mean 19, range $0-200$ ), and with palliative care needs was 5 (mean 7, range 0-50). The median number requiring end of life care per year was 2 (mean 3 , range $0-50$ ), the estimated number of children who died was also 2 (mean 3 , range $0-50)$, and those receiving specialist palliative care team input was 2 (mean 4, range $0-20$ ).

Most had never had formal education in the paediatric palliative care (56.8\%), with some having completed a short course (8.4\%). Areas of future training that were ranked as highly important (percentage of respondents) included: Preparing families for the death of their child $63.8 \%$, Management of the dying child $63.8 \%$, Pain management $57 \%$, Advanced care planning $55 \%$, Other symptom management $47.5 \%$, Communication skills $47.5 \%$, and Practical support $39.2 \%$. Those surveyed were also asked to comment on the challenges of their most recent clinical interactions with children requiring palliative care support, a number of common themes emerged including communication, symptom management and difficulty in coordinating community support.

Conclusions: This survey highlights the learning needs of paediatricians and will inform the development of meaningful education sessions for doctors who are caring for children with palliative care needs.

\section{Abstract number: P01-009}

Abstract type: Poster

\section{Enhancing Perinatal Palliative Care at the Ottawa Hospital in Collaboration with the CHEO Palliative Care Team, Roger Neilson House Hospice, and the Community}

Ashton G. ${ }^{1}$, Ferretti E. ${ }^{1,2,3}$, Reuvers E. ${ }^{1}$, Benoit C. ${ }^{4}$, Van Zanten S. ${ }^{2,3,4}$ Vadeboncoeur $\mathrm{C}^{2,3,4}$

${ }^{1}$ Obstetrics and Newborn Care, The Ottawa Hospital - General Site, Ottawa, Canada, ${ }^{2}$ Pediatrics, CHEO, Ottawa, Canada, ${ }^{3}$ Pediatrics, uOttawa, Ottawa, Canada, ${ }^{4}$ Pediatric Palliative Care, Roger Neilson House, Ottawa, Canada

Background: Detection of life-limiting conditions (LLC) early in pregnancy provides parents months to anticipate death or an infant born with severe challenges. Unanticipated problems after birth can lead to immediate or early death. Intensive care does not always prolong life. Palliative care provides holistic, family centered care, honoring the needs of the infant and family. Women and families desire active involvement in end-of-life discussions and care planning.

Many health care professionals (HCP) are uncomfortable with aspects of palliative care including their knowledge base, counseling skills, and facilitating decision-making. The care of severely ill infants can be challenging for both HCPs and parents. The decision-making process can be overwhelming for families.

Aims: To enhance the quality of perinatal palliative care provided to families experiencing a pregnancy with LLC.

1. Consistent end-of-life care for infants with LLC and support to women and families

2. Comprehensive communication of choices to parents

3. Update educational resources 
Method: Plan-Do-Study-Act model.

Plan: Gather information from women and families experiencing the diagnosis of LLC during pregnancy and from HCPs who care for them. Feedback will be reviewed by two project members.

Do: Develop and implement strategies based on themes identified such as system issues, patient care, and HCP competencies, including staff education, review of patient information, revision of policy, and clarification of liaison relationships

Study:

Outcome Measures:

- Increased patient satisfaction with services offered

- Obstetric team member statements of competency and system improvement.

- $\quad$ Palliative Care statements of system improvement.

Process Measures:

- $\quad$ Palliative care offered to $90 \%$ or more of women who meet inclusion criteria.

- Increased number of consultations to Palliative Care

ACT: Cycle will be repeated based on evaluation outcomes Results of this ongoing process will be presented at the conference

Abstract number: P01-010

Abstract type: Poster

\section{Assessing Outcomes of Pediatric Palliative Care That Are Pivotal to Patients and their Families \\ Ribbers S., Wager J., Zernikow B., Reuther M. \\ Department of Children's Pain Therapy and Paediatric Palliative Care, Witten/Herdecke University, Faculty of Health, School of Medicine, Paediatric Palliative Care Centre and German Paediatric Pain Centre, Children's and Adolescents' Hospital Datteln, Datteln, Germany}

Background: The utilization of patient-centered outcomes is essential in providing high quality healthcare. In pediatric palliative care (PPC), the patients are not the sole focus of attention. Their families are always included within the care setting. Together, the patients and their families constitute the unit of care. To date, no validated multidimensional PPC outcome tool focusing on the entire unit of care exists.

Aim: We aim to develop and validate such a multidimensional, patientand family-focused outcome tool for the use in PPC.

Method: Guided interviews with patients' families and professional carers were conducted to identify potentially relevant outcomes of PPC. The interview transcripts were analyzed using qualitative content analysis. Next, the actual relevance of the identified outcomes was quantitatively rated by patients' families and professional carers (on a scale of 0-10). Based on the outcomes rated as being relevant, the first version of the new outcome tool was drafted. This new measure is now being validated in a large sample of PPC patients (parent and staff ratings) by means of face validity, usability and factor structure.

Results: The seven identified relevant outcome dimensions, namely symptom control, quality of care, empowerment, relief, coping, normalcy and security will be presented. They each consist of 2-15 constituent aspects and are thought to collectively influence the Quality of Life of the unit of care. Each aspect obtained a mean relevance between 5.3 and 9.9 and was thus considered relevant. Preliminary data of the validation process will be presented.

Conclusion: The current data reflect the complexity of PPC. The challenge during the currently ongoing validation process of the assessment tool is to capture this complexity while simultaneously realizing a relatively short and easy to administer assessment tool.
The study is funded by the German Federal Ministry of Education and Research

(funding code: 01 GY1717).

Abstract number: P01-011

Abstract type: Poster

Cancer-Bereaved Siblings' Advice to Peers - A Nationwide

Follow-up Survey

Eilegård Wallin A. ${ }^{1}$, Udo $C^{1,2}$, Kreicbergs U. ${ }^{2,3}$, Lövgren $M^{2,3}$

${ }^{1}$ School of Education, Health and Society, Dalarna University College,

Falun, Sweden, ${ }^{2}$ The Department of Health Care Sciences, Palliative

Research Center, Ersta Sköndal Bräcke University College, Stockholm,

Sweden, ${ }^{3}$ Karolinska Institutet, The Dept of Children and Women's

Health, The Childhood Cancer Research Unit, Stockholm, Sweden

Background and aim: Evidence favors sibling support as a standard of care in pediatric oncology. The aim of this study was to explore cancerbereaved siblings' advice to others with a brother/sister with cancer. Knowledge about this can guide health care professionals (HCPs), families, and others in providing sibling support.

Methods: This study derives from a nationwide survey in Sweden among cancer-bereaved siblings. One hundred and twenty-five of the 174 participating siblings responded to the open-ended question (responserate: $73 \%$ ): "What advice would you give to others with a brother/sister with cancer",. The responses were analysed with content analysis.

Results: The siblings gave 257 pieces of advice which were sorted into four categories. Be together encompassed around half of the advice and related to being with the ill brother/sister, cherishing the time together, supporting him/her, and saying important things before death, e.g., saying goodbye or letting the brother/sister know that he/she will be missed. Communicate openly related to the importance of talking to others in the same situation and to seek professional support if needed. Advice in Let go of guilt encouraged peers to make themselves heard and show feelings even though they might feel guilt when doing so. The category Live life as usual involved advice about the importance of living as normal a life as possible, going to school, participating in leisure activities/hobbies, etc., as a way of taking care of oneself.

Conclusion: The key results in this study and a significant part of all the advice given related to the relational aspect between the ill child and the sibling during the period of illness. They highlighted the importance of HCPs, family, and others facilitating for siblings to spend time together and communicate openly.

Funding: The Swedish Cancer Society, Gålö Foundation, and the Swedish Childhood Foundation.

Abstract number: P01-012

Abstract type: Poster

Use of Medical Cannabis in Paediatric Palliative Care

Reschke F. ${ }^{1}$, Schallner J. ${ }^{2}$, Nolte-Buchholtz S. ${ }^{1}$

${ }^{1}$ Brückenprojekt - Centre of Paediatric Palliative Care, Technical University Carl Gustav Carus, Dresden, Germany, ${ }^{2}$ Department of Neuropaediatrics, Technical University Carl Gustav Carus, Dresden, Germany

Background: Medical Cannabis (MC) has become increasingly legal at the state level in many countries and accessible even to children with serious illness. This led to a high social acceptance and a huge interest in its medical use in paediatric palliative care (PPC) patients. Despite this, there is only little evidence about its use and benefits particularly for children.

Aim: To evaluate the current use and potential benefits and risks of $M C$ use in PPC

Methods: An uncontrolled, monocentric, retrospective observational study was conducted, including paediatric patients suffering from 
life-threatening conditions and receiving $M C$ in between January 1st, 2013 and September 30st, 2018. The study population $(N=48)$ received specialized paediatric palliative care and cannabinoids for any reason. The study focused particularly upon the active ingredients, indications, duration, dosages, benefits and adverse effects.

Results: The patients' diagnoses included complex neurological (32/48), oncological (13/48) and other (3/48) diseases. Spasticity (40/48), emesis $(26 / 48)$ and agitation (25/48) were evaluated as the main indications for the use of MC, which was given orally to all patients either isolated as dronabinol (DRB) or cannabidiol or combined. DRB was given most frequently $(46 / 48)$ in a median dosage of $0.40 \mathrm{mg} / \mathrm{kg} / \mathrm{d}(0.2-0.7 \mathrm{mg} / \mathrm{kg} / \mathrm{d})$. Though in 7 cases no effect was seen, in total a promising therapeutic effect was noticed (41/48). Drowsiness (20/48), nausea (17/48) and dizziness $(9 / 48)$ were the most frequent observed adverse effects. The median duration of treatment was 246 days (5-1728 days). MC was reduced or discharged only in three patients.

Discussion / Conclusion: In this study a benefit for the use of MC was revealed for the bigger part of the patients. The main indication for MC is spasticity due to neurological and oncological diseases. Controlled prospective studies must be conducted to confirm these findings.

Abstract number: P01-013

Abstract type: Poster

Vaccine Preventable Diseases (VPD) in Children's Palliative Care - First Report of the German PalliVac Study Group

Hauch H. ${ }^{1}$, Kimmig A. ${ }^{2}$, Kaestner J. ${ }^{3}$, Rohde M. ${ }^{1}$, Brunder P. ${ }^{4}$, Tretiakowa I. ${ }^{1}$, Lieber K. ${ }^{5}$, Bockenkamp B. ${ }^{6}$, Hagenguth A. ${ }^{7}$, Scheer M. ${ }^{8}$, Berthold D. ${ }^{9}$

${ }^{1}$ Palliative Care Team for Children, University Children's Hospital Giessen, Giessen, Germany, ${ }^{2}$ Palliative Care Program, University Children's Hospital Tubingen, Tubingen, Germany, ${ }^{3}$ Palliative Care Program, University Children's Hospital Jena, Jena, Germany, ${ }^{4}$ Palliative Care, Kinder-Hospiz Dienst Saar, Merchweiler, Germany, ${ }^{5}$ Palliative Care, Björn Schulz Stiftung, Berlin, Germany, ${ }^{6}$ Pediatrics, University Hospital Charite, Berlin, Germany, ${ }^{7}$ SAPPV Brückenteam, DRK HeinrichSchwesternschaft e.V., Kiel, Germany, ${ }^{8}$ Kinder- und Jugendhospiz Loewenherz, Betreuungsnetz für schwerkranke Kinder, Syke, Germany, ${ }^{9}$ Medical Department V, University Hospital Giessen, Giessen, Germany

Background: In Germany, 33 pediatric specialised home palliative care teams (SHPC) provide comprehensive support for children and adolescents with life-limiting diseases. In 2017, a 12 year old non vaccinated boy with severe delay of neurological development and progressive respiratory deficiency suffered from a varicella infection. He suffered from pneumonia and dyspnea. This was the first reported patient with a vaccine preventable disease (VPD) in SHPC according to our knowledge so far. Aims: Description of other cases of VPD to generate the base for the prospective study. These data could allow appropiate counseling related to vaccinations in pediatric palliative care.

Methods: Retrospective analysis of other VPD cases in Germany. Results: 34 physicians ( 32 SHPC and 2 hospices of 12/16 German states) answered. Overall 17 patients with a diagnosed VPD (group 1) were reported. Most frequent VPD was influenza (64\%), varicella (24\%) and others (14\% e.g. diphtheria, pertussis, measles, pneumococcus and meningococcus infection). Suspicion of VPD (group 2) was reported in another 17 cases (influenza $71 \%$, pertussis $18 \%$, rotavirus $18 \%$ ). Average age of both groups was 8,8 years $(+-5,7)$. Distribution of IMPaCCT groups (1-4) was $5 \%$ (1), $0 \%(2), 55 \%$ (3), $40 \%$ (4). Diagnosis was made clinically in $64 \%$, serologic testing in $29 \%$ and PCR in $59 \%$. Severe symptoms (min. 1 to max. 5 points) were dyspnea $(3,4+-1,5)$ and deterioration $(3,8+-1,1)$. Main complications for both groups were admission to intensive care unit (ICU) in $29 \%$, ventilation $20 \%$, death in $6 \%$ and infection of a health care professional in $20 \%$. Physicians agreed it could be helpful to prevent VPD (5= strong to $1=$ no agreement) with a mean score of $4,4+-1,1$.

Discussion: VPD could cause distressful symptoms up to ICU treatment and death. VPD seems to play a certain role in palliative care in Germany.
Further investigation appears to be reasonable. Prospective phase of PalliVac (DRKS: 00015045) will begin in 2019.

Abstract number: P01-014

Abstract type: Poster

Understanding the Education and Support Needs of those Providing Children's Palliative Care in Scotland

Malcolm C. ${ }^{1}$, McGirr D. ${ }^{1}$, Grant $J^{2}$

${ }^{1}$ School of Health and Social Care, Edinburgh Napier University, Edinburgh, United Kingdom, ${ }^{2}$ Children's Hospices Across Scotland (CHAS), Edinburgh, United Kingdom

Aim: To identify the current and future education, learning and continuing professional development requirements of staff and volunteers working in children's palliative care.

Design: Employing a qualitative exploratory design, data was collected through focus groups to gain a better understanding of the following:

1) education, learning and development opportunities currently available;

2) learning and development requirements including any unmet needs;

3) preferred learning methods and approaches; and

4) the wider role of children's hospices in palliative care education.

Results: A total of 79 participants took part in the project - 61 via focus groups and 18 via an online survey - representing a wide range of services and care providers including the NHS (acute and community), children's hospices, the voluntary sector, social care and education. The findings were extensive identifying a number of learning needs with particular importance being placed on communication skills, end of life and bereavement care, managing complex ethical dilemmas, and the emotional impact of working within children's palliative care. Participants proposed a variety of recommendations to inform and influence the delivery of children's palliative care education.

Conclusion: There is a strong appetite for educational development in children's palliative care. This project contributes to the existing evidence base surrounding the requirement for further development of educational initiatives to enhance capacity and capability of the workforce. Moreover, it advocates a national, standardised and accessible approach to ensure children's palliative care education is embedded across all settings.

\section{Abstract number: P01-015 \\ Abstract type: Poster}

Challenges in Receiving Research Ethics Committee Approval for Studies Involving Children and Young People with Lifelimiting Conditions and Life-threatening Illnesses: An Analysis of Research Ethics Committee Minutes and Correspondence with Principle Investigators

Butler A.E., Vincent K., Bluebond-Langner M.

Louis Dundas Centre for Children's Palliative Care, UCL Great Ormond Street Institute of Child Health, London, United Kingdom

Background: Researchers working with children and young people with life-limiting conditions and life-threatening illnesses (CYP with LLC/LTIS) frequently identify research ethics committees (RECs) as a barrier to their research. However, little is known about what concerns RECs in research with CYP with LLC/LTIS.

Aim: To explore the concerns expressed by RECs when reviewing studies involving CYP with LLC/LTIs, and how researchers address these concerns. 
Methods: Data included REC-research team correspondence, specifically: REC decision letters, meeting minutes and researcher response letters. Analysis was conducted using content analysis.

Results: REC decision letters and meeting minutes were available for 77 studies. For 27 studies, response letters from researchers were available. Within the REC decision letters, 298 concerns were raised, 162 of which were agreed to by chief investigators. In addition to general REC concerns relating to research methodology, information sheets, and formatting errors, there were concerns specifically relating to CYP with LLC/ LTIs, such as the need for clinical team involvement in recruitment and appropriate ages for consent/assent.

Conclusions: Commonly, studies involving CYP with LLC/LTIs require changes before they are approved by RECs. Many of these changes, including formatting issues can be resolved easily with careful review from the research team and peers before submission. More detailed consideration is needed for the other concerns, such as consent procedures and recruitment strategies, to ensure the needs of CYP with LLC/LTIs are adequately considered and to ensure that these concerns do not present a barrier to getting research with this population underway.

Funding:The authors disclosed receipt of financial support from The National Institute for Health Research (NIHR) Collaboration for Leadership in Applied Health Research \& Care,Great Ormond Street (GOS) Children's Charity, NIHR-GOSH BRC \&The True Colors Trust.

\section{Abstract number: P01-016 \\ Abstract type: Poster}

\section{Gastrointestinal Bleeding - What to Expect in Home-based Palliative Care of Children \\ Gagnon G., Trocan L., Dechert O., Danneberg M., Hillebrecht M., Heythausen A., Janssen $G$. \\ Paediatric Oncology, Haematology and Clinical Immunology, Medical \\ Faculty, University of Duesseldorf, Duesseldorf, Germany}

Home based palliative care of children has the goal of managing various symptoms like pain or vomiting in the home setting. Many studies have assessed different treatment options. Little is known about the symptom of gastrointestinal bleeding in this group of patients. We aim to describe the risk and outcome of gastrointestinal bleeding in pediatric patients with a life threatening condition.

We conducted a retrospective, observational, monocentric study about children currently being cared for by our palliative care team in a homebased setting. The frequency of gastrointestinal bleeding, risk factors as well as treatment with proton pump inhibitors and the outcome of these patients were assessed.

Among the 64 patients currently under care there were 6 patients with cancer, 58 children were diagnosed with a neurological disease or disease with neurological manifestation. Patients age ranged from 12 month to 22 years. None of the patients with cancer suffered from gastrointestinal bleeding, while 23/58 children with neurological disease (40\%) were affected. 21 Patients had upper gastrointestinal bleeding. Other symptoms present were seizures (20/23) and pain (22/23). 19/23 patients received a proton pump inhibitor, 18/23 were taken Ibuprofen in case of pain or fever. 22 children had gastrostomy tubes. Bleedings could be stopped by starting or increasing the doses of a proton pump inhibitor. No child was referred to the hospital.

Children with a life threatening disease with neurological manifestation have a high risk of upper gastrointestinal bleeding. Treatment with proton pump inhibitor might avoid a clinically significant bleeding with the need of further intervention. Ibuprofen as a pain medication should be avoided in this group of patients.

Abstract number: P01-017

Abstract type: Poster
Parental Dealing with DIPG in Children

Radlanski K., Hartwig M., Kordes U.

Pediatric Hematology and Oncology, Universitätsklinikum Hamburg-

Eppendorf, Hamburg, Germany

Background: Diffuse intrinsic pontine glioma (DIPG) in children is a dismal disease with median overall survival (OS) of nine months, even with primary treatment. Usually palliative care is involved. Open communication about death eases dying for children and surviving for parents.

Aims: This study establishes case reports based on parental confrontation with DIPG. During the course of DIPG designated key points exist. We determine specific needs of parents and children as well as typical problems in interaction between families and care givers. The results lead to recommendations and improvements for existing treatment concepts.

Method: We conducted a problem-oriented guided interview followed by a qualitative content analysis according to Mayring.

Results: 13 parents of 7 children (aged 5-16 years) were interviewed. The children's median OS was 8 months and 4 days. Palliative home care was involved in 5 families.

Palliative care generally met the needs of the examined families. Parents stated overall satisfaction and comfort regarding to most subjects (management of physical symptoms and support, provision of information, family support, patient psychological care).

Communication about death is a relevant inductive category. Out of 7 children it was assumingly spoken openly to only one child. Two families remained in mutual pretense. Two children were actively prevented by their parents to have open communication. In two other cases the concept remains unclear. All parents regret that they have not talked to their children about death.

Conclusion: Parents are less burdened when palliative care is involved in the treatment of their children's DIPG. The overall load of statements about not talking with children about their death meets the relevance of this topic in literature. Concepts need to be established on how to rise attention to the chances that open communication about death offers for both children with life-limiting diseases and their families.

Abstract number: P01-018

Abstract type: Poster

What Challenges Exist for Paediatricians When Advance Care Planning and How Might they Be Overcome?

Nohavicka L. ${ }^{1}$, Harrrop E. ${ }^{2}$

${ }^{1}$ Cardiff University School of Medicine, Cardiff, United Kingdom, ${ }^{2}$ Helen and Douglas House, Oxford, United Kingdom Presenting author email address: laurakemp1304@gmail.com

Background: To provide the best care for patients with life limiting diseases, clinicians must feel competent to complete paediatric advance care plans (pACPs). In order to be competent, doctors and other members of the multidisciplinary team must be aware of the challenges that exist for them personally and professionally when advance care planning.

Aims: To identify the challenges that exist for paediatricians of all training grades who are not intensivists or level 4 trained palliative specialists when creating pACPs.

Methods: A mix methods study conducted within the Oxford Deanery. Paediatric trainees and consultants who met the inclusion criteria within the Deanery were emailed a questionnaire to complete about their experiences with pACPs. Those who completed the questionnaire were offered the opportunity to participate in semi-structured interviews for more in-depth qualitative analysis.

Results: 45 people responded to the questionnaire (response rate $17.8 \%$ ). 25 of those agreed to be interviewed (55.6\%). 11 semi-structured interviews were held. The most frequent challenges highlighted in the questionnaire stage was time, both finding the right time for the conversation (14.2\%) and having enough time to conduct the 
conversation (8.3\%), followed by prognostic uncertainty (12.8\%). The interviews highlighted key themes of the challenges experienced: what, who; when and how. The most prominent challenges were concerns about the timing of pACP conversations, and how to conduct them. Conclusion: pACPS remain an emotive topic for paediatricians. Nonpalliative care paediatricians/non-intensivists have limited exposure to pACPs, both within training and at consultant level. Most prominent amongst the challenges identified were timing of the conversations and uncertainty around the prognosis.

Education and training are potential ways to reduce the impact of these challenges.

\section{Abstract number: P01-019 \\ Abstract type: Poster}

Pediatric Palliative Care and Allogeneic Hematopoietic Stem Cell Transplantation: The Experience of a Peaceful Coexistence de Castro A.C.P. ${ }^{1}$, Kioroglo P. ${ }^{1}$, Delponte V. ${ }^{1}$, Rocha V. ${ }^{2}$, Gomes A.A. ${ }^{2}$, Mafra A.B.B. ${ }^{2}$, Achette D. ${ }^{1}$, Borges A.P.d.S. ${ }^{1}$, de Carvalho L.F.G.L. ${ }^{1}$, Novis Y.A. ${ }^{2}$, Forte D.N. ${ }^{1}$

${ }^{1}$ Hospital Sirio Libanes, Equipe de Suporte e Cuidados Paliativos, São Paulo, Brazil, ${ }^{2}$ Hospital Sirio Libanes, Unidade de Transplante de Medula Ossea, São Paulo, Brazil Presenting author email address: acpcastro@me.com

Background: Hematopoietic Stem Cell Transplantation (HSTC) offers a chance to cure hematological neoplasms, but involves a high risk of complications. Patients experience physical suffering, drawn-out hospitalization and high levels of anxiety and depression, with a decline in quality of life (QOL).

Aims: present the experience of the Palliative Care Team's (PCT) integration to a HSTC unit at a tertiary hospital, following patients, family and the healthcare $(\mathrm{HC})$ team.

Methods: All allogeneic HSCT pediatric patients (from 0 to $17 \mathrm{y}$-o) were followed from Feb. 2016 to Sept. 2018. PCT applied multidimensional evaluation on every patient. The patients were followed by interdisciplinary rounds, fitting the therapeutic care to their needs. When requested, Psychiatry, Chaplaincy and others were active in the process. The HC team was also supported by the PCT through everyday presence at the facilities, individual attendances and didactic meetings.

Results: 29 patients - ranging from $3 \mathrm{~m}-0$. to $17 \mathrm{y}$-o. (median 8) were monitored. 21 had Leukemia $(72,4 \%)$. Due to bad outcomes or disruptive behavior from parents, in 11 cases the focus laid on handling conflicts, through periodic meetings with the family.

Outcome: 11 deaths (38\%), 3 before HSTC; 4 did not transplant; and 14 survivors. When death occurred, interventions to support goals of care decisions, end-of-life care and grief-support were put in place.

Conclusion: Due to a large body of evidence, ASCO recommends the integration of palliative care $(\mathrm{PC})$ to oncologic treatment in patients with advanced cancer. In Onco-hematology, the integration is still incipient. This project shows the scope of action and potential contribution that PC can bring to $\mathrm{HSCT}$, highlighting the role in supporting patients, family and $\mathrm{HC}$ team, and conflict mediation. The integration of PC to pediatric HSCT provided focus on $\mathrm{QOL}$ improvement for patient, family and $\mathrm{HC}$ team, combining interventions with sophisticated multidisciplinary coordination.

Abstract number: P01-020

Abstract type: Poster

Timing Is Everything- Initiating Advanced Care Planning Discussions in Children with Severe Neurological Impairment Lagan N. ${ }^{1,2}$, Kelly S. ${ }^{1,2}$, Mc Donald D. ${ }^{1,2}$, Balfe J. ${ }^{1,2}$

${ }^{1}$ Department of Developmental Paediatrics and Neurodisability, Tallaght University Hospital, Dublin, Ireland, 2 Paediatric and Child Health Department, Trinity College Dublin, Dublin, Ireland
Background: Children with Severe Neurological Impairment (SNI) have a limited-life expectancy as a consequence of their medical complexity and physical disability, secondary to their underlying aetiology. Initiating advance care planning (ACP) discussions early with families supports the development of trusting relationships, and an ethos of shared decision making in addressing realistic treatment goals with an emphasis on quality of life.

Aim: To review the timing of ACP discussions with families of children with SNI with a view to service improvement.

Methods: Retrospective chart review children with SNI attending a neurodisability service

a. who have died in the last ten years

b. who are alive but have ACP discussions documented

Results: Nine children with SNI attending the neurodisability service have died in the last 10 years. Full data was available on $8 / 9$ children. $44 \%$ (4/9) had a diagnosis of Cerebral Palsy, 2 had cerebral malformations, 1 child had Batten's disease, 1 a genetic abnormality, 1 early onset epileptic encephalopathy. All children had epilepsy.

The age of death ranged from 1years 5 month to 15 years 8 month. The number of hospital admissions in year prior to death ranged from 2-6. One child had a previous ICU admission. Timing of APC discussion varied from few days to 10 months before death. $50 \%$ were held in the month before death. $71.4 \%(5 / 7)$ children died at home. Support from palliative care was provided to all.

Current practice shows improvement, $16 \%(8 / 50)$ children attending the neurodisability team with SNI have ACP plans in place. All discussions have occurred in an outpatient setting, not at a time of acute deterioration. ACP hard copy is readily available in the hospital notes and shared with relevant teams.

Conclusion: ACP discussions should be considered for all children with SNI. This retrospective review has prompted service evaluation and further quality improvement in this regard is planned.

Abstract number: P01-021

Abstract type: Poster

DNR in a Pediatric Patient: When to Bring it up?

Gabrani A. ${ }^{1}$, Obaid M. ${ }^{1}$, Hodock K. ${ }^{2}$, Boman J. ${ }^{2}$, D'Mello S. ${ }^{2}$

${ }_{1}^{1}$ Pediatrics, Rutgers New Jersey Medical School, Newark, United States, 2Joseph M. Sanzari Children's Hospital, Hackensack, United States

Aim: Pediatricians often find it difficult to discuss end-of-life (EOL) and comfort care with the families of patients suffering from terminal disease, delaying the transition to comfort care. We report a patient who was placed on comfort case late in her disease.

Case report: An 8 year old girl, born prematurely at 25 weeks, with global developmental delay, short gut syndrome, intractable seizures, G-tube and tracheostomy dependence and hyperbilirubinemia (possible IgG4 Cholangitis), presented with fever, tachycardia and increased tracheostomy secretions for 5 days. She had multiple admissions for infections, jaundice, and decreased feeding tolerance in the past months leading up to this admission. On presentation, her vital signs were: Pulse 147 bpm, Temp 102.6F, BP 100/64 mmHg, respiratory rate 35/minute, oxygen saturation $96 \%$ on room air. Physical exam revealed icteric, nonverbal patient with bilateral upper and lower limb contractures, unilateral micropthalmia, tracheostomy with white secretions, clear lungs, normal S1S2, firm hepatomegaly, hyper-reflexia, and grade 2 sacral ulcers. She was admitted for severe hypernatremic dehydration, Serratia urinary tract infection and end stage hepatic failure. On Hospital day (HD) 10, discussion about EOL and comfort care was initiated with the family due to worsening liver failure and seizures. The family required multiple discussions regarding plans of care and comfort care. On HD 30, medical team and family came to a consensus to provide comfort care,to alleviate discomfort from pain, agitation (with narcotic infusion) and 
air hunger. On HD 50, patient was pronounced dead; cause of death was hepatic failure of unknown etiology.

Conclusion: This patient was an appropriate candidate for palliative care early in disease course, but a late EOL discussion delayed comfort care. This highlights the importance of early initiation such discussion by clinicians, for the best interest of the patient and for familial comfort.

Abstract number: P01-022

Abstract type: Poster

Making the Unvisible More Visible? A Retrospective Study Identifying Children and Adolescents with Complex Chronic Conditions Hospitalized in Brussels from 2010 to 2014 and their Access to Specialized Palliative Care Teams

Friedel M. ${ }^{1,2}$, Gilson A. ${ }^{3}$, Bouckenaere D. ${ }^{4}$, Brichard B. ${ }^{3,5}$, Fonteyne C. ${ }^{6,7}$, Wojcik T. ${ }^{4}$, Mahboub A. ${ }^{1}$, Lahaye M..$^{3,8}$, Aujoulat I..$^{1,9}$

${ }^{1}$ Institut de Recherche Santé et Société (IRSS), Université catholique de Louvain (UCL), Bruxelles, Belgium, ${ }^{2}$ Institut Parnasse-ISEI, Haute Ecole Vinci, Bruxelles, Belgium, ${ }^{3}$ Université catholique de Louvain (UCL), Bruxelles, Belgium, ${ }^{4}$ Fédération bruxelloise Pluraliste de Soins Palliatifs et Continus asbl, Bruxelles, Belgium, ${ }^{5}$ Département d'Oncologie et d'Hématologie Pédiatrique, Cliniques Universitaire Saint-Luc, Bruxelles, Belgium, ${ }^{6}$ Hôpital Universitaire des Enfants Reine Fabiola, Bruxelles, Belgium, ${ }^{7}$ Université libre de Bruxelles, Bruxelles, Belgium, ${ }^{8}$ Pédiatrie, Cliniques Universitaire Saint-Luc, Bruxelles, Belgium, ${ }^{9}$ Faculté de Santé Publique, Université catholique de Louvain (UCL), Bruxelles, Belgium

Background: Complex chronic conditions (CCC) are considered life-limiting conditions requiring palliative care, which is provided in Belgium through paediatric liaison teams (PLTs). Two such teams are operating in the Brussels Region, where 8 hospitals have a paediatric department that admit CA with CCC. The number and characteristics of CA facing such conditions in Brussels are not known.

Aims: To compare over a 5-year period (2010-2014) the number and characteristics of CA with CCC who were admitted in one of the 8 hospitals in Brussels with those who benefitted from paediatric liaison care (PLC).

Methods: All International Classification of Disease-codes corresponding to the list of CCC defined by Feudtner et al. (2001) were extracted from the databases of the 8 hospitals with a paediatric department and the 2 PLTs located in Brussels. Descriptive statistics were used to calculate age and diagnosis categories, frequencies and lengths of hospitalization.

Results: A total of 22,533 CA were identified through hospital databases (group 1), 572 through the databases of the PLTs (group 2) and 384 CA were identified both in the hospital and the PLT databases (group 3). There was no significant difference regarding the distribution of age categories between group 1 and group 2. Regarding the type of diagnosis, cardiologic conditions were most represented in group 1 and neurologic conditions most found in group 2. Frequencies and lengths of hospital stays were superior for CA who benefitted from liaison care than for those who did not, especially for CA affected by cancer, suggesting that CA who are also cared for by the PLTs are those with more severe conditions.

Discussion / Conclusion: Only a small proportion $(1,7 \%, 384 / 22,533)$ of all CA with CCC that were admitted to a hospital benefitted from PLTs in 2010-2014. Our results call for a greater awareness and a more systematic provision of palliative care for children and adolescents with CCC.

Abstract number: P01-023

Abstract type: Poster

Allowing Hospital Death to Be a Choice, Not a Necessity Barbour L.

University of the West of England, Bristol, United Kingdom
Aims: I aimed to explore the various reasons that families might choose the inpatient setting as the place for end-of-life care.

Methods: A paper search was carried out using the EMBASE database, identifying all papers that contained the key words (end-of-life, child, hospital) and derivations of these. The abstracts of papers that sounded relevant were reviewed, and a total of 31 papers were identified for full review. The NICE guidelines for end-of-life care in children were also reviewed.

Results: The review of the papers revealed a number of areas that need to be considered when discussing end-of-life care with parents. Place of death and place of end-of-life care are two important areas to parents, although their importance has perhaps been overemphasised in the past, as most retrospective studies report that parents felt it was the care received rather than the place itself that was most important. When making decisions, parents' decisions about place of death may be influenced by a number of factors, including cultural and societal expectations, previous experience, support networks. Highlighted as key factors to making the experience of death in hospital a better one are the importance of multi-disciplinary team care, having a good symptom care plan and a good relationship with staff.

Conclusion: Whilst traditionally we have considered that home is the preferred place of death, this is not always the case. For some families, the preferred place may been in hospital, and there are a wide range of factors that contribute to this. Collaborating with families to achieve what is best for them is a key component of palliative care, and allowing hospital death to be a choice rather than a necessity, or to be avoided, should be part of this.

Abstract number: P01-024

Abstract type: Poster

'Cold Bedrooms, Cooling Blankets, and Cuddle Cots' Provided by Children's Hospices: Mapping Practice and Exploring Bereaved Parents' Experiences

Hackett J. ${ }^{1}$, Fraser L. ${ }^{2}$, Beresford B. ${ }^{1}$

${ }^{1}$ Social Policy Research Unit, University of York, York, United Kingdom, ${ }^{2}$ Institute of Health Sciences, University of York, York, United Kingdom

Background: The ways parents are supported at the end of their child's life and after death can profoundly affect the grieving process. Within children's hospices cold bedrooms, cooling blankets and cuddle cots are provided to enable families to be close and have unrestricted access to their child after death. Such facilities are thought to support parents in the initial stages of grief. However there is wide variation in practice and almost no evidence to inform parents' decision-making during advance care planning.

Aim: We aim to map practice and explore parents' experiences of using these facilities - in the hospice or at home - and the perceived impact of this on immediate and long-term bereavement.

Methods: This study comprises two stages. First a cross-sectional survey of all UK children's hospices to gather data on previous and current practice, followed by structured telephone interviews with heads of care to identify reasons for inclusion of these facilities within the portfolio of care and support $(n=55)$. Second qualitative interviews with parents $(n=30)$ exploring stories of the early days of bereavement and the perceived role of these facilities on immediate and long-term bereavement. Survey data will be analysed using descriptive statistics, interviews using narrative and thematic approaches.

Results: Here we report preliminary survey results. Current practice regarding both the provision and usage of these cooling facilities is highly variable across children's hospices. For many these facilities were not available for usage in the home. Hospices varied in the number of days which families can use these facilities, ranging from 2 days to entirely flexible, responding to family's wishes. A number of factors affected whether families used these facilities, including ethnicity and religious beliefs. 
Conclusion: Findings have implications for practice within children's hospices and also for statutory services who are beginning to offer this provision.

Abstract number: P01-025

Abstract type: Poster

How and when to Introduce Specialized Palliative Care for Children and Youths with Life-limiting Diseases - Development of Screening Tools for Pediatricians and Palliative Care Teams van Buiren M. ${ }^{1}$, Deckers M. ${ }^{1}$, Felten P. ${ }^{2}$, Hahn L. ${ }^{3}$, Kimmig A. ${ }^{4}$, Meyburg J. ${ }^{5}$, Steinbach D. ${ }^{6}$, Barth M. ${ }^{7}$

${ }^{1}$ Department of Pediatric Hematology and Oncology, Center for Pediatrics, University of Freiburg, Freiburg, Germany, ${ }^{2}$ Center for Pediatrics, University of Mannheim, Mannheim, Germany, ${ }^{3}$ Department of Palliative Care, Medical Center University of Freiburg, Freiburg, Germany, ${ }^{4}$ Center for Pediatrics, University of Tuebingen, Tuebingen, Germany, ${ }^{5}$ Center for Pediatrics, University of Heidelberg, Heidelberg, Germany, ${ }^{6}$ Center for Pediatrics, University of Ulm, Ulm, Germany, ${ }^{7}$ Center for Pediatrics, University of Freiburg, Freiburg, Germany

Background: Identification of children/families profiting from the introduction of specialized palliative care (SPPC) is challenging for primary care doctors as well as pediatric specialists. So far no screening-tool to facilitate this decision has been established.

Aim: Within a multi-center study we aimed to develop a) a screening tool for pediatricians to decide whether to contact a palliative care team (PCT) in a given case and b) a semi-structured interview for PCTs to facilitate shared decision making with patients/families.

Methods:

SIPP - Screening for Indication of specialized Pediatric Palliative care: Experts for pediatric palliative care and different pediatric subspecialties developed the SIPP. To test performance 50 case-vignettes (real cases, different pediatric subspecialties) were designed. These were rated with the SIPP through a) their authors, b) experts of pediatric palliative care not familiar with the case and c) experts of the corresponding subspecialty. SIAPP - Semi-structured Interview for Allocation of specialized Pediatric Palliative Care:

Experts of pediatric palliative care developed a $20-30$ min. semi-structured interview. For more than 80 referred patients in five centers, the SIAPP was used for the first contact between PCT and patient/family. To test inter-rater reliability two SIAP-based interviews were recorded, transcribed, altered as to distinct items and rated by different palliative care experts.

Results: As to the SIPP three items were identified with satisfactory performance criteria: life-expectancy $<12$ months, markedly increased need of support and preference as to intensity of treatment. It proved to be easily used and accepted within the daily routine.

SIAPP provided helpful guidance through the shared decision making process.

Conclusion: SIPP and SIAPP are efficient and transparent tools facilitating the introduction of SPPC.

\section{Abstract number: P01-026 \\ Abstract type: Poster}

The "Children's Life Walk" - An Extraordinary Campaign to Capture Attention for CPC

Kraft S., Bieber A., Werner P.

Bundesverband Kinderhospiz, Lenzkirch, Germany

In summer 2018, the "Bundesverband Kinderhospiz", Germany's national organization for Children's Palliative Care, made their ambitious visions come true: they set off for the "Children's Life Walk", a pilgrim's journey of more than 7000 kilometers, all around Germany, in 132 days. With the objective to raise awareness for children with life limiting illnesses and their families we travelled from one child hospice to the next one.

Far more than 40.000 families in Germany will know the unbearable pain of losing a child - worldwide there are even more than 21 million. Sabine Kraft, Chair of ICPCN and CEO of the Bundesverband Kinderhospiz, is spreading the idea of Children's Life Walks all over the world. Visiting the Congress of ICPCN in Durban in May 2018, she presented our "angeltorches", symbol of the Children's Life Walk, as a gift for every member. While the Bundesverband Kinderhospiz is proud to be planning the second Children's Life Walk for Germany, numerous countries are implementing the idea themselves.

The impact of the very first Children's Life Walk was incredible. It succeeded in gaining hundreds of supporters and volunteers for CPC all over our country who joined us on foot, by bike, ship, plane, carriage, in wheelchairs or even in a hospital bed. Above all, the campaign created an outstanding wave of solidarity with affected families and provided an enormous amount of public attention for CPC. Newspapers, radio stations, internet platforms and social media channels accompanied our campaign over months and helped to make people perceive the situation of affected families and the urgent need for a reliable and highly qualified network of help which was made visible by the Children's Life Walk. Furthermore, the campaign opened up sustainable possibilities of raising funds for CPC - for the Bundesverband Kinderhospiz as well as for our member children hospices, who reported back remarkable benefits by joining the campaign.

Abstract number: P01-027

Abstract type: Poster

\section{Communication in Paediatric Palliative Oncology}

Momber S., Phillips $M$.

Perth Children's Hospital, Perth, Australia

Background and aims: International and national recommendations identify best care of paediatric oncology patients to require exemplary communication within and across the medical and psychosocial multidisciplinary teams (MDTs). Identification and continued review of patient and family goals of care (GoPC) ensures family centred care. To achieve ongoing family focussed best care, changes, aligned with reviews of the child's illness status, must be appropriately communicated to all involved in their care.

Methods: To identify and improve documentation and communication for patients with malignant disease at our institution, audit of all deceased paediatric and adolescent oncology patients for 5 years from January 2012 was performed. Audit identified documentation of medical decision-making including time; persons and place, for the cohort including use of the national Death Review Form.

Results: 96 deceased patient notes were reviewed. Clear documentation of medical decision-making following discussions with parents/carers was found for 75 patients (78\%). However, documentation content was variable; difficult to retrieve from multiple volume notes and filed inconsistently. Gaps in departmental documentation mirrored those across the institution and confirmed the need for a GoPC document to better ensure appropriate communication of complex decision-making for all patients. Development of an acceptable document, and policy, for hospital-wide use proved challenging with wider implementation delayed due to various obstacles despite extensive education. After release of the GoPC form, ongoing audit within the oncology department identifies almost $100 \%$ uptake. Continued education and surveys confirm acceptability aligned with significant improvement in documentation and $100 \%$ retrievability.

Conclusions: A GoPC form ensures best patient care with use encouraged to prompt and document appropriate medical decision-making discussions for paediatric and adolescent patients. 
Abstract number: P01-028

Abstract type: Poster

Good Sleep, Bad Sleep: Increasing the Clinical Applicability of a Sleep Questionnaire for Pediatric Palliative Care - Final results Dreier L.A., Zernikow B., Wager J.

Department of Children's Pain Therapy and Paediatric Palliative Care, Faculty of Health, School of Medicine, Witten/Herdecke University, Witten, Germany

Background: The "Sleep questionnaire for children with severe psychomotor impairment" (SNAKE) identifies sleep problems in children with life-limiting conditions by utilizing parents' rating. It is based on the five scales "disturbances going to sleep"

(1) "disturbances remaining asleep"

(2) "arousal and breathing disorders"

(3) "daytime sleepiness"

(4) and "daytime behavior disorders"

(5) Despite good reliability and validity, the clinical applicability of the SNAKE is limited.

Aims: The aim of the current study is to define cut-off values for the five SNAKE scales, thereby enabling identification of clinically relevant sleep problems. In addition, the change sensitivity will be examined. Lastly, it will be evaluated what constitutes a clinically relevant change defined from the parents' point of view.

Methods: Parents complete the SNAKE at the time of admission to the pediatric palliative care unit. Additionally, a team of experts also assesses the presence of a sleep disorder (gold standard) based on patient observations. Three weeks after discharge, a follow-up survey with the SNAKE and another questionnaire is carried out.

Results: In initial analyses with $n=61$ children, preliminary cut-off values could be defined for the scales "disturbances remaining asleep" and "daytime sleepiness". When the final data $(\mathrm{N}=100)$ are available at the end of December 2018, it is expected that final cut-off values for all scales can be determined through ROC analyses. Furthermore, statements can be made as to whether the SNAKE can detect changes over time and which changes in the sleep behaviour of a child are significant for parents.

Conclusions: It is expected that the final study results will help to optimize the assessment of clinically relevant sleep problems in paediatric palliative care. Results and methodological challenges in the implementation and evaluation of this project will be discussed.

The project is funded through Witten/Herdecke University.

Abstract number: P01-029

Abstract type: Poster

Polypharmacy in Children and Young People with Life-limiting Conditions: A National Observational Cohort Study in England Taylor J. ${ }^{1}$, Jarvis S. ${ }^{1}$, Gibson-Smith D. ${ }^{1}$, Doran T. ${ }^{1}$, Hewitt C. ${ }^{2}$, Hills M. ${ }^{3}$, Fraser L. ${ }^{1}$, Martin House Research Centre

${ }^{1}$ Department of Health Sciences, University of York, York, United Kingdom, ${ }^{2}$ York Trials Unit, University of York, York, United Kingdom, ${ }^{3}$ Martin House Hospice, Wetherby, United Kingdom

Background: Children and young people (CYP) with life-limiting conditions (LLCS) are likely to require multiple different medications during the trajectory of their illness. Polypharmacy, which refers to taking several medications concurrently, is sometimes appropriate but may also occur when several clinicians are involved in a child's care and when medications continue when they are no longer beneficial. Polypharmacy also increases the risk of adverse drug-drug and drug-disease interactions, medication errors and non-adherence, and causes unnecessary burden for families as they manage complex medication schedules.
Addressing what we call problematic polypharmacy is therefore a priority; however, we first need to determine the prevalence and patterns of polypharmacy in this population.

Aims: To describe prevalence and patterns of polypharmacy in CYP with LLCs in England.

Methods: A national observational cohort study in England including all CYP (age 0-25 years) with a diagnosed LLC who are registered with a general practice of up-to-standard data quality contributing data to the Clinical Practice Research Datalink (CPRD) from 01/01/2000 to $31 / 12 / 2015$. Polypharmacy, which for this study will refer to patients who receive four or more different repeat prescriptions, was ascertained using the prescription data in the CPRD per individual per year.

Results: Over the study period the percentage of CYP with LLCs who received polypharmacy ranged from $21-29 \%$. There was little difference by age group with between $22-26 \%$ in each age category having polypharmacy. There was marked difference by diagnostic subgroup with $57 \%$ of those with an underlying respiratory diagnosis experiencing polypharmacy but only $33 \%$ of those with a neurological diagnosis.

Conclusion: This ongoing study shows that polypharmacy is prevalent in CYP with LLCs. Further work to distinguish between problematic and appropriate polypharmacy and how best to manage polypharmacy in this population is required.

\section{Abstract number: P01-030 \\ Abstract type: Poster}

Nationwide Structure for Integrated Children's Palliative Care Schuiling-Otten M. ${ }^{1}$, Huizinga C. ${ }^{1}$, Vallianatos S. ${ }^{1}$, Verhagen E. ${ }^{2}$

${ }^{1}$ Centre of Expertise Children's Palliative Care, Utrecht, Netherlands,

${ }^{2}$ Dutch Association for Pediatrics, Utrecht, Netherlands

Aims: To create a nationwide structure for integrated children's palliative care $(\mathrm{CPC})$ with substantive quality, coordination, and continuity.

Approach taken:

- 2012-2019: academic children's hospitals develop CPC teams which focus on organising integrated care from hospital to home.

- 2013: Guideline 'Palliative care for children'.

- 2014-2018: Development of 7 regional networks integrated CPC with professionals in $1^{\text {st }} / 2^{\text {nd }} / 3^{\text {rd }}$-line care. Focus on coordination and continuity of care close to home and increasing expertise.

- 2017: Individualised children's palliative care plan. This plan, in which advanced care planning is central, is used nationally.

- 2018: A national centre of expertise that provides a basis to preserve the CPC-structure with expertise, information, training and development is realised.

- 2018: Central point to support physicians in medical-ethical and legal dilemmas surrounding the end of life of children.

Results: A nationwide structure for integrated CPC, CPC-teams and -networks coordinate the care in close collaboration with parents and CPC is recognized as a specialized field of care by the government.

Established values since this structure:

1. A comprehensive and timely approach of the palliative process from hospital to home with a focus not only on the medical aspects but also psycho-social, pedagogical, spiritual aspects.

2. Not merely focusing on the ill child, but also on parents and siblings.

3. Focus on health instead of illness, awareness that a child can still be a child.

Conclusions: A model of integrated care, organised closed to home in collaboration with families is implemented nationwide. Both CPC-teams 
as regional networks seem to have an important added value for children, their families and professionals. Furthermore, a process is activated that CPC is so much more than terminal care. The difficult dialogue about ethical dilemmas is still challenging, but open. Five years ago, this was inconceivable.

\section{Abstract number: P01-031}

Abstract type: Poster

\section{Sustainable Innovations in Children's Palliative Care, Based on the Real Patient Story! \\ Huizinga C. ${ }^{1}$, Gosliga F. ${ }^{2}$, den Outer B. ${ }^{3}$, Schuiling-Otten M. ${ }^{1}$}

${ }^{1}$ Centre of Expertise Children's Palliative Care, Utrecht, Netherlands,

${ }^{2}$ Efexis, Amsterdam, Netherlands, ${ }^{3} \mathrm{jb}$ Lorenz, Rotterdam, Netherlands

Aims: In order to improve Children's Palliative Care (CPC), we must understand the needs and wishes of families better. Therefore aims are to gain insight in best practices and bottlenecks in CPC from the perspective of parents, and to evaluate developments from their point of view. Moreover, we aim to translate their perspectives into new initiatives, and gain awareness amongst policymakers on the needs of families regarding CPC. Furthermore, by sharing stories from parents to parents, a form of peer support is created.

Methods: A pilot study among 20 parents was conducted to test the tool's validity. We used the online, narrative collection tool SenseMaker ${ }^{\circledR}$, in which experiences of parents caring for a child in need of CPC were captured. Respondents were asked what is significant about their story; by interpreting his or her narrative, the parent acts as expert. The output was qualitative information, indexed by the sources so that quantitative data emerged without any external interpretation.

Results: During a pilot phase of two months, 17 narratives were collected. The stories contained valuable information about both best practices and bottlenecks. Three main topics emerged: organisation of care, communication and emotional impact. These topics and the feedback from parents were incorporated in a final version. At the time of submission, the final tool is open for use. Parents can share their story online at any time they wish. We expect to report results at this congress.

Conclusion: Based on the pilot results, we were able to design the final version of the tool. In order to be able to translate families' perspectives into policy and to involve them in evaluating current developments, narratives are collected on a continuous basis. To create peer support, an online platform on which the narratives are shared is being developed.

\section{Abstract number: P01-032 \\ Abstract type: Poster}

\section{The Spiritual Dimension in Pediatric Palliative Care: How to Approach it \\ Gavazzoni G. \\ Hospital Universitario de Gran Canaria, Palliative Medicine, Las Palmas de Gran Canaria, Spain Presenting author email address: \\ giulia.gavazzoni@gmail.com}

Background: Approaching spirituality in children with life/threatening conditions considerably influences symptoms control and the way in which the child and his family face the disease.

The task of facing spirituality in children is more complicated than in adults because it is rather difficult for children to express their deepest feelings, and rarely a teenager will invite us to talk about such personal emotions.

Aims: To define a common guideline that instructs the multidisciplinary team how to approach this deep dimension by using a tool or a framework of action to guide and support our children to explore their spirituality.
Methods: Systematic qualitative review. Research strategy: Pubmed, Ovid as electronic databases and analysis of grey literature.

Results: The study of different sources by different combinations of key words revealed 28 articles responding to our inclusion criteria.

As we exposed in SECPAL International Congress 2018 (June 18, Vitoria, Spain) any specific and validated tool was found for the assessment of spirituality in children included in PC programs, but the analysis of these results allow us to draw some general outlines to face this complicated sphere. Discussion: We highlight 6 items to consider when preparing to explore the spirituality of children:

- recognize and analyze the maturational level of the child in order to choose the most appropriate communicative channel.

- all members of the multidisciplinary team can and should participate in the spiritual care of the child

- recognize the spiritual needs of the child

- use a validated tool to guide the interview

- consider behavior patterns as a form of communication of the child

- respect and facilitate the expression of religious beliefs.

Conclusion: This review highlights the necessity to continue training and to rise awareness in order to help patients coping with the disease with active acceptance, taking into consideration that it's not so important what tool we use, bout how we use it.

Abstract number: P01-033

Abstract type: Poster

Bereaved Parents' Use of Fundraising and Media Coverage to Influence the Provision of 'Cuddle Cots' in Paediatric Palliative Care in the UK

Kerr-Elliott T. ${ }^{1}$, Oulton K. ${ }^{2}$, Gibson F. ${ }^{2}$

${ }^{1}$ Louis Dundas Palliative Care Team / Centre for Outcomes and Experience Research in Children's Health, Illness and Disability, Great Ormond Street Hospital, London, United Kingdom, ${ }^{2}$ Orchid, Great Ormond Street Hospital NHS Foundation Trust, London, United Kingdom

In the United Kingdom, there are considerable variations in post-death practices regarding where a deceased child is cared for between the time of death and funeral. Despite evidence demonstrating the importance of continuing to "be with" or "care for" their child after death, the opportunities for families to do so are often limited. Portable "cuddle cots", which allow families to spend time with their deceased child at home, or in hospitals or children's hospices are available in some areas but not in all.

This study investigates how media coverage has represented family's stories and subsequently is influencing practice.

Method: Collaboration with an online monitoring company enabled quantative and qualitative analysis of data from online search results using a variety of search terms. This was corroborated with live monitoring using the search term (cuddle cot/s"). Qualitative content analysis was conducted on articles and retrieved from mainstream and social media referring to parents and the care of their deceased child. Results: Trends emerging from the analysis show a dearth of evidence regarding the use of "cuddle cots" at a service planning or policy level, yet many examples of bereaved parents describe their use in positive terms. Thematic analysis of the content of media stories identified a variety of themes including: having time, parents identified the importance of having time to say goodbye; what helped, seeing the physical deterioration of their child's body was described in helpful terms; decision making, about their child's post-death care; choice, wanting to fundraise for more "cuddle cots" for other families.

Conclusions: There is evidence that some bereaved parents are sharing their experiences of post-death care via the media, subsequently 
targeting their fundraising efforts towards the provision of more "cuddle cots". This is already influencing service provision in some areas which has implications for resources and the nursing workforce.

\section{Abstract number: P01-034 \\ Abstract type: Poster}

Midazolam Use in the Last Two Weeks of Life in a Paediatric Population

Anderson A.-K.1,2, Caroline Menzes Research Team

${ }^{1}$ Royal Marsden Hosptial, Sutton, United Kingdom, ${ }^{2}$ Shooting Star Chase Children's Hospice, Guildford, United Kingdom

Midazolam is a widely used in paediatric palliative care, often as first line for the management of seizures and agitation; at different doses. In the 2017 Association of Paediatric Palliative Medicine (APPM) formulary, Midazolam dosing schedule changed due to concerns that the historical dosing schedule was extrapolated from PICU settings- not from any new published evidence. There was much debate regarding the dose changes with small retrospective case series showing more than $50 \%$ of fitting children at end of life needed a higher midazolam infusion range than the new formulary dosing schedule. It could be argued at a second anticonvulsant may have provided benefit. Midazolam is one of the mainstays of symptom management at end of life and the controversy around dosing does not support and guide prescribers adequately in both prescribing safely and ensuring adequate clinical benefit to children in their dying phase. We therefore undertook a retrospective case review of all children with cancer, under 18years of age at the time of their death, who died under the care of a specialist paediatric palliative care team between the period $1^{\text {st }}$ January 2012 and $31^{\text {st }}$ December 2017 . The aims of the study were to determine the start doses and range, indications, clinical response, adverse events (including time to death after administration) and side effects of Midazolam (all routes) administered in the last two weeks of life. 177 patient records were identified; 15 case notes were excluded (4 to adult and 11 died out of area). 122/162 (75.3\%) received midazolam at some point in the last 14 days of their life. Of the 40 children who did not receive midazolam in the last two weeks, 4 received another benzodiazepine. The remaining data has been collected and is due to be analysed. It is anticipated that this review provides some insights in the interim but furthermore guides the development of a prospective study on the use of midazolam in the children at end of life.

Abstract number: P01-035

Abstract type: Poster

Networking for Children's Palliative Care: Shaping the Past and the Future for Global Palliative Care

Downing J. ${ }^{1,2}$, Murphy M. ${ }^{3}$, Nkosi B. ${ }^{1}$, Boucher S. ${ }^{1}$, Sithole L. ${ }^{1}$, Steel B. ${ }^{1}$, Daniels A. ${ }^{1}$

${ }^{1}$ International Children's Palliative Care Network (ICPCN), Durban, South Africa, ${ }^{2}$ Makerere University, Kampala, Uganda, ${ }^{3}$ University of Bristol, Bristol, United Kingdom

Background: Collaboration \& networking are key to the implementation of the WHA Resolution on palliative care. A global network was set up in 2005 to raise awareness of children's palliative care (CPC), lobbying for global CPC development, \& sharing expertise, skills \& knowledge.

Aims: To review the impact of the network prior to the development of a new strategy for shaping global CPC.

Methods: Mixed methods review:

1) of the 2015-2018 strategic plan;

2) of activities:

3) consultation - 37 stakeholder \& 102 member surveys, \& 11 interviews.
Results: The network has: $>2,000$ individual members $\&>350$ organisations; developed a strong global reputation; a unique role, with a high level of expertise; committed staff; a clear vision; \& made significant progress towards its previous strategy, representing the voice of children \& their families. Achievements include: Communication:e.g.: monthly newsletter, $>11,000$ Facebook followers, 2,475 Twitter \& 265 Instagram; the International Children's edition of ehospice has $>700$ users monthly, \#hatson $4 \mathrm{cpc}$ events in $>25$ countries, reaching $>1.4$ million Twitter impressions. Advocacy:representing the global voice of CPC including global influencing with the WHO \& Multilateral Agencies. Research:developing, implementing \& supporting global CPC research, with $>250$ conference presentations \& 65 papers published. Education:7 elearning courses in 11 languages with 3,200 individuals accessing from 124 countries; Face-to-face training to $>1,000$ participants from 22 countries. Strategic development:The network has contributed to the development of CPC globally, although measuring its impact is challenging.

Conclusion: The review demonstrated the impact of the network with networking being pivotal to the ongoing development of CPC. The network is the only global organisation working towards the global provision of CPC \& is in a great position to drive ongoing changes and shaping the future of global CPC.

\section{Abstract number: P01-036 \\ Abstract type: Poster}

Palliative Sedation in a Regional Paeditric Unit: Frequency of Practice and Characteristics of Patients and Sedation de Noriega Echevarría I., Rigal Andrés M., Navarro Mingorance Á., Puertas Martín V., Pérez Martín M.Á., Martín Jiménez L., Martino Alba R.

Paediatric Palliative Care Unit, Hospital Infantil Universitario Niño Jesús, Madrid, Spain

Background: Palliative sedation (PS) is used to avoid suffering due to refractory symptoms. However, data regarding it's rate of application, characteristics and influencing factors in the paediatric population is still scarce.

Aim: To describe the practice of PS in a Regional Paediatric Palliative Care Unit (PPCU).

Methods: Descriptive retrospective study of the deceased patients attended by a PPCU between Jan 2013-Dec 2017. Epidemiological variables included gender, age at death, type of disease, follow-up time and place of death. In the patients receiving PS specific characteristics were collected including: symptom prompting PS, drugs used, level of sedation, type (continuous vs intermittent) or monitoring (symptomatic vs sedation).

Results: 202 patients were included; 39 were excluded for missing data. Amongst the rest, 20 received PS, 17 performed by the PPCU. 10 patients had oncological diseases and 7 neurological conditions. In 8 patients PS was applied at home and in 9 at the hospital. In 16 patients it was applied in end of life situation (sedation in agony); in 15 it was continuous and in 2 intermittent. The commonest symptoms for the indication of PS were pain (11/17) and dyspnoea (10/17). Midazolam was the commonest drug, used alone $(10 / 14)$ or in combination (4/14). Symptomatic monitoring was performed in all patients with sedation monitoring performed in 4 . No differences were found between the patients that received PS and the ones that did not regarding gender, age, follow-up time or being affected by cancer against other diseases $(p>0,05)$.

Conclusions: PS was practised in $12,3 \%$ of the patients. Pain and dyspnoea were the commonest symptoms prompting it's indication. Midazolam was the drug used in the majority of cases, alone or in combination. In this study, there were no differentiating factors between patients that received PS and patients that did not. 
Abstract number: P01-037

Abstract type: Poster

The Impact of Psycho Social Support to Vulnerable Children and Adolescents Living with Cancer in Increasing Access to Treatment. (New Hope Children's Hostel, a Kawempe Home Care Experience - Uganda)

Gerever N. ${ }^{1,2}$, Guma S. ${ }^{3,4}$

${ }^{1}$ Program Manager, Kawempe Home Care, Kampala, Uganda, ${ }^{2}$ Board Member, Palliative Care Association of Uganda, Kampala, Uganda, ${ }^{3}$ Executive Director, Kawempe Home Care, Kampala, Uganda, ${ }^{4}$ Vice President, Uganda Cancer Society, Kampala, Uganda

Background: Kawempe Home Care $(\mathrm{KHC})$ is a not for profit organization providing holistic care to persons living with HIV/AIDS, TB and Cancer in Kawempe division Kampala City. According to the Uganda Cancer Institute 7,000 children are estimated to develop cancer annually. However Children face many psycho social problems as they seek treatment from the only cancer treatment centre; Limited bed capacity, Lack of food, transport, treatments fees and accommodation make carers lose hope, go back home and stop treatment of their children.

Goal: Increase access to specialized cancer treatment and care for 300 vulnerable children \& adolescents through provision of hostel services in 3 years.

Design and methodology: KHC established a referral \& linkage system with Uganda Cancer institute targeting children below 18 years from less able families. Provided free accommodation, nursing care, Patient Navigator, Psychotherapy, Play therapy, legal aid support, meals, transport and social workers closely attended to children with special needs. Results: Children's Quality of life improved due to availability of quick holistic palliative care services.

Free transport increased service demand and timely treatment access for 302 patients in two years.

Carers being sure that they had where to sleep, sure of at least 3 meals increased assurance to have more several treatment days for their children and possible cure.

Lessons learned. Children with cancer have unique psycho social and emotional needs that need special attention.

Children on chemotherapy tend to eat specific types of food than those who are not on medication.

Majority of Children felt safe and comfortable at the hostel rather than at home during the course of treatment.

Conclusion: Strong Psycho social support system using children's hostel model is a catalyst in increasing access to Care and improves survival rates for children \& adolescents living with cancers in limited resource settings.

\section{Abstract number: P01-038 \\ Abstract type: Poster}

Reaching out to the Disadvantaged: Shaping the Future of Palliative Care in Lesotho and Mozambique

Nkosi B. ${ }^{1}$, Boucher S. ${ }^{2}$, Daniels A. ${ }^{3}$, Steel B. ${ }^{4}$

${ }^{1} \mathrm{ICPCN}$, Advocacy, Johannesburg, South Africa, ${ }^{2} \mathrm{ICPCN}$, Communications, Durban, South Africa, ${ }^{3} \mathrm{CPCN}$, Education, Durban, South Africa, ${ }^{4} \mathrm{ICPCN}$, Administration, Chapter Court, Amery Hill, Alton, Hampshire GUJF, United Kingdom Presenting author email address: busi.nkosi@icpcn.org

Background: Lesotho \& Mozambique are developing countries with high incidents of HIV, Lesotho having the second highest prevalence in the world. In 2017 Lesotho had 16000 children living with HIV whilst Mozambique had 13000 in 2016. Though the actual number of children with other serious illnesses is not known, hospitals see a significant number of these children. For example Maputo Central Hospital in Mozambique registers 9 to 10 children with cancer every month. Most children with serious illnesses die prematurely as they present with advanced disease or cannot afford the cost of treatment.

Aim: To integrate children's palliative care (CPC) into the health care setting to make it universally accessible thus relieving children's suffering in these countries.

Design: Programmes were designed to train health care workers in CPC and integrate training into the government setting.

Results: In Lesotho, 27 health workers were trained in CPC in 2017, followed by training of 10 trainers to cascade training throughout the country. To support integration of CPC, National Guidelines \& a training curriculum were developed along with registers to capture data for M\&E. In March 2018, 19 doctors \& nurses from across the country, were trained in CPC, developing implementation plans supported by technical assistance \& mentoring from trainers

Lessons learned: Education on CPC is a core component of the WHA resolution on PC \& must include ongoing mentorship \& supervision. The philosophy of CPC can be applied in different settings \& the same broad approaches to CPC can be utilised resulting in holistic care of the child \& family. However, these programmes need to be culturally sensitive to be accepted by the communities they intend to serve. Similar programmes have been developed for other countries within sub-Saharan Africa thus shaping the future of CPC within the region.

Funding: This programme was funded by the Open Society Institute of Southern Africa (OSISA)

\section{Abstract number: P01-039}

Abstract type: Poster

Adapting the Dignity Therapy Schedule of Questions for Adolescents, Ages 10 to 18 Years Old

Julião M. ${ }^{1}$, Antunes B. ${ }^{2,3,4}$, Santos A. ${ }^{5}$, Faria de Sousa P. ${ }^{1}$, Sobral M.A. ${ }^{1}$, Runa D. ${ }^{1}$, Chaves P. ${ }^{1}$, Gonçalves $C .{ }^{1}$, Fareleira F. ${ }^{2}$, Silva Soares $D .{ }^{6}$, Chochinov H. ${ }^{7}$

${ }^{1}$ Equipa Comunitária de Suporte em Cuidados Paliativos de Sintra, Lisboa, Portugal, ${ }^{2}$ Centro de Investigação em Tecnologias e Serviços de Saúde (CINTESIS), Faculty of Medicine, University of Porto, Porto, Portugal, ${ }^{3}$ Centro de Estudos e Investigação em Saúde da Universidade de Coimbra, Coimbra, Portugal, ${ }^{4}$ Department of Midwifery and Palliative Care, Florence Nightingale School of Nursing, King's College London, London, United Kingdom, ${ }^{5} \mathrm{PIN}$ - Progresso Infantil (Centro de Desenvolvimento), Porto, Portugal, ${ }^{6}$ Departamento de Cuidados, Paliativos da Unidade Local de Saúde do Nordeste, Bragança, Portugal, ${ }^{7}$ Department of Psychiatry, Manitoba Palliative Care Research, Winnipeg, Canada

Background: Dignity Therapy (DT) is a brief, individualized intervention, providing terminally ill adults an opportunity to conveying life's memories and prepare their legacy, supporting sense of meaning and purpose. Efficacy on adults' quality of life, wellbeing and psychological suffering has been well documented. It is likely that DT could benefit younger people. Protocol adaptation is warranted.

Aim: To adapt the adult Portuguese DT schedule of questions for adolescents (ages 10-18).

Methods: Four stages were followed (June-October 2018) in the first phase:

1) initial adaptation of adult DT schedule of questions for adolescents;

2) expert committee panel analysis on adapted version;

3) DT's original author consult for accuracy;

4) new expert committee consult for final consensus.

The panel was comprised of 20 members (some with common expertise areas), previously introduced to the fundamental aspects of DT and question protocol: 2 pediatricians, 6 adult or pediatric palliative care physicians, 7 family physicians, 6 adult and children psychologists, 3 
pediatric palliative care nurses, 1 child psychiatrist. Comments were sought on clarity, comprehensibility and ambiguity of each item; possible inclusion of other relevant questions or supporting ideas; building on the DT session to improve legacy construction.

Results: DT's original author endorsed the final consensus DT schedule of questions, reinforcing that it captures the fundamental dimensions of DT. There was $100 \%$ agreement on the final consensus version and defined age group. To strengthen the process, a linguistic expert was consulted and no changes were needed.

Conclusion / Discussion. We developed a DT schedule of 9 questions for Portuguese adolescents. It appears to be clear and well-aligned with fundamentals of DT and could add additional value to pediatric palliative care for adolescents, their families and friends. Next, we will test this version with Portuguese adolescents using a qualitative approach.

Abstract number: P01-040

Abstract type: Poster

Barriers and Challenges for the Provision of Pediatric Palliative Care in Mexico

Ramos-Guerrero J. ${ }^{1}$, Grüneberg E. ${ }^{2}$, Pastrana $T^{3}$

${ }^{1}$ Palliative Care Team, Instituto de Seguridad Social y Servicios Sociales de los trabajadores del Estado, Zapopan, Mexico, 2Department for Palliative Medicine, RWTH Aachen University, Aachen, Germany, ${ }^{3}$ Department for Palliative, Medicine RWTH Aachen University, Aachen, Germany

Background: Eighty percent of Mexican children diagnosed with complex chronic conditions, who died, would have benefited from pediatric palliative care (PPC). The development of PPCs in Mexico has its own particularities given the geographical, social, political and cultural characteristics. Although, extraordinary advances have been made in the last decades, PPC in Mexico continues to be a challenge.

Aim: This study aims to explore the barriers and challenges for the provision of PPC in Mexico from the professional perspective of PPC providers. Methods: An on-line survey based on the theoretical frame of Grol and Wensing (2014) was conducted. 22 Mexican physicians and 3 psychologists identified as PPC providers in the entire country were asked to participate.

Results: 22 out of 25 responded (Response rate=88\%, women=61,9\%). $40.9 \%$ pediatricians with PC training, $45.4 \%$ physicians (no pediatrician with PC training), and $13.6 \%$ psychologists. $23.8 \%$ of the participants work in a PPC-team, while $42.9 \%$ work in PC-adult teams, which also attend children. Six key areas were analyzed, presenting the following results: 1) Innovation: with resistance to change, lack of awareness/ understanding about palliative care, commitment. 2) Individual professional: Lack of training and opportunities for training 3) Patient/family: Communication, complexity of the situation, unrealistic expectations 4) Teamwork: Interaction between teams and between levels of care, work overload 5) Organizational context: weak domiciliary network, lack of human resources, lack of medicaments and medical technologies, lack of reimbursement for PPC provision; and 6) Economic and political context: Geographic distribution, poverty, cultural barriers.

Conclusions: Several barriers for the provision of PPC in Mexico were identified. Training in PPC was highlighted as fundamental issue for the advance of PPC in Mexico.

Abstract number: P01-041

Abstract type: Poster

\section{Supporting a Paediatric Ward to Achieve 'A Good Death': A Case}

Study

Marshall S. ${ }^{1}$, Dickie C. ${ }^{2}$, Whelan $\mathrm{N}^{2}$, Prentice $\mathrm{W}^{2}$

${ }^{1}$ Palliative Care, King's College Hospital NHS Foundation Trust, London, United Kingdom, ${ }^{2}$ King's College Hospital NHS Foundation Trust, London, United Kingdom
Aim: The majority of planned paediatric hospital deaths occur in intensive care (PICU). The aim of this abstract is to illustrate some of the issues and challenges involved in achieving 'a good death' on a paediatric ward. Paediatric palliative care is a medical speciality in its own right, however the number of specialist trained teams remains small. This abstract illustrates how skills from an adult palliative care team are able to support staff working in paediatrics.

Methods: A case study is presented.

Results: A was a 7-month-old boy with mitochondrial (multisystem) disease referred to the hospital palliative care team. His condition has a maximal prognosis of 4 years and primarily causes neurological and liver symptoms. As a result, nursing staff were mainly concerned about potentially fatal or post-mortem bleeding.

The referral was to support the paediatric team to:

- $\quad$ Support medical and nursing staff around managing the death of a child.

- $\quad$ Establish ceilings of treatment, including DNACPR and ensuring that A was not taken to PICU.

- Control symptoms.

- $\quad$ Support the family and ensure that their spiritual and cultural wishes around end-of-life care were met.

Conclusion: This case study can be used to inform the practice of palliative care professionals working with paediatric wards.

Key issues were:

- $\quad$ Staff support around documentation of DNACPR and other limitations of treatment documentation

- Nursing staff anxiety around last offices and EOL care

- Anxiety about the cultural needs of families

- Anxiety talking to families about death and dying

- The need for staff debriefs before and after the death

- Consideration of the development of innovative models of care between adult and paediatric palliative care services to facilitate effective support for patients, families and staff.

A died peacefully on the ward 7 days after referral.

Abstract number: P01-042

Abstract type: Poster

A Program of Study to Increase Research Access and Participation by Children and Young People with Life-limiting Conditions and Life-threatening Illnesses and their Families: Barriers to Research Access: Voices, Experiences and Solutions. The BRAVES Project

Butler A.E., Vincent K., Bluebond-Langner M.

Louis Dundas Centre for Children's Palliative Care, UCL Great Ormond Street Institute of Child Health, London, United Kingdom

Background: Previous studies have found clinician gatekeeping to be a major barrier to research participation for children and young people with life-limiting conditions and life-threatening illnesses (CYP with LLC/LTI). Little is known about other barriers to research with CYP with LLC/LTI and their impact.

Aims: BRAVES is a programme of research aiming

1) to explore barriers to conducting research with CYP with LLC/LTI

2) to develop solutions for overcoming these barriers.

Methods: The programme includes five mixed methods work packages (WP):

(1) A systematic review investigating recruitment of CYP with LLC/ $\mathrm{LTI}$ to research 
(2) A survey exploring views of paediatric palliative care (PPC) clinicians on barriers to research with CYP;

(3) A national survey exploring the difficulties and solutions proposed by chief investigators' conducting studies with CYP with LLC/LTI;

(4) An analysis of correspondence between researchers conducting studies with CYP with LLC/LTI and research ethics committees (RECs);

(5) Interviews with REC members to understand their experience of reviewing research with this population.

Results: Findings from three WP completed to date indicate that:

(1) recruitment information is consistently underreported (WP1);

(2) PPC clinicians identify the REC review process; time and resources as well as clinician gatekeeping as barriers to research (WP 2) and chief investigators identify funding, access to families, and ethical and regulatory approval as barriers to research with CYP with LLC/LTI (WP 3).

Conclusion: Clinician gatekeeping is a barrier to research with CYP with LLC/LTIs but other barriers include resources and the ethical/regulatory approval process. Findings from BRAVES will contribute to the development of guidance for overcoming such barriers.

Funding: This research is funded by NIHR CLAHRC,The Health Foundation, GOSH Children's Charity,NIHR-GOSH BRC \&The True Colors Trust.

Abstract number: P01-043

Abstract type: Poster

\section{Yin \& Yang - The Ethos of Paediatric Palliative Oncology}

Phillips $M$.

Perth Children's Hospital, Paediatric \& Adolescent Palliative Oncology, Perth, Australia Presenting author email address: marianne.phillips@ health.wa.gov.au

The diagnosis of cancer in a child results in physical, psychological, social and spiritual needs and symptoms regardless of overall prognosis. International recommendations identify the early integration of palliative care into paediatric and adolescent oncology practise ensures the best outcome for patients and their families.

Pediatric palliative oncology (PPO) is an emerging field, integrating the principles of palliative care into the illness trajectory from the time of diagnosis. High quality assessment, communication and management of symptoms by medical and psychosocial multi-disciplinary teams (MDTs) provide the optimal care to children with cancer.

Through an embedded paediatric palliative oncology expert (EPPOE) model, our institution has provided a statewide oncology service for over 40 years. A strong ethos of clinical trials is supported by an integrated palliative oncology service provided from within the department by specifically trained, qualified medical and nursing leadership to ensure expert guidance and support for all patients and staff. Patients benefit from equitable care through a case-management model provided by specific interdisciplinary team members. Development of departmental assessment and communication tools, utilised in parallel to national frameworks and guidelines, facilitate a continuum of care from diagnosis throughout treatment to outcome for approximately 100 new diagnoses or relapsed cases each year with overall outcomes benchmarked to international standards. Targeted departmental and community-based support is provided, regardless of location, for an average 25 patients annually who require palliative support during end of life phases and evidence-based bereavement follow-up provided to the families. Ongoing involvement in national and departmental research projects with departmental communication and bereavement audits result in an evidence-based encompassing service and holistic care for all patients and their families.
Abstract number: P01-044

Abstract type: Poster

The Use of Simulation Based Education in Interprofessional Paediatric Palliative Care Education in a Tertiary Children's Hospital

Kerr-Elliott T. ${ }^{1}$, Barker F. ${ }^{2}$, Bayliss J. ${ }^{1}$, Brierley J. ${ }^{3}$, Cooke R. ${ }^{1}$, Craig F. ${ }^{1}$, Downie J. ${ }^{1}$, du Pré P. ${ }^{3}$, Moore Brooks D. ${ }^{1}$, Rajapakse D. ${ }^{1}$, Sidgwick P. ${ }^{4}$, Skellett S. ${ }^{3}$, Ward L. ${ }^{1}$, Akers E. ${ }^{5}$

${ }^{1}$ Louis Dundas Palliative Care Team, Great Ormond Street Hospital, London, United Kingdom, ${ }^{2}$ Neonatal Transport Service, Royal London Hospital, London, United Kingdom, ${ }^{3}$ Paediatric Intensive Care, Great Ormond Street Hospital, London, United Kingdom, ${ }^{4}$ Paediatric Intensive Care Unit, Great Ormond Street Hospital, London, United Kingdom, ${ }^{5}$ Clinical Simulation Centre, Great Ormond Street Hospital, London, United Kingdom

Aim: Three highly evaluated education programmes have been developed to address unique and challenging aspects of Paediatric Palliative Care; focusing on communicating with families, compassionate extubation and when a child dies. Each utilises a blended approach based primarily on simulation based education (SBE), led by a multi-professional expert faculty.

Design: All have been developed utilising inter-professional education tenets, with interagency faculty and candidates: crucially, this imparts a richness of experience, reflecting the wider systems supporting this care. A particular focus is on complex communication, managing conflict, advanced care planning, symptom management, bereavement, spirituality, care after death, and organ donation.

Utilising the experiential nature of SBE, the programmes have been developed utilising the following; Training Needs Analysis: professionals' views of the care they deliver, relevant literature, expert perceptions of care delivery and parental feedback. Each course addresses unique aspects of this highly complex and emotive aspect of care delivery. Specialist trained simulation actors perform in parent roles to enable exploration of care and communication: direct quotes are used to deepen the experience.

Learning objectives: Candidates must; demonstrate complex and challenging communication, gain a greater understanding of the range of teams and systems involved, gain practical skills relating to Palliative and End of Life Care and consider elements of Human Factors- each should gain an insight into their own strengths and anxieties around this care.

Results: Evaluation reflects Kirkpatrick Level 2; each programme effectively meets learners' needs; candidates can identify key aspects of practice to adapt.

Conclusion: Simulation is novel in Palliative and End of Life Care education; the programmes described are effective in developing specialist skills and knowledge required to support families and children.

\section{Abstract number: P01-045}

Abstract type: Poster

Specialist Children's Palliative Care Service in Ireland Audit on Malignant versus Non-malignant and Location of Death O'Donoghue E., Hurley I., Jennings V., NiLaoire A.

Palliative Care, Our Lady's Children's Hospital, Crumlin, Dublin, Ireland

Background: Children's palliative care is an evolving speciality internationally. The first consultant paediatrician with a special interest in paediatric palliative medicine was appointed to a national tertiary paediatric centre in Ireland in May 2011 along with a team of 1.5 WTE Clinical Nurse Specialists in Children's Palliative Care. This study undertakes to compare referral patterns from Children with a malignant condition versus a non-malignant condition and location of death. 
Aim: To identify patterns of location of death of Children referred to SPCT and implications on diagnosis and location of death between children with a malignant condition versus non- malignant condition. Methods: The medical records of all children referred to the palliative care service who died between 01/01/2017 to 31/12/2017 were reviewed retrospectively. Data collected included the number of deaths, location of death and diagnose, adhering Categories of life-limiting and life-threatening conditions from Category 1 to 4 as identified by Together for Short Lives

Results: The audit revealed 20 (34\%) of the children's death were identified as Category 1 diagnosis, malignancies, the remainder 38 (66\%) deaths comprised of Category 2,3,4 diagnosis, non-malignant. There was significant discrepancy in the findings on location of death depending on initial Category of illness at diagnosis. Showing Children with Category 1 illness at diagnosis were more likely to die at home $(75 \%)$ rather than in hospital (25\%). The Children with Category 2,3,4 illness at diagnosis data shown as follows the number of Children dying at Home (28\%), Hospital (62\%), Hospice (5\%), Other (5\%, out of the country).

Conclusions: The National Model of Care for Peadiatric Healthcare Services (2015), acknowledges most families wish to care for their Child at home. Supported by the Hospital SPCT and Community Adult SPCT, following the Children and Family, providing the End of Life Care in their location of choice.

Abstract number: P01-046

Abstract type: Poster

Family Life Changes after Diagnosis of Chronic Complex Condition: A Report of a Paediatric Palliative Care Team Cancelinha C. ${ }^{1,2}$, Freitas $F^{1}{ }^{1}$, Seabra S. ${ }^{1}$, Pleno P. ${ }^{1}$, Campos $F^{1}{ }^{1}$, Namora P. ${ }^{1}$, Pedruco A. ${ }^{1}$

${ }^{1}$ Pediatric Department, Coimbra Hospital and University Centre, Coimbra, Portugal, ${ }^{2}$ Faculty of Medicine, University of Coimbra, Coimbra, Portugal

Background: Families of children with chronic complex condition (CCC) experience persistent stress, requiring a constant adaptation process.

Aims: We aimed to evaluate:

i) the number of significant relatives/others directly affected by the disease:

ii) changes in marital status, unemployment or changes to work hours, home relocation and need for psychological support.

Methods: Retrospective and descriptive study using demographic and clinical data of children and adolescents (0-17y) with CCC, referred to a paediatric palliative care (PPC) team between January 2016 and October 2018.

Results: We included 42 patients ( $52 \%$ male) with median age 6,5 years (3 months - 17 years); $40 \%$ classified into group 4 (Together for Short Lives classification); $24 \%$ died during the follow-up. The number of relatives directly affected varied between 1 and 7 (median 3); 21\% of parents divorced/separated after CCC diagnosis. Parents were at significantly increased risk of loss of employment than peers nationally ( $27 \%$ vs $7 \%$ ) and additionally $11 \%$ decreased total number of work of hours. $26 \%$ families relocated homes and 4 children (10\%) needed institutionalization (in 1 case together with mother). 58\% parents needed psychological support and $11 \%$ were referred for psychiatry service. In 5 families $(12 \%)$, siblings needed psychological support (mainly due to behavioral changes and scholar difficulties) and in $9(21 \%)$ other relatives were also referred.

Conclusion: Families of children with CCC experience a high incidence of major life changes. Our results reinforce the importance of multidisciplinary PPC teams, including psychologists and social workers. Understanding these burdens helps health care professionals to provide appropriate anticipatory guidance and support, ultimately improving children/famiIy's quality of life.

Abstract number: P01-047

Abstract type: Poster

The Experiences of Health Care Professionals Caring for Children with a Rare, Life-threatening Neurodegenerative Disease:

Fortune, Hope, Admiration, Peace, Satisfaction and Closure. A Qualitative Study

Olsman E. ${ }^{1,2}$, Peeters C. ${ }^{3}$, Tio N. ${ }^{1}$

${ }^{1}$ Medical Ethics \& Health Law, Leiden University Medical Center, Leiden, Netherlands, ${ }^{2}$ Spiritual Care, Hospice Bardo, Hoofddorp, Netherlands, ${ }^{3}$ Child Neurology, Leiden University Medical Center, Leiden, Netherlands

Background: A rare, life-threatening neurodegenerative disease (RLND) in children not only affects these children and their family members but also their health care professionals. However, the experiences of professional caregivers have not been explored.

Aim: The objective of this study was to explore the experiences of professionals caring for children/adolescents with a RLND.

Design: Semi-structured interviews were conducted with fifteen professionals, which were thematically analysed. Saturation was reached when around twelve participants had been included and member checking with participants was done.

Participants: 15 professionals participated in the study (4 male, 11 female). Five physicians, four nurses and six other professionals were included (mean age, 49; range, 34-66; SD, 9.9).

Results. Six themes were identified.

1) Fortune: participants felt the bad luck of children with a RLND and their parents, while they felt fortunate because of their own (family) lives.

2) Hope: participants wanted to give hope to parents because of the tragedy of the disease.

3) Admiration: they felt admiration for how children coped with their deterioration.

4) Peace: when the child suffered from epilepsy or psychosis, caregivers struggled to bring peace by providing spiritual and biomedical care.

5) Satisfaction: several participants stated how the fine-tuning to (the wishes of) dying patients and their families (had) satisfied them.

6) Closure: in many interviews, interviewees addressed the death of a child and how they found closure, such as by going to the funeral.

Conclusions: The findings show the effect of a RLND in children on their professional caregivers. Several of these themes have been addressed in existential philosophy and spiritual care, which deserves more attention in future studies.

\section{Abstract number: P01-048}

Abstract type: Poster

Attracting Earlier Referrals to a Paediatric Palliative Care Service: The Vocabulary of Enhanced Supportive Care Hemsley J. ${ }^{1}$, Dinsdale A. ${ }^{1}$, Bayliss J. ${ }^{1}$, Wood A. ${ }^{1}$, Walters L. ${ }^{1}$, Downie J. ${ }^{1}$, Vemuri S. ${ }^{1,2}$, Barone G. ${ }^{1}$, McCulloch R. ${ }^{1}$, Bluebond-Langner M. ${ }^{3}$, Henderson E. ${ }^{3}$, Stephenson $A{ }^{1}$

${ }^{1}$ Louis Dundas Centre Oncology Outreach and Palliative Care, Great Ormond Street Hospital for Children NHS Foundation Trust, London, 
United Kingdom, 2Palliative Care Program, The Royal Children's Hospital, Melbourne, Australia, ${ }^{3}$ Louis Dundas Centre Paediatric Palliative Care, University College London Great Ormond Street Institute of Child Health, London, United Kingdom

Background: Previous studies have shown that early specialist palliative care support is of benefit to cancer patients, but that there is resistance amongst cancer specialists to refer to palliative care services before curative treatment options have been exhausted. A recent initiative in adult cancer services has attempted to improve this situation through the implementation of a framework of enhanced supportive care (ESC). The subject is a tertiary level children's hospital with both cancer services and a dedicated specialist palliative care service that agreed to trial this initiative within a paediatric cohort.

Aims: To increase the rate of early referrals by cancer teams to a specialist palliative care service through the implementation of a new shared vocabulary of ESC and "poor prognosis". The services aimed to ensure that patients identified as having a poor prognosis were offered an ESC referral within one week of the new prognosis being communicated to the patient and family.

Methods (design, data collection, analysis): The vocabulary of ESC was integrated into the Principle Treatment Centre (PTC) and an agreed definition of "poor prognosis" was developed in discussions between palliative care and cancer specialists. A "poor prognosis" checkbox was added to routine data collection at ward rounds, with a status recorded for every patient discussed. Those with new poor prognoses were tracked until an ESC referral was offered.

Results: Of the 36 patients identified as having a new poor prognosis between October 2017 and March 2018, 27 (75\%) were offered an ESC referral within the target timeframe.

Conclusion / Discussion: The project demonstrated the feasibility of attracting earlier referrals to a specialist palliative care service through a change of vocabulary and reconfiguration of service. Future work is underway to attract referrals for all cancer patients admitted to the hospital with a high symptom burden, irrespective of prognosis.

Abstract number: P01-049

Abstract type: Poster

Experienced Support when Confronted with End-of-Life

Decisions in the Neonatal Intensive Care Unit: A Nationwide Population Survey among Neonatologists and Neonatal Nurses Beernaert K. ${ }^{1}$, Dombrecht L. ${ }^{1}$, Deliens L. ${ }^{1}$, Cools F. ${ }^{2}$, Goossens L. ${ }^{3}$ Naulaers G. ${ }^{4}$, Chambaere K. ${ }^{1}$, Cohen J. ${ }^{1}$, Consortium N. ${ }^{5}$, End-of-Life Care Research Group

${ }^{1}$ Ghent University \& Vrije Universiteit Brussel (VUB), Gent, Belgium, ${ }^{2}$ Universitair Ziekenhuis Brussel, Brussels, Belgium, ${ }^{3}$ Ghent University Hospital, Gent, Belgium, ${ }^{4}$ University Hospital Leuven, Leuven, Belgium, ${ }^{5}$ Neonatology Intensive Wards Flanders, Gent, Belgium

Background: Most child deaths occur within the neonatal period. Most of these happen in a neonatal intensive care unit (NICU) and often involve difficult ethical decision making that can cause stress and moral conflict.

Aim: This study investigates the support neonatologists and neonatal nurses experience in end-of-life decision (ELD) making at the NICU.

Methods: A mail survey was sent to all neonatologists and neonatal nurses of all eight Flemish Neonatal Intensive Care Units in May 2017, Belgium. Respondents were asked to indicate whether or not they agreed with 7 statements regarding personally experienced support on a 5-point Likert scale. All answers were analysed anonymously. Descriptive analyses were done. We present the combined percentages of point 4 and 5 on the scale.

Results: The response rate was 63\% (52/83) for neonatologists and $46 \%$ (250/527) for nurses. There were $80 \%$ of neonatologists, compared to
$32 \%$ of the nurses, that reported they have the possibility to express concerns with respect to specific ELDs. In case of disagreement with a specific ELD, $53 \%$ of the neonatologists and $65 \%$ of the nurses stated they could choose not to be involved anymore for this case. Half of nurses $(55 \%)$ stated that the physicians do not take their opinion into account when ELDs are made. $70 \%$ of both neonatologists and nurses experienced increased stress when confronted with an ELD. Both neonatologists and nurses (90\%) felt supported by their colleagues. They both experienced insufficient psychological support in their unit for the staff (50\%).

Conclusion: NICU staff experiences support from their colleagues but express a need for more psychological support. Half of the nurses stated that neonatologists do not take their opinions into account regarding ELDs. However, nurses may have complementary knowledge that could be helpful. Further insights on how the psychological support and sharing of relevant opinions between disciplines can be improved at the NICUs.

\section{Abstract number: P01-050}

Abstract type: Poster

Disease and Symptom Characteristics of in Patient Children in a Regional Referral Hospital in Uganda

Esther T. ${ }^{1}$, Juliana A. ${ }^{2}$, Mackuline A. ${ }^{3}$, Emmanuanel L. ${ }^{3}$, Hutt E. ${ }^{4}$ ${ }^{1}$ Nursing, Mbale Regional Referal Hospital, Mbale, Uganda, ${ }^{2}$ Mbale Regional Referal Hospital, Mbale, Uganda, ${ }^{3}$ African Palliative Care Association, Kampala, Uganda, ${ }^{4}$ medicne, university of Colorado, colorado, United States

Background: Children are faced with many health conditions from the growing process as well as increasing incidences of Non-communicable diseases (NCDs) which require palliative care interventions. These conditions are associated with distressing symptoms, which are associated with suffering and negative effects on their lives and family wellbeing.

Aim of the work: To provide onsite capacity building for staffs aimed at improving capacity of hospital staff to assess, document, monitor and manage pain.

Methods: Over a period of 12 months (March 2017 to March 2018), clinical hospital staff of Mbale Regional Referral Hospital, longitudinally assessed and scored pain for in-patients on 6 wards. We also collected demographic and clinical data. The children and their care takers were also asked reasons for their visit to the hospital.

Results: Data collected from the children's ward showed that out of the 532 admitted children,43\% suffered from Non Communicable Diseases(NCDs)including; diabetes-3\%,Sicklecell-6\%, cancers-6\%, cardiac conditions- $5 \%$, kidney condition- $4 \%$ and cerebral conditions-3\%. HIV with co infections was $4 \%$, diarrhea $7 \%$, Malaria $14 \%$ and TB /pneumonia at $21 \%$. The remaining had abdominal pain, Fevers, and anemia. For $55 \%$ of the patients, the main reason for visiting the hospital was pain and fever followed by difficulty in breathing (12\%) and diarrhea $8 \%$.

Other symptoms for visiting the hospital included; body weakness, Cough and running nose.

Conclusion: Over $50 \%$ of children admitted to the hospital suffer from NCDs and other conditions that would benefit from palliative care and should be included in palliative care interventions. There is need for more awareness on improving pain and symptom management for children.

\section{Abstract number: P01-051}

Abstract type: Poster

\section{The Start of our Journey: Support for Adolescents and Young} Adults with Cancer

Wong V. ${ }^{1}$, Tan Y.Y. ${ }^{1}$, Quah S.C.D. ${ }^{2}$, Poon Y.L.E. ${ }^{3}$, Hong J. ${ }^{3}$, Goh W.L. ${ }^{3}, \mathrm{Ng}$ Y.H. ${ }^{4}$, Tan Y.P. ${ }^{4}$, Lee G. ${ }^{5}$, Neo S.H.P. ${ }^{1}$

${ }^{1}$ Supportive and Palliative Care, National Cancer Centre Singapore, SG, Singapore, ${ }^{2}$ Radiation Oncology, National Cancer Centre Singapore, SG, 
Singapore, ${ }^{3}$ Medical Oncology, National Cancer Centre Singapore, SG, Singapore, ${ }^{4}$ Psycho-Oncology, National Cancer Centre Singapore, SG, Singapore, ${ }^{5}$ SingHealth, Singapore, Singapore

Aim: Adolescents and young adults (AYA), defined as individuals aged 15-39, often face various complex issues in this phase of unique emotional, cognitive, and social development. The diagnosis of cancer could be especially distressing at this time, as it can cause disruptions in crucial developmental tasks and feelings of isolation. Support groups are useful in helping patients and families with coping, but this is not available for the AYA population in Singapore. The aim of this study was to explore patients and caregivers' expectations and preferences for an AYA cancer support group.

Approach: A multimethod approach was undertaken. Thirty-six AYA cancer patients and their caregivers were enrolled and randomly assigned into 4 groups, to facilitate discussions and idea generation. Each group was asked to explore what they hope an AYA cancer support group could entail, and was given time to brainstorm before presenting their ideas. At the end of the session, each participant was given a survey form to specify their preferences for support group talks and activities.

Results: The group discussions brought about ideas that revolved around areas of providing support for patients, their parents and caregivers. Incorporating the sharing of experiences on social media platforms was highly favoured. Twenty participants who responded in the post-discussion survey felt that informational talks on caregiver support and financial subsidies would be useful, and expressed interest in going for cooking classes and movie screening as part of support group activities.

Conclusion: Informational and support group activities need to be tailored with relevance to the attendees. Preferences of our AYA cancer patients and their caregivers for support group communication platform and activities were established in this study. This will facilitate the development of a much-needed, dedicated AYA cancer support group that can meet the needs of this unique group in Singapore.

Abstract number: P01-052

Abstract type: Poster

Modifiable Factors for Improving End-of-Life Decision-Making for Neonatologists, Nurses, and Parents in the Neonatal Intensive Care Unit: A Qualitative Study

Piette V. ${ }^{1}$, Deliens L. ${ }^{1}$, Dombrecht L. ${ }^{1}$, Chambaere K. ${ }^{1}$, Cools F. ${ }^{2}$, Goossens L. ${ }^{3}$, Naulaers G. ${ }^{4}$, Cornette L. ${ }^{5}$, Beernaert K. ${ }^{1}$, Cohen J. ${ }^{1}$, Consortium N.I.C.U. ${ }^{6}$, End-of-Life Care Research Group

${ }^{1}$ End-of-Life Care Research Group, Vrije Universiteit Brussel \& Ghent University, Brussels, Belgium, 2Department of Neonatology, University Hospital Brussels, Brussels, Belgium, ${ }^{3}$ Department of Neonatology, University Hospital Ghent, Ghent, Belgium, ${ }^{4}$ Department of Neonatology, University Hospital Leuven, Leuven, Belgium, ${ }^{5}$ Department of Neonatology, General Hospital Sint-Jan BruggeOostende, Brugge, Belgium, ${ }^{6} \mathrm{NICU}$ Consortium, Brussels, Belgium

Background / Aims: Neonatal end-of-life decision-making processes present ethically difficult and stressful dilemmas for medical staff as well as for parents. To improve support in future cases, it is necessary to know what factors complicated or facilitated neonatal end-of-life decision-making for the main actors. This study therefore aimed to identify barriers and facilitators in neonatal end-of-life decision-making as perceived by neonatologists, nurses, and parents.

Methods: We conducted semi-structured face-to-face interviews with 15 neonatologists, 15 neonatal nurses and 12 parents or parent dyads, recruited via five neonatal intensive care units in Flanders, Belgium. All three groups were asked what factors facilitated and what factors complicated previous decision-making. Two researchers independently analyzed the full sample, using content analysis to extract and summarize barriers and facilitators. We retained only barriers and facilitators that could be modified for future improvements.

Results / Discussion: Modifiable barriers and facilitators emerged across three themes. First, preparation and structure facilitated decision-making. This includes having regular (interdisciplinary) meetings to discuss cases and collecting main actors' perspectives on acceptable quality of life in advance. Second, cultivating trust and communication between neonatologists, nurses, and parents facilitated decision-making, e.g. using appropriate language at the parents' level. Third, gathering and transferring knowledge complicated decision-making substantially. Actors indicated insufficient briefing, lack of education, and lack of predictable quality of life in ambiguous cases. Transferring knowledge also facilitated decision-making. For example, neonatologists indicated objective second opinion facilitated decision-making. The barriers and facilitators can ultimately help to improve support in future neonatal decision-making.

Funded by: Research Foundation Flanders.

\section{Abstract number: P01-053}

Abstract type: Poster

PaPaS Score Enables Assessment of Palliative Home Care of Children and Adolescents with Severe Irreversible Non-progressive Neurological Conditions

Dechert O. ${ }^{1}$, Gagnon G. ${ }^{1}$, Trocan L. ${ }^{1}$, Distelmaier F. ${ }^{2}$, Janßen G. ${ }^{1}$, Kuhlen M. ${ }^{3}$

${ }^{1}$ Department of Pediatric Oncology, Haematology and Clinical Immunology, Pediatric Palliative Care Team, University of Duesseldorf, Centre for Child and Adolescence Health, Düsseldorf, Germany, ${ }^{2}$ Department of General Pediatrics, University of Duesseldorf, Centre for Child and Adolescence Health, Düsseldorf, Germany, ${ }^{3}$ Pediatric Oncology and Haematology, Klinikum Augsburg, Augsburg, Germany

Background: According to Together for Short Lives (TfSL), life-limiting conditions (LLCS) in children, adolescents and young adults (CAYAs) are classified into four groups (1-4). In group 4, CAYAs with severe irreversible non-progressive neurological conditions are summarised, who are susceptible to life-threatening complications and hence, premature death. Although these patients constitute the largest group of patients, data on palliative home care (PHC) is still lacking.

Aim: To survey PHC of CAYAs in TfSL group 4 and to analyse differences in subgroups.

Design and methods: Single-centre retrospective cohort study of CAYAs cared for between 01/2013 and 09/2016. Patients were assigned to 5 subgroups according to the underlying condition.

Results: In the study period, 70 CAYAs were cared for, 35 (50\%) were male, $48(68.6 \%)$ presented with cerebral palsy. Median age at referral was 7.8 years (range 0.1-25.0), median duration of care 214.5 days (range 2-2,754). 21 (30\%) CAYAs died within the study period. There was a significant correlation between medical orders for life-sustaining treatment and place of death $(p=.0096)$ and the Pediatric Palliative Screening Scale (PaPaS) score and subsequent density of home visits $(p=.003)$, interruption of $\mathrm{PHC}(\mathrm{OR}=0.79$ [95\%Cl: $0.69 ; 0.90], \mathrm{p}=.001)$ and death $(\mathrm{OR}=1.24$ [95\%Cl: $1.07 ; 1.43], \mathrm{p}=.003)$ respectively. Subgroup analysis revealed significant differences concerning care by a hospice service, supply with oxygen via tracheostoma, mechanical ventilation and symptoms (difficulties in moving, palsy) $(p<.05)$ whereas no differences were determined for other symptoms, drugs and care tools.

Conclusion: Care of CAYAs with severe irreversible non-progressive neurological conditions only slightly differs between subgroups. However, our data indicates that the PaPaS score at referral enables assessment of the course of PHC in these patients. This data needs to be confirmed in larger patient numbers. 
Abstract number: P01-054

Abstract type: Poster

Seizing the Moment: Continuous Infusion Levetiracetam for Seizure Management in Paediatric Palliative Care (PPC) Patel B. ${ }^{1}$, Downie J. ${ }^{2}$, Blackburn K. ${ }^{2}$, Bayliss J. ${ }^{2}$, Bluebond-Langner M. ${ }^{2,3}$ ${ }^{1}$ Louis Dundas Centre for Paediatric Palliative Care / Pharmacy, Great Ormond Street Hospital NHS Foundation Trust, London, United Kingdom, ${ }^{2}$ Louis Dundas Centre for Paediatric Palliative Care, Great Ormond Street Hospital NHS Foundation Trust, London, United Kingdom, ${ }^{3}$ Faculty of Population Health Sciences, Louis Dundas Centre for Paediatric Palliative Care,University College London, Institute of Child Health, London, United Kingdom

Background: Children with CNS malignancy may experience seizures; increasingly managed using oral Levetiracetam. During transition from disease-directed therapy to $\mathrm{PC}$, existing anticonvulsant medication may be maintained, only for as long as oral administration is viable. Upon loss of reliable oral administration, parenteral anticonvulsant therapy choice in PC become limited due to lack of evidence or clinical experience. Phenobarbitone (PHB) or Midazolam (MDZ) have been the therapeutic agents of choice for anticonvulsant therapies in PPC. Many children experience debilitating sedation with these agents. PHB also has a high propensity for drug-to-drug interactions and poor stability with other medication, requiring separate infusion sites. Emerging adult data suggests safety and efficacy of using continuous levetiracetam infusions for end of life seizure management.

Aims: Evaluate safety and efficacy of continuous infusion of Levetiracetam in children.

Methods: A single centre retrospective chart review of children, requiring oral Levetiracetam conversion to continuous infusion. We reviewed safety, in terms of infusion site reactions and adverse events, efficacy. Results: 5 children received continuous infusions of Levetiracetam. $40 \%$ children experienced seizure control to end of life through use of Levetiracetam alone, with $40 \%$ requiring further anti-seizure medication to aid seizure control, 1 requiring PHB following Levetiracetam dose escalation and 1 whom had previously been commenced on MDZ infusion prior commencing Levetiracetam, due to loss of seizure control. Infusion site reaction was observed in 1 child, with no other adverse events noted. Conclusion: This is the first report the use of Levetiracetam in the PPC setting. Our review suggests continuous infusions of Levetiracetam are safe for use in children to provide seizure management at the end of life, meriting continuing research to further evaluate safety as well as efficacy in paediatric end of life care.

\section{Abstract number: P01-055 \\ Abstract type: Poster}

Healthcare Professionals' Attitudes towards Termination of Pregnancy at Viable Stage

Roets E. ${ }^{1}$, Dierickx S. ${ }^{2}$, Chambaere K. ${ }^{2}$, Deliens L. ${ }^{2}$, Roelens K. ${ }^{1}$, Beernaert K.2

${ }^{1}$ Women's Clinic, University Hospital Ghent, Gent, Belgium, ${ }^{2}$ End-ofLife Care Research Group, Vrije Universiteit Brussel (VUB) and Ghent University, Brussel, Belgium

Background: In case of fetal or maternal pregnancy complications at viability, caregivers' attitudes may influence their guidance on parental choice between late pregnancy termination (ITOP) or continuation, with curative or palliative care at birth.

Aims: To study attitudes towards perinatal decisions of staff involved in ITOP practice.

Methods: We conducted an anonymous mail survey among all staff involved in ITOP counselling and/or decision-making of all 8 prenatal referral centres in Flanders, Belgium (March - April 2018).
Results: Response rate is $78 \%(91 / 117)$. None of the caregivers is in favor of prohibiting ITOP. Almost all respondents (98\%) agreed to discuss ITOP multidisciplinarily. A majority agrees to discuss it with at least one physician $(88 \%)$, a neonatologist $(81 \%)$ or an ethics committee $(63 \%)$. In case of a healthy fetus, only $20 \%$ resp. $13 \%$ agrees with ITOP in maternal psychological or socio-economical problems. Most staff agrees to allow for ITOP in lethal (99\%) and in serious (96\%) fetal conditions. In lethal conditions, $98 \%$ and in serious fetal conditions, $86 \%$ agrees to offer parents a choice between postnatal palliative care or feticide.

$89 \%$ of staff mambers agrees with actively ending the life of a neonate with a serious condition and that Belgian law should be changed to facilitate this (88\%). In a hypothetical case with obvious prognosis, more staff agrees to both ITOP and actively ending neonatal life (98\% and 54\% resp.) compared to a less obvious case ( $82 \%$ and $32 \%$ resp.).

Conclusion: ITOP is a valuable option among most caregivers in Flanders with decreasing acceptance according to indication (least for maternal socio-economic problems). The majority prefers to discuss with colleagues, but less with an ethics committee. ITOP is tolerated more than neonatal life-ending, probably as a consequence of current legislation in Belgium although most caregivers prefer a change in Belgian law to facilitate neonatal decisions.

Funded by: Research Foundation Flanders.

\section{Abstract number: P01-056}

Abstract type: Poster

First Steps in Spreading Legal Services Component for Palliative Care in Romania

Simion A. ${ }^{1}$, Dumitrescu M. ${ }^{2}$

${ }^{1}$ Social, Fundatia Hospice Casa Sperantei, Brasov, Romania, ${ }^{2} \mathrm{PR}$, Fundatia Hospice Casa Sperantei, Brasov, Romania

Background: The Palliative Care (PC) patients and caregivers who have benefited of legal support service revealed a continuous need of covering legal issues they met. Access to legal right is often blocked by: bureaucracy, misunderstanding of legal norms, poor communication with public officers and authorities, lack of interest of those who could provide the legal rights. Patients proposed different ways to solve or to decrease the number of their problems: existence of a family lawyer, existence of pro bono legal services, existence of a hospital lawyer. Including a lawyer in PC team, we approached some of patient's proposal concluding that we need to go forward with our actions regarding legal services provided in the frame of PC.

Aim: To maintain the model of legal support for PC patients and caregivers introduced by a specialized PC service in Romania and to extend the created model in other 3 settings.

Results: Between October 2017-October 2018, a partnership was developed to extend the created legal model for PC. There were distinct meetings in three different PC settings. During each meeting, the project team (social workers and lawyer under contract) presented the project, its benefits and discussed the possible ways of replicating it in the PC setting. During implementation period, the project team offered support to the social workers from other 2 PC providers in their actions of finding and working with legal specialists. There were skype meetings with the aim of mentoring, identifying challenges, presenting results they had during this period and establishing next steps in order to continue the collaboration.

Conclusion: This example of integrating legal part into palliative care services is a potential solution for offering legal support, a model of good practice for other $\mathrm{PC}$ services.

This project was co-financed by Open Society Foundations.

Abstract number: P01-057

Abstract type: Poster 
Identifying Key Elements of Pediatric Advance Care Planning (pACP) with the Help of Bereaved Parents and Experienced Care Professionals

Hein K. ${ }^{1}$, Knochel K. ${ }^{1}$, Reimann D. ${ }^{1}$, Zaimovic V. ${ }^{1}$, Borasio G.D. ${ }^{2}$, Führer M. ${ }^{1}$

${ }^{1}$ Center for Pediatric Palliative Care, University Children's Hospital Munich, Munich, Germany, ${ }^{2}$ Service de Soins Palliatifs et de Support, Centre Hospitalier Universitare Vaudois, Lausanne, Switzerland

Background: Research has shown that advance care planning concepts for adults are not directly applicable to the pediatric setting. A specific pediatric advance care planning intervention (pACP) is still missing.

Aim: To develop a pACP intervention for children with life-limiting diseases.

Methods: We selected bereaved parents and (health) care professionals (HCPs) by purposeful sampling and invited them to participate in a oneday transdisciplinary workshop (participatory approach). We assigned participants to one of 3 focus groups (parents, pACP-facilitation, pACPimplementation) to discuss different perspectives and identify needs, barriers and helpful aspects of pACP. Data were analyzed by content analysis.

Results: Main topics identified were

1) Timing,

2) Communication,

3) Decision making,

4) Documentation, and

5) Implementation.

Some participants had difficulties to identify pACP as an explicit process separate from palliative care as a whole. Timing subtopics included parents' readiness and wrong or missed time points. Decision-making was not restricted to the PACP conversations. Parents needed to discuss their decisions as a couple and with significant others. They requested $\mathrm{pACP}$ discussions to be open and non-judgmental. Parents did not want to be urged to decide. They needed to address their fears and to focus on their child's personality and quality of life. Both parents and HCPs wanted to prepare for crises. HCPs emphasized the documentation of parents' preferences of care and emergency plans. All participants perceived $\mathrm{pACP}$ as an iterative process that allows decisions to be adapted to the child's changing condition. Coping with emotional distress and dissent between professionals and parents were perceived as challenging.

Discussion: The analysis of the three focus groups allowed the identification of key elements for a specific PACP intervention. These elements will form the basis for the participative development of a specific PACP framework.

\section{Abstract number: P01-058 \\ Abstract type: Poster}

MyChildrensComfortNet - Development of a Digital Community for Better Communication in Children's Palliative Care (CPC) Vallianatos S. ${ }^{1}$, Schuiling-Otten M. ${ }^{1}$, Noordam C. ${ }^{2}$, Hermans M. ${ }^{2}$, Jacobs-de Leur J. ${ }^{2}$, Tuil W. ${ }^{3}$

${ }^{1}$ The Dutch Centre of Expertise for Children's Palliative Care, Utrecht, Netherlands, ${ }^{2}$ Amalia Children's Hospital at Radboud University Medical Centre, Nijmegen, Netherlands, ${ }^{3}$ Re:Public, Rosmalen, Netherlands

Background: To improve the care for children with a lifelimiting/lifethreatening disease and their families, a nationwide structure for integrated CPC is created. This structure consists of 7 networks and 7 teams in the academic children's hospitals which focus on organizing CPC from hospital to home. Many improvements have been made for quality CPC, but communication is still a problem. Families and professionals indicate the need for better communication between all involved in order to improve CPC and support families.

Aim: We aim to create a personal digital community around families, for better communication and cooperation between family and all professionals involved.

Design, methods and approach taken: To come to the requirements for the digital community we started with a mirrormeeting with parents to hear their experiences and points of improvement. Together with a CPC-team and an experienced designer, we developed MyChildrensComfortNet. It is a web-based program and offers a personal digital community around a child and family in a secured environment. The communication module offers functionalities as texting and video calling. In the information module, both documents and videos can be added. The family determines who is part of the community. After a process of design, testing, and redesign, the tool will be available for all CP-teams/networks in NL.

Results: At the time of submission, the tool is being piloted in a CPCteam with parents. Parents will provide their experience and feedback on improvements. We expect to report the results and further developments of the tool at this congress.

Conclusions: Digital development is a complex process. Our ultimate goal is to achieve better communication and cooperation to support the families. The nationwide structure offers a platform for feedback and further development to reach our goal. The Dutch Centre of Expertise for CPC will provide a basis for ongoing improvements and updates to MyChildrensComfortNet

Abstract number: P01-059

Abstract type: Poster

Psychosocial Support as a Pillar to Improve Adherence to Treatment among Adolescents Living with HIV/AIDS in Dar es Salaam

Kayange A. ${ }^{1,2}$, Kiwanga F. ${ }^{3}$, Manase $F^{4}{ }^{4}$

${ }^{1}$ Uhuru Medical Centre (UMC), Dar es Salaam, Tanzania, United Republic of, ${ }^{2}$ Christian Leadership University (CLU), Cheektowaga, United States, ${ }^{3}$ Ocean Road Cancer Institute, Dar es Salaam, Tanzania, United Republic of, ${ }^{4}$ The Community Centre for Preventive Medicine [CCPmedicine Medical Center], Dar es Salaam, Tanzania, United Republic of

Background: Psycho-social support is an activity that addresses the ongoing psychological and social problems of HIV infected individuals, their partners, families and caregivers. Poor parental care, stigma and discrimination may affect an adolescent and the entire family leading to poor adherence on antiretroviral drugs.

Aims: This abstract demonstrate the role of psychosocial support to adolescents living with HIV, on treatment with un-suppressed viral load in Dar es salaam, Tanzania.

Methods and results: A total of 150 adolescents were found to have more than 35,000 viral copies as baseline viral results. Among these adolescents with highest viral load, only 120 adolescents had their parents signed consent form to allow them participates in the psychosocial activities such as music, drama, art work etc. During this program, psychosocial and adherence sessions were assessed for 5 days. This was followed by an enhanced adherence counseling sessions that was tailored to an individual adolescent. Poor adherence was found to be a possible cause for poor viral suppression. All adolescents were followed up biweekly for 3 months while conducting enhanced adherence counseling in every monthly clinic visit. Among 100 adolescents who were enrolled in the camp, 101 adolescent (67\%) had shown viral suppression whereas 49 (33\%) were not suppressed. Among the 49 adolescents, 6 adolescent (12\%) had maintained high viral load, 17 adolescents (34\%) had viral suppression by $50 \%$ and 26 adolescents $(54 \%)$ had viral suppression by $75 \%$. 
Conclusion: Psycho-social support play a pivotal role helping an adolescent and the family to cope better with illness and dealing more effectively with discrimination. It improves the quality of their lives, and prevents further transmission of HIV infection through an improved drug adherence.

\section{Abstract number: P01-060 \\ Abstract type: Poster}

Supporting Families to Clarify Treatment Preferences for Emergency Conditions in Pediatric Palliative Care

Deinert I., Scholz C., Nolte-Buchholtz S.

Department of Pediatrics, University Hospital Carl Gustav Carus, Technische Universität Dresden, Dresden, Germany

Treatment decisions in pediatric palliative care must be made in accordance with the patient and its parents. In Germany, for emergency conditions a structured form was established to clarify the families' treatment preferences including the following nine parameters: cardiopulmonary reanimation, thorax compression, catecholamine administration, defibrillation, pleural drainage, arterial puncture, mask ventilation, endotracheal suction and intubation. In addition, a dialog protocol is recommended.

The aim of the study was to analyze the factors influencing the families' decisions for medical procedures in case of emergency conditions.

Data of patients treated in the SPPHC (specialized pediatric palliative home care) team from January 2015 to September 2018 were analyzed. The retroperspective monocentric study includes 123 patients (median age 7.3 years, $46 \%$ females). The majority suffered from non-cancer conditions (66\%). Descriptive statistics were performed using IBM SPSS, version 22. Protocols were analyzed by the qualitative content analysis by Mayring (2010).

In the cohort, 46 patients ( $37.4 \%$ ) declared their wishes in a structured form. Out of these, 16 patients (36.4\%) updated the form. The dialog protocol was written for $79 \%$ of all patients. Most of the consultations were performed by a SPPHC team (63.8\%). In addition to the nine procedures mentioned on the form, the majority of the parents ( $91.5 \%)$ added further information. The majority of families of ACT group four patients, with conditions that are irreversible but non-progressive, had a structured form $(62,5 \%)$ in contrast to families of ACT group one patients $(27,9 \%)$ with life threatening conditions like cancer diseases.

Independent of the disease and the prognosis, almost all families needed to further specify their wishes in addition to the information mentioned on the form. A revised version of the form may be necessary to support the families to fully clarify their treatment preferences.

Abstract number: P01-061

Abstract type: Poster

Drafting a Specific Pediatric Advance Care Planning Program in Modular Design to Ensure a Child-focused Communication Process

Knochel K. ${ }^{1}$, Hein K. ${ }^{1}$, Reimann D. ${ }^{1}$, Zaimovic V. ${ }^{1}$, Borasio G.D. ${ }^{2}$, Führer

M. ${ }^{1}$

${ }^{1}$ Center for Pediatric Palliative Care, Ludwig-Maximilians-University, Munich, Germany, ${ }^{2}$ Service de Soins Palliatifs et de Support, Centre Hospitalier Universitaire Vaudois, Lausanne, Switzerland

Background: Previous research analyzed challenges, benefits and barriers of pediatric Advance Care Planning (pACP). However, a common understanding and a consistent approach to $\mathrm{PACP}$ are still missing.

Aims: To develop a framework for pACP based on a participatory approach. Methods: The pACP development process encompassed two transdisciplinary workshops with bereaved parents and stakeholders. In the first workshop, key elements of the PACP process (contents, actors, tools) were identified. In the second workshop, parents and professionals worked in 3 mixed groups, with the task of organizing the key elements of pACP in a timeline and visualize the process on a poster (constellation analysis). Results were systematized and translated into a first draft of the program by the research team.

Results: The pACP guide includes the following modules:

1) Timing: personalized identification of the right time for initiating pACP;

2) Preparation aspects: e.g. building up a trustful relationship, information for participants;

3) Opening discussion: Framing the process and making expectations and aims of participants explicit;

4) Focus on the child: including quality of life, personal wishes and hopes;

5) Medical topics: disease-specific emergency situations and crises discussed in hypothetical scenarios;

6) End of life issues: optional module for the end of the pACP process,

7) Documentation: Emergency and care plans, advance directives, and discussion protocols;

8) Implementation: summarizes and validates documentation and discusses details about implementation;

9) Transversal module: aspects of communication and structural issues relevant to the whole PACP process.

Conclusion / discussion: The PACP program was designed in a participative process with stakeholders who take care of affected children and bereaved parents. Previously identified key elements of pACP could be organized in a flexible modular timeline, thus ensuring a personalized pACP process.

Abstract number: P01-062

Abstract type: Poster

The Magic of Holistic Management of Children Living with

Chronic Illness and Other Associated Conditions: A Case

Observation of a Child Living with HIV/AIDS, Pulmonary

Tuberculosis, Marasmic Kwashiorkor in Tanzania

Kiwanga F. ${ }^{1}$, Manase F. $^{2}$, Kayange A. ${ }^{3}$

${ }^{1}$ Ocean Road Cancer Institute, Dar es Salaam, Tanzania, United Republic of, ${ }^{2}$ Community Centre for Preventive Medicine [CCPmedicine Medical Center], Dar es Salaam, Tanzania, United Republic of, 3Uhuru Medical Centre (UMC), Dar es Salaam, Tanzania, United Republic of

Introduction: Palliative care is an approach that improves the quality of life of sick person and their families through the prevention and relief of suffering by means of early identification and impeccable assessment and treatment of pain and other problems, physical, psychosocial and spiritual.

Patient description: A child RK 15 years old girl, who physically was wasted with multiple skin rashes, febrile and failure to thrive. She was living with a widowed jobless mum who had poor interaction with other family members. Both mother and her daughter were on ARV, and child had stopped school His family had stopped school due to illness and stigma and they could afford only one meal in a day.

Intervention and outcome: A holistic management plan was initiated. Pain assessment and management according to WHO analgesic ladder, anti TB drug initiation, nutritional support with plumpy nuts were provided. Provided family health education on balanced diet, body and environmental hygiene, reassurance to child about her situation. Family meeting conducted that encouraged support from other relatives, encouraged going to school once she is doing better and encouraged mother to join income generating groups. Within a short period of time, the child improved, gained weight, skin rashes and itching stopped and hope of living regained. 
Conclusion: Looking at multiple conditions from a patient is key in ensuring patient with multiple illnesses are thoroughly managed. Communication with a patient helps us getting medical information that will be useful in management plan.

\section{Abstract number: P01-063}

Abstract type: Poster

"Will you Still Visit my Family When I Am Gone?" Participation of a Young Boy with Hypoplastic Left Heart Syndrome in Advance Care Planning: A Qualitative Case Study Knochel K. ${ }^{1}$, Gatzweiler B. ${ }^{1}$, Zaimovic V. ${ }^{1}$, Borasio G.D. ${ }^{2}$, Führer M. ${ }^{1}$ ${ }^{1}$ Center for Pediatric Palliative Care, Ludwig-Maximilians-University, Munich, Germany, ${ }^{2}$ Service de Soins Palliatifs et de Support, Centre hospitalier universitaire vaudois, Lausanne, Switzerland

Background: Disease complexity, psychosocial factors, and individual decisional capacity influence decision-making processes in pediatrics. Ensuring appropriate participation at each developmental stage is essential in order to respect the child's autonomy and to reduce emotional distress for parents and professionals.

Aims: This case study aims

(1) to clarify individual steps of participation in pediatric advance care planning $(\mathrm{pACP})$ in accordance with a growing decisional capacity and

(2) to present how a child- and age-appropriate participation can be put into practice.

Methods: In this qualitative longitudinal case study, the palliative care team accompanied a boy diagnosed with Fontane failure in hypoplastic left heart syndrome from age 9 to 15 years. The study includes a content analysis of field notes and a description of adapted methods and tools used for participation of the child in discussions.

Results: Initially, pACP discussions only involved the parents. The patient was informed in a child-appropriate way before and after discussions. Over time we intensified the multi-professional approach including serial assessments of the child's growing decisional capacity and close collaboration between psychologist and physician. Different tools were used for child-appropriate engagement and support: (1) an individually drawn up "That's me"-book (2) a self-designed memory game (3) a power-watch with self-painted fantasy animals and (4) story-telling by a model (Fridolin, the frog). We analyze setting and topics at different ages and demonstrate the increasing participation of the boy in decisionmaking over the 6-year-period.

Conclusion: Trust, honesty and truthfulness are key elements for engagement of children in advance care planning. Employing a variety of age-appropriate tools and drawing on the specific competencies of all professionals involved is crucial to enable an increasing participation of the child in decision-making processes and advance care planning.

Abstract number: P01-064

Abstract type: Poster

Implementation of a "Sterile Cockpit" to Improve Medication Safety in a Children's Hospice

Balfe J. ${ }^{1,2}$, Cassidy M. ${ }^{1}$, McEnerney N. ${ }^{1}$, Moseley A. ${ }^{1}$

${ }^{1}$ LauraLynn Children's Hospice, Mount Merrion, Ireland, ${ }^{2}$ Discipline of

Paediatrics, Trinity College Dublin, Dublin, Ireland

Background: All healthcare professionals have an obligation to report healthcare incidents and to work towards a safer environment for patients. Medication errors are recognised as a common source of adverse healthcare incidents. Children with life-limiting conditions often have multiple medical co-morbidities each requiring medical intervention and can be on complex medication regimes which increase the risk of medication prescribing, reconciliation or administration errors.

Following a number of low harm medication errors, an improvement in medication safety was identified as a key quality improvement priority in our hospice.

Methodology: Assessment of the problem included measurement of all medication incidents or near-miss incidents between January and September 2017, total $110.15 / 110$ errors related to prescribing.

A cause-and-effect diagram examined the root cause of prescribing errors and in the majority of cases an unsafe environment was identified as a significant contributor to the incident.

Further quality improvement methodology included production of driver diagrams and process mapping of medication prescribing with a view to identifying opportunities for positive change.

Results: Initial Plan, Do, study, Act cycles focused on the establishment of a "sterile cockpit" aimed at minimising interruptions during prescribing. This has contributed to a reduction in prescribing errors from 4 per month to 1 per month on average. There has been an associated improvement in medication safety across the organisation.

Staff and service user education sessions have been implemented with a focus on the use of the Red Apron to reduce interruptions during medication administration this has supported the reduction in medication administration errors.

Conclusion: This quality improvement project has led to a significant reduction in drug related incidents in our hospice. Work in ongoing to embed the learning throughout the service and to ensure continuation of the project.

\section{Abstract number: P01-065}

Abstract type: Poster

Polypharmacy in Children with Life-limiting Conditions; Benefit or Burden?

Balfe J. ${ }^{1,2}$, Cassidy M. ${ }^{2}$

${ }^{1}$ Discipline of Paediatrics, Trinity College Dublin, Dublin, Ireland,

LauraLynn Children's Hospice, Mount Merrion, Ireland

Background: Polypharmacy is most commonly defined as greater than five medications per day and is a recognised to increase the risk of adverse drug reactions and medication errors. Children with life-limiting conditions(LLCs) have complex medical needs and multiple medical comorbidities each requiring pharmacological intervention but research has shown that parents are unsure if any medical intervention has improved their child's quality of life.

This audit aimed to explore the rate of polypharmacy in children with LLCs conditions attending a children's hospice.

Method: Prescription sheets of all children attending for short breaks were reviewed and data recorded on Excel. Data collected included number and frequency of routine and PRN medications. Number of antiepileptic drugs, opiates and adjuvant analgesics was also collected.

Results: The prescription sheets of all (total 106) children currently attending for short breaks were reviewed and confirmed high rates of polypharmacy.

The average number of prescribed regular medication was 8.2 (median 7, range 0-24). 89/106 (84\%) were prescribed greater than 5 medications per day. Medications were administered on average 5.3 times per day (range 0 -13). 78/106 (74\%) of children were prescribed anti-epileptic medications (AEDs), mean number 2.5 (range 1-6) 17/78 (22\%) were prescribed 4 or more AEDS.

$36 / 106$ (34\%) were prescribed at least 1 adjuvant medication, most commonly gabapentin (24.5\%). 28/106 (26.4\%) were prescribed an opiate, $14 / 28$ both regular and as needed and 14 as needed only.

Conclusion: This study confirms high rates of poly-pharmacy in children with LLCs. Complex medication routines are highlighted, these potentially 
increase the risk of medication administration errors and burdensome adverse interactions. Children's Palliative Care aims to improve quality of life and prescribers should continuously review medication regimes to ensure safety and to attempt to reduce any potential harm.

\section{Abstract number: P01-066 \\ Abstract type: Poster}

Impact of Neuropediatric Diseases in Pediatric Palliative Care: An Inventory of the Pediatric Palliative Care Team Südhessen Becker S., Fiedler H., Pietz J.

KinderPalliativTeam Südhessen, Frankfurt, Germany

Background and aim: In contrast to adult Palliative Care neuropediatric diseases are present in a large proportion of the patients supported by pediatric palliative care teams (PPCT). However, the exact amount of these patients in specialized palliative care of children and eol-care at their homes, as well as their symptoms, the challenges in treatment and special requirements are not well described.

Methods: We retrospectively evaluated all patients in our PPCT over a 5 year period. For this purpose, the data of 219 Children stored in the EDP system Pallidoc ${ }^{\circledR}$ was evaluated relating to diagnosis, symptoms, required treatment.

Results: $48.9 \%$ of patients had a neuropediatric diagnosis: $19.6 \%$ preperinatal brain damage, $15.5 \%$ neurodegenerative diseases, $5.5 \%$ neuromuscular diseases, $5 \%$ genetic brain malformations, $3.2 \%$ genetic epilepsy syndromes. A further group of patients with numerous neurological symptoms were patients with brain tumors (16.9\%) and complex malformation syndromes (5.5\%). The most common neurological symptom was epilepsy, which had to be treated in $45 \%$ (98/219). The very annoying symptom of epilepticus status was particularly frequent in pre-/perinatal brain damage, brain tumors, neurodegenerative diseases and genetic epilepsy syndromes. Challenges in treatment included implementation of alternative application routes for antiepileptic drugs, especially in terminal phase when oral or enteral routes are no longer available.

Discussion and conclusions: Cooperation between PPCT and neuropediatric clinics and specialized out-patients ambulances appear critical for defining and communicating approachable aims of therapy (especially as in some patients seizure-free intervalls will not be achieved). Special importance has to be given to advanced team training, especially in antiepilepsy therapy and the acute treatment of epileptic seizures.

\section{Abstract number: P01-067 \\ Abstract type: Poster}

Future Orientation and Advance Care Planning by Parents and Health Care Professionals in Paediatric Palliative Care: A Qualitative Study

Kars M.C. ${ }^{1}$, Verberne L.M. ${ }^{2}$, Fahner J.C. ${ }^{1}$, Sondaal S.F.V. ${ }^{1}$, de Kruijff C.C. ${ }^{3}$, Schouten-van Meeteren A.Y.N. ${ }^{4}$

${ }^{1}$ UMC Utrecht, Utrecht, Netherlands, ${ }^{2}$ Maastricht University Medical Center, Maastricht, Netherlands, ${ }^{3}$ Amsterdam UMC, Amsterdam, Netherlands, ${ }^{4}$ Princess Máxima Center for Pediatric Oncology, Utrecht, Netherlands

Background: Advance Care Planning (ACP) is a process of sharing goals and preferences for future medical care and treatment. Growing evidence suggests that ACP improves patient-clinician communication and increases concordance between preferred and received care in adults. ACP could also improve care of children but still has to find its way in clinical practice.

Aims: This study aimed to explore in which manner parents of children receiving Pediatric Palliative Care (PPC) and involved health care professionals (HCPs) anticipate future care.
Methods: A qualitative interview study using thematic analysis. Results: In total 105 one-time and repeated interviews were conducted with 42 parents ( 24 mothers, 18 fathers) of 24 children and 35 involved HCPs.

Both parents and HCPs anticipated future care, but thoughts were not shared to a large extent. Initiatives were aimed at ensuring the child's quality of (end-of-)life and comfort. Three approaches were identified:

1. ACP involved conversations resulting from an intentionally and goal-directed initiative by either the parent or the HCP. The initiator often framed the content of the conversation to ensure that the other party could connect with his/her point of view.

2. Anticipated care involved preparations to organize care in advance as felt appropriate for anticipated needs.

3. Guidance on the job involved a short-term anticipation and guidance on managing concrete problems or symptoms in the near future, mainly conducted by HCPs to guide parents through situations they felt nog (yet) prepared for.

Anticipated care and guidance on the job could build on earlier ACP-conversations.

Conclusion: Parents and HCPs anticipate future care. ACP often occurs intentionally using a goal-directed approach, which can be better characterized as care negotiation. ACP can guide anticipated care and guidance on the job and as such can facilitate high quality PPC concordant to the child's and the parents' preferences.

Abstract number: P01-068

Abstract type: Poster

PaPaS Scale Used in Daily Clinical Practice in University Hospital Motol in Prague: Are Children Referred to Pediatric Palliative Care Early Enough?

Hrdličková L.1,2,3

${ }^{1}$ Universty Hospital Motol Prague, Pediatric Hematology and Oncology, Prague, Czech Republic, ${ }^{2}$ University Hospital Motol Prague, Pediatric Palliative and Supportive Care Team, Prague, Czech Republic, ${ }^{3} \mathrm{Czech}$ Society for Palliative Medicine, Pediatric Palliative Care Section, Prague, Czech Republic Presenting author email address: lucie.hrdlickova@ fnmotol.cz

Approximately 8,000 children with life-limiting or life-threatening disease are taken care of in University Hospital Motol every year. Multidisciplinary team was established in September 2017 to provide pediatric palliative care in our hospital. The aim of our study was to identify, how early (or how late) do physicians refer patients to palliative care. A secondary objective of the study was to evaluate whether children with different diagnosis are referred to palliative care team at different time points of the illness trajectory.

Pediatric Palliative Screening Scale (PaPaS Scale), an instrument to identify children with palliative care needs, was evaluated in every patient referred to the multidisciplinary team prior to the initial consult. PaPaS Scale evaluation was performed by referring physicians. Patients were then divided into 5 diagnostic groups. Neonates and infants were included in the study.

In total, 38 children with life-threatening and life-limiting disease were referred to the multidisciplinary team in the past 14 months. Median PaPaS Scale score was $25[11,34]$. Patients presented a heterogeneous group of diagnoses: 15 children with cancer, 11 children with neurological disease, 5 with cardiovascular disease, 4 with congenital malformation and 3 children with cystic fibrosis. The highest PaPaS Scale score was identified in children with cystic fibrosis and in cancer patients with median score $28[25,34]$ and 27 [20,34] respectively. Group of patients with neurological disorder and with congenital disease both presented 
with median PaPaS Scale score 21. In patients with cardiovascular disease median PaPaS Scale score of 22 was identified.

Very high score of PaPaS Scale was identified in all diagnostic groups. In conclusion, children are referred to palliative care team relatively late. Strategies to integrate palliative care earlier during the trajectory of illness in pediatric patients must be developed and implemented in our hospital.

\section{Abstract number: P01-069 \\ Abstract type: Poster}

\section{Home Care for End-of-Life AYA Cancer Patients}

Satomi E., Nomura K., Takada H., Shimizu M., Kojima R., Shimizu M., Kiuchi D., Ishiki H.

National Cancer Center Hospital, Tokyo, Japan

Aim: Adolescents and young adults (AYA) with cancer need dedicated physical and psychosocial management and care. Especially advanced patients with severe symptoms and suffering require enough palliative management toward the end-of-life. But their condition may make it difficult to continue home care. The aim of this study is to know the management and care at home of terminal AYA cancer patients.

Methods: A survey was sent to homecare physicians who have advanced AYA patients with cancer referred by National Cancer Center Hospital from 2013 to 2017. Questionnaire covered the demographics and the clinical training of respondents, their clinical homecare-experiences and difficulties of terminal AYA patients with cancer.

Results: Forty out of 75 were responded and we analyzed on data from the 36 homecare physicians. The average age of respondents was 50.1 and their average clinical experiences are 23.1 years. Most frequent management of end-of-life home care for AYA cancer patients included pain control by opioid (96\%) and others were other symptom management including ascites/pleural effusion control, terminal sedation, psychological support for patients and caregivers. The devices used at homecare is central venous port placement, patient-controlled analgisic system, Oxygen, urological catheter, draining tube placement for ascites/pleural effusion, draining tube placement for bowel obstruction including PEG/PTEG. Total number of AYA cancer patients followed by homecare physicians are 52 (1.4 /year/physician) and the total number of death of AYA patients at home are 27 . Homecare physician felt difficulties about advance care planning/end-of-life discussion among AYA cancer patients and caregivers. Conclusion: Homecare of terminal AYA patients are mostly performed as same as hospital. Homecare physicians face difficulties in ACP/EOLd. Further research is necessary about terminal AYA patients with cancer in order to spend good time at home.

Abstract number: P01-070

Abstract type: Poster

Using Social Media in Palliative Care Research - The Pitfalls, the Opportunities

Taubert M. ${ }^{1}$, Norris J.2 ${ }^{2}$ Davies J.M.V. ${ }^{3}$

${ }^{1}$ Velindre NHS Trust Palliative Medicine Department, Cardiff University School of Medicine, Cardiff, United Kingdom, ${ }^{2}$ Digital Legacy

Association, London, United Kingdom, ${ }^{3}$ Velindre University NHS Trust, Cardiff, United Kingdom

Background: Social media (SM) are now part of everyday living. Medical and palliative care research could potentially benefit hugely from the data that SM generate on a daily basis, in particular in exploring sentiment in the wider public on important areas of healthcare, law and social policy. Yet there is still a dearth of researchers who are willing or perhaps able to critique articles that have used metrics involving social media, for instance, sentiment analysis.
Aim: Here, we aim to explore the many potential uses of SM. Firstly, SM can help with generating the right research question at the start of a project. There is virtually no topic in the world that has not been debated online on micro blogging sites or on video messaging sites. A detailed review of existing research on microblog sites like Twitter, or on more specific research-tailored social media outputs such a ResearchGate, can save replication of existing or parallel work. Asking research relevant questions on social media forums can provide an insight into areas of disagreement and uncertainty within key topic areas.

Summary: We analyse the following areas:

- Defining and narrowing down a research question using social media

$\mathrm{SM}$ as a means to recruit into clinical trials

- $\quad$ Ethics/Governance committees and Social Media and the role of Good Clinical Practice

- What the public are saying and information they are accessing on key topics via social media

- $\quad$ Researchers' ability to connect to other researchers anywhere in the world

- $\quad$ SM as a means to disseminate research more widely than just a journal audience

Conclusion: Many researchers are aware that patients and carers get their medical advice from the web and social media, so this provides a very valuable insight into why they think what they think Social media have democratised the way we access information, and this can create opportunities but also many hazards, and delving further into this paradox can be fascinating.

\section{Abstract number: P01-071}

Abstract type: Poster

Can a Care Using Virtual Reality Be Efficacious for Terminal Cancer Patients to Improve Various Symptom Burden? A Preliminary Study

Niki K. ${ }^{1,2}$, Okamoto Y. ${ }^{2}$, Maeda I. ${ }^{3}$, Mori I. ${ }^{3}$, Ishii R. ${ }^{4,5}$, Matsuda Y.4, Takagi

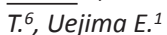

${ }^{1}$ Department of Clinical Pharmacy Research and Education, Osaka University Graduate School of Pharmaceutical Sciences, Suita, Japan, 2Department of Pharmacy, Ashiya Municipal Hospital, Ashiya, Japan, ${ }^{3}$ Gratia Hospice, Gratia Hospital, Mino, Japan, ${ }^{4}$ Department of Palliative Care, Ashiya Municipal Hospital, Ashiya, Japan, ${ }^{5}$ Department of Psychiatry, Osaka University Graduate School of Medicine, Suita, Japan, ${ }^{6}$ Department of Pharmainformatics and Pharmacometrics, Osaka University Graduate School of Pharmaceutical Sciences, Suita, Japan

Background: Some terminal cancer patients wish to "go to a memorable place". However, owing to various symptom burdens, these wishes are often difficult to realize.

Aim: To verify whether simulated travel using virtual reality (VR travel) has impact on patient symptom burden and satisfaction.

Methods: This was a prospective, single-arm study. Twenty participants with far-advanced cancer were recruited. Patients experienced a single session of VR travel using the VR software Google Earth VR ${ }^{\star}$. The primary endpoint was the changes in the Edmonton Symptom Assessment System (ESAS) scores before and after the VR travel. Secondary endpoints were the correlations between the VR experience types (participants who went to memorable places or not) and changes in ESAS scores.

Results: The average age (mean \pm SD) of the participants was $72.3 \pm 11.9$ years. Significant improvements were observed for pain, tiredness, drowsiness, dyspnea, depression, anxiety, and well-being scores (Table 1). Fifteen participants who went to memorable places indicated larger improvement in pain, tiredness, drowsiness, lack of appetite, dyspnea, 
ESAS scores of pre- and post-VR travel.

\begin{tabular}{|c|c|c|c|c|}
\hline $\begin{array}{l}\text { Outcome } \\
\text { Variables }\end{array}$ & $\begin{array}{l}\text { Before VR travel, } \\
\text { Mean } \pm \text { SD (Range) }\end{array}$ & $\begin{array}{l}\text { After VR travel, } \\
\text { Mean } \pm \text { SD (Range) }\end{array}$ & $P$ value & Cohen's d \\
\hline Pain & $2.35 \pm 2.25(0-6)$ & $1.15 \pm 2.03(0-6)$ & .005 & 0.574 \\
\hline Tiredness & $2.90 \pm 2.71(0-9)$ & $1.35 \pm 1.90(0-7)$ & .004 & 0.679 \\
\hline Drowsiness & $2.70 \pm 2.87(0-9)$ & $1.35 \pm 2.30(0-8)$ & .012 & 0.533 \\
\hline Nausea & $0.10 \pm 0.31(0-1)$ & $0.05 \pm 0.22(0-1)$ & .577 & 0.191 \\
\hline Lack of appetite & $2.85 \pm 3.66(0-9)$ & $1.60 \pm 2.60(0-8)$ & .063 & 0.404 \\
\hline Dyspnea & $1.74 \pm 2.73(0-8)$ & $0.35 \pm 0.99(0-4)$ & .022 & 0.684 \\
\hline Depression & $2.45 \pm 2.63(0-8)$ & $0.40 \pm 0.82(0-3)$ & .001 & 1.081 \\
\hline Anxiety & $2.60 \pm 2.64(0-8)$ & $0.80 \pm 1.51(0-6)$ & $<.001$ & 0.858 \\
\hline Well-being & $4.50 \pm 2.78(0-10)$ & $2.20 \pm 1.99(0-8)$ & $<.001$ & 0.976 \\
\hline
\end{tabular}

depression, anxiety, and well-being scores compared to those who did not. No participants complained of serious side effects.

Discussion: This preliminary study suggests that VR can be efficacious and safe for terminal cancer patients to improve symptom burden. Future studies should be conducted with larger samples including a comparison group.

Abstract number: P01-072

Abstract type: Poster

Neuromodulation of Pain by Small Fibre Matrix Stimulation in a Human Surrogate Model Using Capsaicin

Peuckmann-Post V., Dreher K., Rolke R.

Palliative Medicine, Medical Faculty RWTH Aachen University, Aachen, Germany

Background: Pain can be distressing, and its pharmacological management may be insufficient. Further strategies such as non-invasive neuromodulation using small fibre matrix stimulation seem promising in palliative care patients. However, mechanisms of action for this type of electrical stimulation and involved spinal cord projection pathways are unclear.

Aim: We used a human surrogate model for neuropathic pain known to induce central sensitization of dorsal horn nociceptive neurons (widedynamic-range neurons, WDR). We aimed to

(1) show involvement of WDR neurons by reducing capsaicininduced hyperalgesia after $4 \mathrm{~Hz}$ matrix stimulation, and

(2) to determine a sham frequency to be used in future trials.

Methods: We examined $\mathrm{N}=16$ healthy human subjects after conditioning by intracutaneous injection of $50 \mu \mathrm{g}$ capsaicin into the volar forearm, each at $4 \mathrm{~Hz}$ (test stimulus) and $40 \mathrm{~Hz}$ (control condition) matrix stimulation versus no stimulation using a single-blinded, balanced crossover design. Sensitivity to pin prick and light touch to the skin was tested by using quantitative sensory testing (QST) at different time points (30-180 minutes) to characterize mechanical hyperalgesia and allodynia. Statistical testing was done using ANOVA.

Results: Both 4 and $40 \mathrm{~Hz}$ matrix stimulation resulted in significant reduction of mechanical pain sensitivity (MPS) $(p<0.001)$ but not dynamic mechanical allodynia (DMA) compared to 'no stimulation'. Four $\mathrm{Hz}$ stimulation reduced mechanical pain sensitivity more than $40 \mathrm{~Hz}$. Conclusion:

(1) $4 \mathrm{~Hz}$ matrix stimulation was effective in reducing capsaicininduced mechanical hyperalgesia. This finding is consistent with the concept of matrix neuromodulation turning down hyperexcitability of spinal cord WDR neurons. It may be used by palliative care patients in addition to standard analgesic regimens.

(2) $40 \mathrm{~Hz}$ did not serve as an ideal sham stimulation. We suggest to use slightly higher frequencies when performing future RCTs.

\section{Abstract number: P01-073 \\ Abstract type: Poster}

Planning and Evaluating Engagement with Evidence Users

Tieman J. ${ }^{1}$, Lewis V. ${ }^{2}$, Erny-Albrecht $K .^{1}$

${ }^{1}$ Palliative Care, Flinders University, Bedford Park, Australia, ${ }^{2}$ Australian Institute for Primary Care \& Ageing, La Trobe University, Bundoora, Australia

Aim: Availability of palliative care evidence is a necessary, but not necessarily sufficient, condition to support evidence-based practice and evidence informed decision-making. CareSearch is an Australian palliative care evidence resource for health professionals and health consumers. The aim of this study was to develop an Engagement Framework to support increasing awareness and use of this resource.

Methods: Working with an external evaluator, CareSearch has designed an evidence-based framework to trial engagement with different user groups. The Engagement Framework was informed by an examination of different strategies and approaches to engagement and reviewed by the CareSearch advisory groups. The completed Engagement Framework outlines the stages of activities, scope of workshop materials, processes for recruiting and selecting workshop participants, and process and impact evaluation measures.

Results: The Engagement Framework is a structured approach comprising the following stages:

- Context statement for each user groups (aged care staff; allied health professionals; and patients and families)

- Recruitment of "exemplar" users for workshops to provide user perspectives on value and use of evidence

- Action Plans for each user groups for implementation through CareSearch

- Assessment of impact of the Action Plans, and

- Evaluation of effectiveness the Engagement Framework

Conclusions: While evidence retrieval and synthesis activities are critical, we also need to address how to support the use of evidence in practice. Creating a structured approach to engagement will not only enhance how CareSearch engages with intended users but provide us with the ability to evaluate the effectiveness of our engagement. 


\begin{tabular}{llllll}
\hline & & rest & CPT (-MT) & CPT (+MT) & $\begin{array}{c}\text { p-value } \\
(-M T \text { vs. }+ \text { MT) }\end{array}$ \\
\hline Average BP & {$[\mathrm{mmHg}]$} & $79.2 \pm 13.6$ & $89.7 \pm 11.9$ & $85.5 \pm 17.5$ & 0.000 \\
SV & {$[\mathrm{ml}]$} & $91.0 \pm 21,0$ & $85.5 \pm 18.3$ & $83.7 \pm 18.3$ & 0.034 \\
LF/HF & {$[-]$} & $1.90 \pm 2.93$ & $1.44 \pm 1.45$ & $0.90 \pm 0.44$ & 0.000 \\
Breath rate & {$[\mathrm{Hz}]$} & $12.6 \pm 4.7$ & $13.3 \pm 4.9$ & $14.2 \pm 6.2$ & 0.001 \\
\hline
\end{tabular}

LF, HF: low / high frequency domain of HR variability.

Abstract number: P01-074

Abstract type: Poster

Prioritising Evidence - Practice Gaps: Perspectives of Palliative Care Professionals

Tieman J., Damarell R.

Flinders University, Bedford Park, Australia

Background: As palliative care strengthens its evidence base, the potential for evidence -practice gaps grows. To ensure that relevant research evidence is used, we must understand and support processes that translate knowledge into practice.

Aims: To assess specialist palliative care clinicians' understanding of underused evidence, familiarity with knowledge translation strategies, and perceptions about palliative care that might contribute to less-thanoptimal research uptake in practice.

Methods: Palliative care physicians, specialist nurses and allied health completed an online survey distributed through Australia's peak bodies involved with palliative care. The survey contained a mixture of multiple choice and open-ended questions. Ethics approval was received from Flinders University (SBREC 7584).

Results: Forty-three clinicians responded (Allied Health: 35\%; Nurses: 33\%; Physicians: 23\%; Other: $9 \%$ ). All reported familiarity with the term 'quality improvement' but were less familiar with terms such as implementation science or diffusion of innovation.

Respondents identified a range of palliative care issues where they felt research evidence was not being used and other areas where they felt research evidence was needed. Audit and feedback, interactive workshops, and conferences were seen as the most effective mechanisms for instilling change.

$74 \%$ of respondents believed there are aspects of palliative care that make it more difficult than other healthcare areas to effect practice changes. These barriers to translation included variable practices within the specialty and general misconceptions of its purpose.

Conclusions: This study provided insights into perceived evidence-practice gaps within palliative care, reasons for inaction, and effective knowledge translation strategies. Despite a heterogeneity in responses, we believe there is a need for more strategic researcher-clinician collaborations for redressing front-line care issues.

Abstract number: P01-075

Abstract type: Poster

Effects of Music Therapy on Continuously Monitored Heart Rate, Blood Pressure and Cardiac Output - A Pilot Study

Malessa A. ${ }^{1}$, Steigleder T. ${ }^{1,2}$, Metzner S. ${ }^{3,4}$, Callenbach M. ${ }^{1}$, MuellerBohn J. ${ }^{5}$, Heckel M. ${ }^{1}$, Ostgathe C. ${ }^{1}$

${ }^{1}$ Department of Palliative Medicine, University Hospital Erlangen, Friedrich-Alexander University Erlangen-Nürnberg (FAU), Erlangen, Germany, ${ }^{2}$ Department of Neurology, University Hospital Erlangen, Friedrich-Alexander University Erlangen-Nürnberg (FAU), Erlangen, Germany, ${ }^{3}$ Centre for Interdisciplinary Health Research (ZIG), University of Augsburg, Augsburg, Germany, ${ }^{4} \mathrm{Clinic}$ for Psychosomatic Medicine and Psychotherapy, Otto von Guericke University Magdeburg,
Magdeburg, Germany, 5Leopold-Mozart Centre, University of Augsburg, Institute for Music Pedagogy, Music Therapy and Musicology, Augsburg, Germany

Background: Music therapy (MT) is widely used as non-medical treatment in palliative care. Its known effects are mainly reduction in pain perception and increase in well-being and relaxation, assessed by evaluation of the patients' subjective perception. The effects of MT on cardiovascular parameters like heart rate (HR), HR variability, stroke volume (SV) or blood pressure (BP) are much less well understood.

Aims: To assess which physiological parameters are influenced by MT and might serve as surrogate parameters in the evaluation of music therapy.

Methods: $\mathrm{N}=8$ healthy subjects ( 5 female, age 21-28) were examined by gold standard methods (6-channel ECG, impedance and continuous BP measurement). Cardiovascular response and activation of the autonomic nervous system were induced by the cold pressure test (CPT). Hand immersion into ice water was 2 minutes. A music therapist played on an instrument previously chosen by the volunteer (monochord, sansula or ocean drum). The music intervention started 2.5 min before the CPT and ended after $8 \mathrm{~min}$. Data were analysed by 2 -sided T-test with the statistics software SPSS.

Results: Verification of test procedure:

CPT vs. rest: significant increase $(p<0.05)$ in HR and systolic (sBP), diastolic (dBP) and average (aBP) blood pressure

Conclusion: MT reduces the activation of the sympathetic nervous system, as shown by LF/HF, most probably due to a relaxation effect. Average BP, SV and breath rate might be further parameters that are influenced by music therapy and be used also in palliative care patients to evaluate non-medical approaches.

Funding: This study is funded by the German Federal Ministry of Education and Research (16SV7695).

Abstract number: P01-076

Abstract type: Poster

Palliative Sedation in Bavarian Hospice Setting - Interpreting Estimated Sedation Rates. An Online Survey

Klein $C_{.}{ }^{1}$, Heckel M. ${ }^{1}$, Haas $N^{2}{ }^{2}$, Kulla A. ${ }^{3}$, Warter $C .{ }^{2}$, Ostgathe $C^{1}$

${ }^{1}$ Department of Palliative Medicine, University Hospital Erlangen, Friedrich-Alexander University Erlangen-Nürnberg (FAU), Erlangen, Germany, ${ }^{2}$ Master Program Medical Process Management, FriedrichAlexander University Erlangen-Nürnberg (FAU), Erlangen, Germany, ${ }^{3}$ Hospiz der Diakonie Erlangen, Erlangen, Germany

Background: Palliative sedation (PS) is regarded as part of specialised palliative care (PC) treatment. Previous data showed a rapid increase of PS in the last years. In Germany, hospices are institutions of longterm-care, usually run by nursing specialists. Medical service is provided by the family doctors of the patients (pt).So far, no information exists regarding the rates of different forms of PS (mild, deep intermittent (DI), deep continuous sedation until death (DCS)) provided in that setting.

Aims: This study explores the use of PS in Bavarian hospices. 
Methods: An online survey regarding the year 2017 inquired the number of pt, whether and in which form (mild, DI, DCS) PS was performed and its documentation and monitoring. The heads of the service of all Bavarian hospices $(n=19)$ were invited personally to take part in the survey (www.umfrageonline.com, enuvo $\mathrm{GmbH}$ ). Data was analysed descriptively (mean, relative frequency, standard deviation (SD)) using SPSS 21.0 (IBM Corp.).

Results: Of the 19 hospices in Bavaria, n=10 completed the survey. In 2017 the institutions treated between 91 and 152 pt (mean 121 pt, total 1213 pt). 3 out of 10 participants reported that no PS had been performed in 2017 because it had not been necessary. The other 7 participants stated that PS was performed in 3\% to $77 \%$ of all patients (mean $21 \%$ ). Most of the PS had been classified as mild sedation, only three participants reported having performed DCS (2;1;1 cases resp.). Rate of DCS in participating hospices ranged between 0 and $1.4 \%$ (SD $0.68 \%$ ); overall rate of DCS was $0.3 \%$. The use of specific documentation sheets for PS was reported by three of the four hospices with higher rates of PS

Conclusions: PS use varies in Bavarian hospices. Most PS is performed as mild sedation, DCS is extremely rare. Institutions with specific protocols often communicate higher rates of PS. The differentiation of mild /DI / DCS is helpful to understand reported data.

Abstract number: P01-077

Abstract type: Poster

Telephonic Communication in Palliative Care for Better Management of Terminal Cancer Patients in Rural India - An NGO Based Approach

Mandal N.

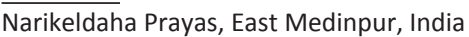

Aim: Due to financial incapability and absence of manpower poor families often fail to carry their advanced cancer patients to the nodal centres. This pilot study will explore whether communication by mobile phone can lessen this burden.

Method: Initially a plan was generated regarding management of an advanced cancer patient in a nodal centre at District Head Quarter. Subsequently every two week a trained social worker attached to nodal centre will follow up and give necessary advice and emotional support to the patients and their families through their registered mobile phone number. Patient's family were also encouraged to communicate with the team by phone in case of fresh complain and urgency in between.

Results: Since last one year, 193 cancer patients were contacted by mobile phone every two weeks to enquire about their difficulties. In 76\% of the situation trained social workers could give necessary advice by phone regarding management of their physical symptoms. Moreover patient's family were really overwhelmed by the emotional support offered by the team over phone. Only $24 \%$ of cancer patients has to attend the nodal centre for expert advice from Palliative Care specialists. Conclusion: This novel approach helped

* In providing regular physical and emotional support to the patients and their families.

* In significantly reducing the financial and manpower problems of carrying patients to the nodal units.

* In improve the quality of life of patients by continuous guidance.

More and more team members can take help of this new strategy for better communication and uninterrupted care.

Abstract number: P01-078

Abstract type: Poster

Which Decision Aids to Support Shared Decision Making Have the Potential to Improve Patient Outcomes in Palliative Lungand Colorectal Cancer Care: Results from a Systematic Review
Meijers M. ${ }^{1}$, Spronk I. ${ }^{1}$, Heins M. ${ }^{1}$, Francke A. ${ }^{1,2}$, Elwyn $G .{ }^{3}$, van Lindert A. ${ }^{4}$, van Dulmen S. ${ }^{1,5,6}$, van Vliet $L .{ }^{1}$

${ }^{1}$ Nivel (Netherlands Institute for Health Services Reserach), Utrecht, Netherlands, ${ }^{2}$ Department of Public and Occupational Health, Amsterdam Public Health Research Institute, Amsterdam UMC, Vrije Universiteit Amsterdam, Amsterdam, Netherlands, ${ }^{3}$ The Dartmouth Institute for Health Policy and Clinical Practice, Darthmouth College, Hanover, United States, “Lung Disease, UMC Utrecht, Utrecht, Netherlands, ${ }^{5}$ Radboud University Medical Center, Nijmegen, Netherlands, ${ }^{6}$ Faculty of Healh and Social Sciences, University College of Southeast Norway, Drammen, Norway

Background: Shared decision-making (SDM) is not always commonplace in palliative lung- and colorectal cancer care, albeit associated with positive patient outcomes. Initiatives to improve SDM are needed, but which decision aids (DAs) are available to support SDM and whether they have the potential to improve patient outcomes is unclear.

Aim: To determine the availability and effectiveness of DAs to support SDM in palliative lung- and colorectal cancer care.

Methods: DAs were collected via a systematic literature search, via experts and via internet searches. Pubmed/Cinahl/Embase/Psychinfo were searched $(03 / 2018)$ for studies published since 2006 focusing on (i) SDM with adult lung/colorectal cancer patients in the palliative phase, (ii)the development/evaluation (on patient outcomes) of DAs to improve information-provision, decision-making, eliciting (treatment) preferences. Data from included DAs was extracted, and a GRADE (quality of evidence) and IPDAS (quality of the tool) score determined.

Results: 12 DAs were included (8 DAs identified via the 1086 uniquely identified articles, and 4 DAs via internet searches/experts). 4 DAs were lung specific, 4 colorectal specific and 4 disease-generic. Most DAs were applicable across the disease trajectory, and usable for all decisions or the choice for supportive care with/without anti-cancer therapy. Effects on patient outcomes were generally weakly positive (if present), with very low-moderate GRADE evidence and a broad range of IPDAS scores (1-7). Discussion: Despite its recognized importance, few DAs are available to support SDM in palliative lung-and colorectal cancer care. Recommended use in clinical care of detected DAs is restricted, as effects were limited, and many DAs were not up-to-date and/or accessible. Further development, evaluation and implementation of existing/new DAs is recommended, with a focus on DAs that are being kept up-to-date with the guidelines and to be implemented in the direct clinical consultation.

Abstract number: P01-079

Abstract type: Poster

Difficulties in Communication between Patients, Family Caregivers and Health Professionals in Palliative Care Lemus P. ${ }^{1}$, Carreño S. ${ }^{1}$, Arias M. ${ }^{2}$

1Faculty of Nursing, Universidad Nacional de Colombia, Bogota, Colombia, ${ }^{2}$ Faculty of Nursing, Universidad de Antioquia, Medellin, Colombia

Aim or goal of the work: With chronic diseases on the rise, there is a growing demand for palliative care. The global landscape of the integration of palliative care into healthcare systems is incipient, which leads to a lack of social awareness of this reality and, consequently, to communication failures, particularly a conspiracy of silence. The aim of this paper was to analyze the concept of conspiracy of silence in palliative care.

Design, methods and approach taken: Literature review was done using Walker and Avant method of concept analysis. Review and synthesis of literature supported the analysis process. Forty-seven articles were analyzed.

Results: The results showed that the conspiracy of silence in palliative care is a communication failure, typical of limited life expectancy prognosis, and involves patients, their families, and health care teams. Lack 
of autonomy, overburden, family malfunctioning and coping, and health care dehumanization are consequences of the conspiracy of silence in palliative care.

Conclusion / lessons learned: Scales to measure this concept as well as interventions that consider important factors identified in this analysis are needed.

Abstract number: P01-080

Abstract type: Poster

Family Members' Experiences of Family Support Conversation in Specialist Inpatient Palliative Care Units. Part1. “ ...Being in Uncertainty..."

El Aamraoui N. ${ }^{1,2}$, Seiger Cronfalk B. ${ }^{2}$

${ }^{1}$ Stockholms Sjukhem Foundation, Stockholm, Sweden, ${ }^{2}$ Ersta Sköndal

Bräcke College, Stockholm, Sweden

Background: The aim of palliative care is to improve the quality of life for patients and their families and to offer them support. Strategies for support can vary but most common is conversations. Conversations are held to inform patients and family members about treatments and progression of illness. During palliative care family members need for support is also emphasized as family support conversation is offered. Still, little is known about family members experiences of such conversations.

Aims: The aim was therefore to describe family members' experiences and expectations of one specific family support conversation during ongoing palliative care.

Methods: A qualitative study with an explorative design. Fifteen individual interviews were carried out with family members after they had participated in a family support conversation within specialist palliative inpatient care. The interviews were analysed using Sally Thorne's interpretive description.

Results: Family members' expectations and experiences varied and are presented in two patterns: Family members' need to gain an understanding of the situation and Factors that can affect their experience of the family support conversation. The overall experience was that family support conversations were assessed as being positive, however, there were some negative aspects or reflections regarding the expectations before the conversations.

Conclusion: The family support conversation was important and appreciated as it was reported that other healthcare settings lacked this routine. The conversation can offer family members support while managing their own situation and in preparation for the patient's impending death. The results provide insight to what family members need and want to be asked by professionals during a family support conversation in palliative care. The results could be applicable in other care settings as well.

Abstract number: P01-081

Abstract type: Poster

Dealing with Desire to Die in Palliative Care: Development of a Semi-structured Interview Schedule

Kremeike K., Frerich G., Romotzky V., Bostroem K., Dojan T., Galushko

M., Voltz $R$.

Center for Palliative Medicine, University Clinic of Cologne, Cologne, Germany

Background: A desire to die (DD) is frequent in palliative care. However, uncertainty remains as to the appropriate therapeutic response. To support health practitioners' (HPs) reactions to a patient's DD, a training program and a first draft of a semi-structured clinical interview schedule with prompts (CISP) has been developed.

Aims: To revise and consent the CISP to deal with a DD for use in everyday clinical practice.
Methods: Within a sequential mixed methods design, 16 palliative patients were invited to participate in semi-structured interviews. Data was analyzed using qualitative content analysis. Moreover, 377 (inter-) national experts and patient representatives were asked to evaluate the CISP in a two-step Delphi survey (A priori consensus criterion: $\geqslant 80 \%$ agreement).

Results: Eleven palliative patients participated in individual in-person interviews and stressed the HP-patient-relationship, the framing of conversation and possible support from external experts to be central aspects of DD conversations.

After modifying the CISP based on these findings, 210/200 experts (Delphi round I/II) rated ten CISP domains:

1) actively building the relationship,

2) proactively addressing $D D$,

3) classification of DD,

4) background and meaning,

5) functions of DD,

6) closure of discussion

7) after discussion;

8) HPs own attitudes and emotions,

9) self-protection and

10) further recommended action.

Except for "proactively addressing DD", all domains reached consensus in Delphi round I. Based on feedback in round I, this part and three further domains with agreement $<90 \%$ were modified. A new domain "usage notes" was added. During round II, "proactively addressing DD" reached an agreement of $83.5 \%$. All other domains reached agreement levels $>90 \%$.

Conclusion/discussion: The CISP can be helpful in identifying DD in palliative patients, proactively addressing and therapeutically approaching them in an adequate manner.

Funding: German Federal Ministry of Education and Research.

Abstract number: P01-082

Abstract type: Poster

End-of-Life Communication in Advanced Cancer: International Trends from a Mortality Follow-back Study between 2009 and 2014

Verkissen M.N. ${ }^{1}$, Penders Y.W.H. ${ }^{2}$, Onwuteaka-Philipsen B.D. ${ }^{3}$, Moreels

S. ${ }^{4}$, Donker G.A. ${ }^{5}$, Vega Alonso A.T. ${ }^{6}$, Van den Block L. ${ }^{1}$, Deliens L. ${ }^{1,7}$ ${ }^{1}$ End-of-Life Care Research Group, Department of Family Medicine and Chronic Care, Vrije Universiteit Brussel (VUB) \& Ghent University, Brussels, Belgium, 2Epidemiology, Biostatistics and Prevention Institute, University of Zürich, Zürich, Switzerland, ${ }^{3}$ Amsterdam Public Health Research Institute, Department of Public and Occupational Health, Expertise Center for Palliative Care, VU University Medical Center, Amsterdam, Netherlands, ${ }^{4}$ Sciensano, Epidemiology and Public Health, Health Services Research, Brussels, Belgium, ${ }^{5}$ NIVEL Primary Care Database - Sentinel Practices, Netherlands Institute for Health Services Research, Utrecht, Netherlands, ${ }^{6}$ Public Health General Directorate, Regional Ministry of Health (Dirección General de Salud Pública, Consejería de Sanidad), Castile and León, Valladolid, Spain, ${ }^{7}$ Department of Medical Oncology, Ghent University Hospital, Ghent, Belgium

Background: End-of-life communication as a key component of good palliative care has received increasing policy attention over the last years in Europe.

Aim: To examine, in three European countries, trends in the frequency of conversations about end-of-life care with cancer patients in general practice.

Methods: Mortality follow-back survey among GPs in representative epidemiological surveillance networks in Belgium (BE) and the Netherlands (NL) in 2009, 2010, 2013 and 2014, and in Spain (ES) in 
2010, 2011, 2013 and 2014. Using a standardized form, GPs reported every deceased patient in their practice. GPs were asked if the diagnosis, preferences for medical treatment in the final phase of life and preferences for a proxy decision-maker had been discussed with the patient. We included all non-sudden cancer deaths.

Results: A total of 2306 decedents were studied (BE: 1233, NL: 729, ES: 344). BE showed a significant increase in the proportion of people with whom the diagnosis was discussed between 2009 and 2014 (84-94\%), which was largest in those aged $\geqslant 85$ and those who lived in a long-term care facility in the last year of life ( 25 percentage point in both groups). Awareness of a preference for medical treatment increased significantly in BE (41-53\%) and NL (62-70\%) between 2009 and 2014. The largest increase was seen in those who died in a palliative care unit or hospice in both BE and NL (21 and 34 percentage point, respectively). BE (20$28 \%)$ and NL (32-52\%) showed a significant increasing trend in awareness of a preference for a proxy decision-maker, which in BE was largest in those who died at home (21 percentage point), and in NL in males (35 percentage point). ES did not show a significant trend for any of these variables over the years.

Conclusion: The increases in the frequency of GP-patient communication on end-of-life care related topics show that significant change is possible in a relatively short time-span, although international differences seem to persist over time.

\section{Abstract number: P01-083}

Abstract type: Poster

\section{An Original Qualitative Study of Patients' Perspective on} Expression of End-of-Life Wishes during Interaction with Nurses in Oncology

Oustalet $A$.

Hôpital Lariboisière Fernand-Widal, Equipe Mobile de SoinsPalliatifs, Paris, France Presenting author email address: anja.oustalet@aphp.fr

Facing cancer, the fear of dying and the desire to be cured do, despite very adverse side effects, usually lead patients to accept treatments. In France the incidence of cancer is 399.500 . Several cancers have become "chronic" diseases, but 150.000 people die from it every year. How and when the turning point in the balance benefit of treatment versus quality of life occur in these patient's trajectory, is little explored by health professionnels.

As we consider nurses to be sentinels assessing the patient's symptoms and the meaning of the treatments pursued, we aimed to explore patients' points of view on expressing their end of life wishes during the informal exchanges in repeated moments of nursing.

In our single-centre, qualitative phenomenological study, an investigator conducted face-to-face interviews with 6 solid cancer tumour inpatients. Their mean age was 68 . The data collected, using five open questions, was analysed by means of the Framework Method.

Six main themes emerged from the analysis. Our result found the patients point of view to express:

1. Refusal to speak of wishes for end of life

2. Difficulties in anticipating their future

3. Preferring the doctor as interlocutor

4. The quality of exchanges between patients and professionals to be impacted by organisational challenges

5. Relying on nurses for practical help

6. To value opportunities for informal exchanges with nurses, it enhance trust and improve quality communication.

Although one patient related the importance of exchanges with the nurse coordinating her trajectory, our study revealed that most patients do not think of nurses as a health professional with whom they can discuss their end of life wishes. To enhance patients' possibility to express their end of life wishes nurses should develop communication skills on the subject. When patients do express end of life wishes during informal exchanges, nurses should advocate them at interdisciplinary meetings.

Abstract number: P01-084

Abstract type: Poster

Making a 'Do Not Resuscitate Decision' on Twitter - A Patient's

Story

Cass K. ${ }^{1}$, Taubert M. ${ }^{2}$

${ }^{1}$ Red Sock Campaign, Cardiff, United Kingdom, ${ }^{2}$ Palliative Medicine Department, Cardiff University School of Medicine, Cardiff, United Kingdom

Background: Cancer patient Keith Cass (KC) met his palliative care consultant Mark Taubert (MT) in 2017. KC has advanced prostate cancer, and it has spread to nearly all the bones in his body. Over several consultations, they talked about KC's wishes should he become very unwell. When MT first mentioned do not attempt cardiopulmonary resuscitation (DNACPR) decisions, and what KC's views on this topic might be, the patient was taken aback. MT explained that many people have a rosetinted view of how successful CPR is, perhaps because of the way it is portrayed on television.

Intervention: MT suggested looking at the TalkCPR website \& videos on http://talkcpr.wales and to search for the \#TalkCPR hashtag on Twitter, to understand more about CPR in palliative care settings. KC followed this advice \& read about what the procedure involves. He was surprised at the low success rates of CPR for patients when cancer has spread.

Lessons learned: At subsequent visits to see MT, KC was clear that he wanted a DNACPR form to be filled in. He specified his views, in particular stating that he would still be keen to be considered for anticancer treatments. The main influence on his views on CPR was the poor success rate and the body damage that would make (unlikely) survival with quality of life almost impossible.

$\mathrm{KC}$ keeps the form with him at all times, and even took it on a holiday to Barbados. He wants healthcare professionals to be aware of his prior wishes, and has told his (grown up) children about the decision. At the time of writing, he is receiving chemotherapy and radiotherapy.

Conclusion: CPR in palliative care is difficult to discuss and some people may find it distressing, but doing so early and having resources online and via social media, facilitates well informed patients/carers, who can then have an intelligent conversation with their healthcare professionals. KC has since written a patient experience article about this for the British Medical Journal.

\section{Abstract number: P01-085 \\ Abstract type: Poster}

\section{The 6S Dialogue Tool to Facilitate Person-centered Palliative}

\section{Care}

Henoch.$^{1}{ }^{1}$ Österlind J. ${ }^{2}$

${ }^{1}$ Sahlgrenska Academy, Institute of Health and Care Science, University of Gothenburg, Göteborg, Sweden, ${ }^{2}$ The Department of Health Care Sciences, Palliative Research Centre, Ersta Sköndal University College and Ersta Hospital, Stockholm, Sweden

Background: Palliative care must be planned in accordance with patients' needs, beliefs and preferences. The 65 person-centered palliative care model aims to co-create care together with patient and family in order to achieve good quality palliative care and a peaceful death for patients. The model includes six concepts that will facilitate to plan a person-centered care for patients in palliative care. The concepts are Self-image, Symptom relief, Self-determination, Social relationships, 
Synthesis, and Strategies. The 6S Dialogue Tool was elaborated to concretize the concepts.

Aim: To explore if the 6S Dialogue Tool covers the meaning of the $\mathrm{S}$-concepts in the $6 \mathrm{~S}$ person-centered palliative care model.

Methods: This is a qualitative study where data was collected with structured questions and patients responded in an open-ended format to the 15 questions in the 6S Dialogue Tool. Forty-six patients in palliative care services in Sweden responded to the 6S Dialogue Tool from May 2015 to August 2016. Responses were analyzed with qualitative content analysis.

Results: Six categories related to the $6 \mathrm{~S}$ concepts were formulated: Selfimage was illustrated of Maintaining everyday life, Symptom relief was related to Challenges in everyday life, Self-determination concerned Maintaining control, Social relations was illustrated by Maintaining selected relations, Synthesis was related to Appraisal of life, and Strategies was illustrated by Appraisal of future.

Conclusion: The responses to the 6S Dialogue Tool questions illuminated the intent and meaning of the 6S-concepts. The 6S Dialogue Tool questions are suitable for obtaining patients' preferences and could be used as an approach in palliative care. Nurses should integrate both 6S-concepts and questions in their approach to obtain the patient's own view of the situation and make it possible to co-create palliative care in dialogue with the patient and improve the possibilities for patients to have an appropriate death

Abstract number: P01-086

Abstract type: Poster

What Influences a Patient's Decisions to Receive Palliative Care as Part of Advance Care Planning? Qualitative Perspectives from Advanced Cancer Patients, Families and Healthcare Professionals in Taiwan

Lin C.-P. ${ }^{1}$, Evans C.J. ${ }^{1,2}$, Koffman J. ${ }^{1}$, Hsu S.-H. ${ }^{3}$, Sheu S.-J. ${ }^{4}$, Harding R. ${ }^{1}$ ${ }^{1}$ Cicely Saunders Institute of Palliative Care, Policy and Rehabilitation, King's College London, London, United Kingdom, ${ }^{2}$ Sussex Community NHS Foundation Trust, Sussex, United Kingdom, ${ }^{3}$ Centre of Research and Development in Community Based Palliative Care, Taipei City Hospital, Taipei, Taiwan, Republic of China, ${ }^{4}$ Institution of Community Health Care, School of Nursing, National Yang Ming University, Taipei, Taiwan, Republic of China

Background: Advance care planning (ACP) is a widely accepted component in palliative care (PC) delivery. However, the concept of ACP is largely derived from Western countries. It remains unclear how well the ACP works in delivering PC in other cultures.

Aim: To explore what influences patient's decisions for PC as part of ACP from perspectives of advanced cancer patients, families and healthcare professionals in northern Taiwan.

Method: In-depth semi-structured qualitative interviews with advanced cancer patients in the last 12 months of life, their families, and healthcare professionals from inpatient oncology and hospice units. Purposive sampling identified participants based on key characteristics including age, gender, cancer type, education level, and disease prognosis. Thematic analysis was conducted. Analytical rigour was enhanced by dual coding. Investigator triangulation was performed for consensus and agreement.

Results: 15 patients, 15 family members, and 15 multidisciplinary healthcare professionals were interviewed. Three types of patient decision-making to receive $P C$ were identified: 1)Receiving $P C$, patients actively chose PC to lessen suffering, reduce medical resource waste and avoid becoming a burden, particularly to family and society; 2)Ambiguity regarding receiving $\mathrm{PC}$, patients were unsure about the relevance of $\mathrm{PC}$ either due to poor knowledge and support system, pacifying family members and physicians, or wishing to complete unfinished business; 3)Inability to make decisions to accept $P C$, patients were unable to make decisions due to medical dominance, distrustful physician/patient relationship, or reduced mental capacity.

Conclusion: Receiving PC as part of ACP in Taiwan is influenced by three forms of decision-making. The presence of power and knowledge imbalance between patient, families and medical staff and distrustful relationship represent key barriers. Strategies to overcome them are required to improve patients and family outcomes.

Abstract number: P01-087

Abstract type: Poster

Speak Now or Forever Hold your Peace? The Extent of Patient Involvement In End-of-Life Care Decision-making at the Emergency Department

Fong E.L.S. ${ }^{1}$, Nadarajan G.D. ${ }^{2}$, Lian W.Q.S. ${ }^{2}$, Ong E.H.M. ${ }^{2}$

${ }^{1}$ Lee Kong Chian School of Medicine, Nanyang Technological University, Singapore, Singapore, ${ }^{2}$ Department of Emergency Medicine, Singapore General Hospital, Singapore, Singapore

Background: Visits by patients with chronic diseases to the emergency department (ED) during their end-of-life (EOL) period are distressing to patients and their loved ones. EOL care discussions (such as decisions on extent of care and resuscitation) are often omitted due to the fast-paced environment of the ED. The prevalence of EOL care discussions sets the baseline for future studies on patient-centred care but has never been studied locally.

Aim: To study the discussion and documentation of EOL care decisions of imminently dying patients presenting to the ED.

Methods: A retrospective study was done on a 3-month period in a tertiary hospital ED. Patients with a documented diagnosis of a chronic disease and die within $72 \mathrm{~h}$ from presentation to the ED were included. EOL discussion characteristics were analysed. Outcomes of interest include proportion of patients with prior EOL care decisions documented and proportion with such discussions initiated in the ED.

Results: Of the 87 deaths studied, $64.3 \%$ were males and the median age was 76 . Only $35.6 \%$ had discussions on EOL care decisions being made with the ED physician and $31.0 \%$ had documentation of such pre-made decisions.

$51.7 \%$ had neither EOL discussions in the ED nor documented EOL care plans previously made. Only 2 patients (2.30\%) had prior advanced care planning (ACP)* discussed and documented.

The median time from registration to death was $13 \mathrm{~h} 26 \mathrm{~min}$.

Conclusion: The majority of patients did not have documented prior EOL decisions, and efforts should be made to encourage such discussions at the point of diagnosis of a life-limiting chronic disease. The ACP is a useful but underutilised tool to facilitate such discussions. ED staff should be trained to initiate such discussions if these are not documented. Given the short survival time, proper EOL care discussion can ensure comfort of patients and loved ones.

*ACP is a structured documentation of one's care preferences in the event of emergencies in Singapore.

Abstract number: P01-088

Abstract type: Poster

Communicating about Death with Adults with Intellectual Disabilities (ID): A UK-wide Survey of Staff Working in Supported Living and Residential Care Settings

Tuffrey-Wijne I. ${ }^{1}$, Lam C. ${ }^{2}$, Todd S. ${ }^{3}$, Bernal J. ${ }^{3}$, Finlayson J. ${ }^{4}$, Taggart L. ${ }^{5}$

${ }^{1}$ Faculty of Health, Social Care and Education, Kingston University \& St George's University of London, London, United Kingdom, ${ }^{2}$ Kingston University \& St George's University of London, London, United Kingdom, ${ }^{3}$ University of South Wales, Cardiff, United Kingdom, ${ }^{4}$ Glasgow Caledonian University, Glasgow, United Kingdom, ${ }^{5}$ University of Ulster, Ulster, United Kingdom 
Background: Previous studies show that many people with ID are not helped to know about death.

Aim: To investigate the extent to which staff talk about death and dying with people with ID who are who are terminally ill or bereaved, and to explore staff needs

Method: Support staff working in supported living and residential settings completed an online survey ( $n=690,64 \%$ response rate). Those who reported that a client had died during the past 12 months ( $n=111)$, was currently terminally ill $(n=41)$, or had been bereaved in the past 12 months $(n=201)$ were asked detailed questions about this person, including their communication. Analysis included descriptive statistics and Chi-squared tests.

Results: Over half of people with ID who were terminally ill were told about their illness, although only $18 \%$ were told that they would die of it; around one in five were involved in advance care planning. The majority of all respondents felt confident talking about death with someone with ID (58\%), working with bereaved clients $(68 \%)$ or working with terminally ill clients (57\%). A third of staff who described supporting a client who had died or was terminally ill or had been bereaved $(n=313)$ found the experience distressing. Most (71\%) had felt well supported in coping with the situation, especially by managers and outside professionals. Around a quarter of all staff had received training in end-of-life and/or bereavement; training levels were slightly higher for those who had supported a terminally ill person.

Discussion: Working with dying or bereaved clients can be distressing, with staff needing significant support. This was an experienced cohort with relatively high levels of staff confidence. Low levels of staff training suggest that confidence is partly due to experiential learning and support. Despite this, communication challenges remain, especially for people with ID with lower levels of understanding; therefore, it appears that there are unmet staff training needs.

Abstract number: P01-089

Abstract type: Poster

Communicating with Patients with Limited Prognoses: Training Skills Is Not Enough

Apondo E. ${ }^{1}$, Handtke V. ${ }^{1}$, Villalobos M. ${ }^{1}$, Siegle A. ${ }^{1}$, Thomas M. ${ }^{1}$, Wensing M. ${ }^{2}$, Krug K. ${ }^{2}$, Deis N. ${ }^{3}$, Juenger J. ${ }^{3}$

${ }^{1}$ Thoraxklinik Heidelberg, Heidelberg, Germany, ${ }^{2}$ Heidelberg University Hospital, Heidelberg, Germany, ${ }^{3}$ The German National Institute for State Exams in Medicine, Pharmacy and Psychotherapy, Mainz, Germany

Background: Current concepts for bettering physicians' communication when breaking bad news aim at improving patient satisfaction by training predetermined skills. A few authors have argued against the prescriptive technicality of a skills-based approach. Studies on difficult consultations from physicians' perspective remain scant and focus on behavioural changes in consultations, largely neglecting their cognitive underpinnings. Aims: To explore how physicians experience the task of fostering hope while delivering bad news at a Comprehensive Lung Cancer Centre.

Methods: Passive observation of 13 consultations, field notes and assessment using the Breaking Bad News Assessment Scale. Semistructured face-to-face interviews with 6 physicians, descriptive phenomenological approach, coding and analysis using the Framework Method.

Results: Four major themes were identified:

1) oncologists have diverse concepts and beliefs about hope's foundations, sources and roles;

2) physicians' reflections on personal experiences and previous consultations impact whether and how prognosis is discussed;

3) physicians forge hope with patients as opposed to merely giving the patients hope;

4) goals set before consultations lead to feelings of success or distress in physicians when reached or missed.
Discussion: This study develops a theory that concretely shows how the perspectives of physicians can affect how they approach difficult consultations. Previous experiences with patients mould and inform the cognitive approaches of physicians to consultations and affect whether and how they communicate prognoses and foster hope. Communication should be more than a skill aimed at reaching patient-based indicators. Incorporating physicians' experiences is an important step in creating a concept that not only changes the behavior of professionals in consultations but also helps physicians creatively find and share the core essence of their relational interactions with patients.

Abstract number: P01-090

Abstract type: Poster

Dealing with Desire to Die in Patients with Incurable Cancer: Recommendations of the German S3 Guideline Palliative Medicine

Kremeike K. ${ }^{1}$, Anne P. ${ }^{1}$, Simon S. ${ }^{1}$, Bausewein C. ${ }^{2}$, Voltz R. ${ }^{1}$, Lindner R. ${ }^{3}$, "Working Group Desire to Di", of the German Guideline Group Palliative Care

${ }^{1}$ Center for Palliative Medicine, University Clinic of Cologne, Cologne, Germany, ${ }^{2}$ University Clinic Munich, Munich, Germany, Institute for Social Work, University Kassel, Kassel, Germany

Background: Desire to die (DD) is not uncommon in palliative patients. Medical advance and improved therapy options do not prevent these wishes, but on the contrary a lot of patients think about their future and ask about the meaning of life. Health practitioners are often confronted with these thoughts.

Aims: To present the current national and international state of knowledge and experience in dealing with DD and to offer orientation and assurance in responding to it in an appropriate manner.

Methods: Development of evidence and consensus based recommendations with the participation of more than 50 professional societies, in particular based on expert input of the German Association for Suicide Prevention and the German Society of Psychiatry, Psychotherapy and Neurology.

PubMed, Embase, and Cochrane Library databases were systematically searched for relevant publications, and selected ones were assessed as recommended by the Scottish Intercollegiate Guidelines Network. Guideline recommendations were developed by the expert panel employing a prescribed consensus process.

Results: The expert panel agreed to 19 statements and recommendations dealing with $D D$ issues like differential diagnosis, potential background and meaning, how to deal with DD, possible options for action and legal framework. The handling of DD within the treatment team, possible support from external experts and the involvement of relatives are also covered in the guideline.

Conclusion / Discussion: The guideline aims to support decision-making in practice and provides systematically developed recommendations on the basis of the best possible evidence and clinical experience of a large number of experts. The recommendations concerning DD aim at helping with (proactively) addressing potential DD as well as dealing with them. Therefore, they should be considered as a contribution to the advancement of multi-professional competence in palliative care.

Funding: German Guideline Program in Oncology (GGPO).

Abstract number: P01-091

Abstract type: Poster

“Lower-Intensity Care Plan?” Searching for a Standard Definition of "Comfort Care"

Kelemen A. ${ }^{1}$, Smith J. ${ }^{2}$, Groninger H. ${ }^{1}$

${ }^{1}$ Medicine, Georgetown University Medical Center, Washington, United States, ${ }^{2}$ Health Education, Marymount University, Arlington, United States 
Background: "Comfort care" is a specific term widely applied throughout clinical literature, organizational policy, and research to indicate some framework of end-of-life care. However, no standard definition of "comfort care" exists, potentially engenders semantic misunderstandings that negatively impact clinical care and communication between providers and patients/families. How is the term "comfort care" applied in clinical literature?

Objective: To understand specific language used to describe "comfort care" in existing clinical literature and to determine any common semantic elements in these definitions.

Methods: We searched PubMed and Embase databases over the period of calendar years 2016 and 2017 for the search term "comfort care." Investigators independently reviewed all publications for "comfort care" term use and, when applicable, performed content analysis on publications that provided any definition of the term "comfort care."

Results: We reviewed 85 unique publications. Four were excluded because the manuscripts did not actually contain the term "comfort care." Of the remaining 81 publications, categories included original research $(n=51)$, case reports (11), commentaries (10), clinical reviews (4), study protocols (3), 1 clinical practice guideline and 1 letter to the editor. Authors provided some kind of definition of the term "comfort care" in 12 publications. Content analysis of these 12 associated definitions revealed two common themes:

a) medical focus on physical symptom management and,

b) reducing/eliminating disease-modifying therapies. Only 2 definitions included any non-medical or non-pharmacological elements ascribed to "comfort care." None included any attention to psychosocial-spiritual interventions as part of "comfort care."

Conclusion: In clinical literature, the term "comfort care" is widely applied but rarely clearly defined. Definitions provided overwhelmingly focus on symptom management and disease-modifying therapies.

Abstract number: P01-092

Abstract type: Poster

Implementation of a Comprehensive Interprofessional Communication Approach for Cancer Patients with Limited Prognosis - A Process Evaluation

Bossert J. ${ }^{1}$, Villalobos M. ${ }^{2}$, Siegle A. ${ }^{2}$, Hagelskamp L. ${ }^{2}$, Jung $C^{2}{ }^{2}$, Deis ${ }^{3}{ }^{3}$, Jünger J. ${ }^{3}$, Wensing M. ${ }^{1}$, Thomas M. ${ }^{2}$, Berger S. ${ }^{1}$, Krug K. ${ }^{1}$

${ }^{1}$ Dept. of General Practice and Health Services Research, University Hospital Heidelberg, Heidelberg, Germany, ${ }^{2}$ Thoracic Oncology, University Hospital Heidelberg, Heidelberg, Germany, ${ }^{3}$ Institut fuer medizinische und pharmazeutische Pruefungsfragen, Mainz, Germany

Background: Sustaining change in service provision when introducing new care concepts into routine palliative care needs to be facilitated by appropriate implementation strategies. Process evaluation can contribute material for long-term sustainability. An innovative patient-centered concept, the Milestone Communication Approach (MCA) has been developed for cancer patients with a limited prognosis ( $<12$ months). Implementation of the MCA was facilitated by an interprofessional communication training for physicians and nurses based on a detailed manual to support implementation in routine care. Training and implementation in one hospital were accompanied by a formal process evaluation.

Aim: To explore acceptance and fidelity during implementation of the MCA.

Methods: Physicians' and nurses' perspectives on the concept of the MCA, their interprofessional training and the implementation process were assessed in qualitative semi-structured interviews. Conversation records of patient encounters were analyzed for implementation fidelity. Results: Preliminary results show medium to high implementation fidelity to the MCA in patient encounters, e.g. therapy $(91-100 \%)$ and organization issues (41-82\%). Participants reported a reduction of physician workload and improved interprofessional collaboration. However, implementation difficulties in clinical care included logistical challenges, e.g. coordinating schedules between service providers and patients. Communication challenges, e.g. discussing poor prognosis and advance care planning, occurred in more than $70 \%$ of patient encounters.

Conclusion: Acceptance of MCA among health professionals increased during implementation. Fidelity of the implementation also improved. However, organizational and training adaptions in the MCA as a consequence of the results were identified to improve long-term sustainability. Funding: Federal Ministry of Health (1504-54401), National Center for Tumor diseases (NCT 3.0, G835), Germany.

Abstract number: P01-093

Abstract type: Poster

Talking about End-of-Life in the Hospital - What is Going on? A Qualitative Case Study Bergenholtz H. ${ }^{1}$, Missel M. ${ }^{2}$, Timm H. ${ }^{3}$

${ }^{1}$ Holbaek Hospital and REHPA (Knowledge Centre for Rehabilitation and Palliative Care), University of Southern Denmark, Holbaek, Denmark, ${ }^{2}$ Rigshospitalet, Copenhagen, Denmark, ${ }^{3}$ REHPA (Knowledge Centre for Rehabilitation and Palliative Care), University of Southern Denmark, Copenhagen, Denmark

Background: End-of-life (EOL) conversations in the hospital should serve the purpose of giving patients the opportunity to deal with future treatment options in advance, and help them clarify their values and wishes before it becomes relevant to make decisions about treatment.

However, it is known that EOL conversations are not performed systematically. This may mean that patients and their relatives do not talk about EOL issues. There is a lack of knowledge about whether this is the case. Furthermore, there is a lack of knowledge about who is responsible for conducting these conversations, and when and under what circumstances they are conducted.

Aim: The purpose of this study was to explore the existing practices with EOL conversations in an acute care hospital setting.

Methods: The study was a descriptive qualitative case study, which included:1. Participatory field studies in the pulmonary medical and surgical wards (two weeks each ward); 2 . Four focus group interviews with health care professionals $(n=14)$ in an acute care hospital. The focus of the interviews was on discovering who tends to be offered EOL conversations in practice, what was the content of the conversations and how are they experienced by the health care professionals.

Findings: The findings showed that EOL conversations are part of daily clinical life, but are conducted unsystematically, with unclear roles, a lack of competencies and under poor environmental and organizational circumstances.

The conversations were initiated by an intuitive sense and the content of the conversations often refers to a "here-and-now" disease status as well as the treatment level for the patient. To a smaller extent, the conversations included thoughts and wishes of the patient and relatives concerning EOL. Patients are often waiting for health care professionals to start the conversation; thus, it becomes the task of professionals to initiate these conversations.

Abstract number: P01-094

Abstract type: Poster

Cross-cultural Education in Palliative Care

Semlali I., Weber 0. , Singy $P$.

Psychiatrie de Liaison, CHUV, Lausanne, Switzerland

Background: Despite the increase of cultural diversity among patients in palliative care, few studies address the challenges relating to clinical 
interactions between migrant patients and clinicians as well as the training needs of the latter.

Aims: This study focuses on caregivers in palliative care. It explores the difficulties they perceive to communicate with linguistically and culturally diverse patients and examines their interest in cross-cultural education.

Methods: We sent an online-survey to every caregiver working in specialized palliative care in Switzerland (French and Italian speaking parts). The first part of the survey features 21 questions about the clinicians' perceptions of difficulties in four domains:

1) language barriers;

2) variability of sociocultural background in patients;

3) insufficient background knowledge in clinicians (e.g. life conditions and residence status of migrants); and,

4) missing institutional resources (e.g. written materials in other languages). The second part asks 17 questions about clinicians' interest in different aspects of cross-cultural training, reflecting the four domains of difficulties.

Results: A total of 204 participants took part on the survey, $49 \%$ were nurses and $81 \%$ were women. A large number of clinicians reported communication difficulties with migrant patients, mainly in relation to missing institutional resources, and variable sociocultural and linguistic codes. Interest in participants for cross-cultural training was very high (70\% were very interested in 15/17 topics).

Discussion: This study shows that palliative care specialists perceive cross-cultural teaching as useful. This attests the feasibility of future training initiatives. The next step of this project is to explore practical ways of including cross-cultural training in palliative care curricula in Switzerland.

Keywords: Communication, palliative care, diversity, cross-cultural education

Funding: Swiss Academies of Arts and Sciences

Abstract number: P01-096

Abstract type: Poster

Are Healthcare Professionals in Norway Confident in Talking about Life-threatening IIIness with Cancer Patients and their Relatives?

Driller B. ${ }^{1,2}$, Skjelvan L. ${ }^{3}$, Knudsen A.K. ${ }^{4}$, Brenne A.T..$^{2,3}$

${ }^{1}$ Cancer Department, Møre og Romsdal Hospital Trust, Ålesund, Norway, ${ }^{2}$ Department of Clinical and Molecular Medicine, European Palliative Care Research Centre (PRC), Faculty of Medicine and Health Sciences, NTNU, Norwegian University of Science and Technology, Trondheim, Norway, ${ }^{3}$ Cancer Clinic, St. Olavs Hospital, Trondheim University Hospital, Trondheim, Norway, ${ }^{4}$ Department of Oncology, Oslo University Hospital and University of Oslo, Oslo, Norway

Background: Discussing end-of-life care preferences at the appropriate time may be of particular value for patients with a progressive disease due to the evident risk of deterioration and dying. In qualitative studies patients with life-threatening illnesses indicated that health care professionals (HCPs) appear uncomfortable talking about death. Research shows that HCPs avoid the conversation because of a fear of destroying patients and relatives hope. Nurses (N) and nurse assistants (NA) are often key front-line communicators in palliative care especially in primary health care.

Aims: We explored how confident HCPs in Norway feel in talking about life-threatening disease with cancer patients and their relatives.

Methods: Physicians (P), N and NA in specialist and community care at two local hospitals and in 22 municipalities in Central Norway were invited by e-mail to answer a structured survey with closed questions about knowledge, skills and attitudes in cancer palliative care. Responses regarding the two relevant topics here were given on a four point verbal scale from strongly disagree $(=1)$ to strongly agree $(=4)$, presented as mean scores.

Results: There was 2184 HCPs invited, 399 (18\%) provided analyzable responses, P; 37 (9\%), N; 217 (54\%), NA; 145 (36\%). Answers to «I feel confident in talking to cancer patients about life-threatening illness", showed mean scores P; 3.5 / N; 3.0 / NA; 2.5 and «I feel confident in talking to cancer patients' relatives about life-threatening illness», mean scores P; 3.5 / N; 2.9 / NA; 2.4 .

Conclusion: Even though we had a low response rate results indicate that HCPs in Central Norway feel relative confident in communication with patients and relatives about life-threatening disease. A selection bias in the sample is possible; highly motivated HCPs might be main responders. Future challenges for education is to talk about end-of-life issues at a pace that patients and relatives can tolerate and in ways that preserve hope.

\section{Abstract number: P01-097}

Abstract type: Poster

The Perception of Persons with Cancer on Starting a Conversation about Palliative Care: A Qualitative Interview Study

Scherrens A.-L. ${ }^{1,2}$, Cohen J. ${ }^{1}$, Mahieu A. ${ }^{2}$, Deliens L. ${ }^{1,2}$, Deforche B. ${ }^{2,3}$, Beernaert K.1,2

${ }^{1}$ End-of-Life Care Research Group, Vrije Universiteit Brussel \& Ghent University, Brussels, Belgium, ${ }^{2}$ Department of Public Health and Primary Care, Ghent University, Ghent, Belgium, ${ }^{3}$ Department of Movement and Sport Sciences, Physical Activity, Nutrition and Health Research Unit, Vrije Universiteit Brussel, Brussels, Belgium

Background: Despite the benefits of timely initiation of palliative care for persons with cancer, it is often initiated late or not at all. Communicative behaviours and patient-centered care are both important determinants for timely initiation of palliative care. However, research is barely focused on patients' communicative behaviours and patients' perspective.

Aim:

1) To understand and explain the behaviour 'starting a conversation about palliative care with a health professional' from the perspective of persons with non-curative cancer by using behavioural theories (eg Theory of Planned behaviour)

2) To formulate a behavioural model for the defined behaviour.

Methods: A qualitative study using semi-structured face to face interviews with 25 persons with non-curative cancer: 13 not (yet) receiving palliative care and 12 already receiving palliative care. Identified determinants related to 'starting a conversation about palliative care with a health professional' were fitted in concepts of behavioural theories (eg 'attitude' of the Theory of Planned Behaviour).

Results: Some participants were positive and others negative towards starting a conversation about palliative care with a health professional. The oncologists and the family physician were the preferred health professionals. Determinants facilitating and hindering the behaviour were identified: awareness (eg perceived health threat); knowledge (eg about palliative care possibilities); attitude (eg association of palliative care with quality of life or death); social norm and influence (eg the relationship with the health professional); and perceived behavioural control (eg self-confidence).

Conclusion: The identified modifiable determinants of our behavioural model can be used to develop more promising interventions promoting palliative care communication initiated by the patient and timely palliative care initiation. 
Abstract number: P01-098

Abstract type: Poster

Discussing Prognosis and Managing Uncertainty in End-of-Life Conversations with Family Members: A Conversation Analytic Study

Anderson R., Stone P., Low J., Bloch S.

Marie Curie Palliative Care Research Department, University College London, London, United Kingdom

Background: When patients reach the terminal phase of their illness effective communication with family members is vital. In particular, clinicians must be able to recognize and sensitively communicate when a patient is likely to die in the coming hours or days.

Aim: To understand how senior, experienced hospice clinicians and relatives/friends of patients discuss prognosis at the end-of-life.

Methods: We have collected 30 audio-recordings of conversations between hospice clinicians and relatives/friends of patients at the endof-life. A conversation analytic approach is taken to examine how relatives request prognostic information and how clinicians manage uncertainty in answering such requests. Conversation analysis goes beyond traditional qualitative approaches to look not just at what is said, but how it is said and the influence of one party's speech on the other. Results: The topic of prognosis was raised by relatives in the majority of cases. When asking for time estimates, relatives displayed an awareness of the likely shortness and uncertainty of the prognosis. Clinicians answered these prognostic requests by referring to 'hours' or 'days'. They highlighted the uncertainty of these estimates, both with their language within the estimates and with explicit statements about the difficulty of prognostication. Prognostic estimates were explained with reference to changes in the patient that were evident to both the clinician and relative.

Conclusion: Uncertainty was present throughout prognostic discussions from both clinicians and relatives. Relatives requested information in a way that gave clinicians permission to be uncertain. Clinicians were able to sensitively give prognostic estimates whilst still highlighting the difficulty of accurately predicting time to death. The strategies used by these experienced clinicians could be used to inform recommendations for other, less experienced clinicians.

This study is funded by the Marie Curie Chair's grant

Abstract number: P01-099

Abstract type: Poster

Facilitating Person-centred Care: Feasibility and Acceptability of the Support Needs Approach for Patients (SNAP)

Gardener A.C. ${ }^{1}$, Ewing G. $^{2}$, Farquhar M. ${ }^{3}$

1 Primary Care Unit, University of Cambridge, Cambridge, United Kingdom, ${ }^{2}$ Centre for Family Research, University of Cambridge, Cambridge, United Kingdom, ${ }^{3}$ School of Health Sciences, University of East Anglia, Norwich, United Kingdom

Background: The Support Needs Approach for Patients (SNAP) is a complex intervention to facilitate delivery of person-centred care in progressive non-malignant conditions, such as chronic obstructive pulmonary disease (COPD).

SNAP consists of:

1) the validated SNAP tool: designed for purpose, comprising 15 evidence-based questions to help patients consider areas where they may need more support

2) the SNAP approach: a needs-led conversation between the patient and healthcare professional (HCP) to express, prioritise and address unmet support needs identified through the patient-completed tool.
Feasibility and acceptability of the SNAP intervention now needs exploring in clinical practice.

Aim: To explore the feasibility and acceptability of SNAP in a range of clinical settings.

Methods: HCPs from three care settings (primary, community and secondary) were recruited to the SNAP pilot in the East of England. SNAPtrained HCPs implemented SNAP with patients with COPD over four months and participated in monthly qualitative interviews to monitor tool use and identify modifiable barriers to SNAP delivery. Purposively sampled patients $(n=24)$ who received the intervention were interviewed about their experience. End of pilot focus groups with participating HCPs explored barriers and facilitators to delivering SNAP in clinical practice. Normalisation Process Theory guided data collection and analysis throughout.

Results: Early results suggest SNAP is acceptable to HCPs and patients with COPD across settings. SNAP enabled new needs-led conversations (including future care conversations) even where patient-HCP relationships were well-established. Data collection and analysis completes in February 2019.

Discussion: SNAP appears feasible and acceptable with potential benefits to patients with COPD and their HCPs across a range of care settings. Findings will inform refinement of SNAP training and indicate whether formal evaluation of SNAP is warranted.

Abstract number: P01-100

Abstract type: Poster

Breakpoint Conversations in Palliative Care - A Study in Progress Melin-Johansson C. ${ }^{1}$, Lövgren M. ${ }^{1}$, Udo C. ${ }^{2,3}$

${ }^{1}$ Institution of Caring Sciences/Palliative Research Center, Ersta Sköndal Bräcke University College, Stockholm, Sweden, ${ }^{2}$ School of Education, Health and Social Studies, Dalarna University, Falun, Sweden, Falun, Sweden, ${ }^{3} \mathrm{CKF}$, Centre for Clinical Research Dalarna, County Council of Dalarna, Falun, Sweden

Palliative care is provided over a long period of time in parallel with curative interventions but sooner or later a breakpoint occurs when curative treatment no longer has an effect. A new state enters and the patients need a palliative breakpoint conversation (PBC). This requires a medical assessment and should be documented in the patients record. PBC are often conducted as a response to patient's unexpected deterioration or not at all. According to the Swedish Register of Palliative Care (SRPC) about 27000 patients in hospitals (year 20142017) did not receive PBC and $50 \%$ of all patients ( $N=500000$ ) PBC were not documented.

Aim: To explore the proportion of patients in all clinical units in Sweden that did not receive $\mathrm{PBC}$ documented in the medical records.

Methods: All clinical units $\mathrm{N}=6000$ in the SRPC that collects data from hospitals, hospices, nursing homes and home care, with an end-of-life questionnaire about palliative care content focusing on the last week of life will be included. We will explore what units documented PBC (answered yes in the questionnaire) or not documented PBC (answered no in the questionnaire). Data analysis is under progress with statistical analysis, and preliminary results will be presented at the conference.

Outcomes: Patients that do not receive PBC may suffer in a greater extent, in comparison with those who get $\mathrm{PBC}$, from pain and other symptoms and have less frequent access to drugs for pain, anxiety and nausea. They die with parenteral nutritional treatment and with pressure ulcers. Patients don't die where they prefer and they die alone. Their relatives don't receive information and are not offered bereavement support.

Discussion: Insufficient documentation about PBC may bring about unnecessary unclarity on how to care for the patient, this uncertainty can be perceived as confusing for both patient and family. A PBC late in the disease can also impair the patients ability to participate in decision-making. 
Abstract number: P01-101

Abstract type: Poster

An Advance Care Planning Conversation Tool for Primary Health Care Professionals Working with Patients with Dementia Pelgrum-Keurhorst M. ${ }^{1}$, Uitdehaag M. ${ }^{1}$, Groen-van de Ven L. ${ }^{2}$, Smits $C .{ }^{2}$ ${ }^{1}$ Research Center of Nursing, Saxion University of Applied Sciences, Deventer/Enschede, Netherlands, ${ }^{2}$ Research Group Innovating with Older Adults, Windesheim University of Applied Sciences, Zwolle, Netherlands

Background and aims: Both professionals and dementia patients are hesitant of initiating advance care conversations and hardly complete advance directives. In this study we developed and evaluated a conversation tool that supports primary health care professionals, in engaging conversations about patients' wishes and preferences for future issues in the context of dementia.

Methods: We developed a conversation tool, based on

(1) relevant literature

(2) semi-structured interviews with 9 people with dementia, 9 relatives, and 4 general practitioners (GP), and

(3) 2 focus group interviews with casemanagers dementia (CD).

Secondly, 3 GPs, 3 geriatricians and $18 \mathrm{CD}$ tested the tool in daily practice while participating in two supervision and peer-to-peer intervision meetings. Semi-structured interviews were conducted with professionals, patients and relatives. We applied qualitative thematic data-analyses with Atlas.ti software.

Results: The tool contains six conversations topics: daily enjoyment, daily care, medical treatment, coping with the dementia and the (nearing) death, financial and legal issues, and the social network. Professionals indicate that the conversation tool provides structure and support in planning and starting conversations. There is variety in the manner of use. The topics were recognizable and appropriate, though professionals also formulated recommendations regarding content and lay-out of the tool. Since the conversation tool has reached its definite format, professionals are highly interested in implementing the conversation tool in daily practice.

Conclusion: Advance care planning in the context of dementia includes issues related to the daily life, and medical and legal issues. The conversation tool provides structure, a visual overview of the important topics and can be used flexibly. The content and lay-out requires small adjustments. This study was funded by the The Netherlands Organisation for Health Research and Development (ZonMW).

Abstract number: P01-102

Abstract type: Poster

The Use of Humour in Palliative Care

Cuervo Pinna M.A. A. ${ }^{1}$, Zapata Macías B. ${ }^{2}$

${ }^{1}$ Palliative Care, SES, Badajoz, Spain, ${ }^{2}$ Extremadura, Badajoz, Spain

Aims: To explore the importance and acceptability of humor to patients and caregivers in a palliative care context, to investigate the relationship between the use of humor and demographic and clinical variables, to examine the acceptance of humor in a doctor-patient interaction and assess it as an instrument of social relationship.

Methods: Qualitative research through a semi-structured interview as a questionnaire. Twenty four patients included in the regional palliative care program and fourteen caregivers were surveyed. Previously, demographic and clinical data were collected. The questions of the interview were about the proposed topics in objectives, they were answered in a structured way. In the last part, participants were also given the opportunitiy to answer a question about humor in a palliative care context.
Results: For most participants, humor was important both prior to (71\%) and during (79\%) their illness experience. In addition, humor helps most patients (79\%) cope with their illness and it is considered a symptomatic relief by $60 \%$. Despite this valuation, the frequency of laughter diminished as patients' illness progressed. 95\% of participants like using humor with their doctors and the most of them (61\%) remembered laughing with a sanitary profesional on their last visit. The vast majority of patients found humor useful to build relationships with their doctors. In the free question, there is consensus about the importance of humor as a therapeutic tool.

Conclusion: It seems that the use of humor at the end of life is a good opportunity to help and accompany the patient, having an important place in the affective and psychological area and assuming better care.

Abstract number: P01-103

Abstract type: Poster

Breast Cancer Patients Preferences for Truth vs Hope is Dynamic and Change during Late Lines of Palliative Chemotherapy Bergqvist J. ${ }^{1,2}$, Strang P.1,3

${ }^{1}$ Department of Oncology and Pathology, Karolinska Institutet, Stockholm, Sweden, ${ }^{2}$ Department of Surgery, Breast Center, Capio St Görans Hospital, Stockholm, Sweden, ${ }^{3}$ Department of Research and Development, Stockholms Sjukhem Foundation, Stockholm, Sweden

Background: Women with metastatic breast cancer often receive many lines of palliative chemotherapy, which might be beneficial but also harmful. Still, little is known about the patients' perception of the patientdoctor communication regarding late lines of non-curative treatment.

Objective: Our aim was, therefore to explore what information the patients perceived and preferred, before continuing with palliative chemotherapy.

Patients and methods: A qualitative study with semi-guided face-to-face interviews with 20 women, $40-80$ years old, on at least their second line of palliative chemotherapy ( 2 nd-8th line). We used a qualitative conventional content analysis.

Results: All women knew they had incurable breast cancer but expressed hope for cure. Patients' definition of a good compassionate doctor was one who gives positive news and leaves room for hope. Ongoing chemotherapy, positive news from the doctors and support from relatives encouraged hope. The women often expressed they accepted chemotherapy to please their doctor and relatives. The informants appreciated the doctor to be honest, but within positive limits. Over time they stopped asking questions afraid of getting bad news, and left more and more treatment decisions to the doctor.

Conclusions: The women's preferences for truth vs. hope in patientdoctor communication changed over time, which increase the risk for continuous late lines of palliative chemotherapy by common collusion. Doctors need to individualise information, help patients make sense of their life and allow hope to endure without further chemotherapy.

Abstract number: P01-105

Abstract type: Poster

\section{Information Resource for Families of Dying Patients - A National} Response

Anderson A.

Irish Hospice Foundation, Healthcare, Dublin, Ireland

Background: Research continues to demonstrate the importance of written information to compliment the verbal communication between healthcare staff and family. In 2014 the Irish Ombudsman report found that poor communication was a feature of almost every complaint received. In 2017, the Voices MaJam Bereaved Relatives Survey Report found that $50 \%$ of respondents did not receive the hospital leaflets outlining what happens after a patient dies. 
Aims and objectives: In response a national booklet was developed to help families understand what is happening when someone they care about is dying in an acute hospital.

Methods: The booklet was developed following a scoping review of existing literature and reports in Ireland and other countries over a period of 18 months and included 2 consultation phases with a total of 61 responses. Patient opinion was obtained via the membership of the Committee overseeing development of the booklet, as well as direct feedback on the content of the two consultations. The majority of feedback was positive, with constructive feedback in relation to tone, images and content referring to clinical intervention and services.

Findings/results: Topic headings in the booklet include: coping with changes in the person who is dying; talking about feelings; talking about beliefs; what to do if someone dies while you are with them and looking after yourself. Specific illustrations were commissioned. A note outlining purpose and use of booklet is available for staff.

Conclusions: The booklet is to be used to augment and not replace communication from healthcare staff on such difficult and sensitive topic. Feedback from patients, families and staff is being sought. An adapted version is recommended for residential and primary care.

Abstract number: P01-106

Abstract type: Poster

Advance Care Planning in Palliative Care in the Hospital;

Experiences of Patients and General Practioners (GPs)

Veltman P.G., van der Werff G.F.M., van de Water C.R.T., Govers A.C., van der Velden A.W.G.

Groningen, Martini Hospital, Groningen, Netherlands

Background: In The Netherlands much effort is made to implement advance care planning (APC) to optimise the continuity and quality of care for palliative patients. At present experiences of patients and GPs with ACP after discharge from the hospital in our region is lacking.

Aim: To gain insight into the experiences of palliative patients and their GPs regarding transmural cooperation in ACP and the documented information including current and future problems.

Methods: Between May and October 2018 palliative patients with life expectancy $<6$ months -and their GPs- were requested to participate in the study in order to elucidate their experiences with ACP and its documentation. Participating patients and GPs were approached by phone for a semi-structured interview one week after discharge. Data collection and analysis took place in an iterative process.

Results: Nine patients gave informed consent and three of them were excluded. At this time six patients -or relatives- and seven GPs were interviewed. Two of the GPs preferred a written response. Analysis shows that patients are satisfied with the ACP communication and transfer. Although all patients like to receive an ACP document, only for one of them this document played a major role after discharge. It is especially important to them to be well taken care of by their GP and district nurse. GPs mention different experiences. Two GPs believe an ACP document should not be initiated at the hospital. Four GPs consider it a good and useful addition to the oral transfer. All GPs indicate a consultation by phone at discharge is most meaningful. Short lines in the collaboration between hospital and GPs are experienced as valuable.

Conclusion: Regarding ACP communication and transfer patients show high satisfaction. Nevertheless the ACP document seems to have little impact on the care. GPs seem to attach particular value to a personal telephone transfer. An ACP document can be supplemental to this.

Abstract number: P01-107

Abstract type: Poster

Implementation of a Patient and Public Involvement Group in the Context of Palliative Care Research - Experiences from Two Projects on Sedation in Palliative Care
Meesters S., Handtke V., Kühnel M.B., Bausewein C., Schildmann E. Department of Palliative Medicine, University Hospital, LMU Munich, Munich, Germany

Background: Patient and Public Involvement (PPI) is an increasingly recognized approach to enhance research quality and relevance. It is defined as research being carried out "with" or "by" members of the public. Although PPI is now required by many research funding bodies, there are no resources to support its implementation in Germany to date.

Aim: To describe our first experiences regarding the implementation of a PPI group for two projects on sedation in palliative care (PC).

Results: The present PPI group consists of 8 members ( 5 relatives of former PC patients, 1 patient, 1 hospice volunteer, 1 student nurse). All were first contacted by a person known to them professionally. Subsequently, they received information via letter and phone call by our research team. All persons approached agreed to participate, except one. An introductory session was organized to get familiar and to present and discuss research basics, information about PPI, PC, the two research projects and potential tasks. Motivations for participation were interest in the topic, gratitude or the wish to benefit others by own experiences. Involvement during the earlier research stages was not possible, as the PPI group started at the same time as the projects. PPI so far comprised participation in the development of participant information leaflets and interview guides, attending project meetings and assisting in pilot interviews. Existing international resources (e.g. "INVOLVE") could only partly be applied due to different structural conditions.

Conclusion: PPI recruitment proved successful, and first involvement helped to improve the research process. However, creating effective and sustainable PPI requires resources and guidance as well as exchange between research groups. A national programme bringing together expertise and experience and providing training and resources regarding $\mathrm{PPI}$, such as "INVOLVE" in the UK, would be desirable.

Funding: Federal Ministry of Education \& Research

\section{Abstract number: P01-108}

Abstract type: Poster

\section{Decisional Control Preferences in the Hispanic Population in the}

\section{Bronx}

Cardenas Mori J.1,2

${ }^{1}$ Montefiore Medical Center, Bronx, NY, United States, ${ }^{2}$ Albert Einstein Medical College, Family Medicine, Bronx, NY, United States Presenting author email address: jhosselini@gmail.com

Context: Hispanic Americans are among the fastest growing minority groups in the United States, and understanding their preferences for medical decision-making and information sharing is imperative to provide high quality end of life care. Studies exploring these decision control preferences (DCPs) are limited and found inconsistent results.

Objectives:

1) To measure DCPs of Hispanic patients in the Bronx.

2) To explore which patient characteristics are related to DCPs.

Methods: This is a cross-sectional survey of 119 cancer patients who self-identified as Hispanic and were waiting at the oncology clinic at Montefiore Medical Center Cancer Center. The relationship between patient characteristics and DCPs was tested using chi-squared tests of homogeneity.

Results: The majority $(63,52.9 \%)$ preferred shared decision-making with their doctors, families or both, while $46(38.7 \%)$ had an active decision-making style. A minority $(9,7.6 \%)$ had a passive decision-making style, deferring to their families, and only $1(0.8 \%)$ deferring to the physician. No demographic characteristics significantly predicted DCPs. The 
majority of patients agreed or strongly agreed that they wanted to hear all of the information regarding their diagnosis $(94 \%)$, treatment options $(94 \%)$, treatment expectations (92\%) and treatment risks and benefits (96\%).

Conclusion: These results confirm our hypothesis that most Hispanic patients prefer either an active or shared decision-making process rather than a passive decision-making process. Most patients prefer disclosure of diagnosis, prognosis and plan.

Abstract number: P01-109

Abstract type: Poster

Collaborative Advanced Care Planning: Improving Palliative Care and Quality of Death with Communication about Values, Life and Death

Leppin N. ${ }^{1}$, Nagelschmidt K. ${ }^{1}$, Koch M. ${ }^{2}$, Knorrenschild J.R. ${ }^{2}$, Timmesfeld $N .^{3}$, Seifart C. ${ }^{2}$, Rief $W^{1}{ }^{1}$, von Blanckenburg $P^{1}$

${ }^{1}$ Psychology, Philipps University Marburg, Marburg, Germany, ${ }^{2}$ Medicine, Philipps University Marburg, Marburg, Germany, ${ }^{3}$ Medical Informatics, Biometry and Epidemiology, Ruhr-University Bonn, Bonn, Germany

Aim: Advanced care planning (ACP) is evidently a useful process to improve end of life care and quality of life in terminal disease phases. The uptake of ACP in palliative patients and caregivers, though, remains unsatisfactory. An interdisciplinary, collaborative concept of advanced care planning (CACP) was therefore developed in a project of the Federal Ministry of Education and Research to identify and reduce barriers that restrain from engaging in ACP. The core elements of this new approach and its effects shall be demonstrated within a case study.

Methods: The case study describes the life course of a patient with metastatic cancer and his wife - before, during and after their participation in the randomized controlled CACP-study. The transcript of the conversations during the intervention was rated for adherence to the manual. Quality of life, communication barriers, inner peace as well as quality of death and dying are analyzed qualitatively and quantitatively. Data was obtained before and directly after the intervention with follow-ups every two months until the patient's death.

Results: Both, patient and his wife, stated that they profited from the intervention and reported improvements in their communication. The dying process was supported by the ACP-documentation: his wish to die at home, in presence of his family and with priestly care, could be fulfilled. Also his preference to rather lose consciousness than suffering from pain was accomplished. Still, there were untouchable topics for the couple, such as discussing wishes for the funeral.

Conclusion: While quality of life took a course typical for the disease, inner peace and partner-communication seemed to be enriched by the intervention. The support from the intervention also proved appropriate in this case to ensure the quality of the dying process. Reducing barriers by talking about values, dignity and individual concerns as well as informing about ACP could be worthy for enhancing ACP uptake.

Abstract number: P01-110

Abstract type: Poster

Development and Evaluation of an Advance Care Planning Program for Professionals in Palliative Care for People with Intellectual Disabilities

Wagemans A..$^{1,2,3}$, Vogel A. ${ }^{1}$, Voss $H_{.}{ }^{4}$, Francke A. ${ }^{4,5,6}$, Metsemakers J. ${ }^{3}$, Courtens . $^{7}$, de Veer $A .^{4}$

${ }^{1}$ Maasveld, Koraalgroep, Maastricht, Netherlands, ${ }^{2}$ Expertise Centre for Palliative Care, Maastricht University Medical Centre, Maastricht, Netherlands, ${ }^{3}$ Department of Family Medicine, Faculty of Health, Medicine and Life Science, Maastricht University, Maastricht, Netherlands, ${ }^{4}$ Netherlands Institute of Health Services Research (NIVEL), Utrecht, Netherlands, ${ }^{5} \mathrm{VU}$ Medical Center, Amsterdam Public
Health Research Institute (APH), Amsterdam, Netherlands, ${ }^{6} \mathrm{VU}$ Medical Center, Expertise Center for Palliative Care Amsterdam, Amsterdam, Netherlands, ${ }^{7}$ Maastricht University Medical Centre, Expertise Centre for Palliative Care, Maastricht, Netherlands

Background and Aim: People with intellectual disabilities (ID) have restricted cognitive and communicative abilities, and therefore encounter difficulties in clarifying their wishes and needs. Professionals in ID-care do not always recognize palliative care needs and lack communication skills to talk about future care in advance. The aim of this study was to develop and evaluate an advance care planning (ACP)-program to train professionals in knowledge and skills about ACP in palliative care for people with ID.

Methods: This study contained five phases. Phase 1 consisted of a systematic review, (medical) file analysis and supplementary interviews, and depth-interviews to discover important aspects of ACP. Findings in phase 1 were translated into an ACP-program in co-creation with professionals, relatives and people with ID (phase 2) and a communication training framework was developed (phase 3). Implementation of the ACP-program and associated training took place in six organizations providing ID-care (phase 4). In phase 5 the program was evaluated by pre-structured questionnaires send to participants 6 months after implementation.

Results: The ACP-program consists of

1. Methodology about important aspects of ACP,

2. ACP communication training, and

3. Consultation about implementation strategies for ACP.

It is based on 10 competencies needed for ACP; identification of the palliative phase, signaling symptoms, communicating, documenting, structurally deploying $A C P$, taking wishes of people with ID into account, collaborating, paying attention to possible dilemmas, reflecting, and regarding ACP as a standard aspect of palliative care.

Conclusion: An ACP program is helpful to make professionals aware of the importance of ACP and improve communication skills. Participants appreciate the course as useful. Research is needed to further investigate the effective elements of the ACP program.

Abstract number: P01-111

Abstract type: Poster

HELEN-44: How do Palliative Care Experts Define an End-of-Life Literate Person?

Cardenas Mori J.1,2, Chuang E. ${ }^{1}$, Kosminsky L. ${ }^{3}$

${ }^{1}$ Albert Einstein Medical College, Bronx, NY, United States, ${ }^{2}$ Montefiore Medical Center, Bronx, NY, United States, ${ }^{3}$ Temple university, Family Medicine, Philadelphia, PA, United States

Background: Communication between health professionals, patients and families is critical to providing high quality end-of-life (EOL) care that matches patient preferences.

Aims: To develop a health literacy assessment for end of life tool (HELEN) for use in patients with serious illness and their caregivers, which will allow for clinicians to more adequately inform and treat their patients in alignment with the patient's goals.

Methods: Delphi study: Three consecutive rounds of surveys were conducted among a panel of palliative care and hospice experts to answer the question of what should be included in EOL literacy and what should be omitted. The list of words/phrase was coded on a 5-point Likert scale. A consensus in this study was defined a priori as an agreement (i.e., "strongly agree" and "agree") by a minimum of $70 \%$ of the experts, consistent with standards in other Delphi studies. Demographic information from the experts was obtained.

Results: 91 experts answered (palliative care physicians (15\%) and nurse practitioners (3\%), nurses from hospice and palliative care wards (4\%), 
palliative care social workers $(77 \%)$ and chaplain (1\%). Most of the respondents work in the US in an academic institution (75\%). From the first list of 125 words/phrases, 44 words/phrases were obtained after using a cutoff of mean score $=4.05$ on the Likert scale.

Conclusion: Disparities in quality of EOL care may be due, in part, to differences in EOL health literacy (HL). The results of this Delphi study will be used to develop the EOL-specific HELEN health literacy evaluation tool. This tool can then be used by the general palliative care research community to better understand the relationship between $\mathrm{HL}$ and quality of EOL care.

\section{Abstract number: P01-112 \\ Abstract type: Poster}

"Misunderstandings Are Virtually Inevitable" The Understanding of 'Palliative' in Oncology and Palliative Care

Schwabe S. ${ }^{1}$, Bradtmöller K. ${ }^{1}$, Jaspers B. ${ }^{1,2}$, Radbruch L. ${ }^{1,3}$

${ }^{1}$ Department of Palliative Medicine, University Hospital Bonn, Bonn, Germany, ${ }^{2}$ Department of Palliative Medicine, Georg-August University Göttingen, Göttingen, Germany, ${ }^{3}$ Palliative Care Centre, Malteser Hospital Bonn/Rhein-Sieg, Bonn, Germany

Background: Palliative care (PC) is an interdisciplinary approach to provide care for patients with life-threatening diseases. Communication within the therapeutic team and between physicians of different medical disciplines is highly important, but a lack of consensus about the term 'palliative' remains a challenge.

Aims: To explore the different understanding of the term 'palliative' in PC and oncology.

Methods: Qualitative guideline interviews with 10 experts from the fields of PC and oncology in Germany were held between Oct 2017 and Feb 2018. Experts were defined as persons with extensive knowledge in oncology or PC, substantiated by authorship of relevant medical guidelines and textbooks, involvement in medical societies and in education and training. An interview guideline was developed focusing inter alia on the understandings of the term 'palliative'. Interviews were audio-recorded, transcribed verbatim and analysed according to qualitative content analysis. Results: The term 'palliative' is used differently in PC and oncology. Differences concern

a) the criteria for the indication for palliative treatment,

b) the focus of the treatment,

c) the goal and

d) the measures.

In oncology, the indication for palliative treatment mainly depends on somatic findings (illness-related). It includes an anticancer therapy with a focus on prolongation of life and quality of life. In PC, aside from the life-threatening illness, the indication for palliative treatment mainly depends on patients' needs (need-related), includes comprehensive care and focuses on symptom control and pain relief.

Conclusion: Different understandings of the term 'palliative' in PC and oncology can be identified on the basis of distinguishing criteria. A clarification about the term 'palliative' is of crucial importance to enable interdisciplinary communication.

The project is funded by the German Federal Ministry of Education and Research (No. 01GY1714).

Abstract number: P01-113

Abstract type: Poster

Inappropriate Interventions and the Value of Positive versus Negative Language at the Terminal Stage

Larkin M. ${ }^{1}$, Hayes E. ${ }^{1}$, O Reilly L. ${ }^{1}$, Gallagher M. ${ }^{2}$, Bourke M. ${ }^{2}$, Waldron $D .{ }^{1}$ ${ }^{1}$ University Hospital Galway, Galway, Ireland, ${ }^{2}$ Galway University Hospital, Galway, Ireland
Background: Many 'routine' interventions in hospital have repercussions for the comfort of patients. The decision to perform them should depend on whether the patient is diagnosed as in a terminal phase. Effective and honest communication surrounding the dying phase has a positive influence on patients and their family.

Aims: A cross sectional study over six-months:

1. To determine if the terminal phase was marked by an actual diagnosis

2. To determine if Resuscitation Status was documented

3. To analyse the health interventions performed and decisions made in the last

4. days of life. Was communication 'positive' or 'negative' in this documentation, i.e. withdrawal of treatment vs discontinuation of futile or potentially harmful treatment. Use of 'critical incident' was utilised to highlight the recording of 'negative' and 'positive' comments made in the patient's notes during the dying phase.

Methods: All patients referred to the Specialist Palliative Care (SPC) service over a six-month period were included.

Results: 250 patients were included in the study, 51 patients died. The actual diagnosis of 'dying' was clearly documented in $52 \%$; the documentation in the charts do not frequently use the word 'dying'. Resuscitation status was documented in $98 \%$ of the charts; family were involved in this decision in $66 \%$ of cases. Interventions were continued in many; steroids in $21 \%$, intravenous fluids in $88 \%$, chemotherapy in $5 \%$ and antibiotics in 57\%. SPC language was found to be 'positive' whereas the language used by the majority of other teams was 'negative' i.e. CPR not possible to sustain quality of living rather than not for CPR.

Conclusion: Interventions near to and up to death need to be addressed. Burdensome and aggressive care can worsen quality of life and limits patients' exposure to palliative care.

Abstract number: P01-114

Abstract type: Poster

Development of an Intervention to Support Lung Cancer Patients and their Clinicians when Considering Systematic Anticancer Therapy: The PACT Study, a Prospective, Multi-centre, Multi-methods, Five-stage, Qualitative Study Nelson A. ${ }^{1}$, Byrne A. ${ }^{1}$, Sivell S. ${ }^{1}$, Longo M. ${ }^{1}$, Noble S. ${ }^{1}$, Lester J. ${ }^{2}$, Anagnostou D. ${ }^{1}$, Sampson C. ${ }^{1}$

${ }^{1}$ Cardiff University School of Medicine, Marie Curie Palliative Care Research Centre, Cardiff, United Kingdom, ${ }^{2}$ Velindre NHS Trust, Cardiff, United Kingdom

Background: The NHS aspires to place patients' needs, wishes and preferences at the heart of clinical decision-making; however, this can be extremely difficult for patients diagnosed with advanced non-small cell lung cancer (NSCLC). NSCLC patients move quickly through the illness trajectory, this poses major challenges for patients, families and clinicians when discussing care pathways and it can lead to futile treatment. The aim of this study was to develop an intervention to support patients, families and clinicians when considering systematic anti-cancer therapy.

Methodology: PACT was a prospective, multi-centre, multi-methods, five-stage, qualitative study. Non-participant observation of MDT meetings determined patients' allocation to treatment. Non-participant observation of patient-clinician consultations explored communication of treatment options and decision-making. Interviews with patients/ companions and clinicians explored perception of treatment options and involvement in decision-making. Mediated discourse, thematic, framework and narrative analysis were used to analyse the data. An expert consensus meeting finalised the content and format of a proposed intervention. 
Results: For NSCLC patients decision-making is a process rather than a single event. Patients' priorities and preferences are not routinely or intrinsically part of the clinical pathway, and rise according to the skills/ attitudes of the clinician and the confidence/awareness of the patient (and family).

Conclusion: A series of missed opportunities alongside the patient clinical pathway emerge and these affect clinicians and patients. Current pathway for NSCLC patients focuses on clinical management at the expense of patient-centred care. Future work will test the feasibility of applying a communication tool held by the patient alongside the clinical pathway.

\section{Abstract number: P01-115 \\ Abstract type: Poster}

\section{Social Campaign for Pain Management in Ukraine: Take Off Pink} Colored Glasses

Shapoval-Deinega K. ${ }^{1}$, Rokhanskiy A. ${ }^{2}$, Tymoshevska V. ${ }^{3}$, Andriishyn L. ${ }^{4}$, Bratzuin $0 .^{5}$

${ }^{1}$ International Renaissance Foundation, Kyiv, Ukraine, ${ }^{2}$ Legal Analysis and Strategies, Kharkiv, Ukraine, ${ }^{3}$ Public Health Program, International Renaissance Foundation, Kyiv, Ukraine, ${ }^{4}$ Mother Teresa Charitable Foundation, Ivano-Frankivsk, Ukraine, 'Sobornist' Charitable Foundation, Kyiv, Ukraine

"Take off pink glasses" the information campaign on access to pain treatment, 2017-2018.

"Who is in pink glasses?" - this is often the leaders of various parts of the health system. If these officials or managers ask: "Do all patients get necessary painkillers if necessary?" - there will be an answer: "Sure!". This answer based on statistical data: in the area of one public clinic service in 2016, opioid analgesics received 3 patients with cancer. It is looks like that health care system fulfilled its mission. In fact that this clinic in the big city serves about 100000 p., and have more that 3 patients with chronic pain. In Ukraine, almost 90000 people die every year from oncological diseases. About $80 \%$ of them suffer from pain syndrome. How many patients with other illnesses suffer from pain - hard to count.

In 2016-2017 human rights activists documented more than 100 stories of palliative care patients around Ukraine and conducted social campaign for pain management.

1. A randomized study showed that in most cases, the goal of treatment for chronic pain was not achieved - the elimination of pain or its significant reduction for a long time.

2. The expected level of successful pain management has not been achieved in $80-90 \%$ of those in need, successful pain management has been achieved in $5-14 \%$ of patients.

3. Painkillers are prescribed formally, without taking into account clinical guidelines.

4. Separate explanations from physicians about fears of causing drug dependence, or fears of provoking respiration in patients, or ignorance of the orders of the Ministry of Health - all this is an excuse that covers the Soviet tradition of medical education: not to prescribe opiates in any case.

The quality of providing palliative care in accordance with WHO standards requires changes in the professional training of doctors, the wide implementation of modern scientific concepts of pain relief in all parts of the training and improvement of medical staff

Abstract number: P01-116

Abstract type: Poster

The Concept of Advance Care Planning According to Patients and Relatives: An Interview Study van der Smissen D. ${ }^{1}$, Rietjens J.A.C. ${ }^{1}$, van Dulmen S. ${ }^{2}$, van Gemert-Pijnen J.E.W.C. ${ }^{3}$, van der Heide A. ${ }^{1}$, Korfage I.J. ${ }^{1}$

${ }^{1}$ Public Health, Erasmus MC, Rotterdam, Netherlands, ${ }^{2}$ Netherlands Institute for Health Services Research (NIVEL), Utrecht, Netherlands, 3University of Twente, Enschede, Netherlands

Background: The EAPC consensus concept, as defined by professionals, describes Advance Care Planning (ACP) as enabling persons to identify, discuss, record and review goals and preferences for future treatments and care. It is useful to know whether patients and relatives conceptualize ACP in a similar way, for example for the provision of ACP information and its findability on the internet.

Aims: To explore what patients and relatives consider important in ACP and how they would search for information about ACP on the internet. Methods: To address our aims, we interviewed 9 patients with chronic diseases and 7 relatives. We used constant comparative analyses to categorize key elements of ACP.

Results: To search information, patients and relatives would use search terms such as 'ACP', 'care/treatment plan', 'disease trajectory', 'advance directive' and names of patient associations. Patients and relatives would appreciate information on what ACP is and why it is important, on how to adapt ACP to individual needs, values and quality of life, on their disease and options for treatment and care, and on how to effectively communicate their preferences. The appointment of a healthcare representative was mentioned by a few interviewees. They also mentioned additional information needs, such as how to get support in ACP, explanation about whom to involve in ACP, encouragement to think about $A C P$, information for relatives, and peer support.

Conclusion / Discussion: Key elements of ACP as mentioned by patients and relatives are in line with the EAPC consensus concept of ACP as defined by professionals. However, patients and relatives also mentioned additional elements, indicating they may have a broader concept of ACP. Including these elements and search terms as indicated by patients and relatives in online ACP information may help to increase its findability and to meet information needs.

Funding: The Netherlands Organisation for Health Research and Development (ZonMw).

\section{Abstract number: P01-117 \\ Abstract type: Poster}

Advance Care Planning among Healthcare Professionals in Asia: A Systematic Review of Knowledge, Attitude and Experience Martina D. ${ }^{1,2,3}$, Kristanti M.S. ${ }^{4}$, Bramer W.M. ${ }^{5}$, Mori M. ${ }^{6}$, Korfage I.J. ${ }^{2}$, van der Heide A. ${ }^{2}$, van der Rijt C.C.D. ${ }^{1}$

${ }^{1}$ Medical Oncology, Erasmus MC, Rotterdam, Netherlands, ${ }^{2}$ Public Health, Erasmus MC, Rotterdam, Netherlands, ${ }^{3}$ Internal Medicine, Universitas Indonesia, Jakarta, Indonesia, ${ }^{4}$ School of Nursing, Universitas Gadjah Mada, Yogyakarta, Indonesia, ${ }^{5}$ Medical Library, Erasmus MC, Rotterdam, Netherlands, ${ }^{6}$ Clinical Laboratory, Seirei Mikatahara General Hospital, Hamamatsu, Japan

Background: Despite universal recognition of the importance of advance care planning $(A C P)$, the spirit of collectivism may impact the uptake of ACP in Asia.

Aims: To gain insight into health care professionals' (HCP) knowledge of, attitude towards, and experience with ACP in Asia.

Methods: We systematically searched 4 electronic databases for quantitative and qualitative studies on ACP in East and South East Asian countries. Anticipating unfamiliarity with the term ACP, we operationalized ACP broadly, also covering discussions about future medical care/treatment and documentation thereof.

Results: Of the 3358 identified studies, 41 were eligible; 36 with a quantitative and 5 with a qualitative design. Studies were conducted mostly in high-income countries: Japan (16), South Korea (13), Singapore (4), 
Hong Kong (4), and Taiwan (3). Twenty nine articles studied ACP documentation, six studied end-of-life care decision making/discussion, and only six used the term ACP. Ten studies addressing HCP's knowledge indicated moderate HCP's awareness of ACP. The 30 studies addressing HCP's attitudes showed that, even though many considered ACP to be important, several HCP's found initiating ACP difficult. This hesitation was partly due to fear of legal consequences and fear of conflicts with family members. HCPs frequently indicated that families have an essential role in ACP. The 20 studies on experiences indicated that the level of actual engagement of HCP in ACP is rather low, and that ACP is mostly initiated at an advanced stage of patient's illness.

Conclusion: Despite an overarching view that ACP may have an important role in care for patients with advanced diseases in Asia, in practice ACP does not occur often. HCPs seem to be reluctant to engage in ACP. The role of the family in ACP is crucial in Asia. However, the existing evidence was highly heterogeneous and limited to a few highincome countries, which limits its generalizability to other Asian countries.

\section{Abstract number: P01-118 \\ Abstract type: Poster}

Skilful Connectedness - A Model of the Regulation between Personal Closeness and Distance in the Clinical Encounter Orellana-Rios C.L. ${ }^{1}$, Becker G. ${ }^{1}$, Schmidt S. ${ }^{2}$

${ }^{1}$ Clinic for Palliative Care, Medical Center, University of Freiburg, Faculty of Medicine, University of Freiburg, Freiburg, Germany, ${ }^{2}$ Clinic for Psychosomatic Medicine and Psychotherapy, Medical Faculty, Medical Center, University of Freiburg, Freiburg, Germany

Background: Providing compassionate care can be challenging for palliative care practitioners (PCPs). An empirically-grounded model describing the factors that contribute to preserve the balance between personal closeness and distance in the professional setting is still lacking.

Objective: To investigate which factors help to regulate closeness and distance while providing compassionate care from the perspective of PCPs.

Methods: Semi-structured interviews were conducted with twentyeight staff members of an interdisciplinary palliative care team in Bonn, Germany. Interviews were transcribed verbatim and analyzed using a reconstructive analysis method.

Results: The common element that addressed the balance between compassionate care and well-being through all interviews was the phenomenon of skilful connectedness. This means having the capacity to establish closeness to patients and their relatives on a level of shared humanity and in a conscious and self-reflective manner. General feelings of love and appreciation for others were seen as a protective factor for burnout in PCPs. Yet, an important feature of connectedness was a sense of coherence regarding closeness in the clinical setting. Participants described various emotional and cognitive strategies that helped them to establish a sense of meaningfulness and manageability. Further factors that helped the staff members to avoid feeling overwhelmed by closeness at work were respect for the autonomy and self-determination of others and themselves as well as mentally sorting work tasks and roles early in the clinical encounter.

Conclusion: This study provides empirical evidence on how PCPs perceive the regulation of closeness and distance in palliative care. Further research is needed in order to ensure external validity of the retrieved skilful connectedness model.

Funding: Maaßen-Stiftungs-gGmbH, Steinen, Germany.

Abstract number: P01-119

Abstract type: Poster

Medical Residents Needs on Communication Skills: What about after a Rotation in a Palliative Care Unit?
Reis Carreira N., Galriça Neto I.

Unidade de Cuidados Continuados e Paliativos, Hospital da Luz, Lisboa, Portugal

Background: Effective communication is a cornerstone in Palliative Care (PC), an important tool for symptomatic control and therapeutic strategy in distressing situations in severely ill patients. Thus, communication skills need to be learned and trained. Rotations in Palliative Care Units (PCU) are a striking opportunity to learn and train those fundamental skills.

Aims: Identify the most common difficulties in the communication process of a residents' group, which learning methods were considered most useful and analyze benefits after a rotation in a PCU.

Methods: We selected a sample of 42 residents who completed a rotation in a PCU since January 2015, on which was applied a survey on communication issues, including resident description, prior felt difficulties, learning methods and uppermost gains in the communication process. Results: $69 \%$ of the residents answered. $76 \%$ had any previous experience in PC. 83\% didn't have pre-graduate communication formation, and $48.3 \%$ attended post-graduate formation. $90 \%$ usually communicate bad news at least 5 times a month. The most difficult situations identified were the "Conspiracy of Silence", "Denial" and "Discussion of End of Life Issues". 69\% felt uncomfortable in managing the patients' emotions. During the rotation, individuals found decisive the active training with patients, tutors coaching and anticipating various scenarios. $100 \%$ felt substantial gains, mainly in the "Conspiracy of silence" and "Discussion of End of Life Issues". It was unanimous the impact of those learnings in clinical practice, mainly by increased ease and security communicating, patient and family satisfaction and easier identification of patient needs.

Conclusion: The communication process is a crucial goal in a PC rotation for medical residents, whom considered the training of these competences extremely useful and profitable, reflecting their own preparation and safety in communication, with beneficial effects on their clinical practice.

Abstract number: P01-120

Abstract type: Poster

Factors Associated with End of Life Discussions with Carers of Adults Aged 75 Years or Older: A Population-Based Mortality Follow-back Survey

Cauldwell K. ${ }^{1}$, Bone A. ${ }^{2}$, Osborne T. ${ }^{1}$, Evans C. ${ }^{2}$

${ }^{1}$ Medical Department, St Joseph's Hospice, London, United Kingdom, ${ }^{2}$ Cicely Saunders Institute of Palliative Care, Kings College London, London, United Kingdom

Background: Informal carers play an important role in caring for older adults. Their preference is for end of life discussions (EOLD) with health care professionals (HCPs) to discuss the possible disease trajectory. EOLD are often inadequate, negatively impacting carer and patient outcomes. Aim: To identify explanatory variables associated with EOLD between carers of patients over 75 years and HCPs.

Methods: Multivariable logistic regression analysis of a populationbased mortality follow-back survey, examining the experiences of a random sample of people dying at home, hospital, hospice or care home. The person registering the death completed the survey.

Results: $443 / 882$ (50.2\%) responded, of which 438 were appropriate for analysis. Most died from non-malignant conditions (76\%). 60.5\% respondents reported EOLD had occurred. The odds of EOLD increased if the deceased had a cancer diagnosis (OR $2.0495 \% \mathrm{Cl} 1.07-3.89$ ), or if in the week before death they were confused (OR $2.4195 \% \mathrm{Cl} 1.35-4.30$ ), bedbound (OR $2.1195 \% \mathrm{Cl}$ 1.27-3.49), or taking painkillers (OR $1.7695 \%$ $\mathrm{Cl}$ 1.06-2.91). The odds increased if they had access to a "Key HCP" (OR $2.7295 \% \mathrm{Cl} 1.67-4.42$ ), or in the 3 months before death contact with a district nurse (OR $1.8095 \% \mathrm{Cl} 1.11-2.91)$, or hospital attendance (OR 
$1.9495 \% \mathrm{Cl} 1.12-3.37)$, and halved if the deceased lived alone compared with a communal environment (OR $0.4995 \% \mathrm{Cl} 0.25-0.95$ ).

Conclusion: It is known that HCPs are less likely to discuss the potential for death if there is prognostic uncertainty. This is borne out by the findings of this study which indicate that EOLD are less likely in the context of non-malignant disease, fewer signs of clinical deterioration, where patients have been living independently prior to death, and have not required access to specific health care.

Thus, a focus for future work may be to identify ways to improve communication in the setting of unpredictable disease trajectories. Funder: NIHR Research for Patient Benefit

\section{Abstract number: P01-121}

Abstract type: Poster

\section{What Is this about? Key Messages Explaining PC by the Professionals: An Ethnographic Study \\ Reigada C. ${ }^{1,2}$, Centeno C. ${ }^{1,2}$, Arantzamendi M. ${ }^{1,2}$ \\ ${ }^{1}$ Institute for Culture and Society, ATLANTES, University of Navarra, Pamplona, Spain, ${ }^{2}$ Instituto de Investigación Sanitaria de Navarra (IdiSNA), Pamplona, Spain}

Background: Palliative Care (PC) it is associated with death, dying, terminal and end of life. A negative public discourse has been identified as a barrier to PC implementation worldwide. A greater understanding of PC is needed to elaborate a proper message for the society. We intend to achieve that through the observation of the micro-culture of PC professional in action.

Aim: To understand what messages on PC are being transmitted by PC professionals in daily clinical practice.

Methods: Focused Ethnography to identify messages (e.g. values, attitudes) transmitted by PC professionals among patients/carers. Participant observation (PO), field notes, document analysis, and informal conversations were used for data collection. A thematic analysis was made was made, following the European Commission recommendation on "Research Ethics in Ethnography/Anthropology".

Results: A total of 250 hours of PO was made between Jun-Sept. 2018 in three PC teams in different regions of Spain. Three key messages were identified (thematic coding):

i) "We are a team"-they do not always introduce themselves using the word PC. Instead, they talk about symptom control, care planning, meaning of life, as their objectives;

ii) "This is about you, let me know"-based on the experience of the patient/carer, conversations show availability, transmitted by emotional attitudes;

iii) "We care about your family"- they wish to know and support the family, almost in a protective way. Interactions seem to be conditioned by the personal experiences, place of care, institutional culture, among others related to team-staff-patient/carers.

Conclusion: The key messages used by PC professionals in their daily interaction could contribute to greater understanding of PC in the society.

Abstract number: P01-122

Abstract type: Poster

Evaluation and Further Development of a Dutch Question Prompt List on Palliative Care to Support Patients and Family Verhoef M.-J. ${ }^{1}$, Sweep B. ${ }^{1}$, de Nijs E. ${ }^{1}$, Horeweg N. ${ }^{2}$, Oomes M. ${ }^{1}$, van Noord-Stijlen J. ${ }^{1}$, Vergroesen $D .{ }^{1}$, Schoof-van der Ham S. ${ }^{1}$, Pieterse A. ${ }^{3}$, van der Linden $Y^{1}$

${ }^{1}$ Centre of Expertise Palliative Care, Leiden University Medical Center, Leiden, Netherlands, ${ }^{2}$ Department of Radiation Oncology, Leiden
University Medical Center, Leiden, Netherlands, ${ }^{3}$ Biomedical Data Sciences, Doctor-patient Decisions, Leiden University Medical Center, Leiden, Netherlands

Background: Patients and family often do not know what to expect of palliative care consultations. Question prompt lists (QPL) help them to gain insight into and express their care needs and questions. The Palliative Care Consultation Team (PCCT) of our institution designed the 'Leidse Gesprekshulp Palliatieve Zorg' (LGP), combining an adapted Edmonton Symptom Assessment Scale and a translated QPL on palliative care ${ }^{1}$, to hand out to patients and family to prepare their PCCT-consultation.

Aims: To evaluate and further develop the LGP according to views of patients, family and health care providers (HCP).

Methods: Evaluation and further development occurred in three phases (2016-2018). 1: PCCT-consultations were recorded. Semi-structured interviews about the use, content and lay-out were conducted with patients who used the LGP and with LGP-naive HCPs. 2: a pilot version of the new LGP was developed based on phase 1. 3: the pilot version was evaluated and adapted in two rounds of semi-structured interviews with patients/family. All transcriptions of consultations and interviews were coded and thematically analyzed by two researchers.

Results: In phase 1, 33 consultations and 22 interviews with patients/ family and HCPs were analyzed. Patients/family experienced the LGP as helpful; it helped structuring thoughts and formulating questions. HCPs considered the LGP as useful and complete. The topics of the LGP were considered helpful and relevant. HCPs had different opinions about the necessity of "sexuality and intimacy". Patients and HCPs indicated the need to add "prognosis" and "questions for family". In phase 2, remarks on most relevant topics, language usage and order were incorporated in the pilot version. In phase 3, the LGP 2018 was finalized.

Conclusion: Patients, family and HCPs consider the LGP as useful in preparation of and during consultations about palliative care. LGP 2018 satisfies current Dutch information needs.

Reference: 1 Clayton J et al Br J Cancer 2003

\section{Abstract number: P01-123}

Abstract type: Poster

How Do Patients Experience Patient-physician Communication about their Limited Life Expectancy? - An Interview Study Owusuaa C. ${ }^{1}$, van der Lent L.G.G. ${ }^{1}$, van der Heide A. ${ }^{2}$, van der Rijt C.C.D. ${ }^{1}$ ${ }^{1}$ Medical Oncology, Erasmus MC Cancer Institute, Rotterdam, Netherlands, ${ }^{2}$ Public Health, Erasmus MC, University Medical Center Rotterdam, Rotterdam, Netherlands

Aim: The Dutch Framework for Palliative Care advises physicians to timely start advance care planning (ACP) in patients with life-limiting diseases. Such communication requires disclosure and discussion of patients' limited life expectancy. We explored patients' experiences with such discussions.

Methods: Medical specialists from three different hospitals included patients with incurable cancer or late-stage chronic obstructive pulmonary disease (COPD), with whom they had spoken about their limited life expectancy. All 14 patients (7 cancer and 7 COPD) had a semi-structured interview with one researcher about their experiences on the topics life expectancy and ACP. The interviews were audiotaped, transcribed, coded, and analysed by two researchers.

Results: All patients were aware of their limited life expectancy. They were often shocked when their life expectancy was indicated with numbers. Patients who had talked about ACP preferred to do so with their general practitioner. ACP conversations were experienced as confronting, but sometimes also as soothing. Some patients with COPD who felt they did not need ACP conversations believed that it is not the responsibility of the pulmonologist to discuss the topic. Most patients agreed that the physician should initiate and guide ACP conversations, but that 
the patient should have control over the content and continuation of such conversations. Factors that facilitated agreeable conversations for the patients were: clear explanations and messages about the disease, prognosis and treatment options, sufficient time, feeling of being heard, adequate preparation by the physician, sensitive non-verbal communication, an open attitude, and the inclusion of relatives.

Conclusion: Overall, patients appreciate honest, personalized and attentive communication on a limited life expectancy. For communication about ACP, sensitive orientation on patients' needs is even more important.

Source of funding: ZonMw

Abstract number: P01-124

Abstract type: Poster

Barriers to End-of-Life Communication in the Family Context Systematic Review of the Perspectives of Persons at the End of Life and their Closed Ones

Nagelschmidt K., Rief W., Von Blanckenburg P.

Philipps-University Marburg, Marburg, Germany

Background: Open communication at the end-of-life (EoL) about, amongst other things, medical decision making, emotional distress and meaning at the EoL can lead to better care and quality of life for the Dying. It can also provide stress relief for the family members and have positive effects on the grief process. These conversations can be emotionally challenging and there seem to be barriers to openly discuss matters of EoL and death. Barriers and facilitators in EoL communication with physicians or general practitioners are systematically studied. Nevertheless, little is known about barriers in the family context even tough the patient and the family are recognized as the unit of care in palliative situations.

Aim: To identify barriers to EoL communication in the family context.

Methods: A systematic literature research using the databases PsycInfo, CINAHL, Embase, PubMed and Web of Science was performed and further articles were identified by hand search. Studies were included if they empirically examined the perspective of persons at the EoL (terminally ill or residents of nursing homes) or their family members on conversations about the EoL (Advance Care Planning, death and dying, EoL preparation and preferences).

Results: 10 qualitative studies and 3 quantitative studies were included. The main perceived barriers can be categorized in personal barriers (e.g. condition of the dying), relational barriers (e.g. family conflict), emotional barriers (e.g. fear of death and dying), cognitive barriers (e.g. belief in positive thinking) and organizational barriers (e.g. difficulty of gathering the family).

Conclusion: Barriers in the EoL communication should be considered in palliative care when talking with the patient-family unit of care. Modification of communication barriers can positively affect EoL care, quality of life and the process of grief. Practical recommendations are discussed.

Abstract number: P01-125

Abstract type: Poster

\section{The Unmet Care Needs of LGBT Individuals around the End of Life \\ Candrian $C$. \\ University of Colorado Anschutz Medical Campus, Aurora, CO, United States}

Background: Hospice and palliative care for people near the end of life is associated with less distress, fewer hospitalizations, and improved caregiver outcomes. There is still a population, however, that does not have consistent access to high quality hospice and palliative care: the lesbian, gay, bisexual and transgender (LGBT) population. The LGBT older adult population has been identified as an international health priority because after encountering significant prejudice throughout their lives, LGBT individuals are known to encounter even more as they grow older. There is a critical need to understand barriers of care among LGBT individuals in order to improve the quality of care and eliminate inequalities in accessing end of life care.

Aims: The aim of this project is to understand the experiences of LGBT individuals when accessing hospice and palliative care.

Methods: Methodologies include the analysis of 30 qualitative in-depth interviews considering hospice and/or palliative care using critical discourse analysis. Interviews were conducted after LGBT individuals met with an admission nurse to decide about hospice or palliative care.

Results: Results from the qualitative interviews include:

1) LGBT individuals experience discrimination within the health care system that leads many to cautiously navigate a heteronormative system;

2) LGBT individuals are more likely to seek care when they know the provider is inclusive of their sexual orientation and gender identity;

3) many LGBT individuals continue to fear that "coming out" will influence the type of care they receive and

4) providers often operate without clear guidance on how to interact with LGBT individuals about care decisions.

Conclusion/ discussion: Developing communication guidelines to help nurses better interact with LGBT individuals when talking about end of life care decisions is a novel next step in order to ensure that LGBT individuals receive care that is in line with their needs and values.

Abstract number: P01-126

Abstract type: Poster

Bereaved Parents' Perceptions of Communication about End-ofLife Care Issues: A Nationwide Study

Lykke C. ${ }^{1}$, Ekholm O. ${ }^{2}$, Schmiegelow K. ${ }^{3}$, Olsen M. ${ }^{3}$, Sjøgren P. ${ }^{1}$

${ }^{1}$ Department of Oncology, Copenhagen University Hospital, Copenhagen, Denmark, ${ }^{2}$ National Institute of Public Health, University of Southern Denmark, Copenhagen, Denmark, ${ }^{3}$ Department of Pediatrics and Adolescent Medicine, Copenhagen University Hospital, Copenhagen, Denmark

Background: Communication between patients, families, and healthcare professionals is an essential component of palliative care.

Aim: To improve communication between healthcare professionals and families this study aims at investigating bereaved parents' perception of communication with healthcare professionals after the loss of a child due to life-limiting diagnoses.

Methods: In the period 2012-2014 a register-based study identified death causes of 951 children 0-18 years of age. Previously used classification of life-limiting diagnoses included 402 children. A self-administered questionnaire assessed the parents' perception of communication with the healthcare professionals after the loss of their child. A nonresponse survey identified reasons for lack of response.

Results: In all, 152 children (38\%) were represented by 193 bereaved parents. In all, $98 \%$ of the parents reported that physicians should immediately disclose, when curatively intended treatment was exhausted. Seventy-nine percent of the parents reported that information about the child's incurable illness was given in an appropriate manner; however, $42 \%$ reported that the information about imminent death was given too late. Finally, $31 \%$ felt they were deprived taking leave with their child in the way they wished and $56 \%$ expressed that their child's death was as "a shock".

Conclusion: Parents want accurate and timely information. However, a substantial number of the bereaved parents reported that healthcare 
professionals communicated too late about palliative care issues. Even though healthcare professionals strive to communicate effectively with dying children and their parents, several barriers on both the side of the parents and the professionals may hinder even the best of intensions. National guidelines in communication and improved education of healthcare professionals should be a part of the future agenda.

The study was funded by the Danish Childhood Cancer Foundation.

Abstract number: P01-127

Abstract type: Poster

Narrative Based Medicine in Palliative Care - Building a Model Simões C. ${ }^{1,2}$, Pinto S. ${ }^{3}$, Magalhães S. ${ }^{4,5}$

${ }^{1}$ Escola Superior de Saúde de Santa Maria, Porto, Portugal, 2Palliative Care Team, Hospital da Luz Arrábida, Vila Nova de Gaia, Portugal, ${ }^{3}$ Escola Superior de Saúde de Santa Maria, Vila Nova de Gaia, Portugal, ${ }^{4}$ Universidade Fernando Pessoa, Porto, Portugal, ${ }^{5}$ Bioethics, Universidade Católica Portuguesa, Porto, Portugal

Background: Narratives are an important issue in human's life. Yet, when the person is seriously and/ or terminally ill, (re)making the personal narrative of life may help to face with illness and suffering. Listening to the patient and the family story helps the health care professional to get a better knowledge about the patient and the family needs. However, there is still poor evidence on the impact of using Narrative Based Medicine (NBM) in caring of Palliative Care (PC) patients.

Aims: To analyse the use of NBM in PC settings.

Methods: Scoping Review on Pubmed, Lilacs and RCAAP, for the terms narrative based medicine, palliative care, medicina narrativa, cuidados paliativos. All English, Portugueseand Spanish papers published between January 2017 and June 2018 were included. Eligibility criteria included studies carried out in PC units with full abstract available. Studies within paediatric PC units were excluded. Results were analysed according to the Qualitative Assessment and Review Instrument.

Results: 16 papers were retrieved and 10 were included in the review. Results highlight a low level of evidence. Most studies are study cases. Preliminary results show that the use of NBM with patients is under well studied. Most studies were conducted with educational purposes, and focus on healthcare narratives about the management of spirituality, patient's autonomy, terminal sedation, patient's body image and healthcare reflections on death and dying.

Conclusion: The use of NBM in PC settings can allow healthcare professionals to improve a best patient centered individualized and integrated care plan. However, research on this issue is still scarce and, when exists, there is a low level of evidence. Further research on the topic must be conducted with stronger and robust study designs.

\section{Abstract number: P01-129}

Abstract type: Poster

Moral and Epistemic Virtues in Anti-oppressive Surrogate Decision-making with Children and Young People with Lifelimiting Conditions in South Korea?

Oh S. ${ }^{1}$, Han J.W. ${ }^{2}$

${ }^{1}$ Durham University, Sociology, Durham, United Kingdom, ${ }^{2}$ Division of Pediatric Hematology and Oncology, Department of Pediatrics, Yonsei University Health System and College of Medicine, Yonsei University, Seoul, Korea, Republic of

Background: While conducting surrogate decision making in South Korea, hidden moral dilemmas which arise towards near the end of life associated with risks, contingencies and the best interests.

Aim: This research aims to answer the question of how moral and intellectual virtues can be identified, the difficulties in the ethos of surrogate decision-making in paediatric palliative care and advocate the development of a model of anti-oppressive surrogate decision-making rather than shared decision-making.

Methods: The researcher employs critical ethnography. Three main methods of data collection are; observation, semi-structured interviews and document analysis. In total, 112 interviews took place. The research underwent full ethical review by the School of Applied Social Science's ethics committee at Durham University and the Institutional Review Boards in Korea receiving full approval.

Results: The emerging themes were identified and elaborated in the following areas:

(1) The involvement of beliefs regarding religion, attitude, culture, and superstition when making the surrogate decision.

(2) Hidden oppression of terminally ill children and young people are linked with political, economic, social and cultural aspects.

(3) Intellectual passion in compassion can pursue to elucidate the surrogate decision-making in oppressive structures, constraining both those who suffer from the oppression and those who take benefits from it.

(4) Commitment in compassion can lead to practical improvements and try to connect both to eradicate oppression and to respect the best interests.

Conclusion: In multi-disciplinary context, the role of palliative care social workers requires virtues in anti-oppressive decision-making that can contribute to the intervention of the role of social work as well as possible solutions for the hidden oppression, luck and beliefs associated with surrogate decision-making.

\section{Abstract number: P01-130}

Abstract type: Poster

\section{Advanced Cancer Patients' Reported Wishes Regarding the Importance of Place of Death Using the Go Wish Game Delgado Guay M., Williams J., Bruera E.}

Palliative, Rehabilitation, and Integrative Medicine, The University of Texas MD Anderson Cancer Center, Houston, United States

Background: The use of Go Wish Game cards (GWG) can be helpful to explore end-of-life (EOL) wishes, such as place of death among others, to help prevent distress associated with the dying process. There is limited data about the importance of advanced cancer patients' wishes related to the place of death.

Methods: This is a secondary analysis of a randomized controlled trial about EOL wishes of 100 advanced cancer patients using a GWG and/or a List of wishes/statements; patients were asked to categorize these wishes as very, somewhat, or not important. After 4-24 hours, the patients were asked to complete the same or other test. To determine the frequency and importance of the wish "To die at Home" among this population using the data of the GWG.

Results: Median age (Inter Quartile Range= IQR): 56 (27-83) years. There was high correlation among the patients who prioritized the wish "To die at Home" as "very important" between test 1 and test $2(13 / 100$ vs. $16 / 100, r=0.67, p<0.0001$ ). Only $38 / 100$ (38\%) patients expressed their wish "To die at Home" as very/somewhat important, compared with $62 \%$ who chose it as Not important wish at the end of life. Age, sex, ethnicity, marital status, religion, education, and cancer diagnosis did not differ significantly among the groups. Patient who selected "To Die at home" as Somewhat Important/Very Important were 10 times more likely to die in Hospice than in Hospital (OR:10, $p=0.045)$. The median survival among the patients who chose "To die at home as very/somewhat important" was 1 month (IQR: 1-4) vs. 3 months (IQR: 1.6-8, $\mathrm{p}=0.145$ ).

Conclusions: The importance attributed to advanced cancer patient's wishes regarding "To die at home" is low compared with other wishes at 
the end of life. GWG is a useful tool to identify/explore these wishes and promote better communication among patients and clinicians. More research is needed.

\section{Abstract number: P01-132 \\ Abstract type: Poster}

Context, Mechanisms and Outcomes in End of Life Care for People with Advanced Dementia: Family Carers Perspective Kupeli N. ${ }^{1}$, Moore K. ${ }^{1}$, Harrington J. ${ }^{1}$, Davis S. ${ }^{1}$, Leavey G. ${ }^{2}$, Candy B. ${ }^{1}$, King M. ${ }^{3}$, Nazareth I. ${ }^{4}$, Jones L. ${ }^{1}$, Sampson E.L. ${ }^{1,5}$

${ }^{1}$ Marie Curie Palliative Care Research Department, University College London, London, United Kingdom, ${ }^{2}$ Bamford Centre for Mental Health \& Wellbeing, University of Ulster, Belfast, United Kingdom, ${ }^{3}$ Division of Psychiatry, University College London, London, United Kingdom, ${ }^{4}$ Department of Primary Care \& Population Health, University College London, London, United Kingdom, ${ }^{5}$ Barnet Enfield and Haringey Mental Health Trust Liaison Psychiatry Team, North Middlesex University Hospital, London, United Kingdom

Background: More people are providing informal care for people with dementia and the role of carers in managing the end of life is challenging. Most people with advanced dementia die in care homes so family carers and health care professionals (HCP) must negotiate how to provide optimal care. Previously we reported a realist analysis of the HCP perspective [1]. Here, we report a similar analysis using family carer data. We aimed to understand the similarities and differences between the two perspectives, how the interdependent roles of carers and HCP can be optimised and make recommendations for policy and practice.

Method: We use the EAPC white paper [2] framework for good outcomes. We applied thematic analysis to qualitative interview data from 14 carers. We used realist methods to describe contexts and mechanisms that may affect outcomes.

Results: Findings mirrored those from HCP data including business driven care homes with low skilled care staff who have demanding workloads and low salaries. Carers reported a context of ad hoc information about services, funding and dementia progression. Not all carers identified dementia as terminal, but many saw the importance of continuity of care and knowledge of the wishes of the person with dementia. New mechanisms included specific resources for improving end-of-life care and barriers to discussing end of life care. Carers identified the importance of comfort, being present, the meeting of basic care needs and feeling the right decisions have been made as death approaches.

Conclusions: Structures for providing information to support carers and trusting relationships between family and HCP are recommended for end-of-life care

\section{References:}

1. Kupeli et al. Context, mechanisms and outcomes in end of life care for people with advanced dementia. BMC Pall Care 2016;15(1):31

2. van der Steen et al. White paper defining optimal palliative care in older people with dementia. Pall Med 2014 28(3):197

Abstract number: P01-133

Abstract type: Poster

The Importance of Relationship Quality between Spouses in Advanced Parkinson's Disease from the Perspective of the Caregiving Partner in Palliative Care Needs of Patients and Family

Arshinoff . $^{1}$, Preston N. ${ }^{2}$, Grinyer A. ${ }^{2}$

${ }^{1}$ Religious and Spiritual Care, Baycrest, Toronto, Canada, ${ }^{2}$ Faculty of Heath and Medicine, Lancaster University, Lancaster, United Kingdom

Introduction: Parkinson's Disease (PD) is a chronic and degenerative illness with a long trajectory involving multiple physical, cognitive, and emotional changes. In contrast to the holistic approach of palliative care, medical visits for PD patients tend to focus on physical symptoms. Little attention is paid to the chronic psychosocial effects of PD, such as relational satisfaction both patient and family. As illness advances, dependence on others occurs; mainly the spouse is the primary caregiver. Despite research on burden of care for PD spouses, the literature reveals little on mutuality, which concerns the quality of the relationship and almost nothing on mutuality in advanced illness. This qualitative study researched mutuality specifically in advanced PD from the perspective of the caregiving spouse.

Aims: To gain insight into the impact of advanced PD on the quality of the marital relationship from the perspective of the caregiving spouse with the goal of developing holistic and palliative care approaches to PD patients and their caregiving spouse.

Method: Twelve caregiving spouses of partners with advanced PD were recruited by purposive sampling. They participated in a single face-toface semi-structured interview in this qualitative hermeneutic phenomenology study that utilised van Manen's approach to data analysis and writing.

Results: Participants' challenges to mutuality included limited enjoyable shared activities, living with a "stranger", little fun, and resentment about PD. Loss of identity, ambiguous loss (the ill person is present but psychologically absent), and searching for meaning are challenges for caregiving spouses caring for an advanced PD partner; nevertheless most participants want to be with their spouse.

Conclusion: Understanding how PD affects the marital relationship is crucial for health professionals to provide a palliative approach to both partners living with this unwelcome long term "intruder" in their lives.

\section{Abstract number: P01-134}

Abstract type: Poster

"The Last Thing I Can Do" - Experiences of the Commanding Character of End-of-Life Informal Care Giving Haan M., Olthuis G., van Gurp J.

Radboud Institute for Health Sciences, IQ Healthcare, Radboud University Medical Center, Nijmegen, Netherlands

Background: In end-of-life care, the focus is on the dying patient. The perspectives of informal caregivers at home are often less visible. Studies show that they feel burdened, being confronted with new and (emotionally) difficult situations and responsibilities. Less clear is what normative aspects constitute this burden and what motivates caregivers.

Aims: We aim to tell the story of taking care of a dying relative. Our scientific results will be incorporated in a graphic novel, aimed at public education. For the authors to develop this book, it is important to know the experiences and normative constituting aspects of informal care giving. What does it mean to take care of a dying relative?

Methods: Up to September 2018, a total of 14 informal caregivers, 12 bereaved informal caregivers and 7 patients with various life-threatening diseases participated in in-depth semi-structured interviews. They were recruited purposively and interviewed at their homes. Supported by ATLAS.ti software, the data were qualitatively analyzed, using a Grounded Theory approach. Data collection continued from Fall 20172018, until saturation was reached.

Results: Preliminary results show the commanding character of informal care giving. Previous experiences with death or care direct one's believes about how to take care. In the present, being overwhelmed by the disease inevitably taking over life, one is commanded more explicitly by the call for help from the dying person and by managing all tasks. The (imagined) future commands with regard to doing the last things right for one's loved one.

Conclusion: Support aimed at unburdening informal caregivers should perhaps not focus only on relief from tasks, but also on normative aspects of care giving. Further research will show whether and how a graphic novel raises public awareness. This might help informal caregivers in 
feeling understood and supported. The research was funded by The Netherlands Organisation for Health Research and Development.

Abstract number: P01-135

Abstract type: Poster

Grief Symptoms in Family Caregivers during the Patient's End-ofLife Period and the Development of Adverse Grief Trajectories. A Population-based, Prospective Study

Nielsen M.K. ${ }^{1}$, Neergaard M.A. ${ }^{2}$, Bidstrup P.E. ${ }^{3}$, Guldin M.-B. ${ }^{1,2}$

${ }^{1}$ Research Unit for General Practice, Aarhus $C$, Denmark, ${ }^{2}$ Department of Oncology, Palliative Care Unit, Aarhus University Hospital, Aarhus C, Denmark, ${ }^{3}$ Survivorship Unit, Danish Cancer Society Research Center, Copenhagen, Denmark

Background: The grief of family caregivers develops differently. We have identified common patterns in five grief symptom trajectories - low (39\%), late (7\%), moderate/decreasing (30\%), high/decreasing (18\%) and high (6\%) grief trajectories - in a prior study based on three measurements of sum scores of the Prolonged Grief-13 (PG-13) scale before and after the patient's death. The high grief trajectory indicates longterm psychological consequences and a need for interventions by health professionals starting during end-of-life care.

Aim: We aim to explore whether a single time-point measurement before the patient's death of adapted prolonged grief criteria was associated with the development of a high grief trajectory.

Methods: A population-based, longitudinal study of 1,735 family caregivers to terminally ill patients participating before (TO), six months after (T1) and three years after the patient's death (T2). We describe the fulfillment of the $P G-13$ criteria at TO (pre-loss version) for family caregivers in specific grief trajectories.

Results: In total, 260 (16\%) family caregivers fulfilled the $P G-13$ criteria at T0 and $52 \%$ of those fulfilling the $P G-13$ criteria had a high/decreasing and $23 \%$ had a high grief trajectory. Furthermore, a group developed a high grief trajectory without fulfilling the pre-loss $P G-13$ criteria (41\%). Conclusion: Most family caregivers who fulfilled the pre-loss $P G-13$ criteria did not develop a high grief trajectory and this assessment failed to identify a large group of family caregivers who developed a high grief trajectory. Pre-loss PG-13 criteria may point at support needs during end-of-life care both for family caregivers with grief trajectories of persistent high grief and those with decreasing symptoms. However, to improve health professionals' identification of family caregivers at risk of developing a persistent high grief trajectory, future research on screening tools is necessary.

Funding: The Novo Nordisk Foundation

Abstract number: P01-136

Abstract type: Poster

Interprofessional Caring for Children who Are Relatives of Cancer Patients in Palliative Care: Perspectives of Doctors and Social Workers

Karidar H., Glasdam S.

Integrative Health Research Department of Health Sciences, Lunds University, Lund, Sweden

Background: A palliative cancer diagnosis in a parent has a major impact on many aspects of patients' children. This article aims to explore how doctors and social workers met children as relatives of a parent with cancer in order to understand the possibilities and difficulties in supporting children in specialized palliative homecare in Sweden.

Methods: Qualitative, semi-structured interviews were conducted, inspired by Bourdieu.

Results: The findings showed that professionals had limited contact with patients' children. Mono- and inter-professional meetings were organising structures for working days. Due to hierarchical positions, doctors often set the agenda for inter-professional teamwork. Doctors seldom met patients' children, only when information about parents' cancer diseases was needed. Social workers were responsible for psychosocial issues, but mostly only special vulnerable families and their children were prioritised. Meetings between children and professionals were conditional on the parents' permission, and even if permission was given, meetings seldom took place.

Conclusion: Doctors and social workers were subject to the structural frame and a medical logic, which limited their interactions with children of the patients. Children were dependent on both their parents and professionals, who had the power to include or exclude them from the parent's illness situation.

\section{Abstract number: P01-137 \\ Abstract type: Poster}

International Consensus Statements Defining Advance Care Planning versus the Reality Experienced by Families of People with Dementia - A Qualitative Study

Beck E. ${ }^{1}$, Mcllfatrick S. ${ }^{2}$, Hasson F. $^{1}$

${ }^{1}$ School of Nursing, University of Ulster, Newtownabbey, United Kingdom, ${ }^{2}$ School of Nursing, University of Ulster, Jordanstown, United Kingdom

Background: Advance care planning (ACP) is a core component fro improving palliative care, yet discussions are rare among people living with dementia.

Aim: To examine family caregivers' of people with dementia understanding, perspectives and experience of engaging in ACP in the long term care setting, and to contrast these with international consensus statements defining advance care planning.

Methods: A qualitative design was adopted for this component of a larger scale sequential explanatory mixed methods study. Semistructured interviews were undertaken between January-March 2016. Thematic analysis was used to elicit key themes. A total of 16 families, identified as next of kin, were recruited across nursing homes in a geographical region of the UK.

Results: Findings reflected that a limited understanding was present, impacting upon all aspects of the advance care planning process. Essentially awareness of the physical decline associated with dementia was absent, in addition to acknowledgement that discussions should precede such. Subsequently, families needed to witness this decline before the onus to engage was created. Lastly, currently ACP discussions are clinically focused and physically driven, resulting in a reactive as opposed to proactive approach to planning.

Conclusions: The quest for a universal definition and conformity as to what represents successful outcomes in ACP, has failed to reflect the unique challenges created by dementia. As a result current definitions do not take into the consideration the particular nuances faced within the context of dementia. Additionally, such definitions fail to reflect the perspectives of those currently expected to undertake the role of decision maker by proxy. Therefore there is an urgent need for international collaboration that addresses the current disparity between international definitions, and the reality experienced by those currently engaging in the process of ACP.

Abstract number: P01-138

Abstract type: Poster

Caregiving in Home Palliative Care: Addressing the Most Important Factors Predicting a Better Quality of Life Varani S. ${ }^{1}$, Raccichini M. ${ }^{1}$, Franchini L. ${ }^{2}$, Ercolani G. ${ }^{1}$, Malerba M.B. ${ }^{1}$, Gengarelli G. ${ }^{2}$, Pannuti R. ${ }^{1}$, Pannuti F. ${ }^{1}$

${ }^{1}$ ANT Italia Foundation, Bologna, Italy, ${ }^{2}$ University of Padua, Padova, Italy 
Background: In Italy there is about $15 \%$ of people who assume the role of caregiver assisting a family member in home palliative care context, exposing them to a strong emotional distress.

Aim: The aim of this study is to evaluate caregivers well-being, in particular if the perceived burden, the preparedness caregiving level and the psychological mood could be considered quality of life (QOL) predictors.

Method: Demographic data of 570 adult informal caregivers assisting cancer patients at home were collected. Caregiver-reported outcomes were measured by Profile Mood of States (POMS), Caregiver Burden Scale (CBS), Preparedness for Caregiving Scale (PCS) and Caregivers Quality of Life Index-Cancer (CQOLC). The Activities of Daily Living (ADL) and the Instrumental Activities of Daily Living (IADL) for patients were also given. A correlation analysis was carried out. A regression analysis was also conducted to assess whether the examinated variables could predict the QOL.

Results: The standardized regression coefficients indicated that depression and perceived burden could be significant predictors of a worse quality of life of caregivers (respectively $\beta=-, 515 t=-16,477, p=, 000 \mathrm{e}$ $\beta=-, 361 t=-12,148, p=, 000$ ); in addition a perceived preparedness for caregiving could predict a better caregiver quality of life $(\beta=, 119$ $t=-4,802 ; p=000)$. ADL and IADL resulted poorer predictors of QOL $(r=, 163, p=000$ e $r=, 208, p=000)$.

Conclusion: Considered psychological factors could significantly predict the quality of life of informal caregivers. The results suggest that it is not so much the functional patient disabilities which negatively impact on caregiver QOL, but mostly their psychological mood and their perceived burden. This study underline also the importance to take in account the psychological needs of the caregivers since often underestimated.

Abstract number: P01-139

Abstract type: Poster

\section{Assuming the Healthcare System: Experience of End-of-Life Care for Families \\ Martín-Martín J. ${ }^{1}$, Pérez-Diez del Corral M. ${ }^{1}$, Olano-Lizarraga M. ${ }^{1}$, Valencia-Gil S. ${ }^{2}$, Saracibar-Razquin M. ${ }^{1}$ \\ ${ }^{1}$ University of Navarra, Pamplona, Spain, ${ }^{2}$ Primary Care Osasunbidea \\ Navarra, Pamplona, Spain}

Background: Currently, terminally ill patients prefer to receive end-of-life care at home, with their loved ones. To enable this, family involvement is essential. But the likelihood of patients receiving the best care without excessive impact on their families depends on the support of the sociosanitary team. The preliminary results on this issue are presented; they are part of broader research on family experience of home care.

Aims: To learn about family experiences of end-of-life healthcare in the home.

Methods: Narrative research was done with seven families who had a terminal patient in the home. Narrative group and individual interviews were completed with family members (7 groups and 18 individuals). Two researchers made a narrative thematic analysis of the data.

Results: The family experiences were grouped into three themes:

1) The lack of care from the healthcare system;

2) Being responsible for care of relative;

3) Lack of support from social system.

The first refers the families' need for care. Health care professionals frequently do not listen to family members' concerns and do not even ask how they are managing in this situation. The second deals with how the responsibility for attending the patient lies with the families and the problems this involves in decision-making, in direct care and even regarding sedation. The third points out the families' lack of knowledge and need for advice when accessing and using the social resources available.
Conclusion: The families assume end-of-life care without the necessary knowledge and support. Health care teams should be more aware of the situation and should include the families in their care. In addition, there should be greater and better-quality social resources, with improved information and methods of access.

\section{Abstract number: P01-140 \\ Abstract type: Poster}

Informal Caregivers' Experiences and Needs within Different Phases of the Palliative Care Trajectory

Mulder F.M.H. ${ }^{1}$, van Rijn M. ${ }^{2,3}$, Flierman I. ${ }^{1,2}$, Buurman B.M. ${ }^{2,3}$, Willems D.L. ${ }^{1}$

${ }^{1}$ Department of General Practice, Section of Medical Ethics, Amsterdam UMC, University of Amsterdam, Amsterdam, Netherlands, '2Department of Internal Medicine, Section of Geriatric Medicine, Amsterdam UMC, University of Amsterdam, Amsterdam, Netherlands, ${ }^{3}$ Faculty of Health, ACHIEVE-Centre of Applied Research, Amsterdam University of Applied Sciences, Amsterdam, Netherlands

Background: Informal caregivers perform a wide range of activities within different phases of the palliative care trajectory (PCT). These informal caregivers often experience an increased caregiver burden and a deteriorated quality of life. Timely and well-organized engagement and support of informal caregivers during the PCT could prevent caregiver burden and increase their quality of life. However the experiences and needs of informal caregivers during the different phases of the palliative care trajectory are unknown.

Aim: To characterize informal caregivers' experiences and needs within different phases of the PCT.

Methods: Qualitative semi-structured interviews, involving 15 informal caregivers of patients aged $\geqslant 70$, were conducted. A thematic content analysis was performed.

Results: Two main themes (enabling support and direct support) were categorized within three phases of the PCT (palliative-, terminal-, and bereavement phase). Participants described that educational support was lacking in the early palliative phase. Because of structured end-oflife care services, needs regarding practical and educational support were often met during the terminal phase. However, emotional support was reported to be lacking during the terminal phase, as well as during the bereavement phase. Overall, participants urged the need for: 1) continuity of care by seeing one care coordinator on a regular base, 2) communication on end-of-life expectations, preferences and needs, especially at an early stage of the palliative phase; and 3) the acknowledgement of the emotional challenges of being an informal caregiver of a patient receiving palliative care.

Conclusion: This study demonstrates that informal caregivers need educational and emotional support depending on the palliative phase of the patient. Incorporation of informal caregivers' needs in a structured palliative care pathway might contribute to improved continuity and coordinated care.

Source of funding: ZonMw

Abstract number: P01-141

Abstract type: Poster

Development of a Leaflet to Support Laycarers in the Administration of Subcutaneous Injections for Home-based Palliative Care Patients

Chua Z.R. ${ }^{1}$, Tan L. ${ }^{2}$, Salim N.H.B.M. ${ }^{2}$, Tan A. ${ }^{2}$

${ }^{1}$ Geriatrics, Khoo Teck Puat Hospital, Singapore, Singapore, ${ }^{2} \mathrm{HCA}$

Hospice Care, Singapore, Singapore

Background: The majority of Singaporean patients prefer to die at home. When terminally ill patients can no longer swallow reliably, non-oral routes of drug administration are essential for effective symptom 
control. The likelihood of patients dying at home often depends on laycarers who may need to administer subcutaneous (SC) injections.

Objective: This study reports the development, trial and evaluation of a combined instructional leaflet and medication administration record (MAR) for use in patients receiving home-based palliative care.

Method: The first draft of the leaflet was trialed at a single satellite centre of a national home hospice service in Singapore. Feedback during the initial implementation phase was used to refine the final design. The original English-version of the leaflet was also translated to Mandarin Chinese (a language commonly spoken in Singapore) and its use expanded across all 5 satellite centres in Singapore. Outcome of primary interest was laycarer confidence in preparation and administration of SC injections to achieve symptom control.

Measurements: Fifty-two laycarers were contacted via phone, with responses collected from $43(82.7 \%)$. Nine laycarers (17.3\%) were either uncontactable or declined to participate. Of the 43 laycarers who agreed to participate, 40 (93.0\%) found the leaflet easy to understand and 35 $(81.4 \%)$ felt that the leaflet gave them confidence in preparing and administering SC injections. In addition, 5 laycarers specifically requested for future versions of the leaflet to be translated into Bahasa Indonesia (a language commonly spoken by domestic helpers working in Singapore). Findings: This leaflet supports laycarers in the preparation and administration of SC injections, thus supporting palliative patients to remain at home. This has important implications for patients, families and health care services. Future considerations would include a translated version to Bahasa Indonesia.

\section{Abstract number: P01-142 \\ Abstract type: Poster}

Mother Voices: "Being the Main Advocate to our Children": The Experience of Jordanian Mothers in the Care of Children with Palliative Care Needs during Hospitalization

Atout M. ${ }^{1}$, Hemingway P. ${ }^{2}$, Seymour $J .^{3}$

1Philadelphia University, Amman, Jordan, ${ }^{2}$ University of Nottingham, Nottingham, United Kingdom, ${ }^{3}$ Sheffield University, Sheffield, United Kingdom

Background: Health and social professionals described themselves as being the target of parents' stress and anger mention that at least some degree of conflict was associated with a wide range of issues in child palliative care. In these situations, the professionals felt themselves powerless and unable to ease the fears and stress of families.

Purpose: To explore the experience of communication in the care of children with palliative care needs, from the perspective of physicians, nurses, and mothers in Jordan. In this paper, the findings concerning mothers' experiences will be presented.

Methods: This study employed a collective qualitative case study approach. It was conducted in three paediatric units in a Jordanian hospital. Each case comprised a child aged 1-12 years, their most involved family carer (mothers), physician(s) and nurse(s). Two data collection methods were employed: participant observation and semi-structured interviews with three categories of participants: mothers, physicians, and the nurses who cared for the children that participated in this study. Findings: The parents described themselves as having the responsibility to protect their children from any physical or psychological harm they faced because of their illness. They sometimes felt that they needed to intervene and advocate for their children's right to receive the best health care available. The mothers were not passive receivers of the treatment given to their children; instead, they were active stakeholders who tried to understand every medical or nursing procedure, and they were curious about any modifications or changes undertaken in their children's treatment plans.

Conclusion: The parents' experiences should be taken into consideration when the medical teams establish the children's treatment plans. The continuous education for professionals, which provides staff with specialised communication skills with children and parents will improve the quality of care provided to them.

Abstract number: P01-143

Abstract type: Poster

Misperceptions of Heart Failure Terminality: Aggressive

Treatments at End of Life

Hupcey J. ${ }^{1}$, Kitko L. ${ }^{2}$, Gilchrist J. ${ }^{3}$

${ }^{1}$ College of Nursing, Pennsylvania State University, Hershey, United States, ${ }^{2}$ College of Nursing, Pennsylvania State University, University Park, United States, ${ }^{3}$ Duke University, Durham, United States

Background: Heart failure (HF) remains a worldwide epidemic, affecting over 26 million adults worldwide. Half of those with HF will die within 5 years of diagnosis. Although the unpredictable trajectory of HF ultimately ends in death, caregivers (CGs) often misunderstand the terminality of HF which may complicate decisions about treatment choices at the end of life (EOL).

Aims: To determine how CG perceptions of the terminality of HF impacted aggressive treatment decisions made at EOL.

Methods: Participants included CGs who were part of a longitudinal parent study on the palliative care needs of persons with Stage D HF with less than a 2-year predicted survival. Utilizing content analysis, CGs of patients who died during study enrollment were divided into two groups based on their perception of HF as a terminal disease. Post death interviews were analyzed to determine group differences in aggressive treatment decisions at EOL.

Results: The analysis included 25 CGs. Therapies considered to be aggressive included endotracheal intubation, LVAD implant, cardioversions, hemodialysis, family initiation of CPR prehospitalization, and insertion of a feeding tube. Only $40 \%$ of CGs $(n=10)$ perceived the terminality of $H F$, while $60 \%(n=15)$ did not. Sixty percent of the $C G s(n=9)$ who did not perceive $\mathrm{HF}$ terminality made aggressive treatment choices at EOL whereas only $30 \%$ of CGs $(n=3)$ who did perceive HF terminality made similar choices. While this appeared to be a trend in the data, it was not statistically significant $(p=14)$. This was most likely due to the small sample size.

Conclusions: The majority of the CGs did not acknowledge the terminality of $\mathrm{HF}$ which often led to aggressive therapeutic choices at EOL and a decreased likelihood of treatment withdrawal. More effective communication with CGs and persons living with advanced HF, with an emphasis on advance care planning and goals of care conversations throughout the HF trajectory is necessary.

\section{Abstract number: P01-144}

Abstract type: Poster

Facilitators of Resilience in Carers for a Midlife Partner with an Incurable Cancer: A Thematic Analysis

Opsomer S. ${ }^{1}$, Pype P. ${ }^{2,3}$, Lauwerier E. ${ }^{2,4}$, De Lepeleire J. ${ }^{1}$

${ }^{1} \mathrm{ACHG}$, Catholic University Leuven, Leuven, Belgium, ${ }^{2}$ Faculty of Medicine and Health Sciences, Ghent University, Ghent, Belgium, ${ }^{3}$ Endof-Life Care Research Group at theVrije Universiteit Brussel and Ghent University, Ghent, Belgium, ${ }^{4}$ Faculty of Psychology and Educational Sciences, Ghent University, Ghent, Belgium

Background: Providing care for advanced cancer patients is often the responsibility of the partner. Being confronted with an incurable cancer diagnosis can be highly disruptive for the patient's partner. However, most carers seem to adapt well and create a healthy path during the process of providing care, linked to the concept of 'resilience'. Resilience is age-related with the elderly exposing higher levels of resilience. It can be assumed that middle-aged partners are more at risk for a non-resilient outcome. Moreover, resilience has been understudied in this age group of carers. 
Aim: To explore what resources make caregiving for a midlife advancedcancer patient a resilient process.

Methods: Nine partners of midlife patients who died at home of cancer were selected purposively and interviewed in depth within the first year following the death of their partner. A thematic analysis utilizing an inductive approach was conducted.

Findings: Resilience was challenged by the partner's abrupt diagnosis of incurable cancer. All participants made use of a set of interacting, carerspecific and context-related resources, facilitating a resilient process and leading to positive feelings or even personal growth. The carers demonstrated individual competences: adaptive flexibility, positivism, a sense of self-initiative, and adaptive dependency, and they relied on their context: cancer-related professionals and long-time relatives. Context and situation were continuously interacting. The resulting dynamics were based on the context-availability, meaningful relationships and the patient's role.

Conclusion: A resilient trajectory results from an interplay between individual and context resources. To build resilience in partners of midlife, advanced-cancer patients, health care professionals should address all available resources. Moreover, they should be aware of being part of the carer's context, a complex adaptive system that can be either resiliencesupporting or -threatening.

Abstract number: P01-145

Abstract type: Poster

Comparative Analysis of Quality of Life and Attachment Style among Cancer and Dementia Informal Caregivers

Papageorgiou N. ${ }^{1}$, Zygogianni A. ${ }^{2}$, Parpa E. ${ }^{3}$, Tsilika E. ${ }^{3}$, Nikoloudi M. ${ }^{3}$ Mystakidou K. ${ }^{3}$

${ }^{1}$ Day Care Center for Adolescents and Young Adults in the Autism Spectrum, Athens, Greece, ${ }^{2}$ Areteion Hospital, Department of Radiology, National \& Kapodistrian University of Athens, Athens, Greece, ${ }^{3}$ Pain Relief and Palliative Care Unit, Department of Radiology, Areteion Hospital, School of Medicine, National \& Kapodistrian University of Athens, Athens, Greece

Background: Caregivers of cancer and dementia patients face burden associated with lower quality of life, psychological distress and unpleasant physical.

Aims: To assess and compare the quality of life and attachment styles between informal cancer and dementia caregivers.

Methods: A comparative prospective cross sectional study consisting of 90 caregivers. Quality of life was measured using the Short Form 36 health survey questionnaire, Experiences in Close Relationships assessed ways to relate to others in close relationships, and the ECOG Performance Status was used to identify cancer patients' general condition.

Results: The unadjusted analysis of subscales between compared groups did not reveal any statistically significant association. There was not any significant difference between the caregivers' groups compared for all scales of the SF-36 questionnaire. The score closest to statistical significance is the physical role $(p=0.076)$, in dementia caregivers.

Conclusion / Discussion: Poor quality of life was found in both populations. Assessing the quality of life among caregivers showed that the impact of caregiving stress on psychological and health functioning was important. Dementia caregivers were more burdened in physical functioning. They were older than cancer caregivers, while old age was associated with worse physical resistance and more physical problems. Cancer caregivers showed more stress in interpersonal relationships than dementia caregivers. Some of the related indicators can be the effect of fear of loss of the loved ones, which in case of cancer caregivers may be experienced as a more immediate danger. An integrated management involving caregivers would reduce the risk of developing psychological distress in cancer caregivers, while by educating dementia caregivers regarding dementia and its progress would help then to better adapt and lessen their burden.
Abstract number: P01-146

Abstract type: Poster

Supporting Family Carers at Hospital Discharge at End-of-Life: A Feasibility Study of the Carer Support Needs Assessment Tool (CSNAT) Intervention

Hall A. ${ }^{1,2}$, Grande G. ${ }^{1,2}$, Rowland C. ${ }^{1,2}$, Ewing G. $^{3}$

${ }^{1}$ Division of Nursing, Midwifery \& Social Work, University of

Manchester, Manchester, United Kingdom, ${ }^{2}$ NIHR Collaboration

for Leadership in Applied Health Research Greater Manchester, Manchester, United Kingdom, ${ }^{3}$ University of Cambridge, Cambridge, United Kingdom

Background: The majority of patients in hospital at end-of-life wish to die at home. Family carers are essential in making this possible, but often feel unprepared and unsupported in the discharge process, which can lead to a breakdown of care at home. The CSNAT intervention is a person-centred approach for assessing and supporting carers to support the patient at home and preserve their own health and wellbeing. It has been developed and validated for home care, and used in more than 25 countries. For hospital discharge, practitioners and carers have recommended that the CSNAT intervention should be introduced to carers in hospital, with a post-discharge community follow-up.

Aim: To test the feasibility of using the CSNAT intervention to support carers during discharge of patients from hospital at end-of-life.

Methods: Case study of implementation of the intervention within one English healthcare organisation. Participants $(n=17)$ were hospital and community palliative care practitioners. We conducted longitudinal pre- and post-implementation qualitative interviews during a 6 month implementation period exploring practitioners' perceptions and experiences of using the CSNAT intervention, observed team meetings, and reviewed implementation procedures. Data analysis using Framework approach.

Results: Facilitators included embedding the CSNAT intervention into clinical and administrative team meetings, and developing a clear mechanism of handover of CSNAT assessments from the hospital team to the community team. Challenges included a lack of time for the hospital team to connect with carers prior to discharge.

Conclusion / Discussion: The CSNAT intervention can be embedded into routine administrative practice in hospital and community palliative care, but structural and procedural constraints within acute care and transition to community can pose challenges requiring further investigation.

Funding: NIHR Collaboration for Leadership in Applied Health Research Greater Manchester

Abstract number: P01-147

Abstract type: Poster

'A Juggernaut Coming': Family Experiences of End of Life Care for People Using Alcohol and Drugs

Yarwood G. ${ }^{1}$, Wright S. ${ }^{1}$, Templeton L. ${ }^{2}$, Galvani S. ${ }^{1}$

${ }^{1}$ Social Care and Social Work, Manchester Metropolitan University, Manchester, United Kingdom, 2Independent Researcher, Bristol, United Kingdom

Background and aims: Family members and care givers (FCGs) of people with substance problems typically live through years of substantial cumulative strain in their family relationships. This makes their experience of providing end of life care and their subsequent bereavement all the more traumatic. This UK based in-depth qualitative study is the first of its kind to explore:

- FCGs' experiences of substance use, health and social care services at the end of their relative's life. 
- FCGs' support needs and the role of both formal and informal support.

Study design, population sample and data collection methods: There were two forms of data collection:

- Secondary data analysis of 102 qualitative interviews with adults bereaved by substance use, recruited from a range of settings in England and Scotland.

- Semi-structured interviews with 15 FCGs recruited from hospices, substance use treatment services and community contacts.

Analysis: Data were systematically analysed using secondary analysis of qualitative data techniques and template analysis.

Results:

1) Many opportunities for providing care to the relative were missed, resulting in multiple interactions with services and unnecessary pain and suffering.

2) Even where death could be anticipated, end of life was rarely explicitly communicated to family members, leaving them distraught and angry.

3) Many families felt guilt and despair that they had not managed to 'save' their family member from an undignified death.

4) FCGs received little direct support at this difficult time.

Conclusion: It is globally recognised that EoL care brings substantial stress into families and care networks but less is known about how much more challenging this is if the individual has alcohol/drug difficulties. This study develops evidence on experiences of FCGs of people who are at end of life with historic/existing substance use problems.

Funding: Big Lottery Fund UK

Abstract number: P01-148

Abstract type: Poster

\section{Carer Support Needs and Quality of Life in Palliative Care: A} Methodological and Empirical Study

Alvariza A. ${ }^{1,2}$, Holm M. ${ }^{3}$, Benkel I. ${ }^{4}$, Norinder M. ${ }^{1}$, Ewing G. ${ }^{5}$, Grande G. ${ }^{6}$, Håkanson C. ${ }^{7}$, Öhlen J. ${ }^{8}$, Årestedt K. ${ }^{9}$

${ }_{1}^{1}$ Palliative Research Centre/Department of Health Care Sciences, Ersta Sköndal Bräcke University College, Stockholm, Sweden, ${ }^{2}$ Dalen Hospital, Capio Palliative Care, Stockholm, Sweden, ${ }^{3}$ Department of Nursing Science, Sophiahemmet University, Stockholm, Sweden, ${ }^{4}$ Department of Geriatric Medicine, Sahlgrenska Academy, University of Gothenburg, Gothenburgh, Sweden, ${ }^{5}$ Centre for Family Research, University of Cambridge, Cambridge, United Kingdom, ${ }^{6}$ Division of Nursing, Midwifery \& Social Care, Faculty of Biology, Medicine and Health, University of Manchester, Manchester, United Kingdom, 'Department of Nursing Sciences, Sophiahemmet University, Stockholm, Sweden, ${ }^{8}$ Institute of Health and Care Sciences and the Centre for Person-Centred Care, Sahlgrenska Academy, University of Gothenburg, Gothenburg, Sweden, ${ }^{9}$ Faculty of Health and Life Sciences, Linnaeus University, Kalmar, Sweden

Background: The Carer Support Needs Assessment Tool (CSNAT) was developed to identify support needs of family carers in the context of palliative care which aims to improve quality of life, not just of patients but also their families.

Aims: This study aims to 1) evaluate validity and reliability of the CSNAT in a sample of Swedish family carers and nurses in a specialised palliative care context, 2) investigate associations between carer support needs and quality of life.

Methods: The study was conducted in four stages.
I: translation of CSNAT to Swedish;

II: cognitive interviews with 8 family carers and 10 nurses;

III: completion of the CSNAT, Preparedness for Caregiving Scale, Caregiver Burden Scale, Quality of Life in Life Threatening Illness- Family Carer Version by 118 family carers (spouses/partners: mean age 68 years; 69 women and 45 men). Evaluation of data quality, construct validity and test-retest reliability;

IV: Investigation of associations between carer support needs and quality of life using linear regression analyses.

Results: CSNAT items were considered relevant and useful to identify support needs and demonstrated sound psychometric properties with satisfactory data quality and few problems with missing data. All items had satisfactory test-retest reliability. Construct validity was supported, as CSNAT items correlated with caregiver burden and preparedness. Associations were found between CSNAT items and seven different domains that represent carer quality of life; carer state, patient wellbeing, quality of care, outlook, environment and finances. Having more support needs was associated with poorer quality of life.

Conclusion: This study adds to the validity of the CSNAT and shows in addition that it is reliable and stable for use among family carers in palliative care. Associations between carer support needs and quality of life suggests that carers' quality of life may be improved by acknowledging and addressing their needs for support.

Abstract number: P01-149

Abstract type: Poster

Back to the Future: Bringing Family Caregivers Centre Stage in a New Cottage Hospice Model of End of Life Care. Considerations from an Action Research Study

Barnes H. ${ }^{1}$, Walshe C. ${ }^{1}$, Turner M. ${ }^{2}$, Hughes S. ${ }^{1}$

${ }^{1}$ International Observatory on End of Life Care, Lancaster University, Lancaster, United Kingdom, ${ }^{2}$ University of Huddersfield, Huddersfield, United Kingdom

Background: A UK hospice is launching a new model of end-of-life provision, Cottage Hospice, responding to demographic and cost challenges. Family members and volunteers will provide the majority of patient care, in a move to 'bring death back to the community'. While presented as an innovation, this could equally be seen as a return to an emphasis on informal and unpaid care.

Aims: To use participatory action research (PAR) to evaluate the development of Cottage Hospice from inception to opening and to disseminate the knowledge gained to inform service innovation in the sector.

Methods: An embedded researcher implemented a PAR research approach in a UK hospice over 29 months. Documentary analysis was conducted using strategic records, internal meeting minutes, press releases and other written materials $(n=120+)$. Semi-structured interviews (participants: $n=36$ ) captured stakeholder views on process and progress over time. A series of action cycles led by a Core Action Group identified key areas for solution focussed change. A patient and carer advisory panel contributed to data analysis.

Results: Some stakeholders embraced this vision, envisaging a constituency of people able and willing to provide end of life care for those close to them. Others remained doubtful about caregiver capacity - suggesting that older spouses might be too frail or unwell while younger family members might have domestic or work commitments - or felt that it would require too large an attitudinal shift. 'Contracting' with family caregivers was seen by some as potentially problematic in practice. These factors could limit take-up and threaten the viability of the initiative. Conclusion: Delivering this new model of hospice care is highly contingent upon the capacity and willingness of family members to provide hands-on care in the final weeks of life. Our research suggests varied views on the proportion of people able to fulfil this role, highlighting a key risk to successful implementation. 
Abstract number: P01-150

Abstract type: Poster

Reducing Psycho-social Pain through Involving Patients and their Families in Economic Empowerment Skills Musimenta R. ${ }^{1}$, Asiimwe Buhiire M. ${ }^{2}$, Spiegel A. ${ }^{3}$, Gerever N. ${ }^{3}$, Guma S. ${ }^{3}$ ${ }^{1}$ Skills Development and Empowerment, Kawempe Home Care, Kampala, Uganda, ${ }^{2}$ Quality Assurance/ Research, Kawempe Home Care, Kampala, Uganda, ${ }^{3}$ Administration, Kawempe Home Care, Kampala, Uganda

Aim: This is to share how economic empowerment skills have contributed towards reducing psycho-social pain for patients and their families. Provision of only medical services to people living with HIV/AIDS, Tuberculosis, and Cancer does not make sufficient impact on the peoples' health status causing psycho-social pain. Due to these challenges, Kawempe Home Care started a paper beads project called Beads for Education (BfE) and due to its success, various projects have been developed like mushroom growing, piggery, recycled paper bag making and tailoring which has empowered 425 patients.

Methods: Selection of the beneficiaries is based on being infected or affected by HIV/AIDS, TB and Cancer in need of economic empowerment. Community volunteers identify patients with psycho-social challenges and are willing to be trained. After training, some are retained at SDE department to work on various projects and others are empowered to start own projects.

Results: 425 patients have been trained and empowered in different skills. Among the 425 patients, 25 were trained in bead making, and 5 were retained and 20 started small businesses with this skill, 300 were trained in piggery and mushroom farming and all were empowered to start their own project by providing them with seedlings and piglets, 55 were trained in paper bag making, all were given start up materials and were encouraged to start their businesses and 45 have been trained in tailoring skills, 15 retained and 30 were empowered to start their own tailoring businesses and $95 \%$ are able to meet their basic needs and educate their children. This has also contributed to KHC's sustainability. Conclusion: Basing on the KHC experience, economic empowerment skills have contributed towards enhancing the economic status of patients and their families, leading to positive living, good drug adherence, and hope thus reducing on psycho-social pain and improving on their quality of life.

\section{Abstract number: P01-151}

Abstract type: Poster

What Is Known from the Existing Literature about Peer Support Services for Carers of Individuals Living with Dementia: A Scoping Review

Carter G., Monaghan C., Santin O.

School of Nursing \& Midwifery, Queen's University Belfast, Belfast, United Kingdom

Background: A large proportion of global society will be affected by either having dementia themselves, or by providing informal care. This caregiving role varies significantly across the course of the dementia trajectory, and by accessing different support methods can help family carers adapt to their new and changing role. Providing informal care can have a negative impact on quality of life, physical and mental health. Nonetheless, current health service provision is lacking any specific statutory service for carers. It has been suggested that interventions focused on peer support may serve a vital role.

Aim: To undertake a knowledge synthesis to determine what exactly is known about peer support services for dementia carers and their modes of delivery to inform research, policy and practice.

Method: A scoping review of peer-reviewed literature was conducted following a five-stage framework. Key academic and nursing/medicine/ social science databases were searched.
Preliminary results: Of 11 databases searched 2508 records were identified. Following title/abstract screening, full text review and removal of duplications 36 studies were included. Results showed that the most common mode of intervention delivery was face-to-face support $(n=24)$ with the remainder $(n=12)$ providing online peer support, with the majority of interventions being psychoeducational including support. The most common intervention components consisted of peer connection; information giving; sharing experiences; practical tips and caregiving skills. The effectiveness of the interventions reviewed needs further analysis with respect to composition, and delivery to investigate optimal formatting of a peer support intervention.

Conclusions: Key findings which require further investigation include what the optimal mode of delivery and composition of a peer support intervention could be for family carers of individuals living with dementia to have the greatest impact on their wellbeing.

Abstract number: P01-152

Abstract type: Poster

Inclusion of Family Caregivers into EOL Care in Poland. From EUPCA Research Project to Public Education Campaigns and Practical Initiatives in Local Communities

Janowicz A. ${ }^{1}$, Krakowiak P. ${ }^{2}$, Legowska $E^{3}{ }^{3}$

${ }^{1}$ Educational Sciences, WSB Gdansk, Gdansk, Poland, ${ }^{2}$ Educational Sciences, Social Work, Nicolaus Copernicus University, Torun, Poland, ${ }^{3}$ WNP AC-UMK, Nicolaus Copernicus University, TORUN, Poland

Purpose: Families have helped especially in PC at home, which dominates in Poland. For generation caring for family member had been seen as sacred obligation, deeply rooted in Christian culture. With fast social and demographic changes in Poland (migration, ageing society,) family caregivers need more support and inclusion, as there are often elderly themselves and feel burden of constant care. Researchers have initiated steps to create effective support system, changes in policy regulations, and engagement of local communities.

Data sources: Research has been conducted during EUPCA Leadership Course in Gdansk as quantitative study (50 families). Consequently the qualitative research has been conducted with 12 families involved in care. It has illustrated different kinds of problems and challenges of family caregivers. Additionally, an electronic database search has been used to collect articles in Polish and international databases.

Implications: Families of PC patients need support on various levels. Both research helped to formulate a set of nationwide campaigns which have been created in cooperation with the media. Meetings of experts, organization of workshops for carers have been performed. Local volunteering, based on hospice experiences has been initiated. Discussions about the rights of caregivers have started and the Day of the Caregiver has been introduced. Conclusion: Knowing and monitoring the needs of caregivers and providing support, especially in home care, are needed for good quality of EOL care. Those people, who stay with patients $24 / 7$, sometimes for many years, need support of PC teams, as well as adequate help from health \& social care systems. Crucial is also help from local communities. All of these can be achieved with involvement of PC teams, while well supported families of caregivers can be great volunteers or ambassadors of PC in their local communities. Care for family caregivers urgently needs empowerment in Poland.

Abstract number: P01-153

Abstract type: Poster

Anxiety Depression Symptoms and Social Support of Informal Caregivers of Palliative Care Patients

Arkin S. ${ }^{1}$, KIlıc Ozturk Y. ${ }^{2}$

${ }^{1}$ Seyhan Governmental Hospital, Family Medicine, Adana, Turkey, ${ }^{2}$ Health Sciences University Izmir Tepecik Training and Research Hospital Palliative Care Clinic, Family Medicine, Izmir, Turkey 
Background: Although the life quality of the caregivers' is one of the main goals for Palliative Care (PC); usually the health needs of family caregivers take less attention.

Aim: To evaluate the psycosocial features of informal caregivers of PC patients at an inpatient clinic.

Material and methods: Caregivers (one carer for each patient) of 109 inpatients at a Hospital PC Clinic between 1 April 2017 and 30 June 2017 were evaluated. The demographic information of the caregiver, Multidimensional Social Support Scale and the Hospital Anxiety Depression Scale were administered. Patients' diagnosis and dependency in daily living activities were recorded. Data were analyzed in SPSS package program. The level of significance was $p<0.05$.

Results: Most (82.6\%) of the caregivers were female and symptoms of anxiety and depression were more common in female. The overall rates of anxiety and depression symptoms were $33 \%$ and $55 \%$, respectively. The gender of the caregivers, and the presence of supporters in care were associated with anxiety $(p=0,022 ; p=0,002)$. Intimacy with the patient, presence of supporters; duration of caregiving were related with depression $(p=0,004 ; p=0,034 ; p=0,02)$. The average score of the caregivers' support was $16,44 \pm 7,27$; special support $14,83 \pm 7,24$; family support $23.3 \pm 5.53$. The mean anxiety score of the caregivers was $8,34 \pm 5,66$ depression score was $8.63 \pm 4.92$. There was a negative weak correlation between social support and anxiety scores $(r=-0.234$, $p=0.014)$; between social support and depression scores $(r=-0.225$, $\mathrm{p}=0.019$ )

Conclusion: Depression and anxiety risk by the caregivers were high. The higher scores at social support perceptions of caregivers were associated with a decrease in the symptoms of anxiety and depression. There is a need for psychosocial support activities (such as training, counseling, and informational materials) to maintain the health and well being of caregivers as well as patients with palliative care needs.

\section{Abstract number: P01-155 \\ Abstract type: Poster}

Understanding Wishes: The Importance of Advance Decisions for Surrogate Family Members

Kitko L., Hupcey J.

College of Nursing, The Pennsylvania State University, University Park, United States

Background: Heart failure (HF) remains a worldwide epidemic, affecting over 26 million adults worldwide. Of those, approximately $10 \%$ are living and dying with Stage D HF. With a five year mortality rate of $50 \%$, both the person living with $\mathrm{HF}$ and their surrogate need to be prepared for end-of-life (EOL) decisions. Few studies have explored if formal advance decisions impact surrogate decision-making at EOL.

Aims: To examine how formal advance decision planning impacts surrogate decision making at EOL.

Methods: As part of a longitudinal study investigating the EOL trajectory of persons living with Stage D HF and their surrogates, 39 surrogates completed qualitative interviews following the death of the person with HF. Surrogates were asked to describe their decision-making experiences and how they were impacted by a previously established advance decision. The data were analyzed via a qualitative content analysis and descriptive statistics.

Results: Of the 39 surrogates, 9 did not have the opportunity to make decisions at the EOL due to the sudden death of the person with HF. Fifteen persons with $\mathrm{HF}$ and their identified surrogate had participated in EOL planning and had a formal advance decision. The majority of these surrogates $(80 \%)$ expressed comfort with the decisions they had to make at the EOL. A statistical difference $(p=.04)$ was found between surrogates to persons with and without advance decisions. Those without advance decisions expressed difficulty with the decision-making process at the EOL.
Conclusions: Surrogates who were able to discuss EOL wishes with their loved ones through the process of creating an advance decision were less likely to experience difficulties making EOL decisions than those who had not participated in formal EOL planning. Future research is needed to determine if the formal advance decision documents were used to make EOL choices or if they were the vehicle that spurred conversations regarding EOL wishes.

Abstract number: P01-157

Abstract type: Poster

Emotional Distress and Caregivers Burden at the End of Life

Limonero J.T. ${ }^{1}$, Gómez-Romero M.J. ${ }^{1,2}$, Maté J. ${ }^{3}$, Mateo D. ${ }^{4}$, Gil F. ${ }^{5}$, Álvarez M. ${ }^{1}$, Cladellas-Pros R. ${ }^{1}$, Montes-Hidalgo J. ${ }^{6}$, Tomás-Sábado J. ${ }^{6}$ ${ }^{1}$ Stress and Health Research Group. Faculty of Psychology, Universitat Autònoma de Barcelona, Bellaterra (Cerdanyola del Vallès), Spain, ${ }^{2}$ Psychology Unit, Egarsat, Mutua Colaboradora con la Seguridad Social no 276, Terrassa, Spain, ${ }^{3}$ Psycho-oncology Care Unit, Catalan Institute of Oncology, L'Hospitalet de Llobregat, Spain, ${ }^{4}$ Palliative Care Unit, Consorci Sanitari de Terrassa, Terrassa, Spain, ${ }^{5}$ Psychooncology Unit. Psychosocial Observatory in Cancer, Catalan Institute of Oncology, L'Hospitalet de Llobregat, Spain, ${ }^{6}$ Research and Scientific Communication Unit, Gimbernat University Schools. Universitat Autònoma de Barcelona, Sant Cugat del Vallès, Spain

Background: Caring for a loved one at the end of life, involve many changes in caregiver's life. Some of these changes are perceived in a negative way by caregivers experimenting burden and emotional distress. Aim: We aim to analyse the relationship between emotional distress and caregiver burden in caregivers of patients at the end of life who receive palliative care.

Methods: 136 caregivers with a mean age of 56.24 ( $S D=14.63$; 84 women, 52 men) of patients at the end of life admitted to palliative care unit answered the scale of Detection of Emotional Distress Caregivers (DED-C) and the Reduced Zarit Scale (RZS) also of demographic variables.

Results: $54 \%$ of caregivers had moderate to severe burden and emotional distress. We observed a positive correlation between emotional distress and caregivers burden $(r=.404 ; p<.01)$. We didn't find any differences between gender in relation to emotional distress or global caregiver burden. Only we observed statically differences in relation to self-care, where women were more worried about this dimension $(t=-2.76 ; p<.01)$.

Caregivers who presented emotional distress showed more perception of burden [global score of reduced Zarit $(t=-2.78 ; p<.01)$ ] and in two of their dimensions: loss of role $[t=-2.17 ; p<.01]$ and caregiver burden $[t=-2.43 ; p<.05]$. We didn't observe statistic differences in the dimension of self-care $(t=-1,48 ; p>.05)$ of the reduced Zarit.

Conclusions: Most caregivers of patients at the end of life presented emotional distress and burden. Taking attention to the relation of emotional distress and caregivers burden could help health professional to prevent this situation by providing psychological and social resources to caregivers and improve their wellbeing and the adaptation to this situation.

Funding: This study was supported, in part, by grant Number PSI201785134-R from the Spanish Ministry of Economy, Industry and Competitiveness (MINECO).

Abstract number: P01-158

Abstract type: Poster

Caregivers Psychological Needs at the End of Life Care for Cypriot Cancer Patients

Tryphonos A. ${ }^{1}$, Bellali T. ${ }^{2}$

${ }^{1}$ Psychosocial, Pasykaf, Nicosia, Cyprus, ${ }^{2}$ Nursing, "Alexander' Technological Educational Institute of Thessaloniki, Thesalloniki, Greece 
Purpose: To explore in depth the lived experience of families with cancer patients through the palliative home care services. The research question that guided the larger qualitative study was: "What were the needs of the caregivers at the terminal stage of the disease and how they have being me",? The purpose of this paper is to describe the psychological needs of the caregivers.

Method: It is a descriptive qualitative phenomenology study. With the use of semi-structured interviews, 10 caregivers of terminal stage cancer patients described their experience from the palliative home care services and focused on their psychological needs. The method by Colaizzi was used for the analysis of the interviews.

Results: The results of this study identified important psychological issues in relation to the palliative homecare needs of the caregivers concerning:

a) to feel safe,

b) to receive encouragement,

c) to be able to pretend,

d) they were shocked upon the diagnosis and unprepared for the caregiver's role,

e) they felt automatically responsible to take up the role of the caregiver.

Caregivers of adult cancer patients lived experience though palliative home care services was a psychologically painful experience and used solution-focused, emotion-focused and meaning focused strategies.

Conclusions: The psychological needs of the caregivers as well as the mechanisms used by the families during this difficult period, have to be taken into account in order for the professionals to design the appropriate psychoeducational programs for the caregivers to increase resilience. Psychosocial contingencies, and contextual factors need to be taken into account in designing adequate interventions for supporting the caregivers.

Abstract number: P01-161

Abstract type: Poster

Changing Priorities in the Care of Children with Cancer: The Experience of Jordanian Parents

Atout M. ${ }^{1}$, Alkharabsheh A. ${ }^{2}$, Thalgi M. ${ }^{2}$, Aldawagreh A. ${ }^{2}$

${ }^{1}$ Philadelphia University, Amman, Jordan, ${ }^{2}$ Royal Medical Services, Amman, Jordan

Purpose: The purpose of the current study is to explore the experience of parenting among parents who care for children with cancer.

Methods: A Phenomenological hermeneutic approach was conducted informed by the philosophy of Martin Heidegger. The study was conducted in one oncology unit a Jordanian hospital. Parents of children aged (6-18) years old or with all types of cancer were considered eligible. A purposeful sampling strategy was adopted to recruit the participants. Maximum variation sampling was employed. Data was collected using semi-structured interviews.

Findings: Thirty-two interviews were collected involving mothers $(n=29)$, fathers $(n=3)$. The parents changed their focus during the care of their children from an initial emphasis on normalising their children's lives, to relieving their physical and psychological discomfort. This change of focus accompanied changes in the parents' understanding of their children's disease over time. They became more realistic regarding the future of their children, and therefore attempted to make their lives as enjoyable and comfortable as possible. Moreover, they evaluated any activity their children performed according to how much it affected their levels of comfort. When these activities resulted in further physical or psychological effort, the parents prioritised the comfort of their children over the benefits of the activities.

Conclusion: The parents' experiences should be taken into consideration when the medical teams establish the children's treatment plans.
Additionally, the continuous education and specialised training for professionals, which provides staff with specialised communication skills and emotional support for children and parents are imperative in improving the clinical practice in the healthcare settings with limited access to specialist palliative care.

Abstract number: P01-162

Abstract type: Poster

Exhaustion, Did You Say "Exhaustion"? A Concept Analysis among Informal Caregivers

Dubech M..$^{1,2}$, Schell M. ${ }^{3,4}$, Lefranc A. ${ }^{1,2}$, French Research Group on

Respite Care

${ }^{1}$ Fondation France Répit, Tassin-La-Demi-Lune, France, ${ }^{2}$ ALQUALINE, Solignac, France, ${ }^{3}$ Fondation France Répit, Lyon, France, ${ }^{4}$ Centre Léon Bérard, Lyon, France

Introduction: The term "exhaustion" is widely used to describe a condition to be avoided for informal caregivers. Nevertheless, "exhaustion" still need to be properly defined. The aim of this review was to achieve a better definition of exhaustion in the specific context of caregiving.

Methods: Health thesaurus HeTOP was searched to identify Defined Concepts Approaching Exhaustion (DCAEs). PubMed database was searched combining the identified DCAEs, "caregiver", and related keywords. Inclusion criteria involved publications mentioning a definition of one or more DCAEs, and/or investigating potential risk factors among informal caregivers. Definitions, risk factors, as well as assessment tools of each DCAE were analyzed.

Results: The identified DCAEs were "burnout", "compassion fatigue" and "chronic fatigue". To date, 38 out of 1468 publications were retained. More than $80 \%$ dealt with burnout. All DCAEs stem from a long-term exposure to stressors combined with chronic individuals' resources depletion. Nevertheless, burnout, compassion fatigue, and chronic fatigue were associated with occupational, relational and physical/mental stressors, respectively. Similarly, the nature of risk factors differed between the DCAEs. Specific assessment tools were listed for each DCAE, from which common dimensions were identified (i.e. physical, emotional, and mental exhaustion). Caregiver exhaustion could be conceptualized using the caregiving stress and coping resources models. Finally, a definition of caregiver exhaustion was proposed.

Conclusions: The DCAEs were not clearly defined and too often employed indiscriminately. Even though distinct, there is a major overlap between the DCAEs, supporting the need to precise definitions. A better understanding of caregiver exhaustion is a necessary step prior to the development of an assessment tool, to better prevent the onset of this state.

Review funded by the MSDAVENIR foundation.

Abstract number: P01-163

Abstract type: Poster

Problems and Needs of Family Caregivers of Patients with Cancer in an Asian Setting: Cultural and Validation of PNPC-c Kristanti M.S. ${ }^{1}$, Effendy C. $^{2}$, Utarini A. ${ }^{2}$, Vernooij-Dassen M. ${ }^{3}$, Engels ${ }^{4}{ }^{4}$ ${ }^{1}$ QQ Healthcare, Radboud UMC, Nijmegen, Netherlands, ${ }^{2}$ Universitas Gadjah Mada, Yogyakarta, Indonesia, ${ }^{3} \mathrm{Q}$ Healthcare, Radboud University Medical Center, Nijmegen, Netherlands, ${ }^{4}$ Pain and Palliative Medicine, Radboud University Medical Center, Nijmegen, Netherlands

Background: Family caregivers have a profound role in caring for cancer patients. In most Asian countries, it is seen as a normal duty. However, little is known about family caregivers' problems and needs, and no instrument to assess their problems and needs is available in any Asian country. 
Aim: The study aims to adapt, translate, and validate the Problems and Needs in Palliative Care-caregiver questionnaire (PNPC-c) for Indonesia. Also, it aims to use PNPC-c new version (PNPC-c-nv) for family caregivers of cancer patients in Indonesia.

Methods: The PNPC- c was cross culturally translated using the guideline of Beaton (2000). Based on a previous qualitative study, some items were added. Content validity was done through team meetings and experts' evaluation with Content Validity Item. Cronbach alpha was used to measure the internal consistency. Chi Square was used to identify differences in proportion of reported problems in some groups.

Results: Through content validity, 7 new items were added. Four hundred and fifty one caregivers participated from outpatient clinics in 3 cities in Indonesia. Cronbach's alpha ranged from $0.65-0.87$. The most frequently reported problems were handling the patient's pain (59\%), fatigue (56.1\%) and a long waiting list (49.9\%). The most frequently reported needs were handling the patient's pain (83.4\%); patient's nourishment (76.9\%), and knowing the physical signs (72.9\%). Older and nonspouse caregivers were more significantly reported on some problems. Conclusions: The PNPC-c-nv was relevant for family caregivers in Indonesia. It may also be useful for other Asian countries, since Asians have the similar tendency in caregiving context. The most urgent reported problems and needs related to patient's, rather than the caregivers' demands. Since needs were more reported, asking for this seems to be more acceptable than mentioning problems in Asian countries. It also reflects the active attitude and provides clues for future care.

\section{Abstract number: P01-164 \\ Abstract type: Poster}

The Experience of Family Caregivers of Patients with Cancer in an Asian Country: A Grounded Theory Approach

Kristanti M.S. ${ }^{1,2}$, Effendy C. ${ }^{2}$, Utarini A. ${ }^{3}$, Vernooij-Dassen M. ${ }^{1}$, Engels.$^{4}$ ${ }_{1}$ QQ Healthcare, Radboud University Medical Center, Nijmegen, Netherlands, ${ }^{2}$ Nursing School, Universitas Gadjah Mada, Yogyakarta, Indonesia, ${ }^{3}$ Universitas Gadjah Mada, Yogyakarta, Indonesia, ${ }^{4}$ Pain and Palliative Medicine, Radboud University Medical Center, Nijmegen, Netherlands

Background: Indonesia has a culture with strong family bonds. The family members of patients with cancer are intensively involved in the caregiving process, both at home and in the hospital. The involvement is considered 'common': a societal and religious obligation. The values underpinning caregiving by the family might influence their perception of caregiving.

Aim: To explore the experiences of family caregivers of patients with cancer in Indonesia in performing caregiving tasks, and to model those experiences.

Design: A grounded theory approach was applied in this qualitative study. The constant comparative method was used for data analysis. A paradigm scheme was used to develop a theoretical model from qualitative data.

Setting/participants: The study was conducted in the outpatient clinics of three hospitals in Indonesia. The participants were family caregivers of patients with cancer.

Results: Twenty four family caregivers participated in the current study. We found 'belief in caregiving' as the core phenomenon. It appeared to be influenced by three contextual factors: Quality of service, financial condition and challenges in caregiving. Belief in caregiving itself influences the actions of the family caregivers: Coping mechanisms, sharing tasks and sacrifices. These actions result in consequences that are physical, psychological, social and financial. Social support as an intervening condition influences the process of the core phenomenon and the actions of the family caregivers.

Conclusion: Belief in caregiving is the major factor in family caregivers' experiences. This model might help palliative care professionals in providing care for caregivers who come from or live in a country where family plays a significant role in caregiving. Specific interventions for family caregivers which take this basic mechanism into account are needed.

\section{Abstract number: P01-165}

Abstract type: Poster

Mapping Financial Support for Family Caregivers of Those at the End of Life across Six Countries

Gardiner C. ${ }^{1}$, Taylor B. ${ }^{1}$, Robinson J. ${ }^{2}$, Gott M. ${ }^{2}$

${ }^{1}$ The University of Sheffield, Sheffield, United Kingdom, ${ }^{2}$ University of Auckland, Auckland, New Zealand

Background: Family caregivers are the main providers of end of life care, but often feel unsupported in their role, particularly with regard to the financial burden of caring. Concerns about how best to support family caregivers have featured on the political agenda of a number of countries in recent years. However, there is limited evidence on the scope and availability of existing financial support for caregivers internationally.

Aim: To identify and compare sources of financial support for family caregivers of those at the end of life across the UK, Australia, New Zealand, Canada, USA and Ireland.

Methods: This study comprised four phases:

(1) palliative care experts from each of the six countries completed an inventory of financial support for carers;

(2) grey literature searches and

(3) academic database searches were undertaken to confirm and extend the survey findings;

(4) a comparative analysis was performed of complete dataset.

Results: Ninety nine survey responses were obtained. Whilst most countries had implemented some form of policy or legislature to support carers, there is little evidence of policy commitments being translated into appropriate financial support for carers. Some form of financial support was available in all six countries but significant issues were identified with access and eligibility. Survey respondents indicated that financial support was in general, insufficient to meet needs, an issue compounded by excessive complexity in process.

Conclusion: Financial remuneration for caregiving is considered to be one of the most important sources of support for informal carers. This study identified that sources of financial support are available but may be inconsistent in implementation. Financial support may not be sufficient to forestall significant financial burden for caregivers. The possibility for policy-makers to learn from other countries' experiences is significant.

\section{Abstract number: P01-166}

Abstract type: Poster

\section{Discharge from Hospice: Relatives and Healthcare Providers} Experiences

Wieles-Griffioen G.A.M. ${ }^{1}$, Evers - Fikken J.C.C.M. ${ }^{2}$, Huisman T.F. ${ }^{2}$, Gootjes J.R.G. ${ }^{1}$, ter Horst - van Oord M.E. ${ }^{1}$, van Nus-Stad J.L. ${ }^{1}$

${ }^{1}$ Hospice Kuria, Amsterdam, Netherlands, ${ }^{2}$ University of Applied Science VIA, Zwolle, Netherlands

Background: In the Netherlands, patients with an estimated live expectancy of less than three month are given an indication for hospice admission. When, during the period in the hospice, their medical situation is improving, they are discharged to go home or into nursing homes. Healthcare providers in the hospice are concerned about the decision to discharge a patient. They are of the opinion that the discharge process is distressing for patients and relatives and also for themselves. They 
believe that the process of being discharged must be done carefully. Little is known about the experiences of relatives and healthcare providers and what they find important during this process.

Aims: What are the experiences of relatives and healthcare providers during the process of discharge. In which way can the process being improved.

Methods: Interview with 4 relatives of patients who were discharged from hospice and a focus group interview with nurses and a social worker from the hospice to investigate the experiences of family and hospice team. Interview with 3 hospices about the discharge process.

Results: Relatives admit that the discharging process is distressing, because legal rules determine the dismissal, not the wishes of the patient. They experience the process within the hospice as going well. Healthcare providers themselves struggle with the other focus, the patient is not dying but their health improved. They experience the communication within the organisation as unsure, not knowing reasons of discharge and not having a step-by-step plan. Some of the other hospices do have a step-by-step plan, some don't.

Discussion/ conclusion: Contrary to what was thought, relatives didn't find the process of discharge distressing, as far as the hospice is responsible for the relocation process. We recommend the organisation to make the procedure more transparent with attention paid to the transition of focus for healthcare providers.

Abstract number: P01-167

Abstract type: Poster

Anticipatory Medication in Home Palliative Care: A Systematic Review of Existing Practices, Challenges and Safeguards Hernández-Marrero P. 1,2,3, Martins Pereira S.1,2,3, Project EnSupCare: Ensuring Home-Based Palliative Care at the End-of-Life: Supporting Family Carers to Manage Pain Medication

${ }^{1}$ Instituto de Bioética, Universidade Católica Portuguesa, Porto, Portugal, ${ }^{2}$ UNESCO Chair in Bioethics, Institute of Bioethics, Universidade Católica Portuguesa, Porto, Portugal, ${ }^{3} \mathrm{CEGE}$, Centro de Estudos em Gestão e Economia, Porto Católica Business School, Universidade Católica Portuguesa, Porto, Portugal

Background: Family carers provide much of the essential care that enables patients to stay and die at home. Anticipatory prescribing is designed to ensure that there is a supply of medication and apparatus needed for family carers to administer them in the patient's home. Little is known about simultaneously promoting effective methods for ensuring the optimal management of anticipatory medications (AM) and supporting family carers when caring for their loved ones at the end of life at home.

Aims: To systematically review the existing literature on AM in home palliative care (HPC) with respect to its clinical, organizational, ethical and legal frameworks, main challenges and safeguards.

Methods: Systematic Review following PRISMA 2009. Databases: PubMed, Web of Science, Scopus, ProQuest, CINAHL Complete, MEDLINE Complete, Nursing \& Allied Health Collection: Comprehensive, Database of Abstracts of Reviews of Effects, Cochrane Database of Systematic Reviews, Cochrane Methodology Register, MedicLatina, Health Technology Assessments, NHS Economic Evaluation Database and EBSCO databases. Search terms: "anticipatory medication" and "palliative care". Inclusion criteria: studies reporting the use of AM in HPC, in English, German, Spanish and Portuguese, full text, references available. Selected articles were independently reviewed by two researchers. The Hawker et al tool was used for quality assessment.

Results: Of the 180 articles retrieved in our searches, 7 were included for analysis. Findings show that nurses have an active role in the prescription and training of family carers in the management of AM in HPC. Some conditions are taken into account for a safe practice of AM. Resourcing concerns, professional expertise and articulation among professionals are perceived as challenges.
Conclusions: Anticipatory medication is a major need and has ethical challenges in symptom control at the end of life at home. Research is needed to ensure optimal management of AM in HPC.

Abstract number: P01-168

Abstract type: Poster

Understanding Ethical Challenges and their Impact on Family Caregivers of Patients with Advanced Cancer

Ullrich A. ${ }^{1,2}$, Theochari M. ${ }^{1}$, Bergelt C. ${ }^{2}$, Marx $G .{ }^{3}$, Woellert K. ${ }^{4}$,

Bokemeyer C. ${ }^{1}$, Oechsle K. ${ }^{1}$

${ }^{1}$ Palliative Care Unit, Center of Oncology, University Medical

Center Eppendorf, Hamburg, Germany, ${ }^{2}$ Department of Medical

Psychology, University Medical Center Eppendorf, Hamburg, Germany, ${ }^{3}$ Department of Palliative Medicine, University Medical Center Goettingen, Goettingen, Germany, ${ }^{4}$ Department of History and Ethics of Medicine, University Medical Center Eppendorf, Hamburg, Germany

Background: Caring for terminally ill and dying patients (PT) can contribute to ethical challenges, but little is known on experiences and impact on family caregivers (FC).

Aims: Understanding ethical challenges of FCs of PT with advanced cancer by exploring their experiences and resulting burden, coping strategies and moral distress.

Methods: Qualitative semi-structured interviews using open-ended questions with 12 bereaved FC. Data analysis using grounded theory and abductive reasoning.

Results: Analyses showed two paths leading to ethical challenges in FC. Challenging situations during the disease trajectory - not necessarily perceived as ethically challenging by FC - either triggered a perceived lack of available choices or a difficult decision-making process. A lack of available choices caused feelings of powerlessness accompanied by anxiety, sadness and confusion. Either then detrimental factors (e.g. no truthful communication with PT) led to paralyzing shock, or resources (e.g. better preparedness) stimulated acceptance of the situation and avoidance of moral distress. In case of difficult decision-making processes, detrimental external factors (e.g. lacking time) caused emotional distress of feeling left alone, helplessness and overwhelm. To overcome experienced obstacles or to make a decision FC used proactive strategies (e.g. social support). Across both paths and independent of FC's burden and strategies, moral distress occurred when FC could not act according to their values (e.g. providing "good" care, respecting PT's will), generating painful emotions. While clinicians' acknowledgment of caregiver role, clarification of ownership of things gone wrong and FC's self-acceptance helped FC to find closure, some identified residual frustration and self-blame.

Conclusion: Findings provide a starting point towards understanding paths leading to ethical challenges in FC and can support clinicians to help minimizing associated emotional and moral distress.

Abstract number: P01-169

Abstract type: Poster

Supportive Nursing Care for Informal Caregivers - A

Retrospective File Study

van Driel A.G. ${ }^{1}$, Becque Y. ${ }^{1}$, Rietjens $J^{2}{ }^{2}$, van der Heide A. ${ }^{2}$, Witkamp F.E. ${ }^{1}$

${ }^{1}$ Nursing, University of Applied Sciences Rotterdam, Rotterdam, Netherlands, ${ }^{2}$ Public Health, Erasmus University Medical Center Rotterdam, Rotterdam, Netherlands

Background: In the Netherlands many patients receive end-of-life care (EoLC) at home from nurses working for homecare organisations (HCO). Informal caregivers (IC), such as spouses and children, play a significant role in facilitating patients to stay at home. Nurses also aim to support these IC, but information on how this is done is lacking.

Aim: To get insight into supportive care for IC as provided by community nurses. 
Methods: Four HCOs were approached to participate in this study. We studied nursing files of patients who received EoLC. A data extraction form was used to collect demographic data of the patient and their IC, and information about supportive nursing care for IC.

Results: We examined nursing files of 59 patients (28 men and 31 women). Mean age of the patients was 75 years (SD \pm 11$)$. They were diagnosed with cancer (63\%), heart failure (10\%) or other illnesses (24\%) (e.g. neurological or lung diseases). Most patients died at home (76\%). Some of them were still alive during this study (14\%). The mean time between assignment for EoLC and death was 50 days ( \pm 76$)$. The number of involved IC was one (41\%), two $(27 \%)$, three $(10 \%)$ or four $(2 \%)$. In most cases informal care was provided by the patient's spouse $(58 \%)$ or child (27\%).

The HCOs used different classification systems to register nursing care. One HCO used NANDA[1], the others used OMAHA[2]. A nursing diagnosis for IC was documented in $68 \%$ of all files (e.g. overburden). One organisation used the Care Giver Strain Index to assess the needs of IC. Nursing files contained little information on which interventions were applied to provide supportive care for IC, nor on what their effect was. Conclusion: In almost two thirds of patients receiving EoLC at home, nurses documented some supportive needs of IC. Documentation of IC related interventions and evaluation of these interventions was scarce, which poses a risk for the continuity of supportive care for IC.

[1] www.nanda.org/

[2] www.omahasystem.org/

Abstract number: P01-170

Abstract type: Poster

Supporting Family Carers of People with Motor Neurone Disease: Adaptation of a Carer Support Needs Assessment Tool (CSNAT) Intervention

Ewing G. ${ }^{1}$, Croke S. ${ }^{2}$, Rowland C. ${ }^{2}$, Grande G. ${ }^{2}$

${ }^{1}$ Centre for Family Research, University of Cambridge, Cambridge, United Kingdom, ${ }^{2}$ Division of Nursing, Midwifery \& Social Work, University of Manchester, Manchester, United Kingdom

Introduction: Family carers play a vital role in supporting people with Motor Neurone Disease (MND); a debilitating condition both physically \& mentally demanding for carers. Timely support from healthcare professionals (HCPs) is crucial to enable them to fulfil their caregiving role and for their own health and wellbeing. Currently there are no UK interventions for this.

Aims:

1. To obtain carers' experiences of caregiving in the context of MND

2. To adapt an existing Carer Support Needs Assessment Tool (CSNAT) intervention to support the needs and situation of family carers of people with MND.

Methods: Stages: 1 Nine carer focus groups $(\mathrm{N}=29)$ and four individual interviews (14 bereaved, 19 current carers) to capture support needs, key points of change during patient's illness, main sources of support; 2 Workshops with carer advisors $(\mathrm{N}=19)$ and HCPs $(\mathrm{N}=24)$ to adapt the CSNAT and procedures for MND carers.

Results:

Stage 1: Carers' experiences included dealing with a devastating diagnosis, a constantly changing situation with heavy dependence on carers \& little consideration of their separate support needs. Carers found the CSNAT appropriate \& relevant: support needs mainly mapped to existing domains. One additional domain for MND carers (managing relationships) identified for further investigation.

Stage 2: integrated HCPs' and carers' views on process of delivering comprehensive, person-centred carer assessment \& support. Endorsement of carer support by HCPs as part of routine care was central to legitimise carer needs, followed by introduction of adapted CSNAT-MND to identify carer support needs \& a dedicated assessment/planning conversation to provide supportive input.

Conclusions: This study provides insights into how MND carers want to be assessed/supported \& how this process may be delivered in routine practice. The CSNAT-MND intervention will be tested in a future Stage 3 feasibility study.

Funder Marie Curie Research /MND Association

\section{Abstract number: P01-171}

Abstract type: Poster

Health-related-Quality-of-Life in End-of-life Patients and their Caregivers

Navarro Verdú P. ${ }^{1}$, Montoya-Juárez R. ${ }^{2}$, García Sanjuán S. ${ }^{1}$, Perpiñá

Galváñ J. ${ }^{1}$, Clement Carbonell V. ${ }^{1}$, Guardia Mancilla P. ${ }^{2}$, Cruz Quintana

F. $^{2}$

${ }^{1}$ Alicante, Alicante, Spain, ${ }^{2}$ Granada, Granada, Spain

Introduction: Health-Related-Quality-of-life ( $\mathrm{HRQOL}$ ) is a multidimensional concept that includes the subjective perception of the impact of health status, illness and treatment, on physical, psychological and social functioning and well-being. HRQoL is an important indicator of the palliative care services for the patients and their relatives.

Aim: The aim of this study was to describe the quality of life for a sample of palliative patients and their family caregivers.

Methods: A cross-sectional design has been carried out at the cities of Alicante and Granada during April-June 2018. Interviews were conducted at home and through a set of questionnaires data about quality of life for patients and caregivers was obtained. For patients the McGill Quality of life Questionnaire and Hospital Anxiety and Depression Scale (HADS) were used; while the SF-12, the Zarit Burden Interview and the HADS were included in the caregivers' assessment. Data was analyzed with the statistical package SPSS version 15.

Results: Mean quality of life perceived by palliative patients was 6.03 and the most frequent symptoms were pain and weakness. Depression was a clinical problem for $33.3 \%$ of patients and $44.8 \%$ of caregivers perceived intense overload. Anxiety was a clinical problem for $44.8 \%$ of caregivers. Depression was more frequent in patients, while anxiety was more intense for caregivers. There was more anxiety in the caregivers of the city of Granada, in comparison with Alicante.

Conclusion: Palliative patients seem to report higher levels of depression, while the caregivers had higher levels of anxiety.

Keywords: palliative care, quality of life, caregivers, anxiety, depression.

Abstract number: P01-172

Abstract type: Poster

Generating Local Evidence for Palliative and End of Life Care: A Strategic Approach to International Partnerships and Services for Pacific Peoples

Dewes O., Williams $K$.

School of Nursing, The University of Auckland, Auckland, New Zealand

Background: The World Health Organization has identified improving palliative and end of life care for older people as an international public health priority. There is little evidence to show that this challenge has been taken on board in research, practice, or policy, either in the Pacific nations or in New Zealand where Auckland has the largest Polynesian population in the world. Demographic projections over the next two decades predict marked increases in the Pacific older age groups. Building the evidence base to inform palliative and end of life care services is vital.

Aim: To investigate the end of life circumstances of Pacific peoples dying at $>65$ years and the experiences and challenges faced by their family carers who carry out the bulk of their care at home. 
Methods: Interviews with 33 family carers (Samoan, Tongan, Cook Is Maori, Other Pacific) and 3 stakeholder focus groups comprising 24 participants were conducted in Auckland. A social constructivist framework underpinned by Pacific health models informed our qualitative study design. Narrative and thematic analyses within specific domains were conducted using nVivo10. A digital storytelling workshop was also conducted for 5 family carers to give meaning and context to the interviews. Results: A range of lived experiences among carers was identified, with most having undergone significant lifestyle changes. Access to services was mixed. Caregiving tasks were extensive and posed a number of challenges including financial stress and logistical difficulties in managing other life commitments. Some carers struggled to maintain their own health and wellbeing due to the relentless nature of the role and range of emotions experienced. Carers reported coping strategies. Informal supports played a key role. Caring for a loved one at home was very important.

Conclusion: Pacific family carers must be involved in the development and provision of palliative and end of life care services for Pacific older adults.

\section{Abstract number: P01-174 \\ Abstract type: Poster}

"It was Peaceful, it was Beautiful". Family Carers' Descriptions of Good End of Life Care in Acute Hospitals for People Dying in Advanced Age

Robinson J. ${ }^{1}$, Gott M. ${ }^{1}$, Black S. ${ }^{2}$, Lisa W. ${ }^{1}$, Wiles J. ${ }^{2}$

${ }^{1}$ School of Nursing, University of Auckland, Auckland, New Zealand,

${ }^{2}$ University of Auckland, Auckland, New Zealand

Objectives: To explore good end of life care for people dying in advanced age in the hospital setting.

Methods: In-depth interviews were conducted with 58 family/whānau (Māori term related to extended family) of 52 people who died $>80$ years. Culturally appropriate methods were used to ensure the safe inclusion of Māori. A questionnaire collected information about health service use at end of life. This presentation presents discussed the experiences of participants who were 'extremely'/'very' satisfied with end of life care delivered in hospital.

Results: The examples of good care described by this subset of participants aligned with Dewar and Nolan's (2013) model of 'compassionate care', which is congruent with Māori understandings of 'aroha' (love). Adapting Nolan and Dewar's (2013) framework we identified the following dimensions of good care:

1) A relationship based on empathy: He ngākau aroha;

2) Effective interactions between participants, over time and across settings: Whakawhanaungatanga;

3) Contextualised knowledge of the patient and family: Tuakiri o ngā tangata Māori; and

4) Staff, patients and families being active participants in their care: Manaakitanga.

Conclusions: Focusing on what is working well had been identified as an important strategy to build the capacity of health services. We used this approach to identify low cost, high impact, actions that are valued by the relatives of people of advanced age who experienced hospital admissions at the end of life.

Reference: Dewar, B., Nolan, M. (2013). Caring about caring. International Journal of Nursing Studies, 50(9), 1247-1258.

Abstract number: P01-175

Abstract type: Poster

Circles of Care: A Follow-up Study to Describe how Support Networks Develop to Satisfy the Caregiving Needs of Patients who Face End-of-Life
Soriano Soto M. ${ }^{1}$, Castro L. ${ }^{2}$, Rubio L. ${ }^{2}$, Parra S. ${ }^{2}$, Krikorian A. ${ }^{1}$

${ }^{1}$ Universidad Pontificia Bolivariana, Medellín, Colombia, ${ }^{2}$ Clínica Somer, Rionegro, Colombia

Background: A solid caregiving network is critical for the wellbeing of patients at the end-of-life (EOL). Little is known about how they develop over time to meet their needs.

Aim: To identify caregiving needs of patients at EOL and how their support networks develop over time.

Methods: Descriptive, prospective study to assess the evolution of the caregiving needs of patients at EOL who attend a palliative care unit. The Barthel and Lawton \& Brody scales were used to assess caregiving needs. Six follow-up telephone interviews were conducted. The number, characteristics and tasks performed by each caregiver at each follow-up were registered. Preliminary results are presented.

Results: $n=93$ patients, mean age 64 (SD16; range 26-93), 52,7\% male, $63,4 \%$ married, and $89,2 \%$ with a cancer diagnosis. Mean initial scores for Barthel were 61,17 (SD36,41; range 0-100) and for Lawton \& Brody were 2,57 (SD2,37; range $0-8$ ). Identified caregivers were 256 , mean age 42,84 (SD15,18; range 13-85), 72,6\% women, 57\% married; 96,5\% were relatives, most commonly sons/daughters (51,6\%) and espouses $(16,04 \%)$. Mean number of caregivers per patient was 2.47 (SD1,51; range 1-8). At each follow-up, caregivers were asked the number of tasks performed, from a total possible of 29 . The overall mean number of tasks was 15 (SD7; range 1-29). At follow-up 1 mean number of tasks was 16,68 (SD7,28; range 1-28); follow-up 2: 15,07 (SD7,04; range 1-28); follow-up 3: 14,30 (SD6,94; range 1-29); follow-up 4: 14,18 (SD6,63; range 2-29); follow-up 5: 14,79 (SD6,80; range 3-29); and follow-up 6: 14,11 (SD6,91; range 2-27). Differences in the number of tasks at each followup showed a statistical difference using Kruskal-Wallis non-parametrical tests ( $x=24,50 ; p<0.001)$.

Conclusion/ discussion: Patients showed a high degree of dependency on caregiving for daily activities and two or more caregivers are needed to satisfy them. It is vital to ensure that the support network grows along the needs detected.

\section{Abstract number: P01-176 \\ Abstract type: Poster}

The Impact of Perceived Competence and Optimism in Reducing the Burden of Informal Caregivers of Patients with Advanced Oncological Disease Limonero J.T. ${ }^{1}$, Palacio C. ${ }^{2}$

1Universidad Autonoma de Barcelona, Barcelona, Spain, 2Universidad Pontificia Bolivariana, Medellin, Colombia

Introduction: Informal caregivers often experience different consequences relate to take care of the patient, such as physical and emotional exhaustion, reducing the global well-being and influencing both the perception of loss of the personal control and in the coping strategies affecting the process of adaptation to the disease context.

Objectives: To describe the role of perceived competence, optimism, post-traumatic growth (PTG) and spirituality in reducing the burden of caregivers of patients with an advanced oncological disease who attended a cancer center in the city of Medellín-Colombia.

Methodology: Descriptive-cross-sectional study. 100 informal caregivers of age $(>18)$, selected by convenience sampling. Scales were administered to assess perceived competence, burden, optimism, spirituality and posttraumatic growth. Descriptive analyzes, a correlation between the variables and linear regression of successive steps were carried out. Results: Correlation analyzes indicated a negative relationship between burden and perceived competence $(p<0.05)$ and with optimism ( $p<$ 0.01 ) and a positive relationship between perceived competence and optimism ( $p<0.01$ ) PTG with spirituality and optimism $(p<0.01)$. The regression analyzes showed that perceived competence and optimism reduce the burden of informal caregivers. 
Conclusion: Enhancing perceived competence and mobilizing optimism as a personal resource in informal caregivers, influences the reduction of burden, thus favoring recovery in terms of the perception of personal control in the face of stress, and the possibility of anticipating positive consequences related to the activities of the role in terms of the detection and coping of physical and emotional needs of patients.

Acknowledge: This study was supported, in part, by grant Number PSI2017-85134-R from the Spanish Ministry of Economy, Industry and Competitiveness (MINECO).

\section{Abstract number: P01-178 \\ Abstract type: Poster}

\section{The Characteristics and the Association with Hospice and} Palliative Care of Terminally-III Cancer Patients according to Family Composition

Park S.M., Hwang S.W.

Family Medicine, St. Paul Hospital, The Catholic University, Seoul, Korea, Republic of

Background: Previous research offers suggestive effects of family composition on health care and marital status may play important roles in shaping end-of-life care.

Aims: The purpose of this study was to investigate the association between family composition of terminally-ill cancer patients admitted to the hospice units and utilization of palliative and hospice care.

Methods: We retrospectively analyzed the medical records of terminally-ill cancer patients who had died in one hospice unit between January 2009 and March 2014. The demographic and clinical characteristics of the patients were examined and the differences related to the patients' marital status were evaluated. We calculated the time interval between cancer diagnosis and hospice admission and the survival period from hospice admission to death and analyzed their association with family composition.

Results: In the group of patients living with their spouses, there were a higher percentage of male sex $(68.1 \%)$ and people who received standard cancer treatment (77.2\%). When divided by the median time of 13 months between diagnosis and admission, there was a higher proportion of single, divorced, separated, or widowed patients in group A $(<13$ months), whereas there was a significantly higher proportion of patients living with their spouses in group $B(>13$ months); $(p<0.001)$. The main decision maker were adult offspring $(59.3 \%)$ in group $A$ and spouses $(50.8 \%)$ in group $\mathrm{B} ;(\mathrm{p}<0.04)$.

Conclusion: Among the characteristics of family composition, the presence of spouse is an important factor associated with admission to the hospice unit. Clinicians need to be aware of the impact of marital status on end- of- life care.This study indicates that it is helpful to understand family composition of terminally-ill cancer patients for an effective utilization of palliative and hospice care.

\section{Abstract number: P01-179 \\ Abstract type: Poster}

\section{Support Groups for Family Caregivers \\ Jørgensen $B$. \\ Aarhus University Hospital, Aarhus, Denmark Presenting author email address: britjoer@rm.dk}

Background: Family caregivers of terminally ill patients need support in order to master the role as relatives and future loss of their loved one. The Palliative Care Team in Aarhus, Denmark plans to offer family caregivers participation in a support group. However, research on whether the family caregivers' need for support is accommodated in a group, has only scarcely been performed in a specialised palliative care setting. Aim: To gain insight into how family caregivers of terminally ill patients experience the support received by the palliative care team: What kind of support needs do they have and can some of the needs be met by participating in a support group?

Method: A qualitative study with a phenomenological-hermeneutic approach based on semi-structured individual interviews with five relatives. Data was analysed using a Ricoeur inspired analytical method.

One male spouse, two female spouses and two daughters participated. Age: $45-70$ years. Four patients died of cancer and one of COPD.

Results: Family caregivers all expressed high satisfaction with the support they got. Four themes emerged concerning support needs:

1. Support to be prepared for the trajectory, including knowledge about the disease and symptom relief.

2. Support to handle changing of everyday life, roles and loneliness.

3. Available, close and continuous relationship with health professionals including availability to get specific guidance as well as being appreciated for what they were doing.

4. Structure and composition of a potential group would have great importance in order to relate to others in the group.

Conclusion: Family caregivers experience a great extent of support by the present individual support, if the support is based on a relationship of empathy and professionalism. An additional support group program can fulfill some specific support needs, particularly the need to meet other caregivers in the same situation, but the composition of the group is important.

\section{Abstract number: P01-180}

Abstract type: Poster

During Agony: Relatives Support Practices of an Interdisciplinary Team in a Palliative Care Unit

Melin M. ${ }^{1}$, Raoux N. ${ }^{2}$, Ouvrard C. ${ }^{2}$, Frasca M..$^{3,4}$, Burucoa B..$^{3,4}$

${ }^{1}$ Palliative Care Unit, CHU Bordeaux, Bordeaux, France, ${ }^{2}$ U1219InsermUniversité Bordeaux, Bordeaux, France, ${ }^{3} \mathrm{CHU}$ Bordeaux, Bordeaux, France, ${ }^{4}$ Université de Bordeaux, Bordeaux, France

Context: Relatives support is an essential axis of palliative cares $(1,2)$ and agony may be extremely challenging (4). Literature yet poorly explored care practices of relatives during agony. Multidisciplinary professional from a palliative care unit (PCU) and researchers performed a qualitative study (PROPAGE) to describe interdisciplinary supportive practices adopted during the agony phase of patients. The secondary objective was to highlight relatives' expectations.

Method: Two focus group (FG) was performed: one by type of person (six groups: physicians, nurses, caregivers, psychologists, other professionals, palliative care volunteers) and one for bereaved relatives. Searchers looked for four categories of practices: frequent consensual, occasional consensual, non-consensual and expected practices (or to develop). Content analysis followed three steps:

1- Verbatim transcript,

2- Identification of practices by FG, then categorization,

3- Critical reading to the PCU teams for approval, potential corrections or additions.

Results: Verbatim identified 211 practices: 195 consensual (148 common, 47 occasional), one non-consensual and 15 expected. Scientific committee developed a classification of these practices in four themes:

1- Interact: "go to... create a relationship";

2- Inform-Communicate-Explain: "announce... answer to questions...";

3- Provide care and ensure comfort: "patient care... needs and pleasure of relatives... support"; 
4- Mobilize interdisciplinary skills: "transmit ...bring together". Moreover, some practices described by relatives matched with those cited by professionals. They also suggested development of some of them.

Conclusion: Such data may allow to improve training of professionals and practices related to interaction theme. Collecting opinions of other PCU teams about this directory is required to validate these results. A consensus method based on an inter-professional group of experts might be then used to establish guidelines about these practices.

Abstract number: P01-181

Abstract type: Poster

Support to Family Carers When Caring for a Family Member at the End of Life at Own Home - Implications for Practice Based on Family Carers Preferences

Larsson M. ${ }^{1}$, Lee S.H. ${ }^{2}$, Grov E.K..$^{3,4}$, Hov R. ${ }^{5,6}$, Olsson C. ${ }^{1}$, Bjuresäter K. ${ }^{1}$, Wilde Larsson B. ${ }^{1,7}$, Kennedy $C^{2}{ }^{2}$

${ }^{1}$ Karlstad University, Karlstad, Sweden, ${ }^{2}$ Robert Gordon University, Aberdeen, United Kingdom, ${ }^{3}$ Institute of Nursing and Health Promotion, Oslo, Norway, ${ }^{4}$ Akershus University College of Applied Sciences, Oslo, Norway, ${ }^{5} \mathrm{Hedmark}$ University College, Elverum, Norway, ${ }^{6}$ Centre for Development of Home Care Services in Hedmark, Hamar, Norway, ${ }^{7}$ Hedmark University College, Elverum, Sweden

Background: Demand for palliative home care across Europe has resulted in an estimated 100 million family carers involved in end of life care. Family carers report unmet health and social care needs and our understanding for their preferences around end of life care are relatively unknown.

Aim: To explore family carers' preferences for support and examine what helps family carers cope during end of life care in their own home.

Methods: Integrative literature review. Ten databases were searched including MEDLINE, EMBASE, CINAHL, Cochrane Library, PsycINFO, AMED, ASSIA, TRIP, ISI Web of Science and Scopus between January 2000 - October 2016. A combination of MeSH and keywords were used. Four pairs of reviewers screened titles and abstracts, extracted data and performed quality appraisal. Data were analysed thematically.

Results: 5348 titles and abstracts were screened and 74 articles were included. Family carers viewed themselves as Being a partner in care and Involvement in decision making and end of life care was important. For family carers their Priority was ensuring the patient support needs were met before they could address their own psychosocial needs. Timeliness of transitions of care through the illness trajectory was crucial. Access to knowledeble health care personnel, information and continuity in contacts contributed to Preparedness for transisions. Knowing what support can be accessed including competent personnel as well as respite care and support groups helped family carers to cope with the illness trajectory.

Conclusions: Health Care Professionals should recognise that family carers want to be involved in decision-making about care at home. Continuity of care, key contacts and support around the clock is fundamental for family carers to feel safe and secure in their caring role. They need information about what to expect to be prepared and respite care in order to cope. Implications for practice and future research will be explored in the presentation.

Abstract number: P01-182

Abstract type: Poster

Collaboration at the End of Life between Professional Care Providers and Family Carers: A Qualitative Study

Vermorgen M. ${ }^{1}$, De Vleminck A. ${ }^{1}$, Van Audenhove C. ${ }^{2}$, Vandenbogaerde I. ${ }^{1}$, Deliens L. ${ }^{1,3}$, Cohen J. ${ }^{1}$
${ }^{1}$ End-of-Life Care Research Group, Vrije Universiteit Brussel (VUB) and Ghent University, Jette, Brussels, Belgium, '2LUCAS Center for Care Research and Consultancy, University of Leuven, Leuven, Belgium, ${ }^{3}$ Department of Public Health and Primary Care, Ghent University, Ghent, Belgium

Background and aim: As family carers increasingly hold a central position in the provision of end-of-life care, a close partnership with formal care services may be needed, especially in the home environment. However, few research initiatives have paid attention to how family carers collaborate with professionals. By examining family carers' experiences, this study aims to gain insight into the aspects that characterize such collaboration.

Methods: Semi-structured interviews were conducted in Flanders, Belgium with 30 family carers of a person with dementia, cancer or heart failure who had a limited life expectancy and lived outside a residential care service. Family carers were contacted through a variety of services and formal care providers, including hospitals and GPs. A phenomenological approach was used to analyze the interviews.

Results: Family carers' experiences mainly centered around seven aspects required from professionals to promote collaboration:

1) clear and transparent communication;

2) awareness for family carers' well-being;

3) continuity in availability of care;

4) expertise with and know-how about how to support both persons with a serious illness and their family carers;

5) a respectful, empathetic and engaged attitude;

6) a match between the actual care delivered and family carers' standards and expectancies about the quality of care; and

7) minimization of slip-ups in care that family carers need to fix.

The absence of any of these could undermine the relationship and impede collaboration.

Discussion: Well-attuned collaboration between professionals and family carers is currently rare, especially outside the context of specialized palliative care. It is only conceivable if family carers are treated as both partners and clients throughout the whole care process.

This study is supported by a grant from the Flemish government agency for Innovation by Science and Technology (agentschap voor Innovatie door Wetenschap en Technologie, SBO IWT nr. 140009).

Abstract number: P01-183

Abstract type: Poster

The Effect of Advance Care Planning on Wellbeing of Family Carers in Six European Countries: Preliminary Results of the ACTION-study

Vandenbogaerde I. ${ }^{1}$, De Vleminck A. ${ }^{1}$, Cohen J. ${ }^{1}$, Verkissen M.N. ${ }^{1}$, Lapeire L. ${ }^{2}$, Ingravallo F. ${ }^{3}$, Wilcock $A .{ }^{4}$, Payne S. ${ }^{5}$, Seymour J. ${ }^{6}$, Kars M.C. ${ }^{7}$, Gronvold M. ${ }^{8}$, Lunder U. ${ }^{9}$, Rietjens J.A.C. ${ }^{10}$, van der Heide A. ${ }^{10}$, Deliens L. ${ }^{11}$

${ }^{1}$ End of Life Care Research Group, Vrije Universiteit Brussel \& Ghent University, Jette, Belgium, ${ }^{2}$ Medical Oncology, Ghent University, Gent, Belgium, ${ }^{3}$ Medical and Surgical Sciences, University of Bologna, Bologna, Italy, ${ }^{4}$ Nottingham University Hospital NHS Trust, Nottingham, United Kingdom, ${ }^{5}$ Division of Health Research, Lancaster University, Lancaster, United Kingdom, ${ }^{6}$ School of Health Sciences, University of Nottingham, Nottingham, United Kingdom, 7 Julius Center for Health Sciences and Primary Care, University Medical Center Utrecht, Utrecht, Netherlands, ${ }^{8}$ Public Health, University of Copenhagen, Copenhagen, Denmark, ${ }^{9}$ University Clinic of Respiratory and Allergic Diseases Golnik, Golnik, Slovenia, ${ }^{10}$ Public Health, Erasmus MC, Rotterdam, Netherlands, ${ }^{11}$ End of Life Care Research Group, Public Health and Primary Care, Vrije Universiteit Brussel \& Ghent University, Ghent \& Brussel, Belgium 
Background: Facilitating advance care planning (ACP) among patients, their family carers (FCS) and health care professionals is essential to improve end of life experiences for both patients and their FCs. Previous studies from the USA show that FCs who received facilitated ACP had improved outcomes in wellbeing (e.g. less anxiety, less depression, etc.), compared with those who did not. However, studies on the impact of ACP on FCs in Europe and especially in oncology settings are missing. The ACTION trial is the first multicentre randomized controlled ACP trial in 6 European countries.

Aim: To examine the effect of ACP on the wellbeing of bereaved FCs in 6 European countries.

Methods: 451 questionnaires were sent to FCs 3 months after bereavement and 163 were returned (response rate: $36.1 \%$; $\mathrm{N}$ control= 93, N intervention $=70$ ). Wellbeing was measured with the Hospital Anxiety and Depression Scale (HADS - range 0-21) and Impact Event Scale (IES range $0-75$ ). A higher score indicates more anxiety, depression, intrusion and avoidance.

Results: No significant differences were found in wellbeing of FCs between groups. The mean scores for the HADS show no significant differences between groups for anxiety (mean score control 7,09 vs. mean score intervention 8,29) and depression (mean score control 6,72 vs. mean score intervention 7,17 ). The mean scores for the IES were all in the same range. Intrusion had a mean score of 21,27 for control vs. 21,38 for intervention; Avoidance had a mean score of 10,34 for control vs. 12,72 for intervention.

Conclusion: Despite previous evidence about improved outcomes for wellbeing in FCs in ACP programs, our ACP intervention did not show differences between groups. Possibly the non-response or cultural discrepancies between the different countries have a part in this. More research is needed to explain what mechanisms are present.

This study is supported by a grant from the Flemish foundation for research (FWO, nr. G034717N)

\section{Abstract number: P01-184}

Abstract type: Poster

\section{Relatives' Experiences towards Continuous Palliative Sedation:}

\section{A Systematic Review}

Oude Veldhuis S. ${ }^{1}$, Uitdehaag M. ${ }^{2}$

${ }^{1}$ Utrecht University, Utrecht, Netherlands, ${ }^{2}$ Research Center for Health, Social Work and Technology, Saxion University of Applied Sciences,

Enschede, Netherlands

Background: At the end of life, continuous palliative sedation (CPS) can be used to relieve the patient's intolerable suffering. Relatives are often closely involved and present during the last days or hours of patient's life. Therefore, guidelines indicate that explicit attention must be paid to the relatives during the sedation process.

Aim: The aim of this systematic review is to gain insight into the experiences of relatives towards CPS.

Methods: PubMed, Embase and CINAHL were searched for eligible articles up to February 2018. Articles were eligible when they focused on the CPS of terminal adults, described experiences of adult respondents and were written in English. Articles about short-term or intermittent sedation and (systematic) reviews were excluded. The methodological quality of the articles was assessed by two independent researchers using the CEBMa tool and COREQ tool.

Results: Three qualitative studies and five survey-studies were included. The methodological quality of the qualitative studies was moderate. Two surveys were of low methodological quality and three of moderate quality. The results of the included articles were integrated and clustered on five main themes: positive experiences, negative experiences, additional and contradictory experiences, contextual factors and image of palliative sedation. Most frequently mentioned topics were relief of the patient's suffering, (dis)satisfaction with information and communication, wondering for other options than palliative sedation and the image that palliative sedation hasten death.
Conclusion: CPS leads to both positive and negative experiences for relatives. Also contextual factors and the image of palliative sedation play an important role in their experiences. Health care professionals can achieve an optimization of the CPS process by improving their communication and information provision towards relatives.

\section{Abstract number: P01-185 \\ Abstract type: Poster}

The Lived Experiences of Parents of Children Recently Diagnosed with Acute Lymphoblastic Leukaemia

Formosa $E$

University of Malta, Msida, Malta Presenting author email address: elaine.c.formosa@gov.mt

Background: The incidence of acute lymphoblastic leukaemia is quite rare, yet it is the most common cancer diagnosis in children. The diagnosis of Acute Lymphoblastic Leukaemia involves an initial long term of hospitalisation period that is followed by continuity of care at home. The parents' role as a care giver changes to include the daily demands of their diagnosed child. There is limited knowledge about the lived experiences and the challenges encountered by the parents following their discharge.

Aim: This study aimed to explore the lived experiences of parents following the first discharge from hospital of their child who had been diagnosed with Acute Lymphoblastic Leukaemia.

Research design: The study followed a qualitative research design using the approach of an Interpretative Phenomenological Analysis.

Method: Data was collected by means of individual semi-structured interviews. The interviews were transcribed and analysed guided by the Interpretative Phenomenological Analysis approach.

Results: Six super-ordinate themes representing the parents' experiences emerged from the data:

(1) Mixed emotional cycle,

(2) Relocations,

(3) Daily struggles of parents caring for children with ALL,

(4) The way forward

(5) A shifting perspective and

(6) Supportive Encounters.

Conclusion: The findings reflected the need for a hospital structured training for health professionals to be proficient in helping parents better manage the transition phase from hospital to home. Findings reflected also the need for more a specialised system as increase in professional therapy such as, play therapists, specialized nurses and peadiatric psychological care to work within this setting. Additionally, the introduction of financial and community nursing support and nursing home care could theoretically reduce the burdens of the parents' encountered as a result of their child's illness.

Abstract number: P01-186

Abstract type: Poster

Early Palliative Care for Those Who Care: A Qualitative Exploration of Cancer Caregivers' Information Needs during Hospital Stays

Preisler M. ${ }^{1}$, Rohrmoser A. ${ }^{2}$, Goerling U. ${ }^{2}$, Kendel $F^{3}$, Baer $K^{1}{ }^{1}$, Riemer

M. ${ }^{4}$, Heuse S. ${ }^{3}$, Letsch A. ${ }^{1}$

${ }^{1}$ Charité - Universitätsmedizin Berlin, corporate member of Freie Universität Berlin, Humboldt-Universität zu Berlin, and Berlin Institute of Health, Medical Department, Devision of Hematology and Oncology, Campus Benjamin Franklin, Berlin, Germany, ${ }^{2}$ Charité - Universitätsmedizin Berlin, corporate member of Freie Universität Berlin, Humboldt-Universität zu Berlin, and Berlin Institute of Health, Charite Comprehensive Cancer Center, Psycho-Oncology, Berlin, 
Germany, ${ }^{3}$ Charité - Universitätsmedizin Berlin, corporate member of Freie Universität Berlin, Humboldt-Universität zu Berlin, and Berlin Institute of Health, Institute of Medical Psychology, Campus Mitte, Berlin, Germany, ${ }^{4}$ Wilfrid Laurier University, Department of Psychology, Waterloo, ON, Canada

Background: The supporting role of caregivers is crucial to patients' care and wellbeing during cancer treatment. Periods of inpatient hospital treatment are common in the cancer trajectory. However, there is insufficient institutional knowledge of caregivers' experiences and information needs in a hospital context.

Aim: The aim of this study is to identify information needs of caregivers in an inpatient oncology unit.

Design: A qualitative approach was used to identify major themes and specific types of information needs. Semi-structured interviews and focus groups with caregivers were conducted, transcribed, and analyzed using a modified Grounded Theory approach.

Setting/participants: The study was conducted in a university oncology department. All together 17 caregivers of patients with advanced cancer were interviewed.

Results: Caregivers' needs during inpatient treatment vary and are largely unmet. Four major themes emerged from the analysis, revealing information needs of caregivers concerning (1) the cancer disease, (2) the patient, (3) the caregivers themselves, and (4) the hospital context. For these themes the most frequently mentioned issues were potential outcomes of the treatment and the related consequences to future dimensions of care and caregivers' life, and about the transition from inpatient to outpatient care.

Conclusion: Caregivers in an inpatient oncology unit have a variety of specific information needs. These needs often remain unmet. Based on our findings we provide recommendations to integrate caregivers into oncology care, which could be realized by early palliative care and should be implemented in clinical practice, guidelines, education, policy decisions, and research.

\section{Abstract number: P01-187}

Abstract type: Poster

A Pet. Fellow or Unnecessary Obligations. The Point of View of Experienced Palliative Care Professionals

Chojnicki M. ${ }^{1}$, Deskur-Śmielecka E. ${ }^{2}$, Bętkowska I. ${ }^{3}$

${ }^{1}$ Department of Biology and Environmental Science, Poznan University of Medical Science, Poznań, Poland, ${ }^{2}$ Chair and Department of Palliative Medicine, Poznan University of Medical Science, Poznań, Poland, ${ }^{3}$ Queen of Apostles Home Care Hospice by Medi Kompleks Sp. z o.o, Wiśniowa, Poland

Background: Current data suggest that pets play a large part in the lives of people receiving palliative care. However, all of the previous studies have been carried out based on an observation of a small number of patients ( $\max 20)$.

The knowledge about the role of pets living with a patient at his/her life's end, is important for hospice personnel. Persons with lower experience are especially prone to make mistakes by insisting on elimination of pets from patient's vicinity.

Aims: Critical assessment of the presence of pets, in the direct vicinity of a patient, in the opinion of experienced physicians and nurses of the hospice staff.

Attempt to form universal recommendations for students and young professionals who begin their work with people receiving palliative care. Method: Interview based on open questions, relating to personal experience with animals accompanying patients in their last weeks of life. The questions addressed the problems of animal care, possibility of zoonoses occurrence and the attitude of the patients towards their own pets.
The interviewed group was composed of physicians and nurses who work in hospice (at least 15 years of professional experience). The survey also included a question about a personal attitude towards pets and about experience of having a domesticated animal.

Results: The authors acquired opinions of 40 experienced employees of working in hospices. The vast majority of interviewed persons did not declare that the presence of an animal was a burden for any patient and did not suggest addressing this topic directly.

However, there were singular cases, where pets were removed from patient rooms.

Conclusions: A common conclusion based on all of the acquired information is confirming a significant role of pets in maintaining mental balance of patients. The decision about removing an animal should be left to the closest relatives and the issue, in the opinion of palliative professionals, should not be addressed directly.

\section{Abstract number: P01-188}

Abstract type: Poster

\section{Hospitalization in Palliative Care, a Space to Resignify Care at the End of Life}

Montecino Bacigalupo C. ${ }^{1}$, Vega P. ${ }^{2}$, Gonzalez R. ${ }^{2}$

${ }^{1}$ Escuela de Enfermería, Universidad de las Américas, Santiago, Chile, ${ }^{2}$ Escuela de Enfermería, Pontificia Universidad Católica de Chile, Santiago, Chile

Aim: The objective of the study was to uncover the experience lived by informal caregivers during the care of their family member in advanced stage of cancer in the home that took them to hospitalize them in a palliative care clinic.

Method: The research was carried out with a phenomenological qualitative methodology based on Husserl, the testimonies of ten participants who lived the experience of hospitalizing their relative in a palliative care clinic, after having taken care of them at home. The information was obtained through in-depth audio-taped interviews transcribed literally protecting confidentiality and ethics.

Results: In the study it was revealed that the participants, after assuming the responsibility of taking care of their family member in the home, began to experience difficulties when exercising alone, overloading themselves and facing with anguish the complications derived from cancer. To which was added, less support from the Program staff. This triggered a crisis in care, which led them to make the decision to hospitalize them. Once inside the palliative care clinic, the caregivers felt supported by the professionals, receiving together with their family member, a specialized and affectionate attention. This allowed us to resignify the experience, feeling that they were once again taking care of their family member with greater dedication and time and, at the same time, feeling them taken care of as people.

Conclusion: The investigation revealed that home care requires continuous accompaniment by the Palliative Care team, directing efforts to improve the quality of life of family members who care for their relatives at the end of their life at home. Therefore, nursing must manage resources and implement innovative interventions for the accompaniment of informal caregivers, strengthening communication and networking at the national level.

Abstract number: P01-189

Abstract type: Poster

Making Sense of Living with and beyond Cancer: A Systematic Review and Narrative Synthesis

Le Boutillier C. ${ }^{1}$, Archer S. ${ }^{2}$, Barry C. ${ }^{1}$, King A. ${ }^{1}$, Mansfield L. ${ }^{3}$, Urch C. ${ }^{1}$

${ }^{1}$ Imperial College London Healthcare NHS Trust, London, United Kingdom, ${ }^{2}$ Imperial College London, London, United Kingdom, ${ }^{3}$ Brunel University London, London, United Kingdom 
Background: The concept of living with and beyond cancer is now emerging in policy and literature, and attention to understanding the needs of those living with and beyond cancer and to develop models of care that meet their needs is required. A call has been made to improve the longterm quality of life for people who live with and beyond cancer and to better understand the experiences and needs of those who have completed primary cancer treatment. Research to explore the personal experience of living with and beyond cancer will begin to address this gap.

Methods: A systematic review and narrative synthesis was conducted to integrate descriptions and models of living with and beyond cancer into an empirically based conceptual framework. The protocol for the review was pre-registered in the PROSPERO database (PROSPERO CRD42017059860). The review question was What does the term 'living with and beyond cancer' mean? All included studies were qualitative, so narrative synthesis was used to integrate descriptions and definitions of living with and beyond cancer into an empirically based conceptual framework.

Results: Out of 2345 papers that were identified and 180 that were reviewed, a total of 73 papers were included. The synthesis consists of three interlinked themes: Adversity - realising cancer, Restoration - readjusting life with cancer and Compatibility - reconciling cancer, resulting in the ARC framework.

Conclusions: Three themes describe the experience of living with and beyond cancer: Adversity, Restoration and Compatibility. The ARC framework provides an empirically informed insight into the adjustment experience of people living with and beyond cancer and can be used to promote the development of person-centred models in palliative and supportive care.

Abstract number: P01-190

Abstract type: Poster

Influence of Short-term versus Long-term Prognosis on Decisions to Admit Patients with Advanced Diseases to Intensive Care Escher M..$^{1,2}$, Nendaz M.2,3, Ricou B. ${ }^{4}$, Scherer $F^{1}$, Hudelson $P^{5}$, Cullati S. ${ }^{1}$, Perneger T. 6

${ }^{1}$ Palliative Care Consultation, University Hospitals of Geneva, Geneva, Switzerland, 2Faculty of Medicine, Unit for Development and Research in Medical Education, Geneva, Switzerland, ${ }^{3}$ Division of General Internal Medicine, University Hospitals of Geneva, Geneva, Switzerland, ${ }^{4}$ Division of Intensive Care, University Hospitals of Geneva, Geneva, Switzerland, ${ }^{5}$ Division of Primary Care, University Hospitals of Geneva, Geneva, Switzerland, ${ }^{6}$ Division of Clincal Epidemiology, University Hospitals of Geneva, Geneva, Switzerland

Background: There are no definitive criteria for admission to intensive care (ICU). Long-term prognosis should be considered along with patient need for life-sustaining therapies and potential benefit from intensive care. Physicians accurately predict short-term survival of patients assessed for ICU and survival prediction is associated with admission decisions. How physicians balance long-term against short-term prognosis is not known.

Aims: Assess the influence of long-term prognosis on admission decisions for patients with advanced diseases.

Methods: Requests for ICU admission for medical in-patients were identified. The internal medicine (IM) physician and the ICU physician involved were asked to estimate the patient's probability of survival if the patient was admitted to the ICU, and if the patient remained on the ward ( $<10 \%, 10-40 \%, 41-60 \%, 61-90 \%,>90 \%)$. Patients were classified as having an advanced disease based on diagnoses in the medical record. The admission decision was recorded.

Results: 201 patients were included, of whom 116 (57.7\%) had an advanced disease. Patients with advanced diseases were as often admitted to the $\operatorname{ICU}(80 / 116,69.0 \%)$ as those without $(60 / 85,70.6 \%)$. In multiple logistic regression, admission was positively associated with a higher probability of survival if in the ICU estimated by the ICU physician (odds ratio (OR) 2.0 per survival category, $\mathrm{p}=0.005$ ) and by the IM physician (OR
2.1, $p=0.003)$, negatively associated with a higher probability of survival if on the ward $(O R=0.28, p<0.001$ for ICU physician, and $O R=0.47, p=0.002$ for IM physician), and was not associated with advanced disease (OR=1.04, $p=0.93$ ).

Conclusions: Admission to intensive care was strongly influenced by the physicians' estimation of patient short-term survival. The recommendation to take long-term prognosis into account for admission decisions is not routinely applied.

Funding: Swiss National Science Foundation, NRP 67 "End-of-life"

Abstract number: P01-191

Abstract type: Poster

Bereaved Family Members' Satisfaction with Care during the Last Three Months of Life for People with Advanced IIIness O'Sullivan A. ${ }^{1}$, Alvariza A. ${ }^{1,2}$, Öhlen J. ${ }^{3}$, Håkanson C. ${ }^{1,4}$

1Palliativt Research Center, Institution of Health Care Science, Ersta Sköndal Bräcke University College, Stockholm, Sweden, ²Palliative Care Unit, Dalen Hospital, Stockholm, Sweden, ${ }^{3}$ Institute of Health and Care Sciences and University of Gothenburg Centre for PersonCentered Care and Palliative Centre, Sahlgrenska Academy, University of Gothenburg, Gothenburg, Sweden, ${ }^{4}$ Department of Nursing Science, Sophiahemmet University, Stockholm, Sweden

Background: Studies evaluating the end-of-life care for longer periods of illness trajectories and in several care places are currently lacking in the Swedish context.

Aims: This study explored bereaved family members' satisfaction with care in several care places, during the last three months of life for people with advanced illness, and associations between satisfaction with care and characteristics of the deceased persons and their family members.

Methods: A retrospective cross-sectional survey design using the VOICES (SF) questionnaire, descriptive statistics and logistic regression was applied. The sample was 485 family members (age range 20-90 years, $70 \%$ women) of persons who died in hospitals in two Swedish health care regions. The deceased persons (age range 27-100) died mainly of circulatory or respiratory diseases, or malignant neoplasm.

Results: Of the family members $77,3 \%$ were satisfied with all care received during the last three months of life, when added together and rated as one. The results show variations in care satisfaction between different care places and care services; $87,2 \%$ of the bereaved family members had a high satisfaction with care in hospices, followed by hospitals $(85,9 \%)$, district nurses $(68,9 \%)$, nursing homes $(63,0 \%)$, specialized home care $(60,0 \%)$ and GPs $(55,6 \%)$. Spouses were more likely to be satisfied with the care than children or other family members. Family members of deceased persons with cancer were more likely to have a higher satisfaction with the care. A lower satisfaction was more likely if the deceased person or the bereaved family member had a higher educational attainment and a length of illness before death for one year or longer.

Conclusions: The satisfaction with care is influenced by the care place/ type of care service, as well as by diagnoses, length of illness, educational attainment and the relationship between the deceased person and the family member.

Abstract number: P01-192

Abstract type: Poster

Medical Professionals Experiences and Attitudes Regarding Terminally III Patients' Do-not-Resuscitation and Withdrawal of Life-sustaining Treatments: A Hospital-based Cross-sectional Study

Chang H.-T., Lin M.-H., Chen C.-K., Yang C., Chiang Y.-T., Shyu C.-H., Lin

H.-J., Chen H.-F., Yang W.-L., Chen Y.-J., Chen T.-J., Hwang S.-J.

Taipei Veterans General Hospital, Taipei, Taiwan, Republic of China 
Background: There is little evidence on medical professionals' (MPs') experiences and attitude regarding do-not-resuscitate (DNR) and lifesustaining treatments (LST) decisions from Asian countries.

Aims: The aims of this study were to evaluate MPs' experiences and attitude regarding DNR and withdrawal of LST.

Methods: This study was a cross-sectional study which was conducted in a tertiary hospital in Taiwan. Medical professionals aged $\geqslant 20$ years old were randomly recruited. Semi-structured questionnaire was administered to evaluate their experiences and attitudes regarding DNR and withdrawal of LST in taking care of terminal patients. Statistical analyses were conducted by SPSS 20.0 .

Results: A total of 150 MPs participated in this study. The mean age was $36.7 \pm 8.7$ years and $80.7 \%$ were female; $83.3 \%$ with an education level of university; $57.3 \%$ single; $35.3 \%$ without religious beliefs; $88.0 \%$ were registered nurses and 9.3\% physicians. 97.3\% respondents had experiences in taking care of terminal patients; $63.7 \%$ had ever initiated DNR discussion with patients while $70.5 \%$ with patients' families $(P<.001)$; $41.1 \%$ initiated withdrawal of LST discussion with the patients' families while $23.3 \%$ with the patients. $28.8 \%$ recommended patients or their families to withdrawal artificial nutrition; $35.6 \%$ vasopressors; and $12.3 \%$ renal replacement therapy. $39.3 \%$ respondents will try to initiate discussion on withdrawal of MV for lessening the suffering of these patients, maintaining patients' dignity and for good death.

Conclusion/ discussion: Majority respondents had ever taking care of terminal patients but less than half ever actively discussed DNR decision and withdrawal of LST with these patients or their families. There are still concerns in DNR decision and withdrawal of LST discussion which need further investigation for a better end-of-life care. (Grants: The Ministry of Science and Technology Taiwan MOST 106-2314-B-075-004 and Yen Tjing Ling Medical Foundation Cl-107-31).

Abstract number: P01-193

Abstract type: Poster

The Role of the General Practitioner as Referrer to Specialized Palliative Care in Germany

Scholten N., Volkert A., Peter S., Pfaff H., APVEL (Hellmich, Pfaff,

Radbruch, Rietz, Rolke, Scholten, Schubert)

IMVR, University of Cologne, Cologne, Germany

In Germany, general practitioners (GPs) play an important role in palliative care $(P C)$, as providers of general outpatient palliative care (AAPV) and as referrers to specialized palliative care (SAPV). The aim of this study was to identify the main reasons for referral of palliative patients to SAPV and to evaluate whether GPs have difficulties in assessing PC needs.

Method: A postal survey was conducted to evaluate SAPV, AAPV, and the role of the GP within the German region of Nordrhein. The questionnaire had been developed based on qualitative research. All GPs in five predefined subregions in Nordrhein ( $n=2,199$ ) were invited to take part in the survey. Overall, $445 \mathrm{GPs}$ returned the survey (response rate: 20.2\%). Results: More than $93 \%$ of the responding GPs seem to know when patients benefit from SAPV. About $57 \%$ stated that SAPV is only necessary in very complex cases. GPs with an extra qualification in PC as well as GPs with more work experience are more likely to support this statement. Severe pain is the most frequently mentioned reason for referral to SAPV (almost $40 \%$ ), followed by neurologic/psychiatric symptoms $(11 \%)$ and respiratory symptoms (6\%), although differences between GPs with and without an extra qualification in PC and GPs can be found. The main reasons why patients were not referred to SAPV, although clinical requirements were fulfilled, were that the patients will do not want to receive SAPV (67\%), informal support is sufficient (45\%), and the GP has palliative competence (37\%).

Conclusion/discussion: An extra qualification in PC has a significant impact on the GP's opinion towards PC, the assessment of PC needs, and the main criteria leading to referral to SAPV.
APVEL is funded by the Innovation Committee of the Federal Joint Committee (01VSF16007).

Abstract number: P01-194

Abstract type: Poster

Multidisciplinary Cooperation in Outpatient Palliative Care from a GP's Perspective

Peter S., Volkert A., Pfaff H., Scholten N., APVEL (Hellmich, Pfaff,

Radbruch, Rietz, Rolke, Scholten, Schubert)

IMVR, University of Cologne, Cologne, Germany

Background: General practitioners and oncologists are important providers of outpatient palliative care (PC) in Germany. Nevertheless, to deliver good medical care a multidisciplinary approach in PC is needed. Little is known about the GP's perspective towards multidisciplinary cooperation in outpatient PC.

Methods: Data collection proceeded within the APVEL project ("Evaluation of specialized outpatient palliative care in North Rhine"). A focus group was conducted with 6 GPs. Content analysis was conducted by using inductive and deductive categorization. Based on the qualitative results, a postal survey of all GPs in five predefined subregions in North Rhine ( $n=2,199$, response rate: $20,2 \%$ ) was performed.

Results: Results from the focus group are that communication between providers in general is very important but does not occur frequently. The frequency depends on the interest and motivation of the PC providers. The cooperation between doctors is based on reciprocal consultations. Sometimes a direct competition between PC professions occurs. Communication of providers is influenced largely by personal factors like interest in $\mathrm{PC}$, motivation, and knowledge. A multidisciplinary approach in outpatient PC is rated as very important but also hard to be implemented. Similar results were found in the survey, where $84 \%$ of the responding GPs stated that they want to stay involved in the patient's treatment, even after referral to specialized palliative care (SAPV). Therefore $33 \%$ are unsatisfied with the SAPV team due to a lack in inclusion. Regarding the readiness to provide information, $75 \%$ are satisfied. Conclusion/discussion: A multidisciplinary approach is optimal for outpatient PC but hard to implement in PC provision. GPs feel committed towards their patients and to a certain degree want to stay involved. This can be challenging for the SAPV team as well as for the GP.

APVEL is funded by the Innovation Committee of the Federal Joint Committee 01VSF16007

Abstract number: P01-195

Abstract type: Poster

ImpaktGer - Implementation of Particularly Qualified and

Coordinated Palliative Care (BQKPMV) in Germany

Mühlensiepen F. . $^{2,3}$

${ }^{1} \mathrm{KV}$ Consult- und Managementgesellschaft $\mathrm{mbH}$, Potsdam, Germany, ${ }^{2}$ Medizinische Hochschule Brandenburg - Theodor Fontane, Neuruppin, Germany, ${ }^{3}$ Universität Witten/Herdecke, Witten, Germany Presenting author email address: felix.muehlensiepen@kv-comm.de

Cave: ImpaktGer is not yet initiated. So far, only preliminary data of the state of Brandenburg is available. This abstract is intended to attract coinvestigators to join ImpaktGer.

Background: By passing the Hospice and Palliative Act (2015), outpatient palliative care in Germany is subject to normative and structural changes. One of the measures settled in the law is the implementation of a particularly qualified and coordinated palliative care (BQKPMV) which is supposed to enhance primary palliative care. BQKPMV is being implemented since October 2017. The conversion will have major impact on German outpatient palliative care.

Methods: The study design contains a mixed method approach: 
1. Delphi-Survey: health care professionals in hospice and palliative care in Germany

2. Secondary data analysis of the Association of Statutory Health Insurance Physicians (Kassenärztliche Vereinigungen)

3. Questionnaire Survey with resident doctors in Germany

Results: Data from the Association of Statutory Health Insurance Physicians (Kassenärztliche Vereinigung) in the state of Brandenburg indicate that 92 out of 4,200 physicians currently participate in BQKPMV. 55 of 92 are family doctors. 45 physicians acquired additional qualification in palliative medicine.

Initial data from qualitative interviews indicate that, palliative care providers judge BQKPMV with great skepticism. Resident doctors assess prerequisites for participation as inappropriate. Interviewees argue that BQKPMV does not increase quality of palliative care. Palliative care providers perceive competitive pressure through implementation of BQKPMV.

Conclusion: Implementation of BQKPMV requires an appropriate process evaluation that generates valid data. So far, only the number of participants is being assessed. Measuring the impact of BQKPMV on care structures requires a more complex study design.

To implement this, I am looking for partners.

\section{Abstract number: P01-196 \\ Abstract type: Poster}

Factors for a Successful Performance: Specialized Outpatient Palliative Care (SAPV) in Germany - Outcomes on the Basis of Interviews with SAPV Teams and Network Partners

Krauss S.H. ${ }^{1}$, Bauer A. ${ }^{1}$, von Hayek J. ${ }^{1}$, Jansky M. ${ }^{2}$, Stichling K. ${ }^{3}$, Schneider W. ${ }^{1}$, SAVOIR Study Group

${ }^{1}$ Center for Interdisciplinary Health Research, University of Augsburg, Augsburg, Germany, ${ }^{2}$ Clinic for Palliative Medicine, University Medical Center Göttingen, Göttingen, Germany, ${ }^{3}$ Institute for General Practice and Family Medicine, Jena University Hospital, Jena, Germany

Background: Specialized outpatient palliative care (SAPV) in Germany is ruled by the Federal Joint Committee directive. Over 10 years after implementation, a broad variety of organizational structures exists.

Aims: Identification of beneficial and inhibiting factors for a successful performance described by SAPV care providers (team members and network partners) from teams with contrasting structures.

Methods: The study is carried out by means of 10 on-site field studies. Over 100 qualitative, problem-centred interviews with SAPV teams members and their network partners as well as ethnographic field notes are included. Results: Different concepts of a successful performance and their effective implementation strategies are linked with corresponding team structures and the surrounding palliative care (PC) environment. General standards and individual ideals are secondary.

All teams have a network of care providers, but the perceived significance and the strength of network ties correspond typically to certain perceptions of quality, e.g. pharmacies or GPs are more or less important for certain teams.

One finding is that teams that are part of a hospital are structurally able/ unable to consider certain factors as criteria for a successful SAPV, whereas independent teams consider other factors as beneficial for a successful SAPV performance.

What is considered a successful performance is produced locally within the specific setting of a SAPV team and its PC environment.

Conclusion: The structural heterogeneity of the SAPV leads to similarly heterogeneous beliefs in what constitutes a successful SAPV performance. Discussion should be focused on the question if the current heterogeneity itself is a central criteria or rather an inhibiting factor for the quality of SAPV.

Funding: The study is part of the SAVOIR consortium which evaluates different aspects of SAPV in Germany and is funded by Federal Joint Committee German Innovations Fund.
Abstract number: P01-197

Abstract type: Poster

\section{Exploring Knowledge Transfer within Palliative}

Care Research

Payne C. ${ }^{1,2}$, Brown M.J. ${ }^{2}$, Guerin S. ${ }^{3}$, Kernohan W.G. ${ }^{2}$

${ }^{1}$ All Ireland Institute of Hospice \& Palliative Care, Dublin, Ireland, ${ }^{2}$ Nursing and Health Research, Ulster University, Newtownabbey, United Kingdom, ${ }^{3}$ University College Dublin, Dublin, Ireland

Background: Despite increasing levels of research being gathered in health settings, there are several challenges to the implementation of evidence into practice, which is required to influence change.Knowledge transfer models in health care are largely unrefined and untested. Between 2013-2017 an Evidence-based Model for the Transfer \& Exchange of Research Knowledge (EMTReK) was developed through an in-depth scoping review and stakeholder feedback on knowledge transfer and exchange frameworks used in health.

Aim: To pilot EMTReK using knowledge created from palliative care research.

Methods: A qualitative approach, with thematic analysis of case documentation, semi-structured interviews and field notes from five case studies of knowledge transfer and exchange in palliative care. Data arising from case studies using the model as a means to transfer knowledge relating to palliative care research to a range of user groups were collected between January 2016 and May 2017.

Results: Analysis supports the core components of the EMTReK model, which focus upon identification of messages to be transferred to defined stakeholders through interactive processes that take account of context. Case study findings show how the model was interpreted and operationalised by participants and demonstrate its impact on knowledge transfer and exchange. Eight themes were drawn from the data: Credibility of the Model; Model Accessibility; Applicability to Palliative Care; A Matter of Timing; Positive Role of Facilitation; Resources for KTE; Enhancing Research Quality; Limitations or Areas for Further Consideration.

Conclusions: The findings indicate that study participants found the EMTReK model

components were useful as a guide to the creation of KTE plans when combined with adequate facilitation and guidance.

\section{Abstract number: P01-198 \\ Abstract type: Poster}

The Challenges of Hospital-based Palliative and End of Life Care: A Case Study of a Nigerian Teaching Hospital Khalid D.S. ${ }^{1,2}$, Bailey C. ${ }^{1}$, Higginbottom G. ${ }^{1}$

${ }^{1}$ School of Health Sciences, University of Nottingham, Nottingham, United Kingdom, ${ }^{2}$ Department of Nursing Sciences, Ahmadu Bello University, Zaria, Nigeria

Background: Nurses in Nigeria face a number of challenges associated with inadequate resources to provide quality care for patients at the end of life. This is evidenced in the palliative and end of life care ranking across the world in 2015 Quality of Death Index by the Economist Intelligence Unit; Nigeria is at the $77^{\text {th }}$ position. There is a dearth of literature relating to palliative and end of life care in Nigeria, only a few sources were found. A systematic review reveals that most of the studies in Sub-Saharan Africa (SSA) where centred around HIV/AIDS, despite other conditions with similar palliative and end of life care needs such as cancer, renal failure, chronic respiratory diseases etc. The study explored the challenges of hospital-based palliative and end of life care in a Nigerian Teaching Hospital.

Method: A qualitative ethnographic case study design was employed. Participant observations and Interviews were used as tools for data 
collection. Data analysis follows an inductive thematic approach using NVivo 11 qualitative data management programme.

Findings: There were 14 cases, each case involved (nurses, patients, and family carers), participants were observed and interviewed. Each interview was preceded by a week of observations. Four overarching themes emerged: Economic factors, Communication problem, Poor condition of service, and Logistics problem.

Conclusion: Financial problem is a major concern among patients and their family carers because healthcare expenses are paid out-of-pocket. While the patients and their family carers expressed dissatisfaction about the care they received, the nurses attributed the poor quality of care to inadequate manpower, decayed infrastructure, logistics problem and poor condition of service.

\section{Abstract number: P01-199 \\ Abstract type: Poster}

\section{The Association between Palliative Care Team Consultation and Hospital Care for Patients with Advanced Cancer: An Observational Study in 12 Dutch Hospitals Brinkman-Stoppelenburg A. ${ }^{1}$, Polinder S. ${ }^{1}$, Olij B. ${ }^{1}{ }^{1}$, van den Berg} B. ${ }^{2}$, Gunnink N. ${ }^{3}$, Hendriks M.P. ${ }^{4}$, van der Linden Y.M. ${ }^{5}$, van der PadtPruijsten A. ${ }^{6}$, Peters E.A. ${ }^{7}$, Roggeveen B. ${ }^{8}$, Terheggen $F^{9}{ }^{9}$, Verhage $S .{ }^{10}$, van der Vorst M.J. ${ }^{11,12}$, Willemen I. ${ }^{13}$, van der Heide $A .^{1}$

${ }^{1}$ Public Health, ErasmusMC, Rotterdam, Netherlands, ${ }^{2}$ Department of Medical Oncology, Haga Hospital, The Hague, Netherlands, ${ }^{3}$ Department of Internal Medicine, OLVG, Amsterdam, Netherlands, ${ }^{4}$ Department of Internal Medicine, Northwest Clinics, Alkmaar, Netherlands, ${ }^{5}$ Center of Expertise Palliative Care, Leiden University Medical Center, Leiden, Netherlands, ${ }^{6}$ Department of Medical Oncology, Maasstad Hospital, Rotterdam, Netherlands, 'Department of Pulmonary Diseases, Norhwest Clinics, Den Helder, Netherlands, ${ }^{8}$ Department of Medical Oncology, ErasmusMC Cancer Institute, Rotterdam, Netherlands, ${ }^{9}$ Department of Internal Medicine, Bravis Hospital, Bergen op Zoom, Netherlands, ${ }^{10}$ Breast Center, Jeroen Bosch Hospital, 's Hertogenbosch, Netherlands, ${ }^{11}$ Department of Internal Medicine, Rijnstate Hospital, Arnhem, Netherlands, ${ }^{12}$ Department of Medical Oncology, Cancer Center Amsterdam, VU University Medical Center, Amsterdam, Netherlands, ${ }^{13}$ Department of Internal Medicine, Elisabeth-Tweesteden Hospital, Tilburg, Netherlands

Background: Many hospitals have established Palliative Care Teams (PCTs) to support clinicians in providing care for patients with advanced disease.

Aims: To study the association between PCT consultation and the content and costs of hospital care in patients with advanced cancer.

Methods: A prospective, observational study in 12 Dutch hospitals including patients with advanced cancer and an estimated life expectancy of less than one year. We compared hospital care during three months of follow-up for patients with and without PCT involvement. Costs of hospital care for both groups were compared while taking into account possible confounding by indication.

Results: We included 535 patients of whom 126 received PCT consultation. Patients with PCT had a worse life expectancy and performance status, and more often had no more options for anti-tumor therapy. Hospital length of stay, use of most diagnostic procedures, medication, and other therapeutic interventions were similar. The total mean hospital costs were $€ 8393$ for patients with and $€ 8631$ for patients without PCT consultation; for patients with PCT consultation within three days of admission these costs were $€ 6543$. When adjusted for baseline differences, differences in hospital costs were not statistically significant.

Conclusions: PCTs are predominantly consulted for patients with a relatively poor prognosis and performance status. Costs of hospital care are lower for patients with than patients without PCT consultation, especially when such consultations occur early during admission. When taking confounding by indication into account, we did not find a statistically significant association between hospital costs and PCT consultation.
Abstract number: P01-200

Abstract type: Poster

Provision of Palliative Care by General Practitioners:

Expenditures and Stressors

Volkert A., Peter S., Pfaff H., Scholten N., APVEL-Study Group: Palliative Medicine Departments of the University Hospitals RWTH Aachen (Prof. Rolke), Cologne (Prof. Voltz) and Bonn (Prof. Radbruch); IMSB, PMV Forschungsgruppe, IMVR (University of Cologne), IfE (PH Heidelberg) IMVR, University of Cologne, Cologne, Germany

Background: In Germany general practitioners (GPs) are gatekeepers within the provision of outpatient palliative care. Not only are they providers of palliative care; they can also refer patients to specialized palliative care teams (SAPV) if the need exists (for example, when there is complex symptomatology or pain). One aim of the study is to identify the expenditures and stressors involved in palliative care in the daily working routine of GPs.

Methods: On the basis of expert interviews and focus groups, we developed a questionnaire, which was sent to 2199 GPs in five pre-defined sub-regions within the German region of Nordrhein. Overall, 445 GPs returned the survey (response rate: 20.2\%).

Results: Although $81 \%$ agree that the care of palliative patients is a key responsibility of general practitioners, $28 \%$ of all responding GPs stated that providing it is too time consuming in their daily routine. Specialized GPs are less likely to support this statement. Thirty-five per cent say that providing adequate palliative care as a GP is not possible. Especially, home visits $(81 \%)$, flexibility and after-hours availability $(76 \%)$ are mentioned and influence the referral of palliative patients to SAPV. Moreover, many GPs are stressed by clashing requirements within their working time $(65 \%)$, their involvement in their patients' emotional suffering (59\%) and the bureaucratic burden (61\%).

Conclusion: Providing palliative care is a time-consuming task, and not all GPs feel that they are meeting their obligations, even if they agree that palliative care is within their sphere of responsibility. They make use of referrals to specialized palliative care teams, which seem not only to provide specialized care but also to substitute for lacking GP resources.

The study was funded by the German Innovation Fund.

Reference: Teasdale E, Drew S, Taylor C, Ramirez A (2008): Hospital consultants' job stress \& satisfaction questionnaire (HCJSSQ). Cancer Research UK London Psychosocial Group, London.

Abstract number: P01-201

Abstract type: Poster

Home Palliative Care Reduces Emergency Department Visits and Hospitalizations

Lam E. ${ }^{1}$, Patterson A. ${ }^{1}$, Lam M. ${ }^{2}$

${ }^{1}$ Markham Stouffville Hospital, Markham, Canada, ${ }^{2}$ Queen's University, Kingston, Canada

Background: Many patients spend significant time in the hospital during the last months of life. In Ontario, Canada, $62.7 \%$ of palliative care patients had an emergency department (ED) visit in their last month of life and $25.8 \%$ spent the majority of their last month in hospital. The Markham Stouffville Community Palliative Care Team (MSCPCT) is a physician team providing home palliative care. A primary goal of MSCPCT is to reduce unnecessary ED visits and hospitalizations.

Aim: To determine the efficacy of MSCPCT in reducing ED visits and hospitalizations for palliative cancer patients. Secondly, to determine which factors predict early referral and home death.

Methods: The records at MSCPCT and Markham Stouffville Hospital (MSH) were accessed to identify all palliative cancer patients who died between November 2014 and October 2017. Patients were divided into three groups; those not seen by MSCPCT prior to death at MSH, those 
followed by MSCPCT who died at MSH and those followed by MSCPCT who died at home. The groups were compared for ED visits, hospitalizations and cancer diagnosis.

Results: 430 palliative cancer patients died at MSH without prior linkage to MSCPCT. 248 MSCPCT cancer patients died at MSH and 194 at home. Least Square Means analysis showed significant reduction in ED visits and hospitalization days in the last 30,60 and 90 days of life when comparing patients who died at MSH who were followed by MSCPCT versus those who were not. Patients with brain cancer and sarcoma were more likely to be referred $(66 \%, 71 \%)$ and those with hematological malignancy and sarcoma were more likely to have a home death $(73 \%, 67 \%)$

Conclusion: In cancer patients who die in hospital, those followed by MSCPCT were significantly less likely to have ED visits and hospitalization days in the last months of life. Certain diagnoses are predictors of early referral and home death.

\section{Abstract number: P01-202}

Abstract type: Poster

Beneath the Surface: A Palliative Care Needs Assessment

Gwyther L., London L., Heap M.

School of Public Health and Family Medicine, University of Cape Town, Cape Town, South Africa

Background: A needs assessment is a recognized planning tool to inform a state's obligation to fulfil the Right to Health. Internationally needs assessment methodologies have been based on mortality rates for anticipated deaths and hospital admissions identified by limited ICD-10 codes.

Aim: To describe the need for palliative care (PC) in South Africa (SA). Objectives:

1) to identify causes of death by ICD-10 code in $S A$ associated with the need for PC;

2) to describe: the number of reported deaths in SA for 2010 associated with the need for PC based on mortality data reported for 2010 .

Methodology: The Burden of Disease (BoD) Research Unit MRC made available SA mortality data for 2010. An expert group reviewed ICD-10 codes to identify diagnoses appropriate for PC in the local setting. Data relating to these ICD-10 codes were extracted from the full BoD data set. Data were analysed to estimate need for PC according to age, gender, place of death, province and diagnosis. An age-standardized mortality rate was calculated by age and by province.

Results: At least 258,268 people who died in 2010 would have benefited from palliative care. This equates to $0.52 \%$ of the total SA population of 49,991,300 (2010); or 698.5 persons per 100,000; or 1 out of every 143 people every year.

Conclusion: Mortality data indicate a large burden of illness needing PC in SA which can inform the National Health Plan and National Palliative Care strategy. However, mortality data may underestimate need, pointing to limitations of this approach. It is suggested that use of one indicator from a validated PC indicator tool - patients with three unplanned hospital admissions for the chronic condition could provide a more robust method of assessing palliative care need.

Abstract number: P01-203

Abstract type: Poster

Limits to Availability of Palliative Care

Gwyther L., London L., Heap M.

School of Public Health and Family Medicine, University of Cape Town, Cape Town, South Africa
Background: Public sector palliative care (PC) services are few in South Africa (SA), limiting the availability of PC. Training of health care personnel in PC is also limited and SA has been identified as using low quantities of morphine.

Aim: To describe availability of PC in SA.

Objectives:

1) to identify PC services:

2) to describe the nature of PC; the demographics of PC beneficiaries; the human resources and level of PC training; availability of morphine.

Methodology: Services providing PC were identified. An on-line survey was distributed to 131 organisations between March and October 2011. The survey explored elements of PC provided by these services by staffing; staff training, and availability of morphine for pain. Responses were captured and analysed in Excel. Data on availability of morphine, employment and training of doctors was imported into Stata. Chi squared test and Odds ratios (with 95\% Confidence Intervals) were conducted to assess statistically significant associations between availability of morphine and

1) employment of doctors; and

2) the training levels of doctors.

Content analysis explored the narrative question on availability of morphine.

Results: 104 PC services responded; $\mathbf{3} \%$ from the public sector services. PC services usually employed nurses. The majority of staff trained in PC were professional nurses. PC services rely on volunteer doctors, social workers and spiritual counsellors. Availability of morphine depends on employment of and level of PC training of doctors. The estimated number of patients who received PC in 2011 was 46,$347 ; 17.94 \%$ of the estimated need.

Conclusion: In SA a number of NGOs (hospices) provide comprehensive PC. Supply is poor in the rural areas, especially access to morphine. Lack of PC training and the use of volunteers indicates that the prerequisite of domestically competitive salaries for trained personnel is not met. PC services in SA fall short of the need.

Abstract number: P01-204

Abstract type: Poster

Is Palliative Care Accessible?

Gwyther L. ${ }^{1}$, London L. ${ }^{2}$, Heap M. ${ }^{2}$

${ }^{1}$ Family Medicine, University of Cape Town, Cape Town, South Africa, ${ }^{2}$ School of Public Health and Family Medicine, University of Cape Town, Cape Town, South Africa

Background: Palliative care (PC) services are lacking in South Africa SA, particularly in rural areas. Even where PC services are available barriers to access may exist and impact on patients' care and right to health. Aim: To describe access to PC for patients with advanced chronic illness in Cape Town where PC services are adequate.

Objectives: To describe the proportion of patients with specified advanced chronic illnesses referred to PC services from three care settings; patient-reported PC outcomes within patients' current care setting.

Methodology: A prospective cohort study conducted over 6-months. The study recruited patients (i) with metastatic cancer; (ii) with HIV with CD4 count of $<200$ cells $/ \mathrm{mm}^{3}$; (iii) with motor neuron disease (MND). A research assistant administered a questionnaire to report demographics and other people or services providing care and the APCA African Palliative Outcome Scale (POS), a validated patient-reported outcome scale to measure care at first visit. Participants were followed up 
telephone, using the APCA POS once a month over the following six -months.

Results: Of the 162 participants 43 died and only nine patients were referred for PC. Most patient outcomes improved for patients with HIV; but there was no improvement in patient-reported outcome measures amongst the oncology and MND patients. A sub-set of patients with HIV and cancer reported high pain scores that did not resolve over the study period. Patient narratives disclosed extensive support from family, friends and faith communities.

Conclusion: Few patients were referred to palliative care services even though a significant number of patients died during the course of the study and the majority of patient outcomes remained poor. With family and friends providing care, the state is evading its obligation to facilitate access to PC. Lack of access to PC raises the question of the violation of patients' right to health.

Abstract number: P01-205

Abstract type: Poster

"Hospice Helped with Acceptance, Love, Care and Compassion". Quality of Palliative Care

Gwyther L., London L., Heap M.

School of Public Health and Family Medicine, University of Cape Town, Cape Town, South Africa

Background: Quality is an essential element of the Right to Health. Its assessment includes the technical aspects of care - structure, process and outcomes; and patient experience of care. South African hospices have an established quality improvement programme of survey, mentorship and accreditation against standards of governance and management.

Aim: To evaluate the quality of care delivered to patients requiring palliative care (PC) in hospice and non-hospice non-governmental NGOs in South Africa (SA).

Objectives:

1) to evaluate the quality of patient care by patient reported outcomes over 4-6 weeks;

2) to explore patient perceptions of the quality of care provided by the NGOs.

Methodology: A prospective cohort study was conducted in19 accredited hospices and 16 non-hospice NGOS in five provinces, selected by stratified sampling. The study selected 16 participants and nine family members per NGO, by convenience sampling. The APCA African POS, a validated palliative outcome scale, evaluated quality of care provided by the NGOs; a questionnaire explored patient experience of care.

Results: 459 patients and 253 family members were recruited. The study showed significant improvement in patient reported outcomes in both hospice and non-hospice sites. Pain, other symptoms and worry improved most in the hospice setting, and spiritual care most for patients in non-hospice NGOs. Content analysis of the narrative questions identified five themes: Quality of care, Place of care, Staff values and attitudes, Information-sharing and Problems experienced by participants. Some participants responded 'with love' to the question: How would you like to be cared for?

Conclusion: NGOs in SA provide quality PC according to elements required to meet the Right to Health. The respect and compassion of NGO carers enhance patients' sense of dignity. Love, not often associated with healthcare, is important to these participants. Is this the ultimate goal for person-centred care?

Abstract number: P01-206

Abstract type: Poster

How is Palliative Care Part of the Right to Health?

Gwyther L. ${ }^{1}$, London L. ${ }^{2}$, Heap M. ${ }^{2}$
${ }^{1}$ Family Medicine, University of Cape town, Cape Town, South Africa, ${ }^{2}$ School of Public Health and Family Medicine, University of Cape Town, Cape Town, South Africa

Background: Palliative care (PC) is an appropriate and compassionate response to the needs of patients with life-threatening illness. International human rights law establishes $\mathrm{PC}$ as part of the right to health. What does this mean in practice? How is PC part of the right to health at the country level of everyday patient experience.

General Comment 14 was used to frame four nested studies investigating the four overlapping elements of the Right to Health: availability, accessibility, acceptability and quality. Study One established the need for PC in South Africa using mortality data for 2010. Study Two examined facilities available to meet the need for PC through survey of 131 PC organisations. Only $\mathbf{3} \%$ of services were located within public sector. The estimated number of patients who received PC during the year was $17.94 \%$ of the need. Study Three investigated access to PC in Cape Town via two pathways

1) referral of patients to $P C$ services and

2) provision of PC in their current care settings.

Few patients were referred to PC services and the majority of patient outcomes remained poor. Study Four evaluated the quality of care delivered to patients requiring palliative care in accredited hospice and nonhospice non-governmental services in five of the nine provinces of South Africa. The results showed significant improvement in patient reported outcome measures.

Conclusion: Palliative care is not yet part of the Right to Health in South Africa. Availability of PC services is insufficient to meet the need for PC. Access is poor even where palliative is relatively well supplied. However, when access is achieved, quality of care is generally good and patient outcomes improve over time. Palliative care values patients as individuals. In South Africa Ubuntu describes the essence of being human, the value and worth of each individual entitling them to respect and dignity - dignity being common foundation to both human rights and palliative care.

Abstract number: P01-207

Abstract type: Poster

Impact of Medical Assistance in Dying on Palliative Care Practice: A Qualitative Study of Physicians and Nurses' Experience Two Years Following Canadian Legislation Hausner D. ${ }^{1}$, Jacob J. ${ }^{2}$, Al-Awamer A. ${ }^{2,3}$

${ }_{1}$ Palliative Care Service, Sheba Medical Center, Oncology Institute, Ramat Gan, Israel, ${ }^{2}$ Department of Supportive Care, Princess Margaret Cancer Centre, University Health Network, Toronto, Canada, ${ }^{3}$ Department of Family and Community Medicine, University of Toronto, Toronto, Canada

Background and aim: Medical Assistance in Dying (MAID) describes an intervention provided by medical practitioners to cause death. In June 2016, MAID became legal in Canada for patients who meet pre-defined criteria. The introduction of MAID has sparked an intense debate about its impact on palliative care (PC). The study aims to qualitatively explore the impact of the introduction of MAID on PC professionals and practice.

Methods: We interviewed PC physicians and nurses who practiced for six months or more in MAID accessible PC settings, before and after MAID legalization. Purposeful sampling was used to recruit participants with diverse personal views and experiences with MAID. Semi-structured interviews were conducted, transcribed and then analyzed using a modified grounded-theory.

Results: Conceptual saturation was achieved after interviewing 21 PC professionals - 11 physicians and 10 nurses. Participants have been 
practicing PC in different inpatient, outpatient and community settings. Different themes emerged from PC professionals' experience with patients requesting MAID. Although participants felt they individually maintained the same practice of PC, changes in management, policies, communication patterns and patients' dispositions were identified. Participants felt that MAID has created opportunities, including increasing patient's choices, improving access to PC, encouraging open dialogue about death and building strong trust in PC providers. Participants also identified challenges as lack of laws clarity, greater demands from patients and families, blocking psychosocial interventions or symptom management, lack of communication skills and added physical and emotional burden. All participants cited a need for additional support resources.

Conclusions: The practice of PC has been affected as a result of the legalization of MAID in Canada. Additional resources are needed to better understand and support PC professionals' distress around MAID.

\section{Abstract number: P01-209}

Abstract type: Poster

Factors Associated with Improvement in Patient-reported Outcomes after Receiving Early Palliative Care

Rodin R. ${ }^{1}$, Swami N. ${ }^{2}$, Pope A. ${ }^{2}$, Hui D. ${ }^{3}$, Hannon B. ${ }^{2}$, Zimmermann $C .{ }^{4}$ ${ }^{1}$ Department of Medicine, University of Toronto, Toronto, Canada, ${ }^{2}$ University Health Network, Toronto, Canada, ${ }^{3} \mathrm{MD}$ Anderson Cancer Center, Houston, United States, ${ }^{4}$ University of Toronto/University Health Network, Toronto, Canada

Background: Integration of early palliative care (EPC) in oncology improves quality of life (QoL) and symptom burden. However, directing EPC to patients most likely to benefit may be more cost-effective and time-efficient than universal implementation.

Aims: To determine predictors of outcome improvement at 4 months among patients with advanced cancer receiving EPC in a cluster randomized controlled trial (RCT).

Methods: We performed a secondary analysis of data from the treatment arm of an RCT conducted between December 1, 2006 and February 28, 2011. Data on demographic factors, treatment status, and ECOG score were collected at baseline. Participants completed measures at baseline and 4 months to assess QoL (Functional Assessment of Chronic Illness Therapy-Spiritual Well-Being [FACIT-Sp] and Quality of Life at the End of Life [QUAL-E]), symptom severity (Edmonton Symptom Assessment System Distress Score [ESAS-EDS], and satisfaction with care (FAMCARE-P16). Multivariable regression analyses were conducted to determine baseline factors associated with improvement at 4 months. Results: A total of 131 patients in the EPC arm completed 4 month measures. Improvement in FACIT-Sp was associated with worse baseline ESASEDS (beta $=0.29, p=0.001$ ). Improvement in ESAS was also associated with worse baseline ESAS-EDS (beta $=0.59, p<0.0001$ ). FAMCARE scores improved most in those living alone (beta $=3.90, p=0.03$ ) and not receiving active chemotherapy (beta $=4.30, p=0.03$ ). There was no factor significantly associated with improvement in QUAL-E. These outcomes were not affected by age, sex, primary tumour site, or education or employment status.

Conclusion: Patients with greater symptom burden were most likely to demonstrate improvement in QoL and symptoms following EPC, while those with greater social support needs had the greatest improvement in satisfaction with care. Prioritizing such patients may be a cost-effective and time-efficient approach to early palliative care.

Funding: CCS 700862

Abstract number: P01-210

Abstract type: Poster

Palliative Home Care Patients: A Special Group? An Empirical Analysis of Palliative Home Care and General Healthcare Service Use in the Last Year of Life
Meyer I., Schubert I., APVEL Consortium

PMV Forschunsgruppe, University of Cologne, Köln, Germany

Background: The German healthcare system comprises different types of palliative care, including three levels of palliative homecare services, of which specialised palliative homecare (German acronym SAPV) introduced in 2008 - is reserved for the most severe cases.

Aims: As part of an ongoing project evaluating palliative homecare provision (in particular SAPV), this paper addresses differences in service use in the last year of life to better understand what distinguishes palliative from non-palliative patients and what differences might exist even within the former group.

Methods: Descriptive analysis of administrative data of all insurants of the AOK Rheinland / Hamburg, aged 18+ and living in the Northrhine region who died between 2014 and 2016 ( $N=90.797$ ).

Results: $30.6 \%$ of the deceased received any type of palliative care in their last year of life, with $6.1 \%$ receiving SAPV. Their share increased from $5.1 \%$ in 2014 to $7.1 \%$ in 2016. SAPV commences 68 days before death on average (median $=22$ days (d), $75 \%$ quantile (q) $=66 \mathrm{~d}, 95 \% \mathrm{q}$ $=315 \mathrm{~d}$ ). Patients on average receive SAPV for 32.5 days (median $=14$ $d, 75 \% q=37 d, 95 \% q=121 d$ ). When looking at service provision from all sectors of the healthcare system in the last year of life, SAPV patients show an average 9.7 transitions from one care provider to another $(75 \%$ $q=12,95 \% q=19)$ compared to $4.5(75 \% q=6,95 \% q=10)$ for nonpalliative patients.

Conclusion: The findings provide an indication that palliative patients differ from non-palliative patients in terms of the complexity of care they receive in the last year of life. The variability in relation to start and duration of SAPV as well as the number of transitions furthermore suggests that there may also be differences within that patient group. This would raise the issue in how far existing services are geared to addressing this complexity and its impacts on patients, in particular in relation to burden of treatment.

\section{Abstract number: P01-211}

Abstract type: Poster

Last Year of Life in Cologne: Hospitals Are the Most Important but Least Valued Checkpoints

Hanke G. ${ }^{1}$, Schippel N. ${ }^{1,2}$, Rietz C. ${ }^{3}$, Strupp J. ${ }^{1}$, Voltz R. ${ }^{1,4,5}$, Cologne

Research and Development Network (CoRe-Net)

${ }^{1}$ Department of Palliative Medicine, University Hospital of Cologne, Cologne, Germany, ${ }^{2}$ Institute of Medical Sociology Health Services Research and Rehabilitation Sciences, University of Cologne, Cologne, Germany, ${ }^{3}$ Mixed Methods Research, University of Education Heidelberg, Heidelberg, Germany, ${ }^{4}$ Center for Integrated Oncology Köln Bonn, University Hospital of Cologne, Cologne, Germany, ${ }^{5}$ Center for Health Services Research, University Hospital of Cologne, Cologne, Germany

Background: Data on transitions during the last year of life beyond diagnoses and care settings are still rare for Germany. The Cologne Research and Development Network (CoRe-Net) is establishing a model region for health services research towards value-based care for vulnerable patients. Aims: To describe transitions into and within the last year of life, the dying phase and how they are valued.

Methods: As part of a mixed methods study we conducted a cross-sectional survey of bereaved relatives in Cologne recruited via institutional records (hospital, care home, hospice, funeral home) and self-selection. Relatives received a modified German version of the VOICES-SF (Views of Informal Carers-Evaluation of Services Short Form) questionnaire. Descriptive statistics were used.

Results: The sample $(n=359)$ was representative for the decedents of Cologne in 2017 regarding sex (48\% male) and age ( $x 76,2$ years (SD $13,1)) .60 \%$ of the relatives reported that the decedent was told he/she was likely to die. Hospital physicians were breaking the news most often 
(58\%) while being rated worst in comparison (30\% , not sensitive'). Each of the five most frequent transitions ( $>85 \%$ of all transitions) included hospitals (to/from home, nursing home, other hospitals). Quality of care rated as 'good' was lowest where care was provided by general hospital units $(74 \%)$ and highest by palliative care units $(94 \%)$ followed by hospices (88\%). Informants were more likely to rate overall care as 'good', if communication was perceived as sensitive and caring $(r=.34, p<.01)$. The place of death was most frequently in hospitals (42\%), followed by home (28\%).

Conclusion: Despite the full availability of palliative and hospice care in Cologne, general hospital units are the most important but least valued checkpoints in the last year of life. Clinical staff should have more support to identify when a palliative care approach is needed.

Funded by the Federal Ministry of Education and Research (BMBF\#01GY1606)

\section{Abstract number: P01-212 \\ Abstract type: Poster}

\section{Dying Well Night and Day: A Mixed-methods Evaluation of} Out-of-Hours Services in Scotland for People with Palliative Care Needs

Mason B. ${ }^{1}$, Murray S. ${ }^{1}$, Kerssens J. ${ }^{2}$, Stoddart A. ${ }^{3}$, Laidlaw S. ${ }^{4}$, Kendall M. ${ }^{1}$, Finucane A. ${ }^{1,5}$, Carduff E. ${ }^{4}$, Moussa L. ${ }^{6}$, Macpherson S. ${ }^{7}$, Ritchie L. $^{8}$, Keen J. ${ }^{9}$, Tucker S. ${ }^{10}$, Haraldsdottir E. ${ }^{11}$, Boyd K. ${ }^{1,10}$

${ }^{1}$ Centre for Population Health Sciences, The University of Edinburgh, Edinburgh, United Kingdom, ${ }^{2}$ Farr Institute Scotland, NHS National Services Scotland, Edinburgh, United Kingdom, ${ }^{3} \mathrm{Clinical}$ Trials Unit, University of Edinburgh, Edinburgh, United Kingdom, ${ }^{4}$ Marie Curie hospice Glasgow, Glasgow, United Kingdom, ${ }^{5}$ Marie Curie Hospice Edinburgh, Edinburgh, United Kingdom, ${ }^{6} \mathrm{PPI}$ representative, Edinburgh, United Kingdom, ${ }^{7} \mathrm{PPI}$ representative, Dumfries, United Kingdom, ${ }^{8}$ University of Abderdeen, Aberdeen, United Kingdom, ${ }^{9} \mathrm{Highland}$ Hospice, Inverness, United Kingdom, ${ }^{10} \mathrm{NHS}$ Lothian, Edinburgh, United Kingdom, ${ }^{11}$ St Columba's Hospice, Edinburgh, United Kingdom

Background: High-quality palliative and end-of-life care (PEoLC) at all times is a top priority for improving outcomes for patients and carers in the UK. Scotland's national datasets provide insights into use of services by people with PEoLC needs.

Aim: Analyse use of out-of-hours $(\mathrm{OOH})$ services in their last year of life by people who died in Scotland in 2016. Explore the decision-making by people with $\mathrm{PEOLC}$ needs when accessing $\mathrm{OOH}$ services.

Methods: We extracted the medical records of everyone who died in Scotland in 2016 from the national register of deaths. This cohort was linked to five urgent care datasets and analysed for every episode of $\mathrm{OOH}$ care recorded during their last 12 months. Cause of death was mapped to a major illness trajectory: cancer, organ failure, frailty. Three qualitative case studies in diverse regions of Scotland with patients, carers, and healthcare professionals explored decision-making.

Results: The records of 56,407 people who died in Scotland were mapped to an illness trajectory and linked to $\mathrm{OOH}$ service use. 53,852 used an $\mathrm{OOH}$ service in their last year of life; 2,796 used an $\mathrm{OOH}$ service more than 10 times during that period. $33.7 \%$ of all services used were during a patient's last month of life. The first point of contact led to different pathways and outcomes. Qualitative data indicated that issues around perceived legitimacy, prior experience and enhanced access to services influenced people's decisions.

Discussion: $\mathrm{OOH}$ services in Scotland may not meet the needs of people in their last year of life. Such users find $\mathrm{OOH}$ services difficult to navigate but need to access them more often as death approaches. Barriers include lack of knowledge, complicated and repetitive phone-based triage systems, and lack of prior identification of the patient for PEOLC. Our findings can inform development of more flexible and targeted care for these people, especially those who are not formally identified as needing palliative care.
Abstract number: P01-213

Abstract type: Poster

Association of Specialist Palliative Care with Health Care Resource Use at End of Life in Cancer Patients

Sinnarajah A. ${ }^{1}$, Earp M. ${ }^{2}$, Murray A. ${ }^{1}$, Wu J. ${ }^{1}$

${ }^{1}$ Department of Oncology, University of Calgary, Calgary, Canada, 2O'Brien Institute for Public Health, University of Calgary, Calgary, Canada

Background: Aggressiveness of care near the end of life (EOL) has increased steadily in Canada. This is concerning as aggressive EOL care, on a population level, is associated with poor quality EOL care. Specialist palliative care (PC) has been shown to help relieve EOL symptoms, improve patient quality of life, and reduce aggressive EOL care.

Aim: Describe the relationship between advanced cancer patient's use of $P C$ and the aggressiveness of EOL care ( $\leqslant 30$ days).

Methods: A population-level retrospective cohort study using administrative data. Adult cancer patients who died in Calgary, Alberta from 2003-2010 were determined from a cancer registry. Data on PC, acute and cancer care were extracted. The primary outcome was PC use (any/ none), evaluated at $>3$ months (early) and 0-3 months (late) before death. Aggressive care (yes/no) was indicated by $\geqslant 1$ of: chemotherapy, $>1$ emergency department visit, $>1$ hospital admission, $>14$ days of hospitalization, an intensive care unit admission $\leqslant 30$ days before death, and hospital death. Logistic regression was performed.

Results: 12064 patients met inclusion criteria; after data clean-up 9029 patients remained for analysis. Most used PC before death (90\%); 45\% had $\geqslant 1$ indicator of aggressive EOL care. Lack of PC increased the odds ratio $(O R)$ of EOL aggressive care for all patients ( $O R=1.4-4.5)$. Relative to patients with early PC, patients with late PC had double the odds of aggressive EOL care; and, for patients with no $\mathrm{PC}$, there was a nearly a 4-fold increase in the odds. A significant interaction between PC use and age at death $(<75 / \geqslant 75)$ was noted, such that a lack of PC in younger patients was associated with greater odds of aggressive care $(\mathrm{OR}=3.3$ ) than older patients $(\mathrm{OR}=1.7)$.

Conclusions: Our main finding is the consistent association between PC and EOL aggressive care. Efforts to ensure earlier PC for all patients, but particularly for younger patients, could improve quality of care for advanced cancer patients at EOL.

Abstract number: P01-214

Abstract type: Poster

The Prevalence of End-of-Life Conditionsin Two Canadian Emergency Department

Brisebois A. ${ }^{1}$, Garrido Clua M. ${ }^{2}$, Rowe B. ${ }^{3}$, End of Life Study Group

Edmonton Alberta

${ }^{1}$ Internal Medicine and Palliative Care, University of Alberta, Edmonton, Canada, ${ }^{2}$ Palliative Care, University of Alberta, Edmonton, Canada, ${ }^{3}$ Emergency Medicine, University of AbderdeenAlberta, Edmonton, Canada

Background: Many patients with end-stage diseases are in need of optimal physical, spiritual, psychological and social care. Despite efforts to provide community care, those with severe symptoms often present to emergency departments (EDs) with these needs unmet.

Aim: The objective of this study was to identify palliative care (PC) patients and document their unmet end-of-life care (EOLC) needs in EDs. This abstract presents preliminary results regarding the demographics and illnesses of PC patients presenting to EDs.

Methods: This 4 month prospective cohort study was conducted in 2 EDs in Edmonton, Alberta. Each of the volunteer physicians identified PC patients and recorded data on their type of illness, unmet PC needs and their PC referral recommendations based on a modified screening tool to identify patients in need for PC. Adult patients presenting to the 2 ED sites 
with life-limiting, chronic conditions including cancer, chronic obstructive pulmonarydisease (COPD), chronic kidney disease (CKD), heart failure (HF), cirrhosis, dementia and/orprogressive central nervous system (CNS) disease, were eligible. Research assistants collected the screening toolsdailyand entered the data. Descriptive analyses were conducted.

Results: A total of 663 PC patients (over 26328 total ED visits) were enrolled from 45 participating physicians. The prevalence of PC patients varied between $1-5 \%$. There was a higher proportion of females $(n=338$, $51 \%)$, with a median age of $76(I Q R=63,85)$. Cancer was the most prevalent $(\mathrm{N}=271,41.3 \%)$, followed by dementia ( $\mathrm{N}=150,22.9 \%)$ and COPD $(\mathrm{N}=107,16.3 \%) ; \mathrm{HF}, \mathrm{CKD}$, progressive CNS disease, and cirrhosis, being the least common (all $<10 \%$ ).

Conclusion / Discussion: Palliative care varied between EDs and cancer remains the most prevalent condition of the PC patients. This study may facilitate the identification of potential deficits in care and serve as a baseline for future studies.

\section{Abstract number: P01-215}

Abstract type: Poster

Congruence between Preferred and Actual Place of Death and its Relationship with Quality of Dying among Terminal-III Cancer Patients: A Multicenter Prospective Cohort Study Chen P.-J. ${ }^{1,2}$, Cheng S.-Y. ${ }^{3}$, Wu C.-Y. ${ }^{4}$, Lin W.-Y. ${ }^{5}$, Ho T.-L. ${ }^{6}$

${ }^{1}$ Department of Family Medicine and Division of Geriatrics and Gerontology, Kaohsiung Medical University Hospital, Kaohsiung, Taiwan, Republic of China, ${ }^{2}$ Marie Curie Palliative Care Research Department, Division of Psychiatry, University College London, London, United Kingdom, ${ }^{3}$ Department of Family Medicine, College of Medicine and Hospital, National Taiwan University Hospital, Taipei, Taiwan, Republic of China, ${ }^{4}$ Department of Family Medicine, Kaohsiung Medical University Hospital, Kaohsiung, Taiwan, Republic of China, ${ }^{5}$ Department of Family Medicine, College of Medicine and Hospital, China Medical University, Taichung, Taiwan, Republic of China, ${ }^{6}$ Department of Family Medicine, National Taiwan University Hospital, Taipei, Taiwan, Republic of China

Background: Place of death (POD) and its relationship with quality of dying (QOD) is a culture-specific issue. The study in East Asia and is insufficient.

Aim: To investigate the relationship between QOD and congruence of preferred and actual POD in Taiwan.

Methods: A prospective cohort study was conducted in three tertiary hospitals. We enrolled the terminal-ill cancer patients who were admitted to palliative care units (PCU) and assessed their comorbidities, preferred POD, symptoms, functional status, etc. Actual POD and QOD were checked during the follow-up. Multivariate analyses was applied to identify the factors influencing the congruence of preferred and actual POD. The score of QOD in different groups were compared.

Results: 341 cancer patients were identified, $62 \%$ of cases chose PCU as the preferred POD, while $19.9 \%$ preferred their own home and $15.5 \%$ was undecided/unknown. The top reasons for patient's preference on POD included availability of EOL care resources $(54 \%)$, influence of family members' preferences (45.7\%), worry about being burden to others (29.3\%). After a 6-months follow-up, 330 patients died, and the actual POD were mainly in PCU (83\%) while only $10.9 \%$ at home. 255 cases (PCU: 228, home: 27) achieved the congruence between preferred and actual POD. In the multivariate analyses, expressed preference for POD was associated with congruence between preferred and actual POD, while the influence from traditional or cultural custom when death happened was associated with the incongruence. Good Death Scale score for those died in PCU is not different from those died at home $(13.35 \pm 1.95$ and $12.82 \pm 2.60$, respectively; $p$ value $=0.2941)$. Good Death Scale score for the patients who had the congruence of POD and those who had the incongruence are similar $(13.38 \pm 2.03$ and $13.24 \pm 2.00$, respectively; $p$ value $=0.6301$ )
Conclusion: The QOD of terminal-ill cancer patients did not differ from the POD and the congruence between preferred and actual POD.

Abstract number: P01-216

Abstract type: Poster

Exploring the Experience of Personal Bereavement for Nurses Working in a Palliative Care Setting and the Experience of Returning to Work

Reed L. ${ }^{1}$, Waghorn M. ${ }^{2}$, Sills E. ${ }^{3}$, Vriens ${ }^{4}{ }^{4}$, Gregory A. ${ }^{2}$, Todd J. ${ }^{5}$

${ }^{1}$ Princess Alice Hospice, Education and Research, Esher, United Kingdom, ${ }^{2}$ St Catherine's Hospice, Crawley, United Kingdom, ${ }^{3}$ Woking Hospice, Woking, United Kingdom, ${ }^{4}$ Phyliss Tuckwell Hospice, Farnham, United Kingdom, ${ }^{5}$ Princess Alice Hospice, Esher, United Kingdom

Background: The impact on palliative care nurses of working in an environment that reminds them of their personal experience of bereavement may have a detrimental effect on their psychological wellbeing ${ }^{1}$. The emotional labour of nursing can be stressful, but when the internal reality of grief is at odds with the external reality of the professional, nurses are at risk of burnout ${ }^{2}$. Little is understood about the impact a personal bereavement has on palliative care nurses. Four hospices joined together to explore the experience of their bereaved nurses.

Aim: To explore the experience of a personal bereavement for nurses working in a palliative care setting through the death of a significant relative or friend.

Outcome: Make recommendations for ways hospices support nurses working in palliative care before and after the death of a significant relative or friend.

Methods: Using a grounded theory approach, 13 nurses were interviewed using a semi-structured approach.

Results: Initial findings suggest that if support and time to grieve is not given at the time of the death, nurses may experience a period of disintegration at a later date. Those experiencing a sudden bereavement may have different needs to those experiencing an anticipated bereavement. Grief and bereavement is a continuum experienced over a prolonged time. Anniversaries, times of stress or resonance with patient diagnosis or family reaction can trigger an emotional response challenging nurses ability to integrate bereavement into their lives and work. Separating the personal from the professional can take time.

Conclusion: Nurses grieve in different ways and managers and employers need to allow flexibility and time for each person to grieve recognising that bereavement continues a long way past the statutory compassionate leave allowance. Recommendations will be shared with Hospice UK to consider a national initiative.

Abstract number: P01-217

Abstract type: Poster

Characterising 'Standard Care' in Randomised Controlled Trials to Understand Added Value of Complex Interventions in Palliative Care

Yorganci E. ${ }^{1}$, Evans C. ${ }^{1,2}$, Yi D. ${ }^{1}$, Gao W. ${ }^{1}$, Barclay S. ${ }^{3}$, Pickles A. ${ }^{4}$, Murtagh F.E.M. ${ }^{1,5}$, Johnson H. ${ }^{1}$, Wilson R. ${ }^{1}$, Higginson I. ${ }^{1}$, Koffman J. ${ }^{1}$ ${ }^{1}$ Palliative Care, Policy and Rehabilitation, Cicely Saunders Institute, London, United Kingdom, ${ }^{2}$ Sussex Community NHS Foundation Trust, Brighton General Hospital, Brighton, United Kingdom, ${ }^{3}$ Primary Care Unit, Department of Public Health and Primary Care, University of Cambridge, Cambrige, United Kingdom, ${ }^{4}$ Clinical Trials Unit, King's College London, London, United Kingdom, ${ }^{5}$ Wolfson Palliative Care Research Centre, Hull York Medical School, University of Hull, Hull, United Kingdom

Background: Standard care provided in control arms is rarely described in intervention trials. Describing standard care is essential to determine intervention benefits, and assess changes in standard care during trials. 
Aim: To examine the standard care provided in a feasibility cluster RCT of a complex intervention to improve care of patients with uncertain recovery.

Methods: Multi-methods in 4 wards in 4 district general hospitals. In each ward:

(i) case note review (CNR) of 20 purposively sampled patients;

(ii) open-ended standard care survey (SCS) at baseline completed by ward staff on communication/planning of uncertain recovery;

(iii) focus group with ward staff. Descriptive statistics and thematic analysis.

Results: CNRs of 80 patients, 4 focus groups ( $N=46$ ), and SCS completed by 23 staff members. From the SCS, structures, processes and outcomes across trial arms were clear and similar, including escalation plans and resuscitation status, supported by $>60 \%$ and $>75 \%$ completion in notes, respectively. The SCS showed medical teams in both trial arms did not recognise deterioration, clinical uncertainty and futile active treatments as reasons for palliative care referral. At baseline, non-cancer patients' palliative care needs were not identified as part of standard care. In a control ward focus group at the end of the trial, referring more non-cancer patients to palliative care was stated. Answers in SCS about involvement of junior, and nursing staff in clinical decision-making and planning were inconsistent. CNRs showed that the medical plan was not documented as discussed with nursing staff for $40 \%$ of patients in control arm.

Conclusion: Common and inconsistent aspects of standard care were identified. Clear processes led to outcome completion. Ward staff's roles in decision-making and identification of clinical uncertainty are not clearly defined or structured in standard care. Examining standard care in RCTs can inform improvements required in service delivery.

Abstract number: P01-218

Abstract type: Poster

\section{Out-of-Hours Specialist and Generalist Palliative Care Service} Provision: A Review of Models and Evidence

McCauley R. ${ }^{1}$, Johnston B. ${ }^{1}$, May P. ${ }^{1}$, McQuillan R. ${ }^{2}$, Power J. ${ }^{3}$, Rabbitte M. ${ }^{4}$, Honohan C. ${ }^{1}$, Mockler D. ${ }^{1}$, Thomas S. ${ }^{1}$

${ }^{1}$ Trinity College Dublin, Dublin, Ireland, ${ }^{2}$ Beaumont Hospital, Dublin, Ireland, ${ }^{3}$ St Francis Hospice, Dublin, Ireland, ${ }^{4}$ All-Ireland Institute for Hospice and Palliative Care, Dublin, Ireland

Background: A majority of people with life-limiting illness would prefer to receive treatment and to die at home, provided there is sufficient supportive care to meet their needs. Access to palliative services outside of office hours is critical to continuity of care, but out-of-hours care is an underdeveloped aspect of services in most countries.

Aims: To establish best-available evidence on out-of-hours palliative care:

(1) which models of care have been employed,

(2) what are the critical components of these, including barriers and facilitators,

(3) what is the published evidence on effectiveness (patient, caregiver outcomes) and cost-effectiveness of these interventions?

Method: To address (1\&2) we identified 16 high-income countries with well-integrated palliative care services and performed a detailed documentary review of policy documents to evaluate models of care. These analyses were supplemented by direct engagement with clinical and policy leaders in each country. To address (3) we performed a systematic search of 10 databases using two independent reviewers at each stage. Returned studies were complemented by grey literature review and snowball sampling. Results of the study highlighted a wide variance in accessibility, equity and quality of out-of-hours palliative care services across the countries.

Results: Key components in service development included communication between in- and out-of-hours specialist and generalist staff, suitable training, addressing cancer exceptionalism, and the need for central reporting systems for efficient services and sufficient anticipatory action. The systematic database search identified two relevant evaluations with limited generalisable evidence.

Conclusion: Out-of-hours palliative care is critical to appropriate and cost-effective treatment of serious illness but services are underdeveloped internationally and there is a weak evidence base to inform their expansion. Further research to address these gaps is needed.

Abstract number: P01-219

Abstract type: Poster

Review of Medication Directives in Discharges from Acute

Hospital to Community Palliative Care Setting

Brennock J., O'Gorman A., Hennessy M., Tipping G.

Department of Palliative Medicine, Our Lady of Lourdes Hospital, Co Louth, Ireland

Background: Medication directives are used by Community Palliative Care Teams (CPCT) to allow planned, safe administration of medications by teams in the community setting for patients with chronic, life-limiting conditions for symptom control (SC) or end of life care (EOLC). This often allows patients to remain in the community and receive care that they may otherwise have had to receive in an in-patient setting.

Aims: The aims of this project were to assess:

- Number of patients who were discharged with a medications directive from an acute hospital setting to CPCT

- Duration of care under CPCT

- Circumstances of any re-admission to acute hospital following discharge with medication directive.

Method: Review of discharged in-patients to CPCT from Jan - Dec 2017 using hospital palliative care team (HPCT) and CPCT databases and medication directive records, hospital electronic database and patient charts. Review was conducted by one person over a two week period and results were tabulated and analysed using simple Excel format.

Results: 47 discharges to CPCT with medical directives were identified (38 EOLC, 9 SC). 2 were re-admitted following discharge with EOLC directive, and all 9 SC patients were readmitted (re-admission date range was 1 - 16 weeks post-discharge). 6 patients discharged with EOLC directive remained under CPCT care at time of review, 3 were discharged from service, with 32 dying under care of СРCT (median days after discharge 8.5). Conclusion: Medication directives helped to facilitate $84 \%$ of patients discharged from acute hospital to CPCT care to remain at home for EOLC. $100 \%$ of SC patients required re-admission but had a varying period in the community prior to re-admission. This clearly demonstrates the importance of medication directives in both HPCT and CPCT for effective palliative care.

Abstract number: P01-220

Abstract type: Poster

Will We Be Prepared for the Palliative Care Needs of the Near Future?

dos Santos C.E. ${ }^{1}$, Cruz R.P. ${ }^{1}$, Machado E. ${ }^{1}$, Hauser F. ${ }^{1}$, Campos L. ${ }^{1}$, Saavedra L. ${ }^{1}$, Klug D. ${ }^{1}$, Zuardi M. ${ }^{1}$, Serafim J.A. ${ }^{1}$, Peixoto Caldas J.M. ${ }^{2}$, Barros N. ${ }^{1}$, Pelin De Nardi S. ${ }^{3}$

${ }_{1}^{1}$ Palliative Care, Hospital Nossa Senhora da Conceição, Porto Alegre, Brazil, ${ }^{2}$ Public Health, Universidade do Porto, Porto, Portugal, ${ }^{3}$ Pain an Palliative Care, Hospital Conceição Group, Porto Alegre, Brazil 
Background: Considering the aging of the population and the increase in life expectancy, establishing a national strategy for care needs becomes imperative.

Aims: To estimate palliative care health care workers and service settings needs for the brazilian population growing projection up to 2040 .

Methods: Population and mortality estimates and projections were obtained from the Instituto Brasileiro de Geografia e Estatística. Service settings were estimated from literature data.

Results: The expected increase in the Brazilian population between 2000 and 2040 is $31.5 \%$. The minimum estimate of patients with palliative care needs was 662,065 in 2000 and 1,166,279 in 2040. Staff required for each hundred thousand inhabitants would increased from 1,734 to 2,282 and the number of nurses needed would increase from 8,586 to 11,294 in this time frame.

Conclusions: The expansion of the network for chronic and non-communicable diseases is necessary, but the qualification of existing human resources at all levels of attention to perform palliative actions can be a feasible alternative to minimize the suffering of the population.

Abstract number: P01-221

Abstract type: Poster

Using Qualitative Methods to Identify the 'Active Ingredients' of a Complex Intervention for Managing Clinical Uncertainty in Acute Hospital Settings

Johnson H. ${ }^{1}$, Evans C. ${ }^{1,2}$, Yi D. ${ }^{1}$, Gao W. ${ }^{1}$, Barclay S. ${ }^{3}$, Pickles A. ${ }^{4}$, Murtagh

$F^{5}{ }^{5}$, Yorganci E. ${ }^{1}$, Wilson R. ${ }^{1}$, Higginson I. ${ }^{1}$, Koffman J. ${ }^{1}$

${ }^{1}$ Cicely Saunders Institute, King's College London, London, United Kingdom, ${ }^{2}$ Sussex Community NHS Foundation Trust, Brighton General Hospital, Brighton, United Kingdom, ${ }^{3}$ Department of Public Health and Primary Care, University of Cambridge, Cambridge, United Kingdom, ${ }^{4}$ Clinical Trials Unit, King's College London, London, United Kingdom, ${ }^{5}$ Wolfson Palliative Care Research Centre, Hull York Medical School, University of Hull, Hull, United Kingdom

Background: Using qualitative methods to identify 'active ingredients' within complex interventions can provide insight into the essential intervention components required to achieve intended outcomes within local settings. The AMBER Care Bundle (AMBER) is a complex intervention aimed at improving the quality of care for patients who are deteriorating, near end of life, and whose situations are clinically uncertain.

Aim: To identify the active ingredients of AMBER required to benefit patients and inform intervention implementation.

Methods: Process evaluation nested within a feasibility cluster RCT. Focus groups with healthcare professionals, semi-structured interviews with patients and relatives, non-participant observations of multidisciplinary team meetings (MDTs) at 2 intervention wards in 2 hospitals in England. Thematic analysis using Framework approach.

Results: 2 focus groups ( 26 health care professionals), 9 non-participant observations, and 12 interviews ( 2 patients, 10 relatives). Active ingredients included:

(i) recognising patients whose situations were clinically uncertain;

(ii) engagement between patients and families and health care professionals in decisions about care and treatment as part of daily practice;

(iii) clear documentation of current and future patient-centred treatment and care plans

(iv) implementation facilitated by a 'champion' to educate and support staff in (i) (ii) and (iii).

Conclusions: Process evaluation is vital in unravelling the active ingredients of AMBER and the requirements for the interventions use in practice. These will inform implementation of the intervention in a full trial, and the methods for wider implementation across groups and settings.
Funding: The ImproveCare - The management of clinical uncertainty in hospital settings is funded by the National Institute for Health Research Health and Technology Assessment (NIHR HTA) programme. Project number: 15/10/17

Abstract number: P01-222

Abstract type: Poster

Feasibility of a Multi-method, Multi-centre, Cluster RCT of a Complex Intervention to Care for Hospital Patients with Clinically Uncertain Recovery: The Improvecare Study Koffman J. ${ }^{1}$, Yi D. ${ }^{1}$, Gao W. ${ }^{1}$, Barclay S. ${ }^{2}$, Pickles A. ${ }^{3}$, Murtagh F. ${ }^{4}$, Yorganci E. ${ }^{1}$, Johnson H. ${ }^{1}$, Wilson R. ${ }^{1}$, Higginson I. ${ }^{1}$, Evans C. ${ }^{1,5}$ ${ }^{1}$ King's College London / Cicely Saunders Institute, London, United Kingdom, 2Department of Public Health and Primary Care, University of Cambridge, Cambridge, United Kingdom, ${ }^{3}$ Clinical Trials Unit, King's College London, London, United Kingdom, ${ }^{4}$ Wolfson Palliative Care Research Centre, University of Hull, Hull, United Kingdom, ${ }^{5}$ Brighton General Hospital, Sussex Community NHS Foundation Trust, Brighton, United Kingdom

Background: The AMBER care bundle was developed to support hospital patients with uncertain recovery. Feasibility evidence is required before evaluation in a definitive trial.

Aim: To examine the feasibility of a multi-method RCT of AMBER and optimise the trial design.

Methods: Multi-method, cluster RCT, included 4 wards in 4 hospitals in England. Prospective, quantitative data collected at 3-time points from deteriorating patients, with uncertain recovery, and at risk of dying during admission (and their proxies). Qualitative components: semi-structured interviews with patients and relatives, focus groups with health professionals and non-participant observations of ward meetings. Primary outcomes comprised: IPOS psychological subscale, and the howRwe, a patient-reported experience measure. Secondary outcomes: functional status (AKPS), EQ-5D-5L, service use data, screening, and recruitment rate. Descriptive statistical analyses and qualitative framework approach with subsequent integration.

Results: $22 \%$ and $5 \%$ of screened patients recruited in intervention and control arms. Data were collected from 65 patients $(51 \%$ men, $40 \%>80 y r s, 54 \%$ non-cancer, AKPS: $=31, \mathrm{SD}=14.6) .25 \%$ died during admission and $36 \% \leqslant 100$ days post discharge. In both trial arms mean baseline IPOS subscale scores identified 'moderate' patient anxiety $(=13$, $95 \% \mathrm{Cl} 12-14)$, and the howRwe identified 'good' care experience (=13, $95 \% \mathrm{Cl} 12-14)$ with minor fluctuations at follow-up. Follow up at 10-15 days was not feasible since $37 \%$ of patients were discharged. Service use collection was feasible. Data from qualitative components identified vital improvements relating to study design including:

(i) revise inclusion criteria;

(ii) comparable ward specialties;

(iii) use acute medical units as earlier recruitment start point;

(iv) revise consent procedures;

(v) shorten questionnaires to minimise patient burden.

Conclusions: With refinements identified using multi-methods, an RCT of AMBER is feasible.

Funding: NIHR HTA

Abstract number: P01-223

Abstract type: Poster

Development of a United Kingdom Palliative Medicine Trainee Research Collaborative: New Knowledge through Networking Schofield G. ${ }^{1}$, Chamberlain $C^{2}{ }^{2}$, Hancock S. ${ }^{3}$, Etkind S. ${ }^{4}$, Robbins S. ${ }^{5}$, Coop H. ${ }^{6}$, Watson R. ${ }^{7}$, Noble S. ${ }^{8}$, Koffman J. ${ }^{4}$, on behalf of the UK Palliative Care Trainee Research Collaborative 
${ }^{1}$ Centre for Ethics in Medicine, Medical School, University of Bristol, Bristol, United Kingdom, ${ }^{2}$ Population Health Sciences, Medical School, University of Bristol, Bristol, United Kingdom, ${ }^{3}$ Lancaster University, Lancaster, United Kingdom, ${ }^{4}$ Cicely Saunders Institute of Palliative Care, Policy \& Rehabilitation Florence Nightingale Faculty of Nursing, Midwifery \& Palliative Care King's College London, London, United Kingdom, ${ }^{5}$ St Peters Hospice, Bristol, United Kingdom, ${ }^{6}$ Royal Wolverhampton NHS Trust, Wolverhampton, United Kingdom, ${ }^{7} \mathrm{St}$ Richards Hospice, Worcester, United Kingdom, ${ }^{8}$ Cardiff University, Cardiff, United Kingdom

Background and aim: Clinical trainee collaboratives harness resource of clinicians in training for research and multicentre audit. Well established in other medical fields in the United Kingdom, collaboratives have demonstrated increased trainee research exposure for trainees in research design, ethics, data collection and analysis, and manuscript preparation. National trainee collaboratives are also involved with international research projects with widespread benefits in terms of scope and reach of research outputs, skills gained and training opportunities. Until now no trainee collaborative has existed specifically for palliative medicine trainees.

Methods: The UK Palliative Care Trainee Research Collaborative (UKPRC) was established in 2017. Modelled on other successful trainee collaboratives, we employed the following steps:

1. Writing and adopting a constitution with shared authorship as a central tenant

2. Identifying a core group of interested trainees

3. Nominating a clinical and research lead

4. Defining a brand; logo, website, email address, newsletter, Twitter profile

5. Identifying a launch project

6. Recognising crucial stakeholders to disseminate information and garner support.

Results: Launched publicly in August 2018, the UKPRC now has active members from $12 / 14$ of the UK training regions. The first national audit project is underway aiming to recruit 40 sites across all sectors in the UK. The UKPRC has a website comprising 192 followers on Twitter and has been discussed in national speciality settings.

Conclusions: Collaboratives strengthen research culture and experience amongst their members, embedding research activity and evidencebased practice from the first year of training. The UKPRC has the potential to conduct research and audit across UK hospice and hospital sectors that will inform evidence-based practice and improve patient care. International collaboration with other national trainee groups globally will only increase these benefits.

Abstract number: P01-224

Abstract type: Poster

How Do Cancer Specialists (CS) and Palliative Care Specialists (PCS) Assess the Integration of Early Palliative Care (EPC) at German Comprehensive Cancer Centers (CCC)? An integrative View of Current Structures and Persistent Challenges

Seibel K. ${ }^{1}$, Bardenheuer . $^{2}$, Mayer-Steinacker R. ${ }^{3}$, Thomas M. ${ }^{4}$, Viehrig M. ${ }^{5}$, Becker G. $^{1}$

${ }^{1}$ Department of Palliative Care, University Medical Center Freiburg, Freiburg, Germany, ${ }^{2}$ Department of Anesthesiology, University Medical Center Heidelberg, Heidelberg, Germany, ${ }^{3}$ Department of Hematology and Oncology, University Medical Center Ulm, Ulm, Germany, ${ }^{4}$ Department of Oncology, University Medical Center Heidelberg, Thoraxklinik Heidelberg, Heidelberg, Germany, ${ }^{5}$ Department of Radiation Oncology, University Medical Center Tuebingen, Tuebingen, Germany
Background: Despite the growing body of clinical research regarding the efficacy of EPC and various calls for its integration into oncological care, there is little health services research that analyses the actual EPC clinical practice by CS and its persistent challenges. Yet, this appears important because only the synergy of both research types can prospectively provide effective EPC.

Aims: To explore the views of CS and PCS regarding the integration of EPC into routine cancer care in outpatient settings and their shared identification of unmet challenges.

Methods: 49 qualitative expert interviews were led at 4 German CCC (39 CS from 6 specialties/10 PCS), transcribed verbatim and a qualitative content analysis was conducted.

Results: CS describe the integration of Palliative Care (PC) in their daily routines, also in early stages, but rarely labelled it as EPC nor referred to shared standards. The results display rather a broad range of assessment and interventions.

Further, both groups identify structural restrictions and vagueness as main challenges.

Structural restrictions include e.g., time restrictions, $\mathrm{CS}^{\prime}$ and patients' avoidance of EPC, the lack of a clinic-wide uniform EPC service, a partial gap in education and experience of CS, and the overlapping EPC tasks of CS/PCS resulting in intraprofessional competition.

Vagueness refers to lacking criteria for the integration of EPC vs. the need for individualised treatment, the nonexistence of a standardised EPC concept, the coexistence of various EPC models, and PC's responsibility to clearly impart her EPC standards.

Conclusion: In addition to the structural factors that need to be addressed by health policy on the meso- and macro-level, this study illustrates the continuing conceptual vagueness of EPC and the crucial need for clarification and standardisation, both within each specialty and mutually. This will provide common ground for a successful EPC integration regardless of the provider.

Funding: R. Bosch Stiftung

Abstract number: P01-225

Abstract type: Poster

Perception and Support for Advance Care Planning among Healthcare Professionals

Harasawa N. ${ }^{1}$, Nagae H. ${ }^{1}$, Sakai S. ${ }^{1}$, Ikeda M. ${ }^{1}$, Moriya H. ${ }^{1}$, Watanabe $K^{2}$

${ }^{1}$ School of Nursing, Tokyo Women's Medical University, Tokyo, Japan, ${ }^{2}$ School of Nursing, Jichi Medical University, Tochigi, Japan

Background: The healthcare professionals (HCPs; doctors, nurses, public health nurses, pharmacists, social workers, care managers, and welfare commissioners) have a role to support advanced care planning (ACP) for citizens.

Aim: This study aimed to identify the perception and support for ACP among HCPs.

Methods: A questionnaire included aspects of ACP, such as, whether HCPs had ever considered ACP, if so, how they wish to share and put their ACP into action. Our study defined ACP as a preferences regarding future medical care based on their life goals and personal values. The questionnaires were distributed to 1319 HCPs working in Kakegawa City in October, 2017. The data were analyzed quantitatively using Fisher test and MannWhitney $\mathrm{U}$ test. The study protocol was reviewed and approved by the Ethics Committee of the Tokyo Women's Medical University.

Results: Nine hundred and seventy one $(73.6 \%)$ completed the questionnaire. The mean (SD) age was 45.3 (14.4) years, $80.2 \%$ were women and $60.2 \%$ were nurses. Of all the participants, $60 \%$ had considered ACP, $46 \%$ had hoped to share their ACP, $34.7 \%$ actually did share, and $3.5 \%$ had the ACP in written form.

The factors related to ACP were experience of learning about EOL $(p<$ $.01)$, volunteer activity experience $(p<.05)$, and had medical and care 
referrals $(p<.05)$. Those who considered ACP and actually talked about it, correlated with the number of consultations $(p<.001)$. Eighty nine (9.2\%) HCPs supported 483 clients in a month, and most of them were nurses.

Conclusion: HCPs' own attitude toward ACP were influenced by their education on EOL care, and having medical and care referrals. It was suggested that education on EOL care was necessary for professionals who play a role in supporting practice of ACP, so that they could be a good consultant to citizens.

Abstract number: P01-226

Abstract type: Poster

\section{Non-referral to Palliative Care: An Attempt to Investigate Major Reasons}

Bora S.

North East Cancer Hospital \& Research Institute, Palliative Oncology, Guwahati, India Presenting author email address: sanghamitrabk@ gmail.com

Background: Cancer is an emerging public health problem in India. Major cancer centres confirm that $2 / 3^{\text {rd }}$ of cancer patients are incurable at presentation and need palliative care. Unequal distribution of services and few palliative care workers continue even after nationwide awareness and steps taken to implement palliative care. Even where services do exist, patients are referred late or not at all. Oncologists care for patients throughout their illness, but little is known of their palliative care referral practices.

Aim: To investigate the reasons for non-referral of cancer patients to palliative care by oncologists in the city of Kolkata, India.

Method: A qualitative approach was adopted to explore in-depth the experiences, practices and attitudes of 13 oncologists using semi-structured interviews. The interviews were audio recorded, transcribed verbatim and analysed using Interpretative phenomenological analysis.

Results: Key themes on reasons for non-referral are: Lack of palliative care service is about lack of palliative care service coupled with lack of knowledge compelling oncologists to seek alternative means of extending care to their patients; Pressure to continue active treatment describes how patients and families force oncologists to continue with futile cancer treatment; Nascent status of palliative care is about palliative care facing various teething problems; Resistance to accept palliative care as a specialty describes resistance from oncologists to accept palliative care as a new specialty.

Conclusion: The study addresses covert manifestations of non-referral to palliative care by oncologists, the findings being consistent with several other studies. It suggests remedies to develop uniform palliative care service in the region. Considering that the patient-oncologist interface is a platform for palliative care information sharing, the study emphasizes the need for education and awareness for both physicians and patients for improving referrals.

Abstract number: P01-227

Abstract type: Poster

\author{
Explaining Possible Under-utilization of Palliative Care \\ Consultation (PCC) Teams \\ van der Ark A., Engel M., Tamerus R., Brinkman-Stoppelenburg A., van \\ der Heide $A$. \\ Public Health, Erasmus MC, Rotterdam, Netherlands
}

Background: Palliative Care Consultation (PCC) teams are involved in only a small minority of patients with an advanced illness. Underutilization PCC teams is suspected by stakeholders, but explanations are often not evidence-based.

Aims: This study explores which factors might influence PCC consultation by physicians.
Methods: A survey study was performed in 2017 among physicians in the Southwest region of the Netherlands, using a random sample $(n=2212)$ complemented by open invitation. Bivariate and logistic regression analyses were performed.

Results: 547 physicians participated. $85.5 \%$ were aware of the availability of a PCC team, while $52.7 \%$ reported to consult it when necessary. In bivariate analyses the use of a PCC team was positively associated with considering palliative care advice important, ever asking colleagues for palliative care advice, use of the Surprise Question, more satisfaction with inter-organizational collaboration, higher number of patients dying per year, being a general practitioner, level of urbanity and higher age. Being a PC expert, considering inter-organizational collaboration important, having experienced inadequate information transfers and gender were not associated with use of the PCC team. In multivariate analysis, considering palliative care advice important $(O R=3.4, p<0.001)$, being a GP as opposed to a clinical specialist $(O R=5.2, p<0.001)$ and age $(\mathrm{OR}=2.0, \mathrm{p}<0.001)$ remained positively associated. Being a palliative care expert became negatively associated $(O R=0.2, p=0.002)$.

Conclusion: About half of the physicians reported to ever consult a PCC team. Unfamiliarity alone does not explain these results. GPs, older physicians, and physicians who feel that it is important to involve experts in palliative care more often consult a PCC team than others. Efforts to increase the use of PCC teams should focus on younger physicians and clinical specialists, and increase awareness of the added value of palliative care expertise.

\section{Abstract number: P01-228 \\ Abstract type: Poster}

Evaluating Palliative Day Care Services: A Full-population Survey among Patients and their Informal Caregivers Dierickx S..$^{1,2}$, Beernaert $K^{1,{ }^{1,2}}$, Verhaert . $^{3}$, Chambaere $K_{.}{ }^{1,2}$

${ }^{1}$ End-of-Life Care Research Group, Vrije Universiteit Brussel (VUB) and Ghent University, Gent, Belgium, ${ }^{2}$ Department of Public Health and Primary Care, Ghent University, Gent, Belgium, ${ }^{3}$ Coda Day Care Centre, Wuustwezel, Belgium

Background: Palliative day care centres remain a marginal service in the palliative care landscape. Presently there are five such centres in Flanders, Belgium where multidisciplinary teams provide care and support for people with life-limiting conditions in a homely surrounding. Additionally, palliative day care centres support the patients' informal caregivers by providing some time-off from informal care duties. A rigorous analysis and evaluation of the service is needed to identify the added value of palliative day care centres and opportunities for improvement.

Aim: This study aims to evaluate palliative day care services from the perspective of patients visiting the palliative day care centres and their informal caregivers.

Method: Questionnaire survey of patients visiting palliative day care centres and their most important informal caregivers. Data will be collected over a period of six months (November 2018 - April 2019) in all five palliative day care centres in Flanders, Belgium.

Results: We estimate a study population of 200 patients and 200 informal caregivers. We will be able to present preliminary results. Sociodemographic characteristics of patients and their informal caregivers will be reported, as well as their quality of life (using the EORTC QLQ-C30) and care needs within the domains of palliative care, and to what extent their needs are met by palliative day care centres. Additionally, patients' and informal caregivers' reasons, expectations and wishes related to visiting palliative day care centres and which problems they encounter in using palliative day care services will also be presented.

Conclusion: The results of this study will inform about the added value palliative day care has for those who use it, about the direction in which to rethink or optimise the palliative day care centres' operation and provision, and about further steps in policy and financing.

Funding: The Belgian Cancer Society "Kom Op Tegen Kanker" 
Abstract number: P01-229

Abstract type: Poster

Problem Issues of Palliative Pediatric Care Organization

Detsyk O., Zolotarova Z., Melnyk R.

Social Medicine and Public Health, Ivano Frankivsk National Medical

University, Ivano-Frankivsk, Ukraine

Background: The presence of a severe ill child causes the number of not only medical but related problems: social, psychological, spiritual, etc. This requires special approaches to providing care both children and their relatives.

Aims: To analyze the shortcomings in the organization of palliative pediatric care.

Methods: It was carried out a survey at health care facilities of IvanoFrankivsk region, which provided medical care for children. 372 relatives of children in need of palliative care were interviewed.

Results: It was established that respondents' children commonly suffered from cerebral palsy (46.3\%) and other diseases of the nervous system $(9.9 \%)$, as well as congenital malformations $(25.2 \%)$. It was quite often to meet: mental and behavioral disorders (7.1\%), tumors (3.6\%), and other chronic diseases (7.9\%). The absolute majority of severe ill children (81.4\%) required physical assistance.

The care for seriously ill children mostly carried out by women (92.2\%) of working age (97.3\%).

Almost all respondents (98.1\%) indicated that they have several years experience of caring for a sick child. Despite this, half of the questioned $(48.0 \%)$ recognized the lack of special knowledge and skills on care.

Respondents indicated the need for support from other family members $(88.7 \%)$, medical $(88.0 \%)$ and social $(74.4 \%)$ workers, psychologists (72.6\%), clergy $(75.0 \%)$, teachers $(72.4 \%)$, and volunteers $(60.5)$.

In addition, the respondents noted a lack of provision to children of medicines (62.1\%) and special care products: diapers (78.2\%), crutches (71.8\%), beds (47.6\%), anti-decubitus mattresses $(68,0 \%)$, and means for facilitating feeding $(69.7 \%)$, toilet (66.2\%), and training (65.5\%).

Conclusion / Discussion: National pediatric palliative health services require significant improvement because it does not satisfy the needs of parents with seriously ill children in medical care and social support, does not meet the principles multidisciplinary approach.

Abstract number: P01-230

Abstract type: Poster

Structures of Specialized Outpatient Palliative Care (SAPV) in Germany - A Nationwide Assessment

Jansky M. ${ }^{1}$, Bergmann L. ${ }^{2}$, Freytag A. ${ }^{3}$, Hach $M .{ }^{4}$, Lehmann $T^{5}$, , Meißner $W^{6}{ }^{6}$, Mühler F. ${ }^{1}$, Parhizkari S. ${ }^{1}$, Schneider W. ${ }^{7}$, Wedding U. ${ }^{6}$, Nauck F. $^{1}$, SAVOIR Study Group

${ }^{1}$ Clinic for Palliative Medicine, University Medical Center Göttingen, Göttingen, Germany, 2Deutsche Gesellschaft für Palliativmedizin, Berlin, Germany, ${ }^{3}$ Institute for General Practice and Family Medicine, Jena University Hospital, Jena, Germany, ${ }^{4}$ Bundesarbeitsgemeinschaft SAPV, Berlin, Germany, ${ }^{5}$ Center for Clinical Studies, Jena University Hospital, Jena, Germany, ${ }^{6}$ Palliative Medicine, Jena University Hospital, Jena, Germany, ${ }^{7}$ Center for Interdisciplinary Health Research, University of Augsburg, Augsburg, Germany

Background: Since 2007, patients with severe advanced life-limiting illnesses and complex symptoms have a right to receive specialized outpatient palliative care (SAPV).

Aim: To assess different organizational structures of SAPV teams in Germany.

Method: In 2017, all SAPV teams were asked to update their organizational structures in the online data base "Wegweiser Hospiz- und Palliativversorgung". It contains voluntary information of 270 SAPV teams. Data is analyzed descriptively; cluster analysis will be used to classify SAPV teams.

Results: 192 teams provided updated data. Teams were mostly nurse $(35 \%)$ or physician $(25 \%)$ led (other professions: $14 \%$; multiple professions: $25 \% ; 1 \%$ missing data (MD)). Teams had 18 nurses (median: 8; range: $2-239 ; \mathrm{MD}: 11 \%$ ) and 10 physicians (median: 8 ; range: $1-52 ; \mathrm{MD}$ : $5 \%$ ). $38 \%$ employed at least one psychosocial profession (chaplain: $23 \%$; social worker: $18 \%$; psychologist: $17 \%)$. Only few teams worked with less than $50 \%$ physicians $(3 \%)$ or nurses $(1 \%)$ formally trained in palliative care (MD 4\%; $8 \%$ resp.). Teams worked with physicians from private practices $(68 \%)$ or employed directly by team $(41 \%)$ or hospitals $(36 \%)$. Nurses were employed by team (56\%), nursing services $(32 \%)$ or hospitals (19\%). Many teams had physicians (58\%) or nurses $(28 \%)$ from more than one structure. Teams cooperated externally with volunteer hospice teams (97.9\%), palliative care units and inpatient hospices (95\%), but also pharmacies $(94 \%)$, nursing homes $(93 \%)$, general practitioners (88\%) and psychosocial carers (psychologists: 66\%; social workers: $72 \%$ ). Conclusion: SAPV teams are highly variable in team size and members' institutions, but mostly work with highly skilled professionals. Their cooperation with different internal and external institutions suggests a high coordination effort. To analyze how organizational structures influence patient care is part of the ongoing research project SAVOIR, funded by Federal Joint Committee German Innovations Fund.

\section{Abstract number: P01-231}

Abstract type: Poster

The Things That Are, and That Should Be: Two Faces of

Transitions in Palliative Care

Ateş G. ${ }^{1}$, Peuten S. ${ }^{2}$, Jaspers B. ${ }^{1,3}$, Hainsch-Müller I. ${ }^{4}$, Aulmann $C^{4}{ }^{4}$, von

Hayek J. ${ }^{2}$, Schneider W. ${ }^{2}$, Radbruch L. ${ }^{1,5}$

${ }^{1}$ Department of Palliative Medicine, University Hospital Bonn, Bonn, Germany, ${ }^{2}$ Center for Interdisciplinary Health Research (ZIG), University of Augsburg, Augsburg, Germany, ${ }^{3}$ Department of Palliative Medicine, University Hospital Goettingen, Goettingen, Germany, ${ }^{4}$ Interdisciplinary Center for Palliative Care, Klinikum Augsburg, Augsburg, Germany, ${ }^{5}$ Centre of Palliative Care, Malteser Hospital Seliger Gerhard Bonn/ Rhein-Sieg, Bonn, Germany

Background and aims: There is a substantial increase of general and specialist palliative care (PC) services in Germany. Quality measures for transitions between care settings and healthcare sectors include communication and coordination issues. This study aims to map PC pathways via analyses of patient records, views of health care professionals (HCPs), patients and caregivers by comparing two different hospital settings and their corresponding networks: $\mathrm{M} 1$ with an integrated $\mathrm{PC}$ model and $\mathrm{M} 2$ with a cooperative PC setting.

Methods: Mixed-methods design: documentary research of 1000 patient records (reference year 2016); expert interviews with $10 \mathrm{HCPs}$, and interviews with patients and caregivers (40) at M1 and M2. Items for documentary research, interview guidelines, codes for analyses and recruiting strategies were consented among $\mathrm{M} 1$ and $\mathrm{M} 2$ researchers. SPSS was used for statistical analysis and MAXQDA for qualitative analysis.

Results: M1: $48 \%$ of the transitions to the PC unit were from other departments of the hospital, $36 \%$ from home; M2: $81 \%$ and $14 \%$. At the PC unit at M1 $55 \%$ of patients died and $62 \%$ at M2. Transitions from PC units were: $26 \%$ home, $11 \%$ inpatient hospice (M1); $18 \%$ home, $10 \%$ inpatient hospice (M2). Transitions were dependent on the organisation of services, cost coverage, cooperation and networking and were experienced differently by HPCs from M1 and M2. Concerning satisfaction with transitions, communication practices and typical problems, the interviews have shown great similarities but also local differences. Differences between HCPs and patients/caregivers will be presented in detail.

Conclusions: Regional differences influenced not only care pathways, but also showed different priorities set by HCPs and patients or 
caregivers regarding transitions. Planning ahead is of great importance to achieve smooth transitions and continuity of care.

Funding: Federal Ministry of Education and Research

Abstract number: P01-232

Abstract type: Poster

Early Palliative Care in Real-world Clinical Practice: Effects on Quality of Life and Symptoms of Newly Diagnosed Incurable Advanced Cancer Outpatients of Comprehensive Cancer Centers (EVI Project) - A Prospective, Multicenter, Mixed-methods, Sequential Control Group Study

Siemens W. ${ }^{1}$, Orellana-Rios C.L. ${ }^{1}$, Schoensteiner S. ${ }^{2}$, Schaekel U. ${ }^{3}$, Kessler J. ${ }^{4}$, Eschbach C. ${ }^{5}$, Viehrig M. ${ }^{6}$, Gaertner J. ${ }^{7}$, Becker G. ${ }^{1}$

${ }^{1}$ Clinic for Palliative Care, Medical Center, University of Freiburg, Faculty of Medicine, University of Freiburg, Freiburg, Germany, ${ }^{2}$ Department of Internal Medicine III, University Hospital UIm, UIm, Germany, ${ }^{3}$ Department of Internal Medicine V, University Clinic Heidelberg, Heidelberg, Germany, ${ }^{4}$ Center of Pain Therapy and Palliative Care Medicine, Department of Anesthesiology, University Hospital Heidelberg, Heidelberg, Germany, ${ }^{5}$ Department of Thoracic Oncology, Member of the German Center for Lung Research (DZL), University Hospital Heidelberg and Translational Lung Research Center Heidelberg (TLRC-H), Heidelberg, Germany, ${ }^{6}$ Department of Radiooncology, Palliative Care Unit, University Hospital of Tübingen, Tuebingen, Germany, ${ }^{7}$ Center for Palliative Care Hildegard, Basel, Germany

Background: Many high quality studies showed effects of early palliative care (EPC) but lack external validity. Real-world clinical practice approaches are urgently needed.

Aims: To report the effect of EPC on patients' quality of life (QoL) and symptoms as secondary outcomes.

Methods: This is a prospective, multicenter, mixed-methods, cohort study with a sequential control group design (DRKS00006162, primary aim: feasibility). Adult outpatients with incurable advanced cancer diagnosed within the last eight weeks were included. The control group (CG) received standard oncology care and was compared to standard oncology care plus "as needed" EPC by a PC physician or PC nurse.

We used the Palliative Outcome Scale (POS) and European Organization for Research and Treatment of Cancer QoL Questionnaire-C30 (EORTC QLQ-C30) at baseline, week 12 and week 24

The data was analyzed with mixed models using R. Semi-structured interviews were conducted with 44 patients and 40 caregivers and analysed using content analysis.

Results: For the CG and the intervention group (IG), 1439 and 1716 patients were screened, 338 and 254 entered the study and 116 (34\%) and $88(35 \%)$ were evaluated as complete-case analysis.

For week 12, the POS score was 9.31 (95\% confidence interval (CI) 8.25 to 10.37 ) in the CG and 9.74 (8.50 to 11.00) in the IG (difference: -0.44 $(-2.45$ to 1.58$))$. The QoL scale of the QLQ-C30 was $60.8(95 \% \mathrm{Cl} 56.9$ to 64.8 ) in the CG and 55.1 (50.5 to 59.8) in the IG (difference: 5.7 (-1.8 to 13.2)). Subjective concepts of QoL were more differentiated in the IG. Conclusion: QoL and symptoms were not different between groups. Possible reasons for this finding are discussed. Qualitative data suggest a more elaborated concept of QoL in the IG.

Further real-world studies are needed to find effective approaches balancing "as needed" versus "systemic" and "uniprofessional" versus "multiprofessional" EPC.

Funding: Robert Bosch Stiftung: 11.5.1364.0055.0.

Abstract number: P01-233

Abstract type: Poster

A New Integrated Healthcare Model: Radiotherapy and Palliative Care (RaP) Outpatient Clinic
Rossi R., Foca F., Pieri M., Micheletti S., Tontini L., Romeo A., Altini M., Nanni O., Maltoni $M$

Istituto Scientifico Romagnolo per lo Studio e la Cura dei Tumori (IRST) IRCCS, Meldola, Italy

Background: Over the last century radiotherapy (RT) has been successfully used to control symptoms caused by cancer. With advances in cancer care, the distinction between curative and palliative goals has become blurred. There is substantial evidence that palliative care (PC) combined with standard cancer care improves patient and caregiver outcomes in terms of quality of life (QoL), survival, use of healthcare services and costs.

Methods: From April 2016 to April 2018 a radiation oncologist and PC physician made 291 joint evaluations in the weekly Outpatient Clinic. Consecutive patients with advanced cancer were assessed for palliative RT and timely referral for PC. Before each clinical visit, the 2 physicians first reviewed the patient's clinical history and imaging studies while a nurse received the patient and administered the Edmonton Symptom Assessment System (ESAS) and a QoL questionnaire (EORTC C 15-Pal). Data on clinical and disease characteristics, RT administered and followup information on date and place of death were inserted in a dedicated database. When palliative RT was not indicated, an appointment was scheduled at the Outpatient Clinic for the following month.

Results: 260 patients were evaluated in the integrated RT and PC Outpatient Clinic. Median age was 69 years. 83 patients had lung cancer and 121 were irradiated for bone metastases. RT was not indicated in 137 patients and 87 patients were referred immediately for home-care or hospice PC. In September 2018142 had died, 96 of whom in a PC setting (hospice or home-care). Median survival of irradiated patients was 10.2 months and median overall survival of the entire group was 8,5 months.

Conclusion: This new integrated approach improved the quality of care and QoL of our advanced cancer patients. The systematic analysis of all data collected will hopefully provide the answer to the many open questions remaining in this challenging healthcare area.

Abstract number: P01-234

Abstract type: Poster

Together We'll Go All the Way: Fast-track Early Integration of Patients with Amyotrophic Lateral Sclerosis (ALS)

Ateş G. ${ }^{1}$, Gasper A. ${ }^{2}$, Jaspers B. ${ }^{1,3}$, Kern M. ${ }^{2}$, Radbruch L. ${ }^{1,2}$

${ }^{1}$ Department of Palliative Medicine, University Hospital Bonn, Bonn, Germany, ${ }^{2}$ Centre of Palliative Care, Malteser Hospital Seliger Gerhard Bonn/Rhein-Sieg, Bonn, Germany, ${ }^{3}$ Department of Palliative Medicine, University Medicine Goettingen, Goettingen, Germany

Background: Interviews and a survey among key healthcare providers for ALS patients in the state North-Rhine Westphalia (2016-2018) have shown a broad range of care needs, shortfalls of healthcare services and care gaps.

Aims: To develop a comprehensive care concept and optimise the regional care network for ALS patients.

Methods: Cross-sectional, interdisciplinary and multiprofessional workshops (WS) with purposefully sampled experts (04-09/2018). The participants were informed of interview and survey results, and addressed the following issues: existence of a specific care path for ALS in their line of work, challenges and interfaces of interdisciplinary cooperation, involvement of palliative care services, and improvement of cross-sectional care. The WS comprised of plenary discussions and group work, were led by 2 researchers and 1 physician. Protocols were send round to the participants for approval.

Results: In 5 WS participated physicians of different specialties, nurses, chaplains, and various therapists $(n=52)$. WS results were categorized into 3 thematic fields: 
i) Multi- and interdisciplinary issues (lack of ALS-specific knowledge among logo-, ergo, physiotherapists, nursing services, physicians; long waiting lists for equipment; certain conditions: ALS and frontotemporal dementia, etc.)

ii) Cross-sectional challenges (no home visits by most medical specialties, lack of psychological support at short notice, crosssectoral communication)

iii) ideas (ALS App, digitalized data documentation to be accessed by all care services involved etc.).

Further outcome: Improved networking of the participants, list of useful resources in the region, e.g. physicians for home visits, and establishment of an ALS coordinator in the regional network.

Conclusion: Further improvement of the care network including ALS expertise programmes and a central register for ALS patients is needed. Funding: Ministry for Labour, Health and Social Affairs of North-Rhine Westphalia

Abstract number: P01-235

Abstract type: Poster

Prevalence of Patients with Palliative Needs in a Portuguese Hospital Medical Ward

Batista S..$^{1,2,3}$, Pereira C. ${ }^{4}$, Sapeta P. ${ }^{1,3}$, Capelas M.L. ${ }^{3,5}$

${ }^{1}$ Escola Superior de Saúde Dr. Lopes Dias / Interdisciplinary Research Unit on Building Functional Ageing Communities, Instituto Politécnico de Castelo Branco, Castelo Branco, Portugal, ${ }^{2}$ Orthopedics ward, Unidade Local de Saúde de Castelo Branco, Castelo Branco, Portugal, ${ }^{3}$ Portuguese Observatory for Palliative Care, Universidade Católica Portuguesa, Lisboa, Portugal, ${ }^{4}$ Medicine ward, Hospital de Cascais Dr. José de Almeida, Cascais, Portugal, 5 Institute of Health Sciences, Universidade Católica Portuguesa, Lisboa, Portugal

Background: The prevalence of patients with palliative needs in Portuguese medical wards is still poorly studied. The early referral to palliative care $(P C)$ is one of the keys to the success of this care, to promote the best quality of life of patients and their families, to planning responses to their needs and to organized the health resources.

Aim: To determine the prevalence of patients with palliative needs in one Portuguese medicine ward. To analyze their profile and to verify how many of these patients are referenced to PC and if not, what are the main reasons.

Methods: Descriptive, observational and cross-sectional study. 17 professionals (physicians and nurses) from one medicine ward were asked about 57 adults patients, in June 2014, using a form where, among other subjects, existed the surprise question "would you be surprised if the patient died within a year?". When the answer was no, they were asked if the patient was referenced to palliative care and if not, they were invited to indicate 5 main reasons from a list of 19.

Results: Of the 57 patients, $49.1 \%$ presented palliative needs. The primary diagnosis was stroke (28.6\%); $7.1 \%$ had oncological disease. $68 \%$ of the patients had two or more comorbidities. Only $5.4 \%$ were referred for PC. The 5 main reasons for patients not being referred were: the patient is still being actively treated for the disease; the symptom control was achieved; there are no resources; risk of being hospitalized in a palliative unit away from the family and the PC was not the best for the patient. Conclusion: There was a high prevalence of patients with palliative needs but only a very few were referred to PC. The profile of these patients emphasizes multiple needs and high complexity, which should be considered in care planning. More education in PC is needed to increase the proportion of patients referred early and to articulate with PC teams.

Abstract number: P01-236

Abstract type: Poster
Concept of Sedation in Specialist Palliative Care - First Results of a Qualitative Interview Study on German Health Care

Professionals' Experiences and Views

Handtke V. ${ }^{1}$, Bozzaro $C^{2}{ }^{2}$, Kremling A. ${ }^{3}$, Schildmann J. ${ }^{4}$, Bausewein C. ${ }^{1}$, Schildmann . $^{1}$

${ }^{1}$ Department of Palliative Medicine, University Hospital, LudwigMaximilians-Universität (LMU) München, Munich, Germany, 2Department of Medical Ethics and History of Medicine, Albert-LuwigsUniversity, Freiburg, Germany, ${ }^{3}$ Institute for History and Ethics of Medicine, Martin Luther University Halle-Wittenberg, Medical Faculty, Halle, Germany, ${ }^{4}$ Institute for History and Ethics of Medicine, Martin Luther University Halle-Wittenberg, Medical Faculty, Halle (Saale), Germany

Background: While sedation is common practice in specialist palliative care (SPC), consensus about terminology, definitions and indication of different types of sedation is lacking in the literature. This heterogeneity is reflected in a wide range of reported frequencies and variations in practice. So far, there are no data regarding professionals' views on these topics for Germany.

Aim: To explore German health care professionals' understanding of sedation in SPC.

Methods: Qualitative semi-structured expert interviews with German SPC health care professionals. Participants were included if they reported experience with sedation in SPC. The audio-recorded interviews were transcribed verbatim and analyzed using framework analysis.

Results: 11 interviews (7 physicians, 4 nurses) in 3 SPC centers between August and October 2018. Interviews revealed difficulties of participants in clearly defining sedation in SPC and distinguishing different types of sedation. All initially understood "palliative sedation" (PS) as deep continuous sedation until death. They described a state of sleep without the ability to communicate. The mentioned goal of the therapy was to achieve a state of relaxation and peacefulness for the patient by administering sedative drugs. Refractory symptoms as indication for PS were present in most accounts, describing PS as measure of last resort, while unbearable suffering was mentioned less frequently. Further inquiry revealed disagreement whether dose, route of administration, length and depth of the sedation were defining criteria of PS. Lighter forms of sedation were not or only reluctantly summarized under the term sedation or PS, but were rather termed "symptom control".

Conclusion: The first findings show divergence from definitions in current guidelines. Further research is needed as a basis to adapt further guidance and training to professionals' experiences and needs.

Funded by the Federal Ministry of Education and Research (SedPall 01GY1702B).

Abstract number: P01-237

Abstract type: Poster

"If the Berlin Wall Can Come Down, Nothing is Impossible": Developing an Inclusive Collaborative Culture of Research amongst Palliative Care Services

Heard C. ${ }^{1}$, Dawson A. ${ }^{2}$, Thomas S. ${ }^{3}$, Carson-Stevens A. ${ }^{4}$, Gleeson A. ${ }^{1}$, Lane J. ${ }^{1}$, Noble S. ${ }^{5}$

${ }^{1}$ Aneurin Bevan University Health Board, Newport, United Kingdom, ${ }^{2}$ Hospice of the Valleys, Tredegar, United Kingdom, ${ }^{3}$ St Davids Hospicecare, Newport, United Kingdom, ${ }^{4}$ Division of Population Medicine, Cardiff University School of Medicine, Cardiff, United Kingdom, ${ }^{5}$ Marie Curie Palliative Care Research Centre, Cardiff University, Cardiff, United Kingdom

Goal of work: A review of palliative care research activity across a regional health board serving a population of 1.1 million identified poor co-ordination, limited engagement with charitable providers and no identifiable strategy. Research activity was predominantly medical with 
little multidisciplinary activity. Challenges included: minimal research expertise, lack of governance systems, limited funding sources, supervisor capacity and recruitment pathways.

Methods: A multidisciplinary steering group representing stakeholders was convened. Representatives from outside the region were co-opted as necessary. Following a SWOT analysis, a three-year strategy was devised an approved at Executive level Agreed outcomes included:

- Education in research methodology and literature evaluation

- Ensure each stakeholder had a designated research lead

- Centralised shared indemnity and sponsorship for all stakeholders

- Access funding for at least 2 research based postgraduate qualifications

- Increase research activity and dissemination.

Results: Targeted training in literature evaluation and research methodology training has been provided to research focussed staff in the statutory and third sector. Twelve healthcare professionals have completed GCP training. Funding in open completion has been secured for two Research based MScs and one PhD through closer working. Two hospices and one acute hospital have recruited over 40 patients to clinical trials. One systematic review has been published. The research activity was recognised by the health board with an award for greatest contribution to research.

Conclusions: A culture of multi-professional cross organisational research can be developed within a relatively short time. Key requirements include high level support from each organisation, strong leadership and a shared vision for improvement. Affiliation with academic institutions is essential especially in accessing expertise that is not available internally.

Abstract number: P01-238

Abstract type: Poster

Quality of Collaboration and Information Transfer in Palliative Care: A Cross-sectional Survey among Physicians in the Southwest Region of the Netherlands

Engel M., van der Ark A., Tamerus R., van der Heide A.

Public Health, Erasmus MC, University Medical Center Rotterdam, Rotterdam, Netherlands

Background: Palliative care is often provided by health care providers from different disciplines and different settings. Continuity in such care is therefore a challenge.

Aims: We explored the quality of collaboration and information transfer in palliative care from the perspective of physicians.

Methods: A survey study was performed in 2017 among physicians in the Southwest region of the Netherlands using a random sample from a professional registry of physicians working in primary care, hospitals and nursing homes $(n=2212)$. We also invited physicians to participate via palliative care networks. Differences in scores for collaboration were tested for statistical significance with Chi-square test.

Results: 547 physicians participated. The mean score (scale 1=very poor to $10=$ perfect) for quality of their collaboration with physicians from other organizations was 7.2, with nursing home physicians reporting the lowest (6.2) and hospital physicians reporting the highest scores (7.5). Younger physicians (age $\leqslant 50$ ) and physicians in strongly urbanized areas more often scored quality of collaboration as sufficient $(\geqslant 6)(\chi 2$, respectively $p=0.04$ and $p=0.05$ ). A majority of physicians reported that adequate palliative care is sometimes $(58.1 \%)$ or often $(20.2 \%)$ hindered by inadequate medical information from other settings. Physicians often miss information about prognosis (42.4\%) and psychosocial aspects (65.3\%). In answering open questions, often reported bottlenecks in collaboration were: difficulties with contacting colleagues in other settings and receiving no or late information about treatment plans and goals.

Conclusion: Physicians indicate that collaboration and information transfer in palliative care can be improved. Hospital physicians seem to be more satisfied than general practitioners and nursing home physicians. Continuity of care could be improved by better communication between physicians, e.g. about a patient's prognosis and treatment plans.

Abstract number: P01-239

Abstract type: Poster

Is Physical Activity Training Feasible among Patients with Metastatic Breast Cancer?

Kokkonen K. ${ }^{1}$, Utriainen M. ${ }^{1}$, Penttinen $H^{1}{ }^{1}$, Vehmanen L. ${ }^{1}$, Kautiainen

H. $^{2}$, Pyykkönen T. ${ }^{3}$, Ristimäki K. ${ }^{3}$, Nikander P. ${ }^{3}$, Saarto T. ${ }^{1}$

${ }^{1}$ University of Helsinki, Helsinki, Finland, ${ }^{2}$ Other, Espoo, Finland,

${ }^{3}$ Helsinki University Hospital, Helsinki, Finland

Background: Active rehabilitation aims to restore and improve cancer patients' functional abilities, physical independence and QoL.

Aims: We accomplished a non-randomized prospective intervention of individual rehabilitation program among patients with newly diagnosed advanced breast cancer (ABC). The primary goal was to investigate the feasibility of physical training at that time(recruitment rate and compliance). The secondary objective was to investigate the physical fitness, QoL and body composition during the program.

Methods: Patients (56) with newly diagnosed ABC participated for 6 months individual exercise training protocol. Physical tests (grip strength, sit-to-stand-test, static and dynamic balance, and 6-min walking distance) and body composition (bioimpedance) were measured at baseline and at 6-mo's. Physical therapist revised the program at 1 and 3 mo's. Patients kept 2-week diary of physical activities and reported QoL (RAND-SF-36, EORTC-QLQ-C30, 15D, rBDI, FACIT, ESAS) 4 times.

Results: The mean age was 61 (33-73) years. 52\% had bone metastases. The overall recruitment rate was $49 \%$ and $82 \%$ of the patients completed the study. Steps per day (mean from 7281 to 6923 ), sit-to-stand test, QoL and depression remined unchanged during the 6 months follow-up. Significant improvement was seen in 6 -min walking test $(p=0.045)$, role physical $(p=0.031)$, vitality $(p=0.033)$, social functioning $(p=0.017)$ measured by RAND, and physical function $(p=0.045)$ and appetite loss $(p=0.004)$ by EORTC-C30. ESAS, balance tests, grip strength and body composition will be presented at the congress.

Conclusion: Individual physical rehabilitation is possible and feasible for motivating patients with advanced disease to exercise and to maintain the functional capacity and QoL during treatments.

Abstract number: P01-240

Abstract type: Poster

Enhancing Outpatient Palliative Care: Outcomes of a Service Review at a Tertiary Cancer Centre

Collins A. ${ }^{1}$, Le B. ${ }^{2}$, Marco D. ${ }^{3}$, Philip J.1,2

${ }^{1}$ Department of Medicine, St Vincent's Hospital, University of Melbourne, Melbourne, Australia, ${ }^{2}$ Parkville Integrated Palliative Care Service, Victorian Comprehensive Cancer Centre, Melbourne, Australia, ${ }^{3}$ Centre for Palliative Care, St Vincent's Hospital, Melbourne, Australia

Aims: This study sought to review the existing Outpatient Palliative Care Service provision at a leading Australian integrated cancer centre, with a view to developing recommendations for future service development.

Methods: A mixed method sequential study was conducted, involving: 
1) A narrative literature review of service models, outcomes and challenges in the delivery of outpatient palliative care;

2) A retrospective cohort study of all new patients referred from 1 July to 31 Dec 2017 followed from first assessment to death; and

3) An exploration of current outpatient palliative care services according to the views of service providers.

Results: Care was provided to 473 patients (mean age 62 years), with predominately gastrointestinal $(20 \%)$, lung $(18 \%)$, breast $(11 \%)$, or hematologic (11\%) cancers. Over the study follow-up (median 106 days, IQR 30, 262), 327 (69\%) patients died, a median of 48 days (IQR 17, 113) after first referral to palliative care. Patients had a median of $3($ IQR 1,4$)$ outpatient visits, with $48 \%$ who were first seen in the outpatient setting. At first assessment, the median AKPS was 70 (IQR 60, 80), and the most common presenting symptom was pain (81\%), with a median severity score of 7 (IQR 4, 9), followed by fatigue (71\%, median 6$)$, insomnia $(73 \%$, median 5$)$, and anorexia ( $73 \%$, median 4$)$. Service providers perceived outpatient palliative care services offered enhanced accessibility, and responsiveness, and facilitated earlier integration of palliative care. Providers also reported some time and resource inefficiencies and discontinuities in care in delivering this model which is flexible according to patient need and demand.

Conclusion: Outpatient service provision enhanced earlier integration of palliative care at an integrated cancer centre and offered perceived benefits to patients. Different models of outpatient delivery that may allow for enhanced capacity and responsiveness across all areas of service provision will be presented.

\section{Abstract number: P01-241 \\ Abstract type: Poster}

Telehealth for Palliative Patient-effectiveness and Costeffectiveness of Home Palliative Care Services with Chronic Illness and their Caregivers

Kabir M.S. ${ }^{1}$, Akhter R. ${ }^{2}$, Afrin S. ${ }^{1}$, Nandi S. ${ }^{1}$, Bithi M.J. ${ }^{1}$, Akhter.$^{1}$

${ }^{1}$ Palliative Care, Hospice Bangladesh Limited, Dhaka, Bangladesh, ${ }^{2}$ Department of Sociology, Criminology, and Anthropology, University of Central Arkansas, Conway, United States

Background: Telehealth is being used increasingly in providing care to patients in the community setting. Most palliative care patients indicate that they would prefer to be cared for, and to die, at home. Telehealth represents one avenue for investigation, offering more regular continuous remote monitoring to highlight changing performance status, and immediate access to resources and information for patients and families.

Aims: To evaluate to pattern of diseases, place of death, effectiveness of the service and cost effective.

Methods: This study was a prospective cohort study of a telehealthbased intervention for community-based patients of a specialist palliative care service living in Dhaka, Bangladesh. Data of 50 patients were analyzed who used Telehealth for home palliative care from January 2018 to July 2018. Patient used mobile app or telephony. Data was collected from Hospice Bangladesh's office back end software.

Results: All of our patients lived in Dhaka metropolitan area except 2 patients. Majority of patent's age were over 60 years. Among patients 53\% were females and $46 \%$ were males. All patients were married. Majority of our study patients suffering from cancer $60 \%$ and age-related problems patient $25 \%$, cardiac problem $7 \%$, renal problem $3 \%$ and others $5 \%$. There were $20 \%$ used only telephony service, $80 \%$ patients used mobile apps. Phone call $(80 \%)$ is more than face to face doctor visit. Those who died during this study $60 \%$ of them died at home, $40 \%$ died in the hospital. The most benefited part of the home telehealth care was reducing continue nursing support (60\%) and reduce economic burden (80\%).

Conclusion: This study has shown that palliative care patients and their careers living in the community were able to use telehealth app trial involving remote monitoring of symptoms. The telehealth model offers new ways of supporting care at the end of life in the community and further applications of these approaches should be investigated.

Abstract number: P01-242

Abstract type: Poster

Changes in Clinical Practice in Response to Randomised

Controlled Effectiveness Trials in Palliative Care

Campbell R. ${ }^{1}$, Agar M. ${ }^{2}$, Clark K. ${ }^{3}$, Phillips J. ${ }^{4}$, Hardy J. ${ }^{5}$, Currow D. ${ }^{4,6}$

${ }^{1}$ Marie Curie Hospice, Belfast, United Kingdom, ${ }^{2}$ University of Sydney, Sydney, Australia, ${ }^{3}$ Northern Sydney Local Health District Cancer and Palliative Care Network, Sydney, Australia, ${ }^{4}$ University of Technology Sydney, Sydney, Australia, ${ }^{5}$ Mater Cancer Care Centre, Brisbane, Australia, ${ }^{6}$ Wolfson Palliative Care Research Centre, University of Hull, London, United Kingdom

Background: Influencing change in medical practice is challenging. Until recently there has been little research assessing the impact in response to randomised controlled trials (RCTs) has on current practice especially in palliative care.

Two recent RCTs examined the effectiveness of octreotide in inoperable bowel obstruction due to cancer or its therapies (IMBO) and ketamine for cancer pain. Follow-up studies assessed change in clinical practice. Both demonstrated a significant self-reported change in prescribing practices: change of $49 \%$ in prescribing octreotide and $65 \%$ in prescribing ketamine. Why have these RCTs influenced practice?

Aims: Discuss why these RCTs have resulted in self-reported change in clinician prescribing practices whilst other methods to influence change in clinical practice have been less successful.

Methods: The nature of these RCTs, the context within which the RCTs took place, assessment of change and dissemination of results are explored.

Results: These adequately powered effectiveness RCTs (as opposed to efficacy RCTs) were developed to address real-world clinical questions with real-world outcomes. Each was arguably the first adequately powered study in the palliative care population. Each had a dissemination plan attached to it once it was accepted by a peer-reviewed journal. These are factors that may influence the significant impact on changes in prescribing.

Conclusions: Research in palliative care is a rapidly expanding field but to date little clinical practice has been shaped by evidence. Both the RCTs suggest high quality clinical research does influence clinicians' decisions. Palliative care clinicians are open and willing to adapt practice based on evidence as it becomes available.

Abstract number: P01-243

Abstract type: Poster

A Systematic Review Describing Components of Palliative Care Interventions Addressing the Needs of People with Dementia Living in Long Term Care: Mapping against EAPC Domains of Optimal Palliative Care for People with Dementia

Kochovska S. ${ }^{1}$, Garcia M. ${ }^{1}$, Bunn F. ${ }^{2}$, Goodman C. ${ }^{2}$, Luckett T. ${ }^{1}$, Parker D. ${ }^{1}$, Phillips J. ${ }^{1}$, Sampson E. ${ }^{3}$, van der Steen J. ${ }^{4}$, Agar M. ${ }^{1}$

${ }^{1}$ IMPACCT (Improving Palliative, Aged and Chronic Care through Clinical Research and Translation), University of Technology Sydney, Sydney, Australia, ${ }^{2}$ University of Hertfordshire, Hatfield, United Kingdom, ${ }^{3}$ University College London, London, United Kingdom, ${ }^{4}$ Leiden University Medical Center, Leiden, Netherlands

Background: People with dementia and palliative care needs require complex interventions to address those needs. The European Association for Palliative Care (EAPC) White Paper offers recommendations for palliative care in dementia and highlights domains integral for this population, thus providing useful guidance to developing such interventions. 
Aim: To describe the components of interventions designed for palliative care for people with dementia in aged care, and determine the extent to which they address the EAPC domains.

Methods: A systematic review with narrative synthesis. Four electronic databases and clinical trial registries were searched for peer-reviewed articles and protocols in English, reporting on palliative care interventions for people with dementia in aged care settings, and addressing EAPC Domains 2 (person-centred) or 3 (setting care goals) and $\geqslant 1$ other domain.

Results: Forty-five included papers, reporting on 24 studies: quantitative $(n=12)$, qualitative $(n=2)$, mixed methods $(n=9)$, and a service outline. Interventions focused on (in order of frequency): supporting family decision-making; providing dementia-specific education and training to staff or family; improving symptom assessment/management; improving care delivery through inter-professional engagement; establishing coordinating roles; and furthering person-centred care. Interventions targeted care home staff $(n=19)$, family members $(n=12)$ and other healthcare providers $(n=4)$.

Discussion: There is increasing sophistication in research on this topic with included studies reflecting all the domains but only one study addressing in its design 10 of the 11 domains. Varying emphasis was accorded to different foci, and the contexts in which interventions were delivered. The review challenges methods and interventions currently used as the underrepresented domains (such as continuity of care, prognostication, and ethical and societal issues) are less amenable to single, education driven interventions.

Abstract number: P01-244

Abstract type: Poster

The Private Home as a Shared Space and Place for Co-care - A Photo Elicitation Study with Palliative Care Nurses

Alvariza A. ${ }^{1,2}$, Mjörnberg M. ${ }^{3}$, Goliath $1 .{ }^{4}$

${ }^{1}$ Department of Health Care Sciences/Palliative Research Centre, Ersta Sköndal Bräcke University College, Stockholm, Sweden, ${ }^{2}$ Capio Palliative Care Unit, Dalen Hospital, Stockholm, Sweden, ${ }^{3}$ Specialized Palliative Home Care Unit, Ersta Hospital, Stockholm, Sweden, ${ }^{4}$ Dep Learning, Informatics, Management and Ethics, Karolinska Institutet, Stockholm, Sweden

Background: The private home is an often preferred place for care at the end of life. The rapid increase in advanced home care, with dying people more often receiving qualified care at home for longer periods, involves a process of institutionalization of the home, as professionals, supplies and equipment previously associated with institutional care may enter in the private home. There a need to get a deeper understanding of how palliative care nurses ( $\mathrm{PCNs}$ ') work to facilitate this process through the patients' illness trajectory.

Aim: Explore experiences and perspectives of PCNs' working in patients' private homes.

Methods: A qualitative photo-elicitation approach was used and data was collected through interviews stimulated by 31 photographs produced by ten PCNs' working in palliative homecare.

Results: PCNs' illustrated their experiences through photographs depicting places with dual purposes; a private home and a care work environment. When the PCNs' entered each home they were prepared to adjust their interactions and actions depending on the inhabitants and their unique environment. This care environment required specific support to patients and family members in balancing self-efficacy and safety of selfcare, and independency. Further, their work involved guiding patients and family members towards and through environmental changes needed to support end of life care at home. The PCNs' experienced the transition time driving between homes as crucial, used to prepare, reflect and recover during their daily work.

Conclusions: Working in these shared areas required mutual confidence and trust between PCNs', patients' and family members' to establish and achieve co-care. The home care environment was used as an intrinsic part of, rather than separate to care provision. The results reflect $\mathrm{PCNs}$ competences in dealing with challenges caring for patients and family members in their own space.

Abstract number: P01-245

Abstract type: Poster

Predictors of Patient-reported Quality of Outpatient Specialized Palliative Care in Germany

Kruschel I., Hammer U., Bergmann D., Gaser E., Wedding U., Meißner

W.

Palliative Medicine, Clinic for Internal Medicine II, FSU Jena, Jena,

Germany

Background: Since 2007 there is a legal right to receive outpatient specialized palliative care (SAPV) for critical ill patients with life-limiting diagnosis. Only general requirements were given by the Federal Joint Committee German Innovations Fund, resulting in a large degree of heterogeneity of SAPV structures and processes in Germany.

Aim: To assess outcome quality of SAPV providers from the patients' point of view.

Method: From May till December 2015 a multi-center, prospective, nonrandomized study was performed to evaluate patient-reported outcomes using a self-provided questionnaire and NCCN Distress-Thermometer. Also structural parameters of providers were captured.

Results: 17 Providers of SAPV in different regions of Germany participate. 371 Patients were included. For 169 patients $(45.5 \%)$ complete questionnaires could be analyzed. Mean duration of care was 22 days, Questionnaires were handed out 10 days after inclusion in SAPV care on average. $80 \%$ of the patients had an oncological diagnosis. Four of the SAPV providers quoted common structural data and were summarized as one. One team did not collect any data, so that analysis refer to 13 teams. Overall patients rate a high degree of satisfaction on average 79.9\%, over all 9 items due to full agreement to Likert scale in the questionnaire. Less accordance is shown on social und mental issues. Mean distress is $5.6(0-10, n=140)$. $79.3 \%$ of the patients reported a distress score of $>=4$ which is considered as the cut-off value needy for assistance. Only in 4 of 13 providers a psychologist supported the team. Correlation coefficient between collaboration of a psychologist and distress is $-0,8$, which mean a strong negative relation.

Conclusion/ discussion: Overall, patients are satisfied with quality of specialized palliative care, although they report a high degree of distress. Structures of teams are very various. Larger studies are needed to examine predictors of quality of care.

Abstract number: P01-246

Abstract type: Poster

What is Renal Supportive Care and How Does it Differ from Palliative Care? Renal Clinicians' Perspectives of Renal Supportive Care, Specialist Palliative Care and Referral Practises Across Australia and New Zealand

Ducharlet K.1,2, Gock H.2,3, Jennifer P.2,4

1Palliative Medicine and Nephrology, St Vincent's Hospital Melbourne, Fitzroy, Australia, ${ }^{2}$ Department of Medicine, University of Melbourne, Parkville, Australia, ${ }^{3}$ Department of Nephrology, St Vincent's Hospital Melbourne, Fitzroy, Australia, 4Palliative Medicine, St Vincent's Hospital Melbourne, Fitzroy, Australia

Background: Patients with end stage kidney disease (ESKD) report high symptom burden, complex care needs and often have a limited prognosis. RSC is a clinical approach aiming to improve quality of life (QOL). Clinicians' perceptions of RSC and its role alongside that of SPC are unknown. 
Aim: To understand renal clinicians' views and referral practises of renal supportive care (RSC) and specialist palliative care (SPC).

Methods: A cross-sectional validated online survey was undertaken between February and June 2018. Participants (renal doctors and nurses) completed demographic details, views and referral practises of RSC and SPC and elements of an "ideal model of RSC."

Results: Of the 440 clinicians completing the survey, $78 \%$ were female, $60 \%$ nurses, $28 \%$ doctors, $70 \%$ metropolitan, $83 \%$ had SPC access, $60 \%$ RSC access with variability in reported activities of current RSC services. Overall, RSC was viewed as improving symptoms and QOL $89 \%$, the same as palliative care in only $22 \%$, more acceptable for patients than palliative care in $80 \%$.

Participants suggested an ideal model of RSC should prioritize; symptom management (98\%), integration between nephrology and community healthcare services (97\%) and community SPC (94\%), renal clinician RSC education (95\%), a designated RSC coordinator (91\%), complex treatment decision making (90\%), defined pathway for conservative patients (86\%).

Clinicians were more likely to consider referral to RSC over SPC for symptoms, $(85 \%$ vs $78 \%, p=0.01)$, withdrawal from dialysis $(94 \% v s 87 \%, p=0.00)$, complex treatment decision making ( $84 \% \mathrm{vs} 59 \%, \mathrm{p}=0.00)$, but referral to SPC was favoured if the patient was dying (76\%vs96\%,p=0.00).

Conclusions: RSC is viewed as acceptable with roles in improving symptoms, QOL, care coordination and clinician education for renal patients. This study revealed opportunities to improve new and existing RSC and SPC services to assist ESKD patients.

Abstract number: P01-247

Abstract type: Poster

Challenges Facing Renal Clinicians for Renal Supportive Care, Palliative Care and End of Life Care, a Qualitative Study

Ducharlet K. ${ }^{1,2}$, Hilton G. ${ }^{3,4}$, Weil J.5,6, Jennifer P. ${ }^{4,5}$

${ }^{1}$ Department of Palliative Medicine and Nephrology, St Vincent's Hospital Melbourne, Fitzroy, Australia, ²Department of Medicine, The University of Melbourne, Parkville, Australia, ${ }^{3}$ Department of Nephrology, St Vincent's Hospital Melbourne, Fitzroy, Australia, ${ }^{4}$ Department of Medicine, University of Melbourne, Parkville, Australia, ${ }^{5}$ Department of Palliative Medicine, St Vincent's Hospital Melbourne, Fitzroy, Australia, ${ }^{6}$ Centre for Palliative Care, St Vincent's Hospital, Fitzroy, Australia

Background: Patients with end stage kidney disease (ESKD) often have a high burden of physical and psychosocial morbidity, associated with frailty and limited prognosis. Renal Supportive Care (RSC) is increasingly recognised as an important approach to improve quality of life for people with ESKD. However perceptions of RSC by nephrology services, the challenges and degree of integration of this approach are unknown.

Aim: To explore current attitudes and experiences of RSC, Palliative Care and end of life care by renal clinicians.

Methods: An exploratory qualitative study was conducted across 3 metropolitan and 2 regional Victorian hospitals in Australia. Focus groups and semi-structured interviews of renal clinicians were recorded and transcribed for thematic analysis using grounded theory by two independent researchers.

Results: Of participants recruited $(n=58)$ there were 35 nurses and 23 doctors with clinical experience $0.5-40$ years.

Themes emerging from these data included:

1) While perceptions and practices of RSC vary substantially, it is perceived as separate from palliative care, useful earlier in disease trajectory and overall more acceptable for patients.

2) ESKD patients have few, readily identified transition points to herald the final phase-of-life therefore, recognition of need for renal supportive care and palliative care is inconsistent.
3) Issues around intensification or continuation of life supporting therapies at the end of life were at times extremely challenging for clinicians, causing moral distress and disruption of relationships between patients, families and treating teams.

Conclusions: Views of RSC vary widely, however it is seen as distinct and more acceptable than palliative care; despite considerable overlap between approaches. There are opportunities to develop synergies between Nephrology and Palliative Medicine earlier in disease trajectory for renal patients given challenges described at end of life.

Abstract number: P01-248

Abstract type: Poster

Dissatisfaction with Care in the Last 3 Months before Death in Older Patients: A Pooled Analysis of 3 Mortality Follow-back Surveys

Miyashita M..$^{1,2}$, Evans C. ${ }^{1}$, Yi D. ${ }^{1}$, Gomes B. ${ }^{1,3}$, Higginson $I .{ }^{1}$, Gao W. ${ }^{1}$ ${ }^{1}$ Cicely Saunders Institute of Palliative Care, Policy \& Rehabilitation Florence Nightingale Faculty of Nursing, Midwifery \& Palliative Care King's College London, London, United Kingdom, 2Department of Palliative Nursing, Health Sciences, Tohoku University Graduate School of Medicine, Sendai, Japan, ${ }^{3}$ Faculty of Medicine, University of Coimbra, Coimbra, Portugal

Background: Dissatisfaction is one of the common measures of quality palliative care. Although mortality follow-back surveys including non-cancer death have been conducted to depict the level of dissatisfaction of care at the end-of-life, few reports have compared cancer with specific disease groups other than the combined non-cancer diagnosis.

Aims: To clarify the level of dissatisfaction of care at the end-of-life by causes of death in older patients and to explore factors associated with the dissatisfaction.

Methods: We conducted a pooled analysis of datasets from the QUALYCARE study, the OPTCare Elderly study and the IARE1 study conducted in the UK. We analysed the data of bereaved relatives of people aged $\geqslant 75$ years old who died of various causes. We asked "whether there were any aspects of care that caregivers felt unhappy within the last 3 months" as a measure of dissatisfaction with care.

Results: We analysed 885 responses from three studies. The causes of death were cancer $(n=516)$, heart disease/stroke $(n=172)$, respiratory disease $(n=104)$, neurological disease $(n=42)$ and dementia $(n=51)$.

Across causes of death, $28-38 \%$ of the caregivers reported dissatisfaction with care $(P=0.18)$. Multiple logistic regression analysis revealed that the people who died from dementia and heart disease/stroke had less dissatisfaction compared to cancer $(O R=0.22, p=0.03$ and $O R=0.42$, $\mathrm{p}=0.006$, respectively). Also, the absence of a reliable key health professional $(O R=2.28, p=0.001)$, female caregiver $(O R=1.71, p=0.02)$, child caregiver $(O R=2.29, p=0.005)$ and higher Palliative Outcome Scale practical problems score $(\mathrm{OR}=1.23, \mathrm{p}=0.001)$ were significantly associated with dissatisfaction.

Conclusion / Discussion: After statistical adjustment of potential confounders, dementia and heart disease/stroke had lower dissatisfaction compared to cancer. Further research to explore the role of the key health professionals and cope with the practical problems of patient/ caregiver would be needed.

Abstract number: P01-249

Abstract type: Poster

Prolonged Overall Survival in Patients with Metastasized Non-small Cell Lung Carcinoma after Combined Treatment of Chemotherapy and Viscum album L. versus Chemotherapy Alone, a Cancer Registry Analysis 
Matthes B. ${ }^{1,2}$, Thronicke A. ${ }^{1}$, Merkle A. ${ }^{2}$, Grah C. ${ }^{1,3}$, Matthes H..$^{1,4}$, Schad $F^{1,2}$

${ }^{1}$ Research Institute Havelhöhe, Hospital Havelhöhe, Berlin, Germany, ${ }^{2}$ Hospital Havelhöhe, Interdisciplinary Oncology and Palliative Care, Berlin, Germany, ${ }^{3}$ Hospital Havelhöhe, Lung Cancer Center and Department of Pneumology, Berlin, Germany, ${ }^{4}$ Medical Clinic for Gastroenterology, Infectiology and Rheumatology CBF and Institute of Social Medicine, Epidemiology and Health Economics CCM, Charité University Medicine Berlin Economics, Charité - Universitätsmedizin Berlin, Berlin, Germany

Background: Stage IV non-small cell lung carcinoma (NSCLC) is one of the most devastating diagnoses worldwide. Mistletoe therapy (Viscum album L., VA) has effectively been applied as an add-on treatment within integrative oncological concepts to improve quality of life. Overall, there is little evidence for add-on VA on survival of cancer patients. However, recent results suggest a beneficial effect $([1,2]$. A multicenter observational health services research study was conducted to compare the combination of chemotherapy and VA (CVA) versus chemotherapy (C) for patients with metastasized NSCLC.

Aims: The primary outcome was the assessment of overall survival.

Methods: Patients with stage IV NSCLC living at least 4 weeks postdiagnosis were non-randomly assigned to groups $C$ (chemotherapy) or CVA (chemotherapy and Viscum album L.). Demographic-, diagnosis-, histology- and treatment-related data were retrieved from the network oncology [3], a clinical cancer registry for health services research (register ID: DRKS00013335). Written informed consent from patients has been obtained prior enrolment; the study has been approved by the ethics committee of the Medical Association Berlin (Eth-27/10).

Results: A total of 158 eligible patients between February 2010 and June 2016 were enrolled. The median OS was 8.0 months for group $C$ patients and 17.0 months for CVA patients (Hazard ratio - HR: $0.52,95 \% \mathrm{Cl}, 0.33$ - 0.83; P = .007; adjusted HR: $0.44,95 \% \mathrm{Cl}, 0.26-0.74, \mathrm{p}=.002$ ). Oneyear survival rates for $\mathrm{C}$ and CVA were $35.5 \%$ and $60.2 \%$, three-year survival rates were $14.2 \%$ and $25.7 \%$, respectively.

Conclusion: The results of the present health services research analysis reveal that the survival is significantly prolonged in patients with metastasized NSCLC treated with a combination of chemotherapy and VA.

References:

1. Troger W, et al. Eur J Cancer 2013.

2. Axtner J, et al. Schad F. BMC Cancer 2016.

3. Schad F, et al. Forsch Komplementmed 2013.

Abstract number: P01-250

Abstract type: Poster

Assessment of Palliative Care Quality of Care from the Point of View of Nurses in General Palliative Care in Germany Escobar Pinzon L.C. ${ }^{1,2}$, Rieger S. ${ }^{2}$, Diehl E. ${ }^{2}$, Schablon A.S. ${ }^{3}$, Nienhaus A. ${ }^{4}$,

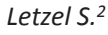

${ }^{1}$ Division 3 Work and Health, BAuA - Federal Institute for Occupational Safety and Health, Berlin, Germany, ${ }^{2}$ University Medical Center of the Johannes Gutenberg University of Mainz, Institute of Occupational, Social and Environmental Medicine, Mainz, Germany, ${ }^{3}$ Competence Center for Epidemiology and Health Services Research for Healthcare Professionals (CVcare), University Medical Center Hamburg-Eppendorf, Hamburg, Germany, ${ }^{4}$ Institute for Health Care Research in Dermatology and Nursing, University Medical Center Hamburg-Eppendorf, Hamburg, Germany

Background: Nurses in the general palliative care supply the majority of palliative care needy patients in Germany.

Aims: The aim of the study* was to examine the assessment of palliative care quality of care from the point of view of nurses in general palliative care (nursing homes and outpatient care) in Germany. The focus was on differences in assessment in the inpatient and outpatient areas as well as other factors.

Methods: In $2017 \mathrm{n}=437$ nurses of the general palliative care in Germany took part in the cross-sectional study (response rate: 17\%). The following question was asked, "How do you assess the palliative care quality of care of patients in your institution / by your employer?" The answer possibilities were: very bad, bad, partly / partly, good and very good. The data were analysed by univariate and bivariate methods (contingency table $\mathrm{p}<0.05$ ).

Results: Over $35 \%$ were 50 years or older, $17 \%$ were 30 years or younger. The majority was female (90\%). $79 \%$ worked in the old / nursing home and $21 \%$ in an outpatient nursing service.

Nurses in nursing homes rated the palliative care quality of care worse than nurses in outpatient care. In addition, nurses, whose facility was located in large cities, assessed the palliative care quality of care more often as good than nurses, whose establishment was located in medium or small towns.

Conclusion / Discussion: The study gives indications of differences in general palliative care in Germany according to the type and location of the institution. In nursing homes, especially in those who are located in medium or small towns, measures must be taken to improve palliative care quality.

\section{Abstract number: P01-251 \\ Abstract type: Poster}

Financial Stress as a Sensor for Emotional and Physical Burden in Lung Cancer Patients

Matthes B. ${ }^{1,2}$, Thronicke A. ${ }^{1}$, Kröz M. ${ }^{1,2,3}$, Oei S.L. ${ }^{1}$, Grah C. ${ }^{1,4}$, Matthes H.1,5, Schad F. ${ }^{1,2}$

${ }^{1}$ Research Institute Havelhöhe, Hospital Havelhöhe, Berlin, Germany, ${ }^{2}$ Hospital Havelhöhe, Interdisciplinary Oncology and Palliative Care, Berlin, Germany, ${ }^{3}$ Institute of Social Medicine, Epidemiology and Health Economics CCM, Charité University Medicine Berlin, Berlin, Germany, ${ }^{4}$ Hospital Havelhöhe, Lung Cancer Center and Department of Pneumology, Berlin, Germany, ${ }^{5}$ Medical Clinic for Gastroenterology, Infectiology and Rheumatology CBF and Institute of Social Medicine, Epidemiology and Health Economics CCM, Charité University Medicine Berlin Economics, Charité - Universitätsmedizin Berlin, Berlin, Germany

Background: It has been acknowledged that pre-treatment general health quality of life (HRQL) is predictive for survival of lung cancer patients. A lack of systematic research on pre-treatment HRQL of lung cancer patients applying integrative oncology (IO) treatment has been detected.

Aim: Patient reported outcomes of patients with lung cancer at time of diagnosis being treated within an Anthroposophic-integrative concept at a certified lung cancer center were evaluated in a real-world observational health services research study.

Methods: Clinical and demographic data were retrieved from the Network Oncology cancer registry. Pre-treatment patient-reported outcomes (PRO) were evaluated (EORTC QLQ-C30 and HADS questionnaires) in people with all-stage lung cancer.

Results: 87 patients were eligible for the questionnaire analysis (median age 68.0 years, IQR 59.0-74.4). Financial burden at first diagnosis was observed as the only statistically significant discriminating PRO variable between patients that would later apply add-on IO treatment. $29.9 \%$ of the total cohort reported financial difficulties. Univariate analysis revealed that self-reported pre-treatment financial difficulty was associated with younger age $(p=0.007)$, pre-treatment pain $(p=0.006)$, anxiety $(p=0.04)$ and feeling of discourage $(p=0.03)$ as well as with the decision for add-on VA therapy $(p=0.007)$ while tumor stage was not an association factor.

Conclusions: Pre-treatment financial problem is an issue in oncology but has until now not recognized for lung cancer patients who are treated within 10 concepts. Financial burden which is significantly more often 
reported in younger lung cancer patients may be a sensor for increased pain and poor emotional outcomes. We suggest physicians to screen for lung cancer patients who are of working age (broadly aged $<65$ years) and/or who report increased pain at the time of diagnosis as they might be at particular risk for financial as well as emotional problems.

Abstract number: P01-252

Abstract type: Poster

Supporting People towards the End of Life: Knowledge, Attitudes and Behaviours of Nurses and Healthcare Assistants Involved in the Provision of Supportive Palliative Care White L., Agbana S., Connolly M., Guerin S.

University College Dublin, Dublin, Ireland

The progression of a life-limiting illness represents a major transition for individuals and families. This paper investigates the knowledge, behaviours, attitudes and general attitudes towards death of nurses and healthcare assistants (HCAs) providing supportive palliative care. A hardcopy survey was used to collect demographic data. Knowledge, attitudes and behaviours were measured using Connolly and colleagues' competency scale. Attitudes towards care of the dying were assessed using the Frommelt Attitudes Towards Care of the Dying (FATCOD) scale (Form A) and attitudes towards death were measured using the Death Attitude Profile - Revised (DAP-R) ${ }^{3}$. A total of 36 practitioners from one service organisation completed the study (nurses $=18$; healthcare assistants= 18 ; female $=32$ ). Responses fell in the lower range of scores for competency variables for both groups. A significant difference was observed between HCAs and nurses on measures of knowledge $(t=-2.718$; $\mathrm{df}=$ $30 ; p<.05)$ and behaviour $(t=-3.576 ; \mathrm{df}=30 ; p<.05)$. Inspection of the means showed that HCAs scored significantly higher than nurses on these variables. No significant relationship was observed between competencies and general attitudes towards death and dying. Results were discussed in relation to strengths and limitations, practical implications and future research suggestions. This research contributes to our understanding of palliative care competencies and general attitudes towards death and dying among nurses and healthcare assistants working in level two palliative care. It has important implications for the education and training of nurses and HCAs working in level two palliative care.

Abstract number: P01-253

Abstract type: Poster

\section{Evaluation of Specialized Palliative Care in Germany: Data of 6756 Outpatients}

Grabenhorst U. ${ }^{1}$, Joist T.M. ${ }^{2}$, Lueg A. ${ }^{3}$, Porz J. ${ }^{4}$, Reichmann J. ${ }^{5}$, Wagner S. ${ }^{6}$, Weckbecker K. ${ }^{4}$

${ }^{1}$ Palliative Care, SAPV linker Niederrhein, Viersen, Germany, ${ }^{2}$ SAPV Cologne, Colgone, Germany, ${ }^{3}$ Palliative Care, SAPV Rheinerft, Brühl, Germany, ${ }^{4}$ Gerneral Medicine, University of Bonn, Bonn, Germany, ${ }^{5}$ Statconsult, Magdeburg, Germany, ${ }^{6}$ Palliative Care, SAPV Cologne, Cologne, Germany

Background: Most people desire dying peacefully at home. To fulfil this last wish, specialized multi-professional teams were implemented in Germany in 2009. Those teams are now spread all over Germany. In the Nordrhein, a region with more than 10 million inhabitants, 21 teams were established. Although the outpatient palliative care teams take care of up to $20 \%$ of the dying people in Germany, there is little data on their work until today.

Aims: The study aims to evaluate the success and effectiveness of the work of outpatient palliative care teams in Germany.

Methods: We analysed the real-life data of 14 of 21 teams in the Nordrhein which looked after 6756 patients in this specific area in 2017. The data of two different software programs for documentation were extracted anonymously from the data base of the individual teams und were merged in one big data pool.

Results: We will present a detailed analysis of the real-life-data of 6756 patients. Thereby, we deliver relevant information about the structure, function and performance of outpatient palliative care teams in Germany. Special attention will be paid to the analysis of parameters like average care duration, location of death and other important features to characterize the work of the teams. $64,2 \%$ of those patients had a carcinoma diagnosis, and $77,5 \%$ of all treated patients died, most of them $97,4 \%$ not in a hospital. On average, the patients were treated for 49, 15 days, with a median duration 21,0 days. And in $86,7 \%$ a GP has been involved in the treatment as well.

Conclusion / Discussion: For the first time, we show the effectiveness and the outcome of patients treated in this new structure in Germany. Our data contributes to further evaluations of specialized palliative care teams. On the basis of this data, guidelines and quality indicators for the health system in Germany must be developed.

Abstract number: P01-254

Abstract type: Poster

Opioid Pathways - Access and Barriers for the Use of Opioids for Medical Purposes in Argentina

März L. ${ }^{1}$, Minatel M.D.I.A. ${ }^{2}$, Pastrana T. ${ }^{3}$

${ }^{1}$ Palliative Medicine, University Hospital RWTH Aachen, Hamburg, Germany, ${ }^{2}$ Asociación Argentina de Medicina y Cuidados Paliativos, Buenos Aires, Argentina, ${ }^{3}$ Palliative Medicine, University Hospital RWTH Aachen, Aachen, Germany

Background: Pain medication forms a cornerstone in palliative care. Although advances in the provision have been made in Argentina, people do not have sufficient access.

Aim: This study analyzes pathways for opioids in Argentina and their barriers.

Method: This qualitative study is based on Actor Network Theory and identifies the key actors on the pathway from importation to the patient. Data collection was done using problem-centered interviews and analyzed based on Grounded Theory. The field work in Argentina was carried out between January 2018 and April 2018.

Results: 39 interviews were conducted using theoretical sampling. 21 physicians, 6 pharmacists, 6 patients and 6 regulators were interviewed. This study identifies and follows the steps in opioid distribution, from manufacture to patient administration, thereby illustrating this dichotomy. The fragmentation of the system, along with the bureaucracy it entails, form the two greatest obstacles for the access. Furthermore, there exist many myths and prejudices against morphine. Therefore, the engagement of single palliative care physicians becomes essential. Conclusion: Opioid pathways constitute a complex process that requires a high level of collaboration between the actors involved in providing access to opioids. This study offers a basis for the improvement of the access to opioids for palliative patients.

\section{Abstract number: P01-255 \\ Abstract type: Poster}

Patient and Public Involvement in Palliative and End of Life Care Research

Johnston B.

University of Glasgow, School of Medicine, Dentistry and Nursing, Glasgow, United Kingdom Presenting author email address: bridget. johnston@glasgow.ac.uk

Background: The evidence base for patient and public involvement in health research and within palliative and end-of-life care is expanding. Moderate evidence has shown that involvement can result in more 
relevant, readable and understandable patient information for research studies. As well as, increased knowledge, skills, confidence, personal support, and emotional and practical demands of members.

The team have recently set up a patient and public involvement (PPI) group at University of Glasgow. Our aim is to meaningfully involve, using novel and creative methods, group members from diverse backgrounds in our palliative and end of life care research.

We have recently been selected as one of 10 NIHR test bed national standards for public involvement in research https://sites.google.com/ nihr.ac.uk/pi-standards/test-beds/test-bed-projects.

This paper will draw our achievements and present novel ways of working with people at the end of life.

Materials and methods: Thematic analysis of group participation and key priorities for research benchmarked against the NIHR test bed standards.

Results: We will present results from a workshop (standard 3)conducted with support from Glasgow School of Art using creative and inclusive methods to co create research ideas with people and their informal carers facing life limiting illnesses. Themes matched against the standards include:

\section{Experiences of care Standard 1, Standard 2}

Participants have identified difficulties experienced in their stories of care and the need for more emotional and practical support.

2. Priority areas for end of life care research standard 2 identified by our group.

3. Different ways are members are involved in our research

4. Ways we communication with our group 9 standard 4)

Conclusions: The importance of patient and public involvement are strongly endorsed by our group we will present ways we are achieving the NIHR test bed standards and plans for future work and engagement.

\section{Abstract number: P01-256 \\ Abstract type: Poster}

Hospice Care, What to Expect? An Exploration of the Expectation of Future Hospice Patients

van Klinken M., de Graaf E., Bressers R., van der Baan F., Teunissen S. Center of Expertise in Palliative Care Utrecht, Dept. General Practice, Julius Center for Health Sciences and Primary Care, University Medical Center Utrecht, Utrecht, Netherlands

Introduction: Hospice care aims to optimize the quality of life of patients and their families in the last phase of life by diminishing physical, psychological, social and spiritual suffering. Expectations are associated with patient satisfaction.

A theoretical framework was used, distinguishing expectations with respect to process, structure and outcomes.

Aim: Explore expectations of hospice care of future hospice patients. Methods: A generic qualitative study was performed from January to July 2018.

Eleven patients with a life expectancy $<1$ year ('surprise question') were enrolled. In addition, two patient advocates were included to gain a broader perspective.

Semi structured interviews were transcribed verbatim, Thematic analysis was performed by 2 researchers independently.

Results: The participants characteristics varied with respect to: gender, age, spiritual beliefs, living area, primary diagnosis, phase of palliation.

Participants expect to be admitted to hospice when death is imminent and dying at home becomes impossible. They expect a homelike environment and the ability to continue living like being at home.
Participants are sure that hospice caregivers are loving and trustworthy. Without doubt patients expect them to provide appropriate care and treatment with the skills and knowledge necessary.

Expectations on outcomes are high, since medical and daily care is provided by competent and highly skilled professionals. Additional support from volunteers is expected specifically in the form of "being there".

Conclusion and discussion: Hospices have the responsibility to ensure nuanced and balanced information on hospice care in the Dutch society to set realistic expectations. Information about the possibilities and aims of hospice care, including short stay admissions, respite care and support at home in collaboration with primary caregivers should be widespread to ensure timely and appropriate hospice care for all patients in need.

Abstract number: P01-257

Abstract type: Poster

Understanding 'Good Care' from the Perspective of Oncology Nurses: An Ongoing Balancing of Proximity and Distance Botman F. ${ }^{1,2}$, Brown P. ${ }^{1}$, Eeden-van Dam E. ${ }^{2}$, Buiting $\mathrm{H}^{2}{ }^{2}$

${ }^{1}$ Medical Antropology, University of Amsterdam, Amsterdam, Netherlands, ${ }^{2}$ Antoni van Leeuwenhoek Hospital, Amsterdam, Netherlands

Background: Advances in oncology increasingly result in protracted disease trajectories for patients with incurable cancer. As a result, most patients with incurable cancer see their nurses regularly and intensively for a longer time period. This can result in the development of deeper relationships, potentially posing emotional strain on nurses.

Aims: We explored the understandings and practices of oncology nurses regarding 'good care' for patients in protracted disease trajectories.

Methods: Qualitative ethnographic study based on twenty days of observations and in-depth interviews, lasting between 15 and 40 minutes, with 13 oncology nurses, at the day-care unit of a Dutch comprehensive cancer hospital. Data-analysis was inspired by grounded theory.

Results: Oncology nurses described their practices of care as involving an ongoing balancing of meaningful proximity and distance towards their patients. Some forms of proximity, in the sense of hearing and getting to know the patient and seeing the patient as a person, contributed to individual, patient-centred care, which was interpreted by nurses as core to good care. Proximal care helped in establishing a nurse-patient relationship involving trust. Consequently, nurses experienced that having a trustful relationship facilitated dealing with patients' feelings, as it opened up the possibility for dialogue. However, while most nurses agreed that proximity was important, the drawbacks to proximity were frequently reported too. Nurses agreed that a certain distance to patients, particularly with incurable cancer, was important to keep nursing doable.

Conclusion: Oncology nurses commonly understand proximal care as contributing to good care, but simultaneously express that keeping some distance is important to keep their professional work feasible. Hence, to ensure good care for patients, nurses continually aim to manage tensions between, and to balance, proximity and distance in caring relationships.

\section{Abstract number: P01-258}

Abstract type: Poster

Experience of Providing Palliative Care in a Rural District in Uganda

Muwanguzi D.1,2

${ }^{1}$ Makerere and Mulago Palliative Care Unit, Medicine, Kampala, Uganda, ${ }^{2}$ Makerere University, Medicine, Kampala, Uganda 
Background: Palliative care (PC) development has made significant progress in many parts of Uganda especially in health facilities however in many rural areas it is difficult to have continuity of care after discharge, especially when people spend most of their time in the community and often prefer to die there yet there are few home based care options. Butemba Health Center which is 160 kilometers from Kampala the capital city started to meet this need to ensure continuum of care and quality of life.

Aim: To develop a palliative care service based at a rural health center to include home based care.

Method:

1. Starting in the HIV clinic the lead clinician followed up patients (PTS) to their homes.

2. Following $P C$ specialist/ leadership training the PC services were fully integrated into the HIV clinic to respond to an increasing number of PC referrals from the community.

3. Negotiation with the District Supervisor led to a sustainable plan. Home visits were supported with a motorbike and follow up by phone.

4. 12 health workers were trained in basic PC services.

Results: It has reduced overcrowding on the ward and unwanted emergencies at outpatient department from an average of 1040 in 2013 to 156 PTS in 2017.

The number of PTS seen at the HIV clinic has reduced from a weekly average of 360 in 2013 to 60 in 2017.

This coordinated model of home care has ensured that bed-bound patients receive PC. The number of home visits has increased from 52 in 2013 to 208 in 2017.

It has provided a good referral system between the facility and the community. The staffs and the family members have a better understanding of PC.

Conclusion: Model of care which combines facility based PC services with home based care services ensures continuity of care.

Effective use of available resources is important in a resource limited settings like Uganda.

Home based care has ensured the equality of care for both mobile and bed-bound PTS.

Abstract number: P01-259

Abstract type: Poster

Attitudes of Primary and Specialized Palliative Care Physicians towards Early Palliative Care

Sorensen A., Le L., Hannon B., Krzyzanowska M., Wentlandt K., Rodin G., Zimmermann $C$.

University of Toronto, Toronto, ON, Canada

Background: Evidence supporting early palliative care is based on trials of specialized palliative care (SPC), but a more sustainable model might involve mainly primary palliative care (PPC) providers.

Aims: To compare characteristics of PPC and SPC physicians, their attitudes toward early palliative care, and their perception of their resources to provide it.

Methods: A survey was distributed by mail and e-mail to Canadian physicians identified by the Canadian Society of Palliative Care Physicians as providing palliative care. SPC were defined as receiving palliative care referrals from other physicians and not providing palliative care only for their own patients.

Results: Of the 531 participants ( $71 \%$ participation rate), 274 (52\%) practiced PPC and 257 (48\%) SPC. SPC physicians were more likely to work in urban centers ( $94 \%$ vs $76 \%, p<0.001$ ) and to provide mainly cancer care ( $84 \%$ vs. $65 \%, p<0.001$ ); $65 \%$ of PPC and $29 \%$ of SPC physicians had no palliative care training. Although almost all physicians favored early palliative care, only half in each group agreed they had sufficient resources to deliver it. Agreement with having sufficient resources to do this was stronger among family physicians (OR, 1.78 [1.15-2.74], those working on teams (OR, 1.75 [1.04-2.84]), and those with greater availability of community/psychosocial support (OR, 1.50 [1.01-2.24]). PPC physicians were more likely to agree that the specialty should be renamed "supportive care" ( $36.2 \%$ vs $20.4 \%, p<0.001)$ and that this would increase patient comfort with early referral ( $47 \%$ vs. $36 \%, p<$ 0.001).

Conclusion: Early palliative care is supported by most SPC and PPC physicians, but both groups lack resources for it. An early palliative care model may most be feasibly provided by team-based family physicians, with select SPC referral. There is disagreement among palliative care providers regarding the value of renaming the specialty.

\section{Abstract number: P01-260}

Abstract type: Poster

\section{Stigmatised Changed 'Social Identity' in Men: Consequences of} Prostate Cancer?

Salifu Y. ${ }^{1}$, Almack K. ${ }^{2}$, Caswell G. ${ }^{1}$, Nottingham Centre for the Advancement of Research into Supportive, Palliative and End-of-life Care (NCARE)

${ }^{1}$ School of Health Scieces, University of Nottingham, Nottingham, United Kingdom, ${ }^{2} \mathrm{School}$ of Health and Social Work, University of Hertfordshire, Hertford, United Kingdom

Background: One in 8 men develops Prostate Cancer (PC); however, the risk is double for black African, Asian and Caribbean men with high mortality.

This study sought to find out how men living with prostate cancer experience the changes in the masculine identity and the possible stigma associated with it and how they react to such changes.

Methods: Twenty-three men with prostate cancer and 23 family caregivers were interviewed.

Focus group and interviews were conducted with 12 healthcare professionals.

Men and the caregivers were involved in two sessions of interviews (repeat interview).

The study lasted for lasted for 6 months.

Thematic Analysis technique was to identify patterned meaning across total of 68 interviews. This was done in iterative manner to allow emergence of themes from within the data.

Three overarching themes were developed from the data.

Findings: This study focuses on the theme 'Stigma and Masculinity changes'.

Prostate cancer is stigmatised as a promiscuous disease.

Incontinence affects men's dignity and led to felt and courtesy stigma.

The changing physical appearance impact on their identity as men and also affect their masculine role as leaders, breadwinners and head of the household.

Prostate cancer leads to reduced sexual activity or impotence that made the men felt 'not man enough' and a feeling of 'living dead'.

Conclusion: Although evidence of supportive family was noted, there were challenges of men living with a 'new' identity due to prostate cancer.

Prostate cancer presents different experiences and coping strategies. This depends on the support available for them, whether or not they experience stigma, the type of treatment option used to manage cancer, their emotional resilience among others. The need to enhance existing informal support by the family. Health and social policies could coordinate and improve the quality of lives of men living with prostate cancer.

Abstract number: P01-261

Abstract type: Poster 
One in Ten Older Adults Aged $\geqslant 45$ Years Report Having No-one to Turn to for Help at the End of Life Apart from Professionals Who are they?

Verne J. ${ }^{1}$, Tapp A. ${ }^{2}$, Nancarrow C. ${ }^{2}$, Morey Y. ${ }^{2}$, Warren S. ${ }^{2}$

${ }^{1}$ Public Health England, Bristol, United Kingdom, ${ }^{2}$ Faculty of Business and Law, University of the West of England, Bristol, United Kingdom

Background: There is increased interest in the impact of living alone and loneliness on people's wellbeing at the end of life and practicality of providing care, especially at home. In a nationally representative sample of 3,590 people aged $>45$ years surveyed on-line about care at the end of life (EoL) $12 \%$ said they had no close family, friends or neighbours that they felt they could turn to for help.

Aim: To investigate the characteristics of the $12 \%$ who feel they have no-one to turn to (NTTT).

Method: Analysis of the sociodemographic characteristics of the NTTT $12 \%$ (441 people) compared with those who did declare family, friends or neighbours (FFN) who they reported could help $88 \%$ (3149) using the results of the online panel survey.

Results: More NTTTs were younger aged 45-69 (77\%) than the FFN (71\%) and still working 37 vs. $26 \%$. The NTTTs were more likely to live in London $19 \%$ and urban areas. The NTTTs were less likely to: be married/partnership, have children and grandchildren, have family living with them or nearby, belong to a local club/society/association, belong to a religion. $52 \%$ of the NTTT said they had never cared for a relative compared with $38 \%$ FFN but of those who had, a higher proportion was for a spouse/ partner 17 vs. $11 \%$ and more likely to have undertaken personal hygiene 38 vs. $29 \%$ and cared $>50$ hours per week 21 vs. $12 \%$. When asked who they would want to care fewer NTTTs would want family $26 \mathrm{vs.51 \%}$ and more professionals 23 vs. $16 \%$ or a care home 23 vs. $12 \%$. Asked why family would not be first choice reasons for NTTTs vs. FFNs included: my family would probably prefer not to be involved $14 \mathrm{v} v \mathrm{vs} .9 \%$ we don't get on 8 vs $4 \%$, I have no family left 13 vs.7\%.The NTTT were less likely to have thought about planning care for themselves 29 vs. $35 \%$.

Conclusions: This study has identified and explored the characteristics of a potentially vulnerable group for EOLC. The results will help professionals identify people needing extra state or volunteer input.

Abstract number: P01-263

Abstract type: Poster

The Past Is Present: Death Systems among the Indigenous Sámi in Scandinavia

Kroik L. 1,2, Lindqvist O. ${ }^{3}$, Stoor K. ${ }^{4,5}$, Tishelman C. ${ }^{2,3,6}$, part of the DöBra Research Program

${ }^{1}$ Umeå University, Dept of Nursing, Umeå, Sweden, ${ }^{2}$ The Center for Rural Medicine, Storuman, Sweden, ${ }^{3}$ Karolinska Institutet, LIME/ Division of Innovative Care Research, Stockholm, Sweden, ${ }^{4}$ Umeå University, Dept of Lanugage Studies, Umeå, Sweden, ${ }^{5} U$ meå University, Center for Sámi Studies-Vaartoe, Umeå, Sweden, ${ }^{6}$ Stockholm Health Care Services, Stockholm, Sweden Presenting author email address: carol.tishelman@ki.se

Background and aim: Kastenbaum articulated the death system concept to examine "the interpersonal, sociophysical, and symbolic networks through which an individual's relationship to mortality is mediated by society." Despite growing interest in Indigenous health, there is virtually no research on the Sámi people of northern Fennoscandia regarding the end-of-life (EoL). We therefore explored experience-based knowledge about EoL issues among the Sámi. We discuss here how symbols of specific Sámi identity are related to these death systems and the extent to which Kastenbaum's conceptualization of death systems is appropriate to Sámi culture.

Methods: The database consists of transcribed interviews with 15 individuals, chosen for their varied experiences with EoL issues among Sámi.
These were first inductively analyzed using a qualitative approach. Kastenbaum's model of death systems, consisting of functions along a time trajectory from prevention to social consolidation after death, and the components people, times, places, and symbols/objects, was applied thereafter. The model provides a cohesive framework for understanding aspects of the death system that were Sámi-specific, Sámirelevant as well as what has been lost and retained over time.

Results: Whereas Kastenbaum differentiated among the components of the death system, we found these were often interrelated among the Sámi. Seasonal changes and relationships to nature instead of calendar time, dominated many aspects of death systems, linking people, places and times. The role of the extended family in enculturation across generations and support throughout the EoL was salient. Numerous markers of Sámi culture, both death-specific and those recruited into the death system, served to strengthen community identity in the EoL.

Conclusions: The Sámi identity and sense of community appeared central to both that which was described as functioning well in death systems, as well as that which was said to be problematic.

Abstract number: P01-264

Abstract type: Poster

Creating Peaceful Spaces? - Hospice Philosophy in Danish

Hospice Practice

Graven V., Timm H.

REHPA, University of Southern Denmark, Nyborg, Denmark

Background: The first Danish hospice opened in 1992 and today there are 20 hospices which are integrated within the main stream healthcare system. The still sparse literature on Danish hospices indicates that hospice philosophy is influencing practice. However there is no research which explores these influences at an institutional level nor how hospice philosophy values fit with mainstream healthcare.

Aim: To explore the nature of hospice philosophy in Danish hospice practice.

Methods: The research is informed by participant observation and interviews with professionals, patients and families at three hospices in different regions of Denmark (May 2017 - February 2018). The role as researcher was balanced between observation of daily activities such as staff meetings and routine practices and active participation in daily activities plus conducting interviews with patients, relatives and staff.

Findings: Hospice philosophical values work in practice as interpreted ideals adapted to: the individual dying person and his/her close ones and evidence based and managerial conditions for care. This means that for example a context of open awareness of dying is balanced with the dying person's beliefs, palliative pain relief is balanced with evidence and the atmosphere of homeliness is balanced with functionality due to medical and labour purposes, spirituality is adapted to different approaches. These interpreted values contribute to creating a peaceful space for dying people and their relatives within the mainstream healthcare system.

Conclusion: In practice hospice philosophy does not operate with fixed values but works as a 'lived' philosophy which shapes and is shaped by practice. Tensions between hospice philosophy, evidence based practice and managerialism as guiding logics for practice should be further explored.

Funded by The Organisation of Danish Hospice Managers.

Abstract number: P01-265

Abstract type: Poster

An Exploration of Patients' Preferences and Priorities for End of Life Care at Tiyanjane Clinic for Palliative Care, Queen Elizabeth Central Hospital, Blantyre, Malawi

Chitani A. 
Queen Elizabeth Hospital Birminghamueen Elizabeth Central Hospital, Medicine, Blantyre, Malawi

Background: For patients to have a dignified death, their wishes should be known and respected. The challenge is that, if conversations about death are avoided by patients, their families and health professionals because talking about death is taboo, it becomes hard to plan and implement preferred care for such patients. The topic is regarded as sensitive and taboo in Malawi because discussing end-of-life care preferences and priorities is taken as prophesying death.

Aim of the study: This study aimed to explore preferences and priorities for end of life care experienced by adult patients with a terminal diagnosis.

Methods: This was a qualitative, descriptive study. The study purposely sought to recruit between 10 and 14 participants until data saturation was reached. Data was collected by means of semi-structured interviews that were recorded and afterwards transcribed verbatim. A thematic framework was used to inform the systematic approach to data analysis. Results: In December 2015 and January 2016, interviews were conducted with 14 adults (six men and eight women). The following 10 themes emerged from the data: Awareness of reason for being at Tiyanjane; reluctance to mention HIV; psychosocial concerns, expressed emotions; legacy; receiving information; decision-making; place of care; place of death; and spiritual concerns.

Conclusion: This research shows that, although choices are limited in Malawi due to a lack of resources, patients need to be given an opportunity to make their own healthcare choices for end of life care. This research has shown that it is not as hard to start end-of-life care conversations as previously thought. Further research needs to explore whether advance directives or legal wills should be offered as part of care for palliative patients in Malawi, as most of the participants highlighted the need to be heard in regard to their wishes being upheld after death

Abstract number: P01-266

Abstract type: Poster

\section{Is Socioeconomic Status a Fundamental Cause of Racial} Differences in End-of-Life Care Use?

Cross $S$.

Duke University, Sanford School of Public Policy, Durham, NC, United States Presenting author email address: sarah.h.cross@duke.edu

Background: De-medicalized dying is now the innovation in end-of-life (EOL) care and evidence indicates that more-educated people are the first to take advantage of medical advances. Research has yet to examine racial differences in EOL care use from this perspective.

Aims: This study builds on fundamental cause and diffusion of innovation theories to examine if education mediates the relationship between Black race and three outcomes, commonly accepted as indicators of EOL care quality: hospice use $(n=1214)$, life support use $(n=917)$, and hospital death $(n=1196)$.

Methods: Using from the Health and Retirement Study 2014 Core and Exit Interviews, I used modified poisson regression to assess the relationship between race and EOL outcomes. I then adjusted for level of education to assess the mediating effect of race on these outcomes.

Results: In unadjusted models, Black race was significantly associated with lower risk of hospice use (unadjusted risk ratio $\mathrm{RR}=.813 ; \mathrm{p}=$ 0.023 ), greater risk of life support use (unadjusted $R R=1.48 ; p=0.001$ ) and greater risk of hospital death (unadjusted $R R=1.29 ; p=0.013$ ), all indicators of poor quality EOL care. Results found that education level mediated the effect of Black race on hospice use $(a R R=.839 ; p=0.060)$ but not on life support use ( $a R R=1.49 ; p=0.001)$ or hospital death $(\mathrm{aRR}=1.29 ; \mathrm{p}=0.014)$
Conclusion: Socioeconomic status (SES), as measured by years of education, appears to be a fundamental cause of racial inequities in hospice use. These findings imply that hospice use, life support use and hospital death are associated with different social factors and that resources may not equally influence different types of care received at the EOL. Quality indicators may ignore cultural differences in patient preferences. Future research is needed to better understand barriers to hospice use among those of lower SES and to examine drivers of EOL care use by non-White Americans.

Abstract number: P01-267

Abstract type: Poster

Between the "Clinic" and the "Home": How Patients and

Families Negotiate Home Care at the Ends of Life Llewellyn H. ${ }^{1}$, Higgs P. ${ }^{2}$, Sampson E.L. ${ }^{1}$

${ }^{1}$ UCL Marie Curie Palliative Care Research Department, London, United Kingdom, ${ }^{2}$ UCL Division of Psychiatry, London, United Kingdom

Background: UK End of Life Care is often centred on supporting dying at home. This includes introducing clinical technologies to the home which make dying at home comparable to hospital safety standards. While this is assumed to challenge the medicalization of death and respect patient preferences, it also blurs lines between clinic and home and introduces major challenges to patients and families.

Aim: To examine the medicalization of domestic space and how patients and families negotiate the challenges of dying at home.

Method: Data are drawn from an ethnography of care and decisionmaking among people with brain tumours (2014-16). This involved participant observation in clinical and domestic settings and repeated semi-structured interviews with 16 patients and their families as they approached death. Data were analysed thematically with focus on the social and spatial contexts of the home and how these changed to accommodate dying.

Results: Dying at home was characterized by divergent assumptions of patients, families and clinicians. Patients and families encountered crises of authority, invasions of privacy, and medicalized environs that undermined as much as sustained their images of a "good death." Amid changes to their domestic spaces, patients and families employed tactics to maintain intimacy, preserve privacy and appropriate new technologies.

Discussion: Dying at home is complex. It might best be considered as dying in a "hybrid space." Hybrid spaces are neither wholly home nor hospital, and unfamiliar to clinicians, patients and families. While the image of "home" is critical to discourses around dying at home, it obscures the moral confusion, negotiation and equivocation inherent in attempts to maintain a safe, familiar and intimate space.

Conclusion: Understanding dying at home through the lens of hybrid space helps disrupt illusions that home is a stable place. Rather than hiding the competing logics of care and disruption, it foregrounds them.

Abstract number: P01-268

Abstract type: Poster

\section{Gender and Diversity at the End of Life}

Araujo Hernández M. ${ }^{1}$, García Navarro S. ${ }^{2}$, Pérez Espina R. ${ }^{3}$, García-

Navarro E.B.

${ }^{1}$ Facultad de Enfermería, Universidad de Huelva, Huelva, Spain

${ }^{2}$ Distrito Sanitario Huelva-Costa-Condado-Campiña, Huelva, Spain,

${ }^{3}$ Hospital Vázquez Diaz, Huelva, Spain

Introduction: Gender has some cultural elements conditioned by roles and stereotypes which are established in different cultures. These stereotypes exist from the start to the end of life, taking a bigger importance in the life processes that it unfolds. 
One of these relevant processes is the end of life. Incomparable experience which generates changes to all different spheres of a human being. The way in which this process is experienced will be directly connected with the biography of the dying person, their family, social and cultural surroundings and gender.

The way in which people go through this process will generate experiences that will make them unique and therefore, creditor of specific and individual needs. The approaches to these specific needs are necessary to offer a care that is sensitive to gender and the needs that this will require. Objectives:

1. Identifying gender influence at the end of life

2. Determining specific needs depending on gender at the final life process

Methodology: The methodology that has been used in order to know the needs of the participants is qualitative. In-depth interviewing was the instrument that was used.

Conclusion: Gender is a crucial element in the final experience of life, which will condition primarily two elements: family planning and worries expressed by the family.

In relation to family planning, the main issue for men is to control and care for the economic situation of the family and their sustenance when they are not there anymore.

For women, the emotional side takes more importance as they worry about who will bring the emotional sustenance to the family in their absence.

Women find it easier to express feelings, which favours anxiety liberation before death, whereas men have cultural castration which is conditioned by gender with regards to expressing feelings, which complicates facing this process.

\section{Abstract number: P01-269 \\ Abstract type: Poster}

The latrogenic Suffering among Advanced Cancer Patients: A Phenomenological Study

Rodríguez-Prat A., We Care, End-of-Life Care

Universitat Internacional de Catalunya, Faculty of Humanities,

Barcelona, Spain Presenting author email address: arodriguezp@uic.es

Background: Research has shown that patients with life-threatening conditions can experience illness as a series of losses: loss of identity, loss of functionality, loss of control and autonomy, etc. This series of losses is derived from the process of decline due to the illness and is usually accompanied by suffering. On top of this suffering, other types of malaise are generated by the clinical context (iatrogenic factors).

Aims: To explore what iatrogenic factors contribute to suffering among advanced cancer patients.

Methods: We conducted semi-structured qualitative interviews using an interpretive phenomenological analysis approach. Eight advanced cancer patients from an oncology unit from Barcelona (Spain) were interviewed. A purposive sample was used. The interviews were recorded and analysed using Atlas.ti v.8.

Results: We identified 6 themes that emerged from the data:

1. The long process of detection of the illness.

2. Lack of understanding of the patient's situation (from others)

3. False promises from healthcare professionals (HPs).

4. Limitations due to medical apparatus.

5. Lack of commitment from healthcare professionals.

6. Inadequate hospital environment for the patient's needs.

Conclusion / Discussion: The impact of the hospital environment on patients is very important for the process of managing the illness. A lack of appropriate attention from HPs and not knowing the consequences of some medical interventions can undermine the perception of dignity and agency in these patients. When looking further at the experiences of patients and being aware of their increased vulnerability could help to design care plans centred on the needs of these patients.

\section{Abstract number: P01-273 \\ Abstract type: Poster}

Cultural Impact on the Palliative Care of Cancer in the World Maincent C. ${ }^{1}$, Masson J. ${ }^{2}$, Quenot J.P. ${ }^{1}$, Aubry R. ${ }^{3}$, Bonniaud P. ${ }^{1}$ ${ }^{1} \mathrm{CHU}$ Dijon, Dijon, France, ${ }^{2} \mathrm{CH}$ Chalon sur Saône William Morey, Chalon sur Saône, France, ${ }^{3} \mathrm{CHU}$ Besançon, Besançon, France

Introduction: In oncology, the end-of-life care faces to many conceptual and organizational difficulties.

Objective: To understand the link between culture and palliative care in oncology and to open up lines of reflection for a better management of the patient at the end of life.

Method: Qualitative study with an approach phenomenologic and ethnographic. Thirteen doctors around the world were interviewed with a questionnaire on eight themes: euthanasia and legislation, palliative care organization, medical training, broncho-pulmonary cancer symptomatic therapy, artificial nutrition and hydration, financial management, medicine traditional and religion.

Results: The cross-sectional analysis brought out 11 sub-themes highlighted by horizontal overlap about the key cultural aspects impacting palliative care. Among other things, we have noted the importance of end of life at home and the place of the family in Asia and South America. Medical training in end-of-life care and drug therapies is essential. In addition, traditional medicines have uniformly left the hospital structures despite an importante interest of patients.

Conclusion: The diversity and complexity of palliative care around the world result from culture, history, political and religious, human and financial means. Many lines of thought are highlighted in order to improve care in France, such as the role of the family, the care of the patient in his entirety, and the acceptance of death.

\section{Abstract number: P01-274}

Abstract type: Poster

Friendship in the Stable Phase of Incurable Cancer van Eijk M. ${ }^{1,2}$, de Vries $D^{1}{ }^{1}$, Buiting $H_{.}{ }^{2}$

${ }^{1}$ Medical Antropology, University of Amsterdam, Amsterdam, Netherlands, ${ }^{2}$ Antoni van Leeuwenhoek Hospital, Amsterdam, Netherlands

Background and aims: The incurable phase of cancer is not only an intense period for patients and close relatives, but can be considered an intense period for friends as well. We explored friends' experiences in supporting patients in the stable phase of incurable cancer. We eventually wanted to explore how social networks can contribute to a patient's well-being.

Methods: Qualitative study based on 14 in-depth interviews with family members and friends of patients living with protracted incurable cancer. Analysis was done using grounded theory.

Results: During all interviews it became clear that participants' experiences with their ill friends had also left its prints on their personal ideas about cancer. Although participants reported that the absence of bad news for some time could result in little urgency to invest in the relationship, uncertainty to a certain extent always remained. Participants emphasized the ever-present knowledge of cancer inside the body as a time bomb. In this time-period, the extent to which a patient was transparent about her own emotions influenced the amount and type of support of friends. Some participants that were included in this study felt 
burdened with or forced in providing more care than they were willing to provide. Nevertheless, participants also indicated that there might be circumstances to step in more than they were initially willing to at some point.

Conclusion: Patients' disease experiences can sometimes be considered unexpectedly helpful for friends as such situations provided options to reflect about their own life. At the same time, friends are constantly negotiating and renegotiating their relationship depending on the severity of the disease, their experiences with previous cancer patients, and personal circumstances in life. Just as close relatives, friends need to be protected against providing more care then they are willing or able to give.

Abstract number: P01-275

Abstract type: Poster

The Impact of Disease Labels on Disease Experience in Patients with Prolonged Incurable Cancer: A Qualitative Study

Busink V. ${ }^{1,2}$, Ho V. ${ }^{3}$, van Tol D. ${ }^{4}$, Buiting H. ${ }^{1}$

${ }^{1}$ Antoni van Leeuwenhoek hospital, Amsterdam, Netherlands, ${ }^{2}$ Clinical

Psychology, VU University Amsterdam, Amsterdam, Netherlands,

${ }^{3}$ Netherlands Comprehensive Cancer Organisation (IKNL), Utrecht,

Netherlands, ${ }^{4}$ University Medical Center Groningen, Groningen,

Netherlands

Background and aims: Advances in medicine have resulted in prolonged disease trajectories, increasingly even in those with incurable cancer. These prolonged disease trajectories have induced discussions about the 'right' medical terminology, and the impact of medical terminologies on patients' well-being. We examined the impact of disease labels on disease experience in patients with prolonged incurable cancer.

Methods: Qualitative study based on participant observations and 33 conversations at the day-care unit in two stages covering a period of 2 years (2015-2017). We especially looked at disease-labeling in an indirect way to avoid possible bias.

Results: Patients ascribed different disease labels to themselves, either on purpose or not. They varied in their preference regarding specific disease labels (e.g. 'chronic', 'palliative', 'stable') in their communication about the incurable nature of their disease. Patients' use of diseaselabels seemed to be related in how they coped with their situation. Patients showing a positive or neutral mood were often more comfortable with the label 'chronic', whereas patients with a more negative mood more often felt they could become trapped in a definition. Some patients preferred not to label their disease at all, as they felt it would make them 'less human'. Although some labels used by healthcare professionals bothered patients, their impact on disease perception seemed low as patients often decided to stick to their own, more 'positive' disease label. Conclusions: Patients' explicit use of disease labels can be part of their coping strategy. Healthcare professionals' use of disease labels, on the other hand, may cause confusion. In a world where the diagnosis of incurable cancer is not a direct death sentence anymore, appropriate use of disease labels by healthcare professionals is warranted: to explore how they can contribute to patient's well-being as well as the decision-making process.

Abstract number: P01-276

Abstract type: Poster

Difficulties in Providing Palliative Care in Rural India (West

Bengal) - Experience of an NGO

Manna A.

Narikeldaha Prayas, Purba Medinipur, India

Introduction: As in any developing countries state of West Bengal in India has a huge burden of cancer patients in advanced stage coming from rural area where awareness regarding the usefulness of palliative care in rather poor.
Objective: Our goal is to give a pain free good quality of life in these advanced stage cancer patients. Objective of this study is to identify the main difficulties in achieving the above goal in a rural village setting in India. Method: Advanced cancer patients in need of palliative care in various villages in of rural India were selected for this study. Their symptoms and managements in that rural surroundings were evaluated by an NGO (under the guidance of a senior palliative care specialist) working in that area. An attempt was made to identify the main obstacles in getting proper palliative care in a rural setting.

Results: Pain, fatigue are the main symptoms effecting these patients. In most patients pain and other symptoms control were grossly inadequate due to lack of properly trained manpower in the rural India. However regular homecare visits by a group of social workers were of immense help in the last few months of life. NGO team was well guided by a palliative care specialist.

Conclusion: There is a wide gap of trained manpower in this filled in rural areas of India. Dedicated groups from rural area itself need encouragement and proper training, so that difficult symptoms can be managed locally along with necessary social and psychological support to these patients.

Abstract number: P01-278

Abstract type: Poster

Palliative Care in Crisis Situations: Catastrophic Disasters and Humanitarian Aid Response

Wilkinson A.,

Palliative Care Edith Cowan University, School of Nursing and Midwifery, Joondalup, Australia Presenting author email address: anne. wilkinson@ecu.edu.au

Both catastrophic disasters and humanitarian crises can have widespread, long-term and often unpredictable negative impacts, causing shortages of safe water, food, shelter, transportation and sanitation as well as the displacement of large numbers of people and the loss of many lives. Routinely ignored in disaster and humanitarian planning are those socially and medically vulnerable populations living in the affected community who are dependent on the existing healthcare system for survival, making them particularly predisposed to the risk of harm or death due to the event. Because of their unique needs, extra steps need to be taken in order to reduce the likelihood that vulnerable populations will suffer injury, disease, or even death. Serious health-related suffering resulting from illness or injury stemming from a natural or man-made crisis could be ameliorated by the inclusion of palliative care services in planning and response activities. While the primary goal of disaster medicine is to maximize the number of lives saved and to ensure the best outcomes for the community as a whole; a holistic and fully compassionate response necessitates a secondary goal of minimizing the physical and psychological suffering of those whose lives may be shortened by a crisis event. In order to effectively provide palliative care services in crisis situations, all care delivery sites, especially triage and alternative care sites, will need clearly articulated protocols, trained staff (including personnel with palliative care, mental health and spiritual counselling skills), supplies (including those necessary for appropriate pharmacologic treatment), and appropriate space for dying patients. Details of recommended steps to incorporate palliative care into disaster and humanitarian aid response will be outlined and discussed.

Abstract number: P01-279

Abstract type: Poster

TalkCPR - A National Project to Evaluate Technology Resources in Do Not Attempt Cardiopulmonary Resuscitation Discussions Taubert M. ${ }^{1,2}$, Edwards S. ${ }^{3}$, Snow V. ${ }^{4}$

${ }^{1}$ Palliative Care Department, Cardiff University School of Medicine, Cardiff, United Kingdom, ${ }^{2}$ Bevan Commission, Swansea University 
School of Management, United Kingdom, ${ }^{3}$ Cardiff University School of Medicine, Cardiff, United Kingdom, ${ }^{4}$ Byw Nawr Live Now Coalition, Cardiff, United Kingdom

Background: A national steering group in Wales pioneered information videos and a website for patients, carers and healthcare professionals, forming part of a quality improvement program. Videos were planned, scripted and produced with healthcare professionals and patient/carer representatives, and were completed with both English and Welsh language versions. The TalkCPR videos promote open discussion about Cardiopulmonary Resuscitation (CPR) \& DNACPR in palliative care situations.

Methods: Patient/carer groups evaluated whether video resources to convey the salient facts involved in CPR and DNACPR decisions for people with palliative and life-limiting illness were acceptable or not. We conducted a mixed-method design service review in five phases to evaluate whether this technological resource could help. After creating video and website materials, they were evaluated by doctors, nurses and a patient/carer group. We also sent out one lightweight TalkCPR video media pad to each practice in Wales. These rechargeable electronic video media pads have communication videos loaded in for easy viewing, especially in areas with poor roaming data coverage.

Results: Videos were demonstrably acceptable to patient \& carer groups and improved healthcare professional confidence. Videos went live on the TalkCPR website and Youtube and are now used in routine practice throughout Wales and the UK.

Conclusion: This is the first time that DNACPR information videos are aimed directly at palliative care patients and carers, to explore this sensitive subject with them, and to encourage them to approach their doctor or nurse about it. The website, app and video media pads were developed by patients, the Digital Legacy Association, Welsh NHS IT services, Welsh Government, the Bevan Commission and the Dying Matters Charity in Wales 'Byw Nawr'. A stepwise implementation approach was used to ensure that content would not be distressing or insensitive to viewers. http://talkcpr.com

Abstract number: P01-280

Abstract type: Poster

A 2-year New Experience of Advance Care Planning Made under Patient Autonomy Act and the Special Elements Found in Eastern Culture

Hsieh W.-T. ${ }^{1}$, Lin Y.-C. ${ }^{2}$

1Palliative Care Center/ Family Medicine, Chi-Mei Medical Center, Tainan, Taiwan, Republic of China, 2 Plastic surgery, Chi-Mei Medical Center, Tainan, Taiwan, Republic of China

Background: Patient Autonomy Act valid on 2019/1/6 in Taiwan provides the legal foundation for special medical refusal rights on life-sustaining treatment(LST) and artificial nutrition and hydration(ANH) under 5 clinical conditions if advance decision(AD) exists. Legal competent people should have once advance care planning(ACP) before signing AD.

Aims: The ACP team includes at least one doctor, one nurse and one social worker or psychologist who completed the ACP classes and got the certification. These regulations are special essentials for ACP compared with other countries. This report want to figure out what are the benefits or limits by performing ACP in this way.

Methods (design, data collection, analysis): Collect the opinions of 2 palliative physicians, 3 nurses, 1 social worker and 1 psychologist who participate in ACP within limited 60-90 minutes. One hundred ACP consultations were completed from May, 2017 to October, 2018.

Results: Almost all clients choose no LST and ANH. People in southern Taiwan focused on the way "not to be treat" more than "want to be treat" during discussion. The nurses found that discussing the caring place and method could arise the deep issues in their value, such as preferring dying in hospital not for themselves but for their families. Social worker and psychologist found that ACP consultation is more likely a strategic consultation to identify the family type and the possible conflict issue when they focus on LST and ANH. Designating one or more health care attorney is rare because Taiwan people used to rely on legal representatives.

Conclusion/ discussion: Lots of time should be spent to help our clients to realize that how their preference be achieved during the uncertain and complex medical process. Social worker and psychologist are important in this kind of consultation because they can figure out the profound conflict. Taiwan people's cultural preference should be studied as the important reference for $A D$ content.

\section{Abstract number: P01-281}

Abstract type: Poster

Defining Sedation in Palliative Care. A Review of Guidelines and Conceptual Analysis

Kremling A., Schildmann J., SedPall

Institute for History and Ethics of Medicine, Martin Luther University Halle-Wittenberg, Halle (Saale), Germany

Background: Differences with regards to definitions of sedation in palliative care have been cited as a problem for empirical research as well as for consensus on good practice.

Aim: To provide a conceptual analysis and systematic comparison of existing guideline definitions and to develop suggestions for improvement on the basis of identified structural and/or content differences of current definitions.

Methods: The working group on normative analysis within the SedPall project extracted definitions of sedation in palliative care from guidelines presented in two systematic reviews $(1,2)$. Following translation a conceptual analysis was performed by applying categories from theory of action ("basic action", "intended consequence", "means", "object" and "ethical property").

Results: Definitions could be extracted from 18 guidelines. "Reduced consciousness" was the only conceptual element that formed part of all analysed guidelines - either as "basic action" or as "intended consequence". Differences exist both in structure and content regarding the "means", "object" (e.g. patients in the last stages of life, dying patients) and the declaration of a subsequent intended consequence (a purpose as for example the reduction of refractory suffering or symptoms). Four guidelines included an ethical property of sedation practice in the definition.

Conclusion: The demonstrated heterogeneity of structure and content provide a challenge for a sound description of sedation practices as well as development of guidance. Strategies for improvement of definitions encompass a distinction with regards to purpose (i.e. empirical research versus regulating practice), identifying essential definitional elements as well as clarifying elements prone to different interpretations.

\section{References:}

1 Abarshi et al. BMJ Support Palliat Care 2017:223-29

2 Schildmann and Schildmann J Pall Med 2014: 601-11

SedPall is funded by the German Federal Ministry of Education and Research

\section{Abstract number: P01-282}

Abstract type: Poster

Early Identification and Timely Support - A Chance to Get it Right for Carers Supporting Someone at the End of Life through the Scottish Government's Adult Carer Support Plan

Swan S. ${ }^{1}$, Meade R. ${ }^{2}$, Carduff E. ${ }^{1}$

${ }^{1}$ Marie Curie, Glasgow, United Kingdom, ${ }^{2}$ Marie Curie, Edinburgh, United Kingdom

Introduction: The Carers (Scotland) Act (2016) places a duty on local authorities to prepare an Adult Carer Support Plan (ACSP) for any carer 
who requests one, or is identified as such. From 2020, this will be assisted by a fast track process for carers of people in the last six months of life. Timely identification of unpaid carers, assessment and support can reduce the overwhelming pressure of caregiving and increase competence, confidence, satisfaction the quality of the care.

Aim: To provide evidence on the supportive needs of carers to inform recommendations regarding the timescale for the creation of fast tracked ACSPs under the Carers (Scotland) Act (2016).

Methods: The study triangulated data from a literature review, qualitative secondary analysis ( $\mathrm{n}=19$ interviews; 3 focus groups) and two primary focus groups with bereaved carers $(n=11)$. Qualitative thematic analysis was used.

Results: There were many missed opportunities to identify carers, for example when there was interaction with services or a change in health status of the carer or cared-for person. Psychological support was beneficial and this extended into bereavement. Peer support and compassion from health professionals were valued. Respite was critical so carers could have time away. Primary care staff and social workers played an essential role, particularly with co-ordination of care and information. The decline to death was often faster than expected.

Conclusion: Health and social care professionals need to take a radical, reactive approach to presume that every patient has a carer who would benefit from identification, and ensure that the carers they identify understand their entitlements. Identification is everyone's responsibility and it should be the ambition of the Carers (Scotland) Act (2016) that this happens early in the illness trajectory. In so doing, rapid assessment and support can be initiated contemporaneously to help carers navigate and cope with an uncertain illness trajectory.

Abstract number: P01-283

Abstract type: Poster

Palliative Care Physicians' Perception on the Practice Related to Advance Care Planning

Pawłowski L. ${ }^{1}$, Modlińska A. ${ }^{1}$, Mróz P. ${ }^{1}$, Pawłowska I. ${ }^{2}$, Wojtacki J. ${ }^{3}$, Wyszadko A. ${ }^{1}$, Lichodziejewska-Niemierko M. ${ }^{1}$

${ }^{1}$ Department of Palliative Medicine, Medical University of Gdańsk, Gdańsk, Poland, ${ }^{2}$ Chair and Department of Pharmacology, Medical University of Gdańsk, Gdańsk, Poland, ${ }^{3}$ Rev. Eugeniusz Dutkiewicz SAC Hospice, Gdańsk, Poland

Background: The implementation of Advance Care Planning (ACP) into Polish medical practice has been insufficient, however selected aspects of ACP are present in national legal regulations. Physicians are obliged to inform patients about diagnosis of the disease, its treatment options, including hospice and palliative care (HPC), and prognosis as soon as it is applicable. Physicians have to consider patients willingness related to the therapy as well as patients' decisions about withholding or withdrawing treatment particularly in the end-of-life situations.

Aim: The purpose of this study was to identify the practice related the ACP prior to the beginning of the HPC.

Methods: A cross-sectional survey was applied. Physicians from Polish HPC centers were asked to complete the questionnaire.

Results: 53 physicians from 16 Polish HPC centers (inpatient and home hospices) participated in this survey (W: $57 \%, \mathrm{M}: 43 \%$, medium age: 47 ). Physicians indicated that the level of patients' knowledge about their disease and treatment at the beginning of palliative care is low. The majority of respondents (68\%) reported that in general patients are informed about the possibility of palliative care too late. Such information is usually provided at the very end of life-prolonging treatment $(60 \%)$ or as late as after an active therapy has been finished $(28 \%)$. In the cases of patients with incapacity, the decisions about the treatment are based on: patients' willingness (51\%) declared earlier, willingness of the patients' family (45\%), people who care for the patient $(55 \%)$ as well as physicians (21\%). Depending on the circumstances some practices cannot be legally accepted. At present Polish patient cannot appoint a healthcare agent. In the opinion of $74 \%$ respondents implementation of such possibility could improve representation of patients interests.

Conclusion: Implementation of ACP into Polish practice needs both new legal regulations and improvement in informing patients by physicians.

No funding

Abstract number: P01-284

Abstract type: Poster

Palliative Care Policy Development: The Case Study of Zambia' Chaila M.J. ${ }^{1,2,3}$, Mbozi P.4,5, Kapambwe S. ${ }^{6,7}$

${ }^{1}$ ACHIEVE Project, Centre for Infectious Disease Research in Zambia (CIDRZ), Lusaka, Zambia, ${ }^{2}$ Livingstone Central Hospital, Livingstone, Zambia, ${ }^{3}$ St Joseph Hospice, Livingstone, Zambia, ${ }^{4}$ Palliative Care, Cancer Diseases Hospital, Lusaka, Zambia, ${ }^{5}$ Zambia Oncology Nurses Society, Lusaka, Zambia, 6 Ministry of Health (Zambia), Lusaka, Zambia, ${ }^{7}$ Women's Cancer Control, Centre for Infectious Disease Research in Zambia (CIDRZ), Lusaka, Zambia

Background: A number of hospices in Zambia were threatened with closure around 2011/12 due to reduced funding. Palliative care (PC) integration, therefore, in any national health system requires a strong political will through the Ministry of Health $(\mathrm{MOH})$ and presence of key stakeholders, like national palliative care associations such as Palliative Care Association of Zambia (PCAZ). Zambia has in the recent years made strides in the integration of PC services within the existing health system. She is committed to achieving universal health coverage across all spectra of care including PC.

Aim: To create a policy environment that encourages PC development in Zambia.

Methods: The government through $\mathrm{MOH}$ held a series of meetings with key stakeholders such as PCAZ that discussed a number of activities that needed to be done to integrate PC.

Results:

- PCAZ became the secretariat to the National PC Technical Working Group which was chaired by $\mathrm{MOH}$

- $\mathrm{MOH}$ provided a platform through which PCAZ and other key partners worked

- The first draft of the National PC Strategic Framework (NPCSF) was developed in 2012

- Zambia developed the 2016-2021 National Cancer Control Strategic Plan prioritizing 4 cancers \& PC. It aims to develop an effective PC service at all levels of health care system \& implement the NPCSF

- Hospices were saved from closure as $\mathrm{MOH}$ provided direct funding to support hospices and placed medical personnel on $\mathrm{MOH}$ payroll

- $\mathrm{MOH}$ created funded PC specialist positions for medical doctors and nurses in government

- $\mathrm{MOH}$ continued to support the training of health care workers in PC

- In 2015, Zambia hosted the MaZaFi Nursing, Cancer and PC Conference. The First Lady of Zambia was Guest of Honour.

Conclusion: It is possible to integrate PC services within the health system in a country like Zambia where the concept of PC is relatively new due to strong government will and institutional frameworks and policies that support its integration.

Abstract number: P01-286

Abstract type: Poster

Visibility of Palliative Care in Health Care Policies for Older People: A Documentary Analysis in 12 Countries

Pivodic L. ${ }^{1}$, Smets T. ${ }^{1}$, Gott M. ${ }^{2}$, Sleeman K.E. ${ }^{3}$, Kodba Čeh H. ${ }^{4}$, Arrue B. ${ }^{5}$, Hrda K. ${ }^{6}$, ten Koppel M. ${ }^{7}$, Cardenas Turanzas M. ${ }^{8}$, Wilson D.M. ${ }^{9}$, Rhee Y. ${ }^{10}$, Nakanishi M. ${ }^{11}$, Lo T.J. ${ }^{12}$, Van den Block L. ${ }^{1}$

${ }^{1}$ End-of-Life Care Research Group, Vrije Universiteit Brussel \& Ghent University, Brussels, Belgium, ${ }^{2}$ University of Auckland, Auckland, New 
Zealand, ${ }^{3}$ King's College London / Cicely Saunders Institute, London, United Kingdom, ${ }^{4}$ University Clinic of Pulmonary and Allergic Diseases Golnik, Golnik, Slovenia, ${ }^{5}$ Age Europe, Brussels, Belgium, ${ }^{6}$ Sue Ryder, Prague, Czech Republic, ${ }^{7}$ VU University Medical Center, Amsterdam, Netherlands, 8University of Texas Health Science Center, Houston, United States, ${ }^{9}$ University of Alberta, Edmonton, Canada, ${ }^{10}$ Dongduk Women's University, Seoul, Korea, Republic of, ${ }^{11}$ Tokyo Metropolitan Institute of Medical Science, Tokyo, Japan, ${ }^{12 N a t i o n a l ~ C a n c e r ~ C e n t r e ~}$ Singapore, Singapore, Singapore

Background: Integrating palliative care in the continuum of care for older people is an important task for public health and clinical practice. It requires a strong policy base, especially in rapidly ageing countries. We studied whether palliative care elements are included in public policy documents guiding health care for older people in 12 countries with steep ageing projections.

Methods: Directed content analysis of public policy documents (legislation, policies/strategies, guidelines, white papers) on health care for older people in Austria, Belgium, Canada, Czech Republic, England, Japan, Mexico, Netherlands, New Zealand, Singapore, Slovenia, and Spain. Country experts identified documents and evaluated inclusion of nine core elements of palliative care using a standardised data extraction form, based on 'essential practices for palliative care' (WHO 2016) and quality indicators for palliative care (Leemans et al. 2017).

Results: We identified 57 policy documents in 12 countries. One country (NZL) addressed all elements of palliative care. At least one document in each country addressed 'communication and care planning', 'coordination and continuity of care' and 'ethical aspects'. 'Care for family' was mentioned in all but one country (CAN). 'Symptom management' was mentioned in all but 3 countries (BEL, CZE, SLO). 'End-of-life care' was addressed in all countries except CAN, ESP, SLO, BEL. Of 10 countries that mentioned palliative care explicitly (all except CAN, AUT), 5 mentioned eligible patients but only one (NZL) specified who should provide palliative care. Policy documents in 5 countries referred to stand-alone palliative care strategies (out of 9 countries with such strategies).

Conclusion: Countries' policies on health care for older people require revision primarily to address end-of-life care, specify which patients should receive and which professionals should provide palliative care, and include references to existing palliative care strategies.

Abstract number: P01-287

Abstract type: Poster

High Level Meeting - Strengthening Health Systems. An

Experience for the Advancement of CPs for Latin America -

Brazilian Example

Fonseca A.C., Soler D.

Latin American Palliative Care, Buenos Aires, Argentina

Objective: To present results about the High Level Meeting Strengthening Health Systems, the meeting with the principal stakeholders in Latin America (LA) about Palliative care (PC).

Method: CP-FeSS was led by the Latin American Association of Palliative Care (ALCP) and partners: the International Association for Hospice and Palliative Care and the Latin American Federation of Associations for the Study of Pain. The Grünenthal Foundation for Palliative Medicine is in charge of financing and non-financial support. Among the objectives of the meeting: to include the PC in the national policies of each country; to make public resources available to enable PC services to be developed at all three levels of care; make public resources available to ensure the essential package of medications; facilitate access to essential medications such as opioid; the Brazilian delegation were composed of representatives of the National Health Agency, of the National Council of State Secretaries of Health and PC physicians belongs to ALCP committees. During the event, there was an exchange of information about the current situation of PC in Brazil mainly the difficulties to delivery opioid.
Results: There was a wide diversity among the participating countries around the PC in LA; scarcity of national policies, lack of basic drug dispensing policies, lack of education; however, from the exchanges of experiences, from the perception that there are similar models and that, above all, there is a great opportunity and need for the development of PCs. At the end, the group signed a compromise document for measures and actions that can reduce the space between the need for PC and access. Lessons learned: A meeting with managers, opinion makers and politicians from different countries can be a strong path for the development of public policies; this model can be considered replicable at different levels of attention and thus contribute to the advances of PCs in different health sectors.

Abstract number: P01-288

Abstract type: Poster

Research on Problems and Countermeasures of Chinese Hospice

\& Palliative Care: Summary of Phased Exploration Based on National Pilot Studies

Li Y.-G. ${ }^{1}$, Luo Z.-W. ${ }^{2}$, Qin Y. ${ }^{3}$, Zhang Z. ${ }^{3,4}$

${ }^{1}$ Graduate School, Chinese Academy of Social Sciences, Beijing, China, ${ }^{2}$ Institute of Industrial Economics, Chinese Academy of Social Sciences, Beijing, China, ${ }^{3}$ Hospice Palliative Care Unit, Beijing Haidian Hospital, Beijing, China, ${ }^{4}$ Hospice Palliative Care Alliance of China Foundation, Eagleville, United States

Aim: To explore the strategy of constructing a high-quality and advanced hospice care service system and management pattern. Hospice care is to provide a soothing service to the end-stage patients who have no use in curative treatment, and to complete their final journey of life with dignity. Problems such as the aging and the high incidence of malignant diseases of China have triggered a large number of needs for hospice care. However, Chinese long-term neglect of "death quality" has caused the ranking of death quality to be almost at the bottom. Therefore, it is urgent and necessary to speed up the development of the hospice care. Methods: From October 2016 to October 2018, the authors participated in a total of 300 patients of national pilot studies about hospice \& palliative care in China, the STOF business model with sustainability, social responsibility \& ethics was taken. Some strategies were adopted to improve the construction of subject, it included the status quo of subject development analyzed with SWOT theory, development objectives formulated according to SMART rules, PDCA rules employed to promote quality improvement of service system, KPI evaluation system established to improve work efficiency.

Results: This paper reviews the current situation of China's hospice care, summarizes the national pilot programs, conditions and relevant policies that have been issued, and reveals the current problems encountered in Chinese hospice care in terms of concepts, funds, teams, and legal safeguards, and then proposes targeted countermeasures.

Conclusion: The issues mentioned above can be addressed through these initiatives: Strengthen publicity and education to establish the right concept of hospice care under Chinese cultural environment; Improve the institutional system to protect the rights and interests of doctors and patients; Adjust the cost standard and improve healthcare security; Optimize talent incentives and build a care team with Chinese characteristics.

Abstract number: P01-289

Abstract type: Poster

Dying Worlds in Austria - The Perspectives of Those Affected on 'Good Dying'

Heimerl K. ${ }^{1}$, Egger B. ${ }^{1}$, Schuchter P. ${ }^{2}$, Lang A. ${ }^{3}$, Frankus E. ${ }^{3}$, Kaelin L. ${ }^{4}$, Dinges S. ${ }^{5}$, Wegleitner K. ${ }^{2}$

${ }^{1}$ Department of Nursing Sciences, University of Vienna, Vienna, Austria, 2Palliative Care and Organizational Ethics, University of Graz, Graz, 
Austria, ${ }^{3}$ Technoscience and Societal Transformation, Institute for Advanced Studies, Vienna, Austria, ${ }^{4}$ Institute for Practical Philosophy/ Ethics, Private catholic University Linz, Linz, Austria, ${ }^{5}$ Institute for Ethics and Law in Medicine, University of Vienna, Vienna, Austria

Background: The professional debate in Austria around the issues of dying, death and grief focusses on controversies about euthanasia and assisted suicide and on the extension of the provision of specialized palliative care. The research aims at filling a gap: it explores the perspectives of those affected on ,good dying'.

Methods: We conducted 30 qualitative research interviews with people that are affected by dying, death and grief, i.e. with incurably and ill patients, with very old persons and with informal care givers, who have accompanied a person at the end of his or her life. We recorded, transcribed and analyzed the interviews. A dispositive analysis was performed as well as an ethical reflection. The results were discussed in three group discussions with professional care givers.

Findings: We identified the following messages of those affected regarding , good dying'

1. Of utmost priority are the caring networks: family networks, professional networks and civic society networks.

2. Although dying is 'never beautiful' on one hand, our interviews partners describe many beautiful and elevating situations on the other hand.

3. It matters for our interview partners to have moments of enjoyment at the end of their lifes.

4. Those affected strive at maintaining a daily routine. This supports their wish for autonomy in a situation in which dependency increases.

5. Dying is not only a physical process, but also a psychological, a social and a spiritual process. Whereas the social and the spiritual dimension are of priority to our interview partners, the physical dimension is attributed with shame, not only for those affected but also for the researchers.

Conclusions: The dying worlds are as diverse as the biographies of the interview partners. If we want to respect the wishes of those affected at the end of their lives we need to strengthen their caring networks - the family, the professional as well as the civic society networks.

\section{Abstract number: P01-290 \\ Abstract type: Poster}

\section{Consumption of Opioid Analgesics for Pain Management in Ukraine 2011-2016}

Datsiuk N. ${ }^{1}$, Shapoval-Deinega K. ${ }^{1}$, Tkachenko O. ${ }^{2}$, Tymoshevska V. ${ }^{1}$ ${ }_{1}^{1}$ Public Health Program, International Renaissance Foundation, Kyiv, Ukraine, ${ }^{2}$ Institute of Analisys and Advocacy, Poltava, Ukraine

The main task of study to analyze the consumption of opioid analgesics in Ukraine. On the basis of established trends, the changes in the availability of pain management for Ukrainians in the context of recent regulatory changes.

Sources of information were the legislation, the data of the licensee's registry for narcotic drugs, the annual reports of the above-mentioned licensees to the State Medical Service, analytical reports of the Monitoring Center, as well as the data of regional departments (offices) of health care of regional state administrations.

With the help of information requests, researchers turned to:

- 282 pharmaceutical companies that have the right to sell opioid analgesics in 518 pharmacies;

- health care facilities;

- healthcare departments of regions which have their own medical institutions.
It was found that during 2011-2015 the use of morphine, tramadol, and fentanyl increased in medical institutions. In 2016, the aforementioned and other opioid analgesics were used less. After analyzing the information received from 67 pharmacies, they recorded an increase in pharmacy withdrawal of morphine drugs in 2011-2015 in most regions of Ukraine. This was largely due to the tablet formulation of an analgesic. In cases of Vinnitsa, Cherkasy, Chernivtsi, Ivano-Frankivsk, Poltava oblasts and Kyiv, it was possible to gather the most complete data on the prescription drugs. Using the WHO methodology for need calculating for morphine, for these regions the provision of "gold standard" - morphine has been calculated. During 2012-2016, its level ranged from $11.2 \%$ to $14.3 \%$.

Conclusions: liberalization of the legislation on the circulation of narcotic substances for medical purposes has not yet led to proper provision of adequate anesthesia for Ukrainians. At the same time, it is positive to increase the prescription morphine release and increase the percentage of use of tablet formulations compared to other forms.

Abstract number: P01-292

Abstract type: Poster

Knowledge and Attitudes of Healthcare Professionals in Primary Care in Mallorca Health District Concerning Living Wills (LW) and Advance Care Planning (ACP)

Llagostera M. ${ }^{1}$, Guillén M. ${ }^{2}$, Leiva A. ${ }^{3}$, Serrano $C .{ }^{4}$, Rotger $A .{ }^{4}$, Pascual A. ${ }^{5}$, Llobera J. ${ }^{3}$

${ }^{1}$ Centre Coordinador del Programa de Cures Pal-liatives Illes Balears, IBSALUT, Palma, Spain, ${ }^{2}$ Atenció Primària Mallorca, IBSALUT, Palma, Spain, ${ }^{3}$ Unitat Investigació. Gabinet Tècnic Atenció Primària, IBSALUT, Palma, Spain, ${ }^{4}$ Centre Coordinador Programa Cures Pal-liatives Illes Balears, IBSALUT, Palma, Spain, 5 Unidad de Cuidados Paliativos, Hospital Sant Pau, Barcelona, Spain

Aims or goal of the work: To explore the knowledge and attitudes of Primary Care healthcare professionals towards LW and ACP in the Mallorca health district.To compare the outcomes among professional groups.To explore patient LW registration among healthcare professionals in Primary Care in Mallorca.

\section{Methods:}

Design: A cross-sectional descriptive study among health care professionals of Mallorca (Spain)

Data collection: We sent letters with an anonymous self-administered questionnaire to 45 health care centers in Mallorca, we received 379 questionnaires from General practitioners $(47,7 \%)$, nurses $(51,7 \%)$ and nurse or GPs residents (0,5\%).

We designed an ad-hoc questionnaire of knowledge, attitudes and practices of LW and ACP in primary care, there were 24 questions, 4 sociodemographics, 10 about LW and 11 about ACP, 12 question were in a likert ordinal scale $(0=$ strongly disagree to $10=$ strongly agree) and 12 were categorical.

Analysis: We performed a descriptive analysis and we reported median and interquartile range (IQR) for ordinal variables and percentages for categoric variables.

Results: $60,5 \%$ of the health professionals had more than 20 years of working experience, $75,0 \%$ were women, $20,3 \%$ received specific training about LW and $6,7 \%$ about ACP, $86,8 \%$ never consult the LW of a patient and $71,9 \%$ didn't know where to consult it, $2,1 \%$ of the health professionals registered their own LW. The health professionals agree with the following statements: -1 know what LW are (median=7 IQR(59)); More training is needed in LW (median=10 IQR(9-10)); I know the differences between LW and ACP (median=2 IQR(2-5)); - I know what ACP is (median=4 IQR(1-6)).

Conclusion: A big number of Primary healthcare professionals of Mallorca don't know what LW and ACP are and their differences. Both things are underused in daily clinical practice. LW few registered by clinicians themselves. Strong educational and informative efforts are needed in heathcare professionals. 
Abstract number: P01-293

Abstract type: Poster

Developing Community Palliative Care Services - An Evaluation of Four Danish Municipalities Raunkiær M., Jarlbæk L., B. Mikkelsen T.

The University of Southern Denmark, Department of Clinical Research, REHPA, The Danish Knowledge Centre for Rehabilitation and Palliative Care, Nyborg, Denmark

Background and questions: The development of community palliative care (PC) may take different approaches. We present results from an evaluation of 4 municipalities' different approaches supporting death at home among people with life-threatening diseases. These approaches included: guidelines for PC discussions (PCD) with families including wishes on place of care and death; tools to identify and assess PC needs (e.g. SPICT, ESAS); developing inter-disciplinary and inter-service collaboration; competency development for professionals. The evaluation questions were: Did the 4 municipalities increase the number of home deaths? What were the families' and professionals' experiences of the developments?

Methods: A mix-methods design was used:

1) a survey of the deaths registered during the municipalities' project period, March 2016 to June 2018;

2) interviews using "User Participation in Quality Assessment model". A descriptive statistical analysis was conducted for the survey and a thematic content analysis for the interviews.

Results: Of the 282 deaths reported in the survey, $62 \%$ died in their preferred place, $54 \%$ died at home and $18 \%$ at hospital. Discussions on preferred place of death were documented in $51 \%$ of cases, with relatives being present $78 \%$ of the time.

38 interviews took place: 4 patients, 13 relatives and 21 professionals. For the family, discussions about place of care and death were a family matter. The professionals found it difficult to initiate talking about place of care and death, but found the PCD guidelines and tools to identify and assess the patients' PC needs, helped to initiate and legitimize PCD. Conclusion: Data from the Death Cause Register showed that $46 \%$ of all deaths in the four municipalities happened at home, in comparison with $54 \%$ in our evaluation. According to the informants the number of home deaths might have been supported by the PCD guidelines and tools.

Abstract number: P01-294

Abstract type: Poster

Feasibility and Predictive Value of Intuitive Frailty Screening in the Elderly: A Pilot Study Veldhoven $C .{ }^{1,2}$, Janssen $A .{ }^{1}$, de Graaf $W^{2}{ }^{2}$, Perry $M .{ }^{3}$, Vissers $K .^{1}$, Engels $Y^{1}$

${ }^{1}$ Radboud University Medical Center, Anesthesiology, Pain, Palliative Medicine, Nijmegen, Netherlands, ${ }^{2}$ General Practice Berg en Dal, Berg en Dal, Netherlands, ${ }^{3}$ Radboud University Medical Center, Geriatrics, Nijmegen, Netherlands Presenting author email address: yvonne. engels@radboudumc.nl

Objective: Identifying frail patients is critical in delivering optimal supportive care. Several diagnostic instruments exist, but none of them is simple enough to screen the entire elderly population of a general practice. Since GPs have an elaborate knowledge of the health and social situation of their patients, they might be able to intuitively estimate their patients' frailty adequately. The goal of this pilot study is to evaluate feasibility and predictive validity of this intuitive frailty screening Methods: We performed a prospective cohort study with medical record review in a general practice in the eastern part of the Netherlands. Two general practitioners classified all patients on their patient list aged $\geqslant 75$ years as not-frail, frail or frail-complex (294 patients; $59 \%$ female; mean age 83 years). The primary outcome was 1 -year healthcare consumption. Secondary outcomes were feasibility, mortality, institutionalization and prescribed number of drugs.

Results: The screening took two hours, ( $<30$ sec per patient). 77 patients (26\%) were classified as frail and $62(21 \%)$ as frail-complex. The latter had a higher healthcare consumption than those classified as not-frail: more home visits (OR 8.0 [95\%-Cl 5.6-11.6], $\mathrm{p}<0.001$ ) out of hours GP contacts (OR 4.4 [2.3-8.3], $\mathrm{p}<0.001$ ), emergency room visits (OR 1.9 [1.0-3.5], $p=0.040$ ) and hospital and nursing home admissions OR 3.9 [2.1-7.3] $p<0.001$. For patients classified as frail, effect sizes were smaller but still significant.

Mortality was higher for the frail-complex group (OR 10.9 (2.2-55.2], $\mathrm{p}=.004$ ) as was institutionalization (OR 29.8 [3.2-276.9], $\mathrm{p}=.003$ ). No statistic difference was found for the group classified as frail. Mean number of drugs prescribed was higher for both frail and frail-complex persons. Conclusions: Intuitive frailty screening by GPs is highly feasible and shows promising predictive characteristics. Further research in more practices is necessary to confirm the findings of this study.

Abstract number: P01-295

Abstract type: Poster

"Home is where I Lay my Head to Rest" - Gentle Transitions in the Last Weeks to Years of Life for Elderly Patients Living in East Toronto, Canada

Joshi R. ${ }^{1}$, Workentin K. ${ }^{1}$, Guo J. ${ }^{2}$, Pham T.-N. ${ }^{1}$

${ }^{1}$ Department of Family and Community Medicine, University of Toronto, Toronto, Canada, ${ }^{2}$ South East Toronto Family Health Team, Toronto, Canada

Aim: To preserve goals of care and self-determination among homebound elderly patients in a primary care setting as they transition between home and hospital more often at end of life.

Approach taken: Transitions in care are stressful for elderly patients, with high risk of fragmentation of care and exposure to unnecessary treatments. Without clear goals of care, patients spend more time in hospital at end of life, with little focus on quality of life and autonomy. Our Family Health Team consists of family physicians with palliative care expertise, working collaboratively with a physician assistant, nurse practitioner, and homecare case manager to provide comprehensive patientcentered primary care to 20,000 patients in East Toronto. A dedicated home-visit program targets frail patients identified by the hospital or homecare agency. Innovative telemedicine technology improves access and safety through remote monitoring. Using patient vignettes, we demonstrate how this team-based approach across sectors creates seamless transitions in care while maintaining a patient-centered focus until the end of life.

Results: Using a team-based approach and telemedicine technology, patients are cared for in their home until their end with fewer hospital re/admissions. When an admission is required, intensive team communication and care coordinated between home and hospital create seamless transitions and preserves patients' goals of care at end of life.

Lessons shared:

1. Providing home-based primary care to frail patients with frequent hospitalizations or multiple comorbidities allows for better patient-centered care to a high needs population at the end of life and preserves patient dignity.

2. Home-bound patients and families benefit from palliative expertise in family physicians.

3. Unnecessary hospitalizations are reduced through intensive team communication between different team providers and across sectors. 
Abstract number: P01-296

Abstract type: Poster

What Factors Make Primary Palliative Care More Positive or Negative - Experiences from Patients, Relatives, GPs, Medical Assistants and Nurses

Ewertowski H., Schneider N., Stiel S.

Institute for General Practice, Hannover Medical School, Hannover, Germany

Background: The vast majority of seriously ill or dying people can be treated within generalist care. Previous research indicates the strengthening of the general practitioners' (GP) role in PC as one top priority for the improvement of palliative care (PC) in Germany. However, how GPs can provide $\mathrm{PC}$ in such a way that it is experienced as positive and successful has not yet been defined.

Aim: To assess and understand factors that lead to positive or negative experiences of primary PC including the perspectives from all those involved.

Methods: In this qualitative sub-study of the project "Primary Palliative Care in General Practice" 16 interviews with patients, relatives, GPs, medical assistants, and nurses were conducted. Following the Critical Incident Technique, the participants were asked to describe a particular positive and negative experience in PC supply. Interviews were analysed according to principles of Grounded Theory.

Results: A lack of appropriate legal regulations and financing of care were experienced as negative factors for PC supply. Especially if care was delivered GP centred, proactively, and continuously, interview partners were satisfied with primary PC. Other facilitating factors were ensuring care at night and on weekends, the feeling that time has been taken, and experiencing safety.

Discussion: The core mission of primary care meets the manners of care for appropriately experienced primary PC well. Yet, strategies have to be developed to reduce negative and strengthen positive influences for PC supply. In further project steps, participatory action research and consensus methods will contribute to an intervention package for GP practices to improve primary PC.

Source of funding: Federal Ministry of Education and Research (BMBF 01GY1610)

Abstract number: P01-297

Abstract type: Poster

A Contribute to the Development of Primary Palliative Care in Italy: Cross-cultural Adaptation and Pre-test of the SPICT ${ }^{\mathrm{TM}}$ - IT Magnani C. ${ }^{1}$, Mastroianni C. ${ }^{1}$, Surdo L. ${ }^{1}$, Goletti M. ${ }^{2}$, Casale G. ${ }^{1}$, The SPICT-IT Study Group: Fanelli R, Latorre P, Stefanelli MC, Colotto M, Di Giacomo A, Pace A, Massaro M, Gentili G, Savarese A, D’Angelo D, Scarlata S, Fucito G, Lora Aprile P, Cancian M and Turriziani A. ${ }^{1}$ Antea Palliative Care Centre, Rome, Italy, ${ }^{2}$ Health Direction, Local Health Authority Rome 1, Rome, Italy

Background: The taskforce group for primary palliative care (PPC) established by the EAPC in 2012 documented the main barriers and opportunities for palliative care (PC) development in the community. Difficult identification of patients with PC needs was described as one of the main barriers. In 2017, the Italian Ministry of Health published the updated "Essential healthcare levels" with special reference to the "PC approach" delivered by General Practitioners (GPs). Currently, validated tools in Italian aimed to identify patients with $\mathrm{PC}$ needs are not available.

Aim: To translate, cross-culturally adapt and pre-test the Italian version of the SPICT ${ }^{\mathrm{TM}}$.

Method: The Beaton recommendations on the process of cross-cultural adaptation of instruments were followed. The focus was on conceptual equivalence, rather than literal equivalence. Content validity was assessed using the Lynn method. Face validity was assessed asking to a sample of GPs to answer some questions on appropriateness and clarity of the SPICT ${ }^{T M}-I T$. They were also asked to use the SPICT ${ }^{T M} M_{-}$IT to identify their patients with PC needs.

Results: In the cross-cultural adaptation phase of the SPICT TM-IT some issues regarding experiential and conceptual equivalences were highlighted. The Scale-Content Validity Index was 0.82. A sample of 41 Italian GPs who provide care for a total of 47.884 persons in the community, identified 966 patients with at least two general criteria and one specific criteria of the SPICT ${ }^{\mathrm{TM}}$-IT ( $2 \%$ of their patients). The SPICT ${ }^{\mathrm{TM}}$-IT was evaluated as clear and suitable for its aim by $97.6 \%$ of the GPs' sample.

Discussion: The SPICT TM-IT resulted to have an acceptable content and face validity. The percentage of patients identified through the SPICT ${ }^{T M}$-IT was comparable to findings from literature. The next step of this project will investigate the impact of a proactive training programme to support GPs in recognizing patients with unmet PC needs.

Abstract number: P01-299

Abstract type: Poster

Evaluation of Palliative Care in a Danish General Practice Larsen A.M. ${ }^{1}$, Andersen M.F. ${ }^{1}$, Neergaard M.A. ${ }^{2}$, Gorlen T. ${ }^{1}$

${ }^{1}$ Søborg Torv Family Doctors, Søborg, Denmark, ${ }^{2}$ Palliative Care Team Oncology Department, Aarhus University Hospital, Aarhus, Denmark

Background: Most terminally ill patients wish to die at home and this requires specific competences in end-of-life-care amongst general practitioners.

Aim: The aim of the study was to quantify and evaluate the palliative trajectories in a Danish general practice that has systematically followed patients with palliative needs over the last five years.

Hypothesis: Number of home-deaths (including nursing homes) over a three-year period was expected to be larger than the national average from 2016 (49\%).

Methods: All patients affiliated with a large (approx. 6500 patients) Danish 4-doctor general practice located in a Copenhagen suburb, who died between 1/1 2015 and 31/7 2018 were identified.

Patients without available relevant information from the medical record and patients who died by accident or suicide were excluded.

The following variables were extracted from the patient files: age, gender, diagnosis, place of death (preferred and actual), number and type of clinical contacts (consultation, phone, e-mail) during the last four months of life, the use of palliative medication and subcutaneous needle and any specialised palliative care.

The study has been approved by the Danish patient safety committee (j.nr. 3-3013-2592/1).

Results: 180 patients were included in the study. The mean age at time of death was 76,5 years and $99(56 \%)$ were women. Nine-ty patients $(50 \%)$ died at home or at a nursing home, 77 patients (43\%) died at a hospital. Two-sample test of proportions showed no significant difference $(p>0.05)$ in home-death as compared to the national 2016 data.

Conclusion: The number of home-deaths in the study population (50\%) was not significantly different from the national average (49\%). The reason for no change in home-death-rate may be due to a different distribution of important variables in the study population. This will be further explored in the final results presented at the 2019 EAPC congress.

Funding: The study has not received any funding

Abstract number: P01-300

Abstract type: Poster

Palliative Care in a Home Care Setting: The Development of a Guideline for District Nurses and Nurse Assistants Verschuur E.M.L. ${ }^{1}$, van der Sande R. $^{1}$, Francke A. ${ }^{2,3}$

${ }^{1}$ HAN VDO, HAN University of Applied Sciences, Nijmegen, Netherlands, 
${ }^{2}$ Amsterdam UMC, Vrije Universiteit Amsterdam, Amsterdam, Netherlands, ${ }^{3} \mathrm{Nivel}$ (Netherlands Institute for Health Services Reserach), Utrecht, Netherlands

Background: In the Netherlands district nurses and nurse assistants have a pivotal role in palliative care at home. Partly due to aging of society, an increase of palliative care is expected. Related to this, we developed a guideline Palliative Care at Home for district nurses and nurse assistants. Methods: This guideline is developed following the standard of the Dutch AQUA-Advisory and Expert Group. To assess the bottlenecks in daily practice, face to face and online focus groups with district nurses and nurse assistants were performed. Seven predefined questions were answered by a systematic international literature review of existing systematic literature reviews and evidence-based guidelines in the field of palliative care at home. The AMSTAR and AGREE II criteria were used to assess the methodological quality.

Results: The 39 recommendations covered 4 themes: support of selfmanagement among patients; identification of the palliative phase and needs assessment; advance care planning and competences of district nurses and nurse assistants. All 4 themes are structured as follows: predefined questions, recommendations, results of the literature review and other considerations. All recommendations were thoroughly discussed in the multidisciplinary project group and advisory group with representatives of key stakeholders in palliative care. This resulted in both evidence-based and consensus-based recommendations. One example of the recommendations is: District nurses and nurse assistants discuss the outcome of the 'surprise question' with the general practitioner if the answer to that question is: No, I would not be surprised. Conclusions: This guideline provide recommendations concerning key aspects of palliative care at home by district nurses and nurse assistants. They should serve as a reference standard for providing palliative nursing care and for collaboration with the interdisciplinary team around patients, in particular with the general practitioner.

Abstract number: P01-301

Abstract type: Poster

\section{Primary and Community Palliative Care in Indonesia: A Scoping}

Review

Perdamaian T. ${ }^{1}$, Murray S.A. ${ }^{2}$

${ }^{1}$ Faculty of Medicine, Duta Wacana Christian University, Yogyakarta, Indonesia, ${ }^{2}$ Primary Palliative Care Research Group, Usher Institute of Population Health Sciences and Informatics, University of Edinburgh, Edinburgh, United Kingdom

Background: Primary and community approach has been proven in extending accessibility and affordability of palliative care in low resource setting. The growing primary health care structure and community initiatives in Indonesia poses an opportunity to expand the palliative care provision in Indonesia.

Aim: To map and understand the extent and nature of research on primary and community palliative care in Indonesia.

Method: A scoping studies framework was used to search both international and Indonesian databases between the year 1990 and 2017. The results were also discussed with some experts in the field as recommended in last step of scoping review.

Results: The search initially identified 261 articles, which further filtered to only 15 original studies. These researches were published since 2011. Ten of them was conducted by nurses and none by physicians. Among the qualitative researches $(\mathrm{N}=7)$, the most common themes found are nurse's attitude, motivation, and expectation in providing primary palliative care, and caregiver's burdens and quality of life. The survey researches $(\mathrm{N}=4)$ mainly discussed the patient's satisfaction on community pharmacist role in palliative drugs dispensing. Meanwhile, the quasi-experimental studies ( $N=4$ ) mostly discussed the role of home care in the continuity of care after hospital discharge.
Discussion: Currently, two major Cities in Indonesia, Jakarta and Surabaya, are developing a network of palliative care. However, research on primary and community palliative care seemed inadequate to support the provision of high-quality early palliative care in low resource setting. The research areas should be addressed to integrate palliative care provision in the community in Indonesia. These include quality care for patients, families and caregivers; the role of multidisciplinary team; and strengthening health system and policies.

Abstract number: P01-302

Abstract type: Poster

Consistency of GP Seen and Regularity of Contact Are Associated with Emergency Care Use for Children and Young People with

Life Limiting Conditions

Jarvis S., Fraser L.

Health Sciences, University of York, York, United Kingdom

Background: Children and young people (CYP) with life limiting conditions (LLC) have complex healthcare needs, visit A\&E departments and have emergency inpatient admissions. There is some evidence that they go straight to hospital, bypassing GP care - improving GP care may reduce this.

Aims: To determine whether regular consultations and consistently seeing the same GP is associated with reduced levels of emergency care. Methods: An extract of GP consultations from the Clinical Practice Research Datalink with linked Hospital Episode Statistics was requested for CYP who had ever had a diagnosis matching either an ICD-10 or READ code framework for LLC. Each year, a variability score was calculated (the variance divided by the mean gap between consultations) and the proportion GP consultations per person that were with the most commonly seen GP was calculated. Numbers of A\&E visits and emergency admissions per person per year were counted. Separate models were developed, for A\&E visits and emergency admissions as the dependent variables; regularity of consultations and consistency of GP seen was the independent variables of interest. Multilevel negative binomial regressions were used as the outcomes were count data clustered by individuals.

Results: Those with higher variability scores had up to $9 \%$ (95\% Cl 2-16\%) more A\&E visits and $12 \%(95 \% \mathrm{Cl} 6-19 \%)$ more emergency admissions than those with the lowest scores. Those with $90 \%$ or more of visits with the same GP had $10 \%(95 \% \mathrm{Cl} 6-13 \%)$ fewer $\mathrm{A} \& \mathrm{E}$ visits and $10 \%(95 \% \mathrm{Cl}$ $7-13 \%$ ) fewer emergency admissions than those with $<40 \%$ of consultations with the same GP.

Conclusions: Scheduling and attending regular GP consultations and ensuring that young people with life limiting conditions have consultations with the same GP as often as possible may have the potential to reduce their use of emergency care services.

Abstract number: P01-303

Abstract type: Poster

Palliative Patients' Use of Potentially Inappropriate Medication: How to Reduce this in General Practice?

Mertens F., Callewaert C., Coppé H., Pype P.

Public Health and Primary Care, Ghent University, Gent, Belgium

Background: Palliative patients' medication use often is extensive. Although the focus of palliative care is the patient's quality of life, drugs for chronic conditions or illness prevention are often continued. Besides the risk of adverse drug effects, the benefits of this medication are unclear. Therefore they are regarded as potentially inappropriate medication (PIM). Nevertheless general practitioners (GPs) continue to prescribe PIMs.

Aim: To explore which PIM is used and to what extent this can be reduced in general practice.

To explore the GPs' barriers to deprescribe PIM. 
Method: Mixed method design: Interventional study in two primary care practices in Belgium: development of a deprescribing protocol, comprising: discussing shared care goals; ascertain patient's drugs; identify PIMs; prioritise drugs for discontinuation; implement a discontinuation regimen; monitor outcomes and adverse effects. Chart review of PIM use pre- and post-intervention. Descriptive statistics were used.

Semi-structured interviews ( $\mathrm{n}: 11$ ) with GPs. Inductive analysis approach. Results: In total 65 patients participated. In both practices, the number of PIM decreased after the intervention from n:126 to n:66 and from $\mathrm{n}: 124$ to $\mathrm{n}: 110$ respectively. Benzodiazepines, antidepressants and proton pump inhibitors were used most often and were deemed difficult to stop out of fear for complications.

Perceived barriers to deprescribe were doctor-related (medication started by specialists, not wanting to take away hope), medication related (previous negative experiences), context related (lack of clear guidelines) and patient-related (patient refused to stop). However, patients were perceived open for deprescribing after being informed.

Conclusion: GPs acknowledge the frequent prescription of PIM. Deprescribing interventions may support GPs in reducing PIMs. Timely and adequate communication with the patient and implementation of deprescribing guidelines may help to overcome barriers of deprescribing.

\section{Abstract number: P01-304 \\ Abstract type: Poster}

Primary Healthcare Professionals View of Implementing a Structured Palliative Plan and Ethical Reflection Alme T. ${ }^{1}$, Driller B. ${ }^{2}$, Olsen L.A. ${ }^{3}$, Dybvik T.K. ${ }^{3}$, Thorsnes S.L. ${ }^{3}$, Alnes R.E. ${ }^{3}$ ${ }^{1}$ Sula Municipality, Cancer Coordination, Sula - Langevåg, Norway, ${ }^{2}$ Cancer Department, Møre og Romsdal Hospital Trust, Ålesund, Norway, ${ }^{3}$ Department of Health Sciences, Norwegian University of Science and Technology (NTNU), Ålesund, Norway

Background: Advanced care planning (ACP) enables palliative patients to define and discuss goals and preferences for future medical treatment and care together with family and health-care providers. A palliative plan summarizes an ACP discussion and provides direction to healthcare professionals reflecting a patient's preferences and current medical condition.

Aims: We explored the use and benefits of a structured palliative plan and ethical reflection in ACP among primary health care professionals.

Methods: In 2015 a structured paper survey was send to all 36 municipalities in the county Møre and Romsdal, Norway. The municipalities were asked to let nurses with special responsibility in palliative care respond. A qualitative content analysis of semi structured interviews of four general practitioners (GPs) gives additional information about the use of palliative plans.

Results: Fifty five nurses from 29 municipalities responded to the survey. Twenty nine $\%$ confirmed the use of palliative plans, $83 \%$ of these experiences better palliative care. Almost all of them feel competent to initiate the ACP process and palliative plan. Sixteen \% said they have periodic ethical reflection, $84 \%$ of them suppose it helps to increase quality and competence in palliative care.

More than $90 \%$ of all nurses assume that patients will benefit from developing a common understanding and procedure for palliative plan and ethical reflection.

Key factors for GPs for a palliative plan to work are involvement, interdisciplinary cooperation and continuity in health care support together with dialogue between patients, relatives and health care professionals. Conclusion: Although a structured palliative plan and periodic ethical reflection is not yet widely used in the county in 2015, nurses and GPs see that it contributes to better palliative care. Using trained nurses to initiate the ACP process and palliative plan can be essential for the implementation process in the municipality.
Abstract number: P01-305

Abstract type: Poster

Integration of Palliative Care in Primary Practice in Greece - Do Health Care Professionals Working in Public Community Settings Really Know How to Care for Patients with Chronic, Life-limiting, Life-threatening Conditions?

Panagiotou I. ${ }^{1}$, Liva E. ${ }^{2}$, Georgiou 0. ${ }^{3}$, Liakopoulos G. ${ }^{4}$, Stoila A. ${ }^{5}$, Skliros $S^{2}$

${ }^{1}$ Local Primary Health Unit, Athens, Greece, ${ }^{2}$ Nemeas Health Center, Nemeas, Greece, ${ }^{3}$ Local Health Primary Care Unit, Pavlou Mela,

Thessalonica, Greece, ${ }^{4}$ Aiginiteion University Hospital, Athens, Greece, ${ }^{5}$ Attikon University Hospital, Athens, Greece

Background: Palliative care patients usually need a community-based care. In Greece, novel, public/local primary health care units (introducing the interdisciplinary team) have been recently expanded nationally. Objective: Our aim was to determine whether health care professionals working in such facilities know how to provide palliative care.

Methods: A survey (available on line only) was created. It investigated the knowledge of palliative care principles and structures, as well as the possibility of delivering such care in the community. The electronic survey was promoted via email to health care managers and to a Facebookteam (with $>2000$ members). Answers, voluntary and anonymous, were automatically sampled electronically.

Results: 156 health care professionals of all ages responded. $66.7 \%$ were women; $64.2 \%$ were physicians and $13.2 \%$ nurses. $22 \%$ worked at rural areas. 76.8\% used the Internet (Scholar Google and PubMed) regularly. $125(80 \%)$ answered knowledge of palliative care, while 105 (68\%) had been actively involved in chronic patients' care.Nevertheless, when asked to describe palliative care principles, only 75 were able to mention the holistic approach and the end-of-life care. Other principles were not mentioned. Awareness of the availability of in-patient and out-patient palliative services was reported by $44.2 \% ; 14.3 \%$ knew the presence of a hospice. All responders stated they knew strong opioids. However, none knew how to use them or had never used them. Responders were, also, unable to talk with patients about end-of-life $(68.2 \%)$ or advanced care planning $(66.4 \%)$. $76 \%$ were unaware of Liverpool care pathway. No collaboration with palliative care experts was mentioned.

Discussion/conclusions: Health care professionals working at new local primary care units in Greece seem to be aware of palliative care. Further education, clinical competencies and collaboration are needed, in order to integrate it clinically, in a national level in Greece.

Abstract number: P01-306

Abstract type: Poster

Why Do Palliative Care Patients Request Deep Sedation at Home in France, and how Is Deep Sedation Conducted? A Multicenter, Observational Case-control Study

Ebert M.-A. ${ }^{1}$, Richard B. ${ }^{1,2}$

${ }^{1}$ Palliative Care Department, $\mathrm{CHU}$ de Montpellier, Montpellier, France, 2Palliative Medicine, University of Montpellier, Montpellier, France

Deep sedation for palliative care patients was legalized in France in 2016. It is a difficult practice because the patients lose their capacity to communicate and are no longer autonomous. This can be stressful for doctors, nurses and caregivers who can feel deeply isolated. We tried to determine the reasons for sedation requests to further anticipate them and maybe avoid them. A secondary aim was to determine how deep sedations were conducted at home.

We conducted a multicenter, observational case-control study in patients who chose to die at home, and who requested deep sedation. All home palliative-care teams and homecare services in an area of 2.5 million people were associated with this study in order to include patients and control patients, and to collect data according to our questionnaire. Data 
including socio-demographics, spiritual faiths, symptoms, prognosis, advance-care directives, medical evaluations, deep-sedation representation and deep-sedation parameters were collected.

20 patients were included over a 3 month-period. 11 had deep sedation and 9 were control patients. The only significant difference between the 2 groups was anxiety. Anxiety was present among $81.8 \%$ of cases vs $22.2 \%$ of control patients ( $p$ 0.0216). There were no significant differences for dyspnea, pain, digestive symptoms, insomnia, depression. 5 deep sedations were started according to advance-care directives. Deep sedation was conducted with midazolam in $90.9 \%$ of patients, clorazepate in $18.2 \%$, and both drugs in $9.1 \%$. Different midazolam doses were used at daytime and nightime in 2 patients. Titration was used in 5 out of 11 patients. Midazolam boluses were necessary in 3 patients.

In this study, anxiety is the only significant factor for deep-sedation requests in home patients. Therefore home medical-teams would focus on anxiety in order to better accompany and treat palliative-care patients who choose to die at home. Home deep sedation is mainly conducted with midazolam.

Abstract number: P01-307

Abstract type: Poster

Palliative and Primary Health Care in Athens, Greece- for which Patients with Chronic, Life-limiting, Life-threatening Conditions is there an Increased Need for Care?

Panagiotou I. ${ }^{1}$, Liva E. ${ }^{2}$, Liakopoulos G. ${ }^{3}$, Stoila A. ${ }^{4}$, Roumeliotou A. ${ }^{4}$, Skliros S. ${ }^{2}$

${ }^{1}$ Local Primary Health Unit, Athens, Greece, ${ }^{2}$ Nemeas Health Center, Nemeas, Greece, ${ }^{3}$ Aiginiteion University Hospital, Athens, Greece, ${ }^{4}$ Attikon University Hospital, Athens, Greece

Background: Patients with chronic diseases usually need a communitybased palliative care; a coordination of care can be ensured, when patients are not hospitalized.

Objective: The aim of the study was to determine the incidence of palliative care patients and their needs for care at a local, primary/family care office, in a community of Athens, Greece.

Methods: A random sample of 605 patients (429 female) was selected, from the family care unit in a suburb of Athens, Greece. Patients with palliative care needs were identified, through a descriptive method; including their informal caregivers.

Results / Discussion: From a total of 605 patients, 153 (25.28\%) had a chronic, life-limiting, life-threatening condition. Their family members were 279 . Only 22 patients had cancer ( 5 survivors, 17 with locally advanced/metastatic disease, receiving oncology treatment), 7 had neurological diseases and 1 end-stage chronic obstructive lung disease. No heart or renal failure patients were identified. On the contrary, 121 patients (79.8\%) were frail elderly (community living persons aged 70 and older) (89 female, mean age 82 , range 71-93). Cancer patients and family did not report unmet needs or increased symptom burden; they considered that palliation was provided by oncologists at tertiary centers. On the contrary, all neurology and the majority of frail elderly patients $(7 / 7$ and $90 / 121$, respectively) reported numerous problems/needs. Restricted activity, depression and anxiety, insomnia, weakness and loss of independency were mentioned by all. Furthermore, more than half (66/121) worried about their financial well-being, suffered from the fear of becoming a burden to their family, as well as from the fear of the way of dying. $92 \%$ of their informal caregivers reported a need for psychosocial and financial support.

Conclusions: Palliative care in Athens could focus on frail elderly. A number of physical and psychosocial needs have to be adequately addressed.

Abstract number: P01-308

Abstract type: Poster
Moving toward Universal Access to Palliative Care: Eliminating Disparity and Improving Access to Palliative Care for People Living with Life Limiting Illness in Telangana, India

Palat G. ${ }^{1}$, Kumari P. ${ }^{2}$, Jagannath J. ${ }^{2}$, Vineela R. ${ }^{3}$, Nethagani.$^{4}{ }^{4}$ ${ }^{1}$ Palliative Medicine, Consultant, MNJ Institute of Oncology and Director, Palliative Care, Asia, Two World Cancer Collaboration, Canada, Hyderabad, India, ${ }^{2}$ Pain Relief and Palliative Care Society, Hyderabad, Hyderabad, India, ${ }^{3}$ Palliative Medicine, MNJ Institute of Oncology and RCC, Hyderabad, India, ${ }^{4}$ Director, MNJ Institute of Oncology and RCC, HYDERABAD, India

People living with life limiting illnesses in India are often out side the gamut of regular health care system. When cure is not possible, they are sent home. It is estimated that in India, only $4 \%$ of the population who need palliative care get it. Most deaths happen in hospitals. This results catastrophic health expenditures and impoverishment. Government of India mandates universal access to health care. Telangana is a state with a population of 35 million. Non-communicable diseases and injuries constitute $72 \%$ of total disease burden, disability and death. Lack of palliative care was identified as a serious issue of disparity.They identified that one of the critical component to eliminate this disparity was to improve access. They proposed to achieve this by integrating palliative care into public health system at all level of care by creating palliative care facilities and by means of home care. They partnered with non- governmental organizations (NGO). Government committed to Infrastructure, human resource and drugs including access to opioid medications. The NGO supported by sharing their expertise and resources.

Results: In the first phase, 8 centers were chosen based on need assessment. All became functional in one year. Creation of these units resulted in touching the lives of 60 in-patients and their families and more than 500 patients in their homes, at given point of time. Morphine was made available in all the centers. There was successful utilization of funds and vast infrastructure available in the public health system. Collaboration with NGO resulted in optimal use of resources. The model was found to be viable, sustainable and replicable. It is proposed to be roll out in all 23 districts of the state.

Conclusion: A public health approach resulted in reducing disparity. A quality and affordable palliative care was made available in the population and helped in reducing catastrophic health expenditures.

\section{Abstract number: P01-309 \\ Abstract type: Poster}

General Practicioners' Evaluation of Specialized Outpatient Palliative Care (SAPV) in Germany

Stichling K. ${ }^{1}$, Krause M. ${ }^{1}$, Ditscheid B. ${ }^{1}$, Hach M. ${ }^{2}$, Jansky M. ${ }^{3}$, Kaufmann M. ${ }^{1}$, Krauss S. ${ }^{4}$, Lehmann T. ${ }^{5}$, Meißner W. ${ }^{6}$, Schulz S. ${ }^{1}$, Vollmar H.C. ${ }^{1,7}$, Wedding U. ${ }^{6}$, Freytag A. ${ }^{1}$, for the SAVOIR Study Group

${ }^{1}$ Institute of General Practice and Family Medicine, Jena University Hospital, Jena, Germany, ${ }^{2}$ German Working Group for SAPV, Berlin, Germany, ${ }^{3}$ Clinic for Palliative Medicine, University Medical Center Göttingen, Göttingen, Germany, ${ }^{4}$ Center for Interdisciplinary Health Research, University of Augsburg, Augsburg, Germany, ${ }^{5}$ Center for Clinical Studies, Jena University Hospital, Jena, Germany, ${ }^{6}$ Department of Palliative Care, Jena University Hospital, Jena, Germany, ${ }^{7}$ Institute of General Practice and Family Medicine, Ruhr University Bochum, Faculty of Medicine, Bochum, Germany

Background: In Germany, since 2007, patients in need of palliation with a particularly high and multifaceted burden of symptoms can seek specialized outpatient palliative care (SAPV) services offered by multi-professional teams. SAPV is meant as an addition to the general outpatient palliative care infrastructure which is predominantly provided by GPs. As the application of SAPV requires a prescription issued mostly by GPs, their evaluation of the necessity and extent of SAPV is crucial. 
Aims: How do GPs evaluate SAPV? What factors determine their evaluation of SAPV? Which SAPV activities do they appreciate most?

Methods: A literature and interview based previously piloted questionnaire was sent to $6,000 \mathrm{GPs}$ in 8 German federal states. By August 2018 1,144 GPs had responded (19.1\%). We performed univariate analyses on the correlation between GPs' self-reported palliative care (PC) activity, their appreciation of SAPV, their PC experience, qualification and access to remuneration. Multiple regression analyses will be added.

Results: Explorative analyses showed a moderate negative correlation between GPs' PC activity and their SAPV appreciation $\left(r_{\mathrm{sp}}=-.204\right.$, $\mathrm{n}=1041, \mathrm{p}<.001)$. We found a positive correlation of the extent of GPs' PC activity with their formal qualification in PC $\left(r_{s p}=.306\right.$, $\mathrm{n}=1067, \mathrm{p}<.001)$, their remuneration of $\mathrm{PC}\left(\mathrm{r}_{\mathrm{sp}}=.338, \mathrm{n}=1110, \mathrm{p}<\right.$ $.001)$ and their experience in $P C\left(r_{s p}=.482, n=1092, p<.001\right)$. At the congress we will present further results based on multiple regression analyses.

Conclusion: The observed inverse relation between GPs' PC activity and the evaluation of SAPV is in line with the concept of SAPV as an addition to the general PC infrastructure. This has implications for the further development of PC in Germany that will be discussed on the conference. Funding: Our study is part of the SAVOIR project which is funded by Federal Joint Committee German Innovations Fund to evaluate SAPV in Germany.

\section{Abstract number: P01-310 \\ Abstract type: Poster}

DNACPR in the Community: A Case Study Analysis

Shanagher D. ${ }^{1}$, Lynch M. ${ }^{1}$, Gregan P. ${ }^{2}, O^{\prime}$ Shea B. ${ }^{3}$, Walls E. ${ }^{4}$, Charnley K. ${ }^{5}$, Ferns M. ${ }^{6}$, Holland N. ${ }^{2}$, MacMahon H. ${ }^{7}$, Marsden M. ${ }^{8}$, Foley $T^{9}$ ${ }^{1}$ Irish Hospice Foundation, Dublin, Ireland, ${ }^{2}$ Blackrock Hospice, Dublin, Ireland, ${ }^{3}$ Trinity College Dublin, Dublin, Ireland, ${ }^{4}$ Waterford University College Hospital, Waterford, Ireland, ${ }^{5}$ AllHPC, Dublin, Ireland, 6 Irish Cancer Society, Dublin, Ireland, ${ }^{7}$ Donegal Hospice, Letterkenny, Ireland, ${ }^{8} \mathrm{HSE}$, Dublin, Ireland, ${ }^{9}$ University College Cork, Cork, Ireland

Background: The pending commencement of the Assisted Decision Making (Capacity) Act 2015 that will provide a legal framework to fully support the use of advance healthcare directives in Ireland has highlighted practical issues in relation to Cardiopulmonary Resuscitation (CPR). Though HSE policy is in place that provides some guidance to staff across settings and specialisms the delivery of CPR is an area that health and social care professionals working in the community are experiencing conflicting practices and distressing situations.

Aims: The aims of this work are as follows:

- To highlight the practical issues re Do Not Attempt Cardiopulmonary Resuscitation (DNCAPR) orders in the community

- To identify gaps in policy and procedures in place regarding DNACPR

- To identify potential new ways of working to address any gaps.

Methods: For the purpose of this work a small collective case study was carried out. Rather than sharing individual stories, six cases were analysed for common themes that outline the issues of relevance in relation to CPR and DNACPR orders in the community. Crowe et al (2011) identify four stages of conducting case studies: definition, selecting, collecting and analysing and reporting.

Results: The following themes were identified via case study analysis:

1. Advance care planning

a. Needs to happen earlier

2. Policy, guidelines, procedures

a. Clarity is needed and new ways of working to be established
3. Collaboration \& Communication
a. Needed across settings and professions
4. Training and education.

Conclusion: Inconsistencies in practice and lack of clear policy and guidelines are of significant relevance and are a gap identified by this work. There is an opportunity for community based health and social care professionals to engage in advance care planning. Efforts should be made to increase and seek collaboration across specialities.

Abstract number: P01-312

Abstract type: Poster

Dying at Home - How to Make it Possible

Beyer S. ${ }^{1}$, Eibel M. ${ }^{1}$, More-Hollerweger E. ${ }^{2}$, Pelttari L. ${ }^{1}$

${ }^{1}$ Hospice Austria, Wien, Austria, ${ }^{2}$ Competence Center for Nonprofit and Social Entrepreneurship, Vienna, Austria

Background: $70 \%$ of Austrian people would prefer to die at home but it's possible only for $27,4 \%$.

In Vienna around 29.000 people use mobile homecare services every year. Almost 3.700 careworkers (nurses and domestic workers) provide these services for many patients who suffer from life limiting conditions. Neither homecare providers nor the careworkers were experienced in hospice and palliative care (HPC) and were lacking knowledge and support in difficult end of life situations.

Four major home care providers in Vienna took part in the project involving more than 2.000 careworkers within 3 years.

Aims: The project aimed to

1) provide support for careworkers when caring for patients with life limiting conditions

2) improve the collaboration with all other professions and cooperation with other home care services (e.g. general practitioners, specialized mobile palliative care teams)

3) change structures and train staff so that dying at home would become possible

Methods: The project was evaluated by the Competence Center for Nonprofit Organization \& Social Entrepreneurship.

Quantitative method

- $\quad$ baseline-survey with all careworkers at the beginning and end of the project: questionnaires were sent out to 1.395 careworkers, $97 \%$ were returned

Qualitative methods

- $\quad$ guideline-based-interviews with 22 patients and relatives

- focus groups with careworkers and managers

Results: Organisational development focusing on hospice and palliative care, introducing new roles within homecare providers and training made possible that:

- $\quad$ careworkers are more confident and better qualified in dealing with patients with life limiting conditions

- careworkers can better assess when dying is foreseeable among patients

- a new curriculum was developed, $60-80 \%$ of all careworkers were trained in 85 workshops

- cooperation with other services improved

- $\quad$ problem areas in homecare setting were pinpointed - many are based on the existing funding structures

- $\quad$ activities for sustainability were ensured. 
Abstract number: P01-313

Abstract type: Poster

Distress of Family Caregivers - Nurses' Perspectives in Outpatient Palliative Care in Vorarlberg, Austria Rizza K., Mathis G.

Bildungshaus Batschuns, Zwischenwasser, Austria

Background: A large part of outpatient caring is done by family caregivers. Their distress and overload often entail hospitalising patients. An international central aim in palliative care is enabling patients to die at home if that is their wish. To meet this, family caregivers should be best supported. Up to now the effectiveness of support in primary outpatient setting has not been investigated in Vorarlberg, Austria.

Aim: This study explores the need of support for family caregivers from nurses' perspectives in outpatient palliative care in Vorarlberg.

Methods: By online survey all 50 community-based nurse services in Vorarlberg were invited to estimate symptoms, needs and problems of family caregivers. The results are part of an online survey concerned with nurses' perspective of outpatient palliative care in Vorarlberg. The response rate was $76 \%$.

Results: Nurses report high distress of family caregivers and $36,8 \%$ $(n=14)$ say that overload is often or always reason for hospitalising patients. Facing dying and death was the highest overload perceived $(89,5 \%, n=34)$ followed by need for counselling and professional assurance $(86,8 \% ; n=33)$, fear of deteriorating and sadness or depressiveness $(84,2 \% ; n=32)$, overload with nursing and fear of crisis $(81,6 \%, n=31)$. Nurses do counselling and advising (very) often $(94,7 \% ; n=36)$ as part of their service.

Discussion: Family caregivers' need is well noticed by nurses. Considering the good deal of time spent on counselling and advising the rate of hospitalisation due to family caregivers' overload and distress seems proportionally high. Support for family caregivers is regarded one of the core competencies in palliative care. Therefore, education level in palliative care and communication skills should be optimised in outpatient palliative care in Vorarlberg.

\section{Abstract number: P01-314 \\ Abstract type: Poster}

\section{Palliative Home Care - Nursing Care Approach Attending Patient Needs}

Coelho S.P. ${ }^{1}$, Capelas M.L. ${ }^{2}$, Sá L. ${ }^{1}$

1 Universidade Católica Portuguesa, Institute of Health Sciences, Porto, Portugal, ${ }^{2}$ Universidade Católica Portuguesa, Institute of Health Sciences, Lisboa, Portugal

Aim: Identify the nursing care needs of home assisted patients requiring palliative care services from primary care centers belonging to a Local Health Unit, the north of Portugal.

Methods: A quantitative, descriptive, cross-sectional, observational study was performed, in which participants were patients enrolled in a group of primary care centers. A stratified random sample was defined per health unit and the data were collected after personal contact at each patient's home at a single moment. An instrument was developed based FACIT-Pal and FACIT-Sp having the license to use the Portuguese version. A question was posed to physicians, asked if they would be surprised in case the patients randomly selected died in a 1 year.

Results: Of the 234 selected patients, 121 of them participated in the study. The majority were women, over 70 years of age, inactive, with low education, and living in their own accommodation. Regarding the patients' needs, the physical dimension was identified by the patients as the most relevant associated with symptoms of pain and asthenia; followed by additional concerns included the fact that patients considered themselves as a burden to the family. At the emotional level, patients reported feeling sad, nervous and concerned about the evolution of their disease. At the spiritual level, patients reported lack of meaning and purpose for living, and an absence of peace of mind. At social and family dimension, patients had a bad relationship with friends, poor proximity to partner or caregiver. Concerning functionality, patients felt low professional fulfillment and general dissatisfaction with their quality of life.

Conclusion: The study is a valuable resource for strategic management of nursing care planning and promoting quality of life. Additionally, can also be used as an instrument for defining a set of interventions in Health System to be developed training requirements, nursing guidelines and cost-effectiveness.

\section{Abstract number: P01-315 \\ Abstract type: Poster}

A Needs Assessment of Global Home Healthcare Nurses' Roles, Satisfaction, and Barriers to Palliative Care Provision Fink R. ${ }^{1}$, Brant J. ${ }^{2}$, Thompson C. ${ }^{3}$, Li Y.H. ${ }^{2}$, Silbermann M. ${ }^{4}$ ${ }^{1}$ General Internal Medicine, University of Colorado Anschutz Medical Campus, Aurora, United States, ${ }^{2}$ Collaborative Science and Innovation, Billings Clinic, Billings, United States, ${ }^{3}$ Palliative Care Consult Service, University of Colorado Anschutz Medical Campus, Aurora, United States, ${ }^{4}$ Middle Eastern Cancer Consortium, Haifa, Israel

Background: The World Health Assembly urges members to build palliative care (PC) capacity as an ethical and public health imperative. Nurses provide PC services in many settings, including the home, and may be the only healthcare professional able to access disparate populations. Identifying current nursing services, resources, satisfaction with, and barriers to nursing practice are essential to build global PC capacity.

Aim: To assess the needs of home health nursing globally encompassing nurses' duties, satisfaction, barriers, preferred learning methods, and country differences related to PC development and capacity. To identify overall and country-specific views and home healthcare provision recommendations with the goal to inform the development of future educational and training activities.

Methods: A validated needs assessment was distributed globally with a cross-sectional survey study design using non-probability sampling.

Results: Convenience sampling comprised 532 home healthcare nurses in 29 countries. Nurses from developing countries performed more duties compared to those from high-income countries, suggesting a lack of resources in developing countries. Significant barriers to providing home care exist: personnel shortages, lack of funding and policies, poor access to end-of-life or hospice services, and decreased community awareness of PC services provided. Lack of time, funding, and staffing coverage are primary educational barriers. In person local meetings and on-line courses were suggested as strategies to promote learning. Conclusion/ discussion: Home healthcare nurses do not have adequate resources to build PC capacity globally, which is so desperately needed. Nurses must be up to date on current evidence and practice within an evidence-based PC framework. Health care policy to increase necessary resources and the development of a multifaceted intervention to facilitate education about PC is indicated to build global PC capacity.

Abstract number: P01-316

Abstract type: Poster

Accuracy of the Surprise Question on Patients with Advanced Chronic Disease in the Primary Care Setting de Sousa P. ${ }^{1}$, Julião M. ${ }^{2}$, Rodrigues A.P. ${ }^{3}$, Nunes $B .^{3}$ ${ }^{1}$ ECSCP Sintra, Rio de Mouro, Portugal, ${ }^{2}$ ECSCP Sintra, Massamá, Portugal, ${ }^{3}$ Departamento de Epidemiologia, Instituto Nacional de Saúde Doutor Ricardo Jorge, Lisboa, Portugal

Background: The need to identify patient with palliative care needs has resulted in the development of many tools. The Surprise Question 
(SQ) "Would you be surprised if this patient were to die in the next year?", is widely used for this purpose. Our country's reform and expansion of its palliative care network increases the need for simple and effective referral instruments. The SQ may achieve this end, but there are no studies measuring its accuracy in our country's Primary Care setting.

Aim: To measure the accuracy of the SQ at 12 months of follow-up, when applied by General Practitioners (GP) to patients with advanced chronic disease.

Methods: In this pragmatic follow up study, we recruited GPs from a General Practitioner Sentinel Network and personal invite. They were asked to select all patients from their patients list with:

1) solid cancer, stage IV;

2) congestive heart failure (CHF) NYHA stages III/IV;

3) chronic kidney disease (CKD), stages IV/V; and

4) chronic obstructive pulmonary disease (COPD), stages III/IV.

They answered the SQ at 12 months for every patient, along with reporting their demographics and chronic conditions from January to April 2017. We measured the diagnostic accuracy properties of the SQ with 95\% confidence interval.

Results: Our sample is composed of $22 \mathrm{GPs}$. A total of 209 patients were included, $34 \%$ suffered from cancer, $33 \%$ of CHF, $20 \%$ of CKD and $16 \%$ of COPD. Of these, $65 \%$ had a "not surprised" response for the SQ at 12 months. We had a follow up rate of $96 \%$. The SQ at 12 months had a sensitivity of $59.0 \%(46.7 \%-71.4 \%)$ and specificity of $70.5 \%(62.9 \%$ $-78.1 \%)$ with corresponding positive likelihood ratio of 2.00 (1.44 - 2.79), and negative likelihood ratio 0.58 (0.42 - 0.80).

Conclusion: The positive predictive value of $46.8 \%$ highlights that the SQ alone, is not an accurate tool. This should inform policy makers and drive researchers to seek alternative tools for patient referral to palliative care services.

Abstract number: P01-569

Abstract type: Poster

Methodology of Economic Appraisal of Palliative Care in the

Absence of a National Cost Database

Maessen M. ${ }^{1}$, Steck N. ${ }^{2}$, Zwahlen M. ${ }^{2}$, Eychmüller S. ${ }^{1}$

${ }^{1}$ Inselspital, Bern University Hospital, University Centre for Palliative Care, Bern, Switzerland, ${ }^{2}$ University of Bern, Switzerland, Institute of Social and Preventive Medicine, Bern, Switzerland

Background: Economic analyses of palliative care services are indispensable for politicians and health care leaders for critically appraise the best use of scarce resources in end of life. Unfortunately, many countries are lacking the required cost data to perform such analyses.

Aim: This Swiss example study demonstrates how economic estimates about the affordability of mobile specialist palliative care teams (MSPCT) for regional governments can be calculated with limited information on palliative care costs.

Methods: In the absence of a national cost database, we searched for the best data available, leading to a 10 -year-old anonymized Swiss health insurance claim database of $56 \%$ of all deceased patients. We performed a literature search and several probabilistic linking and extrapolation steps to provide estimates of the costs and cost reductions of MSPCT on health care costs in 2020. We compared the potential cost reduction with the potential costs (based on four operational MSPCT) for the evaluation of the affordability of local governments' budgets to offer MSPCT in their canton in the future.

Results: Potential total hospital cost savings in 2020 for all cantons were expected to be around 81.5 million CHF (restrictive scenario). The operation of nationwide MSPCT would cost the cantonal administrations about 18.8 million CHF per year. Therefore, the costs are less than a fourth of the expected savings. The difference between costs and savings varies from $60 \%$ in Canton Uri to $25 \%$ in the canton of Geneva.

Discussion: The methodology was successful in providing the information for data driven economical decision making for local governments. The analyses steps can be adjusted for other palliative care interventions or time projections. The development of general applicable roadmaps for the analyses of potential economic impact of palliative care in the absent of national costs databases can support a solid basis for future political decisions.

Abstract number: P01-317

Abstract type: Poster

Art in Health: The MOTH-TalkCPR Graphic Design Collaboration to Help Resuscitation Discussions in Palliative Settings Taubert M. ${ }^{1}$, Salkeld N. ${ }^{2}$, Rudolph A. ${ }^{2}$

${ }^{1}$ Cardiff University School of Medicine, Cardiff, United Kingdom,

${ }^{2}$ Falmouth University, Falmouth, United Kingdom

Background: The 'Talk CPR' project in Wales promotes anticipatory discussions about views on resuscitation for palliative patients. Work in 2017 with graphic design lecturers \& students from Falmouth University, who have their own end-of-life project called MOTH, resulted in videos and graphic designs that facilitate advance care planning discussions, in an 'ethnographical' approach model.

Aim: Explore graphic design use in medical palliative care settings as an effective way of addressing future care planning issues that face people at the end of their lives.

Methods: TalkCPR project leads met art school lecturers and students to describe the issue of resuscitation in palliative settings and tasked them with creating visual and graphic stimuli to encourage frank discussion about future wishes surrounding death, dying and matters such as wishes around cardiopulmonary resuscitation.

This resulted in the students creating artwork that looked at dying from a different angle, compared to the more typical mainstream healthcare approaches of patient information leaflets. Students produced videos, images and photographs.

Results: Students' work was displayed in two art exhibitions with relevance to healthcare, where feedback was sought. Attendees found the information displayed effective, striking and not distressing. Student projects:

- The Plan: J Cook, J Arnold, H Hosegood, S Baker.

- Let's Talk CPR: A Bray-Whitworth, J Edwardes, S Lebaigue.

- The Conversations Project: T Hallas.

- Little questions, big conversations: F Monteiro, P Andruskevicius, A Bright, E Woodman.

- \#talkdeath: M Holmes, L Scholes, T Penrice.

Artwork produced by students is now available on the http://talkcpr. wales site and is being viewed by patients.

Conclusion: Graphic design can help facilitate difficult conversations in healthcare, including those about future care decisions and resuscitation wishes. Art work and a video create by students will be displayed as part of this presentation.

Abstract number: P01-318

Abstract type: Poster

Cost of Specialized Palliative Care in Vorpommern Greifswald - A Strategic Simulation

Schindler C. ${ }^{1}$, Jülich A. ${ }^{1}$, Flessa S. ${ }^{2}$

${ }^{1}$ Department of Internal Medicine C (Haematology, Oncology and Palliative Medicine), University of Greifswald, Greifswald, Germany, 2Department of General Business Administration and Health Care Management, University of Greifswald, Greifswald, Germany 
Background: The aging society will lead to an increasing incidence of fatal diseases. Thus, the growing demand and cost of palliative care will put economic pressure on politics. In particular, it will be challenging to ensure comprehensive medical care in rural areas. However, decisions are frequently not based on evidence as economic data is scarce. This thesis aims to forecast future cost for specialized palliative care in Vorpommern Greifswald.

Method: We analyzed the existing treatment statistics and costs of 464 palliative patients in the General Ward (GW), Palliative Care Unit (PCU), Hospice (HOS) and Specialized Ambulant Palliative Care (SAPV)). These numbers have been extracted from the Data Pool of University Medicine Greifswald from 01.04.2013 to 31.03.2014 and have been analyzed from the funders' perspective. A Markov-Model was used to predict the total cost.

Results: The daily average costs per patient for specialized palliative care amount to $€ 252$. By simulating a distribution of all patients to $\mathrm{GW}$ and PCU in equal parts the costs increase by $198 \%$. Reducing the percentage of patients in PCU to $50 \%$ and GW to $10 \%$ leads to a cost reduction of $52 \%$. Administering ambulant care exclusively via SAPV leads to the lowest cost rate of $€ 106$. Without SAPV the model predicts a cost increase of $107 \%$. This number can be reduced to $72 \%$ if SAPV in nursing homes is included.

Conclusion: Hospitalization without PCU leads to a significant increase in costs. The biggest costs reduction is reached by SAPV in domestic care. Comprehensive domestic care without hospitalization would be cost optimal but not realistic due to different reasons. Consequently, effort has to be invested to create and develop conditions which allow more domestic care to reduce hospitalization, duration of hospital stays and costs for the funders.

\section{Abstract number: P01-320 \\ Abstract type: Poster}

Context, Motives and Evolution of Requests to Hasten Death in France: An Epidemiological Study Trimaille H., Godard-Marceau A., Vidal C., Pazart L., Aubry R. Clinical Investigation Center, University Hospital of Besançon, Besancon, France

Background: In France euthanasia and physician-assisted suicide are illegal, but in some situation, patients near the end of life expressed this kind of request. At the same time, public debate are still ongoing. Yet, there exist currently no empirical data to shed light on these requests.

Aims: Our aim was twofold:

(1) to identify and describe the characteristics and the reported motives of patients who formulate a wish to hasten death, and

(2) to understand how these requests evolve over time.

Methods: This was a prospective cohort study. All patients at the end of life who expressed a wish to hasten death towards a clinician between $01 / 01 / 2017$ and $31 / 12 / 2017$ in a single region could be included. Patients and clinicians gave their consent to participate in the study. Clinicians were asked to fill 3 questionnaires over one week (at day 1, 2, and 7). The study was approved by the regional ethic and national data protection committees.

Results: We identified a total of 147 requests to hasten death. Of these, 22 patients were not eligible, and 60 were eligible but didn't receive the information form. Thus, only 65 patients were included and analyzed (mean age 82 years, 51\% female, 58\% had cancer). Among them, 32 (53\%) had experienced a worsening of their health during the preceding month, 44 (73\%) were hospitalized, and 54 (90\%) expressed difficulties regarding their loss of independence. Also, 51 (85\%) had a antalgic treatment but $34(56 \%)$ were in pain. $41(68 \%)$ had the feeling of being a burden or useless. One week later, 23 out of 65 patients (36\%) reiterated their request.

Conclusion / Discussion: Our study shows that it is possible albeit difficult to conduct research about requests to hasten death in a country where both euthanasia and physician-assisted suicide are illegal. The context and motives of these requests question the way in which patients deal with mental suffering more than physical pain.

Abstract number: P01-321

Abstract type: Poster

The Use of Specialized Palliative Care and Place of Death among Cancer Patients, who Died during 2012 to 2014 in Denmark: A National Cohort Study

Jarlbaek L. ${ }^{1}$, Timm H. ${ }^{2}$

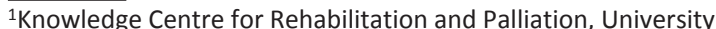
of Southern Denmark, Nyborg, Denmark, ${ }^{2}$ Knowledge Centre for Rehabilitation and Palliation, University of Southern Denmark, Copenhagen, Denmark

Background: How specialized palliative care (SPC) is used and where people die are of interest in the health care system's organization of palliative care. Epidemiological knowledge from population-based linkage studies can supplement with knowledge from randomized clinical trials investigating the relation between SPC and place of death.

Aims: To investigate where cancer patients die in relation to their use of specialized palliative care.

Methods: A cohort study using linkage between the Danish National Patient Registry, the Danish Death Causes Registry and the Cancer Registry. People diagnosed with cancer (cptts), who died during 2012 to 2014 were included. The outcomes were 1; death in hospice and 2; death in hospitals among the remainder in relation to contact with SPC. In Denmark (DK) SPC-units are; hospices, palliative teams and departments.

Results: 60,648 cptts died during 2012-2014; 43,281 died from cancer (c_deaths), while 17,367 died from other causes (notc_deaths). SPC was involved in $42 \%$ of c_deaths and $5 \%$ of notc_deaths. Hospice was place of death in $17 \%$ c_deaths and in $1 \%$ notc_deaths. SPC-teams or departments were involved in $14 \%$ c_deaths and $2 \%$ notc deaths, while $11 \%$ of c_deaths and $2 \%$ of notc_deaths have had contact with SPI prior to death, but not at the time of death. Among 29,852 deceased cptts dying from cancer without SPC involved, 54\% died in hospital, while only $27 \%$ of 5,994 deceased, who had been in contact with a palliative care team or department died in hospital.

Conclusion / Discussion: To our knowledge, this is the first linkage-study to indicate a strong relation between place of death and provision of SPC in DK. One in six patients, dying from cancer, died in hospice. Patients receiving SPC from palliative teams/department died half as frequently in hospital, compared to patients who didn't receive SPC. SPC was provided eight times more often to cancer patients dying from cancer compared to cancer patients dying from other causes.

Abstract number: P01-322

Abstract type: Poster

\section{The London Challenge in Delivering High Quality Palliative and} End of Life Care

Verne J., Pring A., Bowtell N.

Public Health England, Bristol, United Kingdom

Background: Greater London has an estimated population of 8.8 million people. London is very different to the rest of England with greater ethnic diversity $(44.9 \%$ White British, $37 \%$ of the population born outside the UK and $24.5 \%$ outside Europe). London is a young city where the average age is 36.5 . The differences between rich and poor are also extreme with some of the richest and poorest areas in the country. 
Aim: To describe some of the specific challenges for Palliative and End of Life Care (P\&EOLC) in London.

Methods: The indicators in End of Life Profiles and Atlas of Variation in P\&EOLC developed by Public Health England were used to compare the ranking of Clinical Commissioning Groups (CCGs) in London (32 CCGS) with the rest of England (total 209 CCGs)and for local authorities where the indicator was only available for this geography.

Results: 8 of the top 10 local authorities for people $>65$ living alone are in London (40-45\% of $>65 \mathrm{~s}), 7$ of the 10 with the highest $>3$ emergency admissions in the last 90 days of life are in London ( $>10.2 \%$ of those who die), 6 of the 10 CCGs with the greatest \% of patients dying in hospital after an admission of $>8$ days are in London ( $>57.5 \%$ have final admissions of $>8$ days), 8 out of the 10 lowest for death in usual place of residence (home/care home) were in London and 9 of the 10 lowest CCGs for death in care home were in London. 10/10 lowest populations living in care homes were in London reflecting the low rate of provision of beds $(<6)$ per 100 population $>75$ years with $7 / 10$ lowest. Statistical significance of the difference between each London CCG and the England value was calculated with the majority of CCGs showing statistically significant differences for indicators of P\&EOLC.

Conclusions: London faces very different challenges in provision of P\&EOLC compared to the rest of the country not least because of the isolation of elderly people living alone in the capital and the shortage of provision of care home places.

Abstract number: P01-323

Abstract type: Poster

\section{Why Do Nurses Decide to Work in Palliative Care? Results of a Nationwide Study in Germany \\ Rieger S. ${ }^{1}$, Diehl E. ${ }^{1}$, Schablon A. ${ }^{2}$, Nienhaus A. ${ }^{3}$, Letzel S. ${ }^{1}$, Escobar Pinzon L.C. ${ }^{1,4}$ \\ ${ }^{1}$ Institute of Occupational, Social and Environmental Medicine, University Medical Center of the Johannes Gutenberg University of Mainz, Mainz, Germany, ${ }^{2}$ Competence Center for Epidemiology and Health Services Research for Healthcare Professionals (CVcare), University Medical Center Hamburg-Eppendorf, Hamburg, Germany, ${ }^{3}$ Institute for Health Care Research in Dermatology and Nursing, University Medical Center Hamburg-Eppendorf, Hamburg, Germany, ${ }^{4}$ Federal Institute for Occupational Safety and Health (BAuA), Berlin, Germany}

Background: In the literature, the reasons why nurses decide to work in the palliative sector in Germany are hardly investigated.

Aims: The aim of the study* was to examine the reasons why nurses choose to work in palliative care.

Methods: A cross-sectional study (2017) of 1,316 nurses in inpatient and outpatient specialized palliative care in Germany (response rate $39 \%$ ) was used as the data basis. Open questions were asked about the reasons for working in palliative care and what characteristics nurses should possess in palliative care. The data analysis was based on two basic types of Grounded Theory (open and axial coding) with the analysis software MAXQDA.

Results: The average age was about 46 years (SD: 10.2; Range: 20-69 years). The reasons for working in specialized palliative care were: Time for patients and relatives $(n=264)$, better care $(n=227)$, interest in the topic/work/pleasure at work $(n=191)$, individual care $(n=127)$, desire for support $(n=125)$. Empathy $(n=674)$, professional competence ( $n$ $=82)$ and balance $(n=61)$ were named as characteristics which nurses in palliative care should have.

Conclusion / Discussion: The most frequent reasons for working in palliative care were better professional conditions and personal factors. Besides this nurses in palliative care are on average older than nurses in general care.

Empathy also seems to play an important role in this area. The extent to which personal characteristics have an influence on working in palliative care, especially in dealing with stress, should be examined in more detail in further research.

*The study was funded by the Employers' Liability Insurance Association for Medical Services and Welfare Work (BGW). The abstract contains parts of the doctoral thesis of Sandra Rieger.

\section{Abstract number: P01-324}

Abstract type: Poster

Nationwide Hospital Admissions in Children with Complex Chronic Conditions (2011-2015)

Lacerda A. ${ }^{1,2}$, Oliveira G. ${ }^{3}$, Cancelinha C. ${ }^{2,4}$, Lopes S.., 6

${ }_{1}^{1}$ Pediatrics, Instituto Português de Oncologia de Lisboa, Lisboa, Portugal, ${ }^{2}$ Observatório Português de Cuidados Paliativos, Lisboa, Portugal, ${ }^{3}$ Pediatrics, Hospital de Santa Maria, CHULN, Lisboa, Portugal, ${ }^{4}$ Medical Pediatrics, Hospital Pediátrico de Coimbra, CHUC, Coimbra, Portugal, ${ }^{5}$ Escola Nacional de Saúde Pública, Universidade NOVA de Lisboa, Lisboa, Portugal, ' ${ }^{6}$ Centro de Investigação em Saúde Pública, Escola Nacional de Saúde Pública, Universidade NOVA de Lisboa, Lisboa, Portugal

Aim: Due to the epidemiological change in Pediatrics, health systems have a growing interest in complex chronic conditions (CCC). We aimed to evaluate the use of hospital inpatient care by children with CCC in a developed Western European country, with limited recognized pediatric palliative care (PPC) provision in the years studied.

Methods: Epidemiological study using administrative data of admissions to public hospitals within the national health service. We selected hospitalizations within pediatric age limit (0-17y), 2011-2015; excluded healthy newborns and radiotherapy outpatients. Performed descriptive analysis of the annual numbers of admissions, length of stay, costs, and deaths, by CCC category; region of residence and hospital group; used non-parametric tests, $\mathrm{P}<0.001$.

Results: In $15.5 \%(\mathrm{~N}=64.918)$ of the admissions ( $\mathrm{N}=419.927)$ there was at least one CCC code; these represented $29.8 \%$ of hospital days, $39.4 \%$ of costs and $87.2 \%$ of deaths. CCC hospitalizations' expenses were double (median $€ 1467$ vs. $€ 745$ ) and mortality 40 times higher ( $2.4 \%$ vs. $0.06 \%$ ) as those without CCC. These differences were even more significant when there was more than one CCC. Of the CCC hospitalizations: $46 \%$ planned (no CCC $23.2 \%$ ); $64.8 \%$ in tertiary / specialized hospitals (no CCC $27.1 \%$ ); malignant was the most frequent CCC category $(23.0 \%$ ); neonatal had the biggest median cost (€3568, 92924602 ), median length of stay (12 days, 6-41), and number of deaths (43.5\% of total).

Conclusions: In a developed Western European country with scarce PPC provision, CCC are relevant in the activity and costs of pediatric hospitalizations. Recognizing this and integrating PPC since CCC diagnosis are essential to an appropriate hospital use, by developing effective and sustainable alternatives that meet the needs of children, families, and healthcare professionals.

Abstract number: P01-325

Abstract type: Poster

Factors of Access to Palliative Cares in Patients with Cancer: A Literature Review

Frasca M. ${ }^{1,2}$, Bidegain-Sabas A. ${ }^{1}$, Galvin A. ${ }^{2}$, Burucoa B. ${ }^{1}$, Bellera C. ${ }^{2}$, Mathoulin-Pellissier S. ${ }^{2}$

${ }^{1}$ Palliative Medicine Department, Bordeaux University Hospital, Bordeaux, France, ${ }^{2}$ Population Health INSERM U 1219, University of Bordeaux, Bordeaux, France

Background: Palliative cares preserve quality of life regardless disease stage and setting of cares. Guidelines recommend an early access to these cares for newly diagnosed cancer patients with high symptom burden or advanced disease. Literature reflects a constant underuse and a 
late referal to these services. Further understanding of the factors involved in access to palliative care is required for patients with cancer. Aims: Objectives were to identify the described factors and outcomes regarding access to palliative cares in literature and to precise the research unmet needs.

Methods: We performed a systematic review of the last 10-year litterature using MEDLINE and SCOPUS databases. We selected articles focused on both palliative care, cancer patients and access outcomes and collected data on journal, study, factors, timing and setting of access.

Results: The analysis included 56 studies. Most of them set in America $(n=41)$, used a logistic regression $(n=51)$ and included aggregated data $(n=27)$. Only five reported multilevel analyses and none took into account competitive risk of death. Most studied factors were sociodemographic and cancer characteristics $(n=42, n=30)$. Older patients or those with haematological cancers seemed less likely to access to palliative cares ( $O R=0.28-0.99, O R=0.44-0.98)$. Advanced cancer or public coverage usually led to a higher referral. Only rare articles focused on early access or outpatient settings.

Discussion: Studied factors are not multidimensional. While substantial literature exists on sociodemographic and tumor-related factors, psychosocial and existential needs are poorly described. Health policies for oncology-embedded palliative cares should focus on elderly patients and haematological cancers. Further research is required in elderly patients and European population, emphasizing on multilevel and competitive risks models. This review also highlights research needs regarding early referral and outpatient facilities access.

Abstract number: P01-326

Abstract type: Poster

Comparison of End of Life Care Intensity between ChineseAmerican and Caucasian Advanced Cancer Patients at an American Tertiary Medical Center

Ernst E. ${ }^{1}$, Vesel T. ${ }^{2}$, Tybor D. ${ }^{3}$

${ }^{1}$ Tufts University School of Medicine, Boston, United States, ${ }^{2}$ Medicine, Tufts Medical Center, Boston, United States, ${ }^{3}$ Public Health and Community Medicine, Tufts University School of Medicine, Boston, United States

Background: Understanding current ethnic disparities in end of life care (EOLC) intensity is central to improving outcomes for diverse patient populations. Although Chinese-Americans represent one of the fastest growing ethnic groups in the U.S., little is known about their EOLC intensity. Aim: To explore differences in EOLC intensity in the final 30 days of life, and location of death between Chinese-American and Caucasian advanced cancer patients.

Methods: In this exploratory review, we collected data on 48 ChineseAmerican and 48 Caucasian stage IV solid tumor patients at a tertiary medical center who died 2013-2018: indicators of high intensity care from the last 30 days of life including $\geqslant 2$ hospital, $\geqslant 1$ intensive care unit (ICU), and/or $\geqslant 2$ emergency department admissions; cardiopulmonary resuscitation (CPR) administration; and mechanical ventilation; as well as location of death; and whether patients were on hospice at death.

Results: Fewer Chinese-Americans died at home (17\% vs $43 \%$ ) and more died in hospitals ( $49 \%$ vs $36 \%$ ) and nursing homes (17\% vs $5 \%$ ) compared to Caucasians. Hospice enrollment was similar between groups. More Chinese-Americans died in the ICU (15\% vs $7 \%$ ) and received mechanical ventilation ( $17 \%$ vs $8 \%$ ). Mean age at death was 74.2 for ChineseAmericans and 69.1 for Caucasians. More Chinese-Americans had lung cancer compared to Caucasians ( $54 \%$ vs $29 \%$ ). Time between diagnosis and death was less for Chinese-Americans ( 4.42 vs. 7.65 geometric mean months). More Chinese-Americans died within 30 days of diagnosis ( $17 \%$ vs $8 \%$ ).

Conclusion: Our finding that fewer Chinese-Americans died at home deserves further exploration. More Chinese-Americans died in the ICU, received mechanical ventilation, and died within 30 days of cancer diagnosis, suggesting possible disparities in EOLC that deserve further attention. Studies with larger sample sizes are needed to explore differences indicated in this preliminary study.

Abstract number: P01-327

Abstract type: Poster

Social Networks, Social Capital and End-of-Life Care in People with Dementia: A Realist Review

Sawyer J.M., Kupeli N., Sallnow L., Stone P., Sampson E.L.

Marie Curie Palliative Care Research Department, University College London, London, United Kingdom

Background: Transition towards end-of-life is poorly recognised in people with dementia. The responsibility of care often falls on informal carers leading to burnout. The expansion of dementia friendly communities, whilst helping with these issues, poses questions over the role and impact of social networks at the end-of-life.

Aims: To evaluate the role of social networks in caring for people with end stage dementia. Specifically, what are the mechanisms and associated contexts leading to observed health outcomes.

Methods: Conceptualising social networks as complex social interventions, we used realist methodology to identify and explain interactions between context, mechanism and outcome. Searching was iterative and purposive across 3 electronic databases (Medline, EMBASE and Cinhal). All study designs and outcomes were included. Using immersion and interpretation, theoretical models of how social networks work for people with dementia were tested to see if they explained observed outcomes.

Results: We identified 38 articles from 13 countries ranging from 1996 to 2018. Transition in care was the most frequently examined outcome. Where social networks were incorporated into the transition process connections were preserved and more favourable outcomes in terms of independence and autonomy were reported. This was achieved by accessing specialist biographical knowledge, via the social network, relating to the person with dementia. Accessing this knowledge allowed integration of new members to the network whilst preserving the original networks autonomy. 'Success features' were a function of the interaction with the cultural, economic and social context of a social network.

Conclusion: Accessing a social networks specialist knowledge offers a mechanism on which to build upon the therapeutic potential of social capital. By recognising transition towards the end-of-life may be necessary, the focus must be on preserving connections between the person and their surrounding network.

Abstract number: P01-328

Abstract type: Poster

Why Don't the British Talk about End-of-Life Care? A Secondary Analysis of Associated Factors among the General Public Gallagher J.

University of Oxford, Oxford, United Kingdom

Background: Early discussion of wishes for care is a key component of advance care planning and thereby impacting on access to relevant services. In recent years, coalitions such as Dying Matters and the Death Cafe movement have sought to de-medicalise conversations about dying and break the taboo around death. Yet despite these efforts, not much is known which groups of the British public do not typically discuss their wishes for end-of-life care who could benefit from conversation.

Aims: This study aims to:

1. Identify factors associated with having not discussed about any wishes for end-of-life care with another person among the British general public. 
2. Compare factors among different topics: medical care, spiritual care, privacy and peace, dignified care, place of death, and pain relief.

Methods: Data from a randomised, stratified and weighted sample of the British public was analysed. Binary logistic regression was first conducted on participants who had discussed any wish for end-of-life care with another person, and then on individual topics of discussion.

Results: The response rate was 54\%, with 2058 respondents' data analysed. $32 \%$ had discussed their wishes. Associated factors of not discussing wishes for end-of-life care were; being male, younger, born outside the UK, having no experience working in health or social care, being of good health, no recent death of a close person, and being uncomfortable talking about death. Comparison of topics revealed; university education was associated with discussing medical wishes; religion was associated with discussing spiritual care; living alone and higher household income were associated with discussing pain relief.

Conclusion: This is the first study to explore factors in Britain and the first to compare different topics. Given these factors, future consideration on how to engage these populations in conversation, especially those born outside of the UK, may aid a public health approach to endof-life care.

\section{Abstract number: P01-329 \\ Abstract type: Poster}

Palliative Care Culture in Alternative Housing: A Participatory Research Project

Reitinger E. ${ }^{1}$, Dressel G. ${ }^{2,3}$, Wälter R. ${ }^{4}$, Vinke B. ${ }^{4}$, Zybell L. ${ }^{4}$, Völkel M. ${ }^{2,3}$, Pichler B. ${ }^{1}$

${ }^{1}$ Department of Nursing Science, University of Vienna, Vienna, Austria, ${ }^{2}$ Institute for Palliative Care and Organisational Ethics, University of Klagenfurt, Vienna, Austria, ${ }^{3}$ nnstitute for Palliative Care and Organisational Ethics, University of Klagenfurt, Graz, Austria, ${ }^{4}$ Workers' Welfare Association (AWO), Bielefeld, Germany

Background: Staying at home or at places where one can "feel at home" until the end is important for many people living in alternative housing. This needs a diverse range of individual competences, communication, social networks and adequate structures in end of life care.

Aims: The aims are

1) Identifying factors supporting people to live and die in their preferred place and social setting in alternative housing.

2) Increasing of an interdisciplinary, community based palliative care culture.

Methods: The project is designed as participatory research in three steps:

1) Investigation and assessment of needs and demand. A mixed methods study has been conducted throughout the first year.

2) Actions are designed and established collaboratively between provider-organisation and research-team in the second year. Process evaluation is going on.

3) In the third year, an outcome evaluation will be conducted.

Results: Based on the recommendations of the first year, a range of actions have been taken throughout the second year.

1) Regular steering committee meetings have been established.

2) Informative meetings in the community have been conducted.

3) A course for volunteers has been developed and organised.

4) "Last aid courses" have been performed.
5) Palliative care courses for staff have been realised.

6) Networking between members of provider organisations, hospice, specialized palliative care and volunteers as well as with residents and relatives living in the community has been supported. Process evaluation shows that there are rich resources but there is still a high need for information in the community.

Conclusion: Increasing palliative care culture in the community and alternative housing needs complex interventions. Participatory research can offer communicative spaces that support coordinated action, structured communication and trustful relationships. Boundaries of socialand health care systems have to be taken into account.

Funding: Stiftung Wohlfahrtspflege NRW

Abstract number: P01-330

Abstract type: Poster

Future Palliative Care Needs for People Living and Dying with Life-limiting Illness: Results from Ireland

May P. ${ }^{1,2}$, Johnston B.M. ${ }^{1}$, Higginson $1 .{ }^{3}$, Normand C. ${ }^{1,3}$, Ryan K..$^{4,5}$

${ }^{1}$ Centre for Health Policy and Management, Trinity College Dublin, Dublin, Ireland, 2The Irish Longitudinal Study on Ageing (TILDA), Dublin, Ireland, ${ }^{3}$ Cicely Saunders Institute, Kings College London, London, United Kingdom, ${ }^{4}$ School of Medicine, University College Dublin, Dublin, Ireland, ${ }^{5}$ School of Medicine, Trinity College Dublin, Dublin, Ireland

Background: Palliative care needs are growing worldwide due to population ageing and the shifting burden from infectious to chronic disease. Historic estimates that $75 \%$ of people who die have palliative care needs are therefore too low. More sophisticated quantification of palliative care need at end of life and earlier in the disease trajectory is essential for adequate planning of services.

Aims: Our primary aim was to estimate the number of people in Ireland with palliative care need who will die annually to 2046, taking into account population ageing and changing disease patterns. Our secondary aim was to expand these estimates to include quantification of those aged $50+$ with palliative care needs prior to the year of death.

Methods: Data were drawn from three sources: government projections of deaths to 2046, by age and gender; government records of deaths since 2007, by cause, age and gender; The Irish Longitudinal study on Ageing (TILDA) data on prevalence of diseases with palliative care need in the population aged $50+$. We estimated age- and gender-adjusted proportions of need to 2046, incorporating increases in prevalence of serious chronic diseases.

Results: The number of people dying with palliative care need in Ireland will increase from 21,907 (76\% of all deaths) in 2016 to 39,081 (81\%) in 2046. This represents a $78 \%$ absolute increase, compared to $45 \%$ in the United Kingdom, which is explained by Ireland's younger population. Over $50 \%$ of people aged $50+$ will experience advanced cancer, organ failure or dementia, a large majority will not die within a year of diagnosis, and these groups will also grow precipitously due to demographic ageing.

Conclusion: Countries with young populations experiencing the epidemiological shift to chronic disease face profound challenges in service planning. Improved delineation of specialist and generalist need according to disease and proximity to death would assist such planning.

Abstract number: P01-331 Abstract type: Poster

Using Participatory Co-design to Create Supporting End-of-Life Environments in Elder Care

Stranz A. ${ }^{1,2}$, Hilton R. ${ }^{3}$, Westerholm B. ${ }^{4}$, Macarow K. ${ }^{5}$, Nilsson F. ${ }^{4}$, Tishelman C. $2,6,7$, Goliath I. $^{2}$ 
${ }^{1}$ Stockholm University, Stockholm, Sweden, ${ }^{2}$ Karolinska Institutet, Dep Learning, Informatics, Management and Ethics, Stockholm, Sweden, ${ }^{3}$ Stockholm University of the Arts, Stockholm, Sweden, ${ }^{4}$ University of Arts Craft and Design, Stockholm, Sweden, ${ }^{5}$ RMIT, Melbourne, Australia, ${ }^{6}$ Center for Rural Medicine, Storuman, Sweden, ${ }^{7}$ Stockholm Health Care Services, Stockholm, Sweden Presenting author email address: ida.goliath@ki.se

Residential care homes ( $\mathrm{RCHs}$ ) are common places for end-of-life care (EoL) in Sweden. While discourse about dying and death in RCHs often has a negative focus, in this project we work with constructive change processes, which can better support quality of life and death in RCHs. This action research project, part of the DöBra research program, uses a variety of approaches based on the Ottawa Charter for Health Promotion to: develop personal skills; create supportive environments; and reorient health services in relation to EoL care. We aim to both explore and use experiences from the $\mathrm{RCH}$ setting in co-design workshops with residents, family, staff and researchers to prioritize and carry out change processes that promote supportive EoL care environments from these stakeholders' perspectives.

Our transdisciplinary research team works at Sweden's largest RCH through participatory co-design groups bringing together residents, family and staff. Recruitment began in January 2018 at 2 units. To date, there have been 39 participants -16 residents, 3 family members, 14 staff, and 6 researchers have attended one or more of the 13 groups held.

These discussions have enabled residents, often physically and cognitively frail, their relatives, and staff to work together and exchange knowledge. Residents, family and staff have jointly formulated prioritized areas for improvement related to: 'being seen \& heard', 'sense of community', 'meaningful common spaces', and 'bodily care \& sensory experiences'. At present, suggested potential improvements focused on 'being seen \& heard' derived from the groups are being implemented. We found that the aspects participants prioritized related to their impact on identity, relationships, power, and meaningfulness. Expertise, ideas and insights from all stakeholder perspectives in the $\mathrm{RCH}$ setting have proven to be crucial in researching and actualizing change processes with potential to have sustainable impact on the EoL setting.

Abstract number: P01-332

Abstract type: Poster

The Impact of Population Ageing on End-of-Life Care in Scotland: Population-based Projections of Place of Death and Recommendations for Future Service Provision

Finucane A. ${ }^{1,2}$, Bone A. ${ }^{3}$, Evans C. ${ }^{3}$, Meade R. ${ }^{4}$, Gomes B. ${ }^{3,5}$, Warren T. Higginson I. ${ }^{3}$, Murray S. ${ }^{2}$

${ }^{1}$ Marie Curie Hospice Edinburgh, Edinburgh, United Kingdom, ${ }^{2}$ Usher Institute of Population Health Sciences and Informatics, University of Edinburgh, Edinburgh, United Kingdom, ${ }^{3}$ Cicely Saunders Institute of Palliative Care, Kings College London, London, United Kingdom, ${ }^{4}$ Marie Curie, Edinburgh, United Kingdom, 5 University of Coimbra, Coimbra, Portugal, ${ }^{6}$ Scottish Government, Edinburgh, United Kingdom

Background: Due to global population ageing, we are expecting a rise in the number of deaths, with implications for service provision in different care settings. It is essential to examine where future deaths may occur at a national level, to facilitate decision-making regarding future service provision and resource allocation.

Aims: To project where people will die from 2017 to 2040 across all care settings in Scotland, and identify expert recommendations in response to projected trends.

Methods:

1. Population-based trend analysis of place of death using all deaths in Scotland (2004-2016) and projections using simple linear modelling (2017-2040).
2. A consensus group meeting with experts from government, clinical practice and academia, using Transparent Expert Consultation techniques to identify recommendations.

3. Results: Annual deaths in Scotland are projected to increase by $15.9 \%$ from 56,728 in 2016 (32.8\% aged $85+$ years) to 65,756 deaths in 2040 (45\% aged $85+$ years). Between 2004 and 2016, proportions of home and care home deaths increased (19.8\%$23.4 \%$ and $14.5 \%-18.8 \%$ ), while the proportion of hospital deaths declined (58.0-50.1\%). If current trends continue, the numbers of deaths at home and in care homes will increase by $45.7 \%$ and $79.4 \%$, and two-thirds of patients will die outside of hospital by 2040. To sustain current trends, expert recommendations include: 1 ) to increase, equip and sustain the health and social care workforce through education, training and valuing of care work; 2 ) to build community capacity and resilience through informal carer identification and support, and community engagement, and 3 ) to hold a realistic national debate on funding of palliative and end-of-life care.

Conclusion: To sustain current trends, end-of-life care provision in community settings needs to increase by over $60 \%$ by 2040 , otherwise hospital deaths will increase. Investing in a community based health and social care workforce; and supporting informal carers are crucial.

Abstract number: P01-334

Abstract type: Poster

Are we Evolving towards More and Earlier Use of Palliative Home Care? A Trend Analysis Using Population-Level Data from 2010-2015

Maetens A., Beernaert K., Deliens L., Van den Block L., Cohen J., End-oflife care research group

End-of-Life Care Research Group, Vrije Universiteit Brussel \& Ghent University, Brussels, Belgium

Background: The need for increased use and earlier initiation of palliative home care has been advocated in the past decade.

Aim: To investigate time trends in the use of palliative home care support and the timing of its initiation between 2010 and 2015 .

Design: Observational study using routinely-collected health claims data linked with population-level databases.

Setting: All deaths of home-dwelling people with possible palliative care needs (estimated by their underlying cause of death) in Belgium 2010$2015(n=230,704)$.

Measures: We measured the use of a multi-disciplinary palliative home care team, palliative home nursing care, palliative physiotherapy at home, or the allowance for palliative home patients. We calculated actual and standardized (for age, sex and cause of death) rates and median number of days before death of initiating palliative home care support for each year. Trends were also calculated within specific disease groups.

Results: The proportion of home-dwelling people with possible palliative care needs remained stable between 2010 and 2015 (about 36\% of all deaths). The uptake of palliative home care support increased from $31.7 \%$ to $34.9 \%$ (36.0\% standardized) between $2010-2015$. The uptake increased among cancer and organ failure patients, but not among dementia patients. The timing of initiating any type of palliative home care support increased from a median of 41 days to 46 days before death. Dementia patients had the smallest increase and latest initiation compared to the other disease groups, with an increase from a median of 20 to 22 days before death.

Discussion: There is a trend towards more and earlier uptake of palliative home care support. However, this was not the case among dementia patients, and non-cancer patients overall still have lower and later uptake than cancer patients. Policy and practice should focus on making more and earlier access to palliative home care equitable. 
Abstract number: P01-335

Abstract type: Poster

Appropriateness of End-of-Life Care in Dementia: A Populationlevel Evaluation Using Quality Indicators De Schreye R. ${ }^{1}$, Smets T. ${ }^{2}$, Deliens L. ${ }^{2,3}$, Annemans L. ${ }^{4}$, Gielen B. ${ }^{5}$, Cohen J. ${ }^{2}$

${ }^{1}$ End of Life Care Research Group, University of Brussels, Brussels, Belgium, ${ }^{2}$ End of Life Care Research Group, Vrije Universiteit Brussel \& Ghent University, Brussels, Belgium, ${ }^{3}$ Department of Medical Oncology, Ghent University, Ghent, Belgium, 4 Public Health, Ghent University, Ghent, Belgium, ${ }^{5}$ InterMutualistic Agency, Brussels, Belgium

Introduction: People dying from dementia benefit most from a care approach focused on comfort, avoiding overly aggressive treatment of comorbidities. Hitherto, the appropriateness of end-of-life care in people with dementia has not been evaluated for the full population. Using previously validated population-level quality indicators (QIs) for dementia this study aims to 1 ) evaluate the appropriateness of end-of-life care in people dying from dementia in Belgium and 2) to establish relative standards for appropriate end-of-life care in this population.

Methods: We conducted an observational study of all persons deceased from dementia in 2015 in Belgium. Health and medication data were linked to six other administratively collected population-level databases. Qls were calculated using a previously validated set of 28 Qls for end-of-life dementia care. We used the distribution in quality indicator scores across 14 health regions to suggest the best scoring quartile as a relative standard.

Results: Of 11,410 people who died from dementia in Belgium in 2015, 64.2 percent died at home or in the nursing home where they lived. In the last 30 days before death, 46.2 percent were subscribed anti-hypertensives, 26.3 gastric protectors and 38.0 percent NOACs or vitamin $\mathrm{K}$ antagonists. The relative performance standard (based on best scoring quartile) for ED admission could be realistically set below 28.1 percent, for dispension of gastric protectors below 29.5 percent, and for antihypertensives below 51 percent.

Conclusion: For several indicators of inappropriate end-of-life care in dementia, including potentially inappropriate medication, ED and hospital admissions, relatively high percentages were found. The large riskadjusted variation between health regions for several Qls indicates realistic opportunities for quality improvement in end-of-life dementia care. The relative standards we determined can be indicating the direction for quality improvement efforts.

Abstract number: P01-336

Abstract type: Poster

Self-management of Patients with Advanced Cancer: A

Systematic Review of Experiences and Attitudes

van Dongen S. ${ }^{1}$, de Nooijer K. ${ }^{1,2}$, Cramm J. ${ }^{3}$, Francke A. ${ }^{4,5}$, Oldenmenger

W. ${ }^{6}$, Korfage I. ${ }^{7}$, Witkamp E. ${ }^{8}$, van der Heide A. ${ }^{1}$, Rietjens J. ${ }^{1}$

${ }^{1}$ Public Health, Erasmus University Medical Center, Rotterdam, Netherlands, ${ }^{2}$ End-of-Life Care Research Group, Vrije Universiteit Brussel \& Ghent University, Brussel, Belgium, ${ }^{3}$ Erasmus School of Health Policy and Management, Rotterdam, Netherlands, ${ }^{4}$ Expertise Center Palliative Care, Amsterdam Public Health Research Institute, Amsterdam, Netherlands, ${ }^{5}$ Nursing and Caring Research, Netherlands Institute for Health Services Research (NIVEL), Utrecht, Netherlands, ${ }^{6}$ Medical Oncology, Erasmus University Medical Center, Rotterdam, Netherlands, ${ }^{7}$ Erasmus University Medical Center, Rotterdam, Netherlands, ${ }^{8}$ Nursing and Research Centre Innovations in Care, Rotterdam University of Applied Sciences, Rotterdam, Netherlands

Background: Patients with advanced cancer are increasingly expected to self-manage their health and care. However, studies on this topic are heterogeneous regarding self-management strategies and populations examined. We present an overview of self-management strategies of patients with advanced cancer, their experiences with these strategies, and attitudes of relatives and healthcare professionals towards selfmanagement of these patients.

Methods: We systematically searched Medline (Ovid), Embase, Cochrane Central, PsycINFO (Ovid), CINAHL (Ebsco), Web of Science Core Collection and Google Scholar for observational studies on self-management of patients with advanced cancer published until February 2017. Study selection and quality appraisal of included studies were performed by two researchers independently. Data were extracted with a pilottested extraction form.

Results: The search yielded 1342 studies, of which 27 studies (i.e. seven quantitative studies and 20 qualitative studies) were relevant for this review. Twenty-six studies assessed patients' perspectives; two studies addressed perspectives of healthcare professionals. Patients with advanced cancer used self-management strategies in seven domains: medicine and pharmacology, lifestyle, psychology/ mental health, social support, knowledge and information, navigation and coordination, and decision-making. Strategies and experiences with these strategies were highly individual and sometimes ambivalent. Nurses and other healthcare professionals generally perceived self-management as both desirable and achievable if based on sufficient skills and knowledge and solid patient-professional partnerships.

Conclusion: Self-management of patients with advanced cancer spans many domains. Patients use highly individual and sometimes seemingly contradictory strategies. Self-management support can benefit from an individualized approach and embedding in solid partnerships with relatives and healthcare professionals.

Abstract number: P01-337

Abstract type: Poster

Transforming the Approach to Death: Social Approaches and Strategies for Change Noonan K. ${ }^{1,2,3}$, Horsfall D. ${ }^{4}$, Leonard . $^{4}$

${ }^{1}$ Western Sydney University, Penrith (Kingswood), Australia, ${ }^{2}$ Palliative Care, Liverpool Hospital, Liverpool, Australia, ${ }^{3}$ The GroundSwell Project, Woodford, Australia, ${ }^{4}$ School of Social Sciences and Psychology,

Western Sydney University, Penrith (Kingswood), Australia

Aims: Dying well is a social issue, yet there is very little evidence that the social model has been incorporated into end-of-life care practices in Australia. This research aimed to explore the lived experiences of people who, despite the dominant biomedical approach to end of life and deathcare, their work is guided by social or new public health approaches to dying, death and loss.

Design/ methods: Critical social science is concerned with questioning dominant ideologies and taken-for-granted assumptions, and giving voice to alternative viewpoints. In-depth interviews were conducted with 12 people ( 6 institutionally based and 6 community based) who work in end of life and death care. These 'deathworkers' identified as palliative care specialists, nurses, academics, death doulas/end of life workers, artists, community development workers and death educators. Methods of analysis were informed by a critical and interpretive approach using a six phase inductive thematic approach outlined by Braun and Clarke (2006).

Results:

1. Strategies and tactics used for creating social and cultural change are shaped and influenced by workplace setting (i.e.institutional or community) and death literacy

2. The 'siloed' system of end of life care despite its limitations creates 'transitional spaces' that can activate social change practices and activism.

3. Informal or community based work was invisible to the formal healthcare and deathcare systems even though it was 
practiced across, within, and around all of the silos of care, at any stage of illness, including post-death work in preparation for funeral and burial.

Conclusion: If palliative care and other formal service providers are going to adopt social practices such as compassionate communities, a greater acknowledgement of existing community capacity and informal end of life care is needed. If public health approaches to palliative care develop into a social movement, transformative practice, and emergent leadership is needed.

\section{Abstract number: P01-338}

Abstract type: Poster

\section{National Prevalence and Profiles of Children with Complex} Chronic Conditions (2011-2015) Lacerda A. ${ }^{1,2}$, Oliveira G. ${ }^{3}$, Cancelinha C. ${ }^{2,4}$, Lopes S. ${ }^{5,6}$

${ }^{1}$ Pediatrics, Instituto Português de Oncologia de Lisboa, Lisboa, Portugal, ${ }^{2}$ Observatório Português de Cuidados Paliativos, Lisboa, Portugal, ${ }^{3}$ Pediatrics, Hospital de Santa Maria, CHULN, Lisboa, Portugal, ${ }^{4}$ Medical Pediatrics, Hospital Pediátrico de Coimbra, CHUC, Coimbra, Portugal, ${ }^{5}$ Escola Nacional de Saúde Pública, Universidade NOVA de Lisboa, Lisboa, Portugal, ${ }^{6}$ Centro de Investigação em Saúde Pública, Escola Nacional de Saúde Pública, Universidade NOVA de Lisboa, Lisboa, Portugal

Aim: Health systems have a growing interest in children with complex chronic conditions (CCC) and their unmet needs. We aimed to evaluate the prevalence and profile of children with CCC in a developed Western European country, with limited pediatric palliative care (PPC) provision in the years studied.

Methods: Epidemiological study using administrative data of admissions to public hospitals within the National Health Service. We selected admissions within pediatric age limit (0-17y), 2011-2015, with CCC codes; a unique identifier allowed yearly individual follow-up. Descriptive analysis (by gender, age group, CCC category, and region of residence) of: prevalence (per 10.000 children); annual number of admissions (NoA) and length of stay (LoS); children in top 5\% NoA and LoS.

Results: Yearly number of children hospitalized with CCC varied between 7.401 (2011, prevalence 41.2) and $7.673(2015,45.0)$, the highest being 7.760 (2014, 45.0); $21.9-23.8 \%$ had $2+C C C$. In 2015 prevalence varied by gender (male 48.9; female 41.0), age $(<1 y, 314.0 ; 1-5 y, 38.9 ; 6-10 y$, $24.4 ; 11-14 y, 29.2 ; 15-17 y, 35.1)$, CCC category (higher in neurological/ neuromuscular, 9.0; lower in respiratory, 2.8), and region (from 35.5 to 47.2). NoA in 2015: median $=1$; top $5 \% \geqslant 4$ - more frequent profiles were $1-5 y(30.9 \%)$, malignantCCC $(47.7 \%)$ and $2+$ CCC $(70.6 \%), 18.4 \%$ had $\geqslant 9$ admissions. LoS (days) in 2015: median $=6$; top $5 \% \geqslant 70$ - more frequent in < 1y (60.5\%), neonatalCCC (38.8\%) and 2+CCC (63\%), $14.1 \%$ admitted for $\geqslant 151$ days.

Conclusions: In a country with scarce PPC provision, the hospital-based prevalence of children with CCC seems to be higher than reported in other settings. Children with neonatal,malignant, neurological/neuromuscular, and $2+\mathrm{CCC}$ seem to use more hospital resources. These results prompt the need to develop hospital PPC teams that may help implement family empowerment, advance care planning and care in the community, ultimately changing children's place of care and quality of life.

\section{Abstract number: P01-339 \\ Abstract type: Poster}

Is the Number of Specialized PC Services per Population a Good Macro-indicator for Measuring National-level Palliative Care Development?

Arias N. ${ }^{1}$, Garralda E. ${ }^{2}$, López-Fidalgo J. ${ }^{3}$, de Lima L. ${ }^{4}$, Rhee J.Y. ${ }^{5}$, Centeno $C^{3}$
${ }^{1}$ Institute for Culture and Society, University of Navarra, Pamplona, Spain, ${ }^{2}$ University of Navarra, Pamplona, Spain, ${ }^{3}$ Institute for Culture and Society, Universidad de Navarra. Instituto Cultura y Sociedad, Pamplona, Spain, ${ }^{4}$ International Association for Hospice and Palliative Care, Houston, United States, 5 Icahn School at Mount Sinai, New York, United States

Introduction: There is a need to identify adequate indicators to monitor palliative care (PC) development at the country level. Our aim is to find out whether an indicator such as the number of specialized PC Services per population correlates well with the level of global PC development as determined by most frequently cited publications in the field: the World Map (WM) and the Quality of Death Index (QOD).

Method: Secondary analysis of data from 131 countries on specialized PC services per population (provided by the European, African, Latin American and Eastern Mediterranean Regional Atlases) was conducted. A Spearman correlation between this variable and the WM (ordinal variables), and a Pearson analysis for the QOD (quantitative variables) were calculated.

Results: A strong Spearman correlation exists between services per population ( $r h o=0.676 ; \mathrm{P}<0.001$ ) with the $\mathrm{WM}$ categories. A very strong Pearson correlation exists between services per population $(r=0.820$; $P<$ 0.001 ) with the QOD.

Conclusion: The indicator of services per population shows strong correlation both with the $Q O D$ and $W M$, suggesting that this variable is a good indicator to monitor the level of PC development at the country level.

Abstract number: P01-340

Abstract type: Poster

What Do They Know? Examining Young People's Knowledge of Palliative Care Using a Public Health Approach: A Mixed Method Study

Mallon A., Mcllfatrick S., Hasson F., Casson K., Slater P.

Institute of Nursing and Health Research, Ulster University, Newtownabbey, United Kingdom

Introduction: The development of a public health approach to palliative care $(\mathrm{PC})$ requires an informed and engaged public. Whilst research on public awareness of PC has focused on the older population, there is a lack of studies relating to young people. Investigating this population will help to tailor education and policy campaigns to provide the foundations of knowledge, to manage future needs and nurture effective advocacy. Aim: To determine the knowledge and attitudes to PC of young people at university and explore its context and meaning within a public health approach.

Methods: An explanatory mixed methods two-phased design study was undertaken with university students aged 18-29 years. Phase one, an online survey underpinned by the theory of planned behaviour measured, knowledge, beliefs and attitudes to PC $(n=859)$. Phase two employed semi-structured interviews with a purposive sample of survey respondents who agreed to be interviewed $(n=24)$.

Findings: Phase One: $83 \%(n=710)$ of respondents had heard of the term PC. $33 \%(n=282)$ had cared for someone at the end of life, however $64 \%$ $(n=546)$ did not know where to access information regarding PC if required for a family member. Phase Two: some confusion was reported over the remit of PC with participants relating PC exclusively with non curative end of life care, for older people and delivered within a medical model. Prior experience and exposure to PC was an important factor in shaping attitudes and while welcoming the opportunity to discuss PC, it appeared that social norms such as facing mortality stifled debate.

Conclusion: Future public health approaches aimed at engaging young people with PC should ensure the dissemination of early and accurate information. The results of this study suggest that public health initiatives should target the social norm that prohibits open discussion about 
PC, this will help promote public confidence and civic responsibility in relation to death, dying, loss and care.

Abstract number: P01-341

Abstract type: Poster

\section{Live Documentation - A New Technique in Qualitative Palliative Care Research}

Herbst F.A. ${ }^{1}$, Heckel M. ${ }^{2}$, Ostgathe $C^{2}{ }^{2}$, Schneider ${ }^{1} .{ }^{1}$, Stiel S. ${ }^{1}$

${ }^{1}$ Institute for General Practice, Hannover Medical School, Hanover, Germany, ${ }^{2}$ Department of Palliative Medicine, Comprehensive Cancer Center CCC Erlangen-EMN, Universitätsklinikum Erlangen, FriedrichAlexander-Universität (FAU) Erlangen-Nürnberg, Erlangen, Germany

Background: Group discussions with study participants and expert workshops are popular methods for gathering qualitative data in palliative care research. Typically, discussion results are post-processed: audio-recordings are transcribed and transcripts are analysed; handwritten notes are transferred to meeting minutes. The authors aim to introduce the field-tested technique of live documentation.

Methods: Using the live documentation technique, a research team member types threads of a discussion and suggestions for modification directly during the meeting into a prepared draft document. Participants can follow on a screen, instantly propose their adjustments, rework and approve the final document. The live documentation technique was tested in three palliative care research projects:

1) "M-EndoL - MRSA in end-of-life care" (2014-16);

2) "Hospice and Palliative Care Networks in Bavaria: Well Connected - Optimally Cared For! (PallBayNet)" (2015-17); and

3) “Dy@EoL - Interaction at the end of life in dyads of parents and adult children" (2017-20).

Results: In M-EndoL, empirical recommendations were adjusted via live transcript and the final document was approved on the spot in a workgroup meeting (Mai 2016). Within PallBayNet, the final wording of definitions of key terms and examples of realisation was consented in two expert workshops (Nov. 2015 \& Mar. 2017). In Dy@EoL, members of the scientific advisory board reflected in a personal meeting (Jan. 2018) on interview guides, survey questionnaires, and recruitment strategy. In all three projects, post-processing time could be shortened with data already being available in written and approved form.

Conclusions: Live documentation is an appropriate technique to enhance transparency and deepen discussion while gathering qualitative data. The live documentation technique requires a research team member who is capable of following the discussion and who reworks the draft at the same time.

\section{Abstract number: P01-342 \\ Abstract type: Poster}

\section{Oncology Observed: What Ethnography Can Add to the Study of} Transitions to Palliative Care

Letcher Lazo C.J. ${ }^{1,2}$, Bradtmöller K. ${ }^{1}$, Jaspers B. ${ }^{1,3}$, Schwabe S. ${ }^{1}$, Radbruch L. ${ }^{1,4}$

${ }^{1}$ Department of Palliative Medicine, University Hospital Bonn, Bonn, Germany, ${ }^{2}$ Department for the Anthropology of the Americas, Rheinische Friedrich-Wilhelms-University Bonn, Bonn, Germany, ${ }^{3}$ Department of Palliative Medicine, University Medical Center Göttingen, Göttingen, Germany, ${ }^{4}$ Palliative Care Center, Malteser Hospital Bonn/Rhein-Sieg, Bonn, Germany

Background: Research has shown that transitions, i.e. changes in physical locations or providers, are frequent in health care delivery systems.
In this context, transitions to palliative care have received increasing attention in order to improve patients' care. However, although there is now an extensive literature on the subject, research examining transitions to palliative care in practice remains scarce and mainly focusses on caregivers' views.

Aims: The aim of the study was to explore transitions to palliative care in an oncology setting through an ethnographic lens.

Methods: Participant observation was conducted in an oncology ward during a three-month period. The purposive sample consisted of six patients. Inclusion criteria were: no prior palliative care intervention, advanced tumour stage ( 3 or 4), Glasgow Coma Scale $=15$ and/or ECOG $1-3$, able to give informed consent. Detailed field notes formed the basis of the documentary data. Grounded Theory guided data collection, coding, and analysis.

Results: Participant observation led to a rich body of data that provides a fine-grained picture of the transitions studied. For example, a patient's refusal or acceptance of the transition to palliative care is influenced by a complex interplay of various factors that, depending on individual backgrounds and characteristics, can either facilitate or present barriers to the transitional process. Despite their importance in patients' decision-making, some factors identified in this ethnographic study are rarely addressed: the perception of palliative care and Western cultural concepts linking cancer and cancer treatment with metaphors of war and notions of hope.

Conclusions: Participant observation allows researchers to capture the complexity of human behaviour and social interactions. Therefore, it is a powerful tool for analysis of transitional processes and, as such, can contribute new and important insights to the study of this field.

Abstract number: P01-343

Abstract type: Poster

Evaluating the Feasibility of a Novel Population-Based Recruitment Strategy in Bereavement Research with Lesbian, Gay, Bisexual and Heterosexual Individuals Bereaved of a Partner or Spouse

Timmins L. ${ }^{1}$, Harding R. ${ }^{1}$, King M. ${ }^{2}$, Almack K. ${ }^{3}$, Johnson K. ${ }^{4}$, Gao W. ${ }^{1}, Y u$ P. ${ }^{1}$, Yi D. ${ }^{1}$, Pitman A. ${ }^{2}$, Marshall S. ${ }^{5}$, Braybrook D. ${ }^{1}$, Bristowe K. ${ }^{1}$ ${ }^{1}$ Cicely Saunders Institute, King's College London, London, United Kingdom, ${ }^{2}$ Division of Psychiatry, University College London, London, United Kingdom, ${ }^{3}$ Centre for Research in Public Health and Community Care, University of Hertfordshire, Hatfield, United Kingdom, ${ }^{4}$ Social

\& Global Studies Centre, RMIT, Melbourne, United Kingdom,

${ }^{5}$ Department of Palliative Care, King's College Hospital NHS Foundation Trust, London, United Kingdom

Background: Lesbian, gay and bisexual (LGB) people represent a hard-toreach population that face additional barriers and stressors in palliative and bereavement care.

Aims: To evaluate a novel recruitment strategy for LGB and heterosexual individuals recently bereaved of a partner.

Methods: All death registrations in England and Wales in two periods, 9/9/17 - 8/10/17 (1 month) and 9/10/17 - 8/1/18 (3 months) were searched for registrants recorded as a married or civil partner of the decedent. Registrants were invited to complete a survey on bereavement and sexual orientation via letter. Non-respondents were sent a reminder two weeks after invitation. Two waves were completed, a trial wave using period 1 data to identify issues and a larger wave using period 2 data. Decedents had died 9-10 months previously at the time of invitation for wave 1 and 7-10 months for wave 2 .

Results: Of $N=129,973$ registrants in period $1, n=46$ were same gender partners and $n=30,355$ were opposite gender partners. Of $N=$ 36,118 in period 2, $n=126$ were same gender partners and $n=8,748$ were opposite gender partners. All same gender partners and 120 and 360 opposite gender partners were invited for the waves 1 and 2 respectively. $36.6 \%$ of same gender partners and $24.0 \%$ opposite gender 
partners participated. More same gender partners participated in wave $1,63.0 \%$, than wave $2,26.9 \% . n=4$ same gender partners self-reported a heterosexual identity and $n=3$ reported a bisexual identity. The final response from each wave was received within 6 weeks of invitation. Discussion: A high proportion of deaths were registered by a partner. Acceptable response rates were achieved for a postal survey, compared with 19.7-39.3\% in previous studies. A same gender partner sample of a sufficient size for various designs was achieved in a short period. Death registration data are an effective means of recruiting bereaved LGB and heterosexual partners.

Funding: Marie Curie Research Grants Scheme.

Abstract number: P01-344

Abstract type: Poster

Do Indigenous Research Methods Have Anything to Offer Bereaved Western Research Participants?

Williams L., Moeke-Maxwell T., Black S., Wiles J., Gott M.

School of Nursing, University of Auckland, Auckland, New Zealand

Background: Scholars have highlighted a lack of research that focuses on indigenous palliative/end of life care issues. To fill the gap, indigenous researchers have sought to conduct research that will offer a better understanding of their peoples' cultural needs and experiences at the end of life. In New Zealand, indigenous Māori have developed 'Kaupapa Māori' research methods that foreground an indigenous worldview. Included in this worldview are ethical care processes that prioritise generosity, reciprocity and co-operation. What has not been explored, however, is how such an indigenous approach might also benefit non-indigenous research participants.

Aim: To explore how bereaved non-Māori research participants who completed in-person, post-bereavement interviews about a family member's end of life experiences, reacted to the application of Māori ethical care processes during their interviews.

Methods: Cooperative inquiry informed by researcher reflections and field notes provided the data for a thematic analysis, informed by a feminist ethics of care lens.

Results: The isolation of key themes revealed participants views on the essential principles and core practices underpinning the Māori ethical care processes applied in the interviews.

Conclusion/ discussion: Bereaved non-Māori, western research participants for the most part appreciated the application of Māori ethical care processes as expressed in essential principles and core practices. While such procedures have commonalities with feminist research notions of an ethics of care, fundamental differences exist regarding responsibility to foster and maintain relationships with participants.

\section{Abstract number: P01-345}

Abstract type: Poster

\section{Oral History as a Methodology in Exploring the Demise of the Liverpool Care Pathway (LCP) \\ Keeley P. ${ }^{1,2}$ \\ ${ }^{1}$ Glasgow Royal Infirmary, Palliative Care Team, Glasgow, United Kingdom, ${ }^{2}$ University of Glasgow, Centre for the History of Medicine, Glasgow, United Kingdom Presenting author email address: paul. keeley@ggc.scot.nhs.uk}

Aim: To begin an archive of oral history relating to the history of the LCP. Method: Ethical permission was sought from the University's ethical committee and granted in November 2017. Over the period April to June 2018 , I conducted five digitally recorded interviews with senior palliative physicians involved in the implementation or demise of the LCP. The interview consisted of open questions about the experiences of the interviewees and views of the LCP. The contents of the transcriptions were used and only varied from the recordings by having some phrases removed at the request of the interviewee which did not materially alter the meaning

Results: The content of the material offers a valuable means of gaining insight into and analysis of the multifactorial reasons for the demise of the LCP and will be a valuable resource for future historians of palliative care. The interviews as recordings and transcripts will be stored in the Library of the Royal College of Physicians of Glasgow.

Conclusion: Oral history as a methodology is well established in the historian's armamentarium, but there is an obvious methodological paradox. It may be too soon to use this method of historical record gathering. However, over time witnesses to events may be prone to all manner of biases brought on by time's passage: failures of memory, confirmation bias and witness selection by the interviewer. I would argue that having as near contemporaneous record as possible, whilst not unproblematic, is of value. Oral history has been used to great effect in documenting the history of palliative care. My experience of the professionals I interviewed is that their testimony was neither vituperative nor anguished but measured and reflective.

The methodology is clearly feasible and will be extended further to capture the experience of professionals, public, press, policy-makers and politicians in the development and demise of the LCP.

Abstract number: P01-346

Abstract type: Poster

How Can Data-driven Technologies Be Used to Improve the Experience and Outcomes of People Living with Advanced Cancer? Defining 'Priority Areas' to Influence Future Developments in Data-driven Technologies

McGlinchey T. ${ }^{1}$, Mason S. ${ }^{1}$, Nwosu A.C. ${ }^{1,2,3}$

${ }^{1}$ Palliative Care Institute Liverpool, University of Liverpool, Liverpool, United Kingdom, ${ }^{2}$ Royal Liverpool and Broadgreen University NHS Hospitals Trust, Liverpool, United Kingdom, ${ }^{3}$ Marie Curie Hospice Liverpool, Liverpool, United Kingdom

Background: Over the last 5 years data-driven technology has been increasingly promoted in healthcare policy as a driver for quality improvement. Increased investment in the development of data-driven technology presents a new and exciting opportunity to improve in the quality and efficiency of healthcare provision and patient experience, for patients with advanced cancer.

To ensure the healthcare system, and wider society, benefits from this technology, it is important to engage key stakeholders to determine how resources should be best utilised. This project will explore how digital technology can improve the care of people with cancer, by setting 'priority areas' for further research and development, and creating an International Collaborative group of key stakeholders to drive this agenda. Aim: To define 'priority areas' for the future development of data-driven healthcare technologies, to improve the care of people living with advanced cancer.

Methods and Objectives:

- Objective 1: Scoping review to identify existing and future uses, and examples, of data-driven technology to support patients living with advanced cancer;

- Objective 2: Delphi Process (using the CREDES guidance) for consensus on 'priority areas', identified from scoping review, for future development of data-driven technology, to improve the care of patients with advanced cancer;

- Objective 3: Develop International Collaboration to facilitate and support future grant applications.

Results: Priority areas resulting from the scoping review and Delphi will be presented. 
Conclusion: The identified 'priority areas' for data-driven technologies will direct and drive further research to improve the care and experience of people living with advanced cancer. The developed International Collaborative will lead this agenda, to facilitate the conduct of innovative collaborative research to improve palliative care delivery on a global scale.

Abstract number: P01-347

Abstract type: Poster

Palliative Care Research in Italy: A Scoping Review

Mastroianni C. ${ }^{1}$, Magnani C. ${ }^{1}$, Surdo L. ${ }^{1}$, Ramon Codina M. ${ }^{2}$, D'Angelo D. ${ }^{3}$, De Paolis G. ${ }^{4}$, Colotto M. ${ }^{5}$, Petitti T.6, De Marinis M.G. ${ }^{6}$, Casale G. ${ }^{1}$ ${ }^{1}$ Antea Palliative Care Center, Roma, Italy, ${ }^{2}$ Universidad Nacional de Educacion a Distancia (UNED), Roma, Italy, ${ }^{3}$ stituto Superiore di Sanità, Roma, Italy, ${ }^{4}$ International Association for Analytical Psychology, Roma, Italy, ${ }^{5}$ Università Cattolica del Sacro Cuore, Roma, Italy, ${ }^{6} \mathrm{Campus}$ Bio Medico University, Roma, Italy

Background: Palliative Care (PC) research provide patients with appropriate quality care based on the best available evidences. Despite great progresses in PC provision and education, research in PC in Italy remains an under explored domain.

Aim: This review aims to explore the volume, nature and characteristics of the Italian research contribution, to understand to what extent it matches PC priorities reported by international literature.

Methods: A scoping review was conducted following Arksey and O'Malley method. Search was performed by an interdisciplinary team on 6 databases, using relevant PC keywords combined with "Ital*", to set articles published on indexed journals between 2000 and 2017, conducted in Italy by a 1st author (or at least 1 of the authors) affiliated in Italy. The eligible studies were screened independently by 4 reviewers and were classified by topic, type of study and other features.

Results: A total of 5892 articles were retrieved through database search, while 605 were included in the review. Compared to the 2000-2009 decade, from 2010 to 2017 the number of PC publications was almost doubled $(\mathrm{N}=410)$. Italian PC scientific production is characterized by a prevalence of observational studies $(218,38 \%)$, discussion papers $(126,21 \%)$ and a low number of papers about experimental studies $(22,4 \%)$. The top four areas of investigation were symptoms management $(172,28 \%)$, services organization $(132,22 \%)$, last days of life $(81,13 \%)$ and ethics $(90,15 \%)$.

Conclusion: The process of continuous growth in PC clinical and educational area experienced in Italy since 2000 also concerns research. Italian PC research is still focused on describing PC main issues, some research topics reflect the European research priorities, but strong evidences are still missing. Study findings represent a first background for future PC research development in Italy.

Abstract number: P01-348

Abstract type: Poster

Finding 'the Least Worst Time' to Approach Patients and Family Carers about Taking Part in Research: A Qualitative Multiple Case Study Exploring Recruitment Issues in Palliative Care Randomised Controlled Trials (RCTs)

Dunleavy L., Preston N., Walshe C.

Lancaster University, Lancaster, United Kingdom

Background: A trial's success in reaching its target sample size is reliant on health care professionals being actively involved in the recruitment process. However, we know that gatekeeping by clinical professionals is an issue in palliative care research.

Aims: To identify how health care professionals undertake recruitment to palliative care RCTs and the factors that influence the strategies they use and the choices they make during the recruitment process.
Method: Three diverse UK palliative care RCTs were used as 'cases'. In depth semi structured interviews were carried out with 19 professionals involved in the recruitment process from the trials coordinating centre or clinical recruitment sites. Framework analysis was used to analyse interview data and trial documentation. The '6Ps' of the 'Social Marketing Mix Framework' were used as an analytical framework: identifying participants, product, price, place, promotion, working with partners.

Results: Choosing the best time to approach patients and carers (price), explaining the concepts of palliative care and end of life care (promotion) and explaining sensitive aspects of the research process (promotion) is challenging for health care professionals. Well-developed verbal and non-verbal communication skills are required to facilitate the recruitment process (Promotion). How prepared and comfortable health care professionals feel to approach patients and their carers about taking part in research is dependent on their previous clinical experience and training as well as their clinical support networks (working with partners).

Discussion/ conclusion: The communication skills required to approach and discuss palliative care research with patients and their carers must not be underestimated. Those involved in developing trials need to ensure the necessary training and support is in place for clinical professionals involved in the recruitment process, to address professional gatekeeping.

\section{Abstract number: P01-349}

Abstract type: Poster

Challenges of Caring for a Dying Patient from Perspective of Health Care Providers: A Qualitative Interperative Metasynthesis

Tahmasebi M. ${ }^{1}$, Sotoudeh S. ${ }^{1}$, Mahdavi H. ${ }^{2}$

${ }^{1}$ Tehran University of Medical Sciences, Tehran, Iran, Islamic Republic of, 'Iran University of Medical Science - Firoozgar Hospital, Tehran, Iran, Islamic Republic of

Background: Palliative philosophy emphasizes on prevention and relief of physical, psychosocial and spiritual suffering.Peaceful death is one of the primary goals in palliative care which is not always met. Sometimes death of a terminally ill patient turns into a clinical crisis. Identifying the leading causes is necessary to prevent this challenging situation.

Aims: To explorel the challenging circumstances during caring of a dying patient in perspective of health care providers.

Method:

- Design: This study is a qualitative interperative meta-synthesis to provide a comprehensive view of caring for a dying patient from perspective of health care providers.

- Data collection: Searches were performed by using PubMed, MedLine, Google Scholar, ScienceDirect and PsycINFO databases since last 10 years.

- Analysis: Findings of qualitative studies were synthesized through thematic analysis to develope overarching themes.

Results: In total, 8 articles met the inclusion criteria.These are English languaged qualitative studies published between 2009 and 2016. Authors of these studies identified many themes related to dying process and end of life care in perspective of health care providors.Eight overarching themes were extracted: poorly controled symptoms, unexpected death, time and facility constraints, lack of education, inapproprate coping responses, communication difficulties, family conflicts and health care providers' limitations.

Conclusion: The findings of this study shows the importance of reconizing death's around situations to achieve a good death.Precise understanding 
of leading causes of clinical challenges during dying process can help practitioners to prevent them.

Abstract number: P01-351

Abstract type: Poster

Recruiting Dyads of Parents and Adult Children for End-of-Life Research

Gawinski L., Schneider N., Stiel S., Herbst F.A.

Institute for General Practice, Hannover Medical School, Hanover, Germany

Background: Challenges in recruiting patients and family members for end-of-life research have been broadly discussed. Here, specific challenges related to the recruitment of

1) dyads of terminally ill adult children and their parents and

2) terminally ill parents and their adult children are reflected aiming at an adaption of recruitment strategy.

Methods: Within the research project “Dy@EoL - Interaction at the end of life in dyads of parents and adult children" (2017-20), adult children and parents of both dyads are recruited since February 2018 via ambulatory and inpatient palliative care providers. The research team presented the research project in personal contact to eligible patients and family members. If requested by the recruitment partner, they approached patients and family members first. Data on initially approached dyad partner, participation and reasons for (non-)participation were recorded in IBM SPSS Statistics 25.

Results: Overall recruitment rate was $41.4 \%$ ( 7 dyads, 13 single patients, 1 single family member). For dyad 1 we recruited $14.2 \%$ of approached patients. Approaching family members was possible in $28.5 \%$ of all cases and $50 \%$ participated. For dyad 2 we recruited $48.7 \%$ of eligible patients. We approached $51.2 \%$ of family members of which $42.8 \%$ participated. Prominent reasons for participation were the wish to help $(48.2 \%)$ and research interest (24.1\%). Patients' most prevalent refusal reasons were the need for rest $(52.3 \%)$ and strained family relationships (14.2\%). A common reason for patients' exclusion of family members and family members' refusal was protection from extra burden (53.9\%).

Conclusions: The major challenge is to recruit both partners of one dyad. After 3 months of recruitment, the research team decided to adjust the study protocol to include individual patient or family member interviews instead of dyads only because partial data analysis is still feasible. With this adaption, we expect to successfully complete recruitment.

Abstract number: P01-352

Abstract type: Poster

Patient and Public Involvement in Palliative Care Research Gardiner $C$.,

Palliative Care Studies Advisory Group The University of Sheffield, Nursing and Midwifery, Sheffield, United Kingdom

Background: Patient and public involvement (PPI) is an established component of high quality palliative care research. The way patients and public are involved in research differs between and across research studies, however the range of contributions and different models of working are not well documented in palliative care.

Aim: The aim is to document the range and scope of activities of a service user advisory panel - the Palliative Care Studies Advisory Group (PCSAG), established at The University of Sheffield in 2009.

Methods: A narrative summary of PPI activities, documenting the range and scope of contributions to research over the nine years since the PCSAG was established.

Results: Since they were established the group have contributed to 34 research projects and funding applications in the area of palliative and end of life care. These have ranged from substantial multi-million pound international research studies to PhD studies and student projects. In the main, researchers attended facilitated group discussions to request advice on aspects of their research projects, however other models of working have emerged in more recent years. These include group members sitting on project steering committees as co-applicants, contributing to data analysis and presenting at conferences, and developing on-line methods of providing PPI input.

Discussion: The Palliative Care Studies Advisory Group have made a substantial contribution to palliative and end of life care research. The scope and depth of PPI involvement has increased over the years, as researchers and service users have gained confidence in working together. Models of co-working which involve service users as coapplicants have more recently become commonplace as the PPI movement has become more embedded in research. However, little guidance is available to support researchers working with service users in palliative and end of life care, and further research could address this gap.

\section{Abstract number: P01-353 \\ Abstract type: Poster}

Making their Voices Heard - Conducting Qualitative Interviews with Patients with Communication Impairments Schaepe C., Ewers M.

Institute of Health and Nursing Science, Charité - Universitätsmedizin Berlin, corporate member of Freie Universität Berlin,

Humboldt-Universität zu Berlin, and Berlin Institute of Health, Berlin, Germany

Background: In-depth interviewing is a commonly used qualitative methodological approach to gain insight into palliative and hospice patient's experience and perceptions. Although interviewing is widely used, verbally impaired patients are often excluded due to methodological and ethical reasons. Excluding them prevents qualitative research to provide a complete picture of the population. That is the reason why methodological and ethical challenges in conducting interviews needs to be highlighted and discussed.

Aim: To present the challenges in collecting data from home mechanically ventilated (HMV) patients and discuss approaches to include them into future research.

Methods: The authors will draw on insights derived from a three year health services research project, were HMV patients were interviewed. Based on the experiences the main methodological and ethical challenges in conducting interviews with patients with communication impairments will be reported. Drawing from available literature, strategies employed to overcome these challenges will be presented.

Results: Challenges encountered when preparing to interview participants were the reduced speech intelligibility, participants' fatigue, and the inability to give detailed account of their experiences. After ethical and methodological reflections within the research team, a number of strategies to integrate verbally impaired patients into the sample could were identified. Proposed approaches include to increase the sample with a wide variety of different patients, to use an interpreter or augmentative and alternative communication.

Conclusion: Rather than excluding patients with communication difficulties from qualitative research, creative solutions to overcome the challenges and a careful preparation are suggested to make their voices heard in palliative and hospice care research.

The study was funded by the German Federal Ministry of Education and Research.

Abstract number: P01-354

Abstract type: Poster 
'Waiting for Resident Number 4': Experiences of Recruiting Nursing Home Residents Living with Advanced Dementia to a Cluster Feasibility Trial of the Namaste Care Intervention Dunleavy L., Patel S., Preston N., Walshe C., Froggatt K. Lancaster University, Lancaster, United Kingdom

Background: Recruiting nursing home residents living with advanced dementia to take part in research is challenging. Nursing homes have limited or no research infrastructure and staff are often research 'naïve'. Proxy consent via a consultee is also required as residents lack the capacity to consent to take part in research.

Aim: To assess the length of time taken to recruit 32 nursing home residents living with advanced dementia to a feasibility cluster trial of the Namaste Care Intervention.

Method: Trial screening and recruitment data were analysed for the 8 homes involved in the trial. Data collection could only begin in each nursing home when 4 residents had been recruited to the trial.

Results: 2 nursing homes withdrew from the study before completion of recruitment. 1 due to staffing issues and 1 withdrew due to study start delays. The mean number of days between giving out the first consultee pack and to consenting the fourth resident in the 6 remaining homes was 36 days (range 11-55, median 37.5). There was a range of 2-44 days across the 6 homes between the consultee receiving a pack and consent being obtained. Even when the fourth consent had been obtained, there was a delay in commencement of data collection, with an average of 21.5 days (range 8-41, median 15.5) between the fourth resident being consented and the baseline visit due to nursing home staffing issues.

Discussion / Conclusion: Staff intervention and recruitment training and support, obtaining the necessary ethical and organisational approvals, arranging staff and family meetings in the home with patient and public involvement representation and awaiting the return of consultee consent documentation accounted for this lengthy recruitment process. The length of time needed to recruit nursing home residents living with advanced dementia must be factored into the trial design process and information giving to potential recruitment sites.

Funded by: NIHR HTA Programme

Abstract number: P01-355

Abstract type: Poster

Palliative and Hospice Care for People with Profound Intellectual and Multiple Disabilities - Results from a BMBF-funded Project on Ways to Generate Informed Consent and Qualitative Methods with Vulnerable Persons on Sensitive Topics in Health Contexts (PiCarDi-U)

Heusner J., Fricke $C$.

University of Leipzig, Leipzig, Germany

The "Convention of the Rights of Persons with Disabilities" demands the right for everyone to non-discriminatory participation in all health care structures. Therefore people with intellectual and multiple disabilities should be able use all structures and services of palliative and hospice care at the end of their lives.

Currently, in Germany little is known about wishes and needs at the end of life from the perspective of people with intellectual disabilities.

The participatory project PiCarDi-U, funded by the Federal Ministry of Education and Research, concentrates on the perspective of people with intellectual and/or multiple disabilities and their relatives concerning their needs and possibilities of participation in palliative care.

As a part of the project, traditional qualitative survey methods were extended to enable people with intellectual disabilities to take part in the research. In three federal states a total of 36 persons with intellectual disabilities were included. The data collection consisted of 9 group discussions with 4 people each and 18 one-to-one conversations. A pool of different methods adapted to the individual needs for assistance were developed and used to talk about experiences related to dying, death, grief and wishes at the end of life.

The consistent implementation of ethical principles in research with vulnerable groups, especially on sensitive issues such as serious illness and death, requires a comprehensive person-centered process to produce a real informed consent on the contents and procedures of the research process.

At the EAPC World Congress we want to present how informed consent can be achieved, which methods can be used for data collection and to give an overview what this group of people said about their wishes and needs at the end of their lives.

The results can provide important impulses for ethically acceptable access to vulnerable groups of people in connection with sensitive topics in the medical context.

\section{Abstract number: P01-356}

Abstract type: Poster

\section{The Benefits of a Case Study Approach in Hospice Research} saunders $Z^{1,2}$

${ }^{1}$ The Open University, School of Health and Social Care, Milton Keynes, United Kingdom, ${ }^{2}$ Willen Hospice, Milton Keynes, United Kingdom Presenting author email address: zana.saunders@open.ac.uk

This research project was designed to uncover perceptions of hospice care provision within a community and ascertain commonalities and disparities between different community groups.

The method focused on one UK urban community served by one hospice, and incorporated several embedded units of analysis from qualitative data collected via surveys, focus groups, interviews and documents. These included patients, professionals and community members. It also necessitated looking in-depth at the hospice and community history as well as the health geography and demographics of that community. All data sources were analysed thematically as independent units and then a meta-analysis to draw out themes, shaping the discussion and results. The project is in its final stages, and early findings are suggesting that many sections of communities including care professionals are not understanding local hospice provision. They require greater education to enable informed choices about options for people who are nearing end of life. The presentation discusses the benefits of case studies to provide rich, in-depth holistic data. This was particularly beneficial for the local palliative care providers who had not engaged in funded research before, and little was known about their background, their community and their interactions. It discusses different types of case study, and how this design was chosen and managed throughout the project, including the practicalities of generating multiple sources of rich data, and relationship building between a hospice, its community and researchers.

This presentation promotes case studies as a valid alternative to other research methods. It demonstrates how case studies are a possible method for researchers, hospices and palliative care providers to adopt, if they wish to get research active and transform the care provision. Funded PhD Project by UK University and UK based hospice

Abstract number: P01-357

Abstract type: Poster

Palliative Care Research in Portugal: Where Do we Stand? Cunha $T^{1,2}$, Ferreira S..$^{1,2}$, Paula S. ${ }^{1,2}$

${ }^{1}$ Escola Superior de Saúde Dr Lopes Dias, Instituto Politécnico de Castelo Branco, Castelo Branco, Portugal, ${ }^{2}$ AGE.COMM - Interdisciplinary Research Unit on Building Functional Ageing Communities, Instituto Politécnico de Castelo Branco, Castelo Branco, Portugal

Background: Palliative Care (PC) intends, through a bond between the patient, family and PC team, to ease the physical symptoms as well as the emotional and spiritual burden. The ageing of the population and 
increasing incidence of chronic illnesses brings the need for caregivers. Therefore, the healthcare professionals' education is crucial entailing the need for further investigation for efficient caregiving. The adequate development of these professional teams is only possible through investigation. In Portugal, we noticed a gradual increase in studies between the years of 2005 and 2010, however the number of publications on national and international magazines was still low ${ }^{1}$.

Aim: To analyze the evolution of published scientific studies on PC in Portugal in a period of six years and eight months.

Methods: Systematic review of published studies on PC carried out on the Portuguese population from Jan 2011 to Aug 2017. The used databases were B-on, RCAAP, EBSCO, PubMed, ScienceDirect and scientific magazines.

Results: A total of 114 articles were included. We verified an increase in publications, mainly from 2014 onwards. Most of the studies are quantitative and were published abroad. The stand out area of interest in this field was the application of scores and queries. The studied populations concerned mainly the PC patient and the health care providers, and the preferred theme was the burden of the disease on the patient.

Conclusion: There has been a steady increase on publications concerning PC in the Portuguese population especially the PC patient and health care providers. The themes most frequently encountered where of areas described in the National PC Plan. Studies concerning indicators, costeffectiveness/cost-benefit and guidelines of care in PC are still lacking leading to challenges in standardized patient care and networking. ${ }^{1}$ Santos, M. E., \& Capelas, M. L. (2011). Investigação em cuidados paliativos em Portugal. Cadernos de Saúde, Vol 4, no 1, 4, 63-69

\section{Abstract number: P01-358 \\ Abstract type: Poster}

Studying Complex Interventions in Palliative Care: Experiences with the Action Research Method

van der Ark A., Engel M., Tamerus R., van der Heide A.

Public Health, Erasmus MC, Rotterdam, Netherlands

Background: Studying complex interventions poses a challenge to the field of palliative care research. One possible approach is Action Research $(A R)$, which uses a collaboration between research and practice, and aims to contribute to both fields simultaneously. However, experiences with the feasibility of AR in studying complex interventions are limited. Aims: Sharing our experiences with AR in studying complex interventions. Methods: We use the AR approach in 7 projects. Each project team consists of healthcare professionals (multidisciplinary and inter-organizational) and one researcher, and chooses, develops and implements an intervention that aims to improve inter-organizational collaboration in palliative care. A flexible AR protocol was developed to accommodate this bottom-up approach.

Results:

Challenges:

- $\quad$ Preparation time: Team formation and consensus processes took 1 -2 years.

- Collaboration costs: Overcoming differences in speed, language and aims requires effort.

- Role volatility: Aiming to contribute while avoiding overdue influence or ownership.

- Limited control: Team determines project content and planning Strengths: (and outcomes).

- Scientific gains: Close-up, longitudinal insights in inter-organizational processes, intervention mechanisms and context factors.

- Collaboration gains: Prompts inter-organizational collaboration in palliative care, activates stakeholders.
- Intervention gains: Interventions are tailored to the region and locally consolidated.

- Positive externalities: Stimulates self-reflection on practical relevance (researchers) and evidence base (healthcare professionals). Can strengthen ties between science and research.

Conclusions: We perceive considerable challenges in the use of $A R$, probably more so because our research involves multiple complex, interorganizational projects. Despite this, the AR approach provides unique insights into complex intervention development and implementation in palliative care.

\section{Abstract number: P01-359 \\ Abstract type: Poster}

Development and Validation of Search Filters to Find Articles on Palliative Care in Bibliographic Databases

Rietjens J. ${ }^{1}$, Bramer W. ${ }^{2}$, Geijteman E. ${ }^{1}$, van der Heide A. ${ }^{1}$, Oldenmenger $W^{3}$

${ }^{1}$ Public Health, Erasmus MC, Rotterdam, Netherlands, ${ }^{2}$ Medical Library, Erasmus MC, Rotterdam, Netherlands, ${ }^{3}$ Medical Oncology, Erasmus MC, Rotterdam, Netherlands

Background: Palliative care is a relatively young, growing, and multidisciplinary area of expertise. Relevant papers are published in palliative care journals, general medical journals, and discipline specific journals. Healthcare professionals and researchers in this field often have difficulties finding relevant articles. Standardized search filters may help improve the efficiency and quality of such searches, but prior developed filters showed only moderate performance.

Aim: To develop and validate a specific search filter and a sensitive search filter for the field of palliative care.

Design: We used a novel, objective method for search filter development. First, we created a gold standard set. This set was split into three groups: term identification, filter development and filter validation set. After creating the filters in PubMed we translated the filters into search filters for Ovid Medline, Embase, Cinahl, Psychinfo and Cochrane Library. We calculated specificity, sensitivity and precision of both filters.

Results: The specific filter had a specificity of $97.4 \%$, a sensitivity of $93.7 \%$, and a precision of $45 \%$. The sensitive filter had a sensitivity of $99.6 \%$, a specificity of $92.5 \%$, and a precision of $5 \%$.

Conclusions: Our search filters can support literature searches in the field of palliative care. Our specific filter retrieves $93.7 \%$ of relevant articles, while $45 \%$ of the retrieved articles are relevant. This filter can be used to find answers to questions when time is limited. Our sensitive filter finds $99.6 \%$ of all relevant articles, and may for instance help conducting systematic reviews. Both filters perform better than prior developed search filters in the field of palliative care.

Abstract number: P01-360

Abstract type: Poster

Using Twitter to Recruit, Participate and Disseminate Palliative

Care Research: A Rapid Review

Russell S. ${ }^{1}$, Taubert M. ${ }^{2}$, Johnston B. ${ }^{3}$

${ }^{1}$ Independent Researcher, Lymington, United Kingdom, ${ }^{2}$ Palliative Care, Velindre NHS Trust, Cardiff, United Kingdom, ${ }^{3}$ School of Medicine, Denistry and Nursing, University of Glasgow, Glasgow, United Kingdom

Background: There is a paucity of knowledge about the use of the social media networking platform Twitter in palliative care research recruitment, participation or dissemination.To provide clinical practice \& research recommendations, literature was reviewed using a rapid review method to identify, analyse \& synthesize contextual evidence within a shortened time frame. 
Methods: Search strategy over 6 months: electronic databases, grey literature, hand searching of health/palliative care journals. SR/BJ reviewed all titles/abstracts, SR/MT/BJ full text relevance, quality assessment, data extraction \& final texts. Articles in English published Jan 2008 - Oct 2018 reporting use of Twitter for recruitment, participation \& dissemination of research/palliative care research.

Results: Twitter can include studying its own activity \& content or as a research tool with many evaluation metrics. There is a sparsity of reports on its use in palliative care research recruitment, a limited number of its use in research in general and several studies on its use to disseminate research. How it can be used includes to recruit participants to studies, publicize \& disseminate, to provide knowledge (practitioners and public), campaign/communicate on issues $\&$ as a patient \& public involvement platform (research design \& dissemination). Some studies report on its use to support education, create virtual communities of practice, a market place for ideas \& to improve care by providing feedback. Design, ethical \& implementation considerations when using Twitter in research \& guidance in research and practice continues to evolve.

Conclusion: Twitter has its place in palliative care research \& practice but how it is used, the validity of it in recruitment, participation \& dissemination is still developing. The use of Twitter in clinical practice \& research design should be paid the same attention as any other intervention or research process to utilise it ethically, effectively \& efficiently.

\section{Abstract number: P01-361 \\ Abstract type: Poster}

\author{
10 Years of the Cancer Awareness Measure - Time to Move \\ Online? \\ Hudson B.F., Power E., Connor K., Crawford C. \\ Cancer Research UK, London, United Kingdom
}

Background: The Cancer Awareness Measure (CAM) monitors awareness of cancer signs, risk factors, screening programmes and help seeking attitudes among a UK representative sample. The CAM has run biannually since 2008 via the ONS's Opinions and Lifestyle Survey (OLS) utilising face to face interviews. OLS response rates are declining, and costs are escalating.

Aims:

1. To assess the feasibility and implications of moving CAM data collection online

2. to assess changes from 2014 - 2017 in awareness of cancer signs, risk factors and help seeking attitudes in a UK representative sample.

Methods: Three parallel CAM runs were undertaken in 2017; via OLS (face to face) and two online providers; NatCen and YouGov. Weighted comparisons between online and face to face samples were conducted. Multivariable logistic regression $(p<0.05)$ with adjustment was used to assess change from 2014-2107.

Results: Compared to face to face samples $(n=819)$, online samples (YouGov $n=2,066$; NatCen $n=1190$ ) demonstrated higher knowledge and were more likely to endorse barriers. They were also older, more frequent internet users and were more likely to have a partner. No other differences were observed. From 2014 to 2017 awareness of obesity as a potential cause of cancer increased significantly. Recall increased from $10 \%$ to $15 \%$ while recognition of obesity increased from $62 \%$ to $67 \%$. Recall of weight loss ( $21 \%$ to $26 \%$ ) and tiredness ( $12 \%$ to $16 \%$ ) also increased but recall for lump (64\% to $59 \%$ ), bleeding (39\% to $35 \%$ ), and change in bowel/bladder habits ( $32 \%$ to $27 \%$ ) decreased.

Conclusions: Although responses differed by mode of collection, online samples were representative of the UK population and echoed the response pattern observed in OLS data. Future CAM data will therefore be collected online, enabling inclusion of additional items. Apart from notable increases in awareness of the obesity-cancer link, there was little change in awareness and attitudes from 2014 to 2017.

\section{Abstract number: P01-362}

Abstract type: Poster

Appropriate Hospice Care in the Netherlands - A Pilot Study Graaf E., van Klinken M., Koorn R., van der Baan F., Teunissen S. Center of Expertise in Palliative Care Utrecht, Dept. General Practice, Julius Center for Health Sciences and Primary Care, University Medical Center Utrecht, Utrecht, Netherlands

Background: Hospice care in the Netherlands is provided in volunteer driven hospices (VDH), professional driven hospices (PDH) and hospice units in nursing homes (HU). In preparation of a large multicenter study te evaluate appropriate hospice care in the Netherlands, a pilot study was performed.

Aim: To evaluate the appropriateness of the planned study procedures and outcomes of the future multicenter study.

Methods: A pilot study using records of hospice patients, admitted in 2017. A convenience sample of $5 \mathrm{VDH}, 5 \mathrm{PDH}, 5 \mathrm{HU}$ was invited to participate. Per hospice 4 records were randomly selected and 4 records were selected by the hospice.

Primary outcome: availability of patient records

Results: Data were gathered from 104 patient records from 13 different hospices. In 3 hospices the encountered barriers turned out too high to participate, therefore 1 additional hospice was recruited. The availability of patient records differed due to different methods of recording patient information, physicians: $93.1 \%$, nurses, $98.6 \%$ and volunteers $60 \%$.

The main barriers we encountered:

1) multiple records per patient, stored on different locations, and in different care organizations,

2) hospices' lack of experience with research and lack of knowledge of the legislation,

3) denied access to records, communication problems.

Conclusion: The availability of patient records differed per caregiver. Collecting records for a single patient proved time consuming, especially when stored on different locations. To improve communication, and thus participation of all types of hospices, study procedures are adapted:

1) more personal contact,

2) improved information on research, legislation and ethics, and

3) start at higher levels of influence to gain access.

Although a pilot study in preparation of an observational study is not often performed, these results show its importance, specifically in a field with various organizational structures and where research is not common.

Abstract number: P01-363

Abstract type: Poster

Spirituality, Quality of Life and Depression among Patients with Cancer in Lombok, West Nusa Tenggara, Indonesia Rochmawati E., Wiksuarini E., Rahmah $R$.

Universitas Muhammadiyah Yogyakarta, Bantul Yogyakarta, Indonesia

Purpose: Cancer and its treatments can cause complex issues including physical, psychological and spiritual aspects. We aimed to examine the impact of cancer to the spirituality well-being, quality-life and depression among cancer patients in eastern part of Indonesia. 
Methods: Our study was conducted between January-April 2018 among a sample of 100 cancer patients (stadium III and IV) who admitted to a curative setting in the regional public hospital. All the participants filled three self-report questionnaires; the Spirituality Well Being Scale, the European Organization for Research and Treatment of Cancer Quality of Life (EORTC QOL), and the Beck Depression Inventory in addition to socio-demographic data. Pearson correlation was conducted to examine the associations between spirituality and depression, overall quality of life and quality of life domains (functional and symptoms).

Result: All of the participants $(n=100)$ are affiliated with particular religions and most of them always conduct worships. Pearson correlation showed significant positive associations between spirituality and overall QOL ( $p<0.01$ ) and individual functional quality of life domains (physical, roles, emotional, and social). Spirituality was negatively associated with symptoms domains (fatigue, pain, dyspnea, insomnia, loss of appetite and financial difficulties). Higher spirituality was associated with lower depression $(p<0.01)$.

Conclusion: This study confirm that spirituality related with overall quality of life and depression and this reiterates the importance of developing spirituality-based intervention along with cancer care for this population.

Abstract number: P01-364

Abstract type: Poster

Spiritual Needs and Life Satisfaction of Palliative Patients: Lithuanian Experience

Spirgiene L. ${ }^{1}$, Tomkevičiūtè J. ${ }^{2}$, Kaselienè S. ${ }^{3}$, Riklikiené $0 .^{1}$

${ }^{1}$ Faculty of Nursing, Lithuanian University of Health Sciences, Kaunas, Lithuania, ${ }^{2}$ Faculty of Medicine, Lithuanian University of Health Sciences, Kaunas, Lithuania, ${ }^{3}$ Faculty of Public Health, Lithuanian University of Health Sciences, Kaunas, Lithuania

Background: In 1995 physicians and nurses volunteered to formulate the statute of Lithuanian Association of Palliative Medicine considering the needs of terminally ill patients and based on the experience of Western countries. Significant achievement was a decree on the palliative care services issued by the $\mathrm{MoH}$ in 2007. The holistic approach encouraged to pay attention to psychosocial and spiritual needs of patients.

The aim is to provide the results on spiritual needs and their relationship with satisfaction with life in hospitalized palliative care patients.

Methods: 'The Spiritual needs questionnaire' (Büssing 2012) and 'Brief Multidimensional Life Satisfaction Scale' (Bussing et al., 2007) were used. In total, 91 palliative patients participated in the study in 2017.

Results: Needs of active Giving/Generativity had mean score $2.01 \pm$ 0.67 on the scale. Inner Peace needs indicated mean score $1.83 \pm 0.62$. Existential Needs mean score was $1.68 \pm 0.63$ and Religious Needs had mean score $1.61 \pm 0.82$ on the scale.

The majority of patients extremely or strongly agreed with following needs: to feel relation with the family $(92.3 \%)$ and to feel healthy and safe $(84.6 \%)$. The rarest needs were: to read religious books $(29.7 \%)$, to be visited by somebody from religious community $(33.0 \%)$ and to talk with somebody about fears (38.5\%).

Patients' life satisfaction was the lowest for satisfaction with health status $(1.07 \pm 1.23)$, ability to cope with daily living problems $(1.49 \pm 1.62)$ and future ability and plans (1.64 \pm 1.53 ).

Religious and Existential needs were significantly associated with needs of other three groups. Those palliative patients that expressed the needs for inner piece were significantly less satisfied with their life.

Conclusions: Spiritual need for inner peace negatively correlated with life satisfaction of palliative patients. Spiritual needs for palliative patients have to be more carefully addressed in care process at nursing and supportive treatment hospitals.

Abstract number: P01-365

Abstract type: Poster
Dreams and Visions Near Death

Nyblom S., Benkel I., Molander U.

Högsbo Sjukhus, Sahlgrenska University Hospital, Gothenburg, Sweden

Background: Comforting dreams and visions (D\&V) are reported to occur among patients (pat) near death. The experience is most common days-weeks before death and often contains deceased relatives and religious figures. So far only one study has been published in which the pat are asked directly, finding a high prevalence. To our knowledge there are no previous studies conducted in Sweden, a highly secular country, on these types of experiences.

Aims: To find out if pat have comforting dreams and visions near death.

Methods: Pat at hospice and palliative home care are interviewed at 4 consecutive occasions, $\geqslant 1$ week apart.

Inclusion criteria: $>18$ year. Able to understand and speak Swedish. Oriented. Written consent.

Healthcare professionals at hospice and palliative care are invited to focus groups interviews.

All interviews are recorded, transcribed and analyzed with qualitative content analysis.

Results: The results is a hitherto summary of an ongoing larger study and is based on interviews with 16 pat and 2 group interviews. 3 pat were excluded at the first interview due to confusion.

Of the remaining 13 pat, 6 reported comforting $D \& V$, which in most cases included a positive view of death. Those pat lived from 2 days to 3 months after the last interview.

4 out of the 7 pat who reported no D\&V lived $>4$ months after the last interview.

$7 / 11$ healthcare professionals in palliative care had experience of pat with D\&V.

Discussion: Despite few pat included so far the results indicate that comforting D\&V occur near death also in a highly secular country. Pat with no D\&V lived long after the last interview and could have had the experience later. An increased awareness of these experiences among healthcare professionals could be of value in order to support the pat and not mistakenly medicalize.

Conclusion: Comforting dreams and visions are reported directly by palliative patients, including a positive view of death.

Funding: Hjalmar Svensson Foundation.

Abstract number: P01-366

Abstract type: Poster

Principles of Teaching Spiritual Care (SC) in Palliative Care (PC) for Health Care Professionals (HCP)

Gratz M. ${ }^{1}$, Roser $T^{2}$

${ }^{1}$ Hospiz St. Martin, Stuttgart, Germany, ${ }^{2}$ Department of Practical

Theology, University of Muenster, Muenster, Germany

Aims:

secure provision of qualified SC by HCP's

b describe basic and role-specific qualification

c create a teaching framework to limit arbitrariness while maintaining openness for situational circumstances

Design:

a expert meeting of the DGP to reach expert consensus on essential training aims and themes

b hermeneutical approach to identify education-related issues 
Results:

Main curricula themes:

a spiritual needs of PC patients, caregivers and staff

b definition and individual understanding of spirituality

c communication

d religious, cultural, and philosophical diversity

e basic $\mathrm{SC}$ interventions

f rituals

g cooperation with chaplains, providers of religious care

$\mathrm{h} \quad$ integrating $\mathrm{SC}$ in own professional practice

Each theme offers training aims and examples for didactic methods. Training concept has to consider:

a target group: physicians, nurses, social workers, psychologists, different types of therapists, other professionals

b institutions interests, frequency of contact with PC patients, denomination and its unterstanding of spirituality and SC

c main teaching goal: basic information, perception skills, action skills

d providing care in a multireligious and multicultural world

e mono- or multiprofessional learning environment

$f \quad$ given amount of lessons

\section{Quality characteristics:}

a identify training aims and themes for each profession

b differentiate training in multiprofessional course setting and need for monoprofessional specification

c describe participants inpatient or outpatient care setting

d identify the underlying understanding of spirituality and concept of SC

e consider the religious oder philosophical affiliation of the institution and include the expectations of care

$f$ embed training into a project of personnel and organizational development

g base training on present SC literature and research findings

Conclusion: SC trainers will profit from consensualized standards for training non-chaplaincy staff to provide SC in various settings

Abstract number: P01-367
Abstract type: Poster

Dignity-conserving Individual Music Therapy Protocol for Patients Near the End of Life

Sakashita Y. ${ }^{1}$, Nagashima R. ${ }^{2}$

${ }^{1}$ Palliative Medicine, Chiba Cancer Center, Chiba, Japan, ${ }^{2}$ Rehabilitation, Chiba Cancer Center, Chiba, Japan

Introduction: Dignity therapy (DT) based on a dignity model was developed by Chochinov to conserve the dignity of terminal patients. DT is an individualized psychotherapeutic intervention and appears to be effective approach for addressing the existential distress. However, DT is difficult for patients with impaired cognitive function or patients with poor condition, and some patients refuse to participate in DT. On the other hand, individual music therapy can be carried out even in patients with poor condition. However, music therapy has been studied on relief of pain and anxiety, but there are no reports from the viewpoint of reducing the existential distress and conserving dignity. Based on the experience of DT and individual music therapy, we developed dignity-conserving individual music therapy (DMT) protocol incorporating a dignity model. Method: DMT protocol:
1. Introduce DMT to patient by giving introduction form with 5 questions such as "When was the most lively you were in your life.? Is there any song that makes you remember that time?" "What role did you accomplish in your life, what did you accomplish? Is there any song that reminds them? "

2. Patient chooses favorite songs by referring to questions.

3. Music therapist playing electronic piano in hospital room, participants share lyrics and sing along.

4. Facilitator asks the patient to allow the patient to talk freely about the reason why the patient chose the song and episode of life.

Results / Conclusion: Pilot DMT gained popularity from patients and families. Currently we are investigating the feasibility of DMT and the existential distress before and after implementation.

Abstract number: P01-368

Abstract type: Poster

Development of an One-item Question to Assess Spiritual Wellbeing in Advanced Cancer Inpatients in South Korea

Suh S.-Y. ${ }^{1,2}$, Park Y. ${ }^{3}$, Kim S.-H. ${ }^{4}$, Lee E.S. ${ }^{5}$, Park J. ${ }^{6}$, Yoon S.J. ${ }^{7}$, Kim Y.J. ${ }^{8}$, $\overline{\text { Kang B. }}{ }^{9}$, Kwon J.H. ${ }^{10}$, Park K. ${ }^{11}$, Ahn H.-Y. ${ }^{1}$, Hui D. ${ }^{12}$

${ }^{1}$ Dongguk University, Seoul, Korea, Republic of, ${ }^{2}$ Dongguk University Ilsan Hospital, Goyang, Korea, Republic of, ${ }^{3}$ National Health Insurance Service Ilsan Hospital, Goyang, Korea, Republic of, ${ }^{4}$ Catholic Kwandong University International St. Mary's Hospital, Incheon, Korea, Republic of, Inje University Ilsan Paik Hospital, Goyang, Korea, Republic of, ${ }^{6}$ Bobath Hospital, Seongnam, Korea, Republic of, ${ }^{7}$ Chungnam National University, Daejeon, Korea, Republic of, ${ }^{8}$ Seoul National University Bundang Hospital, Seongnam, Korea, Republic of, ${ }^{9}$ Yonsei University College of Medicine, Seoul, Korea, Republic of, ${ }^{10}$ Kangdong Sacred Heart Hospital, Hallym University, Seoul, Korea, Republic of, ${ }^{11}$ Pusan National University Yangsan Hospital, Pusan, Korea, Republic of, ${ }^{12}$ The University of Texas MD Anderson Cancer Center, Houston, United States

Background: Spiritual well-being is important for patients having lifelimiting illness. Functional Assessment of Chronic Illness TherapySpirituality 12(FACIT-Sp 12) is commonly used to assess spiritual well-being(SWB). Shortened versions of question would be helpful to assess SWB in emaciated patients.

Aims: Our goal was to develop an one-item question appropriately in order to assess SWB in advanced cancer inpatients.

Methods: This is a cross-sectional, multicenter study involving adult advanced cancer inpatients from 7 palliative care units in South Korea. Candidates of an one-item question were three questions to check; their peacefulness(Are you at peace?), spirituality(Do you think yourself as a spiritual person?) and religiosity(Do you think yourself as a religious person?). Patients answered three questions by self-rating using numeric rating scales(0-10). We collected data on clinicodemographic characteristics and SWB. SWB was measured by FACIT-Sp 12, comprised of 2 subscales (Meaning/Peace and Faith). Pearson's correlation test was conducted to determine the relationship among three questions, the total score of FACIT-Sp 12 and its subscales.

Results: A total number of 202 patients were enrolled in the study. A good correlation was observed in the questions of spirituality $(r=0.732$, $P<0.01)$ and religiosity $(r=0.708, P<0.01)$ with a total score of FACIT-Sp 12. Question of peacefulness showed a moderate correlation with a total score of FACIT-Sp $12(r=0.505, P<0.01)$. The faith subscale showed a good correlation with the questions of spirituality $(r=0.790, P$ $<0.01)$ and religiosity $(r=0.758, P<0.01)$. All three questions had a moderate correlation with the meaning/peace subscale.

Conclusion: The questions of spirituality and religiosity showed better convergence validity than the question of peacefulness. Therefore, we 
recommend the questions of spirituality or religiosity as an one-item question to assess SWB in advanced cancer inpatients.

Abstract number: P01-369

Abstract type: Poster

"I Didn't Know that This Is Spirituality", A Hermeneutic of Spiritual Care of Persons with Dementia in the Field of Palliative Care Birkholz C.B.

Institut für Lebensbegleitung, Essen, Germany Presenting author email address: birkholz@institut-lebensbegleitung.de

Background: Spiritual care for people with dementia in palliative and dementia care is rarely explored. Four nursing homes, each with a varied background of religious and ideological conviction, took part in the study. There were 23 participants, each of different religions and spiritualities, professions and functions.

Aims: To investigate how carers experience spirituality in their caring relationships with people with dementia. Which challenges do they face in their encounter with dementing behaviour and how do the constructs of spirituality help them?

Methods: The poster will present an interpretive study using logbooks and group discussion to gain data. The 61 logbooks were analysed indepth, using knowledge-based, sociological interpretation. In this way the subjective meaning of the individual person experiencing spiritual care was reconstructed. Its hermeneutic approach is unique.

Results: Apparent subjective concepts of spirituality could be classified into a typology of eight conceptual types of spiritual care. It became evident that the subjective constructs of "spirituality" are independent of the religion of participants in the study. They correspond with role and scope for action. Spiritual care is shown to be fundamentally a process of reciprocal response. The more person-centered the attitude, the more reciprocal and responsive the relationship.

Discussion: These results are contradictory to research on spiritual care that acts out a concept that is split into dualisms: caring versus cared for and needing versus giving.

The developed typology enables insights into the concept of spirituality as a framework for discussing the function of spirituality in caring for people with dementia at the end of their life. It is shown that the organisational logic of nursing homes is contradictory to the logic of palliative care and dementia care. Spiritual care tension areas are identified which need to be discussed in research and practice.

Abstract number: P01-370

Abstract type: Poster

The Development of a Spiritual Conversation Model for Patients with Early Dementia or a Migrant Background Potma M.C. ${ }^{1}$, Haufe M. ${ }^{1}$, Teunissen S.C.C.M. ${ }^{2}$, Leget C.J.W. ${ }^{1}$

${ }^{1}$ Dept. of Care Ethics, University of Humanistic Studies, Utrecht, Netherlands, 2Julius Center Research Program / Centre of Expertise Palliative Care, University Medical Center Utrecht, Utrecht, Netherlands

Background: The development of conversational tools for spiritual care (SC) in palliative care (PC) has international research priority (Selman 2012). In Dutch multicultural society too SC is still the least developed dimension of PC. There is an evidence gap of scientifically developed and evaluated conversational models, especially for people with early dementia and culturally sensitive groups.

Aims: The aims of this empirical study are to develop a conversational model for Dutch patients with

1) early dementia or

2) a Turkish, Surinamese or Moroccan background, their relatives and caregivers, a corresponding culture sensitive practice approach and diverse trainings.
Methods: This paper reports of a sub study of a participatory action research (PAR) approach with 72 patients and relatives in general practices, hospitals and nursing homes. Data collection and analysis of the total study: sensitizing concepts through 15 expert interviews; digital survey with 400 caregivers; 6 focus groups and 3 action groups; 60 observations; 4 co-creation groups; 30 training sessions.

In our sub study qualitative data were collected in 5 focus groups with caregivers, spiritual counselors and dementia case managers and 1 focus group with patients and relatives. After a thematic analysis an existing model for SC was further developed into a consensus-based model for patients with early dementia or a Turkish, Surinamese or Moroccan background.

The model is designed in practical and culturally sensitive language, with images and symbols.

Results: New empirical data on an existing model show the relevance of SC in PC practice and policy. The data analyses of the focus groups highlight the importance of a culture sensitive spiritual model for all Dutch citizens with knowledge from practice itself.

Conclusion: A spiritual conversation tool for patients with early dementia or a migrant background, developed in co-creation with PC practices, is enriched by images and symbols.

Funding: ZonMw

Abstract number: P01-371

Abstract type: Poster

Education and Training in Spirituality: Results from a Crosssectional Study with Portuguese Nurses

Sapage C. ${ }^{1}$, Pinto S. ${ }^{2,3}$

${ }^{1}$ Unidade de Saúde Familiar - Saúde no Futuro / ACES Gaia, Vila Nova de Gaia, Portugal, '2Escola Superior de Saúde de Santa Maria, Porto, Portugal, ${ }^{3} \mathrm{CINTESIS}$ - Center for Health Technology and Services Research, Porto, Portugal

Background: Spirituality is a human life dimension particularly important in Palliative Care (PC). However, theoretical education and training is still scarce.

Aims: To describe the theoretical education and training of Portuguese nurses on Spirituality and Spiritual Care.

Methods: Quantitative, cross-sectional and descriptive study. An intentional sample of 180 nurses working in 3 Community settings and in 4 in-patient's units from 2 Portuguese Hospitals (Pneumology, Medicine, Intensive Care and Oncology) was composed. Inclusion criteria were: to have a nursing diploma and been working as a nurse for more than 6 months. Head Nurses were excluded. Data were collected through a questionnaire composed by: i) sociodemographic issues; ii) education and training in Spirituality/Spiritual care; iii) The Portuguese version of the Spirituality and Spiritual Care Rating Scale $(\alpha=0.75)$. Data were analysed using descriptive statistics (SPSS ${ }^{\circledR}$, version 22 for Windows). The research project was approved by the Ethics Committee of both settings (Community and Hospital).

Results: Participants ( $n=180$; mean age $=33.50$ years; Mean of working time $=12.08$ years) have very few effective theoretical education in Spirituality. Most (46.70\%) failed to define Spirituality. Yet, 59.17\% has basic education in Spirituality, and the majority (51.97\%) works in hospital settings (38.89\% works in oncology, $16.41 \%$ in Medicine, $7.40 \%$ in Pulmonology and $7.69 \%$ in Intensive Care). Only $7.27 \%$ of the nurses who work in the Community have theoretical education on spirituality. This education was obtained within short courses in PC (44.60\%) or in PC master courses $(4.05 \%)$. It was not observed practical training in Spirituality/ Spiritual Care.

Conclusions: There is a need for further theoretical education and practical training in Spirituality. Nurses working in community have less theoretical education on this field. Practical training is an emerging area.

Abstract number: P01-372

Abstract type: Poster 


\section{ExLab - Existential Laboratory}

Baastrup Jørgensen M., Bargfeldt E.

Rigshospitalet, Copenhagen, Denmark

ExLab is an educational initiative for health care professionals bridged between supervision and traditional staff training. The main goal of the course is to better prepare the health care professionals for conversations with patients/next of kin about basic existential/spiritual themes at work when dealing with serious/life threatening disease. In the labs we strive to establish a space and a discourse wherein it is possible to address a wide variety of existential aspects of life.

The framework: Existential support and/or spiritual care can have a profound impact on patient treatment. But a rising demand on staff competence in this area is not necessarily commensurable with the staff's own sense of confidence in addressing the field. On the contrary, studies show that working with the patients' existential challenges can represent a tremendous daily struggle for the health care staff.

The structure: An ExLab course is composed of three two-hour modules - or 'laboratories' - with approx. three weeks between. ExLab is designed for 6-8 staff participants.

In each lab we combine presentations and exercises to adjust the participants' own expectations and beliefs in relation to 'the difficult conversation', and in connection to this, try to address the experienced barriers of the health care participants; i.e.: lack of clarification about own values, lack of a natural language about the existence, experienced lack of time to perform the satisfactory existential support/care, or a lack of knowledge/know-how in connection to existential themes.

Who facilitates? Each ExLab is planned and directed by a lab facilitator, educated in the method through a plentiful collection of exercises designed to inspire the reflective space of the labs.

Behind the project: ExLab was created by Hospital Chaplain Maria Baastrup Jørgensen in 2015. By now over 30 'laboratory leaders' facilitate the labs and more than 70 labs have been completed in a multitude of health care departments.

Abstract number: P01-373

Abstract type: Poster

The Multidimensional Nature of Chaplaincy in Palliative

Care - Literature Review

August H. ${ }^{1}$, Esperandio M.R. ${ }^{2}$

${ }^{1}$ Theology, Pontifical Catholic University of Paraná, Curitiba, Brazil, ${ }^{2}$ Bioethics/Theology, Pontifical Catholic University of Paraná, Curitiba, Brazil

In the last decade, chaplaincy has increased its relevance in palliative care (PC). It is known, however, that there is no single model of chaplaincy and in some countries, where PC are still in their first steps. The purpose of this study is to highlight the focus of empirical research in Brazil and abroad and to point out the main findings on the subject. The method used was the integrative review of the empirical literature in the following databases: Biblioteca Virtual de Saúde, CAPES Portal of Journals, PsycARTICLES, PubMed and SciELO. The search terms in English (and the equivalent in Portuguese) were: 'chaplaincy' AND 'palliative care/spiritual care'. Studies that were not aligned with the subject, double, and not fully available were excluded from the analysis. Initially there were 41 studies, but then 11 were excluded for not meeting the selection criteria. Thus, 30 studies were examined: 29 in English and 1 in Portuguese. The first study was published in 2002; 22 studies (73\%) were published after 2015. The USA is the country with the largest number of publications (80\%). The only one Brazilian study was published in 2015. Six studies used scales for measuring spirituality. The publications can be organized according to the researched population, with some covering more than one category: patients and relatives (12), chaplains (12), health professionals (7), training of professionals (3), hospital managers (2) and researchers (2). The studies show the multidimensional character of chaplaincy and confirm that, in general, patients want spiritual care. Considering the growing relevance of the theme, future research could contribute to the analysis of the spiritual care delivery for patients and their families and in offering possible models of effective spiritual assistance with this population.

Abstract number: P01-374

Abstract type: Poster

Spirituality/Religiosity in Patients and their Family Members' Decision Making In Palliative Care - Integrative Review

Fanini L., Gomes Esperandio M.R.

School of Life Sciences, Pontifícia Uiniversidade Católica do Paraná, Curitiba, Brazil

Spirituality/religiosity has grown steadily into the field of health and markedly, when it comes to palliative care (PC). Palliative care is an approach that tries to identify and assess physical, psychosocial, and spiritual needs from patients and their family members who face challenges related to life-threatening diseases, its aim is to assist and take care of patients and their families to the end. That is why identifying patients' spiritual/religious (S/R) beliefs allows not only for catering for their needs and understanding their preferences for certain treatments and therapies but also understanding their roles in the decision-making process regarding interventions and medical procedures in health. This study aims to verify the link between spirituality/religiosity and decision making within PC in the literature. It is an integrative literature review with bibliographic research done from 2008 and 2018. Database from BVS, CAPES, Pubmed, and SciElo were used. The search terms used were: "palliative care" AND "spirituality" AND "decision making", "patients" and "family members" in Portuguese and English. Investigations that are not related to the topic, duplicated studies, and texts only partially available were excluded from this analysis. At first, 141 studies were taken and 135 excluded for not meeting the selection criteria. From the 6 analyzed papers, 100\% were published in English. Results show that $S / R$ beliefs have a role in the choice for aggressive treatment at the end of life, for instance, the choice for resurrection procedures but, do not play a part in other types of decisions. On the other hand, it was possible to notice some links between S/R beliefs and willingness to withstand the burden of the treatment, and readiness to endure pain and decrease in quality of life. As this review has demonstrated, studies investigating the association between $S / R$ and decisionmaking are needed in order to better understand the role of $S / R$ in $P C$.

Abstract number: P01-375

Abstract type: Poster

How is Spirituality Discussed in Palliative Care Consultations: Do Question Prompt Lists Help?

Best M. ${ }^{1}$, McArdle M. ${ }^{2}$, Huang Y.-J. ${ }^{3}$, Clayton J. ${ }^{3,4}$, Butow P. ${ }^{3}$

${ }^{1}$ Psycho-Oncololgy, University of Sydney, Sydney, Australia, ${ }^{2}$ Dartmouth College, Hanover, United States, ${ }^{3}$ University of Sydney, Sydney,

Australia, ${ }^{4}$ Greenwich Hospital, Sydney, Australia

Background: Patients would like to discuss spirituality more often with their doctors, but feel disempowered to raise the topic, especially at the end of life. Question prompt lists (QPLS) are an aid to assist patients in asking questions.

Aims: This study aimed to examine the frequency and nature of conversations about spirituality in palliative care (PC) consultations, and to assess the impact of a QPL including questions about spirituality, on this discussion. We also aimed to explore the predictors of spirituality discussion.

Methods: We randomised palliative care patients to either receive a QPL 20 minutes prior to a consultation or not. We recorded, transcribed and analysed consultations to examine the frequency and content of spirituality discussions. We conducted logistic regression analysis to investigate the impact of QPL and predictors of spirituality discussion. 
Results: 174 patients participated. Spirituality was discussed in just over half of the recorded consultations. Peace was the topic discussed most often. Patients randomised to receive a QPL discussed spirituality 1.38 times ( $\mathrm{Cl}$ 0.76-2.53) more than those in the control group, but the result was insignificant $(p=0.295)$. Logistic regression identified that first palliative care consultation for the patient and being asked about their concerns by the doctor were significant predictors of a spiritual discussion. Conclusion: Patients are more likely to discuss spirituality in palliative care consultations when it is their first visit, and when their doctor asks them about their concerns. In view of this finding, doctors caring for patients at the end of life should be encouraged to routinely raise spiritual issues.

\section{Abstract number: P01-376 \\ Abstract type: Poster}

Palliative Care Patients' and Informal Caregivers' Contested Beliefs about Optimal Spiritual Care: A Qualitative Inquiry O'Callaghan C. ${ }^{1,2}$, Brooker J. ${ }^{3,4}$, de Silva W. ${ }^{4}$, Glenister D. ${ }^{5}$, Melia A. ${ }^{1}$, Lynch S. ${ }^{2}$, Symons $X^{2}{ }^{2}$, Kissane D. ${ }^{3,6}$, Michael N. ${ }^{1,4,6}$

${ }^{1}$ Palliative and Supportive Care Research Department, Cabrini Health, Melbourne, Australia, ${ }^{2}$ Institute for Ethics and Society, University of Notre Dame, Sydney, Australia, ${ }^{3}$ Szalmuk Family Psycho-Oncology Research Unit, Cabrini Health, Melbourne, Australia, ${ }^{4}$ Faculty of Medicine, Nursing and Health Sciences, Monash University, Melbourne, Australia, ${ }^{5}$ Royal Melbourne Hospital, Melbourne, Australia, ${ }^{6}$ School of Medicine, University of Notre Dame, Sydney, Australia

Background: Spiritual care refers to practices and rituals addressing spiritual/religious concerns. It supports coping with loss and finding hope, meaning and peace. Although integral to palliative care, implementation of spiritual care is challenging.

Aim: To understand palliative care patients' and caregivers' perspectives on how a hospital could optimize spiritual care.

Methods: Patients and caregivers of patients with $\leqslant 12$ month prognosis were recruited from a broader spiritual study via convenience sampling and agreed to opt-in telephone interviews. Participants were given a definition of spiritual care and asked how the hospital assists or could assist them to connect with what they consider sacred or important to their well-being. Interview transcripts were analysed using qualitative description. Descriptive statistics were used for background data.

Results: 30 patients (17 male; mean age 70 -years) and 10 caregivers (6 male; mean age 59-years) participated. 25 identified as Christian and 12 had no religion. Participants described multifaceted and contested beliefs about spirituality. All valued respectful staff who affirmed personhood, that is, each individual's worth, especially when care exceeded expectations. Many queried the tangibility of spirituality but resonated with positive organisational and environmental tones that improved holistic well-being. They stressed the importance of hospitality, that is, how the hospital's welcoming context and skilled care comforted and reassured.

Conclusion: While many palliative care patients and caregivers did not resonate with spiritual care, all described how the hospital's hospitality could affirm their value and strengthen coping. Hospitality may be a useful concept alongside spiritual care in pluralist palliative care settings, recognising that such care encompasses an interplay of generalist and pastoral staff, and organisational and environmental qualities.

Main Funding: Cabrini Foundation

Abstract number: P01-377

Abstract type: Poster

Perceptions and Attitudes at End of Life in Georgia: The Similarities Regardless of the Causes of Limited Life Expectancy - Cancer or Advanced Age
Chikhladze N. ${ }^{1,2}$, Tebidze N. ${ }^{1,2}$, Velijanashvili M.1,2, Khachapuridze K.1,2, Kordzaia D. ${ }^{1,2}$

${ }^{1}$ Ivane Javakhishvili Tbilisi State University, Tbilisi, Georgia, ${ }^{2}$ Georgian National Association for Palliative Care, Tbilisi, Georgia

Background: In developing a model of palliative care, the attitudes, needs and requirements of its beneficiaries recognizing the limited remaining life expectancy need to be met.

Aim: We compared the transcripts of semi-structured interviews of 50 advanced cancer patients and 50 healthy individuals aged over 85 .

Methods: Every respondent was asked to tell about past life and future plans/wishes. The interview tool was based on Grounded Theory with the coding based on Maslow's "Hierarchy of Human Needs". Data comparisons of two groups were tested in bivariate analysis using Pearson's chi-square or Fisher's exact test. Two-sided significance tests were used and $\mathrm{P}<0.05$ was deemed significant. Every respondent was required to speak in Georgian. Also, their physical condition and neurological status had to permit proper understanding of survey requirements.

Results: Studying the interviews the sincerity of the respondents of both groups was noteworthy. Some stories were so personal, that caused to researchers the sense similar to hearing the confession (about the complicated relation with children, behaviors during the conflicts at work, regret because of wrong actions and etc.). It confirms that the survey was conducted in trustful and cordial atmosphere.

In the narratives of both groups the stories consistent with satisfaction of the upper levels of Maslow's hierarchy explicitly prevail: social relation (belonging), love and esteem are very important while realising the end of life. The feeling that after their departure their loved ones will worry remained actual as well.

Conclusion: Our research showed that the end-of-life needs, perceptions and attitudes of people are basically similar, regardless of what determined the sense of limitation of the remaining life - incurable disease or senility. Georgian citizens do not support the excessive institutionalization of death/dying and prefer to die at home, surrounded by family members and relatives.

Abstract number: P01-378

Abstract type: Poster

Spirituality/Religiosity in Medical Decision Making by Patients' Family Members in Palliative Care

Fanini L., Gomes Esperandio M.R.

School of Life Sciences, Pontifícia Uiniversidade Católica do Paraná, Curitiba, Brazil

Palliative Care (PC) is an approach that aims at identifying and assessing physical, psychosocial, and spiritual patients and family members' needs who face life threatening diseases. Despite PC's holistic view, it was in the recent years that more concern with catering for spiritual/religious (S/R) needs of patients and family members was noticed. The goal of this study was to identify the psycho-spiritual needs of family members and their relationship with medical decision making. It is a descriptive qualitative study in which the participants were 12 family members of patients hospitalized in a university hospital in Curitiba, Brazil. Data collection was carried out by means of a social-demographic questionnaire, Spiritual Religious Coping Scale (PARGAMENT, 1997), The Centrality of Religiosity Scale (HUBER \& HUBER, 2012), and interviews. The results point out that the main psycho-spiritual needs of family members are: feeling the presence and strength of God, faith, and the presence of religious people to talk to and offer consolation. These family members were ranked as highly religious. No conflict was identified between medical decisions religious beliefs and conviction because for the family members, suggestions and recommendations given by medical team are interpreted as an expression and manifestation of the power of God therefore not causing any conflict. By following through with physicians' 
guidance, they believe to be complying with God's will. S/R reveals itself as an important component to be integrated into the treatment and should be done in the regard of ethical care. As the present study has shown power impacts the relationship of physician-patient-family in the decision-making process. Given the small number in the sample, more studies are required to confirm the results achieved.

Abstract number: P01-379

Abstract type: Poster

Spiritual Well-being and Religious Commitment of Cancer Patients under Chemotherapy

Martins H. ${ }^{1}$, Domingues T.D. ${ }^{2}$, Caldeira S. ${ }^{3}$

${ }^{1}$ Institute of Health Sciences, Universidade Católica Portuguesa, Lisbon, Portugal, ${ }^{2}$ CEAUL- Centro de Estatística e Aplicações, Universidade de Lisboa, Lisbon, Portugal, ${ }^{3}$ Centre for Interdisciplinary Research in Health, Institute of Health Sciences, Universidade Católica Portuguesa, Lisbon, Portugal

Background: Spirituality is one on the human dimension that cancer has effect on and that needs to be included in healthcare (WHO, 2014). Spirituality comprises religiosity and is critical in overcoming illness and in finding meaning in life (Puchalski, 2012). Religious commitment may be affected in times of crisis, and his research explores its relation with spiritual well-being in cancer patients.

Objective: To analyze the relation between the spiritual well-being and religious commitment of Portuguese cancer patients under chemotherapy.

Method: A quantitative, observational, descriptive, correlational and cross-sectional study was conducted. A convenience sample of 150 cancer patients undergoing chemotherapy in outpatient setting was obtained. Data collection method was a questionnaire comprising the Spiritual well-being (SWB) and Scale and the Belief into Action Scale (BIAC). Data was analyzed using Statistical Package for the Social Sciences (SPSS). This study was approved by the institutional ethics committee. Results: Main participants (55.3\%) aged over 60 years, about $64.7 \%$ were female, married $(68.0 \%)$, and $86.7 \%$ were catholic. The findings suggest that SWB and BIAC have moderate correlation, based on Pearson Correlation of 0.6.

Conclusion: Religious commitment and spiritual well-being have a reasonable relationship in cancer patients under chemotherapy. This may guide nursing care in promoting spiritual and religious practices, according to patients' wishes and beliefs.

References:

Puchalski, C. (2012). Spirituality in the Cancer Trajectory. Annals Oncology, 23(3), 49-55.

World Health Organization. (2014). World cancer report. Geneva: World Health Organization.

Abstract number: P01-380

Abstract type: Poster

Validation of the Duke University Religion Index (DUREL)

in Portuguese Cancer Patients under Chemotherapy: Methodological Study

Martins H. ${ }^{1}$, Domingues T.D. ${ }^{2}$, Caldeira S. ${ }^{3}$

${ }^{1}$ Institute of Health Sciences, Universidade Católica Portuguesa, Lisbon, Portugal, ${ }^{2}$ CEAUL- Centro de Estatística e Aplicações, Universidade de Lisboa, Lisbon, Portugal, ${ }^{3}$ Centre for Interdisciplinary Research in Health, Institute of Health Sciences, Universidade Católica Portuguesa, Lisbon, Portugal

Background: Cancer is considered a public health problem worldwide (Siegel et al., 2018). Cancer patients who frequently participate in religious activities have a lower mortality risk (Li et al., 2016).

More than 100 tools are available to assess different aspects of religiosity, and DUREL relates the brief assessment of the religious involvement
(Hall et al., 2008). This tool has been widely used in research but is not available in European Portuguese, which seems necessary to assess patients' religious/spiritual needs and compare findings.

Objective: To conduct the translation, adaptation and validation of the DUREL in Portuguese cancer patients under chemotherapy.

Method: The study was conducted according to Sousa and Rojjanasrirat (2010), which is designed to be used in healthcare context. Study was approved by the institutional ethics committee.

Results: First, linguistic and conceptual equivalence of the scale was guaranteed. Then, a total of 150 adult cancer patients under chemotherapy was included using convenience sampling. The internal consistency was good (Cronbach $=0.86$ ).

Conclusion: The DUREL-Portuguese version is a reliable and valid measure to assess religiosity in cancer patients under chemotherapy.

References:

Hall, D. E., Meador, K. G., \& Koenig, H. G. (2008). Measuring religiousness in health research: review and critique. Journal of Religion and Health, 47(2), 134-163.

Li, S., Stampfer, M.J., Williams, D.R., \& VanderWeele, T.J. (2016). Association of religious service attendance with mortality among women. JAMA Internal Medicine, 176(6), 777-85.

Siegel, R.L., Kimberly, D.M., \& Ahmedin, J. (2018). Cancer statistics, 2018. CA: A Cancer Journal for Clinicians, 68, 7-30.

Sousa, V., \& Rojjanasrirat, W. (2011). Translation, adaptation and validation of instruments or scales for use in cross-cultural health care research:a clear and user-friendly guideline. Journal of Evaluation in Clinical Practice, 17(2), 268-274.

Abstract number: P01-381

Abstract type: Poster

\section{Spiritual Care in Palliative Care: A Narrative Review of the} Recent European Literature

Gijsberts M.-J.H.E. ${ }^{1,2}$, Olsman E. ${ }^{3,4}$, Liefbroer A.I. ${ }^{5}$

${ }^{1}$ End-of-Life Care Research Group, Vrije Universiteit Brussel \& Ghent University, Brussels, Belgium, ${ }^{2}$ Department of Public and Occupational Health, Amsterdam AMC, VU Medical Center, EMGO Institute for Health and Care Research, Amsterdam, Netherlands, ${ }^{3}$ Section of Ethics \& Law of Health Care, Leiden University Medical Center, Leiden, Netherlands, ${ }^{4}$ Department of General Practice, Section of Medical Ethics, Amsterdam UMC, University of Amsterdam, Amsterdam, Netherlands, ${ }^{5}$ Faculty of Religion and Theology, Vrije Universiteit Amsterdam, Amsterdam, Netherlands

Background: Spiritual care is an intrinsic and essential component of palliative care. However, it remains the least developed and most neglected dimension of research on healthcare and the recent literature in Europe has not been reviewed. Most studies originate from North America. However, it is unclear if findings in these studies can be 'transferred' to spiritual care in palliative care in Europe.

Aims: We aimed to map studies that represent the European perspective on the understanding, the provision, and the effectiveness of spiritual care in palliative care.

Methods: As palliative care is multidisciplinary in nature and because the variety of languages, a multi-source search strategy will be applied. Inclusion criteria were:

(1) an empirical study,

(2) concerning spiritual care provided in the context of palliative care,

(3) published in a peer-reviewed journal,

(4) in 2016 or 2017 . The studies had to be

(5) full-text articles published in English, German, Spanish, Portuguese or Dutch, and

(6) conducted in Europe. Studies were thematically analyzed with a focus on the way in which spiritual care in palliative care was 
understood, on how it was provided, and on the effect it had on the patients' wellbeing.

Results: Having screened half of the studies, we included 36 studies. They were 16 quantitative studies, including several survey studies and some trials, 11 qualitative studies, which were mainly semistructured interviews, and 9 mixed methods studies. The findings suggest that, though participants often emphasized the importance of spiritual care, the evidence for the effectiveness of spiritual care is low since there are hardly RCT's showing its effectiveness. Several studies focused on the attitudes and the development of health care professionals, other than spiritual caregivers, in providing spiritual care.

Conclusion: The final conclusions of all included articles in 2016 and 2017 will be presented at the EAPC Congress in 2019.

Abstract number: P01-382

Abstract type: Poster

\section{Finding a Voice through Poetry in Palliative Care}

Haraldsdottir E. ${ }^{1,2}$, Gilmour $F^{2}{ }^{2}$ Riccobono R. $^{3}$

1Education and Research, St Columba's Hospice, Edinburgh, United Kingdom, ${ }^{2}$ Nursing, Queen Margaret University, Edinburgh, United Kingdom, ${ }^{3}$ School of Modern Languages, University of St Andrews, St Andrews, United Kingdom

Background: Embedded in Cicely Saunders' Philosophy of Palliative Care is open awareness of death whereby patients and families are encouraged to engage with the emotional impact of life limiting illness and express their feelings and thoughts. And for her, health care practitioners in palliative care should have a key role in supporting this to happen. This is still seen as essential part of holistic palliative and end of life care whereby patient and family members are encouraged to find a voice to express their experiences and through this find healing, mirroring person centred care.

Aim: To explore the use of reading and discussing poetry for people in palliative and end of life care and its role in supporting patients, families and health care professionals to explore themes within the poems and voice their own emotions and thoughts.

Methods:

- Narrative review/ critical interpretative synthesis

- A comprehensive search of the following databases was undertaken: CINAHL, Medline, PsycINFO

- Search terms included combinations of; Poetry, Creative Writing, Prose, Narratives, Palliative Care, Grief, Dying, Life Limiting Illnesses, Degenerative IIInesses, Medical Humanities.

- 95 studies were generated with 15 included.

Results: Within the literature, 4 key themes emerged:

- The impact of poetry on the wellbeing of people in palliative and end of life care

- the impact that poetry may have on the wellbeing of health care professionals,

- $\quad$ the power that poetry may have for family members to prepare and cope with bereavement

- $\quad$ The power of poetry for health care professionals to prepare and support people to die in dignity.

Conclusions: Discussing poetry in palliative care may enhance person centred practices and enable people in the end stages of life to connect with others, resonate with themes in the poems and voice their own eelings and experiences.
Abstract number: P01-383

Abstract type: Poster

\section{"Color in the Ward": An Art Therapy Project for Hospitalized} Cancer Patients

Bergianti M.L. ${ }^{1}$, Pellini G. ${ }^{2}$, D'Incà M. ${ }^{3}$, De Panfilis L. ${ }^{4}$, Sacchi S. ${ }^{5}$

${ }^{1}$ Stella Maris, Bologna, Italy, ${ }^{2}$ ANDOS, Reggio Emilia, Italy, ${ }^{3}$ Oncology and Advanced Technologies, Azienda USL - IRCCS di Reggio Emilia, Reggio Emilia, Italy, ${ }^{4}$ Scientific Directorate, AUSL - IRCCS, Reggio Emilia, Italy, ${ }^{5}$ Palliative Care Unit, Arcispedale SMN - IRCCS, Reggio Emilia, Italy

Background/aims: Art Therapy (AT)is an artistic method aimed at helping patients to perceive both themselves and the surrounding environment in a deep manner. As AT can induce positive emotions dealing with inner domain it may be useful to support frail cancer patients (CPs). "Color in the ward" project was born to provide CPs with a "space for soul" during hospitalization in a Clinical Cancer Centre, and give them the message that they can think and feel they are human beings, and not simply their disease.

Methods: The project was originally developed by a 48 y old woman cancer patient and carried on by an art therapist, expert in palliative care. One day a week all patients of an oncological ward were invited to take part in a free session of paintings according to R. Steiner "Stella Maris" method. They painted natural subjects described by the art therapist using thin colours on dry papers. Then thoughts and feelings from participants, as well as from relatives and professionals, were collected in a narrative way by the art therapist.

Results: The project was carried out throughout 12 months. Sessions were attended by 85 participants (of whom 11 relatives). According to what reported by the art therapist, CPs perceived "a break from disease", "a breath during hospitalization", and told "to have spent a pleasant time", "to have coloured a black moment". A patient referred: "these colours warmed my heart". Narratives highlighted participants' sense of gratitude and marvel when looking at their pictures.

Conclusions: AT may constitute a promising approach to take care for hospitalized CPs with an holistic perspective. This can be expecially true for CPs with advanced illness, given AT emphasis on support to frailty and dignity. Finally AT may contribute to relieve patients from suffering nurturing their spiritual dimension of hope.

Funding was provided by ANDOS association.

Abstract number: P01-384

Abstract type: Poster

Unpacking Awkwardness with Patients' Spiritual Struggles among Palliative Care Professionals: A Survey

Haufe M. ${ }^{1}$, Potma M.C. ${ }^{1}$, Leget C.J.W. ${ }^{1}$, Teunissen S.C.C.M. ${ }^{2}$

${ }^{1}$ Care Ethics, University of Humanistic Studies, Utrecht, Netherlands, 2Julius Center for Health Sciences and Primary Care, University Medical Center Utrecht, Utrecht, Netherlands

Background: Patients and their loved ones often grapple with spiritual questions at the end of life. Many healthcare professionals however, feel awkward talking to patients about these issues, especially when patients are from a different cultural background or suffer from early stage dementia.

Aims: Our study examined more closely the awkwardness of palliative care professionals when dealing with spiritual issues of patients and their loved ones. Specifically, it sought to ascertain for these professionals:

1) which aspects of spiritual care they struggled with most and

2) which aspects of spiritual care they would like further assistance with. 
Study design and methods: A survey using stratified sampling, was conducted among palliative professionals working in primary, secondary and tertiary care. The Ars Moriendi model (Leget 2008) was used to gain a clearer sense of the spiritual themes professionals encounter in their dealings with patients and their loved ones.

Results: 400 Dutch palliative professionals participated in the study. Preliminary analysis identified that professionals struggled with aspects such as: how to recognise specific types of spiritual questions, how to start a conversation concerning spiritual matters and how to understand patients cultural or religious influences on their spiritual questions. Professionals would especially like more assistance with recognising specific spiritual questions and knowing how to start a conversation concerning spiritual matters.

Conclusion: The findings of this study give a clearer picture of what exactly palliative care professional are struggling with regarding the spiritual dimension of their work. The results will be used to develop trainingsets for palliative professionals to help overcome spiritual awkwardness, especially with respect to patients from a different cultural background or with early stage dementia.

Funding was provided by the Netherlands Organisation for Health Research and Development.

\section{Abstract number: P01-385 \\ Abstract type: Poster}

\section{Hospital Biography as Spiritual Accompaniment: Quantitative} and Qualitative Results over 2 Years

Joly C. ${ }^{1}$, Verdet M. ${ }^{1}$, Desmonds C. ${ }^{2}$, Olivier V. ${ }^{3}$, Richard M. ${ }^{4}$, Dorange C. ${ }^{5}$, Milewski V. ${ }^{6}$, Brau R. ${ }^{7}$, Dewulf $C^{8}$

1 USP, Centre Hospitalier, Pont Audemer, France, ${ }^{2}$ USP, Centre Hospitalier, Le Havre, France, ${ }^{3}$ Médecine, Centre Hospitalier, Fécamp, France, ${ }^{4}$ Médecine, Centre Hospitalier, Lillebonne, France, ${ }^{5}$ EHPAD, Saint Romain de Colbosc, France, ${ }^{6}$ Oncologie, Centre Hospitalier, Chartres, France, ${ }^{7}$ Fonds pour les Soins Palliatifs, Paris, France, ${ }^{8}$ USP/ EMSP, Centre Hospitalier, Le Havre, France

Background: Hospital biography (HB) consists in proposing to a seriously ill patient to tell his story and to receive it (or a close relative) as a life story written on a handmade book by a craftsman. This approach is fully part of the interdisciplinary work of a hospital team and allows the patient to reinvent himself, to feel alive, to share his reality and view on the world by reviewing his life.

Aims: We evaluate the results of 2 years of HB in five Norman hospitals. Methods: Half-year checkups are established according to a grid of figures and verbatim from the nursing staff collected through questionnaires and oral discussions during college meetings.

Results: From October 2016 to October 2018, thanks to a weekly intervention in each hospital in several departments, we were able to introduce the approach to 358 patients. 44 books are under way, 16 are being crafted and 34 are already finished and have been handed over. Only one finished book could not be given to the patient who died before.

The qualitative checkup shows that the HB values the patient who feels more connected to the world, restores their dignity and ease them. Families "are reassured are less terrified by the idea of losing their relative". The handing of the book is an important moment for the family and can be a way to see the nursing team again, to help the mourning for instance. Conclusion: This new approach is based on the narrative identity and is a renewal for caring practices and allows the team to focus on the spiritual accompaniment and ethic reflection. It must expand to other hospitals and evolve as a scientific procedure (philosophy, sociology,...).

Abstract number: P01-386

Abstract type: Poster

Social and Spiritual Needs of Patients in the Palliative Phase: A Systematic Literature Research
Lormans A.H.M. ${ }^{1}$, de Graaf E. ${ }^{1}$, van der Baan F.H. ${ }^{1}$, Leget C.J.W. ${ }^{2}$, Teunissen S.C.C.M. ${ }^{1}$

${ }^{1}$ Department of Primary Care, Julius Center for Health Sciences and Primary Care, Center of Expertise in Palliative Care, University Medical Center Utrecht, Utrecht, Netherlands, 2 University of Humanistic Studies, Utrecht, Netherlands

Background: Understanding patients' needs is essential for optimizing quality of life and tailoring person-centered care. However, unambiguous knowledge concerning social and spiritual needs is lacking.

Aim: To map the existing knowledge on social and spiritual needs of patients in the palliative phase.

Methods: A systematic review of qualitative and quantitative studies was conducted using five databases: Pubmed, Embase, PsycINFO, CINAHL and Scopus. A four-component search strategy was used: palliative care, social and spiritual dimension, needs, and patient perspective. In addition, snowballing was applied using the reference lists. Eligible studies,

(1) studied social and spiritual needs from a patient perspective,

(2) included patients $\geqslant 18$ years diagnosed with cancer or organ failure, and in the palliative phase of their disease,

(3) were published between 2008-2018 and

(4) were written in English, German or Dutch.

Two reviewers independently assessed titles and abstracts, and subsequently assessed full texts and quality.

Results: Of $>19000$ search results 35 studies were included. Social needs concern independence, relationships, reconciliation, participating in daily life and financial support. Spiritual needs concern existentiality, dignity, autonomy, forgiveness and relationships. These needs show an overlap when it comes to autonomy, reconciliation/forgiveness and relationships. Conclusions: Patients' social and spiritual needs are not always clearly defined. The basis on which these needs are distinguished is not uniform and the way researchers categorize them to be social, spiritual, or even psychological, emotional or sexual is ambiguous. The results give better insight in social and spiritual needs, which could help healthcare professionals develop more focused tools to easier tailor person centered care. Furthermore, research on social needs stays far behind on spiritual needs.

Funding: ZonMw, the Netherlands Organization for Health Research and Development.

\section{Abstract number: P01-388} Abstract type: Poster

\section{A Creative Legacy Work Project}

Ashton W.

Eden Valley Hospice, Carlisle, United Kingdom Presenting author email address: wendy.ashton@edenvalleyhospice.org

Background: Legacy work isn't about death and dying, it's about life and living. It's about making connections and sharing precious moments with the special people in your life. Legacy work provides a unique opportunity to reflect on your life and process through the events and people who shaped it. It can be a means of moving on, not only for you, but for the people around you.

Leaving a Legacy Frame gives your loved ones something to hold on to, something that can provide healing and comfort. It's a reminder of who you were, what you loved, what was important to you and what contributions you have made.

Aim: To enable and support patients to participate in a creative legacy frame project and offer psychological and emotional support.

Methods: Verbally interviewed patients and the wider MDT about their thoughts on the creative arts project. Feedback was positive. Pilot frame completed by social worker. 
Developed a 4 session programme on a once a week basis.

Each session to last approx 1.5 hours.

Ratio of staff to patients is 1:1 or 1:3 in a group setting.

Referrals can be made by any member of Palliative care team.

Results: Participant's felt a personal self fulfilment as it is a short, time limited project that they can manage within the timescales of their illness.

The project was held in the Hospice setting and had positive outcomes.

The participants reflected on life with emotional support from social worker and had a

completed frame to keep and take home.

Conclusion: Participants reported a sense of self worth. That life had been good and they had achieved things to be proud of. A bereaved daughter stated it was the best way to remember her dad.

Socializing with people face-to-face can help reduce depression, anxiety, insomnia and feelings of loneliness.

Art and creative work are a vital human activity and integral to a good quality of life. (Shaw 1999)

The project is ongoing.

\section{Abstract number: P01-389}

Abstract type: Poster

\section{Psychosocial Care in the Hospice}

van Nus J.L. ${ }^{1}$, van den Brink J. ${ }^{2}$, Kruithof J.W.F. ${ }^{2}$, Wieles G.A.M. ${ }^{1}$, Gootjes

J.R.G. ${ }^{1}$

${ }^{1}$ Hospice Kuria, Amsterdam, Netherlands, ${ }^{2}$ Christian University of

Applied Sciences, Ede, Netherlands

Background: Psychosocial care is an important part of palliative care in the hospice. The hospice in this study has a social worker to provide this care and at other hospices a psychosocial worker does this. In this research the psychosocial activities in the hospice are shown, as well as options to develop and improve the care.

Aims: The question is: What did the psychosocial care provided by professionals of the hospice consist of in 2016 and 2017 and how can this care be improved?

Methods: File and literature research have been done. Interviews were conducted with the social worker, spiritual caregiver, nurses and psychosocial workers of other hospices.

Results: The interviews and file research showed that, besides the social worker, the nurses and spiritual caregiver also provide basic psychosocial assistance or psychosocial care to residents and their loved ones. During interviews with external psychosocial workers it became apparent that they focus more on psychosocial care for residents and their loved ones than the social worker does. The social worker spends more time on practical issues. The psychosocial worker, in contrast, spends little time on them or only assists in them. The psychosocial work of the social worker largely consists of care for loved ones, whereas nurses and the spiritual caregiver focus on the residents. Recognizing and discussing signals during the multi-disciplinary discussion is an important task.

Conclusion/discussion: It is recommended to more clearly define psychosocial care: what does it consist of, who executes the tasks, what are the agreements and what should the care achieve? A second recommendation is to hire a volunteer who can assist the social worker with practical issues. The social worker can then, if needed, spend more time on psychosocial care for residents and their loved ones. Opening up discussions with other hospices without social workers can also give more clarity on the role of psychosocial care within the hospice.

Abstract number: P01-391

Abstract type: Poster

Social Worker Profile in Palliative Care in Romania

Simion A., Popa C.

Social, Fundatia Hospice Casa Sperantei, Brasov, Romania
Background: Social Work in Palliative Care (PC) Services is a new, exciting and growing field. The number of social workers in PC is small in Romania. And as such the profile and the area of interventions for a social worker in PC is not well known.

Aims: to frame a profile of social worker in Palliative Care.

Methods: qualitative study done March 2018-September 2018 with 10 social workers from 5 districts, who work in PC. Data collected through semi-structured interview with 11 questions, were recorded, transcribed verbatim and analyzed by 2 researches through open coding.

Results: the average age of respondents is 35 with an average of 9 years in PC. The identified core elements for social worker profile: ADVOCATE on patients behalf and OBTAINING RIGHTS for patients and family. 3 additional elements were identified RESILIENCE in: a) working with authorities who are resistant "the reluctance of institutions that still do not accept social status", "many state employees did not know what palliative care means", b) dealing with urgency of needs of patients with short life spam"the patient has no time to enjoy the legal rights I am struggling to ge", c) creating networks"it was a challenge to make them understand the role of the social worker and to develop a relation"; FLEXIBILITY "new challenges every day you need to pass", and MAINTAINING EMOTIONAL BALANCE IN THE FACE OF SUFFERING. The motivating factors for PC social workers are: work brings benefits for patients' life, appreciation and gratitude from patients, team spirit, new challenges which help social workers to develop. The social worker from PC identified useful personal skills such as transparency, promptness, communication, openness, initiative, trust.

Conclusion: A successful social worker for PC service in a resource restrained setting must be resilient, flexible, emotional mature and see his /her mission as advocating and obtaining patients and families rights.

Abstract number: P01-392

Abstract type: Poster

Pediatric Palliative Care Social Work Competencies: Lessons from the Field on a Work in Progress

Remkes.

University of Minnesota, School of Social Work, St Paul, MN, United States

Pediatric palliative care is a growing and important specialty practice around the world. WHO has described palliative care as a human right. Family and social care is a critical focus for effective pediatric palliative care. Family centered care models emphasize care provision in the appropriate cultural context, and inclusion of diverse family members. This practice is complex and multi-faceted. Social workers are often considered core members of interprofessional teams, yet there is little research and few educational resources to guide and prepare social workers for this important area of practice. Social work training and education is not always available in areas where social work practice is greatly needed. Social work competencies for adult oriented practice have been described (Gwyther et al, 2005; Bosma et al, 2010). However, little guidance exists for pediatric practitioners. This presentation will review the literature and current thinking about pediatric palliative social work through an international lens, drawing on the presenter's practice and teaching experience, as well as feedback from others in the field around the world to begin to describe social work competencies for pediatric palliative care. In this interactive workshop, participants will be offered an opportunity to weigh in on establishing consensus of recommended essential competencies that can guide future research, education, and preparation for practice in diverse settings.

Abstract number: $\mathrm{P} 01-393$

Abstract type: Poster 
Chimpanzees, Chameleons and Guerrillas: Lessons in Leadership from an Embattled Profession

Cullen A.

University of Hull, Social Work, Hull, United Kingdom Presenting author email address: a.f.cullen@2009.hull.ac.uk

Background: This presentation reports findings from a UK PhD study of leadership in social work by a palliative care social worker/manager. The study was motivated by repeated accusations from without and within that the social work profession was lacking in 'leadership at all levels'. The researcher questioned this, based on the achievements of social workers in palliative care, and evidence from earlier research. The study explored how leadership was conceptualised, and if and how it was practiced, by members of 3 social worker participant cohorts: experienced practitioners, organisational managers and national leaders. Participants from end of life services were included in each cohort.

Aim: To find out whether there were distinctive features of social leadership that could be used to develop a model to enhance leadership within and on behalf of a profession.

Method: A qualitative methodology was used. A purposive recruitment strategy drew participants with suitable qualifications and experience for each cohort, from a range of specialist areas, organisations and regions $(n=36)$. Data were obtained through individual, small and large group interviews. Analysis used grounded theory and Framework, using NVIVO.

Results: Social workers at each level showed 3 characteristic traits: 'ethically inspired' (driven by an emotion of compassion and principles of social justice), 'empirically informed' (focus on evidence and rigorous analysis), and 'strategic' (positively adaptive, combining holistic appraisal and tactical agility). These were operationalised in 2 forms of behaviour:

(1) eliciting and sustaining collaboration and

(2) challenging; which proved to be complementary.

\section{Outcomes were:}

(1) influencing decisive change at case, organisation, system and policy levels

(2) enabling others, including service users, to activate their own power.

Discussion: Originating from a palliative care perspective, this model is salient for all palliative care professionals.

Abstract number: P01-394

Abstract type: Poster

What do Palliative Care Social Workers do? A European Study Firth P. ${ }^{1}$, Bitschnau K. ${ }^{2}$

${ }^{1}$ Independent Consultant, St. Albans, United Kingdom, ${ }^{2}$ Hospice Vorarlberg, Feldrick, Austria

Background: Palliative care social workers are essential members of the multi professional teams in palliative care. but, their roles are often misunderstood. This study followed on from the 2014 white paper.The task force has provided a useful link and focus for networking.

Aims: To conduct a pan European survey to consider the differing roles and functions of our colleagues.This was to provide information about common role function and to gain an insight of the state of palliative care social work in Europe.

Methods: The European Association of Palliative Care administration enabled us to contact national associations who were asked to complete an online questionnaire and name a link social worker who distributed part 2.
Part 2 asked about basic data concerning palliative care social work in the participating countries as well as their settings, management, roles, functions and role overlap and education levels.

Results: The results of the survey are currently being analysed. Common patterns :evidence of role overlap with other professions,the importance of advocacy, social justice and human rights, family assessment, practical support and community knowledge Only about half of the responding social workers reported a specialized training in palliative care.

Limitations: The data collection proved difficult because information did not reach social workers.

Conclusion: From a European perspective a mixed picture of palliative care social work is emerging This could be due to the high adaptability of social work, which is used to being as flexible as possible to the needs of its clients. In some countries palliative care social workers were well recognised and had their own specialist groups, in other countries isolation and lack of representation were experienced by many palliative care social workers. This strengthened our belief that some palliative care social workers appeared to have little or no voice in some countries and the need for recognition and focus.

Abstract number: P01-395

Abstract type: Poster

\section{Professional Identity of Hospice Social Workers}

Macková M.

University of Social and Administrative Affairs, Social Science and Social Policy, Havířov, Czech Republic Presenting author email address: phdr. mackova@seznam.cz

Introduction: Social work celebrates a hundred years since the beginning of social work in the Czech Republic, but its position is still uncertain among other scientific disciplines. The fight for recognition of social work's professional status has been influenced by multiple factors including negative public perceptions of the occupation. At present, a professional law on social workers is being prepared and the opinions of the professional public are not uniform for its adoption. Very much discussed is the professional identity and the associated professional growth. This research considers the ways in which palliative social workers perceive themselves, and the profession that they identify with.

Methods: Semi-structured interviews with 5 social workers in Czech Republic were conducted. Participants were required to be curently work at hospice and in previous quantitative research they were characterized by a strong and very strong professional identity. Interview transcripts were thematically analysed.

Findings: Several identity themes emerged during the analysis of the five interviews. The first theme relates to the ways in which palliative social works are motivated for this work. The second, theme corresponds with their self-esteem and last theme addresses the impact of the professional law on the position of palliative social workers and their professional identity.

Conclusion: All participants in the current study believed that professional law may positively influence the public's view of social workers, other professionals, and also social workers themselves. However, many participants seemed to experience levels of self-stigma - and potentially believe they are viewed more negatively by the public than they actually are.

Abstract number: P01-397

Abstract type: Poster

The Practice of Psychologists Working in Palliative Care: A

French Guidelines

Van Lander A. ${ }^{1,2,3}$, Amar S. ${ }^{4}$, Bioy A. ${ }^{5}$, Gautier S. ${ }^{6}$, Delatour A. ${ }^{7}$, Guastella $V^{2}$, Morel $V^{8}$ 
${ }^{1}$ Collège des Psychologues, SFAP, Paris, France, 2Palliative Care, CHU Clermont-Ferrand, Clermont-Ferrand, France, ${ }^{3}$ UPU ACCePPT, Université Clermont Auvergne, Clermont-Ferrand, France, ${ }^{4}$ Psychology, Groupement Hospitalier Nord Essonne, Paris, France, ${ }^{5}$ UFR Psychology, Université Paris 8, Paris, France, ${ }^{6}$ Centre des Massues USP, Croix-Rouge Française, Lyon, France, ${ }^{7}$ SFAP, Paris, France, ${ }^{8}$ Unit of Palliative Care, Centre Hospitalier Universitaire de Rennes, Rennes, France

In 2011 the European Association of Palliative Care Task Force on Education for Psychologists in Palliative Care presented a report on the training and competencies of practitioners working in this field (Jünger, 2011). As a result of this initiative, the French palliative care association (SFAP) decided to make a comprehensive survey of the complex practice of psychology in palliative care. Beyond the diversity of practices a common approach by clinical psychologists can be identified from which a set of basic parameters can be extracted and formulated. This nationwide study, which ran from 2012 to 2016, was organized by the French National College of psychologists.

Objective: The survey aims to serve as a professional reference that will define practice guidelines for psychologists during palliative care treatment and identify what is specific to the psychologist's function and to this particular field of practice.

Methods: The guidelines were drawn up by four groups using the consensus method. It was validated by the board of the SFAP.

Results: The guidelines (SFAP, 2016) state in clear and relevant terms the benefits and limitations of psychologists' clinical practice.

Conclusions: This frame of reference should be considered as a work basis that gives a clear and simple presentation of the common international practice of psychologists working in the field of palliative care. The framework, while reflecting French practices, could serve as a source of debate for psychologists from other countries and lead to the initiation of a new project that would take into account the diversity of practices in

Europe.

Collège des psychologues SFAP (2016). Référentiel des pratiques des psychologues en soins palliatifs. Journal des psychologues.

Jünger S, Payne S.A. (2011). Guidance on postgraduate education for Psychologists involved in Palliative Care. European Journal of Palliative Care, 18(5), 238-252.

Abstract number: P01-398

Abstract type: Poster

"We Will Stay in Touch" A Study and a Follow-up Casestudy (2018) on How the Language of Saying Goodbye- Impact the Griefproces for Young Children 1-2 Years After the Loss of a Parent

Jensen L.A., Gitte Juhl

Nordsjællands Hospital, Onkologisk \& Palliativ Afdeling, Frederikssund, Denmark Presenting author email address: leneeria@yahoo.dk

Background: Early loss of a parent leaves a child vulnerable. This palliative intervention seeks to relief both palliative patients and their children. An earlier study (us, 2016) showed positive results for the patients when writing their children a personal book with focus on the relation to the child.

Aims: By developing a "relational" language and rituals confirming the bond in the proces of saying goodbye, the aim is to strengthen the connection and the attashment, to increase resilience and prevent complicated griefreactions in children.

This intervention: contains different tools:

- the parent writing a book to the child, expresses the significant qualities of their relation and qualities of the child

- the parent expressing: "my love to you feels like." with concrete forms that represents the loveconnection
- the child wishing certain themes to be written about

- the parent and the child makes a common "story" which includes rituals (place, flower, music and artefact).

Methods: The data is collected through 5 empirical follow-up casestudies with bereaved children, exploring the significance around the rituals.

Results: The analysis shows the following results;

- professional facilitation supports the familymembers in the language of saying goodbye

- to begin the intervention in the early stage of the palliative proces strengthen the relational language of saying goodbye

- the rituals is meaningful and soothing for both parents and their children

- rituals can help regulate emotions on grief, loss and hopelessness.

Conclusion / Perspective: There seems to be a great need for psychological support in the proces of saying goodbye and in helping families developing a language that can strengthen the emotional bond, and letting the children "stay in touch" with the deceased. This study points that developing a "relational" language reduces the risk of complicated grief in children. An important perspective and a future research is what attashment-styles means for the griefproces.

Abstract number: P01-399

Abstract type: Poster

Parenteral Nutrition for Advanced Cancer Patients at End of Life: Patients' Attitudes and Motivations

Mathieu-Nicot F. ${ }^{1,2}$, Cornet C. ${ }^{1}$, Cretin E. ${ }^{1}$, Grodard G. ${ }^{1}$, Bouleuc C. ${ }^{3}$, Pazart L. ${ }^{1}$, Aubry R. ${ }^{1,4}$

1 University Hospital of Besançon, Clinical Investigation Center, Insem 1431, Besançon, France, '2University of Bourgogne Franche-Comté, Besançon, France, ${ }^{3}$ Institut Curie, Department of Supportive Care, Paris, France, ${ }^{4}$ University Hospital of Besançon, Department of Palliative Care, Besançon, France

Background: Eating is not just a biological need, it also has a symbolic meaning. In end of life, the introduction of Parenteral Nutrition (PN) for malnourished patients with advanced cancer is a recurring issue. Acceptance or refusal the PN at this stage of the disease seems less guided by evidence-based data than by subjective reasons.

Aims: Understand why a patient in end of life situation would accept or reject PN proposed within a randomized controlled trial.

Method: This qualitative study is ancillary to the "ALIM K" Randomized controlled trial (Efficacy of parental nutrition on quality of life deterioration-free survival among palliative cancer patients). Data were collected through semi-structured interviews, in 13 French centers with patients who accepted or refused PN and their relatives. Data were analyzed using thematic analysis.

Results: The acceptance or refusal of the PN often depends on the presence or absence of a relative: Most of patients who accepted PN have not relatives present in their care while patients who refused have relatives involved in their care. They want to keep mealtimes with their relatives. Patients associate eating by mouth to a "normal function" that gives them the feeling to be a "normal person". The PN would be then perceived as a foreign element in the body causing a real anxiety.

Conclusion: A qualitative approach is necessary to understand better the patients' points of view. Understand motivations for acceptance or refusal PN help us to improve the communication around alimentation. Mealtimes stay for most patients and their relatives a shared quality time that gives a meaning to life.

The project has been funding by the French National Cancer Institute (INCa) through PHRC 2011.

Abstract number: $\mathrm{P} 01-400$

Abstract type: Poster 


\section{Suffering at the End of Life: A Systematic Review}

Limonero J.T. ${ }^{1}$, Casellas-Grau A. ${ }^{2}$, Gómez-Romero M.J.1, ${ }^{1,3}$, Maté J. ${ }^{4}$, Mateo D. ${ }^{5}$, Cladellas-Pros R. ${ }^{1}$, Álvarez M. ${ }^{1}$, Montes-Hidalgo J. ${ }^{6}$, TomásSábado J. ${ }^{6}$, Gil F. $^{7}$

${ }^{1}$ Stress and Health Research Group, Faculty of Psychology, Universitat Autònoma de Barcelona, Bellaterra, Spain, ${ }^{2}$ Psychosocial Observatory in Cancer, Catalan Institute of Oncology, L'Hospitalet de Llobregat, Spain, ${ }^{3}$ Psychology Unit, Egarsat, Mutua Colaboradora con la Seguridad Social № 276, Terrassa, Spain, ${ }^{4}$ Psycho-Oncology Care Unit, Catalan Institute of Oncology, L'Hospitalet de Llobregat, Spain, ${ }^{5}$ Palliative Care Unit, Consorci Sanitari de Terrassa, Terrassa, Spain, ${ }^{6}$ Research and Scientific Communication Unit, Gimbernat University Schools, Univesritat Autònoma de Barcelona, Sant Cugat del Vallès, Spain, ${ }^{7}$ Psycho-oncology Unit, Psychosocial Observatory in Cancer, Catalan Institute of Oncology, L'Hospitalet de Llobregat, Spain

Background: There is currently a considerable amount of studies exploring suffering among patients with terminal illnesses. To date, no systematic reviews have been performed exploring which are the factors that trigger suffering at the end of life in patients diagnosed with chronic illnesses.

Aim: We aim to systematically explore, synthesize, and reach a consensus about those factors which trigger end-of-life suffering in patients diagnosed with advanced chronic illnesses.

Methods: The four major databases in the field (PsyclNFO, Pubmed, Web of Science, Scopus) were explored using relevant terms that enabled the access to relevant literature in accordance to the review focus: 'suffering', 'emotional distress', 'end-of-life', 'advanced illness' and 'terminal illness'. PRISMA guidelines were followed, screening the articles by relevance through their title, abstract, and full text.

Results: From the 1,978 results obtained from databases after removing duplicates, 105 articles finally met the inclusion criteria. Preliminary results show that the most relevant factors that generate suffering among patients at the end of life can be grouped in four big dimensions: physical (e.g. pain, fatigue, dyspnoea), psychological (e.g. fear, depression, hopelessness), social (e.g. family issues, physician-patient communication, isolation) and existential (e.g. unmet spiritual needs, religious fellowships, compromised dignity).

Conclusions: Patients with chronic illnesses at the end of their lives should be attended taking into account the four dimensions that comprise their suffering. Thus, it does not only include the control of their physical symptoms, but also meeting their social, psychological, and existential needs. Multidisciplinary professional teams, therefore, would provide an optimal attendance to these patients.

Acknowledgment:This study was supported by grant Number PSI201785134-R from the Spanish Ministry of Economy, Industry and Competitiveness (MINECO).

Abstract number: P01-401

Abstract type: Poster

Brief Engagement and Acceptance Coaching in Community and

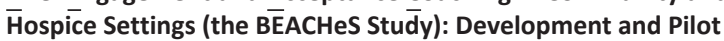
Testing an Evidence-based Intervention to Enhance Wellbeing at Transition into Palliative Care

Swash B. ${ }^{1}$, Norwood S. ${ }^{1}$, Gillanders D. ${ }^{2}$, Finucane A. ${ }^{3}$, Spiller J. ${ }^{3}$, Strachan ${ }^{3}{ }^{3}$, Kreft ${ }^{1} .{ }^{1}$, Millington S. ${ }^{1}$, Hulbert-Williams N. ${ }^{1}$

${ }^{1}$ University of Chester, Chester, United Kingdom, ${ }^{2}$ University of Edinburgh, Edinburgh, United Kingdom, ${ }^{3}$ Marie Curie, Edinburgh, United Kingdom

Background: The transition into palliative care can cause uncertainty, fear and distress, with detrimental effects on quality of life frequently reported. Acceptance and Commitment Therapy (ACT) is a promising intervention for supporting palliative patients, but specific evidence for its effectiveness in palliative cancer populations is limited.
Aims: The aim of this study is to develop and pilot test a brief ACT-based intervention to aid participants in their transition from curative cancer care to specialist palliative care. Our target outcomes are increased quality of life and psychological acceptance.

Method: A multiple-baseline, single-case, non-controlled design is used. We aim to recruit 10 to 20 participants to receive five one-to-one intervention sessions with a trained therapist. Quality of life, distress, and changes in ACT therapeutic processes are self-reported weekly. Acceptance is measured daily via a smartphone app. Qualitative interviews are offered to discuss experiences and acceptability of the intervention

Results. To date, five participants have consented. Three dropped-out due to ill health, leaving two participants at various stages of intervention. One has completed the entirety of the study. While the data show some floor and ceiling effects, an increase in psychological acceptance is shown through intervention, with a slight decrease at follow-up. Importantly, quality of life remained consistently high, despite daily variability in overall self-rated health.

Conclusion / Discussion: Single-case designs are not common in psychosocial oncology, however, they offer a scientific, data-driven approach to intervention development. This study will be completed by March 2019; data so far indicate that ACT may be a beneficial intervention framework for patients transitioning to palliative care. We aim to refine and manualise our intervention for use in future research and clinical services. Funding: This work is funded by Macmillan UK (Grant: 6488043)

Abstract number: P01-402

Abstract type: Poster

Implementation of a Psychosocial Assessment in Specialist Inpatient Palliative Care - Feasibility and First Results

Ullrich A., Bahloul S., Ahrenholz M., Coym A., Bokemeyer C., Oechsle K. Palliative Care Unit, Center of Oncology, University Medical Center Eppendorf, Hamburg, Germany

Background: Assessing patients' (pts) psychosocial burden is highly important for allocating appropriate support during specialist inpatient palliative care (SIPC).

Aims: Evaluating the feasibility of a psychosocial assessment implemented at a SIPC ward, results on pts' burden and its association with time provided by the palliative care team during SIPC (mean minutes/day).

Methods: Over a 1-year period, 428 consecutive pts of a SIPC ward (mean age 67 years, $58 \%$ male, $85 \%$ cancer) were eligible for assessment within 48 hours after admission. Assessment was performed by specialized psychologists/creative therapists using a standardized interview form, including screening for distress (DT), suspected depression (PHQ2) and anxiety (GAD2), and specific need items.

Results: Assessment was implemented in 339 pts (79\%), but could not be performed in 201 (59\%) cases most commonly due to cognitive impairments ( $n=58$ reduced vigilance, 36 disorientation, 23 other), pts' refusal (24) and symptom burden (17). Among 138 pts with completed assessments, $89 \%$ showed clinically relevant (DT $\geqslant 5$ ) and $52 \%$ severe $(\mathrm{DT} \geqslant 8)$ distress, $51 \%$ were suspect for depression (PHQ2 $\geqslant 3$ ) and $50 \%$ for anxiety $(G A D 2 \geqslant 3)$. About half of pts $(53 \%)$ expressed subjective need for psychosocial support. Correlation analyses showed more FC-directed support by nurses/physicians to be associated with pts' depression $\left(r_{s}=.183, p=.035\right)$. Receiving more care by psychologists/creative therapists was related to pts' depression $\left(r_{s}=.197, p=.023\right)$, anxiety $\left(r_{s}=.439, p<.001\right)$ and subjective need for psychosocial support ( $r s=.398$, $p<.001$ ). Higher extent of overall time spent by the SIPC team correlated with pts' severe distress $\left(r_{s}=.212, p=.014\right)$ and anxiety $\left(r_{s}=.297\right.$, $\mathrm{p}=.001$ ).

Conclusion: Distress, suspected depression and suspected anxiety were significant symptoms for more than half of the pts systematically screened. Results suggest barriers for systematic screening, but mainly its potential for adequate psychosocial care. 
Abstract number: P01-403

Abstract type: Poster

Distress, Anxiety, Depression, and Influencing Factors in Patients at Initiation of Specialist Palliative Care - Results from a Multicenter Study

Ullrich A. ${ }^{1}$, Schulz H. ${ }^{2}$, Goldbach S. ${ }^{3}$, Hollburg W. ${ }^{3}$, Rommel A. ${ }^{4}$, Müller

M. ${ }^{5}$, Kirsch D. ${ }^{6}$, Kopplin-Förtsch K. ${ }^{7}$, Messerer J. ${ }^{1}$, Schulz-Kindermann F. ${ }^{2}$, Bokemeyer C. ${ }^{1}$, Oechsle K. ${ }^{1}$

${ }^{1}$ Palliative Care Unit, Center of Oncology, University Medical Center Eppendorf, Hamburg, Germany, ${ }^{2}$ Department of Medical Psychology, University Medical Center Eppendorf, Hamburg, Germany, ${ }^{3}$ Specialist Outpatient Palliative Care Team, PalliativPartner Hamburg GbR, Hamburg, Germany, ${ }^{4}$ Specialist Outpatient Palliative Care Team, Das Palliativteam, Hamburg, Germany, ${ }^{5}$ Palliative Care Ward, Asklepios Hospital Rissen, Hamburg, Germany, ${ }^{6}$ Specialist Outpatient Palliative Care Team Hamburg - West, Hamburg, Germany, ${ }^{7}$ Palliative Care Ward, Agaplesion Diakonie Hospital, Hamburg, Germany

Background: Addressing psychological burden is a core component of specialist palliative care (SPC), but systematic data on its extent and influencing factors are rare.

Aims: Systematic screening for distress, suspect depression and anxiety in patients (pts) entering the local SPC network in an urban German area.

Methods: Within 12 months, all pts entering SPC were screened for study inclusion. Participants completed the Distress Thermometer, PHQ4, sociodemographic and care-related characteristics.

Results: 1713 pts entered SPC, whereof 990 fulfilled exclusion criteria (e.g. cognitive impairment), 280 refused participation and 18 failed to return the questionnaire. Of 425 included pts (mean age 69 years, $45 \%$ female, $75 \%$ cancer), 285 had entered outpatient and 140 inpatient SPC. Mean DT was 7.2 (SD 2.2; range, 0-10) with $89 \%$ indicating clinically relevant $(\geqslant 5)$ and $53 \%$ severe $(\geqslant 8)$ distress. Distress did not differ according to care situation with 7.2 in outpatient SPC $(90 \% \geqslant 5 ; 51 \% \geqslant 8)$ and $7.3(89 \% \geqslant 5 ; 56 \% \geqslant 8)$ in inpatient SPC (all $p \geqslant .05)$. Mean scores were 5.3 for PHQ4 (SD 3.7; range, 0-12), 3.0 for PHQ2 (SD 2.0; range, 0-6), and 2.4 for GAD2 (SD 2.0; range $0-6$ ) with $52 \%$ of pts being suspect for depression ( $\mathrm{PHQ} 2 \geqslant 3$ ) and $38 \%$ for anxiety (GAD2 $\geqslant 3$ ). For pts entering out-/inpatient SPC, rates were $48 \% / 53 \%$ for depression and 38\%/34\% for anxiety, again without showing group differences (all $p \geqslant .05$ ). Multivariate testing of potential influencing sociodemographic/carerelated factors (stepwise linear regression analyses) revealed childlessness ( $\beta=.836, p=.001$ ) as single predictor for higher distress, and younger age $(\beta=-.119, p=.029)$ and non-cancer disease $(\beta=.165, p=.002)$ for higher depression and anxiety.

Conclusion: Psychological burden is relevant in pts entering SPC independent of out- and inpatient care. Pts should be screened for psychological burden, e.g. using ultra-brief measures, DT and PHQ4, with special attention being paid to younger, childless and non-cancer pts.

\section{Abstract number: P01-404}

Abstract type: Poster

\section{"One Last Visit to my Homeland" - Patients Wishes at Initiation} of Specialist Palliative Care

Ullrich A. ${ }^{1}$, Schulz $H{ }^{2}$, Goldbach S. ${ }^{3}$, Hollburg W. ${ }^{3}$, Rommel A. ${ }^{4}$, Müller M. ${ }^{5}$, Kirsch D. ${ }^{6}$, Kopplin-Förtsch K. ${ }^{7}$, Messerer J. ${ }^{1}$, Schulz-Kindermann F. ${ }^{2}$, Bokemeyer C. ${ }^{1}$, Oechsle K. ${ }^{1}$

${ }^{1}$ Palliative Care Unit, Center of Oncology, University Medical Center Eppendorf, Hamburg, Germany, ${ }^{2}$ Department of Medical Psychology, University Medical Center Eppendorf, Hamburg, Germany, ${ }^{3}$ Specialist Outpatient Palliative Care Team, PalliativPartner Hamburg GbR, Hamburg, Germany, ${ }^{4}$ Specialist Outpatient Palliative Care Team, Das Palliativteam, Hamburg, Germany, ${ }^{5}$ Palliative Care Ward, Asklepios
Hospital Rissen, Hamburg, Germany, ${ }^{6}$ Specialist Outpatient Palliative Care Team Hamburg - West, Hamburg, Germany, ${ }^{7}$ Palliative Care Ward, Agaplesion Diakonie Hospital, Hamburg, Germany

Background: Knowledge of patients' (pts) wishes can help palliative care teams' understanding of what matters most to pts and serve as a guide in conducting goals-of-care conversations. Common wishes of palliative care pts have rarely been investigated.

Aims: This multicenter study investigated wishes among pts recently transferred to out- or inpatient specialist palliative care (SPC).

Methods: Within 72 hours after having entered SPC, 425 pts (mean age 69 years, $45 \%$ female, $75 \%$ cancer, $67 \%$ outpatient SPC) completed a survey. Of these, 361 responded to the question if they had a special wish, and, if any, to describe these. Listed items were analyzed using inductive qualitative content analysis to identify key themes of pts' wishes. All transcripts of whishes were coded for presence or absence of each of the nine themes identified.

Results: Of 361 pts, about 243 (67\%) had a special wish they desired to be accomplished. Themes of wishes were desire to travel or visit specific places in $31 \%$, to accomplish soothing or daring activities in $18 \%$, to live longer or regain health in $17 \%$, to achieve better quality of life in $15 \%$, to spend quality time with family and friends in $14 \%$, to experience conditions attributed to a "good death" in $6 \%$, to turn back time in $3 \%$, to solve things before dying in $2 \%$, and others in $3 \%$. Univariate logistic regression analyses revealed higher educational level, inpatient SPC, screening result of suspected anxiety, and higher numbers of practical, emotional and physical problems to be significantly associated with having a wish $(p<.001$ to .015). Multivariate analysis confirmed inpatient SPC $(p=.009)$ and higher numbers of physical problems $(p=.002)$ to increase the likelihood of having a wish.

Conclusion: Palliative care teams should consider asking about pts' wishes to provide preference-sensitive care. It could also be used as a starting point for a personalized approach in supporting pts' good quality of life and dying with dignity.

Abstract number: P01-405

Abstract type: Poster

\section{Efficacy of Yoga Therapy for Patients who Suffer from a Tumor} Disease

Jentschke E.

Universitätsklinikum Würzburg, Comprehensive Cancer Center, Würzburg, Germany

A tumor disease is bringing with it various burdens. Depending on the tumor type, its severety, duration as well as its treatment, those affected have to endure psychological and physical strains which are perceived individually.

For that reason it can be very helpful for tumor patients to consider apart from the usual therapy, furthermore complementary oncological methods like for example yoga therapy.

There is still to be remained a vast requirement for profound research concerning the efficacy of yoga therapy as complementary method. This is especially the case for Germany.

Within this study the following questions should be answered:

- What contribution can yoga do in the processs of coping with the disease?

- Can the practice of yoga help tumor patients to improve sypmtoms like anxiety, depression and fatigue?

- Which coping strategies do tumor patients use?

Method: The patients are consecutively admitted into the study and individually randomized to either the yoga therapy or the waiting control group. 
Included in this study were adults with a tumor disease who voluntarily signed up to take part in the study. The follow-up examination was taken in order to determine the sustainability of possible effects.

Results: So far there are 70 completed datasets of patients available. Yoga therapy improves anxiety compared to the waiting control group. Anxiety, depression and fatigue are less pronounced after 6 months than at baseline. Yoga should be offered to tumour patients to support them in coping with the disease.

Conclusions: Yoga therapy enables a better dealing with a tumor disease.

\section{Abstract number: P01-406 \\ Abstract type: Poster}

\section{Humor in Palliative Care - Preliminary Data}

Linge-Dahl L. ${ }^{1}$, Radbruch L. ${ }^{1,2}$

${ }^{1}$ Department of Palliative Medicine, University Hospital Bonn, Bonn, Germany, ${ }^{2}$ Malteser Hospital Seliger Gerhard, Bonn, Germany

Systematic literature reviews showed a lack of randomized controlled studies evaluating the effect of humor on patients being treated in palliative care units. So far most studies have been qualitative observational studies without control groups. This study evaluated the effects of a humor intervention by questionnaires on cheerfulness, life satisfaction, distress and burden of symptoms. Allocation to groups has been done by groupwise randomization. The humor intervention was a humorous visit from two humor coaches for 15-30 minutes using a multistage model based on the humor training of McGhee. This intervention has been recorded in observational protocols and protocols which have been completed by the humor coaches after the interventions. The control group did not receive an intervention. 27 out of the 70 Patients which have been included in the data so far were able to complete the questionnaires. In the intervention group cheerfulness increased after the intervention (MV 14.00 before and 14.56 after) and bad mood decreased (MV 13.43 before and 11.11 afterwards, $\alpha<0.05$ ). In the control group cheerfulness decreased (MV 13.33 before and 13.20 afterwards) and bad mood increased (MV 12.00 vs. 13.30) but those differences were not significant. Life satisfaction increased in the intervention and the control group and pain was reported less stronger in the second investigation also in both groups but the difference was not significant. The differences between measures and groups were quite small and can only be reported in the sense of means now. It needs to be considered, that most of the patients were not able to complete the questionnaires due to their burden of symptoms. The qualitative data might therefore provide a more detailed insight of the effects of the interventions. A bigger sample is required to get significant results and it needs to be considered that patients in palliative care have a very limited capacity to complete questionnaires and tests.

\section{Abstract number: P01-407 \\ Abstract type: Poster}

\section{Bringing Humour Therapy to Paediatric Palliative Care:}

Preliminary Results from a Scoping Review

Martins T..$^{1,2}$, Marques G. ${ }^{1,3}$, Pinto S. ${ }^{1,3}$

${ }^{1}$ Escola Superior de Saúde de Santa Maria, Porto, Portugal, 2Instituto de Educação, Universidade do Minho, Braga, Portugal, ${ }^{3}$ NursID, CINTESIS Center for Health Technology and Services Research, Porto, Portugal

Background: Humour Therapy (HT) has been considered an important intervention, particularly in paediatric settings, helping to relief suffering and to improve physical and spiritual well-being. Yet, the concept remains unclear and there is some reluctance to move forward with humour in contexts of Palliative Care (PC).

Aims: To develop a HT intervention for children in PC.
Methods: The method proposed by the Medical Research Council for the development and evaluation of complex interventions was used. The Intervention Design includes a Scoping review and a focus group study with experts on the topic (nurses, doctors, professional clowns). Then (Feasibility study), the intervention will be tested and improved in a quasi-experimental study, in a sample of children in PC settings (Pilot Study). Currently, the study protocol is under appreciation by an ethics committee. The Scoping Review was conducted on PubMed, with the query ((("humor"[Title/Abstract]) OR "laugher therapy"[Title/Abstract]) AND "paediatric*"[Title/Abstract]. Data were systematically collected and analysed by 3 researchers.

Results: 27 results were included. The preliminary data analysis suggests that HT is essential in the context of illness, not only for the child but also for their relatives, and healthcare providers. Humour as the potential to prevent and to relief suffering, particularly during invasive procedures, but it requires healthcare professional's education and training. However, very few information is available regarding the use of HT within Paediatric PC services.

Conclusions: HT must be considered in the context of human suffering. Traditionally, life-limiting illness are associated with negative emotions, and anxiety but the humour and the laugher can help to relief suffering, both for Childs, relatives and healthcare providers. Studies in other paediatric settings suggests that the improvement of $\mathrm{HT}$ in PC can bring important benefits.

\section{Abstract number: P01-408 \\ Abstract type: Poster}

Association between Depressive Disorder and Clinical Factors in Mexican Patients with Advanced Cancer in Palliative Care

\section{Service}

Rodríguez-Mayoral $O{ }^{1}$, Rodríguez-Ortíz B. ${ }^{2}$, Peña-Nieves A. ${ }^{1}$, MonrealCarrillo E. ${ }^{1}$, Allende-Pérez S. ${ }^{1}$

${ }_{1}^{1}$ Palliative Care Service, Instituto Nacional de Cancerología, Mexico City, Mexico, ${ }^{2}$ Mental Health, Clínica Especializada Condesa-Iztapalapa 'Dr. Jaime Sepúlveda Amor', Mexico City, Mexico

Introduction: Depressive disorder (DD) is one of the most common mental disorders in palliative advanced cancer patients. It has been one of the most underdiagnosed and untreated disorders in patients at the end of life, as well as the most important factor for the presence of desire to hasten death, a lower functional status, survival and quality of life, a poor therapeutic adherence and worst intensity of physical symptoms.

Objective: To identify the relation of the clinical factors and the presence of depressive disorder.

Methods: We conducted a prospective, cross-sectional and observational study. Patients treated at the palliative care service of the National Institute of Cancerology in Mexico City were included; who agreed to participate in the study and did not have cognitive impairment or treatment with antidepressants. Among the clinical factors used were the intensity of physical symptoms (ESAS), functionality (ECOG) and survival clinical prediction (PPI), then they underwent a semi-structured clinical interview according to the DSM- 5 criteria for the major depressive disorder by a psychiatrist. Depression was defined as a dichotomous variable based on the presence or absence. Univariate and multivariate analyzes were performed.

Results: One hundred patients were included, of them, 69 were women, the median age was 57 years. Twenty-four patients met the criteria for DD. In the univariate analysis, the patients with DD presented a significant more intensity of physical symptoms, lower functionality, and poorer survival clinical prediction, compared to patients without DD. In the multivariate analysis, pain ( $p=0.05, O R 1.266$ ) and constipation ( $p$ $=0.014$, OR 1.344) were significant.

Conclusion: The depressive disorder is associated with a higher intensity of physical symptoms, lower functionality and poorer survival clinical 
prediction. Strategies for the diagnosis and treatment of depressive disorder in patients and palliative care services are required.

Abstract number: P01-409

Abstract type: Poster

Prevalence and Predictors of Burnout and Psychological Distress among Physicians in Specialty Training in Palliative Care: A Japanese Nationwide Study

Matsumoto Y. ${ }^{1}$, Uehara.$^{1,}{ }^{1,2}$, Mizushima A. ${ }^{2}$, Nozato J. ${ }^{3}$, Miyamoto S. ${ }^{4}$, Mori M. ${ }^{5}$, Nishi T. ${ }^{6}$, Kizawa Y. ${ }^{7}$, Morita $T^{8}$

${ }^{1}$ Department of Palliative Medicine, National Cancer Center Hospital East, Kashiwa, Japan, ${ }^{2}$ Department of Palliative Medicine, Juntendo University School of Medicine, Tokyo, Japan, ${ }^{3}$ Department of Cancer Center, Tokyo Medical and Dental University, Tokyo, Japan, ${ }^{4}$ Department of Medical Oncology, Japanese Red Cross Medical Center, Tokyo, Japan, ${ }^{5}$ Palliative Care Team, Seirei Mikatahara General Hospital, Hamamatsu, Japan, ${ }^{6}$ Kawasaki Comprehensive Care Center, Kawasaki Municipal Ida Hospital, Kawasaki, Japan, ${ }^{7}$ Department of Palliative Medicine, Kobe University Graduate School of Medicine, Kobe, Japan, ${ }^{8}$ Palliative and Supportive Care Division, Seirei Mikatahara General Hospital, Hamamatsu, Japan

Background: Previous researches have reported high rate of burnout and psychological distress in health care providers. Because palliative care physicians are placed under a stressful environment where they routinely face patients with significant suffering, we should pay attention to their burnout and psychological distress, especially among younger physicians who may have burnout more easily than older physicians.

Aims: To clarify the prevalence of burnout and psychological distress among physicians in specialty training in palliative care, and to explore predictors of burnout and psychological distress.

Methods: Using a double-envelope method, we sent participation requests with questionnaires for distribution to eligible physicians in specialty training in palliative care, working in the institutes which were certified for palliative care training by the Japanese Society for Palliative Medicine (total number of institutions: 735 in October 2013). Participants were asked to complete the Maslach Burnout Inventory (MBI), K6, participants' characteristics and unmet learning needs. High levels of burnout and psychological distress were identified using cut-off scores of the $\mathrm{MBI}($ emotional exhaustion [EE, $\geqq 27$ ], depersonalization [DP, $\geqq 10]$ and personal accomplishment [PA, $\leqq 33]$ ) and $K 6(\geqq 5)$, respectively.

Results: In total, 253 of 735 institutions (34\%) responded; of 284 physicians, 253 (89\%) responded and 229 were eligible. Overall, $69.4 \%$ of the respondents had burnout: $19.2 \%$ with high $\mathrm{EE} ; 7.4 \%$ with high DP; and $65.9 \%$ with low PA. Thirty percent had psychological distress. In multivariate analysis, high EE, high DP and low PA were significantly associated with psychological distress (Odds Ratio [95\% confidence intervals] of 9.3 [4.4-19.5], 6.3 [2.1-18.8] and 4.0 [1.8-8.6]; $P<0.001, P=0.001$ and $\mathrm{P}=0.001$, respectively).

Conclusion: The majority of physicians in specialty training in palliative care suffered from burnout, which may lead to their psychological distress.

\section{Abstract number: P01-410 \\ Abstract type: Poster}

Crustative Care: A New, Palliative Approach towards Patients with a Severe and Persistent Mental Illness

Moureau L.

KULeuven, Wetteren, Belgium

The crossroads between palliative care and psychiatry are a very interesting though under- researched topic. Several patient groups may be distinguished: people with a mental illness developing a severe somatic illness, palliative patients developing psychiatric symptoms and, e.g. in Belgium, those patients with a severe mental illness 'legally' requesting euthanasia. Another specific, difficult group are those patients with a severe persistent mental illness (SPMI). Our aim is to explore the needs and challenges regarding this last group.

A literature review indicates that these people often underwent years of futile treatment and are at risk of permanent over-treatment or neglect. For them, there are de facto no valid treatment options left. Two observational/explorative studies on the field in Belgium show that most of these SPMI patients possibly end up in care units with fewer and less trained staff, even though their needs are very substantial.

For this group, the recovery approach, which has made a great contribution in mental healthcare with its focus on empowerment, is insufficient by itself. A radical holistic approach with a focus on quality of life and the relief of suffering, rather than continuous treatment, is paramount: 'crustative care' or 'shell care'. A conceptual analysis of this approach shows that it aims to develop a protective, palliative framework for SPMI patients, providing structure in daily life, with high regard for existential questions, attentiveness towards (the acceptance of) loss in all its aspects and qualitative somatic treatment.

By raising awareness in both academic and political circles, 'crustative' care wants to enhance research towards the needs of this group and plead for adequate funds to provide for high-quality care. Also, research is needed regarding the possibilities of a palliative care approach as a valid alternative for the growing number of patients with a death-wish based on unbearable mental suffering.

Abstract number: P01-411

Abstract type: Poster

Animal-assisted Therapy at a University Centre for Palliative Medicine: A Qualitative Interview Study to Review the Efficacy and Feasibility from the Patients' Point of View

Schmitz A. ${ }^{1}$, Beermann M. ${ }^{2}$, MacKenzie C. ${ }^{3}$, Schulz-Quach C. ${ }^{4,5}$ ${ }^{1}$ LVR Clinic of Psychiatry, Psychosomatic and Psychotherapy for Children and Adolescence, Viersen, Germany, ${ }^{2}$ Interdisciplinary Centre for Palliative Medicine, Heinrich Heine University Hospital Dusseldorf, Dusseldorf, Germany, ${ }^{3}$ Institute of Medical Microbiology and Hospital Hygiene, University Hospital, Heinrich Heine University Dusseldorf, Dusseldorf, Germany, ${ }^{4}$ St Christopher's Hospice, London, United Kingdom, Institute of Psychiatry, Psychology and Neuroscience, Department of Psychological Medicine, King's College, London, United Kingdom

Background: A program of Animal-assisted therapy (AAT) was started in June 2014 at the Interdisciplinary Centre for Palliative Medicine of the University Hospital Dusseldorf, Germany, performed by a trained and certified dog-assisted therapy team. To date only limited scientific data is available with regard to feasibility and efficacy of AAT in palliative care. Aims: The present qualitative interview study aims to describe the efficacy and feasibility of AAT from the patients' point of view.

Methods: This study is a qualitative interview study performed from December 2015 through June 2016. Semi-structured, guided interviews were conducted the day after the patient received the first AAT. Data was transcribed verbatim and analysed according to Mayrings approach of qualitative content analysis with an inductive coding process. Data from the electronic patient record was also analysed. The report followed the recommendations of the consolidated criteria for reporting qualitative research (COREQ).

Results: A total of 21 patients were included in the study, of which 14 were able to be interviewed. Seven patients had either died or were cognitively incapable to be interviewed. Eight main categories were established with a total of 186 codes. Distraction was a key category with subcategories such as Distraction from the disease, Self-efficacy, and Engaging of the patient. Other key categories were Feeling of safety and comfort, with the subcategories Reduction of loneliness, Trust and 
closeness, and Calm space, and Feasibility with the leading subcategories of Patient hygiene, and Welfare of the dog.

Conclusion: AAT may constitute a valuable and practical adjunct to the interdisciplinary therapeutic interventions of palliative care in the hospital setting. The results of this study suggest that patients may benefit from AAT particularly in terms of Distraction and Feeling of safety and comfort. These initial results will need to be followed-up by more robust study designs.

\section{Abstract number: P01-412 \\ Abstract type: Poster}

Development of a Mindfulness Integrated Cognitive Behavioural Intervention Program for Cancer Palliative Care Professionals in India

Kaur A. ${ }^{1}$, Sharma M.P. ${ }^{2}$, Chaturvedi S.K. ${ }^{3}$

${ }^{1}$ Mental Health, George Institute for Global Health India, New Delhi, India, ${ }^{2}$ Clinical Psychology, National Institute of Mental Health and Neuro Sciences, Bengaluru, India, ${ }^{3}$ Psychiatry, National Institute of Mental Health and Neuro Sciences, Bengaluru, India

Being a cancer palliative care professional is both challenging and stressful. There has been very less work done on developing practical need based interventions based on challenges perceived by this population. Mindfulness has been used successfully as a therapy alone as well as an adjunct to other techniques. It would be fruitful to develop a need based mindfulness integrated intervention for cancer palliative care professionals working in India. The aim of the study was to formulate the components of Mindfulness Integrated Cognitive Behavioural Intervention program based on the felt needs and professional quality of life of cancer palliative care professionals in India.

The study adopted a mixed-methods cross-sectional design to explore felt needs and professional quality of life. Permissions were obtained from the four cancer palliative care centres and ethical clearance was obtained from the Institute Ethics Committee (Behavioural Sciences) NIMHANS, Bengaluru. A total of 65 participants gave written informed consent for participation in the study and 15 participants gave an additional consent for the semi-structured interviews. Semi-Structured Interview Schedule for Palliative Care Professionals and Professional Quality of Life Scale Version 5 were used as measures. Based on emerging themes using thematic analysis, the components for Mindfulness Integrated Cognitive Behavioural Intervention were formulated.

The cancer palliative care professionals in the present study reported high secondary traumatic stress, moderate to high burnout, and moderate to high compassion satisfaction while the emergent themes were: death\& grief, challenges in practice, self-care strategies, and positive professional experiences. 6 session MICBI program was developed which could address grief, professional quality of life, stress inducing thoughts, self-care, cultivating kindness and compassion at workplace using mindfulness meditation.

\section{Abstract number: $\mathrm{P} 01-413$}

Abstract type: Poster

\section{The Psychological Experience of the Pediatric Oncological Patient in Front of Death: Systematic Review with Narrative Synthesis} Comas-Carbonell E. ${ }^{1}$, Mateo-Ortega D. ${ }^{2}$, Busquets E. ${ }^{3}$

${ }^{1}$ Departament de Ciències Socials i del Benestar, Universitat de VicUniversitat Central de Catalunya, Vic, Spain, ${ }^{2}$ Palliative Care, Consorci Sanitari de Terrassa, Terrassa, Spain, ${ }^{3}$ Ciències de la Salut i el Benestar, Universitat de Vic-Universitat Central de Catalunya, Vic, Spain

Background: The psychological experience of oncological patients in front death is a key issue for adequate psychological care in pediatric palliative care. It is not known how this situation is experienced for the patient and which support he might need.

Aim: Identify scientific evidence about the psychological experience of pediatric cancer patients in front of death:

(1) synthesize the evidence to describe the key elements of the experience and the corresponding needs, and

(2) identify gaps in research.

Design: A narrative systematic review of qualitative, quantitative and mixed-methods research.

Data sources: The databases SCOPUS, WEB OF SCIENCE, PUBMED and PsycINFO were searched from 1986 to July 2018 for publications on the psychological experience of pediatric cancer patients (6-18 years) in front of death. The risk of bias of the articles was assessed with the 'Critical Appraisal Skills Program'.

Results: The search strategy identified 3.528 articles, and twenty of them were included in the review. The oncological pediatric patient confronting death experiences: changes in relationships, thoughts about death, emotional changes, physical symptoms that cause suffering, changes at the spiritual level, and feelings of uncertainty.

Conclusions: The pediatric patient needs to be treated normally, to be informed or involved in treatment decisions, and to have the possibility of shearing thoughts related to death whenever he or she wishes. Hope should be fostered through emotional and spiritual support to reduce uncertainty. Future research should focus on the perspective of pediatric patients, especially at ages 7 to 12 years.

Abstract number: P01-414

Abstract type: Poster

Posttraumatic Growth in Palliative Care

Bernard M., Althaus B., Borasio G.D.

Palliative and Supportive Care Service, Lausanne University Hospital, Lausanne, Switzerland

Background: Psychology research in palliative care is overwhelmingly concerned with the reduction of psychological distress. Little is known about positive psychological factors influencing the wellbeing of patients. We therefore conducted a cross-sectional study to evaluate the association between such positive factors, quality of life (QOL), and distress. Among these factors, posttraumatic growth (PTG) refers to positive changes experienced as the result of a struggle with a traumatic event, such as a life-threatening illness.

Aims: The aims are to evaluate:

(i) the proportion of patients with a PTG,

(ii) which areas of the PTG are most salient and

(ii) the link between PTG, QOL and distress.

Methods: Patients were recruited in a Swiss university hospital. Validated questionnaires were used to measure QOL (McGill Quality of Life Questionnaire Revised), gratitude (Gratitude Questionnaire 6 items, GQ6), psychological distress (Hospital Anxiety and Depression Scale, HADS), and PTG (Posttraumatic Growth Inventory, PTGI). Descriptive analyses and Pearson correlations were performed.

Results: 64 patients participated ( 30 men, mean age $=67$ ). $82 \%$ of patients reported at least a moderate PTG (PTGI total score $\geqslant 45$, [possible range 0-105]. The highest scores were observed in the areas "relating to others" (M=23.4, [0-35]), and "appreciation of life" of the PTGI ( $m=10.6$, [0-15]). "Personal strength" $(M=11.4$, [0-20]), "new possibilities in life" ( $m=12.5,[0-25])$ and "spirituality" (M=4, [0-10]) scored lower. PTGI total score was significantly associated with the depression scale $(r=-.47)$, the total score of the HADS ( $r=-.33)$, and with the GQ- 6 $(r=.40)$. 
Conclusion: PTG occurs frequently in the end of life context, particularly in areas oriented towards the "outside world", which is confirmed by the association with gratitude, defined as an other-oriented emotion. The ambivalent link with the HADS confirms the possibility of a co-existence between PTG and distress and will be discussed.

\section{Abstract number: P01-415 \\ Abstract type: Poster}

The Expression of Hope in Cancer Patients when Engaged in Advance Care Planning: Experiences from the ACTION Study Kodba-Čeh H. ${ }^{1}$, Lunder U. ${ }^{1}$, Bulli F. ${ }^{2}$, Caswell G. ${ }^{3}$, Christensen C.A. ${ }^{4}$, Kars M.C. ${ }^{5}$, Miccinesi G. ${ }^{2}$, Toccafondi A. ${ }^{2}$, Zwakman M. ${ }^{5}$, Preston N. ${ }^{6}$, Rietjens J.A.C. ${ }^{7}$, Pollock K. ${ }^{3}$

${ }^{1}$ Research Department, University Clinic of Respiratory and Allergic Diseases Golnik, Golnik, Slovenia, ${ }^{2}$ Oncological Network, Prevention and Research Institute (ISPRO), Florence, Italy, ${ }^{3}$ Faculty of Medicine and Health Sciences, School of Health Sciences, University of Nottingham, Nottingham, United Kingdom, ${ }^{4}$ Department of Public Health, University of Copenhagen, Copenhagen, Denmark, ${ }^{5}$ Julius Center for Health Sciences and Primary Care, University Medical Center Utrecht, Utrecht, Netherlands, 6 International Observatory on End of Life Care, Lancaster University, Lancaster, United Kingdom, ${ }^{7}$ Department of Public Health, Erasmus University Medical Center, Rotterdam, Netherlands

Background: Concerns of health care professionals that advance care planning (ACP) may diminish hope in patients, usually referring to hope for cure or longevity, is a recognized barrier in ACP initiation. As part of the cluster randomized clinical trial ACTION, a qualitative study explored the perspectives of patients with advanced colorectal or lung cancer and their personal representative (PR) of the ACTION Respecting Choices ACP intervention. This sub-study reports on the expression of hope in relation to the intervention as one of the secondary objectives of a qualitative study.

Aim: To observe hope in patients with advanced cancer involved in ACP. Methods: 23 participants (11 patients \& 12 PRs) from Italy, the Netherlands, Slovenia and the UK were interviewed with no specific focus on hope over 14 weeks after the intervention. A predefined framework based on previously published facets of hope in terminal illness was used to identify relevant data segments. Thematic analysis was then performed.

Results: Participants expressed continuing hopes and expectations for a possible cure or longevity after engaging in the ACP process. These hopes shaped their daily focus (e.g. continue normal life) and engagement in ACP (e.g. if prognosis was poorly understood, ACP was perceived as personally less relevant). Also, ACP had the potential to support hopefulness in an individualized manner through enhancing insight and acceptance of one's limits and possibilities, aspects of control and relationships.

Conclusion: Patients and their PR can simultaneously discuss ACP issues and maintain their hopes for a cure or longevity. Therefore, our results do not support clinicians concerns about disrupting such hopes by discussing end-of-life issues. Additionally, ACP might positively enhance hope through exploring and discussing possibilities for the future. Funding: EU 7th framework

Abstract number: P01-416

Abstract type: Poster

Psychosocial Issues among Adolescents and Young Adults with Sarcoma. The Need of an Interventional Assessment at Greek Hospitals

Dede M. ${ }^{1,2}$, Liakopoulou P. ${ }^{3}$, Mitsimponas N. ${ }^{4}$, Prevezanou M. ${ }^{5}$ ${ }^{1} 1$ st Department of Medical Oncology, Saint Savvas Hospital, Athens, Greece, ${ }^{2}$ Cancer, Education, Research and Clinical Practice Company,
Athens, Greece, '3House for Cancer Patients 'I.D. Kritikos \& E.F. Kritikou', NGO Pnoi Agapis, Athens, Greece, ${ }^{4} 1$ st Oncology Department, Hygeia Hospital, Athens, Greece, ${ }^{5} 2$ nd Department of Internal Medicine, Attikon University Hospital, Athens, Greece

Background: Adolescents and young adults (AYAs) with sarcoma constitute a separate age group between 15-39 years old, who face unique medical, physical, psychosocial, and supportive care.

Aims: The purpose of this review was to identify AYAs' with sarcoma perceptions and expectations of cancer care services before, during and after the treatment and key psychosocial factors impacting quality of life (QoL) at this category.

Methods:.A systematic review was conducted in Greek and English literature (PubMed, Medline) from 2000 to 2018, about studies published or in development referred at psychosocial needs, perceptions and QoL of AYAs with sarcoma. Based on inclusion criteria defined a priori titles, abstracts and full texts were analysed to find relevant studies.

Results: A total of 1519 articles were found with "cancer" reference while 284 related on patients with sarcoma. Ten (10) articles were found that met inclusion criteria. Other relevant articles were tracked through systematic reviews and a further eight (8) studies were included. At the end of the data analysis process, 10 main themes were identified: physical burdens, future prospects, isolation, guilt, mortality, functional repercussion of the disease, creating a positive attitude, healthcare, online social interaction, and survivorship.

Conclusions: Few data exist concerning intellectual and other psychosocial issues for this specific patient population. We consider that it is a major importance of all professionals to assess and record AYAs' with sarcoma perceptions about care services from the time of diagnosis to Survivorship or Palliative Care and End-of-Life. We also suggest the need of specialized programs in hospitals that will comprise a multidisciplinary team. Last but not least the construction of specialized centers for AYAs with sarcoma would help this population in a smooth reintegration into society without feeling marginalized and the promotion of a Survivorship philosophy in Greece.

\section{Abstract number: P01-417 \\ Abstract type: Poster}

Existential Suffering in Palliative Care: A Systematically Conducted Scoping Review

Lucas $\mathrm{H}$.

Instituto S. João de Deus (OHSJD), Palliative Care Unit, Lisboa, Portugal Presenting author email address: hugo.lucas@isjd.pt

Background: The term "existential suffering" (ES), commonly found in the scientific literature on palliative care (PC), has been used to describe a broad spectrum of psychological disorders. It is defined as loss of meaning or lack of clarity in the value of life.

Aims: To identify and analyze the different definitions of ES; to identify and describe the intervention strategies in ES.

Method: A scoping review (PRISMA criteria following Arksey and O'Malley's framework) of published articles using PubMed, MEDLINE, EBSCO-Host, ScienceDirect, PsycINFO, Cochrane and Embase with the search terms: "existential suffering", "existential suffering and palliative care", "existential distress" and "existential distress and palliative care". Selected articles include both systematic reviews and original articles. Results: The search resulted in 14660 articles, of which 23 met the inclusion criteria by addressing a definition of ES and intervention strategies. This review led us to an integrative and comprehensive view of the nature of the construct of ES in PC, in which the following domains were identified: loss of meaning/purpose of life; feeling of hopelessness/ despondency; loss of autonomy or freedom; feeling of burden to others and self-rupture. There are also different intervention strategies in the context of ES in PC. 
Conclusion: There is no generally accepted definition of ES. An association of concepts, such as "existential distress", "demoralization syndrome" and "total pain", is evident. "Spiritual suffering" must be differentiated from ES because is part of this and not a synonym. Some of the most relevant intervention strategies focus on: meaning-centered psychotherapy; dignity therapy and supportive expressive group therapy. This review is important for palliative care professionals caring for end-of-life patients and their families by clarifying and standardizing the concept of ES. Further research is needed to develop effective strategies to address $\mathrm{ES}$ in $\mathrm{PC}$.

\section{Abstract number: P01-418 \\ Abstract type: Poster}

\section{The Role of Volunteers in Quality Palliative Care Delivery} Manna A.

Narikeldaha Prayas, Purba Medinipur, India

Introduction: Here in India almost $75 \%$ of cancer patient die a sad death of neglect due to lack of awareness about palliative care and low economic level. Surveys in India show that two third of cancer patient do not get proper care during the terminal phase of their life. Palliative care through volunteers can make a significant difference in this respect.

Objective: To identify and try to solve, to the extent possible, the main difficulties in giving palliative care to the terminal cancer patients of the area. And evaluate the impact of volunteer's direct care of palliative patients and their families.

Methods: Feedback from patients and their relatives regarding the palliative care they receive from nursing home and from volunteers and compare the two. Also feedback from volunteers regarding their positive and negative experience while delivering palliative care service. Then evaluate the data to compare and improve the quality of service.

Results: We carried out two studies. One study was undertaken in nursing home palliative care and another was in home setting by volunteers. Both studies were in adult palliative care services. Since January 2016, 416 cases were studied to enquire about their experience in both home based care and nursing home care. Both the studies fulfilled our quality appraisal criteria. One found that those families and patients who received home visits from volunteers were significantly more satisfied. The study highlighted the value of the role of volunteers in better satisfaction of patients and their families.

Conclusions: Further research is needed to evaluate the role of volunteers in palliative care and how it can be delivered appropriately and effectively. We also wish to compare our findings with similar studies elsewhere.

Abstract number: P01-420

Abstract type: Poster

\section{The Perspectives on Volunteer-professional Collaboration in} Palliative Care: A Qualitative Study

Vanderstichelen S. ${ }^{1}$, Cohen J. ${ }^{1}$, Van Wesemael Y. ${ }^{2}$, Deliens L. ${ }^{1,3}$, Chambaere K. $^{1}$

${ }^{1}$ Faculty of Medicine and Pharmacy, End-of-Life Care Research Group at the Vrije Universiteit Brussel and Ghent University, Brussel, Belgium, ${ }^{2}$ Palliabru, Brussels, Belgium, ${ }^{3}$ Department of Public Health and Primary Care, Ghent University, Ghent, Belgium

Background: Governments intend to meet resource constraints in professional healthcare by stimulating informal care, including volunteerism. However, very little is known on how volunteers are to be integrated into care, and about current volunteer-professional collaboration. Such insights are relevant for future policy development regarding volunteer efficiency, quality of care and the capacity of volunteer care in supporting services and professionals.
Aim: To explore what constitutes volunteer-professional collaboration around palliative care.

Method: A qualitative study was conducted using semi-structured focus groups with volunteers, nurses, psychologists and family physicians and interviews with patients and family caregivers. Participants were recruited from hospital, home, day care and live-in services.

Results: 79 people participated in the study. Volunteers collaborate most with nurses, less with psychologists, and not with physicians. Volunteer-professional collaboration entailed mutual information-sharing regarding patient conditions and coordinating care provision together, while nurses and psychologists provide emotional and functional support for volunteers. Lack of volunteer-nurse contact, of communication channels, of leadership and of patient-information sharing guidelines were the most prominent barriers to collaboration.

Conclusion: Volunteers are at the frontline of palliative care provision and therefore collaborate intensely with nursing staff, particularly in dedicated palliative care services. However, volunteer collaboration with other professionals in care provision was absent. The presence and availability of nurses was found to be crucial for volunteers, both in their daily functioning as for the realisation of volunteer integration through collaboration.

Funding: was provided by the Agency for Innovation through Science and Technology (I.W.T.) as part of the INTEGRATE-project. [IWT-SBO 140009]

\section{Abstract number: P01-421}

Abstract type: Poster

Volunteers, Family Caregivers and Co-production in End of Life Care - A Big Ask? Exploring Potential Hurdles Using Action Research in the Development of Cottage Hospice

Hughes S. ${ }^{1}$, Barnes H. ${ }^{1}$, Turner M. ${ }^{2}$, Walshe $C .{ }^{1}$

${ }^{1}$ International Observatory on End of Life Care, Lancaster University, Lancaster, United Kingdom, ${ }^{2}$ University of Huddersfield, Huddersfield, United Kingdom

Background: Cottage Hospice is a new model of end-of-life service where care will be provided by family members and volunteers. Volunteers will multi-task, doing domestic cleaning and providing personal care - also overnight. Family caregivers are asked to sign an agreement to provide care and remain on the premises. While these roles offer greater involvement and control, they also represent a significant increase in (unpaid) responsibility and commitment.

Aims: To use participatory action research (PAR) to evaluate the development of Cottage Hospice from inception to opening and to disseminate the knowledge gained to inform service innovation in the sector.

Methods: An embedded researcher implemented a PAR approach in a UK hospice over 29 months. Documentary analysis was conducted using strategic records, internal meeting minutes, press releases and other written materials $(n=120+)$. Semi-structured interviews (participants: $n=36$ ) captured stakeholder views on process and progress over time. A series of action cycles led by a Core Action Group identified key areas for solution focussed change. A patient and carer advisory panel contributed to data analysis.

Results: The research identified enthusiasm and support for the increased autonomy Cottage Hospice offered patients and family caregivers in the dying phase. Concerns about the level of volunteering commitment anticipated and how sustainable this might be for individuals and their wider family were expressed. Views varied on how appealing volunteer roles would be and whether sufficient people with the appropriate skills and attitudes would come forward.

Conclusion: Delivering this innovative model of hospice care is highly contingent upon the capacity and willingness of family members and volunteers to provide hands-on care in the final days and weeks of life. It remains to be seen to what extent the ambitious scale of co-production 
currently envisaged will be embraced by service users and the wider local community.

Abstract number: P01-422

Abstract type: Poster

Political Affiliation and Population Views on the Role of Family and Volunteer Carers to Supplement State Provision in End of Life Care in England

Verne J. ${ }^{1}$, Tapp A. ${ }^{2}$, Nancarrow C. ${ }^{2}$, Morey Y. ${ }^{2}$, Warren S. ${ }^{2}$

${ }^{1}$ Public Health England, Bristol, United Kingdom, ${ }^{2}$ Faculty of Business and Law, University of the West of England, Bristol, United Kingdom

Background: In England politicians along the political spectrum promote volunteering but for different ideological reasons. It is clear that with the aging population, state end of life care (SEOLC) will need to be supplemented by family and volunteer carers. A qualitative study of people's views on the role of volunteers to fill gaps in state provision of end of life care services elicited anger, especially from labour supporters who felt the state should provide.

Aim: To investigate whether declared party political affiliation influences views on supplementary family and volunteer EOLC in addition to state provision.

Methods: An online panel survey of 3,590 adults in England aged 45 years and over through YouGov, purposely sampled to be representative of the population of England with booster samples of those aged 70-79 and $80+$ to permit reliable analysis of these groups. The boosters were weighted to ensure the total sample picture was nationally representative on key demographics for adults in England aged 45+. The online panel survey minimised the bias of socially desirable responding (no interviewers present) and question formats used face-saving techniques. Main political affiliations: Conservatives (C), UKIP (U), Labour (L), Liberal Democrat (LD).

Results: Conservatives (76\%) followed by LD $(72 \%)$ were more likely to think a volunteer model was a good idea compared with UKIP (69\%) Labour (63\%). Labour supporters (65\%) were most likely to think the volunteer model should not be necessary and the state should provide EOLC compared with U (54\%), LD (50\%), C (41\%). Conservatives (56\%) were most likely to think that family should fill the gaps in state EOLC provision compared with U (46\%), LD (44\%), L (41\%).

Conclusions: Political views on volunteering in EOLC are important

a) in how to influence policy makers, dependent on ruling party and

b) how to promote idea to population as messaging may have to be segmented and tailored to their political affiliation.

Abstract number: P01-423

Abstract type: Poster

Volunteers in Palliative Care in Nursing Homes - Providing Quality of Life for Residents

Stølen K. ${ }^{1}$, Raunkiær M. ${ }^{2}$, Frederiksen J. ${ }^{3}$, Jacobsen M.H. ${ }^{4}$

${ }^{1}$ Department of Nursing and Nutrition, University College Copenhagen, Græsted, Denmark, ${ }^{2}$ Department of Clinical Research, SDU, Knowledge Center for Rehabilitation and Palliative Care, REHPA, Copenhagen, Denmark, ${ }^{3}$ Department of Nursing and Nutrition, University College Copenhagen, Copenhagen, Denmark, ${ }^{4}$ Institute for Sociology and Social Work, Aalborg University, Aalborg, Denmark

Background and aims: Volunteers work in palliative care among seriously ill or dying people in nursing homes. Studies and practice indicate that the volunteers attend to tasks and offer services for the nursing home residents and their families that extend beyond or supplement palliative care provided by professionals. The aim of this study is to describe the volunteer activities in a nursing home and how these activities contribute to quality of life for nursing home residents with palliative needs and their families. The study is part of a PhD thesis.

Methods: A qualitative design was used: participation observations of the volunteers when they had tasks in the nursing home, supplemented with individual interviews with volunteers, residents, relatives and professionals. Three different nursing homes, with 70-80 volunteers engaged, were included and 80 hours of participant observations were completed. 11 individual follow up interviews took place: 5 volunteers, 2 residents, 2 relatives and 2 professionals. A thematic content analysis was used.

Results: The volunteers established and provided social fellowships related to for example music, culture, food, nature experiences and physical movement. The activities seemed to contribute to the quality of life for the residents. The activities offered something different from what the professional palliative care could offer, but was limited to those who could come to the common room in the nursing home. The voluntary commitment depended on management and cooperation and recognition from professionals and other volunteers was essential.

Conclusions: The findings showed that volunteer activities are characterized by great diversity and contribute to quality of life for the resident and supplement the palliative care in nursing homes. The activities benefit the resident most when the resident is still mobile. Volunteering demands management, cooperation and recognition. Provisions for the families was unclear.

\section{Abstract number: P01-424 \\ Abstract type: Poster}

\section{Volunteers in End-of-Life Care for Non-oncological Patients - Are} There Distinctions?

Deiseroth L. ${ }^{1}$, Marx G. ${ }^{2}$, Jansky M. ${ }^{1}$, Banse C. $^{1}$, Heiß K. ${ }^{1}$, Nauck F. ${ }^{1}$

${ }^{1}$ Clinic for Palliative Medicine, University Medical Center Goettingen, Goettingen, Germany, ${ }^{2}$ Department of General Practice / Primary Care, University Medical Center Hamburg-Eppendorf, Hamburg, Germany

Background: Volunteers have a fundamental role in end-of-life care and are an essential part of multi-professional palliative care today. The continuous integration of non-oncological patients (NOPs) into palliative care may create distinct challenges and problems for volunteers when supporting these patients and their relatives compared to oncological patients (OPs).

Aims: To assess volunteer support for NOPs and potential distinctions to OPs in Germany.

Methods: Via a national online survey, coordinators of volunteer services were asked to give information of NOP-support in the past and rate factors like age, disease and family regarding their importance to establish volunteer care. Data was analysed using descriptive statistics and qualitative content analysis.

Results: 212 valid data sets were obtained (response rate unknown due to the pyramid scheme of the online survey). $45 \%$ of the participants observed increased NOP-support over the last years (decrease $9 \%$, no change $27 \%$, unknown $17 \%$, non-response rate (NRR) $2 \%$ ). $58 \%$ stated that the support duration for NOPs is longer than for OPs (shorter duration $3 \%$, no difference $23 \%$, unknown $6 \%$, NRR $10 \%$ ). Meanwhile, $50 \%$ weighed the factor "Disease" as a high/rather high influence for the arrangement of volunteer care (low/rather low $41 \%$, no impact $1 \%$, NRR $8 \%)$. "Expected duration of support" was rated with important by $41 \%$ (low/rather low $45 \%$, no impact $1 \%$, not specified $4 \%$, NRR $9 \%$ ) and "Intensity of symptoms" with $38 \%$ (low/rather low $51 \%$, no impact $1 \%$, not specified $2 \%$, NRR $8 \%$ ).

Conclusion: The increase of NOP-support with an extended period of support compared to OPs might lead to a lack of volunteers for urgent requirements, especially for smaller volunteer services with little resources. 
Specific training for volunteers focusing on the longer support, specific challenges and disease specific factors of NOPs might be necessary to ensure satisfaction of both patients and volunteers.

\section{Abstract number: P01-425 \\ Abstract type: Poster}

Together We Can: Developing Resources to Enable Volunteer Support for Families Caring for a Child with a Life-limiting Condition

Scott R. ${ }^{1}$, Chambers L. ${ }^{2}$

${ }^{1}$ School of Education and Social Work, University of Dundee, Dundee, United Kingdom, ${ }^{2}$ Together for Short Lives, Bristol, United Kingdom

Background: Research suggests that $84 \%$ of families caring for a child with a life-limiting condition feel isolated and that they need more domestic support. This abstract reports on a project to develop national resources for the development of community volunteering programmes offering support to such families.

Project aim: To improve family wellbeing and quality of life through volunteer support.

Method: Evidence-informed resources were developed and tested in practice by five pilot project organisations, comprisingchildren's community palliative care organisations, drawn from voluntary and statutory sectors. Three organisations had no previous experience with community volunteering. Resources covered:

- Starting a service: guidance for organisations and volunteers, family information, assessing families needs, model policies and procedures

- Six module volunteer training programme materials, facilitators guide, staff training overview;

- Monitoring and evaluation

The materials were also widely reviewed by children's palliative care organisations with no community volunteering experience to assess the clarity, content and potential value of the resources.

Results: Feedback on the resources was positive in terms of content and ease of use. Gaps were identified and pilot sites contributed additional materials including new sections on planning, recruitment, support and supervision and specific guidance for particular settings. This resulted in the publication of Together We Can, an online resource covering four key areas:

- Engaging families

- Planning your service

- Engaging volunteers

- Running your service.

A virtual community of practice was established to support the development of family support volunteering nationally.

Conclusion: There has been much interest in this first national resource for family support volunteering in children's palliative care in the UK. The flexibility of the materials enabled this to be adapted for use in other settings and countries.

Abstract number: P01-426

Abstract type: Poster

An Innovative Method of Choosing the Best Outcome Tool to Measure Impact of Non-clinical or Volunteer Provided Palliative Care Services: Mapping Key Concepts from a Scoping Review to Contender Tools

Dodd S., Preston N., Payne S., Walshe C.

International Observatory on End of Life Care, Lancaster University, Lancaster, United Kingdom
Background: Volunteer provided or non-clinical palliative care services are common. Measuring service outcomes can be challenging and existing palliative care outcome tools are potentially focused inappropriately on clinical issues.

Aims: To identify key outcomes of non-clinical end-of-life interventions, as perceived by older people, family carers, or health and social care professionals. To map identified key outcome domains to contender outcome measurement tools.

Methods: A scoping review design enabled identification of studies. Inclusion criteria: qualitative research; data from older people, family carers or professionals; focused on outcomes from non-clinical interventions for people with palliative care needs. Databases (EMBASE, CINAHL, MEDLINE and PSYCHinfo) searched to March 2018 using a combination of terms relating to palliative care, qualitative research and a range of non-clinical interventions. Data extracted using $\mathrm{NVivo}^{\mathrm{TM}}$, and thematically coded to identify outcome domains. Domains were mapped onto the content of contender outcome measurement tools, selected from the PCRC instrument library.

Results: 62 included papers focused on interventions such as advance care planning, guided conversations, and volunteer befrienders or compassionate community support. Four overarching domains: greater autonomy and control; improved mental health; enriching relationships; and more involved, informed and considered about care choices. Mapping domains to contender tools revealed issues of relevance and redundancy. Some tools (e.g. WEMWBS) had no redundant questions, but mapped only to two of four domains, others mapped to all domains, but with many additional redundant questions (e.g. WHOQOL-Bref). Tools such as ICECAP-SCM and McGill Quality of Life had high relevance and low redundancy.

Conclusions: Selecting a fit for purpose outcome tool is critical. This innovative review and mapping method may have wider utility in selecting appropriate tools.

Abstract number: P01-427

Abstract type: Poster

\section{Towards a Minimal Dataset of Figures on HPC Volunteering in} Europe Goossensen A. ${ }^{1}$, Pelttari L. ${ }^{2}$, Pissarek $A .{ }^{2}$, Scott $R .^{3}$

${ }^{1}$ University of Humanistic Studies, Utrecht, Netherlands, ${ }^{2}$ Hospice Austria, Vienna, Austria, ${ }^{3}$ University of Dundee, Dundee, United Kingdom

Despite the routine collection of national generic volunteering data, European countries show great differences when it comes to data about national HPC volunteering. Only few umbrella organisations collect data annually. Data collection is important in understanding trends, activities and to inform the further development of HPC volunteering nationally. Consensus about a minimum dataset of figures collected for each European country would support the further development of HPC volunteering. This project aims to present a format for a national minimal dataset on HPC volunteering as implementation action of the EAPC Charter on Volunteering.

We worked towards a minimal dataset for HPC volunteering at a national level by the comparison and analysis of successful national data collections in Austria and The Netherlands, followed by a discuss and agree intervention with 7 country experts.

Examples of data from Austria demonstrate that in 2017 3,541 volunteers ( $85,5 \%$ female) worked 405,092 hours in HPC organisations for adults helping 13.119 patients. 220 volunteers ( $87 \%$ female) spent 14,674 hours in 10 children's volunteer hospice teams helping 187 patients and 357 siblings, parents. Information includes number of:

1) hours worked

2) hours in direct patient care,

3) volunteers, 
4) co-ordinators,

5) patients and family members,

6) place of care,

7) bereavement care.

Dutch data examples show that in 201711,430 volunteers worked $1,425,251$ hours to help 12,351 patients. This data set addresses characteristics of HPC volunteering in 198 volunteering organisations, subdivided over 5 settings, with a $93 \%$ response. Information includes the numbers of:

1) hours worked,

2) clients,

3) volunteers,

4) occupied beds, satisfaction, finances and tasks of volunteers.

Reflection: National data sets are vital for a country as they facilitate planning and show trends. With a minimal data set in every country a clear picture of HPC volunteering would emerge.

Abstract number: P01-429

Abstract type: Poster

Training Peer Supporters for People with Advanced Cancer (PACT): Results from a Feasibility Randomised Controlled Trial Walshe C. ${ }^{1}$, Roberts D. ${ }^{2}$, Calman L. ${ }^{3}$, Appleton L. ${ }^{4}$, Skevington S. ${ }^{2}$, Perez Algorta G. ${ }^{5}$, Croft R. ${ }^{6}$, Lloyd Williams M. ${ }^{7}$, Grande G. ${ }^{2}$

${ }^{1}$ International Observatory on End of Life Care, Lancaster University, Lancaster, United Kingdom, ${ }^{2}$ University of Manchester, Manchester, United Kingdom, ${ }^{3}$ University of Southampton, Southampton, United Kingdom, ${ }^{4}$ Clatterbridge Cancer Centre NHS Foundation Trust, Liverpool, United Kingdom, ${ }^{5}$ Lancaster University, Lancaster, United Kingdom, ${ }^{6}$ Patient and Public Representative, Liverpool, United Kingdom, ${ }^{7}$ Liverpool University, Liverpool, United Kingdom

Background: People with advanced cancer indicate that peer support may be important in maintaining psychological wellbeing. However little is known about how to effectively train peer supporters for such roles. Aims: The overall aim of the study is to determine the feasibility of delivering and investigating a peer mentor intervention to promote psychological wellbeing in people with advanced cancer. Here, we present information on training peer mentors to deliver the intervention.

Methods: A bespoke training module was developed, including content derived from a prior qualitative study with people with advanced cancer. Peer mentors were recruited via advertising. Training was delivered face to face, over two separate days, in group settings. Qualitative interviews with participants post-training and/or post delivering the intervention provided detailed feedback on training, and its implementation in practice.

Results: Peer mentor recruitment was successful ( $n=48$ enquirers, $n=21$ entered training, $n=12$ completed training). Two-day training was held on four separate occasions. Six mentors were matched with people with advanced cancer. Participants volunteered to add a 'human factor' to the cancer experience. They were initially 'a bit apprehensive', despite 'looking forward' to the training. They felt the training was appropriate, reassuring and beneficial. Unexpected benefits included 'becoming friends' with other mentors. Mentors matched with patients found that the role fulfilled their reasons for volunteering. Other benefits included the opportunity to talk about difficult subjects in ways not possible with family or friends and gaining greater insight into their own perceptions of living with cancer.

Conclusion: The bespoke peer mentor training package was appropriate to prepare people to fulfil a peer mentor role. Important learning included factors such as group size, timing of training days, and facilitating group identity.
Funding: NIHR RfPB

Abstract number: $\mathrm{P} 01-430$

Abstract type: Poster

Peer Support to Maintain Psychological Wellbeing in People with Advanced Cancer (PACT): Results from a Feasibility Randomised Controlled Trial

Walshe C. ${ }^{1}$, Roberts $D .{ }^{2}$, Appleton L. ${ }^{3}$, Croft R. ${ }^{4}$, Calman L. ${ }^{5}$, Lloyd

Williams M. ${ }^{6}$, Skevington S. ${ }^{2}$, Grande G. ${ }^{2}$, Perez Algorta G. ${ }^{7}$

${ }^{1}$ International Observatory on End of Life Care, Lancaster University, Lancaster, United Kingdom, 2University of Manchester, Manchester, United Kingdom, ${ }^{3} \mathrm{Clatterbridge}$ Cancer Centre NHS Foundation Trust, Liverpool, United Kingdom, ${ }^{4}$ Patient and Public Representative, Liverpool, United Kingdom, 5University of Southampton, Southampton, United Kingdom, ${ }^{6}$ Liverpool University, Liverpool, United Kingdom,

${ }^{7}$ Lancaster University, Lancaster, United Kingdom

Background: People with advanced cancer indicate that peer support may be important in maintaining psychological wellbeing. However little is known about how to provide such support, or whether it works.

Aims: To determine the feasibility of delivering and investigating a peer mentor intervention to promote psychological wellbeing in people with advanced cancer.

Methods: Two arm randomised controlled trial with qualitative process evaluation. Peer mentors recruited via media/cancer centres. People with advanced cancer recruited via clinics and hospices. Intervention was a proactive introduction to and informal contact with a trained peer mentor for $\leqslant 12$ weeks. Data collection included patient, carer and mentor wellbeing, coping strategy, and social support outcomes (WHOQOL-Bref; EORTC-15 PAL; BriefCOPE; mMOS-SS; PHQ-9) at baseline, 4 and 12 weeks.

Results: Peer mentor recruitment/training was feasible ( $n=48$ enquirers, $\mathrm{n}=21$ entered training, $\mathrm{n}=12$ completed training). Patient recruitment was not feasible. Information packs distributed to eligible patients ( $n=181)$, enquiries $(n=25)$, participants $(n=12$; Intervention (6), Control (6)). Intervention and data collection were feasible. Participants typically male $(I=3, C=4)$, mean age $(I=64.2 \pm 6.2, C=69.6 \pm 9.7)$, married $(I=3$, $C=6)$, living with partner $(I=3, C=6)$, retired $(I=3, C=5)$. Quality of life trends favoured those in intervention e.g. WHOQOL-Bref (Physical) intervention median increased from 46.42 (T0), 55.35 (T4), 58.92 (T12) compared to control median decrease from 73.21 (T0), 39.28 (T4), 21.42 (T12). Attrition and missing data were as expected.

Conclusion: All aspects of the study were feasible, except patient recruitment. Broader recruitment approaches, incorporating (social) media approaches may be beneficial. Outcomes must be interpreted with extreme caution given the small numbers enrolled, but indicate that peer mentor interventions may have benefit and warrant further investigation.

Funding: NIHR RfPB.

Abstract number: P01-431

Abstract type: Poster

Social Importance of Volunteering in Palliative Care in the Netherlands

Notenboom $M$.

VPTZ Nederland, Amersfoort, Netherlands Presenting author email address: info@vptz.nl

Aim: In 201789.400 people died of chronic diseases. In 13,8\% of these cases (12.351 in total) a palliative care volunteer was involved. Their umbrella organization, VPTZ Nederland, does annual research on the social importance of the work of these HPC volunteers. This research helps to strengthen their position. Furthermore, these data form a basis for accountability towards private and governmental funders. 
Design: Umbrella organization, VPTZ Nederland, with 214 member organizations, annually collects the most relevant information. This survey is done by research institute DirectResearch. In 2017 and 2018198 members received the online questionnaire. The response rate was $93 \%$. The survey analyses the activities of VPTZ member organizations by means of descriptive statistics on subjects like organisation, finance, number of clients, hours of care, effort and hours of coordinators and volunteers.

Result: Of the 12.351 people that received care from 11.430 volunteers in $2017,56 \%$ stayed in a hospice, $40 \%$ received support at home and $4 \%$ elsewhere (for instance in a nursing home). In total these volunteers gave 1.425.251 hours of care. The average period of support was just over 3 weeks, in hospices as well as in the clients home. Most clients were referred to VPTZ-volunteers by professional home care organisations (28\%), hospitals (21\%) and general practitioners.

Conclusion: By tracking these data we are able to show the importance of volunteering and follow how it develops over time. Ten years ago there were 6800 volunteers, in 2017 there are 11.430 active volunteers, a growth of $68 \%$. In 20176416 clients received voluntary care. In the meantime this number has grown to 12.351 , an increase of $93 \%$.

Abstract number: P01-432

Abstract type: Poster

Development of a Community Advance Care Planning Program for LGBT Individuals

Candrian C. ${ }^{1}$, Flanagan L. ${ }^{2}$, Jordan S. ${ }^{1}$, Lasker-Hertz S. ${ }^{2}$, Lum H. ${ }^{1}$

${ }^{1}$ University of Colorado Anschutz Medical Campus, Aurora, United

States, ${ }^{2}$ Denver Hospice, Denver, United States

Background: Lesbian, gay, bisexual, and transgender (LGBT) individuals face significant unmet care needs as they age. Compared to heterosexual individuals, LGBT individuals are twice as likely to age as a single person, twice as likely to live alone, 3 to 4 times less likely to have children to support them, and are nearly 3 times more likely to live in poverty. Given the challenges LGBT individuals face as they age, interventions that promote open and inclusive communication and care planning are needed.

Aims: Our aims seek to:

1) develop a LGBT volunteer workforce to facilitate meaningful conversations related to advance care planning (ACP) among older LGBT individuals and

2) increase the quality and quantity of ACP among the LGBT community.

Methods: To train and certify LGBT individuals to facilitate conversations related to ACP among LGBT communities, we leveraged a long-standing community and academic partnership, as well as the development of a community-based stakeholder advisory group (SAG). To evaluate the program's effectiveness among the LGBT population, a combination of qualitative interviews and quantitative surveys were completed.

Results: 85 individuals from diverse backgrounds in the Rocky Mountain Region of the United States have been trained. Further, we developed a 9-person SAG consisting of individuals working in different sectors and who identify as LGBT; the SAG has provided input on volunteer support processes, and preferred language and strategies for talking about ACP among LGBT individuals. Finally, outreach and recruitment strategies for working with community organizations to engage with LGBT communities will be discussed.

Conclusion: This project has created the first of its kind training program for a volunteer workforce of LGBT individuals that are equipped to facilitate and promote quality conversations about ACP. We hope in sharing our lessons learned, others will also seek to meet the growing needs of LGBT individuals around ACP.
Abstract number: P01-433

Abstract type: Poster

Assessment of End-of-Life Renal Patients' Clinical Outcomes, Quality of Life and Quality of Death in a Tertiary Medical Center - A Preliminary Report of Renal Hospice-palliative Care in Taiwan Tsai H.-B. ${ }^{1,2}$, Chao C.-T. ${ }^{3}$, Hung K.-Y. ${ }^{4}$, Chang R.-E. ${ }^{2}$, COGENT Study Group ${ }^{1}$ Division of Hospital Medicine, Department of Internal Medicine, National Taiwan University Hospital, Taipei City, Taiwan, Republic of China, ${ }^{2}$ Institute of Health Policy and Management, College of Public Health, National Taiwan University, Taipei City, Taiwan, Republic of China, ${ }^{3}$ Department of Internal Medicine, National Taiwan University Hospital Bei-Hu Branch, Taipei City, Taiwan, Republic of China, ${ }^{4}$ Department of Internal Medicine, National Taiwan University Hospital Hsin-Chu Branch, Hsinchu City, Taiwan, Republic of China

Background: At present, elderly multi-morbid dialysis patients has increased significantly, but the clinical prognosis is not ideal. This study aims to compare quality of life $(\mathrm{Q} O L)$ and quality of death $(\mathrm{Q} O D)$ between end-of-life (EoL) renal (Gr.A) and other EoL (Gr.B) patients in a tertiary medical center in Taiwan.

Methods: A case-control study was conducted from Oct. 2016 to Jun 2018. The hospice/palliative care (HPC) assessment scale was applied to survey EoL patients in general medical wards. All patients received standardized HPC. Their demographic features, clinical characteristics, McGill QoL questionnaire (MQOL) were analyzed. When the patient was deceased, a phone interview was provided 3 months later for caregiver to assess QoD.

Results: The Gr.A ( $n=42)$ patients are older (mean age:79.0 vs.71.1 years) than $\mathrm{Gr} . \mathrm{B}(\mathrm{n}=42)$, having lower $\mathrm{Hb}$ and platelets. The comorbidities in Gr.A were more congestive heart failure, dementia, diabetes, advanced CKD, while metastatic solid tumor more prevalent in Gr.B Seldom social activities were identifed in both groups. The majority of dialysis vintage was 1-3 months for HD. For peritoneal dialysis (PD) patients, 2 with CAPD, 2 with Extraneal, and 3 added HD once a week. The primary cause of death was uremia (30.56\%6) in Gr.A, septicemia (33.33\% v.s. 3.33\%) more in Gr.A and cancer (6.67\% vs. $16.67 \%$ ) more in Gr.B. For the last 2-week acute events, more heart failure $(30.56 \%$ v.s. $6.67 \%$ ) in Gr.A, and more pneumonia (66.67\% vs.36.11\%) in Gr.B. More dyspnea seen in Gr.B (71.79\% vs.43.59\%), and more cramping (28.21\% v.s. $10.26 \%)$ in Gr.A. The both groups had similiar QoL and QoD. The HPC assessment scale scored over 6 points could predict higher risk of death than those with $3-4$ points $(O R=16,95 \% \mathrm{Cl}$ : 1.25-204.11).

Conclusion: The terminal ill renal patients' QoL and QoD were not inferior to other EoL patients. Hospice-palliative care assessment scale over 6 points can predict grave prognosis of EoL renal patients.

\section{Abstract number: P01-434}

Abstract type: Poster

Exploring the Barriers and Consensus of Implementing Renal Palliative Care among Taiwanese Nephrologists

Tsai H.-B. ${ }^{1,2}$, Chao C.-T. ${ }^{3}$, Hung K.-Y. ${ }^{4}$, Chang R.-E. ${ }^{2}$, Cheng S.-Y. ${ }^{5}$, COGENT Study Group

1Division of Hospital Medicine, Department of Internal Medicine, National Taiwan University Hospital, Taipei City, Taiwan, Republic of China, ${ }^{2}$ Institute of Health Policy and Management, College of Public Health, National Taiwan University, Taipei City, Taiwan, Republic of China, ${ }^{3}$ Department of Internal Medicine, National Taiwan University Hospital Bei-Hu Branch, Taipei City, Taiwan, Republic of China, ${ }^{4}$ Department of Internal Medicine, National Taiwan University Hospital Hsin-Chu Branch, Hsinchu City, Taiwan, Republic of China, ${ }^{5}$ Division of Family Medicine, Department of Internal Medicine, National Taiwan University Hospital, Taipei City, Taiwan, Republic of China 
Background: When the Right to Patient Autonomy Act will be enforced in 2019, the medical staffs should respect the patients' wills of good death. However, the definition of end-of-life renal patients (EoLRPs) is still debated.

Aims: This study aims to establish the indicators of EoLRPs through consensus methods to promote renal palliative care (RPC) in Taiwan.

Methods: We searched databases to collect international guidelines of RPC in 2008-2016 to construct the preliminary indicators. The 14-experts adopts the RAND/UCLA Appropriateness Method to further discuss the appropriateness of these indicators. The questionnaire was then issued by the method of disproportionate stratified random sampling in July to October 2016. We applied exploratory and confirmatory factor analyses to explore the latent variables of EoLRPs. In the end, the mixed research method was used to clarify the barriers and consensus of Taiwanese nephrologists on RPC.

Results: A total number of 337 of 624 nationwide dialysis facilities responded to this survey and 322 nephrologists' questionnaires were analyzed. The distribution of hospital type (HT) vs. outpatient clinic type (OCT) nephrologists (55.6\% vs. $44.4 \%$ ) was representative in Taiwan. More experiences of forgoing dialysis and more medical ethics training within recent one year were seen in HT than OCT nephrologists ( $p<$ 0.001). We analyzed 254 nephrologists about indicators for EoLRPs suitable to receive hospice and dying care and obtained high consensus indicators included "the prolonged mechanical ventilator (PMV) patients receiving dialysis still comatose" and "the PMV patients receiving dialysis with multi-organ failure." Through factor analysis, three latent variables were EoLRPs with ventilator dependence, EoLRPs with multi-morbidities and EoLRPs with full dependence.

Conclusion: To formulate the policy of promoting RPC in Taiwan, high consensus indicators should be given priority, especially for EoLRPs with ventilator dependence.

\section{Abstract number: P01-435 \\ Abstract type: Poster}

\section{Multidisciplinary Guideline 'Palliative Care for Patients with Heart Failure'}

Engels $Y .{ }^{1}$, de Graeff A. ${ }^{2}$, Aertsen M. ${ }^{3}$, van Erp J. ${ }^{4}$, van Leen M. ${ }^{5}$, Lucas C. ${ }^{6}$, Middeljans L. ${ }^{7}$, Starmans R. ${ }^{8}$, Gilsing M. ${ }^{9}$, Tabak R. ${ }^{10}$, Bellersen L. ${ }^{11}$

${ }^{1}$ Anesthesiology, Pain, Palliative Medicine, Radboud University Medical Center, Nijmegen, Netherlands, ${ }^{2}$ Universtiy Medical Center Utrecht, Utrecht, Netherlands, ${ }^{3} \mathrm{NVHVV}$, Amersfoort, Netherlands, ${ }^{4}$ Hart en Vaatgroep, Den Haag, Netherlands, ${ }^{5}$ Verenso, Utrecht, Netherlands, ${ }^{6} \mathrm{NVVC}$, Utrecht, Netherlands, ${ }^{7} \mathrm{NVKG}$, Utrecht, Netherlands, ${ }^{8} \mathrm{NHG}$, Utrecht, Netherlands, ${ }^{9} \mathrm{Netherlands}$ Comprehensive Cancer Organization (IKNL), Utrecht, Netherlands, ${ }^{10}$ PAR Radboudumc, Nijmegen, Netherlands, ${ }^{11}$ Radboud University Medical Center, Nijmegen, Netherlands

Background: Although the mean survival of patients with congestive heart failure (CHF) is even shorter than of patients with cancer and their symptom burden higher, many of them do not receive palliative care. Therefore, we developed a multidisciplinary guideline for patients with CHF NYHA classification III/ IV.

Methods: The guideline was developed with support of Netherlands Comprehensive Cancer Organisation and by a group of experts, representing all relevant professional associations as well as patients. With the help of a survey, the most important barriers were collected from professionals and patients. Next, systematic literature searches were performed and evidence-based conclusions were drawn. Recommendations were based on evidence or, if not available, on expert opinion. A concept guideline was written by the working group and sent to all professional associations involved and patient organisations for comments.

Results: Six topics were selected, explored, described and summarized in recommendations: prognosis, palliative care provision, advance care planning, communication, organisation of care, and medication in the last months of life.

Conclusions: In February 2018, the multidisciplinary guideline for palliative care for patients with heart failure was published and is currently being implemented. As this guideline might also inspire researchers and professionals from other countries, an English summary is available. The recommendations will be discussed during the presentation.

Abstract number: P01-437

Abstract type: Poster

Baseline Quality of Life among Patients with End Stage Kidney Disease in a Resource Limited Setting Bagasha P. ${ }^{1}$, Petrova M. ${ }^{2}$, Biddle J. ${ }^{3}$, Katabira E. ${ }^{1}$, Leng M. ${ }^{1}$

${ }^{1}$ Internal Medicine, Makerere and Mulago Palliative Care Unit, Kampala, Uganda, ${ }^{2}$ Department of Public Health and Primary Care, Cambridge University, Cambridge, United Kingdom, ${ }^{3}$ University of California, San Francisco, United States

Background: At 23\% the prevalence of chronic kidney disease in sub-Saharan Africa is higher than the global prevalence of $16 \%$. With no national healthcare coverage for hemodialysis or kidney transplant, Ugandan patients struggle to pay driving communities into poverty. Studies in developed countries show that patients on hemodialysis may prioritize quality of life (QoL) over survival time. There is a dearth of information about QoL of patients with End Stage Kidney Disease (ESKD) in LMIC.

Aim: Establish a baseline review of QoL, patient outcomes and associated factors for patients with ESKD receiving hemodialysis versus conservative management in a national referral hospital.

Methods: A mixed methods analytical study using the Kidney Disease Quality of Life Short Form, African APCA POS and Renal POS. Clinical, demographic and micro-financial information is collected and patient experiences explored using semi-structured interviews.

Results: 40 patients completed with 6 interviewed. Full evaluation will be presented with scores grouped into mental health, physical health and kidney disease related composite scores. Summary; QoL $91 \%$ felt kidney disease interfered too much with their life. 37\% felt they were a burden to their family, $61 \%$ felt very frustrated with their lives. Average pain score (POS) was 2.4 with $27 \%$ reporting severe or overwhelming pain. Most had only 1 additional symptom with breathlessness highest prevalence (16\%). Extensive co-morbidities present; $90 \%$ hypertension $\& 50 \%$ diabetes. Key themes; disease burden and comorbidities, importance of spirituality, loss of hope and financial constraints "I wish God could take me before I deplete my family's resources'.

Conclusion: Improved QoL is a key indicator of successful chronic disease management. This baseline review will inform a wider mixed methods longitudinal study. Improving this evidence base will inform renal and palliative care services with the aim of improving patient experience.

Abstract number: P01-438

Abstract type: Poster

Integrated Palliative Care Interventions for Patients with COPD: A Systematic Review

Broese J.M.C. ${ }^{1,2}$, de Heij A.H. ${ }^{3}$, Janssen D.J.A. ${ }^{4,5}$, Chavannes N.H. ${ }^{1}$, Engels Y. ${ }^{6}$, van der Kleij R.M.J.J. ${ }^{1}$

${ }^{1}$ Public Health \& Primary Care, Leiden University Medical Center, Leiden, Netherlands, 'Lung Alliance Netherlands, Amersfoort, Netherlands, ${ }^{3}$ Center of Expertise for Palliative Care NoordOost, University Medical Center Groningen, Groningen, Netherlands, ${ }^{4}$ Department of Research \& Education, CIRO, Center of Expertise for Chronic Organ Failure, Horn, Netherlands, ${ }^{5}$ Center of Expertise for Palliative Care, Maastricht University Medical Center, Maastricht, Netherlands, ${ }^{6}$ Anesthesiology, Pain \& Palliative Care, Radboud University Medical Center, Nijmegen, Netherlands 
Background: Despite the high burden of disease and low quality of life, most patients with advanced COPD do not receive palliative care. Therefore, care models are needed with specific attention to the delivery and organization of palliative care in COPD.

Methods: The aim of this systematic review was to assess the content, organization and effect of integrated palliative care interventions for COPD patients. Seven databases were systematically searched for studies evaluating palliative care interventions. Interventions focusing on single components of palliative care, e.g. ACP or symptom management were excluded. Studies were eligible if $>30 \%$ of patients included suffered from COPD or if subgroup analysis data were reported. Study quality was assessed via the Mixed Methods Appraisal Tool. Intervention and study characteristics, as well as outcomes were extracted and analyzed using narrative synthesis.

Results: Of 4532 identified records, 180 full text articles were assessed for eligibility. Twenty-seven articles reporting on 19 integrated palliative care interventions were included. Most interventions contained multidimensional assessment during one or more home visits. Across studies, the criteria for patients to enter the intervention, the timing and total duration differed. Eight interventions were evaluated in a controlled study; three were RCTs. The most frequently used patient effect outcome measure was hospital admission. Two studies reported effects on informal caregiver outcomes and none on healthcare professional outcomes. Mixed effects were found on the various outcome measures identified.

Conclusion: In the last 15 years, an increasing number of palliative care interventions for patients with COPD have been developed, of which the majority contained home visits. We found that little high quality evidence is yet available; more adequately controlled studies and process evaluations are needed.

Funding: Netherlands Organization for Health Research \& Development.

Abstract number: P01-439

Abstract type: Poster

Awareness and Challenges to Advance Care Planning (ACP) among Japanese Doctors and Nurses Involved in Chronic Heart Failure Treatment-Characteristics of Japanese Medicine in this Field as Revealed by a Survey

Higuchi Y. ${ }^{1}$, Yoshida M. ${ }^{1}$, Morita M. ${ }^{1}$, Honjo K. ${ }^{1}$, Tanaka $T_{.}^{1}$, Nakamura M. ${ }^{2}$, Tanaka A. ${ }^{3}$, Tonoki Y. ${ }^{1}$, Sakai C. ${ }^{1}$

${ }^{1}$ Japanese Red Cross College of Nursing, Tokyo, Japan, 2Japanese Red Cross Kyushu International College of Nursing, Fukuoka, Japan, ${ }^{3}$ Showa University, Tokyo, Japan

Background: The initiative for ACP in Japan, though encouraged as a national policy, has yet to identify the specific challenges in relation to chronic illnesses.

Purpose: To clarify awareness to ACP among Japanese doctors and nurses treating chronic heart failure and the challenges they face in practicing ACP.

Methods: A mail survey. We created a 20-item, 6-category questionnaire to ask about awareness and challenges to ACP. The responses were given in four- or five-level Likart scales. The questionnaire was sent to randomly-chosen medical specialists engaged in chronic illnesses and all certified nurse specialists and certified nurses in the field, total 1,855. The 524 addressees, 240 doctors and 284 certified nurses, were providing care for chronic heart failure patients.

Results: The 238 respondents (Response rate: $45.4 \%$ ) included 46 doctors in cardiovascular medicine and 192 certified nurses in chronic heart failure nursing. Regarding the timing to discuss ACP, "When obstacles in daily life arise" (4.23 \pm 0.92$)$ scored high. As to difficulties in implementing ACP, "Coordinating when opinions differ (among family members)" $(4.0 \pm 0.89)$ was most highly recognized. They were most aware of "Major hospitals and local clinics need to work together" as an operational barrier $(4.70 \pm 0.53)$
Discussion: The timing for patient and medical care providers to agree on ACP included at the time when obstacles are found in daily life. This is probably because patients and medical professionals involved in chronic heart failure, which is mainly dealt with drug therapy, are not usually obliged to choose treatment methods or required to make decisions. The necessity of medical institutions and local clinics to work together, identified as the biggest challenge, suggested that relevant information may not be effectively utilized due to the lack of a system in Japan to share medical records between hospitals and clinics.

\section{Abstract number: P01-440 \\ Abstract type: Poster}

Integration of Palliative Care in the Management of Congestive

Heart Failure

Senderovich $\mathrm{H}$.

Baycrest Centre for Geriatric Care, Ontario, ON, Canada

Introduction: Congestive heart failure (CHF) is an increasingly prevalent terminal illness in a globally aging population. Despite optimal medical management, prognosis remains poor - a fact seldom communicated to patients and/or their families. Evidence suggests numerous benefits of palliative care consultation in advanced $\mathrm{CHF}$ but to date, their services remain woefully underutilized.

Objectives: To identify specific challenges to accessing and implementing palliative care in patients with advanced $\mathrm{CHF}$, and to use this information to formulate recommendations for practice.

Methods: Literature review whereby recommendations for practice were formulated on the basis of primary quantitative/qualitative data and consensus expert opinion.

Results: Accessing palliative care services for patients with $\mathrm{CHF}$ remains a challenge for numerous factors including prognostic uncertainty, misconceptions about what palliative care is, and difficulty recognizing when a patient is suitable for referral. Strategies to improve access/ delivery of palliative care to this population include education and proper discussion about prognosis/goals of care. A team-based approach is essential as we move towards a model where symptom palliation exists concurrently with active medical disease-modifying treatment.

Conclusion: Despite evidence that palliative care has a role in improving symptom control and overall quality of life in patients with end-stage $\mathrm{CHF}$, a multitude of challenges exist and this ultimately hinders access to palliative care services. Education to abolish pre-existing misconceptions about the role of palliative care and a movement towards a team-based approach focused on simultaneous palliative and traditional medical care will undoubtedly improve access to, and benefit from, palliative care services in this population.

Abstract number: P01-441

Abstract type: Poster

Factors Associated with Awareness of Palliative Care among Patients with Chronic Obstructive Pulmonary Disease

Sono S., Fumaneeshoat O., Ingviya T.

Family Medicine and Preventive Medicine, Prince of Songkla University, Hatyai, Thailand

Background: Chronic obstructive pulmonary disease (COPD) causes morbidities and mortalities. Despite its ability to alleviate COPD's consequences, palliative care services and awareness worldwide, as well as in Thailand, are currently limited.

Aims:

1. To measure the prevalence of palliative care awareness in COPD patients, visiting outpatient clinics in Songklanagarind Hospital, Thailand

2. To assess factors associated with patients' palliative care awareness. 
Methods: Our study design is a cross-sectional study, conducted in COPD patients, visiting Songklanagarind hospital during the periods from 1st July to 30th October 2018. A paperless questionnaire, asking about demographic factors, COPD assessment test (CAT) score, medications, and palliative care awareness was developed. COPD patients, who met inclusion criteria, were recruited and interviewed by a trained research assistant. Factors, associated with the palliative care awareness were assessed by either; Wilcoxon's or Fisher's exact test and multivariate logistic regression.

Results: The Conbranch's alpha of the questionnaire was $0.78-0.82$. The validity of the questionnaire was evaluated by 3 experts, including 1 pulmonologist and 2 palliative care specialists. Of 90 enrolled patients, they were predominately male $(96.7 \%)$, with a median age of 71 years (IQR: 65-78 years). The median FEV1\% predicted was 60\% (IQR: 44-81\%). 14 patients $(15.6 \%)$ were on home oxygen, and only $22.2 \%$ of patients was aware that palliative care was useful for COPD patients. Only 8 patients $(13.3 \%)$ reported being informed about advance care plans. None of the demographic or clinical factors was associated with palliative care awareness.

Conclusion: Palliative care awareness in COPD patients was quite low which is similar to several studies in the United Kingdom and European countries. Further studies and interventions to boost palliative care awareness and services in our hospital is inevitably needed.

\section{Abstract number: P01-442 \\ Abstract type: Poster}

Palliative Care in Cystic Fibrosis: How Could Pneumologists and Palliative Care Teams Work Together? Chaumier F. ${ }^{1,2,3}$, Arnaud J. ${ }^{1}$, Flament T. ${ }^{1}$

${ }^{1} \mathrm{CHRU}$ de Tours, Tours, France, ${ }^{2}$ UMR INSERM U1246 SPHERE, Université de Nantes, Nantes, France, ${ }^{3}$ UMR INSERM U1246 SPHERE, Université de Tours, Tours, France

Background, aims and method: Cystic fibrosis is a rare congenital pathology responsible for respiratory infections, pneumothorax and chronic respiratory failure. In the absence of a lung transplant, it is an incurable disease whose median life expectancy is 50 years. There is little data in the literature on the partnership between palliative care teams and pneumologists for the management of patients with advanced cystic fibrosis. We report the case of a patient to identify the possible aims of a such collaboration.

Results: A single 35-years-old patient was followed every three months in consultation by a Cystic Fibrosis Reference Center in France. The respiratory failure was severe (FEV1=20\%). In 2017 he refused a lung transplant because he found no meaning in extending his life. He even expressed a wish to hasten death (WTHD). On the proposal of his doctor, he met palliative care physician and psychologist. The patient has expressed his advanced care planning. Advanced directives have been addressed to avoid resuscitation in case of life-threatening complications. Psychological support and medical interviews provided spiritual care. In 2018 the patient had a severe pneumothorax, with failure of a thoracic drainage. Medical care were discussed between respiratory and palliative care teams. He refused again lung transplant but accepted a very risky surgical treatment. The social worker helped him in his administrative procedures in case of death. However the intervention was successful and follow-up is still continued in consultation.

Discussion: We identified with the pneumology team possible ways to collaborate with the palliative care team for patients with advanced cystic fibrosis: end-of-life discussions, wish to hasten death, social support, help with ethical questions, help with the management of rebellious symptoms (dyspnea, pain, anxiety, etc.) and support for caregivers.

Abstract number: P01-443

Abstract type: Poster
Implementing Advance Care Planning for Patients with Heart Failure: A Pilot Study

Coster J. ${ }^{1}$, Reyners A. ${ }^{2}$, de Rooij S. ${ }^{3}$, Drenth D.J. ${ }^{4}$, Beltman F. ${ }^{4}$, van

Veldhuisen D.J. ${ }^{1}$, de Graeff P. ${ }^{2}$

${ }^{1}$ Cardiology, University Medical Center Groningen, Groningen, Netherlands, ${ }^{2}$ Expertise Centre Palliative Care North-East, University Medical Center Groningen, Groningen, Netherlands, ${ }^{3}$ University Center of Geriatric Medicine, University Medical Center Groningen, Groningen, Netherlands, ${ }^{4}$ University Medical Center Groningen, Groningen, Netherlands

Background: Patients with advanced heart failure have a poor prognosis, and often have impaired quality of life. Although the need for advance care planning (ACP) in heart failure is increasingly recognized, it is far from being widely implemented.

Aim: To perform an observational cohort study to assess feasibility of ACP in patients with advanced heart failure.

Methods: Fifty patients with NYHA class III/IV heart failure who had $\geqslant 2$ unplanned hospital admissions in the previous year because of decompensated heart failure will be included upon approval of their primary cardiologist. Patients who are eligible for advanced heart failure therapy are excluded. The intervention consists of ACP conversations and documentation by the cardiology team. Primary outcome is the use of healthcare services within 3 months after ACP conversation. Secondary endpoints are satisfaction of patients and cardiologists with the intervention measured by a short questionnaire. Here, results of the first 19 patients included in the study are presented.

Results: Median age was 80.5 years (range 33-94 years). Advance directives and preferred place of death were documented in all cases. Fifteen patients (79\%) did not want to be readmitted to the hospital. All patients that died within 3 months after the intervention $(n=8,42 \%)$ died at home, in agreement with their wishes. Presentation at the emergency department or hospital readmission did not occur. Patients and healthcare providers were satisfied with the intervention: $4 / 6$ patients $(67 \%)$ would recommend ACP conversations to other patients. The majority of cardiology residents and cardiologists would recommend this intervention to colleagues ( $94 \%$ and $83 \%$, respectively).

Conclusion: Results of the interim analysis suggest that ACP in patients with advanced heart failure is feasible and that both patients and cardiologists are satisfied with the intervention.

Funding: Netherlands Organization for Health Research and Development

\section{Abstract number: P01-444 \\ Abstract type: Poster}

Implementation of a Circuit of Palliative Care in Patients with Advanced Lung Diseases

Gonzalez de Etxabarri S. ${ }^{1}$, Campo M.A. ${ }^{1}$, Salinas M.B. ${ }^{2}$, Arrizabalaga M.J. ${ }^{1}$, Sanz M. ${ }^{3}$, Urzay V. ${ }^{3}$, Arriaga I. $^{2}$

${ }^{1}$ Palliative Care, Basurto University Hospital, Bilbao, Spain, ${ }^{2}$ Respiratory Diseases, Basurto University Hospital, Bilbao, Spain, ${ }^{3} \mathrm{Hospital}$ at Home, Basurto University Hospital, Bilbao, Spain

Background: Interstitial lung diseases(ILD),especially idiopathic pulmonary fibrosis(IPF) have high morbidity, mortality, short survival and great impact on the quality of life, needing early palliative care but most die in a hospital and a minority receives palliative care.

Aim: Palliative care may reduce symptom burden in patients with ILD. This study aims to describe how do we perform palliative care support until the death in patients with ILD. The timing of referral for palliative care and the location of death.

Material and methods: Since October 2015, all patients with symptomatic ILD or with MRC 3-4 were referred to Palliative Care Unit(PCU).We have made a retrospective review of the clinical histories analyzing 
symptom control, the moment of the referral to PCU, admission or control needs in the hospital at home and the place of death.

Results: 63 patients(33 M-30 F), with advanced disease or MRC 2(4), MRC 3(5), MRC 4(54).Average age 79 years(62-95).30 were IPF(17antifibrotic treatment). All patients/families have received diagnostic, disease severity, prognosis and end-of-life information as well as their preference about the place of death in the 151 PCU consultation. 873 control phone calls have been made by the PCU(48\% modifying treatment-reentering); 215 by patients/family members $(87 \%$ modifying treatment-reentering).Hospital admission 139 times(90 Hospital at Home(HH), 18 Hospital Palliative Care unit(HPCU) and 13 Chronic Hospital and 19 in the Respiratory unit(RU)). The follow-up time has been 1-25 months in patients who are alive (median12)and 1-18 months in the death(median 5). 37 have died, 31 progression. 16 in a HPCU, 12 in your home with $\mathrm{HH}$ and 9 in the Respiratory unit, 3 of them were waiting for the transfer to the HPCU.

Conclusion: Palliative care in the ILD should be started early, then be continued through treatment, until death.Palliative care in ILD has made possible the integral control achieving and death at home in $32 \%$ and only $24 \%$ in $\mathrm{RU}$.

\section{Abstract number: P01-445}

Abstract type: Poster

\section{Dialysis Discontinuation and Family Perceptions of End-of-Life} Care

Richards C. ${ }^{1,2}$, Liu C.-F..$^{1,2}$, Hebert P. ${ }^{1,2}$, Ersek M. ${ }^{3,4,5}$, Wachterman M. ${ }^{6,7}$, Green P. ${ }^{1}$, Reinke L. ${ }^{1,8}$, Taylor L. ${ }^{1}, O^{\prime}$ Hare A. ${ }^{1,9}$

${ }^{1}$ Health Services Research \& Development, Veterans Affairs SeattleDenver Center of Innovation for Veteran-Centered and Value Driven Care, Seattle, United States, ${ }^{2}$ Health Services, University of Washington, Seattle, United States, ${ }^{3}$ National Veteran Experience Center, Corporal Michael J. Crescenz Veterans Affairs Medical Center, Philadelphia, United States, ${ }^{4}$ School of Nursing, University of Pennsylvania, Philadelphia, United States, ${ }^{5}$ School of Medicine, University of Pennsylvania, Philadelphia, United States, ${ }^{6}$ Health Services Research \& Development, Veterans Affairs Boston Health Care System, Boston, United States, ${ }^{7}$ Harvard Medical School, Boston, United States, ${ }^{8}$ School of Nursing, University of Washington, Seattle, United States, ${ }^{9}$ School of Medicine, University of Washington, Seattle, United States

Background: People who discontinue dialysis have higher rates of palliative and hospice care and are less likely to die in the hospital as compared to those who do not. To date, little is known about family perceptions of end-of-life (EOL) care related to discontinuity of dialysis. Aims: We sought to compare treatment intensity and family perceptions of EOL care and among people on maintenance dialysis who discontinued dialysis before death with those who continued dialysis until they died.

Methods: We conducted a retrospective study among a cohort of 3,350 Veterans with advanced kidney disease treated with maintenance dialysis who died in a Veterans Affairs facility between 2009 and 2015. We examined the relationship between dialysis discontinuation and familyrating of EOL care using logistic regression, adjusting for patient characteristics. We additionally adjusted for acute hospital and intensive care unit (ICU) admissions, death in the ICU, and receipt of intensive procedures, palliative care, and hospice services near the end of life.

Results: Among the sample, 27.9\% discontinued dialysis treatments. Those who discontinued dialysis were less likely to be admitted to the ICU in the last 30 days, die in the ICU, and receive an intensive procedure, and were more likely to receive palliative and hospice care. Family of patients who discontinued dialysis were more likely to report excellent overall EOL care and had more favorable responses to most other survey items. After additional adjustment for patterns of EOL care, family of those who discontinued dialysis were still more likely to report favorable care for 5 of 11 survey items.
Conclusions: Family ratings of EOL care for Veterans who discontinued dialysis treatments before death are more favorable, even after adjustment for EOL treatment patterns. These findings highlight the importance of defining the role of dialysis discontinuation in shaping quality of EOL care for patients receiving dialysis.

\section{Abstract number: P01-446}

Abstract type: Poster

\section{Withdrawal of Dialysis; End of Life Considerations and Advanced} Care Planning

Prentice J. ${ }^{1,2,3}$, Hetherington L. ${ }^{1}$, Findlay M. ${ }^{4}$, Collidge T. ${ }^{5}$

${ }^{1}$ The Beatson West of Scotland Cancer Centre, NHS Greater Glasgow and Clyde, Glasgow, United Kingdom, '2University of Glasgow, Glasgow, United Kingdom, ${ }^{3}$ Scottish Renal Palliative Collaborative Group, Glasgow, United Kingdom, ${ }^{4}$ Renal, NHS Greater Glasgow and Clyde, Glasgow, United Kingdom, ${ }^{5}$ The Glasgow Renal \& Transplant Unit, NHS Greater Glasgow and Clyde, Glasgow, United Kingdom

Background: Dialysis withdrawal is the commonest cause of death in patients with end stage renal disease aged $>75$. Factors contributing the withdrawal have been described but to what degree advanced care planning $(A C P)$ is initiated in unknown. We sought to describe current practice within a large renal unit.

Methods: We performed a retrospective analysis of patients who died following dialysis withdrawal from 2015-2017. Patient demographics, markers of health, triggers for withdrawal, admissions, timing and degree of ACP discussions were extracted.

Results: 52 patients were included. Median age at death 76 interquartile range (IQR) $(70,81)$ years. Median duration of dialysis was 42 (IQR 10.5, 75) months with $30.8 \%$ dying within one year of commencement of dialysis. Median Charleston co-morbidity score was 8 (IQR 6, 9) with documentation of 'frailty' present in $52 \%$ of the cohort. $25 \%$ of the patients had an acute event triggering withdrawal. ACP was discussed with $34 \%$ of patients with $82 \%$ of patients achieving their preferred place of death where this was documented. Median length of last admission was 24 days (IQR 11, 43) compared with 11 days (IQR 0, 55) if the patient had an ACP (p-value 0.736).

Conclusion: We are not good at initiating timely ACP in deteriorating dialysis patients despite identifying such patients. With an increasingly frail, multi-morbid population the need for these discussions will increase. Early ACP discussions with frail patients starting dialysis may inform later conversations and improve their quality of care. Encouragingly asking about preferred place of death improved the likelihood of achieving a death in the patients preferred location. Identification of poor prognostic markers such as dementia, introducing staff training in palliative care and adopting routine frailty scoring may guide timely conversations in anticipation of deterioration in vulnerable patient groups.

\section{Abstract number: P01-447}

Abstract type: Poster

Early Integration of Palliative Home Care in Standard Care for End-stage COPD Patients (EPIC): Feasibility, Acceptability and Preliminary Effectiveness of a Phase II Pilot Randomised Controlled Trial

Scheerens C. ${ }^{1}$, Pype P. ${ }^{2}$, Van Cauwenberg J. ${ }^{3,4}$, Vanbutsele G. ${ }^{2}$, Joos G. ${ }^{5,6}$, Derom E. ${ }^{5,6}$, Van Belle S. ${ }^{7}$, Deliens L. ${ }^{2}$, Chambaere K. ${ }^{2}$

${ }^{1}$ Ghent University, Ghent, Belgium, ${ }^{2}$ Department of Public Health and Primary Care, End-of-Life Care Research Group, Ghent University, Ghent, Belgium, ${ }^{3}$ Public Health and Primary Care, Ghent University, Ghent, Belgium, ${ }^{4}$ Research Foundation Flanders (FWO), Brussels, Belgium, ${ }^{5}$ Internal Diseases, Ghent University, Ghent, Belgium, ${ }^{6}$ Respiratory Diseases, Ghent University Hospital, Ghent, Belgium, ${ }^{7}$ Medical Oncology, Ghent University Hospital, Ghent, Belgium 
Background: While studies suggest early integrated palliative home care (PHC) to be beneficial for COPD patients, trials testing implementation are scarce.

Aim: To study early integrated PHC for end-stage COPD.

Methods: We pilot tested feasibility, acceptability and preliminary effectiveness during a six-month RCT on early integrated PHC for end-stage COPD. Intervention patients received monthly visits from trained PHC nurses. They offered information leaflets on coping and followed a protocol (disease insight, care planning, symptom, psychosocial, spiritual, carer's support, care and action plan) of which a report was sent to involved health professionals after each visit. Every 6 weeks, we assessed quality of life (SF-36) and care (PACIC), symptoms (CAT-COPD), mood (HADS), caregiver contacts, hospitalisations, living will decision. Intervention patients and their carers, general practitioners (GPS), pulmonologists (PLs) and PHC nurses were interviewed for a post-trial evaluation.

Results: $56 \%$ (39 of 70) of eligible patients participated, with a $20: 19$ intervention-control ratio. $64 \%$ completed the trial. $80 \%$ of intervention patients had at least $3 \mathrm{PHC}$ visits, which were predominantly focusing on disease insight, symptom management and care planning. $100 \%$ of reports of PHC visits were sent to involved health professionals. Most patients and carers were positive about the trial, referring to the ongoing support, helpful breathing exercises and care decisions. Some GPs had doubts about the early timing and were not well aware of the trial, while PLs and PHC nurses thought the trial was acceptable/useful, in particular the leaflets and the protocol. Preliminary effectiveness will be presented at the congress.

Conclusion: Early integration of PHC for end-stage COPD seems feasible and was accepted by patients, carers, PLs, PHC nurses, and to a lesser extent GPs. Future research should address optimization of trial design, care integration, and involvement of GPs.

Abstract number: P01-448

Abstract type: Poster

The Last Phase of Life of Patients with an Implantable Cardioverter Defibrillator (ICD): Results of a Survey among Bereaved Relatives

Stoevelaar R. ${ }^{1}$, Brinkman-Stoppelenburg A. ${ }^{1}$, Bhagwandien R.E. ${ }^{2}$, van

Bruchem-Visser R.L. ${ }^{3}$, Theuns D.A.M.J. ${ }^{2}$, van der Heide A. ${ }^{1}$, Rietjens

J.A.C. ${ }^{1}$

${ }^{1}$ Public Health, Erasmus University Medical Center Rotterdam, Rotterdam, Netherlands, ${ }^{2}$ Cardiology, Erasmus University Medical Center Rotterdam, Rotterdam, Netherlands, ${ }^{3}$ Internal Medicine, Erasmus University Medical Center Rotterdam, Rotterdam, Netherlands

Background: The Implantable Cardioverter Defibrillator (ICD) is effective in treating life-threatening arrhythmias. However, when patients are at the end of life, they might want to deactivate their ICD to avoid shocks. Aims: To study the last phase of life of ICD patients, with regard to decision-making about ICD deactivation and quality of care and dying, from the perspective of bereaved relatives.

Methods: Relatives of patients in two Dutch hospitals who had deceased between 2012-2017 received a written survey in 2017/2018 about the last phase of life of the patient.

Results: In total, 170 (69\%) of 247 invited relatives completed the survey. Relatives had a median age of 62 and were mostly female (78\%), $52 \%$ were the spouse of the patient. Patients died at a median age of 74 and were mostly male (87\%). Thirty-four percent of relatives reported to have discussed ICD deactivation with the patient, and $38 \%$ reported that patients had discussed this with a professional caregiver. In $55 \%$ of patients, the ICD was deactivated. When not deactivated, $24 \%$ of patients had experienced one or more shocks in the last month of life and $10 \%$ on the last day of life, leading to pain $(57 \%)$ and anxiety (52\%) in patients, and to worrying $(78 \%)$ and feelings of helplessness $(57 \%)$ in relatives. Of the relatives, $42 \%$ agreed that the way the professional caregiver had informed them about ICD deactivation was sufficient, and
$52 \%$ agreed that the professional caregiver had provided good emotional support. Overall, the quality of dying was rated with a mean score of 6.4 out of 10 .

Conclusion / Discussion: ICD deactivation was discussed with a minority of patients. ICDs were deactivated in about half of the patients. Shocks in patients with an active ICD in the last month of life were not uncommon, leading to distress in patients and relatives. Timely and recurring conversations about benefits and drawbacks of the ICD have the potential to contribute to quality of life in the last phase of life. Funding: ZonMw.

\section{Abstract number: P01-449 \\ Abstract type: Poster}

Patients' Perspectives on Implantable Cardioverter Defibrillator (ICD) Deactivation at the End of Life: A Qualitative Focus Group Study

Stoevelaar R. ${ }^{1}$, Brinkman-Stoppelenburg A. ${ }^{1}$, Bhagwandien R.E. ${ }^{2}$, van

Bruchem-Visser R.L. ${ }^{3}$, Theuns D.A.M.J. ${ }^{2}$, van der Heide A. ${ }^{1}$, Rietjens

J.A.C. ${ }^{1}$

${ }^{1}$ Public Health, Erasmus University Medical Center Rotterdam, Rotterdam, Netherlands, ${ }^{2}$ Cardiology, Erasmus University Medical Center Rotterdam, Rotterdam, Netherlands, ${ }^{3}$ nternal Medicine, Erasmus University Medical Center Rotterdam, Rotterdam, Netherlands

Background: Implantable Cardioverter Defibrillator (ICD) shocks can negatively influence the last phase of life. Guidelines recommend to timely discuss ICD deactivation with the patient, and to deactivate the device prior to death.

Aims: To examine patients' perspectives on ICD deactivation in the last phase of life.

Methods: Patients were recruited via the national ICD patient association in the Netherlands. Focus groups were conducted using a predefined topic list and were audiotaped and transcribed. Transcripts were analyzed using the constant comparative method.

Results: Forty-one patients participated in a total of five focus groups. Average age was 64 years and $56 \%$ of patients were male. Patients received their ICD on average 6 years before, in $59 \%$ for primary prevention. Several patients expressed a need for more information from their physician about ICD deactivation and some indicated that nurses could play a bigger role in this process. Some other patients were satisfied about how they were informed, or did not want any information, because they did not want to think about the end of life. Although most patients agreed that it would be appropriate to deactivate the ICD on a certain point in the disease trajectory, they had difficulties deciding on the right moment. Possible circumstances in which patients would consider deactivating their ICD in order to avoid shocks were: a terminal stage of their disease, and a diminished quality of life. Arguments against deactivation were also mentioned, and mainly concerned not wanting to give up on life, both for themselves and for their family.

Conclusion / Discussion: There is room for improvement in informing patients about ICD deactivation in the last phase of life.

We found that patients' perspectives towards ICD deactivation were highly personal and sometimes ambivalent. This emphasizes the importance of early and recurring discussions on this topic.

Funding: ZonMw.

Abstract number: P01-450

Abstract type: Poster

Investigation of Optimal Timing for Palliative Care Referral in Patients with Chronic Heart Failure: Predictors for Prognosis and Readmission in Patients with Chronic Heart Failure

Ohmori.$^{1}{ }^{1}$, Imamura $\mathrm{Y}^{2}$, Tagami $\mathrm{K}^{3}$, Kashiwagi H. ${ }^{1}$, Inoue $\mathrm{S}^{4}$

${ }^{1}$ Palliative Care, ASO lizuka Hospital, lizuka, Japan, ${ }^{2}$ Heart Failure Care, 
Aso lizuka Hospital, lizuka, Japan, ${ }^{3}$ Palliative Care, Tohoku University School of Medicine, Sendai, Japan, ${ }^{4}$ Cardiology, ASO lizuka Hospital, lizuka, Japan

Background: Heart failure (HF) is the largest cause of death and suffering, and some international guidelines have acknowledged the importance of palliative care $(\mathrm{PC})$ intervention. It has been indicated that $\mathrm{HF}$ patients with poor prognosis or frequent readmission may require $\mathrm{PC}$, but their predictors have not been clarified.

Aims: This study aimed to identify the factors that would predict death or frequent readmission in HF.

Methods: We retrospectively enrolled 475 consecutive patients (age77.7 $\pm 12.4, M / F=245 / 230$ ) discharged with a principal diagnosis of HF between 2013 and 2015 and observed at a single tertiary medical center. Clinical and laboratory data were collected at discharge and serially at three month intervals. We investigated death or frequent readmissions (more than twice per 6 months), and only the earliest event of them was counted per person.

Results: During a mean follow-up period of 22.2 months, the events were 45 deaths (31 cardiac, 14 non-cardiac) and 47 frequent re-admissions due to worsening HF. Multivariate analysis (COX proportional hazard model) showed that declining estimated glomerular filtration rate (eGFR cut-off value of $<28.7 \mathrm{ml} / \mathrm{min} / 1.73 \mathrm{~m} 2$ ) and age (age cut-off value of $<91$ years old) were independently associated with death or frequent readmissions, however cardiac function or other laboratory data were not. When eGFR fell below $28.7 \mathrm{ml} / \mathrm{min} / 1.73 \mathrm{~m} 2$, events occurred at a mean interval of 345 days.

Conclusion: Age and Serial measurement of eGFR would be useful to predict future readmission and death. It may be useful to determine the appropriate timing for initiating PC.

\section{Abstract number: P01-451}

Abstract type: Poster

The Presence of Palliative Care in a Intensive Care Cardiology Unit. Five-year Experience Report in a Private Hospital in Rio de Janeiro

Fonseca A.C. ${ }^{1}$, Allão C. $^{1}$, Montenegro F. ${ }^{2}$

${ }^{1}$ Study Center, Adventis Silvestre Hospital, Rio de Janeiro, Brazil,

${ }^{2}$ Coronary Unit, Adventis Silvestre Hospital, Rio de Janeiro, Brazil

Background: Heart Disease is one the most cause of death in Brazil. As the focus of treatment is the quality of life, Palliative Care (PC) is essential for the heart disease, essencial cardiac insuficient. Symptom control is one of the focus and therefore the joint monitoring between a PC and the cardiology team is necessary. The share of responsibilities of care, the organization of team, correct technique about PC, safety care plan for the patient and, finally, the satisfaction of the patient and family, all of them are the objectives of this joint monitoring.

Aims: To demonstrate the experience of 5 years of services between the team of Intensive care Cardiology Unit (ICCU) and the PC team.

Method: Retrospective analysis of patients followed between January 2013 and September 2018.

Results: Were reviewed 144 cases; mean age 79 years; Mean PPS of 50; $60 \%$ women; $75 \%$ of patients with ischemic disease with other comorbidities, such as advanced dementia syndrome; an average of 2.5 familiar conferences were held per month, and during the period, weekly activities of technical training of the team of the ICCU, with theoretical support and space for discussion of cases, led by the PC team; $40 \%$ alive and $25 \%$ of families received some kind of support after one month the death.

Discussion: Success is building a relationship of trust between the professionals of each team and organizing the care plan. The biggest challenges were to align schedules both for meetings between professionals and families, to enable them to stay in the unit and to technically train Uco's employees regarding prescription drugs and nursing care.
Conclusion: The partnership in the care delivery contributed to a better control of symptoms, greater safety on the part of the ICCU professionals and the greater satisfaction of the relatives in relation to the proposal of care.

Abstract number: P01-452

Abstract type: Poster

Embedding Advance Care Planning into Everyday Practice for People with Heart Failure

Edmunds L., Hazel K., Brooks J., Hockey D., Cross S., Palfrey C.

Aneurin Bevan University Health Board, Abergavenny, United Kingdom

Aim: To improve palliative and supportive care for people living with heart failure.

Design: Due to the unpredictable disease trajectory of heart failure many patients do not receive supportive palliative care in a timely manner. Heart failure nurse specialists (HFNS) often find themselves trying to provide care but with a degree of uncertainty of patients wishes. Following the death of a patient cared for by the Aneurin Bevan University Health Board (ABUHB), the HFNS reflected on practice and identified that there were issues around communication and Advance Care Planning (ACP).

Method: This led to the development of a program of activities to address these issues.

1. Training was undertaken in basic and advanced communication, ACP e-learning and workshops.

2. A Supportive and Palliative Care Indicator tool (SPICT) was introduced to HFNS assessments to help guide the introduction of ACP.

Approach: Education and peer support were used to introduce SPICT tool in practice helping to identify when to introduce ACP.

Results; Confidence to introduce ACP was assessed pre and post training, a qualitative focus group analysed, and audit of the use of SPICT and ACP in practice undertaken. HFNS confidence increased, the communication skills training provided a framework and the SPICT a structure to their assessment helping identify the need for ACP. There has been an increased use of ACP with heart failure patients and HFNS now consider ACP to be embedded in practice as can be seen from these two quotes from the focus group:

Voice 1- "The ACP is part of the parcel of what I talk about in Heart failure care",

Voice 2- "We've just embedded it (ACP), it's just there now.

Conclusions: Providing a structured education program and tools to support clinical assessment has led to an increase of ACP for people living with failure patients.

\section{Abstract number: P01-453 \\ Abstract type: Poster}

Palliative Care Need in Patients with Advanced Heart Failure Hospitalized in a Tertiary Hospital

Campos L. ${ }^{1,2}$, Orzechowski R. ${ }^{3}$, Galvão A.L. ${ }^{4}$, Nunes T. ${ }^{3}$, Barros N. ${ }^{3}$ ${ }^{1}$ Hospital Nossa Senhora da Conceição, Porto Alegre, Brazil, ${ }^{2}$ Instituto de Saúde Pública, Universidade do Porto, Porto, Portugal, ${ }^{3}$ Serviço de Dor e Cuidados Paliativos, Hospital Nossa Senhora da Conceição, Porto Alegre, Brazil, ${ }^{4}$ Serviço de Cardiologia, Hospital Nossa Senhora da Conceição, Porto Alegre, Brazil

Background: While the number of deaths from heart disease has not changed substantially since 1990 in developed countries, there has been a $66 \%$ increase in deaths in low- or middle-income countries. Because of disease characteristics, patients frequently require Palliative Care (PC) in 
order to relieve suffering and improve the quality of life. Among chronic diseases, Congestive Heart Failure (CHF) has the highest rate of rehospitalization and mortality, which limits the life expectancy.

Objectives: To evaluate the need for palliative care in patients with advanced Congestive Heart Failure (CHF) hospitalized in a cardiology ward.

Methods: Application of the World Health Organization Palliative Needs tool (NECPAL) with the assistant physician, patient and/or caregiver for evaluation of indication of Palliative Care (PC).

Results: 82 patients with a diagnosis of class III/IV Heart Failure or ejection fraction less than or equal to $40 \%$ in echocardiography of the last 12 months were included: Mean age $68 \pm 20$ years, 51 male patients and 31 female patients. Forty-three patients $(52.4 \%)$ were married or in consensual union and ten (12\%) lived alone. The death of 46 patients (56.1\%) in the subsequent 12 months would not surprise their physician, and forty-five patients $(55 \%)$ had palliative care indication according to the NECPAL.

Conclusion: About half of patients hospitalized for class III/IV Heart Failure would have an indication of Palliative Care for the relief of suffering caused by the disease.

Abstract number: P01-454

Abstract type: Poster

\section{Equal Palliative Care for COPD and Lung Cancer Patients? A} Register Study

Strang P. ${ }^{1}$, Adlitzer H. ${ }^{2}$, Schultz T. ${ }^{3}$

${ }^{1}$ Oncology-Pathology, SSH, Karolinska Institutet, Stockholm, Sweden, ${ }^{2}$ RCC Regional Cancer Centre Stockholm - Gotland, Stockholm, Sweden, ${ }^{3}$ Statistics and Development Dept, Stockholm County Council, Stockholm, Sweden

Background: Dying COPD patients have significant comorbidity, and a symptom burden with breathlessness, weakness, fatigue, pain, nausea, anxiety and depression, comparable with the situation for lung cancer (LC) patients. Still they have limited access to palliative care.

Aim: To use regional data registers to study and compare COPD and LC patients as regards care consumption, access to palliative care and, whether access to palliative care translates in diminished need of acute hospital care.

Method: To retrieve data about care consumption from Stockholm County council (covering 2.2 million inhabitants) central data warehouse, for patients who died of COPD or lung cancer during 2014-2017.

Results: Data from 2159 COPD patients and 2588 lung cancer patients were retrieved (total populations) and a preliminary analysis was performed. Whereas only $10.1 \%$ of COPD patients had used any palliative home care service during the last 3 months of life, the corresponding figure for LC patients was $57.8 \%$ of $(p<0.0001)$. For COPD patients who had or had not access to any form of palliative care service, the need of emergency room visits was $48.8 \%$ and $68.3 \%(p<0.0001)$, respectively whereas the corresponding figures for LC patients were $38.7 \%$ and $68.0 \%(p<0.0001)$. As regards hospital admissions during the last month of life for those with or without access to palliative services, $44.7 \%$ and $64.7 \%$ of the COPD patients were hospitalised at least once $(p<0.0001$ ). A total of $13.6 \%$ of COPD patients and $66.0 \%$ of $L C$ patients died at a palliative care service (home care or palliative incare) $(p<$ 0.0001).

Conclusion: Despite a huge symptom burden, COPD patients have significantly less access to palliative care services during the dying phase. For those patients who are enrolled in palliative care services, the utilization of emergency rooms as well as admissions to acute hospitals is significantly lower than for other patients.

Abstract number: P01-456

Abstract type: Poster
Delirium and Distressing Movements: Palliative Sedation Therapy in Patients with Refractory Terminal Delirium when Standard Treatment Options are Limited

Partain D. ${ }^{1}$, Kamdar M. ${ }^{2}$, Zehm A. ${ }^{2}$

${ }^{1}$ Center for Palliative Medicine, Mayo Clinic, Rochester, United States, ${ }^{2}$ Palliative Care and Geriatric Medicine, Massachusetts General Hospital, Boston, United States

Background: Palliative sedation therapy (PST) is used to treat refractory symptoms from terminal illness, including pain and agitated terminal delirium. Little is known about using PST for terminal delirium in patients with advanced movement disorders when traditional palliative therapies are contraindicated. Here, we discuss a case in which PST was used for this challenging clinical scenario.

Aim:

1. Review practice guidelines and indications for palliative sedation therapy (PST).

2. Recognize ethical considerations for using PST for refractory delirium in a patient with a terminal movement disorder that limits treatment options.

3. Develop best practices for implementing PST for managing terminal delirium in the setting of an advanced neurodegenerative movement disorder.

Case: Ms. P was a 59-year-old woman with refractory bipolar affective disorder who developed progressive drug-induced Parkinsonism and akathisia from her anti-psychotics. Despite discontinuation of her medications and use of carbidopa/levodopa, her functional status declined over several months due to dementia with Lewy bodies (DLB). She was admitted to the hospital with refractory Parkinsonism, akathisia, and progressive cognitive decline that left her profoundly debilitated and nutritionally compromised. In the setting of end-stage dementia with limited prognosis, her surrogate decision makers requested comfort-focused care. The patient developed severe terminal delirium. Traditional dopablockade was contraindicated due to Parkinsonism/akathisia and multiple other interventions were unsuccessful. Given her profound refractory delirium and limited treatment options, PST was initiated for comfort.

Discussion: PST is used for refractory terminal delirium, but unique challenges arise when an underlying illness restricts usual treatment options. In this discussion, we review the practice of PST, including indications, current guidelines, and ethics in this difficult situation.

No funding received.

Abstract number: P01-457

Abstract type: Poster

A Systematic Literature Review to Identify and Assess Current Evidence Concerning the Impact of Parkinson's Disease on Mutuality/Quality of Interaction in Marital Couples with the Goal of Developing a Palliative Approach for Patients and their Caregiving Spouse

Arshinoff $R^{1}{ }^{1}$, Grinyer A. ${ }^{2}$, Preston N. ${ }^{2}$

${ }^{1}$ Religious and Spiritual Care, Baycrest, Toronto, Canada, ${ }^{2}$ Faculty of Heath and Medicine, Lancaster University, Lancaster, United Kingdom

Introduction: In contrast to the holistic approach of palliative care, medical visits for Parkinson's Disease (PD) patients focus primarily on physical symptoms with less attention on psychosocial effects of PD. As illness advances, dependence on others occurs, in most cases on the spouse. Little appears in the literature about mutuality, which concerns the quality of the relationship, in PD especially in advanced illness.

Aims: To identify and assess current evidence concerning the impact of PD on mutuality/quality of interaction in marital couples using an integrative systematic review. The goal was to assist in developing holistic and palliative approaches to PD patients and their caregiving spouse. 
Methods: Key terms were Parkinson's Disease; mutuality; spouse; common Law; caregiver, as well as terms depicting relational interactions and emotions were also used. Databases searched were Medline, CINAHL, Embase, CINAHL, PsychInfo, Cochrane, Scopus, and Web of Science.

Results: Twenty-eight studies were included in the synthesis. The seven themes elicited were: mutuality decreases with disease progression; many losses; living with a stranger; length of caregiving time; feeling trapped; optimism as protection of mutuality; need for personal coping strategies; sexuality and intimacy. Most papers focused on clinical symptoms and treatment with some research on burden of care but far less on mutuality. Studies on mutuality focus on early to mid-stage illness with none conducted specifically on advanced illness; as illness advances and patients require a palliative approach to their care, clinicians are challenged to understand palliative needs of the family unit.

Conclusion: This review has indicated a need for work in the area of advanced PD to inform development of palliative approaches for the patient and family to chronic and degenerative illnesses.

Abstract number: P01-458

Abstract type: Poster

\section{The DIgnity in Advanced DEMentia (DAIDEM) Study: Quality of Life and Caregiver Burden of Home-dwelling Persons with Dementia \\ Tay R.Y. ${ }^{1}$, Ali N.B. ${ }^{2}$, Leong I. ${ }^{2}$, Wu H.Y. ${ }^{2}$, Chin J.J. ${ }^{2}$, Lee A. ${ }^{3}$, Koh M. ${ }^{2}$, Hum \\ A. ${ }^{2}$}

${ }^{1}$ Dover Park Hospice, Singapore, Singapore, ${ }^{2}$ Tan Tock Seng Hospital, Singapore, Singapore, 33aint Andrew's Community Hospital, Singapore, Singapore

Background: Advanced dementia patients frequently transition from the home to institution at the end of life as care at home is inadequate in the absence of an integrated geriatric palliative homecare service.

Aims: To examine the baseline quality of life (QoL), symptoms and familial caregiver burden of patients recruited into an integrated geriatric palliative homecare program.

Methods: This is a prospective cross-sectional study. Patients at Stage 7 on the Functional Assessment Staging Tool with pneumonia, albumin $<$ $35 \mathrm{~g} / \mathrm{L}$ and/or enteral feeding were recruited into the program from October 2014 to August 2018. Dementia-specific scales assessed patients' QoL [(Quality of Life in Late-Stage Dementia (QUALID)], symptoms [Pain Assessment in Advanced Dementia (PAINAD), Neuropsychiatric Inventory Questionnaire (NPI-Q), Mini Nutritional Assessment (MNA)] and caregiver burden [Zarit Burden Interview (ZBI)]. Data was described using descriptive statistics. Mann-Whitney $U$ test was used for between group comparisons while Spearman Rank Correlation examined variable relationships.

Results: Among the 340 recruited patients [median age 87 (IQR 83-93) years; Chinese $(86.2 \%)$; female $(71.8 \%)]$, pain $(54.1 \%)$, neuropsychiatric symptoms $(80.2 \%)$ and malnutrition $(92.3 \%)$ were prevalent. The median QUALID score was 23 (IQR 19-27) which correlated with PAINAD and NPI-Q $(p<0.0001)$. Tube feeding increased acute healthcare resources utilization and lowered QoL ( $p<0.0001$ ). Median ZBI score was 25 (IQR 15-36.5) with higher burden experienced by those staying with patients [median=27 (IQR 15-38)] and without domestic help [median=40.5 (IQR 25-49.8)], $p<0.05$.

Conclusion: An integrated homecare service aims to support caregivers to address the needs and QoL of advanced dementia patients at home. Its close collaboration with the hospital and hospice allows seamless transition across the 3 settings if needed. Future studies will examine the clinical outcomes and health economics of this care model.

Abstract number: P01-459

Abstract type: Poster
Challenges of Palliative Care Provision to People with Dementia (PWD) in a Community-based Psychogeriatric Program (CPGP) Tan L.L., Sim A., Ng W., Chan F., Yik P., Noor N., Rahim S.

Psychological Medicine, Changi General Hospital, Singapore, Singapore

Aim: With Singapore's aging population, there has been an increase in PWD requiring palliative care support. CPGP was set up to improve the mental health of older persons who are unable to access hospital or outpatient services. Many of the clients have moderate to severe dementia with unique palliative care needs. A participatory action research was initiated by CPGP staff to explore how home-based palliative care provision to PWD can be improved.

Design: An action research method was adopted to involve staff in processes whereby they have an opportunity to reflect and critique their practice. 6 CPGP staff participated in a recurring helical cycle with problem identification, planning, taking action, collecting data, analysis, reflection and replanning. Four meetings were convened over 6 months to complete the first action cycle. Audio recordings and meeting notes were analyzed to capture reflections and strategies of staff.

Results: The concept of 'palliative care approach' was unclear and staff held different views of whether dementia should be viewed as a terminal illness. There were different considerations of when and how to initiate advanced care planning (ACP). However, there was agreement on the need to assess for readiness to engage in ACP and end-of-life care discussions. A checklist was created to facilitate care planning discussions. Staff was also able to identify individual learning needs and knowledge gaps and sought out training workshops to overcome some of the challenges in their delivery of palliative care support.

Lessons learned: The action research approach was effective in facilitating staff involvement and learning in palliative care provision to PWD. The collaborative nature with its flexibility and circular process supported action with change and improvement. Further analysis of the use of the checklist and group meetings for reflections and learning will help to facilitate the adoption of the palliative care approach in PWD.

Abstract number: P01-460

Abstract type: Poster

Atittudes towards End of Life Care of Patients with Multiple Sclerosis: Cross-sectional Study

Bužgová R. ${ }^{1}$, Kozáková $R .{ }^{1}$, Bar M. ${ }^{2}$, Ressner $P^{2}{ }^{2}$, Škutová M. ${ }^{2}$, Bártová $P{ }^{2}$ ${ }^{1}$ Department of Nursing and Midwifery, University of Ostrava, Ostrava, Czech Republic, ${ }^{2}$ Neurology Clinic, The University Hospital Ostrava, Ostrava, Czech Republic

Background: On the basis of literary research, no research assessing the attitudes and opinions of multiple sclerosis (MS) patients on end-of-life care is available.

Aims: The aim of the research was to determine MS patients' attitudes to end-of-life care, and to compare them according to demographic data, duration and progression of disease.

Methods: 133 patients with MS meeting the following criteria were included in this cross-sectional study: > six months after diagnosis, age $>18$ years, MMSE $\geqslant 24$ points. The ELC-PND (The Attitudes and Opinions of Patients with a Progressive Neurological Disease to End-of-Life Care Questionnaire), containing 33 questions divided into four domains, was used to collect data. For data analysis the Kruskal-Wallis test and the Spearman correlation coefficient were used.

Results: MS patients reported a higher level of agreement $(p<0.001)$ with the idea of control over the end of life than with keeping patients alive at all costs (e.g., introduction of artificial nutrition, artificial pulmonary ventilation). Wanting control of the end of life was reported most often by widowers $(p=0.0389)$. Forty-one percent of patients (most often patients over 40 years $(p=0.044)$; and without university education $(p=0.036)$ agreed with having "medicine available to terminate one's life". Conversely, $24 \%$ of patients would prefer to maintain life at any 
cost. A negative correlation was found between favouring keeping patients alive, and duration of disease $(r=-0.197 ; p=0.023)$. Ninety percent of patients wished to be informed of prognosis and end-of-life care as early as at the onset of the disease. Patients with the disease for longer than seven years $(p=0.324)$ most often favoured full awareness of families from the onset of the disease. Most patients (75\%) wished to die at home.

Conclusion: Communication on end-of-life care in MS patients should become an important part of care when introducing the concept of neuropalliative care in the Czech Republic.

\section{Abstract number: P01-461 \\ Abstract type: Poster}

Palliative Care: Flowchart of Acute Neurological Illness in a University Hospital

Rocha J.A. ${ }^{1,2}$, Carvalho R.T. ${ }^{1}$

${ }^{1}$ Palliative Care, Hospital das Clínicas, University of São Paulo, São

Paulo, Brazil, ${ }^{2} 1$ Hospital das Clínicas, University of São Paulo, São Paulo, Brazil

Background: In devastating brain lesions, uncertainty about prognosis is a determining factor that hinders the management of these patients. The lack of neurological improvement most of the time becomes frustrating for the assistant team and very painful for the family. After long hospitalization, the patient is affected by multiple infections and failure of antibiotic therapy. Therewere, it is necessary to create care planning strategies in acute neurological disease of poor prognosis(DNAMP).

Aims: To elaborate a flow chart that meets these needs.

Methods: The flowchart consists of:

1) Clinical and laboratory evaluation of patients based on established criteria.

2) Define the diagnosis of DNAMP shortly after the first 72 hours of the event, prognosis, therapeutic and care planning in a meeting shared with the family.

3) To Perform an early tracheostomy.

4) To delimit the advanced support of life in a patient with serious sequels without proposal modifying the disease. This includes limiting the use of antimicrobials due to therapeutic failure, standardized in this study as the use of several cycles of antibiotics (staggered up to carbapenem and vancomycin).

5) Define adequate allocation according to the clinical evolution.

Results: The expected results are:

1) Reduce the mechanical ventilation time after the early tracheostomy

2) Reduce the infection associated with mechanical ventilation

3) Reduce the length of ICU stay.

4) Determine adequate allocation depending on the clinical evolution aiming the removal of high complexity beds.

5) Establish the appropriate use of technological resources

6) Reduce prolonged hospitalization costs.

Discussion: The implementation of a flowchart for the management of patients with DNAMP avoids the irrational use of technological resources and promotes treatment proportional to the clinical condition, adequate control of symptoms, better allocation and more humanized care, often meeting the expectations of families.

Abstract number: P01-462

Abstract type: Poster
Efficacy and Safety of Low-dose Morphine in Patients with Amyotrophic Lateral Sclerosis

Nakayama T., Kumakura Y., Kotoda M., lijima T., Nakajima E., Akita K., Matsukawa T.

Anesthesiology, University of Yamanashi, Chuo, Japan

Background: Dyspnea is one of the most stressful symptoms in patients with amyotrophic lateral sclerosis (ALS). Although morphine has been commonly used to mitigate the dyspnea, proper doses of morphine in patients with ALS remain unknown.

Aims: The purpose of this study was to investigate starting and maintenance doses of morphine, and the incidence of side effects of morphine in patients with ALS.

Methods: A retrospective review was conducted in 1966 patients followed by the palliative care team in the University of Yamanashi Hospital between January 2013 and December 2017. A total of 15 patients who presented with dyspnea due to ALS were involved in this study. The doses, efficacy, and incidence of side effects of morphine treatment were analyzed.

Results: Five patients had already used morphine for dyspnea at the time of consultation. Another patient had been prescribed morphine but had not been taking it. The remaining 9 patients started morphine treatment during the study period. The prescribed doses of morphine were effective in 8 out of the 9 patients. The starting doses of morphine for the 9 patients were $3.8 \pm 1.2 \mathrm{mg} /$ day, and the maintenance doses for the 15 patients were $20.7 \pm 20.9 \mathrm{mg} /$ day. Narcosis occurred in 3 out of the 15 patients. One out of the 3 patients presented with narcosis after we prescribed morphine; however, the relationship between narcosis and morphine treatment was not clear. Of the 9 patients who started morphine treatment during the study period, 3 developed drowsiness and another 3 presented with constipation. No patients presented with nausea.

Conclusions: In the present study, morphine treatment effectively attenuated dyspnea in patients with ALS. However, it should be noted that respiratory depression easily occurred in these patients, possibly due to their impaired respiratory muscle function. This study would suggest that morphine treatment for dyspnea in patients with ALS should be started at low doses.

\section{Abstract number: P01-463}

Abstract type: Poster

"Are you Seeing what I'm seeing?" How Does the Literature Conceptualise (dis)Comfort in People with Dementia? A Systematic Review

Hindocha C.N. ${ }^{1}$, Sampson E.L. ${ }^{1,2}$, Moore K.J. ${ }^{1}$

${ }^{1}$ Division of Psychiatry, University College London, London, United Kingdom, ${ }^{2}$ Barnet, Enfield and Haringey Mental Health Trust, London, United Kingdom

Background: Comfort is multifaceted and the main goal of palliative care. Its presence may not be clear, particularly in people with advanced dementia. Comfort is an individual experience that others find difficult to interpret. The term 'comfort' is commonly used but we lack clarity in how it is defined, identified and managed. It is often equated with absence of pain.

Aim: To explore how the current literature conceptualises (dis)comfort in people with dementia.

Methods: Searches were performed on MEDLINE, EMBASE, CINAHL and PsycINFO using keywords for dis(comfort) and dementia. The inclusion criteria included qualitative and quantitative studies with a minimum $50 \%$ of participants who had dementia which measured (dis)comfort in dementia that were published from 1980 to present. The main outcome was dis(comfort). Abstracts were reviewed by the first author and two independent reviewers checked $15 \%$ of abstracts. We used the Critical 
Appraisal Skills Programme checklist for quantitative data and the COnsolidated criteria for REporting Qualitative research. Narrative synthesis was performed due to the heterogeneity of the included articles. Results: We identified 1284 studies from nine countries, 37 met eligibility criteria (five qualitative and 32 quantitative). Two qualitative and six quantitative studies defined (dis)comfort. All definitions of (dis)comfort were based around emotional, physical (the most common theme) and environmental factors. Although the studies measured discomfort, many failed to examine the source of discomfort. We found that a lack of education and poor communication among caregivers, physicians and relatives often contributed to poor detection of (dis)comfort.

Conclusion: Although some studies defined (dis)comfort, the source of it in many people is unknown. Further research is needed to identify sources of (dis)comfort to help improve the wellbeing of people with advanced dementia.

This studentship is funded by Healthcare Management Trust.

Abstract number: P01-464

Abstract type: Poster

Refining the Namaste Care Intervention for People with Advanced Dementia Prior to a Feasibility Cluster Randomised Trial: A Novel Four Stage Process

Walshe C. ${ }^{1}$, Kinley J. ${ }^{2}$, Patel S. ${ }^{1}$, Goodman C. ${ }^{3}$, Bunn F. ${ }^{3}$, Lynch J. ${ }^{3}$, Scott D. ${ }^{4}$, Davidson Lund A. ${ }^{4}$, Stacpoole M. ${ }^{2}$, Preston N. ${ }^{1}$, Froggatt K. ${ }^{1}$ ${ }^{1}$ International Observatory on End of Life Care, Lancaster University, Lancaster, United Kingdom, ${ }^{2}$ Previously of St Christopher's Hospice, London, United Kingdom, ${ }^{3}$ Centre for Research in Primary and Community Care, University of Hertfordshire, Hatfield, United Kingdom, ${ }^{4}$ Patient and Public Representative, Lancaster, United Kingdom

Background: Palliative care can be important for people with advanced dementia, but there are few effective interventions. Namaste Care is a multi-component intervention for people with advanced dementia, delivered in care home, community and hospice settings. Implementation into practice has started, but without evidence of effect being determined.

Aims: To describe the development of an intervention description, manual and training package to support implementation of Namaste Care within the context of a feasibility trial.

Methods: A four stage approach:

i) Collating existing intervention materials and drawing from programme theory developed from a realist review to draft an intervention description.

ii) Exploring readability, comprehensibility and utility with Namaste Care naïve staff.

iii) Using modified nominal group techniques with those with Namaste Care experience to refine and prioritise the intervention implementation materials.

iv) Final refinement with a patient and public involvement panel.

Results: 18 nursing home staff, 1 carer, 1 volunteer and 5 members of our public involvement panel participated. A 16 page A4 booklet was produced, with flow charts, graphics and colour coded information to ease navigation through the document. This was supplemented by infographics, and a training package. The guide describes the boundaries of the intervention and how to implement it, whilst retaining the flexible spirit of the Namaste Care intervention.

Conclusions: Interventions in palliative care can be rapidly developed, poorly specified, or implemented without evidence. This four stage process may have utility for others to adapt or fully specify interventions before testing. This should enhance fidelity, and facilitate practice implementation.
TRIAL REGISTRATION: ISRCTN14948133 FUNDER NIHR HTA

Abstract number: P01-465

Abstract type: Poster

A Trial of Suprascapular Nerve Block for Shoulder Pain in Motor Neuron Disease

Allcroft P. ${ }^{1,2}$, Shanahan E.M. ${ }^{3}$, Glaetzer K. ${ }^{2}$, Gill T. ${ }^{4}$, Hill C. ${ }^{5}$, Graff S. ${ }^{3}$

${ }^{1}$ Flinders University, Adelaide, Australia, ${ }^{2}$ Palliative Care, Southern Adelaide Local Health Network, Adelaide, Australia, ${ }^{3}$ Rheumatology, Flinders University, Adelaide, Australia, ${ }^{4}$ Biostatistics, University of Adelaide, Adelaide, Australia, ${ }^{5}$ Medicine, University of Adelaide, Adelaide, Australia

Background: Shoulder pain is a distressing but under-reported and poorly managed symptom in people with motor neuron disease (MND). Medications utilised for pain management have a range of side-effects, which can have a negative effect on quality of life.

Aims: This study aimed to quantify the prevalence of shoulder pain in people with MND and assess the efficacy of suprascapular nerve block (SSNB) for the management of this symptom.

Methods: The study was made up of two parts. In the first part of the study 49 consecutive patients from a dedicated MND clinic were surveyed as to the presence of clinically significant shoulder pain. The rate of shoulder pain was compared with an age and gender matched cohort. 27 patients with significant shoulder pain were then offered a SSNB to manage the pain. 10 of the patients had bilateral shoulder pain and both were injected making a total of 37 shoulders. The patients were followed for a total of three months, or until death. Shoulder pain was measured using the pain scale (out of 100) of the shoulder pain and disability index (SPADI) and compared with baseline scores and a placebo control group from an earlier study using the same methodology for the nerve block. Results: The prevalence of shoulder pain in the three months prior to the survey was $51 \%$ (Cl $37.5-64.4)$ compared with a rate of pain of $11.7 \%$ (Cl 10.3-31.1) from the community cohort. Following the SSNB there was a statistically and clinically significant improvement of pain scores from baseline (58.4) at week 1 (20.8, p < 0.000), week $6(17.6, p<0.000)$ and week $12(30.4, p 0.001)$ and a clinically and statistically significant improvement compared with the control group across each time interval.

Conclusions: Approximately $50 \%$ of patients with motor neuron disease suffer from shoulder pain. SSNB is a safe, effective therapy for the management of shoulder pain in people with this disease.

Abstract number: P01-466

Abstract type: Poster

What Are the Hopes of Family Caregivers Regarding the Care of Their Family Member with Advanced Dementia?

Figelj M. ${ }^{1}$, Lunder U. ${ }^{2}$, Rawlinson . $^{3}$

${ }^{1}$ Palliative Care, Dr Franc Derganc General Hospital Nova Gorica, Sempeter pri Gorici, Slovenia, 2 University Clinic of Respiratory and Allergic Diseases Golnik, Golnik, Slovenia, ${ }^{3}$ Centre for Medical Education, Cardiff University School of Medicine, Cardiff, United Kingdom

Background: Setting the goals of person-centred care for persons with advanced dementia, is in practice mostly limited to shared decisionmaking with their family caregivers and health care professionals. Family caregivers hopes regarding the care of their family member with advanced dementia can be used for setting the care goals.

Aim: To explore the goals of family caregivers regarding the care of their family member with advanced dementia.

Design: Descriptive qualitative study.

Methods: During 2018 twelve semi-structured research interviews were conducted with primary family caregivers who were providing assistance 
to their family member with advanced dementia (FAST Stage 6 and 7) at home or in a nursing home in north-western part of Slovenia. Purposive sampling with data saturation were used to define the sample. Thematic analysis of verbatim transcribed interviews was performed.

Results: Two overarching themes were identified:

(1) Hoping and striving for comfort;

(2) Decision-making in the world of ambivalence and distress.

Conclusion: Family caregivers hope for comfort-care for their family member with advanced dementia when the patients are in a stable state of the disease. In case of complications of the disease, however, family caregivers hopes are not always directed toward comfort. Ambivalence and distress are two important factors that steer family caregivers' hopes in case of complications of the disease away from their otherwise strong commitment to comfort. The results have implications for practice. Palliative care with expertise in dementia is seen as in line with the concept of good-quality care for persons with advanced dementia from the perspective of family caregivers. Advance care planning could have the potential to reduce the ambivalence and distress of family caregivers and to help them build meaningful hopes for the future care of their family member with advanced dementia.The study was self-funded.

Abstract number: P01-467

Abstract type: Poster

Dying in Neurological Disease - Different Pattern of Needs Ozanne A. ${ }^{1,2}$, Sawatzky $R^{3,4,5}$, Alvariza A. ${ }^{6,7}$, Årestedt $K .{ }^{8,9}$, Håkansson C. ${ }^{10}$, Fürst C.J. ${ }^{11}$, Öhlén J.5,12,13

${ }^{1}$ Department of Clinical Neuroscience and Rehab, Institute of Neuroscience and Physiology, Sahlgrenska Academy at Gothenburg University, Gothenburg, Sweden, ${ }^{2}$ Department of Neurology, Sahlgrenska University Hospital, Gothenburg, Sweden, ${ }^{3}$ School of Nursing, Trinity Western University, Langley, Canada, ${ }^{4}$ St. Paul's Hospital, Centre for Health Evaluation \& Outcome Sciences, Vancouver, Canada, 5 Institute of Health and Care Sciences, University of Gothenburg, Gothenburg, Sweden, 65 . Department of Health Care Sciences/Palliative Research Centre, Ersta Sköndal Bräcke University College, Stockholm, Sweden, ${ }^{7}$ Dalen Hospital, Capio Palliative Care, Stockholm, Sweden, ${ }^{8}$ Faculty of Health and Life Sciences, Linnaeus University, Kalmar, Sweden, ${ }^{9}$ The Research Section, Kalmar County Council, Kalmar, Sweden, ${ }^{10} \mathrm{Head}$ of Department of Nursing Science, Sophiahemmet University, Stockholm, Sweden, ${ }^{11}$ The Institute for Palliative Care, Lund University and Region Skåne, Lund, Sweden, ${ }^{12}$ Palliative Centre, Sahlgrenska University Hospital, Gothenburg, Sweden, ${ }^{13}$ Centre for Person-centred Care, University of Gothenburg, Gothenburg, Sweden

Background: Many neurological diseases are progressive and cause physical and psychological impairments, but motor neuron disease (MND) and tumors in the central nervous system (CNS tumor) often result in more rapid deterioration than other neurological diseases (OND).

Aims: Was to compare symptom prevalence and relief, and palliative oriented care last week of life for patients grouped in MND, CNS tumor, and OND.

Methods: A retrospective study from the Swedish Register of Palliative Care, documenting quality of care indicators during the last week of life in 2011-2012. Multivariable multinomial logistic regression was used to compare the three patient groups.

Results: At the study period, 419 patients with MND, 799 patients with CNS tumor, and 1407 patients with OND were reported as underlying cause of death. Last week of life the "Frequencies of "Partial/no relief" / "symptom prevalence" (\%)" in Shortness-of-breath was 150/220 (68) in MND, 54/100(54) in CNS tumor, and 177/265 (67) in OND; in the same order it was in Anxiety 94/220 (43), 87/261 (33), and 103/319 (32); in
Rattles it was 141/218 (65), 227/462 (49), and 432/774 (56); in Pain it was 57/188 (30), 109/555 (20), and 144/661 (22); in Nausea it was 14/37 (38), $27 / 70$ (39), and 29/54 (54), and in Confusion it was 23/25 (92) 171/196 (87) 148/179 (83).

Patients with CNS tumor had greater odds of having totally relieved pain and rattles relative to partly/not at all relieved symptoms compared to MND and OND. Patients with MND and CNS tumor had greater odds of having end-of-life conversations, a consultation of a specialized palliative team and other external competence, and family members having an after death conversationthan in OND.

Conclusions: All groups experienced symptom distress with variation in symptom relief. Patients with CNS tumor seem to receive better care than patients with MND and OND. Individual care planning is recommended to promote symptom relief regardless of diagnosis.

Abstract number: P01-468

Abstract type: Poster

Analysis of Survival in Patients with Dementia and Enteral Nutrition in a Health Area in the South of Spain

Cristino Agudo B., González Campos L.G.C., Vergara López S., Antiñolo $F$.

Internal Medicine, Hospital Universitario Valme, Sevilla, Spain

Background: Dementia is a syndrome that involves a deterioration of the functions and capacities of the individual, causing an impact on the patient and society in general. One of the most important associated problems are eating disorders, which lead to the frequent use of enteral nutrition. This issue generates ethical controversies.

Objectives: In this paper we analyze morbidity and survival in patients diagnosed with dementia with enteral nutrition in a health area in the south of Seville (Spain).

Methods: Prospective, observational, descriptive study. We analyzed a sample of 110 patients who were treated with enteral nutrition by tube between January and June 2017 a Health Area, all diagnosed with advanced dementia.

Its survival and morbidity are analyzed from the beginning of nutrition with analysis of the functional assessment and nutritional status.

Results: In the population of this study, survival is greater than that described, with a median survival of 111.28 weeks. Patients who survive a first phase survive for long periods of time. Complications are present in less than $40 \%$ of patients. However, the decision-making process regarding the implementation of enteral nutrition continues to present ethical conflicts, since an individualized analysis of the patient and his family must be carried out, with the collaboration of different professionals to carry out the best care.

Conclusions: In the studied population, survival is greater what has been described to date with fewer complications. Future research in this line is necessary to discern the variables that influence the survival of these patients.

Abstract number: P01-469

Abstract type: Poster

How Is Professional "Truth-telling" Perceived by the Carers of Patients with Total Anterior Circulation Stroke? How Does this Influence Hope and Decision-making? Mullin J.1,2, Kendall M. ${ }^{3}$, Koffman J. ${ }^{4}$, Murray S. ${ }^{3}$, Mead G. ${ }^{2,5}$ ${ }^{1}$ Cicely Saunders Institute Of Palliative Care, Policy \& Rehabilitation, Kings College London, London, United Kingdom, ${ }^{2} \mathrm{NHS}$ Lothian, Edinburgh, United Kingdom, ${ }^{3}$ Usher Institute of Population Health Sciences and Informatics, University of Edinburgh, Edinburgh, United Kingdom, ${ }^{4}$ Cicely Saunders Institute of Palliative Care Policy and Rehabilitation, Kings College London, London, United Kingdom, ${ }^{5}$ Geriatric Medicine, University of Edinburgh, Edinburgh, United Kingdom 
Background: Perceived poor communication at the end of life is a primary cause of health care complaint, yet perceptions of informationsharing following stroke - the second largest cause of death worldwide - are little-researched.

Aim: To describe and explore informal carers' experiences of healthcare professional truth-telling in the first 12 months following Total Anterior Circulation Stroke (TACS).

Methods: Secondary analysis of serial semi-structured interviews with carers at 6 weeks, 6 months and 1 year post-TACS, including bereavement interviews. Population: Informal carers of severely dependent adult patients in Lothian with TACS, sampled purposively for maximal variation. Framework approach was used for secondary analysis, using deductive and inductive themes; $10 \%$ of coding was cross-checked, and deviant, negative and extreme cases examined.

Results: 20 transcripts from 15 carers were analysed. All referenced truth-telling by healthcare professionals (HCPs). Carers frequently described constructing truth as a process and HCP omission of information, but not HCP intention to deceive. Most carers reported seeking, persisting or double-checking to find truth. Loss of hope was often reported but usually resulted from perceived loss of dignity or life quality, rather than from information given by HCPs. Carers were more likely to describe frank communication positively if the patient died. Some carers would have made different decisions, had information been shared differently.

Conclusions: Carer perceptions of HCP truth-telling are influenced by carer factors (their knowledge, worldview and prior experience), patient factors (dependency, trajectory and outcome) and factors intrinsic to the HCP or broader environment. Many factors are dynamic, so perceptions of truth-telling are volatile. We propose that frequent communication, acknowledging uncertainty and promoting the construction of truth as a dynamic process, could improve carers' perceptions of communication.

Abstract number: P01-470

Abstract type: Poster

\section{Decision Making of End-of-Life Care for Neurological Patients in an Acute Neurological Ward \\ Jonsdottir G. ${ }^{1}$, Haraldsdottir E. ${ }^{2}$, Sigurdardottir V. ${ }^{3}$, Hjaltason $\mathrm{H}^{3}$,} Jonsdottir $\mathrm{H}^{1}$

${ }^{1}$ University of Iceland, Reykjavik, Iceland, ${ }^{2}$ Queen Margareth University, Edinburgh, United Kingdom, ${ }^{3}$ Landspitali University Hospital, Reykjavik, Iceland

Background: The decision to alter life prolonging treatment into end-oflife care can be challenging for health professionals in acute hospital wards. This is particularly demanding in patients with life-limiting neurologic diseases and severe impairment and symptoms complicating the disease trajectory.

Aim: To gain insight and comprehension into the process of decision making in end-of-life care of patients with neurological diseases in an acute neurological ward at a university hospital.

Design/ method: A retrospective cross-sectional study measuring indicators of decision-making in end-of-life care by a data collection tool developed and tested by the authors. The tool is divided into 7 categories, with items that reflects decision-making, discussion and planning of end-of-life care and care in the last days of life.

Result: 170 patients' electronic health records during 2011 to 2016 were content analyzed. Majority had stroke, ALS or Parkinson disease. Mean age was 77 years, 84 (49\%) were women, 93 (55\%) were married or cohabiting, 125 (74\%) lived at home before admittance to the hospital. 38 patients $(22 \%)$ had been hospitalized $>3$ times the last year before their death and 106 (62.5\%) for more than 7 days before their death. The difference between the number of decisions of end-of-life care before and after implementation of end-of-life registration in the patients' hospital records was non-significant.
Conclusion: Timely decision making of end-of-life care for patients with life-limiting neurologic diseases is/remains problematic for health care professionals in an acute neurological ward.

Abstract number: P01-471

Abstract type: Poster

The Use of Enteral Nutrition in Patients with Dementia: A Structured Review in 2018

Bonilla Roldán M., López Navarro A., Gamboa Antiñolo F.

Internal Medicine, Hospital Universitario Valme, Sevilla, Spain

Background: A common experience suffered by people with dementia is the development of eating difficulties that lead to problems such as malnutrition and weight loss. When presented, the use of enteral nutrition (NE) by nasogastric tube or percutaneous endoscopic gastrostomy may be considered.

Objective: We carry out a bibliographical review prior to a fieldwork. It is intended to answer the following question: What is the indication of enteral nutrition in patients with dementia?

Methodology: Literature review of the Web of Science (WOS), Scopus and Pubmed databases.

MeSH terms: "enteral nutrition", "Dementia”, "nutritional support", "comorbidity", "pressure ulcer", "tube feeding", "disorders", "comorbidity". Articles published between 2008 - 2018 in Spanish, English and German what are available as full text or abstract.

Result: In patients with mild or moderate dementia can reduce the rate of complications and increase the survival rate. NE in advanced dementia does not prevent comorbidities, does not improve nutritional status or survival and increases the mortality rate, the incidence of pneumonia, hospital admissions, suffering and pain, a decrease in quality of life and appearance of UPP.

The discrepancy of the daily clinical practice and the evidence is verified: the generalized use, the clinical experience, the beliefs, the culture, the religious ideas of the professional and the pressure exerted by the family are the main reasons that surround the prescription. It is important to create multidisciplinary teams. Decision making is an ethical challenge for family and professionals.

Conclusion: The use of NE in the mild or moderate stage of dementia can provide benefits. Not so in advanced dementia. Decision making is an ethical challenge. It is important to create multidisciplinary teams.

\section{Abstract number: P01-472}

Abstract type: Poster

Palliative Care Interventions for People with Dementia Living at Home: A Systematic Review

Miranda R. ${ }^{1,2}$, Bunn $\mathrm{F}^{3}$, Lynch J. ${ }^{3}$, Van den Block L. ${ }^{1,2}$, Goodman C. ${ }^{3}$ ${ }^{1}$ End-of-Life Care Research Group, Vrije Universiteit Brussel (VUB) and Ghent University, Brussel, Belgium, ²Department of Family Medicine and Chronic Care, Vrije Universiteit Brussel (VUB), Brussel, Belgium, ${ }^{3}$ Centre for Research in Primary and Community Care, University of Hertfordshire, Hatfield, United Kingdom

Background: There have been increasing calls to improve palliative care for people with dementia living at home, but evidence on how to achieve this remains scant.

Aim: Based on literature, we aimed to study the effects of home palliative care interventions for people with dementia on end of life care outcomes.

Methods: We performed a systematic review of intervention studies relating to specialist home palliative care services and non-specialist home palliative care interventions (i.e. interventions that were not labelled as 'palliative care' but described as aiming to improve care at the end of life). Primary outcome was patient death at home. Secondary 
outcomes included quality of life, symptom control, functional status, institutionalisation and resource use. We searched 4 electronic databases from inception to April 2018 and conducted lateral searches. Two authors assessed the quality of the studies independently.

Results: We retrieved 8 intervention studies, of which 3 relate to specialist palliative care services and 5 to non-specialist palliative care interventions. Five of these studies provided weak evidence, while the rest provided evidence of moderate quality. Synthesis of evidence showed small but statistically significant beneficial effects of home palliative care interventions compared to usual care on improving behavioural symptoms (4 studies), functional status ( 1 study), institutionalisation ( 3 studies) and resource use (2 studies). Evidence on patient death at home (1 study) and pain reduction (1 study) was inconclusive. No study reported on quality of life.

Conclusion: Although home palliative care interventions may improve some end of life care outcomes for people with dementia, there is a clear lack of high-quality evidence which precludes a robust conclusion on their effectiveness. There is thus an urgent need for high-quality studies to evaluate palliative care interventions for people with dementia living at home.

Funding: EU500 INDUCT ga $n^{\circ} 676265$

Abstract number: P01-473

Abstract type: Poster

An Evaluation of a Partnership Model of Hospice Enabled

Dementia Care: A Four Phased Study

McLaughlin D. ${ }^{1}$, Hasson F. ${ }^{2}$, Reid J. ${ }^{1}$, Brazil K. ${ }^{1}$, Rutherford L. ${ }^{3}$, Stone C. ${ }^{4}$, Van Der Steen J. ${ }^{5}$

${ }^{1}$ Queen's University, Belfast, United Kingdom, 2 Ulster Univerisiity, Jordanstown, United Kingdom, ${ }^{3}$ Queen's University, Marie Curie, Belfast Health and Social Care Trust, Belfast, United Kingdom, ${ }^{4}$ Marie Curie Hospice, Belfast, United Kingdom, ${ }^{5}$ Leiden University Medical Center, Leiden, Netherlands

Background: Concerns have been highlighted around the unmet needs of people with dementia and informal carers and internationally it is evidenced that people with dementia infrequently access hospice care. European domains of best practice in end of life care for this population have been developed.

Aim: To evaluate a partnership model of Hospice Enabled Dementia Care, based on the European domains of best practice, between a specialist palliative care hospice provider and a Health and Social Care Trust, in a region of the United Kingdom.

Methods: A formative evaluation framework was used involving four phases.

Phase 1 involved monitoring of service activity and key indicators of success through documentary analysis of anonymised data provided from hospice records. In

Phase 2 a purposive sample of twelve active carers of people with dementia were recruited to semi-structured interviews and in

Phase 3 six focus groups took place with a purposive sample of twenty-eight Health and Social Care Professionals. Other professionals requested telephone interviews $(n=3)$ and one a face-to- face interview $(n=1)$.

Phase 4 involved telephone interviews with five participants who had a policy making or service commissioner role relevant to palliative or dementia care.

Results: One hundred people with dementia were referred mainly for end of life care from May 2016-December 2017. Thirty-eight of the forty people who died achieved their preferred place of care at home. Core combined themes were identified within the qualitative data collected from family carers, Health and Social Care Professionals and policy makers and service commissioners. Core combined themes were: 'Impact of Dementia', 'Value of the Service', 'Information and Learning Needs' and 'Working in Partnership'.
Conclusion: Positive outcomes and impact are apparent from this best practice model of care. This should be replicated given the international relevance and increasing incidence of dementia globally.

\section{Abstract number: P01-474}

Abstract type: Poster

\section{Lost in the Labyrinth: Who Cares?}

Ateş G. ${ }^{1}$, Gasper A. ${ }^{2}$, Jaspers B. ${ }^{1,3}$, Kern M. ${ }^{2}$, Radbruch $L .{ }^{1,2}$

${ }^{1}$ Department of Palliative Medicine, University Hospital Bonn, Bonn, Germany, ${ }^{2}$ Centre of Palliative Care, Malteser Hospital Seliger Gerhard Bonn/Rhein-Sieg, Bonn, Germany, ${ }^{3}$ Department of Palliative Medicine, University Medicine Goettingen, Goettingen, Germany

Background: Patients with amyotrophic lateral sclerosis (ALS) and their carers are faced with complex challenges. Patient-centred support is needed from diagnosis onwards, and will have to increase during the disease. By whom and how comprehensive support should be coordinated, often remains unclear.

Aims: To explore among key healthcare providers (HCP) for ALS patients in the state North-Rhine Westphalia (NRW)

i) the range of needs to be addressed, in order to provide insights into

ii) who formulates and communicates patients' care goals, and

iii) how are care gaps addressed.

Methods: Semi-structured interviews with purposefully sampled experts (10/2016-08/2017) and quantitative survey among palliative care (PC) services (inpatient hospices, PC care services, specialized PC teams and neurological centres - 09/2017-02/2018) in NRW. The research team developed and piloted the interview guideline and the questionnaire. Interviews were audiotaped, transcribed verbatim and thematically analysed using MAXQDA. The questionnaire was descriptively analysed using SPSS.

Results: Most of the $14 \mathrm{HCPs}$ interviewed mentioned that different approaches of treatment and care goals cause serious friction among HCPs. Combined with the results from the survey $(n=196$, response rate $50 \%$ ) the most frequent challenges for the treating teams were coordination $(78 \%)$, information $(77 \%)$, time resources $(75 \%)$ and communication (65\%). Coordination tasks were taken up at random and with different views on its meaning. Differences between services will be presented in detail.

Conclusion: Appropriate solutions for patient-centered care for ALS patients are hardly to be found within the current regional healthcare system. Coordination among the various involved care providers is complex and time-consuming, particularly without a common understanding of care goals. The establishment of a coordinator for ALS patients is of utmost importance.

Funding: Ministry for Labour, Health and Social Affairs of NRW

Abstract number: P01-475

Abstract type: Poster

Living Life to the Full with Duchenne: Including Palliative Care in the Multi-disciplinary Team

Laidlaw S. ${ }^{1,2}$, Carduff E. $^{1}$

${ }^{1}$ Marie Curie Care, Glasgow, United Kingdom, ${ }^{2}$ Cardiff University, Cardiff, United Kingdom

Background: Duchenne Muscular Dystrophy (DMD) is an x-linked, ultrarare neuromuscular condition affecting 1 in 3600-6000 live male births. Life expectancy is increasing but adults are receiving less comprehensive, co-ordinated care compared with those in the paediatric service. Individuals live with an exceptional illness trajectory of prolonged dwindling, frailty and high symptom burden, often have insufficient care in their last years of life, and yet feel that palliative care is not important or relevant to them. 
Aim: To investigate quality of life (QoL) in adults with DMD living in the West of Scotland (WoS) and how it may be improved.

Methods: The Schedule for the Evaluation of the Individual Quality of Life - Direct Weighting (SEIQoL-DW) tool was used to assess the five most important elements that contribute to an individual's QoL - these were then used to guide qualitative interviews with 6 men in the WoS. A thematic analysis was undertaken.

Results: Men living with DMD in the WoS described living good lives but feel "forgotten" due to perceived gaps in their care: poorly co-ordinated and infrequent health care; lack of multi-disciplinary team input and holistic care; and poor or no access to allied health care professionals, for example physiotherapy and psychological support. The lack of support was particularly evident at times of their own illness, on the death of their peers, and living with unbroken uncertainty.

Conclusions: There are numerous opportunities to introduce palliative care gently, as part of the MDT, early in the illness trajectory and continue in a dynamic manner as time elapses and when trigger points arise. This is especially important given that the recognition of the end of life phase is difficult in those with neurological conditions. However, a shift in thinking is necessary to move from "death and dying" to "living well with life-limiting illness" so that the additional of such services is not seen with fear and rejection.

Abstract number: P01-476

Abstract type: Poster

Telemedical Care for Palliative Patients with Neurodegenerative Diseases: Results from a Monocentric Pilot Trial

Weck C. ${ }^{1}$, Lex K. ${ }^{2}$, Lorenzl S. ${ }^{1,2,3}$

${ }^{1}$ Neurologie, Krankenhaus Agatharied, Hausham, Germany, ${ }^{2}$ Pflegewissenschaften, Paracelsus Medizinische Privatuniversität Salzburg, Salzburg, Austria, ${ }^{3}$ Palliativmedizin, Ludwig-Maximilian University Munich, München, Germany

Background: Growing necessity in palliative support is determined for patients with advanced neurodegenerative disorders. In an outpatient setting in Germany specialized palliative care teams (SPC) look after these patients. Usually there is little knowledge in neurodegenerative diseases and there exists no systematic access to a neurological expertise. The project "telemedical care for palliative patients with neurodegenerative diseases (TPNP)" provides a new patient centered care by connecting the Department of Neurology of a University Hospital with the outpatient teams using a videocounseling system.

Aim: To transport expert care for neurological patients cared by outpatient SPC teams.

Methods: A prospective explorative single arm pilot trial was implemented in Bavaria/Germany. 6 outpatient SPC teams got equipped with a mobile telesystem. In any time the opportunity has been given to ask an expert in neuropalliative care at the specialized centre in the hospital (24/7). A mixed methods design was chosen to analyse the impact of the telemedical system. Semistructured interviews were held with the physicians of the specialized outpatient palliative care teams after a trial duration of 9 months.

Results: Over a trial period of nine months we supervised 21 patients of the SPC teams via teleconsultations. The most frequent diagnosis in these patients was motor neuron disease. Evaluation on the first 25 videoconsultations showed that the technical structure allows a reasonable neurological examination at distance in an outpatient setting. The patients and carers acceptance is high. Qualitative interviews with the specialized palliative care physicians indicate a major impact on the quality of work for the SPC teams and on the quality of care for neurological patients.

Conclusion: The system proves to be useful and is well accepted by caregivers and patients. It supplies a structure that can be transported to other disciplines.

Funded by the bavarian StMGP
Abstract number: P01-477

Abstract type: Poster

General Practitioners' Care for Frail Older Patients in the Last Phase of Life - Where Does Geriatric Care End and Palliative Care Start?

Stiel S., Ewertowski H., Krause O., Müller-Mundt G., Schneider N. Institute for General Practice, Hannover Medical School, Hannover, Germany

Background: Delivering end-of-life care for older patients, who often present with frailty, usually requires more than just one care approach. The integration of geriatric and palliative care (PC) could be necessary to relieve suffering and enhance quality of life - with different accentuations at different time points. Nevertheless, this approach conflicts with regulations in Germany since general practitioners (GPs) can remunerate only one approach, even if providing both, geriatric and PC.

Aim: To investigate how GPs experience the fine line between geriatric and PCwhen caring for frail older patients.

Methods: A secondary data analysis of the former prospective qualitative longitudinal study „End of life care for frail older patients in family practice" from 2012-15 (BMBF 01GY1120) was applied within the project "Primary Palliative Care in General Practice" (2016-21). 52 serial case-related interviews with $14 \mathrm{GPs}$ and one focus group were analysed following principles of Grounded Theory.

Results: The differentiation and practical transition between geriatric and PC in GPs' daily practice is perceived to be fluid. GPs have the impression that they care for their patients first according to their needs and reflect afterwards whether it was actually the delivery of geriatric or PC. Therefore, the fine line between both subjects is perceived rather artificial. Mostly they do not consider the mutual exclusion of the remuneration of both approaches and do not choose for the better remuneration in the first place.

Discussion: A large overlap and fluent transition between geriatric and PC are perceived, so that one way of care practice is created. The German remuneration system should adapt to the practical reality and allow for

i) either one joint remuneration or

ii) the compensation of geriatric and palliative care at the same time in one patient.

Source of funding: Federal Ministry of Education and Research: BMBF 01GY1610

Abstract number: P01-478

Abstract type: Poster

Collaborative Solutions to the Challenge of Frailty

BradyJ.

North London Hospice, London, United Kingdom Presenting author email address: jbrady@northlondonhospice.co.uk

The number of people living and dying with frailty is rising. The borough of Barnet has a large frail elderly population and working across primary and secondary care we have seen the impact this is having on stretched services. Living longer is a success story for modern medicine but brings more complex co-morbidities, disability and frailty. Services tend to be designed around single organ diseases. Palliative care needs to consider what role we have to play in addressing the challenges of frailty and multi-morbidity.

We aimed to understand the local needs and identify the role of the hospice. We aimed to improve the continuity of care specifically in relation to advanced care planning and end of life care decisions.

We chaired the frailty hub; a meeting with health and social care that brought together expertise to devise a care plan. This was support by an 
integrated locality team including case management. We provided specific specialist advise but also upskilled the wider generalist workforce in relation to recognising end of life and developing appropriate care plans. We worked alongside the Community Education Provider Network to deliver a series of locality based education events bringing together primary and secondary care. This culminated in a pan borough event and agreed action plan delivered via multi-professional working groups. In the next phase we are piloting a locality frailty model. Also using frailty data we have secured funding to expand the reach of our services beyond specialist care to an enhanced end of life model.

We found the cross collaboration between primary and secondary care has led to a shared understanding of each other. There is a shared sense of ownership and responsibility across the whole patient journey. Improving care for people with frailty and multi-morbidity must involve a shift away from a reactive, disease-focused, fragmented model of care towards one that is more proactive, coordinated and collaborative in nature.

Abstract number: P01-479

Abstract type: Poster

How Do Geriatricians Diagnose Dying in People with Dementia in the Emergency Department?

Sampson E., Humphreys R., Kupeli $N$.

Marie Curie Palliative Care Research Unit, University College London, London, United Kingdom

Background: The palliative care needs of people with advanced dementia (AdvD) are increasingly being recognised. A significant proportion will end their lives in the acute hospital. Hospital clinicians are required to predict imminent death in order to appropriately initiate end of life care. Aims: To establish how accurately geriatricians predict dying in elderly nursing home residents with AdvD who have been admitted to the acute hospital and to understand how these predictions are made.

Design, setting, and participants: 29 geriatricians completed an online prognostication task, with three vignettes based upon real clinical cases of which 14 participated in a semi-structured interview. Thematic analysis was used to identify the contextual factors which influence geriatricians' predictions.

Results: Geriatricians find it difficult to predict dying in patients with AdvD who have been admitted to hospital, regardless of the amount of clinical information available to them. Confidence in their predictions grows as knowledge of the patient increases, but accuracy does not. The accuracy of participants' predictions did not increase as they received more clinical information. Participants became increasingly pessimistic in their predictions for Vignettes 1 ("uncertain dying") and 2 ("anticipated dying").The process of prognostication is complex and is influenced by a variety of clinical and impressionistic contextual factors. Uncertainty in predicting death at each stage of the patient's journey is a key finding.

Conclusions: Predicting dying in elderly nursing home residents with AdvD who present to the acute hospital settings is challenging. Whilst further work is needed to fully understand the process of prognostication and identify markers of imminent dying in this cohort, clinicians also need support in managing prognostic uncertainty and avoiding futile interventions.

\section{Abstract number: P01-480 \\ Abstract type: Poster}

Tools to Manage Clinical Uncertainty in Older People towards the End of Life: Systematic Review

Ellis-Smith C. ${ }^{1}$, Tunnard I. ${ }^{1}$, Dawkins M. ${ }^{1}$, Evans C.J. ${ }^{1,2}$

${ }^{1}$ Cicely Saunders Institute of Palliative Care, Policy and Rehabilitation, King's College London, London, United Kingdom, ${ }^{2}$ Sussex Community NHS Foundation Trust, Brighton, United Kingdom
Background: People are living longer and with increasing multi-morbidity and frailty. Clinical uncertainty is common associated with points of decline and poor outcomes notably nearness to end-of-life. Uncertainty is challenging to manage, resulting in worse outcomes.

Aim: To provide a summary of tools to manage clinical uncertainty in older people across care settings.

Methods: A model of uncertainty underpinned the review. We searched Medline, Psyclnfo, CINAHL and IBSS from 2000 to July 2018. Primary studies reporting the development or evaluation of tools in people aged $50+$ with advanced disease were included. Tools encompassed the patient journey from admission to discharge and beyond to improve assessment, communication and coordination of care. Data were extracted on the tool, theoretical domain of uncertainty, evaluation of processes and outcomes, and implementation requirements in routine care. Quality of studies was assessed using QualSyst. The data were synthesised across the domains of uncertainty.

Results: Of the 15,896 papers identified, 76 were identified for inclusion. Studies included tool development, psychometric evaluation, those to explore the mechanisms of action and implementation requirements, or test effectiveness on patient outcomes. Tools included those for assessment e.g. person-centred assessment, frailty, symptom assessment and functional abilities; those for advance care planning, and/or for supporting communication. The data were synthesised to develop a Theory of Change of how a toolkit may support comprehensive person-centred assessment, communication with patients and family, and continuity of care between settings and at times of transition.

Conclusion: We have identified candidate tools for a toolkit intervention for use in routine clinical care to manage clinical uncertainty in older adults towards the end of life. A Theory of Change explains how such an intervention may work and be implemented, to improve patient and family outcomes.

\section{Abstract number: P01-481 \\ Abstract type: Poster}

\section{Quality Improvement Project Looking into the Prescription of} Steroids at a Palliative Care Inpatient Unit

Alam S.

Mount Vernon Cancer Centre, Palliative Medicine, London, United Kingdom Presenting author email address: sorayya.alam@gmail.com

Introduction: Current guidelines state that steroids in terminally ill patients should be reviewed regularly and stopped if there is no clinical benefit. Patients should have a steroid card, blood glucose monitoring (BMs) and a proton pump inhibitor (PPI). Drug interactions (DIs) and Adverse Drug Reactions (ADRs) should be considered. The purpose of this quality improvement project was to analyse patient records retrospectively and implement improvements when prescribing steroids.

Methods: 20 patient notes reviewed between October and November 2017 in a palliative care inpatient unit at Mount Vernon Cancer Centre, UK. Data collected on the date steroids prescribed, their indication, duration, consideration of ADRs, DIs, monitoring BMs and PPI prescription.

PDSA cycle 1: Development of a steroid prescribing prompt sticker for patient notes.

PDSA cycle 2: Feedback from staff highlighting the need for the sticker to be placed in both the patient's notes and drug chart.

PDSA cycle 3: Adaptation of certain phrases on the sticker i.e. "Duration" to "When steroids started" and the "review date" to "review period". Results:

20 patient notes were reviewed.

11 had no review date documented;

19 patients rarely had a clear indication documented;

9 patients had no duration documented;

10 patients did not have a weaning regime documented and only 10 had their BMs reviewed regularly. 
ADRs and DIs were never documented. A PPI was prescribed in $100 \%$ of patients. After 3 PDSA cycles, the reason for steroids and a weaning strategy increased to $100 \%$. Review period increased to $100 \%$ and BM monitoring also increased to $100 \%$.

Conclusion: Certain aspects of prescribing were done well, however weaning strategies and review dates were infrequently documented and therefore patients were at risk of suffering ADRs from their sustained use. Institution of the adapted sticker showed significant improvement in documentation of the review period and weaning strategies for prescribed steroids.

\section{Abstract number: P01-482 \\ Abstract type: Poster}

Pharmacovigilance in Hospice/Palliative Care: De-prescribing Controlled Release Oxycodone-naloxone

Clark K. ${ }^{1,2}$, Byrne P. ${ }^{3}$, Watts G. ${ }^{4}$, Currow D. ${ }^{5}$, Palliative Care Clinical

Studies Collaborative

1Palliative Care, Northern Sydney Cancer and Palliative Care Network Sydney, Australia, ${ }^{2}$ Northern Clinical School, University of Sydney, Sydney, Australia, ${ }^{3}$ University of Sydney, Sydney, Australia, ${ }^{4}$ University of Newcastle, Newcastle, Australia, ${ }^{5}$ University of Technology Sydney, Sydney, Australia

Controlled release (CR) oxycodone-naloxone is a licenced analgesic, prescribed to reduce opioid-induced constipation severity. This is based on high quality efficacy trials comparing CR oxycodone-naloxone with CR oxycodone alone. Outside of trial settings, insufficient data are available to detail the medication's net benefits and harms. A concern is the possibility that some with liver dysfunction may inadvertently experience systemic exposure to naloxone because of altered first pass metabolism with case reports suggest caution when switching from oxycodone-naloxone to alternative opioids. This phase IV study was conducted to document in clinical settings situations where oxycodone-naloxone is deprescribed. The work was conducted in 13 sites across 2 countries as part of Australia's Palliative Clinical Studies Collaborative pharmacovigilance program. Collected data included the final oxycodone-naloxone dose; reason for ceasing; constipation assessment; alternative opioid, dose \& formulation. Results from 112 patients were analysed demonstrating opioid switching occurred because of inadequate analgesia ( $n=49 ; 43.75 \%)$ or liver dysfunction ( $n=46 ; 41.07 \%$ ). Before switching, the mean 24-hour Oral Morphine Equivalent Dose (OMED) was $60.0 \mathrm{mg}$ with 70 people switched to another controlled release opioid (OMED $58.4 \mathrm{mg})$. At baseline, $52 \%(n=58)$ were constipated compared with $40 \%(n=43)$ a day later $(P=0.01)$. Of those switched due to liver problems, 38 were prescribed equivalent OMED. This work describes a group of palliative care patients who had oxycodonenaloxone ceased. Despite naloxone, at baseline the majority were constipated highlighting in palliative care, this is a complex issue. Unexpectedly with switching the number of constipated patients decreased. Impaired liver function was listed as a frequent concern, but people were converted to a similar OMED, raising questions about how much impaired clearance of naloxone was really affecting poor analgesia.

Abstract number: P01-483

Abstract type: Poster

\section{Suboptimal Medication Adherence in Palliative Care}

Tuffin P. ${ }^{1}$, Eng D. ${ }^{1}$, Agar M. ${ }^{2}$

${ }^{1}$ Palliative Care, Royal Perth Hospital, Perth, Australia, ${ }^{2}$ Palliative and

Supportive Services, School of Medicine, Flinders University, Adelaide, Australia

Background: Adherence to prescribed medication regimens is known to be poor across many groups of patients and medications. Poor adherence results in reduced clinical benefit of therapy, diminished quality of life and wastage of health care resources.
There is limited information about medication adherence of palliative care patients although it would be expected to be high due to joint decision making about treatment and medication choices and symptom focussed medication regimens.

Aims:

- Determine level of adherence to prescribed medication

- Explore factors that may influence these rates

- Measure intentional nonadherence medication rate

Methods:

Design: Prospective cross-sectional study utilising paper-based questionnaires.

Setting/participants: Patients attending a palliative care outpatient clinic of a tertiary teaching hospital.

Data collection: Participants self-reported their adherence by completing the Morisky Medication Adherence Scale 8-Item-Non-Specific Health Condition tool and symptom burden using the Symptom Assessment Scale tool. Clinicians assessed functional status, and demographic, medication and disease information was collected.

Results: Of 49 participants, 8 (16\%) self-reported high adherence, 17 (35\%) moderate and 21 (43\%) poor adherence. Participants reporting high adherence were older, generally in better health (higher functionality and lower symptom load) and more likely to be visiting only one doctor. They were also more commonly taking less doses of medication/day. 20 participants (41\%) were intentionally not adhering to their medication.

Discussion/conclusion: This study found the rate of medication adherence in palliative care patients is lower than the general population. In addition, these patients frequently choose not to take their medications as prescribed. Medication adherence discussion needs to be part of each clinical assessment to optimise symptom management and quality of life for palliative care patients.

Abstract number: P01-484

Abstract type: Poster

Caution Advised - A Case Series of Rotation from Tapentadol to Morphine Sulphate in Cancer Patients

Ní Nualláin A., Ni Laoire A., Twomey M., O Reilly M.

St. Luke's Radiation Oncology Centre Rathgar, Dublin, Ireland

Background: The recommended conversion ratio of Morphine Sulphate to Tapentadol is 1:3.3. Little research exists on the use of Tapentadol for cancer pain, and even less on Tapentadol switching. Mercadante et al suggests the 1:3.3 ratio is acceptable in cancer patients; however, this is based on a small sample size ( $n=37)$.

Case presentation: Two patients attending a tertiary referral radiotherapy centre presented with poorly controlled pain despite regular Tapentadol use. Patient 1 had a diagnosis of high-grade sarcoma and was on Tapentadol 200mg BD. Patient 2 had T1N3 left breast cancer and was on Tapentadol 75mg BD.

Management and outcomes: Patient 1 was rotated from Tapentadol $200 \mathrm{mg}$ BD to MST 30mg BD; despite the conversion ratio suggesting an equivalent dose of MST $60 \mathrm{mg}$ BD. A cautious approach was taken due to the knowledge of the team regarding the uncertainty surrounding Tapentadol conversion ratios. The patient was pain-free 24 hours post rotation but required a further reduction to $20 \mathrm{mg}$ BD due to opioid toxicity. Patient 2 was rotated from Tapentadol 75mg BD to MST 10mg BD and discharged pain-free on $5 \mathrm{mg}$ BD.

Discussion: Tapentadol has a dual mode of action; $\mu$-opioid receptor agonist and norepinephrine reuptake inhibitor. Therefore, the equi-analgesic effect of Tapentadol can be difficult to determine. Morphine Sulphate to Tapentadol conversion ratios in our patients were 1:10 and 1:15 respectively; considerably less than recommended conversion ratios. This highlights the potential risk of opioid toxicity when rotating from Tapentadol to Morphine Sulphate in cancer patients. 
Conclusion: With the increasing use of Tapentadol in non-specialist pain services, further research is necessary to clarify conversion ratios to ensure safe prescribing. Specialist Pain or Palliative Care advice should be sought when switching between Tapentadol and Morphine, particularly at high doses, where the risk of toxicity is greatest.

\section{Abstract number: P01-485 \\ Abstract type: Poster}

Opioid Errors in Patients Followed by a Domiciliary Palliative Care (PC) Team: Results from a Pilot Study

Fernandez Gomez M.C. ${ }^{1}$, Diaz Sierra G. ${ }^{1}$, Berrocal Higuero M.A. ${ }^{1}$, Ortega San Martin A.M. ${ }^{1}$, Peinado Cañaveras M.C. ${ }^{1}$, Del Cura Gonzalez M.I. ${ }^{2}$, Rigal Andres M. ${ }^{3}$

${ }^{1}$ ESAD SUR, SERMAS, Getafe, Spain, 2 UAI, Gerencia de Atencion

Primaria, Madrid, Spain, ${ }^{3}$ UAIPP, SERMAS, Madrid, Spain

Background: Opioids are considered high alert medications by several international agencies. The risk of medications errors with opioids is higher in PC patients due to advanced disease, comorbidities and complex pharmacological regimes.

Aims: To study the frequency of medication errors with opioids and to describe the type, cause and consequence of these errors using the Spanish adaptation of the National Coordination Council for Medication Error Reporting and Prevention taxonomy.

Methods: Observational, descriptive, cross-sectional study. Patients $>18$ years of age, with opioid treatment, followed by a domiciliary PC team between 13th of June and 20th of July 2018, that gave their informed consent were included. Patients in last days of life and those in nursing homes were excluded. Data were collected from interview and from patient's clinical history.

Results: 30 patients were recruited. 50\% were women. Median age was 76. $73 \%$ had oncological disease. Median number of drugs prescribed for each patient was $10.63 \%$ were follow up visits. $80 \%$ of primary caregivers were women older than 50 years old. In $50 \%$ medication was handled by the primary caregiver. $63 \%$ had 2 opioids prescribed. In $76 \%$ the initial prescription was issued by a PC doctor. Main symptoms for opioid prescribing were pain followed by dyspnoea. Most prescribed opioids were fentanyl followed by morphine sulphate and oxycodone. A total of 15 medication errors with opioids were identified in 13 patients. Wrong frequency of administration was the most common type of error, lack of knowledge or training the most common cause of error and the most common consequence was that medication error reached the patient but didn't cause harm.

Conclusions: The frequency of medication errors with opioids (43\%) observed is consistent with the literature reviewed, anyhow due to the sample size, further research including more domiciliary PC teams and a wider geographical area is planned.

Abstract number: P01-486

Abstract type: Poster

\section{Balancing Medication Use in Nursing Home Residents with} Limited Life-expectancy

Paque K.1,2, Elseviers M. ${ }^{2,3}$, Vander Stichele R. ${ }^{2}$, Pardon K. ${ }^{1}$, Vinkeroye C. ${ }^{3}$, Deliens L. ${ }^{1,4}$, Christiaens T. ${ }^{2}$, Dilles T. ${ }^{3}$

${ }^{1}$ Vrije Universiteit Brussel, Brussel, Belgium, ${ }^{2}$ Ghent University, Gent, Belgium, ${ }^{3}$ Antwerp University, Wilrijk, Belgium, ${ }^{4}$ Ghent University Hospital, Gent, Belgium

Background: At the end of life, balancing medications that are needed and beneficial, and avoid medications that may be harmful or have no short-term benefit is important to prevent drug related problems, and improve quality of life.

Aim: The aim of this study is to describe medication use at first and second evaluation, the prevalence of actual deprescribing of medications suitable for deprescribing, and the prevalence of new initiation of potentially inappropriate medications (PIMs) in nursing home residents with limited life-expectancy.

Methods: Medication use was based on a copy of the resident's full medication chart at the time of data collection ( $\mathrm{t} 2$ ) and three to six months before ( $\mathrm{t} 1$ ). The appraisal of appropriateness of medications was done using a list of medications documented as potentially inappropriate and thus suitable for deprescribing, and STOPPFrail criteria.

Results: Residents' ( $n=296$, mean age 86 years, $74 \%$ female) mean number of chronic medications increased from 7.4 (t1) to 7.9 (t2). In 31\% of those using medications suitable for deprescribing, at least one medication was actually deprescribed. In $30 \%$ at least one PIM from the group of selected PIMs was newly initiated. In the subgroup for whom changes in medication use were observed, deprescibing was associated with less new initiations of PIMs ( $n=133, r=-0.477, p<0.001)$.

Conclusion: Medication use remained high at the end of life for nursing home residents with limited life-expectancy. Changes in medication use were observed in a small subpopulation. Our findings indicate that, in this subgroup, medications were carefully balanced. However, deprescribing was still limited.

Abstract number: P01-487

Abstract type: Poster

Discontinuation of Medications at the End of Life. A Population Study in Belgium, Based on Linked Administrative Databases Paque K..$^{1,2}$, De Schreye $R^{1}{ }^{1}$, Elseviers M. ${ }^{2,3}$, Vander Stichele R. ${ }^{2}$, Pardon $K_{.}{ }^{1}$, Dilles T. ${ }^{3}$, Christiaens T. ${ }^{2}$, Deliens L. ${ }^{1,4}$, Cohen J. ${ }^{1}$

${ }^{1}$ Vrije Universiteit Brussel \& Ghent University, Brussel, Belgium, ${ }^{2}$ Ghent University, Gent, Belgium, ${ }^{3}$ Antwerp University, Wilrijk, Belgium, ${ }^{4}$ Ghent University Hospital, Gent, Belgium

Background: Many medications can be considered as potentially inappropriate at the end of life, e.g. because they lack short-term benefit. Discontinuation of these potentially inappropriate medications (PIMs) may improve medication use at the end of life, reduce negative health outcomes, and support and improve quality of life. It is crucial to get an insight in the current prescribing and use of PIMs in this population to determine the need for guidance in this area, e.g. for development of clinical practice deprescribing guidelines.

Aim: To examine PIM use in relation to time before death, to explore if PIMs are discontinued at the end of life, and the factors associated with this discontinuation.

Methods: We conducted a retrospective register-based mortality cohort study of all deceased in 2012 in Belgium, aged at least 75 years at time of death $(n=74368)$, using linked administrative databases. We used STOPPFrail to identify PIMs received during the period of twelve to six months before death (P1) and the last four months (P2) of life.

Results: Mean age was 86 at time of death, $57 \%$ female, $38 \%$ was living in a nursing home, and $16 \%$ was admitted to hospital two years to four months before death. Overall, PIM use was high, and increased towards death for all PIMs. At least one PIM was discontinued during P2 for $20 \%$ of the population, and $49 \%$ had no discontinuation. Being hospitalized in the period before the last four months of life, living in a nursing home, female gender, and a larger number of medications used during P1 were associated with discontinuation of PIMs (respective OR(95\%CI):2.89(2.73-3.06), 1.29(1.23-1.36),1.26(1.20-1.32),1.17(1.16-1.17)).

Conclusion: PIM use was high and increased towards death. Only $20 \%$ had discontinuation and this was related to hospitalization, living in a nursing home, female gender, and the number of medications. More attention for medication use and discontinuation of medications at the end of life is highly recommended.

Abstract number: P01-488

Abstract type: Poster 
A Retrospective Chart Review of the Effectiveness and Indication of Corticosteroids in the Palliative Care Unit

Okamoto Y. ${ }^{1}$, Tanaka I. ${ }^{1}$, Niki K. ${ }^{1}$, Ishii R. ${ }^{2}$, Matsuda Y. ${ }^{2}$, Numata $C .{ }^{3}$

${ }^{1}$ Department of Pharmacy, Ashiya Municipal Hospital, Ashiya, Japan, 2Department of Palliative Care Medicine, Ashiya Municipal Hospital, Ashiya, Japan, ${ }^{3}$ Educational Center for Clinical Pharmacy, Kobe Pharmaceutical University, Kobe, Japan

Terminal cancer patients show various symptoms. Corticosteroids play an important role in symptom control. Their effects on general malaise, anorexia, nausea/vomiting, brain-metastasis-related symptoms, and dyspnea have been reported, but the evidence level is not high. On the other hand, primary adverse reactions include mental disorder, infection, muscular atrophy, osteoporosis, and peptic ulcers. However, symptoms as adverse events, such as the deterioration of a primary disease, are also present. The timing of appearance and type of adverse reaction remain to be clarified. In this study, we investigated the purpose of corticosteroid use in terminal cancer patients admitted to the palliative care unit, its effects, and adverse events.

We conducted a chart survey involving 595 patients admitted to the Palliative Care Unit of Ashiya Municipal Hospital between July 1, 2012, and December 31, 2015.

Concerning the purpose of administration, corticosteroids were used to relieve malaise and anorexia in most patients. The response rate exceeded $70 \%$. With respect to meals, ingestion was possible until 3.9 days before death in corticosteroid-treated patients and until 6.8 days before death in non-corticosteroid-treated patients; there was a significant difference in the interval between the two groups.

As the most frequent adverse event, delirium was observed in $26.5 \%$ of the patients, followed by oral candidiasis (13.8\%). The interval from the start of administration until the appearance of adverse events was about 3 weeks. There was no significant difference in the incidence of delirium related to the presence or absence of corticosteroid administration, but there was a significant difference in the incidence of oral candidiasis. The results suggest that corticosteroids may become safe, effective drugs if the administration period is limited, with a clear purpose of use.

Abstract number: P01-489

Abstract type: Poster

InfoPall - Benefit of a Palliative Care Drug Information Service Remi C., Bausewein C., Hagemann V.

Department of Palliative Medicine, Ludwig-Maximilians-University, Munich University Hospital, München, Germany

Background: Access to information is essential for health care providers to choose the best treatment for an individual patient. Time for reviewing and assessing available evidence is often lacking. Drug information services can assist health care professionals in gathering relevant information.

Aim: The aim of this study was therefore to evaluate the benefit of a specialized palliative care drug information service (InfoPall).

Methods: Webbased survey among all health care providers who requested information from InfoPall. In addition to demographic data, it was asked whether and how the information was transferred into practice and what the result was.Between 07/2017 and 06/2018 invitations to participate and reminders were sent out 8 and 11 days respectively, after the questioner was provided with the information requested. Results: Response rate 119/176 (68\%). $n=64$ (54\%) of participants were doctors, followed by pharmacists $(n=41(34 \%)$ and nurses $n=12(10 \%) .33$ respondents $(28 \%)$ worked in palliative home care teams, $n=29(24 \%)$ on palliative care units and $n=27(23 \%)$ in retail pharmacies. $n=113(95 \%)$ were satisfied with the answer they received from InfoPall. Information was transferred into clinical practice as recommended in $n=65(55 \%)$ and led to a change in patient status in $n=31(26 \%)$; the latter was mostly an improvement. In $29 \%$ and $34 \%$ respectively, there was no change in patient status or it was unclear. 54/60 respondents stated to have conducted an unsatisfiable literature search before contacting InfoPall.

Discussion: The drug information service was well accepted and mostly used by doctors and palliative home care services. For many users, it provided helpful support for decision-making in everyday clinical practice. A specialized palliative care drug information service can be a valuable resource for health care professionals and contribute to increaser drug safety for the benefit of patients.

Abstract number: P01-490

Abstract type: Poster

Evaluation of Midazolam Prescribing Practices of a Large Tertiary Paediatric Palliative Care Centre in the UK: A Retrospective Case Note Review

Downie J. ${ }^{1}$, Patel B. ${ }^{1,2}$, McCulloch R. ${ }^{1}$

${ }^{1}$ The Louis Dundas Centre for Oncology Outreach and Palliative Care, Great Ormond Street Hospital NHS Foundation Trust, London, United Kingdom, ${ }^{2}$ Department of Paediatric Pharmacy, Great Ormond Street Hospital NHS Foundation Trust, London, United Kingdom

Background: Midazolam is a short-acting benzodiazepine with GABApotentiating effects. It has $\sim 80 \%$ bioavailability (buccal route) and a short half-life. It is a popular choice for the management of agitation, dyspnoea and seizures in palliative care. Despite the frequency of its use, there is a paucity of evidence on dosing guidance for the management of end-of-life symptoms (seizures being the exception).

Aims: To describe midazolam prescribing practices within the last month of life.

Methods: A retrospective cohort study performed at the Louis Dundas Centre for Palliative Care, Great Ormond Street Hospital for Children, London. The electronic case records of all CYP (0-18 years) who died between December 2017 and May 2018 were reviewed. Data were collected on the indication, dosing range, route and efficacy of midazolam in the management of agitation, dyspnoea and seizures in the last 4 weeks of life.

Results: 76 deaths occurred within the study period. Of these 58 were included ( 8 excluded due to incomplete details of end-of-life care/10 managed by another centre at end-of-life). Primary diagnoses were $29 \%$ malignant and $71 \%$ non-malignant. End of life care was managed in a variety of settings (home $31 \% n=18$; hospice $12 \% n=7$; and hospital $57 \%$ $\mathrm{n}=33$ ). Midazolam boluses were prescribed in 33 cases (30 agitation; 21 dyspnoea and 12 seizures). The mean bolus dose ( $\mathrm{mg} / \mathrm{kg}$ ) was 0.051 for agitation; 0.05 for dyspnoea and 0.48 for seizures. The buccal route was the most commonly recommended route $89 \%(n=29) .21$ case records contained recommendations for continuous infusions although only 8 required continuous infusion in the last 4 weeks of life. Of these children 3 were managed in intensive care settings, doses were found to be considerably higher in PICU.

Conclusions: Midazolam is frequently prescribed to manage symptoms at the end-of-life however there appears to be some variability in dosing guidance, particularly when comparing intensive care and palliative care practices.

Abstract number: P01-491

Abstract type: Poster

Study on Medication use in Three Resources of Palliative Care of the province of Valencia

Martínez Gomá R. ${ }^{1}$, Oliete Ramírez E. ${ }^{2}$, Martín S. ${ }^{3}$, Fombuena Moreno M. ${ }^{4}$, Gisbert Aguilar A. ${ }^{5}$

${ }^{1}$ Hospital General de Alicante, Departamento de Ginecología y Obstetricia, Alicante, Spain, ${ }^{2}$ Fundación Instituto Valenciano de Oncología (IVO), Unidad de Hospitalización a Domicilio, Valencia, 
Spain, ${ }^{3}$ Fundación Instituto Valenciano de Oncología, Unidad de Hospitalización a Domicilio, Valencia, Spain, ${ }^{4}$ Hospital Doctor Moliner, Servicio de Cuidados Paliativos, Serra, Spain, ${ }^{5} \mathrm{Hospital}$ Clínico Universitario, Servicio de Soporte Hospitalario Cuidados Paliativos, Valencia, Spain

Background: Due to the importance of palliative care in our environment and in a time when attention to patients at the end of life acquires a special social relevance, research in this area becomes relevant in order to optimize resources and guarantee the best care available to patients with a limited prognosis.

Aim: The purpose of this study is to evaluate the drugs prescribed to patients treated in 3 different resources of palliative care in the province of Valencia and to study the most used in each center, in order to analyze similarities and differences.

Method: We conducted a descriptive, retrospective, observational and cross - sectional review of the medical charts of patients admitted to three palliative care resources in the province of Valencia (two basic equipment and a basic unit) died during 2016. We retrospectively analyzed medications on the patients' charts at admission.

Result: A total of 174 patients were included, 53 from Hospital Clínico Universitario de Valencia (30,5\%), 65 from Hospital Dr. Moliner $(37,4 \%)$ y 56 from Fundación Instituto Valenciano de Oncología (32,2\%). The median age of patients was 72.5 years. $46.4 \%$ of women compared to $53.4 \%$ of men. $70 \%$ of the patients studied present two or more medical antecedents of interest (the most common comorbid diseases were hypertension and diabetes melllitus). There were 1.289 prescriptions (the total number of different drugs is 281), the mean number of drugs per patient is 7,41 and the route of administration most frequently is the oral route. For all three centers the most common prescribed drugs were: Generic Fentanyl, Generic Esomeprazole, Clexane, Generic Paracetamol, Generic Dexamethasona and Omeprazole).

Conclusions: Symptom control is the most frequently reason for admission. Although there are differences between the most frequently used drugs among centers, they coincide in the therapeutic groups (ATC Classification), with the 5 most frequent representing $49 \%$ of the prescriptions.

Abstract number: P01-492

Abstract type: Poster

\section{Cannabis Use in a Rural UK Palliative Care Caseload: A 2018}

Survey

Davies L. ${ }^{1}, O^{\prime}$ Connor $A .^{2}$, Hart $C^{2}$

${ }^{1}$ Severn Hospices, Shrewsbury, United Kingdom, 2 Severn Hospices,

Shropshire, UK, Shrewsbury, United Kingdom

Interest in Cannabis as a medicinal product is growing globally; recent scheduling review of Cannabis by the Chief Medical Officer puts forward a strong case for the therapeutic benefit of cannabis-based medicinal products. We have noticed an increased incidence of questions, from patients and carers relating to Cannabis use. More patients appear to confide in us about their use of Cannabis. The incidence of Cannabis use in a palliative care population is little known.

We undertook a sequential survey of fifty patients referred into and triaged as requiring domiciliary review by the Severn Hospice Nurse Outreach team. Each patient was asked at the end of the usual initial assessment whether they would take part in the anonymised survey. Results: 50 consecutive patients referred to the Shropshire community palliative care team (outreach specialist nurses) 36 patients completed the survey.

Of 36 questionnaires completed 8 patients declined completion. 10 patients (almost a third of those completing the survey) had considered using Cannabis, 18 had not.

The study population $(n=36)$ were mainly aged over $70(n=21)$. Gender split was fairly equal. Of those who had considered cannabis $(n=10)$, the main reason was for pain relief $(n=6)$. Other reasons included general wellbeing and treatment/cure. Half of those who had considered cannabis were on chemotherapy. Ages in the 'cannabis curious' group tended to be younger - with $40 \%$ aged less than 70 . Half of these had researched Cannabis using only Media sources.

Conclusions: In a survey of 50 palliative care patients in a rural county of England asked 'Have you considered the use of Cannabis' most had not. Of 10 patients who said they had considered it, the most commonly cited reason was Pain relief.

Abstract number: P01-494

Abstract type: Poster

Thromboprophylaxis Management of Palliative Inpatients in Acute Hospital Setting

Amorim C., Mendes F., Cardoso P.

Intra-Hospital Palliative Care Team, Hospital Beatriz Ângelo, Loures, Portugal

Background: There are no guidelines for the use of thromboprophylaxis in terminal palliative inpatients and its use is controversial. In acute hospital setting, early referral to a specialized palliative care team is essential to discuss prognosis and useful interventions concerning benefits and symptom control.

Methods: Retrospective, unicenter, descriptive study, based on medical electronic records of inpatients receiving specialized palliative care consultation during 5 months at an acute hospital care setting.

Results: Among the 167 inpatients receiving palliative care consultation, this study enrolled the $77(46 \%)$ patients who died during hospitalization. The majority (95\%) were terminal cancer patients, $35 \%$ of them undergoing active chemotherapy. The mean age was 69.6 years (SD + - 11.1) and 68\% were men. Previously to palliative care consultation $36 \%$ of the patients already scored less than 20 in Barthel Index. In $62 \%$ of the cases referral occured only less than 10 days before death. Fifty three percent were receiving prophylactic anticoagulation, most frequently low molecular weight heparin. Despite specialized palliative consultation, at the time of death $36 \%$ were still on thromboprophylaxis.

Conclusions: Thromboprophylaxis is routinely used in acute hospital setting despite patient's prognosis. Terminal palliative inpatients still receive prophylactic anticoagulation and it doesn't seem to have an important impact on inpatient mortality. Given the absence of guidelines about the use of thromboprophylaxis in terminal palliative inpatients, it should be an early and multidisciplinary decision. This should be a strategie to improve a rational policy of thromboprophylaxis prescription and to prevent discomfort and iatrogenic complications.

Abstract number: P01-495

Abstract type: Poster

\section{Die with the Antibiotic}

Cerullo G. ${ }^{1}$, Abreu K. ${ }^{2}$, Moura A. ${ }^{1}$, Lopes A. ${ }^{1}$, Lima D. ${ }^{3}$

${ }^{1}$ Internal Medicine, University Hospital of Algarve, Faro, Portugal,

${ }^{2}$ Family Health Unit - Farol, Faro, Portugal, ${ }^{3}$ University Hospital of

Algarve, Faro, Portugal

Background: One of the principles of palliative care (PC) is based on improving the quality of life (QOL) of patients, avoiding medically futile attitudes. The use of antibiotics does not change the prognosis of endof-life patients, but in certain situations it may confer comfort to the patient.

Aim: This study intends to investigate the use of antibiotic therapy and its circumstances in hospitalized palliative patients in their last three days of life.

Methods: Retrospective, observational and descriptive study. Analysis of the clinical process and pharmacological prescription of the last 3 days of the life in an Internal Medicine Service, from 01/06/2017 to 01/12/2017. 
Results: 95 patients hospitalized during the analysis period, 85 (89\%) were patients with PC needs, according to WHO and NECPAL ${ }^{\circledast}$ criteria. Of these, $55(64.7 \%)$ had infectious disease, $41(74.5 \%)$ had respiratory infections, $10(18.2 \%)$ urinary and $4(7.3 \%)$ another. Of the 85 patients, $78(91.7 \%)$ were on intravenous antibiotic treatment on the last day of life, and of these, 20 (25.6\%) were receiving a broad spectrum antibiotic (2nd antibiotic). On the last day of life, 4 (5.1\%) had a fever temperature $\left(>37.8^{\circ} \mathrm{C}\right)$ and had an antipyretic drug prescribed in SOS, the remaining $74(94.9 \%)$ had no fever, and 35 ( $47.3 \%)$ were on fixed antipyretic treatment.

Discussion: Patients who died in the Internal Medicine Service with needs of PC represent a important percentage and unfortunately are not recognized as having until the last days of life futile pharmacological treatment. Although the patients were on antibiotic treatment, only a few had fever, most of them did not have fever and about half had fixed antipyretic medication, which is the best option for the symptomatic control. Compared with other national studies, we present a higher rate of antibiotic therapy, which leads us to conclude that there is still a long way to go in approaching and monitoring these patients and increase training.

Abstract number: P01-496

Abstract type: Poster

Difficulties in the Communication about the Future - An Obstacle for Self-determination amongst Residents in Palliative Phase in Residential Care Facilities

Schenell R. ${ }^{1,2}$, Ozanne A. ${ }^{1}$, Strang S. ${ }^{1,3}$, Henoch I. ${ }^{1,3}$

${ }^{1}$ Institute of Health and Care Sciences, University of Gothenburg, Gothenburg, Sweden, ${ }^{2} \mathrm{Health}$ Care Unit, Centrum City District Committee, The City of Gothenburg, Gothenburg, Sweden, ${ }^{3}$ Angered Local Hospital, Gothenburg, Sweden

Background: Residential care facilities are common places of death with a high prevalence of palliative care needs. For some residents the ability to make and execute decisions is reduced due to cognitive or physical losses. Despite that many residents do have capacity in decision making they are not always involved in planning for their own end-of-life care.

Aim: To illuminate staffs' experiences of communication in relation to the residents' self-determination in the palliative phase.

Methods: Twenty individual semi-structured interviews with registered nurses, enrolled nurses, and physicians working in residential care facilities were analyzed with qualitative content analysis.

Results: Communication was described as crucial for the self-determination. Both the residents' own abilities to communicate and the support they could receive from others when the ability was reduced were important factors. Insufficient communication within the care team with e.g. a hierarchical communication chain, fear of talking about death, and the different professions' interpretations of the terms palliative phase, do not resuscitate (DNR), and end-of-life discussions, also affected the residents' self-determination, resulting in lack of planning for the future. The lack of end-of-life care planning sometimes resulted in conflicts between the staff and the relatives, hospital admissions, and life prolonging treatments which was quite the opposite of what the staff believed that the residents wanted.

Conclusion/discussion: Not planning for end-of life-care compromised the residents' self-determination and left the staff and the relatives in an uneasy position, when being forced to make decisions without knowing the residents' wishes. By implementing a palliative approach, with a focus on quality of life, early in the residents' illness trajectories, communication about end-of-life care, both within the group of staff and between residents, relatives and staff could be facilitated.

Abstract number: P01-498

Abstract type: Poster
Review of the Documentation of Mild Cognitive Impairment or Dementia on Initial Palliative Care Assessment

Kilbane L. ${ }^{1}$, Cunningham J. ${ }^{2}$

${ }^{1}$ Palliative Care, Our Lady's Hospice and Care Services, Blackrock, Dublin, Ireland, 2Palliative Care, OLH Blackrock, Dublin, Ireland

Background: Greater numbers of older people are accessing Palliative Care Services. Timely diagnosis of Mild Cognitive Impairment or Dementia may improve outcomes in decision making, symptom management and planning for future care.

Aims: Review documentation of Mild Cognitive Impairment or Dementia on Initial Palliative Care Assessment; and use this information to improve patient outcomes.

Standard: All patients over the age of 65 should be screened for cognitive impairment. If this cannot be undertaken due to patient condition, this should be clearly documented.

Methodology: A retrospective cohort study was undertaken of 80 active patients over the age of 65 under the care of Community Palliative Care Services between June 2017 and August 2018.

Data was manually extracted from patient records.

Initial Palliative Care Assessments were reviewed for the completion of 'Orientation- Memory - Concentration Test' (1)

Results: Of 80 patients, 52 were assessed by Palliative Care Registrars and 28 by Clinical Nurse Specialists.

$42.5 \%$ (34/80) of cognitive assessments were completed; $30 \%(24 / 80)$ by medical staff and $12.5 \%(10 / 80)$ by nursing staff.

$57.5 \%(46 / 80)$ of cognitive assessments were not completed; $35 \%$ $(28 / 80)$ by medical staff and $22.5 \%(18 / 80)$ by nursing staff; Of these, $54 \%(25 / 46)$ did not document a reason why cognitive assessment was incomplete.

Conclusion: The completion of cognitive screening on Initial Palliative Care Assessment is sub-optimal, which has implications on overall quality of patient care.

Actions: I have presented my results locally at Journal Club, and Grand Rounds, reaching local and nationwide Hospice and Community services. I have emphasized the importance of early diagnosis of cognitive impairment, and the implications on overall patient outcomes.

Re-audit plan: Re-audit is now in progress to observe for interval improvement in completion of cognitive screening assessments.

Abstract number: P01-499

Abstract type: Poster

GEriatric Trauma and Palliative Care (GET PC): Predictors of Consultation

Radwany S. ${ }^{1}$, Drost J. ${ }^{2}$, Yoder K. ${ }^{3}$, Oravec M. ${ }^{4}$, Birmingham L. ${ }^{5}$, George R. ${ }^{5}$

1Palliative Medicine, The Ohio State University, Columbus, United States, ${ }^{2}$ Senior Services, Summa Health, Akron, United States, ${ }^{3}$ Hospice and Palliative Medicine, Summa Health, Akron, United States, ${ }^{4}$ Internal Medicine, Summa Health, Akron, United States, ${ }^{5}$ Trauma Services, Summa Health, Akron, United States

Background: Studies have identified risk factors for mortality in geriatric trauma patients (GTP), but little information exists regarding who receives palliative care consultation (PCC).

Aims: The objective of this study is to identify factors in GTP associated with inpatient PCC.

Methods: A retrospective observational study identified patients aged $\geqslant 65$ admitted to the trauma service between January 2013 and December 2015. Bivariate analyses using $\chi^{2}$ and t-tests were used to identify covariates associated with having PCC. Covariates with $p<0.25$ were included in a mixed-effects logistic regression model.

Results: 1,343 patients met inclusion criteria. $151(11.2 \%)$ received PCC with an average age was 84.0. In the adjusted model, significant factors 
for PCC included age (OR 1.04), Injury Severity Score (ISS) $=16-24$ (OR 3.36), ISS $\geqslant 25$ (OR 13.10), ICU admission (OR 1.68), and spinal injury (OR 2.53). Dementia was the only comorbidity that remained significant in the adjusted model (OR 2.30). In-hospital mortality rate was $47.7 \%$ for the PCC group.

Conclusion: This study described a GTP population and examined factors associated with receipt of PCC. In-hospital and 30-day mortality were higher in the PCC group than the non-PCC group, suggesting those GTPs with higher risk of mortality were referred for PCC. The predictive model demonstrated that age, injury severity, ICU admission, sustaining a minor fall, sustaining a spine injury, or having dementia were associated with receipt of PCC. Further research should be conducted to determine which patients would benefit most from PCC in the GTP to optimize utilization of palliative care resources.

\section{Abstract number: P01-500}

Abstract type: Poster

To Be Exposed - And Assent to it. Meaning in Bodily Care as Narrated by Older Persons Living in a Nursing Home Holmberg B. ${ }^{1}$, Hellström I. ${ }^{1,2}$, Norberg A. ${ }^{1,3}$, Österlind J. ${ }^{1}$

${ }^{1}$ Ersta Sköndal Bräcke University College, Department of Health Care Sciences/ Palliative Research Centre, Stockholm, Sweden, ${ }^{2}$ Linköping University, Department of Social and Welfare Studies, Norrköping, Sweden, ${ }^{3}$ Umeå University, Department of Nursing, Umeå, Sweden Presenting author email address: bodil.holmberg@esh.se

Background: Due to improved living conditions, developed care and medicines, people world-wide live longer. This comprises an increase in multimorbidity and a bodily frailty that causes many older persons to live in a nursing home (NH) during the end of life. Following this bodily frailty, they may need help with clothing, eating, personal hygiene and transfer. This means that others get access to their body in a way that would not be accepted in other contexts. Thus, the barriers of privacy are moved in a way that may be stressful to an older person at the end of life. In Sweden, survival time in nursing homes is short, as more than half of the admitted older persons die within a year. This indicates a need of care with an early integration of a palliative approach, to meet their complex needs from a holistic view.

Aim: As bodily needs are paramount to many older persons in $\mathrm{NH}$, the aim of this study was to elucidate meaning in bodily care, as narrated by older persons living in a $\mathrm{NH}$.

Methods: A reflective lifeworld approach within the perspective of caring science was applied, using a phenomenological- hermeneutical analysis-method. Twelve older NH residents were interviewed 1-5 times, in all 28 interviews.

Results: From their narratives one main theme emerged: 'To be exposedand assent to it'. The main theme included five themes, 'To have hope in hopelessness', 'To relinquish the body into other peoples' hands, 'To be between power and powerlessness', 'To oscillate between own responsibility and demands', 'To interact with others', and ten subthemes.

Conclusion: The studied phenomenon can be described as to comprise more than practical aid with every-day- tasks, indicating that the whole person is involved when receiving bodily care. Thus, this study can generate new perspectives on bodily care in $\mathrm{NHs}$, and describe its complexity from a caring perspective. This is important to develop a care with a palliative approach, suitable to older persons' needs.

Abstract number: P01-501

Abstract type: Poster

How Palliative Care Is Understood in Older Person Residential Care: An Action Research Study

Molloy U. ${ }^{1,2}$, Phelan $A .^{3}$

${ }^{1} \mathrm{NMHS}$, University College Dublin, Dublin, Ireland, ${ }^{2}$ Community Palliative Care, St Francis Hospice Dublin, Dublin, Ireland, ${ }^{3}$ School of
Nursing Midwifery and Health Sciences, University College Dublin, Dublin, Ireland

Caring for older people in residential care presents significant challenges in relation to the provision of palliative care.

Using Action research, a total of 18 healthcare assistants and 16 registered nurses in two residential older person care units, participated in co-operative inquiry groups (CIGs). Members of the CIG reflected on deaths that occurred on the units over a six-month period. A number of actions took place: a debriefing group, acknowledging the importance of relationships and a sense of loss experienced by staff on the death of a resident, a group reflection on recognising dying and how this might impact on care a resident receives, and a healthcare assistant group which contributed to developing an understanding of the unique role they have in this context. Finally, reflections on the meaning of palliative care, end of life care (EOLC) and person-centred care(PCC) in older person care. Findings were co-created within the CIG.

This study has illustrated that there are still difficulties with understandings of palliative care in older person care, where it is often aligned with dying. EOLC was a more commonly used term. In this study, however, in the context of older person care, person centred care appeared to best describe the philosophy of care and the co-inquirers suggested that palliative care should be an inherent part of this care. Palliative care was often associated with cancer and specialist palliative care teams. 'Living, Loving and Letting go', were the three main themes integrating the many descriptors used to describe caring for older people in residential care within the CIG.

There is a need to value the expertise and experience of the nurses and healthcare assistants caring for older people living and dying in residential care. There is also a need to develop the interdisciplinary roles between older person care and specialist palliative care.

Abstract number: P01-502

Abstract type: Poster

Withdraw of Advanced Life-Sustaining Therapy from the Elderly People - Time of Survival

Moreira F. ${ }^{1}$, Coelho M. ${ }^{2}$, Casseb G. ${ }^{1}$, Carmo A. ${ }^{2}$, Tosi M.C. ${ }^{2}$, Queiroz J.C. ${ }^{2}$, Motta C. ${ }^{2}$

${ }^{1}$ Cuidados Paliativos, Prevent Senior, São Paulo, Brazil, 2Prevent Senior, São Paulo, Brazil

Introduction: A common scenario in developed countries, palliative care is also gaining ground in Brazil. Prioritizing the care and management of the patients' symptoms with poor prognosis brings these individuals comfort and dignity, allowing their death to occur in a natural way. In this process, palliative extubation has proved to be an important tool. In the present study we show 308 patients undergoing palliative extubation; we followed-up these patients' evolution and post-procedure survival time.

Methodology: A retrospective study analyzing patients undergoing palliative extubation over the years 2016 and 2017 in private hospitals in São Paulo.

Results: 308 patients undergoing palliative extubation with mean age of 80 years of age, being $72 \%$ female. The main admission diagnoses were related to infections $(41.5 \%)$, and to the following disorders: neurological disease (31.1\%), coronary artery disease (14.2\%) and oncological disease $(12.9 \%)$. After the procedure, 28 patients $(9 \%)$ died within $6 \mathrm{~h}$, and 48 subjects (15.6\%) died between 6 and 24h; 22 patients (7.14\%) were released from hospital; however, out of these individuals who were discharged from hospital, 12 (3.89\%) died between 30 and 90 days and 8 (2.59\%) died after 90 days (maximum survival time of 109 days).

Conclusion: Based on the results above, it is clear the importance of stimulating this medical practice, since it seeks to rescue not only the dignity of the patients and their relatives, but also the humanity to the health service, since palliative extubation serves as a tool that may 
possibly lead to less aggressive outcomes, avoiding, thus, futile and unnecessary therapy.

Abstract number: $\mathrm{P} 01-503$

Abstract type: Poster

Could Mobile Team Improve Palliative Care in Nursing Homes? Cernesi S. ${ }^{1}$, Taglini A. ${ }^{2}$, Scandone S. ${ }^{3}$, Mele F. ${ }^{4}$, Daniela D.N. ${ }^{4}$, EAPC Primary Care Reference Group

${ }^{1}$ Movimento Giotto, Trento, Italy, ${ }^{2}$ Ausl Modena, Modena, Italy, ${ }^{3}$ Amici per la Vita Association, Modena, Italy, ${ }^{4}$ Nursing Home Casa Serena, Sassuolo, Italy

Background: Deaths occurring in care homes in UK could more than double in the next 25 years if trends continue. Our Mobile team try to struggle this challenge. Team consist of General Practitioner PC expert, Nurse PC expert and Psychologist. We established a mobile team that visit all the public nursing home $(\mathrm{NH})$ regularly.

Objectives: Find target patients. Involvement of the multiprofessional team, Help to define Advanced Care Plan or Anticipate Care Plan. Symptoms control and Therapy modulation. Audit in difficult case. Different kind of intervention (phone call, $\mathrm{NH}$ visit, writing consultation). Method: Peer education. During consultation the key staff be present. We provide a cascade model trainings. Other aime is data collection and promote audit in difficult case.

Results: From May 2018 to September 2018 we have helped to find 56 cases (10 are in charge, 46 are died).We performed $20 \mathrm{NH}$ visit.We perform PC consultation for $8 \mathrm{NH}$. We have done 3 audit for difficult cases. In 3 cases we facilitated the PC specialist, In 3 cases we suggested psychologist. More than $95 \%$ died in $\mathrm{NH}$ with symptom control.We help to define Advanced Care Plan the aim is that every patient have a ACP.

Conclusion: We have to consider $\mathrm{NH}$ as Large diffuse Hospice.Our team seems to be efficient to help to find the case and we have positive feedback from the staff.We have avoided hospitalization in $>90 \%$ of the cases we have been in contact.Most of the problem $(>60 \%)$ is the relation with the caregiver, working with anticipate care. Still difficult to estimate prognosis but most of the target patient died in 5 months. We use Focus group to maintenance this project Is difficult to involve GP. Is important be flexible and disponible.

Abstract number: P01-504

Abstract type: Poster

Palliative Care in General Practice, a Contradiction in Terms? A Systematic Review

Forero R. ${ }^{1,2}$, Hillman $K_{.}^{1,2}$, Gonzalez N. ${ }^{1,3}$, Frasier S. ${ }^{4}$, Williams R. ${ }^{4}$, Keech P. ${ }^{4}$, Chow J. ${ }^{4}$

${ }^{1}$ Simpson centre, UNSW, Liverpool BC, Australia, ${ }^{2}$ Ingham Insitute for Applied Medical Research, Liverpool BC, Australia, '3Liverpool Hospital, Liverpool BC, Australia, ${ }^{4}$ Clinical Innovation and Business Unit, SWSLHD, Liverpool BC, Australia

Background: Management of older patients at the end-of-life by general practitioners (GPs) is presenting a challenge as most people want to die outside hospitals; want to be informed about their current health situation and want to have the opportunity for open and honest discussions about their end of life care needs.

Aims: To review the existing literature to assess the evidence for managing patients and barriers for early identification of end-of-life care needs in the community setting, and areas of improvement such as consultation with patients and implementation of Advanced Care Directives (ACDs) and Advanced Care Plans (ACPs).

Methods: A systematic review of the literature. We used MEDLINE and EMBASE databases to search for articles containing: "End-of-life", "Physicians", "Primary Health Care", "Family practice", "General practitioner", "community resources", "good death", "palliative care",
"Residential facilities", "Aged", "Advance care planning or Advance directives", "Death or dying" and "tools".

Results: The review identified key areas where there is lack of research and/or suboptimal policy implementation. These barriers include lack of skills for end of life communication, difficulties with defining the right time and place; the attitude that it is the patient who should initiate ACP, and fear of depriving patients of hope. We also identified a discrepancy between the perceived quality of life reported by patients and the perceived loss of quality of life by GPs when referring to palliative care. Stronger evidence was found for the following facilitators: the ability to foresee health problems in the future, skills to respond to a patient's initiation of ACP, personal convictions about who to involve in ACP, and a longstanding patient-GP relationship and the home setting.

Conclusion/discussion: The paper concludes with some areas for policy development and discuss strategies for future research in general practice in New South Wales, Australia.

Abstract number: P01-505

Abstract type: Poster

Exploring Meaning and Identity among Terminally-ill Patients through Engagement in Food-related Occupational Therapy in a Palliative Care Ward in Singapore

Wong L.H. ${ }^{1}$, Han P.S. ${ }^{2}$, Ramaswamy A. ${ }^{3}$, Wong C.H. ${ }^{1}$

${ }^{1}$ Geriatric Education and Research Institute, Singapore, Singapore, ${ }^{2}$ Yishun Community Hospital, Singapore, Singapore, ${ }^{3}$ Khoo Teck Puat Hospital, Singapore, Singapore

In palliative care, occupational therapists work on improving quality of life and optimising functional abilities of patients. Terminally-ill adults with deteriorating health experience has less engagement in foodrelated activities. These activities could be important to maintain personal identities and psycho-social needs amongst palliative care patients in an Asian context. Food is often perceived as an act of filial piety, comfort and care amongst the terminally-ill Chinese adults. However, there is limited research exploring the nexus of meaningful food-related occupational engagement, identity maintenance, and meaning among the terminally-ill patients.

This study aims to explore the perspectives of staff and patients on a new food-related occupational therapy programme and the impact on patients' psychological and social needs.

An inductive qualitative approach was adopted using semi-structured interviews for 2 groups. One for palliative in-patients who have participated in food-related therapies and the other for healthcare professionals (HCP) in the palliative ward. Two independent coders analysed data collected for recurrent themes.

17 patients and $12 \mathrm{HCP}$ interviews were conducted. There were 14 themes collected in three main topics; patient's needs, food-related therapies and outcome. Overall, participants expressed that foodrelated therapy provided a sense of familiarity and identity maintenance. It also empowered patients by allowing them to make decisions and increased opportunities for social interaction. Although most HCP expressed the importance in exploring patient's life context, some were unable to cope with the additional workload.

Overall, food-related occupational therapy could be essential in addressing patients' psychological, social and spiritual needs at a palliative care ward in Singapore. Sometimes, illness for terminal patients define their identity. Therapy helps them to see beyond the illness and re-establish their real identity.

Abstract number: P01-506

Abstract type: Poster

Embedding a Palliative Approach in Residential Settings (EPAIRS) Webley J., Edmunds P. 
Regional Palliative End of Life Program, Vancouver Coastal Health Authority, Vancouver, Canada

The EPAIRS project was designed and implemented in response to numbers of residents being transferred to acute hospitals to die.Working with residents, family members and healthcare team members, we identified what mattered most to stakeholders, developing the model. We began with one home, delivering formal education for all health care members regarding palliative approaches to care, and also presenting to resident and family council meetings. It became clear that to change how people died, we had to change the culture of the home and make death and dying more visible. This allows for supports to be put in place that the users consider important rather than assumptions made by health care professionals regarding what was needed. The model was formed to represent a home, with 4, supporting pillars and a clear process for individualised, resident centered communication and planning for care. The model has been implemented at 48 sites across the health Authority (5,703 residential Nursing beds) and education provided to all stakeholders. Each site reports their monthly deaths, place of death, acute transfers in last 3 months of life and date of goals of care (GOC) conversations. Evaluation of the data indicated that quality of the conversation was important, the timing was critical.Monthly reporting allows us to offer just in time supports and review charts when transfers increase at any given time. To date, days spent in hospital in the last 3 months of life has decreased by $45 \%$ since pre implementation (1582 days), acute transfers without admission decreased by $56 \%$ (248 transfers) and deaths in acute by $26 \%$ in one year ( 79 more residents died at home). Transfers to acute were $4 \mathrm{X}$ more likely to occur if GOC occurred less than 2 weeks prior to death. Transfer risk increased again if GOC occurred more than 6 months prior to death with no follow up. Ideal window is 6-26 weeks. Model is now being implemented in other Provinces in Canada through collaboration with CFHI.

\section{Abstract number: P01-507}

Abstract type: Poster

\section{Families' Experiences with End-of-Life Care in Nursing Homes and Associations with Dying Peacefully with Dementia Bolt S. ${ }^{1}$, Verbeek L.M.H. ${ }^{1}$, Meijers J.M.M. ${ }^{1}$, van der Steen J.T. ${ }^{2,3}$ ${ }^{1}$ Department of Health Services Research, Maastricht University, Maastricht, Netherlands, ${ }^{2}$ Department of Public Health and Primary Care, Leiden University Medical Center, Leiden, Netherlands, ${ }^{3}$ Department of Primary and Community Care, Radboud University Medical Center, Nijmegen, Netherlands}

Background: Previous research indicated that only half of the nursing home residents with dementia died peacefully according to their family. It is important to consider experiences with end-of-life care of families of people with dementia.

Aims: To examine families' experiences with end-of-life care for nursing home residents with dementia and associations with dying peacefully. Methods: Data were collected in the Dutch End of Life in Dementia (DEOLD) study between 2007 and 2010 at 34 nursing homes. This secondary analysis investigated after-death family perspectives on 252 nursing home residents with dementia using mixed-methods. We thematically analysed open-ended questions about unpleasant experiences with end-of-life care. We performed multilevel regression analyses with the primary outcome dying peacefully and associations with the unpleasant experiences, families' satisfaction with care and decisionmaking using validated instruments.

Results: Two themes represented families' experiences. 'Neglect' involved facing inaccessibility, disinterest, or discontinuity of relations, and negligence in tailored care and information. 'Lack of respect' involved perceptions of an unfeeling approach, being purposefully disregarded, non-compliance with agreements, and violations of privacy. Unpleasant experiences with end-of-life care were negatively associated with families' perceptions of their relative dying peacefully ( $\mathrm{B}=-.73, \mathrm{Cl}=-1.37 ;-.09)$. Dying peacefully was positively associated with satisfaction with end-oflife care $(\mathrm{B}=.08, \mathrm{Cl}=.05 ; .11)$, decisions $(\mathrm{B}=.16, \mathrm{Cl}=.07 ; .24)$, and the decision-making process $(\mathrm{B}=.04, \mathrm{Cl}=.01 ; .07)$.

Conclusion/ discussion: Families' unpleasant experiences with care concern disappointment through the absence of humane, compassionate care and attention for a loved one nearing death. Authentic attention from physicians and other nursing home staff for resident and family may facilitate memories of a peaceful death.

Abstract number: P01-508

Abstract type: Poster

'The Absent Voice' - The Reality of Advance Care Planning for People with Dementia in Long Term Care Settings. A Mixed Methods Study

Beck E.-R., Mcllfatrick S., Hasson F.

School of Nursing, Ulster University, Newtownabbey, United Kingdom

Background: Advance care planning (ACP) is advocated for people with dementia, yet discussions with this group are rare, particularly in longterm care settings (LTCS).

Aim: To examine the understanding and experience of family caregivers of people with dementia and HCPs, regarding the process of ACP.

Method: A sequential explanatory mixed methods design underpinned by the Theory of Planned Behaviour.

- A cross-sectional survey to nursing home managers $(n=116)$.

- Interviews and focus groups with key health care professionals $(n=49)$. - Interviews with family caregivers $(n=16)$.

Results: All 3 groups demonstrated poor knowledge of ACP. Whilst HCPs and families acknowledged benefits, these were eclipsed by challenges to implementation in this setting. These included: concerns regarding the ability of the person with dementia to engage, the increased role of the family, a lack of context specific guidance, and a knowledge deficit regarding dementia and $\mathrm{ACP}$.

Widespread variations in perspectives included when it should be implemented, who should be responsible and core components. GPs played a key role, whilst nurses remained on the periphery. Families ability was restricted by a limited awareness of dementia, decisional burden due to guilt and anticipatory grief, and limited ability to reflect the wishes of the person with dementia.

Conclusions: ACP is complex within this context, influenced by multiple factors related to the setting, the perspectives of those involved and the context of dementia itself. The LTCS is too late to initiate the process of $\mathrm{ACP}$, with this resulting in a reactive as opposed to proactive approach to decision making. resulting in people with dementia often being outside the process. This is at odds with the true purpose of ACP, and indeed conflicts with international consensus statements that aim to provide greater clarity on defining ACP.

Abstract number: P01-509

Abstract type: Poster

Retrospective Analysis of Hospital Patient in the Mersin City Education and Research Hospital Adult Palliative Care Unit Turgut Ö., Pektas M., Aydinli B., Koçer S., Sagün A.

Palliative Care Unit, Mersin Şehir Eğitim ve Araştırma Hastanesi, Mersin, Turkey

Introduction: Palliative care is a multidisciplinary approach aiming increase at quality of life the patient and patient relative which encountered with life threating ilness problems. This approach as the other cities in Turkey getting across in our hospital 46 bed palliative care unit is starting up. Aim: Taking out the patient profile which uses Mersin city education and research hospital. 
Material and method: 850 inpatient's file which in the palliative care unit between 15.03.2017 and 15.03.2018 analysed retrospective. Age, gender, duration of hospital stay, admission diagnosis, nutritional status and discharge status are analysed.

Findings: 450 of 850 patient attending the research is $\% 52$ female, $\% 47$ male. The patient staying in the Palliative care unit age avarage is 69.24 , duration of hospital stay 15 days. 242 patient (\%28) stayed in the hospital because of malignancy and its complications. 217 patient (\%25) stayed in because of cerebrovascular disease and its sequel. 150 patient (\%17) stayed in because of Alzheimer and dementia. Beside those diagnosis 241 (\%28) patients were hospitalized for diabetes and its complications, COPD, renal failure, posttrauma and for similar endications.162 of patients (\%18) with those diagnosis also have nutrition deficiency and decubitis ulcer.

423 of those patient's (\%50) nutrition was enteral and parenteral feeding. 468 (\%55) were transferred to another department, 282 (\%33) were discharged, 100 (\%11) died.

Discussion / Conclusions: Although palliative care units are common all around the world, in Turkey they are newly founded. Rising in elderly population and chronic disiases all around the worl is rises palliative unit demands. Therefore patient profile should be determined and according to that palliative care units should have necessary equipment and trained staff. Keywords: Palliative Care, Patient Profile, Nutrition

Abstract number: P01-510

Abstract type: Poster

Engaging Residents and Families in Conversations about Advance Care Planning in Long Term Care Home

Kaasalainen S. ${ }^{1}$, Sussman T. ${ }^{2}$, Kulasegaram P. ${ }^{1}$, Thompson G. ${ }^{3}$, SPA-LTC

Team

${ }^{1}$ McMaster University, Hamilton, Canada, ${ }^{2}$ McGill University, Montreal, Canada, ${ }^{3}$ University of Manitoba, Winnipeg, Canada

Background: Despite the high mortality rates in long term care (LTC), most LTC homes do not have a formalized palliative program that includes capacity-building for staff with a focus on advance care planning (ACP). Unfortunately, failure to proactively and pre-emptively discuss and identify end-of-life issues in LTC often leads to costly hospitalizations and creates added stress and burden for family and staff.

Aim: The aim of this study was to explore the perceptions of LTC staff about engaging in ACP discussions with residents and their family.

Methods: This study used a qualitative descriptive design. Data was collected at four LTC homes in southern Ontario. Thirteen focus groups were held with LTC staff (registered staff, personal support workers, support staff) and six interviews with other health care providers and administration. Data were analyzed using thematic content analysis.

Results: Staff reported that earlier initiation of ACP discussion was needed. Lack of understanding of ACP was identified across all participant groups as well as the role of each health care provider in engaging in these discussions. However, staff emphasized the importance of having personal relationships with residents and families and how this can make having these discussions easier.

Conclusions: Our study identified that staff understanding and comfort with ACP discussions influenced their level of engagement in these important discussions. Clearly, education is needed for LTC staff about ACP to improve their comfort and ability to discuss residents' values and wishes across the life trajectory to facilitate more informed decisionmaking for residents and families during end-of-life.

Abstract number: P01-511

Abstract type: Poster

Identification of the Unmet Social Needs of Community-dweller Elderly with Cognitive Impairment Using WHOQoL-OLD Form
Perdamaian T., Widagdo T.M.M., Sigilipoe M.A., Manus W.C., Centre for Research and Service on Gerontology

Faculty of Medicine, Duta Wacana Christian University, Yogyakarta, Indonesia

Background: Frailty is marked by declining cognitive function and social interaction. Palliative approach in frail elderly should include identification of unmet social needs which might help caregivers and social workers to optimise patient's quality of life (QoL).

Aim: This study is aimed to identify unmet social needs in elderly with cognitive impairment using WHOQoL-OLD form.

Methods: A cross-sectional study was conducted in 14 sub-districts of Yogyakarta City, Indonesia, among 377 community dweller seniors ( $\geqslant 60$ years old) in 2017. Cognitive impairment is defined as adjusted-MMSE score $\leqslant 24$. The Indonesian version of WHOQoL-OLD form was administered with researcher assistance. We conducted multilevel modelling with maximum likelihood approach (nlme ver. 3.1-137 in R) to predict whether cognitive impairment would show poorer QoL profile, with subdistricts as random variable. The limit of significance in this study is 0.05 . Results: There were $80(21.22 \% ; 95 \% \mathrm{Cl}=17.27-25.77 \%)$ elderly people classified with cognitive impairment. The average QoL is $83.84 \pm 7.90 \%$ in healthier group, and $80.52 \pm 9.57 \%$ in impaired cognitive group. The presence of cognitive impairment significantly predict overall QoL ( $b=$ $-0,24, \mathrm{t}(362)=-2.47, \mathrm{p}=0,014)$, Autonomy domain $(b=0,05, \mathrm{t}(362)$ $=-2.82, \mathrm{p}=0.005)$, and Social Participation domain $(b=-0,04, \mathrm{t}(362)$ $=-2,98, p=0,003)$ with negative correlation $(r=-0,224,-0,228$, and $r$ $=-0,357$, subsequently). Within impaired cognitive group, MMSE score predict the Sensory domain $(b=-0.01, t(65)=-1,99, p=0,050)$ with negative correlation ( $r=-0,983)$.

Discussion: Elderly with cognitive impairment showed poorer QoL, especially in Autonomy and Social domain, compared to healthier group. In addition, lower MMSE score is associated with poor sensory abilities. This study demonstrated that WHO-QoL OLD form is useful to identify the social needs, independency and sensory decline in elderly which indicate early social support.

\section{Abstract number: P01-512}

Abstract type: Poster

An Investigation of Inpatient End-of-Life Care for Older Australians: A Secondary Analysis of Medical Record Data Bloomer M. ${ }^{1,2,3}$, Botti M.1,3, Hutchinson A. ${ }^{1,2}$

${ }^{1}$ School of Nursing and Midwifery and Centre for Quality and Patient Safety Research, Deakin University, Burwood, Australia, ${ }^{2}$ Centre for Nursing Research, Deakin University and Monash Health Partnership, Monash Health, Clayton, Australia, ${ }^{3}$ Epworth/Deakin Centre for Clinical Nursing Research, Epworth Healthcare, Richmond, Australia

Background: Australian national guidelines were developed to ensure timely, high-quality end-of-life care across inpatient settings.

Aim: To explore family involvement and end-of-life care practices in inpatient settings.

Methods: A secondary analysis of medical record data from two previous studies of inpatients who died in private acute care, public acute care and aged rehabilitation settings in 2016.

Results: Of deceased inpatients $(\mathrm{N}=506)$, the mean age was $76.9 \mathrm{yrs}$ (SD 12.9) and mean length of stay was 11.3 days (SD 12.6). 63.2\% $(n=320)$ were admitted for active treatment and $34.6 \%(n=175)$ for palliation. $66.0 \%(n=334)$ were admitted to acute and $19.4 \%(n=98)$ to palliative care settings. $87.4 \%(n=442)$ had a Limitation of Medical Treatment (LOMT) order. Family consultation about poor prognosis was documented in $72.5 \%(n=367)$ of cases, yet only $46.6 \%(n=236)$ were referred to specialist Palliative Care and $48.0 \%(n=243)$ had care guided by a dying care pathway. Family were present at $54.7 \%(n=277)$ of deaths. 
A LOMT was more likely for patients admitted for palliation $(\chi 2(2)=0.67$, $\mathrm{p}<0.0001)$, as was evidence of family consultation $(\chi 2(2)=41.55, \mathrm{p}<$ $0.0001)$. Referral to specialist Palliative Care $(\chi 2(2)=65.43, p<0.0001)$ and dying care pathway use $(\chi 2(2)=88.92, p<0.0001)$ were also more likely for patients admitted for palliation.

Family were more likely to be present if death occurred between 0801$2200 h r s$ than overnight $(\chi 2(1)=22.13, p<0.0001)$ and when there was evidence of family consultation about poor prognosis $(\chi 2(1)=21.35, p<$ $0.0001)$. Family presence at death was also more likely following a referral to specialist Palliative Care $(\chi 2(1)=11.34, p=0.001)$ and when a dying care pathway was used $(\chi 2(1)=4.58, p=0.040)$.

Conclusion: End-of-life care practices could be improved through routine specialist Palliative Care referral and care pathway use. These strategies may result in improved communication of prognosis and impending death, greater family involvement and presence at death.

Abstract number: P01-513

Abstract type: Poster

Specialist Palliative Care Services for Older People in Primary Care: A Systematic Literature Review

de Nooijer K. ${ }^{1}$, Penders Y.W.H. ${ }^{2}$, Pivodic L. ${ }^{1}$, Van Den Noortgate N. ${ }^{3}$, Pype $P^{4}{ }^{4}$, Van den Block L. ${ }^{1}$

${ }^{1}$ Family Medicine and Chronic Care, Vrije Universiteit Brussel (VUB) \& Ghent University, Brussels, Belgium, ${ }^{2}$ Biostatistics and Prevention Institute, University of Zürich, Zürich, Switzerland, ${ }^{3}$ Geriatric Medicine, Ghent University Hospital, Ghent, Belgium, ${ }^{4}$ Public Health and Primary Care, Ghent University Hospital, Ghent, Belgium

Background/aims: There is wide recognition that older people with incurable conditions should have access to specialist palliative care services. However, it remains unclear what these services for older people in primary care should entail. Based on current literature, we aim to identify to whom, when, and how specialist palliative care in primary care is provided, which outcomes are identified and how this can be improved.

Methods: A systematic review was conducted according to the PRISMA guidelines, using Embase, Medline, Web of Science, Cochrane, Google Scholar, PsycINFO, and Cinahl ebsco databases. Quality appraisal and study selection of included studies were performed by two researchers independently. Patients' characteristics, intervention features, outcome data and suggestions for improvement were retrieved.

Results: Ten articles were relevant for this review. Three with a qualitative design, three with a quantitative design, three with a mixed-method design, and one narrative review. Referral criteria to specialist palliative care services were mainly based on patient characteristics, such as prognosis. Specialist palliative care services involved multidisciplinary professionals who provided supportive holistic care, through home visits or by phone in varying intensity. The outcomes of specialist palliative care were mainly on the level of patients and/or relatives, such as increased self-confidence and symptom control. Specialist palliative care services could be improved regarding the information flow between care professionals, greater in-depth palliative care knowledge, identification of a skilled key-worker and support for relatives.

Conclusions: There is limited evidence regarding specialist palliative care services for older people in primary care, future studies are needed to increase the evidence-base. In addition, this review pinpoints the importance of comprehensive reporting of interventions and the need for consensus-based outcome measures.

Abstract number: P01-514

Abstract type: Poster

What Do People Want at the End-of-Life? A Mixed Methods Study on Older People's Preferences and Knowledge about Advanced Care Plans
Hanson S. ${ }^{1,2}$, Nielsen D.3,4,5, Brabrand M. 1,6, Ryg J.7,8, Forero R. ${ }^{2,9}$ ${ }^{1}$ University of Southern Denmark / Institute of Regional Health Research, Esbjerg, Denmark, ${ }^{2}$ Simpson Centre for Health Services Research, University of NSW, Liverpool BC, Australia, ${ }^{3}$ Center for Global Health, University of Southern Denmark, Odense, Denmark, ${ }^{4}$ Migrant Health Clinic, Odense University Hospital, Odense, Denmark, ${ }^{5}$ Health Sciences Research Center, University College Lillebaelt, Odense, Denmark, ${ }^{6}$ Emergency Department, Hospital of South West Jutland, Esbjerg, Denmark, ${ }^{7}$ Institute of Clinical Research, University of Southern Denmark, Odense, Denmark, ${ }^{8}$ Department of Geriatrics Medicine, Odense University Hospital, Odense, Denmark, ${ }^{9}$ Ingham Insitute for Applied Medical Research, Liverpool BC, Australia

Background: What matters at end-of-life (EOL) is poorly investigated with limited evidence about preferences and knowledge about advance care plans (ACPs).

Aims: To clarify what matters at EOL along with treatment preferences for the older population in Denmark.

Methods: A mixed methods study was undertaken using purposive and snowball sampling with people $>65$ years. All interviews and focus groups were analyzed using NVivo 11 . The analysis comprised two levels: Systematic Text Condensation (STC) and Theoretical Analysis (TA). The STC was used to generate hypotheses and to identify major themes. The TA was then used to generate a theoretical framework based on the four-dimension EOL (4D EOL) trajectories.

Results: Five focus groups with active older people, and 9 in-depth interviews with nursing home residents and older people admitted to emergency department (ED) were conducted between November 2016 and June 2017.Thematic saturation was reached within the first focus group. Three major themes emerged: (1) Being independent (2) Handling the end of life, and (3) Conditions in everyday life. In relation to the 4D EOL trajectories (psychological, physical, social and spiritual) we also achieved theoretical saturation. All 4Ds were well illustrated, especially the psychological and social dimensions by participants coping with chronic or serious illness, cognitive impairment and EOL experiences.

Discussion/ conclusion: Our study supports the hypothesis that the 4D EOL trajectories play an important role in population preferences and ACP utilization. Fluctuation in health and how individuals cope requires an EOL plan that is adjustable to time and the individual's needs. Our methodological approach revealed that the themes were very important for future research. Our findings will be used to generate tools to measure and improve our understanding about the dynamic process of EOL preferences in the older population.

Funded by the Region of Southern Denmark.

Abstract number: P01-515

Abstract type: Poster

The Cross-cultural Development and Implementation of a Complex Intervention to Improve Palliative Care in Nursing Homes: The PACE Steps to Success Programme

Payne S. ${ }^{1}$, Hockley J. ${ }^{2}$, Van den Block L. ${ }^{3}$, Onwuteake-Philipsen B. ${ }^{4}$, Kylanen M. ${ }^{5}$, Szczerbinska K. ${ }^{6}$, Gambassi G. ${ }^{7}$, Pautex S. ${ }^{8}$, Froggatt K. $^{1}$, PACE

${ }^{1}$ International Observatory on End of Life Care, Lancaster University, Lancaster, United Kingdom, ${ }^{2}$ Palliative Care, University of Edinburgh, Edinburgh, United Kingdom, ${ }^{3}$ Palliative Care, Vrije Universiteit Brussel \& Ghent University, Brussels, Belgium, ${ }^{4}$ Public and Occupational Health, Universitair Medische Centra, Amsterdam, Netherlands, ${ }^{5}$ Welfare, The Ageing, Disability and Functioning Unit, National Institute for Health and Welfare, Helsinki, Finland, ${ }^{6}$ Sociology of Medicine, Jagiellonian University Medical College, Krakow, Poland, 7 UOC Medicina Generale, Universita Cattolica del Sacro Cuore, Rome, Italy, ${ }^{8}$ Community, Geriatric and Palliative Care, University Hospital of Geneva, Geneva, Switzerland 
Background: Little research has investigated cross-cultural adaptation and implementation of complex interventions in palliative care, taking account of health and social care systems, legal and regulatory policies, and cultural norms.

Aims: To present the cross-cultural adaption processes used to implement the PACE Steps to Success programme in nursing homes (NHs).

Methods: The PACE Steps to Success programme was implemented in the PACE cluster randomised control trial in 7 European countries, in 6 languages. A three stage approach was used,

1) preparation of resources;

2) train-the trainers model; and

3) cascading support during implementation.

All stages were underpinned by cross-cultural adaptation, including recognising cultural norms and sensitivities, and languages. Collated evidence from minutes of international meetings, appraisal of training delivered, interviews with those delivering the intervention and providing and/or receiving support, is used.

Results: $78 \mathrm{NHs}$ participated in the trial, with half randomized to the intervention, 3638 nurses/care assistants were identified. 1-3 'country trainers' (total $n=16$ ) facilitated the intervention. A three stage process was used to guide the cross-cultural adaptation and implementation. Adaptation of English training resources for three different groups of staff, involved simplification of content, a review in each country, and forward and backwards translation. Training was initially provided in English to 'country trainers' who cascaded it in local languages. Support was cascaded via country trainers to staff in $\mathrm{NHs}$.

Conclusions: There is little guidance on how to adapt complex interventions developed in one country and language to international contexts. This process for cross-cultural adaptation and implementation of a complex educational and development intervention may be useful to others seeking to transfer quality improvement initiatives to other contexts.

Abstract number: P01-516

Abstract type: Poster

"Our Goal is to Manage Care for Older Patients Like Sailing in Calm Water." Integration of Palliative Care for Older Patients in Primary Care in Germany - The General Practitioners' View Falter S., Tetzlaff F., Schneider N., Müller-Mundt G.

Institute for General Practice, Hannover Medical School, Hanover, Germany

Background: International research results reinforce the relevance of general palliative care for older people with life-limiting illnesses as well as the key role of general practitioners (GPs) in this field.

Aims: This study aimed to explore palliative care practice in primary care and decision making processes underlying the initiation of palliative care (PC) for older patients in Germany.

Methods: As part of the explorative phase of an intervention study we conducted qualitative manual guided interviews with GPs. Data analysis was performed using an inductive-deductive approach of qualitative content analysis.

Results: 13 qualitative interviews, among them two tandem interviews, were conducted with 15 GPs (8 men, median age 53, range 39 to 69 ) in two care regions in Lower Saxony, Germany in spring 2018.

According to the GPs' insight of and strategies to provide PC the analysis shows that the GPs have a holistic understanding, but almost limit PC to terminal phases of life limiting illnesses. Preventing suffering, standing by, networking as well as supporting and preparing family members for loss are viewed as their core responsibility to ensure steady and calm care. But only a minority follows a multidisciplinary and pro-active approach to respond to physical, psychosocial and spiritual needs early in the illness trajectory. The data reveals that enabling factors to initiate and perform early palliative care (EPC) are a comprehensive qualification in $\mathrm{PC}$, a team integrated approach and functioning regional service networks.

Conclusion / Discussion: While owing a holistic PC approach GPs tend to deny palliative care needs in early phases of illness trajectory in life limiting illnesses. Promoting a comprehensive understanding of EPC as well as caring networks seems to be essential.

Funding: The research project is funded by the German Federal Ministry for Education and Research (BMBF, no. 01GY1710).

Abstract number: P01-517

Abstract type: Poster

What Factors Ultimately Influence the Place of Death of Care Home Residents? A Qualitative Study of the Interaction between Care Homes and General Practitioners in the South Wales Valleys

Jones I.R. ${ }^{1}$, Harris D.G. ${ }^{2}$

${ }^{1}$ Glyn Ebwy Surgery, Ebbw Vale, United Kingdom, ${ }^{2}$ Medicine, Cardiff

University, Cardiff, United Kingdom

Background: In Wales, over $50 \%$ of care home patients at end of life die in hospital. Initiatives such as advance care planning aim to reduce hospital admissions from care homes at end of life, but improvements have been slow.

Aims: This study explores why some care home patients are admitted to hospital at end of life, and why similar patients are looked after in their care homes.

Methods: The study is a retrospective, thematic analysis of interviews with General Practitioners (GPs) and care home staff. The interviews were based on care home deaths occurring in 6 GP surgeries, 6 nursing homes and 6 residential homes in the South Wales valleys between 01/09/2016 and 31/08/2017.

Results: Fifty-seven hospital deaths were discussed, along with a similar number of care home deaths. Overall, $78 \%$ of patients died an expected death in their care homes.

Main themes around hospital admissions were emergency ambulance requests by care homes (33\%), arranged admissions by GPs $(21 \%)$, and family request (14\%). Variation was found in the way care homes and GPs managed frail and declining patients, and residents were sometimes admitted to hospital against the wishes of care home staff and families. Other issues identified were difficulties in accessing services, lack of reflection, and conflicting roles.

Conclusions: Most patients in the study died in their care homes, but service provision was fragmented. The author suggests that GPs, Intermediate Care, Ambulance Services, GP Out of Hours, palliative care teams and care homes work together closely to address the issues. Also suggested are regional groups to co-ordinate and monitor end of life care in care homes. National accreditation should be introduced for end of life care in care homes, and regulatory bodies (such as Care Inspectorate Wales) should inspect care homes accordingly.

This research fills a gap in our knowledge, and the findings can be applied internationally.

The study was funded by the lead author, Dr lan Jones.

Abstract number: P01-518

Abstract type: Poster

Talking about Patient's Wishes for Levels of Treatment? Development of a Danish POLST

Tuesen L.D. ${ }^{1,2}$, Bülow H.-H. ${ }^{3}$, Jensen H.I. ${ }^{1,2}$

${ }^{1}$ Department of Anaesthesiology and Intensive Care, Vejle and Middelfart Hospital, Vejle, Denmark, ${ }^{2}$ Institute of Regional Health Research, University of Southern Denmark, Odense, Denmark, ${ }^{3}$ Department of Intensive Care, Holbaek Hospital, Holbaek, Denmark 
Background: Old, medically frail and/or chronically ill patients are often admitted to the hospital without decisions about levels of treatment. This may lead to inappropriate care. An American model, the Physician Orders for Life-Sustaining Treatment (POLST), delineates what specific treatment should be administered for a specific patient, in instances of serious illness.

Aim: To test a Danish POLST model to ensure that patient's wishes for levels of treatment are known and documented.

Methods: A Danish POLST model was developed and a pilot test was performed in hospital wards, nursing homes and at general practitioners. Patients were eligible for inclusion if death was assessed as likely within 12 months. The patient and his/her physician discussed patient values and preferences, and the POLST model was filled in. Family members and/or nursing staff could participate. After one week, participants received a questionnaire about their experiences with the conversation and the POLST model. Furthermore, interviews were conducted with a selected group of the participants.

Results: 25 patients, seven physicians, nine family members and six nurses from seven different settings were included in the questionnaire survey; response rates $92-100 \%$. The result showed that $80 \%$ within all groups of participants found the POLST model to a very high or high degree was useful for discussion about levels of treatment. To gain a deeper understanding of the POLST experience, interviews were conducted with four patients, three physicians, three family members, and four nurses. Interview results highlighted four areas: "Having information", "The need for support", "How stories act" and "Common agreement".

Conclusions: Also in Denmark, the POLST model helps to facilitate conversations about wishes for levels of treatment. Most patients, physicians, families, and nurses found that the POLST model to a high degree was usable.

The main source of funding: The Novo Nordic Foundation.

Abstract number: P01-519

Abstract type: Poster

\section{A Pilgrim's Journey - When Parkinson's Disease Comes to an End in Nursing Homes \\ Lex K.M. ${ }^{1}$, Lorenzl S. ${ }^{1,2}$}

${ }^{1}$ Institut für Pflegewissenschaft und -praxis, Paracelsus Medizinische Privatuniversität Salzburg, Salzburg, Austria, ${ }^{2}$ Neurologische Klinik und Poliklinik, Klinikum der Universität, Interdisziplinäres Zentrum für Palliativmedizin, München, Germany

Background and Purpose: It is now more common knowledge, that patients suffering from advanced Parkinson Disease (PD) have unmet palliative care needs. Furthermore, the burden of relatives is high and little help can be provided, when patients are cared for at home. Therefore, we have investigated how well PD patients are cared for in Salzburg nursing homes, when they are in the advanced and even end stage of the disease. To answer this question, we conducted this mixedmethods interdisciplinary, explorative study investigating patients and relatives as the unit of care.

Methods: We used validated scores and assessment instruments to verify resident's diagnosis. Residents were eligible if they were previously diagnosed with PD (Hoehn \& Yahr stage IV or V) and living in a nursing home. We conducted ethnographic interviews with family members to gain insight into palliative care needs.

Results: Out of 29 patients screened, only 9 fulfilled the criteria of idiopathic PD. The mean duration of illness has been 9 years. All patients were bedridden or wheelchair-bound. Pharmacological treatment has been on a minimum number of drugs. No resident had a feeding tube. With 5 families we conducted ethnographic family interviews. The following codes emerged from the interview data:

- Strong closeness in the family

- Improvement of Quality of Life while living in the nursing home

- No burdensome care for family members
- $\quad$ Relatives fear what might happen and believe that the patient will still live for a long time.

Conclusions: In the end-of life phase in PD it is advisable to maintain considerate nursing care and to treat patients timely and minor medically. Patients had a high quality of life and relatives did not suffer from burdensome care. It has been evident, that the present quality of life is due to the responsible work of the nurses at the Salzburg residential homes indicating that nursing homes might be a good place for patients with advanced PD.

\section{Abstract number: P01-520}

Abstract type: Poster

Pain Intervention for People with Dementia in Nursing Homes (PID): Recognition of Pain Events in Routine Nursing Home Care

Volken $T^{1}{ }^{1}$, de Wolf-Linder S. ${ }^{2}$, Zigan N. ${ }^{2}$, Braun A. ${ }^{2}$, Spichiger $\mathrm{F}^{2}$, Bosshard G. ${ }^{3}$, Koppitz A.L. ${ }^{2}$

${ }^{1}$ School of Health Professions, Institute of Health Science, Zurich University of Applied Sciences, Winterthur, Switzerland, ${ }^{2}$ School of Health Professions, Institute of Nursing, Zurich University of Applied Sciences, Winterthur, Switzerland, ${ }^{3}$ Clinic for Geriatric Medicine and University of Zurich, Centre on Ageing and Mobility, University Hospital Zurich, Zurich, Switzerland

Background: In the last months of life, about $50 \%$ of the Nursing Home $(\mathrm{NH})$ residents are diagnosed with dementia in Switzerland. Their pain is often inadequately treated because it may manifest and present itself in different ways (e.g. as behavioral disorders). This makes it difficult for nursing staff to recognize palliative care needs such as pain in People with Dementia (PwD).

Aims: To train nursing staff to use a systematic pain assessment tool to improve pain management in PWD with palliative care needs, living in a NH.

Methods: In a quasi-experimental nurse-led intervention trial, we used a 5 knots cubic spline logistic mixed effect growth curve model to evaluate pain events over a period of 147 days and adjusted for age, gender, number of previous pain events, death, and $\mathrm{NH}$.

Results: 164 PwD (72\% women) with an average age of 85.5 years (SD 8.5 ) contributed 20,084 observations. The nursing staff documented on average 5.3 (SD 7.6, Range 0-53) pain events per PwD. Overall, the probability of experiencing a pain event declined substantially, particularly in the first 49 days. The respective Odds Ratios (OR) of experiencing a pain event between days 49-98 (T1) and 98-147 (T2) compared to days 0-49 (TO) were $O R=0.54(p<0.0000)$ and $O R=0.43(p<0.0000)$. The trajectory of experiencing pain events in $\mathrm{NH} 1$ and $\mathrm{NH} 2$ were similar and matched the overall pattern. $\mathrm{NH} 3$ showed no statistically significant decline of the marginal probability to experience a pain event between T0 and T1 $(p<0.1360)$ and T2 $(p<0.087)$ respectively.

Conclusion: The use of consecutive systematic pain assessments by nursing staff in $\mathrm{NHs}$ have been shown to increase recognition of pain in PwD. Continuous support and advice on how to implement individual pain action plans and strategies to reinforce systematic pain management skills for nursing staff is critical in order to sustain palliative care in $\mathrm{NH}$.

Abstract number: P01-521

Abstract type: Poster

What do Portuguese Health Care Professionals from a Nursing Home Think about Artificial Hydration in Palliative Care? Results from a Qualitative Study

Silva C. ${ }^{1}$, Martins J.C. ${ }^{2}$, Pinto S. ${ }^{3,4}$

${ }^{1}$ Centro Social de Paramos, Paramos, Portugal, ${ }^{2}$ Escola Superior de Enfermagem de Coimbra, Coimbra, Portugal, ${ }^{3}$ Escola Superior de 
Saúde de Santa Maria, Porto, Portugal, ${ }^{4}$ CINTESIS - Center for Health Technology and Services Research, Porto, Portugal

Background: Artificial Hydration $(\mathrm{AH})$ is an important method to the administration of fluids in several contexts, including in Palliative Care (PC). This theme is also relevant in Nursing Homes, where each year many elderlies with $\mathrm{PC}$ needs are admitted.

Aims: To explore health care professionals' perceptions' from a Portuguese Nursing Home about the use of AH in elderlies with PC needs.

Methods: Qualitative study using in-depth, semi-structured and face-toface interviews, from January to March 2018, according to the Consolidated Criteria for Reporting Qualitative Research. Interviews were tape-recorded, transcribed verbatim and analysed using interpretative content analysis. 27 Healthcare professionals, from a Portuguese Nursing Home were consecutively recruited and interviewed. The study was previously analysed by an insdependent ethics committee, and the participants informed consent was obtained.

Results: Participants ( $n=27$; mean age $=46.10$ years \pm 9.10 years) have advanced training in geriatrics $(n=20)$, and 6 have basic training in PC. Three themes emerged: $\mathrm{PC}$ knowledge, $\mathrm{AH}$ in $\mathrm{PC}$, and Compassionate Care. Participants associate PC to terminal patients in suffering and highlight the need for physical attention. AH is perceived as a basic care, associated with the humanization of care. The interruption of $\mathrm{AH}$ is perceived as a way to precipitate death. Yet, participants believe that the decision-making process related with $\mathrm{AH}$ is a responsibility of healthcare professionals, and the patient/family is not evolved on this issue. $\mathrm{AH}$ reflects the concerns in caring for the person, even when there is "nothing to do", and can strength the sense of connectedness among patients, relatives and healthcare providers.

Conclusion: The study provides a general overview about the use of $\mathrm{AH}$ in elderly people with PC needs in Portuguese Nursing Homes. Findings suggests the need for training in both $\mathrm{PC}$ and $\mathrm{AH}$.

Abstract number: P01-522

Abstract type: Poster

Comparison of Experience of Care during the Last Year of Life in Elderly Patients who Died by Assisted and Non-assisted Suicide in Geneva: Results from a Retrospective Study 2010-2016

Dieudonné Rahm N. ${ }^{1}$, Burkhardt S. ${ }^{2}$, Pautex S. ${ }^{3}$

${ }^{1}$ Palliative Care, Hôpitaux Universitaires de Genève, Collonge Bellerive, Switzerland, '2Legal Medicine, Hôpitaux Universitaires de Genève, Geneva, Switzerland, ${ }^{3}$ Palliative Care, Hôpitaux Universitaires de Genève, Geneva, Switzerland

Background: People 65 years and older have the highest rates of assisted-suicide. Recent research suggests that care may be experienced as a burden that impacts on requests for assisted suicide. Strategies for assisted-suicide prevention can benefit from knowledge concerning care received by individuals who opt to carry out assisted suicide.

Aims: The aim of this study was to compare the experience of care received during the last year of life by patients aged 65 years or older who carried assisted and non-assisted suicide.

Methods: Data from 359 death certificates over a study period of seven years, were investigated. Data sources were results of forensic investigations and hospital charts. For deaths classified as suicide, the presence of the following factors was registered: type and year of suicide, gender, age, marital status, psychiatric and somatic diseases, admissions at hospital, emergency room visits, surgery and palliative care received.These factors were cross-tabulated with whether a suicide was assisted or not. Associations were calculated by using $\mathrm{Chi}^{2}$ and Fisher's exact test. Multivariate logistic regressions were performed to investigate the association between assisted suicide and hospital care during the last year of life, adjusting for diagnoses.
Results: Women living alone represented more than half of the cases. The assisted suicide rate was $75 \%$. Somatic diseases were associated significantly with assisted suicide. Emergency department visits $(p=0.003)$, hospitalizations $(p=0.01)$ and surgery $(p=0.04)$ were associated with assisted suicide, independently of patients' comorbidities. A minority was admitted in a palliative care unit.

Conclusion: Intense care is associated with assisted suicide in elderly people. This factor should be further explored in people who express wishes to hasten death. Advance care planning and earlier referral to palliative care could help slow down end-of-life intensity of care.

\section{Abstract number: P01-523}

Abstract type: Poster

\section{Providing Compassionate End of Life in Residential Care Centres} Steele $\mathrm{K}$.

Irish Hospice Foundation, Healthcare Department, Dublin, Ireland Presenting author email address: kate.steele@hospicefoundation.ie

Background: Each year in Ireland over 7,000 people die in residential settings. CEOL, a quality improvement (QI) programme assists RCCS to identify, reflect and implement changes to enable delivery of compassionate end-of-life care to the benefit of residents, families and staff.

Aims and Objectives: To provide the best possible end of life care for people living in residential care centres and to enable staff to continuously review, reflect and improve the end of life care they provide. Objectives include raising of the awareness and role of quality EOLC at an individual staff level, across the culture and the practices of the residential care centre.

Methods: This QI programme works on a 3 year cycle, initially providing onsite training over a period 6 months, with sustainability and ongoing training provided through attendance at regional CEOL networks. To date 2 evaluations (A Journey of Change Evaluation, 2017 \& CEOL Programme Evaluation, Midlands RCCs, 2018) have underpinned the programme content and development. Through the CEOL reviews (death reviews) and bereaved relatives questionnaire the CEOL Group identifies and introduces the care practice, system and environmental changes needed so that continuous improvements in end of life care are prioritised and implemented.

Findings and Results: The CEOL programme develops staff skills and confidence. It builds capacity to identify and change end-of-life care from within the RCC. The programme is tailored to the specific needs of each care centre and it is through the reflective process of remembering and reviewing the end-of-life care provided that areas of change are identified and action taken.

Conclusions: This quality improvement model provides a formal framework to embed ongoing developments in EOLC. Further in-depth evaluation is recommended to include views of bereaved relative's and links to other initiatives for more coherent approach.

Abstract number: P01-524

Abstract type: Poster

\section{The Importance of Physiotherapy in Palliative Care} Kokot M. ${ }^{1}$, Mikołajczyk M. ${ }^{2}$, Durmała J. ${ }^{1}$

${ }^{1}$ Department of Rehabilitation, Medical University of Silesia in Katowice, Katowice, Poland, ${ }^{2}$ Department of Therapeutic Rehabilitation Pediatric Subvdepartment at the Department of Therapeutic Rehabilitation, Leszek Giec Upper-Silesian Medical Centre of the Silesian Medical University in Katowice, Katowice, Poland

Background: In the past, rehabilitation was seen as a treatment that would lead to full recovery. Such a concept of rehabilitation in the aspect of using it in palliative care may seems paradoxical. 
Currently, physiotherapy is an important element of palliative care. The aim of physiotherapy in palliative care is to improve the quality of life of patients, by helping them achieve maximum efficiency and independence and reduction of unpleasant symptoms. Physiotherapeutic techniques such as healing massage, active exercises, respiratory therapy and manual lymphatic drainage are highly appreciated by patients.

In the perspective of demographic changes and epidemiological, the number of people in need of palliative care which includes physiotherapy, will grow rapidly. The development of palliative care, with its many aspects, is considered one of the priority tasks of health policy.

Aim: The aim of the study was to present the significant role of physiotherapy in palliative care on the basis of a review of current literature.

Method: The article reviews 15 items of the current medical literature of the MEDLINE, Pub Med and Scopus databases.

Results: Based on a review of the literature, it was observed that physiotherapy had a statistically significant effect on the improvement of locomotion and self-care, and reduced the severity of dyspnea, anxiety and depression associated with the disease.

Conclusions:

1. The aim of physiotherapy in palliative care is the interaction between various spheres of life - physical, mental, social and professional, and the patient's achievement of an optimal level of efficiency.

2. In palliative care patients, the physiotherapy aims should be realistic and to be achieved in a relatively short period of time.

3. There is a lack of literature devoted to physiotherapy in palliative care that would fulfill an educational role and was a guide and support for therapists caring for chronically ill patients.

Keywords: Palliative care, physiotherapy

Abstract number: P01-525

Abstract type: Poster

People Living with Advanced Dementia in Nursing Care Homes: Baseline Results from a Feasibility Trial of the Namaste Care Intervention

Froggatt K. ${ }^{1}$, Patel S. ${ }^{1}$, Dunleavy L. ${ }^{1}$, Preston N. ${ }^{1}$, Walshe C. ${ }^{1}$, Perez

Algorta G. ${ }^{1}$, Burnside G. ${ }^{2}$, Best A. ${ }^{2}$, Hardwick B. ${ }^{2}$, Jackson C. ${ }^{2}$

${ }^{1}$ International Observatory on End of Life Care, Lancaster University, Lancaster, United Kingdom, ${ }^{2}$ Liverpool University, Liverpool, United Kingdom

Background: Many people with advanced dementia live and die in nursing homes. The quality of life, care and dying experienced is variable. Namaste Care is a multi-sensory programme of care for people with advanced dementia. With limited evidence of Namaste Care's efficacy, there is a need to conduct a feasibility study, prior to a definitive trial.

Aims of the trial: To establish if it is acceptable and feasible to:

1. Deliver the Namaste Care intervention in the context of a trial, and

2. Identify clinically meaningful outcome measures to support a definitive trial.

Here we provide descriptive baseline data on trial participants.

Study participants: People with advanced dementia (FAST score 6/7) living in a nursing home in NW England.

Methods: Feasibility study, comprising of a parallel, two-arm, multi-centre cluster controlled randomised trial with a process and economic evaluation. Eight nursing homes were randomly allocated to the intervention (delivered at nursing home level) or control arm of the study. Data collection at baseline, 2, 4, 24 weeks. Primary Outcome measures: CAD-EOLD and QUALID. Secondary clinical outcome measures: symptom presence, agitation, and residents' activity using actigraphy. ISRCTN14948133.

Results: Participants $(n=32)$. Baseline data return $100 \%(n=32)$ from 6 sites (2 sites withdrew). Participants' median age 82.4 years, IQR 78.086.9; $53.1 \%$ male; $56.3 \%$ married; $96.9 \%$ white ethnicity; $94 \%$ FAST score 7. Median time since admission 1.6 years, IQR 0.8-3.2. No significant differences between genders for CAD-EOLD or QUALID. Agitation/aggression present in $72 \%(n=23)$ residents, and depression/dysphoria in $47 \%$ $(n=15)$ residents.

Discussion / Conclusion: This study is the first study in UK nursing homes to examine Namaste Care in a trial context, and to describe the characteristics of those who receive this intervention. This population experiences a range of symptoms, and has fair quality of life scores and levels of comfort.

\section{Abstract number: P01-526 \\ Abstract type: Poster}

Experiences and Needs of Integrating Home Telehealth Model for Older Persons Living with Hemodialysis: A Mixed Methods Research

Pungchompoo W. ${ }^{1}$, Parinyajittha S. ${ }^{2}$, Pungchompoo S. ${ }^{3}$

${ }^{1}$ Faculty of Nursing, Chiang Mai University, Chiang Mai, Thailand,

2Faculty of Medicine, Chiang Mai University, Chiang Mai, Thailand,

${ }^{3}$ Faculty of Engineering, Rajamangala University of Technology

Srivijaya, Songkhla, Thailand

Background: The integrating home telehealth into the holistic end of life care for older persons living with hemodialysis with nurse oversight is still limited in Thailand.

Aim: To explore the experiences and needs of integrating home telehealth model for older persons living with hemodialysis.

Methods: A mixed methods exploratory sequential design was used. The quantitative phase,the purposive sampling was used to collect data from 100 older persons living hemodialysis. The instruments were included the VOICES (View of Informal Carers Evaluation of Service-ESRD/Thai version) questionnaire, the 9-items Thai Health Status Assessment questionnaire, Demographic data form and Thai-Stress Evaluation Form (ST5). Data were analysed by using Statistical package SPSS version 17. In the qualitative phase, the semi structure interview schedule and purposive sampling were used to collect data from 20 older persons living with hemodialysis. Interviews were digitally recorded, transcribed and analysed by using framework analysis.

Results: Experiences of patients at home were reported. $31 \%$ of patients rated pain and edema were the main suffering symptoms. $25 \%$ and $15 \%$ of patients reported breathlessness and nausea/vomiting. $90 \%$ reported nurse(s) did not visit at home. $53 \%$ considered as "very difficult" for the physician to visit patents at home. $90 \%$ of patients in this study were found stress at medium level. The overall 9-Thai quality of life scores were within the normal level. Needs of participants at home were identified:

1) medical management (symptoms, medications, and nutrition),

2) activity and role management,

3) emotional management,

4) spiritual support

5) telehealth (VDO visiting, telephone counselling, web based education/monitoring).

Conclusion: The integrating home telehealth model is required in order to enhance quality of care, improve quality of life and may lead to reduce cost of treatment.

Supported by: National Research Council of Thailand

Abstract number: P01-527

Abstract type: Poster 
Redesigning Specialist Palliative and Hospice Care to Meet the Needs of Older People

Nicholson C. ${ }^{1,2}$, George R. ${ }^{2}$, Pace V. ${ }^{2}$, Richardson $\mathrm{H}^{2}$

${ }^{1}$ Florence Nightingale School of Nursing, Midwifery and Palliative Care, King's College London, London, United Kingdom, ${ }^{2}$ St Christopher's Hospice, London, United Kingdom

Older people with multimorbidities/frailty will dominate palliative care in coming decades. For them, dying can be slow and unpredictable. We draw on current theory and evidence to develop a framework that assists services and communities to attune to their needs.

Design: We used stepped framework construction, eight clinicians and service users reviewed and refined initial findings.

- to scope the palliative/geriatric literature and define focus, inclusion and exclusion criteria for a proactive palliative care model (Seymour 2004). Documents from 102 research, review, policy papers and grey literature were included.

- Face-validity testing extended to a wider consultation across care sectors, care commissioners and providers.

Findings: Built around emergent concerns of recipient older people and their family as care partners, the framework identifies three intervention levels that challenge us to work differently

1. As clinicians through systematising full assessment across palliative and older people domains, parallel planning, early ACP conversations, rehabilitative palliative care; proactive engagement to respond to incremental change; Access to advanced palliative practice competent to stop as well as start treatments and working across relevant specialties.

2. As services in partnership with the broader care system: develop new referral criteria, triggers and pathways; PROMs across organisational boundaries, co-location of services, community based rapid response teams, cross boundary working into care homes

3. As support to wider societal change:-recognise and encourage community participation with and by older people: intergenerational activities, attend to legacy as well as advance care planning, strengthen community assets

Conclusions: The Age Attuned Hospice framework offers a flexible structure to guide organisations seeking to become more responsive and integrated to the needs of older people, who currently do not receive specialist palliative care.

Abstract number: P01-528

Abstract type: Poster

Feasibility of a Transitional Palliative Care Pathway for Older Patients: Challenges and Lessons Learned from a Mixed-method Study

Flierman I. ${ }^{1,2}$, van Rijn M. ${ }^{1,2,3}$, de Meij M. ${ }^{4}$, Niezink D.M. ${ }^{5}$, Buurman B.M. ${ }^{2,3}$, Willems D.L. ${ }^{1}$

${ }^{1}$ Department of General Practice, Section of Medical Ethics, Amsterdam UMC, University of Amsterdam, Amsterdam, Netherlands, ${ }^{2}$ Department of Internal Medicine, Section of Geriatric Medicine, Amsterdam UMC, University of Amsterdam, Amsterdam, Netherlands, ${ }^{3} \mathrm{ACHIEVE}-\mathrm{Centre}$ of Applied Research, Amsterdam University of Applied Sciences, Amsterdam, Netherlands, ${ }^{4}$ Onze Lieve vrouw Gasthuis, Amsterdam, Netherlands, ${ }^{5}$ Amsterdam UMC, location VU University, Amsterdam, Netherlands

Background: A transitional care pathway (TCP) could improve care for older patients at last months of life. However, barriers exist, such as unidentified palliative needs, suboptimal collaboration between care settings and fragmented palliative care. The PalliSupport care pathway aims to overcome these barriers.
Aim: To determine the feasibility of a TCP for older patients with palliative care needs.

Design: A mixed-method feasibility study was conducted at one hospital with affiliated primary care facilities in the Netherlands. Patients were $\geqslant 60$ years, acutely hospitalized and in need of palliative care.

Intervention: The intervention consisted of

1) training on early identification of the palliative phase and end of life conversations;

2) involvement of a transitional palliative care team during admission and post-discharge;

3) intensified collaboration between care settings through work protocols.

Outcomes: Inclusion rate, protocol adherence, possibility of data collection and patient burden. Experiences of professionals with the TCP were assessed through qualitative interviews.

Results: The inclusion rate was lower than anticipated (8 instead of 50 patients). Misunderstandings about palliative care, uncertainty in professionals' roles and difficulties in initiating end-of-life conversations were identified as barriers to inclusion. While in all cases the steps of the protocol were followed, the timing was not according to predetermined guidelines because it did not fit daily practice. Data collection was feasible and patients experienced low burden. The training program was well received and both primary and secondary care professionals found the intensified collaboration beneficial for patient care.

Discussion: Based on the findings, the TCP protocol has been modified: the training program now has a stronger focus on introducing palliative care to patients, inclusion criteria were adjusted and work protocols were made to better fit daily practice.

Funding: ZonMw

\section{Abstract number: P01-529 \\ Abstract type: Poster}

What Do Key Stakeholders Consider to Be Important in a Model of Dementia Palliative Care: A Multi-country Survey

Fox S. ${ }^{1}$, Drennan J. ${ }^{2}$, Guerin S. ${ }^{3}$, Kernohan $G .{ }^{4}$, Murphy A. ${ }^{5}$, Robins A. ${ }^{1}$ Timmons S. ${ }^{1}$

${ }^{1}$ Centre for Gerontology and Rehabilitation, University College Cork, Cork, Ireland, ${ }^{2}$ School of Nursing and Midwifery, University College Cork, Cork, Ireland, ${ }^{3}$ School of Psychology, University College Dublin, Dublin, Ireland, ${ }^{4} \mathrm{School}$ of Nursing and Health Research, Ulster Univerisiity, Antrim, United Kingdom, ${ }^{5}$ Department of Economics, University College Cork, Cork, Ireland

Background: The Model for Dementia Palliative Care Project aims to develop a service delivery model for community-based dementia palliative care. While palliative care needs of people with dementia are often poorly addressed, recent developments and national guidelines are promising (Fox et al., 2018). Numerous dementia palliative care services exist internationally, however not much is known about what the people who provide these services would deem to be the most important aspects in the provision of dementia palliative care. The aim of this study was to identify what key stakeholders consider to be important in a model of dementia palliative care.

Methods: A web-based survey was developed, piloted $(n=5)$, and revised. It was distributed electronically within five healthcare jurisdictions, i.e. Republic of Ireland, Northern Ireland, England, Scotland, and Wales. The target population was academics, healthcare professionals, and policy-makers with an interest in dementia and/or palliative care. Results: In total, 113 complete responses were received. The majority of respondents were female ( $86 \%)$. The stakeholders felt that the three most essential general aspects of a good model of palliative care for dementia were: "Care for People at all Stages of Illness" (77\% considered 'essential'), "Information Continuity" (75\% considered 'essential') and 
"Defined Pathways for Specialist Service Input" ( $58 \%$ considered 'essential'). In addition, good communication between palliative care and dementia care teams was perceived to be core to a successful model, with a key worker needed to facilitate this communication, and to support families e.g. around care transitions.

Conclusion: Consistent aspects of a 'good' model were identified. This research complements other studies ongoing within The Model for Dementia Palliative Care Project, to inform the development of a new feasible and acceptable model for dementia palliative care.

Abstract number: P01-530

Abstract type: Poster

Experiences, Expectations and Needs of Palliative Care Teams towards Transitional Care for Older Patients with Palliative Care Needs

van Rijn M. ${ }^{1,2}$, Baks N. ${ }^{3}$, Flierman I. ${ }^{1,3}$, Willems D.L. ${ }^{3}$, Buurman B.M..$^{1,2}$

${ }^{1}$ Department of Internal Medicine, Section of Geriatric Medicine, Amsterdam UMC, University of Amsterdam, Amsterdam, Netherlands, ${ }^{2}$ Faculty of Health, ACHIEVE-Centre of Applied Research, Amsterdam University of Applied Sciences, Amsterdam, Netherlands, ${ }^{3}$ Department of General Practice, Section of Medical Ethics, Amsterdam UMC, University of Amsterdam, Amsterdam, Netherlands

Background: Palliative care for older patients in the Netherlands is suboptimal which results in negative outcomes, such as frequent hospital readmission at the end of life. A transitional palliative care team can contribute to prevention of readmission at the end of life.

Aim: Exploring the experiences, expectations and needs of palliative care teams towards transitional care for older patients with palliative care needs.

Methods: Qualitative semi-structured interviews were conducted, involving 15 members of two hospital based mulitdisciplinairy palliative care teams. A thematic content analysis was performed.

Results: Four main themes were identified:

1) Education; Participants experience a lack of interprofessional education in palliative care. Furthermore most participants have a lot of experience in palliative care, however without having completed a specific training in palliative care.

2) Consultation; Requests for consultation of the palliative care team are limited and often during the last days of life. Main barriers are: sub-optimal recognition of the palliative phase and non-familiarity with the expertise of the team.

3) Team; Participants feel a shared responsibility to deliver optimal palliative care, however they feel limited in time because being a member of the palliative care team is often an additional task.

4) Future perspectives; all participants feel the need to work as a transitional team, however, with multiple professionals involved, continuity and coordination is a concern.

Conclusion: This study demonstrates that members of palliative care teams experience the need for transitional palliative care. However, multiple barriers exist. Interprofessional education and structured organizational interventions might help to overcome these barriers.

Funding: ZonMw

Abstract number: P01-531

Abstract type: Poster

\section{Exploring End of Life Decision Making with People with Mild}

Dementia

Davies N. ${ }^{1,2}$, Rait G. ${ }^{1}$, Sampson E.L. ${ }^{2,3}$

${ }^{1}$ Department of Primary Care and Population Health, University College London, London, United Kingdom, ${ }^{2}$ Marie Curie Palliative Care Research
Department, University College London, London, United Kingdom, ${ }^{3}$ Barnet Enfield and Haringey Mental Health Trust Liaison Team, North Middlesex University Hospital, London, United Kingdom

Background: Many people with dementia do not have an advance care plan, decisions regarding their care and treatment are often left to their families. There is limited work exploring the views of people with dementia about end of life and what factors they would like their family to consider when making decisions.

Aim: The aim of this study was to explore with people with mild dementia the factors they would like family caregivers to consider when making decisions on their behalf at the end of life.

Methods: Ten semi-structured interviews were conducted with people with mild dementia in England. Participants were recruited through general practices, memory services and an online research register. Interviews were analysed using thematic analysis.

Results: Most participants had not discussed end of life or advanced dementia with either professionals or their family. Many felt these discussions were not needed or only needed to be discussed once, however the experience of the interview often changed this view with participants acknowledging a need for such discussions. The key considerations participants wanted their family to consider when making decisions about their health and care on their behalf included: the level of awareness they have, for example are they still aware who their family are and where they are living; the need to minimise the distress on the family; include them as an individual with dementia in the decisions if possible; consult with professionals to reduce the emotional strain on family caregivers.

Conclusions: Despite participants not considering discussions about end of life, they were keen to take part in this study. It is important to talk to people with dementia about end of life, highlighting the role and the decisions family caregivers have to make at the end of life may be a way to encourage discussions. These findings are contributing to the development of a decision aid to support family caregivers.

Abstract number: P01-532

Abstract type: Poster

Palliative Care in the Brazilian Amazon: Invasive vVersus Non Invasive Care in Private Health Services

Borges R. ${ }^{1}$, Campos M. ${ }^{2}$, Chaves A. ${ }^{1}$, Barrios P. ${ }^{3}$, Fuzita W. ${ }^{4}$, Anjos C. ${ }^{4}$

${ }^{1}$ Hospital Adventista de Manaus, Manaus, Brazil, ${ }^{2}$ Santa Casa São Carlos, São Carlos, Brazil, ${ }^{3}$ Palliative Nurse, Hospital do Servidor Público Estadual, São Paulo, Brazil, ${ }^{4}$ Clinical Oncologist, Sensumed Oncologia, Manaus, Brazil

Objective: To describe the knowledge of medical providers inside an Oncology clinic and a school hospital,both private network, in the city of Manaus-AM,about palliative care(PC)in the clinical pratice,through the indication of invasive or non-invasive measures in health care.

Methods: Cross-sectional,descriptive and observational study.After signing the Free and Informed Consent Form,the Physicians answered questions about a hypothetical clinical case throught multiple choice responses.The clinical case described a patient with advanced chronic disease, with no modifying therapy,in end of life process.The questions involved aspects related to nutrition, venous access and hospitalization in Intensive Care Unit.

Results: 31Physicians of many specialties were included.About $67,7 \%$ considered insufficient their PC's knowledge, and none was unaware of this modality of care.Almost half of them defined communication with family and caregivers as the greatest difficulty in initiating/ indicating PC,29\%techinal knowledge and9,7\%lack of specialized multidisciplinar team.After analyzing the responses of the applied clinical case, we observed that in the categories of venous access(central venous access)and nutrition(enteral/parenteral),the prevalence amog invasive procedures among surgeons were higher when compared to clinical doctors.Clinicians were even less invasive in the indication of Intensive Unit 
Care hospitalization(HR1;Cl95\%).In addition, physicians who declared absence of spirituality practice had a higher prevalence of invasive measures when compared to those who had some spiritual belief. Conclusion: It's evidente that the lack of PC preparation,especially the lack of training in communication and the difficulty to predict the evolution of the patients,are barriers in indication and strengthening of PC in our region and country. Further research is needed to elucidate whether medical spirituality can influence the indication of invasive measures. The study was funded by authors.

Abstract number: P01-533

Abstract type: Poster

\section{A Community of Practice for Residential Aged}

Care - Partnerships Across Community and Specialist Palliative

Care

Hughes $R .{ }^{1}$, Sneesby L. ${ }^{1}$, Culver J. ${ }^{2}$, Chand $A .{ }^{1}$

${ }^{1}$ Palliative Care, Calvary Mater Newcastle, Waratah, Australia, ${ }^{2}$ Anglican Care, Newcastle, Australia

Background: Despite existing programs and tools to enable end of life care (EOLC) in place for residents of Australian residential aged care facilities (RACFs), there remains a high rate of hospital attendance and death. Analysis of details of death of RACF residents has demonstrated $14.1 \%$ die whilst on hospital leave with a further $14.4 \%$ attending hospital within 1-30 days of death (AIHW 2014).

Aims: A collaborative of residential aged care staff, specialist palliative care and aged care specialist personnel has been established to develop a locally applicable model of care for RACF in the Greater Newcastle Area of NSW, Australia to provide EOLC in place through community engagement, staff capability and networked clinical support frameworks.

Methods:

- Scoping exercise to Identify and refine relevant assessment tools and escalation pathways. - Identify RACF staffing structures, supports, capabilities and confidence to identify end of life

- Identify and link active and latent stakeholders currently in place.

- Explore local opportunities for community engagement and development

- Develop an engagement and education strategy comprising accessible language, community supports and networking.

Results:

- Personal care assistants comprise the largest cohort of RACF staff.

- The language and presumed knowledge in existing EOLC tools may not reflect or suit the training and capability of the predominant staffing component of RACF.

- Personal care assistants, through established relationships, familiarity with residents and families are the priority cohort for enablement and support in facilitating care in place for RACF residents.

Conclusion: A patient centred model of palliative and end of life care delivered in place in RACF may be achieved through partnerships, appropriate language and training of RACF staff. Further work is underway to improve communication pathways, patient experience, staff training and family experience and bereavement.

Abstract number: P01-534

Abstract type: Poster

Digital Innovation in Aged Care: Developing a Palliative Care Dashboard
Tieman J., Bhattarai P.

Flinders University, Bedford Park, Australia

Background: A digital dashboard is an information tool used to describe and document key activities or indicators relevant to a service, organisation or work group. It enables the captured data to be summarised and presented in ways that can help monitoring, reporting or decision-making. This experimental work is part of the End of Life Directions in Aged Care (ELDAC) project. A palliative care dashboard could enhance care outcomes for residents and home care clients by monitoring performance, providing reports and supporting continuous quality improvement.

Goal of the work:

- To identify reportable elements that could be included in a prototype dashboard

- To document design elements that could improve the functionality of a dashboard

Approach taken: To inform a co-design workshop underpinning the development of the prototype palliative care dashboard for use within aged care, the following activities were completed:

- Survey of staff on what are common tasks and electronic data captures relating to palliative care

- Identification of elements from the ELDAC Care Framework that could be used as care indicators in the dashboard

- Alignment of proposed indicators to the Aged Care Quality Standards that guide care within the aged care sector in Australia

- Development of use case specifications to describe those involved in care activity, components of care activities and interdependencies within the system, and

- Review of desired characteristics for dashboard usability.

Results: This study has identified a set of data elements that appear to be applicable and feasible for inclusion in a prototype dashboard dealing with palliative care in aged care. These dashboard items could be used to create reports that highlight how care is being delivered within the organisation.

Lessons learned: There is a lack of automated data relating to palliative care activity in aged care. Being able to describe activity in order to create data collection can be challenging.

Abstract number: P01-535

Abstract type: Poster

Relevance and Viability of End-of-Life Standards in Spanish Nursing Homes: Are Staff and Primary Care Workers at the Same Point?

Burgos Sánchez J. ${ }^{1}$, Moya Dieguez R. ${ }^{2}$, Rola Morilla G.L. ${ }^{3}$, Herrero-Hahn R. ${ }^{4}$, Esteban Burgos A.A. ${ }^{4}$, Puente-Fernández D. ${ }^{4}$, Mota-Romero E. ${ }^{5}$, Montoya-Juarez R. ${ }^{4}$

${ }^{1}$ Servicio Andaluz de Salud. UGC Armilla (Cullar Vega), Granada, Spain, ${ }^{2}$ Residencia Entreálamos, Granada, Spain, ${ }^{3}$ Residencia Fray Leopoldo, Granada, Spain, ${ }^{4}$ Departamento de Enfermería, Universidad de Granada, Granada, Spain, ${ }^{5}$ UGC Salvador Caballero, Servicio Andaluz de Salud, Granada, Spain

Background: Nursing home staff and primary care workers need to work together in order to build up a strong end-of-life care in these facilities. Scarce attention has been paid to what both groups think it is relevant and viable regarding this topic.

Aims: Compare the relevance and the viability given by own staff and primary care professionals regarding end of life care standards in nursing homes. 
Methods: Descriptive cross-sectional study. Professionals from 8 Spanish nursing homes and primary care nurses and physicians linked to these facilities rated the 16 National Institute of Healthcare Excellence standards for palliative care for adults (UK) regarding their relevance and viability in nursing homes. Non parametric (U-Mann-Whitney) test were used to compare ratings of both groups.

Results: 58 professionals were included (37 from nursing homes). Most of them were women $(81,4 \%)$, nurses $(62,1 \%)$, and have a mean of 10,8 years $(S D=8,4)$ of work experience. For both groups more relevant standards were related to the last days of life and post-mortem care. No statistical differences were found in relevance between both groups. Regarding viability the most rated standards in both groups were related to post-mortem care and communication. Several statistical differences were found between both groups regarding viability. Nursing home professionals think that standards regarding evaluation of end-of-life needs $(p=.01)$, bereavement $(p=.00)$ spiritual $(p=.00)$ and family care $(p=.00)$ are easier to achieve than primary care workers.

Conclusion / discussion: Both groups agreed in which standards are relevant, but disagreed in the viability to achieve them. This could contribute to a misunderstanding between nursing homes and primary care about what kind of end-of-life care could be provided and what resources are needed in these centres.

Abstract number: P01-536

Abstract type: Poster

Relevance, Viability and Achievement of End-of-Life Standards in Spanish Nursing Homes

Cano Garzón G.M. ${ }^{1}$, Moeda Adesla L. ${ }^{2}$, Hermoso Sabio A. ${ }^{3}$, EstebanBurgos A.A. ${ }^{4}$, Herrero-Hahn R. ${ }^{4}$, Puente-Fernandez D. ${ }^{4}$, Montoya-Juarez $R^{4}{ }^{4}$, Mota-Romero E. ${ }^{5}$

${ }^{1}$ Residencia María Zayas, Belicena, Spain, ${ }^{2}$ Residencia Nuestra Señora de Fonseca, Peligros, Spain, ${ }^{3}$ UGC La Zubia, Servicio Andaluz de Salud, Cajar, Spain, ${ }^{4}$ Departamento de Enfermería, Universidad de Granada, Granada, Spain, ${ }^{5}$ UGC Salvador Caballero, Servicio Andaluz de Salud, Granada, Spain

Background: To develop interventions in end-of-life care for nursing homes, it is necessary to know what is viable to achieve and what it is achieved in these facilities.

Aims: Evaluate the relevance, viability and achievement of end-of-life care standards in nursing homes.

Methods: Descriptive cross-sectional study. Professionals from 8 Spanish nursing homes rated the 16 National Institute of Healthcare Excellence standards for palliative care for adults (UK) regarding their relevance, viability and achievement degree in nursing homes. Spearman non-parametric analysis was performed to test correlation between variables for every standard.

Results: 37 professionals were included. Most of them were women $(91,9 \%)$, nurses $(62,2 \%)$, and have a mean of 8 years $(S D=5,9)$ of work experience. The most relevant standards for nursing homes were related to post-mortem care and last days of life. By the other hand more viable standards were related to post-mortem care and communication. The most achieved standards for nursing homes were related to information given to families ( $M=4,18 ; S D=0,84)$, and post-mortem care $(M=4,61 ; S D=0,84)$. The poorest achieved standards were related to patient preferences management $(M=3,52 ; S D=1,19)$, individualized care plans $(M=3,36 ; S D=1,12)$ and psychosocial care $(M=3,27 ; S D=1,14)$. For most standards, there is a positive and strong relationship between relevance and viability, but not between the achievement and the relevance.

Conclusion / Discussion: Professionals perceived that nursing homes are strong in caring in the last moment of life and just before death. These facilities need to broad the focus to include the identification and follow up of palliative care needs and patients preferences management.
Abstract number: P01-537

Abstract type: Poster

Challenges to Providing Palliative Care in Nursing Homes:

Results from Action Research with Care Staff

Lemos Dekker N. ${ }^{1}$, Bagchus C. ${ }^{2}$, Pasman R. ${ }^{2}$, Onwuteaka-Philipsen B. ${ }^{2}$,

Achterberg $W^{1}{ }^{1}$, van der Steen J. ${ }^{1}$

1Public Health and Primary Care, Leiden University Medical Center, Leiden, Netherlands, ${ }^{2}$ Public and Occupational Health, Expertisecenter for Palliative Care, Amsterdam UMC, Vrije Universiteit Amsterdam, Amsterdam, Netherlands

Background: Early identification of the palliative phase is considered to contribute to good palliative care that resonates with the needs of the patient. The multidisciplinary nature of nursing home care requires that the disciplines pursue common care goals. What are, against this background, the challenges of delivering quality palliative care in nursing homes?

Methods: Challenges to provide palliative care were explored using action research with care staff of 10 nursing homes in the Netherlands. We report results from focus groups and individual interviews with nursing staff, elderly care physicians and other disciplines, and observations in the nursing homes.

Results: Knowledge of palliative care differed between the nursing homes. When the palliative phase starts was often debated in terms of whether it only concerns the final days of life, or starts with admission to the nursing home. An important obstacle emerged in the communication between care staff, when reporting was poor or reports were not read thoroughly, and between care staff and the physician, when opinions on the provision of palliative care differed. Also retaining or transferring knowledge was a challenge with high turnover of nursing staff and elderly care physicians in training.

Discussion and conclusion: Because the difference between regular nursing home care and palliative care is not always clear, and because there are various interpretations of what the palliative phase might entail, staff is unsure what, and when, should be identified. Further, when the palliative phase has been identified, organizational challenges arise in the communication between care staff, physicians, and family, and in fostering and passing on available knowledge. We underline the importance of supporting nursing home staff both in the knowledge necessary to identify the palliative phase and in the organizational structures to provide palliative care accordingly.

Abstract number: P01-538

Abstract type: Poster

The Advantages of a Joint Work of Psychology and

Phonoaudiology in Palliative Care: Report from Rio de Janeiro, Brazil

Fonseca A. ${ }^{1}$, Legrumber M. ${ }^{2}$, Aragão $E^{1}$

${ }^{1}$ Adventis Silvestre Hospital, rio de janeiro, Brazil, ${ }^{2}$ adventis Silvestre Hospital, rio de janeiro, Brazil

Introduction: The elderly are the fastest growing segment in Brazil, causing a direct impact on health services, mainly due to chronic diseases such as dementia syndrome. The presence of depressive symptoms in elderly people with dementia can generate worse clinical outcomes, such as an increased risk of bronchoaspiration, malnutrition and death. Objectives: To describe outcomes of joint attention between psychology and speech therapy in patients with advanced dementia.

Methodology: Descriptive study, performed in a hospital in the city of Rio de Janeiro, Brazil.

Results: 159 records were reviewed between January 2017 and July 2018. The mean age was 87.2 years; $52 \%$ female; Mean PPS 30 ; for 
dementia, use of the FAST scale, whose mean result was $6 c ; 85 \%$ of the group was lightly malnourished. Use of the FOIS and Yesavage scales, with a mean score of 3 for the risk of bronchoaspiration and 8 for the risk of depression, respectively; $5 \%$ of the group bronchospasted; $85.7 \%$ of patients were discharged from the hospital, $12 \%$ with gastrostomy and $14.3 \%$ died in the hospital. Of those, $85 \%$ had a palliative approach.

Discussion: The interface allowed families to express questions and anxieties about the patient's diet and nutritional status. The construction of space by the psychologist and, in parallel, the support of the palliative physician, generated an environment of trust and certainty of the decisions made, with reduced emotional claudication about the clinical picture and the difficult decisions due to the irreversible state of the health condition, especially in relation to the adaptation to the alternative route of feeding.

Conclusion: The interface between psychology and speech therapy are enabled the development of multidisciplinary techniques, tools capable of embracing the complexity of this profile of patients and their families.

Abstract number: P01-539

Abstract type: Poster

We're "Lucky" to Have a Good GP: A Qualitative Study of General Medical Practioner (GP) End of Life Care in Residential Aged Care Facilities

Boyd M., Balmer D., Frey R., Te Arai Palliative Care \& End of Life

Research - New Zealand

School of Nursing, The Unviersity of Auckland, Auckland, New Zealand

Background: Over one third of all deaths in New Zealand occur in residential aged care (RAC). Providing effective, high-quality end of life care in RAC is an important medical responsibility, yet little is known about the role played by general practitioners (GPs) in RAC palliative care delivery.

Aim: To explore the GP role in providing end of life care in residential aged care from multiple perspectives.

Methods: Data was collected from in-depth interviews with GPs, RAC staff and bereaved family members. Analysis utilised a qualitative descriptive approach. The study was part of a larger mixed -methods study into end of life care in residential aged care facilities (representative sample of 61 facilities across New Zealand, with data collection from 286 deaths). Interviews were conducted with 17 GPs providing medical services to RAC facilities, as well as interviews from 113 RAC staff and 33 bereaved families.

Results: The main qualitative themes included:

1) GP age and life experience shaped perceptions and skill for end of life care in RAC

2) Most GPs only had on the job experience with no formal training in dementia or end of life care prior to working in RAC,

3) GPs were 'surprised' at the satisfaction they gained in providing RAC services,

4) GP's ability to trust registered nurses assessment and clinical expertise is key,

5) family expectations often don't match what GPs can offer,

6) Hospice was used sparingly and only as 'back up'.

Conclusion: For many GPs, time and cost constraints preclude a more active and in-depth involvement in the care of for those dying in RAC facilities and their families. Specific training for end of life care in RAC, especially for those with dementia, currently appears to be ad hoc and informal. A more formal approach to prepare and support GPs for RAC end of life care is needed. Innovations in the traditional GP model of care could improve services for those dying in RACs.
Abstract number: P01-540

Abstract type: Poster

What are the Challenges in Providing Palliative and End of Life Care for Prisoners in England and Wales?

Burtonwood J. ${ }^{1,2}$, Forbes $K^{2}{ }^{2}$

${ }^{1}$ School of Medicine, Cardiff University, Cardiff, United Kingdom, 2Palliative Medicine, University Hospitals Bristol NHS Foundation Trust, Bristol, United Kingdom

Background: Prisoners approaching the end of life (EoL) are thought to have significant unmet palliative care (PC) needs. Nevertheless, the topic has attracted relatively little research; that which exists suggests PC-delivery in prison is challenging. Much of the research is international and not directly generalisable to the UK prison system. Thus, despite a need for PC in this population, there is a lack of evidence to guide its implementation.

Aims: To explore the challenges of providing palliative and EoL care for prisoners in England and Wales.

Methods: A thematic analysis was conducted of Prison Ombudsman reports on deaths in prisons across England and Wales throughout 2016-17.

Results: One hundred reports were analysed:

- $\quad$ Prisoners had complex care needs which were challenging to manage in the prison environment. Dedicated PC facilities often addressed these needs but were also associated with negative effects such as social isolation.

- $\quad$ Advance care planning (ACP) for this population was complex and often undertaken too late to be able to identify or effect prisoners' wishes.

- $\quad$ Therapeutic relationships with staff and fellow prisoners were common where families were estranged.

- Prognostic uncertainty had significant ramifications for terminally-ill prisoners; early release on compassionate grounds (ERCG) in particular, was less likely to be successful for conditions with unpredictable clinical trajectories.

- $\quad$ Significant variation in palliative care exists between prisons.

Conclusions: Multiple challenges were identified and changes to current PC practice and provision are needed to meet them. Timely ACP, specific to prison care, is suggested; particularly given the incidence of loss of decision-making capacity in prisoners with no advocates. Policy regarding ERCG should be revisited as the current criteria appear to favour those with predictable disease courses such as is seen in malignant disease. Part-funded by a bursary from Health Education England

Abstract number: P01-541

Abstract type: Poster

'Hotel 6' and 'Don't Forget the Rice Pudding': Becoming Prisonwise. (A Reflection on Joint Working between Prison and Local Specialist Palliative Care Teams)

Royce C. ${ }^{1}$, Poolman M. ${ }^{2}$, Chapman R. ${ }^{3}$, Roper J. ${ }^{4}$, Meade . $^{3}$, Bond R. ${ }^{5}$, Forbes J. ${ }^{5}$, Foster A. ${ }^{1}$, Roberts $C .{ }^{1}$, Jones $K .{ }^{4}$, Newman S. ${ }^{4}$

${ }^{1}$ Specialist Palliative Care Team, Betsi Cadwaladr University Health Board, Wrexham, United Kingdom, ${ }^{2}$ North Wales Centre for Primary Care Research, Bangor University, Wrexham, United Kingdom, ${ }^{3}$ Custodial Services, HMP Berwyn, Wrexham, United Kingdom, ${ }^{4}$ Health and Wellbeing Services, HMP Berwyn, Wrexham, United Kingdom, ${ }^{5}$ Clinical Services, Nightingale House Hospice, Wrexham, United Kingdom

Background/aim: Her Majesty's Prison (HMP) ' $\mathrm{B}$ ' is a new closed adult rehabilitation establishment in Wales, and can hold 2,106 category $C$ 
men. It is the second largest prison in Europe. Healthcare is provided by local statutory services, and replicate enhanced primary care with no inpatient facilities.

To support HMP B men in the last year of their lives, it was imperative that a strong partnership develop between the prison healthcare and custodial teams, the statutory specialist palliative care team and local hospice. All are committed to ensuring that the men and those important to them, consistently and equitably receive timely, dignified and compassionate palliative care.

Approach taken: Since HMP B opened in 2017, work comprised joint meetings and training sessions. More recently, we cared for our first joint patient, who died at the local hospice. Monthly clinical multi disciplinary team (MDT) meetings were set up. At present, we are benchmarking services against UK national guidelines (Dying Well in Custody Charter, April 2018).

Wide-ranging lessons:

1. Interpreting what is already known about prison palliative care in the context of the unique healthcare provision arrangement at HMP B.

2. The importance of comprehensive information governance arrangements to facilitate safe, yet effective communication between the prison healthcare and custodial teams, men and their families. Procedures following a death in custody have additionally illuminated this aspect.

3. Ensuring seamless extension of security arrangements when men are transferred to healthcare settings outside HMP B was sharply in focus, as was implications for how best to train clinical and ancillary staff in the large local health board regarding security considerations.

Next steps: Monthly MDT meetings will continue in parallel to benchmarking, and will shape actions and services for the future.

No specific funding was recieved for this work.

Abstract number: P01-542

Abstract type: Poster

Prisoners: End of Life Care for those who Do Not Have a Choice to Die at Home

Panozzo S. ${ }^{1}$, Philip J. ${ }^{2,3}$, Bryan T. ${ }^{4}$, Lethborg C. ${ }^{5}$

${ }^{1}$ St Vincent's Hospital Melbourne, VCcC Palliative Medicine Research Group, Melbourne, Australia, ${ }^{2}$ University of Melbourne, Department of Medicine, St Vincent's Hospital, Melbourne, Australia, 3Victorian Comprehensive Cancer Centre, VCCC Palliative Medicine Research Group, Melbourne, Australia, 'St Vincent's Hospital Melbourne, Palliative Medicine, Melbourne, Australia, ${ }^{5}$ St Vincent's Hospital Melbourne, Social Work, Melbourne, Australia

Background: Australia has a growing prisoner population with an increasing number of older prisoners. Unique circumstances of incarceration mean that prisoners do not have a choice to die at home, placing added significance to hospital care at the end of life.

Aim: To describe end of life care (EOLC) for hospitalised prisoners and explore perspectives of health care professionals (HCP) providing this care.

Methods: This mixed methods study involved a retrospective medical record audit of prisoners who were admitted and died in a tertiary hospital setting from July 2014 to June 2016. The audit extracted items from the final 3-months of life including: clinical details; access and timing of palliative care; hospital use; and place of death. Focus groups with HCPs were conducted until saturation.

Results: 13 male prisoners aged 39-93yrs (median 58yrs) were identified, with most having a cancer diagnosis and dying $20+$ years earlier than non-prisoner patients. Most had multiple vulnerabilities including mental health (54\%) and illicit drug use (38\%). Total median length of stay (LOS) was 61 days, more than double non-prisoner patient LOS. Prisoners were more likely to have $\geqslant 1$ hospital admissions ( $n=11 ; 85 \%$ ). Ten (77\%) prisoners received access to palliative care services. 7 died in the high security hospital ward (54\%). 54 HCPs identified key themes in providing EOLC for prisoners, including: importance of seeing the person; knowing their story; constraints on provision of care and place of death; late acceptance of dying; delayed involvement of palliative care. Conclusion: This study demonstrated prisoners experience multiple vulnerabilities, spend 1 or more of their final months of life in acute hospital care and receive palliative care at a similar rate to other patients. HCPs revealed key insights to EOLC for prisoners that can inform service provision to improve prisoners' EOLC experiences.

Source of funding: Inclusive Health Innovation Fund, St Vincent's Health Australia

Abstract number: P01-543

Abstract type: Poster

\section{An Educational Intervention to Promote Staff Confidence and Care Planning for People Experiencing Homelessness with Palliative Care Needs: A Pilot Study \\ Field $H_{.}{ }^{1,2}$, Hudson $B .{ }^{2,3}$, Shulman C. ${ }^{2,3}$, Stone $P^{3}{ }^{3}$ \\ ${ }_{1}^{1}$ Palliative Medicine, Cardiff University, Guildford, United Kingdom, ${ }^{2}$ Pathway, London, United Kingdom, ${ }^{3}$ Marie Curie Palliative Care Research Department, University College London, London, United Kingdom}

Background: The average age of death for people experiencing homelessness is approximately 30 years younger than that of the housed population, dying from potentially treatable conditions with limited access to healthcare. Many have complex health needs but remain in homeless hostels where non-medically trained staff support them.

Aim: To assess the impact of a short educational intervention on hostel staff confidence in discussing deteriorating health and treatment options with homeless hostel residents.

Methods: A mixed methods pilot study to explore the impact of face-toface educational sessions with written resources on hostel staff's confidence in having conversations with residents about their health wishes. A novel questionnaire was used before and after the intervention. All staff from 2 homeless hostels in London were eligible to participate. Eligible hostel residents were those identified by the multi-disciplinary team (specialist nurse, hostel staff and GP registrar) as potentially nearing the end of their lives (using the surprise question).

Results: Baseline questionnaires completed by 6 hostel residents and 18 staff members showed staff did not feel discussions with residents about their health preferences were occurring, or that staff needs as carers were being met. Follow-up questionnaires two months post-intervention revealed increased discussions occurring (60\% from $27 \%$ ), improvements in carers needs being met (40\% from $0 \%$ ) and increased coordination of care (70\% from $66 \%$ ).

Conclusion: Hostel staff described benefiting from a short educational intervention around supporting residents with deteriorating health but need more support in discussing end-of-life care preferences with residents. All patients should be given the chance to clarify their wishes surrounding the end-of-life and this right should extend to the homeless. This is a feasible intervention which may increase both staff confidence and the number of discussions occurring.

Abstract number: P01-544

Abstract type: Poster

The Impact of Art Therapy on the Physical and Mental Symptoms of Terminally III Patients in Hospices Marini J., Bazzan C., Gallucci M. Hospice, Hospital Policlinico San Martino, Genova, Italy 
Introduction: This study explored the impact and effectiveness of art therapy on the physical and mental symptoms of terminally ill patients in Hospices. Art therapy is a non-verbal method of offering assistance and mediation-based therapy. It's a combination of therapeutic treatments that use artistic expression as the main instrument to improve personal wellbeing and the quality of life, so it's appropriate as a form of palliative care.

Goals: To assess the potential effectiveness of art therapy in the three dimensions of aesthetic perception: the happiness, associated with the joy during and post activity; the satisfaction, associated to creation of something; the beauty, associated with the aesthetic satisfaction and the reduction of frequent symptoms. In particular, seven symptoms (pain, anxiety, wellbeing, tiredness, drowsiness, sadness and depression) were analysed through patient self-assessment based on the Edmonton Symptom Assessment System (ESAS), used before and after the art therapy session, and the Harmonic Cube used after the art therapy session.

Method: Open, monocentric, non-randomised experimental study, done on terminal patients in the advanced stages of the illness, organised in a selection phase and a treatment phase.

Conclusions of the study: This study, showed an improvement in all seven of the parameters analysed; the most notable improvement was with depression $(-1.2 ;-21 \%)$, while the least notable result was with tiredness $(-0.4 ;-7 \%)$. In addition, an ample standard deviation was noted, which may suggest subjective variability in the patients' response to art therapy. The data gathered indicates that one art therapy session may be sufficient to improve the parameters measured (tiredness, drowsiness, depression, anxiety, wellbeing and pain), although further research is required. Finally, it was noticed that the patient's technical ability has no effect on whether or not he/she can participate in and benefit from an art therapy session.

Abstract number: P01-545

Abstract type: Poster

Developing Integrative Medicine Project in the Hospice and Other Polyclinic Departments

Bazzan C., Bonini L., Cosulich E., Ferrari D., Pilastri P., Gallucci M. Hospice, Ospedale Policlinico San Martino, Genova, Italy

Integrative Medicine is defined by the National Center for Complementary and Integrative Health $(\mathrm{NCClH})$ as "a large group of medical and health care systems, practices, and products that are not typically considered to be a part of conventional medicine, and are used in conjunction with the same."

This goes in a direction in which the "core" of the therapy lies in the integration of mind and body, and focuses on improving the quality of life, making it particularly pertinent as a form of palliative care. Integrative Medicine is based on the use of the arts, creativity, materials, instruments and artistic languages as intermediaries in the relationship created with patients, and is generally not used for care in terms of healing. The aim of

this project is to associate clinical therapy on the basis of Conventional Medicine with humanistic Integrative Medicine, by working in a multidisciplinary team. The goal is to improve the quality of life, to permit the patient to adapt to his/her illness and to help friends/relatives improving their end life perception and experience.

Integrative Medicine sessions have being held throughout the week in this hospital's Hospice for the last two years: Art therapy, Music therapy, Meditation and Massage Techniques for in-patients and their friends/ relatives.

Multidisciplinary meetings identified criteria of eligibility and use of protocols on specific therapy areas sharing therapy goals for patients and relatives.

The purpose is to develop creative potential and promote the capacity of human beings to adapt to the world, using their own resources on the basis of the context they are in, a process that lets people acquire greater control over their perception of their health and improve it.

The project has been developed entirely in Hospice and going to propose also in different care setting.

Abstract number: P01-546

Abstract type: Poster

Challenges and Opportunities for Integrating Palliative Care into Humanitarian Health Interventions Among Health Workers Serving Refugee Communities in Uganda

Buyinza N. ${ }^{1}$, Ryan C. ${ }^{2}$

${ }_{1}^{1}$ nstitute of Hospice and Palliative Care, Hospice Africa Uganda, Kampala, Uganda, ${ }^{2}$ Hospice Africa Uganda, Kampala, Uganda

Introduction: The overarching need for palliative care across Africa remains a mammoth task basically because priority is given to preventative and curative services. Whether refugees make a significant contribution to national economy or not, they are largely excluded from social services mainly because they are often considered either to be criminals or illegal settlers.

Aim: To explore health workers' Challenges and Opportunities for integrating palliative care services into humanitarian health interventions in Uganda

Methods: A cross sectional qualitative study applying Focus Group Discussions with health care workers working with UNHCR implementing partners within refugee operational areas in Uganda.

We recruited a total of 36 participants and conducted three focus group discussions with 12 participants each. Participants were stratified into three categories (Nurses, Clinical Officers and Doctors). Analysis was approached by data familiarization, identification of thematic frame works, indexing, charting and interpretation.

Results: No humanitarian organization providing health interventions among refugees were providing palliative care services. This reflects the global situation where very many health workers engaged in humanitarian development are neither trained in palliative care nor offering it.

Respondents experienced challenges unique to working with refugee communities including disjointed referral systems, language barrier, limited palliative care knowledge, infrastructural restrictions and limited morphine access.

Conclusion: Findings indicate that access to palliative care services for refugees in Uganda falls far short of even the host community needs. There is need for an improved referral system, additional skilled human resource work force for palliative care, a health systems strengthening approach for improved availability of oral liquid morphine and access to quality palliative care services within refugee host districts.

Abstract number: P01-547

Abstract type: Poster

„My Daughter Is my Advocate" - Social Marginalisation of Migrants with Severe Illness in Palliative Care

Owusu-Boakye S. ${ }^{1}$, Banse C. ${ }^{1}$, Schade F. ${ }^{1}$, Jansky M. ${ }^{1}$, Marx G. ${ }^{1,2}$, Nauck F. ${ }^{1}$

${ }^{1}$ Clinic of Palliative Medicine, University Medical Center Goettingen, Goettingen, Germany, ${ }^{2}$ Institute of General Medicine, University Medical Center Hamburg-Eppendorf, Hamburg, Germany

Background: Migration background in Germany determines how migrants utilise health care benefits in Germany, e.g. utilisation of specialised palliative care.

Aims: To analyse experiences of migrants with advanced cancer in the German health care system. To explore the social relevance of family caregivers during care giving process. Understanding the mechanisms of social marginalisation. 
Method: Qualitative design, narrative interviews with patients $(n=21$, $12 \mathrm{w} / 9 \mathrm{~m}$, Age $28-79$ ) and family caregivers ( $2^{\text {nd }}$ generation of immigrants, $n=7,1 \mathrm{~m} / 6 \mathrm{w}$, Age 29-50). Data analysed using Grounded Theory. Results: Socio-cultural exclusion is experienced, if patients have the feeling that they can't communicate with their health care professionals because of their language skills, or if they feel mistreated because of their background or their attributed culture. This may result in existential fear. In these cases patients are dependent on their children which can advocate their parents' needs in clinical consultations. The descendants feel a high responsibility for their ill parents because with through migration they offered them chances of social advancement in Germany. With this responsibility, they are burdened to take care of their parents' lives, because their parents' wellbeing depends on the descendants' adequate decision making.

Conclusion/discussion: Experience of culturalisation and discrimination can compromise equal medical treatment of migrants and their family caregivers in palliative care situations. The $2^{\text {nd }}$ generation of immigrants are burdened to take care for their severely ill parents while coping with their own daily life. The vulnerable position of family caregivers can be overlooked by health care professionals, if descendants are mostly seen as interpreters, not as persons with their own feelings and needs. Therefore professional interpreters and psychosocial support may benefit families during palliative treatment.

\section{Abstract number: P01-548 \\ Abstract type: Poster}

\section{Palliative Care for Elderly South Asian Immigrants: A Systematic} Review

Shabnam J. ${ }^{1}$, Timm H. ${ }^{1}$, Nielsen D. ${ }^{2}$, Raunkiær M. ${ }^{1}$

${ }^{1}$ University of Southern Denmark, Knowledge Centre for Rehabilitation and Palliation, Nyborg, Denmark, ${ }^{2}$ University of Southern Denmark / Institute of Regional Health Research, Odense, Denmark

Background: Palliative care that meets the specific cultural needs of elderly immigrants is a priority, to provide equitable palliative care services to all in society.

Aim: The aim was to summarize published research on experiences and perspectives of palliative care among elderly South Asian immigrants, family members, health providers, and society, in relation to culture of the country of origin.

Methods: A systematic review in accordance with PRISMA guidelines was conducted in February 2018, searching PubMed, CINAHL, PsychINFO and EMBASE databases. Key words were palliative care, immigrants and South Asia.

Inclusion criteria: Studies describing original research in peer-reviewed journals, providing information on palliative care of south Asian immigrants and published between 2000 and 2018. Thematic synthesis was used to analyse data.

Results: Of the 87 articles meeting the inclusion criteria, 30 articles were included in the review: qualitative (24), quantitative (5), and mixed methods (1). Studies identified that role of family members and communication with health providers was important for patients' access, use and satisfaction with the existing palliative care services in the country of residence. Studies suggested that culturally appropriate care towards elderly immigrants can improve communication with health providers and thereby facilitate access, use and satisfaction with palliative care services among immigrant families. It points towards the importance of family involvement and culturally appropriate palliative care.

Conclusion: Studies involving palliative care and South Asian immigrants are limited in number and are mainly qualitative. However, considering the multi-ethnic nature of the world today, findings related to the importance of family involvement and culturally appropriate palliative care may be applicable to other immigrant populations beyond South Asians immigrants.
Abstract number: P01-549

Abstract type: Poster

The Role of Intercultural Mediation in End-of-Life Care

García-Navarro E.B. ${ }^{1}$, Araujo Hernández M. ${ }^{1}$, Pérez Espina R. ${ }^{2}$, Fernández Romero R.R.I. ${ }^{3}$, García Navarro S. ${ }^{4}$

${ }^{1}$ Universidad de Huelva, Facultad de Enfermería, Huelva, Spain, ${ }^{2}$ Hospital Vázquez Diaz, Huelva, Spain, ${ }^{3}$ Universidad de Huelva, Huelva, Spain, ${ }^{4}$ Distrito Sanitario Huelva-Costa-Condado-Campiña, Huelva, Spain Presenting author email address: bego.garcia@denf.uhu.es

The care at the end of life, as Vivanco (2013) refers, requires an approach of active compassion that treats, comforts and supports patients who have been made in the process of dying progressively. Such care is sensitive to personal, cultural and spiritual values, practices and practices that include support for family and friends until the period of grief. Cultural beliefs and practices are aspects that take importance in the care and decisions that are taken in the process of dying.

Aims: Identify the perceptions of palliative care professionals in relation to the management of patients from diverse cultures and their own cultural interests.

Know the influence of the figure of the mediator as a facilitator in the decision making of the patient and the family at the end of life.

Methodology: Approach of methodological pluralism, accompanied by the triangulation of data and information

1. Secondary sources: bibliographical and administrative sources.

2. Primary sources of qualitative analysis, via in-depth interview, carried out to different types of profiles: Health professionals working with palliative patients and intercultural mediators

3. Observation.

The sample made for the first group of informants for the second year. The saturation of the speech was what determined the number of participants, ( 7 and 5 respectively)

Results: coding of the categories exposed by the nursing professional informants consulted and confrontation with those generated by the intercultural mediators.

conclusion.

Intercultural mediation is a tool that can facilitate communication with our patients, the writing of the introduction in health institutions for the realization of relevant cultural care in the field of palliative care.

Abstract number: P01-550

Abstract type: Poster

\section{Influence of Cultural Diversity at the End of Life: A}

Phenomenological Study

García-Navarro E.B. ${ }^{1}$, Pérez Espina R. ${ }^{2}$, García Navarro S. ${ }^{3}$, Araujo

Hernández M. ${ }^{4}$

${ }^{1}$ Facultad de Enfermería, Universidad de Huelva, Huelva, Spain,

${ }^{2}$ Hospital Vázquez Diaz, Huelva, Spain, ${ }^{3}$ Distrito Sanitario Huelva-CostaCondado-Campiña, Huelva, Spain, ${ }^{4}$ Universidad de Huelva, Huelva, Spain

In reference to Durkheim, culture is a spider web full of senses that give meaning to your life. When we associate this concept with the final process, the need arises to accompany this moment in the most competent way by taking into account meanings and needs, as well as cultural practices thereof to ensure the care provided is culturally competent. The acceptance of and respect for cultural differences, the sensitivity to understand how these differences influence relationships with people, and the ability to offer better results in cultural encounters are essential requirements in order for cross-cultural care in nursing to be consolidated. 
The objective of this work is to identify the perception, as well as the coping of the patient at a late stage of life and how to determine the culture in this process.

To do this, we used a methodological strategy of qualitative descriptive character. The data was collected through an in-depth interview and the analysis of the data was carried out with the theories of Taylor and Bogdan. Results contemplate 6 categories (migratory process, migratory mourning, family - mother, community, faith and illness). In these categories it is stated that cultural competence at a late stage of life is essential to understand death from the current perspective to the current globalisation. To do this, we must delve into various forms of coping in the late stages of life and thus be able to respond to the needs of patients based on comprehensive, empathetic and holistic care.

Finally, we can conclude that both therapeutic relationship and communication broaden cultural knowledge, even though in order to do so, it will also be necessary to gain cultural competence, increase human resources with diversity knowledge and improve intercultural sensitivity in any health process, emphasising its cultural component in the final life process.

Keywords: nursing, cultural, care at a late stage of life, cultural competence.

Abstract number: P01-551

Abstract type: Poster

Development, Implementation and Evaluation of Palliative Care Education for Health Care Workers, Village Health Teams and Family Caregivers Providing Healthcare for South Sudanese Refugees in Adjumani District, Uganda

Opia V. ${ }^{1}$, McGannon M. ${ }^{2}$, Nalutaaya F. ${ }^{3}$, Purewal G. ${ }^{2}$, Bagasha P. ${ }^{3}$, Grant

L. ${ }^{4}$, Downing J. ${ }^{3}$, Leng M. ${ }^{2,4,5}$

${ }^{1}$ Peace Hospice, Adjumani, Uganda, ${ }^{2}$ Cairdeas International Palliative Care Trust, Aberdeen, United Kingdom, ${ }^{3}$ Makerere and Mulago Palliative Care Unit, Internal Medicine, Kampala, Uganda, ${ }^{4}$ Edinburgh University, Global Health Academy, Edinburgh, United Kingdom, ${ }^{5}$ Makerere University, Palliative Care Unit, Kampala, Uganda Presenting author email address: dr@mhoira.net

Background: Palliative care (PC) and refugee care are national priorities in Uganda, however little is known about integrating PC into humanitarian settings. Adjumani district has 260,000 refugees, with 240,000 host community. A PC integration programme supported by a rapid system appraisal revealed significant gaps in service provision \& need for capacity building.

Aim: To address training \& capacity needs within refugee settlements by developing PC education pathways for Health Care Workers (HCW) who work in health centres serving refugee settlements, Village Health Teams (VHT) and Family Caregivers (FC).

Methods: Training for 30 HCW's ( 5 days) and 75 VHT ( 3 days) was developed \& delivered. Initial evaluation comprised pre/post course knowledge testing \& pre/post course confidence ratings, plus post course evaluation \& sample interviews. 3 month post course follow-up evaluation will be undertaken including qualitative evaluation for the VHTs. Training for 150 FC has been developed for delivery November 2018.

Results: Initial evaluation reveals an increase in knowledge \& confidence for HCW's by 2 out of 5 VAS scale points. VHTs identified improvements including confidence to identify PC patients, and communicate more effectively. HCWs interviewed revealed new skills, improved ability to care for patients and families and increased satisfaction. 'It modeled my character, my behaviour is quite different from before I had the knowledge' Full comprehensive qualitative and quantitative results will be presented including impact on practice.

Conclusion: In order to adopt a universal health coverage approach for chronic disease care in in humanitarian situations strengthening capacity is crucial. This work is being done in partnership with key stakeholders including humanitarian agencies, the Ministry of Health and should also have wider relevance in fragile contexts. A baseline needs assessment \& health systems review is also in process to offer further evidence.

\section{Abstract number: P01-552 \\ Abstract type: Poster}

Improving Adherence to Art for Prevention of Symptoms and for Better ART Clinical Outcome at Bujubuli HC III, Kyegegwa District, Western Uganda

Byamukama P. ${ }^{1,2}$

${ }^{1}$ African Humanitarian Action, Health -Palliative Care, Kampala, Uganda, ${ }^{2}$ Palliative Care Association of Uganda, Health -Palliative Care, Kampala, Uganda Presenting author email address: byamupolycarp@ yahoo.com

Setting: Bujubuli Health center III is a government aided health facility located in Kyaka II refugee settlement, Kyegegwa District. The health center also receives financial support from UNHCR and serves an estimated catchment population of 97266 people majority (72266) of which are refugees.

Problem: By June 2017, 1506 patients were receiving ART at Bujubuli health center with about $41 \%$ of them taking more than $95 \%$ of their prescribed ARV medicines and a similar proportion (40.5\%) were clinically well as defined by body weight, absence of new opportunist infections and functional status.

Intervention: The team decided to initiate quality improvement efforts targeted towards adherence to ART. During the month of august 2017 the team started issuing of adherence cards to all ART clients and taught them how to use them for self monitoring of adherence. Individualized ART adherence counseling sessions for poorly adherent clients were introduced to solve client specific also adherence problems.

Results: With the introduction of the above mention changes, the proportion of patients adherent to ART doubled, increasing from $41 \%$ in June 2017 to $84 \%$ by the end of 2017, similarly the proportion of clients clinically well raised by more than $100 \%$ in the same period. While working on improving the process of adherence to ARVs for ART clients the team discovered that appointment keeping was a problem to some of the clients who were not adhering well to medicines.ART clients seen to have good Clinical outcome due to client's adherence to ART treatment since June 2017 up to December 2017.

Lessons learnt:

1. Applying QI approaches to a particular process of HIV care is helpful in identifying problems with other related processes of care.

2. Good adherence for ART promotes good health and prevents signs and symptoms (pain, Diarrhea, Vomiting and others) associated with HIV/AIDS.

Abstract number: P01-553

Abstract type: Poster

Attitudes to Palliative Care and the Care of People with Intellectual and Developmental Disability in Europe and Africa Oliver $D .{ }^{1}$, Forrester-Jones $R .^{2}$

${ }^{1}$ Tizard Centre, University of Kent, Rochester, United Kingdom, ${ }^{2}$ Social and Policy Sciences, University of Bath, Bath, United Kingdom

Background: The services for palliative care, and the awareness and understanding of the role of palliative care, vary across the World. The involvement of palliative care services with people with Intellectual and developmental disability (IDD) has also been found to be different in different countries. 
Aims: The aim of this study was to compare the attitudes of health care professionals towards palliative care in countries in Europe and Africa, and in particular the attitudes regarding the provision of palliative care for people with intellectual and developmental disability (IDD). Methods: Focus groups were held at conferences of health care professionals - in South Africa, Portugal and Switzerland. The discussions were facilitated by the researcher, using semi-structured, open-ended questions. Results: Diverse attitudes towards palliative care were found to exist between the three countries.

In Switzerland there was greater awareness of palliative care, although this was often associated with end of life care. The care of people with IDD was variable.

In Portugal there was less understanding of palliative care and there was limited discussion of the topics of death and dying, with patients and families often having unrealistic expectations of treatment. People with IDD were often cared for within institutions, although some were cared for at home.

In South Africa there was awareness of palliative care but people with IDD were viewed as "different" and there were few resources to help in their care.

Discussion: Diverse attitudes and practices concerning palliative care exist between countries, affecting the development of services and reflecting wider and deeper attitudes towards people with IDD. Recommendations for future practice are proposed.

\section{Abstract number: P01-554}

Abstract type: Poster

Health Care Utilization at 6 Months Before Death among Patients with COPD and Lung Cancer

Kuo L.C. $^{1}$, Lin C.C. ${ }^{1,2}$

${ }^{1}$ School of Nursing, College of Nursing, Taipei Medical University, Taipei, Taiwan, Republic of China, ${ }^{2}$ School of Nursing, Li Ka Shing Faculty of Medicine, The University of Hong Kong, Pokfulam, Hong Kong

Background: Chronic obstructive pulmonary disease (COPD) and lung cancer patients shared the similar symptoms at the end-of-life. However, until today still lack large sample size study to investigate their medical utilization in their end-of-life.

Aims: Our study aim was to explore the end-of-life resource use in patients with COPD and comparing to lung cancer patients in the last 6 months of their lives.

Methods: Using a Taiwan National Health Insurance Research Database (NHIRD), we performed a nationwide retrospective cohort study of patents with acute exacerbation of COPD $(n=19,442)$ or lung cancer $(n=$ 3,427) who died between 1997 and 2013.

Results: Decedents with COPD were more likely to be admitted to an ICU (58.86\% versus $29.59 \%$ ), to have a longer length of stay in ICU (15.73 days versus 9.70 days), to receive vasopressor agents and treated with more invasive and life supportive procedures; were less likely to use palliative care services at an inpatient palliative care unit (3.32\% versus $14.50 \%$ ) or at home (1.45\% versus $8.11 \%)$, and less likely to receive opioids and benzodiazepines when compared to decedents with lung cancer during the last 6 months of life. Also, decedents with COPD were treated with more aggressive care during the last one month of life. Average total medical cost during the last 6 months of life were approximately 1,358 USD higher for decedents with COPD than for decedents with lung cancer.

Conclusions: The high use of health care resources in patients with COPD at the end-of-life suggests a focus on prolonging life, a tendency toward aggressive care, and might indicate a intensified need for palliative care in these patients. A need for limiting potentially inappropriate care and improving the quality of end-of-life care in patients with COPD by providing palliative care is urgent.
Abstract number: P01-555

Abstract type: Poster

Living with and Dying from Advanced Head and Neck Cancer: A Scoping Review to Assess Experiences and Unmet Needs Mayland C. ${ }^{1,2}$, Mason S. ${ }^{2}$, Doughty H. ${ }^{2}$, Ho M. ${ }^{2}$, Peddinti P. ${ }^{2}$, Chada P. ${ }^{2}$, Rogers S. ${ }^{3,4}$, Jack B. ${ }^{5}$, Hubbard C. ${ }^{6}$, Macareavy D. ${ }^{3}$, Dey P. ${ }^{7}$

${ }^{1}$ Department on Oncology and Metabolism, University of Sheffield, Sheffield, United Kingdom, ${ }^{2}$ Palliative Care Institute, University of Liverpool, Liverpool, United Kingdom, ${ }^{3}$ Aintree University Hospital, Liverpool, United Kingdom, ${ }^{4}$ Edge Hill University, Liverpool, United Kingdom, ${ }^{5}$ Evidence-Based Research Practice Centre, Edge Hill University, Ormskirk, United Kingdom, ${ }^{6}$ Aintree Park Group Practice, Liverpool, United Kingdom, ${ }^{7}$ Faculty of Health and Social Care, Edge Hill University, Ormskirk, United Kingdom

Background: Although studies have examined the integration of palliative care to routine oncological care, none have specifically involved head and neck cancer patients, who have especially complex needs due to: symptom burden; risk of catastrophic events and psychological concerns affecting patients and families.

Aim: To systematically explore and synthesize current literature on the needs and experiences of patients with advanced head and neck cancer.

Methods:

Design: A scoping literature review using systematic methods (re: Joanna Briggs Institute).

Data collection: Electronic databases (MEDLINE (Ovid), EMBASE and CINAHL) were searched from January 1996-2017; keywords/subject headings for 'Palliative care' and 'head and neck neoplasms'.

Inclusions: Empirical studies focused on needs, experiences or views of adults with advanced head and neck cancer/professionals supporting this population, irrespective of setting or study design; articles published in English. Exclusions: case reports; focus on children or survivorship. References of included papers were screened.

Analysis: Data extracted using a specific proforma (setting, country, population, study design and findings). Findings were tabulated and narratively synthesised into themes.

Results: From 928 identified papers, 42 formed the final data set. Main themes: diverse and multiple symptoms (with specific focus on psychological support); issues with communication (differing patient and family needs; transfer of information between healthcare professionals); and high prevalence of 'intensive' interventions in the last month of life. Conclusions: Increased understanding about the complexity of needs should help inform future models of integrated care to better meet the needs of patients with advanced head and neck cancer. In view of the high level of medical interventions, particular focus is needed on advance care planning and upskilling of healthcare professionals in different care settings.

\section{Abstract number: P01-556 \\ Abstract type: Poster}

Palliative Care - Beliefs and expectations about palliative treatment in patients with lung cancer - an explorative study with a mixed methods approach

Ikander $T^{1}{ }^{1}$, Raunkiær M. ${ }^{2}$, Hansen $O .^{1}$, Dieperink $K .^{1}$

${ }^{1}$ Department of Oncology, Odense University Hospitals, Odense, Denmark, ${ }^{2}$ Knowledge Center for Rehabilitation and Palliative Care, REHPA, Nyborg, Denmark

Background: Most incurable cancer patients are offered palliative chemotherapy in order to ease symptoms and improve quality of life. However, life-prolonging palliative chemotherapy is not always the answer, especially if the treatment itself is demanding and associated 
with harsh side-effects making daily life worse and affecting the patients' quality of life. Patients and their relatives may choose to receive palliative chemotherapy for various reasons, which may compromise optimal quality of life. International studies have found that patients and their relatives have high expectations of becoming cured by palliative chemotherapy. We want to examine if this is true for Danish cancer patients as well.

Aims: The aim of this study is to clarify Danish lung cancer patients and their relatives beliefs and expectations when receiving palliative chemotherapy.

Methods (design, data collection, analysis): Patients and relatives will complete a manageable and adapted short questionnaire of four questions of their beliefs and expectations about palliative chemotherapy. The patients will furthermore fill in the quality of life questionnaire Functional Assessment of Cancer Therapy - General. The questionnaire is handed out to patients prior to their first and second course of palliative chemotherapy in the outpatient clinic, and before the consultation in the follow up clinic. The relatives will only answer the questionnaire prior to the first course of treatment.

Results: Preliminary results so far include 37 patients with lung cancer and 32 relatives. An interim analysis is expected to be ready at the beginning of 2019.

Conclusion / Discussion: We expect to deliver new knowledge on the beliefs and expectations of Danish cancer patients and their relatives when receiving palliative chemotherapy.

Abstract number: P01-557

Abstract type: Poster

Delivering Palliative and End-of-Life Care to People with Alcohol and Drug Problems: Challenges and Opportunities

Galvani S., Dance C., Wright S.

Social Care and Social Work, Manchester Metropolitan University, Manchester, United Kingdom

Background: Increasing longevity of the UK population, increasing alcohol-related harm among older drinkers, and an ageing cohort of long term drug users, indicate that the number of people with alcohol or drug (hereafter 'substance use') problems in palliative and end of life services will grow. However, no guidance is available for professionals on how best to work with this growing group of people. This is not surprising given the lack of evidence reflecting the experiences of the professionals who work with them. This research sought to establish the experiences, knowledge and attitudes of professionals working in hospice and substance use services.

Aims: A core aim of this research was to establish the challenges and opportunities professionals face in supporting people with problematic substance use and terminal illness.

Methods (design, data collection, analysis): Data were collected using focus groups and individual interviews. Template Analysis was used to ensure the transparency and quality of the coding process given it encompasses both a priori and grounded coding.

Results: Key challenges included: the multiplicity of needs that people present with; symptom and pain management; the misuse of pain medication; people using a range of additional substances to self-medicate; difficulties talking about substance use or end of life care; a lack of clear care pathways and partnership working; and stigmatising attitudes from other professionals. Professionals also identified frustration, emotional stress and concerns about personal safety. They identified positive team support as helping them to cope with the strain.

Conclusion / discussion: Professionals reported a range of challenges on clinical/practical levels, as well as emotional challenges. However, they showed positive and empathetic attitudes towards this group of people. More guidance is needed to support professionals given the anticipated increase in people presenting with both issues in future.
Abstract number: P01-558

Abstract type: Poster

The Prognosis in Palliative Care Study II (PiPS2): Factors

Affecting Recruitment of Patients with Advanced Cancer into a

Multicentre, Prospective, Observational Cohort Study

Kalpakidou A.K. ${ }^{1}$, Todd C. ${ }^{2}$, Omar R.Z. ${ }^{3}$, Keeley V. ${ }^{4}$, Griffiths J. ${ }^{2}$, Spencer

K. ${ }^{2}$, Vickerstaff $V^{1}{ }^{1}$, Christodoulides $K^{1}{ }^{1}$, Perry R. ${ }^{5}$, Katsampa D. ${ }^{1}$, Todd-

Fordham F. ${ }^{1}$, Stone P. ${ }^{1}$

${ }^{1}$ Marie Curie Palliative Care Research Department, Division of Psychiatry, UCL, London, United Kingdom, ${ }^{2}$ School of Nursing, Midwifery and Social Work, University of Manchester, Manchester, United Kingdom, ${ }^{3}$ Department of Statistical Science, UCL, London, United Kingdom, ${ }^{4}$ Derby Teaching Hospitals NHS Foundation Trust, Derby, United Kingdom, ${ }^{5}$ Marie Curie Hospice, Solihull, United Kingdom

Background: The PiPS2 study is a large prospective multi-centre observational study to validate prognostic tools in palliative care patients with advanced cancer. The study requires a sample size of 1,778 patients. Many palliative care studies fail to reach their planned sample size. Therefore, we set out to rigorously monitor recruitment processes in order to identify (and remedy) any potential bottle-necks in recruitment. Aims: To identify barriers to recruitment to a large multicentre palliative care study in order to inform future studies.

Methods: Comprehensive screening logs were maintained at all recruiting sites. The logs recorded whether patients were eligible for the study; whether they were approached by the study team; and whether or not consent was obtained for enrolment. The reasons for patients' ineligibility, inaccessibility or refusal to consent were documented.

Results: 17,059 patients were screened across 27 participating palliative care services. Of those, $4,673(\approx 27 \%)$ were ineligible for participation in the study primarily due to non-cancer diagnosis. Of the 12,386 eligible patients, $9,089(\approx 73 \%)$ were not approached by the researchers, the most common reason being that approach was deemed inappropriate by the clinical staff. Other reasons included delayed referrals, early patients' death or discharge. Of the 3,297 patients who were approached by the researchers, $1,462(\approx 44 \%)$ refused participation in the study mainly because of feeling too unwell, experiencing severe distress and having too many competing priorities. Approximately $15 \%(n=1835)$ of patients who were potentially eligible were enrolled in the study.

Conclusion: Our findings demonstrate that recruiting large numbers of patients to a palliative care study may predominantly depend upon the willingness of staff to approach patients rather than on their eligibility for study participation.

Abstract number: P01-559

Abstract type: Poster

Palliative Management of a Pregnant Patient with Pancreatic Cancer: A Case Report

Nicolas-Casem M.E., Flores B.A.

Section of Supportive and Palliative Medicine, Far Eastern University

- Nicanor Reyes Medical Foundation (FEU-NRMF), Quezon City,

Philippines

Pancreatic cancer is the third leading cause of cancer death with the lowest five-year survival rates among solid tumors. It is rare before the age of forty-five making it rare in pregnancy. There were thirteen cases of pancreatic cancer during pregnancy reported, of which none were diagnosed during the first trimester. Only one case was documented involving an intraductal papillary tumor. Pancreatic cancer in pregnancy presents with persistent abdominal pain, posing a diagnostic and therapeutic management dilemma for the physician. Treatment options to control the malignancy and to provide symptom control become an ethical issue due to considerations of harm to the developing fetus. Median 
survival of individuals with pancreatic cancer is 8 to 12 months, which may provide enough time to carry a pregnancy to term. The objectives of this study are:

(1) to discuss a rare case of Intraductal Papillary Carcinoma of the Pancreas in a pregnant woman,

(2) to discuss the contribution of palliative care, and

(3) to present ethical issues in the management of the patient.

We present a thirty year - old, multi-gravid at 9 weeks age of gestation who presented with persistent abdominal pain and was eventually diagnosed with intraductal papillary carcinoma of the pancreas. She was managed by a multidisciplinary team including palliative care specialists. Given the complex situation, the contribution of palliative care in this case is essential. Palliative care is geared towards the prevention and provision of relief of suffering through adequate pain and symptom management and supportive measures to achieve the best quality of life for patients and their family. Control of suffering from pain was achieved for the patient as well as best supportive care for her and her loved ones through the provision of continuity of care, constant communication regarding management goals, coordination across services, throughout the illness, during the end of life and thru bereavement.

\section{Abstract number: P01-561 \\ Abstract type: Poster}

HOPEVOL: Hospice Care Appropriate to the Wishes and Needs of Patients in the Palliative Terminal Phase

van Klinken M., Koorn R., van der Baan F., de Graaf E., Teunissen S. Julius Centrum, UMC Utrecht, Utrecht, Netherlands

Introduction: In the Netherlands, hospice care is provided in professional-driven hospices and volunteer-driven hospices. To date, insight is lacking whether the care provided in hospices is appropriate to meet the patient's needs and wishes.

Aim: Gain insight into the characteristics of patients admitted in hospices, their needs and wishes and the care they receive.

Method: A retrospective patient record study of 1500 hospice patients in the Netherlands. Preliminary results are shown for the first 104 patients.

Inclusion criteria: Deceased, adult patients admitted in hospice in 2016-2017.

Outcomes and analysis: Patient characteristics: demographics, disease characteristics, multidimensional symptoms, needs and wishes. Care characteristics: measurement instruments and multidimensional care.

Analyses: Descriptive statistics.

Results: Preliminary results of 104 patients (mean age 78,2 years, $56,9 \%$ woman, $78 \%$ cancer diagnoses) from 13 hospices show that initiative for admission is mostly taken by the patient or their family (53.5\%). Reasons for admission were described as physical symptoms (79\%), preferred location of dying (16\%) and social problems (15\%). Patients were mostly admitted for last-resort (96\%) and most patients were in the phase of symptom palliation (89.9\%).

Problems that were most described were delirium (29\%), decubitus $(23 \%)$ and edema (18\%). On average, 6 interventions were performed in the first 72 hours per patient. Most were within the physical dimension $(65 \%)$ and least in the spiritual dimension (1.2\%). Of the 11 different measurement tools identified, a performance scale (38\%) and a symptom diary (19\%) were most frequent used.

Conclusion: A preliminary overview of hospice patients and the care they receive was presented. The study is work in progress. Additional data and a comparison between organizational structures of hospices will be presented during the congress.

Funding: ZonMw, the Netherlands Organisation for Health Research and Development.
Abstract number: P01-562

Abstract type: Poster

Decision Making for People with Disabilities and Palliative Care Needs: Lessons from Implementing Legislation in Two Health Care Systems

Rabbitte M. ${ }^{1}$, Boylan J. ${ }^{1}$, Guerin S. ${ }^{2}$

${ }^{1}$ Research, All Ireland Institute of Hospice and Palliative Care, Dublin, Ireland, ${ }^{2}$ Psychology, University College Dublin, Dublin, Ireland

People with a disability at end of life may need support in making decisions in relation to their care planning. New laws are in place in the Republic of Ireland and Northern Ireland to ensure people have a right to support in making these decisions; however they have not been fully implemented in practice and policy. The aim of this paper is to identify key challenges for health and social care services on the island of Ireland to make sure that people can access appropriate help in making decisions. A seminar to achieve all-island interdisciplinary engagement on this topic brought together 70 people from 32 different organisations (academic, clinical, legal, not-for-profit) to reflect on this topic. The expert speakers represented a range of disciplines including health research, health sciences, psychiatry, nursing, psychology, law, social work, sociology, education and health care provision; and conversation panel(s) included a person with disability and a family carer. Thematic analysis of the recordings of the expert speakers' presentations identified a number of core topics and lessons. Core topics in implementation around assessing capacity, understanding the legal impact, balancing rights of person and health care professional, and need to build awareness so that people with disability can plan ahead. Key lessons from the seminar included how involving people with disabilities and carers, providing knowledge and expertise on legislation and involving settings which were more advanced in implementation, can identify potential for interdisciplinary palliative care research and opportunities for funding to support evidence-based practice.

Abstract number: P01-563

Abstract type: Poster

Exploring Access to Palliative Care for Socioeconomically Disadvantaged Groups: A Systematic Review French M., Preston N., Keegan T., Anestis $E$.

Faculty of Health and Medicine, Lancaster University, Lancaster, United Kingdom

Background: Evidence suggests that people who are socioeconomically disadvantaged are often less likely to be referred to palliative care than more advantaged populations. However, the reasons for this are not well understood. 'Candidacy' - the idea that eligibility for services is negotiated between patients and providers but constrained or facilitated by social, organisational, and other conditions - has been used to explore differences in health service use between socioeconomic groups. The model has yet to be applied to palliative care but may improve understanding of the relationship between access to this care and socioeconomic position.

Aims: This review aims to explore the relationship between socioeconomic position and access to palliative care in the UK, focusing on which factors are important for influencing access and how.

Methods: This is a mixed-methods narrative synthesis on access to palliative care and socioeconomic position in the UK. Databases AMED, Medline, Embase, CINAHL, SocIndex, and Academic Literature Search were searched along with grey literature sources for articles about access to palliative care and socioeconomic position.

Results: Searches retrieved 3760 studies; 29 were included. Most reported quantitative results; very few used qualitative methods. There was contradictory evidence about whether socioeconomically disadvantaged groups 
are less likely to access palliative care in the UK, possibly explained by differences in study settings and how researchers measured receipt of care or socioeconomic position. The way services are organised and information is acquired may interact in a way that exacerbates difficulties accessing care for some patients.

Conclusion: Sparse data on why socioeconomic position might influence access to palliative care limits any definitive conclusions about the usefulness of the 'candidacy' model. Available evidence suggests its applicability to palliative care warrants further exploration.

Abstract number: P01-564

Abstract type: Poster

“Lean In, Don't Step Back".... The Views, Experiences and Expectations of People with Severe Mental Illness on Palliative and End of Life Care

Jerwood J. ${ }^{1,2}$, Phimister D. ${ }^{3}$, Holliday N. ${ }^{1}$, Ward G. ${ }^{4}$, Coad J. ${ }^{1}$

${ }^{1}$ Centre for Innovative Research across the Lifecourse, Coventry University, Coventry, United Kingdom, ${ }^{2}$ Research and Innovation, Birmingham and Solihull Mental Health Foundation Trust, Birmingham, United Kingdom, ${ }^{3}$ School of Nursing, Midwifery and Health, Coventry University, Coventry, United Kingdom, ${ }^{4}$ Research, Royal College of Occupational Therapists, London, United Kingdom

Background: People with severe mental illness (SMI) have a life expectancy of up to twenty years less than the general population. They also experience poor physical health and higher rates of many life-limiting conditions. Research on the specific needs of people with SMI in palliative and end of life care is extremely limited and focuses on describing barriers to care from clinician's perspectives. The limited research that has been carried out originates outside the UK in the USA, Canada and New Zealand. The views of patients with both SMI and a life limiting and carers have not been included in previous research studies.

Aims: To carry out a series of interviews with patients (and their carers) who have both SMI and a terminal condition to better understand their experiences, views and preferences in palliative and end of life care, with a further aim to use these findings to improve the quality and accessibility of palliative and end of life care for this marginalised patient group. Methods: Semi-structured, in depth interviews were carried out with a small sample of patients and carers. A thematic analysis was then undertaken using the interview transcripts and key themes drawn from the data. Findings: Six themes were developed through the thematic analysis Treating the Whole Person, Stigma and Prejudice, Carers - 'ignored experts', Experiences of Palliative and End of Life Care, Impact of Late Referral and Improvements to Care.

Conclusions: This study offers novel understanding of the views, experiences and expectations of a group of patients often marginalised and excluded from palliative and end of life care - people with SMI and terminal conditions. The themes developed from the interview data offer valuable insights into how access to, and quality of, palliative and end of life care can be improved for people with SMI. The findings are of interest to clinicians, policy makers, patients and carers.

Abstract number: P01-565

Abstract type: Poster

Uncovering the Invisible: Conditions of Dying and End-of-Life Care for Persons with Intellectual and Multiple Disabilities in Germany

Schäper S., Schroer B., Thimm A.

Department of Social Services, Catholic University of Applied Sciences, Muenster, Germany

Background: In palliative care research in Germany, there is low scientific regard on persons with intellectual disabilities. This research gap results from the special demographic development in Germany: Due to the murders of persons with disabilities in the Nazi regime, disability services are now facing experience of end-of-life care increasingly. The research project explores the situation in three differently structured Federal States in Germany (Saxony, North Rhine-Westphalia, Berlin).

Methods: A preliminary study explored residential transitions in the end of life by a secondary analysis of data from local welfare authorities. The current study analyzed these data according to death rates. An onlinesurvey investigated numbers of deceased service users and conditions of end-of-life care in 800 residential services. Semi-structured interviews with staff members complemented the insight into organizational structures and strategies as well as professional concepts with a special focus on participation.

Results: The lack of capacity in disability services to cope with changing needs indicates residential transitions to nursing homes. At the same time, the death rate in disability services is increasing. The conditions of end-of-life care depend on the history of welfare politics in the different parts of Post-war Germany, the type of residential institutions, the actual strategic direction of social politics, the managerial decisions of the services and the professional capacity and attitudes.

Discussion: The study allows an overview on conditions of dying for persons with intellectual disabilities in Germany and shows conceptual working steps and needs of capacity building to ensure the enjoyment of the highest attainable standard of care for persons with disabilities, the state parties are responsible for according to the UN-CRPD (Art. 25). More research is outstanding on participation and self-determination in the end of life for this vulnerable group.

Abstract number: P01-566

Abstract type: Poster

Characteristics of the Patients of Hospice Balade: A Unique Hospice for People with Limited Access to Hospice Care Echteld M.A. ${ }^{1,2}$, Tielen L. ${ }^{2}$, van de Graaf G. ${ }^{2}$, Kolen M. ${ }^{2}$

${ }^{1}$ End of Life Care Research Group, Avans University of Applied Sciences, Breda, Netherlands, ${ }^{2}$ Prisma Foundation, Waalwijk, Netherlands

Background: People with intellectual disabilities (ID), psychiatric problems, dementia, and substance abuse problems have limited access to hospice care. Due to ageing, palliative care is gaining in importance in these groups. However, palliative care in these people is highly complex and requires special knowledge and skills. Balade, a special hospice for people with limited access to regular hospice care, was started in The Netherlands.

Aims: To show the characteristics and care needs of the patients admitted to the hospice.

Methods. A cross-sectional observational study was carried out. Data from all patients admitted to the hospice in 2017-2018 are shown. Oneway ANOVA was used to compare mean age and number of days in care among patient groups.

Results: Balade is a six-bed high care hospice with a specially trained multidisciplinary staff: a hospice ID physician, nurses, social workers, psychologists, and a spiritual counselor. 41 patients were admitted to the hospice, of which 32 died in the hospice. $27 \%$ were women and mean age at admission was 67 years. Most patients had ID (55\%), 16\% had psychiatric problems, $13 \%$ dementia, $11 \%$ were socially disadvantaged, and $5 \%$ had substance abuse problems. Two patients were admitted for day care only and one patient received respite care. 6 patients are still alive. Primary diagnosis was cancer in $68 \%$ of the patients, dementia in $18 \%$, organ failure in $9 \%$, and multiple diagnoses in $5 \%$. All emerging symptoms could be controlled. Mean admission duration was 40 days, but the range was substantial: 1-224 days. Patients with dementia and socially disadvantaged patients were significantly older than other patients and were admitted for less days (ns).

Conclusion: Symptoms at the end of life were controlled, but behavior and communication characteristics of the patients presented significant 
challenges. The hospice is a good example for palliative care in a population with special needs.

Ballentine J.

Northern Ireland Hospice, Adult Services, Belfast, United Kingdom Presenting author email address: joanneballentine@nihospice.org

Background: Currently over 1,400 deaths a year in N. Ireland, directly attributable to dementia, yet dementia is rarely considered within a palliative care context, with limited attention directed towards the person's or carers psychological, social, emotional \& end of life needs. To address this need, Northern Ireland Hospice developed an innovative service, based on collaborative working across voluntary and statutory organisations. This multi-component approach included: 1) a purpose- built dementia friendly facility; 2) an enhanced hospice/ dementia specialist team; 3) a day hospice service which provided opportunities for nurse specialist assessment, creative therapies directed towards cognitive stimulation and promoting well-being for the person, and support for their carers who also attend; 4) palliative focused education for practitioners working in a wide range of settings, underpinned by the certificate programme in holistic dementia care.

Aim: To profile the outcomes from the Hospice Enabled Dementia Partnership pilot in terms of service design, experiences of carers, health and social care professionals and to highlight recommendations for service development, education and practice.

Results: The project has now been evaluated by Queens University Belfast leaving us well placed to highlight, at conference, the value of different components within the service model in improving access to and management of specialist palliative care for people with dementia and their carers as well as palliative dementia education.

Conclusion: Palliative Dementia Care is an important area for service users and the results from the project have shown how other areas can embrace care for this group of patients and their carers while making recommendations for teams, commissioners and policy makers which are considering developing integrated approaches between palliative and dementia care. Areas for future research will also be highlighted.

Abstract number: P01-568

Abstract type: Poster

Is More Palliative Care Needed on Swiss ICUs? A Survey among Professionals

Affolter B. ${ }^{1}$, Schefold J. ${ }^{2}$, Müller E. ${ }^{3}$, Eychmüller S. ${ }^{1}$

${ }^{1}$ Palliative Care, University Hospital of Bern, Bern, Switzerland,

${ }^{2}$ Intensive Care, University Hospital Bern, Bern, Switzerland, ${ }^{3}$ Palliative

Care, University Hospital Freiburg, Freiburg, Switzerland

Background: Despite of a high mortality and morbidity of Intensive Care patients our Palliative Care (PC) consulting service in a University Hospital is rarely called to the Intensive Care Unit (ICU).

Aim: To assess the view of ICU doctors and nurses on availability, needs and use of palliative care service in ICU.

Methods: We performed a survey in $10 \mathrm{ICU}$ s from the German speaking part of Switzerland. The biggest unit had 60, the smallest 8 beds. The survey was sent either electronically or in paper format to 1041 professionals. Among others, the survey consisted of 9 questions regarding needs, cooperation and availability of PC services in their hospital. Analysis was descriptive.

Results: 442 (42.5\% response rate) professionals completed the survey. Of the respondents $82 \%$ were nurses, $18 \%$ were ICU specialists or residents working in ICU. $48 \%$ of the participants have access to a PC consulting service, $56 \%$ to a PC ward. Six percent of the participating ICU staff have no institutional PC and $18 \%$ did not know about the PC services in their hospital. The majority of the respondents $(64 \%)$ had the impression that PC specialists were too rarely involved, $10 \%$ just right, while only two persons thought too often. $25 \%$ refrained from any judgement. $51 \%$ rate cooperation with a PC service as being necessary, and $35 \%$ as being urgently needed, whereas $13 \%$ judge cooperation to be rather unnecessary and $1 \%$ as not needed at all. Nurses considered the need for a cooperation with PC as more urgent than ICU doctors.

Conclusion: In this survey ICU experts confirmed the impression, that PC experts were rarely involved on ICUs in German speaking Switzerland. Most professionals share the need for palliative care, nurses more so than doctors. The fact that almost $20 \%$ did not know about access to PC in their hospital and $6 \%$ had no PC team available suggests that specialised PC is still not commonly implemented in Swiss ICUs.

\section{Poster Exhibition}

(Poster Set 2)

Oncology and Haematology

Breathlessness

Fatigue / Weakness / Cachexia

Bereavement

Pain

Other Symptoms

Assessment \& Measurement Tools

Audit \& Quality Improvement

Development and Organisation of Services

Education

Ethics

Health Economics

International Developments

\section{Abstract number: P02-001}

Abstract type: Poster

Diagnostic Experiences and Diagnosis Coping in Patients with Pancreatic Cancer - An Empirical Study Focusing on the Qualitative Realities of Lives of People Affected

Ristau P.

Protestant University of Applied Sciences Darmstadt, Department of Nursing and Health Sciences, Darmstadt, Germany Presenting author email address: kontakt@patrick-ristau.de

Every year, up to 19,000 people in Germany are diagnosed with pancreatic tumours, more than $95 \%$ of them with adenocarcinoma - the most aggressive type with the highest cancer-specific mortality and a 5 -year survival rate of approx. 10\%. Delayed diagnosis is associated with a worse outcome.

This empirical study focuses on individual diagnostic experiences and coping strategies. Factors that influence successful coping are identified. Furthermore, it is investigated whether already known coping models can be applied to this disease.

After a call to participate in cooperation with a nationwide self-help group (SHG), theoretical sampling was based on sociodemographic and disease-specific parameters. Twelve participants were recruited and completed an approx. 60-minute problem-oriented, guided interview. Conversations were digitally recorded and then content-semantically transcribed. Ten of these data sets were finally evaluated based on the Grounded Theory Methodology.

Data saturation was achieved regarding the living environment and situation of people affected, but not regarding coping. The credibility and dependability quality criteria of qualitative research could be fulfilled; transferability and confirmability could be partially fulfilled.

Treatment processes vary, but overall they are sluggish and unsatisfactory. Supply disruptions often occur across sector boundaries. SHG therefore often play a major role. Social isolation of people affected was identified as a key phenomenon.

Coping is often difficult and probably impossible due to rapid disease progression. Currently published theories are not easily applicable. 
Affected people need personal attention and firm contact persons with sufficient time. The high demand for information needs to be addressed adequately. Contact with SHG should be structurally engaged. The Ethics Committee of the German Society of Nursing Science assessed ethical and legal admissibility of the research project.

Abstract number: P02-002

Abstract type: Poster

End-of-Life Care from the Perspectives of Patients with Cancer and Nurses

Terzi K., Kapucu S.

Internal Medicine Nursing, Hacettepe University, Ankara, Turkey

Aim: The aim of this study was to determine the views of patients with cancer and their nurses regarding end-of-life care and to contribute to the care needs of patients.

Method: This was a phenomenological study. In-depth interview technique was used. The study was conducted between 05.09.2017 and 22.01.2018 at Hacettepe University Hospital. Study sample consisted of 15 patients with cancer at the end-of-life and 15 nurses who care for them. Data were collected using a data collection form introducing socio-demographic characteristics and a semi-structured interview guide.

Results: A content analysis of the data was conducted using the induction method, and then, themes and sub-themes were identified. For patients with cancer at the end-of-life, 6 themes and 4 sub-themes were identified. These themes were "end of life, desperation, painless death, increased needs, feeling loved and quality care." 4 themes and 3 subthemes were identified for nurses. These themes were "increased care needs, providing quality death, multidisciplinary team approach and providing support." In general, patients and nurses defined end-of-life care as a time when painless death is near, physical and psychological needs increase and team collaboration is absolutely necessary.

Conclusion: It was also found that although nurses want to provide the necessary care, they are not able to do so due to excess workload. Thus, the result of this study will be shared with nurses and related institutions, and necessary training and cooperation will be planned to improve the quality of care provided to patients with cancer at the end-of-life by healthcare professionals.

Keywords: Cancer, death, end-of-life care, nurse, patient, patient needs

Abstract number: $\mathrm{P} 02-003$

Abstract type: Poster

Early Palliative Care for Patients with Metastatic Melanoma: Is it Really so Difficult to Achieve?

Prod'homme C. ${ }^{1,2}$, Touzet L. ${ }^{1}$, Le Berre R. ${ }^{2}$, Templier C. ${ }^{3}$, Cobbaut J.-P. ${ }^{2}$, Mortier L. ${ }^{3}$

${ }^{1}$ Soins Palliatifs, Centre Hospitalier Universitaire de Lille, Lille, France, ${ }^{2}$ Medical Ethics Center, ETHICS-EA 7446, Lille, France, ${ }^{3}$ Dermatologie, Centre Hospitalier Universitaire de Lille, Lille, France

Introduction: New targeted drugs and immune therapies reported since 2010 for metastatic or unresectable melanoma (MM) have shown improved survival in randomized trials. In the north of France, new targeted drugs and immune therapies are continued even when MM progresses, as dermatologists have the impression that they may slow progression.Palliative care by specialized palliative care teams for these patients has therefore become more complex.

Aim: To question the benefits of targeted drugs and immune therapies associated with specialized palliative care in patients with advancedstage $\mathrm{MM}$ and altered general state of health.

Methods: Three single-center trials were simultaneously conducted in the University Hospital of Lille:
- A qualitative study based on focus group of dermatologists and palliative care clinicians, nurses and psychologists, exploring professional practices concerning the patient

- A qualitative study based on individual interviews to investigate patient's perceptions of prolonging treatment when their MM progresses

- A quantitative cohort study exploring the survival and morbimortality of patients whose targeted drugs and immune therapies were continued, associated with specialized palliative care when MM progresses and general state of health is altered

Results: collaboration between palliative and dermatologic teams improves early palliative care among patients treated for MM. Results are preliminary but tend to show that the variability of the representations of treatment among physicians and patients sometimes leads to various hopes and misunderstandings that may hinder quality palliative care. Like chemotherapy, it seems that the question of discontinuing targeted drugs and immune therapies must be addressed by health professionals and patients when MM progresses. Interrupting treatment can sometimes trigger palliative care that is more suited to the wishes of the patient at the end-of-life.

Abstract number: P02-004

Abstract type: Poster

Acute Oncology Service $=$ Acute Palliative Service ? Early Palliative Care Assessment Results from a Pilot Project in South East Wales

Taubert M. ${ }^{1}$, Frazer R. ${ }^{2}$

${ }^{1}$ Velindre NHS Trust Palliative Medicine Department, Cardiff University School of Medicine, Cardiff, United Kingdom, ${ }^{2}$ Velindre University NHS Trust, Cardiff, United Kingdom

Background: The National Chemotherapy Advisory Group report 2009 recommends an acute oncology service (AOS) in every UK hospital with an emergency department. Patients discussed in Acute Oncology Service settings are often very unwell, at the start of their cancer journey, and may have multiple challenging symptoms.

Aims: Will earlier palliative care intervention in AOS meetings result in an increase in palliative care involvement? As a comparative baseline we used data from Royal Sussex County Hospital, where an acute admission AOS data collection was carried out: in 53 patients with cancer, palliative care were involved in four cases (7.5\%).

Methods: As part of this project, our palliative care team started to attend AOS meetings at Velindre Cancer Hospital on a daily basis. After initial embedding, a strategy to collect data was designed and an audit cycle was carried out.

Results: Through integration of the palliative care team into Acute Oncology Service meetings, key areas of advance care planning were addressed and discussions with patients were planned. Of 100 patients assessed during the AOS reporting period, $80 \%$ were not known to a palliative care team/provider. Of all patients analysed, $28 \%$ required no palliative input, $29 \%$ were signposted to another palliative care team, $27 \%$ received same day face to face palliative care review and $16 \%$ required verbal advice only to a generalist team member. Advance care planning discussions in the sample of patients who needed some palliative care input were held within a two week time frame in $61 \%$ of cases.

Discussion: Significant findings included large population (80\%) unknown to palliative care services at AOS entry point, but with high level of unmet need. $72 \%$ had palliative care needs. $29 \%$ had received their cancer diagnosis within the last month.

Conclusion: AOS meetings appear to be a valid entry point for referral to palliative services, despite many AOS patients being at the start of their cancer diagnosis. 
Abstract number: P02-005

Abstract type: Poster

Time-dependent Patient-reported Outcomes as Predictors of Patient Survival of Lung Cancer

Chang W.-P. ${ }^{1}$, Lin C.-C. ${ }^{2}$

${ }^{1}$ Nursing Department, Taipei Medical University-Shuang Ho Hospital, Ministry of Health and Welfare, New Taipei City, Taiwan, Republic of China, ${ }^{2}$ School of Nursing, The University of Hong Kong, Pokfulam, Hong Kong

Background: Patient-reported outcomes (PROs) can be used to accurately and directly gauge patients' disease-related symptoms.

Aims: The purpose of this study was to investigate the association between PROs and survival periods among patients with lung cancer considering time-varying covariates.

Methods: A total of 86 patients with confirmed diagnoses of lung cancer were recruited, and a prospective study design was adopted. PROs were collected five times for each patient: before treatment and $6,12,24$, and 48 weeks after treatment. The results were analyzed using time-dependent Cox regression.

Results: Average follow-up duration was 14.3 months; 75 participants died over the course of the follow-up period and 11 survived. The median survival period was 15.0 months (range: 0.6-49.7 months). Bivariate time-dependent Cox regression indicated that the basic attribute variables of significance were cancer diagnosis, stage of cancer, pretreatment pneumonia, and type of treatment. The significant PRO variables were sleep disorders, anxiety, depression, weight loss, chest tightness, and ease of breathing. Thus, we analyzed the predictive power of these 11 variables. Multivariate time-dependent Cox regression revealed that only stage four lung cancer (hazard ratio $[\mathrm{HR}]=7.33, p=$ .029 ) and weight loss (HR $=0.76, p=.006)$ were significant.

Conclusion: Sleep disorders, anxiety, depression, chest tightness, and ease of breathing in PROs were associated with the survival periods of lung cancer patients; however, controlling for time, stages, and other PROs revealed that weight loss is the only independent factor associated with lung cancer patient survival.

Abstract number: P02-006

Abstract type: Poster

Palliative Care Referral, Hematologists' Perceptions: A

Multicentric Qualitative Study

Tricou C. ${ }^{1,2}$, Munier S. ${ }^{2}$, Phan-Hoang N. ${ }^{2}$, Albarracin D. ${ }^{3}$, Perceau-

Chambard E. ${ }^{2}$, Filbet M. ${ }^{1,2}$

${ }^{1}$ Claude Bernard Lyon 1 University, Villeurbanne, France, ${ }^{2}$ Palliative Care, Lyon Sud University Hospital, Pierre-Benite, France, 3Psychologie, Universite de Poitiers, Poitiers, France

Objectives: Early palliative care leads to meaningful improvements in physical and psychosocial symptoms, as well as quality of life, in patients with advanced cancer. Patients with hematological malignancies, despite a high level of distress, continue to have less access to palliative care services. The aim of this study was to identify hematologists' perceptions of palliative care, as well as barriers to patient referral.

Methods: We used a qualitative grounded theory methodology. Twentyfour medical hematologists involved in clinical practice from 2 French centers in Lyon: the Lyon Sud University Hospital and the Léon Bérard Cancer Center were included. The interview guide questions aimed to establish the clinical situations which triggered referral to palliative care, and how participants perceived palliative care.

Results: Data saturation was reached after 14 interviews. The data analysis highlighted 4 themes. The aim of palliative care was clearly identified as alleviating severe suffering. Palliative care was identified as a separate specialty, and respondents expressed the need for collaboration. Early intervention was perceived as beneficial to avoid certain situations such as hospitalization or emergency department visits at the end-of-life. The main barrier to palliative care referral remained the negative connotations associated with the term "palliative", which was overwhelmingly associated with the end of life.

Conclusion: Our results suggest that the principal barrier for palliative care referral is the term "palliative care"; hematologists would prefer "supportive care" instead.

Abstract number: $\mathrm{P} 02-007$

Abstract type: Poster

\section{A Modern Way of Living Well while Dying}

Arantzamendi M.1,2, García-Rueda N. ${ }^{1,3}$, Carvajal A. ${ }^{2,3}$, Robinson C.A. ${ }^{1,4}$

${ }^{1}$ ATLANTES, Universidad de Navarra. Instituto Cultura y Sociedad,

Pamplona, Spain, ${ }^{2}$ IdiSNA, Instituto de Investigación Sanitaria de Navarra, Pamplona, Spain, ${ }^{3}$ Departamento de Enfermería de la Persona Adulta, Universidad de Navarra. Facultad de Enfermeria, Pamplona, Spain, ${ }^{4}$ Faculty of Health and Social Development, British Columbia, Kelowna, Canada

There is a growing body of research focused on select aspects of people's experiences with advanced cancer but a dearth of research attending to the process of living with advanced cancer across the trajectory towards death.

Aims: To explain the process of living with advanced cancer and to determine the applicability of the Theory of Living Well with Chronic Illness (Robinson, 2017).

Method: Qualitative. The study is a secondary analysis of 22 transcripts of interviews with purposefully selected advanced cancer patients using the analytic method of constant comparison.

Result: The refined theory explains that living with advanced cancer is an iterative process comprised of 5 interconnected phases (Struggling, Accepting, Living with advanced cancer, Sharing the illness experience, and Reconstructing life) that revolve around a core concept of Awareness of Dying, which was not present in the original theory. This awareness varies from awareness of the possibility of dying, to accepting the possibility of dying, to acceptance that "I am dying." How participants positioned their Awareness of Dying influenced the 5 phases of living with advanced cancer and was a process unto itself. Through Accepting, in some measure, participants moved forward in their lives as they integrated advanced cancer into daily living, shared the experience, and, at times, were able to live life rather than living an illness. The participants did a remarkable job of living well with an uncertain future colored by Awareness of Dying.

Conclusion: This study offers an integrative explanatory theory of living well with dying and refines the original theory. The refined theory enables us to understand the findings of previous research in a new and integrated context; challenging traditional awareness of dying. Living with advanced cancer involves an iterative process shaped by the awareness of dying that requires deep engagement, time and effort and shows that it is indeed possible to live well.

Abstract number: P02-009

Abstract type: Poster

Antineoplastic Therapy (AT), Chemotherapy and / or Targeted Therapy, in Patients with Advanced Cancer Attended in a Palliative Care Hospital Program (PCP)

Dorantes Romandía R..$^{1,2}$, Guell Perez E. ${ }^{1,2}$, Fariñas Balaguer O..$^{1,2}$, Pascual Lopez A. ${ }^{1,2}$

${ }^{1}$ Palliative Care Unit, Hospital Sant Pau, Barcelona, Spain, ${ }^{2}$ Autonomous University Barcelona, Barcelona, Spain

Background: Concerning patients with advanced cancer, it's recommended to combine the administration of AT with the early intervention of palliative care teams. 
Aims: Analyze the frequency of patients receiving AT since the inclusion in our PCP, describe the types of AT and the characteristics and survival of patients.

Methods: The retrospective descriptive study included patients with advanced cancer who died in the first semester of 2017 after their inclusion in our PCP. Identification of patients who received AT in the month before to their inclusion in PCP and those who followed AT after inclusion. Analysis of clinical, sociodemographic variables, information received about treatment and survival. The data of patients who received AT after being included in PCP are described.

Results: Between January and July 2017, 310 patients with advanced cancer attended by our palliative care team died. 33\%(102)had received AT during the month prior to inclusion in PCP. According to the evaluation of the palliative team, only $54 \%$ of the patients with AT knew the palliative intention of the treatment. $10 \%$ of patients $(31 / 310)$ received AT during their PCP care; 25 patients continued to receive AT after inclusion in PCP and in 6 was started it after inclusion. The median age was 64.2 years, corresponding $52 \%$ to males. The predominant tumor locations were: lung $(16 \%)$, urothelial $(16 \%)$ and bone-muscle $(13 \%) .71 \%$ were treated with chemotherapy, $16 \%$ with targeted therapies and $13 \%$ with the combination of both. $45 \%$ of the patients were receiving the first line of treatment, $26 \%$ the second line and the remaining ones (29\%), 3 or more lines. The mean survival of the patients treated in PCP was 178.6 days, median 134 days. $13 \%$ of patients received AT in the last 15 days of life.

Conclusions: The number of patients in PCP who receive AT is small. The data suggest that a significant number of these patients have a survival expectancy greater than six months and can benefit from shared care.

\section{Abstract number: P02-010 \\ Abstract type: Poster}

When Disrupted Brain Meets Exhausted Team: Study on Patients with Brain Tumors and the Team's Challenge for Exhaustion and Ethical Issues

Leners J.-C.

Physician in Hospice, Ettelbruck, Luxembourg

Background: For more than 7 years the 15 beds hospice is also taking care of patients with primary brain tumors. The number of these rather young aged group is slowly increasing year by year and we are confronted to a very huge variety of neuro-psychiatric signs. In daily team meetings the higher burden for care is often discussed.

Aims: The behaviour is very challenging with verbal or physical outbreaks, wandering, escapes or apathy, and the team is confronted with legal aspects like guardianship or ethical aspects in end of life decisions. For these reasons a retrospective study on all our admissions with this primary diagnosis was undertaken.

Methods: The medical files are all scanned documents including the socio-demographic data as well as ICD10 codes and all medical prescriptions. These documents were all analyzed in order to find mean dosage of relevant drugs, but also the extra-time spend in personal care.

Results: A total of 85 patients was admitted over the years. Mean age 63 , with a higher proportion of men (62\%). The mean dosage of dexamethasone as well as for the main psycho-tropic drug was higher; for morphine in end stage of the disease it was lower, compared to the other patients.

Conclusion / Discussion: In relation to other patients admitted in our hospice, we found a $18 \%$ higher proportion of sedative drugs used in our setting, which was not surprising, and compared to the time spend in individual relation : patient/nurse with these patients, we considered an extra-time dedicated to them of possible one hour/day/patient. This has to be integrated in future care plans in order to protect the team from a higher sick leave.
Abstract number: P02-011

Abstract type: Poster

Developing a Collaborative, Multi-disciplinary Approach to the Supportive Care of Patients Undergoing External Beam Radiotherapy to the Perineum

Burtonwood J. ${ }^{1}$, Snook A. ${ }^{2}$, McCoubrie R. ${ }^{1}$

${ }_{1}^{1}$ Palliative Medicine, University Hospitals Bristol NHS Foundation Trust, Bristol, United Kingdom, ${ }^{2}$ Radiotherapy, University Hospitals Bristol NHS Foundation Trust, Bristol, United Kingdom

Background: External beam radiotherapy (EBRT) to the perineum is a common treatment for vulval and anal cancers. It is associated with a significant burden of early treatment-associated symptoms including skin toxicity, diarrhoea and pain. These symptoms often escalate throughout treatment and peak weeks after cessation of therapy. Symptoms can reduce quality of life and may result in premature cessation of therapy. Where supportive care (SC) of treatment-related side effects is delayed, disjointed or reactive, symptom control efforts may lag behind the escalating symptoms. Thus a proactive, multi-disciplinary team (MDT) approach at an early stage of treatment is required. Key to this approach is improvement in anticipation of symptoms and knowledge of SC amongst the MDT.

Aims: To improve the knowledge and confidence of the MDT in the early provision of SC to patients undergoing EBRT to the perineum.

Methods: Educational sessions for MDT members were used to assess unmet educational needs. These were addressed through a quality improvement project (QIP). A guideline-based approach to deliver early $\mathrm{SC}$ was subsequently developed. The effectiveness was assessed through feedback from the MDT.

Results: The QIP process, MDT feedback and SC-guideline are presented. Input from oncology, palliative care, radiotherapy, occupational therapy and gynaecology staff was sought. Thus, improvements to early recognition, prompt treatment, appropriate escalation and enhanced follow-up were made. Local guidelines for use of topical morphine for refractory symptoms were also developed in response to MDT feedback.

Conclusions: SC of patients undergoing EBRT to the perineum is complex and requires effective, early MDT intervention. This QIP improved the knowledge, early involvement and collaborative working of the MDT in the management of this patient group.

Abstract number: P02-012

Abstract type: Poster

An Assessment of the Integration of Palliative Care in the Caring of Cancer Patients in Selected Oncology Clinics in the Ethekwini District in Kwazulu-natal

Sithole Z.

Hospice Palliative Care Association of South Africa, Advocacy, Pinelands, South Africa

Background: There is a concern at present about the integration of oncology and palliative care services in South Africa and whether or not cancer patients are able to access quality palliative care.

Aim of the study: The aim of this study was to assess the integration of palliative care in the caring of cancer patients in the selected oncology clinics.

Methodology: A qualitative, explorative, descriptive and contextual research design was used to guide this study. The study was participative in nature and employed a focus group methodology. The participants in this study were professional nurses working at selected sites in public urban oncology clinics for more than three months. Two focus groups were conducted within one month of each other with participation from 16 oncology nurses. 
Results: Findings indicated that most participants understood palliative care as end of life care when a patient is beyond curative treatment. Nurses saw their role in the referral process as a passive one this was seen as a medical decision. Nurses acknowledged poor communication between the oncology clinics and hospices. Only one oncology nurse who participated in the study trained in palliative care. All participants showed an interest in becoming more knowledgeable in this area and improving relationships between oncology clinics and hospice

Conclusion: While it's clear that the development of palliative care has come a long way in South Africa, the results of this study show how far it still has to go in terms of achieving full integration with mainstream oncology clinics. The key finding from both sites was that palliative care was defined as end-of-life care only. This definitional confusion among oncology nurses continues to be a major barrier leading to poor access and late referral to palliative care services.

Abstract number: P02-013

Abstract type: Poster

Improvement of Therapeutic Strategies Using an "Aid to

Decision-making Form"(ADF) in Cancer Patients

Viant L., Legoupil C., Laurent C., Ducreux M., Laurent S., Benhamou E., Dauchy S., Blot F.

Palliative Care, GustaveRoussy, Villejuif, France

Background: Anticipating therapeutic strategies for acute worsening in cancer patients is mandatory. Therefore, an "Aid to Decision-making Form" (ADF) has been implemented in a large comprehensive cancer center (1).

Aims: Reported in the ADF, the value of care stratification has been assessed to establish if it can be predictive in relation to level of realized care and patient's outcome.

Methods: This prospective epidemiological study included inpatients transferred from a selected oncologic unit to another between January and May in 2017. All characteristics of medical care, including advance directives and information on shared decisions, were recorded up to 6 months after inclusion. The study was approved by the Internal Ethics Committee.

Results: Out of 206 patients included, 184 (89\%) had an ADF filled, of which 118 (64\%) had a stratification of Intensive Care (IC) or To Discuss ICU Admission, 61 (33\%) a Non-Invasive Medical-Exclusive Comfort Care and $5(3 \%)$ no information. The ADF also recorded the involvement of the palliative care team, the sharing of the decision with patient and/or relatives and the presence of advance directives (filled out in respectively 29,14 and $7 \%$ of the ADF).

Among the 206 patients, 35 had an acute medical event during their hospitalization and 29 (83\%) had an ADF filled with care stratification. Among them, 4 were followed, 18 were downgraded (of which 7 with IC decision didn't have treatment in ICU, nevertheless they survived). For 7 of them, information was missing.

The 6-month mortality rates were respectively $24 \%, 61 \%$ and $76 \%$ for patients stratified as IC, To Discuss ICU Admission and Non-Invasive Medical - Exclusive Comfort Care.

Discussion / conclusion: Care stratification in ADF could be appropriate for subsequent therapeutic behaviors. Its use would help to limit aggressive attitudes. In order to reach such an aim, ADF forms fulfilling have to be improved.

(1) EAPC 2017, abstract P01-004.

Abstract number: P02-014

Abstract type: Poster

Are Advanced Palliative Care for All Cancers Equally Complex? Assessment in a Hospital-at-Home Unit

Gregori Guillem L. ${ }^{1}$, Gallart Gómez P. ${ }^{1}$, Cortés Coll P. ${ }^{1}$, Llisterri Sánchez P. ${ }^{1}$, Almansa Gimeno M. ${ }^{1}$, Mancheño Álvaro A. ${ }^{2}$, Pascual-Pla F.J. ${ }^{2}$,

Sáez-Álvarez E. ${ }^{1}$, Ferrer Piquer R. ${ }^{1}$, Roa Mompó J. ${ }^{1}$, Martínez Granero
A. ${ }^{1}$, Martin-Utrilla S. ${ }^{1,2}$, Grupo de Investigación en Cuidados Paliativos (GRICPAL-UCV)

${ }^{1}$ Universidad Católica de Valencia, Valencia, Spain, ${ }^{2}$ Fundación Instituto Valenciano de Oncología (IVO), Valencia, Spain

Background: Advanced palliative care at home allows comprehensive care of the patient with cancer. The assessment of the levels of complexity of care can be used to estimate the socio-sanitary resources required. Aim: Assess the complexity of palliative care at home in different types of cancer.

Method: A descriptive, prospective study was carried out in a hospitalat-home unit of a cancer center. Patients diagnosed with advanced stage solid tumors requiring advanced palliative care at home were included. The IDC-Pal tool was used to assess the complexity of the palliative care needs of each patient.

Results: A total of 140 patients, 70 men and 70 women, were included in the study, over two different periods of three months each. The mean age was 69.12 years $(S D=12.92)$. Breast cancer (35), urological (33) and digestive (31) were the most frequent types of cancer. 111 patients $(79,3$ $\%)$ presented at least one criterion of high complexity. The median complexity criteria was 6.

- $\quad$ Sudden change in performance status and determining role of the patient in his/her socio-family environment were the most frequent criterion in breast and digestive cancer, but symptoms difficult to control had a higher incidence in urological cancer.

- Existence of comorbidity of difficult control and severe constitutional síndrome were more frequent in breast cancer.

- $\quad$ Family or caregivers not competent for care and complex interventions like radiotherapy stood out in urological cancer. Spiritual suffering and maladaptive emotional coping are more frequent in breast and digestive cancer.

Conclusion: Although most of the most frequent complexity criteria in cancer are similar, in our study differences between the different types of tumors are appreciated. More specific studies are needed to know what are the needs of advanced palliative care for each tumor, but these results can help stakeholders and managers to decide where to use the social and health resources that should be promoted.

Abstract number: P02-015

Abstract type: Poster

CRP and Weight Loss Are Independent Prognostic Variables in Patients with Newly Diagnosed Advanced Colorectal Cancer - A Retrospective Analysis

Sallaberger E. ${ }^{1}$, Simanek R. ${ }^{2}$, Obenaus L. ${ }^{2}$, Sliwa $T^{2}{ }^{2}$, Kaufmann $H^{3}{ }^{3}$, Keil $F^{2}$

${ }^{1} 1$ st Medical Department, Medical University of Vienna, Vienna, Austria, 23 rd Medical Department, Hanusch Krankenhaus, Vienna, Austria, 33rd Medical Department, SMZ Süd Kaiser-Franz-Josef-Spital, Vienna, Austria

Background: Weight loss (WL) in cancer patients is multifactorial and associated with a poor prognosis. WL is the defining factor for cachexia, often accompanied by systemic inflammation and represented by increased C-reactive-protein (iCRP) levels. Data on body-mass-index (BMI) in patients with colorectal cancer (CRC) indicate a correlation between higher $\mathrm{BMI}$ and better outcome.

Aims: Aim was to investigate, whether patients with newly diagnosed CRC lose weight. Additionally, we wanted to find out whether clinical and/or laboratory risk factors (RF) for WL exist and if RF for WL also impact overall survival (OS).

Methods: Retrospective data analysis. Weight was documented in the chemotherapy-subscription-program. A WL of $\geqslant 5 \%$ compared to the 
first entry was considered as clinically significant WL. Multivariate Cox regression analysis was performed to identify statistically significant RF for WL and OS.

Results: 232 patients with advanced CRC (median age 71.0 years [interquartile range (IQR) $61.3-77.0$ years]), $57.8 \%$ male, $42.2 \%$ female, treated between Nov08 and Sep16 at 2 different departments were analyzed. Within 12 months, the cumulative probability (CP) of $\mathrm{WL}>5 \%$ was $41.6 \%$ (95\% Cl 34.1-49.1), within 48 months $62.3 \%$ (95\% Cl 51.5-73.1). RF for WL were iCRP (hazard ratio (HR] 3.45, 95\% confidence interval [CI] 2.24-5.29, $\mathrm{p}=0.000$ ), but not age, sex, BMl, right-sided-tumor, use of cetuximab. Median survival was 24.2 months (IQR 9.8-37.1 months), 101 patients (43.5\%) died, 53 (22.8\%) within the first year. RF for OS were iCRP (HR $1.69,95 \% \mathrm{Cl} 1.07-2.66, \mathrm{p}=0.025)$ and use of cetuximab (HR $1.72,95 \% \mathrm{Cl}$ 1.07-2.79, $\mathrm{p}=0.026$ ), but not $\mathrm{WL}$, age, sex, BMI and right-sided-tumor. Conclusion/ discussion: WL is common in advanced CRC patients. WL had no impact on survival in our patient population. iCRP, probably descriptive for systemic inflammation, is a RF for WL. iCRP and use of cetuximab are RF for reduced OS. WL was not considered by the chemotherapy prescribing clinician.

Abstract number: P02-016

Abstract type: Poster

\section{Quality of Life in Men with Metastatic Prostate Cancer in their Final Years before Death \\ Holm M. ${ }^{1}$, Doveson S. ${ }^{1,2}$, Wennman Larsen A. ${ }^{1,2}$, Fransson P. ${ }^{3}$ ${ }^{1}$ Department of Nursing Sciences, Sophiahemmet University, Stockholm, Sweden, ${ }^{2}$ Karolinska Institutet, Stockholm, Sweden, ${ }^{3}$ Department of Nursing, Umeå University, Umeå, Sweden}

Background: Metastatic prostate cancer (mPC) is a life-threatening illness with potentially severe symptoms and reduced functioning, and hence, a palliative care approach may be beneficial to this group. Over time, new life-prolonging treatments have been developed for men with mPC. However, in this late phase of the disease, it is also important to consider the balance between quality of life (QoL) and life prolongation, particularly because there may be side effects involved with life-prolonging treatments.

Aim: The aim of this study was to evaluate QoL, functioning and symptoms in men with $\mathrm{MPC}$ during their final years before death.

Methods: In total, 190 deceased men with metastatic prostate cancer were included from a larger, long-term prospective study. Validated questionnaires asking about participants' QoL, functioning and symptoms were retrospectively analysed after the patient's death. The men were stratified into three groups, based on how much time that had passed between completion of the last questionnaire and their death; $<$ 6 months, $6-18$ months and $>18$ months before death. The men's results were compared to established clinically significant threshold values. Further, ratings were compared between men who died before and after 2006.

Results: Men with mPC reported low QoL before death compared to threshold values as well as low functioning and high levels of symptoms like pain and fatigue. The group of men that were $<6$ months before death rated their QoL, functioning and symptoms significantly worse than the two other groups. Men who died after the year 2006 reported lower QoL and functioning and more pain and fatigue than those who died before 2006.

Conclusion: Men with MPC have unmet needs with regards to QoL and symptoms. A palliative care approach, alongside possible life-prolonging treatments, that focuses on QoL and symptom relief, may serve as an important frame to give the best support to these men in their final years of life.

Abstract number: P02-017

Abstract type: Poster
Assessing the Utilization of Palliative Care Decision for Cancer Patients during the Last Year of Life at a Finnish University Hospital: A Retrospective Cohort Study

Hirvonen O. $^{1,2}$, Leskelä R.-L. ${ }^{3}$, Grönholm L. ${ }^{4}$, Haltia O. ${ }^{5}$, TyyneläKorhonen K. ${ }^{6}$, Rahko E. ${ }^{7}$, Lehto J.T. ${ }^{8,9}$, Saarto T. ${ }^{4,10}$

${ }^{1}$ Oncology and Radiotherapy, Turku University Hospital, Turku, Finland, ${ }^{2}$ Clinical Oncology, University of Turku, Turku, Finland, ${ }^{3}$ Nordic Healthcare Group, Helsinki, Finland, ${ }^{4}$ Palliative Care, Helsinki University Hospital, Helsinki, Finland, ${ }^{5}$ Tuusula Health Care Centre, Tuusula, Finland, ${ }^{6} \mathrm{Clinical}$ Oncology, Kuopio University Hospital, Kuopio, Finland, ${ }^{7} \mathrm{Clinical}$ Oncology, Oulu University Hospital, Oulu, Finland, ${ }^{8} \mathrm{Clinical}$ Oncology, Palliative Care Unit, Tampere University Hospital, Tampere, Finland, ${ }^{9}$ Palliative Care, University of Tampere, Tampere, Finland, ${ }^{10}$ Palliative Care, Comprehensive Cancer Center, University of Helsinki, Helsinki, Finland

Background: To avoid aggressive treatments at end-of-life (EOL) and to provide good palliative care (PC), physicians need to terminate life prolonging anti-cancer treatments and define the palliative goal of treatment in time. The decision of PC and a referral to PC outpatient unit enable advance care planning and help in avoiding hospital-bound treatments at EOL.

Aims: This single center study assesses the utilization and timing of PC decision in the last year of life, and its' effect on anti-cancer treatments near death.

Methods: Patients with cancer diagnosis (ICD-10 C00-C96) treated in the tertiary central hospital and deceased during $1^{\text {st }}$ an $2013-31^{\text {st }} D e c$ 2014 were identified in the hospital database $(N=2737)$. Of these patients, 992 were randomly selected to this study. The decision and onset of palliative goal of care (termination of life-prolonging cancerspecific treatments and focusing on PC) was searched in the patient records.

Results: Palliative care decision was defined in $82 \%$ of the patients in the last year of life: $40 \%$ during the last month and $23 \%$ during the last two weeks, respectively. The median time of PC period was 46 days. In patients with head \& neck, CNS and gynaecological cancer the lack of PC decision was more frequent. The percentages of patients with PC decision and duration of PC period correlated positively with increasing age. If the PC decision was made less than one month prior to death, only 22 $\%$ visited palliative unit, while $40 \%$ received radiotherapy and $59 \%$ systemic cancer treatment during the last month of life. If the decision was made earlier, the corresponding figures were $62 \%, 20 \%$ and $1 \%$, respectively.

Conclusion: Decision for PC was frequently made for cancer patients but this occurred late in approximately every second patient. Thus, anticancer treatments were continued close to the death, suggesting no benefit on survival nor relieving symptoms. These observations indicate that the early integration of PC is needed.

\section{Abstract number: P02-020}

Abstract type: Poster

Profile of Patients Undergoing Palliative Radiotherapy: A Singleinstitute Study from a Tertiary Care Oncology Center Mariam L.

National Institute of Cancer Research and Hospital, Radiation Oncology, Dhaka, Bangladesh

Background: Palliative radiotherapy (PRT) has significant role for palliation of symptoms in patients with advance and metastatic cancer and constitutes nearly $60 \%$ of the workload in developing country like Bangladesh.

Aims: The aim is to study patient, disease, and treatment related characteristics in loco-regionally advanced and metastatic malignancies treated with palliative Radiotherapy. 
Setting and design: This was a retrospective observational study in a tertiary care government institute with academic and research potential.

Methodology: The hard copy of medical documents, and radiotherapy (RT) treatment charts were retrieved and studied.

Observations: A total of 100 patients were included in the study over 6 months who received PRT to control of symptoms in advance primary site and metastatic site. Out of 100 patients 52 patients received 20 Gray (Gy) in 5 fractions, 38 patients received 30 Gy in 10 fractions PRT to the metastatic site and 10 patients received hypo-fractionated $30 \mathrm{~Gy}$ in 10 fractions PPR to Primary site for symptomatic relief.The most common indication for PRT was to relief pain from painful bone metastases cases, and the next common indication was palliative whole-brain RT for brain metastases and third one to stop bleeding.

Conclusion: PRT forms an integral and important aspect of palliative care to the major number of patients with metastatic disease those need treatment for symptomatic relief. Short course of PRT in outdoor setting with minimum expenditure is a preferred mode of treatment in developing country to control pain and other symptoms and improve the quality of life of these distressed patients.

Keywords: Cancer, metastatic disease, palliative care, palliative radiotherapy, symptomatic relief

Abstract number: P02-021

Abstract type: Poster

Dying in Intensive Care Unit with Cancer: Patients with Hematologic Malignancy Are at Higher Risk

Bordier V. ${ }^{1}$, Fawoubo A. ${ }^{1}$, Economos G. ${ }^{1}$, Bernard L. ${ }^{2}$, Munier S. ${ }^{1}$ Perceau E. ${ }^{1}$, Tricou C. ${ }^{1}$, Filbet M. ${ }^{1}$

${ }^{1}$ Palliative Medecine, Centre Hospitalier de Lyon-Sud, Hospices Civils de Lyon, Pierre Benite, France, ${ }^{2}$ Statistique, Centre Hospitalier de LyonSud, Hospices Civils de Lyon, Pierre Benite, France

Background: In our tertiary hospital between 2010 and 2015, the number of cancer patients' dying in ICU increased from $42(8 \%)$ to 88 (17\%) out of 500 of the total cancer deaths, mainly due to the number of patients with hematologic malignancy.

Objective: To compare the cancer patient's population who died in ICU with the patients with solid tumours and those with hematologic malignancy.

Design: Retrospective study.

Methodology: Medical records of all cancer patients who died in ICU in 2015 were reviewed and sociodemographics and clinical characteristics, palliative care (PC) referral, date of last chemotherapy, number and length of hospitalisations including emergencies and ICU one's were collected.

Results: The patients were mostly male $(62,6 \%) 64,56$ years old (SD $12,54)$, and $53(60 \%)$ had a hematologic malignancy, mostly acute myeloid leukemia, and $16(18 \%)$ had undergone a bone marrow transplantation. The ICU transfer is due to respiratory failure, or septic shock. Patients were at an advanced stage of cancer, and those with a hematologic malignancy $(28 \%)$ had at least one relapse. Cancer patients who died in ICU in 2015 had better access to PC than those in $2010 \quad(p=0.01)$, but the first contact with PC occurred in ICU for $60 \%$ of patients. None had advance directive. Patients with hematological malignancy had less access to $P C(p=0.03)$, spent longer time hospitalized in their last year of life (81days/32 days, $\mathrm{p}<0.001)$ and had a higher risk of dying in ICU $(\mathrm{p}<$ $0.0001)$ than those with solid tumours.

Conclusions: Patients with hematologic malignancy were more likely to receive aggressive end-of-life care than patients with solid tumours. If during a bone marrow transplantation a transfer to ICU is not futile, in this study most of patients were at an advanced stage of leukemia. More collaboration is needed to prevent aggressive treatment during the end of life.
Abstract number: P02-022

Abstract type: Poster

Prevalence and Clinical Relevance of Malnutrition among Older Adults with Cancer

Harneshaug M. ${ }^{1,2}$, Bye A. ${ }^{3,4}$, Kirkhus L. ${ }^{1,2}$, Jordh $\varnothing y$ M. ${ }^{1,2,5}$

${ }^{1}$ Centre for Old Age Psychiatry Research, Innlandet Hospital Trust, Ottestad, Norway, ${ }^{2}$ Institute of Clinical Medicine, Faculty of Medicine, University of Oslo, Oslo, Norway, ${ }^{3}$ Department of Nursing and Health Promotion, Faculty of Health Sciences, OsloMet, Oslo Metropolitan University, Oslo, Norway, ${ }^{4}$ European Palliative Care Research Centre (PRC), Department of Oncology, Oslo University Hospital and Institute of Clinical Medicine, University of Oslo, Oslo, Norway, ${ }^{5}$ Cancer Unit, Innlandet Hospital Trust, Hamar Hospital, Hamar, Norway

Aim: Older cancer patients are at particular risk of malnutrition, a common, often overlooked condition associated with worse outcomes, impaired quality of life (QoL) and increased mortality. We sought to report the prevalence of malnutrition among older patients with a new or newly relapsed cancer diagnosis, and explore the clinical relevance.

Methods: Prospective, observational multicenter study with patients $\geqslant 70$ years referred for systemic cancer treatment. Patient Generated Subjective Global Assessment Short Form (SGA-SF) was used for malnutrition screening, identifying those at risk (RM) or not at risk (NRM). RM was further divided into a group of malnourished patients (MN) using GLIM criteria. Those not MN were well-nourished (WN). QoL was measured by EORTC QLQ-C3O. Groups were compared using T-test, $\mathrm{X}^{2}$ and Mann-Whitney U test.

Results: The SGA-SF placed 178 (66\%) of 269 included patients in the RM group. Comparing the RM and NRM groups, mean age was 76.9 and 76.7 years $(p=.7), 53 \%$ and $61 \%$ were males $(p=.2)$, and $36 \%$ vs $29 \%$ received curative treatment. In the RM group, 91 (34\% of total population) were $\mathrm{MN}$. Age and gender did not differ significantly between the MN and WN groups. More patients in the MN group received palliative treatment ( $80 \%$ vs $63 \%$ ) and had more often lung or $\mathrm{Gl}$ cancer rather than colorectal cancer. The MN group had a significant higher $\mathrm{C}$-reactive protein $(35$ vs $9, p<.05$ ) and lower albumin levels ( 38 vs $42, p<.05$ ), clinically and statistically significant poorer physical function (pf) (mean 61 vs 78,p< .05 ) and overall QoL (mean 50 vs 71, $p<.05$ ), more fatigue, pain and dyspnea $(p<.05)$. Median survival was 358 days and 885 days for the MN and the WN group, respectively.

Conclusion: The majority of older cancer patients were at risk of malnutrition. About one third was malnourished. These patients had poorer pf and QoL and a higher symptom burden and should be thoroughly considered for supportive measures. Funded by Innlandet Hospital trust.

Abstract number: P02-023

Abstract type: Poster

Depression and Physical Symptoms Severely Affect Older Patients' Physical Function during Cancer Treatment Kirkhus L. ${ }^{1,2}$, Harneshaug M. ${ }^{1,2}$, Saltyte Benth J. ${ }^{1,2,3}$, Rostoft S. ${ }^{2,4}$, Grønberg B.H. ${ }^{5,6}$, Bergh S. ${ }^{1,7}$, Jensen Hjermstad M. ${ }^{2,8}$, Selbæk G. ${ }^{1,7,9}$, Bruun Wyller T.2,4, Jordhøy M. ${ }^{1,2,10}$

${ }^{1}$ Centre for Old Age Psychiatry Research, Innlandet Hospital Trust, Ottestad, Norway, ${ }^{2}$ Institute of Clinical Medicine, Faculty of Medicine, University of Oslo, Oslo, Norway, ${ }^{3} \mathrm{H} \varnothing \mathrm{KH}$ Research Centre, Akershus University Hospital, Lørenskog, Norway, ${ }^{4}$ Department of Geriatric Medicine, Oslo University Hospital, Oslo, Norway, ${ }^{5}$ Cancer Clinic, St. Olavs Hospital, Trondheim University Hospital, Trondheim, Norway, ${ }^{6}$ Department of Cancer Research and Molecular Medicine, NTNU, Norwegian University of Science and Technology, Trondheim, Norway, ${ }^{7}$ Norwegian National Advisory Unit on Ageing and Health, Vestfold Hospital Trust, Tønsberg, Norway, ${ }^{8}$ European Palliative Care Research Centre (PRC), Department of Oncology, Oslo University Hospital, Oslo, 
Norway, 9 Institute of Health and Society, Faculty of Medicine, University of Oslo, Oslo, Norway, ${ }^{10}$ Cancer Unit, Innlandet Hospital Trust, Hamar Hospital, Hamar, Norway

Aim: Independency and quality of life (QoL) are highly prioritized outcomes in older adults, and closely interconnected with physical function (PF). The aim of this study was to identify remediable factors affecting the PF trajectories of older cancer patients during treatment.

Methods: Prospective, multicenter observational study including 306 cancer patients $>70$ years referred for systemic treatment (new cancer diagnosis or first relapse after curative treatment). Patients were assessed by a modified geriatric assessment (mGA) comprising comorbidity, regular medications, physical performance, cognition, nutritional status, and depression. PF and physical symptoms were assessed at baseline, 2, 4 and 6 months by the EORTC Quality of Life Questionnaire C-30. The PF trajectory was assessed by estimating linear mixed model. The impact on PF of the mGA components (assessed at baseline) and patients' physical symptom scores during follow-up was investigated by bivariate and multiple linear mixed models.

Results: A total of 288 patients, mean age 76.9 years, were available for analyses, $68 \%$ received palliative treatment. There was a statistically significant non-linear decline in PF (8.9 points; $p<0.001)$ from baseline to 6 months. Multiple linear mixed models showed that depressive symptoms and poorer physical performance at baseline were significantly associated with a poorer PF trajectory, as was also more pain, dyspnea, appetite loss and sleeping disturbances during follow-up. These associations were independent of age, gender, performance status, type of cancer, stage of disease and treatment (palliative/curative).

Conclusion: Treatment of depression and targeted palliation of physical symptoms during the disease trajectory seem paramount to maintain older patients' PF in both palliative and curative settings. Funded by Innlandet hospital trust.

Abstract number: P02-024

Abstract type: Poster

\section{Assessing Quality of Life in Oncology Practice: First Results of a Pilot Study \\ Vasconcelos de Matos L., Graça J., Malheiro M., Barata J., Placido A., Martins A \\ Medical Oncology, Hospital Sao Francisco Xavier, Centro Hospitalar Lisboa Ocidental, Lisbon, Portugal}

Evidence has shown that patient-reported outcomes (PROs) regarding health related quality of life (HRQoL) provide valuable outcomes in clinical practice. Also, PROs can guide clinical decisions to assure that patientcentered care is provided through the trajectory of cancer care. We've aimed to study the feasibility of measuring HRQoL in oncology practice, understand its impact in facilitating physician-patient communication and analyse its predictive use. We've conducted a prospective, descriptive study in a tertiary central hospital. From January to June 2018, patients were asked to complete the European Organization for Research and Treatment of Cancer Quality of Life Questionnaire-Core 30 (QLQ-C30) in consecutive outpatient visits, delivered to the physician prior to each consultation. Included 37 patients (median age 66 y; 70\% female), 30 (81\%) with metastatic disease and $25(68 \%)$ receiving tumor-directed-therapy. Mean of functional and symptom scores at baseline were 70.6 (Cl 95\% 64.6-76.6) and 27.7 (Cl95\% 22.1-33.4), respectively. In following visits $(17,44 \%)$, mean deterioration in functional and symptom scores were -21.1 [Cl 95\% -40.5 to -5.5] and 16.6 [Cl 95\% 4.5-29.5]. Worsening of both scores preceded in all cases evidence of cancer progression. Functional scores improved in 12 patients (71\%) (10.7, Cl95\% 5.1-16.2), and symptom scores in 11 (65\%) (-12.0, Cl 95\% -17.4 to -7.8). In all these, a medical intervention was undertaken. Also, in 29 (78\%) patients were identified issues otherwise not verbally referred. At 3-month follow-up, $6(16 \%)$ patients had died. Patients' perceptions on HRQoL domains are often not perceived by clinicians, leading to insufficient approach of issues that negatively impact patient's wellbeing. PRO's systematic use may be crucial in providing early identification of complexity, with adequate provision of structured palliative care. Furthermore, PROs may counterpart other routine clinical methods to monitor cancer progression.

\section{Abstract number: $\mathrm{P} 02-025$}

Abstract type: Poster

Who Goes to the Hospice? Data from a Brazilian Public Hospice for Cancer Patients

Ayres B. ${ }^{1}$, Zeni E. ${ }^{2}$, Neto R. ${ }^{3}$, Chiba T. ${ }^{4}$

${ }^{1}$ NACE, Hospital São Camilo, Cotia, Brazil, ${ }^{2}$ ICESP - HC - Faculty of Medicine, University of São Paulo, São Paulo, Brazil, 3Psychology, HCOR, São Paulo, Brazil, 'P Palliative Care, ICESP - HC - Faculty of Medicine, University of São Paulo, São Paulo, Brazil

Background and aim: Hospice care is associated with improved quality of life, healthcare cost reduction and lower rates of complicated grief. Over 100 million people will need palliative care services, yet less than $8 \%$ will have access to them. There is need for discussion regarding the development of the field in Brazil and for spreading the work of our hospices. This study aimed to characterize the patients attended at a public Brazilian hospice for cancer patients.

Methods: Internment information was pulled from electronic records regarding the period between March 1st, 2016 and July 31st, 2018. Data collected concerned gender, age, diagnosis, length of stay, outcome (decease/discharge/evasion). Calculations of average and median were obtained through the online tool Google Sheets.

Results: From a total of 1120 admissions, 1058 resulted in decease, 61 in discharge and 1 evasion. Average stay time was 17.2 days (1 241) and the median was 9 days. 478 admissions (42.68\%) lasted 7 days or less. 14 patients were admitted twice. Out of the 1106 patients, 637 were male (57.59\%). Average age was 65.2 years and age median was 65 (19 101). Most prevalent diagnosis were lung cancer (146), head and neck (133), bowel and rectum (110), gastric (80), breast (69), esofagus (63), central nervous system (58) and prostate (55). 52 diagnosis were considered unreliable and excluded.

Discussion: Our data collection showed that lung cancer was the most prevalent $(13.79 \%)$, similar to the national data ( $12.62 \%$ of cancer deaths). There was however a smaller prevalence of frequent neoplasms, such as breast and prostate, and markedly higher of head and cancer $(12.57 \%)$ if compared to the national mortality $(3.7 \%)$, suggesting a connection to the higher need for complex care. There was a large number of short admissions, suggesting late referral, with a possible negative impact on care quality. Another impact is the reduced use of a hospice facility as a strategy for health care cost reduction.

\section{Abstract number: $\mathrm{P} 02-026$}

Abstract type: Poster

Filipino Patients' Awareness of their Prognosis, Palliative Care Services, and Factors Affecting Choice of Complimentary and Alternative Medicine over Standard Anti-cancer Treatment Manalo M.F., Palliative Care

The Medical City, Augusto P. Sarmiento Cancer Institute, Pasig, Philippines Presenting author email address: lizamanalomd@gmail.com

Introduction: Recent researches document serious problems in medical care at the end of life, especially for those diagnosed with advanced and terminal-staged cancer. Communication regarding diagnosis and prognosis often is poor; many seriously ill patients and physicians do not discuss care at the end of life. Many patients suffer significant pain in their 
final days. So there is a need to focus on relief of suffering, psychosocial support, and closure near the end of life.

Fifteen years after the establishment of the first supportive, palliative, and hospice care services and fellowship training program in the state-run University of the Philippines-Philippine General Hospital (UP-PGH), many patients with advanced disease still do not receive palliative care; while,some are referred too late in the course of their disease to benefit from treatment.

At the same time, more and more oncology patients are looking for complimentary and alternative medicine, choosing them over standard anticancer treatment.

Objectives: To determine advanced cancer patients' awareness of their prognosis, palliative care services, and factors affecting choice of complimentary and alternative medicine over standard anti-cancer treatment Methods: Sixty patients with advanced cancer at the Cancer Institute, The Medical City are interviewed using the previously validated instrument called Asian Patients Perspectives Regarding Oncology Awareness, Care, \& Health (APPROACH).

Results: Most advanced cancer patients are not aware of their prognosis. Most believe they will be cured and have 10 years or so to live. Majority have not been told about about palliative care by their attending physician. Most prefer to try complimentary and alternative medicine believing these are safe and will cure them of cancer.

Conclusions: Physician education regarding disclosure and palliative care is crucial. Patient education about anti-cancer treatments, palliative care, and CAM is vital.

\section{Abstract number: P02-027 \\ Abstract type: Poster}

Feasibility and Acceptability of the Early Palliative Home Care Embedded in Cancer Treatment (EPHECT) Intervention Dhollander N. ${ }^{1}$, Smets T. ${ }^{2}$, De Vleminck A. ${ }^{2}$, Van Belle S. ${ }^{3}$, Pardon K. ${ }^{2}$, Deliens $L^{2,4}$

${ }^{1}$ End-of-Life Care Research Group, Vrije Universiteit Brussel (VUB) and Ghent University, Ghent, Belgium, ${ }^{2}$ End-of-Life Care Research Group, Vrije Universiteit Brussel (VUB) and Ghent University, Brussels, Belgium, ${ }^{3}$ Department of Medical Oncology, Ghent University Hospital, Ghent, Belgium, ${ }^{4}$ Department of Public Health and Primary Care, Ghent University Hospital, Ghent, Belgium

Background: To support the early integration of palliative home care (PHC) in cancer treatment, the EPHECT intervention was developed and pilot tested with 32 advanced cancer patients in Flanders, Belgium. The intervention consisted of systematic consultations with a PHC team, supported by a semi-structured conversation guide. Telephone-based contact was used to facilitate transmural collaboration between home and hospital, with the general practitioner (GP) as coordinator of care.

Aim: To determine feasibility and acceptability of the EPHECT intervention as perceived by the patients, family carers and healthcare professionals involved.

Methods: We conducted interviews with patients and family carers $(n=15)$, oncologists and GPs $(n=9)$ and a focus group with the PHC team. Data were analysed using thematic analysis.

Results: Most intervention components were found feasible and acceptable, except for 'transmural collaboration' and 'GP as coordinator of care'. Healthcare professionals mentioned problems with being reachable by phone and reported that it was difficult to estimate when contact was needed. According to GPs and the PHC team, the responsibilities and task allocations of the different professionals remained unclear. The intervention had no perceived effect on the involvement of GPs, according to patients, family carers, the PHC team and GPs. Some patients and family carers reported that the information shared by different professionals was sometimes inconsistent.

Conclusion: Telephone-based contact seems to be insufficient to facilitate transmural collaboration and to guarantee continuity of care in a model of early PHC embedded in cancer treatment. Furthermore, the role of the GP might have to be reconsidered, as most of them were rarely involved in the care trajectory. Based on these results, we are able to further refine the intervention model before implementing it in a phase 3 randomized controlled trial for the evaluation of its effectiveness.

Abstract number: P02-028

Abstract type: Poster

Chemotherapy versus Targeted Therapy in the Last Month of Life in Patients with Advanced Lung Cancer. A Patient File Study in the Netherlands

Mieras A. ${ }^{1}$, Becker-Commissaris A. ${ }^{2}$, Pasman H.R.W. ${ }^{3}$, OnwuteakaPhilipsen B.D. ${ }^{3}$

${ }_{1}$ Pulmonary Disease, Public and Occupational Health, Expertise Center for Palliative Care, Amsterdam UMC, Vrije Universiteit Amsterdam, Amsterdam, Netherlands, ${ }^{2}$ Pulmonary Disease, Expertise Center for Palliative Care, Amsterdam UMC, Vrije Universiteit Amsterdam, Amsterdam, Netherlands, ${ }^{3}$ Public and Occupational Health, Expertise Center for Palliative Care, Amsterdam UMC, Vrije Universiteit Amsterdam, Amsterdam, Netherlands

Purpose: Chemotherapy in the last month of life for patients with advanced lung cancer is often considered as aggressive end of life care. Targeted therapy is a relative new treatment of which not much is known yet about use in the last month of life. We examined what percentage of patients received chemotherapy or targeted therapy in the last month of life in the Netherlands.

Methods: In total, 1322 patient files were drawn from 10 hospitals across the Netherlands, 3 academic and 7 non-academic hospitals. Patients had to meet the following eligibility criteria: advanced lung cancer; died between 1-6-2013 and 31-7-2015. Demographic and clinical characteristics of the patients were obtained from the medical files of included patients.

Results: From these 1322 patients, 813 (61\%) patients received chemotherapy $(52 \%)$ or targeted therapy $(9 \%)$, of whom $232(29 \%)$ patients received this treatment in the last month of life. When treatment was given, targeted therapy was given more often in the last month of life than chemotherapy ( $72 \%$ versus $21 \%$, OR $9.22,95 \% \mathrm{Cl} 4.85-17.51)$. When treatment was given in the last month of life, this treatment was started in the last month of life in $54 \%$ in case of chemotherapy and in $17 \%$ in case of targeted therapy.

Conclusion: Chemotherapy and especially targeted therapy was received by a substantial percentage of patients with advanced lung cancer in their last month of life. Especially when chemotherapy is started shortly before death this can be seen as aggressive care. Targeted therapy is said to have less side effects and does not need hospital visits. Therefore it is debatable whether it should be considered as aggressive treatment. However, when still receiving it until shortly before death, this might hamper preparing for death.

Abstract number: P02-029

Abstract type: Poster

Understanding Complexity of Patients Referred to Hospital Palliative Care Teams: The Role of Haematological Cancer Patients and Symptom Profile

Steinberger K., Ramsenthaler C., Haberland B., Bausewein C. Department of Palliative Medicine, University Hospital Munich, Munich, Germany

Background: Hospital palliative care teams (PCTs) are a relatively new form of PC provision in Germany.

Aim: To describe the level of complexity in referred patients and how this relates to activities performed by hospital PCTs. 
Methods: Prospective observational study within a German university hospital. All consecutively referred patients to the hospital PCT in four months were recruited. Data were collected on clinical characteristics like phase of illness, performance status (AKPS) and symptom profile with the Integrated Palliative care Outcome Scale (IPOS). Intensity of care regarding number of professional groups involved, time per patient and activities of the team were scored using a standard form. Analysis involved modeling of differences in complexity between haematological and other cancers and deceased versus non-deceased patients.

Results: 200 patients ( $M: 69.8$ years, SD: $12.5,57.5 \%$ men) were included in the analysis, comprising $74 \%$ oncological (with $16.2 \%$ haematological cancer) and $20.5 \%$ non-cancer diseases. Half of patients had either unstable or deteriorating disease. The median AKPS was 40 (IQR: 20-50) with $76.5 \%$ having an AKPS score below 60 . Thirty-six patients (18\%) died while under the care of the hospital PCT. Complexity in terms of symptom profile was related to haematological disease versus other cancers and vital status of patients. Patients with haematological cancers presented with more psychological and joint physical and psychological symptoms $\left(X^{2}=9.6, p=0.008\right)$. Cancer type was related to a different intensity (total amount of care time) and activity profile with more advanced care planning and consulting the ward team and patients regarding further care options than in other conditions.

Discussion: Complexity of hospital PCT patients is related to intensity of care provided, and further associated with type of cancer and vital status. Further research should focus on the generalisability of findings beyond tertiary trusts.

Abstract number: P02-030

Abstract type: Poster

\section{Nosocomial Bacteriemias Associated with the Use of} Intravascular Catheters in Palliative Cancer Patients Pascual Pla F.J. ${ }^{1}$, Martin Utrilla S. ${ }^{2}$, Mancheño Alvaro A. ${ }^{3}$, Garcia Lozano $T^{3}{ }^{3}$, Bosca Mayans C. ${ }^{4}$, Ferrandis Olmos A. ${ }^{5}$, Iranzo Tatay A. ${ }^{3}$, Cañado $Y_{.}{ }^{3}$, Pardo Sere . $^{3}$, Samper Hiraldo $F^{3}{ }^{3}$, Santamaría García $P^{3}{ }^{3}$, de Luna Sanchez $F^{3}$

${ }^{1}$ Emergency Department, Fundacion Instituto Valenciano de Oncologia, Valencia, Spain, ${ }^{2}$ Palliative Care, Fundación Instituto Valenciano de Oncología (IVO), Valencia, Spain, ${ }^{3}$ Fundación Instituto Valenciano de Oncología (IVO), Valencia, Spain, ${ }^{4}$ Hospital Universitari i Politécnic La Fe, Valencia, Spain, ${ }^{5}$ Fundacion Instituto Valenciano de Oncologia, Valencia, Spain

Background: Infections related to central venous (CVC) and peripheral (CVP) catheters are complications with a high prevalence and possible serious consequences.

Aims: To determine the catheter-related bacteraemia (CRB) rate in ambulatory and hospitalized palliative cancer (PCP) patients and establish possible relationships between the type of track or the channeling site, the current status of the catheter \& establish possible preventive measures.

Methods: Prospective observational study from 2010 to 2017. Patients who presented fever with positive bacteremia after blood extraction from the catheter were included. Level of severity was determined by Simplified Acute Phiological and Score II score (SAPS). The infection rate that was intended to be determined was the BRC for 1,000 days of CVC. Results: 286 CVC were analyzed in 97 patients ( 7 of them 2 episodiums and 3,3$)$. In $87 \%$, the access route was a Port a Cath (PAC), in $12 \%$ the jugular vein and $1 \%$, a PICC. In critical patients, where a zero bacteremia protocol was followed, there were 16 cases of infection, three patients two episodes, two of them by MARSA. In the remaining patients, the bacteraemia rate per 1000 days of CVC could not be determined. The mean time between catheter insertion and infection was 10 days (range: 4-21) and Gram positive were isolated in most cases, followed by Gram negative and Candida. SAPSII average of 26.
Conclusions: We found a low rate of BRC in $\mathrm{PCP}$, with a high proportion of CVC in locations with lower risk of infection, despite not having found in the sample analyzed a higher rate of infection depending on the the canalized way The place of canalization, operating room in front of the plant, is related to a lower rate of BRC. The existence of a team of professionals trained and dedicated to the management of catheters is associated with a lower incidence of infectious complications, so this study allowed us to identify aspects that can be improved.

Abstract number: P02-031

Abstract type: Poster

Challenges of Integration of Palliative Care and Oncology Analysis of Multi-professional Healthcare Providers Views Missala I. ${ }^{1}$, Preisler M. ${ }^{1}$, Behzadi A. ${ }^{2}$, Kirschner P. ${ }^{3}$, Schmidt M. ${ }^{1}$, Auschra C. ${ }^{4}$ Letsch A. ${ }^{1}$

${ }^{1}$ Med. Department for Hematology and Oncology, Campus Benjamin Franklin, Charité - Universitätsmedizin Berlin, Berlin, Germany, ${ }^{2}$ Med. Department for Hematology, Oncology and Tumorimmunology, Campus Virchow Clinic, Charité - Universitätsmedizin Berlin, Berlin, Germany, ${ }^{3}$ Urology Practice Pankow, Berlin, Germany, ${ }^{4}$ Department of Management, Inter-Firm Cooperation, Freie Universität Berlin, Berlin, Germany

Background: According to national and international guidelines patients with advanced cancer benefit from palliative care (PC) early in the disease course parallel with tumor specific therapy. Unfortunately, this is still not general practice, limited by various difficulties and challenges.

Aims: To examine different health care professional's views of challenges of integrated palliative cancer care (IPCC).

Design/ methods: A multi-professional group of health care providers $(n=35)$ answered a survey questionnaire consisting of 11closed and open items.

Results: $80 \%$ of the participants were in favor of IPCC. In contrast $20 \%$ refuse IPCC at the time of first diagnosis or due to a lack of knowledge and capacity of the existing structures or due to patient denial. The most frequently mentioned benefits of IPCC were improvement of patient's quality of life, individualized and need-oriented therapies, control of symptoms, multi-professional teamwork and improvement of patients' and relatives' care. With respect to triggers, specialization and implementation, $40 \%$ of the professionals identified a need for specialized palliative care if complex symptoms occur, $27 \%$ at first diagnosis and $18 \%$ on request of the patient. The majority stated that the need of general or specialized PC is best identified by a systematic assessment of patients and relatives demands. In order to achieve more differentiated and individualized therapy concepts $1 / 4$ of the participants considered intensive talks with patients important and $1 / 6$ mention patient's autonomy and education as essential. An unclear treatment goal or lack of time or uncertainty in the patient-physician communication were mentioned as main communication barriers.

Conclusion/ discussion: Most healthcare providers agree with the importance of IPCC, naming comparable challenges. Careful evaluation of difficulties, listening to multi-professional views and exchanging best practice are likely to lead to a more effective implementation of IPCC.

Abstract number: P02-032

Abstract type: Poster

Gap between Referral of Early Palliative Care Patients in Study Settings and in Clinical Practice

Zwahlen S., Eychmüller S.

University Center of Palliative Care, University of Bern, Inselspital University Hospital, Bern, Switzerland

Background: Specialist guidelines recommend the integration of early Palliative Care (ePC) into the standard procedure of oncology, based on the results of randomized controlled (RCT) ePC trials. 
Aim: This study investigated whether the recommendations on ePC are being implemented in daily practice at a University Hospital in Switzerland. Having finished an ePC trial restricted to six tumor entities in early 2018, the aim was to compare referral rates of ePC patients identified by screening during the trial period with referral rates of an identical population by physicians in daily practice in the post-study period.

Methods: We gathered data by extraction from 218 medical records of inpatients and outpatients seen consecutively from April to September 2018 by the hospital palliative care (PC) team. Data was collected in October 2018. As in our ePC trial, patients with an ECOG of 0-2 and a first PC contact within a 16-week interval after diagnosis of a cancer no more amenable to curative treatment were defined as ePC patients.

Results: A total of 218 patients with all types of tumor entities were referred to a first PC contact between 01.04.2018 and 30.09.2018. Nineteen of these patients met the ePC criteria, resulting in $3.2 \mathrm{ePC}$ patients referred to $\mathrm{PC}$ per month. For the subpopulation studied in the ePC trial, the referral rate during the study period was 5.2 patients per month with 2.5 patients consenting to participate in the study compared to a referral rate of $0.5 \mathrm{ePC}$ patients per month to the $\mathrm{PC}$ team in clinical practice after completion of the study.

Conclusions: Compared to the recruitment period of a RCT for ePC, only $20 \%$ of an identical population got in contact with ePC in 'real life' during post-study period. Measures such as systematic screening can not only improve recruitment rates in studies, but may also help to integrate ePC into standard oncology care. Further research needs to explore how ePC can gain access to daily practice.

\section{Abstract number: P02-033 \\ Abstract type: Poster}

\section{Sexual Dysfunction in Women with Advanced Cancer - A Cross} Sectional Study

Campos L., Barros N., de Nardi S., Hauser F., Caldas J.M.

Serviço de Dor e Cuidados Paliativos, Hospital Nossa Senhora da Conceição, Porto Alegre, Brazil

Background: Sexual dysfunction refers to conditions that interfere with the ability to engage in sexual function or disrupt the full sexual response cycle. To date, very few original studies evaluated sexual concerns in patients with advanced cancer.

Objective: To evaluate sexual dysfuncion rate in women with breast, gynecologic and colorectal advanced or metastatic cancer.

Method: Sexual dysfunction was evaluated by Female Sexual Function Index (FSFI), a multidimensional instrument. Thirty-six patient who had been diagnosed locally advanced or metastatic cancer from june 2017 to december 2017 were included.

Results: Age: $56.47 \pm 11.21$; cancer type: cervical: 2 (5.6\%), colorectal: 9 (25\%), endometrial: 2 (5.6\%), breast: 17 (47.2\%), ovary: 6 (16.7\%). Seven patients $(20 \%)$ underwent colostomy or ileostomy. Twenty patients (55.6\%) had sexual partner and among them, four reported no sexual intercourse in the last 30 days. Eight women in the group who had sexual partner $(42,1 \%)$ and four in the group who didn't $(25 \%)$ reported masturbation practice. The median FSFI final score for patients who had sexual partner was $14.77 \mathrm{p} 25 \% 2$ and p75\%: 27.10. Only five patients didn't present sexual dysfunction (FSFI cutoff score $>=26$ ). Only three patients reported that a health care team member asked about any sexual problem during treatment or follow-up.

Conclusion: Sexuality is an important issue for quality of life. Health professionals should be prepared to bring up this subject in a natural way, referring patients to sexual or psychological therapy when necessary.

Abstract number: P02-035

Abstract type: Poster
Efficacy of Immediate Release Oxycodone for Dyspnea in Cancer Patient: Cancer Dyspnea Relief (CDR) Trial

Yamaguchi T. ${ }^{1}$, Matsuda Y. $^{2}$, Matsuoka H. ${ }^{3}$, Hisanaga $T^{4}$, , Osaka I. ${ }^{5}$, Hiroaki W. ${ }^{6}$, Maeda I. ${ }^{7}$, Imai K. ${ }^{8}$, Tsuneto S. ${ }^{9}$, Wagatsuma ${ }^{10}{ }^{10}$, Kizawa $Y_{.11}$

${ }^{1}$ Palliative Care, Konan Hospital, Kobe, Japan, ${ }^{2}$ Psychosomatic Internal Medicine, Kinki-Chuo Chest Medical Center, Sakai, Japan, ${ }^{3}$ Psychosomatic Medicine, Kindai University, Osaka-Sayama, Japan, ${ }^{4}$ Palliative Medicine, Tsukuba Medical Center Hospital, Tsukuba, Japan, ${ }^{5}$ Palliative Medicine, Shizuoka Cancer Center, Nagaizumi, Japan, ${ }^{6}$ Palliative Care, Komaki City Hospital, Komaki, Japan, ${ }^{7}$ Palliative Care, Gratia Hospital, Minoh, Japan, ${ }^{8}$ Hospice, Seirei Mikatahara Hospital, Hamamatsu, Japan, ${ }^{9}$ Palliative Medicine, Kyoto University, Kyoto, Japan, ${ }^{10} \mathrm{Clinical}$ Trial and Clinical Epidemiology, Tsukuba University, Tsukuba, Japan, ${ }^{11}$ Palliative Medicine, Kobe University, Kobe, Japan

Background: Guidelines recommend morphine as the first line pharmacological treatment for cancer dyspnea. However, trials with other opioids have not been performed. Our aim was to demonstrate the non-inferiority of oxycodone to morphine for relieving dyspnea in cancer patients.

Methods: We conducted a multicentre, open-label, parallel-group, randomized control trial. We randomly and equally assigned cancer patients on regular oxycodone who developed dyspnea to get a single dose of oral immediate-release oxycodone or morphine. We evaluated the change in dyspnea intensity (numeric rating scale: 0-10) and adverse events after the medication administration.

Results: This study was pre-maturely terminated. All 17 enrolled patients (8 using oxycodone, 9 using morphine) completed evaluations. In the oxycodone group, dyspnea intensity decreased 1.75 points [ $95 \%$ confidence intervals $(\mathrm{Cl}), 0.72-2.78]$ at $60 \mathrm{~min}$ and 1.50 points $(95 \% \mathrm{Cl},-0.11$ to 3.11 ) at $120 \mathrm{~min}$. In the morphine group, dyspnea decreased 1.33 points $(95 \% \mathrm{Cl}, 0.41-2.25)$ at $60 \mathrm{~min}$ and 1.00 point $(95 \% \mathrm{Cl},-0.08$ to 2.08 ) at $120 \mathrm{~min}$. The differences did not fulfil the non-inferiority requirement. Although two and four patients in morphine group developed drowsiness at 60 and 120 minutes, no patient in oxycodone group developed significant adverse events.

Conclusions: Although we did not show the non-inferiority of oxycodone, oxycodone may have some effectiveness for cancer dyspnea without significant safety concern.

\section{Abstract number: P02-036 \\ Abstract type: Poster}

Cognitive and Behavioural Strategies for the Management of Episodic Breathlessness - A Delphi Survey with International Experts

Schlösser K., Eisenmann Y., Simon S.T.

Palliative Medicine, University Hospital of Cologne, Cologne, Germany

Background: Episodic breathlessness (EB) is characterized by a severe worsening of breathlessness intensity that goes beyond usual fluctuations. Episodes are usually short, therefore non-pharmacological strategies seem most promising to be beneficial. Which non-pharmacological strategies - alone or in combination - might be most effective and feasible remains unclear.

Aim: The Delphi survey selects and determines different non-pharmacological strategies for EB in order to develop a brief cognitive and behavioural intervention ( $\mathrm{CBI}$ ) for the management of $\mathrm{EB}$.

Methods: Using an online Delphi survey comprising three rounds international, multidisciplinary experts in breathlessness summarized and determined non-pharmacological strategies. The a priori target agreement for close-ended questions was $70 \%$.

Results: Experts ( $n=41 / 87 ; n=48 / 85 ; n=37 / 85$ ) consented 15 of the 31 collected non-pharmacological strategies. Based on the panelists' opinion 
the final version of the $\mathrm{CBI}$ composed the following characteristics: individually tailored intervention, high proportion of communication, short duration, involvement of carers, use of the Breathing-Thinking-Functioning Model (Spathis et al., 2017). Consensus upon the delivery of the subsequent strategies within the $\mathrm{CBI}$ was reached: handheld fan, forward lean, diaphragmatic breathing, distraction, pursed lips breathing, long breaths out and relaxation training.

Conclusion: Using the consented non-pharmacological strategies the CBI was developed with a focus on the balancing act of individualization and standardization of the intervention. The $\mathrm{CBI}$ will be tested in a single-arm therapeutic explanatory trial (phase II) starting in January 2019.

The study is founded by the Federal Ministry of Education and Research.

Abstract number: P02-037

Abstract type: Poster

Oral Morphine Drops for Rapid Treatment of Breathlessness in Palliative Cancer Patients. A Randomized, Double Blind, Crossover Trial of Morphine Sulfate Oral Solution vs. Morphine Hydrochloride Oral Solution (Red Morphine Drops)

Aabom B. ${ }^{1}$, Laier G. ${ }^{2}$, Christensen P.L. ${ }^{2}$, Karlsson T. ${ }^{2}$, Jensen M.-B. ${ }^{2}$, Hedal $B .^{3}$

1Palliative Unit, Zealand University Hospital, Roskilde, Denmark, ${ }^{2}$ Zealand University Hospital, Roskilde, Denmark, ${ }^{3}$ Hospice Sjaelland, Roskilde, Denmark

Background: Breathlessness is frequent in palliative patients. Opioids are the only pharmacological agents with sufficient evidence in palliation of breathlessness. The exact mechanisms is only partly understood moreover which opioid and especially which formulation (buccal, nasal, tablets) to use, is undergoing intensive research. In Denmark, main recommendation (based on expert opinion) is morphine hydrochloride oral solution i.e. red morphine drops (RMD). RMD is an off-label solution of morphine, alcohol and red color (cochenille) described since 1893 (Pharmacopoea Danica). In 2015, the Danish Medicines Agency increased focus on offlabel medicines and recommended as an alternative: Morphine Sulfate Oral Solution. However, in the clinical setting, our palliative patients told us, that RMD was better.

Aims: To analyze onset of action between the two types of morphine drops

Methods: The study was an investigator-initiated, randomized, double blind, crossover trial. Participants were adult outpatients at Palliative Unit, Zealand University Hospital. The recruitment took place in one year from July 2016 to July 2017. Each patients were tested 2 times (crossover) with 3-7 days wash-out period in-between, using 2 minute walks test ( 2 MWT) as a standardized test to provoke breathlessness. Primary outcome measure were VAS (0-10) across eight time points.

Results: Fifty-four out of 270 patients had breathlessness $>1$ (EORTC), seventeen patients meet inclusion criteria and twelve patients completed the study. A repeated measurements linear mixed model showed overall significant difference between groups $(p=0.0106)$. RMD proved superior in the first minute $(p=0.0103)$ and in a complementary survey 20 minutes following the test $(p=0.0085)$.

Conclusion/discussion: The instant effect of RMD might be the reason for patients reporting us that RMD are more powerful. The results highlights the importance of short onset of action and selfadministration in case of breathlessness.

Abstract number: P02-038

Abstract type: Poster

Understanding How Decisions to Present to the Emergency Department Are Made by Patients with Acute-on-Chronic Breathlessness, their Family Carer and Clinician Hutchinson A. ${ }^{1}$, Barbette C. ${ }^{2}$, Galvin K. ${ }^{3}$, Twiddy M. ${ }^{1}$, Johnson M. ${ }^{1}$ ${ }^{1}$ Hull York Medical School, University of Hull, Hull, United Kingdom,
2Pulmonology, Ospedale S.Maria degli Angeli, Pordenone, Italy, ${ }^{3}$ School of Health Sciences, University of Brighton, Brighton, United Kingdom

Background: Chronic breathlessness is common in cardiorespiratory conditions and is frequently associated with emergency department (ED) presentation when the patient experiences acute-on-chronic breathlessness. Breathing Space is a concept combining patient coping, help-seeking and clinician responsiveness to breathlessness in addition to disease-management.

Aim: To explore whether Breathing Space may help explain the decision by patients with acute-on-chronic breathlessness to present to the ED.

Methods: Secondary mixed-methods analysis of patient self-report survey, case note and interview data. We used an in-depth case-history approach to synthesise these source data from eight representative patients who presented to the ED due to acute-on-chronic breathlessness. Patient-interview data were also linked with interview data from their family carers and clinicians.

Results: Secondary analysis of eight survey and case note reviews, combined with analysis of eight patient interviews (four with a carer) and six clinician interviews was conducted. The Breathing Space concept was useful in understanding the individual patient's decision to attend the ED. Having a clinician who both understands the impact of breathlessness on their patient and offers ways of managing breathlessness in addition to treating the underlying disease seems to be important. Clinicians responsive to breathlessness were able to encourage a disengaged patient (with restricted Breathing Space) to move towards a more adaptive way of living with breathlessness (greater Breathing Space). The converse was seen when clinicians had a sole focus on diseasedirected treatment.

Conclusion: The Breathing Space concept may be useful for clinicians caring for people with chronic breathlessness. Early assessment and management of the Breathing Space of the patient (and carer) may help improve the patient's (and carer's) quality of life and the management of acute-on-chronic breathlessness crisis.

Abstract number: P02-039

Abstract type: Poster

Pulmonary Pre-habilitation: Self-management Programme for Adults with a New Diagnosis of Lung Cancer

McGreevy B., Adults with a New Diagnosis of Lung Cancer NHSCT, Antrim, United Kingdom Presenting author email address: bernie. mcgreevy@northerntrust.hscni.net

Aim: To set- up a 'breathlessness service'. The ideal group to start with was those with Lung Cancer as up to $90 \%$ will experience breathlessness as a consequence of their cancer or treatment. To provide education and allow them to practice techniques before they became breathless in a proactive approach. Provide service-users the tools to self-manage a distressing symptom. Promote the vast benefits of physical activity at all stages of cancer.

Design: Working group established - Macmillan Physiotherapist, Lung Cancer Nurse Specialists (LCNS) and patient representative from Lung Cancer Support Group.

CONTROL GROUP - adults who had received a new diagnosis of Lung Cancer received the normal Lung Cancer information pack and a pedometer to encourage physical activity.

PILOT GROUP attended 2 workshops in rolling programme so after diagnosis everyone had access a LCNS or Clinical Specialist Physiotherapist within 7 days.

Workshops ran in waiting time from diagnosis until oncology appointment and/or commencing treatment/surgery. Prior to our service clients were advised to 'self-manage' the side-effects of the disease and its treatments, with telephone support from the LCNS. This high quality 
new service empowers patients to self-manage whilst providing them with better outcomes and an improved patient experience.

Results: $63 \%$ control group had a hospital admission compared with $21 \%$ of pilot group (43\% when including those who passed away in hospital following admission for end of life care).

Macmillan Move More Project referrals, patients physically more active ( $36 \%$ of pilot group patients made contact with Move More co-ordinator, compared with $0 \%$ in control group).

$100 \%$ of attendees scored programme $10 / 10$ and would recommend to others.

$100 \%$ of attendees felt they benefitted from programme.

Patient co-produced programme/Pathway from diagnosis

Message: Prehabilitation pathway for an often overlooked cohort with complex needs.

Abstract number: P02-040

Abstract type: Poster

Acupuncture for Breathlessness in Advanced Diseases: A Systematic Review and Meta-analysis of its Effectiveness von Trott $P .{ }^{1,2}$, Ramsenthaler $C^{1}{ }^{1}$, Koffman J. ${ }^{1}$

${ }^{1}$ Department of Palliative Care, Policy \& Rehabilitation, King's

College London / Cicely Saunders Institute, London, United Kingdom,

${ }^{2}$ Gemeinschaftskrankenhaus Havelhöhe, Berlin, Germany

Background: Non-pharmacological approaches are effective strategies for difficult to palliate breathlessness. Whilst acupuncture is effective for dyspnoea in early-stage chronic obstructive pulmonary disease (COPD), little is known about its effects in patients with advanced malignant and non-malignant diseases.

Aim: To identify and examine the evidence of acupuncture on breathlessness in advanced malignant and non-malignant diseases.

Methods: Systematic literature review of randomised controlled trials of acupuncture, acupressure and Acu-Tens in five databases (MEDLINE, PsycINFO, Embase, Web of Science, Cochrane trial registers) until October 2018. Included were adult participants with at least $25 \%$ having advanced disease including cancer, COPD, chronic heart failure and other. Primary outcome was severity of dyspnoea on visual analogue or Borg scale. Secondary outcomes included quality of life, function and acceptability. Data were pooled using a random effects model of standardized mean differences.

Results: We included 12 studies that identified a trend towards improved breathlessness severity $(S M D=-0.37(95 \% \mathrm{Cl}-0.82,-0.08$; $\left.p=0.11 ; I^{2}=77 \%\right)$. A significant effect was observed in the subgroup of advanced COPD patients $\left(\mathrm{SMD}=-0.58\left(95 \% \mathrm{Cl}-1.09,-0.06 ; \mathrm{p}=0.03 ; \mathrm{I}^{2}\right.\right.$ $=69 \%$ ) and in those with a treatment duration of at least 9 days. For quality of life, a trend towards effectiveness of acupuncture was shown. Exercise tolerance measured by the 6 minute walk test improved significantly in the acupuncture group $(S M D=0.40(95 \% \mathrm{Cl}$ $\left.0.05-0.74 ; p=0.02 ; I^{2}=35 \%\right)$ ). Regarding acceptability, no differential drop-out was observed.

Conclusions: The trend towards effectiveness of acupuncture on breathlessness severity in patients with advanced disease could only be shown for severe COPD patients or a longer treatment duration. The methodological heterogeneity, low power and potential morphine-sparing effects of acupuncture as add-on should be further addressed in future trials.

Abstract number: P02-043

Abstract type: Poster

Fatigue as a Stressful Cofactor in Uro-oncological Patients with Advanced Cancer Disease

Draeger D.L., Mueller M.M., Fritz E.-M., Kernig K., Milerski S., Hakenberg O.W.

Department of Urology, Medical Center of University Rostock, Rostock, Germany
Background: Cancer-associated fatigue ( $\mathrm{CrF}$ ) is a common complication in cancer patients (78-96\%), characterized by exhaustion, weakness, and decreased performance. It is perceived by patients as a heavy burden. The exact pathogenesis is unknown, but a multifactorial genesis is assumed. In all stages of tumor treatment (diagnosis, therapy, follow-up and palliation), fatigue has an important role for the patient. So far, there are hardly any studies on fatigue in uro-oncology.

Aim: Using a standardized questionnaire, we examined the cancerrelated fatigue syndrome in uro-oncological patients with advanced disease.

Methods: Analysis of metastatic cancer patients $(n=77)$ with locoregional and/or distant metastases undergoing outpatient or inpatient systemic treatment. The patient cohort was evaluated with a standardized questionnaire for the assessment of physical and functional aspects of CrF (FACT-F).

Results: CrF was described in 58\% of the cases (range "slightly" to "very affected"). Pain and/or nausea/vomiting were reported in 34\% and $29 \%$, respectively. In $52 \%$ these symptoms were not reported to the attending physician. Only $15 \%$ of patients received treatment or counseling regarding the management of a CrF. In $45 \%$ symptom control was assessed as insufficient. The median FACT-F score was 21 (range 0-53). Multivariate analysis showed a correlation between $\mathrm{CrF}$ and quality of life, depression, dyspnea, weight loss, inappetence and analgesic use.

Conclusions: Cancer-associated fatigue in uro-oncology is a serious disorder of physical well-being, with adverse physical, mental and social consequences. Symptoms of $\mathrm{CrF}$ are associated with shorter survival time. It correlates with a reduced quality of life as well as decreased treatment compliance.

Abstract number: P02-044

Abstract type: Poster

Cancer Related Fatigue (CRF) - Motivation and Barriers of Physical Activitiy

Frikkel J. ${ }^{1}$, Guyot M. ${ }^{1}$, Kasper S. ${ }^{1}$, Metzenmacher M. ${ }^{1}$, Beckmann M. ${ }^{2}$, Hense J. ${ }^{1}$, Teufel M. ${ }^{2}$, Schuler M. ${ }^{1,3}$, Tewes $M .{ }^{1}$

${ }^{1}$ West German Cancer Center, Department of Medical Oncology, University Hospital Essen, Essen, Germany, ${ }^{2}$ Department of Psychosomatic Medicine and Psychotherapy, University Duisburg Essen, Essen, Germany, ${ }^{3}$ German Cancer Consortium (DKTK), Partner Site University Hospital Essen, Essen, Germany

Background: Despite the large impact of physical activity (PA) in CRF, only $30 \%$ of patients (pts) with advanced cancer are physical active. Currently, only little is known about motivation and barriers of PA in a palliative setting.

Aims: This prospective study investigates barriers and motivation for PA in pts with tiredness (T) or weakness (W).

Methods: From May 2017 to August 2018, all pts treated at the outpatient unit of the West German Cancer Center were screened by the new MInimal DOcumentation System. Pts reported moderate or severe T or W were eligible and were asked to complete a questionnaire included items about demographics, FACT-F-Score (FS), PHQ8-Score (DS), motivation for PA, physical and social barriers. A follow up was done after 3,6 and 12 months.

Results: 151 of 440 eligible pts agreed to participate in the study. Median age was 61 (range 26-83), most pts had metastatic cancer (138; 91\%) of lung $(46 ; 30 \%)$, gastrointestinal tract $(33 ; 22 \%)$ or breast $(29 ; 19 \%)$. Pts had a moderate FS $(30 ; 4-48)$ and a low DS $(7 ; 0-19)$. We found a strong correlation between severe fatigue (low FS) and depression (high DS) $(p<.001, r=-0.685)$. Most pts were aware of the positive impact of PA on their quality of life (QoL) $(89 ; 59 \%)$ and of the course of their disease (92; $61 \% ; p<.001)$. But only $25 \%$ of pts reported to be physically active on a low intense level. Almost half of pts $(72 ; 48 \%)$ reported no or low motivation for PA, and only $38 \%$ reported to be interested in an exercise program. Cancer Therapy $(110,73 \%), \mathrm{T}(99,67 \%)$ and W $(93,62 \%)$ were 
most reported barriers for PA. No significant social barriers were ticked by the pts. No significant correlation was identified between DS and motivation for PA. Data for follow up will be presented at the EAPC. Conclusion: The motivation of pts with CRF for PA was very low, although most pts were informed about the positive impact of PA on QoL and course of disease. Cancer therapy, $\mathrm{T}$ and $\mathrm{W}$ were the most common barriers to $P A$.

Abstract number: $\mathrm{P} 02-045$

Abstract type: Poster

3-STEPS: A Study of an Individual Physical Activity Intervention in Advanced Cancer Patients on a Specialist Palliative Care Ward Blum D. ${ }^{1}$, Jensen W. $^{2}$, Schuetz $C{ }^{2}$, Wannke L. ${ }^{2}$, Ketels G. ${ }^{2}$, Bokemeyer $C .{ }^{2}$ Oechsle K. ${ }^{1}$

${ }^{1}$ Palliative Care Unit, Department of Oncology, Hematology and Bone Marrow Transplant, UKE Hamburg, Hamburg, Germany, ${ }^{2}$ UKE Hamburg, Hamburg, Germany

Background: Physical activity is beneficial in cancer patients (pts), but studies with highly standardized activity interventions reported little accrual and high attrition rates in the palliative care setting.

Aim: The aim was to test the feasibility and acceptance of an adapted three-step activity intervention ("3STEPS") in advanced cancer pts and its efficacy.

Methods: All pts admitted to a specialist palliative care ward (sPCW) of a tertiary comprehensive cancer center between June 2017 and May 2018 were screened by a physiotherapist for a three-step activity intervention consisting of STEP 1: two low-threshold initial tests (basic assessment/ Tinetti-Test) to screen for physical capacity and patient's willingness to participate. Study intervention included STEP 2: low-intensity individually adapted activity intervention and STEP 3: a home-based training after admission. Physical function and global quality of life (QoL) were assessed by the EORTC-QLQ-C30 and the NCCN-Distress Thermometer was used at study start and ward discharge; home care training adherence was controlled four weeks later by phone.

Results: Of 438 pts admitted to the SPCW, a basic assessment could be performed in 248 pts (57\%) and 125 of them (50\%) underwent a Tinetti test. Reasons for non-participation in STEPS 2 and $3(n=119)$ were: cognition (37), physical (34); no interest (32), no German language (13). In 6 pts (median age 59 years; 3 female), the intervention was started, but two of them could not get the full intervention. Four pts completed the intervention and two of them continued with the home based training. In all 4 pts, an improvement in QoL, physical function and distress was measured.

Conclusion: Despite systematic screening, only in 6 pts could be accrued to the individually adapted activity intervention, which showed an effect in this few selected pts. Physical performance and cognition of the pts admitted to a SPCW was even lower than expected.

Abstract number: P02-046

Abstract type: Poster

\section{Fighting Fatigue Together}

Craig A. ${ }^{1}$, McGreevy B. ${ }^{1}$, Sommerville L. ${ }^{1}$, Mellon A. ${ }^{1}$, Adams N. ${ }^{1}$, McCullough J. ${ }^{2}$

${ }^{1}$ Macmillan Unit, Antrim, United Kingdom, ${ }^{2}$ Antrim Area Hospital, Antrim, United Kingdom

Background: Cancer related fatigue (CRF) is the most prevalent distressing symptom in cancer at all stages of the illness. UK/ International Guidelines recommend a self-management, multi-disciplinary group approach. No regionally agreed model meeting these guidelines or local service.

Goal: Establish a co-produced, interactive, multidisciplinary, evidence based (EB) group cancer related fatigue (CRF) programme for adults/ teenagers \& young people (TYP) / significant others. Develop this into an on-line resource for replication across the UK.

Design/ methods: MDT working group established across oncology \& palliative care sectors in Health Trust. EB Practice researched/ existing services scoped. Service users involved - recording DVD of experiences Pilot programmes ran with adults, followed by TYP in partnership with Clic Sergent - evaluated using questionnaires/Fatigue Symptom Inventory (FSI) validated tool.

Results: Using FSI all but one improved in levels of fatigue and in all general activity and enjoyment of life improved significantly. Mixed results on concentration and social relations.

Qualitative information reinforced a group environment -support and normalisation gained from other participants was a major factor. Having a partner/ friend attending was affirmed. Across participants, both service users \& family/friends, the course was rated 9 or 10/10.

Comments included "This is normal and can be improved" (Adult), "It all makes sense now" (Teenager)

The length of programme was shortened and administration support was required to sustain the programme.

An on-line programme providing all resources, presentations \& guidance, downloadable for free, launched in partnership with a hospice \& palliative care national website learning platform. This provides any MDT with a template to replicate this programme \& adapt to their area.

Lessons learned: People with CRF, at any stage of their illness, can be empowered to make sense of and self-manage this debilitating symptom.

Abstract number: P02-047

Abstract type: Poster

\section{Foot Care, Anyone?}

Lin C.L., Chuang H.J., He Y.S.

St. Mary's Hospital, Luodong, Yilan, Taiwan, Republic of China

Background: Having quality sleep is crucial to ordinary people to obtain sufficient energy needed for their daily activities. It is even more important for those terminally ill cancer patients because they need good sleep to preserve strength and energy to help cope with problems related to their illnesses.

Aims: The study is aiming at determining whether Foot Care enhances our terminal cancer patients' sleep.

Methods:

1. Pour warm water with the temperatures around $41-43$ degrees Celsius into a bucket.

2. Patients soak their feet in it for $15-20$ minutes with the water above their ankles.

3. Dry their feet and follow with a 5 - 10 minute foot massage.

4. The process is conducted between 19:00 and 20:00 and repeated for three consecutive days.

5. After 3 days, each patient is asked whether the process has helped their quality of sleep.

6. They can choose from three answers:

(1) Very beneficial

(2) Somewhat beneficial

(3) Totally not beneficial.

Results: Our study ran from January 1, 2015 to December 31, 2017, involving 340 patients.

About 70\% (238) of the patients chose (1) - Very beneficial, and about $15 \%$ (51) chose (2) - Somewhat beneficial, while the rest $15 \%$ (51) answered (3) - Totally not beneficial.

Conclusion / Discussion: The study shows that $85 \%$ of terminally ill cancer patients feel the process of Foot Care has either very much or somewhat helped with their quality of sleep. 
For the remaining $15 \%$ feeling otherwise, it's possible either their responses to external stimulants are not as sharp due to their advanced diseases, or the process is performed too early before their bedtime to have any effects.

Based on this positive results, we strongly believe the effectiveness of using foot care to enhance terminal patients' sleep, and wish to advocate that it not only be continued but also be expanded.

Abstract number: P02-048

Abstract type: Poster

\section{Cachexia in End Stage Kidney Disease (ESKD): Results from a Cross Sectional Analysis}

McKeaveney $C^{1}{ }^{1}$, Adamson G. $^{2}$, Davenport A. ${ }^{3}$, Farrington $K^{4}{ }^{4}$, Fouque D. ${ }^{5}$, Mallett J. ${ }^{2}$, Maxwell P.6,7, Mullan B. ${ }^{8}$, Noble H. ${ }^{1}, \mathrm{O}^{\prime}$ Donoghue $D .{ }^{9}$, Porter S. ${ }^{10}$, Seres D.S. ${ }^{11}$, Shields J. ${ }^{6}$, Slee A. ${ }^{3}$, Whitman M. ${ }^{12}$, Reid J. ${ }^{1}$ ${ }^{1}$ Queen's University, Belfast, School of Nursing and Midwifery, Belfast, United Kingdom, ${ }^{2}$ Ulster Univerisiity, Psychology, Coleraine, United Kingdom, ${ }^{3}$ University College London, Faculty of Medical Sciences, London, United Kingdom, ${ }^{4}$ East and North Hertfordshire NHS Trust, Renal Medicine, Stevenage, United Kingdom, ${ }^{5}$ University of Lyon, Department of Nephrology, Lyon, France, ${ }^{6}$ Belfast Health and Social Care Trust, Renal Services, Belfast, United Kingdom, ${ }^{7}$ Queen's University, Belfast, School of Medicine, Dentistry and Biomedical sciences, Belfast, United Kingdom, ${ }^{8}$ Northern Health and Social Care Trust, Renal Services, Antrim, United Kingdom, ${ }^{9}$ University of Manchester, Faculty of Biology, Medical and Health, Manchester, United Kingdom, ${ }^{10}$ Bournemouth University, Social work and social sciences, Poole, United Kingdom, ${ }^{11}$ NewYork-Presbyterian/ Columbia University Irving Medical Center, Medical nutrition, New York, NY, United States, ${ }^{12}$ NIHR Newcastle Biomedical Research Centre, Newcastle upon Tyne, United Kingdom

Objective: Definitions of cachexia, a muscle wasting condition, in chronic illnesses are required to reflect specific nuances associated with each disease. Research indicates that muscle wasting is common among persons with ESKD. However, the absence of a definition for cachexia in an ESKD population makes it particularly difficult to study the incidence of cachexia or potential treatments.

Aim: To determine if patients with ESKD experience (and to what degree) the known characteristics associated with cachexia.

Method: This a longitudinal study which has recruited 106 adult chronic haemodialysis patients attending two Regional Nephrology Units within the U.K. Patients have been followed every two months over 12 months and measurements of: weight; lean muscle mass (bioelectrical impedance, mid upper arm muscle circumference -from measures of mid arm circumference and tricep skin fold thickness); muscle strength (hand held dynamometer); fatigue; anorexia, bio-chemistry and quality of life collected.

Results: Longitudinal data collection is ongoing however a CrossSectional Analysis has been completed.Patients who met the primary criterion (weight loss) of Evans et al. (2008) definition for cachexia compared to those that did not, had significantly poorer outcomes for muscle strength ( $p=.001)$, muscle depletion $(p=.012)$ and anorexia $(p=.007)$. However, regardless of weight loss, all ESKD patients demonstrated poorer muscle strength, decreased haemoglobin, greater fatigue and inflammation.

Conclusion: Outcomes provide new robust insight into the body composition of ESKD patients however further evaluation of recommended target cut-offs for cachexia is needed. According to Evans et al. (2008) grip strength, anorexia and fatigue were useful whereas the presence of abnormal bio-chemistry was less useful. This study demonstrates much needed data to inform the development of a clinical phenotype for cachexia in ESKD and the initial refinement of a disease specific definition.
Abstract number: P02-049

Abstract type: Poster

"FAB REHAB" an Evaluation of a Fatigue and Breathlessness Programme for Palliative Care

Kelleher K. ${ }^{1}$, Leo $\mathrm{O}^{1}{ }^{1}$, Leahy $\mathrm{H}^{2}{ }^{2}, \mathrm{O}^{\prime}$ Mahony $\mathrm{S}^{1}{ }^{1}$, McVeigh J. ${ }^{3}$, Kiely $\mathrm{F}^{4}$ ${ }^{1}$ Physiotherapy, Marymount University Hospital and Hospice, Cork, Ireland, ${ }^{2}$ Nursing, Marymount University Hospital and Hospice, Cork, Ireland, 3Physiotherapy, University College Cork, Cork, Ireland, ${ }^{4}$ Medecine, Marymount University Hospital and Hospice, Cork, Ireland

Aims: The aim of this study was to evaluate the effectiveness of the FAB programme for the palliation of breathlessness and fatigue in life limiting illness. The primary objective was to explore the impact of the intervention on Quality Of Life. The study also explored the impact of the FAB programme on hope, functional performance, self-perceived breathlessness and fatigue.

Methods: This was a single arm, pre-post intervention study. Ethical approval was obtained. Participants attended eight supervised group exercise sessions and were asked to commit to an additional weekly home exercise session. The exercise comprised aerobic and resistance training tailored to each individual. The MDT delivered education sessions on exercise promotion, mindfulness, aromatherapy and management of dyspnoea, fatigue, anxiety, medications and constipation. Outcome measures used were: McGill Quality of Life QuestionnaireRevised, Herth Hope Index, Brief Fatigue Inventory, 30 second sit-tostand (a measure of functional performance) and Borg Rate of Perceived Exertion.

Results: From September 2016 to April 2017, 63 participants were recruited. Complete data was collected on 31 participants (49\%), reflecting the high attrition rate in palliative care research. The FAB programme resulted in significant improvements in breathlessness $(p=0.017)$ and functional performance $(p=0.003)$. While there was no significant improvement in hope $(p=0.375)$, fatigue $(p=0.056)$ or $Q O L(p=0.238)$, the results of this study are positive.

Conclusion: A MDT education and exercise programme in community palliative care demonstrated improvements in breathlessness and function and shows positive trends in other domains. These results add to the evidence that non-pharmacological interventions have an important role in managing these symptoms and may enhance quality of life in this patient cohort. Qualitative research will explore this further.

\section{Abstract number: P02-050}

Abstract type: Poster

Impact of Depression, Anxiety and Malnutrition on Survival of Patients with NSCLC: Highlighting the Need for Early Assessment Kovacevic T. ${ }^{1,2}$, Zaric B. ${ }^{1,2}$, Bokan D. ${ }^{1,2}$

${ }^{1}$ Institute for Pulmonary Diseases of Vojvodina, Sremska Kamenica, Serbia, ${ }^{2}$ Medical Faculty University of Novi Sad, Novi Sad, Serbia

Background: Prevalence of depression and/or anxiety among non-smallcell lung cancer (NSCLC) patients is high. Weight loss and cachexia are common problems in oncology and lung cancer patients are at high malnutrition risk.

Aims: Aim of this study was to examine the impact of depression and/or anxiety and malnutrition risk on overall survival (OS) of patients with NSCLC.

Methods: NSCLC patients rated themselves on the Hospital Anxiety and Depression Scale (HADS). For assessment of malnutrition risk Malnutrition universal screening tool (MUST) was used. Included patients were followed up to five years. Statistical analysis was performed with significance set at $\mathrm{p}=0.05$.

Results: Out of total 108 patients, $75.9 \%$ were male and $24.1 \%$ female, average age 61 , with diagnosed lung adenocarcinoma (60.2\%). Depression 
and/or anxiety were observed in $42.6 \%$, malnutrition risk in $35.1 \%$ and combined burdens in $16.7 \%$ of patients. Man are prone to moderate and women to mild depression $(p=0.021)$. City area inhabitants are more prone to anxiety $(p=0.008)$ and their median OS is shorter $(p=0.004)$. Patients with depression have shorter OS compared with patients with anxiety and without psychiatric disorders $(p=0.041)$. Patients with higher education have significantly higher malnutrition risk $(p=0.006)$. OS of patients with malnutrition risk combined with psychiatric disorder was 5.6 months which is significant when compared to other groups $(p=0.000)$. Median OS of patients with depression and/or anxiety is shorter when compared with patients without psychiatric disorder $(p=0.014)$. One-year OS is significantly shorter in group of patients with malnutrition risk combined with psychiatric disorder (0.006).

Conclusion: NSCLC is often accompanied with depression and anxiety and malnutrition risk all influencing QoL and OS. Addressing these burdens should be of great importance in order to provide the best possible care of lung cancer patients and improve treatment outcome.

Abstract number: P02-051

Abstract type: Poster

\section{Comfort Feeding in Patients with Advanced Cancer: A Secondary} Analysis of the Randomized ALIM-K Study

Grodard G. ${ }^{1,2}$, Anota A. ${ }^{3,4}$, Bouleuc $C^{5}{ }^{5}$, Cornet $C^{1}{ }^{1}$, Royer-Garabige $V^{6}$, Mathieu-Nicot . $^{1}$, Cretin E. ${ }^{1}$, Pazart L. ${ }^{1}$, Aubry R. ${ }^{1,7}$, ALIM-K Study Group ${ }^{1}$ Clinical Investigation Center, Inserm 1431, University Hospital of Besançon, Besançon, France, ${ }^{2}$ University Hospital of Besançon, Department of Dietetics, Besançon, France, ${ }^{3}$ Methodology and Quality of Life in Oncology Unit, Clinical Investigation Center, Inserm UMR 1098, University Hospital of Besançon, Besançon, France, ${ }^{4}$ French National Platform Quality of Life and Cancer, Besançon, France, ${ }^{5}$ Department of Supportive Care, Institut Curie, Paris, France, ${ }^{6}$ Department of Dietetics, Institut Curie, Paris, France, 7 University Hospital of Besançon, Department of Palliative Care, Besançon, France

Background: In malnourished patients at the palliative stage of cancer, the goal is not systematically the optimal diet. Caregivers define their diet like "comfort feeding".

Aims: To evaluate the diet of malnourished patients with solid tumors at the palliative stage.

Method: Secondary analysis from a prospective multicenter randomized control trial evaluating the efficacy of parenteral nutrition vs oral feeding in 13 cancer centers in France (ALIM-K). This analysis included 111 patients ( $n=48$ with parenteral nutrition and 63 with oral feeding), of whom 109 received a dietary consultation at baseline. Diet-related outcomes were monitored during the first 60 days of follow-up using a food questionnaire. A descriptive analysis was done.

Results: At baseline, among 109 patients 69.7\% described anorexia, 45\% nausea and vomiting, $31.2 \%$ constipation, $30.3 \%$ xerostomia. The average difference between energy requirement and energy intake was 872 calories per day $(S D=516)$. The average difference between needs and protein intake was 34 grams per day $(S D=27)$. During the first 60 days of follow-up, 43 patients had at least two measures of the pleasure to eat: $43 \%$ were stable, $25 \%$ improved and $32 \%$ deteriorated without difference between the two arms ( $p$-value $=0.53$ ). Patients had on average 0.8 snack per day (0.59 parenteral arm vs. 0.99 oral feeding arm, p-value 0.006 ) and 2.49 vs 2.74 meals per day respectively ( $p$-value=0.12). Overall in the two arms, 83 patients on 109 reported taking oral nutritional supplements at least once, and only 26 patients took high level of protein and energy supplements (mean 1.91 unit per day).

Conclusion: Although dietary intakes were reduced, patients maintained a traditional and social meal pattern. Three quarters took oral nutritional supplements. Parenteral nutrition didn't influence eating habits.

This study was funded by the French National Cancer Institute (INCa) through PHRC 2011.
Abstract number: P02-053

Abstract type: Poster

Associations between Family Communication and Psychological Health in Parentally Cancer-bereaved Children and Adolescents Weber M. ${ }^{1}$, Alvariza A. ${ }^{1,2}$, Kreicbergs U. ${ }^{1,3}$, Sveen J. ${ }^{1,4}$

${ }^{1}$ Department of Caring Sciences, Palliative Research Centre, Ersta Sköndal Bräcke University College, Stockholm, Sweden, ${ }^{2}$ Capio Palliative Care, Dalen Hospital, Stockholm, Sweden, ${ }^{3}$ Dept of Women and Child's Health, Karolinska Institute, Stockholm, Sweden, ${ }^{4}$ Department of Neuroscience, Psychiatry, Uppsala University, Uppsala, Sweden

Background: Children and adolescents risk developing poor psychological health following a parent's death from cancer. Family communication is a known protective factor for these children and adolescents. Evidence for association between family communication and psychological health exists in other populations but there is a lack of studies examining this relationship in children and adolescents following a parent's death from cancer using both parent and adolescent reports.

Aim: To examine associations between family communication and psychological health of children and adolescents following a parent's death from cancer.

Methods: An online-survey study conducted 2-4 years post-loss. The Parent and Adolescent Communication Scale, Strengths and Difficulties Questionnaire, and Prolonged Grief Disorder Child were completed for three groups: parent reports for children, parent reports for adolescents, and adolescent self-reports. Associations were examined using Spearman's Rho.

Results: According to the parents, communication with their children was moderate in quality. Children and adolescents had good psychological health. More open communication was associated with less hyperactivity and more problem communication was associated with more conduct and emotional problems in children ages 4-11. Parent reports for adolescents indicated that more open communication and less problem communication were associated with more prosocial behavior. Adolescent self-reports indicated low quality communication with their parent and poor psychological health. Better quality communication was associated with fewer conduct problems. Prolonged grief was associated with emotional problems, but not with communication for all three groups.

Conclusions: There was an association between family communication and children's and adolescent's psychological health, indicating that focusing on family communication may be a useful target of clinical intervention for parentally bereaved families.

Abstract number: P02-054

Abstract type: Poster

What Are the Carers' Understanding of Meaningful Involvement in Supporting People with an Intellectual and/or Developmental Disability (IDD) when Someone of Significance to them Is Dying? Oliver D. ${ }^{1}$, Hedayioglu J. ${ }^{2,3}$, Marsden S. ${ }^{4}$

1University of Kent, Tizard Centre, Canterbury, United Kingdom, ${ }^{2}$ Research Department, Kent Community Health NHS Foundation Trust, Ashford, United Kingdom, ${ }^{3}$ Centre for Health Services Studies, University of Kent, Canterbury, United Kingdom, ${ }^{4}$ Maidstone and Malling Learning Disability Team, Kent Community Health NHS Foundation Trust, West Malling, United Kingdom

Background: Including individuals with an intellectual and developmental disability (IDD) in end of life decisions and having health conversations has been highlighted as a research priority (Tuffrey-Wijne et al. 2016). Experiencing the death of someone close is likely to affect an 
individuals' well-being and involving individuals in end of life conversations is important.

Aims: To explore professional carers' views and experiences of preparing and supporting people with an IDD when they are facing the death of a significant person.

Methods: Semi-structured interviews were conducted with 8 paid carers working across two shared living accommodation areas where a recent death of a resident had occurred. Interviews were professionally transcribed, coded and analysed using thematic analysis.

Results: The main themes found are:

Supporting residents

- Conversations about death and dying with residents were rare with focus on other words or things.

- Returning to normality was often seen as best way to support residents.

Training needs

- Carers expressed a wish for greater training and support

- Carers expressed difficulty in knowing how much some residents understood about recent bereavements

- Personal needs

- Paid carers often need support in dealing with their own grief.

Discussion: Paid carers show clear dedication and regard for the residents they care for but are not sure how to meaningfully involve them in the full journey of the bereavement process.

Conclusion: There is a clear need for bereavement support and training for paid carers to enhance their ability to cope themselves and to feel more equipped to support residents.

Ref: Tuffrey-Wijne I et al. Developing research priorities for palliative care of people with intellectual disabilities in Europe: a consultation process using nominal group technique. BMC Palliative Care. 2016; 15:36 DOI 10.1186/s12904-016-0108-5

Abstract number: P02-055

Abstract type: Poster

Bereavement Groups Facilitating the Future for Significant

Others

Näppä U., Björkman-Randström K.

Nursing, Mid Sweden University, Östersund, Sweden

Background: When death strikes, the period of illness ends for the deceased but bereavement and grief have just begun for the significant others. When death is not imminent and the patient has had a palliative care team, bereavement support is routine. Bereavement groups (BG) exist in varying forms, but research following up on people's experiences of participation has been scant.

Aim: To describe significant others' experiences of participation in BG. Methods: Social workers and deacons at a hospital in Sweden arranged BG according to a concept used by the Swedish Church. Bereaved significant others of patients who had received palliative home-care were invited to participate. They were asked to provide written comments on their opinions of the role of the BG in their processing of grief at two occasions after losing their loved one. A qualitative content analysis with a deductive approach was performed using the predetermined structure of the $6 \mathrm{~S}$, a nursing model of care for a good death developed by Ternestedt, Andershed, Eriksson, and Johansson (2002).

Results: The comments $(n=45)$ provided a deeper understanding of the meaning of participation in BG. Over the course of five meetings findings of the 6S: self-image, self-determination, social relationships, symptom control, synthesis and summation, and surrender overall, showed that the groups contributed to a feeling of being seen by others and increased understanding of one's own identity. The structure of the meetings represented an essential journey for coping with grief. However, over time, some participants continued to grieve.

Conclusion: The BG resulted in an overall decrease in grief for most participants, but whether the effect persists over time is unknown. Significant others may continue to experience complicated grief. To help these persons, those working with the bereaved must identify those still experiencing complicated grief. To do so, further contact must be made beyond six months post-death.

Abstract number: P02-056

Abstract type: Poster

Who Provides Bereavement Support in the Community and Which Sources Are Perceived Helpful? Evidence to Strengthen the Compassionate Communities Approach Aoun S. ${ }^{1,2}$, Breen L. ${ }^{3}$, Rumbold B. ${ }^{1}$, White.$^{3}$, Kellehear A. ${ }^{4}$

${ }_{1}^{1}$ La Trobe University, Melbourne, Australia, ${ }^{2}$ Perron Institute for Neurological and Translational Science, Perth, Australia, ${ }^{3} \mathrm{Curtin}$ University, Perth, Australia, ${ }^{4}$ Bradford University, Bradford, United Kingdom

One of the modern public health paradoxes is the neglect of the bereaved though bereavement is a universal experience. This study is about determining who provides bereavement support in the community, what sources are perceived to be the most or least helpful and for what reason and identify the empirical elements for optimal support in developing any future Compassionate Communities approach.

Bereaved people were recruited from databases of funeral providers in Australia via an anonymous postal survey. Sources of support (informal, community and professional) were categorized according to the Public Health Model of Bereavement Support; most helpful reasons were categorised using the Social Provisions Scale, and least helpful were analysed using a thematic approach.

678 bereaved people responded to the survey. The most frequently used sources of support were in the informal category such as family, friends, funeral providers. While the professional category sources were the least used, they had the highest proportions of perceived unhelpfulness and the lowest proportions of unhelpfulness were in the informal category. The types of helpful support were: Attachment, Reliable Alliance, Social Integration and Guidance. Those for least helpful support were: Insensitivity, Absence of Anticipated Support, Poor Advice, Lack of Empathy and Systemic Hindrance.

A public health approach should be adopted to support the majority of bereaved people as this support is already provided in informal and other community settings by a range of people already involved in the everyday lives of those recently bereaved. These everyday 'assets' who care for the majority of the bereaved should also be supported, without the overreach from professional services. This study has provided further evidence for the need to strengthen the Compassionate Communities approach, not only for end of life care for dying patients but also along the continuum of bereavement support.

Abstract number: P02-057

Abstract type: Poster

Bereaved Caregivers/Family Members' Perceived Quality of Dying and Death in Intensive Care: A Narrative Systematic Review

Lo M.L. ${ }^{1}$, Huang C.C. ${ }^{2}$, Tang S.T. ${ }^{1}$

${ }^{1}$ Chang Gung University, Taoyuan, Taiwan, Republic of China, ${ }^{2}$ Chang Gung Memorial Hospital at Linkou, Taoyuan, Taiwan, Republic of China

Background: Quality of end-of-life (EOL) care in intensive care units (ICUs) is highly valued, but studies on this topic have not been systematically reviewed. This study aimed to fill that gap. 
Methods: Four electronic databases ( 2018) were searched for studies on EOL-care quality in ICUs. We included studies that measured caregivers' perceptions of patient experiences by the most commonly used Quality of Dying and Death (QODD) questionnaire to synthesize its six domains by frequency and experience. The best quality of care is rated by a frequency of most/all of the time. The quality of experience is rated on a 0-10 Likert scale.

Results: We retrieved five articles (2005-2018) on patients admitted to an ICU for $>48-72$ hours, with an average stay of 6-8 days. On average, 161 (SD=115) (range 50-346) caregivers were assessed by the QODD 3-4 weeks post bereavement. Best quality of care (prevalence) was moment of death $(87 \%)$, family concerns $(84 \%)$, treatment preferences $(68 \%)$, symptoms and personal care (62\%), preparation for death (58\%), and whole-person concerns (49\%). Experience ratings (average [SD]) were moment of death (8.50 [0.70]), family concerns (7.09 [0.84]), wholeperson concerns (7.01 [2.34]), treatment preferences (6.84 [0.48]), preparation for death (6.67 [1.36]), and symptoms and personal care (6.46 [1.33]). The best quality items were $78 \%$ of patients had religious visits (rating: 8.05), pain under control most/all of the time in $76 \%$ of patients (rating: 8.04), 84\% of patients had enough time for family to visit (rating: 7.69 ), but only $48 \%$ of patients breathed comfortably (rating: 5.36 ) and $44 \%$ of patients had an opportunity to discuss EOL-care issues (rating: 6.99).

Conclusions: The two domains rated as having poorest EOL-care quality in ICUs are symptoms and personal care as well as preparation for death, highlighting specific needs for adequate symptom management and facilitating caregivers' death preparation to improve quality of EOL care in ICUs.

\section{Abstract number: P02-058 \\ Abstract type: Poster}

Prolonged Grief is Associated with Different Factors During the Child's Illness for Mothers and Fathers

Pohlkamp L., Kreicbergs U., Sveen J.

Department of Health Care Sciences, Palliative Research Centre, Ersta

Sköndal Bräcke University College, Stockholm, Sweden

Background: The death of a child is one of the most painful events a parent may experience. Bereaved parents are known to be at an increased risk for intense and prolonged grief responses such as Prolonged Grief Disorder (PGD). More knowledge is needed to understand mothers' and fathers' experiences of their children's disease trajectory and possible associations to prolonged grief in order to offer better support to grieving parents.

Objective: The aims of this study were to assess symptoms of PGD in bereaved mothers and fathers and to examine possible contributing factors to parent's grief.

Methods: A Swedish population based survey including 133 mothers and 92 fathers who had lost a child to cancer 1-5 years previously. Bivariate and multiple regression analyses were conducted to assess the associations between prolonged grief and independent variables.

Results: Mothers had significantly higher PGD symptom levels than fathers. Suffering of mothers was associated with their child's pain, anxiety and with not being able to talk within the family, whereas the suffering of fathers was associated with lack of information from health care staff and with the feeling of having too much responsibility in the care of the child.

Conclusions: Mothers and fathers reported differences in factors associated with their grief following the death of their child. The results suggest that mothers and fathers would benefit from different kinds of support during their child's illness, which could be considered when developing pediatric palliative care practice.

Abstract number: P02-059

Abstract type: Poster
Care for the Minors after Death of Dear One. Review of International Literature Regarding Methods of Support of Bereaved Children and Teenagers

Legowska E. ${ }^{1}$, Paczkowska A. ${ }^{2}$, Krakowiak P. ${ }^{2}$

${ }^{1}$ Educational Sciences, Ac Umk, Social Work, Nicolaus Copernicus

University, Torun, Poland, ${ }^{2}$ Educational Sciences, Social Work, Nicolaus Copernicus University, Torun, Poland

Purpose: This literature review has examined different forms of therapies used for bereaved children and teenagers who are affected after loss as family members and caregivers. The negative symptoms associated with end of life care and main problems of bereavement among minors will be presented, and main groups of techniques and methods will be analyzing in this presentation. Different group of strategies and methods will be presented.

Data sources: An electronic database search was used to collect articles for this literature review in the following databases: MEDLINE, Academic Search Complete, CINAHL Complete and psychINFO, Caresearch, PubMed, PMC, EBSCO - sociology-social-work; Social Science Database. Evaluated research - both qualitative and quantitative, as well as all existing reports regarding different kinds of techniques used with minors experiencing losses have been reviewed and will be presented.

Implications: Minors in families towards the end of life could be still not adequately supported in their grief, so it is important to know different approaches and techniques, in which children can participate in these therapies in meaningful ways. Knowledge about forms of grief therapies could open those who use only certain strategies in grief and bereavement to new approaches. That information could be a useful care tool for caregivers of those experiencing loss, as well as for PC team members and researchers.

Conclusion: Analysis of various forms of grief therapies and techniques used to help minors in their grief can effectively help those who help, opening them for new dimensions in order to better meet needs of young bereaved. Authors do hope to show complete list of support tools for caregivers of those minors in bereavement process, who should be cared by PC teams and supported also by schools and local communities.

\section{Abstract number: P02-060 \\ Abstract type: Poster}

'Ability to Cope with Grief' and 'Quality of Life and Mental Wellbeing': Two Core Outcomes for Evaluating Bereavement Support in Palliative Care

Harrop E. ${ }^{1}$, Scott H. ${ }^{1}$, Seddon K. ${ }^{1}$, Fitzgibbon J. ${ }^{1}$, Morgan F. ${ }^{1}$, Pickett S. ${ }^{2}$, Byrne A. ${ }^{1}$, Sivell S. ${ }^{1}$, Nelson A. ${ }^{1}$, Longo M. ${ }^{1}$

${ }^{1}$ Marie Curie Research Centre, Cardiff University, Cardiff, United Kingdom, ${ }^{2}$ Swansea Centre for Health Economics, Swansea University, Swansea, United Kingdom

Background: Palliative care has an established role in the provision of bereavement support. However, the evidence base is limited by a lack of consistency in the outcomes used to evaluate services, which makes it difficult to compare approaches and draw conclusions on 'what works best'. Core Outcome Sets (COS) represent the 'minimum that should be measured and reported' in research into specific conditions or services [www.comet-initiative.org]. The aim of this study was to use a stakeholders' perspective to develop a COS for evaluating bereavement support in palliative care for adults who have lost adults through terminal illness.

Methodology: A list of outcomes relevant to bereavement support was created following a systematic review of the quantitative and qualitative literature. At a consensus day 21 stakeholders discussed what they considered to be the most important outcomes and compared these to the lists generated from the review. These lists and discussions informed a two round DELPHI survey $(n=238)$ designed to reach consensus on which outcomes/outcome dimensions should be included in the COS. At a final 
consensus day participants ranked the relative importance of the items which reached consensus in the survey.

Results: Results suggest that the two most important outcomes are 'Ability to cope with grief' and 'Quality of life and mental wellbeing'. A number of core dimensions to explore when measuring these outcomes were also identified. Examples of coping dimensions included; 'Acceptance of grief experiences as normal' and 'Ability to find balance and channel grief'. Examples of quality of life and wellbeing dimensions included; 'Relationships with friends and family' and 'Sense of meaning and purpose in life.

Conclusion: No available validated measures were identified which cover all core dimensions, although some have better 'fit' than others. Further work is being planned to develop suitable measurement tools for future research and evaluation.

\section{Abstract number: P02-061}

Abstract type: Poster

\section{Bereavement Care Following Biocide Disaster and the Role of Social Work \\ OhS. \\ Durham University, Sociology, Durham, United Kingdom Presenting author email address: seunghoon.oh@durham.ac.uk}

Background:.The Korea Centres for Disease Control reported in 2018 that 6174 suspicious cases of Humidifier Disinfectant were used as a toxic chemical in South Korea. The total number of deaths was 1,357 , and 4,817 people suffered lung damage and a wide range of rare diseases. Moreover, according to Supporters for Health and Rights of People in Semiconductor Industry, the number of workers' illnesses is 368 and deaths toll hits 134 from electronic industry in South Korea.

Purpose: This article aims to identify the social worker's role in antioppressive practice, coping with the loss of a loved one and oppression. Methodology: This research in social work based on critical ethnography observation, semi-structured interviews, video analysis and document analysis. This methodology helps to understand the effects of the loss of a loved one made by biocide disaster and the process of the role of social workers. In total, 12 interviews took place.

Results: The emerging themes were identified and elaborated in the following areas:

(1) This bereavement care following the biocide disaster is related to existing oppression regarding political, economic, social, and cultural aspects in South Korea.

(2) The role of palliative social work should require the alleviation of not only disaster-related grief but also the oppression regarding local, national, and international levels.

(3) Focusing on the patient and their family members can help to eradicate anti-oppressive practitioners, their oppression, dealing with their guilty feeling in securing bereavement care and embracing the concepts of participation and empowerment.

Conclusion: An anti-oppressive practice implemented in South Korea exemplifies the ways of addressing the concealed information. It implies that resilience requires cooperation and communication across multiple organisations, professionals, and community groups, and promotes healthy communities.

Abstract number: P02-062

Abstract type: Poster

Burial or Cremation? The Significance of Choices: A Mixed

Methods Rural Study

Rainsford S. ${ }^{1}$, Phillips C. ${ }^{1}$, MacLeod R. ${ }^{2}$, Wilson D. ${ }^{3}$
${ }^{1}$ Medical School, Australian National University, Canberra, Australia, ${ }^{2}$ Hammond Care, Greenwich, Australia, ${ }^{3}$ Faculty of Nursing, University of Alberta, Edmonton, Canada

Background: One factor associated with the "good death" is being prepared for one's death. Funeral planning is one aspect of this preparation. Few studies describe the funeral rituals or interment preferences in rural settings.

Aim: To describe the current funeral rituals and modes of interment for decedents in one Australian rural region; to explore the funeral and interment preferences of rural people living with life-limiting illness and their family caregivers.

Methods: Ethics approved mixed methods study. Quantitative component: cross-sectional survey of all deaths ( $1^{\text {st }}$ February 2015 to $31^{\text {st }}$ May 2016). Data were analysed using descriptive statistics and logistic regression; dependent variable was interment practice - cremation or burial. Qualitative component: ethnographic methodology; 2 patients with terminal illness and 4 bereaved family caregivers were interviewed. Interviews were semi-structured, audio recorded, transcribed, and analysed thematically.

Results: 224 decedents were identified; $90(40 \%)$ cremations were recorded; $106(47.5 \%)$ of the funeral services were conducted in a church. Funeral planning was important with preferences for method of interment often aligned with preferences for the final place of deposition of the body. Burial was common due to strong family traditions, generational connections to historic cemeteries, the desire to maintain a sense of belonging even after death, and the inconvenience of attending out-of-region crematoriums. Conversely, cremation was cost effective and provided opportunity for some to postpone the interment and to scatter ashes at personally-significant places. Funerals are an important community event.

Conclusion: Despite limitations, this study begins to address a gap in the literature. Rural perspectives and practices do not necessarily reflect the urban voice. Further studies are required to better understand the significance of choices as this may influence the bereavement process.

\section{Abstract number: $\mathrm{P} 02-063$ \\ Abstract type: Poster}

Bereavement Program Evaluation - A Research Literature

Review to Establish a Method for Future Program Evaluations Wilson D.M. ${ }^{1}$, Errasti-Ibarrondo B. ${ }^{2}$

${ }^{1}$ Faculty of Nursing, University of Alberta, Edmonton, Canada, ${ }^{2}$ School of Nursing, University of Navarra, Pamplona, Spain

Background: A wide range of bereavement programs are often available now to grieving people, with these designed and offered by well-meaning lay people and organizations or by professional organizations and professionals with expertise in grief counselling or with other relevant skills and knowledge gained through accredited educational programs. All bereavement programs may be beneficial to the people who access them, but it is also possible that some are harmed by the program they attend.

Methods: As there is no apparent requirement that these programs be evaluated and also no standard evaluation method, a scoping literature review of bereavement support or grief recovery program evaluations was undertaken to identify evaluation methods and assess their apparent efficacy for evaluating program impacts or outcomes.

Results: A total of 44 research articles published in 2000-18 were reviewed to reveal considerable variability in evaluation methods, with multiple data collection methods employed in $61.4 \%$ of the studies. A self-devised questionnaire or survey was most often used to collect data (54.5\%), followed by qualitative interviews $(36.4 \%)$, previously-developed data collection instruments, specifically 29 different grief inventories or depression scales (36.4\%), as well as objective data collection measures such as health services utilization data (13.6\%), focus groups 
(4.5\%), observation (4.5\%), and written diary or document analysis (4.5\%). Evaluation data were usually collected at only one point in time (77.3\%), typically upon or shortly after program completion.

Conclusions: Bereavement program evaluation needs development. Recommendations are made in light of these findings, including research to determine which ones of the 29 established tools are more efficacious for collecting relevant, reliable, and comparable data.

Abstract number: P02-064

Abstract type: Poster

Review of Literature Regarding Atypical Methods of Support of Bereaved Children and Teenagers. Initial Research of Grief Support through Activities with 'Scientific Tools' in Poland Legowska E. ${ }^{1}$, Krakowiak P. ${ }^{2}$ ${ }^{1}$ AC UMK, Social Work, Nicolaus Copernicus University, Torun, Poland, ${ }^{2}$ Social Work, Nicolaus Copernicus University, Torun, Poland

Purpose: This literature review has shown different forms of therapies used for bereaved children and teenagers. Apart from typical forms of help, there are innovative, atypical and unusual forms of assistance, which have been researched and presented.

Data sources: Personal experience of one of researchers, being medical physicist, teacher and hospice volunteer working with bereaved children as well as initial research from scientific activities as tools for bereaved minors have been presented. An electronic database search was used to collect articles for this literature review in the following databases: MEDLINE, Academic Search Complete, CINAHL Complete and psychINFO, Caresearch, PubMed, PMC. Evaluated studies were qualitative and quantitative, as well as all existing reports regarding different kinds of techniques used with bereaved minors experiencing difficulties after losses.

Implications: Nowadays especially young people should be supported in difficult times, such as loss, grief and mourning. There are many techniques to work with bereaved children, and the more tools are used, the better. This presentation has shown existing forms of support, stressing in this report especially new, atypical and unusual methods of support and care. Usage of classes with 'scientific tools' as a way of support for bereaved children and adolescents has been presented.

Conclusion: Analysis of existing good practices to support bereaved children with proposals for new forms can effectively help in working with young people. This may not only broaden the horizons of therapists and specialists, but also open up to a new dimension of work with bereaved children and young people in local communities.

Abstract number: P02-066

Abstract type: Poster

Education and Support after Death for Bereaved Minors in Schools and Local Communities as an Important Task of PC Teams. Comparison of Activities in the UK, Ireland, and Poland Krakowiak P. ${ }^{1}$, Paczkowska A. ${ }^{1}$, Legowska E. ${ }^{2}$

${ }^{1}$ Educational Sciences, Social Work, Nicolaus Copernicus University,

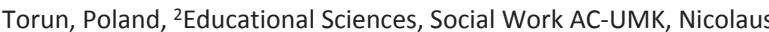
Copernicus University, TORUN, Poland

Purpose: To identify educational initiatives and ways of support for bereaved minors after death in schools and local communities. Good cooperation between PC and schools, in health education and early intervention resulted in creation of integrated models of support in Great Britain and Ireland. In Poland, this process has started but has been not yet sufficiently documented. The results of research of the first comprehensive program for bereaved children in Poland shows the ways in which PC can promote education about end of life and bereavement in schools.
Data sources: Research from Poland has focused on school staff (over 500 people) from about 100 educational institutions in one of the major cities in Poland. To compare similar initiatives in the UK and Ireland an electronic database search has been used to collect articles for this literature review in the following databases: MEDLINE, Academic Search Complete, CINAHL Complete and psychINFO, Caresearch, PubMed, PMC. Implications: To recognize effects of loss among children, it is important for families and teaching institutions to work together with PC team. Crucial are also early intervention measures towards people in grief, as well as early preventive actions in schools. Children should recognize death as natural element of every human life and develop solidarity with bereaved. This pilot study identified elements useful for teachers in education process and show areas of possible cooperation of schools and PC teams.

Conclusion: Analysis of the available data has shown importance of ongoing education for teachers, and the early prevention helping avoid complications in the grieving process. In addition steps towards teachers' professionalization in support of bereaved children are needed. Research shows how PC team in Poland has build awareness of the needs of young orphans in local communities. Actions should be taken to move it from regional to the national and international levels.

Abstract number: P02-067

Abstract type: Poster

Overlooked Bereavement Process

Bernot M. ${ }^{1}$, Ozbič P. ${ }^{2}$, Podlesnik K. ${ }^{3}$, Ebert M. ${ }^{4}$

${ }^{1}$ Acute Palliative Care, Institute of Oncology Ljubljana, Ljubljana,

Slovenia, ${ }^{2}$ in a process of change, Maribor, Slovenia, ${ }^{3}$ Duhovno

Prosvetni Center Dom sv. Jožef, Celje, Slovenia, ${ }^{4}$ Institute of Oncology

Ljubljana, Ljubljana, Slovenia

Most people manage to cope with their grief on their own if they have some healthy habits,social support.Anyway, in a holistic view everyone should be given information about the bereavement process.Some of them required the professional intervention,because needs become complex.

The aim of the research was overviewing the current situation on bereavement process and grief identification.

A quantitative method was used.Data were collected with a structured questionnaire (literature review-based on professional, scientific sources),from October 2017 to March 2018.We included 162 experts, involved in different fields of palliative(health centres, hospitals, nursing homes, hospice) $25 \%$ physicians, $43 \%$ register nurses, $9 \%$ nurse assistant, $7 \%$ social workers, $2 \%$ psychologists, others $14 \%$. The average years of work was: under 20 years $45 \%$ and between 11 to 20 years $32 \%$.

The majority of respondents' $52 \%$, are dealing with death and dying 10 to 49 times/year. $46 \%$ of them not offer any support. If complex bereavement is identified, just $38 \%$ would send the bereaved to others experts. If they provide support, this happens in $27 \%$ less than 9 times/year. Mostly this support is when death happen $37 \%$, and less often before and after the death.The most frequent time of bereavement support is first month. They do not collect any data about the bereavement process or care.The respondents do not use any of the risk assessment tools and do not use any of the recommendations. They agree that bereavement is mostly unrecognised $80 \%$. According to our respondents the most common reasons for that are: lack of time, professionals, staff, funds and knowledge.

The results of the study show the necessity to talk about the bereavement. The tools for empowerment the professionals were identified: education, workshops, provision of literature, Letters/cards to family, telephone support as well as Bereavement Counselling, identify support groups, referral to psychiatrist or psychological support.

Abstract number: P02-068

Abstract type: Poster 
Spouse Caregivers Perception of the End Stages of Life and Death of the Patient at Hospital: An Exploratory Qualitative Study during the Mourning Period

Evin A., Nizard J., Maillard B.

Centre Fédératif Douleur, Soins de Support, Ethique Clinique, Centre Hospitalier Universitaire de Nantes, Nantes, France

Background: Cancer patients have multifaceted needs, including symptom management, medication administration, emotional support and assistance with personal care. Family caregivers have their own emotional responses to the patients' prognosis, and may require coaching and emotional support themselves.

Aim: To adequately help family caregivers of cancer patients in palliative phase, clinicians need to understand the complexity of the problems associated with cancer patients illness that family caregivers experience. We examine the spouse caregiver perception of the final stages of the cancer and mourning period.

Design: An exploratory, qualitative study was conducting employing narrative interviews among spouse caregivers.

Setting: Interviews were conducted in person, at the hospital, or at home, using a semi structured protocol. Interviews were conducted among 15 spouses caregivers contacted in writing three months after the death of the patient.

Results: Four themes were identified : impact of the disease on the patient's body ; temporal discontinuity in the care pathway ; the need for hope ; integration and symbolization of losses. Some themes reflected distress experienced spouse caregivers and revealed important points in the trajectory of care that have the potential for negative effects.

Conclusion: The spouse caregivers implements processes of integration of losses and their own defense mechanisms in the face of this inevitable end of life and death reality. Particular interest should be given to developing guidelines for caregiver assessment, identifying resources, interventions that provide direct practical and psychological support to assisting spouses of end-of-life patients. More attention is needed to reduce the distress of the family at the end of life and during the first month of bereavement period.

Abstract number: P02-070

Abstract type: Poster

Development of a Successful and Reproducible Bereavement Support Day for Families O'Gorman B. ${ }^{1}$, Portnoy S. ${ }^{1}$, Victor J. ${ }^{1}$, Craig F. ${ }^{2}$

${ }^{1}$ Life Force Team - Community Paediatric Palliative Care, Whittington Hospital, London, United Kingdom, ${ }^{2}$ Louis Dundas Centre Oncology Outreach and Palliative Care, Great Ormond Street Hospital for Children NHS Foundation Trust, London, United Kingdom

Aim: To develop a bereavement support day for families cared for by a community paediatric palliative care team that:

- Provides families a protected space to remember their child and receive support

- Enables families to connect with others who have experienced the death of a child, creating a supportive community

- Reconnects families with members of the team who supported them through end of life.

Design, method and approach: Established in 2003, our team has supported 132 bereaved families. We developed a community bereavement day that was different to the memorial ceremonies offered by other services. The day has a strong social focus with structured bereavement work and a short candle lighting activity, as well as play activities for children. Shared meals and conversation are a central part of the day. The bereavement work is focused around a creative activity, with each attendee going home with something they have created in memory of their child/sibling. Each day is evaluated and telephone follow up is offered to each family after the day.

Results: On average 16 families attend the day, some every year. Families attend for the whole or part of the day. Both quantitative and qualitative evaluation will be presented. Positive feedback from families includes the opportunity to meet other families and to see staff who cared for their child. Benefits for staff, and more newly bereaved parents, include seeing that families can reach a place where grief is less raw and they can live alongside their loss. Challenges for the day include the significant amount of planning and preparation, and ensuring adequate staff numbers to support the families.

Conclusions: We have created a successful 1 day bereavement program that we believe can be re-produced by other palliative care services.

\section{Abstract number: P02-071}

Abstract type: Poster

Bereavement Attention in Palliative Care: Comparison between Spain and Other European Countries

Lacasta M. ${ }^{1}$, Keegan $0 .{ }^{2}$, Murphy I. ${ }^{3}$, Monroe B. ${ }^{4}$, Benkel I. ${ }^{5}$, Guldin

M.-B. ${ }^{6}$, Limonero J.T.

${ }^{1}$ Palliative Care Unit, Hospital Unviersitario la Paz, Madrid, Spain, ${ }^{2}$ rish Hospice Foundation, Dublin, Ireland, ${ }^{3}$ Bereavement \& Family Support Services, Marymount University Hospice, Cork, Ireland, ${ }^{4}$ Honorary Professor International Observatory, Lancaster University, Lancaster University, United Kingdom, ${ }^{5}$ Palliative Section, Gothenburg University Hospital, Gothenburg, Sweden, ${ }^{6}$ Palliative Care, Aarhus University Hospital, Aarhus, Denmark, ${ }^{7}$ Stress and Health Research Group. Faculty of Psychology, Universitat Autònoma de Barcelona, Bellaterra (Cerdanyola del Vallès), Spain

Background: Palliative care (PC) in Spain and Europe includes attention to bereavement. Public health models of support are recommended. These services vary between countries.

Aims: Compare bereavement care in PC services in Spain (SP) with other countries in Europe (EU) regarding public health models and NICE framework.

Method: Descriptive study. An online survey was used to describe current support. This survey was sent to 56 national associations of 32 countries. This survey was based on previous research. Comparisons between SP and other European Countries are made using Fisher's exact test or the test of Chi-Square. IBM SPSS Statistics were used.

Results: 56 (15\%) answers from PC in SP versus 314 (85\%) EU answers. $41(73 \%)$ offer a bereavement service in Spain versus $261(83 \%)$ in the other countries. Bereavement care varies: Volunteer Bereavement Care Service in the Community, EU 50\% vs SP $20.5 \%(p<.05)$; Bereavement groups in the community, $51 \%$ EU vs $49 \%$ SP ( $p>.05)$; Professional advice, $55 \%$ EU vs $49 \%$ SP ( $p>.05)$; Mental health services in the community, $52 \mathrm{EU}$ vs $41 \%$ SP ( $>>.05)$ and Information from internet EU $17 \%$ vs $10 \%$ SP $(p>.05)$. Bereavement care is provided by Volunteer services, EU $59 \%$ vs $26 \%$ SP ( $p<.05)$; Church $40 \%$ EU vs $11 \%$ SP $(p<.05)$; Services offered by the national health service, $52 \mathrm{EU}$ vs $69 \mathrm{SP}(p>.05)$ and by Professional payment services $47 \%$ EU vs $40 \%$ SP ( $p>.05)$.

Conclusions: The range of bereavement service options in palliative care are similar in EU and SP. However, in EU more bereavement services are offered by voluntary services than in SP, where state support is evident.

Abstract number: P02-072

Abstract type: Poster

A Randomized, Double-blind, Non-inferiority Study of Hydromorphone Hydrochloride Sustained-release Tablets versus Oxycodone Hydrochloride Sustained-release Tablets for Cancer Pain: Efficacy and Safety in Japanese Cancer Patients 
Saito Y. ${ }^{1}$, Tsuneto S. ${ }^{2}$, Aruga E. ${ }^{3}$, Takahashi H. ${ }^{4}$, Uemori M. ${ }^{5}$

${ }^{1}$ Faculty of Medicine, Anesthesiology, Shimane University, Shimane, Japan, ${ }^{2}$ Human Health Sciences, Graduate School of Medicine, Kyoto University, Kyoto, Japan, ${ }^{3}$ Palliative Medicine, Teikyo University School of Medicine, Tokyo, Japan, ${ }^{4}$ Clinical Development Department, Daiichi Sankyo CO,. LTD, Tokyo, Japan, ${ }^{5}$ Biostatistics \& Data Management Department, Daiichi Sankyo CO., LTD, Tokyo, Japan

Background: Hydromorphone is a standard opioid analgesic for cancer pain that is not yet approved in Japan, where options for opioid switching are limited.

Aims: To confirm the efficacy and safety of hydromorphone sustainedrelease tablets in opioid-naïve cancer patients with moderate to severe cancer pain.

Methods: This was a multicenter, joint, active-controlled, randomized, double-blind, parallel-group, non-inferiority study where hydromorphone tablets or oxycodone tablets was orally administered for 7 days. The initial dose of hydromorphone and oxycodone hydrochloride was $4 \mathrm{mg} /$ day and $10 \mathrm{mg} /$ day, respectively, and the dose adjusted as necessary. Efficacy was evaluated as the intergroup difference (95\% confidence interval $[\mathrm{Cl}]$ ) of the least squares mean by analysis of covariance, using the baseline visual analog scale (VAS) as a covariate for change in VAS score at treatment completion/discontinuation in the full analysis set.

Results: Non-inferiority of hydromorphone versus oxycodone was confirmed, with an intergroup difference $(95 \% \mathrm{Cl})$ in the least squares mean of $-0.4 \mathrm{~mm}[-5.9,5.0])$ for change in VAS scores, which was below the upper limit of the $95 \% \mathrm{Cl}$ at $10 \mathrm{~mm}$, the non-inferiority limit determined at the time of planning. Adverse events occurred in $80.7 \%(71 / 88)$ patients in the hydromorphone group and $83.7 \%(77 / 937)$ in the oxycodone group. The most frequently observed adverse events were nausea, vomiting, somnolence, diarrhea, and constipation. The results were similar to those of a comparative study of hydromorphone and oxycodone immediate-release formulations reported by us in EAPC2016. ( intergroup difference $(95 \% \mathrm{Cl})$ in the least squares mean of $-3.4 \mathrm{~mm}$ $[-9.8,3.1])$

Conclusion: The efficacy and safety of hydromorphone once-daily tablets are equivalent to those of oxycodone tablets.

This study was funded by Daiichi Sankyo Co., Ltd.

Abstract number: P02-073

Abstract type: Poster

Hydromorphone Hydrochloride Injection for Management of Cancer Pain: An Open-Label, Multi-Center Study for Japanese Cancer Patients

Saito Y. ${ }^{1}$, Tsuneto S. ${ }^{2}$, Aruga E. ${ }^{3}$, Takahashi H. ${ }^{4}$, Kakurai Y. ${ }^{5}$

${ }^{1}$ Faculty of Medicine, Anesthesiology, Shimane University, Shimane, Japan, ${ }^{2}$ Human Health Sciences, Graduate School of Medicine, Kyoto University, Kyoto, Japan, ${ }^{3}$ Palliative Medicine, Teikyo University School of Medicine, Tokyo, Japan, ${ }^{4}$ Clinical Development, Daiichi Sankyo CO. LTD., Tokyo, Japan, ${ }^{5}$ Biostatistics \& Data Management Department, Daiichi Sankyo CO., LTD, Tokyo, Japan

Background: Hydromorphone is a standard opioid analgesic for cancer pain that is not yet approved in Japan, where options for opioid switching are limited.

Aims: To confirm the efficacy and safety of hydromorphone injection in opioid-naïve and opioid-tolerant patients with moderate to severe cancer pain.

Methods: This was an open-label multicenter study for hydromorphone injections. Eligible patients were moderate to severe cancer pain under hospitalized. The initial dose of hydromorphone was $0.5-$ $1.0 \mathrm{mg} /$ day for opioid-naïve patients and equivalent to $1 / 8$ of morphine injection for opioid-tolerant patients, respectively. And the dose adjusted as necessary after 24 hours from initial dose. The efficacy endpoint was changed in VAS score from baseline to completion or discontinuation of treatment. Summary statistics were calculated for VAS scores at baseline, at the completion or discontinuation of treatment in the full analysis set. Rescue medication was investigated until Day 7 and evaluated efficacy and safety. The treatment period was up to 29 days ( 28 days in the study treatment and 1 day is a follow-up).

Results: Seventy-one patients were enrolled, of which 70 were included in the safety analysis population and 69 were included in the FAS. The mean VAS was decreased from $59.5 \mathrm{~mm}$ to $34.1 \mathrm{~mm}$ in opioid-naïve patients and from $32.4 \mathrm{~mm}$ to $21.4 \mathrm{~mm}$ in opioid-tolerant patients at the completion or discontinuation of treatment. The mean change in VAS for both groups was $-12.3 \mathrm{~mm}(95 \% \mathrm{Cl}[-17.8,-6.7] \mathrm{P}<$ $0.001)$. The efficacy of rescue medication was improved by $80.1 \%$ $(149 / 186)$ on pain relief assessment at 10 minutes after dosing. Adverse events occurred in $40.0 \%(28 / 70)$ patients, and the most frequently observed adverse events were somnolence, nausea, constipation, and vomiting.

Conclusion: The efficacy and safety of continuous infusion of hydromorphone injection were confirmed.

This study was funded by Daiichi Sankyo Co., Ltd.

Abstract number: P02-074

Abstract type: Poster

The Electronic Medical Record Can Be Used to Measure

Palliative Care Team Impact on Pain Scores

Burns B., Ferris F., von Gunten C.

Hospice and Palliative Medicine, OhioHealth, Columbus, United States

Background: It is challenging for team members to record patient-level data that enables measurement of clinical impact. We wondered if the electronic medical record, where floor nursing routinely records pain scores, could be used to measure the palliative care team's impact on pain.

Aim: Measure the change in pain scores for patients seen by specialist palliative care teams in general community hospitals.

Methods: A large health system of 11 hospitals with an aggregate of 2,000 beds serving a population of 3.5 million people uses the EPIC electronic medical record. In 2015, first and last pain scores recorded by nursing for all hospitalized patients seen by a palliative care team over a 12 -month period were measured. There was no difference. An education program in conjunction with individual physician feedback on breakthrough opioid dosing intervals yielded a change from zero to $80 \%$ adherence. Then, the comparison of first and last pain scores for all patients seen by palliative care and receiving opioids was repeated for a subsequent 12 month period in 2017-2018.

Results: For all hospitalized patients receiving morphine, oxycodone, or hydromorphone via IV, SC, SL, GT, or PO routes, the first mean pain score was 5 and the last mean pain score was $3(n=2,984, p<0.0001)$. When the analysis was repeated for patients with pain score $\geqslant 6$, the first mean pain score was 8 and the last mean pain score $5(n=1,315, p<$ 0.0001).

Conclusion: The electronic medical record showed a $40 \%$ improvement in mean pain scores. When only patients with severe pain were compared, there was also a $40 \%$ improvement. We conclude the data routinely recorded in the electronic medical record can be used to measure important outcomes of specialist palliative care teams in general hospitals. This methodology overcomes the barrier of clinical palliative care team members recording data on their own with the potential bias of patients reporting what the palliative care team wants to hear.

Abstract number: P02-075

Abstract type: Poster 
The Relief Therapy: The Use of Virtual Reality for Pain and Distress Management in Home Palliative Care Setting Varani S. ${ }^{1}$, Sichi V. ${ }^{1}$, Pluchino P. ${ }^{2}$, Ercolani G. ${ }^{1}$, Roganti $D .{ }^{1}$, Gengarelli G. ${ }^{2}$, Pannuti R. ${ }^{1}$, Pannuti F. ${ }^{1}$, Gamberini L. ${ }^{2}$ ${ }^{1}$ ANT Italia Foundation, Bologna, Italy, ${ }^{2}$ University of Padua, Padova, Italy

Background: About 2 out of 3 oncological patients feel pain and approximately $40-80 \%$ of them suffer from breakthrough cancer pain (BTCP). Some studies confirm the virtual reality efficacy during pain management in oncological hospital context. But not so much studies were conducted about the use of virtual reality (VR) at home.

Aim: This pilot study assesses the feasibility of the VR for pain and distress management in home palliative care setting testing which kind of virtual reality scenarios the patients prefer.

Method: At the beginning of the study, a focus group was conducted to investigate what kind of stimulation could be more pleasant for patients. Naturalistic scenarios with a relaxing music were preferred by patients, as well as art museums settings. After this phase 4 videos were chosen. The pilot study involved 58 cancer patients, followed by a psychologist who showed them the 4 videos during 1 week. At the beginning and at the end of each session, patients were asked to complete the Edmonton Symptom Assessment Scale (ESAS) to assess pain and other symptoms, and The Hospital Anxiety and Depression Scale (HADS) to assess anxiety and depression.

Results: The qualitative results showed that the $73 \%$ of the patients that used the VR had a positive impression of the technique. The quantitative analysis obtained with the ESAS assessment showed, just after the first session, a significant reduction of perceived pain, fatigue levels and anxious symptoms $(p<.01 ; p<.01 ; p<.05)$. The reduction persist also in the 4 st session. A reduction in anxiety $(p<.01)$ and depression $(p<$ .001) measured with HANDS scale was observed as well.

Conclusion: These positive results suggest that this innovative technique could positively impact on pain, anxiety and depression in patients in home care. This study underlines the applicability of virtual reality in home care setting, opening the way to additional studies in this context.

\section{Abstract number: P02-076 \\ Abstract type: Poster}

Attitudes and Perceptions of Morphine in Patients with Advanced Cancer and their Family Caregivers: A Qualitative Study

Ho J.F.V. ${ }^{1,2}$, Yaakup H. ${ }^{1,3}$, Low G.S.H. ${ }^{1}$, Wong S.L. ${ }^{4}$, Tho L.M. ${ }^{4}$, Tan S.B. ${ }^{5}$

${ }^{1}$ Supportive and Palliative Care Service, Sunway Medical Centre, Bandar Sunway, Malaysia, ${ }^{2}$ School of Medicine, Cardiff University, Cardiff, United Kingdom, ${ }^{3}$ Palliative Medicine, Universiti Kebangsaan Malaysia Medical Centre, Kuala Lumpur, Malaysia, ${ }^{4}$ Oncology, Sunway Medical Centre, Bandar Sunway, Malaysia, ${ }^{5}$ Palliative Medicine, University Malaya Medical Centre, Kuala Lumpur, Malaysia

Background: The prevalence of undertreated cancer pain remains high and suboptimal control of cancer pain adversely affects quality of life. Common barriers to adequate cancer pain management related to opioid analgesics include fear of addiction and side effects.

Aim: This study investigated the attitudes and perceptions of morphine in advanced cancer patients and their family caregivers to gain a deeper understanding of the barriers related to using morphine for cancer pain.

Method: This is a qualitative study involving adult opioid naïve advanced cancer patients and their caregivers using purposive recruitment and semi-structured individual interviews for data collection. Interviews were transcribed verbatim and analyzed using thematic analysis.

Results: Data saturation was reached after 18 patients and 13 of their family caregivers were interviewed $(n=31)$. Attitudes and perceptions towards morphine were similar in both groups and were influenced by past experiences. The most prevalent themes in morphine perceptions were that morphine was a strong analgesic, associated with end stage of illness and a recreational drug. Most participants agreed to future morphine use mainly to reduce patient's pain and suffering and because of the trust in their doctor to act in their best interest. Many preferred morphine as a last resort due to concerns with side effects and addiction. Attitudes of the caregivers were not found to have a significant impact on the respective patient's decision for morphine use.

Conclusion: Morphine perceptions directly affected the attitudes towards future morphine use. Despite some negative perceptions of morphine, most participants accepted morphine for future use to achieve cancer pain control. Focused patient and caregiver education addressing morphine misperceptions might increase acceptance, adherence and compliance to opioid analgesics, resulting in better control of cancer pain.

\section{Abstract number: $\mathrm{P} 02-077$ \\ Abstract type: Poster}

Collaboration between Palliative Medicine and Pain Medicine in Patients with Serious Illness: Results of a National Survey of Palliative Medicine Physicians

Partain D. ${ }^{1}$, Santivasi W. ${ }^{2}$, Wu K. ${ }^{3}$, Kamdar M. ${ }^{4,5}$, Carey E. ${ }^{1}$, Tilburt J. ${ }^{6}$, Mackey R. ${ }^{1}$, Moeschler S. ${ }^{7}$, Strand J. ${ }^{1}$

${ }^{1}$ Center for Palliative Medicine, Mayo Clinic, Rochester, United States, ${ }^{2}$ Department of Medicine, Mayo Clinic, Rochester, United States, ${ }^{3}$ Center for Palliative Medicine, Mayo Clinic, Scottsdale, United States, ${ }^{4}$ Department of Palliative Care and Geriatric Medicine, Massachusetts General Hospital, Boston, United States, ${ }^{5}$ Division of Anesthesia Pain Medicine, Massachusetts General Hospital, Boston, United States, ${ }^{6}$ Division of General Internal Medicine, Mayo Clinic, Rochester, United States, ${ }^{7}$ Division of Pain Medicine, Mayo Clinic, Rochester, United States

Background: Pain is the most common symptom in advanced cancer patients, and evidence suggests that $10-30 \%$ of these patients will have inadequate analgesia with pharmacologic therapies alone. Interventional pain techniques can provide improved analgesia and/or reduced side effects. Little is known about the collaboration between palliative medicine and pain medicine physicians in the care of patients with advanced cancer in the United States.

Aims: To review collaborative practice patterns between palliative care and pain medicine physicians.

Methods: A 30-question survey was sent to 1000 randomly selected physician members of the American Academy of Hospice and Palliative Medicine. This survey-based research received IRB exempt status (ref\#16-004079).

Results: The response rate was $52.6 \%(n=526)$. Survey responders had initial board certification primarily in internal medicine $(56 \%)$ or family medicine $(28 \%)$, had generally not completed a palliative medicine fellowship (66\%), and practiced for a median of 9 years (range 1-38 years). Most palliative medicine physicians (95\%) felt that interventional pain management was important in the care of their patients and $44 \%$ of responders thought that $>20 \%$ of their patients would benefit from consultation with a pain specialist. However, most palliative medicine physicians $(66 \%)$ made $<10$ referrals to pain medicine clinicians per year. Very few referrals were made for intrathecal drug delivery systems (IDDS); $86 \%$ of respondents made $<5$ referrals for IDDS annually.

Discussion: While palliative medicine physicians see pain medicine physicians as being able to offer important services to patients with a serious illness such as advanced cancer, most refer $<10$ patients annually for consultation. Opportunities exist to connect palliative medicine teams to pain physicians and further research is needed to clarify barriers to collaboration for seriously ill patients.

A donation from a private benefactor funded part of this study. 
Abstract number: P02-078

Abstract type: Poster

Can We Justify a Two Week Inpatient Intrathecal Drug Delivery

Trial in Cancer Patients with a Limited Prognosis?

Evans R., McGhie J., Sommerville L., Mitchell A.

Beatson West of Scotland Cancer Centre, NHS GGC, Glasgow, United Kingdom

Background: The value of and how to conduct an intrathecal drug delivery (ITDD) trial remains contentious. An inpatient trial results in a two week admission when prognosis is often short. However in patients who benefit from the ITDD trial there is potential to improve pain control and thus quality of life with permanent ITDD. There are very limited studies looking at the validity of an ITDD inpatient trial phase in patients with a malignant diagnosis.

Aim: To evaluate the trial phase used by the Interventional Cancer Pain Service (ICPS) by comparing those patients who proceed to permanent pump implantation following an inpatient trial with those patients who did not.

Methods: Retrospective analysis of data collected through the trial ITDD phase of 54 patients between 2007 and 2018. We compared BPI (Brief Pain Inventory) scores and oral morphine equivalent (OME) doses before during and at the end of a trial between those who proceeded to have a permanent pump inserted and those who did not.

Results: Of the 54 patients 44 (81.5\%) went onto to have permanent ITDD and $10(18.5 \%)$ had the intrathecal medication stopped. Pre procedure BPI scores between the groups were similar and both groups showed an improvement on day $1-2$ post op. This improvement was sustained in those who proceeded and was not in those who did not proceed, the scores were significantly different by the end of the trial. OME doses were not significantly different, but both groups showed an initial reduction with those who did not proceed appearing to require higher doses by the end of the trial.

Conclusions: A trial saved $18.5 \%$ of patients the lifelong implications of having a permanent pump. The initial reduction in pain scores and OME observed in both groups suggests that in this patient population a trial of longer than 2 days is needed. Further work is needed to see if the trial period could be shortened and to evaluate the impact of improvement in function on the decision to proceed or not.

\section{Abstract number: P02-079}

Abstract type: Poster

Pain Management by Doctors and Nurses at One District Hospital with Palliative Care Unity in Rwanda

Theoneste N. ${ }^{1,2}$

${ }^{1}$ Kibagabaga District Hospital, Internal Medicine, Kigali, Rwanda, ${ }^{2}$ Rwanda Ministry of Health, Clinical Services, Kigali, Rwanda Presenting author email address: nsabitheo@gmail.com

Background: Pain management is a critical component of comprehensive care for patients with serious illness and doctors and nurses are key mediators of effective pain management.No prior studies measuring knowledge,attitudes and practice of managing pain among doctors and nurses in Rwanda. Yield information shapes effective intervention to address this important component of palliative care.

Objective: Establish baseline data on knowledge,attitudes and practice of pain management in patients with advanced disease.To investigate the specific barriers to effective pain management.

Methods: A cross section, descriptive study, aimed at evaluating the knowledge, attitudes and practice of pain management among doctors and nurses at District hospital was conducted, using a validated questionnaire.
Results: A total of 28 doctors and 107 nurses were included in the study. The results revealed a widespread knowledge deficit and poor attitude focused in the area of pain assessment and pharmacological management of pain. Even though $92.0 \%$ of doctors and $88 \%$ of nurses in our study strongly believed that pain relief is an essential part of care for patients with advanced disease, a large number of respondents incorrectly answered questions regarding the specific management of pain. This included $43 \%$ of respondents stating that vital signs are the best indicator of the presence of pain, and $63.7 \%$ of respondents failing to recognize that aspirin and other none steroid anti-inflammatory drugs are effective treatment for bone pain. Knowledge deficits were also present for the use of morphine, as $60.8 \%$ of respondents did not know the time to peak effect of morphine, $54.2 \%$ did not know the usual duration of analgesia of IV morphine, and $35.5 \%$ did not know how to correctly convert IV morphine to PO morphine.

Conclusion: A widespread knowledge deficit and poor attitude of doctors and nurses regarding pain management were noted. Further education and quality improvement initiatives are needed.

Abstract number: P02-080

Abstract type: Poster

\section{Sustained Efficiency of Perineural Analgesia in Cancer-Related Pain: a Case Report \\ Boden A. ${ }^{1}$, Fuzier R. ${ }^{2}$, Gilbert $0 .{ }^{2}$, Pouymayou J. ${ }^{2}$, Pierre S. ${ }^{2}$, Caunes- \\ Hilary N. ${ }^{1}$ \\ ${ }^{1}$ Supportive Care, Institut Claudius Regaud, Toulouse, France, \\ ${ }^{2}$ Anesthesiology, Institut Claudius Regaud, Toulouse, France}

Background: Continuous regional analgesia can be an effective treatment for cancer pain and an alternative to opioids. However, required catheterization duration and sustained analgesic effects after catheter removal have not been yet clarified.

Aims: Use of peripheral blocks in cancer pain is based upon anecdotal evidence, and reporting new cases may help the development of this technique.

Methods: We report the case of a 66 years old woman currently treated in a French cancer institute for a relapsed metastatic squamous cell carcinoma of the cervix.

Results: The patient presented pain due to osteolytic lesions of the left foot and tibia, which generated complete lower limb functional impotence (WHO Performance Status 4). Pain treatment included acetaminophen, prednisolone, tramadol and oxycodone, which have been poorly efficient and tolerated. Due to pain, she took up to 8gr of acetaminophen per day.

As a second line treatment by carboplatin, paclitaxel and bevacizumab has been started, we implanted a popliteal sciatic catheter for continuous perineural analgesia (ropivacaine $6 \mathrm{mgr}$ per hour). Efficiency was observed 15 minutes after local anesthesic injection with a numeric rating scale (NRS) scored 0/10. All the oral treatments have progressively been stopped within a few days.

The perineural analgesia has been maintained 62 days before removal. The positron emission tomography (PET) showed at this time a partial metabolic response of the osteolytic lesions. No pain has been observed within the 21 days after catheter removal, with NRS remaining scored $0 / 10$. Only acetaminophen was prescribed on demand and the patient was able to walk progressively.

Conclusion/ discussion: This case highlights sustained pain efficiency several days after perineural analgesia catheter removal, despite partial metabolic response on PET. Furthermore, this technique may also allow opiate saving. As there are no controlled studies available in the literature, these datas need further investigations.

Abstract number: P02-081

Abstract type: Poster 
Tailored Treatment for Breakthrough Cancer Pain (BTcP): Is it an Open Issue?

Magnani C. ${ }^{1}$, Mastroianni C. ${ }^{2}$, Calvieri A. ${ }^{2}$, Giannarelli D. ${ }^{3}$, Dardeli A. ${ }^{2}$, Restuccia M.R. ${ }^{2}$, Eusepi G. ${ }^{2}$, Surdo L. ${ }^{1}$, Casale G. ${ }^{2}$

${ }^{1}$ Research Department, Antea Palliative Care Unit, Rome, Italy, ${ }^{2}$ Antea Palliative Care Centre, Rome, Italy, ${ }^{3}$ Biostatistic, Regina Elena National Cancer Institute, Rome, Italy

Background: International guidelines on BTCP treatment are not completely univocal. Study findings reported that some factors should always be considered to tailor BTcP treatment.

Aim: The main aim of this study was to assess any relationship between some variables (setting of assistance, BTCP characteristics, oral mucositis etc.) and BTcP pharmacological treatment in a sample of terminal cancer patients, in the real clinical practice.

Method: A prospective, cross-sectional study was carried out. Patients were recruited if they had BTCP. Data on baseline pain, BTCP, oral and nasal mucosa conditions were registered. These data were summarized through mean and SD if related to quantitative variables and by absolute frequencies and percentage when referred to qualitative variables. The $\chi^{2}$ test was used.

Results: Among the 1180 patients assessed for eligibility, 149 were recruited. Mean age was $71.5 \pm 12.9$. Short-Acting Oral Morphine (SAOM) for BTCP treatment (59.1\%) was related to home care setting of assistance ( $p=0.004)$. Intravenous Morphine (IV) was related to continuous baseline IM infusion ( $p<0.0001$ ). Transmucosal Immediate-Release Fentanyl (TIRF) was related to patients' self-sufficiency in taking medication $(p=0.001)$ and inpatients setting $(p<0.0001)$.

Discussion: BTCP has a negative impact on quality of life. Different formulations are now available to tailor BTCP treatment. A guide to help clinicians in this choice is not available yet but some variables should be considered. From this study findings oral mucositis was not considered as a discriminating factor when choosing the formulation for BTCP. Outpatients received more frequently SAOM, this might be due to difficulties in TIRF titration. Patients' self-ability to take medications influenced the clinicians' decision, probably because TIRF administration requires patients' active collaboration. Future larger studies should be conducted to guide clinicians in choosing a tailored BTCP treatment for each patient.

\section{Abstract number: P02-082}

Abstract type: Poster

Painful and Panting: Under- and Over- Treatment of Symptoms in Patients with Imminent Death at the Emergency Department Fong E.L.S. ${ }^{1}$, Nadarajan G.D. ${ }^{2}$, Lian W.Q.S. ${ }^{2}$, Ong E.H.M. ${ }^{2}$

${ }^{1}$ Lee Kong Chian School of Medicine, Nanyang Technological University, Singapore, Singapore, ${ }^{2}$ Department of Emergency Medicine, Singapore General Hospital, Singapore, Singapore

Background: Patients with chronic diseases most commonly present to the emergency department (ED) with pain or dyspnoea during their end-of-life (EOL) period. There are documented tendencies to under- or over- treat symptoms: oxygen $\left(\mathrm{O}_{2}\right)$ therapy could be unnecessary and analgesia could be neglected. The baseline pattern of symptomatic treatment is crucial for future studies on patient-centred care but has never been studied locally.

Aim: To study the pattern of symptom control given to imminently dying patients presenting to the ED.

Methods: A retrospective study was done on a 3-month period in a tertiary hospital ED. Patients who did not present with a cardiac arrest, had a diagnosis of a chronic disease and died within 72 hours were included. Patterns on symptom management were analysed using patient records. Outcomes of interest were pre- $\mathrm{O}_{2}$ therapy saturation level $\left(\mathrm{SpO}_{2}\right)$, analgesia coverage and waiting time.

Results: A total of 59 patients with $67.8 \%$ males were studied. The median age was 75 . Amongst the $59,39.0 \%$ complained of pain while $57.6 \%$ received $\mathrm{O}_{2}$. Thirty-four patients, including 2 with COPD, received $\mathrm{O}_{2}$, of which $67.6 \%$ complained of dyspnoea. Of the 32 non-COPD $\mathrm{O}_{2}$ recipients, only $46.9 \%$ had pre- $\mathrm{O}_{2}$ therapy $\mathrm{SpO}_{2}$ below $94 \%$. Of the 23 patients who had pain, only $34.8 \%$ received analgesia. The median waiting time for delivery was $1 \mathrm{~h} 52.5 \mathrm{~min}$.

Conclusion: Patients expect pain relief within 30 min, which this ED fell short of. A model involving administration of non-opioid analgesics at triage could be considered to shorten waiting time. A large proportion of patients with pain were not given analgesia. Efforts should be made to ensure that such patients are not denied analgesia.

A majority of non-COPD patients were given $\mathrm{O}_{2}$ despite $\mathrm{SpO}_{2} \geqslant 94 \%$, which is not indicated regardless of dyspnoea. Unnecessary therapy causes discomfort from prongs or masks. Room air and non-pharmacological treatments like position adjustment and breathing exercises could be encouraged.

Abstract number: P02-083

Abstract type: Poster

Better Methods of Pain Management for Frail Older Cancer Patients in the Surgical Intensive Care Unit

Huang B.W.-Y., Lai Y.-L.

Hospice and Palliative Care Center, Mackay Memorial Hospital, New Taipei City, Taiwan, Republic of China

Background: With technological advancements, more frail older cancer patients are undergoing surgery and need surgical intensive care unit (SICU) care. Post -operation optimal pain management can provide a more stable hemodynamic status which both shorten length of hospital stay and reduced medical cost.

Aims: We aimed to determine the better pain management method for acute pain control in frail older SICU cancer patients.

Methods: This retrospective cohort study included a chart review of patients treated in a 26-bed SICU from April 2011 to September 2012. Patients who were less than 75years, unconscious, uncooperative, or post-brain surgery were excluded. The primary aim was to compare visual analogue scale (VAS) (0-100) scores between three different pain

VAS score under 3 different conditions and patient satisfaction with 3 different methods of pain management.

\begin{tabular}{lcccc}
\hline & $\begin{array}{l}\text { Pethidine/NSAIDs } \\
(\mathrm{P} / \mathrm{N})(\mathrm{n}=50)\end{array}$ & PCA $(\mathrm{n}=23)$ & PCEA $(\mathrm{n}=28)$ & \\
\hline VAS-Rest & $28.57 \pm 12.80 \psi$ & $21.36 \pm 8.34 \psi$ & $11.92 \pm 8.95$ & $<0.001$ \\
VAS-Movement & $46.43 \pm 12.46 \psi$ & $41.82 \pm 10.07$ & $34.07 \pm 10.83$ & $<0.001$ \\
VAS-Coughing & $54.05 \pm 17.40$ & $61.36 \pm 10.82$ & $53.08 \pm 12.58$ & 0.106 \\
Patient satisfaction & $4.03 \pm 0.28 \psi$ & $4.05 \pm 0.21 \psi$ & $4.33 \pm 0.48$ & 0.001 \\
\hline
\end{tabular}

WSignificant difference $(p<0.05)$ compared to PCEA 
management methods. Pethidine/NSAIDs $(\mathrm{P} / \mathrm{N})$, intravenous patientcontrolled analgesia (PCA), and patient-controlled epidural analgesia (PCEA), under different conditions (rest, movement, and coughing). The secondary endpoints were patient satisfaction.

Results: We chart reviewed 1872 patients and 1771 were excluded. VAS results presented as mean \pm SD. Data analysis using ANOVA with Scheffe post hoc test. The average age was $81.6 y e a r s$, and $53.5 \%$ were male. At rest, the PCEA group exhibited significantly lower pain scores (11.92 \pm 8.95$)$ compared with the other two groups. During movement, the PCEA and PCA showed no difference; both exhibited lower pain scores than the $\mathrm{P} / \mathrm{N}$ group $(46.43 \pm 12.46)$. While coughing, no difference between one another. The PCEA group showed highest patient satisfaction (4.33 \pm 0.48$)$.

Conclusion: PCEA appears to be a better choice for pain management of frail older SICU cancer patients.

Abstract number: P02-084

Abstract type: Poster

Pain Intensity and Addictive Behavior. Does Nicotine Addiction Matter? Analysis of a Sample of Advanced Cancer Patients Attended in an Outpatient Clinic (OPC) in a Teaching Hospital in Catalonia

Canal-Sotelo J. ${ }^{1}$, Barallat-Gimeno E. ${ }^{2}$, Lopez-Ribes J. ${ }^{3}$, Arraras-Torrelles N. ${ }^{3}$, Gonzalez-Rubio R. ${ }^{3}$, Trujillano-Cabello J. ${ }^{4}$

${ }^{1}$ Supportive Palliative Care Team, Hospital Universitari Santa Maria, Gestió de Serveis Sanitaris, Lleida, Spain, ${ }^{2}$ University of Lleida, Lleida, Spain, ${ }^{3}$ Hospital Universitari Santa Maria, Gestió de Serveis Sanitaris, Lleida, Spain, ${ }^{4}$ Hospital Universitari Arnau de Vilanova, Lleida, Spain

Background: Addictive behavior can be a sign of bad prognosis regarding pain control in cancer patients.

Aims: To describe the population characteristics and to compare the different variables studied according to the level of nicotine addiction (Fagestrom test $\geqslant 7$ points).

Methods (design, data collection, analysis): Prospective,descriptive study carried out during 2015 in a single institution. Patients were recruited at the first visit at the OPC. Inclusion criteria. Age $>18$ years,signed the informed consent, diagnosed of advanced cancer and with a history of smoking $>5$ years in their lifetime. Variables studied: Sociodemographic and clinical variables (cancer diagnosis, Brief Pain Inventory, the Doleur Neuropathique-4 questionnaire, CAGE test, need for opioid rotation, Morphine Equivalent Daily Dose and adjuvant analgesics prescribed. The Edmonton Classification System for Cancer pain (ECS-CP) was used to assess pain features. Descriptive analysis (mean, median, standard deviation and interquartile interval) were used when appropriate. Chi-square and Kruskas-Wallys test were used to compare different groups.

Results: 211 patients were recruited. 153 (72, 5\%) with a Fagestrom test $\geqslant 7$ points. Nicotine addicted population was younger $62,4 \pm 10$ vs $67,9 \pm 13, p=0,002$, included more men $90,8 \%$ vs $77,6 \%, p=0,010$, with less pain improvement prior to our intervention $47,1 \pm 30$ vs $56,9 \pm 33$, $p=0,036$ and showed higher scores in the worst pain in the past $24 \mathrm{~h}$ $7,0 \pm 2$ vs $6,2 \pm 2, p=0,020$. The nicotine addicted population presented with a higher $\%$ of the Incident pain feature ( $83 \%$ vs $70,7 \%, p=0,047$ ) and in the total ECS-CP score $2,6 \pm 1$ vs $2,4 \pm 1, p<0,001$ ). Functional interference in daily living activities (normal work, sleep and relations with other people) are significantly altered in the nicotine addicted population.

Conclusions: A history of nicotine addiction measured with the Fagestrom test ( $\geqslant 7$ points) can act as a marker of bad prognosis in cancer pain presentation.

Abstract number: P02-085

Abstract type: Poster
What Do Palliative Cancer Patients Want in Regard to the Management of their Pain

Pascual Pla F.J. ${ }^{1}$, Martín Utrilla S. ${ }^{2}$, Mancheño Álvaro A. ${ }^{1}$, Boscá Mayans C. ${ }^{3}$, Carratalá A. ${ }^{4}$, Egido A. ${ }^{1}$, Contel E. ${ }^{1}$, Cañado Y. ${ }^{1}$, Santamaría P. ${ }^{1}$, Peña M. ${ }^{1}$, Quiles B. ${ }^{1}$, Ruiz P. ${ }^{1}$

${ }^{1}$ Fundación Instituto Valenciano de Oncología, Valencia, Spain, 2Palliative Care, Fundación Instituto Valenciano de Oncología, Valencia, Spain, ${ }^{3}$ Hospital Universitari i Politécnic La Fe, Valencia, Spain, ${ }^{4}$ Centro de Salud Sant Vicent dels Raspeig, Alicante, Spain

Background: Pain is one of the most frequent symptoms among palliative cancer patients (PCP), although little is known about what they want from the control and management of their pain. Measuring the effectiveness of pain management is a challenge because it is a subjective phenomenon and a multifactorial process. Determining how we currently define whether a PCP with pain due to advanced cancer has controlled pain (or not) is important, particularly from the patient's perspective.

Aims: We aimed to explore how PCP describe pain control and what they want in the management of their pain.

Methods: Qualitative study using direct interviews in a sample of 56 patients with advanced cancer treated in the Emergency Department of an oncological hospital from January to December 2017.

Results: Interviews were conducted with patients who consulted for poor pain control. Four themes emerged: maintaining independence through the management of drugs; modify the expectations of pain control; role of health professionals; and meaning of pain in the context of advance planning decisions (APD).

Conclusions: Patients developed better with fentanyl and tapentadol than with morphine and anti-inflammatories and determined if their pain was controlled, 'in case they could or could not perform different activities and maintain relationships with family or friends'. The numerical scale does not seem to be useful for patients to measure whether they are able to perform these activities or maintain a sense of control and / or independence. The support of professionals in setting the objectives, tasks, activities and information in the management of drugs for PCP with pain can be useful to enable them to determine by themselves what they want to manage their pain. Pain control in the context of APD perceive it as a guarantee for the maintenance of person's dignity in the process of their attention at the end of life.

Abstract number: P02-086

Abstract type: Poster

Inpatient Methadone Rotation for the Relief of Cancer Pain in a Singapore Hospital: An Observational Study

Wong J. ${ }^{1}$, Tan C. ${ }^{1}$, Hum A. ${ }^{2,3}$

${ }^{1}$ Pharmacy, Tan Tock Seng Hospital, Singapore, Singapore, ${ }^{2}$ Palliative Medicine, Tan Tock Seng Hospital, Singapore, Singapore, ${ }^{3}$ Palliative Care Centre for Excellence in Research and Education, Singapore, Singapore

Methadone is a useful option in the treatment of cancer pain. It ameliorates both nociceptive and neuropathic pain, is inexpensive, has a high oral bioavailability of more than $80 \%$ and does not have known active metabolites. Despite its advantages, methadone use is complicated due to a long and variable half-life. Its potency also varies according to a patient's opioid tolerance prior to rotation. Various methods for rotating from another opioid to methadone have been proposed in mostly Caucasian populations. These differ in equianalgesic ratio as well as the duration to convert to the full dose of methadone. There is little published data on the effectiveness of rotation methods in Asian populations.

In our institution, we implemented a protocol combining the "Stopand-go" for low morphine equivalent daily doses (MEDD) and a 3-day rotation method for higher MEDD, utilizing an equianalgesic ratio of 
10:1. This study aims to describe our experience with opioid rotation to methadone in a predominantly Asian population.

A retrospective review of patients initiated on methadone for cancer pain from June 2015 to June 2018 will be conducted. In this study, we discuss in detail the reasons for methadone rotation, the initial and final conversion MEDD:methadone ratio achieved in our patients, the time to achieve improvement in pain control (defined as a decrease in pain score of $\geqslant 1$ or a pain score of $\leqslant 3$ in 3 consecutive reviews), as well as the adverse effects encountered with methadone use.

Abstract number: P02-087

Abstract type: Poster

Predictors of Severe Pain among a Sample of Advanced Cancer Patients with a Lifetime History of Smoking Behaviour Canal-Sotelo J. ${ }^{1}$, Lopez-Ribes J. ${ }^{2}$, Barallat-Gimeno E. ${ }^{3}$, Arraras-Torrelles N. ${ }^{2}$, Gonzalez-Rubio R. ${ }^{2}$, Trujillano-Cabello J. ${ }^{4}$

${ }^{1}$ Supportive \& Specialist Palliative Care Services, Lleida, Spain, ${ }^{2}$ Home Care Team, Lleida, Spain, ${ }^{3}$ University of Lleida, Lleida, Spain, ${ }^{4}$ Hospital Univesitari Arnau de Vilanova, Lleida, Spain

Background: Addictive behavior can be a sign of bad prognosis regarding pain control in cancer patients.

Aims: To determine which variables predict severe cancer pain (VAS $\geqslant 7$ ). Methods (design, data collection, analysis): Prospective, descriptive study carried out during 2015 in a single institution. Patients were recruited at the first visit at the OPC. Inclusion criteria. Age $>18$ years, signed the informed consent, diagnosed of advanced cancer and with a history of smoking $>5$ years in their lifetime. Variables studied: Sociodemographic and clinical variables (cancer diagnosis, Brief Pain Inventory -BPI-, the Doleur Neuropathique-4 test-DN4-, need for opioid rotation -ROP-, Morphine Equivalent Daily Dose -MEDD- and adjuvant analgesics prescribed. The Edmonton Classification System for Cancer pain (ECS-CP) was used to assess pain features. Descriptive analysis (mean, median, standard deviation and interquartile interval) were used when appropriate. Chi-square and Kruskas-Wallys test were used to compare different groups. Multivariate logistic regression was performed to determine OR with IC $95 \%$.

Results: 211 patients were recruited. 136(64. 5\%)with a pain score $V A S \geqslant 7$. Higher pain scores were found in younger patients $(63.2 \%$ vs $44 \%, p=0.007)$. Functional interference -BPI- was found to be statistically significant in all the parameters evaluated. DN4 scores, the MEDD on the first visit and when was under control and the need for ROP were higher in the group presenting with higher pain scores( $4.3 \pm 2$ vs $3.3 \pm 2$, $\mathrm{p}=0.003 ; 45.6 \pm 59$ vs $21.1 \pm 32, \mathrm{p}<0.001 ; 92.7 \pm 91$ vs $54.5 \pm 59, \mathrm{p}<$ $0.001 ; 29.4 \%$ vs $14.7 \%, p=0.019$, respectively).In the multivariate analysis, the variables age $<65$ years [OR (IC95\%) 1.98 (1.10-3.79), $p=0.022$ ] and the Fagestrom test score $>6$ [OR (IC95\%) 2.01 (1.15-3.56), p=0.016], were found.

Conclusions: Age and higher levels of nicotine addiction can act as predictors to severe pain in a sample of advanced cancer patients with a smoking lifetime history.

Abstract number: P02-088

Abstract type: Poster

Is there Room for "Nicotine Addiction" Item into the "Addiction Behaviour" Feature of the Edmonton Classification System for Cancer Pain?

Canal-Sotelo J. ${ }^{1}$, Lopez-Ribes J. ${ }^{2}$, Barallat-Gimeno E. ${ }^{3}$, Gonzalez-Rubio

$\mathrm{R}^{2}{ }^{2}$, Arraras-Torrelles N. ${ }^{2}$, Trujillano-Cabello J. ${ }^{4}$

${ }^{1}$ Supportive \& Specialist Palliative Care Services, Lleida, Spain, ${ }^{2}$ Hospital Universitari Santa Maria. Gestió de Serveis Sanitaris, Lleida, Spain, ${ }^{3}$ University of Lleida, Lleida, Spain, ${ }^{4}$ Hospital Universitari Arnau de Vilanova, Lleida, Spain
Background: The Edmonton Classification for Cancer Pain (ECS-CP) includes addictive behavior to alcohol as a feature of bad prognosis for pain control

Aims: To investigate if the inclusion of the nicotine addiction behavior into de ECS-CP adds more complexity to pain outcomes.

Methods (design, data collection, analysis): Prospective, descriptive study carried out during 2015 in a single institution. Patients were recruited at the first visit at the OPC. Inclusion criteria. Age $>18$ years, signed the informed consent, diagnosed of advanced cancer and with a history of smoking $>5$ years in their lifetime. We included clinical and Sociodemographic variables. The Edmonton Classification System for Cancer pain (ECS-CP) was used to assess pain features and one point for each feature present was given. The cognitive impairment was specifically excluded. Descriptive (mean, median, standard deviation and interquartile interval) and univariate (chi-square and Kruskas-Wallys) were used. The CHAID (Chi-Squared Automatic Interaction Detection) methodology was used to find the cut-off for the different nodes ( $\leqslant 1$ point; 2-3 points; 4 points). We construct the ROC curve (ABC IC 95\%).

Results: 211 patients were recruited. $153(72.5 \%)$ with a lifetime history of addiction to alcohol, nicotine or both. The total sum on the ECS-CP pain features was $2.6 \pm 1$, median $3, \mathrm{IQ}(2-3)$, for the addicted group and 2.4 \pm 1 , median 2, IQ (1-2); $\mathrm{p}<0.001$. The ROC curve IC 95\%) for the ECS-CP without including and including addiction to nicotine were 0.64 (0.56-0.71) and 0.67 (0.59-0.74).

Conclusions: The inclusion of the addiction to nicotine feature measured by the Fagestrom test into the addictive behavior of the ECS-CP adds more sensitivity and specificity to the test mainly when the sum of the ECS-CP features is in the node 1 ( $\leqslant 1$ point).

Abstract number: P02-089

Abstract type: Poster

Palliative Care Health Needs Assessment of Breast Cancer Patients Attending the Oncology Clinics of a Private Hospital in Southwest Nigeria

Akande S.

NNPC Hospital, Lagos Medical Zone, Lagos, Nigeria Presenting author email address: seakande@yahoo.com

Background: Breast cancer remains the commonest malignancy affecting women in Nigeria. Like majority of cancers in Africa, it is diagnosed at an advanced stage when palliative care is often the only choice of treatment. The identification of the palliative care needs particular to each environment, can be a starting point in the development of a strategy for improvement of care and health service delivery.

Methods: The demographics, presenting complaints, diagnosis and palliative care needs of 46 participants and 35 family carers were assessed using the African Palliative Care Association, African Palliative Outcome Scale.

Results: The participants' ages ranged from 29 to 82 years. The largest proportion of participants were aged $61-70$ years $(28.3 \%)$. Pain and physical symptoms were the highest palliative care needs of participants. The psychological and spiritual domains were the highest met needs. Information needs were the highest among the family carers. Conclusion: These domains can be explored in the development of a structured palliative care programme.

Abstract number: P02-090

Abstract type: Poster

Pain, Fatigue and Anorexia in Patients with Advanced Hepatobiliary Cancers Referred to Palliative Care in a Tertiary Care Cancer Centre in a Developing Country: A Retrospective Analysis 
Singh P., Deodhar J., Tiwari A.K., Ghoshal A., Damani A., Muckaden M.A.

Palliative Medicine, Tata Memorial Hospital, Mumbai, India

Background: Hepatobiliary carcinoma (HBC) is a common malignancy worldwide. Patients with advanced HBC usually report multiple concurrent symptoms in palliative care (PC) settings. Pain \& other symptoms like fatigue \& anorexia are often distressing in patients with advanced $\mathrm{HBC}$ and may have severely negatively affect their quality of life. This study aims to examine prevalence \& severity of pain, fatigue \& anorexia in advanced HBC patients referred to PC services in a tertiary cancer centre in a developing country.

Aims and Objectives:

1. To examine the prevalence \& severity of pain in patients with advanced HBC.

2. To determine the frequency $\&$ severity of fatigue $\&$ anorexia in patients with advanced $\mathrm{HBC}$.

Method: It was retrospective observational analysis of electronic \& paper medical records of advanced HBC patients newly referred to specialist PC services between 1/11/ 2016 to 31/10/2017. Incomplete records were excluded. Variables noted were sociodemography, performance status (on Eastern Cooperative Oncology Group ECOG), disease \& treatment related factors \& presenting symptoms (as recorded on the Edmonton Symptom Assessment Scale ESAS).

Results: 317 patient records were analyzed. Female patients were more common (172,54\%). The mean age was 53 years (SD11.4), gall bladder cancer being most common (219,69\%). 146 (46\%) had ECOG 2, 228 patients (72\%) have Child Pugh score A, \& 79 (25\%) patients underwent therapeutic procedures. Pain was present in $277(87 \%)$ patients \& almost half had moderate. Fatigue was seen in $267(57 \%)$ patients, majority having 0-3 score on ESAS. 185 patients (59.2\%) had anorexia, \& mainly of mild severity. The median scores for pain, fatigue, anorexia is 4,1 and 1 respectively.

Conclusion: Pain, fatigue \& anorexia were commonly seen in advanced HBC patients newly referred to specialist PC services. Assessment of symptom burden \& severity is important for comprehensive management of advanced $\mathrm{HBC}$ patients.

Funding received: Nil

Conflict of interest: Nil

Abstract number: P02-091

Abstract type: Poster

Healing Hands: Exploring the Impact of Reiki Therapy, Therapeutic Touch, and Healing Touch upon Patient Quality of Life in Palliative and Hospice Care - A Systematic Review Morse E. ${ }^{1,2}$

${ }^{1}$ Hosparus Health Louisville, Social Work, Louisville, KY, United States, ${ }^{2}$ University of Louisville, Kent School of Social Work, Louisville, KY, United States Presenting author email address: info@evamariemorse.com

Background: Palliative and hospice patients experience a variety of symptoms impacting quality of life, (QOL) including pain, distress, and anxiety. Reiki Therapy, Therapeutic Touch (TT) and Healing Touch (HT) are biofield therapies where practitioners channel energy through their hands, with or without touch, to reduce symptoms and improve wellbeing. Palliative and hospice organizations use these therapies however, little research has demonstrated their impact on QOL.

Aims: To conduct a systematic review of the impact of Reiki Therapy, TT, or HT on QOL when compared to standard of care among palliative/hospice care patients.

Methods: Eligibility criteria included peer-reviewed publications, in English, from 1999 to 2018. Search terms included patient primary diagnoses: cancer, dementia, heart disease, lung disease, stroke, coma, kidney disease, liver disease, amyotrophic lateral sclerosis (ALS), HIV/ AIDS. Databases included: CINAHL, MEDLINE, PsychINFO, Embase, and Ovid. Studies were assessed regarding design, methodology, sampling and demographics, measurement validity, rigor, and application to palliative/hospice care practice.

Results: Ten studies are included: one meta-analysis, five experimental, one mixed-method, two quasi-experimental, and one qualitative. The majority assessed cancer patients. Mean sample size was $n=42$. Participants were predominately white and female. Five studies utilized Reiki Therapy and had the highest level of rigor. Three studies used TT, and two used HT, and had the lowest rigor overall. Reiki Therapy was found to reduce pain and anxiety and improve overall QOL. Outcomes related to TT were conflicting and widely varied, while outcomes of HT suggested improved mood, and reductions in nausea and fatigue.

Conclusion/ discussion: Interventions were delivered in a range of settings suggesting applicability to residential, outpatient and inpatient environments. Reiki Therapy had the greatest impact across outcomes.

\section{Abstract number: P02-092}

Abstract type: Poster

"Total Pain" in End-of-Life Cancer Patients: The Necessity to Implement Pharmacological Treatments with Psycho-socioSpiritual Interventions

Botto R., Bovero A., Digiovanni Y., Fonti I., Torta R.

Department of Neuroscience, University of Turin, Turin, Italy

Background: "Total pain" is patients' bio-psycho-social-spiritual suffering. Adequate care should be provided to alleviate patients' pain.

Aims: To analyze bio-psycho-social-spiritual pain levels and to observe the effectiveness of the pharmacological treatments in end-of-life cancer patients.

Methods: Cross-sectional methodology was adopted. Sample included 382 end-of-life cancer patients receiving palliative care in hospital (237) and in hospice (145), with Karnofsky Performance Status $\leqslant 50$ and life expectancy of few weeks. During the first psychological consultancies, patients' personal and medical data were gathered and a set of validated rating scales assessing pain, anxiety, depression, quality of life, and coping styles was administered to patients. Descriptive statistics were performed using SPSS. Results: $83.5 \%$ of sample took analgesics, $40.6 \%$ antidepressants and $49 \%$ anxiolytics. $61 \%$ of sample had absent, $23 \%$ mild, $13 \%$ moderate, and only $3 \%$ severe physical pain. However, $71.2 \%$ and $76.4 \%$ of sample had respectively clinically relevant anxious and depressive symptomatology. Most of patients reported moderate levels of physical, socio-familiar and emotional well-being, and low levels of functional well-being; moderately used problem- and emotion-focused coping styles; had moderate/high meaning- and peace- related spiritual well-being, but the faith-related one was low.

Conclusion: Physical pain resulted well controlled for almost all the patients. Nevertheless, most of sample showed psychological distress, and not high quality of life. The importance of implementing pharmacological treatments with psycho-social-spiritual interventions aimed at reducing psychological symptoms, promoting quality of life, and potentiating adaptive coping styles seems to be evident. So, combining pharmacological and psychotherapeutic treatments could be desirable. As patients' pain is considered total, "total care" should be guaranteed, to treat all patients' suffering dimensions.

Abstract number: P02-093

Abstract type: Poster

Effect of Palliative Radiotherapy on Pain Management in Axial Bone Metastasis: A Retrospective Study of a Portuguese Palliative Care Unit 
Moutinho A. ${ }^{1}$, Koch Jamal S. ${ }^{1}$, Pinto Machado J. ${ }^{2}$, Sá Araújo V. ${ }^{3}$, Costa I. ${ }^{3}$, Ferraz Gonçalves J. ${ }^{3}$

${ }^{1}$ Hospital de Chaves - CHTMAD, Chaves, Portugal, ${ }^{2}$ Internal Medicine, Centro Hospitalar de Tondela-Viseu, Viseu, Portugal, ${ }^{3}$ Palliative Care, Instituto Português de Oncologia do Porto Francisco Gentil, Porto, Portugal

Introduction: Bone and specially vertebral metastasis are a known cause of impairment of function and pain of difficult control. Radiotherapy is a known adjuvant to this pain. The purpose of this study is to evaluate the evolution of the analgesia before and after axial palliative radiotherapy. Material and methods: Retrospective study of patients submitted to antialgic palliative radiotherapy after admission between 2013 and 2017 in a Portuguese Palliative Care Unit. Evaluation of pain control 48 hours before, 1 week and more than 4 weeks after the treatment. The data were submitted to uni and multivariable descriptive statistic analysis, with $\mathrm{p}$ value $<0.05$.

Results: Our population had $n=70$ patients, with $65.5 \%$ males. Lung tumors were the most prevalent $(21.4 \%)$, with $21 \%$ of cases being diagnosed with bone metastasis at the time of cancer diagnosis. Dorsal spine was the most frequent $(54.3 \%)$. Single fraction 8Gy radiotherapy (RT) schemes were the most used (65.7\%), and $73 \%$ of them occurred within the first month of bone metastasis diagnosis. Before radiotherapy, $92.9 \%$ of patients were medicated with an opioid with average of 170.2 oralmorphine-equivalents (OME), and $48.6 \%$ had an adjuvant drug. At this time the need of rescue analgesia was present in $75.7 \%$ with $349.8 \mathrm{OME}$ in average. At the first week post-RT $97.1 \%$ of patients had opiods on their prescription with an average of $204.9 \mathrm{OME}, 63.8 \%$ needing rescueanalgesia, on average $2376.6 \mathrm{OME}$, and $55,1 \%$ being medicated with an adjuvant. One month post-RT $95.3 \%$ of patients were medicated with an opioid, with medium of $222.3 \mathrm{OME}$, and $41.9 \%$ needing rescue analgesia with $20.5 \mathrm{OME}$, being $55.8 \%$ medicated with an adjuvant. 27 patients died during the time of study.

Conclusions: Palliative radiotherapy is an option regarding pain management in axial metastasis when combined to the correct management of analgesia, and can spare opioid in these cases.

Abstract number: P02-094

Abstract type: Poster

Coordination: Integral Oncological Support Team / Clinic of Pain. Pain Management Priorization

Rodriguez Mesa D. ${ }^{1,2}$, Bella Romera S. ${ }^{3}$, Moreno Lizandra N. ${ }^{1,2}$, Bottaro Parra D. ${ }^{1}$, Arquez Pianetta M. ${ }^{1,2}$, Torres Royo L. ${ }^{1,2}$, Vidal Rodriguez A. ${ }^{1,2}$, Gumà Padrò J.1,2

${ }^{1}$ Oncology, Hospital Sant Joan, Reus, Spain, ${ }^{2}$ Oncology, Institut Català D'oncologia, Reus, Spain, ${ }^{3}$ Anesthesiology, Hospital Sant Joan, Reus, Spain
Materials and methods: The Integral Oncological support team of our hospital has created a pain integral valoration comitee, in order to priorize the evaluation, management and treatment in cases with complicated pain approach.

The comitee is composed by: an anestesiologist, a palliativist, a nurse, a psycooncologist; and it meets weekly. A retrospective analyse has been done, from January to June 2017 ( 6 months). The variables revised were: age, sex, neoplasia, pain type and interventionist technique proposed. Results: 87 patients have been evaluated, $57 \%$ women, age ranged between 30-89 years old. 62 patients $(71 \%)$ received some interventionist technique. The interventions registered in those patients were: Epidural, caudal, facet and dorsal infiltration, trigger points, botulinic toxin, celiac plexus, epidural catheter, intrathecal catheter, neurostimulator, popliteal catheter, capsaicin at $8 \% .90 \%$ of the patients experimented pain control improvement, making possible an opioid and/or coanalgesics diminuisement of dose. Palliative nursing followed all the patients (control of toxicity, efficacy...).

Conclusions: The multidisciplinar evaluation helps to priorize cases with difficult pain approach and simplifies the access of patients to anesthesic and antialgic interventional techniques, which aid to get to a pain control and decreases opioid toxicities risk.

Future projects: To incorporate a traumatologyst and a radiation oncologyst in the integral comitee. To register patients evaluation sistematically.

Abstract number: P02-095

Abstract type: Poster

EGFR-inhibition for Neuropathic Cancer Pain

Cameron M.G., Kersten C.

Center for Cancer Treatment, Sørlandet Hospital Trust, Kristiansand, Norway

Aims: Malignant invasion of nerves may lead to severe, treatment-refractory neuropathic cancer pain (NCP). Novel treatment alternatives are urgently needed to palliate this despairing condition. Based on repeated observation of rapid relief of NCP after single doses of intravenous epidermal growth factor receptor inhibitor (EGFR-I), we have successfully treated patients with treatment-refractory NCP with off-label EGFR-Is. Pain research is complicated by endpoint subjectivity. Patients with advanced cancer are heterogeneous, with complex clinical pictures, hampering feasibility of standard drug-trial procedures. The aims of the study are to further understanding of the EGFR-I/NCP association and facilitate planning drug trials.

Methods: Investigator-initiated, explorative, systematic observational study of patients with cancer and neuropathy receiving any human epidermal growth factor receptor inhibitor. A variety of evaluation tools assess symptoms and test feasibility for this population. Choice of method is based on what is ethical, feasible and clinically relevant for each patient. Results:

Preliminary findings (ongoing study) after a singe treatment for the 8 patients with NCP given an EGFR-I (panitumumab).

\begin{tabular}{|c|c|c|c|c|c|c|}
\hline $\begin{array}{l}\text { Age/gender } \\
\text { Underlying lesion } \\
\text { Functional status }\end{array}$ & $\begin{array}{l}\text { Best possible capture } \\
\text { of pre-treatment } \\
\text { "worst NCP last } 24 \\
\text { hours" on a 0-10 } \\
\text { NRS within } 7 \text { days of } \\
\text { treatment }\end{array}$ & $\begin{array}{l}\text { Best possible } \\
\text { capture of "worst } \\
\text { NCP last } 24 \text { hours" } \\
\text { on a 0-10 NRS } \\
\text { within } 14 \text { days of } \\
\text { 1st treatment }\end{array}$ & $\begin{array}{l}\text { Was } \\
\text { secondary } \\
\text { target met? }\end{array}$ & $\begin{array}{l}\text { Patients' } \\
\text { satisfaction } \\
\text { with } \\
\text { treatment }\end{array}$ & $\begin{array}{l}\text { Physician } \\
\text { assessment: } \\
\text { Clinical benefit } \\
\text { / risk }\end{array}$ & $\begin{array}{l}\text { Feasibility issues: } \\
\text { Major hurdles for a } \\
\text { conventional drug } \\
\text { trial }\end{array}$ \\
\hline $\begin{array}{l}\text { 63/F Gluteal } \\
\text { sarcoma ECOG } 3\end{array}$ & $\begin{array}{l}8 \text { (single } \\
\text { measurement at } \\
\text { treatment start) }\end{array}$ & ND & ND & ND & $\begin{array}{l}\text { No beneficial } \\
\text { effect / No toxicity }\end{array}$ & $\begin{array}{l}\text { - Short baseline } \\
\text { - Missing data } \\
\text { - Rapidly progressive } \\
\text { disease Confounding } \\
\text { treatment }\end{array}$ \\
\hline $\begin{array}{l}\text { 73/M Rectal } \\
\text { cancer ECOG } 2\end{array}$ & 6.7 (avg of 7 days) & ND & ND & ND & $\begin{array}{l}\text { Significant benefit } \\
\text { / No toxicity }\end{array}$ & - Missing data \\
\hline $\begin{array}{l}\text { 73/M Colon } \\
\text { cancer ECOG } 1\end{array}$ & 6.6 (avg of 7 days) & 3.7 (avg of 7 days) & $\begin{array}{l}\text { Goal met: } \\
\text { Better sleep }\end{array}$ & Better & $\begin{array}{l}\text { Significant benefit } \\
\text { / Grade } 2 \text { skin } \\
\text { toxicity }\end{array}$ & $\begin{array}{l}\text { None: potential drug } \\
\text { trial participant }\end{array}$ \\
\hline
\end{tabular}


Table. (Continued)

\begin{tabular}{|c|c|c|c|c|c|c|}
\hline $\begin{array}{l}\text { Age/gender } \\
\text { Underlying lesion } \\
\text { Functional status }\end{array}$ & $\begin{array}{l}\text { Best possible capture } \\
\text { of pre-treatment } \\
\text { "worst NCP last } 24 \\
\text { hours" on a 0-10 } \\
\text { NRS within } 7 \text { days of } \\
\text { treatment }\end{array}$ & $\begin{array}{l}\text { Best possible } \\
\text { capture of "worst } \\
\text { NCP last } 24 \text { hours" } \\
\text { on a } 0-10 \text { NRS } \\
\text { within } 14 \text { days of } \\
\text { 1st treatment }\end{array}$ & $\begin{array}{l}\text { Was } \\
\text { secondary } \\
\text { target met? }\end{array}$ & $\begin{array}{l}\text { Patients' } \\
\text { satisfaction } \\
\text { with } \\
\text { treatment }\end{array}$ & $\begin{array}{l}\text { Physician } \\
\text { assessment: } \\
\text { Clinical benefit } \\
\text { / risk }\end{array}$ & $\begin{array}{l}\text { Feasibility issues: } \\
\text { Major hurdles for a } \\
\text { conventional drug } \\
\text { trial }\end{array}$ \\
\hline $\begin{array}{l}\text { 31/F Cervical } \\
\text { cancer ECOG } 1\end{array}$ & $\begin{array}{l}10 \text { (single } \\
\text { measurement at } \\
\text { treatment start) }\end{array}$ & $\begin{array}{l}1 \text { (single } \\
\text { measurement } \\
\text { after } 2 \text { days) }\end{array}$ & $\begin{array}{l}\text { Goal met: } \\
\text { Reduce } \\
\text { analgesics }\end{array}$ & $\begin{array}{l}\text { Much } \\
\text { better }\end{array}$ & $\begin{array}{l}\text { Significant benefit } \\
\text { / Grade } 1 \text { skin } \\
\text { toxicity }\end{array}$ & $\begin{array}{l}\text { Short baseline } \\
\text { Confounding } \\
\text { treatments }\end{array}$ \\
\hline $\begin{array}{l}\text { 70/F Pancreatic } \\
\text { cancer ECOG } 4\end{array}$ & $\begin{array}{l}10 \text { (single } \\
\text { measurement at } \\
\text { treatment start) }\end{array}$ & 0 (avg of 3 days) & $\begin{array}{l}\text { Goal met: } \\
\text { Reduce } \\
\text { analgesics }\end{array}$ & ND & $\begin{array}{l}\text { Significant benefit } \\
\text { / No toxicity }\end{array}$ & $\begin{array}{l}\text { - Short baseline } \\
\text { - Somnolent; short } \\
\text { survival } \\
\text { - Confounding } \\
\text { treatments Mixed } \\
\text { pain }\end{array}$ \\
\hline $\begin{array}{l}\text { 59/M Lung } \\
\text { cancer ECOG } 3\end{array}$ & $\begin{array}{l}10 \text { (single } \\
\text { measurement at } \\
\text { treatment start) }\end{array}$ & 6.7 (avg of 7 days) & $\begin{array}{l}\text { Goal met: } \\
\text { Better sleep }\end{array}$ & $\begin{array}{l}\text { Much } \\
\text { better }\end{array}$ & $\begin{array}{l}\text { Significant benefit } \\
\text { / Grade } 1 \text { skin } \\
\text { toxicity }\end{array}$ & $\begin{array}{l}\text { - Short baseline } \\
\text { - Confounding } \\
\text { treatments } \\
\text { - Mixed pain Unable } \\
\text { to distinguish } \\
\text { non-NP }\end{array}$ \\
\hline $\begin{array}{l}\text { 89/F Uterine } \\
\text { cancer ECOG } 3\end{array}$ & $\begin{array}{l}9 \text { (single } \\
\text { measurement at } \\
\text { treatment start) }\end{array}$ & 0 (avg of 4 days) & $\begin{array}{l}\text { Goal met: } \\
\text { Reduce } \\
\text { analgesics }\end{array}$ & $\begin{array}{l}\text { Much } \\
\text { better }\end{array}$ & $\begin{array}{l}\text { Significant benefit } \\
\text { / No toxicity }\end{array}$ & $\begin{array}{l}\text { - Short baseline } \\
\text { - Confounding } \\
\text { treatments } \\
\text { - Mixed pain } \\
\text { - Unable to } \\
\text { distinguish non-NP }\end{array}$ \\
\hline $\begin{array}{l}\text { 68/M Lung } \\
\text { cancer ECOG } 4\end{array}$ & $\begin{array}{l}10 \text { (single } \\
\text { measurement at } \\
\text { treatment start) }\end{array}$ & 5.7 (avg of 6 days) & $\begin{array}{l}\text { Goal met: } \\
\text { Get out of } \\
\text { bed }\end{array}$ & $\begin{array}{l}\text { Much } \\
\text { better }\end{array}$ & $\begin{array}{l}\text { Significant benefit } \\
\text { / No toxicity }\end{array}$ & $\begin{array}{l}\text { - Short baseline } \\
\text { - Delirium; poor } \\
\text { follow-up } \\
\text { - Mixed pain } \\
\text { - Rapidly progressive } \\
\text { disease }\end{array}$ \\
\hline
\end{tabular}

$\mathrm{F}=$ female; $\mathrm{M}=$ male; $\mathrm{ECOG}=$ Eastern Cooperative Oncology Group; NRS = numeric rating scale; avg = average; ND = not done; * = according to the Patient Global Impression of Change scale.

Conclusions: Preliminary findings strongly support EGFR-Is' potential to be of significant benefit to a range of patients with treatment refractory NCP, warranting further study. Findings also reinforce the difficulty of using conventional drug trial endpoints and designs in this population. Flexibility and innovative research methods must therefore be considered for pivotal trials.

Abstract number: P02-096

Abstract type: Poster

The Current Usage of Adjuvant Analgesics for Refractory Cancer Pain in Japan: Nationwide Cross-sectional Survey

Tagami K. ${ }^{1}$, Matsuoka H. ${ }^{2,3}$, Ariyoshi K. ${ }^{4}$, Oyamada S. ${ }^{4}$, Koyama A. ${ }^{2}$, Kizawa Y.5 ${ }^{5}$ Inoue A. ${ }^{1}$

1Palliative Medicine, Tohoku University School of Medicine, Sendai, Japan, ${ }^{2}$ Psychosomatic Medicine, Kindai University Faculty of Medicine, Osaka, Japan, ${ }^{3}$ Faculty of Health, University of Technology Sydney, Sydney, Australia, 4JORTC Data Center, Tokyo, Japan, ${ }^{5}$ Palliative Medicine, Kobe University Graduate School of Medicine, Kobe, Japan

Background: One third of patients with cancer pain have not received adequate analgesia with analgesics. Adjuvant analgesics is added in those patients; however strong evidences to support the practice are poor. One of the reasons is few studies investigated the effectiveness of adjuvant analgesics for cancer pain according to each pathophysiology of cancer pain.
Aim: We aim to clarify the current usage of adjuvant analgesics for refractory cancer pain which responds poorly to opioids. Especially, we present it according to each pathophysiology of cancer pain.

Methods: This study was a cross-sectional questionnaire-based survey. Two hundred and eight certified palliative care specialists (cPCS) were surveyed via an online.

Results: A total of 87 (42\%) cPCS responded. Of patients with refractory cancer pain, the median proportion that the CPCS gave adjuvant analgesics was $36 \%$. When an adjuvant analgesic was administered to patients with refractory cancer pain, median dosage of opioids after dose titration was $60 \mathrm{mg}$ (calculation of morphine equivalent daily dose). Pregabalin was the highest frequency of use in a year by cPCS [mean (standard deviation): $59.5 \%$ (27.3)]. Dexamethasone/betamethasone was the second [30.4\% (27.9)], and duloxetine was the third [22.6\% (21.7)]. Most cPCS considered pregabalin was effective for malignant painful radiculopathy and corticosteroids was effective for pain by cancer-related spinal cord compression (93.1\%, 97.3\%, respectively).

Discussion: Many cPCS may select analgesic adjuvant according to pain's pathophysiology or cancer pain syndrome of each case. The large prospective study is now planned to suggest the effectiveness of adjuvant analgesics for cancer pain from the view point of the pathophysiology of cancer pain.

Acknowledgements: We are grateful for support from the 2017 Japan Agency for Medical Research and Development award (Innovative Clinical Cancer Research; Grant No.: 17ck0106328h0001). 
Abstract number: P02-097

Abstract type: Poster

Safety Data on Intravenous Opioid Dosing Diminishes Unwarranted Clinical Variation in Opioid Prescribing

Geiger-Hayes J. ${ }^{1}$, Burns B. ${ }^{2}$, Von Gunten C. ${ }^{1}$, Lookabaugh B. ${ }^{1}$, Poth A. ${ }^{2}$, Ferris $F^{1}$

1Palliative Care, OhioHealth, Columbus, United States, ${ }^{2} \mathrm{OhioHealth,}$ Columbus, United States

Background: We hypothesized that fear of causing respiratory depression affects IV opioid prescribing by palliative medicine clinicians. We previously reported no correlation between naloxone administration and Q15min PRN IV opioids.

Aim: A quality goal for our palliative medicine group practice is to diminish clinically unwarranted variation in practice across an 11-hospital system serving a population of 3.5 million over 20 US counties.

Methods: We retrospectively reviewed opioid orders written by palliative medicine clinicians from January through December 2017. The number of orders for IV opioids written Q15min PRN were then split into 6 month groups and statistically compared. The naloxone safety data was shared halfway through the year. Individual prescriber data was shared every other month.

Results: IV opioid dosing for 21 physicians and 12 nurse practitioners were measured. Prior to safety education, $67 \%$ of all IV PRN opioid orders were Q15min PRN for the physician group. After safety data was shared, $75 \%$ of all IV PRN opioid orders were Q15min PRN. For the nurse practitioner group, $46 \%$ of the IV PRN opioid orders were Q15min PRN in the pre-safety education period. After safety data was shared, the nurse practitioner group increased to $54 \%$ ( $p<0.0001$ for both groups). There was an increase in physician prescribing Q15min PRN from 6 to 13 at or above $80 \%$, and nurse practitioners stayed the same.

Conclusion: Reporting the lack of association between the dosing interval of Q15min PRN and administration of naloxone is associated with a further decrease in unwarranted clinical variation. We previously reported that giving physicians and advanced practice nurses information about their patterns of prescribing in relation to their peers diminished variation. This supports the assertion that anxiety about causing respiratory depression with pharmacologically based dosing regimens is assuaged with safety data from the same institution.

Abstract number: P02-098

Abstract type: Poster

\section{New Approaches in Cancer Pain Management: The Concept of Multimorphic Pain}

Lemaire A. ${ }^{1}$, Burnod A. ${ }^{2}$, George B. ${ }^{3}$, Maindet C. ${ }^{4}$, Gilles A. ${ }^{5}$, Minello C. ${ }^{6}$ ${ }^{1}$ Oncology and Medical Specialties Department, Centre Hospitalier de Valenciennes, Valenciennes, France, ${ }^{2}$ Institut Curie, Department of Supportive Care, Paris, France, ${ }^{3}$ Pain Consultation, Hopital Saint Louis, Paris, France, ${ }^{4}$ Pain Center, CHRU de Grenoble, Grenoble, France, ${ }^{5}$ Pain Center, Clinique de I'Orient, Lorient, France, ${ }^{6}$ Pain Center, Centre George François Leclerq, Dijon, France

The last few years have been marked by the emergence of early palliative care, and it has been clearly substantiated by evidence. Many authors have highlighted different models for early integration of palliative care in oncology, from as early as the cancer diagnosis. These integrative models in cancer have demonstrated their positive impact in terms of patient quality of life, management of symptoms, and optimization of care pathways, regardless of the management method (outpatient, in hospital, home care).

Pain medicine fits into this early palliative care dynamic perfectly and has also undergone its own series of determining changes, both in terms of pathophysiological knowledge, diagnosis or treatment options (be they drug-based, interventional or non-drug-based).

As witnesses to these changes, we have created a group of French clinical doctors with different, complementary initial specialisations and means of operating. This group has tried to put forward its vision of cancer pain by cross-referencing views, clinical experience and the international literature.

It is thus through the concept of multimorphic pain that we will approach the particular aspects of cancer pain management, both in terms of assessment and treatment (in future issues), as cancer pain is constantly subject to disruptive elements leading to unbalanced pain control. This modelling highlights the specificities of cancer pain, as a nosological entity in its own right that requires an interdisciplinary, multimodal and dynamic approach, as well as personalised management. The fundamental dimensions that support this multimorphic pain model are the care pathway in its entirety (diagnosis, cure, relapse, palliative care), as well as the cancer treatments that have an impact on pain (chemotherapy in the broadest sense, surgery, radiotherapy). Finally, identifying the disruptions to this dynamic model is a major key to understanding and providing optimal management for our patients.

\section{Abstract number: P02-099 \\ Abstract type: Poster}

\section{A Picture Paints a Thousand Words}

Reilly L. ${ }^{1}$, Hayes E. ${ }^{1}$, Larkin M.-A. ${ }^{1}$, Waldron D. ${ }^{1}$, Ravindrarasan S. ${ }^{2}$ ${ }^{1}$ Galway University Hospital, Galway, Ireland, ${ }^{2}$ Palliative Medicine, Galway University Hospital, Galway, Ireland

Background: The essence of good clinical practice is obtaining a comprehensive history. However, it can be difficult to achieve this goal when a patient is unable to express their symptoms. Therefore, thorough clinical assessment is paramount.

Case Presentation: A fifty year old lady, with a history of Sheehan's syndrome, was referred to Palliative Care for symptom control at end of life. At initial assessment, she was in distress and obvious pain. She was semiconscious and investigations revealed multi-organ failure. A detailed list of differential diagnoses was compiled and an initial management plan of sub-cutaneous pump with low dose opioid and a centrally acting antiemetic was commenced. However, she required repeated doses of rescue opioid and anti-emetic in the following 24 hours. At this time a detailed collateral history from her husband revealed that weeks before her decline she had been 'pinching' her chest wall. A photograph of her chest wall showed how that she was deeply bruising herself in an area that she could not herself visualize.

Management and Outcomes: The photograph identified a dramatic dermatomal vesicular rash and bruising at the left thoracic level 5 and 6 . She was unable to take oral acyclovir. We commenced carbamazepine 250 mgs suppositories BD, as a membrane stabilizer. Within hours of commencing carbamazepine she settled and had a comfortable death.

Discussion and Learning Points: This case demonstrates the importance of a detailed clinical assessment and collateral history in the non-verbal patient for prompt and effective management of patients' symptoms. Appropriate prescribing based on an accurate diagnosis will limit adverse effects and ensure optimal response to therapy.

Conclusion: Careful clinical assessment will guide best practice at end of life.

Abstract number: P02-100

Abstract type: Poster

Delirium Prolonged for Three Days Following Addition of One Dose of $\mathbf{5} \mathbf{~ m g}$ Methadone to Ongoing Opioid

Ikegaki J. ${ }^{1}$, Kizawa Y. ${ }^{2}$

${ }_{1}$ Pain and Palliative Medicine, Hyogo Cancer Center, Akashi, Japan, 
2Department of Palliative Medicine, Kobe University Graduate School of Medicine, Kobe, Japan

It was recently reported that favorable pain relief could be achieved by adding low-dose methadone (AdOn), precluding the need to switch to another opioid. Not only is it easy, but an excellent analgesic effect is reported. However, we herein report a case of severe adverse effects associated with AdOn.

Case report: A 65-year-old man had been diagnosed with mediastinal thyroid papillary carcinoma 3 years ago. After surgery, anticancer drug treatment had been continued. One month ago, he began to suffer chest pain due to the fifth thoracic vertebrae and sternal metastasis. Although oral oxycodone was started, it was ineffective. The patient was referred to the palliative care team. After the first visit, titration by oxycodone injection was started. When the dose exceeded $120 \mathrm{mg} /$ day, methadone $5 \mathrm{mg}$ was added. After the first dose in the evening, he became restlessness, aggressive and wandering. Disorientation disorder continued for up to three days while antipsychotics were administered. Oxycodone injection was changed to oral oxycodone again, and other drugs were administered similarly except methadone. This patient survived for six months after this event.

Discussion: Since methadone is not only a $\mu$ receptor agonist but also an $\mathrm{N}$-methyl-d-aspartate (NMDA) antagonist, an analgesic effect is highly expected. We expected similar benefits in this case, but the result was unexpected, with delirium continuing for three days. The key mechanism involved here seems to be NMDA antagonism rather than increased action on $\mu$ receptors. NMDA antagonism is thought to be related to opioid tolerance. We suspect that adding methadone diminished the opioid tolerance and abruptly intensified the effects of oxycodone, which had previously not worked well. AdOn may thus suddenly lead to an overdose of oxycodone and cause adverse events, as seen in this case.

Adding a small amount of methadone is not always safe.

Abstract number: P02-101

Abstract type: Poster

Role of Dipeptiven in Treatement of Oralmucositis and Releiving Pain Following Chemotherapy and Radiotherapy in Patients with Cancer

Nafae $W$.

AAOC, Palliative, Alexandria, Egypt Presenting author email address: waleedhamdynafae@gmail.com

Background: Many cancer patients reciving chemotherapy with or without radiotherapy to head and neck region are suffering from sever mucositis with sever dysphagia and odynophagia.

Purpose: We performed this study to determine the safety and efficacy of L-alanyl-L-glutamine in the treatment of mucositis in patients with cancer receiving chemotherapy and radiotherapy.

Methods and materials: One hundred patients with different types of cancer were treated with chemotherapy and radiotherapy were asked to participate.

Sixty patients received the chemotherapy schedules and had febrile neutropenia or even pancytopenia with grade two to three mucositis and so were assigned to receive either intravenous

L-alanyl-L-glutamine ( Dipeptiven vial) every 24 hours or 12 hours according to severity and creatinine clearance.

Fourty patients were on radiotherapy to head and neck region and so got grade two to three mucositis so received also Dipeptiven in the same way as with chemotherapy toxicity.

Results: Mucositis was assessed by the Objective Mucositis Score (OMS) and the World Health Organization (WHO) grading system. There was a significant improvement in mucositis and pain developed in patients received L-alanyl-L-glutamine.

No adverse effects related to the drug or the infusions were noted in all patients.
Conclusion: For patients with cancer suffering from moderate to sever mucositis due to Chemotherapy or Radiotherapy, intravenous L-alanyl-Lglutamine may be an effective measure to decrease and treat the mucositis and alleviating pain during swallowing.

Abstract number: P02-102

Abstract type: Poster

\section{See your Pain - A Review}

Maia R. ${ }^{1}$, Aparicio A.I. ${ }^{1}$, Mina M. ${ }^{1}$, Martins E. ${ }^{2}$, Clara M.D.J. ${ }^{3}$

${ }^{1}$ USF 'A Ribeirinha', ULS Guarda, Guarda, Portugal, 'USF 'Beira Rio', Aveiro, Portugal, 3USF 'A Ribeirinha', Guarda, Portugal

Background: Pain is a subjective experience, influenced by exogenous and endogenous factors. The simplest, one-dimensional scales are of little value in patients with communicative difficulties. Aging is often associated with multiple illnesses, including dementia. Difficulties of communication, leads to poor pain management.

Aim: The Importance to recognize pain in elderly patients with communicative difficulties; knowing the different tools and scales used to evaluate pain in this patient; recognize these tools in clinical practice; improve communication strategies between doctor-patient-family/caregiver.

Method and Results: A search was performed on the PubMed database with the keywords "pain and methods of pain assessment and communication", published in the last 5 years. Two hundred and seventy-one articles were found, being selected only 22 . Among the excluded articles, 249 did not meet our criteria. Among selected articles, 11 referred the different advantages and disadvantages of each scale used; 3 about the evaluation methods for pain, 3 to the different signs of pain, and 1 to the importance of caregiver in different methods to diagnose pain.

Conclusion: Pain, patient and tools have different dimension to be considered in order to analyze different dimensions such as: social, psychological, behavioral, cognitive and individual. These articles demonstrated a statistically significant importance between scales applied and detection of pain. An early approach to pain in noncommunicative patients is essential to improve our clinical results. It is important to carry out new studies in order to validate different scales, signs and symptoms that better define pain for early diagnosis.

\section{Abstract number: P02-103}

Abstract type: Poster

\section{The Comparison of Clinical Characteristics of Cancer Pain}

Patients with or without Pain Catastrophizing

Matsuda Y., Hakata S., Fujino Y.

Anesthesiology \& Intensive Care Medicine, Osaka University Graduate School of Medicine, Osaka, Japan

Background: Pain Catastrophizing is an important psychological factor of pain. In chronic non-cancer pain population, many studies showed the characteristics of pain catastrophizer and the influence of pain catastrophizing for the aggravation of pain. However, there was no study about the characteristics of cancer pain patients who catastrophized about pain.

Aim: The aim of this study was to compare clinical characteristics of cancer pain patients with or without pain catastrophizing.

Methods: 100 patients with cancer-related pain were employed in this prospective study. We corrected patient's demographic data (age, gender, primary site, physical status (PS), opioid dose) and four questionnaires data (Pain Catastrophizing Scale (PCS), the short form of Brief Pain Inventory (BPI), Hospital Anxiety and Depression Scale (HADS), and European Organization for Research and Treatment of Cancer Quality of Life Questionnaire Core 15 Palliative (EORTC QLQ-C15-PAL)). We compared the corrected data between the patients who catastrophized pain 
(PCS score $>=30$ ) and the patients without pain catastrophizing (PCS $<$ 30). The date was statistically analyzed with chi-square test and MannWhitney $\mathrm{U}$ test.

Results: There was no deference in age, gender, primary tumor site, PS, opioid dose per day, pain intensity score (BPI) and the score of physical function, nausea/vomiting, dyspnea, appetite loss, constipation (EORTC QLQ-C15-PAL) between the groups. However, pain interference score (BPI), HADS score, and the score of global QOL, emotional function, fatigue, pain, insomnia (EORTC QLQ-C15-PAL) were significantly worse in the patients who catastrophized pain than the patients without pain catastrophizing

Conclusion: Our study indicated that pain catastrophizing in cancer pain patients was not associated with the exacerbation of pain intensity and the increase of opioid dosage, however, might decrease the activity of daily living and quality of life regardless of physical condition.

Abstract number: P02-104

Abstract type: Poster

How Do We Measure the Effects of Opioids on the Cognition of Older Adults with Cancer and Chronic Non-cancer Pain? A Systematic Review

Pask S. ${ }^{1}$, Dell'Olio M. ${ }^{2}$, Murtagh F.E.M. ${ }^{1}$, Boland J.W. ${ }^{1}$

${ }^{1}$ Hull York Medical School, University of Hull, Wolfson Palliative Care Research Centre, Hull, United Kingdom, ${ }^{2}$ Hull York Medical School, University of Hull, Academy of Primary Care, Hull, United Kingdom

Background: Pain is poorly managed in older adults with cancer/chronic non-cancer conditions, in part because of concerns about adverse cognitive effects from opioids. There is little consensus on how to measure cognitive effects of opioids in this population.

Aim: To identify, appraise and synthesise evidence on screening tools/ neuropsychological assessments to assess older adults' cognition.

Methods: Protocol followed the Preferred Reporting Items for Systematic Review and Meta-Analysis Protocols (PRISMA-P). Searches of MEDLINE, EMBASE, PsycINFO, CINAHL, Cochrane Library and Web of Science, from inception to April 2018. Randomised controlled trials, quasi-experimental studies and observational studies of adults aged $\geqslant 65$ taking opioids, using a screening tool and/or neuropsychological assessment, were included. Primary outcome of interest: screening tools/neuropsychological assessments used. Study eligibility, data extraction and quality assessment were independently performed in duplicate.

Results: Searches returned 3,370 unique papers, of which 12 met inclusion criteria. Six studies adopted screening tools to assess cognitive impairment and five used neuropsychological assessments; only one study used both. For screening tools, the Mini Mental State Exam (MMSE) was most frequent (5 studies) and for neuropsychological assessment, the Trail Making Test (3 studies). There was little consistency in choice/timing of tests. Multiple neuropsychological assessments are reported to accurately capture different cognitive domains that may be useful in detecting the effects of opioids, whilst the accuracy and sensitivity of the MMSE has been debated.

Conclusion: Existing tools are limited if used in isolation and a combination of screening/neuropsychological assessments is necessary to determine global cognitive effects of opioids in older adults with cancer/ chronic non-cancer pain. Validity and reliability of these tools in this population requires further investigation.

Abstract number: P02-105

Abstract type: Poster

Oral Methadone Administration for Cancer Patients with Neuropathic Pain due to Neoplastic Brachial Plexopathy Matsuda Y. ${ }^{1}$, Okayama S. ${ }^{2}$, Yoshikawa $Y^{3}{ }^{3}$, Ishii R. ${ }^{4}$, Okamoto Y. Hashimoto M.5, Tanaka I. ${ }^{5}$
1Department of Palliative Care Medicine, Ashiya Municipal Hospital, Ashiya, Japan, ${ }^{2}$ Palliative Care Unit, Takarazuka Municipal Hospital, Takarazuka, Japan, ${ }^{3}$ Department of Palliative Care Medicine, Takarazuka Municipal Hospital, Takarazuka, Japan, ${ }^{4}$ Department of Palliative Medicine, Ashiya Municipal Hospital, Ashiya, Japan, ${ }^{5}$ Department of Pharmacy, Ashiya Municipal Hospital, Ashiya, Japan

Background: The brachial plexus nerves originate in the cervical (C5-C8), and first thoracic (T1) spinal nerves, and innervate the muscles and skin of the chest, shoulder, arm and hand. Brachial plexus injuries can occur as a result of shoulder trauma and inflammation. Malignant tumors can also cause neoplastic brachial plexopathy (NBP). Refractory neuropathic pain is the most common symptom of NBP. Methadone which is a synthetic opioid with high efficacy as an opioid-receptor agonist and $\mathrm{N}$-methyl-D-aspartate (NMDA)-inhibitory effects may play a role of pain relief.

Aims: To assess whether oral methadone administration exhibits safety and efficacy against neuropathic pain due to NBP.

Methods: In 2 hospitals, 105 patients with cancer pain were prescribed oral methadone between April 2013 and July 2017. Among those, NBP was diagnosed in 4 cases without brain or cervical spine metastasis. The clinical aspects of these patients were analyzed retrospectively.

Results: All cases had no indication for operation due to advanced cancer. Three cases received radiation therapy before methadone administration, but there was insufficient effect. One case refused radiation therapy. All cases had both nociceptive and neuropathic pain. Methadone was initiated from among the other opioids due to refractory pain using the stop-and-go method as shown in Table 1. NRS pain scores were decreased in all cases, and no severe side effects were detected.

The pain control by methadone was considered successful in Case 1-3, though insufficient in Case 4.

Conclusion: Although more studies are necessary, oral methadone administration may be a safe analgesic method for the patients with neuropathic pain due to neoplastic brachial plexopathy.

Table 1

\begin{tabular}{lllllll}
\hline Case & Age & Sex & Cancer & $\begin{array}{l}\text { MEDD } \\
\text { mg/day }\end{array}$ & $\begin{array}{l}\text { Methadone } \\
\text { mg/day }\end{array}$ & NRS \\
\hline 1 & 65 & M & Sarcoma & 100 & $15 \rightarrow 20$ & $9 \rightarrow 3$ \\
2 & 62 & M & Sarcoma & 120 & $15 \rightarrow 20$ & $8 \rightarrow 3$ \\
3 & 38 & F & Breast & 660 & $45 \rightarrow 60$ & $9 \rightarrow 4$ \\
4 & 60 & M & Lung & 90 & $15 \rightarrow 20$ & $7 \rightarrow 5$ \\
\hline
\end{tabular}

Abstract number: P02-106

Abstract type: Poster

Ketamine in Context of Oncologic Palliative Care - A Challenge Experience of a Palliative Care Service

Martins Cunha O. ${ }^{1}$, Ferraz-Gonçalves J. ${ }^{2}$

${ }^{1}$ Faculty of Medicine of Porto University, Porto, Portugal, ${ }^{2}$ Medicina Porto, Porto, Portugal

Introduction: Ketamine is a dissociative anesthetic agent which has analgesic properties in subanesthetic doses, and it has been used as an adjuvant treatment for opioid-refractory cancer pain. However, few studies have used the drug in a palliative care population.

Aims: The aim of this study was to assess the use of ketamine in a palliative care center.

Methods: Retrospective study for thirty months in an oncologic adult palliative careservice of patients undergoing treatment with ketamine. Patients were evaluated in the three days before and during the week 
after the introduction of ketamine. Results/Discussion - Twenty patients were included- 9 women and 11 men. Ages varied from 26 to 78 years, median of 54. ketamine was used as an adjuvant treatment for opioidrefractory cancer pain in 18 , and in two to treat depression. In the first group all patients had unbearable pain and $22 \%(4 / 18)$ showed signs of opioids adverse effects; $73 \%(13 / 18)$ had mixed pain. The average dose of ketamine was $85 \mathrm{mg} /$ day in continuous intravenous or subcutaneous infusion in $90 \%$ of patients. They were treated with high doses of opioids- equivalent to $480,5 \mathrm{mg}$ /day of oral morphine. In $70 \%$ the pain was relieved. The dose of opioid decreased in $12 \%$ and the rescue opioid dose in $53 \%$. Improvement in pain was found in an average of 4,8 days. All received neuroleptics with or without benzodiazepines. The psychotomimetic side effects in one patient forced to discontinue ketamine. In the second group the patients demonstrated an improvement in mood as the main response to a single dose of ketamine.

Conclusion: Despite being a promising therapeutic approach in palliative care units more research must be carried out to define the role of ketamine in Palliative Care.

Abstract number: P02-107

Abstract type: Poster

Long-term Use of Methadone for Refractory Cancer Pain: A Cohorte Follow-up Study

Fawoubo A., Tricou C., Bordier V., Economos G., Ruer M., Munier S., Perceau E., Filbet $M$.

Palliative Medecine, Centre Hospitalier de Lyon-Sud, Hospices Civils de Lyon, Pierre Benite, France

Background: Methadone is used in last option line for refractory cancer pain.

Aims: Our aim was to assess the long-term effects of methadone on cancer pain intensity, and on the mood and quality of life of the patients.

Methods: After methadone rotation, pain intensity were assessed on Day 0 (D0), Day 7 (D7), Day 14 (D14), Day 21 (D21), Day 28 (D28) using a visual analogue scale (VAS) over the last 8 days, the neuropathic pain questionnaire (DN4) and the Neuropathic Pain Symptom Inventory (NPSI) for the neuropathic component. Moods and quality of life were assessed using HADS, ESAS and the EORTC QLC30.

Results: Out of 46 patients included, 38 patients were analyzed. They were men(71\%) aged 61 years old (48-74), mostly with an head and neck, lung or pancreatic cancer. They received 422 mg (104-1200)morphine orale equivalent by day before methadone switch and needed an average dose of $32.5 \mathrm{mg} /$ day at D 0 and $39 \mathrm{mg} /$ day at D28 of methadone. Pain intensity decreased, especially from day 14 . At day $28,50 \%$ of patients had low intensity pain and $31.25 \%$ of patients showed a decrease in EVA of 20 points or more. The intensity of the different components of neuropathic pain decreased of 2 points: $53 \%$ for compression, $29 \%$ for burning, $24 \%$ for allodynie.Confusion (20\%) and sweating (43\%) increased significantly the first week, sleepiness increased to $56 \%$ $(p=0,08)$ on D28. Anxiety decreased significantly from $55 \%$ at D0 to $33 \%$ at $D 28(p=0.006)$ on ESAS. There was no significant change in HADS or quality of life during the follow-up.

Conclusion: The results suggests a delayed action of methadone from D14 to D28 related to the anti NMDA action. The analgesic action and few sides effects of methadone could allow its early use in the first line for cancer pain with neuropathic component.

Abstract number: P02-108

Abstract type: Poster

Neuromodulation Using Matrix Stimulation in Cancer Pain - Methodology, Safety and Effectiveness

Mücke M. ${ }^{1}$, Tils M. ${ }^{2}$, Conrad R. ${ }^{3}$, Kravchenko D. ${ }^{2}$, Cuhls $\mathrm{H}^{2}$, Radbruch

L. ${ }^{2,4}$, Marinova M. ${ }^{5}$, Peuckmann-Post V. ${ }^{6}$, Rolke R. $^{6}$
${ }^{1}$ Center for Orphan Diseases, University of Bonn, Bonn, Germany, ${ }^{2}$ Department of Palliative Medicine, University of Bonn, Bonn, Germany, ${ }^{3}$ Department of Psychosomatics, University of Bonn, Bonn, Germany, ${ }^{4}$ Center for Palliative Care, Malteser Hospital Bonn/RheinSieg, Bonn, Germany, ${ }^{5}$ Department of Radiology, University of Bonn, Bonn, Germany, ${ }^{6}$ Department of Palliative Medicine, Medical Faculty RWTH Aachen University, Aachen, Germany

Background: Low-frequency electrical stimulation (LFS) is a stimulation paradigm utilized to induce a normalization of prior sensitized central projection neurons, implicating a synaptic long-term depression of the nociceptive system.

Aims: This feasibility study addresses the applicability of a matrix electrode to reduce the ongoing pain intensity of cancer patients by applying LFS using electrical currents similar to TENS (transcutaneous electrical nerve stimulation).

Methods: Matrix low frequency stimulation $(4 \mathrm{~Hz})$ was applied to the skin within the 'Head's zones' referring to the tumour localization of cancer pain patients. Pain at baseline was compared to a 3-day treatment interval consisting of 5 minutes of matrix stimulation in the morning and evening followed by a 3-day follow-up period without therapy. Main outcome parameters included numeric rating scale values (rating scale $0-100$ ), painDETECT, HADS, and German pain questionnaire as well as the daily opioid consumption calculated as the oral morphine equivalent (OME).

Results: Twenty patients with cancer pain (aged $64.4 \pm 10.3$ years; 9 women) were examined. In the majority of patients the pain was classified as nociceptive. Mean pain reduction under matrix therapy was $30 \%$ under stable daily controlled-release opioid doses between 177 and 184 $\mathrm{mg} /$ day (OME). Seventeen patients (85\%) were responders to matrix therapy defined by a pain reduction of at least $30 \%$, four responders experienced a pain reduction of over $50 \%$. Apart from short-term erythema, there were no side effects.

Conclusion: Findings are consistent with the concept of synaptic longterm depression in cancer pain induced after conditioning LFS using a matrix electrode. Despite the short but well-tolerated treatment duration of $2 \times 5 \mathrm{~min} /$ day, effects persisted throughout the 3-day follow-up. Cutaneous neuromodulation using LFS by a matrix electrode has been shown to be a safe intervention to effectively reduce cancer pain in palliative care patients.

Abstract number: P02-109

Abstract type: Poster

Pharmacoepedemiological Study on the Prescription of Strong Opioid Antalgics to Patients Affected with Metastatic Bone Cancer: Comparison before/after the Qualification of the Status as Palliative

Guastella V., Van Lander A., Tarot A.

CHU Clermont-Ferrand, Clermont-Ferrand, France

Background: Oncologic diseases are largely spread in palliative care. The identification of a palliative situation is very often done too late in the evolution of the disease. Among symptoms, pain is one of the most important and it distorts the quality of life. Bone metastatic location creates the most severe pain intensity. Moreover, in many studies we observe that pain is taken care of by practitioners in very heterogeneous ways. It must be one of the priorities to improve the quality of life. So, we ask why?

Aims: We perform a study to assess the impact of an early palliative identification on the prescription of analgesic strong opioid to argue the real interest of the identification to optimize the comfort and by consequence the quality of life.

Methods: It is a pharmaco-epidemiological study, on the basis of data, the DCIR (Données de Consommation Inter Régimes) which processes exhaustive data. The studied main assessment criterion is the exposure 
time in analgesic strong opioid expressed in OME (Oral Morphine Equivalents)/day, three months before then three months after an identification of palliative situation at the patient's reached of a cancer with at least an bone metastatic location.

Results: We were able to include 112189 patients affected by a cancer with bone metastatic location in palliative situation between 2012 and 2016. Among these patients, 38399 did not die during period. Among these patients $17 \%$ have an institution of strong opioid to $90.4 \mathrm{mg}, 19.8$ $\%$ have an increase, going of $99.6 \mathrm{mg}$ to $142.3 \mathrm{mg}, 32 \%$ have no opioid before or after and $31.1 \%$ have opioid before and not after the palliative identification.

Conclusion: These results show a difference of analgesic care further to the identification of a situation as being palliative. It questions because the prescription of strong opioids must be adapt to the pain and not to the recognition of a palliative situation.

\section{Abstract number: P02-110}

Abstract type: Poster

Improvement of Breakthrough Cancer Pain in Advanced Patients Attended in Palliative Care Units

Canal-Sotelo J. ${ }^{1}$, Maradey P. ${ }^{2}$, Núñez Viejo M. ${ }^{3}$, Guardia Mancilla P. ${ }^{4}$ Gutiérrez Rivero S. ${ }^{5}$, Raja Casillas I. ${ }^{6}$, Herrera Abián M.7, López Bermudo C. ${ }^{8}$, Tuca Rodríguez A. ${ }^{9}$

${ }^{1}$ Hospital Universitari Santa Maria. Gestió de Serveis Sanitaris, lleida, Catalonia, Spain, ${ }^{2}$ Hospital Sant Joan de Déu, Palma de Mallorca, Spain, ${ }^{3}$ Complejo Hospitalario Universitario de Orense, Ourense, Spain, ${ }^{4}$ Hospital Universitario Virgen de las Nieves, Granada, Spain, ${ }^{5}$ Hospital Universitario Virgen del Rocío, Sevilla, Spain, ${ }^{6} \mathrm{Hospital}$ Virgen de la Salud, Toledo, Spain, ${ }^{7}$ Hospital Universitario Fundación Jiménez Díaz, Madrid, Spain, ${ }^{8}$ Medical Department, Angelini Farmacéutica S.A, Barcelona, Spain, ${ }^{9} \mathrm{Hospital}$ Clínic i Provincial, Barcelona, Spain

Background: Breakthrough cancer pain (BTcP) in advanced cancer patients is highly prevalent. Its adequate management will determine quality of life outcomes. The CAVIDIOPAL study was designed to evaluate quality of life in advanced cancer patients with BTcP attended in Spanish palliative care units.

Aims: This is a subanalysis of the CAVIDIOPAL study that focuses on the pharmacological approach to BTcP.

Methods: Prospective, observational and multicenter study carried out during 2016-17. Main inclusion criteria: age $>18$ years, agreed to participate and signed the informed consent, diagnosed of advanced cancer and with BTCP according to the Davies algorithm. Data regarding BTcP management and outcomes were collected at days $0,8,15$, 28 and at 3 months follow-up. Clinical and sociodemographic variables were collected. Descriptive analysis (mean, median and standard deviation) were used to describe the population and variables. Univariate analysis (T-Student test) was used to compare selected variables.

Results: 99 patients were recruited from 8 palliative care services. Mean of age $69 \pm 13$ years. 61 patients were men $(61.6 \%)$. Pancreatic $(N=16$; $16.2 \%)$ and lung cancer $(\mathrm{N}=13 ; 13.1 \%)$ were the most prevalent. Mean of basal VAS of pain was $4 \pm 1.9$ on day 0 and $1.8 \pm 1.3$ on month $3 ; p<$ 0.001 . Fentanyl based formulations were used in 45 patients (45.5\%) to treat basal pain. Mean of VAS for BTcP on day 0 was $8 \pm 1$ and $4 \pm 2.4$ on month $3 ; p<0.001$. During the study, 38 patients $(38.8 \%)$ needed a change in the pharmacological approach for BTcP on day 8 (26 change in the dose and 12 swap drug) and 2 (2.1\%) on the month 3 (both change of dose). Rapid onset opioids based on the lowest available doses of sublingual fentanyl were the most used to treat BTCP in this real clinical practice study.

Conclusions: BTcP can be successfully treated using low fentanyl doses when the basal pain was adequately managed. An accurate and individualized pharmacological approach is also mandatory.
Abstract number: P02-111

Abstract type: Poster

Prevalence, Management and Risk Factors of Opioid-induced Constipation in Cancer Pain: A Nationwide, Cross-sectional Study in Korea

Koh S.-J. ${ }^{1,2}$, Hong Y.S. ${ }^{3}$, Lee K.T. ${ }^{4}$, Kim S. ${ }^{5}$, Lee K.H. ${ }^{6}$, Shin S.-H. ${ }^{7}$, Choi M.Y. ${ }^{8}$, Sym S.J. ${ }^{9}$, Oh H.-S. ${ }^{10}$, Lee S.N. ${ }^{11}$, Song E.-K. ${ }^{12}$

${ }^{1}$ Department of Hematology and Oncology, Ulsan University Hospital, Ulsan, Korea, Republic of, ${ }^{2}$ Ulsan University College of Medicine, Ulsan, Korea, Republic of, ${ }^{3}$ Department of Medical Oncology, Seoul St. Mary's Hospital, Catholic University of Korea, Seoul, Korea, Republic of, ${ }^{4}$ Department of Hematology/Oncology, Sooncheonhyang University Cheonan Hospital, Cheonan, Korea, Republic of, ${ }^{5}$ Department of Medical Oncology, Chungnam National University Hospital, Daejeon, Korea, Republic of, ${ }^{6}$ Department of Hematology/Oncology, Yeungnam University Medical Center, Daegu, Korea, Republic of, ${ }^{7}$ Department of Hematology/Oncology, Kosin University Gospel Hospital, Busan, Korea, Republic of, ${ }^{8}$ Department of Hematology/Oncology, Inje University Busan Paik Hospital, Busan, Korea, Republic of, ${ }^{9}$ Department of Medical Oncology, Gachon University Gil Hospital, Incheon, Korea, Republic of, ${ }^{10}$ Department of Hematology/Oncology, GangNeung Asan Hospital, Gangneung, Korea, Republic of, ${ }^{11}$ Department of Internal Medicine, College of Medicine, Ewha Womans University, Seoul, Korea, Republic of, ${ }^{12}$ Department of Internal Medicine, Chonbuk National University Medical School, Cheonju, Korea, Republic of

Background: Although opioid therapy is the mainstay treatment for moderate-to-severe cancer pain, opioid-induced constipation (OIC) remains as a major side effect which makes patients suffer.

Aims: We investigated the incidence, management, and risk factors of OIC among cancer pain patients in Korea.

Methods: We performed a cross-sectional analysis of 2395 patients with cancer pain from 30 teaching hospitals across Korea. Data was extracted from patient charts and questionnaires. Clinical characteristics, incidence and management of adverse events (AEs) were assessed.

Results: The most common opioid-related AE was constipation (29.7\%). Constipation had a high impact on patients' daily activities, with $69.8 \%$ of patients reporting severe interference with their daily activities ( $\geqslant$ Grade 3 interference). The incidence of OIC was higher with opioid dose increase, but similar across different routes of administration. OIC incidence was reduced with prophylactic laxatives versus no laxatives; patients with OIC using laxatives therapeutically tended to have more severe symptoms than those with prophylactic use. Opioid use for $>6$ days was also significantly associated with OIC.

Conclusion: Our findings revealed high OIC incidence among patients with cancer-related pain in Korea. Special consideration is recommended for patients at increased risk of OIC, such as those requiring increased opioid doses or long-term opioid use. Proper management of OIC is critical, and may be improved with the appropriate use of prophylactic laxatives, while considering the unique circumstances and sensitivities of each patient. Patients may also benefit from more specific and innovative therapies such as agonist/antagonist combinations, as described in guidelines.

Abstract number: $\mathrm{P} 02-112$

Abstract type: Poster

Transcutaneous Electrical Nerve Stimulation (TENS) for Pain Management of Inpatients with Advanced Cancer - A Randomized Controlled Pilot Cross-over Trial Siemens W. ${ }^{1}$, Boehlke C. ${ }^{1}$, Offner K. ${ }^{2}$, Becker G. ${ }^{1}$, Gaertner J. ${ }^{3}$ ${ }^{1}$ Clinic for Palliative Care, Medical Center, University of Freiburg, Faculty of Medicine, University of Freiburg, Freiburg, Germany, ${ }^{2}$ Department of Anesthesiology and Critical Care, Medical 
Center - University of Freiburg, Faculty of Medicine, University of Freiburg, Freiburg, Germany, ${ }^{3}$ Center for Palliative Care Hildegard, Basel, Germany

Background: Pain is a leading symptom on acute palliative care units. Transcutaneous electrical nerve stimulation (TENS) can be used as nonpharmacological treatment for pain relief. However, the evidence base is inconclusive.

Aims: To evaluate efficacy and safety of TENS.

Methods: We conducted a blinded, randomized placebo-controlled cross-over trial (NCT02655289) on an acute palliative care unit. Adult inpatients with cancer pain of $\geqslant 3$ on a Numerical Rating Scale (NRS, $0=$ no pain; $10=$ worst pain) in the last 24 hours were included. Intensitymodulated high TENS (IMT) was compared to placebo TENS (PBT) based on non-perceptible low electric current. Patients used each TENS mode individually during 24 hours with a 24 hour washout phase. The primary outcome was the change of average pain intensity during the last 24 hours measured by NRS. Patients, therapist, outcome assessor and biometrician were blinded.

The data was evaluated according to international recommendations using the software $R$.

Results: We screened 632 patients and 25 were randomized (sequence IMT-PBT: 13 and PBT-IMT: 12). Finally, 11 patients in IMT-PBT and 9 in PBT-IMT completed the study $(\mathrm{N}=20)$. Baseline pain was $<4.3$ on the NRS.

Average pain did not differ when comparing the difference IMT minus PBT for change scores $(-0.2,95 \%$ confidence interval $(\mathrm{Cl})-0.9$ to 0.6$)$ and post-treatment scores $(0.3,95 \% \mathrm{Cl}-0.2$ to 0.8$)$. However, we observed statistically significant changes within groups: IMT -0.9 (-1.4 to -0.4), PBT $-0.8(-1.4$ to -0.1$)$. Seven patients $(35 \%)$ desired a medical prescription for TENS.

No adverse events were related to TENS except for an uncomfortable feeling caused by the current $(n=1)$.

Conclusion: This pilot study revealed no differences between IMT and PBT for average pain intensity but considerable changes within the groups. TENS use was safe in cancer patients. One third of patients requested a prescription indicating a perceived benefit by TENS.

Funding: None

Abstract number: $\mathrm{P} 02-113$

Abstract type: Poster

Opioids' Prescriptions: Exploring Medical Residents'

Representations at the University of Bordeaux

Ducoin M.-H., Burucoa B., Saussac C., Lafaye F.

$\mathrm{CHU}$ de Bordeaux, Bordeaux, France

Background: Pain management is a priority in palliative care because it has a huge impact on quality of life. However, many medical residents have difficulties with the prescription of strong opioids though they are highly indicated in intense pain.

Aim: The aim of our study was to explore the medical residents' representations expressed toward opioids' prescription and their links to professional practices in order to improve them.

Methods: A descriptive qualitative monocentric method was chosen. Semi-structured interviews were conducted with oldest medical residents from the University of Bordeaux. These interviews were leading by interviews' frame. This frame was built from a brainstorm grid of representations' patterns about opioids' prescriptions ; theoretical knowledges, experiences and beliefs. The transcription of the interviews called verbatim were analyzed with a software named NVivo.

Results: 12 semi-directed interviews were conducted before data saturation was achieved. Pain measurements were subjective, prescribing modalities were poorly knew. Mentorship and palliative care team's help were factors that positively influenced the opioids' prescription. Fears and reluctance were broadly based on the addictive risk and side effects of opioids.

Discussion: The resident's difficulties about opioids' prescriptions were similar to those of the medical literature. The prescription context is influenced by historical opiophobia and confusing American news (opioid crisis) that confronts to misuse and drift. Pain management skills are acquired through relevant academic training and quality mentorship.

Conclusion: The evolution of mentalities evolves in a positive way with a more reasoned and adapted prescription of opioids. Acculturation, fieldwork, and innovative teaching methods and materials can ultimately improve practice.

Abstract number: P02-114

Abstract type: Poster

Prevalence and Characteristics of Breakthrough (Episodic) Pain in Cancer Patients Treated in Poland

Brzqkała J. ${ }^{1}$, Leppert W. ${ }^{2}$, Davies A. ${ }^{3}$

${ }^{1}$ Pallmed Leading Sue Ryder House, Bydgoszcz, Poland, ${ }^{2}$ Laboratory of Quality of Life Research Chair and Department of Palliative Medicine, University of Medical Sciences, Poznan, Poland, ${ }^{3}$ Department of Supportive \& Palliative Care, Royal Surrey County Hospital, Guilford, United Kingdom

Aims of the study: To assess the prevalence and characteristics of breakthrough/episodic pain (BTP) in cancer patients treated at Inpatient Palliative Medicine Units and at home (Home Hospices) by the Breakthrough Pain Assessment Tool (BAT) in Poland.

Patients and methods: Eligible patients were recruited from adult cancer patients treated at home (Home Hospices) and at Inpatient Palliative Medicine Units. In a cross sectional survey patients were asked to complete several questionnaires: Cognitive Assessment Scale (CAS), Edmonton Symptom Assessment System ESAS-revised (ESAS-r), Karnfosky, Memorial Pain Assessment Card (MPAC) as well as BAT. Clinical and demographical data were also gathered.

Results: In a cross - sectional survey out of 384 patients treated at home $303(78.9 \%)$ suffered from pain and $173(57.1 \%)$ declared BTP. The results among patients treated at Inpatient Palliative Medicine Units were similar - out of 62 patients $50(80.1 \%)$ stated that they have experienced pain, and $45(72.6 \%)$ were subjected to BTP.

In the majority [62(35.8\%)] of treated at home patients, BTP lasted 15-30 minutes. The intensity of the most severe episode was described by 120 patients $(69.3 \%$ ) as $7-10$ in Numerical Rate Scale (NRS), whereas the typical episode intensity was assessed by 91 patients (52.6\%) as 5-7 in NRS. As far as frequency of BTP is concerned, $30(17.3 \%)$ patients declared less than 1 episode a day, 51 (29.4\%) -2 episodes, 47 (27.1\%) 3-4 episodes and $45(26.0 \%)$ over 4 episodes a day. The results within inpatient group were comparable.

Conclusions: Pain and BTP are phenomena of high prevalence which may be well described by BAT. As far as BTP characteristics is concerned, BTP intensity is in most cases severe and majority of patients experience 1-4 BTP episodes per day and its average duration is 15-30 minutes. BAT seems to be useful tool in every day clinical practice.

Abstract number: P02-115

Abstract type: Poster

Improvement in Control Pain with Adhesive Capsaicin Dermal Patch $8 \%$ in Peripheral Neuropathy Induced by Chemotherapy in Patients with Multiple Myeloma: A Single-centre Seven Cases Serie

Llorens-Torromé S. ${ }^{1}$, Moreno-Alonso D. ${ }^{1}$, Izquierdo-Trigo P. ${ }^{1}$, Corcoy de Febrer B. ${ }^{1}$, Galiano M. ${ }^{1}$, Duran A. ${ }^{1}$, Serrano-Bermúdez G. ${ }^{1}$, Mayoral V. ${ }^{2}$, Serrano A. ${ }^{2}$ 
1Palliative Care Service, Catalan Institute of Oncology (ICO), Barcelona, Spain, ${ }^{2}$ Department of Anesthesiology, Critical Care and Pain Clinics, Bellvitge University Hospital (HUB), Barcelona, Spain

Background: Capsaicin is a highly selective agonist of transient receptor potential vanilloid-1 (TRPV-1).The efficacy of the adhesive capsaicin dermal patch in benign peripheral neuropathy pain (PNP), such as diabetic neuropathy or postherpetic neuralgia, has been described in the literature.The patch provides a high concentration(8\%) directly in the painful area, and could be administrated with other analgesic agents.On the other hand there is scant evidence of its efficacy in PNP induced by chemotherapy (CT). Multiple myeloma (MM) patients suffer from PNP induced by first line agents such bortezomib or talidomida, emerging the need of new approaches and effective treatments.

Aim: To describe the improvement in control pain of an application of a capsaicin patch in patients with PNP induced by $\mathrm{CT}$ in patients with MM. Methods: Seven adult patients diagnosed with MM and affected by PNP induced by bortezomib or talidomida attending to palliative care outpatient clinic and who underwent application of adhesive capsaicin dermal patch.

Results: We collected data from 2 women( $28.5 \%)$ and 5 men (71.5\%), with a median age of 67.5 years.All were diagnosed with $\mathrm{MM}$ and $7(100 \%)$ received bortezomib and $2(28.5 \%)$ received bortezomib plus talidomida. The median visual analog scale (VAS) of pain pre-patch application was 7/10.The concomitant analgesics were: 5 (71.4\%) patients received opioids with median daily oral morphine dose(OMD) of $106 \mathrm{mg} /$ day, $5(71.4 \%)$ gabapentinoids and $1(14.2 \%)$ antidepressants.The median VAS of pain post-patch application was $2 / 10$, with improvement in analgesics requirements $(O M D=94 \mathrm{mg} /$ day). The performance status improved slightly in $3(42.8 \%)$ patients, and remained stable in the rest.A topic anesthetic cream application was need in all patients, and 1 (14.2\%) needed an extra analgesic dose during the administration.

Conclusions: The application of adhesive capsaicin dermal patch $8 \%$ seems to provide relief to patients with MM suffering from PNP induced by chemotherapy.

\section{Abstract number: P02-116 \\ Abstract type: Poster}

\section{Cancer Pain Guidelines - Are They Worth the Paper They Are} Written on?

Johnston B. ${ }^{1}$, Ryan K. ${ }^{2}$, Ruth M. ${ }^{3}$, Connolly M. ${ }^{2}$, O'Connor L. $^{2}$, Larkin P. ${ }^{4}$

${ }^{1}$ The Centre for Health Policy and Management, Trinity College Dublin, Dublin, Ireland, 2 University College Dublin, Dublin, Ireland, ${ }^{3}$ Mater

Misericordiae University Hospital, Dublin, Ireland, ${ }^{4}$ Lausanne University Hospital, Lausanne, Switzerland

Background: Pain is common among cancer patients at any stage of the disease and treatment can be suboptimal. National guidelines for pain management were introduced in Ireland in 2015 to support healthcare professionals treating patients with cancer-related pain.

Aim: The aim was to determine the range and severity of pain, the burden associated with pain and how pain influences referral to specialist palliative care (SPC) team.

Methods: A national cross-sectional survey was carried out. Inclusion criteria were adult patients with capacity to provide informed consent, aware of their diagnosis and $>2$ days post-operative. The survey comprised several tools including the Brief Pain Inventory and the Breakthrough Pain Assessment Tool. Additional data were extracted through chart review. Data were summarised and significance of variations were determined using appropriate tests. Logistic regression was used to explore the relationship between pain and referral to SPC.

Results: 491 patients participated. 54\% were female. 54\% reported cancer-related pain: $23 \%, 29 \%$ and $7 \%$ reported their pain as mild, moderate and severe, respectively. $19 \%, 18 \%$ and $17 \%$ stated that pain interfered mildly, moderately or severely with life. Breakthrough pain was common:
$54 \%$ experienced at least one episode in the last 24 hours. However, only $25 \%$ were receiving SPC and $36 \%$ taking step 2 or 3 analgesics. Patients with pain were more likely to have been referred to SPC than those reporting no pain (odds ratio mild: $1.86, p=0.03$; moderate: $2.1, p=0.006$; severe: $3.7, p=0.001$ ); however, only moderate or severe pain interference were associated with referral (moderate: $O R=1.9, p=0.03$; severe: $\mathrm{OR}=2.2, \mathrm{p}=0.01$ ).

Conclusion: Twenty years after publication of WHO guidelines and three years after publication of national guidelines in Ireland, pain in cancer patients remains poorly managed and negatively affects quality of life. There is an urgent need for improved implementation strategies.

Abstract number: $\mathrm{P} 02-117$

Abstract type: Poster

Improved Quality Control with Subcutaneous (SC) Infusion Pump

Csikos A., Lukacs M.

Hospice-Palliative, University of Pecs Medical School, Pécs, Hungary

Objectives: The subcutaneous (SC) infusion pump are routinely used in palliative care for the SC infusion of drugs for pain and symptom control. The infusion can lead to patient well-being and the potential for sub-optimal symptom control. This study will outline the use of continuous SC infusion pumps, known as syringe drivers, including their benefits and drawbacks in a palliative care context. At the Clinical Center of the University of Pecs are carrying out trials of the recently launched SC infusion pump in the inpatient and home palliative care. The SC infusion pump is often set at $\mathrm{ml} /$ hour with the rate set at 24-hour period.

Methods: The study is a retrospective, quantitative analysis. Through a purposive sampling we analyzed records of tumor- and terminal stage patients in 2017, who were involved in the service of the Pécs-Baranya County Hospice Foundation and met enroll criteria. We were used descriptive statistics. Syringe driver recording forms were retrieved from case notes of consecutive patients who received medication via a syringe driver. The pain was measured on a numerical scale, and other symptoms and well-being were documented on ESAS scale.

Results: During the presented five case report the continuous infusions help improving well-being, pain and other symptoms (for example: vomiting, nausea, dehydration) relief. Based on the cases we found that the rate of pain decreased by $30 \%$, the number of vomits decreased by $20 \%$, and the patient's well-being showed $15 \%$ increase.

Conclusions: The introduction of syringe drivers revolutionised pain control with added benefits for the patient and profession-als.

Abstract number: P02-118

Abstract type: Poster

The Pattern of Tramadol Use for Cancer Pain Control in the Absence of Oral Morphine in a Lower-income Setting Alsirafy S. ${ }^{1}$, Abdelhafeez A. ${ }^{1,2}$, Azab R. ${ }^{1}$

${ }^{1}$ Kasr Al-Ainy School of Medicine, Cairo University, Palliative Medicine Unit, Kasr Al-Ainy Center of Clinical Oncology \& Nuclear Medicine, Cairo, Egypt, ${ }^{2}$ Cairo University, Cairo, Egypt

Background: Tramadol may be the only available/accessible opioid for cancer pain control in lower-income countries. A group of countries is calling for the international control of tramadol and others are advocating against that to maintain the access to what may be the only opioid available for cancer pain control in some countries. Data is limited on the use of tramadol in the absence of oral morphine.

Aim: To describe the pattern of tramadol use for cancer pain control in patients with advanced cancer in the absence of oral morphine in a lower-income setting.

Methods: Retrospective review of the medical records of patients with cancer pain referred to a palliative medicine unit (PMU) in Egypt during 
the oral morphine shortage crisis. During the study period, from January to June 2017, available opioids were tramadol and transdermal fentayl.

Results: The medical records of 88 eligible patients were reviewed. The median number of PMU visits was 3. Thirty-two (36\%) patients were receiving an opioid upon referral to the PMU and in 29 of them (91\%) the opioid was tramadol. Overall, tramadol was used in $78(89 \%)$ of patients either regularly or as needed for breakthrough pain. In 40 (46\%) patients, tramadol was the only prescribed opioid and strong opioids were not prescribed. A strong opioid (transdermal fentanyl) was administered in $35(40 \%)$ patients after receiving tramadol due to uncontrolled pain and in another $6(7 \%)$ as front line.

Conclusions: Tramadol is used for cancer pain control in the majority of cancer pain patients in the absence of oral morphine in a lower-income setting. However, in almost half of patients, tramadol is not enough to control cancer pain and patients need a strong opioid. While advocating against the international control of tramadol may help cancer pain patients, more efforts should be done to make the cheap oral morphine available and accessible. In the absence of oral morphine, many patients may use an expensive opioid formulation.

Abstract number: P02-119

Abstract type: Poster

Prevalence of Breakthrough Cancer Pain and its impact on Quality of Life in Cancer Patients at Regional Cancer Centre, India: A Cross-sectional Study

Choudhary N., Mishra S., Bhatnagar S., Gupta N., Kumar V. Onco-Anesthesia and Palliative Medicine, All India Institute of Medical Sciences, New Delhi, India

Background: Breakthrough cancer pain is commonly seen in cancer patients with higher pain intensities and poor quality of life. Although efficacious treatment of breakthrough cancer pain is available, its clinical management remains unsatisfactory, indicating the need of proper assessment guidelines.

Objective: To find the prevalence rate of breakthrough cancer painand its impact on quality of life in cancer patients.

Methods: The cross-sectional study was done on patients of $>18$ years of age, whose background pain was well controlled on analgesics.Then study subjects were assessed using the study questionnaires. The semistructured proforma were used to obtain the socio-demographic and clinical details about the patient. The Breakthrough pain assessment tool were applied next to assess for prevalence and pattern of breakthrough pain in cancer patients. The WHOQOL-BREF questionnaire was used in recording information about the impact of Breakthrough pain in patients quality of life.

Results: A total of 400 patients were recruited in this study, out of which $156(39 \%)$ patient had breakthrough cancer pain. 56\% were female, maximum (65\%) in age group 30-60 years of age. Most common location of Breakthrough cancer pain was abdomen (31\%) followed by low back pain (18\%). $38 \%$ of patient had at least $1-2$ times breakthrough pain per day. Incidental pain was the most common reason $(41 \%)$ followed by spontaneous pain (37\%). Overall quality of life score was 1.8 , showing very poor quality of life in patients having breakthrough pain. Mean score of overall health satisfaction was 2.1, showing dissatisfaction. Quality of life domains showed lower scores, meaning patient with breakthrough cancer pain experience poor quality of life not only affecting their physical health but also psychological, social and environmental.

Conclusion: There is a big lacuna in understanding the depth of breakthrough pain which leads to poor quality of life.

Abstract number: P02-120

Abstract type: Poster
"Palliative-D" - Can Vitamin D Supplementation Reduce Symptom Burden for Palliative Cancer Patients? Helde Frankling M..$^{1,2}$, Bergqvist J. ${ }^{3}$, Nordström M. ${ }^{4}$, Classon C. ${ }^{2}$, Sandberg C. ${ }^{4}$, Björkhem Bergman L. ${ }^{2,5}$

${ }^{1} \mathrm{NVS}$, Karolinska Institute, Älvsjö, Sweden, ${ }^{2} \mathrm{ASIH}$ Stockholm Södra, Älvsjö, Sweden, ${ }^{3}$ Department of Surgery; Breast Centre, Capio St Görans Hospital, Stockholm, Sweden, ${ }^{4}$ Stockholms Sjukhem Foundation, Stockholm, Sweden, 5 NVS, Karolinska Institute, Huddinge, Sweden

Background: Vitamin D plays an important role in bone health and calcium homeostasis. Increasing evidence indicate effects also on immune-, nervous- and cardiovascular systems. Vitamin D supplementation to patients with suboptimal serum levels of vitamin $D$ may have beneficial effects on pain, infections, and well-being without any significant side effects. Vitamin D status is measured as 25-hydroxyvitamin D (25-OHD), and serum levels of $25-\mathrm{OHD}$ below $50 \mathrm{nmol} / \mathrm{L}$ are considered to be insufficient. Cancer patients generally have lower $25-\mathrm{OHD}$ levels than healthy controls.

Aim: The primary objective is to test the hypothesis that vitamin $D$ supplementation for 12 weeks can reduce opioid consumption in palliative cancer patients. Secondary objectives are to investigate if infections and fatigue can be reduced, and quality of life improved. We will also study effects on 25-OHD in serum after intervention, as well as genetic polymorphisms involved in vitamin $\mathrm{D}$ metabolism.

Method: In a pilot study, 39 palliative cancer patients were supplemented with cholecalciferol $4000 \mathrm{IU} /$ day for twelve weeks. A significant decline in opioid dose was seen after one month, and antibiotic consumption was significantly lower after three months compared to controls. Based on these findings we designed a randomized, double blind, placebo-controlled multicenter trial comprising 254 adult palliative cancer patients recruited from advanced palliative home care centres. Patients with $25-\mathrm{OHD}$ levels $<50 \mathrm{nmol} / \mathrm{L}$ are randomly assigned to 12 weeks of treatment with cholecalciferol or placebo. The study started in October 2017 and will finish in Dec 2019.

Results: In Oct 2018 we have screened 252 patients, and randomised 120. 57 patients have completed the study, 95 patients have reached Visit 1.

Discussion: Vitamin D might play a role in relieving palliative cancer patients' symptoms. We hope that the ongoing RCT will give us sound evidence regarding the use of cholecalciferol in palliative care.

\section{Abstract number: P02-121}

Abstract type: Poster

The Importance of Dental Care in the Treatment of Oral Candidiasis in a Patient with Uterine Cervix Cancer under Exclusive Palliative Care

Trevisani D., Massaiochi Tanimoto H., Silva Moreira Macari K., Aparecida Caliente E.

Barretos Cancer Center, Barretos, Brazil

Patient MGO, female, 56 years old presenting cervical cancer with locoregional invasion under exclusive palliative care, hospitalized at the São Judas Tadeu Hospital (Barretos-SP / Brazil) to control abdominal pain. During the hospitalization period, the patient received care from the multiprofessional team, which included the dental surgeon. When evaluated by the dental surgeon, he reported pain and discomfort in the oral cavity, and on physical examination, the presence of detachable white plaques were observed in all buccal cavity, which when removed, showed an erythematous bed on the back of the tongue and compatible oral mucosa with buccal candidiasis, which resulted in mucosal irritation due to local infection and fissures in labial commissures. The patient and companion were provided with oral hygiene instructions, as well as for the treatment of oral infection performed the mechanical removal of 
dental biofilm, lingual saburra and buccal candidiasis with gauze and chlorhexidine $012 \%$. The patient was instructed to use mouthwash with chlorhexidine $012 \%$ three times a day and nystatin 100,000 IU four times a day for a period of five days and for fissures lipid hydration with essential fatty acid -AGE associated with low power laser therapy was used. Three days after starting the treatment, the patient already presented significant improvement of the discomfort and in the oral condition. It was concluded that dental care is essential in patients under exclusive palliative care, preventing and treating oral problems such as opportunistic infections, which are commonly related to hyposalivation and accumulation of oral biofilm caused by drugs used to control pain, deficiency in oral hygiene and presence of poorly adapted prostheses. Dentistry in palliative care is instituted with the aim of providing comfort and quality of life to patients with chronic diseases that do not present a chance of cure with currently available therapies.

Abstract number: P02-122

Abstract type: Poster

\section{Methadone and Cancer Pain: A Reflex(ion) of a Palliative Care} Service

Freitas S., Gonçalves F.

Instituto Português de Oncologia do Porto Francisco Gentil, Oporto, Portugal

Introduction: The treatment of pain constitutes one of the main goals of palliative care in patients with advanced cancer. Although methadone is used for the treatment of chronic pain, as an effective and inexpensive strong opioid, is less used in many pain and palliative services due to its unpredictable half-life and potential toxicity.

Aim: The purpose of this work was to describe the prescription and outcomes of methadone as a second-line treatment for refractory cancer pain to "classic" opioids in a tertiary palliative care unit.

Methods: Retrospective review, for a six-year period, of medical patients records, from an oncologic palliative care service, described with cancer pain undergoing treatment with oral methadone. Data collected include disease and pain characteristics, initial opioid type, doses and outcomes. Results: Half of the cases prescribed with methadone, as a second-line treatment, had mixed pain (58\%) followed by neuropathic pain with $31,6 \%$. This refractory pain was due to mostly head and neck cancer with locally advanced disease $(53 \%)$. In $68 \%$ of inpatients had for first linetreatment morphine, followed by buprenorphine TD (16\%), with an average of equivalent dose of oral morphine about $387 \mathrm{mg}$ per day. After introducing oral methadone the most patients $(89 \%)$ had pain improvement at fourth day. At final the minimum dose required was $7,5 \mathrm{mg}$ and maximum dose was $90 \mathrm{mg}$. About $47 \%$ of inpatients reported somnolence and myoclonias.

In $32 \%$ of patients went to home after symptomatic control, keeping oral methadone. The others inpatients, $68 \%$, had death as outcome, most everything for advanced disease.

Conclusion: The refractory pain with "classic" opioids could be common in palliative care services due the high prevalence of complexity of these patients. Methadone is an eligible opioid to treat cancer pain, mostly in experienced teams, providing good pain relief.

Abstract number: P02-123

Abstract type: Poster

Validity and Reliability of the Hindi Version of the Patient Assessment of Constipation Quality of Life (PAC-QOL) Questionnaire in Cancer Patients on Opioids for Pain Management at Tertiary Care Center, India Mishra S. ${ }^{1}$, Singh R. ${ }^{1}$, Satapati S. ${ }^{2}$, Bhatnagar S. ${ }^{1}$

${ }^{1}$ Onco-anaesthesia and Palliative Medicine, IRCH, AlIMS, New Delhi, India, ${ }^{2}$ Psychiatry, AllMS, Ansari Nagar, New Delhi, India
Objective: To assess the validity and reliability of PAC-QOL (Patient Assessment of Constipation Quality of Life) questionnaire translated in Hindi language for cancer patient at tertiary care centre, India.

Material and methods: This isa prospective, cross sectional observational study. The patients who were on opioid treatment ranging from last two weeks to six months and had witnessed change in their baseline bowel habit since the treatment with opioids were enrolled for study. The patient's demographic details, duration of opioid treatment, class of opioid, dose, laxatives use etc. and other relevant information were documented. Severity of constipation was assessed by the physician as per CSS (Constipation Scoring System) questionnaires \& quality of life by filling a self-administered PAC-QOL questionnaire performa by patient. At Follow up visit after two weeks same procedure was repeated. The psychometric properties of the PAC-QOL determined in terms of reliability and concurrent validity.

Results: Internal consistencywas assessed in all 152 patients. Cronbach's alphawas $>0.7$ for the overall score and ranged from 0.56 to 0.79 for four subscales, showing a strong internal consistency for overall score. For the test-retest study only 30 patients were available $\&$ the intraclass correlations coefficientwas calculated for the overall \& for subscales showing a moderate degree of positive correlation between PAC-QOL score at first visit and second visit within mean interval of $17.7 \pm 5$ days. For determining the concurrent validity between PAC-QOL and CSS, the value of Pearson correlation coefficients ranged between 0.47 and 0.84 $\& p<0.001$ for overall score and four subscales showing significant correlation between the scores.

Conclusion: The currently available data and its statistical analysis suggest that PAC-QOL in Hindi version is reliable and also valid.

Abstract number: P02-125

Abstract type: Poster

The Management of Constipation in Palliative Care

Monnery D. ${ }^{1}$, Cooper M. ${ }^{1}$, Ayre R. ${ }^{2}$, Cureton S. ${ }^{2}$, Devlin L. ${ }^{3}$, Cookson T. ${ }^{3}$, Owens C. ${ }^{3}$, Schofield S. ${ }^{3}$, Sudworth G. ${ }^{3}$, Hyland C. ${ }^{3}$, Edmunds L. ${ }^{1}$, Waters L. $^{1}, \mathrm{Scott} A .^{4}$

${ }^{1}$ Clatterbridge Cancer Centre NHS Foundation Trust, Wirral, United Kingdom, ${ }^{2}$ Bridgewater Community Healthcare NHS Foundation Trust, Wigan, United Kingdom, ${ }^{3}$ Willowbrook Hospice, Prescot, United Kingdom, ${ }^{4}$ Marie Curie Hospice, Liverpool, United Kingdom

Constipation is a common symptom in palliative care, resulting in physical, psychological, and social problems affecting quality of life. The lack of a clear definition of constipation can contribute to difficulties in reaching a diagnosis. Contributory factors to developing constipation may include poor dietary intake, physical inactivity, underlying disease and its treatment. As not all of these are reversible in this population, laxative prescribing practice is highly variable, depending on prescriber preference and trial and error.

Aims: To develop evidence-based guidance on the diagnosis and management of constipation in palliative care patients.

Methods: A regional audit of clinical practice was combined with a systematic literature review in July 2017. Databases searched included Medline, Embase, CINAHL and Cochrane. Evidence was compiled to form the strongest evidence to support new guideline construction.

Results: 148 clinical audit forms and 91 professional surveys were returned. Assessment for reversible factors was inconsistent, with the commonest factor assessed being reduced mobility in $70 \%$. Prescribing practice was highly variable. Professional opinions varied greatly in approach to management and most respondents felt unconfident using peripheral mu opioid receptor antagonists to treat opioid induced constipation.

The literature review revealed 11 original research articles applicable to this population and clinical question. These revealed strong evidence for senna and lactulose (both as monotherapy and in combination). In 
addition strong evidence for naloxegol for opioid induced constipation was found. Evidence of non-efficacy was found for sodium docusate, although in the audit it was the joint most commonly used medication. Conclusions: These findings combined with patient representatives' input led to the construction of a new NICE accredited regional guideline to support standardised assessment and management of constipation in palliative care.

Abstract number: P02-126

Abstract type: Poster

What Do We Know about Whether Complementary Therapies Improve Anxiety, Pain and Quality of Life in Palliative Care: A Systematic Review of Randomised Trials

Candy B. ${ }^{1}$, Armstrong M. ${ }^{1}$, Flemming K. ${ }^{2}$, Kupeli N. ${ }^{1}$, Stone P. ${ }^{1}$, Vickerstaff V. ${ }^{1}$, Wilkinson S. ${ }^{3}$

${ }^{1}$ University College London, London, United Kingdom, ${ }^{2}$ University of York, York, United Kingdom, ${ }^{3}$ University of Liverpool, Liverpool, United Kingdom

Background: In palliative care (PC) complementary therapies (CT) are popular. In other settings evidence on their effect is mixed, and there are questions about their inclusion in clinical guidelines. The need to understand CT's benefits in PC has been identified as a clinical and public priority.

Aim: To undertake a systematic reviews in PC on massage, aromatherapy, and reflexology to assess whether these therapies impact in the short-term on patient quality-of-life, anxiety and pain.

Methods: Trial registers and eight databases including Medline were searched. Trial quality was assessed using Cochrane standards and the quality of evidence for each outcome was judged using GRADE. Metaanalysis was considered.

Results: Within 22 trials, involving 1,956 participants, 12 evaluated massage, 8 aromatherapy and 6 reflexology. None of the trials took patients' views into acoount in their development. Overall trials were at an unclear risk of bias. Many had small samples. Heterogeneity prevented meta-analysis. In comparison with usual care, another therapy or an active control, evidence on the impact of massage and aromatherapy on pain, anxiety and quality-of-life was mixed, although most trials found no difference (low to very low quality evidence). There was some evidence (very low quality evidence) that, when compared to an active control, reflexology improved these outcomes.

Conclusions: The short-term impact of aromatherapy and massage was unclear. For reflexology, some positive outcomes were found. Our findings, though, are limited because of trial quality, statistical imprecision, and little overlap in the evaluations between trials prevented us providing a more precise estimate of impact. None of the trials took into account patients' views on trial delivery and measurement. In the provision of $\mathrm{CT}$ in $\mathrm{PC}$ and its place in clinical guidelines, further trials that are rigorous in method and informed by how patients want and value CT could provide clearer answers.

Lam J.C.Y. ${ }^{1}$, So W.K.W. ${ }^{2}$, Ling G.C.C. ${ }^{2}$, Lai T.T.K. ${ }^{3}$, Choi K.C. ${ }^{2}$, Law P.T.W. ${ }^{2}$, Law B.M.H. ${ }^{2}$, Lam L.P.L. ${ }^{4}$, Kwok A.O.L. ${ }^{4}$, Chan C.W.H. ${ }^{2}$

${ }^{1}$ Palliative Care Unit, Caritas Medical Centre, Hong Kong, China, ${ }^{2}$ Hong Kong Chinese University, Hong Kong, China, ${ }^{3}$ Society of Promotion of Hospice Care, Hong Kong, China, ${ }^{4}$ Caritas Medical Centre, Hong Kong, China

Objectives: To assess the feasibility and acceptability of a caregiver-delivered massage intervention for Chinese patients with advanced cancer, and its effectiveness in lowering patients' symptom severity and enhancing their caregivers' preparedness and competence in caregiving. Its effects on patients' and caregivers' perceived distress were also examined.

Methods: Forty-nine dyads of advanced cancer patients and their family caregivers, recruited from a local public hospital, were randomized into either the intervention group or wait-list control group. Patients were subjected to a three-week massage program delivered by their caregivers. Data were collected at baseline and one week post-intervention. Symptom severity, perceived distress, QOL, preparedness and competence in caregiving, and satisfaction level were assessed by self-report. Salivary cortisol level was additionally used as an objective measurement for perceived distress.

Results: The recruitment and retention rates of the intervention were $27 \%$ and $55 \%$ respectively. Patient death and unwillingness of patients to be massaged were the most common reasons for dyads failing to complete the intervention. The intervention was moderately acceptable to the dyads. No significant difference $(p>0.05)$ was observed among all the outcome measures between dyads in the intervention and control groups.

Conclusions: With the low recruitment and retention rates, our caregiver-delivered massage intervention appeared not feasible among Chinese patients with advanced cancer, despite its acceptability among the participants. Its effectiveness cannot be demonstrated owing to the small sample size utilized. A similar study on a larger scale is warranted.

Abstract number: P02-129

Abstract type: Poster

Which Is More Useful for the Treatment of Opioid-induced Constipation Caused by Controlled-release Oxycodone: The New Drug Naldemedine Tosylate or Switching to Transdermal Fentanyl? (An Observational Study)

Hirayama T. ${ }^{1,2}$, Budo R. ${ }^{1}$, Yamazaki M. ${ }^{2}$, Suzuki A. ${ }^{2}$, Homma M. ${ }^{1}$, Ohta

$\overline{T .}{ }^{1}$, Hiratsuka K. ${ }^{1}$, Hayashi N. ${ }^{3}$, Kanai A. ${ }^{4}$, Atsuda K. ${ }^{1,2}$

${ }^{1}$ Department of Pharmacy, Kitasato University Hospital, Sagamihara, Japan, ${ }^{2}$ School of Pharmacy, Kitasato University, Tokyo, Japan, ${ }^{3}$ Department of Anesthesiology, Kitasato University School of Medicine, Sagamihara, Japan, ${ }^{4}$ Research and Developmemt Center for New Medical Frontiers, Kitasato University School of Medicine, Sagamihara, Japan

Background: In 2017, naldemedine tosylate (NT) was launched in the United States and Japan for the indication of opioid-induced constipation (OIC). We compared the effects on defecation between additional treatment with NT (NT group) and switching from oxycodone to transdermal fentanyl (TDF group) in patients who were receiving controlledrelease (CR) oxycodone.

Methods: The study group comprised hospitalized patients who received CR oxycodone for at least 3 days from June 2014 through February 2018 and then consecutively received NT or TDF for at least 3 days. Patients who had colorectal cancer and patients who used more laxatives were excluded.

Primary endpoint: The proportion of patients in whom the number of bowel movements during the 3 days after starting the study treatment increased by at least 1 time as compared with that during the 3 days before starting the study treatment (responders).

Secondary endpoints: the use of laxatives, the use of analgesics.

Results: The study group comprised 23 patients who received NT and 40 patients who received TDF. The mean dose of CR oxycodone before the study treatment was $27.2 \pm 17.1 \mathrm{mg}$ in the NT group and $31.7 \pm 26.0 \mathrm{mg}$ in the TDF group. The proportion of responders was significantly higher in the NT group $(82.6 \%, 19 / 23)$ than in the TDF group $(57.5 \%, 23 / 40 ; p=$ 0.04 ). The proportion of patients with constipation ( 1 or less bowel movement per 3 days) before starting treatment was significantly higher in the NT group $(100.0 \%, 17 / 17)$ than in the TDF group $(57.5 \%, 17 / 25 ; p$ $=0.01)$. The proportion of patients in whom the number or the dose of laxatives was decreased was $57.1 \%(12 / 21)$ in the NT group and $66.7 \%$ $(20 / 30)$ in the TDF group. This difference was not significant $(p=0.49)$. The number of rescue doses administered did not differ significantly between before or after treatment (NT: $p=0.53$, TDF: $p=0.42$ ). 
Conclusions: NT was suggested to be more effective than TDF for the treatment of OIC caused by CR oxycodone.

Abstract number: P02-130

Abstract type: Poster

The Effect of Palliative Rehabilitation on Mood in Newly Diagnosed Advanced Cancer Patients: A Secondary Analysis of a Randomized Controlled Trial

Nottelmann L. ${ }^{1}$, Jensen L.H. ${ }^{2}$, Petersen M.A. ${ }^{3}$, Vejlgaard T.B. ${ }^{4}$, Groenvold $M^{3,5}$

${ }^{1}$ Department of Oncology, Palliative Team, Vejle Hospital, University of Southern Denmark / Institute of Regional Health Research, Vejle, Denmark, ${ }^{2}$ Department of Oncology, Vejle Hospital, University of Southern Denmark / Institute of Regional Health Research, Vejle, Denmark, ${ }^{3}$ the Research Unit, Department of Palliative Medicine, Bispebjerg Hospital, University of Copenhagen, Copenhagen, Denmark, ${ }^{4}$ Palliative Team, Vejle, Denmark, ${ }^{5}$ Department of Public Health, University of Copenhagen, Copenhagen, Denmark

Background: Patients newly diagnosed with advanced cancer face lifealtering circumstances which may affect quality of life (QoL) and mood. We recently showed that early integration of palliative rehabilitation in standard oncology care improves QoL.

Aim: To explore the effect of palliative rehabilitation on mood.

Methods: Adults diagnosed with non-resectable cancer within the last eight weeks were randomized 1:1 to standard oncology care or standard care plus palliative rehabilitation. The intervention was two consultations and a 12-week option of contacting a specialized palliative care team, if needed. Additionally, participants could be offered a multidisciplinary group program combining a patient/caregiver school with physical exercise, supplementary individual consultations, or both. Assessments at baseline and after six and 12 weeks were made with the EORTC QLQ-C30 "emotional function" (EF) scale (scored using item response theory T-scores for optimal responsiveness; European mean $=50, S D=10$ ) and the Hospital Anxiety and Depression Scale (HADS) (standard scoring). Change was measured as area under the curve (AUC) across the 12 weeks adjusted for baseline prognostic variables. As a sensitivity analysis the analyses were repeated for the change from baseline to 12 weeks.

Results: 288 patients were randomized. The absolute group difference in EF across the 12 weeks (AUC) was $3.0(0.7 ; 5.3) p=0.0112$. The absolute group difference $(A \cup C)$ for depression was $0.8(-0.1 ; 1.7) p=0.0828$ and $0.5(-0.5 ; 1.5) p=0.3076$ for anxiety. The change from baseline to 12 weeks showed a group difference of $2.2(0.2 ; 4.1) \mathrm{p}=0.0299$ for $\mathrm{EF}, 0.7$ $(-0.1 ; 1.5) p=0.1031$ for depression, and $0.7(0.0 ; 1.5) p=0.0522$ for anxiety. All differences favoured palliative rehabilitation.

Conclusion: Palliative rehabilitation significantly improves emotional function in newly diagnosed advanced cancer patients whereas only a non-significant trend was observed in relation to anxiety and depression.

Abstract number: P02-131

Abstract type: Poster

An Open Label, Randomised Controlled Feasibility Study to Evaluate whether Nasal Fentanyl Alone and in Combination with Buccal Midazolam Give Better Symptom Control to Dying Patients when Compared with Standard as Needed Medication Perkins P. ${ }^{1,2}$, Parkinson A. ${ }^{3}$, Akyea R. ${ }^{4}$, Husbands E. ${ }^{2}$

${ }^{1}$ Sue Ryder Leckhampton Court Hospice, Cheltenham, United Kingdom, 2Palliative Medicine, Gloucestershire Hospitals NHS Foundation Trust, Cheltenham, United Kingdom, ${ }^{3}$ Research, Sue Ryder Leckhampton Court Hospice, Cheltenham, United Kingdom, ${ }^{4}$ Division of Primary Care, University of Nottingham, Nottingham, United Kingdom
Background: Many patients want to be able to stay at home at the end of life. Patients invariably become unable to take oral medication as part of their terminal phase and symptoms are usually controlled using subcutaneous medication. There have been no studies examining the use of nasal fentanyl or buccal midazolam to control symptoms at the end of life.

Aims: To establish how best to conduct a definitive randomised controlled trial to determine whether the administration of nasal fentanyl and buccal midazolam by families and carers rather than standard as needed breakthrough medication administered by healthcare professionals for patients dying at home leads to faster and better symptom control and fewer district nursing visits.

Methods: A mixed methods feasibility open label randomised controlled trial was conducted comparing the efficacy of nasal fentanyl and buccal midazolam by families and carers rather than standard as needed breakthrough medication administered by nurses for terminally ill patients in a specialist palliative care unit. Partway through the study a 3rd observational arm was introduced where buccal midazolam alone was used as study drug. The primary outcomes were whether recruitment and randomisation were possible; assessment of withdrawal and drop-out; and whether the proposed trial methods were sufficient.

Results: The study methods were acceptable to patients and families and study drugs were well tolerated. There was much missing data for the quality of life outcome measures but no data missing with regards to how long medication doses controlled symptoms before symptoms recurred.

Conclusion: Participation in such a study in a hospice population was acceptable to patients and families. The results will help planning of a future community study.

Abstract number: P02-132

Abstract type: Poster

Gabapentin and Pregabalin for Management of Pain Manifestations in Children with Severe Neurological Impairment

- A Retrospective Study

Collins A. ${ }^{1}$, Mannion R. ${ }^{1}$, Broderick A. ${ }^{1}$, Hussey S. ${ }^{1,2}$, Devins M. ${ }^{1}$, Bourke B. 1,2

${ }^{1}$ Our Lady's Children's Hospital, Crumlin, Dublin, Ireland, '2UCD, Dept of Paediatrics, Dublin, Ireland

Background: Pain, irritability and feeding intolerance are common symptoms affecting quality of life in children with severe neurological impairment (SNI). There are few management options for the many children in whom a specific organic aetiology is not found. We performed a retrospective study to explore the use of gabapentin and pregabalin for symptom control in children with SNI.

Methods: A retrospective study was performed involving patients currently attending the palliative care and gastroenterology department of a tertiary level paediatric hospital in Ireland. Children with SNI who had received or were currently receiving treatment with gabapentin for irritability, vomiting and pain of unknown origin were included. Information was gathered from medical and nursing documentation.

Results: There was a total of 42 patients included in this study. The documented reason for commencing gabapentin was irritability in $62 \%$ of patients with a further $28.6 \%$ commenced for pain of unknown origin. There was an overall improvement in symptoms in $76.3 \%$ of children on gabapentin, with increased gastrointestinal symptoms in only one patient. Gabapentin treatment reduced irritability in $71 \%$ of patients. Pregabalin replaced gabapentin as a second line agent in 12 children. Some degree of benefit was reported in 9 of these children with 3 of them having a substantial improvement of symptoms on pregabalin. Adverse effects of both medications were rare.

Conclusions: This study supports the use of gabapentin and pregabalin in symptomatic management of pain manifestations in children with SNI. 
Abstract number: $\mathrm{P} 02-133$

Abstract type: Poster

\section{A National Cross-sectional Survey of Constipation Management} in Cancer Centres in Ireland

Ryan K. ${ }^{1}$, Johnston B. ${ }^{2}$, Connolly M. ${ }^{1}$, O'Connor L. $^{1}$, Larkin P. ${ }^{3}$

${ }^{1}$ University College Dublin, Dublin, Ireland, ${ }^{2}$ Trinity College Dublin, Dublin, Ireland, ${ }^{3}$ Lausanne University Hospital, Lausanne, Switzerland

Background: In Ireland, national guidelines were introduced for constipation management in 2015. Following their introduction, information is lacking on impact and treatment efficacy.

Aims: To describe the range and severity of constipation symptoms and evaluate treatment efficacy in patients attending Cancer Centres.

Methods: A national cross-sectional survey was carried out. The survey comprised several tools including the Constipation Assessment Scale (CAS). Details on diagnosis and analgesic use were extracted from chart review. Data were summarised using descriptive statistics. Significance of variations for continuous data were determined using t-tests. Logistic regression was used to identify factors associated with laxative consumption.

Results: 491 patients took part. $54 \%$ were female. $25 \%$ were receiving specialist palliative care (SPC). $36 \%$ were taking step 2 or 3 analgesics and $39 \%$ were taking prescribed laxatives. $75.4 \%$ were constipated, with $41.3 \%, 24.3 \%$ and $9.8 \%$ reporting mild, moderate and severe constipation, respectively. The mean CAS score of SPC patients was 5.3; significantly higher than those not receiving SPC (mean 3.2, $p<0.001$ ). Logistic regression showed that increased constipation severity was strongly associated with taking a prescribed laxative and also with self-medicating for constipation. Age and gender were not significant predictors. Laxative use was suboptimal: $36.1 \%$ were not taking laxatives despite symptoms; $40.2 \%$ had symptoms despite laxatives. Controlling for constipation severity, SPC patients were 2.99 times more likely to be taking a laxative than those who did not receive SPC (OR 2.99, 95\% Cl: $1.77-$ $5.06 ; \mathrm{P}<0.001)$

Conclusion: Constipation is common in cancer patients in Ireland. Despite guidelines, management remains suboptimal- even in patients receiving SPC. This study highlights the need for improved guideline implementation and provides a baseline for tracking progress. Implementation strategies are provided.

Abstract number: P02-135

Abstract type: Poster

The Development of a Framework to Personalise Hydration Management in Cancer Care: The Use of Non-invasive Technology to Evaluate Fluid Status and Dehydration-related Symptoms

Nwosu A.C. ${ }^{1,2,3}$, Stanley S. ${ }^{3}$, McDougall A. ${ }^{3}$, Mayland C.R. ${ }^{1}$, Mason S. ${ }^{1}$, Westwell F. ${ }^{4}$, Bell J. ${ }^{5}$, Cox T.F. ${ }^{6}$, Varro A. ${ }^{7}$, Ellershaw J.E. ${ }^{1,2}$

${ }^{1}$ University of Liverpool, Palliative Care Institute, Liverpool, United Kingdom, ${ }^{2}$ Academic Palliative \& End of Life Care Department, Royal Liverpool and Broadgreen University Hospitals NHS Trust, Liverpool, United Kingdom, ${ }^{3}$ Marie Curie Hospice Liverpool, Liverpool, United Kingdom, ${ }^{4}$ Royal Liverpool and Broadgreen University Hospitals NHS Trus, Liverpool, United Kingdom, ${ }^{5}$ Royal Liverpool and Broadgreen University Hospitals NHS Trust, Liverpool, United Kingdom, ${ }^{6}$ Department of Statistics, University of Liverpool, Cancer Trials Unit, Liverpool, United Kingdom, ${ }^{7}$ University of Liverpool, School of Physiological Sciences, Liverpool, United Kingdom

Background: The role of hydration in causing or alleviating suffering in advanced cancer is poorly understood. The evidence for the efficacy of clinically assisted hydration in advanced cancer is inconclusive. Bioelectrical impedance vector analysis (BIVA) is an accurate validated method of assessing hydration status. Previous work with BIVA demonstrates significant relationships with hydration status, symptoms and survival in advanced cancer. However, further work is needed to study these associations in the dying.

Aim: This feasibility study aimed to develop the necessary methodology to evaluate hydration and its relationship with clinical symptoms and quality-of-life in dying cancer patients.

Methodology: An observational study of thirty patients with advanced cancer in a hospice and hospital-based specialist palliative care inpatient unit. An advanced consent methodology was used to conduct assessments in the dying phase. Assessments involved hydration (BIVA), symptoms, physical signs, quality of life, myoclonus and survival. Family-caregivers experiences of hydration were evaluated via questionnaire.

Results: The feasibility aspect of the study was successful in recruiting patients and caregivers across the research sites. The overall recruitment rate for patients was $60 \%$ (30 out of 48 approached agreeing to participate) with six (20\%) of those recruited receiving a further hydration assessment in the dying phase of their illness. Eighteen caregivers completed questionnaires.

Conclusion: It is feasible to use BIVA to assess hydration in the dying. This study will support the next phase of the study which include recruitment from additional palliative care units. The outcomes from this work will help to identify hydration-associated variables to support development of a clinical hydration assessment tool. Ultimately, this will help to develop a framework to clinically assess and manage hydration states patients with cancer.

Abstract number: P02-136

Abstract type: Poster

Virtual Reality in Palliative Care: A Project to Determine the Feasibility of Using Innovative Technology in Hospital and Hospice Settings

Roughneen S. ${ }^{1}$, Mills M. ${ }^{2}$, McGlinchey T. ${ }^{3}$, Mason S. ${ }^{3}$, Chapman L. ${ }^{2}$, Khodabukus A. ${ }^{1}$, Jenions L. ${ }^{4}$, Warriner K. ${ }^{5}$, Nwosu A.C. ${ }^{1,2,3}$

${ }^{1}$ Academic Palliative \& End of Life Care Department, Royal Liverpool and Broadgreen University Hospitals NHS Trust, Liverpool, United Kingdom, ${ }^{2}$ Marie Curie Hospice Liverpool, Liverpool, United Kingdom, ${ }^{3}$ University of Liverpool, Palliative Care Institute, Liverpool, United Kingdom, ${ }^{4}$ Royal Liverpool and Broadgreen University NHS Hospitals Trust, Intensive Care Unit, Liverpool, United Kingdom, ${ }^{5}$ Royal Liverpool Global Digital Exemplar Programme, Liverpool, United Kingdom

Background: Previous studies demonstrate that Virtual Reality (VR) is beneficial in certain clinical scenarios (e.g. distraction therapy for pain management). There is little evidence about the benefits and feasibility of VR distraction therapy in palliative care inpatient settings.

Aims: This project aims to determine the feasibility of using VR distraction therapy in specialist palliative care hospital and hospice inpatient settings.

Method: This project was conducted over 3-months. Samsung Gear VR headsets were used to deliver the VR experience. Participants were recruited from a hospice and two wards in a University teaching hospital (the Specialist Palliative Care Inpatient Unit and the Intensive Care Unit). Participants used VR distraction therapy for 10 minutes. Following the VR session, the participants were asked questions about their experience. Data about their experience and adverse effects were recorded. Results: Twenty-one individuals (15 palliative care patients, 2 relatives and 4 staff members) participated in the project. All participants had a positive experience of VR and indicated that they would like to use this again. One (4.7\%) found the headset heavy to use; otherwise, no complications were reported. All participants stated that they believed that VR could be beneficial in the management of symptoms.

Conclusion: Our data suggests that it is feasible to use VR distraction therapy in hospital and hospice settings. Further work is needed to examine whether VR distraction therapy is effective as a method to improve symptom management in palliative care. 
Abstract number: P02-137

Abstract type: Poster

"Constipation is a Simple Thing": Exploring Healthcare Professionals' Perspectives of Managing Constipation within Specialist Palliative Care: A Qualitative Study

Mcllfatrick S. ${ }^{1}$, Muldrew D. ${ }^{1}$, Carduff E. ${ }^{2}$, Clarke M. ${ }^{3}$, Coast J. ${ }^{4}$, Finucane A. ${ }^{5}$, Graham-Wisener L. ${ }^{3}$, Larkin P. ${ }^{6}$, MacArtney J. ${ }^{7}$, McCorry N. ${ }^{3}$, Slater P. ${ }^{1}$, Watson M. ${ }^{8}$, Wright E. ${ }^{9}$, Hasson $F^{1}$

${ }^{1}$ Institute of Nursing and Health Research, Ulster University, Newtownabbey, United Kingdom, ${ }^{2}$ Marie Curie Hospice, Glasgow, United Kingdom, ${ }^{3}$ Queen's University Belfast, Belfast, United Kingdom, ${ }^{4}$ University of Bristol, Bristol, United Kingdom, ${ }^{5}$ Marie Curie Hospice, Edinburgh, United Kingdom, ${ }^{6}$ Lausanne University Hospital, Lausanne, Switzerland, ${ }^{7}$ Marie Curie Hospice, Solihull, United Kingdom, ${ }^{8}$ Hospice UK, London, United Kingdom, ${ }^{9} \mathrm{HSC}$ R\&D, Belfast, United Kingdom

Introduction: Constipation is a major problem, and significant concern, for many patients towards the end of life who are receiving specialist palliative care (SPC). Whilst clinical guidelines have been developed, there is a dearth of evidence regarding healthcare professionals (HCPs) experiences of constipation management within such settings.

Aim: To explore HCPs perspectives of managing constipation within SPC settings.

Methods: Six focus groups were undertaken with $27 \mathrm{HCPs}$ (doctors $\mathrm{n}=7$, nurses $n=14$, pharmacists $n=1$ and other $n=5$ ) in three SPC units across the UK from November 2016 to January 2017. The focus groups examined knowledge, clinical practices, and barriers and facilitators. All participants were female and the mean length of experience within SPC settings was 8 years. Analysis was underpinned by Braun and Clark's framework for thematic analysis.

Results: Constipation was reported as simple to manage, however, whilst the majority discussed the physical impact of constipation, very few participants identified the social and psychological implications for the patient. Strength in pharmacological management was evident, with discussions of drug choices dominating the conversation. The implementation of lifestyle modifications was rarely discussed across the focus groups, however, difficulties were reported in their implementation. Finally, a need for education regarding the underlying physiology of constipation was recognised.

Conclusions: The simplicity of HCPs' reporting of constipation is incongruent with the complexity of this common and distressing symptom. In line with clinical guidelines, this research identifies the need to move SPC staff towards a holistic approach to constipation management that recognises the physical, psychological, and social impact. This transition in approach, and supporting staff to further enhance clinical knowledge, can be facilitated by interprofessional education.

Funder: Marie Curie

\section{Abstract number: $\mathrm{P} 02-138$ \\ Abstract type: Poster}

Variability in Constipation Management in Specialist Palliative Care: Findings from a Multi-site Retrospective Case Note Review Muldrew D. ${ }^{1}$, Carduff E. ${ }^{2}$, Clarke M. ${ }^{3}$, Finucane A. ${ }^{4}$, Graham-Wisener L. ${ }^{3}$ Hasson F. ${ }^{1}$, Larkin P. ${ }^{5}$, McCorry N. ${ }^{3}$, Slater P. ${ }^{1}$, Mcllfatrick S. ${ }^{1}$

${ }^{1}$ Institute of Nursing and Health Research, Ulster Univerisity, Newtownabbey, United Kingdom, ${ }^{2}$ Marie Curie Hospice, Glasgow, United Kingdom, ${ }^{3}$ Queen's University Belfast, Belfast, United Kingdom, ${ }^{4}$ Marie Curie Hospice, Edinburgh, United Kingdom, ${ }^{5}$ Lausanne University Hospital, Lausanne, Switzerland

Background: Constipation is one of the most common symptoms in patients receiving specialist palliative care (SPC), contributing to considerable physical and psychological suffering for patients. Whilst clinical guidelines are available on the management of constipation for people with advanced cancer in specialist palliative care (SPC) settings, the implementation of these guidelines in clinical practice is unclear.

Aim: To examine current clinical management of constipation for patients with advanced cancer in SPC settings.

Methods: A multi-site retrospective case-note review was undertaken, consisting of 150 patient case-notes from three SPC units across the United Kingdom between August 2016 and May 2017. Descriptive statistics were used to compare clinical practices across three SPC sites.

Results: A physical exam and bowel history was recorded for 109 patients (73\%). Site variations were most evident in multidisciplinary team level of involvement in assessment, patient education, non-pharmacological strategies, and management strategies for opioid induced constipation and bowel obstruction. Ninety-six percent of case notes recorded pharmacological interventions compared to $73 \%$ recording lifestyle alterations. Sodium docusate and Senna were the preferred laxatives across all sites, however, $33 \%$ of patient charts recorded no information on the titration of laxatives.

Conclusion: Variations in assessment and management strategies were evident across sites. Further education is needed to equip HCPs with the necessary knowledge and skills to assess and manage constipation. More focus is required on the implementation and recording of nonpharmacological approaches to management.

Funder: Marie Curie

Abstract number: P02-139

Abstract type: Poster

Which Symptoms and Problems Do Cancer Patients Admitted to Specialized Palliative Care Report in Addition to Those Included in the EORTC QLQ-C15-PAL?

Rojas-Concha L. ${ }^{1,2}$, Hansen M.B. ${ }^{1,2}$, Petersen M.A. ${ }^{1}$, Groenvold M. ${ }^{1,2}$

${ }^{1}$ Research Unit, Department of Palliative Medicine, Bispebjerg Hospital, University of Copenhagen, Copenhagen, Denmark, ${ }^{2}$ Department of Public Health, University of Copenhagen, Copenhagen, Denmark

Background: Patients in palliative care are willing to answer short instruments, like the EORTC QLQ-C15-PAL, assessing the most relevant symptoms and problems (S/Ps). However, patients may experience other S/Ps not covered by such instruments. Therefore, to identify which other $S / P s$ patients experience, in addition to those already included in the EORTC QLQ-C15-PAL, we developed a brief instrument to supplement this questionnaire named WISP (Write In three Symptoms/Problems), allowing patients to report 1-3 additional S/Ps and their severity.

Aim: To explore the nature, prevalence and severity of S/Ps reported on WISP by cancer patients admitted to specialized palliative care (SPC) in Denmark.

Materials and methods: A register-based study with data from the Danish Palliative Care Database. This study included adult cancer patients admitted to the 43 SPC units in Denmark in 2016, who reported $\mathrm{S} / \mathrm{Ps}$ on WISP. The S/Ps were categorized qualitatively, grouping S/Ps already included in the EORTC QLQ-C15-PAL in existing categories (e.g. 'pain in the neck' categorized as 'pain'), and establishing new categories for $S / P s$ not included. The prevalence and severity of $S / P s$ categories were also calculated.

Results: Of the 5,744 patients who completed the EORTC QLQ-C15-PAL, $1,788(32.8 \%)$ reported at least one S/P using WISP. A total of 2,470 S/Ps were reported; $28.1 \%$ of them were already covered by the EORTC QLQC15-PAL and $71.9 \%$ were new. All S/Ps were grouped into 61 categories. The most prevalent S/Ps reported on WISP in decreasing order were pain, edema, dizziness, impaired physical or emotional function, cough and sweats. Overall, $85 \%$ of S/Ps reported were rated as 'quite a bit' or worse.

Conclusion: The WISP instrument strongly improves the recognition of $\mathrm{S} / \mathrm{Ps}$ by combining standardisation with individualisation. We recommend its use alongside the EORTC QLQ-C15-PAL and potentially also alongside the EORTC QLQ-C30. 
Abstract number: P02-140

Abstract type: Poster

"Ultrasound Head is a Stethoscope of the Present Age" Dyspnea Diagnosis Using Ultrasound in Hospice Patients Osowicka M., Mroz P., Modlinska A., Gorzewska A.

Department of Palliative Medicine, Medical University of Gdansk, Gdansk, Poland

Introducion: Palliative medicine requires specialized, holistic care for a patient. In a difficult clinical situation (such as dyspnea), ultrasound performed directly at the patient's bed provides the opportunity to determine its causes (diagnose fluid, pneumothorax, atelectasis or pneumonia). FASP (Focused Assessed Sonography in Palliative Care) is a protocol designed for the assessment of palliative patients. A screening assessment of the lower lung, pleural and abdominal cavities is performed for six basic applications of the ultrasound head.

Aim: The aim of the study is to assess the utility of portable ultrasound in the diagnosis of dyspnea in hospice patients.

Materials and methods: We included 30 hospice patients with dyspnea (at least 4 on the Borga scale) with good verbal and logical contact. Ultrasound examination was performed using a portable ultrasound device in accordance to the FASP protocol.

Conclusions: Preliminary results indicate the usefulness of lung and pleural ultrasonography in diagnosing dyspnea in hospice patients. The FASP protocol allows to exclude the most common pulmonary causes of dyspnea without moving patients to the hospital. Ultrasound examination in palliative medicine has tremendous diagnostic value, facilitates further proceedings and helps patients in home and stationary hospice.

Abstract number: P02-141

Abstract type: Poster

Subcutaneous and Intravenous Access Devices in Palliative Care: How to Choose? A Literature Review Magnani C. ${ }^{1}$, Mastroianni C. ${ }^{2}$, Bordin $\mathrm{F}^{3}$, Calvieri A. ${ }^{2}$, Carinci $P^{4}$, , Conti F. ${ }^{5}$, Corona G.V. ${ }^{6}$, Espino M. ${ }^{2}$, Surdo L. ${ }^{1}$, Valle A. ${ }^{7}$, Casale G. ${ }^{2}$ ${ }^{1}$ Research Department, Antea Palliative Care Unit, Rome, Italy, ${ }^{2}$ Antea Palliative Care Centre, Rome, Italy, ${ }^{3}$ I.N.I. Hospice, Grottaferrata (Rome), Italy, ${ }^{4}$ Albachiara Hospice, Lanciano, Italy, ${ }^{5}$ University Policlinico Tor Vergata, Rome, Italy, ${ }^{6}$ Palliative Care Unit, Local Health Authority Basilicata, Potenza, Italy, ${ }^{7}$ F.A.R.O. Fundation, Turin, Italy

Background: In Palliative Care (PC), when the oral route of administration cannot be used, the preferred alternatives are the intravenous (IV) and the subcutaneous (SC). The choice between the IV and the SC route for drugs and fluids administration may be troublesome. Further, if the IV route is preferred, the clinician should select the most appropriate VAD to position. In literature, guidelines on SC and IV access devices selection criteria in PC are not available.

Aims: To describe evidences related to the selection criteria for SC and IV access devices (short and intermediate-term) in PC and to highlight the most controversial topics.

Method: The PRISMA criteria were used. Three databases were searched for observational studies, reviews and guidelines, published from 1998. Two independent reviewers screened the articles, assessed them for eligibility and extracted data regarding directions for SC and IV accesses decision making in PC. Recent guidelines on VADs were also included (Infusion Therapy Standard of Practice 2016, Royal College of Nursing 2016 and CDC Atlanta 2011).

Results: In total 1770 studies were screened, 246 abstracts were reviewed and 19 papers were included. Literature reported that the SC route should be preferred to the IV if patients are uncooperative and agitated, if they refuse the IV route or as a bridging technique while IV access is being attempted. Further, the IV route should be always preferred if a
VAD is already positioned and if patient has specific conditions that might limit the SC route effectiveness (anasarca, peripheral hypoperfusion etc). Discussion: Directions for SC and IV access devices positioning in PC are not univocal. However, literature reported that different factors related to patients (illness trajectory, performance status, expectancy of life etc) setting of assistance and prescribed medication should be always considered. Further research providing specific direction for SC and VADs decision making in PC is needed.

Abstract number: P02-143

Abstract type: Poster

\section{Existential Well-being in Patients with Cognitive Dysfunction in} Palliative Care

Boelsbjerg H.B. ${ }^{1}$, Kurita G. ${ }^{2}$, Sjøgren P. ${ }^{2}$, Hansen N.V. ${ }^{3}$

${ }^{1}$ Department of Sociology, University of Copenhagen, Copenhagen K, Denmark, ${ }^{2}$ Palliative Research Group, Department of Oncology, Rigshospitalet Copenhagen University Hospital, Copenhagen, Denmark, ${ }_{3}^{3}$ Interacting Minds Centre, Aarhus University, Aarhus, Denmark

Background: There are existential concerns related to cognitive dysfunction among patients with advanced cancer, as it impacts experience of meaning, values and satisfaction in life. Knowledge regarding the influence of cognitive changes on existential well-being may help to optimize palliative care $(\mathrm{PC})$ and improve quality of life.

Aims: To analyze how patients in PC experience aspects of cognitive dysfunction in relation to existential well-being.

Methods: Interview-based investigation of 13 adult patients with advanced cancer in PC, who were assessed in a previous study using five neuropsychological tests (Feb 2013 - Jul 2015). A structured interview guide conducted the assessments, exploring the relationship between cognitive changes, existential concerns and values. Analysis of cognitive dysfunction was performed considering the percentage of agreement between interview content (subjective experience) and the neuropsychological tests (objective knowledge). Subjective experiences were analyzed through Interpretative Phenomenological Analysis to identify interferences on existential well-being.

Results: More than half of the patients reported problems with cognitive function or had poor performance on the neuropsychological tests. A high agreement was noted between the interview content and 3 neuropsychological tests $(92 \%-100 \%)$, while a moderate agreement was observed with 2 tests $(54 \%-77 \%)$. Cognitive dysfunction prevented the patients to practice existential values related to meaning and purpose in life.

Conclusion: Cognitive dysfunction was detected by neuropsychological testing and a high/moderate agreement with patients' report was observed. Cognitive changes seemed to interfere with existential values and well-being of the patients.

Abstract number: P02-144

Abstract type: Poster

A Comparison of Normal Saline Mouthwash and Mouthwash Based on Tea Solution from Salvia Officinalis in Palliative Care: A Randomized Controlled Trial

Monsen R. ${ }^{1}$, Herlofson B. B. ${ }^{2,3}$, Gay C. ${ }^{1}$, Fjeld K.G. ${ }^{4}$, Hove L.H. ${ }^{5}$, Saghaug E. ${ }^{1}$, Slaaen J. ${ }^{1}$, Sundal T. ${ }^{1}$, Tollisen A. ${ }^{1}$, Lerdal A..$^{1,6}$

${ }^{1}$ Lovisenberg Diaconal Hospital, Oslo, Norway, ${ }^{2}$ Department of Oral Surgery and Oral Medicine, Faculty of Dentistry, University of Oslo, Oslo, Norway, ${ }^{3}$ Department of Maxillofacial Surgery and Hospital Odontology, Cancer Clinic, Oslo University Hospital, Oslo, Norway, ${ }^{4}$ Institute of Clinical Dentistry, Faculty of Dentistry, University of Oslo, Oslo, Norway, ${ }^{5}$ Department of Cariology and Gerodontology, Faculty of Dentistry, University of Oslo, Oslo, Norway, ${ }^{6}$ Institute of Nursing Science, Department of Health and Society, Faculty of Medicine, University of Oslo, Oslo, Norway 
Background: A mouthwash solution made from the herb Salvia officinalis (SO) has been used in a hospice for many years to improve patients' oral health. Yet, there are few clinical studies evaluating the effect of such interventions within palliative care.

Aims: This study examines the efficacy of a SO-based herbal mouthwash in comparison with normal saline (NS) mouthwash on oral health and oral symptoms.

Methods: This randomized controlled trial was conducted from February 2014 to October 2016 in an inpatient hospice unit. The SO $(n=44)$ and NS $(n=44)$ groups followed the same procedures, with data collected before and after a 4-day intervention. Items from European Organisation for Research and Treatment of Cancer Quality of Life Questionnaires Oral Health 17 (QLQ-OH17, rated 1-4) and a numeric rating scale (NRS, rated $0-10)$ assessed patients' self-reported oral problems. A dentist performed a clinical examination before and after the intervention using Oral Mucositis Assessment Scale (OMAS) and assessing plaque and dry mouth. $\mathrm{P}<0.05$ was considered statistically significant and Cohen's $d$ values $\geqslant 0.40$ was considered clinically meaningful.

Results: Of the 88 cancer patients included (mean age 63.9, SD 10.6), 73 completed the study. At baseline, 78\% reported dry mouth on QLQ-OH17, and $80 \%$ reported dry mouth on NRS (rating $>4$ ). Both treatment groups had improved QLQ-OH17 mean scores after the intervention $(p<.001)$, with dry mouth ratings showing particular improvement in the $\mathrm{SO}$ group $(p=0.036 ; d=0.75)$. NRS ratings of dry mouth also improved in the SO group $(p=0.045 ; d=0.63)$. OMAS erythema scores improved after intervention for the NS group $(p=.022, d=0.75)$, but not the SO group. Plaque on tongue $(p=.003)$ and teeth $(p<.001)$ was also reduced in both groups. Conclusion: This study detected no significant differences between SO and NS mouth rinses in the treatment of oral problems, but showed that systematic assessment and oral care significantly improve oral health.

Abstract number: P02-145

Abstract type: Poster

Successful Mixture of Levetiracetam Continuous Subcutaneous Infusion with Glycopyrronium in End of Life Care

Freiherr von Hornstein W. ${ }^{1}$, Pinheiro P. ${ }^{2}$, Lynch S. ${ }^{1}$, Wilson M.P. ${ }^{1}$

${ }^{1}$ HSE Dublin North East, Specialist Palliative Care Service Cavan \& Monaghan, Cavan, Ireland, ${ }^{2}$ Department of Internal Medicine, Cavan General Hospital, Cavan, Ireland

Background: Seizures can be a challenging symptom in specialist palliative care. In recent years Levetiracetam as a continuous subcutaneous infusion (CSCl) has become a well-established treatment option for patients unable to take oral anticonvulsive medications. In end of life care Glycopyrronium as an antimuscarinic is frequently needed to reduce upper airway secretions and is administered as a CSCl via a syringe driver. Aims: The aim of this study was to assess whether Levetiracetam could be mixed as a solution with Glycopyrronium in end of life care symptom management to reduce the number of syringe drivers needed.

Methods: 14 Patients referred to a Specialist Palliative Care Service showing seizure activity were analysed.

Noted:

- Demographic data

- Diagnosis

- Symptoms

- Medications

- Indications for anticonvulsive and glycopyrronium treatments

- Results

- Outcome

Results: The patients showed seizure activity originating from malignant and non-malignant underlying diseases. The $\mathrm{CSCl}$ daily Levetiracetam (100 mg/ml solution) dose ranged from 500 to $2000 \mathrm{mg}$. Levetiracetam could successfully be mixed with Glycopyrronium (range 1 - 31 days, median 3 days). The most frequent daily dose for Glycopyrronium was $1.2 \mathrm{mg}$. The subcutaneous infusion site remained functional from 1 up to 12 days (median 3 days).

Conclusion / discussion: Levetiracetam $10 \%$ solution can safely be mixed and administered with Glycopyrronium for continuous subcutaneous infusion. The used combination allowed effective symptom management in a practical manner. The mixture did not show any visible alterations of the solution and was well subcutaneously tolerated.

Abstract number: P02-147

Abstract type: Poster

What is the Prevalence of Patulous Eustachian Tube in Patients with Advanced Cancer?

McGuigan H. ${ }^{1}$, Isherwood R. ${ }^{2}$

${ }^{1}$ Cardiff University, Cardiff, United Kingdom, ${ }^{2}$ Strathcarron Hospice, Denny, United Kingdom

Background: Under normal resting conditions, the Eustachian Tube is held closed by the pressure of the surrounding tissues. A Patulous Eustachian Tube (PET) is one that remains abnormally open causing external auditory stimuli to seem muffled as well as causing an abnormal awareness of the person's own breathing and speech.

Aim: To assess the prevalence of PET of patients with advanced cancer who are hospice in-patients or who attend day hospice.

Methodology: All patients with cancer who were in-patient or who attended day hospice at an independent hospice in central Scotland over one week were invited to participate in the study. The seven-point Eustachian Tube Dysfunction Questionnaire (ETDQ-7) was used to identify those with Eustachian tube dysfunction (ETD) who then underwent otoscopy and tympanometry to assess for PET.

Results: There were 36 participants in the study. 12 were male and 24 were female. Their ages ranged from 52-92 (mean= 70.7). The most frequent primary cancer diagnoses represented were breast $(22.2 \%)$, bowel (16.7\%), oesophageal (13.9\%), prostate (13.9\%) and lung (11.1\%). Of the 36 participants 14 where identified as having ETD and seven (19.4\%) were identified as having PET.

Conclusion: This study suggests that PET is significantly more common in patients with advanced cancer than in the general population. PET is an under recognised condition that may be causing symptom burden in patients known to specialist palliative care and further research is warranted in this area.

Abstract number: P02-148

Abstract type: Poster

"Talking through a Fog": The Impact of Hearing Dysfunction Related to Patulous Eustachian Tube on Hospice Patients with Advanced Cancer

McGuigan H. ${ }^{1}$, Isherwood R. ${ }^{2}$

${ }^{1}$ Cardiff University, Cardiff, United Kingdom, ${ }^{2}$ Strathcarron Hospice, Denny, United Kingdom

Background: Under normal resting conditions, the Eustachian Tube is held closed by the pressure of surrounding tissues. A Patulous Eustachian Tube (PET) is one that remains abnormally open causing external auditory stimuli to seem muffled as well as causing an abnormal awareness of the person's own breathing and speech.

Aim: To explore the impact of hearing dysfunction related to PET on hospice patients with advanced cancer.

Methodology: Patients with PET were identified using the Eustachian Tube Dysfunction Questionnaire (ETDQ-7), otoscopy and tympanometry. Semi-structured interviews were conducted. Interviews were transcribed verbatim to allow analysis of themes. Data was analysed by two researchers using qualitative description. 
Results: Semi-structured interviews were conducted with five participants. Themes emerged including the impact of PET symptoms on communication; particularly in a group setting, adaptation to hearing impairment, stigma around deafness, ageing and hearing aids, and a perceived lack of healthcare professional understanding.

Participants spoke of a clear start to their symptoms and a variety of healthcare professional responses. Many had tried hearing aids in the past but these were found not to be of benefit. Fears expressed included a fear of deafness or that hearing loss may be a symptom of brain metastases. Conclusion: In patients with advanced cancer PET can contribute to overall symptom burden in many ways. PET and its symptoms are underrecognised in patients with advanced cancer and there is a need for better healthcare professional understanding in this area.

Abstract number: P02-149

Abstract type: Poster

Depression, Anxiety and Quality of Life in a Palliative Population: A Comparative Study across Different Settings - Community and Hospital

Reilly L. ${ }^{1,2}$, Salmon C. ${ }^{2}$, Doherty D.A. ${ }^{2}$, Waldron P.D. ${ }^{2}$, McMahon E. ${ }^{3}$

${ }^{1}$ Palliative Medicine, Galway University Hospital, Galway, Ireland, ${ }^{2}$ Galway University Hospital, Galway, Ireland, ${ }^{3}$ Psychiatry, Galway University Hospital, Galway, Ireland

Background: Palliative care patients may experience fluctuating mood due to the nature of their diagnosis. Management plans often focus on keeping physical symptoms at bay, which may result in the patient's mental health being overlooked.

Aim: Understanding adjustment and depressive disorders in this population better may be an important target for improved quality of life.

Methods: Patients in the Galway University Hospital and Galway Hospice Day Centre were approached in this ethically approved cross-sectional study. The scales used were Hospital Anxiety and Depression Scale (HADS), the short form of the Adjustment Disorder-New Module scale (ADMN-6) and the EQ-5D to measure quality of life. The HADS focuses on the patient's recent emotional responses experienced whereas the EQ-5D is a 5-item validated scale which explores functioning in activities of daily living and related quality of life.

Findings: Of all patients approached, 30 agreed to participate in the study. Adjustment disorder- New module 6 (ADNM-6) highlighted $63.13 \%$ of patients whose situation was a burden to them, only $36.84 \%$ reported any change to their sleeping habits. $52.63 \%$ of patients admit to 'supressing' their feelings with others while but only $15.79 \%$ of participants say they have withdrawn from their friends and family. The Quality of Life questionnaire reported $42.11 \%$ of patients to be moderately depressed. Finally, when asked to rate their current health on a scale, a mean of 56 was obtained.

Conclusion: Working with an acutely ill cohort has its challenges. This pilot study will deepen our understanding of the psychological and overall care requirements of palliative care patients. The data collected suggests that end-of-life patients require mental health care to complement their physical management. This will result in an improvement of the overall wellbeing of HSE palliative patients, in both community and inpatient based settings.

Abstract number: P02-150

Abstract type: Poster

Serum Cholinesterase is an Index of Adverse Events of Diarrhea during Treatment with Naldemedine

Hiraide T..$^{1,2}$, Kurita D. ${ }^{2}$, Hirano M. ${ }^{2}$, Muramatsu M. ${ }^{2}$, Shida H. ${ }^{2}$, Shibata

$\overline{K^{2}{ }^{2}, \text { Hori } K^{2}}{ }^{2}$, Yamada Y., ${ }^{1,2}$

${ }^{1}$ Department of Medical Oncology, Hamamatsu University School of Medicine, Hamamatsu, Japan, ${ }^{2}$ Palliative Care Team, Hamamatsu University School of Medicine, Hamamatsu, Japan
Background and Aim: Naldemedine is a novel peripherally acting muopioid receptor antagonist for treating opioid-induced constipation (OIC). Diarrhea is the most frequently observed adverse event associated with naldemedine use. We aimed to elucidate the clinical factors associated with diarrhea in patients with OIC who were treated with naldemedine.

Methods: We retrospectively enrolled 108 (cancer: 96, non-cancer: 12) patients who received opioids and were administered naldemedine from September 2017 to August 2018 at our hospital. Diarrhea was defined as at least three loose or liquid bowel movements per day. Clinical factors such as age, sex, performance status, bowel movement, opioids, and blood test were analyzed through patient medical records. The study protocol was approved by the institutional ethical committee.

Results: The study population comprised 67 men and 41 women (aged 25-87 years; median age, 68 years), and performance status (ECOG-PS) 0-1/2/3-4 were 61/35/12. In total, 86 and 22 patients were administered strong and weak opioids, respectively. Approximately 10 to 840 (mean, 34) $\mathrm{mg} /$ day of opioid in terms of oral morphine was administered. The median duration of naldemedine administration was 36 (range, 1-273) days. Adverse events were observed in 20 patients. Out of 17 patients (16\%) who developed diarrhea, 4 patients experienced severe diarrhea (>10 bowel movements/day). A significant difference in serum cholinesterase levels was observed between the diarrhea and non-diarrhea groups ( $p=0.008)$, and a level of $201 \mathrm{U} / \mathrm{L}$ showed high sensitivity $(92 \%)$ and specificity $(79 \%)$, with an area under the curve of 0.848 by receiver operating characteristic curve analysis.

Conclusion: Serum cholinesterase was a predictive biomarker of diarrhea in patients who received naldemedine. Further prospective studies are warranted.

\section{Abstract number: P02-151 \\ Abstract type: Poster}

Diminishing Drinking at the End of Life: A Concept Analysis Pettifer A. ${ }^{1,2}$, Hughes S. ${ }^{1}$, Froggatt K. ${ }^{1}$

${ }_{1}^{1}$ nternational Observatory on End of Life Care, Lancaster University, Lancaster, United Kingdom, ${ }^{2}$ Coventry University, Coventry, United Kingdom

Background and aim: Most dying people drink less and often cease drinking. Supporting family members who witness this can be challenging for healthcare professionals. A literature review of family members' experiences of this process has shown multiple terms that describe concepts relating to it. However, there is a no agreed term specific and meaningful for clinicians and family members alike. A conceptual analysis of diminishing drinking was undertaken to explore how it was understood in the past and could be conceptualised in future.

Design, methods and approach taken: Rodgers' evolution method (2000) was used to analyse the concept of diminishing drinking. This inductive analysis method focuses on how concepts have developed dynamically over time and within contexts to indicate direction for future research and practice.

CINAHL and Medline databases were searched using free text terms for 'diminishing drinking' and synonyms. Hand and pearl searches were also conducted and all types of indexed literature included. Sampling ensured all geographical and professional domains were represented: $\mathrm{N}=30$ papers. Findings were analysed to identify further surrogate terms, antecedents, attributes, examples and consequences of the ways diminishing drinking has been conceptualised.

Results: Several terms are used to describe diminishing drinking in the literature including: dehydration, declining hydration, oral intake and nourishment. These terms describe concepts that both include or overlap with diminishing drinking. 'Terminal dehydration' is identified as the foundational concept. Professionals and families use different terms reflecting their foci of concern. A conceptual typology of diminishing drinking is proposed. 
Conclusion: Different understandings of the terms around diminishing drinking held between professionals and families may shape communication between the two groups. The proposed conceptual typology may aid communication between professional and family caregivers.

Abstract number: P02-152

Abstract type: Poster

Allied Health Professionals (AHPs) in Specialist Palliative Care Better Living Matters: Making Every Moment Matter Grimes C. ${ }^{1}$, Murdock $C^{2}{ }^{2}$, Craig A. ${ }^{3}$, the Specialist Palliative Care Allied Health Professionals Forum

${ }^{1}$ Public Health Agency, Ballymena, United Kingdom, ${ }^{2}$ South Eastern Trust, Community Specialist Palliative Care Team, Newtownards, United Kingdom, ${ }^{3}$ Northern Health and Social Care Trust, Macmillan Unit, Anrtrim, United Kingdom

Background: In 2015 the Specialist Palliative Care AHP forum was founded. It was established to provide a network of AHPs working in specialist palliative care, an opportunity for AHPs to share and develop evidence based practice and a link between clinicians and commissioners. One of the early work strands within the forum was to develop a document to support clinicians working within palliative and end of life care by clearly outlining the AHP interventions that are required to address some of the symptoms identified by service users and carers as being necessary to achieve better quality of life.

Aims: The main aim of the publication was to outline evidence based practice for symptom management which could be used across all sectors and settings to promote better living for clients.

Methods: The forum members worked together to gain a consensus on current clinical practice across all disciplines and sectors supported by comprehensive literature searches. This was then edited and circulated widely for consultation before being published and available on line. Results: This tool will direct AHPs to assist their clients to be proactive in managing these often distressing symptoms and live life as fully as possible. The Forum brings together four AHP professions from all sectors, with representation on the strategic palliative care programme. They have changed clinical practice from uni and setting specific to inter professional practice across the person's palliative care journey, through a whole system approach to improve patient experience, maximise resource allocation, avoid duplication and ensure equity across the region.

Conclusion: AHPs help make every moment matter for people with specialist palliative care needs. This project ensured that clinicians work together in a whole system, evidence based approach to increase awareness of the unique role of AHPs and their impact promoting selfmanagement and independence to ensure best quality of life possible.

Abstract number: P02-153

Abstract type: Poster

Physiotherapy as an Adjuvant Treatment for Severe Symptoms in Advanced Cancer and Non-cancer Home Based Patients Canal-Sotelo J., Llobera-Solanas M., Cano-del Olmo K., Mejon-Casas O., Gabernet-Foix S., Fauria-Garcia Y., Moreno-Iglesias I., Caceres-Becerra M. Hospital Universitari Santa Maria, Gestió de Serveis Sanitaris, Lleida, Spain

Introduction: Advanced diseases are associated with increased fatigue, increased muscle weakness, and reduced physical functioning, among other severe symptoms, all of which negatively impact quality of life. Physiotherapy addresses the physical and functional dimensions of the patients' suffering.

Methods: Retrospective and descriptive study based on patient's medical files review attended by two home care teams during 2017 in a rural area in Catalonia.
Results: 967 patients were evaluated on 2017 and 178 (18\%) were referred for physiotherapy assessment. 86 patients $(46 \%)$ were considered to be in a late stage of their illness ( $78 \%$ cancer; $22 \%$ non-cancer). Age $\geqslant 80$ years in the $47 \%$.

The main symptoms assessed were respiratory ( $35 \%$ where $72 \%$ on cancer patients); Asthenia (49\% where $95 \%$ in cancer patients); reduced mobility ( $84 \%$ where $75 \%$ in cancer patients) and other symptoms ( $32 \%$ where $85 \%$ in cancer patients).

$72 \%$ of the patients evaluated ( $\mathrm{N}=128$ ) presented with $\geqslant 2$ symptoms. Conclusions: Loss of mobility and the cluster asthenia/respiratory symptoms were the most prevalent indication for physiotherapy interventions.

Pain was not a significant reason to address the patient for physiotherapist evaluation. The pharmacological approach is still considered the cornerstone for patients receiving home-based care.

The level of satisfaction perceived by patients, relatives and the rest of sanitary and non-sanitary professionals involved in the patient's followup was high.

Abstract number: P02-154

Abstract type: Poster

Is Combination Laxatives with Naldemedine Needed for Opioidinduced Constipation? A Single-center Retrospective Study

Kumakura Y., Shimizu M., Nakajima K., Mochizuki M., lijima T., Nakayama T., Nakajima E., Akita K., Ishiguro H., Kotoda M., Matsukawa T.

University of Yamanashi, Chuou, Japan

Background: Naldemedine is a new agent as peripherally acting muopioid receptor antagonist and has been used to treat opioid-induced constipation (OIC). Because of its strong effect, we often prescribe only this agent for OIC and had mitigated suffering from constipation. However, some patients who receive naldemedine need other additional laxatives.

Aims: The aim of this study was to investigate the rate of the patients who need to combine other laxatives with naldemedine.

Methods: Seventy-nine patients were prescribed with naldemedine for OIC in University of Yamanashi Hospital between June 2017 and September 2018. Retrospective chart reviews were conducted in these patients. We investigated patients' characteristics (cancer/non-cancer, morphine equivalent dose of prescribed opioid, etc.), the rate of the patients for whom laxatives except for naldemedine were prescribed at the beginning and after that.

Results: Fifty-eight patients presented with cancer, and the other 21 patients did not. Morphine equivalent daily doses of these patients were $111.7 \pm 59.1 \mathrm{mg}$. Forty-four patients (55.7\%) were prescribed with only naldemedine at the beginning. Sixteen patients could not continue using naldemedine because of diarrhea (7), abdominal pain (3), nausea (1), and other side effects. In 43 patients (68.3\%), a combination with other laxatives and naldemedine was needed. Fourteen patients who were taking with naldemedine at the beginning needed additional laxatives. One patient who initiated naldemedine with other laxatives ceased other laxatives during the follow-up period.

Conclusion / Discussion: Patients with constipation have various causes. The present results indicates that it is difficult to achieve sufficient effects in the management for those patients with naldemedine alone. Additional laxatives may be needed. We should carefully use naldemedine and other laxatives, since the frequency of adverse events due to naldemedine are relatively high.

Abstract number: P02-155

Abstract type: Poster

The German S3 Palliative Care Guideline for Nausea and Vomiting in Adult Patients with Incurable Cancer 
Benze G. ${ }^{1}$, Stamer U. ${ }^{2}$, Goldhammer E. ${ }^{3}$, Doll A. ${ }^{4}$, Wirz S. ${ }^{5}$, Behlendorf T.6, Kahl C. ${ }^{7}$, Montag T. ${ }^{4}$, Bausewein C. ${ }^{8}$, Voltz R. ${ }^{4}$, Simon S.T. ${ }^{4}$, Maier B. ${ }^{9}{ }^{9}$, On behalf of the German Guideline Group Palliative Care ( $d t$.: Deutsche Leitliniengruppe S3-Palliativmedizin)

${ }^{1}$ Palliative Care, University Medical Center Goettingen, Goettingen, Germany, ${ }^{2}$ Klinik für Anaesthesiologie und Schmerztherapie, Inselspital, University of Bern, Bern, Switzerland, ${ }^{3}$ University of Muenster, Muenster, Germany, ${ }^{4}$ University of Cologne, Cologne, Germany, ${ }^{5}$ GFO Kliniken Bonn, Bonn, Germany, ${ }^{6}$ Krankenhaus Martha-Maria HalleDölau, Halle (Saale), Germany, ${ }^{7}$ Klinikum Magdeburg, Magdeburg, Germany, ${ }^{8}$ University of Munich, Munich, Germany, ${ }^{9}$ Med. Klinik III, St. Josefs-Hospital Wiesbaden, Wiesbaden, Germany

Aim: The German Guideline Palliative Care for patients with incurable cancer is part of the German Guideline Program in Oncology. The aim of the chapter "nausea and vomiting" was to develop evidence and consensus based treatment recommendations for patients suffering from nausea and vomiting due to other causes than chemotherapy and radiotherapy. Methods: A systematic review based on database searching of Medline, Embase and Chochrane Library was carried out for assessment tools, pharmacological and non-pharmacological treatment. Based on the identified evidence and clinical experience when evidence was lacking key recommendations were formulated and consented through a structured process with experts of various medical fields and specialist palliative care professionals.

Results: No high level scientific evidence for the management of nausea and vomiting could be identified. Numeric Rating Scale, Minimal Documentation System and Integrated Palliative Outcome Scale are suitable tools for symptom assessment. Individual care is a basic principle e.g. concerning mouth care, nutrition and relaxation techniques. If vomiting persists despite of adequate therapy, a naso-gastric tube should be offered to the patient. Antiemetics should be chosen etiology-based. In case of opioid-induced nausea or vomiting dopamine antagonists such as metoclopramide or haloperidol are recommended. Dexamethasone is effective in case of brain metastases causing brain pressure. Serotonine antagonists can be used if treatment with other antiemetics, such as metoclopramide or neuroleptics, is insufficient. If monotherapy is not successful, a combination of antiemetics with different receptor affinity should be used.

Conclusion: The overall strength of evidence for treatment of nausea and vomiting due to reasons other than chemotherapy and radiotherapy in patients with incurable cancer is low. More well-designed studies in palliative care patients are needed for evidence-based recommendations.

Abstract number: P02-156

Abstract type: Poster

Electrochemotherapy in the Treatment of Ulcerated Malignant Tumors: Results from the InspECT Registry

Kunte C. ${ }^{1,2}$, Moir G. ${ }^{3}$, Bechara F. ${ }^{4}$, Orlando A. ${ }^{5}$, Matteucci P. ${ }^{6}$, Mowatt D. ${ }^{7}$, Clover J.8,9, McCaul J. ${ }^{10}$, Mascherini M. ${ }^{11}$, Gehl J.12,13, Muir T. ${ }^{14}$, Sersa G. ${ }^{15}$, Groselj A. ${ }^{16}$, Odili J. ${ }^{17}$, Giorgione R. ${ }^{18}$, Campana L. ${ }^{19,20}$, Bertino G. ${ }^{21}$, Curatolo P. ${ }^{22}$, Banerjee S. ${ }^{23}$, Kis E. ${ }^{24}$, Quaglino P. ${ }^{25}$, Liew S.H. ${ }^{26}$, De Terlizzi F. ${ }^{27}$, Grischke E.-M. ${ }^{28}$, International Network for Sharing Practice in Electrochemotherapy (InspECT)

${ }^{1}$ Dermatosurgery and Dermatology, Artemed Fachklinik Munich, Munich, Germany, ${ }^{2}$ Department of Dermatology and Allergology, Ludwig-Maximilian University Munich, Munich, Germany, ${ }^{3}$ Department of Cutaneous Medicine \& Surgery, The Royal London Hospital \& QMUL, Bart's Health NHS Trust, London, United Kingdom, ${ }^{4}$ Department of Dermatologic Surgery, St. Josef Hospital, Ruhr-University Bochum, Bochum, Germany, ${ }^{5}$ Department of Plastic and Reconstructive Surgery, Southmead Hospital, North Bristol NHS Trust, Bristol, United Kingdom, ${ }^{6}$ Department of Plastic Surgery, Castle Hill Hospital, Castle Hill, United Kingdom, ${ }^{7}$ Plastic Surgery Department, Christie Hospital
NHS Foundation Trust, Manchester, United Kingdom, ${ }^{8}$ Department of Plastic Surgery, Cork University Hospital, Cork, Ireland, ${ }^{9}$ Cancer Research@UCC, Western Gateway Building, University College Cork, Cork, Ireland, ${ }^{10}$ Maxillofacial/Head \& Neck, Bradford Institute for Health Research, Temple Bank House, Bradford Royal Infirmary, Bradford, United Kingdom, ${ }^{11}$ Department of Surgical Sciences and Integrated Diagnostics (DISC), Polyclinic Hospital San Martino, Genoa, Italy, ${ }^{12}$ Center for Experimental Drug and Gene Electrotransfer (C*EDGE), Department of Clinical Oncology and Palliative Care, Zealand University Hospital, Roskilde, Denmark, ${ }^{13}$ Department of Clinical Medicine, Faculty of Health and Medical Sciences, University of Copenhagen, Copenhagen, Denmark, ${ }^{14}$ Plastic Surgery Department, James Cook University Hospital, Middlesbrough, United Kingdom, ${ }^{15}$ Department of Experimental Oncology, Institute of Oncology Ljubljana, Ljubljana, Slovenia, ${ }^{16}$ Department of Otorhinolaryngology and Cervicofacial Surgery, University Medical Centre Ljubljana, Ljubljana, Slovenia, ${ }^{17}$ Department of Plastic Surgery, St. Georges University Hospitals NHS Foundation Trust, London, United Kingdom, ${ }^{18}$ Dermatologic Clinic, University of Piedmont, Novara, Italy, ${ }^{19}$ Veneto Institute of Oncology IOV-IRCCS, Padova, Italy, ${ }^{20}$ Department of Surgery, Oncology, and Gastroenterology (DISCOG), University of Padova, Padova, Italy, ${ }^{21}$ Department of Otolaryngology Head Neck Surgery, University of Pavia, IRCCS Policlinico San Matteo Foundation, Pavia, Italy, ${ }^{22}$ Department of Dermatology and Plastic Surgery, Dermatologic Clinic, University of Rome "La Sapienza", Rome, Italy, ${ }^{23}$ Division of Surgery and interventional Science, University College, London, United Kingdom, ${ }^{24}$ Department of Dermatology and Allergology, University of Szeged, Szeged, Hungary, ${ }^{25}$ Dermatologic Clinic, Department of Medical Sciences, University of Turin, Turin, Italy, ${ }^{26}$ Merseyside Regional Burns and Plastic Surgery Centre, Liverpool, United Kingdom, ${ }^{27}$ IGEA S.p.a., Carpi, Italy, ${ }^{28}$ Department of Gynecology, University Hospital of Tübingen, Tübingen, Germany

Background: Electrochemotherapy (ECT) is an effective local treatment for cutaneous tumors and metastasis. Treatment involves the administration of chemotherapeutic drugs followed by delivery of electrical pulses to the tumour. The aim of this study was to investigate the effectiveness of ECT in ulcerated versus non-ulcerated cutaneous tumors and metastases.

Methods: 22 cancer centres in the International Network for Sharing Practices on Electrochemotherapy consecutively and prospectively uploaded data to a database. ECT consisted of intratumoural or intravenous injection of bleomycin, followed by application of electric pulses under local or general anaesthesia.

Results: 716 patients with ulcerated (452) and non-ulcerated (264) cutaneous tumors and metastases were identified from the database with a follow-up of at least 45 days.

Non-ulcerated lesions (overall response $86 \%$ ) significantly responded better than ulcerated lesions (overall response $79 \%, \mathrm{p}=0.0493$ ). In large lesions complete response (CR) versus non-CR between the two groups is statistically significant $(p=0.0395)$.

Prior to ECT, patients with ulcerated lesions have significantly more pain. Immediately after ECT pain is significantly raising in non-ulcerated tumors, whereas in ulcerated lesions it remains stable. With increasing time since ECT the difference in pain intensity between the groups is decreasing becoming not significant after 120 days of follow-up. Odor, suppuration, bleeding and ulceration is improving over time after ECT in ulcerated tumors.

No serious adverse events were reported, and the treatment was in general very well tolerated.

Conclusion: ECT is a highly effective local treatment for cutaneous metastases and tumors, with no severe adverse effects. An intense perioperative pain management in non-ulcerated lesions prior to ECT seems to be mandatory since pain raises after treatment. ECT improves quality of life in patients with ulcerated tumors. 
Abstract number: P02-157

Abstract type: Poster

Wounds in Palliative Care: A Cross Sectional Study on Nurses' Knowledge about Kennedy Terminal Ulcers

Maia M.1,2, Pinto S.M. ${ }^{3,4}$

${ }^{1}$ VivaMais - Segurança e Saúde do Trabalho, Oporto, Portugal, ${ }^{2}$ Hospital de S. Martinho, Oporto, Portugal, ${ }^{3}$ Escola Superior de Saúde de Santa Maria, Oporto, Portugal, ${ }^{4}$ CINTESIS - Center for Health Technology and Services Research, Oporto, Portugal

Background: The Kennedy's Terminal Ulcers (KTU) are a particular type of wounds that most often appear at the end of life. Training and research about are still very limited and KTU are often confused with pressure ulcers, which represents an important threat to effective care. Aims: To explore nurses' knowledge about KTU and to analyze if it depends on the education and/or on the professional experience in Palliative Care (PC).

Methodology: Quantitative, cross-sectional and descriptive study. An intentional sample of 51 nurses from Palliative Care (PC) and non-PC settings were primarily recruited through snowball sampling. Eligibility criteria include to have a nursing diploma and been working as a nurse for more than 6 months. Data were collected through a self-filling form focusing on socio-demographic variables, PC training/practice and knowledge about KTU. Descriptive statistics and non-parametric tests were performed to compare differences between groups (SPSS ${ }^{\circledR}$, version 21.0). The research project was approved by an Ethics Committee and the participants informed consent was obtained.

Results: Participants ( $n=51$; mean age $=30.82$ years; working in hospital settings $42.00 \%$ ) have very limited knowledge about KTU. Only 10 nurses have advanced training/practice in PC. Yet, 47 nurses $(70.00 \%)$ have advanced training in Wound Care. Most $(68.63 \%)$ was completely unaware of the concept of KTU. Among those who know the concept (12.38\%), most were unware both about the etiology (68.75\%), and the particular characteristics of the lesion (56.25\%). As the experience time increases to 2-3 years, knowledge about KTU is higher $(X 2(6)=18,022$, $\mathrm{p}=0,001$ ).

Conclusions: The knowledge about KTU is very limited both for nurses working in PC and in non-PC settings. Data highlight the need to develop educational programs and to carry out a more in-depth research about this issue.

\section{Abstract number: P02-158 \\ Abstract type: Poster}

Wounds in Palliative Care: A Systematic Literature Review about Kennedy Terminal Ulcers

Maia M.1,2, Pinto S..$^{3,4}$

${ }^{1}$ VivaMais - Segurança e Saúde do Trabalho, Oporto, Portugal, ${ }^{2}$ Hospital de S. Martinho, Oporto, Portugal, ${ }^{3}$ Escola Superior de Saúde de Santa Maria, Oporto, Portugal, ${ }^{4} \mathrm{CINTESIS}$ - Center for Health Technology and Services Research, Oporto, Portugal

Background: Wounds are often a major discomfort factor for patients, particularly in Palliative Care (PC). The Kennedy's Terminal Ulcers (KTU) are a particular type of wounds whose etiology and appearance is associated with the terminal phase. Yet, effective information and research about KTU are still limited.

Aims: To analyze the scientific evidence produced regarding the study of KTU.

Methods: Systematic literature review on PubMed, Web of Science, EBSCO, Scielo, The Cochrane Database of Systematic Reviews and JBI Database of Systematic Reviews, for the terms kennedy terminal ulcer, end of life ulcer and terminal ulcer. All Portuguese, Spanish, and English papers published until December 31, 2017 were included. The studies should clearly indicate study design, aims, sample and results. Only opinion papers, proceedings and research protocols were excluded. Results were analysed according to the Preferred Reporting Items for Systematic Reviews and Meta-Analyses.

Results: 3617 studies were retrieved and 11 studies were included in the review. Most $(n=10)$ are case studies, and only 1 retrospective cohort study was found. The first study was published in $2009(n=3)$, followed by $2016(n=3)$ and $2014(n=2)$. No studies were found in 2011 or in 2015. Most of the studies were developed in the USA $(n=9)$, but 2 studies were conducted in Spain. The majority focus on elderly patients (minimum age >: 69 years; maximum age: 92 years). Only 1 study case was found in pediatric age. Although the low studies evidence level, there is consensus on some issues. Data highlight these wounds appears suddenly and represents a sign of eminent death. The sacrococcygeal and posterior region of the legs are the most common areas.

Conclusion: The low number of studies and the low level of evidence calls our attention to the limited research about KTU. However, this an important area, and further robust study designs are needed.

\section{Abstract number: P02-159 \\ Abstract type: Poster}

\section{A Rare Case of Insulinoma - latrogenic Symptoms versus} "Natural Causes"

Banting M., Curtis P.

University Hospital Southampton NHS Foundation Trust, Southampton, United Kingdom

A 61 year old inpatient with insulinoma and liver metastases required dexamethasone $4 \mathrm{mg}$ qds, diazoxide $150 \mathrm{mg}$ tds, Lanreotide and a $20 \%$ glucose infusion (2.4 litres per day) for hypoglycaemia.

Despite this she deteriorated mostly due to fluid overload, worsening despite furosemide infusion. She expressed a wish to stop treatment. After discussion with the patient, her oncologists, family and legal team (concerns about depression on mental capacity) we agreed to stop active treatment and manage 'symptomatically'.

Once a $24 \mathrm{hr}$ subcutaneous infusion of oxycodone $10 \mathrm{mg}$, midazolam $10 \mathrm{mg}$ and levomepromazine $25 \mathrm{mg}$ treated her pain, breathlessness and distress, she was able to articulate her wishes again.

She repeated a desire to stop life prolonging intervention but felt paralysed to decide to stop the glucose infusion that was keeping her alive. She was terrified of another hypoglycaemic seizure and didn't want to 'disappoint' family.

After much discussion, I made the decision to reduce the glucose. With her agreement, and her family's, glucose dose was halved, oral drugs stopped and steroids reduced. She was fully aware this would shorten her life. After the decision she thanked us as we 'gave her permission' to stop so she did not feel guilt at letting her family down.

She died peacefully that night with family present who thanked us as they had seen her struggling but found it very hard to 'give permission to let go'.

This case highlights challenges of managing a patient with iatrogenic symptoms caused by keeping her alive. Without the glucose she would have died of hypoglycaemia and yet it was the concomitant fluid overload that contributed to her severe symptom burden.

This patient's problems were unusual, complex and severe. We achieved her wishes by making decisions others felt unable to, supporting her family, taking burden from other professionals, and most importantly, by advocating for a patient who only felt able to open up to us.

Abstract number: P02-160

Abstract type: Poster

Death Rattle: Possible Factors of Influence on Perception of Relatives 
van Esch H.J. ${ }^{1,2,3}$, Lokker M.E. ${ }^{3}$, Rietjens J. $^{3}$, van Zuylen L. ${ }^{1}$, van der Rijt C.C.D. ${ }^{1}$, van der Heide A. ${ }^{3}$

${ }^{1}$ Department of Medical Oncology, Erasmus MC Cancer Institute, Rotterdam, Netherlands, '2Laurens Cadenza, Rotterdam, Netherlands, ${ }^{3}$ Department Public Health, Erasmus MC, Rotterdam, Netherlands

Background: Death rattle is a frequently occurring symptom in the last phase of life. The perception of death rattle of relatives has been found to vary. We studied which factors may influence this perception.

Methods: We performed a qualitative study with a phenomenological approach. Data were collected through semi-structured interviews with relatives of patients who had died and for whom healthcare providers had recorded that DR had occurred in the dying phase. In these interviews questions were asked about the occurrence of DR and other symptoms in the last days of life, care and treatment, and information that was given about DR in the dying phase. Interviews were recorded anonymously, transcribed and thematically analyzed.

Results: Nineteen family members of 17 patients were interviewed. DR was experienced as a distressing symptom by most relatives and was negatively influenced by longer duration and higher intensity of DR itself and by a higher symptom burden of other symptom, e.g. pain or dyspnea. Previous experiences with DR could influence the current perception of DR either positively ("I recognize the symptom: I can handle it because death is not far away") or negatively ("I recognize the symptom: I cannot handle this noise again"). The experience of DR does not seem to be influenced by the amount and quality of information given about the symptom or by actions from the nursing staff to reduce the death rattle and physical discomfort.

Conclusion: Death rattle is a stressful symptom and is influenced by more factors than the sound alone. Influencing factors relate to the symptom itself (duration/intensity), to the burden of other symptoms and to previous experiences, but not to given information to relatives for this symptom.

Abstract number: P02-161

Abstract type: Poster

\section{Symptom Clusters in IPF}

Seppälä S.S. ${ }^{1,2}$, Rajala K. ${ }^{2,3}$, Lehto J.T. ${ }^{4,5}$, Sutinen E. ${ }^{2}$, Kautiainen H. ${ }^{6,7}$, Saarto T.2,3, Myllärniemi M.2,8

${ }_{1}^{1}$ Pulmonary Diseases, Päijät-Häme Central Hospital, Lahti, Finland, ${ }^{2}$ Faculty of Medicine, University of Helsinki, Helsinki, Finland, ${ }^{3}$ Comprehensive Cancer Center, Department of Palliative Medicine, Helsinki University Hospital, Helsinki, Finland, ${ }^{4}$ Department of Oncology, Palliative Care Unit, Tampere University Hospital, Tampere, Finland, ${ }^{5}$ Faculty of Medicine and Life Sciences, University of Tampere, Tampere, Finland, ${ }^{6}$ Primary Health Care Unit, Kuopio University Hospital, Kuopio, Finland, ${ }^{7}$ Folkhälsan Research Center, Helsinki, Finland, ${ }^{8} \mathrm{Heart}$ and Lung Center, Department of Pulmonary Medicine, Helsinki University Hospital, Helsinki, Finland

Background: Idiopathic pulmonary fibrosis (IPF) is a progressive lung disease with a poor prognosis. Lack of research on IPF symptoms and symptom clusters makes symptom-centered care difficult.

Aim: To find out, if there are symptom clusters in IPF patients, and if so, do they affect HRQOL.

Methods: We sent two questionnaires (RAND-36 and ESAS) to 300 Finnish IPF patients of which 245 answered. We performed exploratory factor analysis on the results to search for potential symptom clusters. Result: We found three symptom clusters: the emotional cluster (including depression, anxiety, insomnia, loss of appetite, and nausea), the pain cluster (pain at rest or in movement), and the respiratory symptoms cluster (shortness of breath, cough, tiredness, and loss of wellbeing). Correlation was strong within the clusters $\left(\rho_{\mathrm{T}} 0.78-0.85\right)$. The emotional cluster had the strongest correlation with mental health and vitality, the pain cluster with bodily pain, and the respiratory symptoms cluster with vitality, general health and physical functioning.
Conclusion: Physicians should assess IPF patients' respiratory symptoms, mental health, and pain, since these may be linked with other important symptoms and impaired HRQOL.

Funding: This sub-study was funded by The Research Foundation of the Pulmonary Diseases (Keuhkosairauksien tutkimussäätiö).

Abstract number: P02-162

Abstract type: Poster

Comparison of the Effects of Diuretics on Pedal Edema in

Patients with Cancer or Cirrhosis of the Liver: A Retrospective Single Facility Study

Nakagawa S. ${ }^{1}$, Okamoto Y. ${ }^{2}$, Tsunoda N. ${ }^{2}$, Tsuji K. ${ }^{1}$, Ishida S. ${ }^{1}$

${ }^{1}$ Department of Pharmacy, Kobe Gakuin University, Kobe, Japan,

2Department of Pharmacy, Ashiya Municipal Hospital, Ashiya, Japan

Background: In 2013, tolvaptan (T) became the first treatment drug to be approved for use against "fluid accumulation in cirrhosis of the liver for which other diuretic drugs were ineffective." However, there are no studies investigating the effectiveness of T on pedal edema.

Aims: The primary outcome of this study was to reveal the effectiveness of diuretics including T on pedal edema, and the secondary was to reveal whether there was a difference in the subjective assessments by patients(Pt) and the objective assessments by physicians(MD) of the effect.

Methods: Subjects were 107 hospitalized patients suffering from cancer or cirrhosis of the liver treated with tolvaptan (T), loop diuretics (L), or spironolactone (S) at the Ashiya hospital between 2015 and 2017. Patient characteristics, diuretic usage status, reason for discontinuation, diuretic effectiveness, adverse events(AE), urine volume, and clinical test values were retrospectively investigated using medical records.

Results: There were 76 subjects (average age: 73.6 , male: 36 , female: 40). The rates of improvement were: T: Pt 71.4\%, MD 57.1\%, L: Pt 60\%, MD 59.3\%, S: Pt unclear, MD 57.1\%, L+S: Pt 83.3\%, MD 65.6\%, T+L: Pt $75 \%, \mathrm{MD} 71.8 \%, \mathrm{~T}+\mathrm{L}+\mathrm{S}$ : Pt $60 \%, \mathrm{MD} 76.9 \%$. Excluding the $\mathrm{S}$ group, urine volume increased in $20 \%$ to $60 \%$. Regarding the occurrence of $A E$, in $\mathrm{T}, \mathrm{T}+\mathrm{L}$, and $\mathrm{S}$ dry mouth was seen in $30 \%$. There was no significant difference in changes in clinical test values between the medications.

Discussion: The objective of edema treatment is to alleviate symptoms and improve QOL. Therefore, it is important that symptoms improve according to the patient's subjective assessment. Though there was no statistically significant difference between the effectiveness of the drugs, the ratio of $\mathrm{Pt}$ for which there was improvement in symptoms was relatively high at over $70 \%$, in both T monotherapy and the T combined with other diuretics. It is necessary to collect additional data and evaluate the effect of T on pedal edema.

Abstract number: P02-163

Abstract type: Poster

Epidemiological Profile of the Malignant Bowel Obstruction in a Public Palliative Care Unit in Uruguay

Mendez A., Piriz G.

Servicio de Medicina Paliativa, Hospital Maciel, Montevideo, Uruguay

Background: Malignant bowel obstruction (MBO) is a frequent complication in patients with advanced cancer in Palliative Care services, especially in ovarian and colorectal cancer (CRC). Therapeutic options are scarce: surgery, endotumoral prosthesis(EP) and medical treatment (MT). Many patients end their lives with palliative sedation.

Aim: To know the epidemiological and clinical profile of patients with $\mathrm{MBO}$ in the unit.

Methods: Descriptive, analytical and retrospective study through a database revision from July 2004 till July 2018.

Results: The MBO was present in 129 out of the 5911 total treated patients $(2.2 \%)$, with a mean age of $64.63 .8 \%$ were women. The more frequent etiologies were: CRC $39.2 \%$, gynecological tumors $24 \%$ (ovary 
$38.7 \%$, cervix cancer (CC) $35.5 \%$ and endometrium $25.8 \%$ ), gastric $8.5 \%$, pancreas $4.6 \%$ and breast $3.9 \%$. For the CC the mean age was 55 , and for other gynecological tumors 73 years. At the time of admission, $65 \%$ were ECOG 3-4, 79\% had metastases and $47 \%$ peritoneal carcinomatosis. An $83 \%$ received $\mathrm{MT}$, $9 \%$ surgery, $1 \% \mathrm{EP}, 5 \%$ surgery and $\mathrm{MT}$, and $2 \% \mathrm{EP}$ and MT. 39\% required Palliative sedation, and of these, an $88 \%$ had metastases, $65 \%$ had an ECOG 3-4, and $56 \%$ had peritoneal carcinomatosis. Discussion: We found a slightly lower incidence of MBO (2.2\% vs 3-15\%) if compared to international publications. The etiology is similar in men, but in women the high incidence of CC as well as the early age of appearance draws our attention, which is consistent with the high incidence in our country. The low surgery performances can be due to poor functional status during admission, peritoneal carcinomatosis, and the presence of metastasis. In these patients, palliative sedation was more frequent.

Conclusion: Characteristics of our population are similar to international publications, except for the CC, a type of tumor very frequent in Uruguay. Palliative sedation was more frequent in patients with metastasis, poor functional status and peritoneal carcinomatosis.

\section{Abstract number: P02-164}

Abstract type: Poster

Cancer Patients' Symptom Burden and Health-related Quality of Life (HRQoL) at Tertiary Cancer Center from 2006 to 2013: A Cross-sectional Study

Kokkonen K. ${ }^{1}$, Tasmuth T. ${ }^{1}$, Lehto J. ${ }^{2}$, Kautiainen H. ${ }^{3}$, Elme A. ${ }^{4}$, Jääskeläinen A.-S. ${ }^{4}$, Saarto $T^{1}$

${ }^{1}$ Oncology, University of Helsinki, Helsinki, Finland, ${ }^{2}$ Oncology, University of Tampere, Tampere, Finland, ${ }^{3}$ Other, Espoo, Finland, ${ }^{4}$ University of Helsinki, Helsinki, Finland

Background: Cancer and its treatments affect patient's HRQoL.Alongside with prolonged survival, there is some evidence of cancer patients' symptom burden and decreasing HRQoL. Real-life data concerning the symptom prevalence and $\mathrm{HRQ}$ oL during cancer treatments are required to elucidate the need for earlier palliative.

Aims: The aims of this study were 1) to observe the symptom burden and HRQoL among cancer patients in different stages of the disease and 2) to investigate whether there is any change over a 7-year period.

Methods: This prospective cross-sectional study was carried out in 2006 and repeated in 2013. The primary outcome measurements were main symptoms and HRQoL by European Organization for Research and Treatment of Cancer Quality of Life (EORTC QLQ-C30) questionnaire.

Results: Altogether 581 patients responded (response rate 49\% in 2006 and $54 \%$ in 2013). The disease was local in 51\% and advanced in $49 \%$ of the patients. The HRQoL was significantly lower, except in emotional and cognitive functions, and the symptom burden more severe among patients with advanced compared to local cancer. The most prevalent symptoms were fatigue ( $93 \%$ and $85 \%$; moderate/severe $22 \%$ and $9 \%$ ), pain ( $65 \%$ and $47 \%$; m/s $16 \%$ and $5 \%$ ), and insomnia ( $64 \%$ and $60 \%$; m/s 20 and $21 \%$ ), respectively. No changes in HRQoL or symptoms were found in the 7-year study period.

Conclusion: The symptom load was higher in patients with advanced disease during the oncological treatments, but also patients with local disease suffered from moderate or severe symptoms. No significant changes were seen during the seven years in HRQoL highlighting the need for early integrated palliative care and psycho-oncological support during cancer treatments.

Funding: No

Abstract number: P02-165

Abstract type: Poster

Parents' Lived Experience Caring for a Child with Breathlessness Receiving Palliative Care
Hemsley J. ${ }^{1}$, Gibson F. ${ }^{2,3}$

${ }^{1}$ Louis Dundas Centre Oncology Outreach and Palliative Care, Great Ormond Street Hospital, London, United Kingdom, ${ }^{2}$ Haematology / Oncology, Great Ormond Street Hospital, London, United Kingdom, ${ }^{3}$ Child Health and Cancer Care, University of Surrey, Guildford, United Kingdom

Background: Parents consistently report breathlessness as one of the most frequent and distressing symptoms experienced by their child at the end of life. Assessment and management of breathlessness often falls to parents on a day-to-day basis especially when their child is being cared for at home, but there is a paucity of research exploring parents' experience of caring for a child with breathlessness receiving palliative care.

Aim: To explore the lived experience of parents caring for a child or young person with breathlessness receiving palliative care support. To show how parents perceive their child's experience of breathlessness, identify what influences parents' ability to support the child and explore the impact of breathlessness on the wider family.

Methods (design, data collection, analysis): A qualitative prospective design using a phenomenological methodology guided the study. Semistructured, in-depth interviews were conducted with a purposive sample of 10 parents of 9 children with breathlessness receiving palliative care from a tertiary specialist palliative care team. Data were analysed using a descriptive phenomenological process aiming to understand meanings by identifying themes and patterns to formalise a consistent description of the lived experience of parents caring for a child or young person with breathlessness receiving palliative care.

Results: Six key themes emerged: identifying breathing as a problem; living with breathlessness; managing episodes of breathlessness; impact of breathlessness on the whole family; breathlessness means living with the possibility of death and coping with breathlessness.

Conclusion: The essence of parents' lived experience of caring for a child with breathlessness is characterised by periods of stability interspersed with sudden and unpredictable episodes of acute breathlessness that brings the possibility of their child dying imminently into sharp focus.

\section{Abstract number: P02-166 \\ Abstract type: Poster}

Who Uses Marihuana for What ? Results of Survey on Complementary and Alternative Medicine (CAM) Use among Patients with Advanced Cancer

Wojtacki J. ${ }^{1,2}$, Pawłowski L. ${ }^{2}$, Pawłowska I. ${ }^{2}$, Lichodziejewska-Niemierko M.

${ }^{1}$ Rev. Eugeniusz Dutkiewicz SAC Hospice, Gdańsk, Poland, ${ }^{2}$ Medical University of Gdansk, Gdansk, Poland

Background: Marihuana is used to control symptoms of advanced malignant disease and side effects of anticancer therapies. The aim of the study was to obtain information about the patient population who used marihuana as CAM therapy.

Methods: Semi-structured questionnaire about the usage of CAM was presented to all consecutive patients of Palliative Care Outpatient Clinic, rev. Eugeniusz Dutkiewicz SAC Hospice, Gdańsk, Poland (2016-2018). For statistical analysis Pearson's Chi-square Test was applied.

Results: The final analysis included 181 patients (female: $69,6 \%$, median age: 63 , range: $24-94 \mathrm{yrs})$ with advanced cancer; 101 patients $(55,8 \%)$ used at least one CAM therapy. Among them were 11 (10,9\%) patients who declared marihuana use for 1 to 23 months (median: 3,5). Marihuana users were younger in comparison to non-marihuana patients: $59,7+12,8$ versus $62,4+12,7(p=0,04)$. They also more frequently refused traditional anticancer therapy than the rest of CAM users $(37,5 \%$ versus $8,0 \%, p=0,009)$. Seven out of 11 patients noticed some improvement in symptoms control following marihuana usage. 
Moreover, patients who had a medical practitioner in their close family more frequently used marihuana than patients who declared other CAM therapies usage $(p=0,02)$. What is interesting, primary malignancy location, gender, education level, marital status, economic situation, duration of foreign stay of patients or their relatives, frequency of worship participation did not influence marihuana use.

Conclusions: Marihuana is scarcely used for relieving symptoms in patients in Poland. Medical and health-care education appears to promote usage of various marihuana formulas.

Abstract number: P02-167

Abstract type: Poster

Depressed Mood in Hospice Inpatients - A Cross-sectional Study van der Baan F. ${ }^{1}$, de Graaf E. ${ }^{1}$, Gootjes J. ${ }^{2}$, Teunissen S. ${ }^{1}$ ${ }^{1}$ Center of Expertise of Palliative Care, University Medical Center Utrecht, Utrecht, Netherlands, ${ }^{2}$ Hospice Kuria, Amsterdam, Netherlands

Background: Quality of life is limited in patients experiencing depressed mood. Detecting mood changes in hospice patients is challenging as classic depressive symptoms are difficult to distinguish from physical decline as part of advanced disease. To support this diagnostic process, we studied whether the presence of other symptoms may predict the presence of depressed mood.

Aims: To study the association between patient reported symptoms, well-being and value of life, on perceived depressed mood in patients recently admitted to a hospice.

Methods: A cross-sectional study in 15 hospices in the Netherlands was conducted, including data from patients admitted between January 2015 - February 2018. A Dutch adapted translation of the Edmonton Symptom Assessment System, the Utrecht Symptom Diary (USD), was used to assess symptom burden on a 0-10 point numerical scale. All patients completed the USD as part of their routine care and gave permission to use the data for research purposes. In a multivariate linear regression model the association between anxiety, fatigue, well-being and value of life on depressed mood was analyzed.

Results: 367 patients were included, 53\% women, median age $71 \mathrm{y}$. Most patients (90\%) had cancer. The prevalence of depressed mood (score $>0$ ) was $46 \%$ at admission. Anxiety $(b=0.4795 \% \mathrm{Cl}[0.37-0.56])$, decreased well-being $(b=0.3395 \% \mathrm{Cl}[0.22-0.44])$ and decreased value of life $(b=0.1795 \% \mathrm{Cl}[0.09-0.25])$ were independent predictors for depressed mood, corrected for gender, age and diagnose. These factors explained $44 \%$ of the variance in depressed mood. Fatigue was not associated with depressed mood.

Conclusion: It is recommended to be attentive to depressed mood when anxiety, a low level of well-being and value of life are expressed by hospice patients. Assessing the value of life as integral part of a symptom diary is a unique quality of the USD. We show how communication may contribute to the understanding of a patient's depressed mood.

Abstract number: P02-168

Abstract type: Poster

What is the Purpose of the Massage Used by Rehabilitation Therapists for Terminal Cancer Patients?

Nishiyama N. ${ }^{1}$, Matsuda $\mathrm{Y}^{2}$, Okamura H. ${ }^{3}$

${ }^{1}$ Rehabilitation, Ashiya Municipal Hospital, Ashiya, Hyogo, Japan, 2Palliative Medicine, Ashiya Municipal Hospital, Ashiya, Hyogo, Japan, ${ }^{3}$ Hiroshima University, Hiroshima, Japan

Purpose: When we performed rehabilitation for terminal cancer patients, various programs were available based on the needs of individual patient, and massage was one of common programs. We aimed to clarify how massage was used in the rehabilitation for terminal cancer patients.
Method: This was the retrospective observational study using chart review. Subjects were terminal cancer patients who were hospitalized in the palliative care unit or general wards of our hospital for last 3 years, not taken anti-cancer treatment, and taken rehabilitation for more than 2 weeks until death. We investigated the purpose and frequency of massage at the beginning of rehabilitation and 3 weeks before death.

Results: 109 patients (ave. age: 76 y.o., women: 54\%) were enrolled. Massage was used for $73,79,90,96 \%$ of the patients at the beginning, 3-, 2-, and 1-week before death, respectively. The purpose of massage at the beginning was only "palliation of physical symptom "including pain and fatigue" in 12 cases (15\%), and "both symptom palliation and maintain / improvement of activities of daily living (ADL); physical exercise, gait training, sit out of bed, etc." in 68 cases (85\%).

At the period close to death, "maintenance of peripheral circulation", "prevention and palliation of pain due to immobility" and "family-care during last days" were added as the purpose of massage.

Discussion: Massage was used at high frequency as rehabilitation for terminal cancer patients.

When patients had physical symptoms at the beginning, palliating these symptoms by massage at first might enable to be performed the following rehabilitation programs for maintaining / improving ADL. The purpose of rehabilitation has spread not only "for patient" but also "for family" in the period close to death.

Massage during this period is expected to be an approach to improve the quality of life of terminal cancer patients and families, and lead to improve the quality of palliative care for them.

\section{Abstract number: P02-169}

Abstract type: Poster

\section{Malignant Fungating Wound Care: What's New?}

Aparicio M. ${ }^{1,2}$, Bragança J. ${ }^{3,4}$, Cunha T. ${ }^{5}$

${ }^{1}$ St Christopher's Hospice, London, United Kingdom, ${ }^{2} \mathrm{ICS}$, Universidad de Navarra, Pamplona, Spain, ${ }^{3} \mathrm{H}$ ospital da Luz, Lisboa, Portugal, ${ }^{4}$ Escola Superior de Enfermagem de Lisboa, Lisboa, Portugal, ${ }^{5}$ Unidade Local de Saúde de Castelo Branco, Castelo Branco, Portugal

Background: The malignant fungating wounds (MFW) are well defined in literature. The prevalence ranges from 5 to $10 \%$ in patients with advanced cancer. These are an indicator of poor prognosis. Although infrequent, these lesions cause an enormous physical and psychological impact to the patient, the family and caregivers. The care of a person with MFW is a challenge.

Aim: To gather new treatment approaches to MFW.

Methods: Integrative literature review of international databases and international guidelines since 2010.

Results: From a total of 437 papers, 17 met the inclusion criteria. The majority were British (41\%), 47\% were published in 2011 and seven had empirical methodology. We included ten international guidelines.

The most researched symptom was malodour: when compared, metronidazole and green tea showed similar efficacy; the same happened when comparing honey and silver bandages; coffee and essential oils are good complements as environmental measures. Mohs' paste was effective in bleeding control in breast cancer patients. There was no consensus on the approach of necrosis, but sharp debridement is not recommended. Regarding itching, transcutaneous electrical nerve stimulation is largely recommended in opposition to antihistamines.

Dressings with sealed edges, with high absorbability and fluid storage, allied to the capacity to evaporate fluids are recommended for exudate control.

To control the symptoms of MFW, antibiotic treatment of infection may be advisable.

Pain should be addressed according to the WHO ladder.

Conclusion: We found some new strategies that can help our daily care of patients with MFW. Most papers were based on case reports. The use 
of proper tools, documentation, scientific based care and training for professionals is recommended.

Abstract number: P02-170

Abstract type: Poster

Prevalence of Constipation in Advanced Disease in the Home Setting and its Relationship with Other Health Problems: A Cross-sectional Survey

Rente J. ${ }^{1}$, Gomes B. ${ }^{2,3}$, de Brito M. ${ }^{3}$, Gonçalves E. ${ }^{4}$, Frias E. ${ }^{5}$, Lopes Ferreira P. ${ }^{6}$, Gonçalves L. ${ }^{7}$

${ }^{1}$ Hospital Dr. Francisco Zagalo, Ovar, Portugal, ${ }^{2 K i n g ' s ~ C o l l e g e ~ L o n d o n, ~}$ London, United Kingdom, ${ }^{3}$ Universidade de Coimbra, Coimbra, Portugal, ${ }^{4}$ Centro Hospitalar São João, Porto, Portugal, ${ }^{5}$ Unidade Local de Saúde de Matosinhos, Matosinhos, Portugal, ${ }^{6}$ Centro de Estudos e Investigação em Saúde da Universidade de Coimbra, Coimbra, Portugal, ${ }^{7}$ Unidade Local de Saúde do Nordeste, Bragança, Portugal

Background: Constipation is a challenging symptom, difficult to manage at home when severe, and with scarce evidence about how it relates with others.

Aim: We aimed to determine the prevalence and severity of constipation in advanced disease at home, and its relationship with other symptoms and patient's health state.

Methods: Secondary analysis of cross-sectional face-to-face survey data from patients and their family caregivers in four home palliative care teams (Portugal, Apr'2015-May'2017). Patient experience of ten symptoms/problems in the three days prior to interview was self- or proxyreported (when the patient could not report), using the POS-S (Palliative care Outcome Scale - Symptoms, 0-4). They also reported quality of life (EQ-5D Visual Analogue Scale, 0-100) and functional status (PPS: Palliative Performance Scale, 0-100) on the interview day. We used POS-S values 2-4 (moderate-overwhelming) to determine prevalence, Pearson's $\mathrm{Chi}^{2}$ to compare cancer and non-cancer patients, and Spearman's rho to test for correlations $(p<0.05)$.

Results: We included 100 patients $(55.0 \%$ male, median age $75,67.0 \%$ cancer). The prevalence of constipation was $36.0 \%$. Among constipated patients, $52.8 \%$ experienced severe to overwhelming levels. There were positive associations with nausea ( $r h o=0.201 ; p=0.045$ ) and mouth problems ( $r h o=0.201 ; p=0.045)$, and a negative association with functional status ( $r$ ho $=-0.239 ; p=0.019$ ). There was no relationship with quality of life.

Conclusion: One in three patients were constipated, half severely. The relationship with gastrointestinal symptoms and low functional status suggests a need for better recognition and management of this symptom.

Abstract number: P02-171

Abstract type: Poster

Comparison of Croatian Experience of Palliative Sedation at Home with Others - A Systematic Review

Kustura A. ${ }^{1}$, Fumic-Dunkic L. ${ }^{2}$, Hoic L. ${ }^{1}$, Sabljic Z. ${ }^{2}$

${ }^{1}$ Health Care Center Zagreb, Zagreb, Croatia, ${ }^{2}$ University Hospital

Center 'Sisters of Mercy', Zagreb, Croatia

Background: Palliative sedation (PS) is a procedure in palliative care that has been used in hospitals as a common procedure. However, palliative sedation at home is not so distributed option. There is a general lack of guidelines and experience that consequently causes insufficient use of palliative sedation at home. Croatian experience is similar.

Aims: To compare Croatian experience of PS at home with other's experience.

Methods: A systematic review of literature was performed using MEDLINE and NICE databases with search terms palliative sedation at home, terminal sedation at home.
Results: When compared with other's experience, our main cause for PS was delirium, convulsions and pain which does not differ from other studies.

Concerning medication, recommendation is to use as a first line opioid medication if patient has pain. Second line are benzodiazepines, firstly midazolam subcutaneously administered. Adjustment of medication was done in almost all cases until adequate symptom control. Most common diagnosis was lung cancer. Our experience was limited to head throat and neck cancer, followed by breast cancer. Regarding artificial hydration, results differ. In Croatia artificial hydration usually continues until the end, but with lowered administration through time. Palliative sedation is carried out by different experienced healthcare workers. In Croatia it can be administered only under supervision of experiences anesthesiologist with cooperation of mobile palliative team who handles supervision at home.

Conclusion: Our experience shows that our main limitations come from logistic and technical difficulties of the healthcare system. Other important limiting factor is family of patients where inadequate communication and cooperation which prevent application of palliative sedation at home.

Furthermore, this has shown a growing agreement for research in this field and for further efforts and investments in order to further improve palliative sedation at home.

Abstract number: P02-172

Abstract type: Poster

Should Patients Recruited in Mirtazapine for Anorexia Trials Be Screened for Depression?

Atallah C., Abel Aal H., El-Sherif W., Farag D., Abdelhafeez A., Alsirafy S. Palliative Medicine Unit, Kasr Al-Ainy Center of Clinical Oncology \& Nuclear Medicine, Kasr Al-Ainy School of Medicine, Cairo University, Cairo, Egypt

Background: Anorexia is a common symptom among patients with advanced cancer that may cause significant distress for patients and their families. One of the drugs that are under investigation for anorexia is the tetracyclic antidepressant mirtazapine. Depression is common as well in advanced cancer patients and patients may experience anorexia and depression simultaneously.

Aim: To screen cancer patients with anorexia for depression before recruitment in a mirtazapine for anorexia clinical trial.

Methods: Adult advanced cancer patients with anorexia of $\geqslant 4$ severity on a 0 to 10 numerical scale were assessed for depression using the depression subscale of the Hospital Anxiety and Depression Scale (HADS). According to the HADS-Depression subscale score, patients were grouped into normal ( 0 to 7 ), borderline case ( 8 to 10 ) and case (11 to 21 ).

Results: The median anorexia score of 42 eligible patients was 7 (range: 4-9). According the HADS-Depression subscale score, 14 (33\%) patients were normal, $24(57 \%)$ borderline case and $4(10 \%)$ case. There was significant positive correlation between the anorexia score and the HADSDepression subscale score (Spearman's rho $=0.711, p<0.001$ ). The mean anorexia score differed significantly between the three HADSDepression groups. It was $5.6 \pm 0.75,6.9 \pm 0.65$ and $7.5 \pm 1.29$ in the normal, borderline case and case groups; respectively $(p<0.001)$.

Conclusions: Depression is common in cancer patients with anorexia. This should be taken into consideration while designing and interpreting clinical trials investigating the effect of mirtazapine on anorexia.

Abstract number: $\mathrm{P} 02-173$

Abstract type: Poster

Evidence-based Guideline for Palliative Care of Malignant Wounds of Adult Patients with Incurable Cancer 
Doll A. ${ }^{1}$, Uebach B. ${ }^{2}$, Protz K. ${ }^{3}$, Vasel-Biergans A. ${ }^{4}$, Buentzel J. ${ }^{5}$, Schaperdoth E. ${ }^{6}$, Pralong A. ${ }^{1}$, Simon S. ${ }^{1}$, Voltz R. ${ }^{1}$, Bausewein C. ${ }^{7}$, Krull E. ${ }^{8}$, German Guideline Group Palliative Care (dt.: Deutsche Leitliniengruppe S3-Palliativmedizin)

${ }^{1}$ Department of Palliative Medicine, University of Cologne, Köln, Germany, ${ }^{2}$ Center of Palliative Care, University of Bonn, Bonn, Germany, ${ }^{3}$ Wound Center Hamburg, Hamburg, Germany, ${ }^{4}$ Diakonissenkrankenhaus, Stuttgart, Germany, ${ }^{5}$ üdharz Klinikum Nordhausen, Nordhausen, Germany, ${ }^{6}$ Wound and Ostomy Care, University of Cologne, Köln, Germany, ${ }^{7}$ Center of Palliative Care, Univercity of Munich, Munich, Germany, ${ }^{8}$ Adiuvantes-SAPV, Landshut, Germany

Aim: The German Association of Palliative Care developed an evidencebased guideline about the management of malignant wounds in adult patients with incurable cancer. There is a lack of evidence-based guidelines about malignant wounds and a confusing wide range of available wound dressings. The goal of the guideline is to reduce the suffering of patients with malignant wounds and stabilize or improve their quality of life.

Methods: The guideline is constructed following the German Instrument for Methodological Guideline Appraisal (DELBI): A systematic review was carried out for guidelines, reviews and specific studies. A wound expert group discussed the research findings and suggested recommendations which were adapted and consented by representatives of 62 medical and health professionals associations.

Results: The guideline has 34 recommendations, thereof nine (26\%) are evidence based with an evidence level from $2+$ to 4 (according to SIGN). The assessment chapter comprises specific assessment tools for malignant wounds, odor and wound-related quality of life. Three recommendations address the psychosocial support of patients and their family caregivers. We aim to reduce the impact of the wound on their emotional wellbeing, caregiver burden and social participation. The pain recommendations focus on a preventive atraumatic dressing change, positioning, systemic pain medication (anticipated, rescue and baseline) and local application of morphine or local anesthetics. The guideline gives recommendations on odor and exudate management with different interventions (e.g. metronidazol, active coal and antiseptic dressings) At last the recommendations on prevention and management of bleeding (antifibrinolytica, haemostyptica) are vital for patients and caregivers.

Conclusion: This guideline is one of the first evidence-based and consented guideline on malignant wound care and has the potential to improve the palliation of patients who suffer from there malignant wounds.

Abstract number: P02-174

Abstract type: Poster

Frequency of Extrapyramidal Symptoms in Cancer

Patients who Receive Palliative Care: A Multi-center, Observational Study

Ishiki H. ${ }^{1}$, Hamano J. ${ }^{2}$, Nagaoka H. ${ }^{2}$, Iwase S. ${ }^{3}$, Yoshinobu M. ${ }^{4}$, Tokoro A. ${ }^{4}$, Sakashita A. ${ }^{5}$, Kizawa Y. ${ }^{5}$, Izumi H. ${ }^{6}$, Matsuoka H. ${ }^{6}$, Oyamada S. ${ }^{7}$ ${ }^{1}$ National Cancer Center Hospital, Tokyo, Japan, ${ }^{2}$ University of Tsukuba Hospital, Ibaraki, Japan, ${ }^{3}$ Saitama Medical University, Saitama, Japan, ${ }^{4}$ National Hospital Organization Kinki-Chuo Chest Medical Center, Osaka, Japan, ${ }^{5}$ Kobe University, Hyogo, Japan, ${ }^{6}$ Kindai University Hospital, Osaka, Japan, ${ }^{7}$ Department of Biostatistics, JORTC Data Center, Tokyo, Japan

Aims: We conducted this proof-of-concept study to estimate the feasibility and sample size for an interventional study for extrapyramidal symptoms (EPS). We investigated the frequency of EPS in patients with cancer receiving palliative care.

Methods: This multi-center, prospective, observational study included cancer patients who
1) are receiving palliative care from a palliative care team or in a palliative care unit,

2) have a cancer diagnosis, and

3) are aged $\geqslant 20$ years. We investigated the frequency of EPS and medications used. The primary outcome was the overall DrugInduced Extrapyramidal Symptom Score (DIEPSS).

Results: Between November 2015 and October 2016, 149 patients from 5 centers in Japan were enrolled. The median age was 67 years (range, 21-88). There were 81 males (54.4\%). Cancer types included: lung, 55 (36.9\%); upper gastrointestinal tract, 5 (3.3\%); hepatobiliary, 19 (12.8\%); breast, 12 (8.1\%); head and neck, 10 (6.7\%); gynecologic, 10 (6.7\%); and genitourinary, $10(6.7 \%)$. The median Karnofsky performance status was 60.0 (20-100). Most patients (86.6\%) did not have delirium. Thirty-nine (26.2\%) patients received EPS-inducing medications: metoclopramide, 17 (11.4\%); prochlorperazine, 12 (8.1\%); haloperidol, 7 (4.7\%); risperidone, 5 (3.4\%); olanzapine, 4 (2.7\%), and chrolpromazine, 3 (2.0\%). EPS was found in $4(2.7 \%)$ patients with a cut-off score of 5 points for parkinsonism 5 -items of DIEPSS.

Conclusions: Few patients in this population had EPS $(<3 \%)$, so an interventional study is not feasible. However, medications that cause EPS are often used in palliative care, so a longitudinal study is warranted.

Abstract number: P02-175

Abstract type: Poster

Management of Problematic Opioid Use in Palliative Care: A Scoping Review

Lau J. ${ }^{1}$, Mazzotta P. ${ }^{2}$, Kevork N. ${ }^{3}$, Rho H. ${ }^{4}$, Fazelzad R. ${ }^{3}$, Sud A. ${ }^{4}$, Baxter

S. ${ }^{5}$, Cory S. ${ }^{6}$, Smith A. ${ }^{7}$, Henderson D. ${ }^{8}$, Furlan A. ${ }^{9}$, Zimmermann C. ${ }^{10}$, Ryan $S^{1}$

${ }^{1}$ Palliative Care, Princess Margaret Cancer Centre, Toronto, Canada, 2Palliative Care, Temmy Latner Centre for Palliative Care, Toronto, Canada, ${ }^{3}$ University Health Network, Toronto, Canada, ${ }^{4}$ University of Toronto, Toronto, Canada, ${ }^{5}$ Canadian Hospice Palliative Care Association, Ottawa, Canada, ${ }^{6}$ Canadian Virtual Hospice, Winnipeg, Canada, ${ }^{7}$ Centre for Mental Health and Addiction, Toronto, Canada, ${ }^{8}$ Canadian Society of Palliative Care Physicians, Truro, Canada, ${ }^{9}$ Toronto Rehabilitation Institute, Toronto, Canada, ${ }^{10}$ Princess Margaret Cancer Centre, Toronto, Canada

Background: Opioids are essential medicines in palliative care, but are susceptible to problematic use.

Aim: To identify strategies to manage opioid misuse, abuse and diversion (OMAD) in palliative care populations.

Methods: Comprehensive searches were conducted of electronic databases from inception to March 2018, as well as reference lists and grey literature. We included studies involving adults with diagnoses in three main disease groups: advanced cancer, neurodegenerative diseases and end-stage organ diseases. There were no restrictions on country of origin, language or publication status.

Results: Of 26, 412 records independently screened by two investigators, 20 studies investigated management of OMAD in the palliative care setting. Major themes were identified:

1. administrative policies;

2. interdisciplinary team approach;

3. use of clinical tools to guide opioid prescribing;

4. specific opioid prescribing practices (e.g. rotation, opioid choice);

5. adjuvant medications and interventional analgesia; and,

6. non-pharmacologic strategies.

Most studies were conducted in the USA ( $n=13)$, in outpatient clinics $(n=8)$, and in cancer $(n=17)$. Study designs were: case reports $(n=11)$, 
retrospective cohort $(n=3)$, prospective cohort $(n=1)$, quality improvement study $(n=1)$, randomized control trial $(n=1)$ and survey $(n=1)$.

Conclusion: There is limited evidence around optimal OMAD management in palliative care. Patients with concurrent problematic opioid use and uncontrolled symptoms secondary to life threatening illnesses are often challenging to manage. High quality evidence is needed to provide symptom management and optimize quality of life for individuals with life threatening illnesses and OMAD.

Abstract number: P02-176

Abstract type: Poster

Validity and Reliability of the Scale of Uncertainty in Illness in Family Caregivers of Patients in Palliative Care in Colombia Carreño S. ${ }^{1}$, Arias M. ${ }^{2}$

${ }^{1}$ Faculty of Nursing, Universidad Nacional de Colombia, Bogota, Colombia, ${ }^{2}$ Faculty of Nursing, Universidad de Antioquia, Medellin, Colombia

Background: The scale of uncertainty in illness has been used in several studies in caregivers of patients with acute and chronic pathologies. In this regard studies have shown that measuring and intervening uncertainty in the caregiver is important to improve their quality of life.

Aims: To perform the adaptation and validation of the scale Uncertainty in illness -Family Caregiver version, in family caregivers of patients in Palliative Care in the Spanish language and the Colombian context.

Methods: A methodological study was carried out with family caregivers of patients in palliative care of various pathologies. An initial review of the scale and facial validation was carried out with 20 family caregivers. The construt validity and internal consistency was carried out with a sample of 290 caregivers.

Results: The facial validity of the instrument obtained a high index of acceptability. The content validity showed coherence, clarity and relevance of the instrument. A confirmatory factor analysis was carried out and resulted in 4 factors confirming the dimensions of the instrument in its original version. However, 5 items were eliminated because they were not adjusted to the dimensions found. In the internal consistency was found a Cronbach's alpha of 0.72 this score is an acceptable global value. Conclusion: The scale of uncertainty in illness is an adapted and reliable scale to use with family caregivers of people in palliative care in the Colombian context. Being able to use this scale that is valid and reliable allows to have a deeper understanding of the uncertainty of the caregiver and to generate strategies for its management.

Abstract number: P02-177

Abstract type: Poster

\section{Validity of the Distress Thermometer as a Hetero-assessment Tool \\ Van Lander $A .{ }^{1}{ }^{1}{ }^{2}$, Tarot $A .{ }^{1}$, Vennat $B .{ }^{2}$, Guastella $V^{1}$ \\ ${ }^{1}$ Palliative Care, $\mathrm{CHU}$ Clermont-Ferrand, Clermont-Ferrand, France, 2UPU ACCePPT, Université Clermont Auvergne, Clermont-Ferrand, France}

Background: The distress of patients suffering from a terminal illness can lead to a state of despair and requests for euthanasia or assisted suicide. It is a major challenge for palliative care workers. The Distress Thermometer (DT) is recommended by the National Comprehensive Cancer Network (Holland, 2007) as a means of assessing distress more easily. It is available as a self-assessment tool, but for a wider use in palliative care it would need to be implemented in the form of a heteroassessment (Van Lander, 2015).

Aim: We performed a study to assess the validity of the DT used on a hetero-assessment basis in palliative care.

Method: Hetero-assessments were performed by teams of healthcare providers, doctors and psychologists working in three palliative care centers. The primary objective was agreement between patient assessments and those of the health professionals, as calculated by Lin's concordance coefficient with a threshold (alpha) of 5\%. Eligible patients were aged 18 or older, suffering from a severe disease in the palliative phase, and with a sufficient level of awareness to give their consent. A total of 51 patients were recruited, of whom $55 \%$ were male, with a an everage age of 65.8 [39-90].

Results: 364 self-assessments and 467 hetero-assessments were performed. Lin's concordance coefficient was $0.46[0.38 ; 0.54]$. At the first assessment, it was $0.61[0.44 ; 0.79]$. Cohen's kappa coefficient was 0.52 with a concordance rate of $79.6 \%$. Sensitivity was $82.9 \%$ [66.4-93.4] and specificity $71.4 \%$ [41.9-91.6]. After three assessments, distress was underestimated, especially by doctors.

Conclusion: DT as a hetero-assessment tool has good validity in early care management.

Holland J et al. The NCCN guideline for distress management: a case for making distress the sixth vital sign. J Natl Compr Canc Netw 2007;5: 3-7. Van Lander A. Apports de la psychologie Clinique aux soins palliatifs. Erès, 2015

\section{Abstract number: P02-178 \\ Abstract type: Poster}

\section{The Quality of Dying and Death of Advanced Cancer Patients in} Palliative Care

Gutiérrez Sánchez D. ${ }^{1}$, Gómez García R. ${ }^{1}$, Martín Roselló M. ${ }^{1}$, Cuesta Vargas A.I. ${ }^{2}$

${ }^{1}$ Cudeca Foundation, Málaga, Spain, ${ }^{2}$ Departamento de Fisioterapia, Instituto de Investigación Biomédico de Málaga (IBIMA), University of Málaga, Málaga, Spain

Background: The quality of dying and death is regarded as a goal to reach in end-of-life patient healthcare. The prevention and relief of suffering is essential to achieve the well-being and quality of death of patients who face life-threatening diseases. The evaluation of this construct can be useful in clinical and research settings, the quality of dying and death being an area where new knowledge is needed.

Aims: To determine the quality of dying and death in an advanced oncology population, and to analyse its association with suffering and the quality of care at the end of life.

Method: This is a cross-sectional study. Data were collected from an advanced oncology population in palliative care $(\mathrm{N}=72)$ between January and October 2016. An analysis was performed of the association between quality of dying and death and suffering and the quality of care at the end-of-life. For the evaluation of the quality of dying and death, the Spanish version of the Quality of Dying and Death Questionnaire was used. Suffering was assessed with the Mini-Suffering State Examination and the quality of end-of-life care was evaluated with the Palliative Outcome Scale.

Results: Seventy-two family care providers who care for advanced cancer patients were included in this study. The mean total score of the Spanish version of the Quality of Dying and Death Questionnaire was 64.56 (SD \pm 20.97 ). Quality of dying and death was negatively correlated with suffering and positively correlated with quality of care.

Conclusion: Quality of dying and death in an advanced oncology population in palliative care is positively related to the quality of care at the end of life and negatively related to suffering. Palliative care services can contribute to achieving satisfactory levels in the quality of dying and death of advanced cancer patients.

Abstract number: P02-179

Abstract type: Poster

Palliative Care Assessment Tools for Older Adults: A Review Dakessian Sailian S., Abu-Saad Huijer H., Dhaini S., Adra M. Faculty of Nursing, American University of Beirut, Beirut, Lebanon 
Background: The global population of people aged 60 years and older is expected to more than double. The prevalence of multiple chronic diseases among individuals is substantial among older adults. Therefore, palliative care remains a central part of healthcare services provision whether in the community or institutions. Assessing older adults' palliative care needs is the first step to delivering quality comprehensive care. Objectives: This review aims to describe existing assessment tools used to measure palliative care needs of older adults, their scope of use, content, and psychometrics.

Methods: A systematic literature search in electronic databases CINAHL, MedLine OVID, PsychInfo, and Academic Search Complete, was conducted for the years 2000 to 2016 by two independent researchers. Tools for older patients with palliative care needs regardless of their chronic condition or setting were included. Non-English literature, tools that are disease specific, such as dementia, or those filled solely by caregivers were excluded.

Results: The initial search yielded 942 articles. 17 articles met the inclusion criteria, describing 9 palliative care assessment tools used for older patients in various settings. They were the (POS), (RAI-PC), (MQLS), (ASPE), (NEST), (MQOL), (MQOL), (ESAS), (m-QOLC-E), (SAS \& GWB) integrated.

Conclusion: This review increases understanding of existing tools to assess older patients with palliative care needs. It can be concluded that no ideal tools are available to allow care providers to detect palliative care needs in older adults. The POS and RAI tools demonstrated better comprehensiveness and sensitivity to change in comparison to others.

Abstract number: P02-180

Abstract type: Poster

Quality Assurance in Specialized Palliative Home Care (QUAPS) A Questionnaire's Feasibility

Berghaus D. ${ }^{1}$, Schütz A. ${ }^{2}$, Hammer U. ${ }^{1}$, Gaser E. ${ }^{1}$, Wedding U. ${ }^{1}$, Meissner $W^{1}$

${ }^{1}$ Klinik für Innere Medizin II - Abteilung Palliativmedizin, Universitätsklinikum Jena, Jena, Germany, ${ }^{2}$ Tumorzentrum, HeliosKlinikum Erfurt, Erfurt, Germany

Background: For adequate quality assurance in "Specialized palliative home care" (SAPV) in Germany, structure and process parameters are not sufficient. Asking the patients and their relatives for their assessment is crucial. A Jena focus group developed the QUAPS questionnaire for this assessment of outcome parameters which was tested in two studies.

Aims: Evaluation of the feasibility of a questionnaire for patients, relatives and SAPV teams.

Methods: The questionnaire collects outcome parameters on a Likert scale and the distress of respondents with the numeric rating scale (NRS). In the pilot study QUAPS I, it was tested in three SAPV teams. The survey was simplified in the follow-up study QUAPS II where 17 teams were included. Both studies were conducted in an ex-post-facto design. Results: In QUAPS I, complete datasets could be obtained for 43 out of 308 requested cases. In QUAPS II, 169 complete datasets resulted from 371 requested cases.

The SAPV staff assessed the QUAPS II survey as being organizationally feasible. The questionnaire shows good internal consistency and high approval for the requested aspects of SAPV care. Ceiling effects occurred. More than $70 \%$ of the respondents reported a distress score $>5$, which is considered to require treatment.

Conclusion/ discussion: The simplified survey in QUAPS II resulted in a higher rate of complete datasets. The detected ceiling effects restrict the statement of the survey. Biases like social desirability cannot be ruled out. In the future, a combination of different questionnaires (e.g. Integrated Palliative Outcome Score (IPOS) and QUAPS) should be explored.
Abstract number: P02-181

Abstract type: Poster

Developing and Validating a PROgnostic Model Incorporating Function and Symptom Burden (PRO-MAC) in Advanced Cancer Lee C.S. ${ }^{1}$, Hum Y.M.A. ${ }^{1}$, Yee C.M. ${ }^{1}$, Koh Y.H.M. ${ }^{1}, \bar{W}$ U H.Y. ${ }^{1}$, Wong K.Y.Y. ${ }^{2}$, Low Z.J.C. ${ }^{1}$

1Palliative Department, Tan Tock Seng Hospital, Singapore, Singapore, ${ }^{2}$ Epidemiology Department, Tan Tock Seng Hospital, Singapore, Singapore

Background: Prognostication is acknowledged to be an inexact science, with physicians having difficulty distinguishing patients who will live less than 6 months. Simple, accessible, well validated prognostic models which are generalizable are urgently needed to objectively identify patients who would benefit from an interdisciplinary collaboration with palliative care.

Aim: The primary objective of this study is to develop and validate a prognostic risk model for patients with advanced cancer.

Methods: The PROgnostic Model in Advanced Cancer (PRO-MAC) model was developed by selecting functional, symptom and disease burden predictors and using stepwise regression selection procedures to develop the multivariate model. Numeric scores were assigned based on the relative weight of the independently predictive prognostic variables in the final multivariate Cox regression model.

Results: The Palliative Performance Scale Score, Edmonton Symptom Assessment System summative score, number of sites of metastasis, serum albumin, and total white blood cell level were significant in multivariate analysis. The area under the curve using the final averaged prognostic model ranged between 0.6875 to 0.7506 . Patients in the validation subsets were classified into 3 risk groups. The average survival of patients in the lowest risk Group 1(0-1.5 points) was 79.5 days (reported 63-96 days), Group 2 (2-5.5 points) was 43 days (39-47 days), and the high-risk Group 3 (6-10.5 points) was 13.8 days (11-17 days).

Conclusion: The PRO-MAC model takes into account patient and disease-related factors, stratifying patients based on weighted scores into 3 distinct groups. PRO-MAC may help physicians advise patients according to their prognosis and facilitate shared decision making, improving patient centered outcomes.

Abstract number: P02-182

Abstract type: Poster

The Acceptability and Utility of Accelerometers to Estimate Physical Activity and Sleep Characteristics in People with Advanced Dementia: A Feasibility Cluster Randomised Trial (NAMASTE)

Perez Algorta G., Patel S., Dunleavy L., Preston N., Walshe C., Froggatt $K$.

Division of Health Research, Lancaster University, Lancaster, United Kingdom

Background: Identification of valid, reliable and clinically relevant measures of quality of dying is challenging. Self-reports and/or proxy reporting by professional or family carers has limitations. Objective measurement of general functioning evaluated with accelerometers may be a viable addition. Accelerometers research use is increasing, but not yet considered as an outcome measure for people with advanced dementia at the end of life.

Aim: To determine the acceptability and utility of accelerometers as a proxy outcome of quality of dying in people with advanced dementia, and to estimate physical activity and sleep characteristics.

Methods: People with advanced dementia ( $n=32$ participants) resident in nursing homes $(n=6)$, wore a wrist band accelerometer (GENEActiv) for up to 28 days. We estimated levels of physical activity and sleep characteristics 
(duration and efficiency). Descriptive analyses and hierarchical linear models described and estimated activity and sleep. Acceptability of the accelerometer was ascertained by length of time worn and feedback from the resident, family or care staff.

Results: The accelerometer was acceptable: 30 (94\%) participants wore the actigraph continuously following the protocol (28 days). On average, measurements were available for 23.4 days $(S D=2.8)$. No adverse effects were reported. Participants were generally inactive: $98 \%$ of each day had low levels of activity $<50$ ENMO (moderate physical activity is $100)$; the ENMO mean was $8.5(S D=4.1)$. The estimated mean sleep duration was 7.6 hs $(s e=.41)$, and sleep efficiency was $68 \%(85 \%$ is healthy population target).

Conclusions: The accelerometer was acceptable for wearing, but data on activity demonstrated an inactive population with indication that their sleep was not as healthy as it could be. It may be difficult to measure changes arising from an intervention using activity and sleep as a focus in this population.

Abstract number: P02-183

Abstract type: Poster

\section{Physical Activity and Mobility Assessment on a Specialist}

Palliative Care Ward

Blum D. ${ }^{1}$, Jensen W. ${ }^{2}$, Schuetz $C .{ }^{2}$, Wannke L. ${ }^{2}$, Ketels $G .{ }^{2}$, Bokemeyer.$^{2}$, Oechsle K. ${ }^{1}$

${ }^{1}$ Palliative Care Unit, Department of Oncology, Hematology and Bone Marrow Transplant, UKE Hamburg, Hamburg, Germany, ${ }^{2}$ UKE Hamburg, Hamburg, Germany

Background: Performance status, physical activity and mobility are key determinants of care strategy and prognosis in palliative care, but different assessment methods are in use by different professions.

Aim: Comparing four different assessment methods and testing a specialist physiotherapist assessment.

Methods: All patients (pts) admitted to a specialist palliative care ward (sPCW) of a tertiary comprehensive cancer center between June 2017 and May 2018 were screened using a physiotherapeutic basic-assessment resulting in "mobilization possible" or "not possible". In pts with "possible", mobility was assessed by the Tinetti-mobility test (TT; 0-28 points) by a physiotherapist. A comparison with routinely weekly physician assessment (ECOG-Performance Status; 0-5 points), nurse-led mobility assessment (ePA-AC $=$ result oriented nurse assessment for acute care; $0-12$ points), and subjective patient reported mobility (0-4 points, $0=$ best) was performed. Spearman-correlations between assessment measurements were calculated.

Results: In total 438 pts were admitted to the sPCW during a year. In 248 pts, a basic assessment was done ( $135+, 78$-; 35 declined) and 125 performed a TT. In this group, median age was 63 years (range; $22-95$, SD 15 , 5); 55 were female, 70 male. Types of cancer were 39 gastrointestinal, 27 lung, 20 urogenital, and other 26; non cancer 26 . Median length of stay was 13 days (SD 7). 48 pts were discharged at home, 31 to a nursing home/hospice, 34 died on the ward. Mean TT score was 12 points (SD 9), mean ePA-AC: 8.5 (SD 2.2), mean ECOG: 3 (SD 0.9), Mean self-report 3 (SD 1.1). Correlations between assessment methods were low to moderate and significant for the TT with all other methods but self-report.

Conclusion: In around a quarter of pts on a palliative care ward, a Tinetti test could be performed. A multiprofessional assessment approach for low activity and bed-bound pts might be helpful to define care strategy in this population.

\section{Abstract number: P02-184 \\ Abstract type: Poster}

\section{The Reliability and Validity of Czech Version of IPOS}

VIčková K., Loučka M.

Center for Palliative Care, Prague, Czech Republic
Background: The main goal of palliative care is to improve quality of life so providers of palliative care need to measure it. Questionnaire IPOS (Integrated Palliative Outcome Scale) is one of the tools with good reliability designated to measure quality of life of patients in palliative setting. In the Czech Republic the measurement with good reliability had been missing so we conducted the standardization of IPOS to Czech. The aim of this study is to present the results of the standardization and the good reliability and validity of Czech version of IPOS.

Methods: We collected data from IPOS from 131 patients in 3 hospitals and 3 hospices in the Czech Republic and conducted psychometric analysis of Czech version of IPOS. The inclusion criteria was: Czech mother tongue, use of palliative care and cognitively healthy person. After that we sent questionnaire about the use of IPOS with open-ended question to providers of palliative care involved in this study to assess the face validity of IPOS.

Results: Czech version of IPOS has good reliability $(\alpha=0,79)$ and the items have optimum variability (the average correlation with sum scale $=0,5)$. Part of the sample $(n=18)$ completed also ESAS and the correlation with IPOS is moderate, but not significant $(r=-0,42, p=0,08)$. Providers of palliative care $(n=6)$ agreed in questionnaires that the questions of IPOS cover all problems that patients or relatives are dealing with and all aspects which are important for their quality of life.

Discussion: Czech version of IPOS was found to have good reliability and validity. The constructed validity is confirmed by correlation with ESAS. However, this correlation is not significant because of low number of patients but the tendency is evident. The results from the questionnaires completed by providers showed that Czech IPOS has good face validity.

\section{Abstract number: $\mathrm{P} 02-185$}

Abstract type: Poster

\section{Pilot Testing of the European Association for Palliative Care (EAPC) Basic Dataset}

Sigurdardottir K.R. ${ }^{1,2,3}$, Hjermstad M.J. ${ }^{2}$, Filbet M. ${ }^{4}$, Tricou C. ${ }^{4}$, McQuillan R. ${ }^{5}$, Costantini M. ${ }^{6}$, Autelitano C. ${ }^{7}$, Bennett M.I. ${ }^{8}$, Haugen D.F. ${ }^{1,9}$ ${ }^{1}$ Regional Centre of Excellence for Palliative Care, Western Norway, Haukeland University Hospital, Bergen, Norway, ${ }^{2}$ Department of Oncology, European Palliative Care Research Centre (PRC), Oslo University Hospital, and Institute of Clinical Medicine, Oslo, Norway, ${ }^{3}$ Department of Internal Medicine,Sunniva Centre for Palliative Care, Haraldsplass Deaconess Hospital, Bergen, Norway, ${ }^{4}$ Department of Palliative Care, Centre Hospitalier de Lyon-Sud, Hospices Civils de Lyon, Lyon, France, ${ }^{5}$ St Francis Hospice, Dublin, Ireland, 6 Scientific Directorate, Azienda USL-IRCCS di Reggio Emilia, Reggio Emilia, Italy, ${ }^{7}$ Palliative Care Unit, Azienda USL-IRCCS di Reggio Emilia, Reggio Emilia, Italy, ${ }^{8}$ Academic Unit of Palliative Care, University of Leeds, Leeds Institute of Health Sciences, School of Medicine, Leeds, United Kingdom, ${ }^{9}$ Department of Clinical Medicine K1, University of Bergen, Bergen, Norway

Background: Important patient characteristics are inconsistently and insufficiently reported in studies in palliative care. This fact precludes generalization of results. On this background, the EAPC Basic Dataset was developed. It consists of 31 core demographic and disease-related variables: a patient form with 17 items and a health care personnel form with 14 items.

Aim: To pilot test the EAPC Basic Dataset in cancer patients and health care providers in palliative care settings to assess acceptability, comprehensibility, and feasibility, and adapt the dataset accordingly.

Method: International multi-centre study at 9 study sites in 5 European countries. Quantitative and qualitative methods were used. Patients and health care personnel completed the EAPC basic dataset on paper, followed by a standard structured interview.

Results: 191 patients (of 544 screened) and 190 health care personnel participated. The patients' mean age was 67.6 years $(25-90), 75 \%$ had 
performance status $\geqslant 60$. Median time for completion was 5 minutes for patients, 7 for health care providers. The most challenging item for patients was ethnicity. Health care providers found the items weight loss, principal diagnosis, additional diagnoses, and stage of the non-cancer disease most demanding.

Whether or how to include the item ethnicity will require a decision at the national level. Diagnosis will be changed from ICD-10 codes to a predefined list. Weight loss and stage of the non-cancer disease will be removed. The pilot study led to minor rewording of some items, improvement in response options, and shortening of the dataset to 29 items.

Conclusion: Piloting the first version of the EAPC Basic Dataset confirmed that it is acceptable and feasible to use, and that patients and health care personnel understand the questions in a consistent manner. The pilot testing has led to improvements with regard to clarity and more suitable response options. The new version is now subject to further testing.

Abstract number: P02-186

Abstract type: Poster

Right Patient, Right Approach, Right Time: A Decade of MDT assessment for Procedural Interventions for Intractable Cancer Pain

Mitchell A. ${ }^{1}$, McGhie J. ${ }^{2}$, Somerville L. ${ }^{1}$, McGinn G. ${ }^{3}$, Owen M. ${ }^{3}$, Dymond E. ${ }^{1}$, English R. ${ }^{1}$, Horne K. ${ }^{1}$

${ }^{1}$ Beatson West of Scotland Cancer Centre, Glasgow, United Kingdom, ${ }^{2}$ Pain Management, Stobhill Hospital, Glasgow, United Kingdom, ${ }^{3}$ Pain Management, Victoria Infirmary, Glasgow, United Kingdom

Background: Many cancer patients will experience pain during their illness. A small proportion benefit from a targeted intervention to control refractory cancer related pain. It is just as important to avoid inappropriate interventions in patients with limited life expectancy as it is to ensure those who might benefit receive timely, appropriate treatment.

Since 2007 the Interventional Cancer Pain Service (ICPS) has offered a multidisciplinary team (MDT) assessment process for patients.

The aim is to ensure that where appropriate patients are offered one of several available procedural interventions, and if not appropriate, patients are reassured that they are not 'missing out' by continuing to receive standard palliative analgesia.

Aim: To analyse the outcomes of the ICPS MDT assessment process over 10 years.

Methods: Data were collected on 392 patients who were assessed between 2008 - 2018.The assessment outcomes were categorised into those offered an intervention and those that were not, and the reasons for the decision. Data were also collected on patient demographics and cancer diagnosis.

Results: Of the patients assessed, 187 (48.2\%) were female and 205 were male $(51.8 \%)$. The average age was 63 years. Following assessment, 181/392(46\%) patients were offered an intervention, while 40/392 (8\%) patients were deemed unfit for any intervention. In 142/392 (36\%) patients, an intervention was not felt to be in the patient's best interests for a range of reasons, e.g. there were psychosocial concerns, or review from another specialty was required.

Conclusion: The ICPS offers a holistic assessment process, which ensures not only that patients who have potential to benefit from an intervention are offered this option, but also that patients for whom an intervention has potential to do more harm than good, have this option discounted.

'It is far more important to know the person who has the disease, than what disease the person has.' Hippocrates

Abstract number: P02-187

Abstract type: Poster
"It Hurts when I Walk". Moving on from Static Pain Scores to Functional Pain Scores

Somerville L., Mitchell A.

Beatson West of Scotland Cancer Centre, NHS GGC, Glasgow, United Kingdom

Background: Intrathecal Drug Delivery (ITDD) has been shown to be of benefit in managing uncontrolled pain in cancer patients. This modality allows patients to significantly reduce systemic medications thereby decreasing side effects. Improved function and better quality of life can also be achieved. In our service patients undergo a multi-disciplinary team assessment and, if suitable, proceed to a trial period of ITDD. Patients will then proceed to permanent ITDD, if there is a sustained improvement in pain and/or function. The Brief Pain Inventory (BPI) is used as a static measure of pain and self-reported function. In addition, we also use a dynamic assessment tool for function, the Functional Pain Assessment Tool (FPAT), to guide clinical decision-making and support patient goal setting.

Aim: To assess the utility of the FPAT in determining the benefit of an ITDD trial.

Method: A retrospective analysis of 21 patients who received ITDD for uncontrolled cancer pain. Information was gathered 1 day pre operatively and 13 days into ITDD trial. BPI and FPAT scores were analysed and compared.

Results: During the analysis period, 15 patients proceeded to permanent device and 6 stopped at the end of the trial. The results show FPAT scores mirror the change in BPI scores for both groups. The pain score dropped on average by -4.2 points versus -1.3 points at day 13 for permanent versus trial only patients; FPAT total dropped by 7.5 points (from 18.0 to 10.5 ) for those who proceeded versus 4.2 points (20.5 to 16.3 ) for the trial only group. It should be noted that BPI pain interference scores were often incomplete, particularly in trial only patients.

Conclusion / Discussion: Dynamic assessment of pain and function was felt to positively inform clinical decision making when assessing the trial phase of ITDD. While changes in the FPAT score mirrored changes in conventional tools, further work is needed to assess if the FPAT can reliably be used in isolation.

Abstract number: P02-188

Abstract type: Poster

Transcultural Validation of the French Version of the Modified Edmonton Symptom Assessment (ESAS): ESAS12-F

Tricou C. ${ }^{1}$, Ruer M. ${ }^{2}$, Nekolaichuk C. ${ }^{3,4,5}$, Sigurdardottir K.6,7,8, PerceauChambard E. ${ }^{2}$, Bruera E. ${ }^{9}$, Filbet M. ${ }^{2,10}$

${ }^{1}$ Palliative Care, Claude Bernard Lyon 1 University/Lyon Sud University Hospital, Pierre-Benite, France, ${ }^{2}$ Palliative Care, Lyon Sud University Hospital, Pierre-Benite, France, ${ }^{3}$ Palliative Care, University of Alberta, Edmonton, Canada, ${ }^{4}$ Palliative Care, Grey Nuns Community Hospital, Edmonton, Canada, ${ }^{5}$ Palliative Care, Covenant Health, Edmonton, Canada, ${ }^{6}$ European Palliative Care Research Centre, Department of Cancer Research and Molecular Medicine, University of Science and Technology, Trondheim, Norway, ${ }^{7}$ Palliative Care, Haraldsplass Deaconess Hospital, Bergen, Norway, ${ }^{8}$ Palliative Care, Haukeland University Hospital, Bergen, Norway, ${ }^{9}$ Palliative Care, MD Anderson Cancer Center, Houston, United States, ${ }^{10}$ Claude Bernard Lyon 1 University, Villeurbanne, France

Background: Cancer-related physical symptoms can decrease patients' overall quality of life and are often under-diagnosed. The Edmonton Symptom Assessment Scale (ESAS) is widely used in palliative care for cancer patients to easily assess cancer patients' symptoms. It has been often modified, adding symptoms and explanations, and translated into many languages. The European Association of Palliative Care research team developed a database, which included the modified 12 item ESAS-r as the symptom assessment tool. 
Aim: The purpose of this study was to achieve the translation and crosscultural validation in French of the 12 item ESAS-r, the ESAS12-F.

Methods: A French version of the ESAS-r was developed using a standardized forward and backward translation method. Patients completed the ESAS12-F and provided feedback on the translation. Forty-five patients with advanced cancer, followed by the palliative care team from the Lyon Sud University Hospital in France were recruited.

Results: Eighty-nine percent of patients considered the ESAS easy to understand. They highlighted some concerns more about the tool itself than the translation: the time line "now", the difficulty to quantify a symptom in a numerical evaluation. Some items (sleep and appetite) needed to be re-read and for some others (digestive and psychological symptoms, and well-being) to be re-ordered in the questionnaire.

Conclusion: The ESAS12-F is well accepted and easy to use for the cancer patients. The next step is to carry out a psychometric validation of the definitive version of the ESAS12-F.

\section{Abstract number: P02-189 \\ Abstract type: Poster}

Patients Benefit from Using the Integrated Palliative Care Outcome Scale During Specialized Palliative Home Care Högberg C. ${ }^{1,2}$, Alvariza A. ${ }^{1,2}$, Beck I. ${ }^{3,4}$

${ }_{1}^{1}$ Dalen Hospital, Capio Palliative Care, Stockholm, Sweden, ${ }^{2}$ Department of Health Care Sciences/Palliative Research Centre, Ersta Sköndal Bräcke University College, Stockholm, Sweden, ${ }^{3}$ Institute for Palliative Care, Lund University and Region Skåne, Lund, Sweden, ${ }^{4}$ Department of Nursing and Integrated Health Sciences, Kristianstad University, Kristanstad, Sweden

Background: The Integrated Palliative care Outcome Scale (IPOS) is intended to provide a multidimensional view on patients' situation. IPOS can thus be used with the purpose of assessing patients' perceptions of their functional status and wellbeing. However, few studies have undertaken the patients' perspective when exploring experiences of using IPOS.

Aim: To explore patients' experiences of using the Integrated Palliative care Outcome Scale during specialized palliative home care.

Method: The study had a qualitative approach and to be included patients should have used IPOS twice in a setting where IPOS was recently implemented. The patients were recruited from three different specialized palliative home care settings. Interviews were performed with 10 patients, 7 men and 3 women with a mean age of 72 years. A majority of the patients were diagnosed with incurable cancer. Data were analyzed using interpretive description as it is described by Sally Thorne.

Result: The patients experienced that using IPOS enabled opportunities for them to reflect upon their wellbeing and life situation. Doing so with a nurse present was enriching as this could provide new perspectives. They also expressed that IPOS facilitated discussions between them and the nurse about matters of importance. The patients believed that the use of IPOS entailed secure care as it facilitated for nurses to make correct assessments of patient care needs. Thus, IPOS helped to plan the care according to their specific needs. This made them feel confident that the care provided was adapted to them, giving a sense of security. They also believed that using IPOS secured that information was passed on correctly from the patients to the health care professionals by eliminating misinterpretations.

Conclusion: The result indicates that the patients in the study experienced that using IPOS was beneficial to them. It can be concluded that IPOS with advantage could be used in specialized palliative home care.

Abstract number: P02-190

Abstract type: Poster
The "Liverpool Care Pathway" (LCP) in a German Oncology Hospital Setting: A Qualitative Study of Doctor and Nurse Perceptions

Rother M.

University of Applied Sciences Munich, Munich, Germany Presenting author email address: mar.rother@gmail.com

Background: The dignified care and support of dying patients in the last days of life are the concerns of palliative medicine and care. Concepts and guidelines can be used for support in the delivery of that care. The Liverpool Care Pathway (LCP) is such an instrument. However, there are hardly any related empirical surveys in Germanspeaking countries.

Aim: The aim of the study is to find out how the staff of an oncological ward stands in relation to LCP guidelines, how helpful it is in the day-today running of the station, and what could be improved. The research question is: What attitudes do nurses and doctors of an oncological ward have toward the LCP treatment path and which optimisation options or opportunities for improvement do they see?

Method: A qualitative study design using a partially standardised interview guideline with 10 staff members was chosen. The interviews were recorded on tape, transcribed, and evaluated using the Mayring approach of qualitative content analysis.

Results: The evaluation of the LCP of seven nurses and three doctors with an average work experience of six years and an average age of 30 years is heterogeneous. The overall rating is 5.6 on a scale of 0 (not helpful at all) to 10 (very helpful). The training was mostly positive. The LCP can provide guidance and safety for participants with little working experience. Some weak points, however, could be found, such as in teamwork and documentation. In addition, some allegations by nurses to the doctors are mentioned, such as reluctant behaviour when deciding to apply the LCP. Some participants did not deem it to be necessary to use the LCP at all.

Conclusion: When used, the LCP needs revision and updating. This can be achieved with the help of a working group. Teamwork also needs to be improved.

Keywords: terminal care; palliative medicine; palliative care; Liverpool Care Pathway; oncology; staff perceptions.

Abstract number: P02-191

Abstract type: Poster

PSICPAL PROGRAM: Recommendations for the Psychosocialspiritual Care Assessment of Persons with Advanced Chronic Conditions and Life-limited Prognosis

Mateo-Ortega D. 1,2,3, Gomez-Batiste X.1,2, Limonero J.-T.4, Maté-Méndez

J.4,5, Beas E. ${ }^{1}$, González-Barboteo J. ${ }^{6}$, Barbero E. ${ }^{7}$, Ela S. ${ }^{1}$, Research

Group on Stress and Health

${ }^{1}$ Q Qualy' Observatory, WHO Collaborating Centre for Public Health Palliative Care Programs, Institut Català D'Oncologia, L'Hospitalet, Spain, ${ }^{2}$ Palliative Care, University of Vic, Central University of Catalonia, Vic, Spain, ${ }^{3}$ Palliative Care Service, Consorci Sanitari de Terrassa, Terrassa, Spain, ${ }^{4}$ Research Group on Stress and Health, Faculty of Psychology, Autonomous University Barcelona, Bellaterra, Spain, ${ }^{5}$ Psychooncology Unit, Institut Català D'Oncologia, L'Hospitalet, Spain, ${ }^{6}$ Palliative Care Unit, Institut Català D'Oncologia, L'Hospitalet, Spain, ${ }^{7}$ Social Work Unit, Institut Català D'Oncologia, L'Hospitalet, Spain

Aim: The PSICPAL Program seeks the promotion of quality psychosocial and spiritual care for persons with chronic and/or advanced illness and their families as its main goal.

Design: The PSICPAL comprises a wide range of activities, including the design and validation of tools to assess needs, training activities, research activities (descriptive, therapeutic), and the design, implementation and evaluation of programs of psychosocial and/or spiritual care. It addresses the following aspects: 
- To determine the prevalence of psychosocial needs in health, socio-health and social services and screening purposes

- To identify persons with psychosocial and/or spiritual needs

- To obtain detailed description of psychosocial and/or spiritual needs

- To monitor patients' needs and the intervention performed by professionals

- To refer patients for an specialized approach, if necessary

Results: 3 specific tools published in literature, which were designed and validated for this specific type of population:

1. The questionnaire for the Detection of Emotional Distress (DME), for the screening and identification of psychosocial needs.

2. The questionnaire for the Detection of Emotional Distress of main caregivers (DME-C), for the screening and identification of psychosocial needs of caregivers.

3. The Psychosocial and Spiritual Needs Evaluation scale (ENP-E), to assess and systematically monitor the psychosocial needs.

Specific training program: 30 ETCS postgraduate training, specific workshops.

5 specific publications in scientific literature and 2 specific textbooks.

Conclusion: Addressing the emotional, spiritual and social needs of chronic or end-of-life (EOL) patients allows comprehensive, specific, individualized and effective palliative care for both patients and their loving ones. The routine use of the PSICPAL would allow clinicians to monitor such needs over time and improve effective PC approach.

Abstract number: P02-192

Abstract type: Poster

The Initial Validation of a Patient Orientated Compassion Measure: Exploratory Factor Analysis

Sinclair S. ${ }^{1}$, Hack T. ${ }^{2}$, Maclnnis C. ${ }^{1}$, McClement S. ${ }^{2}$, Russell L. ${ }^{3}$, Cuthbertson L. ${ }^{4}$, Jaggi P. ${ }^{1}$, Boss H. ${ }^{1}$, COMPASS Research Team ${ }^{1}$ University of Calgary, Calgary, Canada, ${ }^{2}$ University of Manitoba, Winnipeg, Canada, ${ }^{3}$ University of Victoria, Victoria, Canada, ${ }^{4}$ Providence Health, Vancouver, Canada

Background: The COMPASS study is a 4 year, multi-centred study to develop a patient reported compassion measure for patients living with an incurable, life-limiting illness. To inform the current work we validated the transferability of the patient compassion model in non-cancer populations, established the content validity of the initial item pool with Subject Matter Experts and Patient Advisors and conducted cognitive interviews with patients.

Aims: We aim to report the initial construct validity and reliability of the current 54-item compassion measure. This will inform the refinement and administration of the final measure for the confirmatory factor analysis stage.

Methods (design, data collection, analysis): A diverse group of 303 patients with life-limiting illnesses were administered the compassion measure. A subsample of 65 of these patients were re-administered the measure within a 24 period to assess test-retest reliability. An exploratory factor analysis was conducted to assess whether underlying latent factors exist within the data. A principal axis factor extraction method with oblique rotation was used to assess the factor structure of the data and refine the measure.

Results: Participants were from 4 care settings (acute [n $=105]$, residential $[n=129]$, hospice [ $n=55]$, homecare [ $n=13]$, undisclosed $[n=1]$ ) and ranged from 22 to 103 years of age. Scale items demonstrating relatively lower test-retest reliability were dropped prior to factor analysis. The principal axis factor extraction method employed in conjunction with parallel analysis revealed a single factor of compassion explaining a large proportion of variance. The scale was further refined based on factor loadings and inter-item correlations. The initial scale demonstrated strong internal reliability.

Conclusion/ discussion: The revised compassion measure will undergo further testing utilizing Confirmatory Factor Analysis. Convergent and Divergent validity will also be assessed.

Abstract number: P02-193

Abstract type: Poster

\section{What Matters to You?}

Irvine J. ${ }^{1}$, Spiller J. ${ }^{2}$, Finucane A. ${ }^{2}$

${ }^{1}$ University of Edinburgh, Edinburgh, United Kingdom, ${ }^{2}$ Marie Curie Hospice Edinburgh, Edinburgh, United Kingdom

Background: The "What matters to you?" question is designed to enable patient care to be increasingly aligned with each patient's personal values. The point of departure is that values-clarification plays an important role in palliative care for clinical staff and their patients' journeys, hence the need to assimilate and execute its devices effectively.

Aims: Explore staff perspectives on the role of understanding patient values and their interaction with clinical practice in a palliative care setting.

Methods: The current study was a service evaluation investigating the practical application of "What matters to you?" as a proxy question for values identification, using a retrospective case-notes review and focus group discussion with 12 clinical staff. Data was analysed using a qualitative method, Thematic Analysis.

Results: Doctors recorded "What matters to you?" information most frequently $(52 \%)$, nurses second (36\%). Focus group results indicated widespread understanding of the importance of values to staff personally and person-centred care but revealed varying approaches to electronic documentation. Multidisciplinary team meeting electronic records were found to be less useful than intended. Quality of evidence regarding community documentation of "What matters to you?" information being transitioned to the inpatient setting was, overall, positive. Casenotes analysis gave rise to patient values core themes including family, being at home and general health.

Discussion: This evaluation found extensive agreement regarding the positive impact of using the "What matters to you?" question to elicit patient values and its use in enhancing person-centred care efforts in this aspect of the patient journey. Implications for hospice practice will be discussed, including clarification of electronic documentation practices, increased healthcare assistant access to electronic records and regular updating of the nursing handover sheets.

This study received no funding.

Abstract number: P02-194

Abstract type: Poster

Measuring Quality of Life in Life-threatening Illness: Content Validity and Response Processes of MQOL-E and QOLLTI-F in Swedish Patients and Family Carers/Caregivers

Axelsson L. ${ }^{1}$, Alvariza A. ${ }^{2}$, Carlsson N. ${ }^{3}$, Cohen S.R. ${ }^{4}$, Sawatzky R. ${ }^{5}$, Årestedt $\mathrm{K}^{3}{ }^{3}$

${ }^{1}$ Department of Nursing Science, Sophiahemmet University, Stockholm, Sweden, ${ }^{2}$ Department of Health Care Sciences Palliative Research Centre, Ersta Sköndal Bräcke University College, Stockholm, Sweden,

${ }^{3}$ Faculty of Health and Life Sciences, Linnaeus University, Kalmar, Sweden, ${ }^{4}$ Departments of Oncology and Medicine, McGill University, Montreal, Canada, ${ }^{5}$ School of Nursing, Trinity Western University, Langley, Canada

Background: The McGill Quality of Life Questionnaire - Expanded (MQOL-E) and the Quality of Life in Life-Threatening Illness-Family 
Carer/Caregiver version (QOLLTI-F) are developed for possible use with patients, irrespective of diagnosis, facing the end of life and their family carers. MQOL-E expands MQOL-Revised by adding the domains of Environment, Cognition, (Feeling like a) Burden, and Health Care. Contemporary measurement validity theory emphasizes the importance of examining response processes while completing translated measurement instruments i.e. how people interpret and respond to questions in each new context.

Aims: To examine content validity and response processes during completion of MQOL-E and QOLLTI-F among Swedish patients with lifethreatening illness and family carers.

Methods: The study was conducted in two stages:

(I) translation

(II) cognitive interviews with 15 patients and 9 family carers.

Patients had life-threatening illness such as advanced heart failure, advanced chronic obstructive pulmonary disease, end-stage kidney disease or advanced cancer.

Results: MQOL-E and QOLLTI-F corresponded respectively to relevant and important areas for patients' and family carers' quality of life. The instruments were easy to understand although some items needed more reflection. Some changes were made to resolve issues of translation. Responding was an opportunity to express feelings and participants expressed that reflecting over their situation while answering questions was valuable and meaningful to them.

Conclusion: The study contributes important validity evidence about response processes and results show that the instruments are valid and useful for the intended purpose at end of life. MQOL and QOLLTI-F may support conversations on matters of importance for quality of life between patients and/or family carers and health care professionals.

The study was funded by the Medical Research Council of Southeast Sweden.

Abstract number: P02-195

Abstract type: Poster

Exploring Supportive Care Needs and Quality of Life of Ambulatory Greek Advanced Breast Cancer Patients Undergoing Chemotherapy

Psychogyiou A. ${ }^{1,2}$, Katsaragakis S. ${ }^{2}$, Lemonidou C. ${ }^{2}$, Mantziou V. ${ }^{2}$, Kaklamanos J.2. Patiraki E. ${ }^{2,3}$

${ }^{1}$ Oncology, General Oncology Hospital of Athens St Savvas, Athens, Greece, ${ }^{2}$ Nursing, National and Kapodistrian University of Athens, Athens, Greece, ${ }^{3}$ Palliative Care Unit Galilee, Spata, Greece

Background: Given the increasing length of Advanced Breast Cancer $(A B C)$ patients' survival, enhancing quality of life and reducing supportive care needs is crucial for quality cancer care. This study aims to investigate the association of supportive care needs (SCN) with quality of life (QOF) of Greek ambulatory $A B C$ female patients undergoing chemotherapy.

Methods: A convenient sample of 132 ambulatory $A B C$ female patients receiving chemotherapy (response rate $97 \%$ ) consented to participate. From July 2017 to April 2018, they completed the Greek translated Short-Form Supportive Care Needs Survey Questionnaire (SCNS-SF) and the European Organization in Research and Treatment of Cancer (EORTC QLQ C30 and Breast Module BR23). The SCNS-SF34 is a 34 items 5 point Likert type scale divided into 5 domains (Cronbach's a: .745 - .934). The statistical significance level was set at 0.05 .

Results: The patients' median age was 61.5 years. Most of them had a partner $(60.6 \%)$ and children $(84.1 \%)$. The most frequently unmet patients' needs were found in the Psychological (P) (59.5 \pm 30.1$)$, followed by the Physical and Daily Living (PDL) $(57.6 \pm 29.4)$ and Health System and Information domain (HSI) $(49.5 \pm 24.8)$. Patients reported the least unmet supportive care needs in the Sexuality domain $(10.9 \pm 26.2)$. Worse Global and most of the Functional and Symptoms
QOF subscales outcomes were statistically significant correlated with unmet $\mathrm{P}$ ( $\mathrm{p}<.0001$ to .027$)$, PDL ( $p<.0001$ to .013$), \mathrm{HSI}$ ( $p<.0001$ to $.030)$ and Patient Care \& Support domain $(p<.0001$ to .030$)$. The EORTC QLQ C30 Fatigue subscale was statistically significant correlated with all SCN domains ( $p<.0001$ to .003) except Sexuality.

Conclusion/Discussion: Ambulatory patients with $A B C$ undergoing chemotherapy had many unmet supportive care needs strongly correlated with low quality of life. Health care providers should routinely monitor the quality of life and supportive care needs of these patients to ensure timely and effective cancer care.

Abstract number: P02-196

Abstract type: Poster

Reasons for Requesting Urgent Care in Palliative Patients Martin-Marco A., Taberner-Bonastre M.T., Lafuente-Sanchis P., GirbesMari R., Tarraso-Gomez L., Clep C., Flor-Bustos L., Duart-Clemente C., Torrijos-Pastor A.

Hospital Universitario de La Ribera, Alzira, Spain

Introduction: We wanted to know the reasons why palliative patients request urgent attention.

Material and method: We carried out a descriptive longitudinal study in which all patients previously labeled as palliative and who requested medical attention in the emergency department.

We collected the patients treated from January 1 to September 31, 2017.

Results: During the nine months in which data were collected, 693 episodes were found, corresponding to 241 patients attended.

$57.1 \%$ of episodes were from cancer patients, which means 396 episodes, compared to 297 episodes in which non-oncological patients were treated $(42.9 \%)$.

Regarding the symptoms presented by patients who come to the emergency room, the most frequent are respiratory, which overall represent $22.8 \%$ (158 episodes), with dyspnea being the most frequent with $20.49 \%$ ( 142 episodes) followed very far by the cough with $1.73 \%$ (12 episodes).

After the respiratory symptoms were general in $17.7 \%$ (123 episodes) and pain in $15.6 \%$ (108 episodes) the most frequents. Among generals, the deterioration of the general state, the feeling of dizziness, falls and agitation are more frequent. Regarding pain, the most frequent location has been at the abdominal level.

A contingency analysis was performed using the chi-square test, finding statistically significant differences with $p<0.001$ between the frequency of presentation of symptoms in the group of cancer patients versus non-cancer patients; the most frequent symptoms being dyspnea in the group of non-oncological patients and pain in oncological patients.

Discussion: We determined the most frequent causes of urgent care requests in palliative patients and the difference of presentation according to whether they are oncological or not.

Abstract number: P02-197

Abstract type: Poster

Estimating Prognosis to Enhance Patient Care - Identification of a Suitable Prognostic Tool for Use in Hospice

Taylor A.G.S., Breen C.M., McCann M.-A., McAuley J.

Antrim Area Hospital, Macmillan Unit, Antrim, United Kingdom

Background: Physicians' prognostic estimates have proven to be more accurate than assessment without physician input. Assessment of prognosis is important to provide patients with a better understanding of their expected survival, enabling informed medical and social decisions. 
Aims: To identify a prognostic tool suitable for palliative patients admitted to hospice that is easy to use, accurate and appropriate.

Methods: Three validated prognostic tools for patients with advanced cancer were identified: Palliative Prognostic Index (PPI), a prognostic nomogram and Prognosis in Palliative care Study (PiPS) Prognosticator. Data was collected prospectively for patients admitted to hospice for 3 months. Physicians completed a survey focusing on ease of use and appropriateness of the tools. Descriptive statistics were generated using Microsoft Excel.

Results: 30 patients were admitted to hospice. Prognostic tools were completed in 20 as 10 were accurately felt to be in the last few days of life and did not have bloods performed. 2 remain alive.

The PPI was felt to be the easiest tool to use. There were no data omissions and no additional blood tests were required. Survival was closest to predicted median in $56 \%$.

The nomogram required additional blood tests and the tool was felt to be complex and confusing. It was omitted for 4 patients due to incomplete data. The prognostic ranges were appropriate but accuracy was low at $28 \%$.

The PiPS-B (with bloods) was simple to complete, but required additional assessments and investigations. The predicted survival range was correct for $61 \%$.

Conclusion: The PPI was felt to be the easiest tool to use. It was successful at predicting median survival in $56 \%$. Additional assessments and investigations for the nomogram and PiPS-B were drawbacks. The PPI was identified as our preferred prognostic tool for use in our hospice to assist with the difficult conversations and decisions around prognostication. This may change as better tools become available.

Abstract number: P02-198

Abstract type: Poster

\section{The Usefulness of Integrated Palliative Care Outcome Scale} (IPOS) for the Evaluation of Terminal Cancer Patients Nakajima N.

University of the Ryukyus, Division of Community-based Medicine and Primary Care, Okinawa, Japan Presenting author email address: nakajy@med.u-ryukyu.ac.jp

Aim: The Japanese version of Support Team Assessment Schedule (STAS-J) was developed in 2004 and has been widely used in our daily practice. The Japanese version of Integrated Palliative care Outcome Scale (IPOS-J) is now under-development. The purpose of this study was to evaluate the usefulness of IPOS (translated to Japanese) for terminal cancer patients in clinical practice.

Methods: This was a prospective observational study. Study subjects were terminal cancer patients who were admitted to our hospital for palliative care for last 2 years.

We performed the initial evaluation in our daily practice by STAS-J and IPOS ( 3 types of evaluations: by patient, by family, and by staff) within a week after admission, and then repeated once a week. We performed

1) the evaluation by STAS-J and IPOS (by staff) in order to compare the score in similar items, and

2) that of IPOS (3 items) in order to grasp the difference between each item.

Then we investigated the modification of patient's care and the change of the evaluation by patient.

Results: We performed these evaluations for 62 cases. There were no case that the score of the similar item became estranged more than 1 point between STAS-J and IPOS (by staff). The number of the cases which the score by patient was higher more than 1 point compared with "by staff" in the following items: pain, other symptoms, anxiety, depressed feeling, anxiety of family, understanding by family, sufficient information, were 2, 4, 5, 4, 4, 6, 4 cases, respectively (overlapped). Based on these results, care plans were modified in $70 \%$ of the cases, and IPOS score (by patient) on the next evaluation were improved in more than half of cases.

Conclusion: Concerning the evaluation by staff, similar results will be acquired by IPOS and by STAS-J. We will be able to grasp the difference of the evaluation between 3 items by using IPOS, and this will lead the improvement of the quality of palliative care.

Abstract number: P02-199

Abstract type: Poster

Evaluation of the Use of the Liverpool Care Pathway in Dutch Nursing Homes

Klapwijk M.S., Lemos Dekker N., Achterberg W.P., van der Steen J.T. PHEG, Leiden University Medical Center, Leiden, Netherlands

Background: The Liverpool Care Pathway (LCP), a multidisciplinary tool for the dying phase, is used in several countries and settings. The literature indicates that there are both positive and negative experiences regarding its use.

Methods: We draw on three studies to examine use and experiences with the LCP in nursing homes in the Netherlands.

(1) A 4-month ethnographic study (2015) was conducted in a nursing home with 11 psychogeriatric units to assess experiences with the LCP in dementia care.

(2) The SigMa action research (data collection 2018-2018) with nursing staff on usefulness of tools including the LCP is being conducted in 10 nursing homes.

(3) Inspired by this work, we developed an online survey with 9 questions regarding use and experiences with the LCP. We will send the survey to all physicians of 33 care organisations with nursing homes in three regions in the north, west and south of the Netherlands to examine any differences in use and experiences between care organisations and between different wards (dementia or other). We will interview nursing home staff with particularly positive experiences and nursing home staff with very negative experiences.

Results: The ethnographic work showed that staff appreciated the LCP as a tool for communication but complained about duplication of work. Further, there was a mismatch in the care for people with dementia. The action research showed that the tool is often available but not used due to its complexity. We will present additional figures regarding use and any differences between dementia and other wards from the third study, the online survey.

Conclusion: The Liverpool Care pathway is still used in Dutch nursing homes (despite negative publicity, especially in the UK) and is perceived as a helpful tool for communication and a reminder of care goals. However, in its current form, it is ill adapted for the nursing home environment and for use with people with dementia.

Abstract number: P02-200

Abstract type: Poster

A German Version of the Palliative Performance Scale as an Instrument for Survival Estimates

Mosich V. ${ }^{1,2}$, Andersag M. ${ }^{3}$, Watzke H. ${ }^{4}$

${ }^{1}$ Palliative Consulting Team, Hospital of Saint John of God, Vienna, Austria, ${ }^{2} \mathrm{CS}$ Hospiz Rennweg, Caritas Socialis GmbH, Vienna, Austria, ${ }^{3}$ Medizinische Universität Wien, Vienna, Austria, ${ }^{4}$ Klinische Abteilung für Palliativmedizin, Medizinische Universität Wien, Vienna, Austria 
Background: The original English version of the palliative performance scale (PPS) has been used for two decades to describe the functional status of palliative patients. Based on clinical parameters PPS helps to estimate the survival time of patients with life-threatening illnesses: the higher the functional status the longer the survival. This is interesting for patients, their family caregivers and health care professionals in order to plan for care. Various German versions of the PPS are availible but so far for none has been shown if it can differentiate the survival of palliative patients in a validated way.

Aims: This study was performed to find out if a German version of the PPS can help to predict the survival of palliative patients.

Methods: The performance state of 771 consecutive patients of a palliative consulting team in an Austrian tertiary care hospital was evaluated using a German version of the PPS. The survivaltime of 377 patients was unknow, so performance status and lifetime of 394 patients were analyzed retrospectively. Kaplan-Meier-curves were drawn and tested for differences using log-rank-test.

Results: Average PPS of the patients of a palliative consulting team who thereafter died in the same hospital was $38,1 \%$, average PPS of patients lost for follow up was $48,9 \%$. Differences in time of survival could clearly be shown for all included categories of PPS. Kaplan-Maier-curves showed significant differences in the log-rank-test: $p<0,001$.

For example $66 \%$ patients of the PPS category $=10 \%$ survived longer than 1 day, $24 \%>2$ days and $4 \%>30$ days. In contrast, $93 \%$ of patients of the category PPS $=40 \%$ survived more than 1 day, $69 \%>2$ days and $21 \%>$ 30 days. Patients of the category PPS=70\% lived even longer: $100 \%>1$ day, $91 \%>7$ days and $74 \%>30$ days.

Conclusion: This German version of the PPS is a useful and valid instrument to estimate survival time of hospitalized palliative patients using just clinical information.

Abstract number: P02-201

Abstract type: Poster

Dry Mouth - A Missing Symptom in Multiple Assessment Tools? Monsen R. ${ }^{1}$, Herlofson B.B. ${ }^{2,3}$, Gay C. ${ }^{1}$, Dahl M. ${ }^{1}$, Saghaug E. ${ }^{1}$, Slaaen J. ${ }^{1}$, Lerdal A. ${ }^{1,4}$

${ }^{1}$ Lovisenberg Diaconal Hospital, Oslo, Norway, ${ }^{2}$ Department of Oral Surgery and Oral Medicine, Faculty of Dentistry, University of Oslo, Oslo, Norway, ${ }^{3}$ Department of Maxillofacial Surgery and Hospital Odontology, Cancer Clinic, Oslo University Hospital, Oslo, Norway, ${ }^{4}$ Institute of Nursing Science, Department of Health and Society, Faculty of Medicine, University of Oslo, Oslo, Norway

Background: Tools for assessing multiple symptoms with minimal burden are critical to effective symptom management for patients in palliative care. The Edmonton Symptom Assessment System (ESAS) is such a tool and an integral part of palliative care in Norway. Our first translated version added three symptoms, dry mouth, constipation and pain on movement. With this ESAS, dry mouth was identified as one of the most prevalent symptoms. In 2010, the revised ESAS-r was published and recommended to replace the Norwegian version, without assessing dry mouth, and the category "other problem" (OP) was introduced and exemplified by constipation.

Aims: This study explores to what extent the ESAS-r OP category might be assessing dry mouth by comparing the number of patients reporting dry mouth in the original ESAS and as an OP on the ESAS-r.

Methods: This study was a retrospective study at an inpatient hospice unit. Demographics and the first ESAS (items scored 0-10) were obtained from medical records.

Results: In total 376 patients were registered; 184 in 2013 (59\% female, median age 65) and 192 in 2016 (57\% female, median age 66). In 2013, dry mouth was the third highest rated symptom (mean 4.3, SD 3.1) after tiredness and appetite, and $83 \%$ rated $\geqslant 1$. Seventy-three $\%$ reported constipation rated $\geqslant 1$ (mean 3.5, SD 3.1). In 2016, 19\% reported an unspecified OP (mean 5.2, SD 2.9), 17\% reported constipation (mean 4.8, SD 2.7 ) and only $7(<4 \%$ ) reported dry mouth (mean 5.7, SD 3.0).

Conclusion: ESAS is an important clinical tool and it is therefore crucial that the most common and severe symptoms are included. The OP category may have introduced bias, as constipation now seems to be reported at the expense of other prevalent symptoms, such as dry mouth. Thus, the core elements may need to be reviewed in future ESAS versions to ensure that patients' most severe symptoms are being adequately assessed so that they can be optimally treated.

Abstract number: P02-202

Abstract type: Poster

Measuring the Burden on Fa3mily Caregivers of People with Cancer: Cross-cultural Translation and Psychometric Testing of the Caregiver Reaction Assessment Indonesian Version

Kristanti M.S. ${ }^{1,2}$, Effendy C. ${ }^{2}$, Utarini A. ${ }^{2}$, Vernooij-Dassen M. ${ }^{1}$, Engels $Y^{3}{ }^{3}$

${ }^{1}$ QQ Healthcare, Radboud University Medical Center, Nijmegen, Netherlands, ${ }^{2}$ Universitas Gadjah Mada, Yogyakarta, Indonesia, ${ }^{3}$ Pain and Palliative Medicine, Radboud University Medical Center, Nijmegen, Netherlands

Background: The Caregiver Reaction Assessment (CRA) is considered one of the best instruments for measuring the multidimensional burden of family caregivers. To date, there is no available validated instrument to assist health care professional in measuring caregivers' burden in Indonesia.

Aim: The aim of this study was to translate the CRA from English into Indonesian (CRA-Indo), and to conduct psychometric testing of the CRAIndo with family caregivers of people with cancer.

Methods: A cross-cultural translation and psychometric testing were conducted. Construct validity was determined through exploratory factor analysis to measure the internal structure. Cronbach's alpha was used to measure the internal consistency of the instrument.

Results: A total of 451 respondents were involved in the study. Of those, 40 were involved in the feasibility testing. Five factors from the original instrument were confirmed with an explained variance of $52 \%$. Almost all items in the CRA-Indo appeared to have a similar structure to the original version. The internal consistency ranged between .63 and .75 . Conclusions: The CRA-Indo has the same structure with the original one. It appeared to be feasible, valid and reliable for measuring the burden of family caregivers of patients with cancer in Indonesia. The CRA-Indo is a valid tool to measure family caregivers' burden. The availability of the CRA in several languages also enhances the opportunity to conduct international comparative studies on family caregiver burden.

Abstract number: $\mathrm{P} 02-203$

Abstract type: Poster

Comparison between Patient-reported and Clinician-reported Outcome: Validation of the Integrated Palliative Care Outcome Scale-Staff Version

Sakurai H. ${ }^{1,2}$, Miyashita M. ${ }^{3}$, Morita T.4, Imai K.4, Miyamoto S. ${ }^{5}$, Otani

H. ${ }^{6}$, Oishi A. ${ }^{7}$, Kizawa Y. $^{8}$, Matsushima . $^{2}$

${ }^{1}$ Palliative Therapy, Cancer Institute Hospital of JFCR, Tokyo, Japan,

${ }^{2}$ Tokyo Medical and Dental University, Tokyo, Japan, ${ }^{3}$ Tohoku University Graduate School of Medicine, Health Sciences, Sendai, Japan, ${ }^{4}$ Seirei Mikatahara General Hospital, Hamamatsu, Japan, 5Japanese Red Cross Medical Center, Tokyo, Japan, ${ }^{6}$ National Kyushu Cancer Center, Fukuoka, Japan, 7University of Edinburgh, Edinburgh, United Kingdom, ${ }^{8}$ Kobe University, Kobe, Japan

Background: The Integrated Palliative care Outcome Scale (IPOS) is a streamlined outcome measure newly developed to comprehensively 
evaluate physical symptoms, emotions, psychological state, spiritual needs, medical information, and availability of various aids during the preceding three days. There are patient and staff versions of IPOS. The patient version is used as a patient-reported outcome, while the staff version, a clinician-reported outcome, is used to evaluate severely diseased patients who cannot respond on their own.

Aims: The goal of this study is to assess the reliability and validity of IPOS-staff version in adult Japanese cancer patients.

Methods: This is a multicenter, cross-sectional observational study. We assessed the missing values, prevalence, criterion validity, intra-rater and inter-rater reliability. Patients provided responses to IPOS-patient version, two medical staff provided responses to IPOS-staff version.

Each participating site commenced the study following approval by its ethical review board. All participants gave written consent.

Results: One hundred forty-two patients and their staff were enrolled at six palliative care facilities. Missing values accounted for $0 \%$ in most of items to $3.5 \%$ in family anxiety item and share feelings item. The prevalence of symptoms recognized by staff was $12.0-91.2 \%$. The range of correlation coefficients with IPOS-patient version as a gold standard was 0.099-0.670. The intra-class correlation coefficient ranged from 0.0330.769 (absolute values) in inter-rater reliability, and 0.721-0.954 in intrarater reliability.

Conclusion/discussion: The criterion validity and inter-rater reliability are low in family related items. The scale is useful in assessing physical, psychological, social, and spiritual symptoms and in measuring outcomes of adult cancer patients by defining the context of use, the objectives of use, and the interpretation.

Abstract number: P02-204

Abstract type: Poster

\section{Inter-rater Reliability of the Phase of Illness Tool in Paediatric} Palliative Care

Burke K. ${ }^{1}$, Coombes L. ${ }^{1}$, Petruckevitch A. ${ }^{1}$, Anderson A.-K. ${ }^{1,2}$

${ }^{1}$ The Royal Marseden NHS Foundation Trust, Children and Young Persons Unit, London, United Kingdom, 2Shooting Star Chase Children's Hospice, Guildford, United Kingdom

Background: The 'Phase of Illness' (POI) tool describes a patient's condition and family circumstances using the five categories stable, unstable, deteriorating, dying and deceased. It can be useful for funding and resource allocation. Additionally it can determine the suitability of a patient's care plan in meeting their needs with 'time in the unstable phase' used as an outcome measure to assess the performance of palliative care services. Substantial inter-rater reliability (IRR) (kappa=0.67, with 2 raters) and moderate IRR (kappa=0.52, with 2-7 raters) has been shown when professionals use the tool to categorise adults; however there is a lack of reliability studies within paediatric palliative care (PPC). The aim of this study was to explore the IRR when assigning paediatric patients to POI.

Methods: Prospective data was collected from Jun-Nov 2017. A specialist palliative care team in England independently categorised all PPC patients under their care into a phase of illness during weekly multidisciplinary meetings.

Results: Nine palliative care professionals (one doctor and eight nurses) undertook 981 joint assessments of 80 PPC patients (mean age 8.9 years; 42 males and 38 females). The most common diagnoses were malignancy (47.5\%), neurological (22.5\%) and congenital (15\%). Kappa values were: overall $=0.51$, stable $=0.63$, unstable $=0.26$, deteriorating=0.46 and dying=0.39.

Conclusion: The overall 'moderate' level of agreement is similar to adult studies which use more than two raters, suggesting the POI tool may have a role in a PPC population for funding and resource allocation. However, agreement for the unstable phase was low and further research is needed to explore the reasons behind the uncertainty in this category before it is applied as an outcome for service delivery in PPC.
Children with palliative care needs are distinct from their adult counterparts and evidence is needed to ensure the tools we use with this unique population are appropriate.

Abstract number: P02-205

Abstract type: Poster

Validation of the SF-8 Scale in Argentinian Palliative Care Professionals: Assessing Perceived Health with Competitive Models and its Relationship with Professional Quality of Life Galiana L. ${ }^{1}$, Oliver A. ${ }^{1}$, De Simone G. ${ }^{2}$, Linzitto J.P. ${ }^{2}$, Grance G. ${ }^{2}$, Sansó $N^{3}$

${ }^{1}$ Department of Methodology for the Behavioral Sciences, University of Valencia, Valencia, Spain, ${ }^{2}$ Pallium Institute Latinoamerica, Buenos Aires, Argentina, ${ }^{3}$ Department of Nursing and Physiotherapy, University of the Balearic Islands, Palma de Mallorca, Spain

Background: Although the profound progress that have taken place during the 20th and 21st centuries in health professions, human side is still key, and the professional the most used tool. Because of that, it is of paramount importance to maintain professionals' levels of well-being and health. Several factors have shown to affect their health, including burnout (BO), compassion fatigue (CF) and compassion satisfaction (CS). Aims: The aim is twofold:

1) to offer evidence on the psychometric properties of the SF-8, an instrument design to assess perceived health; and

2) to study the relation between $\mathrm{BO}, \mathrm{CF}$ and $\mathrm{CS}$ and palliative care (PC)professionals' health.

Methods: 273 Argentinian PC professionals were surveyed with the support of Instituto Pallium Latinoamérica and Asociación Argentina de Medicina y Cuidados Paliativos. $80.8 \%$ of participants were women. Mean age was 43.41 (SD=9.69). Analyses included a set of four structural equation competitive models to test the factorial structure of the SF-8, all based in previous literature results. With the best fitting structure, a multiple indicators multiple causes (MIMIC) model was estimated, to study the relations between BO, CF and CS and professionals' health. Results: The best fitting model was the one with two dimensions of health, physical (items 1-5) and psychological (items 6-8): $\chi^{2}(19)=74.180$ $(p<.001), \mathrm{CFI}=.968, \mathrm{TLI}=.953, \mathrm{RMSEA}=.110[.084-.137], \mathrm{SRMR}=.047$. All factor loadings were statistically significant. The MIMIC model fit was also adequate $\left(\chi^{2}(37)=135.839(p<.001), \quad C F I=.946, \quad T L I=.919\right.$, RMSEA $=.106[.087-.125]$, SRMR=.055), with BO, CF, and CS explaining $25.6 \%$ of physical and $44 \%$ of mental health.

Conclusion: Evidence of this work posits the SF-8 as an appropriate instrument to measure PC professionals' health in two factors, physical and mental health, being the latest one specially affected by their levels of BO, FC and SC. These dimensions are needed to be cared for maintaining healthy professionals.

Abstract number: P02-206

Abstract type: Poster

Specific Tool to Identify and Assess Psychosocial and Spiritual Needs in End-of-Life Patients

Mateo-Ortega D. 1,2,3, Limonero J.T.4 , Maté-Méndez J. 4,5, Beas E. ${ }^{6}$, González-Barboteo J.7, Barbero E. ${ }^{8}$, Ela S. ${ }^{9}$, Buisan M. ${ }^{10}$, Gómez-Batiste $X^{2,6}$, Research Group on Stress and Health. Faculty of Psychology. Autonomous University of Barcelona, Bellatera (Spain)

'The 'Qualy' Observatory. WHO Collaborating Centre for Public Health Palliative Care Programmes, Institut Català D'oncologia, L'Hospitalet, Spain, ${ }^{2}$ Chair of Palliative Care, University of Vic- Central University of Catalonia, Vic, Spain, ${ }^{3}$ Palliative Care Service, Consorci Sanitari de Terrassa, Terrassa, Spain, ${ }^{4}$ Research Group on Stress and Health. Faculty 
of Psychology, Universitat Autònoma de Barcelona, Bellaterra, Spain, ${ }^{5}$ Psychoncology Unit, Institut Català D'oncologia, L'Hospitalet, Spain, ${ }^{6}$ 'Qualy' Observatory. WHO Collaborating Centre for Public Health Palliative Care Programs, Institut Català D'oncologia, L'Hospitalet, Spain, 7Palliative Care Unit, Institut Català D'oncologia, L'Hospitalet, Spain, ${ }^{8}$ Social Work Unit, Institut Català D'oncologia, L'Hospitalet, Spain, ${ }^{9} T$ The "Qualy" Observatory. WHO Collaborating Centre for Public Health Palliative Care Programs, Institut Català D'oncologia, L'Hospitalet, Spain, ${ }^{10}$ Poverty and Health Programs, Obra Social 'la Caixa', Barcelona, Spain

Background: Most screening instruments to detect emotional distress in cancer patients differentiate between those with and without depression or anxiety. Clinicians are obliged to use instruments that only indirectly assess suffering.

Aims: The main aim it's to develop an instrument to better assess and monitoring psychosocial needs and emotional distress.

Methods: The scale was developed through a seven-stage process:

1) literature review;

2) expert panel establishment;

3) discussion and agreement on the most relevant dimensions of psychosocial care;

4) description of key indicators and consensus-based questions to evaluate such dimensions;

5) assessment of the scale by external palliative care (PC) professionals;

6) evaluation by expert patients;

7) analysis of scale's psychometrics properties.

Results: Only 12 specific questions composed the tool to assess psychosocial and spiritual needs.

On the factorial analysis, four factors (emotional-wellbeing, social support, spiritual, and information) explained $58.4 \%$ of the variance. This scale has a sensitivity of $76.3 \%$, a specificity of $78.9 \%$ and the cut off is 28 .

5 key questions discriminate better than others. Scores $>4$ for any of these questions become possible warning signs of psychosocial-spiritual needs.

1 question for healthcare professionals indicate if they observed 6 external signs of distress in the patient. It allows detect quickly that the patient should undergo a specialized evaluation.

Discussion: The ENP-E was developed including the perspectives of patients and professionals.

The routine use of this scale would allow clinicians to provide a systematic, holistic assessment of psychosocial needs and to monitor them over time.

This would, in turn, permit comprehensive, highly-individualized interventions to improve effective PC approach. 5 key questions allow specific interventions in psychosocial or spiritual suffering.

Abstract number: P02-207

Abstract type: Poster

The Professional Self-care Scale (PSCS) in Spanish and Chilean Palliative Care Professionals: Measurement Invariance and Latent Means

Oliver A. ${ }^{1}$, Galiana L. ${ }^{1}$, Reyes E. ${ }^{1,2}$, Tomás J.M. ${ }^{1}$, Rudilla D. ${ }^{3}$, Sansó $N .{ }^{4}$ ${ }^{1}$ Department of Methodology for the Behavioral Sciences, University of Valencia, Valencia, Spain, ${ }^{2}$ Universidad Autónoma de Chile, Santiago, Chile, ${ }^{3}$ Air Liquide Healthcare - Hospital Universitario La Princesa, Madrid, Spain, ${ }^{4}$ Department of Nursing and Physiotherapy, University of the Balearic Islands, Palma de Mallorca, Spain

Background: Accomplishing self-care activities plays an important role in helping palliative care professionals to cope with daily emotional demands. Holistic practice of self-care is a key aspect for maintaining professional quality of life, being an important preventive measure to protect them from burnout and compassion fatigue. It is important to provide health organizations with adequate indicators and measures to monitor and promote professionals' self-care. This adequacy rests in instruments' validity and reliability, but also in its invariance across populations. Measurement invariance is needed for accurate group comparisons.

Aims: The aim of the study was to offer evidence on the Professional Self-Care Scale (PSCS) invariance across two countries, Spain and Chile, while carrying out latent mean comparisons.

Methods: 385 Spanish and 193 Chilean palliative care professionals answered the online survey $(77.55 \%$ and $84.46 \%$ were respectively women). A measurement invariance routine was developed, which included:

1) configural or baseline model;

2) metric or weak invariance model;

3) scalar or strong invariance model; and

4) model with constrained correlations among factors.

As measurement invariance held, latent means were also constrained and tested for differences.

Results: The PSCS was found to be scalar invariant across groups, retaining the model with constrained factor loadings, items' intercepts and correlations among factors: $c^{2}(93)=326.439(p<.001), C F I=.988, T L I=.991$, RMSEA $=.095[.084-.107]$, SRMR $=.080$. Latent mean differences were found for physical self-care, with statistically significant higher levels in Spain.

Conclusions: The study offers preliminary evidence on the PSCS' measurement invariance across two samples of palliative care professionals from countries with fairly different health-care systems. This scale should be studied in more countries, aiming new evidence for its use in cross-cultural monitoring of professionals' of self-care.

Abstract number: P02-208

Abstract type: Poster

Healthcare Professionals' Experiences of Using IPOS in the Care Encounter

Gruvebäck A. ${ }^{1}$, Törnquist K. ${ }^{1}$, Olsson Möller U. ${ }^{2}$, Rasmussen B. ${ }^{3,4}$, Beck I. $2,3,5$

${ }^{1}$ Department of Nursing Science, Sophiahemmet University, Stockholm, Sweden, ${ }^{2}$ Faculty of Health Science, Department of Nursing and Integrated Health Sciences, Kristianstad University, Kristianstad, Sweden, ${ }^{3}$ Institute for Palliative Care, Lund University and Region Skåne, Lund, Sweden, ${ }^{4}$ Faculty of Medicine, Department for Health Sciences, Lund University, Lund, Sweden, ${ }^{5}$ Faculty of Medicine, Department of Clinical Sciences Lund, Lund University, Lund, Sweden

Background: Integrated Palliative care Outcome Scale (IPOS) is a patient reported outcome measure (PROM) developed to be used for identifying physical, psychological, social and spiritual needs and problems. Although the use of PROMs in the care encounter has been described, little is known about healthcare professionals' experiences of using IPOS in these encounters. In 2017, IPOS was implemented to be used, preferably as a conversation tool, in specialized palliative care units in southern Sweden.

Aim: To describe healthcare professionals' experiences of using IPOS in the care encounter with patients in specialized palliative care.

Method: Through purposive sampling, five focus group interviews were conducted with 18 informants (nurses) in four specialized palliative care units. Data was analysed using qualitative content analysis.

Preliminary Results: The informants experienced the use of IPOS both as helpful but also challenging. Several prerequisites for using IPOS were 
described, including an established relationship with the patient; a habit of using IPOS; and a peaceful and quiet environment. Using IPOS gave an opportunity to understand patients' care needs and to guide interventions. Even if the informants experienced that most patients and their families were satisfied with IPOS in the care encounter, it was a challenge for some to address the psychosocial questions and to handle patients who did not like or couldn't manage questionnaires.

Conclusion: The informants expressed a desire to bring patients' own experiences of their needs and problems to the forefront. They experienced that the use of IPOS could facilitate this, if challenges were addressed. The difficulties when addressing psychosocial questions and facing patients' resistance require training and supervision.

The main source of funding of the study are Mats Paulsson, Foundation for Research,

Innovation and Societal Development, and Kristianstad University.

Abstract number: P02-209

Abstract type: Poster

Trusted Assessor: Initiating a Rapid Discharge Process from an Acute Hospital Setting to a Nursing Home Environment for Palliative Patients with Fast Track Funding

Sheldrick M. ${ }^{1}$, Abbott U. ${ }^{2}$, Wright $\mathrm{H}^{3}{ }^{3}$

${ }^{1}$ Palliative Care, Social Worker, Barking Havering and Redbridge University Hospital Trust, Romford, United Kingdom, ${ }^{2}$ Palliative Care, OT, Barking Havering and Redbridge University Hospital Trust, Romford, United Kingdom, ${ }^{3}$ Palliative Care, Team Leader, Barking Havering and Redbridge University Hospital Trust, Romford, United Kingdom

Background: It was evidenced in the trust that patients with fast track funding agreements were waiting on average 12 days before placement to a nursing home. In March 2017 NHS Improvement introduced The Rapid Improvement Guide for Trusted Assessors. This gave the Palliative Care Team the framework to introduce the Trusted Assessor model of care.

Aim: To ensure that palliative care patients are assessed and transferred in a timely manner once NHS funding is agreed and nursing home identified by the family.

Method: We commenced a programme of visiting the homes to explain the process; following this we completed a series of joint assessments within the hospital environment. This allowed us to build our working relationships to ensure that trust was gained, and allowed The Palliative Care Team insight into the information and knowledge that the home would require to support a successful transfer.

Results: Data for the first 37 patients who participated in The Trusted Assessor Scheme identified that 9/10ths of patients were transferred within 24 hours with the remaining patients within 48 hours.

Conclusion: It has become evident that The Trusted Assessor programme has been very successful. There has been overwhelming positive feedback from patients, families and nursing home professionals. Due to this innovative process and its success, members of the Palliative Care Team have presented this pilot at local and national NHS improvement meetings as well as The Healthy London Partnership. The Trusted Assessor Framework has now been agreed to support patients that are funded by Social Services/CCG as it has shown to be a successful approach to discharge planning.

We are continuing to increase a portfolio of nursing homes that participate in the Trusted Assessor Scheme, whilst striving to maintain patient care as our focus and priority.

Abstract number: P02-210

Abstract type: Poster

Pain Assessment Tool for Children and Young Adults Boucher S. ${ }^{1}$, Downing J.2,3
${ }^{1}$ Communication, International Children's Palliative Care Network (ICPCN), Hillcrest, South Africa, ' Management, International Children's Palliative Care Network (ICPCN), Kampala, Uganda, ${ }^{3}$ Health Sciences, Makerere University, Kampala, Uganda

Background: Effective pain management relies on impeccable assessment. However, research indicates an under assessment of pain in children. Those caring for children with pain may not have convenient access to pain scales \& responses are not always recorded. Children are not always able to remember specific details such as the time \& duration of the pain, its intensity, location, type \& what may have helped to relieve it or make it worse.

Aim: To develop \& introduce a digital tool for assessment \& recording of a child's pain levels.

Method: Working with an App designer to develop a Pain Assessment Tool suitable for use with children from 3.5 years of age to young adulthood. The child adopts their own avatar \& records date, time, position, intensity, type $\&$ characteristics of their pain. They can use the faces, numbers or hand pain scales to record their pain intensity $\&$ can easily access a record of responses which can be used by their team of health professionals. After an extended process of design, testing, redesign \& bug fixing, the app was launched $\&$ can be downloaded free on both Apple \& Android devices.

Results: To date the app has been available for download for 13 months. An online questionnaire for app users has been created \& responses will be reported on at the conference. Initial results demonstrate the utility of the App, with one user reporting "It is a lifesaver. It is quick \& easy to use, has a user-friendly interface \& efficient. I am no longer forgetting any important details of my pain on a day-to-day basis. It comes with me to all my appointments enabling me to get the best treatment possible",

Conclusion: App development is a complex process requiring careful planning \& research. The app is a useful tool for regular \& accurate assessment \& recording of a child's pain levels leading to consistent \& effective pain management. Improvements \& updates to the app will be ongoing.

Funding: App development was funded by Krebsallianz

Abstract number: P02-211

Abstract type: Poster

Development and Validation of the PaP Score Nomogram Scarpi E. ${ }^{1}$, Nanni $0 .{ }^{1}$, Maltoni M. ${ }^{2}$

${ }^{1}$ Unit of Biostatistics and Clinical Trials, Istituto Scientifico Romagnolo per lo Studio e la Cura dei Tumori (IRST) IRCCS, Meldola, Italy,

2Palliative Care Unit, Istituto Scientifico Romagnolo per lo Studio e la Cura dei Tumori (IRST) IRCCS, Meldola, Italy

Background: An existing and validated palliative prognostic (PaP) score predicts survival in terminally-ill cancer patients, assigning patients to 3 different risk groups according to a 30-day survival probability:

group $\mathrm{A},>70 \%$;

group $B, 30 \%-70 \%$; and

group $\mathrm{C},<30 \%$.

Aims: We tested the hypothesis that the prediction of terminally-ill cancer patient survival can be improved if traditional predictors of the PaP score are used within a prognostic nomogram to predict survival at 15 , 30 and 60 days.

Methods: Three cohorts of consecutive terminally-ill cancer patients were used: one $(n=519)$ for nomogram development and internal validation, and a second $(n=451)$ and third $(n=549)$ for external validation. Multivariate Cox proportional hazards regression analyses modeled dypsnea, anorexia, Karnofsky performance status, clinical prediction of survival, total WBC, and lymphocyte percentage. The predictive accuracy of the nomogram was quantified with Harrell's concordance index. Internal 
validation relied on 200 bootstrap re-samples. Calibration plots were generated to explore the performance characteristics of the nomogram at 15,30 and 60 days.

Results: The nomogram had a good predictive performance, with a bootstrapped corrected concordance index of 0.74 (95\% Cl 0.72-0.75), and showed good calibration. The internal validation demonstrated virtually no departures from ideal prediction. In the external validation cohorts, the accuracy (and 95\% confidence interval) of nomogram at 15, 30 and 60 days was $74 \%$ (70-77), 89\% (85-92) and 72\% (68-76), respectively. Median survival was 32 days (29-34) for development cohort and 22 days (19-24) for validation cohort.

Conclusion: We developed a more accurate tool than the PaP Score to predict the probability of survival at 15,30 , and 60 days in terminally ill cancer patients. This tool could greatly facilitate clinical care decision making at the end of life.

Abstract number: P02-212

Abstract type: Poster

Development of an International Version of the Integrated Palliative Care Outcome Scale for Dementia (IPOS-Dem) to Support Person-centred Assessment of People with Dementia: Transparent Expert Consultation

Ellis-Smith $C^{1}{ }^{1}$, Hodiamont $F^{2}{ }^{2}$, Bausewein $C^{2}{ }^{2}$, van der Steen J.T..$^{3,4}$, Klapwijk M.S. ${ }^{3}$, Higginson I.J. ${ }^{1}$, Evans C.J. ${ }^{1,5}$

${ }^{1}$ King's College London, Cicely Saunders Institute of Palliative Care, Policy and Rehabilitation, London, United Kingdom, ${ }^{2}$ Ludwig-Maximilian University Munich, Department for Palliative Medicine, Munich, Germany, ${ }^{3}$ Leiden University Medical Center, Department of Public Health and Primary Care, Leiden, Netherlands, ${ }^{4}$ Radboud University Medical Center, Nijmegen, Netherlands, ${ }^{5}$ Sussex Community NHS Foundation Trust, Brighton, United Kingdom

Background: Assessment of a person's wishes and needs to inform care (person-centred assessment) is essential to improve quality of life in people with dementia.

Aim: To refine the Integrated Palliative care Outcome Scale for Dementia (IPOS-Dem) to form an internationally acceptable version.

Methods: A Transparent Expert Consultation involving a workshop with nominal group technique and consensus survey. Participants were teams involved in the development and translations of IPOS-Dem, clinicians and academics, and Patient and Public Involvement (PPI) members. Questions related to the validity and reliability of person-centred proxy assessment. We synthesised all findings to identify key recommendations for an international version of IPOS-Dem.

Results: The workshop included 27 participants comprising clinicians and academics from palliative care, geriatrics and psychiatry, and PPI members. 179 individual recommendations were generated. Key findings related to

1. importance of person-centred assessment to inform 'best' care despite challenges in proxy assessment of subjective experience;

2. 'best' respondent to inform reliable assessment including selfreport, family member, care staff and/or all involved in care;

3. increased focus on positive aspects e.g. 'What is going well?';

4. importance of items on subjective concerns e.g. 'What matters to me?' and removal of less common items e.g. nausea;

5. requirement for guidance to support assessment of subjective experience;

6. use of IPOS-Dem across trajectory and care settings;

7. digital format.

Conclusion: Person-centred assessment of experiences of people with dementia is essential to inform best care despite inherent challenges of proxy assessment. Recommendations to improve valid and reliable person-centred proxy assessment include choice of respondent, guidance on use and importance of subjective items. Consensus survey results will be used to develop a new internationally valid and reliable version of IPOS-Dem.

\section{Abstract number: P02-214 \\ Abstract type: Poster}

The Integrated Palliative Care Outcome Scale for Patients with Palliative Care Needs: Factors Related to and Experiences of the Use in Acute Care Settings

Lind S. ${ }^{1}$, Wallin L. ${ }^{2,3,4}$, Fürst C.J. ${ }^{5,6}$, Beck I. $.5,6,7$

${ }_{1}$ Palliative Research Centre, Department of Health Care Sciences, Ersta Sköndal Bräcke University College, Stockholm, Sweden, ${ }^{2}$ Department of Neurobiology, Care Sciences and Society, Division of Nursing, Karolinska Institutet, Flemingsberg, Sweden, ${ }^{3}$ School of Education, Health and Social Science, Dalarna University, Falun, Sweden, ${ }^{4}$ Department of Health and Care Sciences, Sahlgrenska Academy, University of Gothenburg, Gothenburg, Sweden, 5 Institute for Palliative Care, Lund University and Region Skåne, Lund, Sweden, ${ }^{6}$ Faculty of Medicine, Department of Clinical Sciences, Lund University, Lund, Sweden, ${ }^{7}$ Faculty of Health Science, Department of Nursing and Integrated Health Sciences, Kristianstad University, Kristianstad, Sweden

Background: Patients with palliative care needs are cared for in a diversity of healthcare settings. The use of patient-reported outcome measures (PROM), such as the Integrated Palliative care Outcome Scale (IPOS), has an important meaning in providing palliative care. However, little is known about implementation of IPOS.

Aims: To explore factors that contributed to/hindered patients with palliative care needs having their symptoms assessed using IPOS and explore health care professionals experiences of using IPOS.

Method: An explorative design with a mixed method approach was used. An implementation intervention including education and clinical use of IPOS with support from facilitators was carried out in three acute care settings. Data was collected through review of the presence of IPOS in patients' health records, notes made during the education and 19 semi-structured interviews. Statistics and content analysis were used.

Results: A total of 309 patients were eligible to be offered assessment of symptoms with IPOS. Of these, 69 (22\%) had completed IPOS. Patients with chronic diseases who had completed IPOS was significantly higher $(p=0.001)$ than for those with cancer diseases. A significant positive association was found between health care professionals' participation in training sessions and completed IPOS. The experiences of IPOS were split in two categories: "IPOS acting as a facilitator" and "barriers for use of IPOS". IPOS was described to facilitate improvement of quality of care and the teamwork. Barriers as insecurity how to approach severely ill patients and difficulties in finding new routines for the use of IPOS were described.

Conclusion / Discussion. The results indicate a need of high degree of presence in the training to achieve successful implementation of IPOS. Nevertheless, the use of IPOS could contribute to improvement of quality of patients with palliative care needs in acute care settings.

Abstract number: P02-215

Abstract type: Poster

\section{Wound Management Algorithm}

Cazan $R .^{1}$, Pancu A. ${ }^{2}$, Horeica $R^{3}$

${ }^{1}$ Home Care, Hospice Casa Sperantei, Brasov, Romania, ${ }^{2}$ Inpatient Unit, Hospice Casa Sperantei, Brasov, Romania, ${ }^{3}$ Adult Nursing, Hospice Casa Sperantei, Brasov, Romania

Introduction: One of the main challenges that palliative care (PC) professionals are confronted with is the management of chronic wounds (CW), like pressure sores and fungating tumors. By definition, $\mathrm{CW}$ are considered 
the wounds that have a history of more than 3 months of existence. The development of a clinical algorithm it supposes to synthetize the already existing knowledge and to elaborate specific recommendations for a proper, evidence based, nursing practice.

Goal: To elaborate an instrument that will help PC professionals, particularly nurses, in the process of caring for patients with CW.

Material and method: Literature review in regard to the management of CW. PC nurse experts meeting in which relevant articles and guides have been analyzed. Development of the CW management algorithm.

Results and conclusions: We review a number of 36 article and had 2 meeting to establish algorithm. The CW management algorithm is divided in 4 sections:

1. Evaluation of the patient and the wound - which contains: diagnosis, age, nutritional status, medication, the effect of daily living activity, the effect on body image. The evaluation of the wound contains: location, type of wound, skin damage, smell, exudate (quality and quantity), presence of hemorrhage, the surrounding tissue;

2. Establishing realistic objectives of care: prevention of the expansion of the existing wound and of the appearance of new wounds; control of symptoms related to the wound; maintaining quality of life; wound healing;

3. Interventions

4. Re-evaluation

Pilot study in an inpatient PC service for a period of 6 month after implementing algorithm showed that $\mathrm{CW}$ management brings benefits both to patients and professional by the complex evaluation, the unitary approach in caring for the CW, by commonly agreed on the objectives of care.

Keywords: palliative care, chronic wounds, pressure sores, fungating tumors, type of dressings

Funded by BMS Foundation

Abstract number: P02-216

Abstract type: Poster

What Screening Tools Have Been Used To Identify Delirium in Hospitalised Cancer Patients, what is Known about the Epidemiology of Delirium in this Setting and what Remains Uncertain? A Scoping Review of the Literature

Sands M.B. ${ }^{1}$, Wee I. ${ }^{2}$, Agar M. ${ }^{3}$, Vardy J. ${ }^{4,5}$

${ }^{1}$ Prince of Wales Clinical School, University of New South Wales, Bondi Beach, Australia, ${ }^{2}$ Singapore University Medical School, Singapore, Singapore, ${ }^{3}$ Australian University of Technology, Sydney, Australia, ${ }^{4}$ Concord Cancer Centre, Repatriation General Hospital, Sydney, Australia, ${ }^{5}$ Medical School, University of Sydney, Sydney, Australia

Background: Delirium is an important syndrome associated with poor outcomes for cancer patients, carers and staff. Some data exist, however cancer in-patients, in the acute hospital setting have not been a focus in the literature.

Objectives: We investigated 4 research questions related to this setting:

1. Which instruments are most commonly used to detect delirium?

2. Which reference standards were used to determine rates of delirium?

3. What are the incidence and prevalence of delirium? and

4. What is the rate of reversibility of delirium?

Methods: MEDLINE, EMBASE, CINAHL, PsycINFO, SCOPUS databases were searched from January 1st 1996 to August 12th 2017. Delirium, cancer, in-patient oncology and delirium detection, were the four key concepts for the search. Hand search articles, plus data base search returns were included in title and abstract screening, followed by full text review.

Results: Of 896 unique studies; 91 were retained for full text review. Twelve studies were eligible for final data abstraction and synthesis. 4 delirium screening tools and 1 group of cognitive tests were used for delirium detection; 2 diagnostic criteria and 1 diagnostic algorithm, were used as reference standards. Two studies presented incidence rates; $7.8 \%$ (unscheduled admissions), and $16.5 \%$ respectively. Prevalence rates ranged from $18 \%$ to $33 \%$ for general medical or oncology wards ( 3 studies); Acute Palliative Care Units (APCU) $42 \%$ to $58 \%$ (4 studies); and in the setting of older cancer patients: $21.5 \%$ and $57 \%$. Three studies, in two settings, reported delirium reversibility; $26 \%$ and $49 \%$ (both APCU) and $30 \%$ (older patients with cancer). Study quality was constrained by risk of bias in patient selection, and uncertain generalisability.

Conclusion: Our review has identified important knowledge gaps. Future studies with optimal selection of diagnostic reference standards, will better address identified knowledge gaps.

Abstract number: $\mathrm{P} 02-217$

Abstract type: Poster

Implementing Palliative Care Tools in Nursing Homes through Action Research: Starting from the Needs and Preferences of Care Staff

Bagchus C. ${ }^{1}$, Lemos Dekker N. ${ }^{2}$, Pasman H.R.W. ${ }^{1}$, Onwuteaka-Philipsen

B.D. ${ }^{3}$, Achterberg W.P. ${ }^{2}$, van der Steen J.T. ${ }^{2}$

${ }^{1}$ Amsterdam Public Health Research Institute, Expertise Center for Palliative Care, Amsterdam UMC, VU University Medical Center, Amsterdam, Netherlands, ${ }^{2}$ Department of Public Health and Primary Care, Leiden University Medical Center, Leiden, Netherlands, ${ }^{3}$ Amsterdam Public Health Research institute, Expertise Center for Palliative Care, Amsterdam UMC, VU University Medical Center, Amsterdam, Netherlands

Background: Many evidence-based palliative care tools are available for use in long-term care settings to recognize the palliative and terminal phase, to monitor symptoms and identify needs. However, they may be used rarely. Little is known about care staff preferences for particular tools. Therefore, we aimed to identify tools tailored to staff needs in nursing home practice.

Methods: Using action research, we engaged nurse aids as co-researchers, and next, the multidisciplinary team of 10 nursing homes in the Netherlands. Two anthropologists facilitated a cyclic process of selecting, implementing, evaluating and adjusting tools, using a mix of mostly qualitative methods.

Results: Tools were available in all participating nursing homes, but they were not being used systematically or not at all. All of these tools had been implemented in a top-down manner. As a result, nurse aids generally believed tools are there for the benefit of other professionals and they perceived that they add to the already substantial administrative workload. However, they do feel an urgency to improve palliative care. Most care teams expressed the need for tools to identify when change in care is needed and to verify their subjective observations. The Prospective Prognostic Planning Tool visualizing change, Palliative Performance Scale and Surprise Question seem to meet these needs. Nurse aids value the simplicity of these tools and an overview of a resident's overall condition at a glance. Piloting its use, teams experienced that the tools facilitated communication within the (multidisciplinary) team, a key need identified in initial focus groups.

Discussion: Limited use of palliative care tools in nursing home practice does not imply that there is no perceived need for such tools. Our research demonstrates that tools can be helpful instead of being perceived as just adding to workload when they fit the needs and preferences of people on the work floor, probably increasing sustainable use. 
Abstract number: P02-218

Abstract type: Poster

Assessing the Quality of Care in SOPC from Different Perspectives: Approach and Lessons Learned in the ELSAH-study Kuss K. ${ }^{1}$, Seipp H. ${ }^{1}$, Hach M. ${ }^{2}$, Becker D. ${ }^{2}$, Gruber D. ${ }^{3}$, Engler J. ${ }^{3}$, Erler A. ${ }^{3}$, Boesner S. ${ }^{1}$, Haasenritter J. ${ }^{1}$

${ }^{1}$ General Practice/Family Medicine, Philipps-University Marburg, Marburg, Germany, ${ }^{2}$ Professional Association of Specialized Outpatient Palliative Care in Hesse, Wiesbaden, Germany, ${ }^{3}$ Institute of Genera Practice, Goethe-University Frankfurt, Frankfurt, Germany

Background: There is no consensus how to monitor quality of care in the special context of specialized outpatient palliative care (SOPC) adequately, beyond structural data. Patient-reported outcome measures have increasingly gained attention to assess the quality of palliative care. Aims: To compose, to pilot, to implement, and to evaluate a patientcentered set of instruments, focusing on process-and outcome-quality over the complete course of care.

Methods: We applied different methods and a multi-step process with sequential Mixed-Methods Designs. Based on findings (qualitative methods) to further define the term "quality of care" in the context of SOPC, we chose a preliminary set of instruments taking into account several dimensions. The set was tested in a pilot run in five SOPC teams.

Results: We identified six main elements to characterize quality of care. Therefore, we will present the elements and the designated instruments to capture them. The set of instruments consists of internationally recommended and prevalent instruments, complemented by adapted ones. We will discuss aspects of feasibility and practicability derived from the pilotphase, as well as indispensable adaptations. These aspects were gathered by focus group discussions and continuous feedback of the piloting SOPC teams. Conclusion / Discussion: There are several challenges regarding patient reported outcome measures, short duration of courses of care, as well as dealing with sensitive questions. The early integration of SOPC teams, patients and their relatives, in the adaptation process of suitable instruments might help to overcome the challenges and lessons learned. The standardized application of instruments might directly influence the care process of patients in palliative situations and might allow assessing quality of care in SOPC based on process- and outcome-evaluation. The study is funded by the Innovation fund of the Federal Joint Committee of Germany (grant no.: 01VSF16006).

Abstract number: P02-219

Abstract type: Poster

Evaluation of an Inpatient Palliative Care Service Using the Integrated Palliative Care Outcome Scale (IPOS) is Feasible: A Prospective Observational Study
Spickermann M., Lenz $P$.

Department of Palliative Medicine, University Hospital of Münster, Münster, Germany

Background: With spreading availability of palliative care (PC), outcome measurements play a crucial role to secure high quality and to define parameters for interventional studies. Additionally, due to the increasing number of patients and limited resources $P C$ teams need intelligent tools like e.g. the Integrated Palliative Care Outcome Scale (IPOS) to allocate the multiprofessional team according to the individual needs.

Aims: The aim of study was to evaluate the quality of PC delivered by an inpatient service and to check the feasibility of the IPOS in an inpatient setting. Methods (design, data collection, analysis): Mono-centre observational prospective study, inpatient PC service, University Hospital of Münster, Germany. Patients of age with PC needs receiving specialised PC. Observational period: interval of 7 days. Primary endpoint: symptom burden, measured by IPOS. Secondary endpoint: health-related quality of life (HRQOL), measured by EQ-5D-5L questionnaire.

Results: In the observational period ( 8 months) a total of 49 patients with a balanced distribution of sexes, an average age of 66,5 years and mostly malignant diseases (98\%) were included and of these 47 patients completed the observational period. The most frequent physical symptoms were fatigue (78\%) and pain (65\%). The psychological symptoms named most frequently were family anxieties (96\%) and own anxiety (91\%). Symptom burden were reduced on average about 6,9 points on the overall IPOS score (maximum amount 68 points) from 27,7 to 20,3 points within 7 days. All subscale scores also decreased. HRQOL was very low at timepoint of inclusion and showed only a slight increase (not significant).

Conclusion: The inpatient PC service reached a significant reduction of symptom burden measured by the IPOS which is practicable and useful in the acute setting. However, data collection and analysis mean an additional effort for the inpatient PC teams.

Abstract number: P02-220

Abstract type: Poster

\section{Development of a Prognostic Model for Terminally III Cancer} Patients

Vilches-Aguirre Y. ${ }^{1}$, Alonso-Babarro A. ${ }^{1}$, Lacasta-Reverte M. ${ }^{1}$, FeliuBatlle J.' ${ }^{2}$, Diez-Porres L. ${ }^{1}$, Varela-Cerdeira M. ${ }^{1}$, Madero-Jarabo R. ${ }^{3}$, Perez-Manrique T. ${ }^{1}$, Ybarra-Huesa C. ${ }^{1}$, De La Torre-Cañaveras M.I. ${ }^{1}$ ${ }^{1}$ Palliative Care Unit, Hospital Universitario La Paz, Madrid, Spain, ${ }^{2}$ Medical Oncology Unit, Hospital Universitario La Paz, Madrid, Spain, ${ }^{3}$ Biostatistics Service, Hospital Universitario La Paz, Madrid, Spain

Background: Predicting prognosis in advanced cancer patients is important. Our aim was developing a prognostic model to predict survival in patients with cancer admitted to Acute Palliative Care Unit (APCU).

Variables in prognostic model.

\begin{tabular}{|c|c|c|c|c|}
\hline $\begin{array}{l}\text { Significant Variables } \\
\mathrm{N}=678\end{array}$ & $\beta$ Coefficient & $\mathrm{B}(\mathrm{SE})$ & $\mathrm{HR}(95 \% \mathrm{IC})$ & $\mathrm{p}$ \\
\hline Dyspnoea & 0.023 & 0.014 & $1.02(0.99-1.05)$ & 0.067 \\
\hline Fatigue & 0.083 & 0.019 & $1.09(1.05-1.13)$ & $<0.001$ \\
\hline SGA (Nutritional Status) & 0.118 & 0.071 & $1.13(0.98-1.30)$ & 0.098 \\
\hline Change in Barthel Index & 0.004 & 0.001 & $1.004(1.00-1.01)$ & 0.002 \\
\hline Delirium (yes) & 0.378 & 0.09 & $1.46(1.23-1.74)$ & $<0.001$ \\
\hline Leukocytes $\left(\times 10^{3} / \mathrm{mm}^{3}\right)$ & 0.013 & 0.004 & $1.01(1,01-1,02)$ & 0.001 \\
\hline $\mathrm{CRP}(\mathrm{mg} / \mathrm{L})$ & 0.002 & 0 & $1.002(1.001-1.003)$ & $<0.001$ \\
\hline Sodium (mmol/L) / < 125/ & $0.653 / 0.474 /$ & $0.391 / 0.172 /$ & $1.92(0.89-4.13) / 1.61(1.15-2.25) / 1.10$ & $0.095 / 0.006 /$ \\
\hline $129-125 / 130-134 />145 /$ & $0.094 /-0.167$ & $0.097 / 0,242 /$ & $(0.91-1.33) / 0.85(0.53-1.36) /$ & 0.33 / 0,492/ \\
\hline LDH (UI/L) & 0.00024 & 0 & 1.00 (1.00-1.00) & $<0.001$ \\
\hline
\end{tabular}


Method: Prospective study including all cancer patients admitted (January 12 - April15). Length of time from admitted to death was our main variable. Up to 60 variables were analyzed. Significant variables in the UV analysis were used in the Cox Regression Model and, by selection method by forward steps, selecting the model that best fit the data (lower Akaike information criterion). We chose the variables most interesting from a clinical and prognostic point of view.

Results: Out of 1294 admissions, 959 patients included. Median overall survival:20 days. Variables included are in Table.

The prognostic model included performance status (Barthel Index), nutritional status (SGA), two symptoms (dyspnea, fatigue), two signs (heart rate,edemas), cognitive status (delirium) and four analytical parameters (leukocyte, C-reactive protein, sodium and lactate dehydrogenase levels).

The model had a Harrell concordance index of 0.70 , showing good calibration. This allowed us to establish 4 well-defined risk groups with survival medians of $7,18,37$ and 73 days.

Conclusions: We have built a prognostic model using only available clinical and analytical information which predicts survival time accurately at 15,30 , 60 and 90 days (AUC 0,76-0,79), with a Harrell concordance index of 0.70 .

Abstract number: P02-221

Abstract type: Poster

\section{Reflexology in Palliative Care: Remove the Placebo Effect and} What's Left?

Douglas-Jones A. ${ }^{1}$, Dawson A. ${ }^{1}$, Noble S. ${ }^{2}$

${ }^{1}$ Hospice of the Valleys, Tredegar, United Kingdom, ${ }^{2}$ Cardiff University, Marie Curie Palliative Care Research Centre, United Kingdom

Background: The placebo effect may result in a $40 \%$ clinical improvement following administration of a non-active treatment. Complementary therapies such as reflexology are commonplace in palliative care services yet their evidence base is supported by poorly constructed research and subjective clinical outcomes. Cynics have attributed patient reported responses to the placebo effect.

Methods: Retrospective data analysis of hospice out patients pre and post symptom targeted reflexology using using patient completed POS-S evaluation tool. Mean symptom difference (MSD) and percentage response (PR) were calculated for each symptom. Placebo adjusted response PAR was calculated (PR-40\%) with PAR of $20 \%$ or above being considered significant.

Results: 100 cancer patients received a total of 360 treatment sessions for 8 symptoms.

Conclusion: Adjusting patient reported response for placebo effect suggests reflexology may have a positive effect on constipation and fatigue. Constipation has long been reported to respond to reflexology and can be objectively evaluated with validated outcome tools. A feasibility study to conduct a randomised control trial to compare reflexology with placebo for constipation is in development.

Symptoms and placebo adjusted responses to reflexology.

\begin{tabular}{lllr}
\hline Symptom & MSD & PR & PAR \\
\hline Pain & 0.74 & $24 \%$ & $<0$ \\
Constipation & 1.5 & $80 \%$ & 40 \\
Insomnia & 0.8 & $40 \%$ & 0 \\
Distress & 0.8 & $47 \%$ & 7 \\
Neuropathy & 0.8 & $47 \%$ & 7 \\
Fatigue & 1.6 & $80 \%$ & 40 \\
Dyspnoea & 0 & $0 \%$ & 0 \\
Poor & 0.25 & $17 \%$ & $<0$ \\
appetite & & &
\end{tabular}

Abstract number: $\mathrm{P} 02-222$

Abstract type: Poster

End-of-Life Prognostication in Acute Hospital Palliative Patients: Is the Palliative Performance Scale Useful?

Cohen K., Paz E.

Palliative Care Service, Emek Medical Center, Afula, Israel

Background: Prognostication is the ability to evaluate outcome and expected course of a medical condition based on knowledge and experience. Special emphasis is given to prediction of life expectancy in end-oflife situations, as prognosis directs treatment approach, policy regarding tests and procedures, and discharge planning. Israel, a Western-oriented country in the Middle East, is characterized by diverse population. The health system serves, and comprises, immigrants and members of three religions. End of life care is a very sensitive issue, necessitating prognostic assessment aids. The Palliative Performance Scale (PPS) is a reliable tool designed to measure physical status in palliative care. Research found it correlates well with survival time in outpatient cancer settings. Little is known about efficacy in non-cancer patients

Aim: This study examined PPS use as a tool estimating life expectancy in patients with varied life-threatening diseases who required palliative consult during hospitalization in an acute regional hospital in northern Israel.

Method: A retrospective study examined records of patients assessed by the Palliative Consult Team (PCT), using PPS, Jan.2015 - Sep.2018. Variables were initial PPS score, age, gender, ethnic origin, diagnosis and survival time. Patients were monitored six months from initial assessment.

Results: A total of 557 patients' records (339 solid tumors, 43 hematologic cancer, 175 non-cancer) assessed by PCT using PPS, were included in the study. Findings showed PPS to be a significant predictor of survival for three groups, with no ethnic differences

Discussion: This research supports use of PPS by clinicians estimating prognosis in cancer and non-cancer patients. Findings support utilization in acute hospitalized patients in Israel's diverse population. While PPS is well known in palliative care, this was the first study to investigate its use in Israel. Results encouraged authors to develop the official PPS Hebrew version

Abstract number: P02-223

Abstract type: Poster

Use of Routine Data for Estimating Need in Palliative Care in Ukraine: Towards Development of Reliable Methodology Gorbal A. ${ }^{1}$, Nasridinov R. $^{1}$, Sukhovii $0 .{ }^{1,2}$, Shapoval-Deynega K. ${ }^{3}$, Tymoshevska V. ${ }^{3}$, Bratsyun O. ${ }^{4}$, Ukrainian Center for Social Data ${ }^{1}$ Ukrainian Center for Social Data, Kyiv, Ukraine, ${ }^{2}$ Centre for Mental Health and Monitoring of Drugs and Alcohol of the Ministry of Health of Ukraine, Kyiv, Ukraine, ${ }^{3}$ nternational Renaissance Foundation, Kyiv, Ukraine, ${ }^{4}$ Shupyk National Medical Academy of Postgraduate Education, Kyiv, Ukraine

There is no official medical statistics on provision of palliative care in Ukraine, or estimating its need. Aim of the study was to develop a more reliable methodology of estimating need in palliative care (PC) using available routine data (death causes, disease prevalence, etc). Using available methodologies and recommendations (by WHO, WPCA, UNICEF, ICPCN), as well as by Ministry of Health; and data on death causes (for adults) and disease prevalence (for children, $\mathrm{MoH}$ medical statistics), we carried out initial model of the need in PC, according to which, over 340000 adults and almost 255000 children required palliative care in 2016. But these figures were not reliable. We identified serious flaws in state registration of death. Diseases like Alzheimer or Parkinson are almost not registered as cause of death in Ukraine, while official rate of cardiovascular deaths is off the scale (highest official rate of death globally) - raising concerns of mass 
falsifications of death records. Next iterations of the study included: identification of available routine data sources, removal of potentially unreliable data from calculation (such as significant share of "cardiovascular deaths"); carrying out group discussions and individual consultations with experts on specific disease groups and coefficients to be applied; finalization of methodology; acquiring data and calculating the need, desegregated by region and some hospital districts. Data sources: forms 10, 12 of medical statistics, registered death causes, TB registry, National Cancer Registry. Total of 12 adult disease groups and 10 children disease groups identified. According to new methodology, 245334 adults and 84967 children required PC in 2017 in Ukraine. As EHRs have not proliferated in Ukraine yet and doctors' understanding of PC is low, using routine data is important for estimating resources needed and bringing attention to the issue. As data sources change, methodology should be updated and improved.

Abstract number: P02-224

Abstract type: Poster

Linguistic and Cultural Adaption of the Integrated Palliative Care Outcome Scale (IPOS) for the Greek Population

Anagnostou D. ${ }^{1,2}$, Katsaragakis S. ${ }^{3}$, Patiraki E. ${ }^{3,4}$, Panagiotou I. ${ }^{4}$, Tserkezoglou $A{ }^{4}$

${ }^{1}$ Faculty of Medicine, Division of Human Health Sciences, Kyoto University, Kyoto, Japan, ${ }^{2}$ School of Medicine- Marie Curie Palliative Care Centre, Cardiff University, Cardiff, United Kingdom, ${ }^{3}$ Nursing, National and Kapodistrian Univesrity of Athens, Athens, Greece, ${ }^{4}$ Palliative Care Unit Galilee, Spata, Greece

Context: The Integrated Palliative Care Outcome Scale is a patient-centered outcome measure developed after merging the Palliative care Outcome Scale and the Palliative care Outcome Scale-Symptoms. The need for a patient-reported outcome measure in the Greek context led to this study.

Aim: To translate and culturally adapt IPOS to the Greek population.

Methods: A four phases- sequential study, which included verification of conceptual equivalence, double forward- backward translations and conceptual cognitive debriefing. Focus group interviews, used 'think aloud' and 'verbal probing' techniques. Interviews were audio-recorded, transcribed verbatim and thematically analyzed using predefined categories. Purposely sampled 6 patients and 9 health professionals from a palliative care service in Athens. The tool was then refined by consensus.

Results: The Integrated Palliative Care Outcome Scale was well accepted by both patients and health professionals. Overall comprehension and acceptability of the scale were good. The comprehension and judgement challenges identified in the pre-final version were successfully resolved in the cognitive interviewing phase. Six out of the ten translated items of the scale were modified after cognitive debriefing. Comprehension difficulties were identified with specific terms (e.g. lack of energy/feeling depressed) and with some answer options. Severity of symptoms and not their impact was a common difficulty. A judgement challenge was reported in relation to 3-days recall and fluctuation of symptoms. Layout concerns in relation to length of questions were also stated. All questions were considered important and none as inappropriate.

Conclusion: This study demonstrated face and content validity and acceptability of the Integrated Palliative Care Outcome Scale in the Greek context. Cognitive Interviewing proved valuable in refining concepts within the specific cultural context. Psychometric validation of the refined tool is underway.

Abstract number: P02-225

Abstract type: Poster

Validation of the Hospital One-year Mortality Risk (HOMR) or HOMR-NOW! Tool in a Respiratory Outreach Population
Kodsi A. ${ }^{1}$, Sands M.B. ${ }^{1,2}$, Messum S.-J. ${ }^{3}$, Dimitri A. ${ }^{4}$, Murray N. ${ }^{4}$, GallegoLuxan $B .{ }^{5}$

${ }^{1}$ Palliative Medicine, Prince of Wales Hospital, Randwick, Australia, ${ }^{2}$ University of New South Wales, Sydney, Australia, ${ }^{3}$ South East Sydney Local Health District, Sydney, Australia, ${ }^{4}$ Respiratory and Sleep Medicine, Prince of Wales Hospital, Randwick, Australia, ${ }^{5}$ Australian Institute of Health Innovation, Macquarie University, Macquarie, Australia

Background: Symptom management, advance care planning and family support is important for patients with severe chronic respiratory illnesses. Predicting survival for these patients is also useful in guiding resource allocation and planning service delivery as they historically have high care needs but low palliative care utilisation. Prognostication, however, remains difficult with available assessment tools and clinical triggers for referral may be less apparent in non-cancer settings. Using administrative data abstraction methods, the HOMR and HOMR-Now! tools have been validated in large Canadian cohorts to predict 1 year survival. Use of these tools may improve equity and efficiency of palliative care needs assessment and may be useful as an initial screening tool to guide further evaluation.

Aim: Our primary aim is to test the validity of the HOMR tool in patients with life threatening respiratory illness in an existing Australian tertiary hospital respiratory outpatient program.

Methodology: This is a retrospective validation study of the HOMR tool for all referrals to the respiratory outreach service in a Sydney tertiary referral hospital over a 10 year period. The HOMR is a twenty-point tool and the HOMR-NOW! is a subsequent version allowing real-time assessment at the point of care. Referral to the service will be the anchor point for validation of HOMR predicted versus observed survival. Observed survival will be construed at 12-14 months from anchor point using mortality data linkage from state registries. Our primary end-point is calibration between predicted and actual survival. Secondary end-points include risk of death and the ability of the HOMR score to predict vital status is assessed using the Receiving Operating Curve (ROC) and Bier score.

Results to follow: In a 2015 pilot study there were 182 patients referred to the program. We found 46 patients had a $30 \%$ or greater risk of death which is a feasible number for future interventional study.

Abstract number: P02-226

Abstract type: Poster

Validation of a New Tool to Identify Patients Requiring General or Specialized Palliative Care (ID PALL) in Acute Care Settings Teike Lüthi F. ${ }^{1}$, Bernard M. ${ }^{1}$, Gamondi $C^{2}{ }^{2}$, Vanderlinden $K^{1}{ }^{1}$, Ramelet A.-S. ${ }^{3}$, Borasio G.D. ${ }^{1}$

${ }^{1}$ Service de Soins Palliatifs et de Support, Centre Hospitalier Universitaire Vaudois, Lausanne, Switzerland, ${ }^{2}$ Ente Ospedaliero Cantonale, Bellinzona, Switzerland, ${ }^{3}$ Faculté de Biologie et de Médecine - FBM, Institut Universitaire de Formation et de Recherche en Soins IUFRS, Lausanne, Switzerland

Background: Early identification of patients requiring palliative care (PC) is a major public health concern. A growing number of tools exist to help professionals to identify these patients, but none of them has been thoroughly assessed for criterion validity so far. In addition, available tools do not differentiate between patients in need of general vs specialized PC and are primarily intended for physicians' use. A new tool, ID PALL ${ }^{\odot}$, was thus developed to address these issues.

Aim: To establish the validity and reliability of the newly developed tool to identify general and specialized palliative care patients in acute care settings.

Method: This validation study is conducted in 7 acute care medical units of two Swiss hospitals. The tool is completed by bedside nurses in a convenience sample of $\mathbf{2 0 0 0}$ patients hospitalized in internal medicine units 
from day 2 to 4 . For the inter-rater reliability, the patients' physicians also fill in ID PALL, independently. Sample size was calculated based on a sensitivity of 0.9 with $5 \%$ precision. The same patients are also independently assessed by paired (nurse/physician) experts in palliative care to establish criterion validity.

Results: 1530 patients have been included so far between March and October 2018 (47\% women, median age 72 yrs, 23\% oncological patients). Preliminary analyses show high sensitivity and a moderate specificity for the identification of general PC needs; specificity appears to be higher for the identification of specialized PC needs. Data collection will be completed by the end of 2018, and final results, included inter-rater reliability, will be reported at the congress.

Discussion: Our preliminary results suggest that ID-PALL may be more precise in identifying patients in need of specialised PC than those in need of general PC. These results await confirmation and further statistical analyses are required to identify the reasons for this variance and the discriminatory power of each item.

Abstract number: $\mathrm{P} 02-227$

Abstract type: Poster

\section{Developing and Implementing a Clinical Algorithm for}

Lymphedema Care

Ancuta C. ${ }^{1}$, Buzea L. ${ }^{2}$

${ }^{1}$ Education and Development, Hospice Casa Sperantei, Brasov, Romania, ${ }^{2 H}$ Home Care Service, Hospice Casa Sperantei, Brasov, Romania

Introduction: There are challenges for Palliative Care (PC) services that don't have specialized personnel in lymphedema care, and a standardized algorithm of care is needed.

Goal: To develop an instrument that will help PC professionals, particularly nurses, in the process of caring for patients with lymphedema. This algorithm is the instrument intended to help and guide medical professionals in the process of evaluation, management and decision making. Method: Literature review in regard to the management of lymphedema. PC nurse experts meetings. Development of the lymphedema management algorithm. Implementation and monitoring

Results: Following the literature review 15 relevant articles and 4 guides have been retrieved as relevant and 5 meetings with PC nurse experts were organized. The lymphedema care algorithm was structured in 4 sections:

1. diagnosis and evaluation - shows the steps for making a diagnosis and establishing the stage of the lymphedema;

2. interventions divided into intensive and maintenance interventions, applied in accordance to the lymphedema stage;

3. monitoring of the lymphedema at regular intervals, following certain indicators;

4. evolution of lymphedema. The strategy for implementing the algorithm was: meetings with clinical leaders for presenting the algorithm, education processes of appropriate colleges, piloting the algorithm, discussing the pilot results at conference and other professional meetings, incorporating the algorithm in routine procedures. After piloting process, the dissensions in applying the algorithm were: indicators for monitoring and consecutive number days of care. The major's changes in clinical practice were: improvement in establish the stage of lymphedema and in management.

Conclusions: Using the algorithm help us to organize the assessment and interventions in management of patients with lymphedema. Funding for the algorithm development was provided by BMS foundation.

Abstract number: P02-228

Abstract type: Poster
Validation of a Colostomy Care Algorithm

Ghiran C. $^{1}$, Balan A. ${ }^{2}$, Burlacu . $^{3}$

${ }^{1}$ Hospice Casa Sperantei, Brasov, Romania, ${ }^{2}$ Home Care, Hospice Casa Sperantei, Brasov, Romania, ${ }^{3}$ Palliative Care, Pneumology Hospital, Brasov, Romania

Background: One of the main challenges that patients with advanced colon cancer are confronted with, is the management of colostomy. Our hospice provides a stoma therapy service that operates both in acute care and palliative care services in the aria; the average annual number of consultations for the last 5 years is 307.8 consults annually. Interaction with nurses from services has motivated us to develop a colostomy care algorithm to be implemented in both services.

Aim: To develop and validate in clinical practice a colostomy care algorithm.

Method: The instrument was developed based on literature review and nurses expertise in care for colostomies. Alongside the algorithm, 5 clinical vignettes were prepared. Meetings with groups of registered nurses practicing in acute and palliative care settings were held. Following written consent, respondents answered demographic questions and worked individually a plan of stoma care intervention for each of 5 clinical vignettes. A brief algorithm tutorial followed and then the individual care plans designed were reanalyzed using the algorithm.

Results: 13 major sources have identified for the development of the algorithm; 42 nurses attended the meetings from 3 clinical settings; oncology, surgery and palliative care. $100 \%$ of participants have acknowledged the need and usefulness of the algorithm and 5 suggestions have been made for improvement of the algorithm.

Conclusions: The algorithm purpose is to facilitate a uniform approach to colostomy care, to improve the quality of health care and decrease the use of unnecessary or harmful interventions. It should be used with regard to clinical judgement to determine the plan of care for each patient. More specific result will be presented in the poster.

The main source of funding for this study comes from a Bristol-Myers Squibb Foundation's project

Keywords: Algorithm colostomy, clinical decision instrument, validation.

Abstract number: P02-229

Abstract type: Poster

A Palliative Approach to Care in Geriatric Mental Health: The Utility of ESAS and Patient Dignity Inventory in Geriatric Mental Health

Grossman D. ${ }^{1,2}$, Grief C. ${ }^{3}$, Berall A. ${ }^{4}$, Gardner S. ${ }^{5}$

${ }^{1}$ Family and Community Medicine Division Palliative Care, North York General Hospital, Toronto, Canada, ${ }^{2}$ Department of Family and Community Medicine, Division of Palliative Care, University of Toronto, Toronto, Canada, ${ }^{3}$ Psychiatry, Baycrest Centre for Geriatric care, Toronto, Canada, ${ }^{4}$ Research, Baycrest Centre for Geriatric care, Toronto, Canada, ${ }^{5}$ Research, Baycrest Centre for Geriatric Care, Toronto, Canada

Background: Older adults with multiple medical morbidities often have significant symptom burden affecting physical functioning. Unfortunately these symptoms are often not identified in older adults with mood disorders. We explore the utility of palliative care tools such as the Edmonton Symptom Assessment Scale (ESAS) and Patient Dignity Inventory (PDI) in geriatric mental health programs in order to identify the burden of symptoms and level of patient dignity.

Methods: Participants were recruited from an inpatient geriatric mental health unit and an outpatient day hospital for mood and related disorders. Self-reported rating scales tracking mood, anxiety and somatic symptoms were completed, including the ESAS and the PDI. Demographic characteristics were gathered from a retrospective chart review. 
Descriptive summaries included percentages, means, standard deviations (SD) and Pearson correlation coefficients. Longitudinal cross-sectional model used generalized estimating equations and an exchangeable working correlation (WC) matrix to adjust for repeated measures within patient across time.

Results: Data were obtained for 33 English speaking patients (inpatients $\mathrm{N}=17$, outpatients $\mathrm{N}=16$ ) with a mean age of $76.5(\mathrm{SD}=6.1), 69.7 \%$ female and $48.5 \%$ married.

At baseline, prevalence of ESAS symptoms were often moderate to severe. The top physical symptoms were tiredness, pain, drowsiness, and shortness of breath. Poorer dignity scores were significantly correlated with increased symptom burden $(p<.0001)$. Total PDI scores decreased across time but higher PDI scores were associated with higher total ESAS scores $(p<0.0001, W C=0.71)$.

Conclusions: The ESAS and PDI revealed the high physical symptom burden and loss of dignity experienced by older adults in geriatric mental health inpatient and outpatient settings. Incorporating a palliative care lens and providing a palliative approach may have relevance to patients in geriatric mental health settings.

Abstract number: P02-230

Abstract type: Poster

\section{Quality at the End of Life - Results of the Prospective PaRoLi}

Study

Muscheites W., Sewtz C., Große-Thie C., Murua Escobar H., Kriesen U., Junghan $\beta C$.

Department of Internal Medicine, Clinic for Hematology, Oncology and Palliative Medicine, Rostock University Medical Center, Rostock, Germany

Background: Caring about quality of life $(\mathrm{Q} o \mathrm{~L})$ is a core principle in palliative care $(\mathrm{PC})$.

QoL assessment can work as an outcome measure on behalf of the patients (pts) as well as a quality marker in evaluating PC.

Aims: In order to assess QoL and the effectiveness of PC we conducted a prospective questionnaire-based study including pts admitted to an interdisciplinary PC ward of a university medical center.

Methods: From 5 - 12/2013 consecutive PC pts were enrolled. Mood and QoL were assessed after admittance (P1), before discharge (P2) and 2 weeks after discharge (P3) using the Hospital Anxiety and Depression Scale (HADS-D) and the Functional Assessment of Chronic Illness Therapy (FACIT-Pal). The FACIT-Pal evaluates QoL in a PC context. It consists of 5 subscales: physical (PWB), social, emotional and functional (FWB) wellbeing as well as a particular PC subscale (PalS).

Results: From a total of 206 pts, 102 consented participation (49.5\%). At P1 94 pts (92.2\% of all living participants) completed FACIT-Pal, at P2 53 pts (80.3\%) and at P3 30 pts (56.6\%). FACIT-Pal total score (TS) showed a significant increase between P1 and P2 $(p<0.001)$. That increase became apparent in every subscale, though significant changes were evident in PWB and PalS only $(p<0.001 ; p=0.010)$. Between P2 and P3 FACIT-Pal TS showed a significant decrease $(p=0.001)$. A decline could be found in all subscales, but only PWB, FWB and PalS reached significance ( $p=0.005 ; p=0.006 ; p=0.001$ ).

Conclusions: QoL studies in PC pts are feasible despite high drop-out rates. Most of the living participants were interviewed. QoL improved significantly during an in-patient stay on a PC ward. After discharge a significant deterioration was seen. These findings resemble the previously reported results of HADS-D in our cohort. Our study shows that in-patient PC has a stabilizing effect on QoL, but discharge management and following out-patient PC have to be evaluated in future studies. The authors have nothing to disclose.

Abstract number: P02-232

Abstract type: Poster
Predicting 90-day Mortality and Service Use in Communitybased Palliative Care

Hermans $K^{1}{ }^{1,2}$, Guthrie D. ${ }^{3,4}$, Jakda A. ${ }^{5}$, Declerca A. ${ }^{2}$, Cohen J. ${ }^{1}$, Hirdes J. ${ }^{6}$

${ }^{1}$ End-of-Life Care Research Group, VUB, Brussels, Belgium, ${ }^{2}$ LUCAS, KU Leuven, Leuven, Belgium, ${ }^{3}$ Department of Kinesiology and Physical Education, Wilfrid Laurier University, Waterloo, Canada, ${ }^{4}$ Department of Health Sciences, Wilfrid Laurier University, Waterloo, Canada, ${ }^{5}$ Department of Family Medicine, McMaster University, Waterloo, Canada, ${ }^{6}$ School of Public Health and Health Systems, University of Waterloo, Waterloo, Canada

Background: Palliative care needs are often recognized too late in the disease trajectory, which hinders the provision of quality palliative care. The key to early identifying palliative care needs and timely initiating a palliative care approach lies in home care. There is an emerging need for models to predict mortality and service use in the home care setting.

Aims: This study aims to evaluate a model combining the interRAl Changes in Health, End-Stage Disease, Signs, and Symptoms Scale (interRAI CHESS) with other interRAI Palliative Care (interRAI PC) items as compared to the Palliative Performance Scale (PPS) in order to predict the risk of 90-day mortality and the number of nursing days in community-based palliative care clients in Ontario, Canada.

Methods: This is a population-based retrospective observational study, using data from 80261 interRAI PC and PPS assessments that were collected in Ontario between 2011 and 2018. Logistic regressions were used to evaluate the relationship between the predictor variables and 90-day mortality and the number of nursing days. $\mathrm{X}^{2}$ was calculated in order to test for goodness of fit. The c-statistics obtained from the models were used to provide information on the explanatory power of the models and their components. Survival was displayed using KaplanMeier curves.

Results: Our results show the interRAI CHESS of the interRAI PC to be a good predictor of 90 -day mortality (c-stat $=.683 ; \mathrm{p}<.0001$ ) and the number of nursing days ( $c$-stat=.607; $p<.0001$ ) in palliative home care clients as compared to the PPS (c-stat=.692; $p<.0001$ ). The interRAI CHESS even scores better than the PPS in predicting 90-day mortality when combined with other interRAI PC items (c-stat=.723; $p<.0001$ ).

Conclusion: This model, combining the interRAI CHESS with other interRAI PC items, is a good predictor of 90-day mortality and service use in community-based palliative care patients.

Abstract number: P02-233

Abstract type: Poster

Development and Implementation of a Mobile Phone Application to Improve Palliative Home Care in Resource-limited Settings

Carrasco Gimeno J.M. ${ }^{1}$, Serrano Pons J. ${ }^{2}$, Bouësseau M.-C. ${ }^{3}$

${ }^{1}$ APLICA Investigación y Traslación. Soc. Coop. Mad., Madrid, Spain, ${ }^{2}$ UniversalDoctor, Barcelona, Spain, ${ }^{3}$ Service Delivery and Safety, World Health Organization, Geneva, Switzerland

Background: Most of palliative care (PC) patients in low-middle income countries are living at home cared by their family and/or community caregivers, with few modalities to regularly report on the status of patients to their PC team.

Aim: To develop and implement an app for caregivers of PC patients and a web-based dashboard for PC clinical teams.

Design: An advisory group consisting of IT professionals, clinic staff interacting with family members and patients was involved in the design of:

1) an app based on the APCA African Palliative Outcomes Scale;

2) a dashboard, to collect the information sent by caregivers through the app. 
The study was implemented in three sites (India, Uganda and Zimbabwe) through a quantitative descriptive analysis of the use of the app and dashboard, and a qualitative evaluation by nine semi structured interviews per site.

Results: The app includes:

1) recommendations for the use of the app;

2) information about PC

3) information about the symptoms to be reported;

4) nine questions to be answered using Likert scales.

The web-based dashboard allows to sort the information by each item (patient, level of pain, feelings, etc.), to get information about when the report was filled and to record the action taken by the clinical team (none; phone call; home visit). Two sessions were conducted in each site with PC teams on the use of the app and the dashboard, and on how to instruct 25 family and 25 community caregivers to be recruited. Clinical staff interacting with the caregivers during the implementation found the app and the dashboard useful and feasible to be integrated in their practice.

Conclusions: The development of this tool demonstrated the information to be provided to caregivers and the IT functions requested to support the communication between caregivers and clinical team in the context of home-based PC.

Funding: The True Colours Trust; World Health Organization.

Abstract number: P02-234

Abstract type: Poster

Prevalence of Life-Limiting Diseases, and the Need for Palliative Care at Six Hospitals

Gafer N. ${ }^{1,2}$, Mursi M. ${ }^{3}$, Khair Allah M. ${ }^{4}$, Ali H. ${ }^{5}$, Siddig S. ${ }^{2}$, Harding R. ${ }^{1}$, Davila Quintana C.D. ${ }^{6}$

${ }^{1}$ King's College London / Cicely Saunders Institute, London, United Kingdom, ${ }^{2}$ Khartoum Oncology Hospital, Khartoum, Sudan, ${ }^{3}$ East Oncology Center, Gedarif, Sudan, ${ }^{4}$ Ministry of Health (Sudan), Khartoum, Sudan, ${ }^{5}$ National Cancer Institute, Medani, Sudan, ${ }^{6}$ Universidad de Las Palmas de Gran Canaria, Las Palmas, Spain

Background: Palliative Care needs can be identified using a validated tool. The need is enormous especially in low income countries. The need is for cancer and non-cancer patients.

Aims: To identify the number of adults with life-limiting illnesses; to describe them according gender, age, socioeconomic status; to generate evidence on their palliative care needs using a validated tool (integrated African POS)

Methods: This is a one-day census across several hospitals using a the tool. Demographic data, and diagnoses were also collected. Medical students were trained how to use the tool.

Only in-patients with a documented life-limiting illness (according to their charts) were consented to fill the IAPOS.

Results: A total prevalence of life-limiting diseases of $46 \%$ of in-patients at the general hospitals ( $48 \%$ of the census). Consent rate $99 \% .5 \%$ of patients excluded due to their morbid situation.

Gender Distribution: Males 49\%; Females 51\%; Age distribution: mean 48 years; SD 10.9 years; Diagnoses: Cancer $60 \%$ of cases; others $40 \%$; $59 \%$ know their diagnosis, 33\% don't know; and 9\% are misled; while $36 \%$ only know their prognosis; $53 \%$ don't know; $11 \%$ are misled.

Prevalence of high scores (4 and 5 out of 5 ) of the IAPOS: Pain 42\%; Dyspnoea 10\%; Fatigue 39\%; Nausea $9 \%$; Vomiting 5\%; Poor Appetite 25\%; Constipation 13\%; Sore/Dry Mouth 8\%; Drowsiness 9\%; Poor Mobility $33 \%$; Others $24 \%$; $35 \%$; Inability to share with others $17 \%$; Feeling life is worthless $25 \%$; Lack of Peace $16 \%$; Lack of help to family $28 \%$; Lack of information (carer): $23 \%$; Lack of confidence in caring for the patient (carer) 11\%; Family Anxiety 50\%.
Conclusion: This is the preliminary analysis of the data. The striking outcomes are the high pain scores and the family anxiety. While $59 \%$ of patients know their diagnosis, only $36 \%$ know the prognosis. For a mere $54 \%$ the prognosis was not discussed at all.

The total IAPOS score followed a normal distribution curve with a mean of 31 (considerably high) and a SD of 15.

Abstract number: P02-235

Abstract type: Poster

Translation into Spanish, Cross-cultural Adaptation and Validation of an Advance Care Planning Self-efficacy Scale Lasmarías C. ${ }^{1,2,3}$, Aradilla-Herrero A. ${ }^{4}$, Subirana-Casacuberta M. ${ }^{5}$, Ela $S^{1,2}$, Delgado S. ${ }^{6}$, Gómez-Batiste X. ${ }^{1,2,3}$

${ }^{1}$ The Qualy Observatory-World Health Organization Collaborating Center for Public Health Palliative Care Programs, Institut Català D'oncologia, L'Hospitalet de Llobregat, Spain, ${ }^{2}$ Chair of Palliative Care, Universitat de Vic-Universitat Central de Catalunya, Vic, Spain, ${ }^{3}$ Catalonia Chronic Care Research Group, Universitat de Vic-Universitat Central de Catalunya, Vic, Spain, ${ }^{4}$ Escuela Universitaria de Enfermería Gimbernat, Universidad Autonoma de Barcelona, Sant Cugat del Vallés, Spain, ${ }^{5}$ Consorci Hospitalari de Vic, Vic, Spain, ${ }^{6}$ St Luke's Hospice, London, United Kingdom

Background: Advance Care Planning (ACP) explores patients' values, desires and expectations to plan their care. Barriers for healthcare professionals to start it include fear to talk about end of life, lack of communication skills, specific timings. Self-efficacy (SE) is a key factor that impacts learning about ACP. Measuring SE would show the impact of training to acquire ACP competencies. The Advance Care Planning Selfefficacy (ACP_SE) scale of 17 items was validated in 2017; there are not similar validated tools in Spanish.

Aims: To make a Spanish cross-cultural adaptation and validation of the ACP_SE scale.

Methods (design, data collection, analysis): The ACP_SE scale was forwardbackwards translated. 10 ACP local experts tested it for clarity and comprehensibility. For the validation, we designed a survey with 4 dimensions: sociodemographic variables; knowledge and self-perception on ACP; type of patients attended; 4 scales to the predictive validity: ACP_SE (Baughman, 2017); Trait Meta-Mood Scale 24 (Salovey and Mayer, 1995); Personal Competence scale (Wallston, 1992); Coping with death Scale (Bugen, 1980). Participants: 5,500 professionals from 4 scientific societies: Palliative Care (PC); Geriatrics and Gerontologic; PC physicians; PC nurses. Reliability will be determined by intraclass correlation coefficients, the measurement will be compared by T'Student and internal consistency by Cronbach's $\alpha$. Testretest reliability will be quantified with a 4-week interval.

Results: After the translation/adaptation process, the scale ACP_SE has 19 items. New variables include to involve patients in ACP and to register ACP adequately. In a pilot study with 47 professionals, the consistency was $\alpha=0.909$. The results will confirm the scale's validity and show how professionals rate the ACP process.

Conclusion / discussion: A validated ACP_SE scale in Spanish will allow measuring the impact of training programs designed to implement ACP in the Spanish-speaking context.

Abstract number: P02-236

Abstract type: Poster

Symptoms and Palliative Care Concerns in Patients with Heart Failure Explored with the Integrated Palliative Care Outcome Scale (iPos)

Roch C. ${ }^{1}$, Palzer J. ${ }^{1}$, Störk S. ${ }^{2}$, Frantz S. ${ }^{2}$, van Oorschot B. ${ }^{1}$

${ }^{1}$ Interdisciplinary Centre Palliative Care, University Hospital Würzburg, Würzburg, Germany, ${ }^{2}$ Dept. of Internal Medicine I, University Hospital Würzburg, Würzburg, Germany 
Background: Admissions to the hospital may initiate the supportive and palliative care phase in patients with heart failure with the need for a comprehensive needs assessment.

Aim: To explore symptoms and palliative care concerns and to evaluate the knowledge about palliative care and the acceptance of iPOS in heart failure patients.

Methods: Survey of 100 heart failure patients in the first days of hospitalization (22.03.2018 - 10.08.2018) with the iPOS ( 3 day recall period, 17 questions with a five-point Likert scale (0-4), clinically relevant item values $>2$ ) and two additional questions regarding knowledge about palliative care and the acceptance of the iPOS.

Results: 60 male and 40 female patients with a mean age of 75.5 years (medium: 78.5 years). The mostly named clinically relevant item was "family anxiety (about the patient)" (62.0\%), followed by "dry mouth" (44.0\%), "poor mobility" (43.0\%), "dyspnea" (40\%), "lack of energy (37.0\%), "anxiety" (30.0\%),"pain" (28.0\%), "drowsiness" (23.0\%), "inability to share feelings" (21.0\%) and "feeling depressed" (21.0\%). 32.0\% of respondents knew about palliative care and $68.0 \%$ said they did not know this subject. $91.0 \%$ agreed that the iPOS is suitable for heart failure patients.

Conclusion / discussion: The iPOS was well accepted. Palliative care concerns are very common, most often regarding caregivers needs, oral care and symptoms of general impairment. Comprehensive multidisciplinary assessment especially in regard to psychosocial and family concerns as well as a close cooperation of cardiology providers and palliative care specialists seems to be necessary.

Abstract number: P02-237

Abstract type: Poster

Quality Monitoring for Palliative Care in Older People: Adapting the Q-PAC Indicator Set for Nursing Homes

De Schreye R. ${ }^{1}$, Dupont C. ${ }^{1}$, Cohen J. ${ }^{1}$, De Ridder M. ${ }^{2}$, Van den Block L. ${ }^{1}$, Deliens L.1,3, Leemans K. 1, $^{1,2}$

${ }^{1}$ End of Life Care Research Group, Vrije Universiteit Brussel \& Ghent University, Brussels, Belgium, ${ }^{2}$ Department of Radiotherapy, University Hospital Brussels, Brussels, Belgium, ${ }^{3}$ Department of Medical Oncology, Ghent University, Ghent, Belgium

Introduction: In Belgium as in many countries, a large number of people are admitted to nursing homes as they approach the end of life. Most nursing home residents suffer from progressive life-limiting conditions, increasing the need for high-quality palliative care in this setting. In order to guide nursing homes in quality improvement, a self-evaluation instrument is needed to monitor the quality of the palliative care they provide.

Aim: To adapt the Q-PAC quality indicator set, originally developed for specialized palliative care, to the nursing home context

Methods: First, we identified candidate quality indicators by comparing the Q-PAC set with recent literature and testing the candidate quality indicators for relevance in stakeholder interviews. Next, we organized an expert consultation round according to the RAND/UCLA method. The experts, representing practice, research and policy making, scored all indicators for appropriateness on a scale from 1 to 9 . The indicators for which no consensus was reached in the home assignment, were taken into group discussions. Only indicators with positive consensus among the experts were retained for the final quality indicator set.

Results: The expert panel selected a final set of 18 quality indicators, across 7 domains: physical aspects of care, psychological and social aspects of care, autonomy, communication with residents, communication with and care for relatives, communication between health care professionals, and circumstances surrounding death. Compared to the original Q-PAC set, there is less emphasis on physical aspects of care and more on autonomy.

Conclusion: We were able to adapt the existing Q-PAC quality indicator set for specialized palliative care to a comprehensive set for palliative care in nursing homes residents. These indicators are ready to be tested for feasibility and usefulness in the Flemish nursing homes.

Source of funding: Flemish Government, project number AZG/WEL/ TPKO/2018/20180215

Abstract number: P02-238

Abstract type: Poster

Complex Psychological Assessment in Highly Vulnerable Patients at a Specialised Palliative Care Unit - A Feasibility and Acceptance Study

Schulz-Quach C. ${ }^{1,2}$, Schmitz A. ${ }^{3}$, Fetz K. ${ }^{4}$

${ }^{1}$ St Christopher's Hospice, London, United Kingdom, ${ }^{2}$ Institute of Psychiatry, Psychology and Neuroscience, Department of Psychological Medicine, King's College, London, United Kingdom, 'LVR Clinic of Psychiatry, Psychosomatic and Psychotherapy for Children and Adolescence, Viersen, Germany, ${ }^{4}$ Chair of Research Methodology and Statistics, Faculty of Health, Witten/Herdecke University, Witten, Germany

Background: The core aim of palliative care is to treat patients holistically, acknowledging that the psychological dimension of a human being is a central aspect of quality of life. To address these issues it is important to understand patients' complex psychological condition as detailed as possible. Current research adds to this by advocating a more complex screening methodology to assess psychological phenomena in severely ill and dying patients.

Aims: To investigate the feasibility of a complex routine-assessment of psychological parameters (CRAT) within a vulnerable group of palliative care patients

Methods: The CRAT test battery included 9 validated psychological questionnaires (NCNN-Distress Thermometer, Peace of Mind-Questionnaire, Beck Depression Inventory - Fast-Screen, Demoralization-Scale, Existential Anxiety Questionnaire, State Trait Anxiety Inventory, Posttraumatic Stress Scale, NEO-FFI, Palliative Outcome Scale). The assessment was performed within 24 hours of admission to inpatient care using a tablet computer and concluded by an open-questions feedback survey. Reasons for early termination were recorded. Statistical analysis was conducted using IBM SPSS statistics version 21. ICD-10 diagnoses were independently assigned by two medical doctors specialized in medical psychotherapy.

Results: 140 patients (female: 52.9\%, male: $47.1 \%$ ) participated (inclusion rate $80.5 \%$ ). The dropout rate was $10.5 \%$. Patients averagely needed 85.6 minutes to fill out CRAT ( $S D=21.8$ ). Most frequent reasons for early termination were insufficient vigilance $(n=20 ; 48.8 \%)$ and emotional distress ( $n=10 ; 24.4 \%$ ). Most patients needed support for filling out the questionnaire ( $\mathrm{n}=81 ; 60.9 \%)$.

Conclusion: CRAT was implemented in clinicial routine care. From our results, CRAT appears feasible in a majority of patients. However, level of administration assistance and the length of assessment time were two limiting and challenging factors.

Abstract number: P02-239

Abstract type: Poster

QoD-LTC and QoD-LTC-C Scales. Usefulness, Setting where They Could Be Used and Preferences of Experts for the Spanish Context

Puente-Fernández D. ${ }^{1}$, Navarro -Sánchez A. ${ }^{2}$, García-Sanjuán S. ${ }^{3}$, Jimeno-Uclés R. ${ }^{4}$, Cabañero-Martínez M.J. ${ }^{3}$, Fernández-Alcántara M. ${ }^{3}$, Mota-Romero E. ${ }^{5}$, Roldán-López-de-Hierro C.B. ${ }^{6}$, Montoya-Juárez R. ${ }^{7}$ ${ }^{1}$ Universidad de Granada, Granada, Spain, ${ }^{2}$ Hospital San Rafael, Hermanos San Juan de Dios, Granada, Spain, ${ }^{3}$ Department of Nursing, University of Alicante, Alicante, Spain, ${ }^{4}$ Nursing Home EDADES, Jaén, Spain, ${ }^{5}$ Centro de salud Salvador Caballero, Granada, Spain, ${ }^{6}$ Department of Statistics and Operations Research, Faculty of Medicine, University of Granada, Granada, Spain, ${ }^{7}$ Department of 
Nursing, Faculty of Health Sciences, University of Granada, Granada, Spain

Background: No validated scales have been used to assess Quality of Death in Long Term Care Settings in Spain. The Quality of Death in Long Term Care (QOD-LTC) is an 11 items scale that is used in all decedents from the perspective of relatives and professionals. The QOD-LTC-C is a longer version with 23 items, only for cognitively intact decedents.

Aims: Assess if QoD-LTC and QOD-LTC-C scales are applicable to the Spanish context.

Methods: QOD-LTC and QOD-LTC-C were translated to Spanish and backtranslated to English in order to have a double-checked version. These versions were submitted to an expert-panel according to Delphi methodology. Thirteen experts with clinical or research experience in gerontology or palliative care rated the scales in consecutively 3 rounds. They were asked to rate the overall scales in a 5 Likert scale from 1 (nothing) to 5 (totally) in four dimensions: "How these scales contribute to measure quality of death", "The scales measure every aspect that need to be assessed", "Are they useful", "Are the scales applicable in other contexts such as chronic hospitals". Mean, SD and \% of 4 and 5 values were calculated for third round.

Results: The experts consider that QOD-LTC $(100 \%$ of $4 / 5 ; M=4,61$; $\mathrm{SD}=0,50)$ and $\mathrm{QOD}-\mathrm{LTC}-\mathrm{C}(92,3 \% ; \mathrm{M}=4,53 ; \mathrm{SD}=0,66)$ measure quality of death. $84,6 \%$ of experts consider that both scales measure every aspect of quality of dying, but the medium was higher in longer version $(M=4,38 ; S D=0,76)$ regarding the shorter one $(M=4,23 ; S D=0,92)$. Experts consider that QOD-LTC $(100 \% ; M=4,53 ; S D=0,51)$ and QOD-LTC-C $(92,3 \% ; M=4,38 ; S D=0,65)$ are useful. According to experts opinion, $\mathrm{QOD}-$ LTC $(100 \% ; M=4,69 ; S D=0,48)$ and QOD-LTC-C $(92,3 \% ; M=4,46 ; S D=1,12)$ might be used in chronic hospitals.

Conclusion: The QoD-LTC scale and QoD-LTC-C in their Spanish version are considered as useful, applicable and potentially might be use in nursing homes and chronic hospitals. The shorter version has more acceptance among Spanish experts.

Abstract number: P02-240

Abstract type: Poster

Translation and Psychometric Assessment of the German Version of the Death Attitudes Profile-revised

Schulz-Quach C. ${ }^{1,2}$, Jansen J. ${ }^{3,4}$, Fetz K. ${ }^{5}$

${ }^{1}$ St Christopher's Hospice, London, United Kingdom, ${ }^{2}$ Institute of Psychiatry, Psychology and Neuroscience, Department of Psychological Medicine, King's College, London, United Kingdom, ${ }^{3}$ Lukaskrankenhaus Neuss GmbH, Medical Clinic II, Neuss, Germany, ${ }^{4}$ Interdisciplinary Centre for Palliative Medicine, Heinrich Heine University Hospital Dusseldorf, Dusseldorf, Germany, ${ }^{5}$ Chair of Research Methodology and Statistics, Witten/Herdecke University, Witten, Germany

Background: In Germany, only limited data is available on death attitudes and existing measurements are complex and time consuming, and data on psychometric properties are limited. The Death Attitude ProfileRevised (DAP-R) captures attitudes towards dying and death. The measure consists of 32 items, which are assigned to 5 dimensions (Fear of Death, Death Avoidance, Neutral Acceptance, Approach Acceptance, Escape Acceptance). It has been translated and tested in several countries, but no German version exists to date.

Aims: This study reports the translation of the Death Attitudes ProfileRevised (DAP-R) into German (DAP-R-GR) using cross-cultural adaption process methodology and its psychometric assessment.

Methods: The DAP-R was translated following guidelines for cultural adaption. A total of 216 medical students of the Heinrich Heine University Duesseldorf participated in this study. Interrater reliability was investigated by means of Kendall's W concordance coefficient. The internal consistency of the DAP-R-GR Scales was assessed with
Cronbach's alpha coefficients. Split-half-reliability was estimated using Spearmann-Brown coefficients. Content validity was assessed by means of confirmatory factors analysis (CFA). All statistical analyses were performed using SPSS 24 and AMOS 22.

Results: The items showed fair to good interrater reliability with W-values ranging from .30 to .79 . Internal consistency of the five subscales ranged from .61 (Neutral Acceptance) to .94 (Approach Acceptance). Split-half reliability was good with a Spearman-Browncoefficient of .83. Results of CFA slightly diverged from the original scale. Conclusion: Our results suggest an overall good reliability of the German version of the DAP-R. DAP-R-GR promises to be a robust instrument to establish normative data on death attitudes for the use in German speaking countries.

\section{Abstract number: P02-241 \\ Abstract type: Poster}

Integrated Palliative Care Outcome Scale (iPOS) as a Tool to Identify Complex Palliative Care Needs - First Results of iPOSassessment in a Palliative Care Team

Roch C., Noeske L., van Oorschot B.

Interdisciplinary Centre Palliative Care, University Hospital Würzburg, Würzburg, Germany

Background: It is consent that palliative care should be early integrated into cancer care. How to identify patients needing specific support by palliative care specialists is an ongoing debate. The integrated palliative outcome scale (iPOS) is a well-established questionnaire to identify palliative care needs and is used to assess palliative care needs in the first consultation of patients assigned to the specialized palliative care team (PCT) at University Hospital Würzburg.

Aim: To distinguish patients in need of longer PCT-support from patients with less complex palliative care problems only once advised before further general palliative care.

Methods: We analyzed the iPOS from all inpatients of the PCT (01.01.2018 until 29.10.2018) from electronically patients records. Two groups were built: those who were supported by the palliative care service and those who had only one consultation. A t-test on the summary score from all 17 items (Likert-scale $0-4$, range $0-68$ ) and from the 10 single symptom items was performed.

Results: 538 patients were assigned to the PCT, 376 of them were supported during hospital stay $(69.9 \%)$ and 162 were once advised $(30.1 \%)$. 439 patients had cancer (CP, 81.5\%), 91 patients another disease (OP, $18.5 \%)$. There were no differences between cancer patients and otherregarding support or advice (CP $71.1 \%$ vs. OP $67.3 \%$ support, $p=.165$ ). In supported patients, the iPOS summary score was significantly higher than in advised patients (SP: mean 27.01, SD 9.565, OP: mean 24.45, SD $11.921, p=.027)$. Regarding the single symptom items, there was no significant difference between supported or advised patients.

Conclusions: The iPOS summery score distinguishes supported from advised patients. Further analyses, especially of the psychosocial palliative care concerns and iPOS-screening in the unselected palliative patient, are necessary on the way to identify a cut-off.

Abstract number: P02-242

Abstract type: Poster

Spirituality and Religiosity: Changes in the Adaptation of the QoD-LTC-C Scale to the Spanish Context. Delphy Methodology Puente-Fernández D. ${ }^{1}$, Esteban-Burgos A.A. ${ }^{1}$, Herrero-hahn $R .{ }^{2}$, GarcíaCano M.P. ${ }^{2}$, Hueso-Montoro C. ${ }^{3}$, Montoya-Juárez R. ${ }^{2}$, Roldán-Lopez-deHierro C.B. ${ }^{4}$

${ }^{1}$ University of Granada, Granada, Spain, ${ }^{2}$ Department of Nursing, University of Granada, Faculty of Health Sciences, Granada, Spain, ${ }^{3}$ Department of Nursing, University of Granada, Faculty of Health 
Sciences, Jaén, Spain, ${ }^{4}$ Department of Statistics and Operations Research, University of Granada, Faculty of Medicine, Granada, Spain

Background: The WHO includes Spirituality in the definition of palliative care. This sphere plays an essential role in the end of a person's life. Numerous assessment tools include this sphere, including Quality of Dying in Long-Term Care settings scale.

Aims: Determine which concept of spirituality would be most appropriate for the adaptation of a questionnaire.

Methods: The QOD-LTC-C was translated to Spanish and back-translated to English in order to have a double-checked version. This version was submitted to an expert-panel according to Delphi methodology. Thirteen experts with clinical or research experience in gerontology or palliative care rated all items of the scale in consecutively 3 rounds. In the first round experts a potential cultural conflict emerged for the item "[RESIDENT] was at peace with God", so the researchers propose an alternative item "[RESIDENT] was spiritually at peace". Both items were rated in two consecutively rounds using a Likert scale from 1 (nothing) to 5 (totally) in four dimensions: Relevance for quality of dying, clarity, same meaning according to English version and adequacy to Spanish context. Mean, SD and \% of 4 and 5 values were calculated for all dimensions and both items in Delphi third round.

Results: The original translation (referring to God) was rated lower in relevance for quality of dying (60\%;M=4.0;SD 1.22$)$, a clarity (91\%; $M=4.69 ; S D=0.63)$, but higher in same meaning (100\%;M=4.69). The item "[RESIDENT] was spiritually at peace" obtained a higher relevance $(84,6 \% ; \mathrm{M}=4.54 ; \mathrm{SD}=0.77)$ and clarity $(84,6 \% ; \mathrm{M}=4.46 ; \mathrm{SD}=0.77)$, but obviously lower same meaning (61.6\%;M=3.85; $\mathrm{SD}=1.28)$. "[RESIDENT] was spiritually at peace" was considered more applicable than "was at peace with God" to Spanish context by $92,3 \%$ of the experts.

Conclusion: As spirituality is a wider concept than religiosity, it is necessary to adapt evaluation tools in order to avoid words like "God" that could lead to misunderstandings.

Abstract number: P02-243

Abstract type: Poster

Early and Proactive Assessment of Palliative Care Needs in Oncology and Haematology: Afeasibility Study

Luque-Blanco A., Cruz-Sequeiros C., Cáceres-Muñoz A., Izquierdo-Gómez J., Moral-Torres A., Riera-Tornés G., Rodríguez-Morera A., Porta-Sales J. Support and Palliative Care Service, Catalan Institute of Oncology (ICO), Girona, Spain

Background: Published studies support the benefits of Palliative Care (PC) early intervention. The right point to introduce PC needs assessment is still under debate. Admission could be considered a "crisis point" in the Patients (Pts) disease trajectory and the right moment to assess PC needs, but acceptability by Pts needs to be probe.

Aims: To assess feasibility in terms of Pts' acceptance of an early and proactive Palliative Care Needs Assessment (PCNA) in the inpatient cancer Pts.

Methods: Cross-sectional study enrolling Pts admitted to the oncologyheamatology ward despite their disease type and stage. Within day 2-4 after admission a nurse-led PCNA was conducted including: symptoms, functional, nutritional, emotional and social needs. Exclusion criteria: foreseen stay $<5$ days; impending death and haematopoietic stem cell transplant. Pts were asked to assess confidentially the assessment appropriateness using a 4-point Likert scale ( $1=$ No appropriate; $2=$ Little; 3=Quite; 4=Completely). The PCNA was considered acceptable if $\geqslant 80 \%$ of the participating Pts scored $\geqslant 3$. Sample size of 139 eligible subjects we will be able to estimate a participation rate of $90 \%$ to within a $95 \% \mathrm{Cl}$ of $+/-5$. All Pts signed the informed consent.

Results: A total 239 Pts were admitted and 159 fulfilled the inclusion criteria. 146 (92\%) were evaluable, of those $2(1.4 \%)$ scored $2,33(22.6 \%)$ scored 3, and $111(76 \%)$ scored 4 . Refuse to participate $13(8 \%)$ Pts. Appropriateness (scores $3+4$ ) for Pts with haematological and non-haematological malignancies were $95.8 \%$ vs. $99.2 \%(P=0.3)$, and advanced 99.1\% vs non-advanced $97.1 \%(P=0.4)$.

Conclusion / Discussion: Early after admission and proactive nurse-led PCNA is perceived as highly appropriate by $98.6 \%$ Pts admitted to a standard acute oncology-haematology ward, despite the type of malignancy and stage. This results support the feasibility of Early and Proactive Assessment of Palliative Care Needs in an acute oncology setting.

Abstract number: P02-244

Abstract type: Poster

Translation and Cultural Adaptation of the QOD-LTC and QODLTC-C Scales to the Spanish Context

Puente-Fernández D. ${ }^{1}$, Perpiñá-Galváñ J. ${ }^{2}$, Clement-Carbonell V. ${ }^{3}$, Ruiz-

Miralles M.L. ${ }^{2}$, Cruz-Quintana F. ${ }^{4}$, Montoya-Juárez R. ${ }^{5}$, Roldán-Lopez-deHierro C.B. ${ }^{6}$

${ }^{1}$ University of Granada, Granada, Spain, ${ }^{2}$ Department of Nursing, University of Alicante, Alicante, Spain, ${ }^{3}$ Department of Health Psychology, University of Alicante, Faculty of Health Sciences, Alicante, Spain, ${ }^{4}$ Department of Personality, Assessment and Clinical Treatment., University of Granada, Faculty of Psychology, Granada, Spain, ${ }^{5}$ Department of Nursing, University of Granada, Faculty of Health Sciences, Granada, Spain, ${ }^{6}$ Department of Statistics and Operations Research, University of Granada, Faculty of Medicine, Granada, Spain

Background: No validated scales have been used to assess Quality of Death in Long Term Care Settings in Spain. The Quality of Death in Long Term Care (QOD-LTC) is an 11 items scale that is used in all decedents from the perspective of relatives and professionals. The QOD-LTC-C is a longer version with 23 items, only for cognitively intact decedents.

Aims: Assess if QoD-LTC and QoD-LTC-C scales are applicable to the Spanish context.

Methods (design, data collection, analysis).

QOD-LTC and QOD-LTC-C were translated to Spanish and back-translated to English in order to have a double-checked version. These versions were submitted to an expert-panel according to Delphi methodology. Thirteen experts with experience in gerontology or palliative care rated the scales in consecutively 3 rounds. Three dimensions were evaluated through a 5 points Likert scale from 1 (nothing) to 5 (totally): Relevance for quality of dying, clarity and adequacy to Spanish context. Experts proposed alternatives to items in order to adapt the scale to Spanish context. Consensus was established for a cut-point of $80 \%$.

Results: The response of 13 experts was obtained. The average age is 43 years with an average professional experience of 10.8 years.

A total of 27 items were evaluated. Consensus was obtained on all items for relevance, clarity and adequacy. Regarding the same meaning, only 4 required minor modifications in their translation to make them clearer. The experts also considered that it was necessary to add some items, one for control of pain and other to other symptoms at the end-of-life. An improvement is observed in the level of consensus of all the items in each round, finding a total agreement in 18 items (second) and 19 (third). The rest remained very close to the general consensus in the 3 dimensions.

Conclusion: QoD-LTC and QOD-LTC-C scales translation and adaptation are useful for the Spanish context. With the possible addition of two items related to symptom control.

Abstract number: P02-245

Abstract type: Poster

Validation of the Hungarian Version of the Professional Quality of Life Scale. An Opportunity to Improve the Well-being of Hospice Care Workers 
Kegye A. ${ }^{1}$, Takács S. ${ }^{2}$, Zana Á. ${ }^{1}$, Hegedüs $K .{ }^{1}$

${ }^{1}$ Institute of Behavioural Sciences, Semmelweis University, Budapest, Hungary, ${ }^{2}$ Department of General Psychology and Methodology, Károli Gáspár University of the Reformed Church in Hungary, Institute of Psychology, Budapest, Hungary

Background: Over the past 27 years, hospice and palliative care have gradually integrated into the Hungarian healthcare system. Key elements in the improvement of hospice care include a contribution to the retention of hospice health care workers via the targeted development of mental health promotion.

Aims: To improve the physical and mental health of workers by examining the psychometric characteristics and factor structure of the Hungarian version of the ProQOL5 questionnaire, which measures compassion fatigue and satisfaction, secondary traumatization and burnout.

Methods: A cross-sectional, anonymous, paper-based questionnaire was administered to hospice workers. To examine the factor structure of the measurement scale, explorative factor analysis was conducted (analysis of the main component, Varimax rotation). We employed the CES-D Depression Scale and the shortened form of the Maastricht Vital Exhaustion Questionnaire, the Beck Depression Scale, and the WHO General Well-Being Scale.

Results: 188 questionnaires were returned (female $86.2 \%$, male $13.8 \%$ ); 94 nurses, 31 physicians, and 63 persons working in other positions (psychologists, physiotherapists, pastors, coordinators). The internal consistency of the 4 scales created was acceptable according to the Cronbach-alpha values: Emotional overload resulting from work (ALFA $=$ $0.818)$, Satisfaction with work (ALFA $=0.869$ ), Compassion satisfaction $(A L F A=0.705)$, Secondary traumatization (ALFA $=0.656)$. The correlation of our measurement scales with the standardised scales for external validity exhibited sufficient strength and direction.

Conclusion: The validation of the Hungarian version of the ProQOL5 questionnaire and its practical application may contribute to a greater emphasis on compassion satisfaction and satisfaction with work. Furthermore, it may lend to the development of effective interventions and training programs focusing on the well-being of hospice workers, thus providing better care.

\section{Abstract number: P02-246 \\ Abstract type: Poster}

Spanish Translation, Cultural Adaptation and Validation of the New Version of the Demoralization Scale (DS-II) through a Multicenter Study in Advanced Cancer Patients

Belar A. ${ }^{1}$, Arantzamendi M. ${ }^{1}$, Rodríguez-Nuñez A. ${ }^{2}$, Santesteban Y. ${ }^{3}$, Martínez M. ${ }^{4}$, López-Saca M. ${ }^{5}$, Consigli S. ${ }^{6}$, López-Fidalgo J. ${ }^{1}$, Centeno C. $1,4,7$

${ }^{1}$ Universidad de Navarra. Instituto Cultura y Sociedad, Pamplona, Spain, ${ }^{2}$ Pontificia Universidad Católica de Chile, Santiago, Chile, ${ }^{3}$ Hospital San Juan de Dios Pamplona, Pamplona, Spain, ${ }^{4}$ Clínica Universidad de Navarra, Pamplona, Spain, 5 Universidad Dr José Matías Delgado, El Salvador, El Salvador, ${ }^{6} \mathrm{Hospital}$ Universitario Austral, Buenos Aires, Argentina, ${ }^{7}$ Instituto de Investigación Sanitaria de Navarra (IdiSNA), Pamplona, Spain

Background: Demoralization is a state of existential distress in patients with advanced illness.DS-II is an evaluation tool for this syndrome, which has recently been reformulated.

Aim: To obtain a Spanish version of DS-II and check its psychometric properties in advanced cancer patients in Spain and some LatinAmerican countries.

Methods: Following a translation-back translation process, a pilot test was carried out to verify its validity. A validation study was then undertaken, including advanced cancer patients attended by five palliative care teams in Spain, Argentina, Chile and El Salvador. Patients completed
DS-II(es), Hospital Anxiety and Depression Scale (HADS) and Edmonton Symptom Assessment System-revised (ESAS-r). Reliability was studied according to internal consistency; construct and concurrent validity with HADS and ESAS-r; discriminant validity using The Karnofsky Performance Status scale; feasibility, with response ratio and time required. Cut off points were established, and sensitivity and specificity were studied.

Results: The Spanish version of DS-II was obtained. 150 patients completed the validation study. Cronbach's Alpha for DS-II was 0.88 , for the sense and purpose subscale it was 0.83 and for coping ability, 0.79 . A confirmatory analysis using structural equation models with their corresponding latent constructs showed that all the items correlated positively on their subscales and on the overall scale. DS-II (es) correlated significatively with $\operatorname{HADS}(\mathrm{rho}=0.73, \mathrm{p}<0.001)$. Cut-off points at scores 10 and 20 out of 32 were stablished. The scale showed high sensitivity $(81.97 \%)$ and specificity $(80.90 \%)$. The prevalence of demoralization was $33 \%$ in our sample.

Conclusion: The Spanish version of the new Kissane DS-II demoralization scale has been shown to be valid, reliable and feasible with adequate psychometric properties when tested on advanced cancer patients.

Founding: Project funded by the Spanish Ministerio de Sanidad y Consumo, PI18/01703.

Abstract number: P02-247

Abstract type: Poster

\section{A 'SEDAPALL 'Classification of Sedative Practices in Palliative} Care Validation of Clinical Vignettes by Experts' Consensus in 2017-18

Bidegain-Sabas A. ${ }^{1}$, Burucoa B. ${ }^{1,2}$, Guirimand $F^{3}$, Devalois $B .{ }^{4}$

${ }^{1}$ Hôpital Saint André - CHU Bordeaux, Bordeaux, France, ${ }^{2}$ Université de Bordeaux, Bordeaux, France, ${ }^{3}$ Maison Médicale Jeanne Garnier, Paris, France, ${ }^{4}$ Centre Hospitalier de Pontoise, Pontoise, France

Background: A workgroup of the French Society of Support and Palliative Care ("Société Française d'Accompagnement et de soins Palliatifs" or "SFAP") has elaborated a classification called "SEDAPALL" describing the different sedative practices that can be used in palliative care. The classification follows three criteria: duration, depth and consent, and employs sixteen illustrative clinical vignettes which can be classified according to SEDAPALL.

Objective: The purpose of this study was to validate the precise wording of the clinical vignettes and their associated answers.

Methods: The Delphi consensus method was applied. Twelve specialists (physicians and nurses) from different Palliative Care departments in France, were solicited to participate to this study. During the first phase, they were asked to classify according to SEDAPALL the sedative practices illustrated in the sixteen clinical vignettes. During the following phases, the level of agreement of each expert with the wording of the vignettes and associated answers (such as elaborated by the SFAP's workgroup), as well as the level of agreement among such experts, were measured in order to evaluate the consensus and validate the clinical vignettes.

Results: Consensus was reached for fifteen of the sixteen vignettes and their answers during the second phase. Consensus for the remaining vignette was reached during the third phase after its rewording.

Discussion: The questions raised by the experts in their comments related to the intents and the indications for sedation and medication described in the vignettes. These vignettes simply serve as illustrations, not as recommendations.

Conclusion: All sixteen clinical vignettes enabling the use and thorough understanding of the SEDAPALL classification were validated. They will facilitate a better transmission of the SEDAPALL classification.

Abstract number: P02-248

Abstract type: Poster 
Is the Surprise Question Useful Instrument in General Internal Medicine Setting? A Prospective Study

Loucka M. ${ }^{1}$, Tučková A. ${ }^{1}$, Houska A. ${ }^{1,2}$, Havrda M. ${ }^{3}$

${ }^{1}$ Center for Palliative Care, Prague, Czech Republic, ${ }^{2} 1$ st Faculty of Medicine, Charles University, Prague, Czech Republic, ${ }^{3}$ University Hospital Kralovske Vinohrady, Prague, Czech Republic

Background: Surprise question ("Would I be surprised if this patient died in the next year?") is one of the most widely used triggers for palliative care inpatient consultation. However, its clinical feasibility is limited by the lack of supportive evidence for its validity outside cancer care setting. Aims: To test the 6-month version of the surprise question in the context of general internal medicine department and to compare the difference in correct estimates between physicians and nurses.

Methods: This was a prospective study, conducted at a general internal medicine ward at a large university hospital. All new patients were assessed by admitting physicians and admitting nurses (mostly without any palliative care training), using 6-month version of the surprise question. In total, 385 patients were assessed by physicians and 377 by nurses. Patients or their next of kin were contacted in 6-month follow-up by research administrator/nurse checking on the health status of the patient. Results: Sensitivity of the 6-month surprise question was $73 \%$ and specificity $72 \%$ when estimated by physicians and $57 \%$ and $77 \%$ when estimated by nurses. Chronic heart failure was the only condition, at which physicians were not better in predicting correct outcome (correct outcome predicted in $50 \%$ of patients). There was no significant difference in number of correct estimates between patients with cancer and other diagnoses $(p=.08)$.

Conclusion: The 6-month surprise question is a valid tool for advance care planning in the general medicine setting and can be used by clinicians to facilitate implementation of palliative care.

This work was supported by the Czech Science Foundation grant No. 17-26722Y.

Abstract number: P02-249

Abstract type: Poster

\section{Assessing Social and Spiritual Needs of Hospice Patients, a} Feasibility Study

Graaf E., Lormans T., De Leeuw M., Van der Baan F., Teunissen S. University Medical Center Utrecht, Center of Expertise in Palliative Care Utrecht, Dept. General Practice, Julius Center for Health Sciences and Primary Care, Utrecht, Netherlands Presenting author email address: e.degraaf@umcutrecht.nl

Background: Hospice care aims to optimize quality of life by diminishing physical, psychological, social and spiritual suffering. In routine hospice care, the social and spiritual dimensions are less assessed and reported. To support the structural assessment of these dimensions, the four-dimensional Utrecht Symptom Diary (USD4D) was developed based on the core Utrecht Symptom Diary (Dutch ESAS) and the Ars Moriendi model.

Aim: Evaluate the demand, practicality and acceptability of the USD4D to assess social and spiritual needs of hospice patients.

Method: A feasibility study with an exploratory mixed-method design was conducted from January to June 2017 in the Netherlands.

Quantative phase: Demand was defined as the frequency of completed USD4D items by 157 hospice patients admitted to hospice in 2016.

Practicality was defined as the frequency the social and spiritual dimensions are reported in the records from 30 hospice patients admitted in 2016 to 6 hospices using the USD4D in routine care.

Qualitative phase: Practicality and acceptability were evaluated in 4 focusgroup interviews with multidisciplinary teams using the USD4D in routine hospice care.

Results: Patients completed the USD4D 2.8 times on average, every 9 days. During admission, the social- and spiritual dimension were described resp. 14 and 15 times on average per record, predominantly by nurses. Discussing the USD4D with patients contributes to an indepth exploration of the spiritual dimension. Integration of the USD4D in daily care depends on nurses' competences, level of gate keeping and interdisciplinary collaboration.

Conclusion: Symptom assessment of the social and spiritual dimension by means of the USD4D is feasible in terms of demand, practicality, and acceptability. Implementation strategies of the USD4D should focus on the development of competence of professionals and interdisciplinary collaboration to optimize the integrated use of the USD4D and ameliorate quality of life of hospice patients.

Abstract number: P02-250

Abstract type: Poster

Is an Online Application to Assess Symptom Severity Feasible in Hospice Care?

Graaf E., Lormans T., Heij S., Van der Baan F., Teunissen S.

Center of expertise in Palliative Care Utrecht, dept. General Practice, Julius Center for Health Sciences and Primary Care, University Medical Center Utrecht, Utrecht, Netherlands

Background: Patient reported outcomes are the gold standard to assess symptom intensity. The Utrecht Symptom Diary application (USD-app) is an eHealth self-assessment tool to measure symptom intensity.

Aim: To evaluate the demand, acceptability and integration of the USDapp as a basis for symptom management in hospice care.

Method: This sequential, explanatory mixed-method feasibility study was performed from February to June 2017 in the Netherlands. Nurses working in the 14 hospices using the USD-app in January 2017 were eligible to participate. The main outcomes were:

1) demand of the USD-app for hospice patients,

2) acceptability of the USD-app for hospice nurses and

3) integration of the USD-app in symptom management in daily hospice care.

Data were collected using

1) a self-designed questionnaire and

2) focus groups. Data analysis was performed using descriptive statistics and content analysis respectively.

Results: 80/151 nurses were enrolled. On average the app was used for $332 / 413$ patients, mostly with help from a nurse. Overall $63 \%$ of nurses were satisfied with the USD-app. The use of the app supported deploying appropriate interventions and improved insight into the course of symptoms and the patients' well-being. The development of alternative answering scales besides the numerical scale was suggested in all focus groups. Some nurses indicated that patients could not use the app, but, this was not discussed with a patient.

Conclusion: The USD-app in hospice care is feasible in terms of demand and acceptability. Integration of the USD app in daily care could be ameliorated by

1) improving knowledge and competences in multidimensional symptom management,

2) raising awareness of gatekeeping attitudes of caregivers and

3) support from all members of the multiprofessional team to integrate the use of the USD app.

Future research should evaluate the use of additional scales in the USDapp fitting the patients preferences and abilities. 
Abstract number: P02-251

Abstract type: Poster

Risk Assessment and Diagnostic Tools to Assess Problematic Opioid Use in Palliative Care: A Scoping Review Lau J. ${ }^{1}$, Mazzotta P. ${ }^{2}$, Kevork N. ${ }^{3}$, Rho H. ${ }^{4}$, Fazelzad R. ${ }^{3}$, Sud A. ${ }^{4}$, Baxter S. ${ }^{5}$, Cory S. ${ }^{6}$, Smith A. ${ }^{7}$, Henderson D. ${ }^{8}$, Furlan A. ${ }^{9}$, Zimmermann C. ${ }^{1}$ ${ }^{1}$ Palliative Care, Princess Margaret Cancer Centre, Toronto, Canada, ${ }^{2}$ Temmy Latner Centre for Palliative Care, Toronto, Canada, ${ }^{3}$ University Health Network, Toronto, Canada, ${ }^{4}$ University of Toronto, Toronto, Canada, ${ }^{5}$ Canadian Hospice Palliative Care Association, Ottawa, Canada, ${ }^{6}$ Canadian Virtual Hospice, Winnipeg, Canada, ${ }^{7}$ Center for Addiction and Mental Health, Toronto, Canada, ${ }^{8}$ Colchester East Hants Health Center, Truro, Canada, ${ }^{9}$ Toronto Rehabilitation Institute, Toronto, Canada

Background: Opioids are essential medicines in palliative care, but are susceptible to problematic use.

Aim: To identify whether there are validated opioid misuse, abuse and diversion (OMAD) risk stratification and diagnostic tools that can be used in palliative care populations.

Methods: Comprehensive searches were conducted of electronic databases from inception to March 2018 as well as reference lists and grey literature. We included clinical research studies involving adults with diagnoses in three main disease groups:advanced cancer, neurodegenerative diseases and end-stage organ diseases. There were no restrictions on country of origin, language or publication status.

Results: Of 26,412 records independently screened by two investigators, 37 studies investigated the use of at least one risk assessment and diagnostic tool in the palliative care setting. The main tools used were: CAGE $(n=8)$, CAGE-AID $(n=4)$, Opioid Risk Tool $(n=8)$, screener and Opioid Assessment for Patients with Pain (SOAPP, $n=2$ ), SOAPP-Short Form (SOAPP-SF, $n=6$ ), SOAPP-revised (SOAPP-R, $n=1$ ), and urine drug tests (UDT, $n=16)$. Most studies were conducted in the USA ( $n=36)$, in outpatient clinics $(n=28)$, and in cancer $(n=31)$. Study designs were: retrospective cohort $(n=15)$, prospective cohort $(n=4)$, case report $(n=4))$ and case control $(n=3)$; 3 were surveys, 5 quality improvement and 2 "other" designs. No study validated the CAGE, CAGE-AID, ORT, SOAPP and SOAPP-SF for use in palliative care and none used comparators to ensure accuracy of the tools' scores and interpretation. The frequencies of positive scores: CAGE and CAGE-AID score, $14 \%$ to $19.4 \%$; ORT "high risk", $6 \%$ to $50 \%$; SOAPP R, SOAPP, and SOAPP-SF, $9.3 \%$ to $46 \%$; and abnormal UDT, $8.6 \%$ to $73 \%$.

Conclusion: There are no risk assessment and diagnostic tools validated to assess for OMAD in palliative care and most studies are limited to cancer outpatients. High quality studies are needed to assess for problematic opioid use in palliative care.

Abstract number: $\mathrm{P} 02-252$

Abstract type: Poster

Psychometric Validation of the Integrated Palliative Care Outcome Scale in Greek: Pilot Study

Katsaragakis S. ${ }^{1}$, Patiraki E. ${ }^{1,2}$, Deskou F. ${ }^{1,2}$, Xenikou M. ${ }^{2}$, Avgetidou H. ${ }^{2}$, Palourgiotou G. ${ }^{2}$, Tserkezoglou A. ${ }^{2}$, Anagnostou D. ${ }^{3,4}$

${ }^{1}$ Nursing, National and Kapodistrian Univesrity of Athens, Athens, Greece, ${ }^{2}$ Palliative Care Unit Galilee, Spata, Greece, ${ }^{3}$ Faculty of Medicine, Division of Human Health Sciences, Kyoto University, Kyoto, Japan, ${ }^{4}$ School of Medicine, Marie Curie Palliative Care Center, Cardiff University, Cardiff, United Kingdom

Background: The Integrated Palliative Outcome Scale (IPOS) is a well accepted valid and reliable instrument worldwide.

Aim: To test the psychometric characteristics of the culturally adapted Greek IPOS
Methods: Data was collected in a palliative care service. Inclusion criteria: cancer diagnosis, $>18$ years old, able to communicate. Participants completed the IPOS, the Greek validated European Oncology Research and Treatment of Cancer-Palliative (EORTC-PAL) and Functional Assessment of Cancer Therapy-General (FACT-G), at admission $\left(\mathrm{T}_{0}\right)$ and one week later $\left(\mathrm{T}_{1}\right)$. A health care professional (HCP) completed separately a proxy version of the above scales at $\mathrm{T}_{1}$. Demographic and clinical characteristics were also collected.

Results: 29 patients (response rate 61.7\%) completed data collection. 15 HCPs completed the scales on the same day with patients. There was almost no missing data for IPOS assessments for both patients and HCPs. Internal consistency assessed with Cronbach's alpha varied from .698 $\left(\mathrm{T}_{0}\right)$ to $.702\left(\mathrm{~T}_{1}\right)$ (patient's version) and .699 (HCP's version). Most IPOS items were medium to strongly correlated with similar EORTC-PAL (rho patient $\mathrm{T}_{0} .37-.88, \mathrm{~T}_{1} .37-.84$, HCP .37-.91) and FAGT-G (rho patient $\mathrm{T}_{0}$ -.39 to $-.72, \mathrm{~T}_{1} .-37$ to -.71 , HCP -.34 to -.81$)$ subscales $(p<.050)$ except of Sore or Dry Mouth, Family Anxiety, Information and Practical Problems for both patients and HCPs. Although overall IPOS patient's scores improved at $\mathrm{T}_{1}(\mathrm{p}=.012)$ (mean $\mathrm{T}_{0} 27.8$ vs $\mathrm{T}_{1} 23.3$ ) statistically significant improvement was found only at Shortness of Breath $(p=.004)$, Vomiting $(p=.030)$, Family Anxiety $(p=.028)$ and Information $(p=.033)$. Strong correlation ( $p<.026$, rho: .041-.091) was found between patients' and HCPs' all IPOS item category responses (0, 1-2, 3-4) except Information. The Cohen's к varied: .089- .023.

Conclusion: This pilot study showed overall acceptable internal consistency, interrater and criterion validity for the IPOS Greek version but with limitations to address.

Abstract number: P02-253

Abstract type: Poster

Developing an Evidence Base for Community Palliative Care Discharge Criteria through an Audit Cycle

Davies J.M.V., Capel M., Rees S.

City Hospice, Cardiff, United Kingdom

Background: Community Palliative Care discharge criteria are utilised frequently throughout the United Kingdom in identifying patients suitable for discharge, often with individual organisations possessing their own criteria. A literature review of the evidence base for these criteria shows robust evidence of a valid criteria is lacking.

Aims: To complete an audit cycle looking at discharges from City Hospice, a South Wales Community Palliative Care team.

Audit/Reaudit standard: $100 \%$ of patients discharged from the service should be done so in line with the service Discharge Policy (DP).

Further information was collected on post-discharge outcomes at 3 months (re-referral to the service, acute admissions and deaths). Through this, validation of the DP in identifying appropriate patients for discharge may be possible.

Methods:

Initial audit: Retrospective case note review of all discharges over 3 month period $(n=37)$. Based on audit results, a Discharge Criteria Checklist (DCC) to be used for each patient discharged from the service was produced.

Reaudit: Retrospective case note review of all discharges over 3 month period ( $n=25), 1$ month following implementation of Discharge Criteria Checklist (DCC).

Results: Compliance with DP was $89 \%$ in initial audit, improving to $100 \%$ in the reaudit.

In the reaudit, $76 \%$ of discharges utilized the DCC.

Using pooled data from both audits, the following data was for patients 3 months post discharge was true:

1. $82 \%$ were alive.

2. Mean palliative prognostic index was low (2.3). 
3. $29 \%$ had been admitted acutely, all appropriate admissions on case-note review.

4. Only 1 patient was re-referred within 3 months.

Conclusion: The compliance with the DP improved with implementation of DCC.

An evidence-base for Community Palliative Care Discharge Policies is lacking: post-discharge outcomes of this audit cycle validate City Hospice's DP as appropriately identifying patients suitable for discharge.

Abstract number: P02-254

Abstract type: Poster

Advance Care Planning Evaluation: A 3-fold Plan for the Future Johnstone R. ${ }^{1}$, Poolman M. ${ }^{2}$, Usborne C. ${ }^{3}$, Pottle J. ${ }^{3}$, Hiscock J. ${ }^{4}$ 1Palliative Care Department, Betsi Cadwaladr University Health Board, Caernarfon, United Kingdom, 2Primary Care Research, Bangor University, Wrexham, United Kingdom, ${ }^{3}$ Betsi Cadwaladr University Health Board, Wrexham, United Kingdom, ${ }^{4}$ Primary Care Resaerch, Bangor University, Wrexham, United Kingdom

Advance care planning (ACP) is a patient driven discussion about wishes and preferences for future care. Discussions are documented in a patient held ACP document. An ACP is of particular relevance if disease progression impacts on capacity or capability of the patient to speak for their self. Evaluating ACP as a complex intervention requires a mixed method, multi-study approach assessing impact on patients, those important to them, healthcare professionals, service development and delivery. A three part evaluation was developed with two parallel

Aims:

i) Evaluate the impact of ACP education on practice and to inform the continued provision of ACP training.

ii) To understand the unanswered questions relating to $\mathrm{ACP}$ and the research challenges these raise. Awareness of ACP amongst the general public and healthcare professionals is varied. To support the introduction of ACP discussion opportunities a series of 24 half day ACP workshops were provided, training 363 healthcare professionals from a variety of clinical backgrounds and settings.

Results: Immediate feedback indicated understanding (98\%) skills (89\%) and confidence (86\%) were increased. Six months later we evaluated the transfer of knowledge from the classroom to the clinical setting. Utilizing ACP workshop registers a 14 item electronic survey was distributed to all workshop attendees. The response rate was $19 \%$ and indicated that ACP understanding, skills and confidence were increased. Transfer of ACP knowledge into practice was positively indicated by $68 \%$ of respondents with barriers identified as: "staff shortages", "not enough time", "not my role to do thi",. Exploration of the survey outcomes was done by inviting survey respondents to participate in a focus group session concerned with ACP. Twenty two participants were identified with 19 agreeing to attend a focus group.

Conclusion: Combined outcomes will support training provision and inform development of future ACP evaluation and research.

Abstract number: P02-255

Abstract type: Poster

Improving On-call Handover in a Hospice Inpatient Unit

Soutar D., Lundy E.

Belfast Health and Social Care Trust, Belfast, United Kingdom

Aim: The Royal College of Physicians (RCP) has identified poor handover as a major, preventable cause of patient harm. In the non-resident on-call setting, the accuracy and efficiency of the handover becomes even more important as this information will often play a vital role in initial decision-making.

The aim was to highlight weaknesses and inconsistencies in the handover process and implement changes in line with RCP guidance.

Design, methods and approach taken: An anonymous questionnaire was distributed to all physicians on the on-call rota. This questionnaire sought opinions on the practice of good handover. We also provided a free text space for other suggestions on how handover could be improved upon.

A new electronic handover document was then designed after reviewing the RCP guidance and results from the questionnaire. This document was implemented for a 6-month trial period. Following this, a second questionnaire was distributed to glean opinions on the impact of the changes.

Results:

$>60 \%$ felt patient care had improved.

$>70 \%$ of physicians felt their job was made easier by the new format.

$54 \%$ felt that the document tended to get over-crowded with irrelevant information.

$100 \%$ agreed that direct consultant input is useful when populating the template; previously this was informally sought.

Free text responses highlighted education on good handover practice and how to populate the document as vital.

Conclusion: These results led to the introduction of an updated document template as well as a set of handover guidelines that were disseminated and included in the induction packs for new physicians. Consultant ward rounds were identified as opportunities to discuss important points to be added to the handover.

Through this project the handover process has been brought into line with RCP recommendations. Specifically via standardisation of the process and documentation, providing handover training and education, and allowing for audit and collection of feedback from participants.

Abstract number: P02-256

Abstract type: Poster

THINK Delirium: A Step-wise Approach to Recognising and

Managing Delirium in Palliative Care

Monnery D. ${ }^{1}$, Schofield S. ${ }^{2}$, Powell P. ${ }^{2}$

${ }^{1}$ Clatterbridge Cancer Centre NHS Foundation Trust, Wirral, United

Kingdom, ${ }^{2}$ Willowbrook Hospice, Prescot, United Kingdom

Background: In terminal cancer patients, the prevalence of delirium may be as high as $85 \%$ of which $50 \%$ may be reversible. However, this depends upon correct diagnosis, which is often complicated in patients who are dying and commonly mistaken for terminal agitation. We have previously contributed to the formation of a NICE-accredited regional guideline to aid diagnosis and evidence-based management of delirium. However further action has been needed to update our local practice.

Aims: To improve the quality of care for patients with delirium in our hospice.

Methods: We designed and implemented a new quick reference guide for the assessment and management of delirium including the Confusion Assessment Method (CAM) for routine patient screening and a step chart approach to holistic management, including evidence-based medications. We also designed and delivered an education package to all clinical staff and involved patients in the design of a new information leaflet. A completed audit loop was undertaken to measure impact.

Results: Our education package resulted in an $81 \%$ improvement in all clinical staff confidence in assessing and managing delirium. Knowledge of guidelines around delirium diagnosis and management reached $91 \%$ 3 months after training delivery with $73 \%$ reporting being up to date with their training needs ( $14 \%$ previously). The of the CAM to diagnose delirium increase from $0 \%$ to $87.5 \%$ of patients with delirium 
and appropriate prescribing reached $90 \%$, with medications used more judiciously after non-pharmacological therapies. The patient information leaflet has been positively commented on by patients and relatives and subsequently adopted by other regional healthcare providers.

Conclusions: The combination of a step-wise approach to diagnosis and management of delirium combined with an education package and patient information leaflet has significantly improved care for patients with delirium in our hospice.

Abstract number: P02-257

Abstract type: Poster

\section{Safe Prescribing when Starting Strong Opioids}

Webber N., Flynn S., Halley A.

Royal Marsden Hospital, London, United Kingdom

Background: Opioids are central to the management of cancer pain. Effective and safe titration of opioids is paramount. Prescribers should adhere to NICE guidelines ${ }^{1}$ (2012), and guidance from the Department for Transport and the Faculty of Pain Medicine. The latter address the responsibility of prescribers with respect to new drug driving legislation that came into force in England and Wales in 2015.

Aim: To examine strong opioid prescribing practice against national guidelines for opioid use in palliative care and current best practice guidance on opioid use and driving.

Method: A retrospective audit of adult out-patients initiated on strong opioids at a tertiary cancer centre. Data was collected from the electronic records of 50 patients who attended out-patient clinics in October 2017. Results: Morphine Sulphate IR/MR was used first line in $72 \%$ of patients. The initial maximum possible daily dose of strong opioid prescribed exceeded NICE recommendations in $44 \%$ of cases. $25 \%$ of patients received appropriate breakthrough doses. Prophylaxis for nausea and constipation was prescribed in $26 \%$ and $32 \%$ of cases respectively. All patients received a strong opioid information leaflet; verbal communication about strong opioids was evidenced in $18 \%$ of notes. Driving status was checked for $6 \%$ of patients. Counselling on driving and concurrent opioid use for these patients was not complete.

Discussion: Robust changes have been implemented to improve adherence to the guidelines in the key areas highlighted in this audit. Prescription forms have been amended to include prompts for prophylactic prescribing. The opioid information leaflet now empowers patients to monitor their daily opioid use, be aware of signs of toxicity, and seek medical advice when appropriate. It also now reflects current recommendations on strong opioids and driving. The pharmacy department are revising procedures to review the initial maximum possible daily dose of strong opioid prescribed.

Abstract number: P02-258

Abstract type: Poster

\section{What Happens to Palliative Patients who Come to Emergency Department?}

Martin-Marco A., Taberner-Bonastre M.T., Lafuente-Sanchis P., TarrasoGomez M.L., Girbes-Mari R., Clep C., Flor-Bustos L., Duart-Clemente C., Torrijos-Pastor A., Marques-Aguilar E.

Hospital Universitario de La Ribera, Alzira, Spain

Introduction: We wonder what happens with palliative patients who need urgent attention. To answer this question, we collected all the episodes of emergency department admissions that occurred in the first nine months of 2017 in the health area of La Ribera, both at the primary and hospital levels.

Material and method: In total we collected 693 episodes of which 452 occurred in patients who required urgent attention on more than one occasion.
Results: Only one of the patients who required assistance on more than one occasion died compared to the 163 patients who died within 10 days after their only urgent visit. Regarding the admissions that occurs from the emergency department, in practice we did not find statistically significant differences ( $p$ 0.107) with regarding to the number of visits they had made to the emergency department (141 admissions among those who requested attention on more than one occasion compared to 88 in the group that only came once). In which we did find significant differences was in the place of death, since proportionally more patients die at home (94 of 459 admitted versus 65 of 164 who died in the hospital, with a statistical significance of $p=0.02$ ).

Discussion: The fact of having many consultations in the emergency services does not seem to indicate greater severity, since many more patients die among those who only consult once and also were admitted much less. Regarding the place of death, it is more frequent to die at home than at the hospital, which seems logical since it is desirable for this type of patient. In summary, the patients who most frequent visit emergencies departments are the least severe and those who need less hospital admissions.

Abstract number: P02-259

Abstract type: Poster

Differences in the Requirements of Urgent Care in Palliative Patients Based on whether They Are Oncological or Not Martin-Marco A., Taberner-Bonastre M.T., Lafuente-Sanchis P., Clep C., Tarraso-Gomez L., Magri-Mari R., Flor-Bustos L., Sanchis-Aguilar P., Torrijos-Pastor A., Duart-Clemente C.

Hospital Universitario de La Ribera, Alzira, Spain

Introduction: Emergency departments are seen by the population as a way to obtain health care quickly. This means that there is a high demand by general population. Our objective was to determine the use of resources for urgent medical care among palliative patients and to determine the differences between oncological and non-oncological patients. Methods: All adult patients who came to the hospital and primary care of La Ribera health department during the first nine months of 2017 were collected.

Results: During this period, 88,204 hospital emergencies were attended, of which 484 episodes were from palliative patients, representing an incidence of 5.49 per thousand episodes.

In primary care, 209 episodes of palliative patients were evaluated with respect to 182,083 total episodes, with an incidence of 1.15 per thousand inhabitants.

Of the total hospital emergency episodes in general population, they required admission $(11,217)$; there were 229 episodes of palliative patients that required admission, which means that $2.04 \%$ of hospital admissions in palliative patients were made from emergency department.

$57.1 \%$ of the episodes were from oncological patients, which means 396 episodes, compared to 297 episodes of non-oncological episodes (42.9\%).

We tried to find differences in terms of mortality and need for admission between the group of cancer patients and non-cancer patients, for which contingency tables were made to calculate the $\chi 2$ statistic. We find a value of $\chi 2$ of 5,7 with a $p=0,016$ in favor of a greater mortality of oncological patients; of the total number of palliative patients assessed (241), 164 died, representing $68 \%$ of the patients attended. Of these, $107(65.2 \%)$ were cancer patients and $57(34.7 \%)$ non-cancer patients.

Abstract number: P02-260

Abstract type: Poster

Palliative Care Patients in the Emergency Department Bergbrant I.-M., Carling L. 
Department of Palliative Care, Sahlgrenska University Hospital, Gothenburg, Sweden

Background: One aim of Advanced Medical Attendance at Home (AMAH) is to create security for patients and family. This includes the confidence that acute and complex medical problems can be handled at home. Despite this, patients in such care sometimes call at the Emergency Department (ED).

Aim: Our aim was to increase knowledge about why and when patients in AMAH call at the ED and thus find measures to minimize these visits. Method: We reviewed the medical records of patients enlisted in AMAH in two Swedish cities $2017(n=722)$. We analysed the following information:

- Who took the initiative to call at the emergency department

- What caused the visit

- Did the patient receive inpatient care, and if so, for how long

Result: $16 \%(n=115)$ of the enlisted patients called at the ED one or more times. $50 \%$ of the visits were due to recommendation from the palliative care consultant, $10 \%$ was initiated by another care-giver. In the remaining $40 \%$ the patient or family called at the ED without prior contact with the AMAH team. The most common causes for ED visits were acute abdominal conditions, dyspnoea and general deterioration. $60 \%$ of the visits led to inpatient care (median length 5 days). Our estimation is that $20 \%$ of the visits initiated by patient/family could have been avoided, had they contacted the AMAH team for assessment. Conclusion: Many ED visits could have been avoided through treatment at home. Thus it is an ongoing obligation to create confidence in the competence of the AMAH team among patient and family. The team has a pedagogic task to explain how acute and complex problems can be handled at home. However, inpatient care is occasionally unavoidable. It would then be beneficial if palliative care patients could be transferred directly to a suitable ward instead of spending time in the ED.

Funding: The study was funded by the Department of Palliative Care, Sahlgrenska University Hospital

Abstract number: P02-261

Abstract type: Poster

Measuring the Impact of the Irish Cancer Society's Night Nursing Service

Ferns M., Madigan M.

Irish Cancer Society, Dublin, Ireland

\section{Background:}

The night nursing service supports the patient and family through end of life care in the home setting. It is a free service funded by donations, with each patient entitled to 10 nights of care. A night nurse visits the patient's home between $11 \mathrm{pm}$ and $7 \mathrm{am}$.

Aims

The report aimed to evaluate and develop a greater understanding of the impact of the Society's palliative services. It also aimed to develop a greater understanding of how to improve our palliative services.

Methods:

Respondents from the Night Nursing service who had used the service between $1^{\text {st }} A$ pril and $31^{\text {st }}$ October 2016 were asked to complete a paper based survey with quantitative and qualitative questions. In February 2017, we sent a survey to 380 families and friends of people who had used the Night Nursing service between $1^{\text {st }}$ April and 31st October 2016. We received a total of 163 responses which represented a $43 \%$ response rate.

Data was exported from survey monkey into an excel file. Scaled data was collapsed and combined to show one percentage by calculating an average of 'agree strongly' and 'agree slightly' for most scaled questions.
For the qualitative questions, themes, key words and phrases as well as patterns were identified from the data.

Results: There is clear evidence that patients prefer to die at home surrounded by family and friends, with $86 \%$ of respondent's requesting to die at home. $88 \%$ of families felt less anxious and more in control of caring for the patient as a result of the presence of a Nigh Nurse. Respondents felt that extra nights would have been beneficial.

\section{Conclusion:}

The data shows that the night nursing service is vital in providing end of life care to patients in their home. It was clear that the nurses excelled in guiding families through the care of patients in the home setting. It was evident from the responses that people want to die at home surrounded by family and friends and they advocated for an increase in nights of care.

\section{Abstract number: P02-262 \\ Abstract type: Poster}

\section{Care of the Dying in a Teaching Hospital Documentation of NFR} Guthrie C. ${ }^{1}$, Aggarwal G. ${ }^{2}$, Lee J. ${ }^{1}$

${ }^{1}$ Concord Hospital, Palliative Care, Sydney, Australia, ${ }^{2}$ Concord Centre for Palliative Care, Palliative Care, Sydney, Australia

Background: All too often medical clinicians fail to recognise that patients are in the final phase of their lives. This commonly results in patients being put through unnecessary and invasive procedures and tests, in an attempt to prolong their lives. At this time the focus should be on excellent symptom management so that their quality of life is optimised and preserved.

Aim: The aim of this study was to look at the incidence of documentation of Not for Resuscitation (NFR) in all deaths on the ward after admission to hospital.

Methods: A retrospective chart review was conducted at Concord Repatriation General Hospital, a major Australian teaching hospital. Data was collected from 150 consecutive deaths between Jan and June 2011. This included if patients were considered to be dying at the time of admission, if and when these patients were made NFR prior to death, and the designation of the medical staff having the discussion about resuscitation.

Results: The median age of patients was 82 years with $61 \%$ males and $39 \%$ females. On admission $68 \%$ were considered to be dying, $24 \%$ were admitted with an NFR, however, prior to death, $97 \%$ had an NFR order in place. $30 \%$ of these patients were made NFR in the last 48 hours.

Discussion: The issues around the care of the dying patient are complex and numerous. One of the main issues involves the over treatment of patients, subjecting them to futile treatments, because we often fail to recognise dying. The economic and human cost of these interventions should be questioned. As with a previous audit completed on patients who died in 2001/2002 it was found that more than half of the conversations around NFR were had by junior doctors who have not had adequate training/experience to do so.

Abstract number: P02-263

Abstract type: Poster

Patients, Relatives and Health Care Professionals (HCP) Views of the Terminology "Palliative Care Clinic" in an Acute Teaching Hospital

Hlaing S., Holtom N., Grant L.

Palliative Medicine, Norfolk and Norwich University Hospital, Norwich, United Kingdom

Objectives: To ascertain whether the terminology 'Palliative Care Clinic' had an impact on health care professional's(HCP) willingness to refer patients and whether this varied according to the age of the patient, 
whether patients had any reservations about accepting referral. $\mathrm{HCP}$,patient and relatives views regarding rebranding the clinic.

Method: Consultants and CNS's caring for adult patients with life limiting illnesses completed a semi-structured questionnaire using illustrative case studies.7 clinical scenarios were given.Each was further subdivided into two different age ranges(40-80). For each scenario HCP were asked to rank their preferred name from a drop down list of the following:Supportive CareTeam (SCT), PalliativeCareTeam (PCT), Palliative and Supportive Care Team (P\&SCT),Symptom Management and Supportive Care Team (SM\&SCT),Symptom Management and PalliativeCareTeam (SM\&PCT). They were also asked:Referral to PCT can take away hope for patients and their families?The possibility of taking away hope influence the referral?Team should be rebranded?

Also patients and relatives attending PCT follow up clinic completed an interview questionnaire. What best describes the role of the outpatient clinic from the options listed as above? Whether they had any reservations about being referred to the palliative care team/clinic?Whether they would prefer the clinic to be rebranded from the same list of option.

Results: A majority of HCP believed the terminology "Palliative Care" would deter them from referring certain patients.80\% felt that team should be rebranded. Patients and relatives felt the terminology'Palliative Care'had negative connotation and did not reflect their experience of the support gained from the attending clinic.

Conclusion: Overwhelming support from HCP,patients and relatives to rebrand the clinic.As a result of this study the Palliative Care clinic has been rebranded'The Symptom Management and Supportive Care Clinic' which best describes its role.

Abstract number: P02-264

Abstract type: Poster

Palliative Care at the Front Door

Keith R., Anderson R., Hilston K., Thomson G., Ross C., Harvie K. Palliative Medicine, NHS Lanarkshire, Lanarkshire, United Kingdom

Aim: $75 \%$ of people are admitted to hospital during the last year of their life. We aimed to improve the standard of palliative care provided to patients presenting to the acute hospital by providing an early holistic review by the Hospital Palliative Care Team (HPCT) in the Acute Medical Receiving Unit (AMRU). We anticipated that earlier review would lead to earlier discharge and patients being more likely to achieve goals of dying at home.

Method: We adjusted our reactive model of responding to telephone referrals by proactively attending AMRU each morning to seek out patients with palliative care needs suitable for review. We gave advice on symptom control, facilitated discharge planning and organised appropriate, timely community follow up. Data was collected over an eight week intervention period and compared to the corresponding eight weeks from the previous year.

Results: Length of time from admission to HPCT review reduced from a median of 13 to 4 days. Median length of hospital stay for these patients reduced from 26 days to 8 days with $36 \%$ being discharged directly from AMRU. Number of patients reviewed within $48 \mathrm{hrs}$ of admission increased from $17 \%$ to $52 \%$. The number of investigations performed reduced. All referrals were appropriate and required some kind of intervention from HPCT. Patients were less likely to be readmitted to hospital following discharge and they were more likely to die at home than in the acute sector.

Conclusion: Early HPCT review on admission to acute hospital was correlated with shortened hospital stay which is important for patients with a life-limiting disease. It was associated with increased likelihood of dying at home which can be a goal for some of our patients. Potential cost savings with reduced hospital stays could offset increased staff input.
Abstract number: P02-265

Abstract type: Poster

Opioid Prescribing and Review in 'Chronic' Cancer: A Snapshot of Practice

Downham E., Radley A., Mitchell A.

Beatson West of Scotland Cancer Centre, Palliative Medicine, Glasgow, United Kingdom

Background: Cancer survivorship is improving. Chronic Cancer Pain is now a recognised diagnosis. Long-term opioids lack evidence of efficacy and are associated with significant adverse effects. Opioids may be started appropriately but there are limited cues for treatment review. Guidelines for the management of chronic non-malignant and chronic cancer pain focus on avoiding or reducing opioid use.

Aims: To describe the incidence of opioid use and frequency of review of opioid prescriptions in patients living with 'chronic' cancer, and to raise awareness of the potential adverse effects of long-term opioids.

Methods: We conducted a retrospective electronic case note review of patients attending the metastatic breast cancer clinic over three consecutive weeks. Inclusion criteria were patients diagnosed with metastatic or locally advanced disease for $\geqslant 1$ year. Data on demographics, time since diagnosis, regular long-acting opioid prescriptions, and review of opioid therapy were collected.

Results: Of 135 patients, $76 \%$ had a diagnosis $\geqslant 1$ year. $18 \%$ of patients were prescribed long acting regular opioids. The most commonly prescribed opioid was Morphine. The mean Oral Morphine Equivalent (OME) dose was $85 \mathrm{mg} / 24 \mathrm{hrs}$ (range $10-200 \mathrm{mg}$ ). The mean length of time on opioids was $16 \mathrm{mths}$ (range $1 \mathrm{mth}-5 \mathrm{yrs}$ ). Of 20 clinic attendances, $40 \%$ had a documented opioid therapy review.

Discussion: Mean OME dose/24hrs is within our local chronic non-cancer pain recommendation for long-term opioid dosing. Documented opioid therapy review falls short of published guidance. The oncology team may be best placed to identify who chronic cancer patients are and undertake regular review of opioid therapy according to prognosis. Our study population was broadly defined and doesn't differentiate patients with chronic cancer from those who may be close to the end of life. Nonetheless, this snapshot of practice will assist a future study of opioid use in a more specifically defined chronic cancer population.

Abstract number: P02-266

Abstract type: Poster

Inpatient Assessment and Management of Constipation - Are we Getting it Right?

Eason R. ${ }^{1}$, Halley A. ${ }^{2}$

${ }^{1}$ Princess Alice Hospice, London, United Kingdom, ${ }^{2}$ Palliative Medicine, The Royal Marsden Hospital, London, United Kingdom

Introduction: Constipation is a common condition that affects people in all age groups, with complications that can cause significant physical and psychological distress. Validated assessment tools and gold standards for management of constipation are not available due to the lack of robust clinical trials. We conducted an audit to compare local practice of constipation assessment and management to current best practise, aiming to identify areas for improvement and to raise awareness of the significant problem of constipation in inpatients. Our 8 standards were derived from accepted Palliative Care Guidelines and focussed on history of constipation, daily bowel assessment and prescription of laxatives.

Methods: Data were collected retrospectively from 28 inpatients on general Oncology wards from medical and nursing notes, and medication and bedside charts, in Summer 2018.

Results: Unfortunately, only 1 of the 8 standards for good practise was met. Generally, management of constipation and prescription of laxatives was adequate, especially with concurrent use of opioid medication. 
However, documentation from medical and nursing staff of both assessment and initial and ongoing management was inconsistent and incomplete, and often assumed a level of knowledge of the patient's condition on the part of the reader. The reasons behind these results are likely multi-factorial but may include a lack of confidence in assessing bowels and treating constipation and a lack of understanding of its importance. Recommendations: Following the presentation of this audit we recommended the inclusion of dedicated constipation teaching in the Junior Doctor teaching timetable, with signposting to relevant guidelines, and a review into the use of bowel charts on the ward, to encourage daily use. We are also now in the process of updating the constipation section of our local Symptom Control Guidelines in line with current Palliative Care guidelines.

\section{Abstract number: P02-267 \\ Abstract type: Poster}

Inappropriate Use of Naloxone in a Hospital Setting: Compromising Patient Safety

Goryaeva M., Jamali A., Ali N., Cox S., Caulkin R.

Chelsea and Westminster Hospital, London, United Kingdom

Background: Life threatening opioid toxicity is a rare but recognised complication of opioid use in palliative patients. Local, national and formulary guidelines exist which advise that Naloxone should only be administered to patients with chronic opioid use if they have a significantly depressed respiratory rate (RR), with a reduced consciousness level and/or hypoxia and that doses should be lower than in those with acute opioid overdose. No such clinical guidelines existed in our Hospital Trust.

In 2014, NHS England released a patient safety alert on inappropriate use of Naloxone in patients with chronic opioid use, due to risk of precipitating intense pain and withdrawal, leading to arrhythmias, pulmonary oedema and cardiac arrest.

Aims: To audit the use of Naloxone for opioid toxicity in patients with chronic opioid use as per external guidelines.

Methods: Data was collected retrospectively over 3 months for all adult hospital inpatients on regular opioids that were given Naloxone. Clinical notes/observation charts were reviewed for reason for naloxone use, $\mathrm{RR}$, oxygen saturation, consciousness level and dose of Naloxone given. Results: Naloxone was administered 21 times to 9 patients.

Naloxone administration compared with recommended guidance parameters.

\begin{tabular}{lrcc}
\hline $\begin{array}{l}\text { Guidance } \\
\text { parameters met }\end{array}$ & $\mathrm{RR} \leqslant 8$ & $\mathrm{SaO}_{2} \leqslant 85 \%$ & $\begin{array}{l}\text { Reduced } \\
\text { consciousness level }\end{array}$ \\
\hline No & $18 / 21$ & $18 / 21$ & $8 / 21$ \\
Yes & $2 / 21$ & $2 / 21$ & $11 / 21$ \\
Not Recorded & $1 / 21$ & $1 / 21$ & $2 / 21$ \\
\hline
\end{tabular}

In $18 / 20$ cases Naloxone was administered inappropriately. In 11/18 of these, an incorrectly high dose of Naloxone $(400 \mathrm{mcg})$ was used; In 3/18 a Naloxone infusion was used inappropriately.

Conclusion/discussion: A reduced consciousness level often triggered Naloxone use despite absence of respiratory depression or hypoxia, even though an alternative cause for this was always identified. It appeared there was a lack of knowledge of the safety risk of Naloxone. Trust Guidelines have been developed which are being disseminated through education sessions and a repeat audit will then be conducted.

Abstract number: P02-268

Abstract type: Poster
Organ Procurement from Deceased Donors in Brain Death: Perception by Relatives of Their Own Support during Stay in Intensive Care Unit

Nicolas-Robin A. ${ }^{1}$, Alluin R. ${ }^{2}$, Barbaret $C .{ }^{3}$, Tremellat $F^{4}$

${ }_{1}$ Pediatric Palliative Care Mobile Team, University Hospital Robert Debré (APHP), Paris, France, ${ }^{2}$ University Hospital of Nancy, Nancy, France, ${ }^{3}$ University Hopsital of Grenoble, Grenoble, France, ${ }^{4}$ University Hopsital of Nice, Nice, France

Background: In France, organ procurement (OP) from a deceased person requires the testimony of non-opposition from his relatives. These circumstances mean they are at high risk of post-traumatic stress syndrome. Their experience depends on the quality of the information they receive about brain death and the quality of their own support by the care team. Aim: To analyze the perceptions of relatives facing the stay in ICU of a severely brain injured patient, about their own support during the different steps of resuscitation, before and after brain death and during the specific resuscitation of the organ donor.

Methods: Qualitative prospective study. Six months after death, one relative was proposed with a semi-structured interview. Verbatim were analyzed manually, to bring out the conceptual frameworks.

Results: Eight interviews were conducted. The analysis has identified 3 themes that can influence the experiences of relatives:

- Temporality of resuscitation: for the intensivists, it is defined by the loss of hope of patient's survival, and then the move to exclusive organ resuscitation; for relatives, it is defined by the announcement of the patient's death

- Information to relatives: before the occurrence of brain death, medical explanations seem to be clear. During organ resuscitation, information becomes lessened.

- Quality of support: by caregivers, it is nonexistent; it comes from their own relatives. But this is not a source of any claim, considering that caregivers' job is to the patient exclusively.

Conclusion: The relatives whose testimony is required for a OP do not feel supported by the intensive care team during the different steps of resuscitation. Although this feeling does not seem negative, we think that this information could allow an awareness of caregivers to offer better care to them. A possible impact of this change of caring attitude towards them on the incidence of post-traumatic stress syndrome and on the rate of OP remains to be evaluated.

Abstract number: P02-269

Abstract type: Poster

Strong Opioid Substitution: A Multi-site Evaluation of Equianalgesic Ratios and Audit of Clinical Practice McDonald R. ${ }^{1}$, McLintock S. ${ }^{1}$, Hanchanale S. ${ }^{2}$, Casey M. ${ }^{1}$, Privett-Cook ${ }_{\text {H. }}{ }^{2}$, Griffiths A. ${ }^{3}$, Dickman A. ${ }^{2}$, Coackley A. ${ }^{3}$

${ }^{1}$ Marie Curie Hospice Liverpool, Liverpool, United Kingdom, ${ }^{2}$ Royal Liverpool and Broadgreen University NHS Hospitals Trust, Liverpool, United Kingdom, ${ }^{3}$ Clatterbridge Cancer Centre NHS Foundation Trust, Liverpool, United Kingdom

Background: Strong opioid substitution to a different route or a different opioid is common in palliative care. While the dose of the substituted opioid is an individualised clinical judgement, accurately calculating the equianalgesic dose is key to safely prescribing the new opioid. Variation in the use of equianalgesic ratios had been noted across the region. Aim: Within a regional palliative care audit program in the North West of England to

1. Evaluate opioid substitution practice in hospice, hospital and community settings and audit against current regional guidelines

2. Update regional standards and guidelines for opioid substitution 
Mean Conversion Ratios Compared to Equianalgesic Ratios Recommended by Regional Guidelines.

\begin{tabular}{llllc}
\hline Original Opioid & New Opioid & $\begin{array}{l}\text { Recommended } \\
\text { Equianalgesic Ratio }\end{array}$ & $\begin{array}{l}\text { Mean Conversion } \\
\text { Ratio }\end{array}$ & $\begin{array}{l}\text { Number } \\
\text { of Cases }\end{array}$ \\
\hline Morphine oral & Morphine subcutaneous & $2: 1$ & $2.1: 1$ & 27 \\
Oxycodone oral & Oxycodone subcutaneous & $1.5: 1$ & $1.8: 1$ & 32 \\
Morphine oral & Oxycodone oral & $1.5: 1$ & $1.8: 1$ & 62 \\
Morphine subcutaneous & Oxycodone subcutaneous & $1: 1$ & $1.5: 1$ & 22 \\
Oxycodone oral & Alfentanil subcutaneous & $20: 1$ & $19.9: 1$ & 13 \\
Oxycodone subcutaneous & Alfentanil subcutaneous & $15: 1$ & $11.7: 1$ & 13 \\
\hline
\end{tabular}

Reported conversion ratios compared with equianalgesic ratios recommended by regional guidelines.

\begin{tabular}{llll}
\hline Original opioid & New opioid & $\begin{array}{l}\text { Recommended } \\
\text { equianalgesic ratio }\end{array}$ & Reported conversion ratios \\
& & $2: 1$ & $95 \%$ use $2: 1$ \\
Morphine oral & Morphine subcutaneous & $1.5: 1$ & $67 \%$ use $2: 1,25 \%$ use $1.5: 1$ \\
Morphine oral & Oxycodone oral & $1.5: 1$ & $71 \%$ use $2: 1,26 \%$ use $1.5: 1$ \\
Oxycodone oral & Oxycodone subcutaneous & $59 \%$ use $30: 1,35 \%$ use equianalgesic table or guidelines \\
Morphine oral & Alfentanil subcutaneous & $30: 1$ & $37 \%$ use $7.5: 159 \%$ use equianalgesic table or guidelines \\
Morphine oral & Hydromorphone oral & $7.5: 1$ & $21 \%$ use $100: 1,54 \%$ use equianalgesic table or guidelines \\
Morphine oral & Fentanyl transdermal & $100: 1$ &
\end{tabular}

Methods:

1. Systematic literature review

2. Multi-site retrospective case note analysis

Results: 18 articles were identified to inform the update of the regional standards and guidelines.

285 patient records were analyzed across 21 different sites. Patients had either undergone a change in route or a change to a different strong opioid. $49 \%$ were in hospital, $33 \%$ in specialist palliative care units and $18 \%$ in the community. Data for substitutions with 10 or more cases are shown in the table below.

Conclusion: Mean conversion ratios for the majority of substitutions do not reflect regional, national or international guidance, likely due to the use of a Morphine to Oxycodone equianalgesic ratio of 2:1. The impact on conversions from subcutaneous Oxycodone to Alfentanil, where higher than recommended doses of Alfentanil were prescribed, is particularly concerning. Further work is required with stakeholders across the region to increase awareness of this and maintain patient safety.

Abstract number: P02-270

Abstract type: Poster

\section{Opioid Substitution: A Survey of Healthcare Professional's Practice}

McDonald R. ${ }^{1}$, McLintock S. ${ }^{1}$, Hanchanale S. ${ }^{2}$, Casey M. ${ }^{1}$, Privett-Cook $H^{2}{ }^{2}$, Griffiths A. ${ }^{3}$, Dickman A. ${ }^{2}$, Coackley A. ${ }^{3}$

${ }^{1}$ Marie Curie Hospice Liverpool, Liverpool, United Kingdom, ${ }^{2}$ Royal Liverpool and Broadgreen University NHS Hospitals Trust, Liverpool, United Kingdom, ${ }^{3}$ Clatterbridge Cancer Centre NHS Foundation Trust, Liverpool, United Kingdom

Background: Strong opioid substitution to a different route or different opioid is common in palliative care. The dose of the substituted opioid should be an individualised clinical judgement but accurately calculating the equianalgesic dose is a key step in safely prescribing the new opioid. Variations in professional practice had been noted across the region. Aim: Within a regional palliative care audit program in the North West of England to
1. Evaluate the opioid substitution practice of healthcare professionals (HCPs) in hospice, hospital and community

2. Update regional guidelines for opioid substitution

Methods:

1. Systematic literature review

2. HCPs working in palliative care who prescribe or recommend medications

Results:

18 articles were identified to inform the updated regional guidelines. 82 HCPs from 24 clinical sites completed an online survey of practice. $100 \%$ of respondents $(n=78)$ reported Morphine is their oral strong opioid of choice. $95 \%(n=73)$ reported Morphine or Diamorphine is their parenteral strong opioid of choice.

Reported conversion ratios compared to recommended equianalgesic ratios are summarised in the table below.

Conclusion: When converting Morphine to Oxycodone or oral Oxycodone to subcutaneous Oxycodone the majority of HCPs used a 2:1 ratio, rather than the 1.5:1 ratio recommended by regional, national and international guidelines. This may lead to higher than recommended doses when converting Oxycodone to Morphine, subcutaneous Oxycodone to oral Oxycodone or Oxycodone to Alfentanil. Further work is required with stakeholders across the region to increase awareness of this.

Abstract number: P02-271

Abstract type: Poster

Palliative Prognostic Index in Estimating Survival in Patients with Advanced Cancer in a Tertiary Palliative Care Setting - A Clinical Audit

Tiwari A.K., Rao S., Deodhar J., Salins N., Muckaden M.A., Damani A., Ghoshal A.

Department of Palliative Medicine, Tata Memorial Hospital, Mumbai, India

Background: Prediction of survival helps to support clinical decision making, preparation for the future and enabling timely referrals to hospice care. To enable this goal a palliative prognostication tool is required. 
Aim: To evaluate the utility of Palliative Prognostic Index in estimating survival in patients with advanced cancer in a palliative care setting. Methods: Medical records of adult patients, with advanced cancers registered at a tertiary palliative cancer centre, who died between August and September 2014, either at home or hospital were audited. Patients who died in Intensive Care Unit or whose records were incomplete were excluded from the study. Retrospective scoring of Palliative Prognostic Index (PPI) at the point of first contact was done and survival was estimated as ( $\leqslant 3$ weeks; $>3$ weeks $<6$ weeks; $>6$ weeks). The scorer was blinded to the date of first contact and death of the patient. The actual survival time was then calculated by another investigator. The recorded symptom burden at the first visit using Edmonton Symptom Assessment Scale (ESAS) was noted. Correlation between estimated and actual survival was analysed and its significance studied using the chi-square test. Results: The concordance between the estimated survival using the PPI and the actual survival was analysed in 132 of 139 patients. In the $\leqslant 3$ weeks, it was $55.4 \%$; in the $>3$ to $<6$ weeks, it was $80 \%$; and $96 \%$ in the $>6$ weeks group. Among the symptoms on the ESAS, fatigue and loss of well-being were more severe in patients with survival $\leqslant 3$ weeks.

Conclusion: The results of our study indicate that PPI is a useful tool in assessing survival in patients with advanced cancer in palliative care setting and should be incorporated as part of regular assessment. Funding: No funding from any source.

Abstract number: $\mathrm{P} 02-272$

Abstract type: Poster

Longing for Peace: Waiting Times for Admission and Palliative Care Referral in End-of-Life Patients during their Last Hours Fong E.L.S. ${ }^{1}$, Nadarajan G.D. ${ }^{2}$, Lian W.Q.S. ${ }^{2}$, Ong E.H.M. ${ }^{2}$

${ }^{1}$ Lee Kong Chian School of Medicine, Nanyang Technological University, Singapore, Singapore, ${ }^{2}$ Department of Emergency Medicine, Singapore General Hospital, Singapore, Singapore

Background: Emergency department (ED) visits by patients with chronic diseases during their end-of-life (EOL) period are distressing to patients and loved ones. Early ward admission and referral to palliative care could enhance patient comfort during their last hours. The waiting time for admission and referral in this population has never been studied locally. They are important indicators of patient care and are crucial for measuring the impact for future action plans to expedite these processes.

Aim: To study the quality of EOL care given to imminently dying patients presenting to the ED using waiting time for ward admission and palliative specialist referral as indicators.

Methods: A retrospective study was done on a 3-month period in a tertiary hospital ED. Patients who had a documented diagnosis of a chronic disease, were admitted into the ward and died within 72 hours of presentation to the ED were included. Outcomes of interest include waiting times for admission and palliative referral.

Results: Of the 58 patients studied, $69.0 \%$ were males and the median age was 75.5 . The median waiting time for a bed was $1 \mathrm{~h} 51 \mathrm{~min}$, while the median time from registration to death is $21 \mathrm{~h} 1.5 \mathrm{~min}$. Only 16 patients (27.6\%) had a referral made to a palliative care specialist before death. Of all referrals made, $6.25 \%$ were made in the ED, with a median waiting time of $2 \mathrm{~h} 31 \mathrm{~min}$. Otherwise, the median waiting time for palliative referral made in the ward is $19 \mathrm{~h} 20 \mathrm{~min}$.

Conclusion: Identifying patients requiring EOL care and referral to palliative specialists is not routinely done in the ED despite it being the best setting for expedited admission. Doing so is shown to reduce the waiting time for referral to a palliative care specialist compared to referrals being made in the ward after admission. Early admission and referral improve the comfort of patients and their family, given the short median survival time of less than a day. This may be achieved via screening tools at the ED.
Abstract number: P02-273

Abstract type: Poster

Rekindling Primary Care's Relationship with Advance Care

Planning: A Quality Improvement Project

Naughton J.P. ${ }^{1}$, Williams $H^{2}{ }^{2}$, Gleeson $A .^{3}$

${ }^{1}$ Primary Care, Aneurin Bevan University Health Board, Newport, United Kingdom, ${ }^{2}$ Division of Population of Medicine, School of Medicine, Cardiff University., Cardiff, United Kingdom, ${ }^{3}$ Palliative Medicine, Aneurin Bevan University Health Board, Newport, United Kingdom

Background: In 2017, a local priority setting exercise amongst stakeholders in palliative care identified gaps in the documentation and communication of Advance Care Planning (ACP) as barriers to high quality palliative care. We designed and evaluated an electronic template-based intervention to empower primary care teams to overcome these challenges.

Aims: We aimed to increase recording and communication of ACP discussions. This was via

(1) the development of an electronic palliative care recording and reporting toolkit and

(2) evaluation using Quality Improvement (QI) methods.

Methods: Six primary care practices were recruited for a 6 month QI project. Practices were supported through educational sessions and facilitation from the study team. Utilising regular feedback and repeated PDSA cycles, the template was adjusted to maximise usability and impact. Monthly reports with comparative statistics were shared with practices. We collected continuous data over a 12 month period ( 6 months baseline data and 6 months intervention data). Data was collected via the template's inbuilt reporting module, participant interviews, the research team's observations and practices' after death reviews. Run charts were utilised to correlate data trends with the timing of interventions such as monthly feedback reports and education sessions. Thematic analysis was applied to interviews and feedback from project participants.

Results / Conclusion: A tenfold increase in documentation of preferred place of death was demonstrated during the study period. Documentation of resuscitation wishes increased by $42 \%$ and documented anticipatory prescribing increased 9-fold.

Further work will establish the impact on patient outcomes and apply lessons learned to scale-up efforts. Challenges to overcome include: engaging primary care teams in ACP; the design and scalability of local e-solutions for managing palliative patients; and adequately resourcing this work to ensure sustainability.

Abstract number: P02-274

Abstract type: Poster

Management of Refractory Breathlessness in Patients with Advanced Disease and Incurable Illness: A Multi-centre Regional Audit of Practice

Roberts J. ${ }^{1}$, McDougall A. ${ }^{2}$, Benson S. ${ }^{3}$, Smith E. ${ }^{2}$, Cooke M. ${ }^{4}$, Hayle C. ${ }^{5}$ ${ }^{1}$ Marie Curie Hospice Liverpool, Liverpool, United Kingdom, ${ }^{2}$ Wirral Hospice, Wirral, United Kingdom, ${ }^{3}$ Clatterbridge Cancer Centre NHS Foundation Trust, Wirral, United Kingdom, ${ }^{4}$ Merseycare, Liverpool, United Kingdom, ${ }^{5}$ Wirral University Hospital NHS Trust, Wirral, United Kingdom

Aim:

1. Audit clinical practice of pharmacological and non-pharmacological management of breathlessness in patients with advanced disease against regional standards. 
2. Use audit outcomes to update regional standards and guidelines

Methods:

1. Systematic literature review examining evidence for non-pharmacological and pharmacological management of breathlessness in patients with advanced, incurable illness.

2. Survey practice of Health Care Professionals (HCPs) working in specialist palliative care

3. Multi-centre retrospective case note review of management of breathlessness in patients with advanced incurable illness receiving specialist palliative care.

Results: 82 HCPs participated in the survey. $49 \%$ of HCPs report using oral morphine instant release (IR) as required as the first line opioid regime for breathlessness and $8 \%$ report using oral morphine modified release (MR) first line. $30 \%$ of HCPs reported using a maximum of $30 \mathrm{mg}$ of oral morphine in 24 hours (or equivalent) for breathlessness management. $15 \%$ and $21 \%$ of respondents report using a maximum total daily dose of $10 \mathrm{mg}$ and $20 \mathrm{mg}$ of oral morphine (or equivalent) respectively. 170 sets of case notes were reviewed. Of those patients prescribed an opioid for refractory breathlessness, $67 \%$ were prescribed oral morphine IR. Less than $10 \%$ of the patients commenced on an opioid for breathlessness were commenced on a MR preparation.

Results showed that in $77 \%$ of cases the final total daily dose of opioid to manage breathlessness was less than $30 \mathrm{mg}$ of oral morphine (or equivalent).

Conclusion: Despite current evidence base for the use of MR opioids for breathlessness first line, the majority of clinicians audited use IR opioids. The data suggests that over a third of HCPs working in specialist palliative care may not be utilizing an optimal dose of opioid for breathlessness in keeping with current evidence. Improvement is required in prescribing for patients with refractory breathlessness in the region.

Abstract number: P02-275

Abstract type: Poster

Making a Difference: The Benefit of Using Volunteers in

Obtaining Views on Care Data

Harrison C., Schrikker T., Barclay D.

St. Wilfrid's Hospice, Eastbourne, United Kingdom

Background: We introduced Views on Care (VoC), part of the Outcome and Assessment Complexity Collaboration suite of measures, on the inpatient unit (IPU) with data collected by nursing staff. To facilitate nonbiased feedback from patients within the community, where this initiative had not commenced, the hospice introduced the role of quality and feedback volunteer. Latterly the role has been extended to the IPU. There is no published evidence to suggest that volunteers are active in gaining feedback on this outcome measure.

Aim: To evaluate the introduction and experience of quality and feedback volunteers in collecting $\mathrm{VoC}$ data.

Methods: Four individuals from the current volunteer workforce were identified with an interest in this new role. Training was provided which included simulated patient scenarios, confidentiality and communication skills enabling volunteers to be confident in approaching and questioning patients at end of life. VoC feedback was collected via telephone or face-to-face interaction and inputted to the patient's electronic records supported by the Quality and Improvement Lead of the hospice. Feedback from the volunteers on their experience was gathered in a focus group.

Results: The volunteer experience was universally positive. Volunteers felt privileged that patients were able to speak openly and honestly about the quality of care received and felt their contribution to improving service delivery was acknowledged. For completeness, we present the VoC data: $74 \%$ of patients had improved quality of life from hospice interventions and $91 \%$ gained benefit from hospice services. This venture has successfully expanded the volunteer role and helped to inform and shape future service development.

Conclusion: The supported introduction of quality and feedback volunteers within the hospice has been positive and enabled the roll out of the $\mathrm{VoC}$ measure in all clinical settings.

Abstract number: P02-276

Abstract type: Poster

Palliative Care and Aggressiveness of Cancer Care near the Endof-Life: Variations in Practice Call for Efforts towards Quality Improvement

Colombet I. ${ }^{1,2}$, Bouleuc C. ${ }^{3}$, Piolot A. ${ }^{4}$, Vilfaillot A. ${ }^{5}$, Voisin-Saltiel S. ${ }^{6}$, Vinant P. ${ }^{1}$, EFIQUAVIE Study Group

${ }^{1}$ Palliative Care Team, Assistance Publique-Hôpitaux de Paris - Hôpital Cochin, Paris, France, ${ }^{2}$ Public Health, Paris Descartes University, Paris, France, ${ }^{3}$ Palliative Care Team, Institut Curie, Paris, France, ${ }^{4}$ Equipe Mobile de Soins Palliatifs, Assistance Publique-Hôpitaux de Paris Hôpital Henri Mondor, Paris, France, ${ }^{5} \mathrm{Clinical}$ Research Unit, Assistance Publique-Hôpitaux de Paris - HEGP, Paris, France, ${ }^{6}$ Equipe Mobile de Soins Palliatifs, Institut Gustave Roussy, Villejuif, France

Background: Aggressiveness in end-of-life care has been widely described in patients with cancer, and its reduction is a major challenge for end-of-life care quality in oncology. French data published on this topic are limited to macroscopic measures from nation-wide hospital activity data.

Aims: To determine the intensity of end-of-life cancer care, at the point of care level, from exhaustive data combining administrative databases and hospital clinical records; to evaluate its variability across hospital facilities and its associations with timely introduction of palliative care (PC).

Methods: We selected a series of 997 decedents from a cohort of patients hospitalised in 2010 diagnosed with metastatic cancer in 3 university hospitals and 2 comprehensive cancer centres, combining hospital activity and nationwide mortality databases, completed with data collected from hospital records.

Results: Overall (min-max) indicator values as reported by the centres (the facility providing care rather than the place of death), were as follows: $16 \%(8 \%-25 \%)$ patients received chemotherapy within 14 days of death, $16 \%(6 \%-32 \%)$ had $\geqslant 2$ admissions to acute care, $6 \%(0-15 \%)$ had $\geqslant 2$ emergency visits and $18 \%$ (4\%-35\%) had $\geqslant 11$ intensive care unit admission(s). Only $53 \%$ of these patients met the PC team, and the median (min-max) time between the first intervention of the PC team and death was 41 (17-112) days. Introduction of PC $>30$ days before death was independently associated with lower intensity of care; centre of care was associated with all indicators.

Conclusions: Aggressiveness of end-of-life cancer care is a reality, but highly variable across centres. This validates the use of indicators and calls for a real change in conceptions to integrate PC in oncology and more systematic evaluation of practice. Disseminating a quality audit-feedback cycle should contribute to a shared view of appropriate end-of-life care objectives, and foster action for improvement among care providers.

Abstract number: P02-277

Abstract type: Poster

Fall Incidents in Hospice Care: Why Does it Happen and How to Prevent

Wieles-Griffioen G.A.M. ${ }^{1}$, Verbree P.P. ${ }^{2}$, Wesseling E. ${ }^{2}$, Gootjes J.R.G. ${ }^{1}$, ter Horst - van Oord M.E. ${ }^{1}$, van Nus-Stad J.L. ${ }^{1}$

${ }^{1}$ Hospice Kuria, Amsterdam, Netherlands, ${ }^{2}$ University of applied science VIAA, Zwolle, Netherlands 
Background: Falling incidents regularly occur in the hospice and have a negative influence on the quality of life of the patients. Falling prevention therefore is important in hospice care. In literature, interventions and guidelines are described to reduce the amount of falling incidents and the risk of falling. However, these interventions are often not applicable in the terminal palliative phase.

Aims: Which hospice patients have an increased risk of falling, which interventions are currently employed, and which interventions described in literature are applicable for the hospice. Which clinical interventions can be used by healthcare providers to prevent falling incidents.

Methods: A mixed methods approach with analysis of files of 48 hospice patients with falling incidents between 2014-2017 for clinical pictures, medical and nursing aspects. Semi structural interviews with 5 hospice nurses. Literature research into interventions to prevent falling.

Results: From analysis of the files, it became clear that toilet use plays a major role at the moment of falling. Other risk factors include medication use, reduced cognitive capabilities, and the desire to retain autonomy. Almost all patients showed pain symptoms, exhaustion and reduced mobility. Interviews reveal that nurses in the hospice react reactively, despite being aware of the risk of falling. After a first falling incident, interventions are put in place to prevent further incidents.

According to literature, patients should be treated by a multidisciplinary team, and a risk analysis with accompanying plan must be made for each individual patient. The falling history in the anamnesis has a predicting value.

Conclusion/ discussion: Improvement can be found in education for nurses about risk factors for falling, and establishing preventative measures. We recommend a risk analysis for falling during the intake and for patients with a higher risk the proactive use of preventive interventions.

Abstract number: P02-278

Abstract type: Poster

The Use and Spread of a Swedish Palliative Care Guide - An Update

Duarte A. ${ }^{1}$, Birgisdottir D. ${ }^{1,2}$, H Rasmussen B. ${ }^{1,3}$, Sallerfors B. ${ }^{1,2}$, Fürst C.J. ${ }^{1,2}$

${ }^{1}$ Region Skåne, Institute of Palliative Care, Lund, Sweden, ${ }^{2}$ Department of Clinical Sciences, Lund University, Lund, Sweden, ${ }^{3}$ Department of Health Care Sciences, Lund University, Lund, Sweden

Aim: To describe the dissemination of the Swedish Palliative Care Guide (S-PCG) since the release in 2016.

Background and methods: S-PCG is a structured and person-centred support for adult patients with palliative care needs regardless of the time remaining in life, age, diagnosis or were the care is given. S-PCG aims to assure the quality of care throughout the care trajectory from a curative approach to supportive and end-of- life care and after the death of the patient. S-PCG is an evidence-based support based on documents and guidelines issued by the Swedish health care authorities, the Swedish Palliative Care Registry and the experience of Liverpool Care Pathway. Patients and families have participated through interviews and meetings including patient organizations. A multidisciplinary advisory committee reviewed the content, feasibility and structure. Three pilot tests were conducted in different care settings before the release of version 1.0 in 2016. Units with intention to implement S-PCG have to make a registration as a user through the website of the coordination centre. Results: Since the release in October 2016, a total of 227 units have registered. The care settings include 166 municipal nursing homes, 47 palliative care services and 14 hospital units (September 2018).

Digital versions of S-PCG is under development in hospital and palliative care settings. The content of the S-PCG has been approved congruent with international recommendations for the care of the dying person by International Collaborative for Best Care for the dying Person.

Conclusion: After extensive development, S-PCG is available for use since October 2016 with a revised version (2.0) since September 2017.
The majority of users were seen in municipal nursing homes, but all forms of care are represented. The rapid influx of registered units may reflect the need for a tool to support personalized palliative care. Funding: The Kamprad Family Foundation

Abstract number: P02-279

Abstract type: Poster

Improving and Aligning Delirium Clinical Practices on a Palliative Care Unit through the Development, Implementation and Evaluation of a Modular Clinical Practice Guideline

Bush S.H. ${ }^{1,2,3}$, Skinner E. ${ }^{4}$, Kabir M. ${ }^{2}$, Grassau P.A. ${ }^{1,2,5}$, Parsons H.A. ${ }^{1,2,3}$, Tierney S. ${ }^{3}$, Lawlor P.G. ${ }^{1,2,3}$

${ }^{1}$ Department of Medicine, Division of Palliative Care, University of Ottawa, Ottawa, Canada, ${ }^{2}$ Bruyère Research Institute, Ottawa, Canada, ${ }^{3}$ Bruyère Continuing Care, Ottawa, Canada, ${ }^{4}$ Ottawa Inner City Health, Ottawa, Canada, ${ }^{5}$ School of Social Work, Carleton University, Ottawa, Canada

Background: Delirium is common in palliative care patients and causes significant patient, family and healthcare provider distress.

Aim: We developed a delirium clinical practice guideline (CPG) for our palliative care unit (PCU) in order to: improve delirium detection; increase use of nonpharmacological interventions; align antipsychotic practice in light of recent trial evidence; and improve communication within the interprofessional (IP) team and with patients and families.

Methods: An IP CPG development team synthesized core delirium information for a delirium 'Starter Kit' presentation and online self-learning modules (SLMs) on screening, communication tips, and nonpharmacological and pharmacological interventions. Clinical staff were invited to evaluate the CPG via an online survey. A bilingual patient and family delirium information leaflet was created with IP team input.

Results: The Starter Kit was delivered in a 2 week period as multiple group face-to-face sessions to $>120$ participants: $100 \%$ of PCU physicians and IP staff; $77 \%$ of nurses; 10 nursing students; PCU pharmacist, ward clerks and porter; and over 30 volunteers. The SLMs were subsequently rolled out, with the pharmacological SLM last. Completion rates of the 4 online SLMs by the clinical team ranged from $73-100 \%$.

The online evaluation survey response rate was 35\% (26/75) across PCU physicians, pharmacist, nurses, and IP staff. Most either agreed or strongly agreed that the training was sufficient for them to follow the CPG (79-100\% across all respondent groups), that it was easy to learn (74-100\%), and that they intend to consistently follow the CPG in the next 3 months (50-100\%). Team members have found the delirium information leaflet to be a useful resource for families.

Conclusion: Development and implementation of the CPG took time, requiring a committed team and management support. Ongoing efforts are needed to ensure CPG sustainability and timely updating.

(Funded by Bruyère AMO Incentive grant)

Abstract number: P02-280

Abstract type: Poster

Factors Influencing Preferred Place of Death for People with Advanced Illness Living in Malawi: Practice Inquiry

Kwaitana D.P. ${ }^{1}$, Wagland R. ${ }^{2}$

${ }^{1}$ College of Medicine, University of Malawi, Blantyre, Malawi, ${ }^{2}$ Faculty of Health Sciences, University of Southampton, Southampton, United Kingdom

Background: Decisions on place of death for patients with advanced illness are often ethically challenging. Many people die in institutions despite expressing preference to die at home. There is also consensus from developed nations that home is the preferred place of death for people with advanced illness. Despite the wealth of clinical experience, 
there is a dearth of evidence to inform policy and practice on preferred place of death in patients with advanced illness in Sub-Saharan Africa. Malawi needs to find its own end of life care approaches to redress this balance.

Aim: This study was aimed at establishing factors influencing preferred place of death for people with advanced illness in Malawi.

Methods: Practice inquiry was used as a research method. A situational analysis was done through literature review to understand the palliative care environment where services are delivered in Malawi. This was followed by a retrospective compilation of structured reflection of patient narratives based on my extensive experience caring for patients with advanced illness. Reflections were from clinical practice as a nurse between 2004-2016 at two health centres and two central hospitals in respectively. Common themes were identified and discussed as part of critical reflection adopting a qualitative content analysis approach.

Results: Economic factors were among the major reasons influencing choice for a place of death where most patients and relatives expressed challenges sustaining hospital stay. Other factors included lack of satisfaction with available services in institutions, advanced condition of the patient coupled with no hope for a cure and varying social problems. Conclusions: The liberty to make choices is at the heart of autonomy. It is therefore important for Malawi to increase opportunities for care and death at home for patients who cannot sustain hospital stay based on several perceived factors.

Funding: Commonwealth Scholarship Commission

Abstract number: P02-281

Abstract type: Poster

Audit of the Management of Constipation in Adult Palliative Care Patients at Galway University Hospital by the Palliative Care Team

Reilly L., O'Riordan J., Ravindrarasan S., Mannion E. Galway University Hospital, Galway, Ireland

Background: Constipation is one of the most common symptoms experienced by adult patients with advanced illness. The initiation of a bowel regimen early in the course of opioid therapy is considered to be gold standard practice.

Aims of audit: To examine the adherence of the Palliative Care team to the standards defined in the National Clinical Programme for adult Palliative care patients in relation to the management of constipation for patients receiving opioids.

Standard: National Clinical Programmed for Pall Care- submitted to National Clinical Effectiveness Committee.

Methodology: The medical notes of all inpatients under the care of the palliative team in Galway University Hospital on Jan $22^{\text {nd }} 2018$ were examined with respect to the following:

identify those patients on opioids, ascertain if bowel regime was prescribed on day opioid was commenced and assess if stimulant laxative was initial component of bowel regime.

Results:

Cycle 1: Medical notes of 16 patients were examined. In ten cases the laxative regime was commenced the day the opioid was commenced and in three cases this was done a number of days after opioid initiation. In the three remaining cases no bowel regime was commenced. This reflects a $62.5 \%$ rate of compliance with 5.1 of the audit standard.

Cycle 2: The medical notes of 14 patients were examined on the $5^{\text {th }}$ March 2018. In this case only nine patients, $64 \%$ were commenced on a bowel regime the day the opioid was commenced. In all cases patients were commenced on a stimulant laxative initially; $100 \%$ compliance with 5.2 of the audit standard.

Recommendations \& action plan: It is recommended that all members of the Palliative Care Team at Galway University hospital be familiar with the National Clinical Programme for Palliative Care guideline 10. These guidelines will be presented at a journal club slot to ensure satisfactory adherence.

Abstract number: P02-282

Abstract type: Poster

On Wheels!: Audit of Community End of Life Care Nursing Charnock L. ${ }^{1}$, Massam J. ${ }^{2}$, Groves K. ${ }^{1,3}$, Collins M. ${ }^{3}$

${ }^{1}$ Queenscourt Hospice, West Lancs, Southport \& Formby Palliative Care Services, Southport, United Kingdom, ${ }^{2}$ Queenscourt Hospice, Southport, United Kingdom, ${ }^{3}$ Lancashire Care NHS Foundation Trust, Southport, United Kingdom

Background: High quality, compassionate, palliative care, is vital to patient and family experience at end of life. The district nursing (DN) workforce has been identified as pivotal in co-ordinating and delivering end of life care at home.

Investment in DN training, development \& support would enable more people to cope well at home, increase those able to die at home, prevent unnecessary hospital admissions and have a positive effect on family experience and bereavement.

The TRANSFORM team,created in 2014 to educate, support and empower health professionals, patients and families, across hospital, care home and community settings, in excellence of end of life care, supports district nurses through opportunistic \& planned teaching, role modelling difficult conversations, \& development of appropriate plans for care.

Audit: Three audit cycles were carried out Nov 16-Jan 18, to measure end of life care delivered across each of four local district nursing neighbourhoods.

Method: Each neighbourhood audited 10 sets of patient's notes $(n=40)$ against 36 standards drawn from the local District Nurse in Last Year of Life Model, which recognises the need for increasing DN input and support as patients' condition deteriorates. (Based on North West End of Life Model encompassing the five national key enablers for End of Life Care \& Gold Standards Framework standards).

Results: Results were collated for each neighbourhood, RAG rated \& compared to $1^{\text {st }} \& 2^{\text {nd }}$ audit cycles, to assess improvement in the number of standards met in $80-100 \%$ of patients. $61 \%$ of all standards had improved across the whole district nursing service in 12 months from audit cycle 2 to 3 with up to 6 standards being met at $100 \%$ consistently across last 2 audit cycles.

Conclusion: Results were fed back to each DN neighbourhood \& Trust management. An action plan specific to each neighbourhood's learning needs was developed with the team. Ongoing TRANSFORM input will role model, develop, train and educate.

Abstract number: $\mathrm{P} 02-283$

Abstract type: Poster

A Snapshot Survey - Deaths in Single Rooms and Number of Family Rooms in Hospitals

Brennan J., Anderson A.

Irish Hospice Foundation, Dublin, Ireland

Research evidence suggests that there is a positive impact on the experience of end-of-life care when family rooms are available and when death occurs in a single room. In $201044 \%$ of hospital deaths occurred in single rooms. Over the last 10 years the provision and development of family rooms in hospitals has increased. There is currently no national collection of the data regarding the number of deaths in single rooms or family rooms available in acute hospitals. There are huge challenges in acute hospitals in regard to facilitating death in a single room with low numbers of single rooms available and competing demands for infection prevention and control purposes. Anecdotal evidence suggests a significant number of deaths are occurring in single rooms. 
Objectives:

- To gather a national snapshot of the number of patients that died in a single room in an acute hospital during the month of September 2018

- To gather information about the number of family rooms available in acute hospitals in September 2018

- To create a standardised data collection form and support for acute hospitals to participate in the survey

Method: All acute hospitals were invited to participate in the survey on a voluntary, anonymous basis. Data collection was via an online form. Data collection was for the month of September 2018 only. Results will be analysed, anonymised and presented to the Acute Hospital Network in November 2018.

Results: A high response rate (25/36 hospitals) suggests a willingness from the acute hospitals to participate in the survey, however they also suggest that there is currently no routine or uniform way of collecting this data within the acute hospital system. Final survey results will be available in November 2018. Preliminary results suggest the percentage of deaths occurring in single rooms has improved since 2010.

Conclusions: Results of the survey will be used as a benchmark and also to inform further work on metrics for end-of-life care in acute hospitals.

\section{Abstract number: $\mathrm{P} 02-284$}

Abstract type: Poster

\section{Draining Experience!: Re-audit of Hospice Pleural Aspiration} Groves K.

West Lancs, Southport \& Formby Palliative Care Services, Queenscourt Hospice, Southport, United Kingdom

Background: Hospice pleural aspiration has been undertaken for the last 27 years, following training by local chest physician. When the requirement for ultrasound (US) guided aspiration was introduced, a US machine was purchased and staff training undertaken. The British Thoracic Society issued guidance for pleural drainage in 2003 \& standards were adapted for use in respect of pleural aspiration in hospice. An audit undertaken in 2015 showed 36 procedures undertaken over a period of 3 years. 14 of 19 standards were $100 \%$ met; 2 met in $97 \%$ \& 2 in $94 \%$. Following this there were changes to the documentation template, patient information leaflet and consent form to ensure the capture of missing items.

Method: Retrospective review of all pleural aspirations undertaken since the last audit, drawn from electronic clinical record. Documentation of the procedure was audited against 19 standards identified.

Results: 16 procedures for 8 patients identified. 18 of 19 standards were $100 \%$ met compared to 14 at last audit. In two procedures, aspiration of fluid at the end of instilling local anaesthetic was not confirmed by documentation. On eprocedures failed to obtain more than $10 \mathrm{ml}$ of fluid despite initial aspiration of fluid via syringe and needle prior to procedure. There were no complications of pneumothorax, pain during procedure, haemorrhage or visceral injury during any of the procedures. Conclusions: Almost all the standards are now comfortably met. Further documentation changes and reminders to clinicians undertaking procedure should ensure thorough documentation. New doctors to the service, need to be thoroughly trained and supervised. Out of hours procedures should continue to take place when essential for symptom management, but undertaken by most experienced clinician.

Abstract number: P02-285

Abstract type: Poster

Reflections on the National Summary of Patient Activity Data for Adult Specialist Palliative Care Services in Ireland
Shanagher D. ${ }^{1}$, Foley S. ${ }^{1}$, Reaper Reynolds S. ${ }^{2}$, Toft S. ${ }^{2}$

${ }^{1}$ Irish Hospice Foundation, Dublin, Ireland, ${ }^{2} \mathrm{HSE}$, Dublin, Ireland

Background: Minimum Data Set (MDS) Data is being collected since 2012 from community specialist palliative care (SPC) settings and specialist inpatients units. In 2015, a report was commissioned to track the development of the delivery of SPC services in these settings. This MDS data is vital for the further development of SPC services in Ireland. Aims: The aims of this work are as follows:

- To plot trends in MDS data over time

- To identify gaps in SPC services

- To identify areas where planning and improvement could improve SPC services.

Methods: An analysis of MDS data gathered from 2012 - mid 2016 was carried out. HIPE data and HSE financial data as they apply to SPC was also accessed

Results: The data indicates that even though more people are accessing SPC services, unequal access to SPC inpatient units persists across the country. There is still a gap between the number of people with cancer versus a non-cancer diagnosis accessing SPC services in Ireland.

Conclusion: Although improvements in the provision of SPC services has taken place in recent years there is more that can be done. Funding deficits and regional disparity with regard to accessing inpatient units needs to be eliminated. Disparities in access to SPC for people who don't have cancer needs to be resolved and MDS in acute hospitals needs to be gathered so that KPIs can be established and the end of life care experience for those who die in acute hospitals can be improved.

Abstract number: P02-286

Abstract type: Poster

Lessons Learned about Implementation and Continuation from a Nationwide Quality Improvement Program in Dutch Palliative Care

de Veer A. ${ }^{1}$, Voss H. ${ }^{1}$, Fleuren M. ${ }^{2,3,4}$, Francke A. ${ }^{1,5,6}$

${ }^{1}$ Netherlands Institute of Health Services Research (Nivel), Utrecht, Netherlands, ${ }^{2}$ Amsterdam Public Health Research Institute, Amsterdam UMC, VU Amsterdam, Amsterdam, Netherlands, ${ }^{3}$ Department of Clinical, Neuro and Developmental Psychology, VU University Amsterdam, Amsterdam, Netherlands, ${ }^{4}$ Verpleegkundigen \& Verzorgenden Nederland (Dutch Nurses Association), Utrecht, Netherlands, ${ }^{5}$ Amsterdam Public Health research institute, Amsterdam UMC, VU Amsterdam, Amsterdam, Netherlands, ${ }^{6}$ Amsterdam UMC, location VU, Expertise Center for Palliative Care Amsterdam, Amsterdam, Netherlands

Background: The systematic uptake of evidence- or practice-based tools or methods in palliative care practices is important but also challenging. Therefore, the Dutch National Quality Improvement Programme for Palliative Care was launched, involving 76 quality improvement projects in which tools or methods were implemented. Examples of these so called best practices were the Gold Standards Framework, an instrument identifying palliative care needs, the STEM-inspirational cycle about tailored communication in end-of-life care, and a specialist palliative care consultant.

Aim: To give insight into the implementation and the continuation of the best practices 2 to 6.5 years after the start of the projects, the applied implementation strategies and factors that facilitated or impeded implementation and continuation.

Methods: An online questionnaire was sent to the contact person of the 76 quality improvement projects (response $83 \%$ ). In addition, five qualitative interviews were performed to gather additional in-depth information about the implementation and continuation of the best practices. 
Results: Sustainable application of the best practice was attained in $61 \%$ of projects. In these projects eight implementation strategies were applied more often $(p<.05)$ compared to the projects the best practice was not implemented. Examples of strategies are giving regularly feedback to the professionals about their use of the best practice and integration in electronic reports. Factors influencing implementation most often related to the organization, such as management support, available time, financial support, personnel changes, and a person who takes the implementation lead. Professionals influenced successful implementation, in particular with their enthusiasm, motivation and mutual support.

Conclusion: The organization plays a decisive role in implementation. Engaging the management team in implementation trajectories from early onset is of utmost importance.

Funding: ZonMw

Abstract number: P02-287

Abstract type: Poster

\section{Deciding Right First Time: Audit of Oxycodone Prescribing as an} Alternative to Morphine

Finnegan C. ${ }^{1}$, Groves K.E. ${ }^{2}$

${ }^{1}$ Lancashire Care NHS Foundation Trust, West Lancs, Southport \& Formby Specialist Palliative Care Team, Southport, United Kingdom, ${ }^{2}$ Queenscourt Hospice, Southport, United Kingdom

Background: National UK guidance on prescribing opioids for pain in palliative care recommends morphine/diamorphine as first line (NICE cg140). Locality wide prescribing data in an area in the north of England showed increased prescribing of oxycodone preparations.

Aim: To establish:

- Does opioid prescribing for palliative care patients follow national guidance?

- Where oxycodone is prescribed, is a clear rationale for use documented?

Design / Methods: A retrospective case note review of patients known to an integrated cross boundary Specialist Palliative Care Team (SPCT) who were prescribed oxycodone.

Results: Of the 105 case notes reviewed, the oxycodone prescription was initiated by the local SPCT in $71(68 \%)$ with only $69(66 \%)$ documented as previously taking morphine.

The rationale for prescribing oxycodone was documented in 73/105 (70\%); this was more likely if oxycodone initiated by the local SPCT ( $87 \%$ c.f. $33 \%)$.

The most common indication for prescribing oxycodone was renal impairment. Where renal impairment was the indication for oxycodone, eGFR was well documented $21 / 25$ (84\%), however overall documentation of eGFR was poor $(58 / 105,55 \%)$.

There was limited evidence that attempts were made to manage potentially treatable side effects of morphine prior to an opioid switch (14/40, $35 \%)$.

Laxatives were usually co-prescribed where appropriate $(82 / 93,88 \%)$, however only $20 \%$ were documented as receiving written information at the time of commencing opioids.

Conclusion / Lessons Learnt: Oxycodone is being used as a first line opioid in a small proportion of patients. Although there is a clear rationale for this when initiated by local SPCT, this may not be the case when initiated by other professionals. Prescribers are now routinely contacted to explore the rationale for prescribing oxycodone. This facilitates future clinical decision making and is an educational opportunity.

Renal function was not routinely recorded by SPCT. Education is ongoing to promote this.

Abstract number: P02-288

Abstract type: Poster
Preferences of End-of-Life Patients and their Caregivers about their Place of Death

Navarro Verdú P. ${ }^{1}$, Fernández Alcántara M. ${ }^{2}$, Montoya-Juárez $R .^{2}$, Ruiz

Miralles M.L. ${ }^{1}$, García Caro M.P. ${ }^{2}$, Cabañero Martínez M.J. ${ }^{1}$

${ }^{1}$ Alicante, Alicante, Spain, ${ }^{2}$ Granada, Granada, Spain

Introduction: Place of death is an important aspect end-of-life care. It is one of the core indicators of quality in Palliative Care and an essential issue when death approaches for patients and relatives. Previous research indicates that home is the most frequently desired place of death for patients and relatives.

Aim: The aim of this study was to describe the preferred place of care and death in a sample of palliative patients in two different regions in Spain. In addition, the level of congruency between the patient and the family caregiver was also considered.

Method: A cross-sectional design using self-report measured was carried out in two cities of Spain (Alicante and Granada) during April-June 2018. Interviews were conducted at home using an ad-hoc questionnaire. The preferences of patients and caregivers regarding place of care and place of death were assessed. Data was analyzed with the statistical package SPSS version 15.

Results: Home was the preferred place for death for the majority of patients (63\%) and family members (84.6\%). It was also the most chosen place for care (89.7\% - 96.6\%). The agreement and congruency between patients and caregivers was $93.1 \%$ for the place of care and $62.1 \%$ for place of death.

Conclusion: Home was the most frequently chosen place for care and death by patients and family caregivers.

Keywords: palliative care, place of death, home death, preferred place of death, congruency.

Abstract number: P02-289

Abstract type: Poster

Attitude and Knowledge towards Resuscitation of Patients with Advanced Tumor Diseases at the Palliative Care Unit Unseld M., Gager G.M., Adamidis F., Kitta A., Watzke H.H., Masel E.K. Medical University of Vienna, Vienna, Austria

Aims: The aims of the study were to examine a) patients' attitude and knowledge towards the topic of resusciation b) patients' information about their illness, and c) patient's concept of advance care planning. Methods: The study was based on a qualitative methodology, comprising 18 semistructured interviews with advanced cancer patients admitted to the palliative care unit of the Medical University of Vienna. Interviews were conducted during the first week after admission to the unit, recorded digitally, and transcribed verbatim. Data were analyzed using NVivo 12 software, based on content analysis.

Results: The results revealed four themes:

(1) desire or decline of resuscitation,

(2) education about illness

(3) information about advance care planning, and

(4) stay at the the palliative care unit.

The data showed that most patients did not receive information about the topic of resucitation concerning their own situation and a high percentage of the patients ( 7 out of 18) desired resuscitation. The education about one's own illness was perceived as good by 16 out of 18 patients. There was a lack of knowledge about advance care planning, while 5 out of 18 patients had initiated advance care planning on their own. All patients perceived their stay at the palliative care unit very positive.

Conclusion: The results of the present study reveal that there is a lack of knowledge concerning resuscitation and advance care planning in patients suffering from advanced cancer diseases. Seven out of 18 
patients at the palliative care unit expressed a desire for resuscitation. Five out of 18 patients that declined resuscitation had initiated advance care planning on their own. The stay at the palliative care unit was perceived as very positive by all patients.

Abstract number: P02-290

Abstract type: Poster

Intrathecal Drug Delivery: Training Needs of Hospice Medical and Nursing Staff

O'Kane R. ${ }^{1}$, Black C. ${ }^{2}$, Parsons $C^{1}$

${ }^{1}$ School of Pharmacy, Queen's University Belfast, Belfast, United

Kingdom, ${ }^{2}$ Marie Curie Hospice, Belfast, United Kingdom

Background: Intrathecal drug delivery (ITDD) is a safe and effective drug delivery method for patients receiving palliative care in whom other forms of analgesia have been ineffective. However, this technique has not been used in one hospice in a region of the United Kingdom in recent years.

Aims: To assess the knowledge, confidence and competence of hospice medical and nursing staff in ITDD and determine their training needs, with a view to reintroducing ITDD.

Methods: Two questionnaires were devised; one for medical staff and one for nursing staff, to assess knowledge, confidence and competence in ITDD. Questions also elicited participants' learning preferences for training. Questionnaires were piloted and distributed to all medical and nursing staff in February and March 2018, as part of a quality improvement project.

Results: Twenty-two healthcare professionals completed the questionnaire (10 doctors and 12 nurses; response rates of $63 \%$ of doctors and $46 \%$ of nurses respectively). Analysis of responses suggested that while doctors demonstrated a greater knowledge of ITDD, neither healthcare profession rated themselves as very confident or competent in this technique. Most participants expressed a preference for demonstrations in ITDD as a way to meet their learning needs.

Conclusion / Discussion: Due to staff turnover and the introduction of a new pump system, medical and nursing staff do not have up-to-date knowledge or confidence in ITDD. Education and training are therefore required prior to reintroduction of ITDD as a drug delivery method in the hospice, which must include practice-based learning and teaching incorporating demonstrations of this under-utilised drug delivery technique. These findings have informed the development of training materials for hospice medical and nursing staff; delivery and evaluation is currently ongoing.

Funding: The authors received no specific grant from any funding agency to undertake this work.

Abstract number: P02-291

Abstract type: Poster

How to Create a National Quality Framework for Palliative Care? Experience from the Netherlands

Boddaert M.S.A. ${ }^{1,2}$, Dijxhoorn A.F.Q. ${ }^{1,2}$, Douma J.1,2,3, Larkin P.J. ${ }^{4,5}$, Huijgens P.C. ${ }^{1}$, van der Rijt C.C.D. ${ }^{3,6}$, Vissers K.C.P. ${ }^{3,7}$

${ }^{1}$ Netherlands Comprehensive Cancer Organisation (IKNL), Utrecht, Netherlands, ${ }^{2}$ Association for Palliative Care in the Netherlands (PZNL), Utrecht, Netherlands, ${ }^{3}$ Palliactief, Amsterdam, Netherlands, ${ }^{4}$ Université de Lausanne, Lausanne, Switzerland, ${ }^{5}$ Centre Hospitalier Universitaire Vaudois, Lausanne, Switzerland, ${ }^{6}$ Oncology, Erasmus University Medical Center, Rotterdam, Netherlands, ${ }^{7}$ Department of Anaesthesiology, Pain and Palliative Medicine, Radboud University Medical Center, Nijmegen, Netherlands

Background: In the Netherlands, palliative care (PC) is part of a national program for the improvement of quality of life and care for patients with life-threatening conditions. Hence, the Ministry of Health encourages healthcare professionals and affiliated organizations to provide generalist PC, supported by palliative care specialists, when required. Here we present the process of development of a national quality framework for PC, the requisite consensus-building and main topics of content.

Aim: To create a broadly supported consensus based quality framework for PC for patients with a life-threatening condition or frailty and their families, based on their values, wishes and needs.

Method: Analogous to Dutch national guideline procedures, a working group comprising mandated representatives of patients and their families, scientific societies of various healthcare providers and health insurers formulated the key question: "What do we agree the criteria for quality of palliative care in the Netherlands should be". Constraint analyses for PC in Dutch primary and secondary care settings and a literature search were carried out. Six core documents were used in a Delphi method to build the framework with the working group and stakeholder organizations were kept informed at management level. The whole process took one year of preparing and building relationships and two years of convening, discussing content and consulting peers.

Results: A quality framework, including a glossary of terms endorsed by organizations representing patients and their families, general practitioners, elderly care physicians, medical specialists, nurses, social workers, psychologists, spiritual caregivers and health insurers was launched. In addition, a summary for patients and families and a list of recommended measurement instruments were developed.

Conclusion: A continuous whole-of-sector approach is a feasible way to develop a national consensus and literature based quality framework.

\section{Abstract number: P02-292 \\ Abstract type: Poster}

Guideline for the Use of Subcutaneous Furosemide in the Management of Patients with End-stage Heart Failure Henson L.A., Beighton J.E., Wanklyn S., Khan S.A.

Palliative Care, Guys and St Thomas' NHS Foundation Trust, London, United Kingdom

Introduction: Furosemide administered via the subcutaneous route is a recognised option for managing fluid overload in patients with endstage heart failure (ESHF). There is, however, limited evidence to guide its use and resultant widespread variation in clinical practice.

Aim: To develop an evidence-based clinical guideline for the use of subcutaneous Furosemide (off-label) in the management of patients with ESHF.

Methods: A literature review was conducted to identify any studies or existing clinical guidelines relating to the use of subcutaneous Furosemide. All specialist palliative care teams in London $(n=15)$ were also contacted to gather further information about local practice/policy. Findings from the review were used to develop a draft guideline that is being further refined by feedback from key stakeholder groups (comprising experts in palliative care, heart failure and renal supportive care). Results: 13 studies and six clinical guidelines were identified. We found consistent, but low-quality (case reports/series) evidence for the safety and effectiveness of subcutaneous Furosemide in patients with ESHF. Among the six guidelines identified, two were specific to community settings and one relevant only to patients with stable symptoms. Information regarding Furosemide titration, patient monitoring and use of diluent varied across guidelines. Four of the six guidelines were past their review date.

Conclusions: We have developed an up-to-date, best-practice clinical guideline for the use of subcutaneous Furosemide in patients with ESHF. Our guideline is relevant to healthcare professionals in community, hospital and hospice settings. It encompasses the prescribing of subcutaneous Furosemide for patients with acute symptoms of fluid overload as well as for those with stable disease. Key considerations for administration, monitoring and the use of a medicine 'off-label' are also included. 
Abstract number: P02-293

Abstract type: Poster

Review of End of Life Discharges from Acute Hospital under a National Rapid Discharge Protocol in Ireland Brennock J., O'Gorman A., Hennessy M.

Department of Palliative Medicine, Our Lady of Lourdes Hospital, Co Louth, Ireland

Background: $74 \%$ of Irish people express a wish to die at home, yet only $25 \%$ who die annually die at home. The National Rapid Discharge Protocol (NRDP) guidelines were developed to facilitate the transition of care of patients who are within the last 24-48 hours of life to die at home in accordance with their wishes or those of family members, where this is safe and appropriate to do so.

Aims: The aims of this review were to:

$\S \quad$ Ascertain the number of discharges under NRDP from a single hospital palliative care team over a defined time period

$\S \quad$ Ascertain the interval between discharge and death

$\S \quad$ Review cases where the patient was re-admitted to hospital.

Methods: Discharges under NRDP were recorded for a defined time period, detailing the date of discharge and the community palliative care team (CPCT) to which the patient had been referred. Follow up by phone confirmed date of death (if applicable), duration of time under the care of CPCT, discharges from care of CPCT, and re-admission to hospital. Data was collected by one person and analysed using simple Excel software.

Results:

$\S \quad 15$ patients were discharged under NRDP over a 9 month period

$\S \quad 12$ patients died at home. 1 patient was re-admitted and subsequently died in acute hospital setting. 2 patients are still alive, with 1 remaining under the care of CPCT and 1 discharged from services.

$\S \quad$ The median duration of care under CPCT for patients who died was 4.5 days (mean 21.7).

Discussion: The majority of patients were correctly identified as being in the actively dying stage and the transfer home to die was an appropriate activation of NRDP. At present the NRDP is most often used by hospital palliative care teams but it is hoped that it will become a more prevalent tool in the wider medical community to facilitate the wishes of the dying to spend the last days of life in their own home.
Abstract number: P02-294

Abstract type: Poster

Assessing Ease of Use of an App Based Triaging Tool to

Coordinate Care

Ramakrishnan K. ${ }^{1}$, Padmanabhan S. ${ }^{2}$, Abraham C. ${ }^{3}$

${ }^{1}$ Care Synergist, Norfolk, United States, ${ }^{2}$ Healthabove60, Chennai, India, ${ }^{3}$ College of William and Mary Mason Business School, Williamsburg, United States

Background: There is growing interest in the use of technology to optimize patient care and experience.

Aims: To conduct a pilot survey to study the ease of use and impact of an app and web-based platform: Q life.

Methods: A survey was conducted through a questionnaire which was taken by the triage team and field staff. The questionnaire included a set of 6 questions covering ease use of the application, care coordination, communication, quality of care and experience. The survey was taken by the team after enrolling 50 patients in the Care Synergist Q Life application.

Results: Remarkable acceptance was seen amongst the clinical team which was previously using a manual workflow model. $75-100 \%$ of the clinical team agreed and $25-50 \%$ of the clinical team strongly agreed to the ease use of the app-based triaging and care coordination platform. In addition, responses to other questions reflecting ease of use, care coordination, communication, quality of care and experience of using the new technology were in favor of the platform overall, the platform was deemed to be very user friendly by the clinical team.

Discussion: $Q$ life offers a user-friendly platform for clinical teams to improve care delivered to palliative care patients. Real time data availability capturing, live dashboards and informed decision making can provide the foundation elements for care agencies to optimize care and grow.

Abstract number: P02-295

Abstract type: Poster

\author{
An Evaluation of Compassionate End of Life Care in Residential \\ Care Centres \\ Steele K., Lynch M. \\ Healthcare Department, Irish Hospice Foundation, Dublin, Ireland
}

Background: Each year in Ireland over 7,000 people die in residential settings yet there is no formal framework to sustain quality delivery of

50 Enrollment Survey.

\begin{tabular}{|c|c|c|c|c|}
\hline & $\begin{array}{l}\text { Strongly Disagree } \\
\text { disagree }\end{array}$ & $\begin{array}{l}\text { Neither agree } \\
\text { nor disagree }\end{array}$ & Agree & $\begin{array}{l}\text { Strongly } \\
\text { agree }\end{array}$ \\
\hline It is not complex to use & & & $75 \%$ & $25 \%$ \\
\hline I was able to easily use the tool immediately after the training & & & $50 \%$ & $50 \%$ \\
\hline
\end{tabular}

I give quicker responses to patient as a member of the clinical team after using

$100 \%$ the tool compared to communication before using the tool

"I give clearer answers to the patient as a member of the clinical team about what to do to for their care after using the tool compared to communication before using the tool

The tool has helped me avoid having to patients go to the emergency room at

least once since starting using it

The tool gives me a way to manage patient's pain better 
end of life care (EOLC) in these settings.A quality improvement programme was developed to enhance the EOLC that residents receive whilst also enabling and empowering staff to continuously review, reflect on the EOLC they deliver. The programme objectives are to improve quality and raise awareness of EOLC across the culture and practices within the residential setting.

Aims and objectives: The evaluation of the programme was aimed at 9 residential settings within an established network. It sought to establish if participating in the programme enabled positive change in EOLC for the resident, family member and staff as well as identifying the overall benefits of participating in the programme.

Methods: A telephone interview, using a structured questionnaire was carried out in the participating centres. The questionnaire consisted of open ended and closed questions. Analysis of the responses included identifying and grouping common themes. All participants were sent the amalgamated research findings for review and verification. 7 sites participated in the telephone interview, 2 declined for operational reasons. Findings/ results: All seven respondents reported that EOLC had positively changed for the residents, families and staff. Benefits noted included advance care planning with residents, communication with residents and families. Improvements in care practices included: care at the time of death; care after death; preferred place of death; accessing specialist palliative care and GP links.

Conclusions: Educational initiatives alone are unlikely to improve EOLC delivered by staff in residential care. This quality improvement model provides a formal framework to embed ongoing developments in EOLC in this setting. Further in-depth evaluation is recommended to link to other initiatives for more coherent approach.

\section{Abstract number: P02-296 \\ Abstract type: Poster}

Towards a Comprehensive Quality System for Palliative Care in The Netherlands

van der Velden A.W.G. ${ }^{1}$, Witkamp F.E. ${ }^{2}$, van Esch T.E.M. ${ }^{3}$, Francke A.L. ${ }^{3}$, van der Heide A. ${ }^{2}$, Engels Y. ${ }^{4}$, Reyners A.K.L. ${ }^{1}$

${ }^{1}$ University Medical Center Groningen, Groningen, Netherlands,

${ }^{2}$ Erasmus University Medical Center, Rotterdam, Netherlands,

${ }^{3}$ Nivel (Netherlands Institute for Health Services Reserach), Utrecht, Netherlands, ${ }^{4}$ Radboud University Medical Center, Nijmegen, Netherlands

Introduction: In the Netherlands, care organisations aim to increase the quality of palliative care in the coming years. A reliable and comprehensive system to monitor this quality is lacking.

Aim: To explore the possibility of a comprehensive quality monitoring system by evaluation

1) the most relevant aspects for the Dutch public

2) existing tools used in recently granted palliative care projects funded by the Netherlands Organisation for Health Research and Development

3) continuous registrations of healthcare data.

Methods: The items, assessed by a survey, that are deemed relevant regarding quality of palliative care for the Dutch public are the base of this project. All patient/family oriented outcomes in the granted projects were inventoried. The usability and linking possibilities of existing continuous data sources with routine healthcare registrations through desk research and stakeholder interviews was investigated. Finally, determination of the possibility to integrate these components for practical use was studied.

Results: Based on the Dutch public, 26 most relevant items were determined, of which relief of pain and anxiety, making own decisions, attention to personal wishes and meaningful life stand out. To study symptoms, needs, experiences with care and with quality of life\&dying in the 44 granted projects, 66 patient/family instruments were used, 7 instruments for real-time registration, 29 for data-extraction from patient records. Few instruments were used more than once. Seven health care data sources contain relevant palliative care data and can be interconnected. These data concern domains like 'structure and process', 'physical care' and 'psychological care'. Only 6 out of the 26 relevant items are addressed in these registrations.

Conclusion: Items regarding pain relief and making own care decisions were most relevant for the Dutch public. However, future efforts are needed to retrieve this information from research and routine care registrations.

Abstract number: P02-297

Abstract type: Poster

Improving Palliative Care through Interdisciplinary Diffusion: Effect of Regular Pallative/Radio-oncological Ward Rounds Oertel M. ${ }^{1}$, Schmidt R. ${ }^{2}$, Eich H.T. ${ }^{1}$, Lenz P. ${ }^{2}$

${ }^{1}$ Department of Radiation Oncology, University Medicine Muenster, Münster, Germany, ${ }^{2}$ Department of Palliative Care, University Medicine Muenster, Muenster, Germany

Background: Many patients receiving radiotherapy (RT) present palliative care (PC) needs with increasing intensity in the course of their disease.

Aims: The aim of study was to evaluate regular pallative/RO ward rounds to increase awareness for palliative care and improve professional training in RT.

Methods: Singe-centre retrospective study, inpatient PC service, radiooncology department. Data were analyzed from 05/01/2015 to $05 / 31 / 2018$. Weekly regular ward rounds were conducted under participation of the PC senior physician, residents in RO and nursing staff. Procedural characteristics of the palliative consult service (PCS) were collected retrospectively and surveys were distributed to residents in RO.

Results: During the study period, 415 patients received RT under surveillance of the PCS (14\%). The time from admission to integration of PC was $8 \pm 12$ days, the length of stay under surveillance of the PCS was 19 \pm 15 days. Cooperation between the PCS and the Department of RO is much appreciated as shown by a survey: 10 residents in radiation oncology (5 male, 5 female; $25-30$ years(y): 5, >30-35y: 2, >36y: 3) answered after having fulfilled their obligatory ward rotation. Of these, 9 out of 10 (1 not answered) have known about the PCS and have involved it, mostly frequently (8: frequently; 1 : rarely). Typical indications for consultation were (multiple answers possible): pain (8), coordination of further care (hospice, palliative care unit), nausea/vomiting (7), psycho-oncological help/treatment for patients (7), music therapy (5) and coordination of physiotherapy (4). 9 out of 10 participants ( 1 unknown) evaluated the cooperation to be enriching and useful to broaden knowledge in palliative care.

Conclusion: In RO a high percentage of patients are in need for specialized palliative care. Regular ward rounds are highly effective to improve palliative care knowledge for residents in $\mathrm{RO}$ and provide time shortage for initiation of PC.

\section{Abstract number: P02-298}

Abstract type: Poster

Principles of Care for the Dying Patient - Audit of Use in a Tertiary Referral Cancer Centre

Halley A., Ramsay J., Johns H., Kane G., Stephen A., Stevens A.-M. Symptom Control and Palliative Care, The Royal Marsden NHS

Foundation Trust, London, United Kingdom 
Background: The Leadership Alliance for the Care of Dying People set out an approach with focus on achieving the five priorities of care. Our trust developed an electronic version of the London Cancer Alliance document, The Principles of Care for Dying Patients. This audit set out to determine the number of expected deaths who had a document in place and if completed, adherence to the UK NICE (The National Institute for Health and Care Excellence) Quality Standards for care of adults in the last days of life (NG31).

Methods: Prior to the UK National Care at the end of life Audit, the hospital palliative care team undertook an audit of care of patients in the last days of life for deaths from $1^{\text {st }}$ April 2017 to $31^{\text {st }}$ March 2018. There were 230 deaths across the trust. Deaths in the intensive care unit and paediatric deaths were excluded. 177 case record reviews were undertaken by the palliative care team.

Results: $65 \%$ of patients who were recognised as dying had a principles of care document initiated.

NICE Quality Standard 1: $100 \%$ of patients had daily review to assess for changes in dying phase ie. nearing death, stabilising or recovering.

NICE Quality Standard 2: $53 \%$ of patients had capacity to be able to discuss, and review an individualised care plan (ICP). $97 \%$ of cases showed documented evidence of a family discussion to ensure communication of recognition of dying and development of ICP.

NICE Quality Standard 3: - 94\% of patients had appropriate anticipatory medications prescribed.

NICE Quality Standard 4 - 68\% of patients had documented evidence of discussion of hydration status.

Conclusion: The Principles of Care Document when used adheres to the Nice Quality Standard. The regular education of the multi-disciplinary team in caring for dying patients continues to focus on use of the document to direct excellent care of the dying.

Abstract number: P02-299

Abstract type: Poster

\section{Audit of Effectiveness of In-patient Clinical Management Escalation Plan \\ Vincent C., Day P., Chauhan A. \\ John Eastwood Hospice, Mansfield, United Kingdom}

Background: In 2017, a formal escalation planning process for the clinical management of patients at a hospice in-patient unit was initiated in response to the inappropriate transfer of a patient from the hospice to the acute setting out-of-hours. Our initiative involves formation of an escalation plan (EP) in advance consisting of one of three categories; supportive hospice care, hospice escalation or hospital escalation. The process encourages clinicians to empower patients and carers to be involved in this aspect of advance care planning, and for it to be documented clearly to ensure appropriate treatment is offered, especially out-of-hours.
Aims: This study aims to answer the question 'Does advance escalation planning and documentation improve patient care?'

Methods: Data was collected retrospectively of 50 cases of deaths and discharges from the in-patient unit 1 year after the escalation plan process was commenced. A staff questionnaire was also included. Medical records were reviewed for details of treatment escalation events that occurred or were considered, and this was compared to the EP at the time.

Results: The process was being used effectively with $94 \%$ of patient's having an EP documented. Most escalation events were intended to improve symptom control. $4 \%$ were appropriately transferred from the hospice to the hospital for in-patient care. Escalation events matched the EP in $88 \%$ and where they didn't match, this involved patient choice or a rapid change in the patient's condition. The staff who engaged with the staff questionnaire reported finding the EP helpful.

Conclusion: This audit shows overall positive results of engagement and clinical outcomes of this initiative. The process has enabled improved focus on escalation plans as part of wider advance care planning. Plans are formed in advance, documented, and clinical judgment is used in individual situations to ensure appropriate clinical treatments are offered.

Abstract number: $\mathrm{P} 02-300$

Abstract type: Poster

Identification of Relevance and Feasibility of Registration Items Regarding Quality of Palliative Care: A Delphi Study among Dutch Experts

van der Velden A.W.G. ${ }^{1}$, Reyners A. K.L. ${ }^{1}$, Francke A. L. ${ }^{2}$, van der Heide A. ${ }^{3}$, Engels $Y^{4}$

${ }^{1}$ University Medical Center Groningen, Groningen, Netherlands, ${ }^{2}$ Nivel (Netherlands Institute for Health Services Reserach), Utrecht, Netherlands, ${ }^{3}$ Erasmus University Medical Center Rotterdam, Rotterdam, Netherlands, ${ }^{4}$ Radboud University Medical Center, Nijmegen, Netherlands

Introduction: In the Netherlands a reliable and comprehensive system to get insight in the quality of palliative care is lacking.

Aim: Selection of a limited set of items that should be registered in care files out of a list of aspects of palliative care that are considered most important by the Dutch public.

Methods: A first Delphi round was conducted among a panel of Dutch palliative care experts. They were asked to rate the relevance and feasibility of registration of the 26 most relevant items in palliative care according the Dutch public. Consensus that items are relevant or feasible was obtained if items had a median rating of 8 or 9 on a 9-point Likert scale, without disagreement (meaning $\leqslant 30 \%$ of the ratings was $\leqslant 3$ ).

Results: One Delphi round with 42 experts resulted in consensus that $21 / 26$ items are relevant. For 10 of these 21 items, registration was

Table 1. 10 relevant items that are considered feasible to register.

\begin{tabular}{lll}
\hline $\begin{array}{l}\text { Items relevant for good palliative } \\
\text { care }\end{array}$ & $\begin{array}{l}\text { Relevance, median } \\
\text { score panel (1-9) }\end{array}$ & $\begin{array}{l}\text { Feasibility, median } \\
\text { score panel (1-9) }\end{array}$ \\
\hline no pain and no dyspnea & 9 & 9 \\
recording of desired care & 9 & 9 \\
no confusion & 8 & 8 \\
residency & 9 & 8 \\
end-of-life information & 8 & 8 \\
shared decision making & 8 & 8 \\
information about treatment options & 8 & 9 \\
designated contact person & 9 & 9 \\
family present at conversations & 9 & 9
\end{tabular}


considered feasible (table 1). No consensus was reached about feasibility of some items deemed very relevant for the Dutch public and experts like attention to personal wishes (7), meaningful life (7) and making own health-care decisions (6.5).

A second Delphi round will be conducted to achieve consensus about a limited and feasible final set of items.

Conclusion: Palliative care experts agreed that most of the aspects of palliative care that are considered important by the Dutch population are relevant to asses quality of care. However, less than half of these items are deemed feasible to register in care files.

\section{Abstract number: $\mathrm{P} 02-301$}

Abstract type: Poster

\section{Domains of End-of-Life Care for Critically III Patients in Intensive Care Units: A Systematic Review}

Chiou Y.-G. ${ }^{1}$, Wei T.-Y. ${ }^{2}$, Tang S.-T. ${ }^{3}$

${ }^{1}$ Chang Gung University/Chang Gung Memorial Hospital at Kaohsiung, Kaohsiung, Taiwan, Republic of China, ${ }^{2}$ Chang Gung University/Chang Gung Memorial Hospital at Linkou, Taoyuan, Taiwan, Republic of China, ${ }^{3}$ School of Nursing, Chang Gung University, Taoyuan, Taiwan, Republic of China

Background: A dignified death is a very important quality indicator for critically ill patients in intensive care unit (ICU). Thus, it is necessary to explore the quality of end-of-life (EOL) care for critically ill patients in ICUs. Although the concepts and indicators of the quality of EOL care in ICUs have been proposed, they have not been integrated yet. If they might be done, the most valued domains will be recognized to improve the quality of EOL care. This study explores the domains of quality of EOL care identified in the literature for critically ill patients in ICUs through a systematic review.

Methods: Five common electronic databases were searched for articles published in 1979-2018 by the key words of "critically ill patients", "end-oflife", "ICU" and "quality of EOL care." Eligible articles must identify domains or elements of quality of EOL care in ICUs. Each article was examined by two reviewers independently based on the Robert Wood Johnson Foundation Critical Care End-of-Life peer Workgroup proposed specific domains for patients and families.

Results: Out of the 5259 searched items, 43 from 103 potential articles met the inclusion criteria. There are 18, 21 , and 4 articles of expert consensus, questionnaire surveys, and medical record reviews, respectively. Six domains were synthesized: patient and family-centered decision making $(n=40,93 \%)$, communication with patients and families $(n=37,86 \%)$, emotional and practical support for patients and families $(n=36,84 \%)$, symptom management and comfort care $(n=35,81 \%)$, spiritual support for patients and families $(n=23,53 \%)$, and continuity of care $(n=6,14 \%)$. Conclusion: This systematic review highlights strong consensus on the domains of quality of EOL care in ICUs. Patient and family-centered decision making and communication with patients and families are the most important two domains to improve quality of EOL care in ICUs but spiritual support and continuity of care were less emphasized.

\section{Abstract number: $\mathrm{P} 02-302$}

Abstract type: Poster

\section{IT (Information Technology): Contribution to Palliative Care Development \\ Marđetko R. ${ }^{1}$, Dumbović N. ${ }^{2}$}

${ }^{1}$ Centre for Coordination of Palliative Care in the County of Medimurje, Home Care Centre Cakovec, Cakovec, Croatia, ${ }^{2}$ Centre for Coordination of Palliative Care in the County of Varazdin, Home Care Centre Varazdin, Varazdin, Croatia

Although Croatia is at the early stages of structured palliative care development, information technology has been used in healthcare for a long
time.The use of well-established medical software has been recognized as an opportunity for better connection and coordination of different services providing care for the same patient. Not only it helps creating continuity of care for patients but also encourages professionals to recognize patients with needs for palliative care and refer them to palliative care services. Technologies already familiar to professionals cause less resistance and effort in introducing changes. Software also enables professionals to record and value their work. Workshops with all stakeholders regarding the palliative care technology improvement resulted in better understanding of palliative care and increased non-medical activities in palliative care development. Beside the benefits of information technology the whole process of implementation faces challenges; financing new software solutions, interest of various professionals in collaboration, recognition of patients with palliative care needs, balance between amount of information input and time consumption etc. One of the IT companies that already has software implemented within primary health care has created a new software for palliative care. It allows linking services and institutions that may use different software solutions by different providers. Software has been used in more that 10 regions in Croatia with the same approach but tailored to the needs and resources of each local community. All the regions are in the process of involving new stakeholders creating a broad network of services working together. Although evaluation on the national level will be possible after certain time, the software is suitable for constant improvements.

\section{Abstract number: $\mathrm{P} 02-303$ \\ Abstract type: Poster}

\section{End of Life Essentials: Toolkit Evaluation}

Hutchinson C., Tieman J., Devery K.

Flinders University, Bedford Park, Australia

Background: Many Australians will receive care at some point during the last year of their life in a hospital, and many may end up dying in hospital. Clinical opportunities for quality of life can be optimised during the last 12 months of life. The End-of-Life Essentials project provides e-learning modules and implementation resources within a toolkit for hospital clinicians.

Aims: This study sought to assess the effectiveness of the action-orientated toolkit in supporting behaviour change in relation to quality endof-life care in acute hospital settings.

Methods: Following a review of literature on end-of-life care practices and an examination of toolkit considerations, My Toolkit was drafted. The toolkit contained checklists of sixty suggested actions relating to the project. Feedback on content, structure and format was sought from internal and external reviewers. A mixed method evaluation study comprising an online survey and telephone interviews was designed. Ethics approval was received from Flinders University (SBREC 7568).

Results: 428 toolkits were posted to 13 doctors, 309 nurses, 66 allied health and 40 "other" staff during mid 2017. 65 toolkit recipients completed an online survey ( $92 \%$ female, $77 \%$ nurses). Ten phone interviews were conducted using purposeful sampling ( $100 \%$ female, 7 nurses, 3 allied health).

Overall, survey participants responded very positively to My Toolkit (93.7\% indicated content was relevant to their profession and $95.2 \%$ found the resources easy to navigate). $59.3 \%$ reported implementing a toolkit action within 4 weeks.

Interview participants saw communication regarding end-of-life matters as the major area in which the toolkit could support quality end-of-life care practice.

Conclusion: Toolkit users found value in the toolkit content and format. The survey and interview data indicates that the toolkit led to selfreported changes to workplace practice.

The End of Life Essentials Project is supported by the Department of Health. 
Abstract number: $\mathrm{P02}-304$

Abstract type: Poster

Interpreters' Experience of Working with Healthcare Professionals during End of Life Discussions with Culturally and Linguistically Diverse Patients in Perth, Western Australia Knight E. ${ }^{1}$, Schenker $Y^{2}{ }^{2}$, Hughes . $^{1}$

${ }^{1}$ Sir Charles Gairdner Hospital, Nedlands, Australia, ${ }^{2}$ University of Pittsburgh, Pittsburgh, United States

Background: The provision of high quality palliative care to culturally and linguistically diverse groups of patients requires collaboration between health care professionals and interpreters. Poor preparation and support of interpreters placed in this role may lead to emotionally burdensome and ethically challenging experiences.

Objectives: The objective of this study was to explore the experiences of interpreters from Perth, Western Australia who have interpreted for end of life care discussions, and to use the results to guide the education of healthcare professionals working in this area.

Methods: An electronically administered survey was distributed to interpreters in Perth to assess the frequency that briefing and debriefing meetings were held between healthcare professionals and interpreters, and whether the expectations of the interpreter's role were discussed. Results: $17 \%$ of interpreters reported having a briefing meeting and only $7 \%$ reported having a debriefing meeting, in their experience of interpreting for end of life care discussions. 13\% of interpreters reported clarifying their role prior to the consultation. The lowest frequencies were observed in the outpatient setting and when doctors were the primary healthcare professional.

Conclusions: To improve the collaboration between healthcare professionals and interpreters and better support interpreters as part of the healthcare team in palliative care, education regarding recommendations on how to achieve this would be most constructively aimed at doctors working in the outpatient setting.

Abstract number: $\mathrm{P} 02-305$

Abstract type: Poster

Standard Operating Procedures (SOPs) for Palliative Care in the German Comprehensive Cancer Center Network - An Evaluation of the Implementation Status

Loedel S., Gahr S., Ostgathe C.

Department of Palliative Medicine, University Hospital Erlangen, Friedrich-Alexander University Erlangen-Nürnberg (FAU), Erlangen, Germany

Background: In order to improve the care of palliative patients in the German Comprehensive Cancer Centers (CCC) and to set quality standards, 15 Standard Operating Procedures (SOPs) had been developed and published by the CCCs. It was unknown to what extent these symptom-, treatment pathway- and process-related SOPs are implemented in the clinical routine in CCCs currently funded by the German Cancer Aid (DKH). Aim: The aim was to gain more information about the accessibility, state of implementation, perceived practicability and comprehensibility and storage location of the developed SOPs, as well as how employees became aware of these.

Methods: Between April and July 2018 an online-based quantitative survey was conducted inviting 125 health professionals in palliative care of all 16 locations of the CCCs. Based on the questions to the SOPs conclusions on implementation status and further themes could be drawn. The data were analyzed descriptively using SPSS.

Results: Response rate was $52.8 \%$ whereby at least one person from each CCC took part. More than half of the respondents (57.6\%) know about the free availability of SOPs on the homepage of the CCC network. The implementation status of SOPs in the CCCs varies from $22.7 \%$ to
$48.5 \%$. SOPs were most frequently noticed through recommendations from colleagues, departmental team meetings or a hint from the chief physician. They are mainly placed in the intranet, in the digital Quality Management (QM) portal and in the paper-based QM manual. The most practice-related and understandable SOPs are "Respiratory distress of an adult palliative patient", "Palliative sedation" and "Acute state of confusion". Reasons for obstacles to use are mainly time factors and a lack of knowledge of existence and availability.

Conclusions: In practice, the increase of knowledge about the SOPs can mainly be achieved through internal efforts, e.g. through systematic training or discussion of SOPs in a Jourfix of the management team.

Abstract number: P02-306

Abstract type: Poster

Chart Review: Documentation of Drugs with Sedative Effects and Indications - Results from a Pilot Test

Kurkowski S., Torres Cavazos J.L., Ostgathe C., Heckel M., Klein C.

Department of Palliative Medicine, University Hospital Erlangen, Friedrich-Alexander University Erlangen-Nürnberg (FAU), Erlangen, Germany

Background: A medical indication is the prerequisite and legitimation for therapeutic interventions and should be documented. Regarding Palliative Sedation (PS), in many cases indication is the only delimiter to differentiate between PS as an extraordinary treatment option and usual symptom-oriented treatment. The prescription of drugs with -primary or secondary- sedative effects (DSE) may have ethical and legal implications e.g. regarding patients' autonomy. Hence robust documentation of indications is paramount.

Aim: This pilot study wanted to test the feasibility of chart review regarding administration of and indications for DSE.

Methods: In a pilot test prior to a comprehensive study (SedPall) on clinical practise of sedation in Palliative Care, medical charts of one palliative care unit and one specialized outpatient palliative care team were analysed regarding administered DSE as well as its indication. Data analysis was performed using SPSS 21.0 (IBM corp).

Results: A total of 38 patient records ( $n=21$ female, $n=17$ male) were screened. Average age was 69 years (20-94 years). Mean duration of treatment was 18 days (2-156 days). In total 351 DSE (regular and on-demand) were recorded. In 77 of those (22\%) no indication could be found in the documentation. Administered drugs were: buprenorphine, fentanyl, haloperidol, hydromorphone, levomepromazine, lorazepam, melperone, midazolam, pipamperone, quetiapine and risperidone. Documented indications included agitation, anxiety, delirium, dyspnea, nausea/vomiting, pain. Conclusions: Chart review regarding DSE and indication is feasible for the majority of cases. However, it has to be taken into account that in more than one out of five DSE, the indication could not be retrieved in the documentation. Concerning justification of the treatment and in order to facilitate research, improvement of documentation is recommended.

Funding: The study is funded by the Federal Ministry of Education and Research (01GY1702A).

Abstract number: P02-307

Abstract type: Poster

Prescribing of Continuous Parenteral Infusions for Symptom Relief in Dying Patients: Reassuring but Needs Further Investigation

Burns E., Davis C.

Palliative Medicine, NHS University Hospital Southampton Trust, Southampton, United Kingdom

Aim: To explore the use of continuous parenteral infusions for symptom relief in dying patients. 
Methods: Casenote review of acute hospital in-patients who died in April 2018 recording location, diagnosis and hospital palliative care team (HPCT) involvement. For those who died on an infusion: rationale for starting infusion, duration and drugs were recorded.

Results: 86 casenotes reviewed. 34/86 (40\%) patients died on an infusion.

$21 / 60(33 \%)$ dying of non-malignant conditions and 13/26 (50\%) dying of cancer were on an infusion. 29/49 (59\%) known to HPCT, 5/37 (15\%) not known to HPCT, 9/14 (64\%) patients on an oncology ward and 8/28 (29\%) on a care of the elderly (COTE) ward were on infusions.

$30 / 34(88 \%)$ initial infusions included an opioid (12 morphine, range 5-30mg, median 10mg; 18 oxycodone, range 5-20mg, median $5 \mathrm{mg}$ ) and $33 / 34$ (97\%) died on an opioid (12 morphine, range 5-30, median 10mg; 21 oxycodone, range 5-50mg, median $5 \mathrm{mg}$ ); 19/21 (90\%) on oxycodone were known to HPCT. Midazolam and anti-secretory drugs were the next most frequently used drugs.

Median infusion duration 2 days (range 0-13); 1 day (range 0-3) if not known to HPCT and 2 days (range 0-13) for those who were.

Conclusion: Patients with non-malignant conditions and those on COTE wards were less likely to die on an infusion. Not all patients with cancer nor all those known to HPCT died on an infusion but patients on an infusion were more likely to be known to HPCT and to be on it for longer. The data suggests possible reticence to prescribe continuous infusions for symptom relief in dying patients on COTE wards. Poor documentation around reasons for commencing infusions precluded qualitative analysis.

Further investigation of the reasoning behind opioid choice is required. Doses of opioids were low and did not substantially increase possibly related to short duration of infusions.

We propose a prospective study to further examine any differences in prescribing by generalists and palliative care specialists.

Abstract number: P02-308

Abstract type: Poster

Clinical Decisions Trees in Palliative Care: A Novel Method to Translate/Present Guidelines

Tilma M. ${ }^{1}$, Borggreve B. ${ }^{1}$, Dermois $M .{ }^{1}$, de Graeff A. ${ }^{2}$

${ }^{1}$ Netherlands Comprehensive Cancer Organisation, Utrecht, Netherlands, ${ }^{2}$ Department of Oncology, University Medical Center Utrecht (UMCU), Utrecht, Netherlands

Background: The development and application of Clinical Practice Guidelines (CPG) is resource intensive and time consuming. In CPGs it's difficult to find the right recommendation for this specific patient. To address this problem, we developed a novel methodology to represent CPGs into clinical decision trees (CDTs) that are practical and easy to use, as demonstrated in oncology care.

Aim: We aimed to explore CDTs potential for palliative care guidelines and to develop a CDT for the CPG: Ileus.

Methods: The CPG was translated into CDTs. CDTs are represented by nodes, branches and leaves, representing data-items (population characteristics, e.g. live expectation, ), data-item values (e.g. $<=4$ weeks) and recommendations (e.g. explorative surgery), respectively. CDTs are developed for each step in the care pathway (e.g. diagnoses, treatment, follow-up). CPG Ileus existing of 35 items.

Filling in patient characteristics (data-items and values), a path through a tree, results in a recommendation. The collection of all data-items serve as a standardized clinical vocabulary (scv) for implementation in e. g. electronic health record (EHRs), which is a requirement for computer assisted CPG implementation.

Results: The CPG Ileus is successfully translated into 21 separate CDTs, such as "non-surgical treatment of ileus". These trees are driven by 35 unique data-items, that composed the scv. Decision trees were integrated in an decision support application (www.oncoguide.nl). With the scv as a fundamental component for implementation, the application is ready for EHR connection.

Conclusion / lessons learned: Palliative care CPG focused on symptom control can be translated into CDTs and can be used as a basis for clinical decision support systems. The standardized clinical vocabulary extracted from the CDTs facilitates CPG implementation in EHRs and serves as a requirement for a closed-loop learning cycle from clinical practice to guideline development and research.

Abstract number: P02-309

Abstract type: Poster

\section{Palliative Care Consults and Outcomes in the Emergency} Department

Cardenas Mori J. ${ }^{1,2}$, Huvane B. ${ }^{1}$, Chuang E. ${ }^{1,2}$, Mitchell L. ${ }^{1}$, Genereux C. ${ }^{1}$ ${ }^{1}$ Montefiore Medical Center, Bronx, United States, ${ }^{2}$ Albert Einstein Medical College, Bronx, United States

Adults with chronic illnesses visit the ED several times in their last months of life, with over half of older Americans presenting in their last month and many dying in the ED.

Objectives: Determine the overall outcomes of PC consult in the ED. Evaluate hospice referral from ED

Methods: This is a retrospective cohort chart review study at ED Weiler Hospital Campus at Montefiore Medical Center from April 2017 to September 2018. The Weiler ED serves approximately 75,000 visits per year. Patient demographic,clinical data and quality indicators QI were abstracted from the medical record. QI include: Reason for ED visit, reason for palliative consult PC, hospice referral, Code status discussion, and admission or discharge status.

Results: Over the 18-month study period, there were 105 PC consults seen in the ED. The primary reason for consult was goals of care (GOC) discussion ( $n=68,65 \%)$, followed by end-of-life (EOL) care $(n=19,18 \%)$ and pain management $(n=12,11 \%)$. Five patients $(5 \%)$ were discharged directly from the ED with hospice services. An additional 27 (26\%) were discharged with hospice services after a mean 8.4 day hospital stay. Eleven patients (10\%) were terminally extubated, 3 in the ED. Thirty-one patients (30\%) died in the hospital, 8 of whom died in the ED. Forty-eight patients (46\%) had a code status change to DNR during hospitalization, $31(30 \%)$ had a DNR order on arrival to ED.

Conclusion: Patients receiving PC consultation in the ED were at high risk for death during admission. About $5 \%$ of these consults resulted in discharge directly from the ED with hospice services, representing a significant reduction in burdensome hospital admission and unnecessary health care costs. The palliative care service was also able to facilitate code status change and to perform timely terminal extubation for those patients with comfort-oriented GOC. The main limitation of this study is that it is an observational retrospective design and causality cannot be determined.

Abstract number: P02-310

Abstract type: Poster

Can They Get More Satisfaction Depending on "Where"? A Survey among Patients in Different Settings

Leon Loza G.A. ${ }^{1}$, Arraiza B. ${ }^{1}$, Ruiz de Gaona Lana E. ${ }^{2}$, Calvo Espinos C. ${ }^{1}$

1 Palliative Care Unit, Hospital San Juan de Dios Pamplona, Pamplona, Spain, ${ }^{2}$ Hematology and Hemotherapy, Fundacion Hospital Calahorra, Calahorra, Spain

Introduction: There is few research about satisfaction of relatives of palliative care patients, and even less comparing both home care setting and hospitalized.

Aim: To analyse the perception of the relatives of inpatients of a palliative care unit, compared with those in home care settings. 
Method: Retrospective study among relatives of patients deceased during 2017. We designed a phone survey developed by an independent researcher, scoring each ítem from very well to very bad in a Likert scale. The informed consent was collected at the beginning of the interview. Results: 191/405 deceased at home (50\%) and 95/230 among hospitalized $(41 \%)$ joined the study. About home care program, satisfaction was very good overall (93.7\%), with a great perception of coordination with primary care $(89 \%)$ and oncology $(91 \%)$. Very well rated the phone assistance (93\%, felt compained), described as fast enough $(92 \%)$ and resolutive $(93 \%)$. Number of visits were very well rated $(92 \%)$. All the professionals were very well valued (nurses the best), specially symptom control and information management by physicians $(92 \%)$ and helpfulness of psychological assistance. Patients perceived themselves wellinvolved in advance care planning (94\%), as so the family (93\%). All the respondents valued our bereavement letter very well. Correlation model showed that there was no relation between more visits $(-0.13)$ or more days in programme $(-0.24)$ and more possible satisfaction. About hospital setting, the overall satisfaction was also very good (88\%), being the volunteers (93\% very well) and physiotherapist $(92 \%)$ the best rated. Coordination between services was also well $(74 \%)$, as well as the bereavement letter (94\%). Correlation model at the hospital was statistically significant between more days admitted and more possible satisfaction (0.13)

Conclusion: There is a very good level of satisfaction among our population, a little bit more in some topics in home care setting. More research is needed.

Abstract number: P02-311

Abstract type: Poster

Transformation through Quality Improvement - Improving Experience and Outcomes for those in the Last Year of Life in an Acute Hospital Setting

Mann T. ${ }^{1}$, Bennetts J. ${ }^{2}$, Shadat R. ${ }^{3}$, Caulkin R. ${ }^{4}$, Dumble K. ${ }^{5}$, Carter J. ${ }^{6}$, Arumugam J.7, Beer $\mathrm{H}^{2}{ }^{2}$, Kennard C. ${ }^{8}$, Hasnat M. ${ }^{8}$, Charles H. ${ }^{8}$, Turner M. ${ }^{8}$, Collis E. ${ }^{2}$, Stirling L.C. ${ }^{2}$

${ }_{1}^{1}$ Palliative Care, Rennie Grove Hospice Care, Tring, United Kingdom, ${ }^{2}$ Camden, Islington ELiPSe and UCLH \& HCA Palliative Care Service, Central and North West London NHS Foundation Trust, London, United Kingdom, ${ }^{3}$ Palliative Care, Royal Free Hospital NHS Trust, London, United Kingdom, ${ }^{4}$ Palliative Care, Chelsea and Westminster Hospital, London, United Kingdom, ${ }^{5}$ St Joseph's Hospice, London, United Kingdom, ${ }^{6}$ Barts Health NHS Trust, London, United Kingdom, ${ }^{7}$ North Middlesex University Hospital, London, United Kingdom, ${ }^{8}$ University College London Hospitals NHS Trust, London, United Kingdom

Background: Approximately one in three people in hospital are in the last year of life. Studies suggest that over $60 \%$ of people prefer to die outside hospital, but in London less than $48 \%$ do so. The delivery of coordinated excellent care of patients who might be in the last year of life requires engagement and support of staff of all professions.

Aim: To transform end of life care in a central London acute hospital trust.

Design/methods/approach: A Quality Improvement programme was initiated in 2014, with executive oversight and measurement. A multiskilled team delivered several initiatives including various Trust wide staff training interventions, the development and roll out of an individualised care plan for the last days of life, a bereaved carers survey, review of complaints and incidents and use of the London Electronic Palliative Care Coordination System. Training centres on empowering staff to recognise those in the last year of life, initiate compassionate conversations, provide high quality care and collaborate. A key aspect was not to provide a new clinical service but to initiate a cultural shift with staff championing discussions and high quality care themselves.

Results: Over 4800 staff have been trained in 32 clinical areas. Staff report increased confidence in the recognition of and communication with those who may be in the last year of life $(11 \%$ and $21 \%$ respectively). Complaints related to end of life care have fallen from 6.33 to 1 / quarter ( $84 \%$ reduction). Over $92 \%$ of bereaved carers report excellent/ good care of their loved one. The average length of stay of admissions during the last year of life has fallen from 10.1 to 9.1 days.

Conclusions/lessons learned: The provision of a team to facilitate and empower staff to provide excellent care for those in the last year of life is a high impact intervention that improves patient and carer experience and hospital use. Embedding the team within the Trust ensures sustainability and ongoing improvement.

Abstract number: P02-312

Abstract type: Poster

Does your Services Meet the Standards of Palliative Care? The Australian Experience

Reed K.

Palliative Care Australia, Griffith, Australia

Aim: Palliative care services are delivered in a variety of settings across Australia to enable patients living, and dying, with life limiting illness to have appropriate, timely and compassionate quality care. Palliative Care Australia (PCA) is the peak body for palliative care delivery, services and patients and their carers throughout Australia.

Working with the government, it sets the standard by which services can be assessed and mapped against the Australian Commission of Safety and Quality in Health Care for evidence in organisational or service accreditation.

Design, methods and approach: The first National Palliative Care Standards was launched in 2005 and the National Standards Assessment Program or NSAP, was developed in 2009 to assess services against the standards. While enjoying initial success, there was a high attrition rate with time poor services finding the workload of NSAP too burdensome. A formal evaluation was undertaken and with further funding, NSAP has been re-designed and in its place is a new online portal through the PCA website called Palliative Care Self Assessment or PaCSA.

Results: Services can now assess themselves against the latest addition of the Standards launched in 2018. The assessment generates a quality improvement action plan to recognise service achievements and provide guidance in building capacity in service delivery and workforce development.

Conclusion: PaCSA is available for all levels of services delivering palliative care and through continual evaluation and sector engagement, enables and strengthens services in their ability to meet organisational needs, community needs and above all, provide improved patient outcomes.

Abstract number: P02-313

Abstract type: Poster

Clinical Characteristics and Time of Referral to Palliative Care Service in a Teaching Hospital in Accra, Ghana

Edwin A. ${ }^{1}$, Tweya $H^{2}{ }^{2}$, Ibrahim M. ${ }^{3}$, Addo Opare-Lokko E. ${ }^{3}$, Boateng A. ${ }^{3}$, Corquaye $0 .^{3}$

${ }^{1}$ School of Medicine, University of Health and Allied Sciences, Ho, Ghana, ${ }^{2}$ The International Union against Tuberculosis and Lung Disease, Paris, France, ${ }^{3}$ Palliative Care Service, Korle Bu Teaching Hospital, Accra, Ghana

Background: Palliative care is most effective when it is integrated early into patient care. Cancer patients often face difficult problems such as physical distress, existential issues, and poor coping. Palliative care can address these issues and improve patients' quality of life significantly. A Teaching Hospital in Accra, Ghana established a Palliative Care Service (PCS) in August 2012 to provide inpatient, outpatient and homecare services. 
Aim: To analyze the clinical characteristics of patients and time of referrals to the Palliative Care Service in the context of potentialbenefit from effective palliative care

Methods: We collected data on patients who received palliative care from August 2012 to December 2016. Data included in the analysis was that of patients with complete data, malignant disease, and were seen by the PCS from first presentation to death.

Results: Total number of patients seen was 471 ,but only 302 fulfilled the inclusion criteria for our data analysis. $68.2 \%$ were females and $31.8 \%$ were males.All of them had metastatic malignancies. $31.1 \%$ of the patients were in the age range of $25-49$ years while $45.7 \%$ were in the 50-69 years age range. The reasons for referral were palliative care (57.9\%), symptom management (15.2\%), supportive care (8.6\%), pain management $(7.6 \%)$, end of life care $(6.6 \%)$, and counseling $(4 \%)$. $56 \%$ were seen as outpatients and $44 \%$ as inpatients. The mean time on the PCS was 35.59 days. $44 \%$ of patients died within 15 days of referral. Another $40 \%$ died in the subsequent 45 days. Thus, $84 \%$ of patients died within 60 days of referral. Only $5.6 \%$ of the patients spent more than 121 days on the PCS. $43.4 \%$ died at home while $56.6 \%$ died in hospital.

Conclusion: Despite the availability of a PCS in the KBTH, patients with cancer are often not able to access palliative care services until late in their disease trajectory because of late referrals. However, our study showed that even late referrals derive some benefit from palliative care.

Abstract number: P02-314

Abstract type: Poster

The Management of Malignant Hypercalcaemia in a Cancer Centre: A Retrospective Review

Savage E. ${ }^{1}$, Agapinaki E. ${ }^{2}$, Burley L. ${ }^{2}$, Gough N. ${ }^{1}$

'Palliative Care Department, Guy's and St Thomas' NHS Foundation Trust, London, United Kingdom, 2Pharmacy Department, Guy's and St Thomas' NHS Foundation Trust, London, United Kingdom

Background: Hypercalcaemia of malignancy (HCM) occurs in up to $20 \%$ of cancer patients; usually a poor prognostic indicator associated with advanced disease and significant symptom burden. Literature suggests HCM is treated inadequately in $>50 \%$ of cases and there are currently no universally accepted UK guidelines. We observed wide variation in HCM investigation/management in our unit prompting this review.

Aim: To review HCM investigation/management against locally identified standards.

Methods: Patients with a cancer diagnosis and corrected calcium (CCa) $>2.65$ admitted between July-October 2017 were identified with an automated electronic patient record search. Case notes and drug charts were reviewed and practice compared against locally identified standards.

Results: 69 patients were identified; 22 excluded due to identifiable non-malignant causes of hypercalcaemia. 47 patients were identified with $\mathrm{HCM}$, with presentation median CCa2.85 mmol/L(Range 2.65-4.11). In the knowledge of $\mathrm{HCM}$, clear symptom assessment was documented in $25 / 47(44 \%)$. Potentially contributing medications were rationalised in $36 / 47(77 \%)$. $37(78 \%)$ had clearly documented rationale for treating HCM or not. $37(78 \%)$ had hydration status assessed and documented. IV fluids were started appropriately in all patients $(\mathrm{n}=16)$ with $\mathrm{CCa}>3$ as per the agreed standard. However, of the 16, 6 received Hartmanns solution (containing calcium) rather than recommended normal saline. A bisphosphonate/denosumab was given in 18 cases of which $85 \%$ were deemed appropriate compared to our standards. During admission with HCM, $36 / 47$ cases were referred to palliative care. $30 / 47$ have since died with median time to death from first presentation of HCM 36 days(Range 2-420).

Discussion/conclusion: Identified standards were largely adhered to but HCM symptom assessment was poorly documented, with wide variety in IV fluid type used, requiring clarification. Agreed UK HCM management guidelines are needed and in progress.
Abstract number: P02-315

Abstract type: Poster

Practicability of the Quality Indicator "Opioids and Laxatives" Proposed in the German S3-Guideline "Palliative Care for Patients with Incurable Cancer"

Kögl K., Roch C., Cirac M., van Oorschot B.

Interdisciplinary Center Palliative Care, University Hospital Würzburg, Würzburg, Germany

Background: The German S3-Guideline Palliative Care for Patients with Incurable Cancer proposes the QI "Proportion of patients with incurable cancer and opioids without osmotic or propulsive laxatives, quality goal: low" to evaluate the quality of palliative care.

Aim: To evaluate this QI in cancer patients during their stay in a specialized palliative care unit.

Methods: Data were collected retrospectively from patients' files. Daily oral laxatives as well as periodical rectal interventions were summarized to "laxatives". The QI was analyzed regarding the third day on the palliative care unit (T1) and day 3 before dismissal (T2). Reasons for nonuse were taken from the file. Gastrointestinal reasons like a malignant intestinal obstruction, acute abdominal symptomatic, ileostoma or diarrhoea were summarized to "GI-problems".

Results: 253 files from cancer patients in 2015 were analyzed. 185 patients were treated with opioids at $\mathrm{T} 1$ either for pain control or for alleviation of dyspnea $(185 / 253,73.1 \%) .70$ patients with opioids had no laxative (70/185, 37.8\%). At T2 193 patients had opioids (193/253, $76.2 \%)$ and 81 patients had no laxative $(81 / 193,41.9 \%)$. Reasons for the nonuse of laxatives were "terminal phase" ( $n=22$ in T1 (22/70, 31.4\%), $\mathrm{n}=32$ in T2 (31/81, 39.5\%)), "Gl-problems" ( $\mathrm{n}=20$ in T1 (20/70, 28.5\%, $\mathrm{n}=26$ in T2 (26/81, 32.0\%), "opioids not regular" ( $\mathrm{n}=4$ in T1 and $\mathrm{n}=3$ in T2 $(4 / 70,5.7 \%$ and $3 / 81,3.7 \%)$ and "patient refuses" ( $n=1$ in T1 (1/70, 1.4\% and $\mathrm{n}=4$ in $\mathrm{T} 2(4 / 81,4.9 \%)$. Regarding $\mathrm{T} 2$, significant more deceased patients received opioids without laxative $(51 / 82$ deceased patients, $62.1 \%$ vs. $30 / 111$ other patients, $27.0 \%, \mathrm{p}<.001)$.

Conclusion: The QI was easy to assess using patients' files. Because of cancelling prescription of laxatives in dying patients and the very individual decisions regarding laxatives in patients with GI-problems the target group should be specified before implementing this QI in clinical practice.

\section{Abstract number: P02-316}

Abstract type: Poster

\section{Referral to Palliative Care in an Oncological Center}

Ferraz Gonçalves J.A., David A., Rodrigues A., Pereira C., Pais D., Neves

E., Marques L., Condesa M., Edra N., Silva N., Badim S.

Instituto Português de Oncologia do Porto Francisco Gentil, Porto, Portugal

Background: A palliative care service (PCS) was found in 1994 in a Portuguese oncological center with about 10000 new patients per year. Now the service has 40 beds and it is a very busy one. At that time there was only 3 palliative care physicians and 2 advanced medical trainees in palliative care.

Aim: To study how the referral process to palliative care is now.

Methods: A study of all patients referred to the service in 2016 was carried out. The referral forms of all patients filled out in 2016 by doctors of other services were studied. The document includes information such as demographic data, treatments previously undergone, the motive for referral and to whom the information was delivered.

Results: In 2016, 1177 patients were referred to palliative care, 682 (58\%) were male and the median age was 68 years ( 20 to 96$)$. The more frequent primary tumors were: colorectal - 164 (14\%); lung - 148 (13\%); head and neck - $123(11 \%)$. The ECOG performance status was recorded in $1048(89 \%)$ of the patients: 0 - $7(1 \%) ; 1-61(6 \%) ; 2-145(14 \%) ; 3$ 
- 462 (44\%); 4 - 373 (36\%). 906 (77\%) were admitted to the PCS. The most frequent reason for non-admission was death in 249 (21\%). The record of the first contact with the PCS it was through the: hospital supportive team - $570(48 \%)$; outpatient clinic - $161(14 \%)$; inpatients - 155 $(13 \%)$; and home care - 20 (2\%). When recorded to whom the information was delivered at the referral: to the patient - 136 (12\%); family - 303 (26\%); both - 679 (58\%); nobody - 19 (2\%).

Conclusion: Patients are still referred to palliative care in a very late phase in the evolution of their disease, as deaths before admission and the poor performance status of most patients. The non-admission of some patients may have been due to high volume of patients comparatively to the number of doctors of the PCS. The level of information delivered to patients is far from being satisfactory.

Abstract number: $\mathrm{P} 02-317$

Abstract type: Poster

Prospective Audit of Remote Prescribing Practice in Two Sue Ryder Inpatient Hospices

Moretto E. ${ }^{1,2}$, Whyte S. ${ }^{3}$, Sutherland A. ${ }^{1,2}$, Drake R. ${ }^{1}$, Borthwick A. ${ }^{1,2}$ ${ }^{1}$ Sue Ryder Duchess of Kent Hospice, Reading, United Kingdom, ${ }^{2}$ Royal Berkshire NHS Foundation Trust, Reading, United Kingdom, ${ }^{3}$ Sue Ryder Nettlebed Hospice, Nettlebed, United Kingdom

Background: Inpatient hospices use remote prescribing more frequently than other specialities/care settings. The medical team noted that requests for remote prescribing appeared high and variable between units. An offsite prescription procedure had recently been revised and implemented. We designed a prospective audit to identify the reasons for remote prescribing and whether these could be pre-empted/reduced. We also audited the process of remote prescribing in terms of documentation and discussions between professionals and with patients.

Aims:

1. To identify remote prescribing practice, which drugs were prescribed.

2. To identify if remote prescriptions could have been pre-empted or managed with prn medications.

3. To identify if off site prescription procedure was being followed regarding documentation of discussions of nurses and doctors, and discussions with patients.

4. To identify if the remote prescription, and prescription on drug charts complied with off site prescription procedure and NMC guidance and standards on remote prescribing.

Methods: Prospective audit of first 10 remote prescriptions on each unit from November 2017.

Results:

1. $7 / 19$ prescriptions were for analgesia, $4 / 19$ for anticipatory medications, 9/19 medications were all different drugs (1/20 stop neuro obs).

2. $17 / 19$ the medication could have been pre-empted.

3. There was clear documentation of reason for requesting remote prescription (16/19), but records of documentation of nursing discussion with patients re indication for new drug and possible side effects were low.

4. Documentation on remote prescription was variable, and not reaching standards in procedure.

Conclusion / lessons learned: 17/19 remote prescriptions could have been pre-empted.

Remote prescription documentation needs to be improved in particular noting patient allergies.

Documentation of discussion with patients by nurses, and doctors when next in unit needs to improved.
Abstract number: P02-318

Abstract type: Poster

The Importance of Quality Indicators in Palliative Care Services

Rezgiene L.R.

Siauliai Palliative Care Center, Siauliai, Lithuania

Bacground: Palliative care is a complex of measures for improving the quality of life of people with a chronic progressive illness and their relatives. Lithuania is one of the major emerging economies of the Eastern Europe with inherent transformations in changing society. The increasing average future life expectancy, aging population, growing number of chronic non-communicable diseases and even the advancement of medical technology require a different approach from the health service providers towards palliative care services.

Aim: To assess the availability and timeliness of palliative care.

Methods: Analysis of palliative care services in Lithuania during 2017. Survey of 750 palliative patients.

Results: 2916 people receved paliative care services, $72.5 \%$ of all patients were treated in the inpatient nursing hospital department, $33.4 \%$ - received outpatient palliative care services, i.e. home and day care services.

$80.2 \%$ of patients and / or their relatives reported that they had learned about palliative care from their doctors, $5.4 \%$ found the information in the media, 10.6\% heard about it from their acquaintances and $3.8 \%$ did not remember the source of information.

No patient was aware of palliative care services at the onset of the disease and was not informed about the palliative care options.

Since the start of home palliative care $30 \%$ of patients died in a week, $27 \%$ deceased in a month. $43 \%$ - in a year. In nursing hospitals $68.8 \%$ patients died in 30 days, $17.4 \%-31-60,13.8 \%-60$.

Conclusions: The analysis of the services provided and targeted patient surveys suggest that the evaluation of palliative care organization and strategy requires the introduction of criteria that reflect a systematic approach towards palliative care and help to develop an adequate palliative care strategy.

Palliative care quality criteria might include the timeliness, availability, continuity and sustainability of palliative care services.

Abstract number: P02-319

Abstract type: Poster

Development of a Tool for Internal Quality Review of Palliative Care

Jansen-Segers $M^{1}{ }^{1,2}$, de Wit $A^{1,3}$, Giesen $M^{1,2}$, Boddaert M..$^{1,2}$, Fransen

H.P. ${ }^{1,2}$, Meertens D. ${ }^{4}$, van Marion P. ${ }^{5}$, Schoonheim-Lunenborg H. ${ }^{6}$ ${ }^{1} \mathrm{PZNL}$ - Association for Palliative Care in the Netherlands, Utrecht, Netherlands, ${ }^{2}$ Netherlands Comprehensive Cancer Organisation (IKNL), Utrecht, Netherlands, ${ }^{3}$ Fibula, Platform of Regional Palliative Care Networks, Utrecht, the Netherlands, Utrecht, Netherlands, ${ }^{4}$ Palliative Care Network Roermond and Surroundings, Roermond, Netherlands, ${ }^{5}$ Palliative Care Network Zeeland, Vlissingen, Netherlands, ${ }^{6}$ Palliative Care Network Drenthe, Hoogeveen, Netherlands

Background: In the Netherlands, palliative care (PC) is part of a national program for the improvement of quality of life and care for patients with life-threatening conditions. Here we present the process of developing a tool for internal quality review in organizations or PC networks and its main topics of content.

Aim: To develop a self-assessment tool for healthcare organizations and PC networks, based on the recently launched Dutch national quality framework for palliative care.

Method: The tool was developed in collaboration with 3 PC networks. The items of the tool are based on essential elements of the national quality framework. Within these elements, items address patient care, organization of PC and collaboration with other organizations. To test 
the feasibility of the tool a pilot study was performed and the tool was made available both digitally and on paper.

Results: Users of the tool reported that discussing the questions helped to create awareness for PC. Answering the questions created an overview of the quality of PC provided and generated information for targeted improvement. Participants welcomed the fact that the outcomes are specifically meant for internal improvement without attachment of a quality label or accreditation. They expected this to provide a lower threshold for organizations to use the tool. Pilot-organizations indicated the intention to repeat the internal quality review to assess if improvements were made.Some questions were unclear and were adapted for the final version of the tool.

Conclusion: This internal quality review tool for PC helps healthcare organizations to get an overview of the quality of PC provided in their organization or network and generates information for targeted improvement of PC based on the national quality framework.The digital application makes it possible to use the outcomes of the tool for nationwide benchmarking, to share outcomes in the PC network and to work together in improvement of PC.

Abstract number: P02-320

Abstract type: Poster

\section{Aggressiveness of Cancer Treatment at the End of Life in a Single} Centre

Ferraz Gonçalves J.A. ${ }^{1}$, Luís M. ${ }^{1}$, Lisboa M. ${ }^{2}$, Machado J. ${ }^{3}$, Redondo P. ${ }^{1}$ ${ }^{1}$ Instituto Português de Oncologia do Porto Francisco Gentil, Porto, Portugal, ${ }^{2}$ Hospital de Chaves - CHTMAD, Chaves, Portugal, ${ }^{3}$ Hospital de Viseu, Viseu, Portugal

Background: Aggressiveness of cancer care near the end of life is an important issue, with concerns about potential overuse of treatments with limited clinical benefit and a negative impact on QoL. Likewise, diagnostic and invasive procedures need to be carefully considered in patients with a limited prognosis, as they may cause more harm than benefit.

Aims: To characterize the aggressiveness of end of life cancer care using existing administrative data.

Methods: We performed a consecutive case-series study analysing administrative data from 2852 cancer decedents during 2017 in a single cancer centre. Information regarding systemic anti-neoplastic therapy, radiotherapy, invasive procedures, emergency department visits, hospitalization and imaging studies during the last month of life was retrieved. Results: 426 patients underwent chemotherapy treatment in their last month of life (LML), in a total of 1421 sessions. 99 patients (23\%) of these received a single session of chemotherapy. 139 patients were submitted to radiotherapy treatment in their LML.

91 patients underwent a surgical procedure. 413 red blood cell transfusions were performed.

1181 patients visited the emergency department or an acute oncology service in LML. 78 patients were admitted to an ICU. A total of 1477 hospital admissions were recorded.

$511 \mathrm{CT}$ scans and 92 MRIs were performed during the LML; no PET scan was performed during the LML in our sample.

Conclusion: Our retrospective analysis of existing administrative data allows for a characterization of aggressiveness of cancer care in the LML at our centre. The data retrieved provides a first step for further studies comprising previously described frameworks with indicators of aggressiveness.

Abstract number: P02-321

Abstract type: Poster

Integration of Specialized Palliative Care Services in Cancer Patients at a University Hospital

Roch C. ${ }^{1}$, Schmitt T. ${ }^{2}$, Flentje M. ${ }^{3}$, van Oorschot B. ${ }^{1}$

${ }^{1}$ Interdisciplinary Centre Palliative Care, University Hospital Würzburg,
Würzburg, Germany, ${ }^{2}$ Administration, University Hospital Würzburg, Würzburg, Germany, ${ }^{3}$ Dept. of Radiotherapy, University Hospital Würzburg, Würzburg, Germany

Background: C. Earle et al. (2005) defined benchmarks for the use of hospice and/ or specialized palliative care services (SPC) in cancer patients using administrative data. This approach promises a high quality of data without any additional surveys.

Aim: This study aims to evaluate the integration of SPC in our university hospital. Quality goals were defined using the adapted Earle-criteria: $\geqslant 55 \%$ of all in-hospital deceased patients should have SPC-contact and less than $8 \%$ should have SPC-contact shorter 4 days before death.

Methods: Electronic health records of in-hospital deceased patients being of full age and without any diagnosis of intoxication or accident during their last stay were analyzed (timeframe: from 1.1.2010 until 30.9.2018). Results: $30,3 \%$ deceased had SPC-contact (1.903/6.270 patients). Between 2010 and 2018 an increase from $25.6 \%$ to $33.4 \%$ was observed. In 847 cases the SPC-contact was initiated shorter 4 days before death (13.5\%). Significantly more cancer patients (CP) had SPC-contact compared to other patients ( $61.3 \%$ vs. $12,3 \%, p=.005)$. Since 2012 more than $55 \%$ of deceased CPs had SPC-contact. $22.4 \%$ of them had SPC-contact 3 days or less before death, a slightly increasing number over time $(12,4 \%$ in 2010 vs. $26,1 \%$ in 2018).

Conclusion / discussion: Only in cancer patients the quality goal was reached. Initiation of SPC-contact was often very late. Shorter duration of hospitalization and later admission of final patients as a consequence of effective outpatient care might be a reason. Nevertheless, more information on the interaction of different care strategies and efforts to improve early SPC-contact in patients with life-threatening illness seems to be necessary.

Regarding all deceased patients with SPC-contact 3 days or shorter between 2010 and 2018, an increase was observed (12,6\% vs 16,7\%, $\mathrm{p}=.1$ ).

There was a slight, but non-significant increase in cancer patients with late SPC-contact $(12,4 \%$ in 2010 vs. $26,1 \%$ in $2018, p=.1)$.

Abstract number: $\mathrm{P} 02-322$

Abstract type: Poster

Improving End of Life Care at Home Provided by Domiciliary

Teams Using the GSF Domiciliary Care Programme

Thomas K., Noakes S., Armstrong Wilson J.

The Gold Standards Framework Centre CIC, London, United Kingdom

Aim: Domiciliary care workers (DCWs) play an important role in supporting people to remain at home as they near the end of life, especially those with dementia and frailty. Despite this DCWs contributions frequently go unrecognised and few receive specific training in end of life care. Focussed training enables DCWs to improve the quality of care provided for people who choose to remain at home and promotes better co-ordinated cross boundary care.

Method: The GSF Domiciliary Care training programme focuses on enabling generalist frontline staff in end of life competencies. The Train-theTrainer cascade programme works with Domiciliary Care Agency trainers using action based interactive learning and reflective practice in 6 modules, accompanied by the GSF virtual learning zone, animations and resources. Comparative before and after qualitative and quantitative evaluation is intrinsic. We present an evaluation of progress of several teams using GSF training in end of life care, some delivered by hospices. Results: Homecare workers in various areas of the country show improvements in

- Confidence and competence of home care staff

- Communication, working relationships and collaboration with primary care 
- More Advance Care Planning discussions with service users

- Encouragement and empowerment of staff to advocate for their service users resulting in improved communications with other professionals

- Increased awareness of the knowledge they already possessed and future training needs

Conclusion: This highlights the important role that DCWs play and the importance of empowering care staff who interact with the service users on a daily basis.

The GSF Domiciliary Care Training Programme boosts the confidence and competence of care workers, improves collaboration and coordination with others and helps to improve care for more people nearing the end of life at home - which is for most the best place to be.

Abstract number: P02-323

Abstract type: Poster

GSF in Jersey - Findings from the first whole system islandwide Gold Standards Framework (GSF) programme in Jersey delivering proactive person-centred end of life care Thomas K. ${ }^{1}$, Armstrong Wilson J. ${ }^{1}$, Edwards G. ${ }^{2}$

${ }^{1}$ Gold Standards Framework Centre CIC, London, United Kingdom, 2Jersey Hospice Care, Jersey, United Kingdom

Background: Jersey is a small island near England with a 104,000 population and an independent health jurisdiction. To improve integrated end of life care across the whole island the GSF Whole System programme was introduced over 3 years, focussing on improving care in each setting plus the integrated cross boundary whole system culture of care.

Aim: An evaluation of the introduction of GSF to all health and social care providers in Jersey.

Method: We report on the introduction of GSF as a quality improvement programme for the whole island of Jersey used in a variety of settings, aspiring to proactive personalised care for people in the last years of life. This programme involved introducing GSF in a planned 3-year programme with multiple settings and cohorts. Evaluations include intrinsic comparative quantitative measures to teams in care homes, primary care, hospitals +28 qualitative semi-structured telephone interviews assessing changes in perception, culture and outcomes.

Results: Evaluations indicate significant progress in several key areas in many settings including earlier identification, more offered advance care planning discussions + reduced hospital admissions, and deaths. Qualitative findings found encouraging positive response, better coordinated care, greater integration across boundaries and a 'culture change' across the whole island.

Conclusions: Findings from the 3-year island-wide staged approach to introducing GSF to all settings are encouraging, suggesting that it has catalysed significant improvements in integrated end of life care across the whole island.

Though used piecemeal in many areas, and together in 9 cross boundary care sites, use of GSF across a whole system in a similar staged approach might be feasible elsewhere and lead to similar positive results.

Abstract number: P02-324

Abstract type: Poster

Can We Give Dying Hospital Patients Better Care? Findings from the first GSF Accredited Hospital Wards Thomas $\mathrm{K}^{1}{ }^{1}$, Armstrong Wilson $\mathrm{J}^{2}$, Clifford C. ${ }^{3}$

${ }^{1}$ Gold Standards Framework Centre CIC, Shrewsbury, United Kingdom, ${ }^{2}$ Gold Standards Framework Centre CIC, London, United Kingdom, ${ }^{3}$ University of Birmingham, Birmingham, United Kingdom

Aims: A third of hospital patients are in their last year of life, almost 50\% of people die in hospital and most do not die where they choose. Poor end of life care in UK hospitals has received much recent attention and is recognised as a key area for improvement. With earlier recognition and a more proactive approach more might receive better care, be discharged home earlier, with fewer hospital deaths.

Gold Standards Framework (GSF) is a quality improvement programme delivering proactive person-centred population-based care for people in the last years of life in all settings. We report on the introduction and impact of the GSF Acute Hospitals Programme with findings from the first accredited and re-accredited wards three years on.

Method: The structured 2-year GSF Hospital training programme includes training, tools, support and evaluation at organisational, patient and staff level, including measures of progress and attainment and some independent evaluations. GSF encourages teams to identify patients earlier (aiming for the 30\% figure where appropriate) assess their needs (offering all initial advance care planning discussions) and proactively planning care aligned to preferences, enabling more to die at home.

Results: Evaluations indicate significant progress in several key areas in many hospitals and a 'culture change' on the wards. GSF Accredited wards show embedded good practice including earlier identification (average $35 \%$, three years on an average of $44 \%$ for three of the wards originally accredited) more proactive needs-based approach, more offered advance care planning discussions (75-95\%), better communication with GPs, reduced hospitalisation and improved more appropriate referrals to palliative care specialists.

Conclusions: Findings from the first accredited wards show significant changes with proactive, better coordinated, person-centred care. A key factor is earlier recognition which is being sustained by the GSF reaccredited wards.

\section{Abstract number: P02-325}

Abstract type: Poster

Improving Handover in an In-patient Hospice Using a Human

Factors Approach to Patient Safety

Matthews A., Gould T., Gurung S., Nash S.

Sue Ryder St John's Hospice, Beds, United Kingdom

Background: Sue Ryder St John's Hospice is a 15-bed in-patient hospice. A multi-disciplinary handover of all patients is held at 9am attended by nursing, medical, family support and allied health professionals. Staff became concerned that handover was both too long and failing to allocate responsibility for tasks.

Staff recognised this as a patient safety issue and decided to use a Human Factors approach. We identified the Paediatrics Situational Awareness For Everyone (SAFE) project as potentially having significant crossover to a palliative care environment, being based around large multi-disciplinary teams, complex interventions, unwell patients who may lack capacity, and families in crisis.

Aims: To use a Human Factors approach to improve patient handover.

Methods: A Plan Do Study Act cycle was undertaken. Using the SAFE Toolkit in the Plan phase, the SAFE Huddle Observation Tool was adapted to Palliative Care, and an audit of our handover was undertaken for 9 days.

In the Do phase, a new Script and Rules were created for handover, inspired by the SAFE Huddle. This was re-audited for 9 days.

In the Study phase the results were analysed and in Act, final changes were made to our script.

Results: The average length of handover reduced from 1 hour to $30 \mathrm{~min}$ utes. Critical information discussed improved for: diagnosis (49\% to $100 \%$ ), whether patients were having observations ( $45 \%$ to $100 \%$ ), and daily OACC scores (55\% to $100 \%$ ). Patients were identified as 'watchers' if they were having complex interventions or needed early medical review. Each patient task was allocated to an individual staff member. Conclusion: Using a script made a time saving in handover while improving the quality of information discussed. Patient safety was improved by the creation of a formal process for identification of complex patients. 
This is a novel adaptation of paediatric format. Other tools in the SAFE project and in Human Factors are likely to have significant benefits to patient safety in palliative care.

Abstract number: P02-326

Abstract type: Poster

Health Care Professionals' Perceptions of Palliative Care Quality in a Combined Acute Oncology-palliative Care Unit - Improvements Are Needed Regarding Information, Participation, Continuity and Existential Needs

Olsson C. ${ }^{1}$, Eriksson E. ${ }^{2}$, Rognsvåg M. ${ }^{2}$, Larsson M. ${ }^{1}$

${ }^{1}$ Department of Health Sciences, Karlstad University, Karlstad, Sweden, ${ }^{2}$ Department of Oncology, Central Hospital, County Council of Värmland, Karlstad, Sweden

Background: The number of persons with life-threatening illnesses who need palliative care $(\mathrm{PC})$ is expected to increase as people are living longer, due to advances in medical treatment and technology. There are major shortcomings in PC and regional differences in accessibility, quality, symptom relief, involvement and support for close relatives. Evaluation of quality of care $(\mathrm{Q} o \mathrm{C})$ is important to guide improvements and the use of healthcare professionals' (HCP) perspective means that fragile patients and their close relatives are not burdened.

Aim: To investigate how HCPs in PC perceive QoC regarding perceived reality (PI) (given care) and subjective importance (SI) (how care should be).

Method: Cross-sectional study, descriptive and analytical design. Data collection, February 2018 with the four dimensional questionnaire Quality from the Patient's Perspective Palliative Care (QPP-PC) among $\mathrm{HCP}$ in a combined oncology-palliative care unit (total survey) in Sweden. Response rate of $53 \%(n=41)$.

Results: HCP scored statistically significant higher regarding SI compared to $P R(p \leqslant .00)$ in all four dimension; medical-technical competence, physical-technical conditions, identity-oriented approach and sociocultural atmosphere. Information (PR mean 2.78 SD .57; SI mean 3.66 SD $.34 ; p \leqslant .00$ ), participation (PR mean 2.84 SD .70; SI mean 3.52 SD .54; $p \leqslant .00$ ), continuity (PR mean 2.61 SD .74; SI mean 3,34 SD .59; $p \leqslant .00$ ), and spiritual/existential needs (PR mean 2.73, SD .64; SI mean 3,34, SD $.87 ; \mathrm{p} \leqslant .00$ ) were factors respondents scored lowest values in PR compared to SI. Cronbach's $\alpha$-values, moderate to high.

Conclusion: These findings highlight palliative QoC problems in units with mixed care focus, i.e. acute, supportive and end of life care. Strategies to ensure palliative care when needed has to be developed and implemented in acute care settings to avoid futile interventions.

\section{Abstract number: P02-328 \\ Abstract type: Poster}

Safety Patient Culture in Palliative Care: Professionals' Perception for the Implementation of Safe Practices Pop Csog B. ${ }^{1}$, Martín Fuentes de la Rosa M.A. ${ }^{2}$, Romero Gabino V. ${ }^{3}$, Ramos Gonzalez M. ${ }^{4}$, Lopez García M. ${ }^{5}$, Rodríguez Pérez B. ${ }^{6}$, Flores Belmonte J.7, Alonso Ruiz M.T. ${ }^{8}$, Redondo Moralo M.J. ${ }^{9}$, Bonino Timmerman $F^{9}$

${ }^{1}$ Servicio Extremeño de Salud, Hospital Campo Arañuelo, Navalmoral de la Mata, Spain, 2Servicio Extremeño de Salud, Hospital de Mérida, Mérida, Spain, ${ }^{3}$ Servicio Extremeño de Salud, Hospital San Pedro de Alcántara, Cáceres, Spain, ${ }^{4}$ Servicio Extremeño de Salud, Hospital Virgen del Puerto, Plasencia, Spain, ${ }^{5}$ Servicio Extremeño de Salud, Hospital Vegas Bajas, Don Benito-Villanueva, Spain, ${ }^{6}$ Servicio Extremeño de Salud, Servicios Centrales, Mérida, Spain, ${ }^{7}$ Servicio Extremeño de Salud, Hospital de Zafra, Zafra, Spain, ${ }^{8}$ Servicio Extremeño de Salud, Hospital de Merida, Merida, Spain, ${ }^{9}$ Servicio Extremeño de Salud, Hospital Perpetuo Socorro, Badajoz, Spain
Introduction: Fundamental quality of care 's dimension is culture of patient safety. This is understood as structures and procedures that minimize risks of adverse events or damages derived from healthcare. It is necessary to know the perception and culture about safety patient by professionals of Regional Palliative Care Program of Extremadura (PRCPEx) for the implementation of safe clinical practices that minimize risks of incidents and adverse effects in patients in palliative care that are attended at home.

Aim: Measure the perception that professionals of Support Teams of PRCPEx have on safety culture of patient and identify strengths and weaknesses, as well as aspects on which they should act to improve them and monitor their evolution.

Method: Descriptive study of the safety climate of the professionals of the Palliative Care Support Teams and analysis of dimensions, considered opportunities for improvements for the safety of patients treated in the PRCPEx. They were adapted and used questionnaires on Patient Safety validated by the Health Ministry, the Survey on Patient Safety Culture Hospital and the Medical Office Survey on Patient Safety Culture. Results: Sample: doctors, nurses, psychologists and social workers who work in PRCPEx.

Response rate: doctors, $72.2 \%$, nurses, $62.5 \%$, psychologists, $85.7 \%$, social workers, $100 \%$.

Main dimensions strengths: frequency of events reported, $81.2 \%$, expectation and actions of coordination of the ESCP, 79.3\%, teamwork in ESCP, $63.7 \%$.

Main dimensions area of improvement: problems in shift changes, 52\%, staffing, 50\%, support in hospital management in security, $49.2 \%$.

Conclusions: Promoting Safety Patient Culture in professionals of PRCPEx is a fundamental strategy to improve the safety of patients with palliative care needs seen in health, social care or home centres.

It is necessary that professionals have a level of patient safety culture to be able to successfully implement safe clinical practices that minimize risk of Adverse Events.

\section{Abstract number: P02-329}

Abstract type: Poster

"Safe Use" of the Medication at Home: Terminal Patients' Caregivers Educational Training

Redondo Moralo M.J. ${ }^{1}$, Alonso Ruiz M.T. ${ }^{2}$, Martín Fuentes de la Rosa M.A. ${ }^{2}$, Rodríguez Pérez B. ${ }^{3}$, Bonino Timmerman F. ${ }^{1}$, Lopez García M. ${ }^{4}$, Ramos Gonzalez M. ${ }^{5}$, Romero Gabino V. ${ }^{6}$, Pop Csog B. ${ }^{7}$, Flores Belmonte J. ${ }^{8}$ ${ }^{1}$ Servicio Extremeño de Salud, Hospital Perpetuo Socorro, Badajoz, Spain, ${ }^{2}$ Servicio Extremeño de Salud, Hospital de Merida, Mérida, Spain, ${ }^{3}$ Servicio Extremeño de Salud, Servicios Centrales, Mérida, Spain, ${ }^{4}$ Servicio Extremeño de Salud, Hospital Don Benito Villanueva, Don Benito-Villanueva, Spain, ${ }^{5}$ Servicio Extremeño de Salud, Hospital Virgen del Puerto, Plasencia, Spain, ${ }^{6}$ Servicio Extremeño de Salud, Hospital San Pedro de Alcántara, Cáceres, Spain, ${ }^{7}$ Servicio Extremeño de Salud, Hospital Campo Arañuelo, Navalmoral de la Mata, Spain, ${ }^{8}$ Servicio Extremeño de Salud, Hospital de Zafra, Zafra, Spain

Introduction: Caregiver is the fundamental support in process of palliative homecare. They are responsible for patient care.

Informal caregivers ensure administration of prescribed-drugs considered high risk medications (HRM) but they are not trained and that requires special alert. They also decide drug administration in critical situations.

That, there is a risk of fear and prejudice by the caregiver in use of those medications

Aim: Design and implement a caregiver training program with support materials for safe use of medicines at home.

Method:

Focal Group with Expert Caregivers, for knowing experiences lived by caregivers of deceased patients who had overcome 
mourning phase. Topics discussed: first contact with palliative care team, symptoms and treatments, organization of drugs, use and administration, adverse effects, reasons for end/abandon treatment, worries/prejudices

- Elaboration of information and training materials for safe use of medications for caregivers. Professionals of Quality Group of Regional Palliative Care Program Extremadura, worked with the "design thinking methodology"

They design and develop teaching materials to train caregivers in management and safe administration of medicines at home. They select the most appropriate/accessible materials for caregivers and other professionals involved in the care of the ill patients

Results: From interviews of expert caregivers: palliative team waited as the solution. Patients had uncontrolled symptoms. Caregiver's fears: appearance of AE. Fear's caregivers: negative of patient to take treatment. Failure to administer prescribed dosages/confusion with use drugs. Resolution of doubts/action against difficulties. Telephone assessment and regular homecare visits

From ideation session: it was agree audiovisual and infographics materials to train caregivers.

Conclusions: There is evidence of the need to train caregivers throughout the patient's care process with the means available to us.

Abstract number: $\mathrm{P} 02-330$

Abstract type: Poster

Implementation of a National Strategic Plan for the Development of Palliative Care in a European Country: Impact on a Public Palliative Care Unit

Lucas H., Gil L., Almeida R., Miguel S., Branco A.S., Viegas S., MartinsBranco D.

Clínica São João de Ávila, Instituto São João de Deus, Lisboa, Portugal

Background: The implementation in Apr'17 of a Strategic Plan for the development of Palliative Care (SPPC) in Portugal changed the referral criteria for palliative care units (PCU) of the Continued and Integrated National Care Network (CINCN) and generated a clinical perception of increased complexity of cases admitted to PCU-CINCN.

Aim: To analyze the impact of SPPC implementation on a PCU-CINCN.

Methods: Retrospective cohort study of all patients admitted to a PCUCINCN and discharged until $28^{\text {th }}$ Feb'18. Patients unable to apply scales were excluded. Two groups were generated based on date of informed consent (IC) for admission (24 ${ }^{\text {th }}$ Apr'17): GI - pre-SPPC; GII - post-SPPC. We used Mann-Whitney_U test for comparison of medians and chisquare test for comparison of frequencies (SPSS 23.0). The level of statistical significance was $5 \%$.

Results: We identified 119 patients, $45 \%$ male $(n=54)$, median age 75 yo, $84 \%$ with cancer diagnosis $(n=100)$. Gll patients $(n=59)$ had lower median (days) of: i) time from IC to admission (14 vs 23); ii) length of stay (13 vs 62.5); iii) time to first family conference (FC) (8 vs 11.5 ); iv) scales on admission: Palliative Performance Scale (35 vs 50 ) and Zarit scale (11 vs 16$)$. We found no differences on the overall value of Palliative Outcome Scale (GII 17 vs GI 16) and Edmonton Symptom Assessment Scale ( 28 vs 26 ). GII patients ranked higher ESAS subscales of pain ( 3 vs 1 ), tiredness ( 5 vs 3 ) and drowsiness ( 5 vs 3 ).

Conclusion: Patients admitted to this PCU after the SPPC implementation had higher clinical complexity and shorter prognosis. We assume that caregivers had less exhaustion over time, since time from referral to admission is lower after SPPC. The time to first FC reflects the organizational changes in response to the increased needs of patients and families. This study analyses the impact of a legislative change in Portugal and highlights the importance of PCU in the end-of-life.
Abstract number: P02-331

Abstract type: Poster

Systemic Anticancer Therapy at the End-of-Life: Has Something Changed after 15 Years?

Ferraz Gonçalves J.A. ${ }^{1}$, Luís M. ${ }^{2}$, Machado J. ${ }^{3}$, Lisboa M. ${ }^{4}$, Redondo P. ${ }^{1}$

${ }_{1}^{1}$ nstituto Português de Oncologia do Porto Francisco Gentil, Porto, Portugal, 2Palliative Care Unit, Portuguese Institute of Oncology, Porto, Portugal, ${ }^{3}$ Hospital de Viseu, Viseu, Portugal, ${ }^{4}$ Hospital de Chaves CHTMAD, Chaves, Portugal

Background: Some years ago, we published a study on the use of chemotherapy at the end of life in a Portuguese oncology center in patients with solid tumors who died in 2002 [1]. Since then a considerable evolution in systemic anticancer therapies occurred, not only in chemotherapy but also with the introduction of other systemic therapies such as immunotherapy and targeted therapy.

Aim: To study how close to death those therapies are being used and if there was any change in that aspect relatively to what happened 15 years ago.

Methods: analysis of the administrative data of all patients older than 18 with solid tumors followed in an oncology center who died in 2017, looking for data of the aggressiveness of treatment at the end of life. Here we present those data and compare them with data of the 2002 study. For that comparison we analyzed hormone therapy and other systemic therapies - OST (chemotherapy, immunotherapy and targeted therapy).

Results: During 2017, 1974 patients with solid tumors have died, 1203 (61\%) were male and the median age was 69 years (19-97). The most frequent tumors were - lung - 359 (18\%); colorectal - 266 (14\%); stomach - 247 (13\%); breast - 217 (11\%); head and neck -215 (11\%). 281 (14\%) underwent systemic anticancer therapy: hormone therapy - 61 (3\%); and OST - 220 (11\%).

Discussion / Conclusion: In 2002, 13\% underwent chemotherapy in the last month of life, a percentage close to the $11 \%$ of OST found in this study. Therefore, this aspect of the practice of medical oncologists at this hospital remained essentially stable in the last 15 years.

References:

1. Ferraz Gonçalves, Carmen Goyanes. Use of chemotherapy at the end of life in a Portuguese oncology center: Support Care Cancer 2008;16:321-327.

Abstract number: P02-332

Abstract type: Poster

A Retrospective Review of Subcutaneous Alfentanil Infusions in the Hospital In-patient Palliative Care Setting

Pollok F., Wanklyn S., Gough N.

Palliative Care, Guys and St Thomas' NHS Foundation Trust, London, United Kingdom

Background: Alfentanil is a strong opioid used in palliative care (PC) as an alternative analgesic to conventional opioids e.g. morphine. It is mainly administered as a subcutaneous infusion via syringe pump and indications for use include (i) renal impairment as its metabolites are inactive and (ii) rotation from a more conventional opioid in order to improve analgesia / reduce side effects. In UK hospitals, alfentanil is used relatively infrequently hence the need to audit its use against accepted standards to minimize potential for drug errors and patient harm.

Aims: To evaluate the use of subcutaneous alfentanil infusions for pain in the PC setting in an acute UK hospital.

Methods: Patients prescribed an alfentanil infusion from $1 / 7 / 16$ to $31 / 12 / 17$ were identified using the pharmacy dispensing system. Patient notes were reviewed and practice was compared against standards from accepted guidance. 
Results: One-hundred and three patients commenced a subcutaneous alfentanil infusion: 84 case notes were available for review. 45/84 (54\%) of patients had cancer (most commonly gastro-intestinal); frailty with renal impairment was the commonest non-cancer diagnosis. Alfentanil was started after PC team consultation in $96 \%(81 / 84)$ of cases and a clear starting indication was documented in $85 \%(71 / 84)$. Alfentanil was commenced in 48 patients (57\%) due to renal impairment (eGFR $<$ $30 \mathrm{~mL} / \mathrm{min}$ ) with opioid switch/ rotation due to intolerable side effects the next commonest indication.

Alfentanil dose was prescribed in micrograms in 54\% (45/84) of cases and milligrams in $46 \%$. Alfentanil was prescribed PRN in $70 / 84$ (83\%) cases and oxycodone in $10 \%$. A PRN subcutaneous anti-emetic was prescribed in $94 \%$ of cases though only a minority had concurrent prescription of a laxative (23\%).

Conclusion: Subcutaneous alfentanil infusion use largely conforms to accepted standards but prescribing in either micrograms or milligrams can cause confusion and increase the chance of errors.

Abstract number: P02-333

Abstract type: Poster

An Analysis of the Quality of Advanced Care Plan and DNACPR Documentation Prior to the Introduction of the ReSPECT Process Smith C., Roulston G., Parmar K., Wilkinson E., Henderson S.

St Peter's Hospital, Ashford and St. Peter's Hospitals NHS Foundation Trust, Surrey, United Kingdom

An Advanced Care Plan (ACP) allows discussion and documentation of patient preferences for their future medical care which is not limited to resuscitation only. Despite national guidance, there remains poor communication and inconsistent documentation of ACP and DNACPR decisions, potentially resulting in inappropriate treatment and hospital admissions for patients. ReSPECT (Recommended Summary Plan for Emergency Care and Treatment) is a newly introduced emergency care plan which aims to help support advanced care discussions and record recommendations. We aimed to assess the quality of ACP and DNACPR documentation at a UK District General Hospital before introducing the ReSPECT form.

Retrospective data was collected on all patients from two elderly care wards in March 2018 through case note review. Section headings on the ReSPECT form were used as the basis for collection. Data was also collected on ACP/DNAR decision communication on discharge documentation.

87 patients ( 52 male and 35 female). $25 \%$ (22) had ACP and $72 \%$ (63) had DNACPR agreed. Those with DNACPR, 79\% (50) had discussion documented in clinical notes, and $57 \%$ (36) on discharge documentation. Those with ACP agreed, 68\% (15) of discussions were documented in clinical notes, $95 \%$ (21) had ACP on discharge documentation completed by junior doctors.

ACP and DNACPR decisions are individual to each patient and our initial data shows they are not always clearly documented. Interestingly, ACP decisions are found more on discharge summaries than recorded in medical notes. Discharge summaries are completed by junior doctors and therefore need support by clear senior decision making in the notes. This is currently missing for $27 \%$ of ACP decisions and highlights that discussions required for the ReSPECT process are not currently routinely documented. The ReSPECT form alongside web-based training was introduced in October 2018. The next step is to re-audit and assess its impact on documentation and communication.

\section{Abstract number: $\mathrm{P} 02-334$}

Abstract type: Poster

Coordinate My Care (CMC) Advance Care Planning (ACP) Process, Underpinned by Digital Technology that Transforms Riley J., Droney J.

Imperial College London, London, United Kingdom
Aims: How service was developed and is run $\mathrm{CMC}$ is an ACP service, its Pan London (9 million). Patients initiate plans on line, myCMC. Clinical details are added by clinician who approves CMC PLAN. Once approved, plan is share digitally with all the urgent care services including GPs, ambulance, medical 24/7 hotline (111) and emergency departments. The service involves training healthcare \& social care professionals in creating ACPs. CMC is web-based. Plans can be created and edited across the acute and community settings. Patients can view plans on their smartphones.

Design \& methods:

- Stakeholder engagement; 111, Ambulance service, GPs, hospices \& hospitals

- Design of web based, secure, encrypted, scalable electronic solution with interoperability road map

- Development of a training module including: identify \& consent patients, sensitive conversations, create a care plan, DNAR

- Information and Clinical Governance - Quality improvements

- Barriers to disruptive technologies

- Funding

- Artificial Intelligence - analysis of large datasets

Results: $(29 / 08 / 2018)$

$57,905 \mathrm{CMC}$ records created. Diagnosis: cancer $26.3 \%$, non-malignant $73.7 \%$ (23\% Dementia).

Urgent care accesses 15,711 .

Deaths: 25,130 , deaths in PPD 75\%. Home 37\%, Care Home 27\%, Hospice $16 \%$, Hospital $19 \%$, other $1 \%$

Professionals creating records: GP $68 \%$, community nurses $4 \%$, acute $14 \%$, hospice $14 \%$.

Factors predicting dying in the preferred place with $95 \%$ confidence interval PPD 1.80 (1.61- 2.01) and Not for Resuscitation 1.84 (1.64- 2.06)

Conclusions: $81 \%$ of CMC deaths occur OUTSIDE hospital (47\% deaths IN hospital 2016 National data) and 75\% die in their PPD, avoiding unnecessary hospital admission. CMC, a shared decision making process and digital sharing of ACPs with urgent care services represents a 'paradigm shift' in palliative care service delivery that is appropriate for every country because of decreasing availability of funds and increasing age of populations worldwide. ACP should become routine practice.

\section{Abstract number: P02-335}

Abstract type: Poster

Providing Holistic Integrated Palliative Care in Kampala, Uganda: A Case Study of a 17 Year Old with Advanced Squamous Cell Carcinoma

Billett $H$.

Makerere and Mulago Palliative Care Unit, Kampala, Uganda

Background: Across Africa approximately 9.67 million people are in need of palliative care (PC). Cancer patients contribute significantly to this number as many have advanced incurable disease at presentation. The Makerere/Mulago Palliative Care Unit (MPCU) was formed in 2008 and offers a liaison PC service to inpatients in Mulago Hospital in Uganda's Capital Kampala. Many patients seen in Mulago Hospital present with advanced illness that is not amenable to cure, but their quality of life can be improved by accessing PC.

Case presentation: After sustaining a significant burn aged 7 this 17 year old patient developed an itchy lesion on her right elbow which broke down into a fungating wound. After 5 months she was eventually diagnosed with a Marjolin's ulcer and advanced squamous cell carcinoma. Limited health service resources, expensive transport and rural home location were identified as factors contributing to the delay in diagnosis. At Mulago Hospital the MPCU referral came from a PC link nurse. The teams input included coordinating palliative radiotherapy, guiding the 
use of oral liquid morphine for pain management and providing psychological and spiritual support to the patient and family. Palliative Care Link Nurse Training and the MPCU Volunteers Programme where key in the identification and provision of holistic PC for this patient.

Conclusion: Recognising the factors that contribute to a delay in diagnosis identifies them as potential targets for future intervention. Providing culturally appropriate holistic PC in countries with limited resources is an ongoing challenge. The model developed by the MPCU offers a number of innovative and workable examples of how this can be done in similar settings.

\section{Abstract number: P02-336}

Abstract type: Poster

Enhanced Supportive Care Prevents Unplanned Hospital Admissions on a Regional Level

Monnery D., Benson S., Griffiths A., Cooper M., Watson A., Cadwallader

C., Hampton-Matthews J., Coackley A.

Clatterbridge Cancer Centre NHS Foundation Trust, Wirral, United Kingdom

Background: Enhanced Supportive Care (ESC) promotes earlier implementation of supportive and palliative care within cancer care to prevent and manage adverse effects of cancer and its treatment. This can improve the quality of life for patients, potentially lengthen survival and promote better care at the end of life. Our centre has joined a three year national pilot in establishing ESC for 4 primary tumour sites and measuring the impact on patients and services.

Aims: Evaluate the impact of ESC delivered within our tertiary cancer centre on unplanned hospital admissions within and beyond our Trust. Methods: We designed a new service using an outpatient clinic model to offer a review for all patients at the time of diagnosis of incurable disease. Initially we offered referrals to patients with Upper GI, Head and Neck, CNS and Melanoma primary tumour sites. Patients who accepted received an initial appointment with a palliative care doctor and then were followed up routinely and linked in with local services upon completion of treatment by a palliative care CNS. We measured the rate of unplanned admissions to our trust and 4 surrounding acute trusts. Admission rates for patients with primary tumour sites accessing ESC were compared against all other tumour sites.

Results: The number of patients seen in ESC has increased from 53 in $2016 / 17$ to 399 in 2017/18. After year one of the pilot, annual acute unplanned admissions to our trust and the 4 surrounding acute trusts for patients attending ESC fell by $2 \%$, compared to a $14 \%$ increase for non-ESC patients. After 2 years, annual admissions for ESC tumour sites had dropped $22 \%$, compared to a $13 \%$ increase for all other primary tumour sites.

Conclusions: ESC successfully reduces unplanned hospital admissions. This benefit is wider than just the Trust where the patient is seen, benefitting the regional healthcare economy. This finding supports the development of an ESC service for all primary tumour sites in our region.

Abstract number: $\mathrm{P} 02-337$

Abstract type: Poster

\section{An Exploration of the Referral Patterns of a Community} Palliative Care Team to Specialist Palliative Care Outpatient Physiotherapy for Community Based Individuals with Advanced Cancer

Cahill F. ${ }^{1}$, Guinan E. ${ }^{2}$, Connolly D. ${ }^{3}$

${ }_{1}^{1}$ Physiotherapy Department, St. Francis Hospice, Dublin, Ireland, ${ }^{2}$ Faculty of Health Science, Trinity College Dublin, Dublin, Ireland, ${ }^{3}$ School of Occupational Therapy, Trinity College Dublin, Dublin, Ireland

Physiotherapy (PT) is a core discipline within specialist palliative care (SPC) contributing to the management of common symptoms of advanced cancer including fatigue, breathlessness and decreased mobility. However, referral rates to PT for community individuals range from 24-32\%.This study aimed to describe the differences in the profile of individuals referred to PT and those not. It explored the community palliative care teams (CPCT) understanding of the role of physiotherapy in SPC. A mixed method approach was used. A retrospective chart audit extrapolated the profile of 38 individuals referred to PT and 102 not referred, using a specifically designed tool. Focus group interviews occurred with members of the CPCT. A significant difference in the average age of both groups was found, with younger individuals referred to PT $(p=0.009)$. A significant difference in the palliative performance scale between groups was identified, with higher performance status referred to PT $(p=0.035)$. Themes identified from the focus groups were referral to PT, expertise of physiotherapists within SPC services and development of SPC PT services. Reasons for referral included symptom management and a stepping-stone to other SPC services. Concerns were discussed that influence decisions not to refer to PT. Decreased mobility and breathlessness were the main reasons for referral identified from the chart audit and focus groups. Participants identified that they did not consider a referral to PT in response to symptoms such as fatigue or pain. This highlights knowledge gaps regarding the role of SPC physiotherapy in the management of other symptoms. This research supports the need to develop a referral pathway to SPC PT in response to symptoms and consequences of illness. It highlights areas in which SPC physiotherapists need to provide education to those who refer to physiotherapy, including the management of symptoms other than decreased mobility and breathlessness.

Abstract number: P02-338

Abstract type: Poster

Does Informational and Relational Continuity Improve Outcomes for People Receiving Palliative Care? A Mixed Methods Rapid Review

Hudson B. ${ }^{1,2}$, Best S. ${ }^{1}$, Stone P. ${ }^{2}$, Noble B. ${ }^{3}$

${ }^{1}$ Marie Curie, London, United Kingdom, ${ }^{2}$ Marie Curie Palliative Care Research Department, Division of Psychiatry, UCL, London, United Kingdom, 33. Department of Oncology, Academic Unit of Supportive Care, The University of Sheffield, Sheffield, United Kingdom

Background: Continuity of healthcare is important, but is often lacking, as recognized by the James Lind Alliance Palliative and end of life care Priority Setting Partnership. Continuity may become increasingly valued as a person ages, develops co-morbid conditions or their health deteriorates.

Aims: To identify qualitative, quantitative and mixed methods literature exploring the impact of relational and informational continuity on preferred place of death, hospital admissions and satisfaction for patients receiving palliative care.

Design: A mixed methods rapid review. PUBMED, PsychINFO, CINAHL were searched to identify original peer reviewed, qualitative, quantitative or mixed methods studies, published in the last 10 years exploring the impact of continuity of care for palliative patients. Qualitative data was analysed using methods outlined by the Cochrane Qualitative and Implementation Methods Group. Meta-analyses for quantitative data were planned.

Results: Eighteen eligible papers were identified (11 qualitative, 6 quantitative, 1 mixed methods). In all, 1,951 patients and 190 family caregivers were recruited across included studies. Meta-analyses were not possible due to heterogeneity. Two studies described positive impact on facilitating preferred place of death. Four described a reduction in avoidable hospital admissions. No negative impacts were reported. Poor continuity of care was related to delays and difficulties in accessing support, increasing patient and carer burdens and feelings of vulnerability. In contrast, good continuity of care was related to feeling confident, known and supported by care providers.

Conclusions: The evidence for the impact of continuity on place of death and hospital admissions is limited, but promising and highlights the 
impact that continuity can have. Methods for enhancing and recording continuity should be considered in the design and development of future interventions to support patients receiving palliative care.

Abstract number: P02-339

Abstract type: Poster

Challenges of Sign Language Interpreters in their Work with Seriously III and Dying Deaf People. A Qualitative Investigation Ruszynski A.M. ${ }^{1}$, Orellana-Rios C.L. ${ }^{2}$, Becker G. ${ }^{2}$

${ }_{1}^{1}$ Protestant Diakonissenhaus Berlin Teltow Lehnin, Teltow, Germany, ${ }^{2}$ Department of Palliative Medicine, University Hospital Freiburg, Freiburg, Germany

Background and aim: Sign language interpreters (SLI) enable communication between hearing and deaf people. They play an essential role in the lives of deaf people. Little is known about the challenges faced by SLIs when translating in palliative contexts and being confronted professionally and personally with the issues of dying and death. With the aim of identifying possible challenges, a qualitative study was carried out.

Method: Guideline-based interviews with six SLI were carried out, transcribed and evaluated by content analysis. The interview partners were recruited via the Federal Association of Sign Language Interpreters Germany e.V. and selected by means of Theoretical Sampling. In addition to questions about the translation process, questions were also asked about the impact on both the professional role and the personal area.

Results: The results demonstrate the complexity of the professional, ethical, and personal needs that SLIs face before, during, and after a work assignment in a palliative setting. In addition to structural challenges that generally complicate the translation process, the relationship shaping to patients, their relatives and hearing health professionals as well as dealing with their own emotional consternation are central challenges. Professional ethics conflicts can also arise.

Conclusion: Working in palliative contexts places complex demands on the work, the role and the person of the SLI. The completed qualifications often fail to prepare for these challenges; Strategies for dealing are very individual and opportunities for reflection are insufficient. Specific training and supervision offers are considered desirable.

Abstract number: P02-340

Abstract type: Poster

Adequate Allocation of Resources in Critical Areas: Ethical and Operational Challenges in End-of-Life Palliative Approach

Rocha J.A., Crispim D.H., Franck E.M., Santos A.B.B., Jales S.M.C.P., Costa F.F.S., Carvalho R.T.

Palliative Care, Hospital das Clínicas, University of São Paulo, São Paulo, Brazil

Background: We highlight the work of a palliative care team in a university hospital focused on the ethical and operational challenges of this endeavor.

Aims: The care advanced planning is established at a decision-making meeting shared with the patient/family and aims to establish:

1) Empathic communication of news bad;

2) Understanding the beneficence or not of the use of advanced life support;

3) Understanding the values and preferences of the patient/ family;

4) Definition of better allocation of patients.

Methods: A retrospective descriptive study by database analysis. Results: Between 2012-2016, 3851 patients were evaluated by the palliative care consutation group. Of these patients, 1526 (62.7\%) were in the ER and 907(37.3\%) in the ICU. Of the interned in ICUs 432(47.6\%) effectively managed to be withdrawn, but $475(52.4 \%)$ remained in critical areas. Of those who remained, 189(39.8\%) were very severe but in $286(60.2 \%)$ there was still a clinical condition for this, however, this did not occur. This was due to:

1) failure to wean from advanced life support 146(51.1\%);

2) clinical operating factors $126(44.1 \%)$ and

3) lack of vacancy $14(4.8 \%)$

Clinical operational factors are: clinical worsening of the patient in the last hours, lack of personnel or family to follow the transfer, lack of ambulance and communication failure.

Discussion: Advanced care planning and adequate allocation of resources are part of the routine work of the palliative care team. This process is complex and involves multi-variable management that begins with an ethical deliberation between assistant staff, palliative care team and patient/family and extends to organizing and managing patient transfer to palliative care beds who are often far from the place of detention.The final objective will be the patient/family reception and the union of efforts of several sectors aiming to promote quality and dignity in the end-of-life.

Abstract number: P02-341

Abstract type: Poster

Telehealth Use in Palliative Care Is Being Described but Not

Evaluated - A Systematic Review

Hancock S. ${ }^{1}$, Preston N. ${ }^{1}$, Jones H. ${ }^{2}$, Gadoud A. ${ }^{1}$

${ }^{1}$ Lancaster University, Lancaster, United Kingdom, ${ }^{2}$ St Catherine's

Hospice, Preston, United Kingdom

Background: Telehealth has been proposed as a solution to meeting the needs of the growing palliative care population. A review in 2010 by Kidd et al highlighted that there was an awareness of telehealth in palliative care but a lack of evidence-based research to support its use.

Aims: The aims of this review were to describe the use of telehealth in palliative care in the UK and to examine how these telehealth initiatives are evaluated. A secondary aim was to compare the telehealth initiatives described against the UK government's 'digital service standard'.

Methods: Five databases (MEDLINE, EMBASE, CINAHL, Psychinfo and Cochrane register) were searched using pre-defined terms. Hand searching of conference literature, thesis databases and citation tracking was also conducted. Screening of title and abstract followed by full text review was conducted by two reviewers against inclusion criteria. Data was extracted from included studies by two reviewers.

Results: 3807 records were screened resulting in 30 included studies. There were a wide variety of telehealth applications described with the number of home telemonitoring initiatives increasing from the 2010 review. Other studies aimed to support patients and carers, particularly out of hours, through the use of telephone or video advice lines. There were three randomised controlled trials, but most studies were qualitative in design. The lack of homogeneity in study design and small sample sizes makes comparison and drawing conclusions difficult. Only one paper met the criteria of a digital service standard.

Conclusion: There has been an increase in the number of telehealth interventions for palliative care in the UK, however there remains a lack of evaluation of these services. This review is unable to draw any conclusions on the benefits of using telehealth in palliative care and these findings indicate that further work is needed to evaluate telehealth services, particularly for out of hours palliative care.

\section{Abstract number: P02-342}

Abstract type: Poster

\section{Access to early Palliative Care}

Koekoek B.I. ${ }^{1}$, Breugem J.A. ${ }^{2}$

${ }^{1}$ Network of Palliative Care Oost-Veluwe, Apeldoorn, Netherlands,

${ }^{2}$ General Practice Van Ingen - Breugem, Apeldoorn, Netherlands 
Aims: Palliative care was administered to approximately 800 patients in Oost-Veluwe in the Netherlands in 2016. Often the need for palliative care was recognized too late. A lack of cohesion, along with a missing central role for the patient and their wishes and needs, often occurred in this form of care. Defining and prioritising this resulted in a transmural palliative care pathway; with the goals of early identification, continuous guidance and attention to patient's needs.

Design: Specification of the care pathway: recognising signs, identifying palliative phase by a doctor, guidance by a palliative nurse, using a wish book, end-of-life conversation, identifying terminal phase, organisation of the dying process and aftercare. Based on the model by Lynn \& Adamson and peoples wishes in the region.

Methods and approach:

- $\quad$ informing medical specialists and GP's about identification

- $\quad$ setting up a referral system

- training 21 palliative nurses at 11 home care organisations

- developing a wish book with patients

- coordination by network coordinators

- $\quad$ setting up a registration and evaluation system

- $\quad$ start care pathway 1 January 2018

Results: 1 October 2018:

- 76 patients included, $53 \%$ via GP, $47 \%$ via specialist

- 30 patients passed away, among them were:

- $90 \%$ oncological patients

$40 \%$ used the wish book

- 24 died at home; the preferred place for 23 of them

Conclusion: Various organisations are capable of cooperating together. Identifying the palliative phase is often late.

This pathway fulfils needs: enthusiastic professionals and satisfied patients. Continued guidance is guaranteed.

Lessons learned:

- training in identification of doctors remains a point of attention

- $\quad 90 \%$ of those who died were oncological patients, other palliative patients deserve the same level of care

- $\quad$ not everyone is comfortable discussing their own wishes and needs

- continuing support, the aim is to support at least $10 \%$ more patients each year

Sources of funding: No funding was used.

Abstract number: P02-343

Abstract type: Poster

\section{What Do Doctors Want from Out of Hours Specialist Palliative} Care?

Matthews R. ${ }^{1}$, Tomlinson S. ${ }^{1}$, Corbett L. ${ }^{1}$, Johnson S. ${ }^{2}$, Bullock R. ${ }^{3}$, Perkins $P^{1,4}$

${ }^{1}$ Gloucestershire Hospitals NHS Foundation Trust, Cheltenham, United Kingdom, ${ }^{2}$ Wye Vale NHS Trust, Hereford, United Kingdom, ${ }^{3}$ Worcestershire Acute NHS Trust, Worcester, United Kingdom, ${ }^{4}$ Sue Ryder Leckhampton Court Hospice, Cheltenham, United Kingdom

Background: There is debate about the optimum structure for services supporting patients with palliative care needs out of hours $(\mathrm{OOH})$. In the UK, there is wide variation in the specialist palliative care (SPC) services offered. In Gloucestershire, healthcare professionals currently have access to a telephone advice line, but no face-toface assessments.

Aims: The primary objectives were:
1. evaluate $\mathrm{OOH}$ SPC services currently available to hospital patients in Gloucestershire;

2. determine the level of support hospital doctors think they require to manage palliative patients $\mathrm{OOH}$.

Methods: An anonymous questionnaire was sent to doctors of all grades in Gloucestershire Hospitals NHS Foundation Trust to establish their understanding of the services currently available and the level of SPC support required $\mathrm{OOH}$. We followed up with email reminders.

Results: 104 doctors replied. Only 30 had a correct understanding of the $\mathrm{OOH}$ SPC services available; 15 incorrectly thought that there was support from visiting SPC healthcare professionals. 49 had contacted the $\mathrm{SPC}$ team $\mathrm{OOH}$ at some point in their career, with the most common reasons relating to symptom control (33\%) and medication advice (31\%). $98 \%$ of participants found the advice they had received helpful. 76 participants agreed that an $\mathrm{OOH}$ telephone advice line was required, but of these, nearly half (46\%) felt that additional support from visiting healthcare professionals was needed. $12.5 \%$ of participants felt that visiting SPC professionals were required instead of an advice line. $62 \%$ of participants believed that, with better education, clinicians would be less likely to require $\mathrm{OOH}$ SPC support.

Conclusions: Doctors valued the advice received from the $\mathrm{OOH}$ SPC team, but there was a lack of understanding about the precise services available. Despite this satisfaction, some felt that a visiting service would be of benefit. The majority thought that better SPC education would lead to less reliance on $\mathrm{OOH}$ SPC services.

Abstract number: P02-344 Abstract type: Poster

Effectiveness of a Multidimensional Primary Care-based Intervention on Early Identification and Resource Utilization of Palliative Care Patients: A Cluster Randomized Clinical Trial Llagostera M. ${ }^{1}$, Serrano C. ${ }^{1}$, Leiva A. ${ }^{2}$, Sansó N. ${ }^{3}$, Bulilete O. ${ }^{2}$, Benito E. ${ }^{1}$, Esteban E. ${ }^{4}$, Alvarez C. ${ }^{4}$, Corredor M.T. ${ }^{4}$, Taltavull J.M. ${ }^{4}$, Martin-Roselló M.L. ${ }^{5}$, Llobera J. ${ }^{2}$, Primary Care Research Unit, Balearic Public Health Service, Balearic Islands Health Research Institute (IdISBa) ${ }^{1}$ Centre Coordinador Programa Cures Pal-liatives Illes Balears, IBSALUT, Palma, Spain, ${ }^{2}$ Unitat Investigació. Gabinet Tècnic Atenció Primària, IBSALUT, Palma, Spain, ${ }^{3}$ Infermeria i Fisioteràpia, Universitat Illes Balears, Palma, Spain, ${ }^{4}$ Gerencia Atenció Primària Mallorca, IBSALUT, Palma, Spain, ${ }^{5}$ Biomedical Research Institute (IBIMA), Cudeca Foundation, Benalmadena, Spain

Background: Early identification of patients eligible for PC is beneficial, by avoiding aggressive interventions and by reducing unnecessary suffering.

Aims: To assess the efficacy of a multidimensional intervention based in empowerment-training of a primary care team-leader in PC patient's early identification and to reduce "aggressive" end-of-life-care.

Methods: A two-arm cluster randomized controlled trial including primary care centres(Mallorca-Spain). Subjects: 3835 deceased patients in 18-month evaluation period. 30 primary care centres were randomized to intervention group (IG) or control (CG). IG: 15 team leaders were created, recieving $42 \mathrm{~h}$ training course, to promote $\mathrm{PC}$ training among their colleagues; improve symptoms and psychological management; and incorporate an integral management of patients. The CG provided usual care.

Measurements: early identification ( $>90$ days before death); positive PC identifier and non-aggressive care indicators: Do not present in the last 30 days before death: $\geqslant 2$ visits in hospital emergency service, $\geqslant 2$ admissions in hospitals, patients with stays in acute hospital over 14 days, admission in ICU or die in hospital.

Results: A total of 3835 all-causes deceased were analyzed; 404 (10.5\%) identified as NECPAL positive: $12.7 \%$ IG vs $8.5 \%$ CG. Early identification 
was superior in IG:3.5\% vs $1.6 \%$ in $\mathrm{CG} p<0.001$. Adjusted by cluster - OR 2.28 (CI95\% 1.48-3.52). Do not present: $\geqslant 2$ visits to the hospital emergency department ( $C G=90.4 \%$ vs IG $=90.8 \%$ ); $\geqslant 2$ acute hospital admission (CG $=92.6 \%$ vs IG $=94.2 \%$ ); stays in acute hospital $>14$ days (CG $=80.7 \%$ and IG $=82.9 \%)$; ICU (CG $=93.5 \%$ and IG $=93.5 \%)$; Hospital Death ( $C G=28.4 \%$ and $I G=31.2 \%$ ).

Conclusion: Training and empowering a primary health care team is effective in early identification of patients with PC needs, nevertheless the absolute risk reduction is low. The intervention it was also effective in the reduction of some non-aggressive indicators in the last 30 days of life.

\section{Abstract number: $\mathrm{P} 02-345$}

Abstract type: Poster

Operation Integration - Utilising Nominal Group Technique (NGT) to Promote Community Engagement Tracey G. ${ }^{1,2}$, Bates U. ${ }^{3}$, Connell T. ${ }^{3}$, Voltz R. ${ }^{2}$

${ }^{1}$ Blackrock Hospice, Our Lady's Hospice \& Care Services, Dublin, Ireland, 2European Palliative Care Academy, Cologne, Germany, ${ }^{3}$ Our Lady's Hospice \& Care Services, Dublin, Ireland

Background: Provision of palliative care(PC) is widely reported as inequitable at local \& national level.Access to \& uptake of PC services by communities is influenced by a myriad of factors, with individuals in marginalised groups least likely to engage.Specialist palliative care services(SPC) have a duty to connect with local community groups to ensure services are configured to local need.

Aims and Objectives: To undertake a process of local community engagement with selected cohorts to enhance understanding \& heighten awareness of the role of SPC. To inform \& implement short-medium term organisational change in response to the above process.

Methods: Informed by scoping of literature \& demography, local community representatives from mainstream $\&$ under-represented groups underwent purposive sampling to partake in a consultative process.Local ethical approval was sought \& obtained.A meeting was convened. NGT was employed to facilitate problem identification, solution generation \& enable decision making.Suggestions were ranked by participants $\&$ collated by facilitators to inform development of initiatives to enhance the profile of SPC within the community.

Results: Fifteen community representatives from diverse groups were invited to partake. Response rate was $60 \%(n=9)$. Each participant volunteered five suggestions to a single question which were ranked thereafter.Following interrogation by facilitators, emergent themes included community outreach, novel communication strategies,embracing diversity \& new organisational initiatives.These proposals were harnessed to drive strategy to impact upon current $\&$ future service delivery $\&$ to heighten individual, team \& organisational response to the need for innovative engagement with marginalised groups.

Conclusion: NGT proves a valuable tool to establish initial \& continuing local community engagement.This provides a road-map to initiate reconfiguration of service delivery tailored to local requirements.

Abstract number: P02-346

Abstract type: Poster

Can I Die at Home? A Service Evaluation Comparing the Preferred and Actual Place of Death Known to a Specialist Community Palliative Care Service

Ali M., Capel M., Rees S.

City Hospice, Cardiff, United Kingdom

Background: Place of death is an important aspect of end of life care. The majority of people would prefer to die at home and the stated intentions of both statutory and voluntary healthcare providers aim to support this. The achievement of an individual's preference is considered a quality marker of good end of life care. AIMS This service evaluation compared patients' preferred (PPD) and actual place of death. Patients are known to change their PPD during their illness trajectory. Having dynamic care plans and discussions enable changes to be recorded reflecting patient preferences.

Methods: The key worker for each patient who died completed a 'proforma'. All deaths of patients ( $n=4344$ ) known to the specialist palliative care service 2009-2017 were examined to compare the actual place of death with the preferred place of death.

Results: Between 2009-2017 74.9\% of patients who expressed a wish about their preferred place of death were able to achieve this preference. $71.3 \%$ of patients achieved their chosen preference which was home, $77.1 \%$ hospice, $90 \%$ nursing home and $82.6 \%$ residential home $28.7 \%$ patients either refused to discuss or no preference was elicited for place of death. Of those patients who either declined to express a preference or none was identified the majority of these died in hospital.

Conclusion: The majority of patients PPD is home. The factors identified which have congruence with individuals achieving PPD are:

1. Identifying PPD

2. Having anticipatory medication in place

3. Having community nurses involved in care delivery

4. Having informal carers involved in care delivery (family, friends or carers)

5. No acute events which are unplanned e.g. falls, sepsis Every year there has also been an increase in non-malignant disease referrals reflecting a new need in the community to adequately recognise and prepare for their unpredictability of the trajectory of end-of-life events.

Abstract number: $\mathrm{P} 02-347$

Abstract type: Poster

Interprofessional Collaboration in ICU-Palliative Care: The Challenges and What Works?

Poi C.H. ${ }^{1}$, Yow F. ${ }^{2}$, Ong W. ${ }^{2}$, Hum A. ${ }^{1}$, Koh M. ${ }^{1}$

${ }^{1}$ Palliative Medicine, Tan Tock Seng Hospital, Singapore, Singapore,

${ }^{2}$ Nursing Department, Tan Tock Seng Hospital, Singapore, Singapore

The complexity of the clinical needs of critically ill patients necessitates a team approach to deliver care that encourages effective interprofessional communications and collaboration. Since October 2013, integration of palliative care services had been gradually incorporated in stages into various ICUs our hospital. Engagement of key stakeholders including ICU directors, intensivists, nurse leaders and members of hospital leadership, social work department were done to develop ICU-palliative care initiative.

Our study aims to examine ICU professionals' perceptions of challenges and key elements that makes interprofessional collaboration in ICUPalliative care work.

From September2016 to July2017, 13 focus groups were conducted with 54 ICU professionals (nurses, doctors, allied health professionals) from 4 ICUs. The discussions were audio-recorded, transcribed and analyzed thematically. Challenges includes:

1) Receptiveness of families to end-of-life(EOL) care discussion

2) Sustainability and potential burnout of team members providing EOL care

3) Concerns on deskilling ICU team with over-reliance on palliative team to provide EOL care.

Elements which makes this collaboration works includes:

1) Sharing common vision in care delivery 
2) Proactive approach in early involvement \& identification of patients with palliative needs

3) Team approach in care delivery

4) Consistency within the team in care provision

5) Ability to engage \& support interprofessional team

6) Ability to address families in both medical \& emotional needs

7) Ability to complement care with intensivist

8) Being present, committed \& available

9) Good communications between various teams in ICU

This study helps us assess how palliative care team can collaborate more effectively with other ICU healthcare professionals to care for the dying. It provides insights as to how palliative care team can engage various ICU healthcare professionals including important key stake-holders to enhance care in ICU.

Abstract number: P02-348

Abstract type: Poster

\section{Video Consultations in Palliative Care: A Systematic Integrative Review \\ Jess M., Timm H., Dieperink K.B.}

REHPA, The Danish Knowledge Centre for Rehabilitation and Palliative Care, Nyborg, Denmark

Background: Access to palliative care remains inadequate worldwide. Video consultations are a promising approach to address challenges in palliative care provision. The technology enables real-time interactions between patients, relatives and palliative care professionals at a distance. However, no attempts have been made to review the evidence solely on video consultations in palliative care settings.

Aims: To gain insight into:

1) the advantages and disadvantages;

2) barriers and facilitators, when implementing video consultations in general, and specialized palliative care from the perspective of patients and relatives, healthcare professionals, and society.

Methods: A systematic search was carried out in Pubmed, Embase, Cinahl and Psychinfo using terms related to 'video consultations' AND 'palliative care', published from 2005 - 2018. Titles and abstracts were screened followed by full article screening. Additionally, reference lists were hand searched. Included were primary studies involving video consultations between patients and/or relatives of all ages, and professionals in palliative care. The review followed the PRISMA 2009 guidelines and Whittemore \& Knafl, 2005. The studies' quality was assessed in accordance with Hawker et al., 2002.

Results: The search resulted in 813 articles. 39 articles met the inclusion criteria: mixed methods $(n=14)$, qualitative $(n=10)$, quantitative $(n=10)$ and case studies $(n=5)$. Data analysis resulted in six themes addressing advantages/disadvantages and facilitators/barriers. These were:
1) Redesign of care;
2) communication;
3) user perceptions;
4) technology;
5) privacy issues; and
6) economic implications.

Conclusion: The use of video technology in palliative care is feasible and has advantages as well as disadvantages. Evidence is however limited, and mainly deals with specialized palliative care and cancer patients. Future research should focus on general palliative care and patients with a non-cancer diagnosis.
Abstract number: P02-349

Abstract type: Poster

The Use of Personal Narratives in Hospital Based Palliative Care Interventions: A Systematic Integrative Review Roikjaer S.G. ${ }^{1,2,3}$, Missel M. ${ }^{4}$, Bergenholtz H.M. ${ }^{2,5}$, Schønau M.N. ${ }^{4}$, Timm H.U. ${ }^{2,3}$

${ }^{1}$ Medicine 2, Cardiology, Naestved, Slagelse and Ringsted Hospitals, Slagelse, Denmark, 2Palliative Research, Knowledge Center for Rehabilitation and Palliative Care, REHPA, Copenhagen, Denmark, ${ }^{3}$ Clinical Research, University of Southern Denmark, Odense, Denmark, ${ }^{4}$ Thoracic Surgery, Rigshospitalet, Copenhagen, Denmark, ${ }^{5}$ Medicine and Surgery, Holbaek Hospital, Holbaek, Denmark

Background: Studies show that patients suffering from a life-threatening illness (LTI) experience unmet psychosocial and existential needs. Narrative interventions have shown promise in managing these problems, but no synthesis on the use of narrative interventions exclusively in palliative care in a hospital setting have been made.

Aims: The aim is to review the use of personal narratives in palliative care interventions conducted in a hospital based setting for people suffering from LTI, in order to strengthen general palliative care practices.

Method: A systematic search was conducted in Pubmed, Embase, SCOPUS, Cinahl, SocINDEX and PsychInfo for primary research articles published up to June 2018. Full-text articles were critically assessed against the eligibility criteria followed by a discussion of research rigor using CASP by two reviewers. In case of discrepancies, a third reviewer was included. Papers were included if they presented findings from primary research on narrative interventions conducted in a hospital setting that included adult patients with a LTI in need of palliative care. The study reports on findings in adherence to PRISMA where applicable, and also follows the guidelines of Whittemore and Knafl.

Results: Out of 966 search hits we included 24 studies: 8 qualitative, 14 quantitative and 2 mixed-method. Among these, 6 sets of articles reported on the same intervention and population. Articles reported on: Dignity therapy, Legacy building, Outlook, Life Review and Short Term Life Review. Data analysis resulted in five themes on the use of narrative interventions: Core principles, Theoretical framework, Content of narrative, Outcome of narrative and Acceptability/feasibility.

Conclusion: Narrative interventions are feasible in a hospital setting, and evidence supports clinical application of narrative interventions for people suffering from a LTI as a means of enhancing their life. However, the evidence for statistical significance is limited.

Abstract number: P02-351

Abstract type: Poster

Challenges for a Successful Cooperation between Specialised Palliative Care and Geriatric Nursing Homes

Czudnochowski D. ${ }^{1}$, Lindwedel-Reime U. ${ }^{2}$, Kuhlberg J. ${ }^{1}$, König P. ${ }^{2}$, Topp J. ${ }^{1}$

${ }^{1}$ Palliativnetz Freiburg, Freiburg i. Br., Germany, ${ }^{2}$ Hochschule

Furtwangen University, Furtwangen, Germany

Multimorbidity rates are increasing, especially among elderly people living in nursing homes. Although palliative care (PC) approaches to this challenge are on the rise in Germany, the number of cooperations between nursing homes and specialised PC teams is still limited and much potential remains unexploited. The aim of this study was to identify the underlying reasons for this from the perspective of a PC team. In addition, practical solutions were to be found on how such a team can successfully cooperate with nursing homes. Therefore, the study followed two research questions: Firstly, what are the main problems that hinder cooperations between a PC team and nursing homes? And 
secondly, how does a PC team avoid these problems in order to institutionalise care standards in their partner institutions?

In a two-year ethnographic study at a specialised PC team in southern Germany, 25 interviews were conducted. The study further entailed observation of workflows and team meetings. The analyses were performed using an inductive qualitative approach to reconstruct problems from the perspective of the PC Team.

The most important disruptive elements for a successful cooperation the study identified were a lack of standardized communication cultures, conflicting workflows and lacking knowledge about PC. Specialised PC in geriatric nursing homes can succeed if a stable personal relationship between the PC team and the nursing staff already exists and responsibilities in the care situation are clearly defined. However, it might be unsuccessful if the work of the PC team is perceived as external criticism of the daily work. In order to establish new care standards and relevant knowledge, the PC team attempts to be aware of existing working routines. In sum, a process to improve cooperation must translate the needs and workflows of each profession. The study suggests that to establish PC standards in geriatric homes, suitable public relations work is necessary.

Abstract number: P02-352

Abstract type: Poster

Why Palliative Patients Are Readmitted to Hospital within 30

Days of Discharge: An Analysis of Communication between

Hospital and Community Teams

Modi M. ${ }^{1,2}$, Galbraith S. ${ }^{1}$

${ }^{1}$ Palliative Medicine, Addenbrooke's Hopsital, Cambridge University Hospitals, Cambridge, United Kingdom, ${ }^{2}$ Palliative Medicine, Arthur Rank Hospice, Cambridge, United Kingdom

Aim: While information is available on the speed of the discharge process from hospitals for palliative patients, there is less known about what happens after discharge. This project aims to evaluate discharge processes in patients who were identified as 'rapidly deteriorating' or 'in a terminal phase of their illness' and were subsequently entered on a pathway to enable accelerated discharge.

Method: 30 palliative patients readmitted within 30 days of discharge were compared with 36 not readmitted over an 8-month period.

36 measures of quality were analysed including: discharge destination, additional care provided, inpatient palliative care specialist input, discharge summary and palliative care letter sent to GP and care home, patient prognosis, ongoing management plan after discharge, recommended plan in the event of deterioration and whether readmission may be appropriate.

Results: Data of 336 palliative patients with planned discharge was analysed. 98 were excluded as had died before planned discharge. 30 of the remaining $238(12 \%)$ were readmitted within 30 days. Of these, 6/30 (20\%) stated hospital as their preferred place of care/death.
Conclusion: There was an increased risk of readmission within 30 days for patients with poor documentation of: prognosis, ongoing management plan and recommended plan in event of deterioration. Insufficient care at home appeared to be a significant cause of readmission ( $43 \%$ of patients). Overall, only $12 \%$ of patients were readmitted and a wider audit will be needed to confirm these findings. However, our audit suggests improvement in certain documentation and access to increased care in the community may prevent unplanned readmissions.

\section{Abstract number: P02-353 \\ Abstract type: Poster}

Challenges of Promoting Uniformity in Programs within a Healthcare System

Li Y. ${ }^{1}$, Morris D. ${ }^{2}$, Verga S. ${ }^{1}$, Hunt L. ${ }^{1}$, Worthley K. ${ }^{1}$, Bleznak A. ${ }^{1}$, Bharadwaj P. ${ }^{1}$

${ }^{1}$ Sentara Medical Group, Norfolk, United States, ${ }^{2}$ Eastern Virgina Medical School, Norfolk, United States

Background: Hospitals with palliative care programs within a healthcare system have variations. These variations need to be studied to ensure the delivery of similar services across different hospitals, both clinically and operationally.

Aims: To capture operational data of inpatient programs to better understand the variations within a 12 hospital healthcare system; and to identify major contributing factors so as to minimize variation.

Methods: We developed a "palliative care navigator" in the electronic medical record (EMR) of our system to extract data. Seven of the twelve hospitals are on the same EMR in 2016. We collected the following data for comparison and analyses:

1. hospital type and size, number of hospital admissions/discharges/deaths, program staffing status;

2. new consults numbers, reason for consults (symptoms management vs. goals of care discussions), patient age distribution, consults initiated from emergency department (ED), and overall service penetration rate;

3. percentage of cancer vs. non-cancer patients.

Results: Significant variations were found across all parameters among hospitals within the same healthcare system. Hospital size varied from 145 to 525 beds; hospital admission numbers from 8,430 to 25,675 / year; staff structure from one RN only to a team composed of MD, NPs, RN and SW; the number of new consults from 269 to 1,249/year; symptom management from $2.4 \%$ to $37.8 \%$; goals of care discussions from $62.2 \%$ to $97.6 \%$; percentage of consult originating from ED from $1.27 \%$ to $5.76 \%$. In addition, the overall palliative care penetration rate ranged from $3.2 \%$ to $7 \%$. The percentage of cancer patient was from $19.44 \%$ to $35.28 \%$.

Table 1. Key quality measures for patients readmitted and not readmitted.

\begin{tabular}{lll}
\hline $\begin{array}{l}\text { Quality measures documented in } \\
\text { discharge communications }\end{array}$ & Readmitted & Non-readmitted \\
\hline Prognosis & $19 / 30(63 \%)$ & $29 / 36(81 \%)$ \\
Ongoing management plan & $21 / 30(70 \%)$ & $33 / 36(92 \%)$ \\
Recommended care plan for deterioration & $15 / 30(50 \%)$ & $23 / 36(63 \%)$ \\
Place of discharge & $25 / 30(83 \%)$ discharged to own home, & $16 / 36(44 \%)$ discharged to own home, \\
& $3 / 30(10 \%)$ discharged to care home, & $17 / 36(47 \%)$ discharged to care home, 3/36 \\
Change in care need & $2 / 30(6 \%)$ discharged to hospice & $(8 \%)$ discharged to hospice/other hospital \\
& $13 / 30(43 \%)$ needed an increase in & \\
& care for second discharge &
\end{tabular}


Discussion: Palliative care is a relatively new and rapidly growing field. Multiple interdependent factors affect the daily operation of a program. Practical and reliable benchmark data need to be identified to help organizations establish new programs and make appropriate investments to ensure its healthy growth.

Abstract number: P02-354

Abstract type: Poster

Physician-assisted Suicide - A Survey in German Hospices and Palliative Wards

Juelich A., Buchhold B., Medgyasszay F.B., Schmidt C., Krueger W. University of Greifswald, Greifswald, Germany

Background: Euthanasia, in the sense of physician assisted suicide, is widely endorsed in surveys among the general population. Only few patients in palliative situation want the physician-assisted suicide if the symptoms are well-alleviated. The knowledge and attitude of nurses and doctors in hospices and palliative care units on euthanasia has hardly been investigated.

Aims: Survey of attitude and knowledge of medical professionals on German palliative wards and in hospices on the topic of euthanasia. Their assessment of the education and suggestions for improvement.

Methods: Questionnaire study on all German hospices and palliative wards on the subject of physician-assisted suicide (2625 questionnaires were sent to 215 hospices and 310 palliative wards). The questionnaires were created with the program EvaSys.

Results: The response rate totaled $44.76 \%$ (hospices $39.53 \%$, palliative wards $48.38 \%$ ). Of the respondents, $78.52 \%$ have over 10 years of professional experience in the palliative field. When asked, "Has your education sufficiently prepared you for the topics of death and positions on euthanasia" $61.88 \%$ of doctors, $58.56 \%$ of nurses and $63.04 \%$ of therapists responded with "no". As improvement possibilities were named:

Suggestions or requests for better knowledge about euthanasia.

\begin{tabular}{llll}
\hline Proposal & $\begin{array}{l}\text { Doctors } \\
/ \%\end{array}$ & $\begin{array}{l}\text { Nurses } \\
/ \%\end{array}$ & $\begin{array}{l}\text { Therapists } \\
/ \%\end{array}$ \\
\hline $\begin{array}{l}\text { regular palliative medical } \\
\text { training }\end{array}$ & 89,6 & 86,6 & 87,8 \\
$\begin{array}{l}\text { palliative additional training } \\
\text { (Palliative Care) }\end{array}$ & 57,9 & 68,1 & 70 \\
$\begin{array}{l}\text { at least one nurse with palliative } \\
\text { additional training on each ward } \\
\text { palliative medical consultation } \\
\text { possible at any time (palliative } \\
\text { medical council) }\end{array}$ & 85,2 & 60,1 & 62,2 \\
\hline
\end{tabular}

Conclusion: The medical staff in German hospices and palliative care units has long professional experience. They do not consider their education on euthanasia sufficient. Improvements can be made through targeted training, increasing the number of palliative care professionals, and palliative counseling (consultation).

Abstract number: P02-355

Abstract type: Poster

Physiotherapeutic Intervention in Early, Integrated Specialized Palliative Care and Rehabilitation

Skov Zellweger B., Hansen G., Steiner H.

Palliative Team, Lillebaelt Hospital, Vejle, Denmark
Background: Early palliative care integrated in oncological treatment is recommended by WHO and ASCO. International studies indicate that early palliative intervention improves control of symptoms and enhances quality of life. The Palliative Team at the Oncological Department at Vejle Hospital conducted the study "Palliative rehabilitation program" offering patients individual relief of symptoms, education of patients and relatives in groups as well as individually tailored physical training programs.

Aim: The aim is to uncover if training increases the physical capacity in patients diagnosed with an incurable cancer disease - and to examine how patients experience taking part of a training programme.

Method: Through both quantitative and qualitative methods data are collected during introductory and final physical tests as well as through patient reported statements. 93 patients participate in groups in the rehabilitation program.

Each patient is provided with an individually tailored training program and objectives are set regarding the patient's mobility, strength and endurance. A physiotherapist and nurse are responsible for the training which takes place one hour every week during 12 weeks. The introductory and final tests consist of a Chairstand Test, the 6 Minute Walk Test and measurement of Handgrib. At the end of the program the patients conduct a written evaluation. Data are collected via SurveyXact and a content analysis is performed based on the patient reported statements.

Results: 91 patients participated in the study, 62 patients completed the programme. This group has improved or maintained the physical performance on all three parameters, e.g. $68 \%$ improved their walking distance.The patients report: "My physical capacity has increased significantly ..."

"I am capable of more than I think".

Conclusion: Even though the patients are going through palliative treatment a systematic training initiative can improve the patients' physical parameters.

Abstract number: P02-356

Abstract type: Poster

Cottage Hospice: A Threat to the Prevailing Paradigm? Findings from a Participatory Action Research Study

Hughes S. ${ }^{1}$, Barnes H. ${ }^{1}$, Turner M. ${ }^{2}$, Walshe C. ${ }^{1}$

${ }^{1}$ International Observatory on End of Life Care, Lancaster University, Lancaster, United Kingdom, ${ }^{2}$ University of Huddersfield, Huddersfield, United Kingdom

Background: Cottage Hospice is a new model of end-of-life service with care provided mainly by family caregivers and volunteers. An ageing demographic and increasing sector costs require innovative service delivery designs. This new service offers more choice, but could be viewed as a threat to existing hospice care, de-professionalising death and reducing specialist provision.

Aims: To use participatory action research (PAR) to evaluate the development of Cottage Hospice from inception to opening and to disseminate the knowledge gained to inform service innovation in the sector.

Methods: An embedded researcher implemented a PAR research approach in a UK hospice over 29 months. Documentary analysis was conducted using strategic records, internal meeting minutes, press releases and other written materials $(n=120+)$. Semi-structured interviews (participants: $n=36$ ) captured stakeholder views on process and progress over time. A series of action cycles led by a Core Action Group identified key areas for solution focussed change. A patient and carer advisory panel contributed to data analysis.

Results: Participants struggled to envisage the nature of Cottage Hospice and its interactions with existing hospice services and the local healthcare economy. Stakeholder views varied. Some saw the development as an intermediate option between 'traditional' hospice care and death at 
home. Others welcomed the creative potential of a concept disruptive of the status quo, while some feared it might reduce the quality of end-oflife care, as a trade-off for cost-savings.

Conclusion: While once at the leading edge of innovation in UK palliative care, hospices have become an established cornerstone of end-of-life provision. Innovation may be welcomed, but can also give rise to confusion and concern among users of hospice care and the hospice workforce alike. As such, careful management of change, robust communication systems and 'buy in' from stakeholders at all levels will be needed.

Abstract number: P02-357

Abstract type: Poster

\section{Persistent Challenges of Home Based Palliative Care for Children} Staras K. ${ }^{1}$, Juodaite Rackauskiene A. ${ }^{2}$, Ciurlionis M. ${ }^{2}$

${ }^{1}$ Mykolas Romeris University, Vilnius, Lithuania, ${ }^{2}$ Vilnius University, Vilnius, Lithuania

The care of seriously ill children at home requires competence and resources while providing the best possible support to patients near the end of life. Primary healthcare aims for an early integration of palliative care in order to improve quality of life and prevent suffering. Moreover, solicitude for patients and their relatives at homecare is one of the main aspects of the role of palliative care team. The objective of this review is to explore the barriers to successful homecare for children in Lithuania.

We conducted a multimethod analysis focused on healthcare professionals and families providing care for children with conditions that require palliative care. Legislation data and experience based opinions were gathered and analized to point out issues that burden home based palliative care for children.

The analysis made it possible to clarify the following results: unpreparedness of families to deliver palliative care; lack of professionals' role in palliative care limited by services that are provided only on working days; incomplete recognition of specific aspects that differentiate pediatric palliative care from adult palliative care; insufficient state reimbursments for medications as well as medical equipment. Moreover, it was also recognized by professionals and families that there is room for improvement to ensure the safe and effective transition of children patients from hospital to home.

Palliative care professionals and family caregivers describe children palliative care at home as person-centered ensuring high levels of access and quality. However, the implementation of far reaching palliative care and planning of sufficient professional support to patients and families are needed. It is essential to find a way to catalyze the active role of the family in the palliative care process as well as to help overcome interinstitutional barriers. Furthermore, the process of change can be facilitated by adapting laws to arrising healthcare novelties.

Abstract number: P02-358

Abstract type: Poster

Evaluation of a Hospice Based Ambulatory Blood Transfusion Service for Patients with Cancer

Steele T., Mills M., Richardson L., Taylor T., Nwosu A.C., Chapman L. Marie Curie Hospice Liverpool, Liverpool, United Kingdom

Background: Red blood cell (RBC) transfusion is frequently used in palliative medicine for symptomatic anaemia. Admission for this intervention is burdensome to patients and institutions, whilst home services are not always available or appropriate. Many patients report poor experiences of undergoing transfusions in the emergency department of acute hospitals.

Aim: To evaluate a newly established ambulatory RBC transfusion service based in a hospice day therapy unit.
Methods: The service specification was developed in conjunction with local acute and cancer hospitals. Transfusions during the first four months of the service were retrospectively reviewed. A tool was developed to assess patient experience and resource requirement.

Results: From $1^{\text {st }}$ June to $30^{\text {th }}$ September 2018, 12 transfusions were performed on 6 patients. Four patients were previously known to the hospice services. Four patients were transfused once, one 3 times, and one patient 5 times. Haematinic investigations were carried out in 2 patients, both suggested iron deficiency. Four patients were receiving concurrent chemotherapy. Haemoglobin values $(\mathrm{g} / \mathrm{L}$ ) prior to transfusion were $<70$ $n=1,70-80 n=5,80-90 n=4,90-100 n=1$. Symptoms were documented for $9(75 \%)$ referrals. The majority of patients received two units of RBCs (1 unit, $n=5 ; 2$ units, $n=7)$. Symptomatic response was documented for 8 (67\%) transfusions. Complications included bleeding from a wound in one case, and practical delays in two cases. Data collection is continuing. Conclusions: This work demonstrates the feasibility of an ambulatory blood transfusion service delivered in a hospice day therapy unit. Further optimisation is required to meet current national guidance on RBC transfusion for palliative care patients. Data collection is ongoing including assessment of patient experience, adherence to nationally approved guidelines, economic implications of the service and its potential role in broadening access to hospice services.

Abstract number: P02-359

Abstract type: Poster

The Reflection Room: Participatory Art Installations to Move us from Death-denying to Death-discussing

Holyoke P. ${ }^{1}$, Stephenson $B{ }^{2}$

${ }^{1}$ SE Research Centre, Markham, Canada, ${ }^{2}$ Religious Studies, Memorial University of Newfoundland, St. John's, Canada

Background: Advances in medicine, the rise of a funeral industry and the increased availability of hospitals have distanced people from the direct experience with death. As a result, thinking about dying and death is something we tend not to do and those who promote advance care planning would like us to do more. There are efforts like Death Cafés that encourage dialogue about dying but they are designed for people ready to discuss death.

Aim: The aim of this study was to investigate whether an intervention enabling recording and reading of personal experiences of dying and death could produce an increase in comfort in discussing and planning for death, and whether the intervention's design elements had an influence.

Methods: We used a participatory action research approach and installed versions of The Reflection Room in 37 public and healthcare settings across Canada. Each room had an open invitation to read other peoples' stories of dying and death and to leave a personal "reflection". We analyzed the stories thematically and by setting type, and conducted a survey of Room visitors to gauge changes in attitudes to and comfort with death. Results: In general, the intervention increased comfort with discussing death with families among some but not all visitors and resulted in some advance care planning. Sombre, calming installations encouraged less participation than brighter installations with stories framed and mounted as art pieces. Healthcare providers used the installations more for personal reflection than professional. Shared stories included regrets and gratitude, advice to others, and messages for the dead.

Conclusion: Providing carefully designed spaces for people to record publicly their experiences with dying, to take time to learn about others' experiences and to share stories for others to read appears to move people from death-denying to death-discussing. It also enabled processing of unresolved grief, and brief memorializing.

Study funding: SE Health

Abstract number: $\mathrm{P} 02-360$

Abstract type: Poster 
Integrating Palliative Care in Neurological ICU

Koh T., Poi C.H., Ong W., Yow F., Koh M., Gu C., Wong Y.-L., Tan H.L., Li L. Tan Tock Seng Hospital, Singapore, Singapore

Background: Studies have shown that there is a significant role in integrating palliative care into NICU care.

Aims: Our study aims to evaluate the outcome of early palliative care intervention in the setting of neurosurgical ICU in a tertiary hospital using a common screening tool.

Methods: This is a prospective study that identifies patients referred to Palliative care during their NICU admission. Demographics, progress and outcomes of patients collected.

The Palliative Care Team did regular rounds in the NICU and patients were referred according to a referral criteria. A total of $89 \mathrm{NICU}$ patients were referred to palliative care between January 2016 and December 2017.

Results: The most common reason for referral was extensive intraventricular haemorrhage (65.2\%-58/89), followed by consideration of mechanical ventilator withdrawal $(53.9 \%-48 / 89)$.

ICU mortality was high: $20 \%(20 / 89)$ survived the ICU admission; $23.6 \%(21 / 89)$ died in ICU, $53.9 \%(48 / 89)$ died in general ward. $71.9 \%$ (64/89) of patients passed away on comfort care status. $69.7 \%(62 / 89)$ patients with no meaningful prospect of recovery had ventilator withdrawal in accordance to patients/surrogate wishes.All patients had DNR code after palliative care involvement. During the post-ICU period, $4.5 \%$ (4/89) patients transited home with hospice homecare, $24.7 \%$ ( 22/89) patients to comfort care room- a single room specifically designed for families to spend quality time with the dying patient.60.7\% (54/89) patients had medical social worker involvement. The mean number of family conferences held in ICU was 5 .

Conclusion: NICU-Palliative care collaboration confers significant benefits to both patients and families, providing them with continuous support and engatement extending to post ICU period where providing good terminal care and coordination are essential. The synchronous work between ICU team and palliative team care helps to establish and continue inter-discplinary team based care both inside and outside ICU.

Abstract number: P02-361

Abstract type: Poster

Survey of Patient \& Caregiver Population Looking at Attitudes towards End of Life Care in Gibraltar

James B.M. ${ }^{1}$, McKenna G. ${ }^{2}$, Ayre $D .^{2}$

${ }^{1}$ Department of Medicine, St Bernard's Hospital, Gibraltar, Gibraltar, ${ }^{25}$ South Barracks Road, Cancer Relief, Gibraltar, Gibraltar

Background: End of life care in Gibraltar is evolving however little is known about the cultural influences and attitudes locally.

Aim: To look at attitudes towards end of life and palliative care among patients and caregivers in Gibraltar.

Design: A prospective study using a self-administered, anonymous questionnaire was designed based on previous work by the PRISMA group. Surveys were made available in the hospital and the Cancer Relief Centre and people were invited to complete the survey during a 2-week period in November 2015.

Results: 95 people completed the survey of which $31 \%$ were male and $69 \%$ female; $27 \%$ patients, $11 \%$ relatives and $62 \%$ hospital workers.

Life \& Symptom Priorities:

The majority of respondents ranked the most concerning symptoms at the end of life as being in pain (52.8\%), breathlessness (29.7\%), a burden $(18.7 \%)$ and being alone (11\%).

Preferred Place of death: $54 \%$ wanted to die at home and interestingly $37 \%$ chose a specialist palliative care unit (even though Gibraltar doesn't have one). The least preferred place to die was hospital $41 \%$ or nursing home $38 \%$.
Care Priorities: Only 5\% chose extending life as being the most important care priority, $53 \%$ chose improving quality of life and $40 \%$ felt both equally important.

Conclusions: The results mirror existing studies from European countries however there are differences reflecting the nature of the close-knit, small community (35000) where family ties and religious links are very important.

The $5 \%$ choosing to extend life is fundamental for health care policy makers to understand as funding and investment in services focused on treatment intensive options.

The development of palliative care services needs to be evidence based and specific to the local population. We aim to extend this study to an online population survey to capture a wider demographic of the community and use it to develop an End of Life Care Strategy for Gibraltar.

Abstract number: P02-362

Abstract type: Poster

Integrating Palliative Care and Intensive Care: Different

Concepts and Organizational Models Based on a Mixed-methods Study on Professionals' Perspectives

Martins Pereira S. ${ }^{1,2,3}$, Teixeira C.M. ${ }^{1,4,5}$, Carvalho A.S. ${ }^{1,2,3}$, Hernández-

Marrero P. ${ }^{1,2,3}$, Project InPalln: Integrating Palliative Care and Intensive Care

${ }^{1}$ Instituto de Bioética, Universidade Católica Portuguesa, Porto, Portugal, ${ }^{2}$ UNESCO Chair in Bioethics, Institute of Bioethics, Universidade Católica Portuguesa, Porto, Portugal, ${ }^{3}$ CEGE: Centro de Estudos em Gestão e Economia, Porto Católica Business School, Universidade Católica Portuguesa, Porto, Portugal, ${ }^{4}$ Hospital de Santo António, Centro Hospitalar do Porto, Porto, Portugal, ${ }^{5}$ ICBAS: Instituto de Ciências Biomédicas Dr. Abel Salazar, Universidade do Porto, Porto, Portugal

Background: The integration of palliative care (PC) in intensive care (IC) requires a careful consideration of concepts and organizational models. Aims: To understand professionals' perspectives about the integration of PC and IC; to assess what organizational models are preferred by these professionals.

Methods: Nationwide mixed-methods study, combining interviews and a questionnaire. Concepts and organizational models were explored during interviews. A questionnaire built de novo complemented this by assessing 8 organizational dimensions with an 11-point Likert scale. A combination of purposive and snowball sampling was applied until reaching theoretical saturation. 38 professionals working in Portuguese PC and IC units participated in this study. A thematic analysis was performed to the transcripts of interviews and descriptive analyses to quantitative data.

Results: Professionals had different concepts of integration. While intensivists considered the integration as a way of improving end-of-life care in IC units, PC professionals defined it as a way of promoting patientcentered care. In terms of organizational models, PC professionals defended a consultation model, introducing the role of a specialized PC team in articulation with the IC team. IC professionals preferred an education model, fostering their own competences in PC. Responses given to the questionnaire showed that the organizational dimensions most valued by both PC and IC professionals were patient-centered care and PC needs assessment in IC.

Conclusions: InPalln is the first research study addressing the integration of PC in IC in Portugal. The combination of research methods provides a greater understanding yield. Findings show differences in concepts and preferred organizational models for integration between IC and PC professionals. Tailored strategies are needed to foster the successful integration of PC in IC.

Acknowledgments: Fundação Grünenthal and Fundação Merck, Sharp and Dohme. 
Abstract number: P02-363

Abstract type: Poster

The Beneficial Impact of Schwarz Rounds for Staff: 3 Years' Experience at a Tertiary Referral Cancer Centre McNulty M., Droney J., Stevens A.-M., Halley A.

Royal Marsden NHS Foundation Trust, London, United Kingdom

Background: Schwartz Rounds (SR) aim to provide a structured forum for staff to discuss the psychosocial and emotional issues of work in healthcare. Patient safety is linked with staff wellbeing, with potential associations between burnout and medical mistakes, and possible impact on effective organisational response to change. These issues are particularly relevant to the NHS and other healthcare systems at this time.

Aims: This service evaluation sought to explore the relevance and impact of SRs for healthcare staff.

Methods: Retrospective analysis of feedback forms from staff who attended SRs from March 2015 to June 2018 at a tertiary referral cancer centre. Descriptive statistics were used to describe the attending staff and their agreement with statements about their experience. Thematic analysis of free text comments was conducted.

Results: 1091 feedback forms were analysed from 28 SRs with 3449 attendees (31.6\% response rate). 963 (88\%) agreed with the statement "Today's Round will help me work better with my colleagues". The main staff groups represented were: Nursing (36\%), AHP's (29\%) and administration (17\%). Doctors represented $7 \% .71 \%$ respondents disclosed if they had attended a SR before. Of those, $49 \%$ had been to between 1 and 5 SR, while $43.5 \%$ had never attended. $21 \%$ of respondents gave comments which were analysed for qualitative data. $81 \%$ positive, $3 \%$ negative, $4 \%$ neutral and $12 \%$ other comments were made. Keywords used by staff to describe the SR's emerged; insightful, reflection, interesting, inspiring, sharing, supportive. Poignant comments described relief from feelings of isolation and having been given "the courage to keep going".

Conclusion: Our evaluation of 3 years' experience demonstrates that SRs are highly acceptable to those staff who attend, as a means of interactive and supportive reflection. Qualitative data revealed that most staff described SRs in positive terms. Our evaluation supports evidence that SRs can be a source of support for staff.

Abstract number: P02-364

Abstract type: Poster

\section{A 3-year Follow-up of Palliative Care in a Rural Hospital in} Tanzania

Pöyhiä R.T. ${ }^{1,2}$, Jaatinen A. ${ }^{3}$, Mtega A. ${ }^{4}$

${ }^{1}$ Kauniala Hospital, Kauniainen, Finland, ${ }^{2}$ Oncology/Palliative Medicine, University of Turku, Turku, Finland, ${ }^{3}$ Huittinen Health, Huittinen,

Finland, "Palliative Care, llembula District Designated Hospital,

Ilembula, Tanzania, United Republic of

Aim: This study was carried out to assess how the PC recommendations have been implemented in llembula District Designated Hospital (IDDH), a rural hospital in Njombe Region in Tanzania.

Design, methods and approach taken: The performance of PC was assessed twice a year using standard questionnaires, in observations and in discussions. The availability of liquid morphine, the only strong opioid in the country was specifically monitored. The attitudes of the staff were studied using a standardized questionnaire delivered to the participants in the staff meetings once a year.

Results: IDDH had composed a very detailed plan of PC in 2012-2017. The PC team consisted of a co-ordinator, assistant co-ordinator, 2 doctors, 3 nurses, a chaplain, a social worker, a car driver, a treasurer and volunteers. The team cared patients both in the hospital, at homes and in weekly visits to 17 surrounding villages, delivered medications for free of charge and provided psychosocial support. A total of 17135 patients were registered in PC in 2014, 12718 in 2015, 15446 in 2016 and 4068 in 2017. The most patients had HIV. Morphine was poorly available. Two foreign NGOs provided continuous financial and educational support. 27-33 staff members responded to the questionnaire in 2014 and 2017, respectively. Staff learned to deal with dying, but still a strong need for support remained. The staff reported of high satisfaction with PC functions.

Conclusion: Multiprofessional palliative care can be extended also to a rural hospital in a very poor area such as Ilembula. There is still need for help from international organizations in education because of the lack of professionals and teacher in PC.

Abstract number: P02-365

Abstract type: Poster

Impact, Challenges and Limits of Inpatient Palliative Care Consultations - Perspectives of Requesting and Conducting Physicians

Coym A., Ullrich A., Kanitz A., Puls N., Blum D., Bokemeyer C., Oechsle K. Universitätsklinikum Hamburg-Eppendorf, Hamburg, Germany

Background: Inpatient palliative care consultations (IPCC) are implemented increasingly in routine care in German hospitals. However challenges, limitations and impact are rarely analyzed.

Aims: Qualitative analysis of the perspectives of physicians who request IPCC regularly and of palliative care specialists performing IPCC.

Methods: Semi-structured interviews with 10 palliative care specialists and 9 other physicians. Data analysis was performed using content analysis by Mayring.

Results: IPCC seem to improve patients' symptom burden and discharge management as well as enhance coping in patients and family caregivers. IPCC transfers knowledge and competences to other medical teams, which leads to psychological relief. Challenges in implementing IPCC emerge especially when physicians feel that they themselves have the competence to deal with symptoms/problems (overconfidence) or are in doubt of the benefit. In contrast some physicians are worried about not having managed a palliative situation satisfactory and are in fear of losing credibility when another team is included. Some of these aspects also apply to patients and family caregivers, so that they refuse IPCC. Another factor is lack of time: considering/requesting IPCC can be a problem. IPCC can be difficult in realization due to lack of an adequate setting, or meeting a patient who has not been informed about the IPCC. Limited time and staff capacities also lead to difficulties. Implementation of recommendations can be challenging due to the ward staff who might not agree with the proposed approach, not having time to implement it, or due to patient's refusal. Regular wards also have less multidisciplinarity, less expertise in palliative care and do often not collaborate with palliative home care teams.

Conclusion: IPCC show various positive effects in supporting physicians and patients, but are also limited due to structural problems, lack of knowledge, insecurity, and skepticism by the requesting physicians.

Abstract number: P02-366

Abstract type: Poster

What Are the Opportunities for Cancer Care and Related Research in Primary Care? A Qualitative Study of the Views of Patients, Carers and Practice Staff

Clark J. ${ }^{1}$, Amoakwa E. ${ }^{2}$, Blenkinsopp J. ${ }^{3}$, Reedy F. ${ }^{4}$, Johnson M. ${ }^{1}$ ${ }^{1}$ Wolfson Palliative Care Research Centre, Hull York Medical School, Hull, United Kingdom, ${ }^{2}$ UCEN Manchester, Manchester, United Kingdom, ${ }^{3}$ Newcastle Business School, Northumbria University, Newcastle, United Kingdom, ${ }^{4}$ School of Health and Social Work, University of Hull, Hull, United Kingdom 
Background: Research to identify the role of primary care in cancer care is important. However, trials in primary care are difficult.

Aims: To understand how patients, families and primary care clinicians view their role in cancer care and opportunities for cancer primary care research.

Methods: Qualitative study embedded within a feasibility cluster randomised controlled trial of a primary care based needs assessment intervention. The Supportive Cancer Needs Survey (SCNS) was the main outcome. In-depth interviews with trial participants (patients, carers) and focus groups with primary care staff explored views on cancer care in primary care and experiences of participation in the trial. Data were recorded, transcribed verbatim and analysed thematically by three independent researchers.

Results: We interviewed 15 cancer patients, 3 carers and conducted 4 focus groups $(n=11)$. Three themes were generated:

1) Satisfaction with current care,

2) Key time-points for improved cancer care,

3) Information and research.

Satisfaction with current care amongst patients was high in spite of reported high levels of unmet needs on the SCNS indicating low expectation. Most patients undergoing cancer treatment were unsure when they should access primary care. Patients and carers highly valued proactive contact from their practice following diagnosis although this was perceived as unusual. Post-oncology discharge was a key time-point for information needs and support by patients and clinicians. Patients were reluctant to consider palliative care in spite of palliative care needs - indicating low understanding. Some felt therapeutic benefit from completing study measures. In spite of positive attitudes to cancer research by consented participants, some had poor understanding of study procedures.

Conclusion: Patients are satisfied with cancer primary care despite unmet needs. Palliative care is poorly understood by many who may benefit. Research participation may benefit some patients.

Abstract number: P02-367

Abstract type: Poster

Timing of Referral to Specialist Palliative Care in a Teaching Hospital in Ghana

Owusu-Ansah M. ${ }^{1,2,3}$, Opoku R. ${ }^{4,5}$, Spangenberg K. ${ }^{1,6}$, Dzaka D. ${ }^{7}$, Owusu Appiah M. ${ }^{1}$, Anyane G. ${ }^{3}$, Oncology

${ }^{1}$ Family Medicine, Komfo Anokye Teaching Hospital, Kumasi, Ghana, 2Palliative Care, Ghana College of Physicians and Surgeons, Accra, Ghana, ${ }^{3}$ Palliative Care, Ghana College of Nurses and Midwifery, Accra, Ghana, ${ }^{4}$ Medicine, Komfo Anokye Teaching Hospital, Kumasi, Ghana, ${ }^{5}$ Palliate care, Ghana College of Nurses and Midwifery, Kumasi, Ghana, ${ }^{6}$ Family Medicine, Ghana College of Physicians and Surgeons, Kumasi, Ghana, ${ }^{7}$ Family Medicine/Oncology, Komfo Anokye Teaching Hospital, Kumasi, Ghana

Introduction: The over-arching objective of palliative care is to improve the patient's and family's experience of chronic illness and their quality of life. An essential pre-requisite is a timely referral to specialist palliative care. In low-and middle-income countries where the burden of chronic diseases continues to rise, a relatively low number of patients are known to receive palliative care but the timing of referral has been inadequately explored.

Aim: The aim of this study was to determine the timing of referral for specialist palliative care in a teaching hospital in Ghana.

Material and methods: A retrospective cohort design was used. Data was obtained from the registry of the palliative care team of Komfo Anokye Teaching Hospital (KATH). Records that included dates of first referral and date of death were considered suitable for inclusion in the study. Of the 78 entries made between August 2016 and September 2018 , 39 of them met this criterion. SPSS Version 20 was used to analyze the data.

Results: Majority were female and were less than 60years of age. Ninety percent had advanced malignancy and for $72 \%$ no specific reason for palliative care was stated apart from the expression 'for palliative care'. Majority (62\%) died after spending less than four weeks under the care of the palliative care team and $69 \%$ had only one palliative care consultation before death.

Conclusion: Patients were referred late for palliative care services at KATH, thus limiting the benefits of improvement in quality of end-of-life care. Physician education and sensitization is required to improve referral practices.

\section{Abstract number: P02-368 \\ Abstract type: Poster}

The Concept of a "Palliative Emergency Bed" in Cologne, Germany - A Retrospective 2-year Analysis

Joist T.M. ${ }^{1}$, Wagner S. ${ }^{1}$, Wille-Friederichs A. ${ }^{1}$, Buscham H. ${ }^{2}$, Balboni C. ${ }^{2}$, Schlesinger $A .^{2}$

${ }^{1}$ General Medicine, Cologne, Cologne, Germany, ${ }^{2}$ Internal Medicine, Marien-Hospital, Cologne, Germany

With the development of specialized ambulatory palliative care structures (SAPV) more and more patients in Germany can die at home. Within the process of dying at home the caring social structures e.g. family are often overwhelmed by this task. And within a couple of hours the care at home collapses.

This patients have to be transferred to a hospital, although they are terminally ill.

Due to missing structures in the hospital sector palliative care patients respectively dying patients are misdiagnosed and misled by regular hospital structures. In our case we developed a working base between an ambulatory specialized palliative care net (Palliative Care Team SAPV Cologne) and a non-university hospital (St Marien Hospital, Köln) without a palliative care unit but palliative care expertise in the city of cologne that led to the concept of the "palliative emergency bed”.

The mean idea is that a patient can be transferred to a hospital at any time within a very short time without undergoing unnecessary diagnostic and routine procedures. The patient and the family can benefit of a professional medical structure of a hospital embedded in a professional palliative structure.

Our presented data shows a range of 24 months retrospectively. From the time between 1.1.2016 to 31.12.2017 47 patients were led in the "palliative care bed". Within the following 5 days 22 patients died. Of the left 25 patients 15 were transferred into a hospice. 4 people were transferred at home. The other people were led into different hospitals or a nursing home.

A cooperation between a specialized ambulatory palliative care team and a hospital with palliative expertise is a useful and effective combination in handling moribund patients and their overwhelmed families in a severe crisis. Effective palliative structures are necessary in dealing with moribund patients in an ambulatory sector and should be increased.

Abstract number: P02-369

Abstract type: Poster

The Establishment of 20 Danish Hospices during

25 Years - What's the Story?

Timm H., Kjær T., Graven V.

REHPA, University of Southern Denmark, Nyborg, Denmark

Background: Since 1992 twenty hospices have been established in Denmark. The first hospices were self-financed private institutions 
founded in religious organizations, which is a unique status within the public Danish health care system in a highly secularized society. Since 2004 , all hospices have to have an agreement with the regional authorities to have public support and to perform specialized palliative care. In 2018 Denmark had 250 hospice beds, but as this was not due to public health care planning, what actually caused the establishment of those hospices?

Aim: To report first phase findings on the history of the establishment of the Danish hospices at a micro- and meso-level; what is the story behind the establishment of each hospice and are there some common circumstances that explain why these 20 hospices were founded?

Methods: Methods included document analysis and informal interviews with key informants. Yearbooks, homepages and other documents concerning every Danish hospice were read and content analyzed to uncover the story behind the establishment of each hospice. Former and actual leaders, chairpersons etc. were interviewed when documents did not give a full picture.

Results: Main factors at a micro- and meso-level in explaining establishment of the hospices are;

1) lay peoples' negotiation with local politicians and authorities

2) economic resources and

3) health professionals' arguments.

As well as these factors, the story of the establishment of each hospice seems to be unique. This will be illustrated by three hospice case studies.

Conclusion: At the micro level the story of each Danish hospice is unique. At the meso-level, looking across the stories of the 20 hospices, there are some common factors mainly concerning NGO pressure and funding. Explanations related to a macro-level should be further explored.

Abstract number: P02-370

Abstract type: Poster

\author{
Evaluation of Outpatient Palliative Care in Istrian County - \\ Croatia \\ Kovacovic B. ${ }^{1}$, Franivovic Markovic J. ${ }^{2}$, Ivancic A. ${ }^{1}$, Katacic ${ }^{3}{ }^{3}$, Grozic \\ Zivolic S. ${ }^{3}$ \\ ${ }^{1}$ Palliative Care, Istrian Primary Care Health Services, Pula, Croatia, \\ ${ }^{2}$ Palliative Care, Salus Policlinic, Pula, Croatia, ${ }^{3} \mathrm{Health}$ and Social Care, \\ Istrian County, Pula, Croatia
}

Organized palliative care (PC) began in Istria with mobile palliative teams (MPT) before a national strategy was even formulated. We chose an model of service that would help a family physician provide PC. The original MPT (1 full-time Oncologist, 2 nurses, and 12 part time medical and social workers) had multiple roles that included providing services, educating (healthcare providers, volunteers, social workers, and general public), providing palliative interventions, rented medical equipment, and participating in the regional PC system. From 2012 to 2016, MPT helped 2251 patients and provided 19,044 services and interventions in the homes of patients throughout the county of Istria, providing an adequate number of patients and services for analyzation.

Aim: To evaluate the work of the MPT on the county level. We have done qualitative analyses of the care offered in a four-year period.

Strengths: County provision of regional organization, coordination, and co-financing of the PC system. Easier entrance into the Croatian national insurance, resulting in the inclusion of medical professionals and nonmedical care. General PC taught in the educational systems. Rented medical equipment for patients.

Weaknesses: Except for palliative medicine, many underdeveloped PC segments. Poor recognition of the palliative patient by general practitioners. Confusion regarding "extended" vs "palliative" care. Nonparticipation in EU projects. No social workers are involved exclusively in PC.
Threats: PC is not considered an attractive medical field. Little incentive for education and clinical practice. Fluctuation of employees.

Opportunities: Increased public awareness, development of PC on the national level, new financial partners for hospice, EU funding, and participation in international academic conferences.

Conclusion: The organization and functioning of PC system is a dynamic process that needs continuous evaluation and development on the regional and national levels.

Abstract number: P02-371

Abstract type: Poster

Who are the Key Workers Responsible for Care Coordination in Switzerland? A Nationwide, Mixed-method Approach Schweighoffer R. 1,2, Reeves E. 1,2, Degen E. 3, Liebig B. 2,3

${ }^{1}$ Applied Psychology, FHNW University of Applied Sciences, Olten, Switzerland, ${ }^{2}$ University of Basel, Basel, Switzerland, ${ }^{3}$ FHNW University of Applied Sciences, Olten, Switzerland

Good coordination of health care services is particularly important in the context of palliative care (PC) services. However, previous literature has revealed that there is a fragmented approach to PC provision across Switzerland. The present study therefore seeks to address this gap, with particular attention to identifying the key workers responsible for coordination in PC. Qualitative interviews were therefore conducted with health care providers in PC in four cantons, namely Vaud, Ticino, BaselCity and Luzern $(\mathrm{N}=56)$. Expert interviews have been analysed using framework analysis. First results indicate that there are inconsistent approaches to coordination and that there is ambiguity amongst professionals regarding who the key workers for coordination in PC are. This might be due to a lack of formalized approach to coordination. Regarding the quantitative part, a questionnaire was designed for both primary and specialized palliative care bases $(\mathrm{N}=800)$. The survey has been distributed via email and sent to professionals from the national health care registry in German, French and Italian. The questionnaire is based on Bainbridge's conceptual framework for healthcare services, while items were also drawn from subsections of the Job Satisfaction Survey. Response data gathered from the survey was being analyzed statistically for objective measures about which actors see themselves as "Key workers" and for assessing the subjective value of Case Managers (CM) among providers. Preliminary results indicate, that many different professionals view themselves as «key workers» and "coordinators» in PC, while, in some cases, a pre-destined CM or Coordination Office is able to facilitate procedures in PC. Findings will be of interest to health care practitioners, policy makers and researchers involved in the evolution of coordinative practice, as they imply a need for standardizing approaches to coordination in Switzerland in order to improve coordination of PC services.

Abstract number: P02-372

Abstract type: Poster

"PLAN B" - The Impact of a Structured Palliative Care Documentation and Communication Approach of End-of-Life Decisions on Nursing Activities in a Tertiary Hospital Fliedner M., Felber S., Hagemann M., Eychmueller S.

University Center for Palliative Care, University Hospital Bern, Bern, Switzerland

Aim: Based on an external audit we found relevant room for improvement regarding documentation and communication of treatment adaptations and end-of-life (EOL) decisions. The Palliative Care (PC) center initiated the interprofessional and interdisciplinary project "PLAN B" (Prognosis, Life domain inventory, Advance care planning, Network documentation) to 1) clarify processes and responsibilities regarding clinically relevant EOL decisions, 2) ensure availability of these decisions $24 \mathrm{~h} / 7 \mathrm{~d}$ to all healthcare professionals, 3) coordinating care with external 
care providers. Little is known about the impact on patient related nursing time (direct care) and time devoted to discharge planning (indirect care).

Approach: For a 6-month period, the project under the lead of the PC center started in 5/18 in oncology/PC, neurology, internal medicine, thoracic surgery and pneumology inpatient units respectively. Physicians and nurses were trained in identifying patients (complex PC condition being discharged supported by a specialist PC nursing team / family doctor), documenting EOL decisions and coordinating care after discharge. Nursing activities were measured with a Swiss tool $\left(\operatorname{tacs}^{\circledR}\right)$ to document care activities before and after PC involvement in relation to patient data.

Results: So far, 48 patients were allocated to "PLAN B". Most patients (54\%) were discharged from the PC unit followed by internal medicine. Interprofessional discussions contributed to a better documentation of EOL decisions. Based on tacs ${ }^{\circledR}$, a longitudinal analysis of the impact of nursing showed a clear shift from direct to indirect care activities.

Conclusion / lessons learned: Thorough discussions of treatment adjustments and documentation of EOL decision are relevant for nurses since they often are the first at the patients' side to carry out these decisions. Triggered by "PLAN B", the focus of nursing care activities changed from a diagnosis-driven care toward a pragmatic anticipatory preparation of care.

Abstract number: P02-373

Abstract type: Poster

Recommendations for the Delivery of Advance Care Planning for Cancer Patients in Taiwan: Result of a Transparent Expert Consultation

Lin C.-P. ${ }^{1}$, Chen P.-J. ${ }^{2,3}$, Yang C.-H. ${ }^{4}$, Hou M.-F. ${ }^{5}$, Koffman J. ${ }^{1}$, Evans C.J. ${ }^{1,6}$, Harding $R^{1}$

${ }^{1}$ Cicely Saunders Institute of Palliative Care, Policy and

Rehabilitation, King's College London, London, United Kingdom, ${ }^{2}$ Department of Family Medicine, Kaohsiung Medical University Hospital, Kaohsiung, Taiwan, Republic of China, ${ }^{3}$ Division of Geriatrics and Gerontology, Kaohsiung Medical University Hospital, Kaohsiung, Taiwan, Republic of China, ${ }^{4}$ Department of Family medicine, Kaohsiung Medical University Hospital, Kaohsiung Medical University, Kaohsiung City, Taiwan, Republic of China, ${ }^{5}$ Department of Surgery, Kaohsiung Medical University Hospital, Kaohsiung, Taiwan, Republic of China, ${ }^{6}$ Sussex Community NHS Foundation Trust, Sussex, United Kingdom

Background: Implementation of advance care planning (ACP) is a priority in non-Western countries with rapidly ageing populations and increasing cancer prevalence, such as Taiwan. Most work on ACP is conducted in Western countries. This limits transferability.

Aim: To generate evidence-based clinical recommendations on ACP for people with cancer in Taiwan.

Methods: Transparent expert consultation comprising modified nominal group technique incorporating focus group discussion followed by an online consensus survey (scale 1-9, low to high agreement). Participants comprised experts representing palliative care, law and academia in Taiwan. Descriptive data analysis and collation of narrative discussion were conducted.

Results: 17 participants attended the consultation and generated 45 recommendations, rated in the online survey by 37 participants. 18 recommendations had strong agreement (median, $\mathrm{M} \geqslant 8$ ) and high level of consensus (interquartile range, IQR $\leqslant 2$ ). Experts agreed top 5 recommendations:

1) the decision makers in ACP must be the individuals themselves not families ( $M=8$, IQR8-9);

2) understanding of disease prognosis and preferred end-of-life (EOL) care should be discussed through ACP ( $M=9$, IQR8-9);
3) care should be provided by a multidisciplinary professional team, and physicians were considered as the leader ( $M=9$, IQR 8-9);

4) the importance of training programmes for the public to improve death literacy, and healthcare staff to enhance skills in EOL care and communication ( $M=, 9$ IQR7.25-9);

5) organisation 'buy-in' and policy support is essential to change practice ( $M=9$, IQR7-9).

Conclusion: ACP is suggested to encompass multi-professional care, which is led by physicians, to meet individual's preferences. Key requirements to implement ACP are raising public awareness and staff training on enhanced communication skills, combined with, institutional and policy support for change in practice. Future research is needed to test the optimal ACP model.

\section{Abstract number: P02-374}

Abstract type: Poster

How Can We Achieve Person-centred Care for People Living with HIVAIDS? A Qualitative Study of Healthcare Professionals and Patients in Ghana

Abboah-Offei M. ${ }^{1}$, Bristowe K. ${ }^{1}$, Koffman J. ${ }^{1}$, Vanderpuye - Donton N.A. ${ }^{2}$, Ansa G. ${ }^{3}$, Cottrell L. ${ }^{1}$, Etkind S. ${ }^{1}$, Abas M..$^{4}$, Higginson I. ${ }^{1}$, Harding R. ${ }^{1}$ ${ }^{1}$ Cicely Saunders Institute of Palliative Care, Policy \& Rehabilitation, King's College London, London, United Kingdom, ${ }^{2}$ West African AIDS Foundation, Accra, Ghana, ${ }^{3}$ University of Ghana Hospital, Accra, Ghana, ${ }^{4}$ Health Services \& Population Research, King's College London, London, United Kingdom

Background: People living with HIV (PLWHIV) experience problems and concerns that require holistic assessment and person-centred care. Person-centred approach will facilitate active involvement of PLWHIV in making decisions about their care.

Aim: To explore PLWHIV and healthcare professionals (HCP) views on what constitutes person-centred care, including which care structures, processes and outcomes matter to PLWHIV, in order to develop a framework of person-centred care to improve their care quality, retention and outcomes.

Methods: PLWHIV and HCP in a community setting were recruited for semi-structured interviews. Purposive sampling was used based on PLWHIV $\geqslant 20 y r s$, diagnosed $\geqslant 6$ months, with cognitive ability to consent; $\mathrm{HCP}$ in HIV care $\geqslant 6$ months. Sampling continued until thematic saturation was achieved when no new themes emerged that were relevant to the aims of this study. A deductive six-phase thematic analysis was undertaken to understand what constitutes person-centred care for PLWHIV.

Results: Interviews were undertaken with 24 PLWHIV (median age 32.5, $50 \%$ female) and 15 HCP (median age 28, 53\% female). PLWHIV discussed distance travelled, burden of disclosure, stigma of having a sign up saying 'HIV clinic', non-involvement in care and care not delivering what matters to them. HCP discussed their lack of skills to undertake holistic assessment and expressed uncertainty about how to practice person-centred care. These views informed a person-centred care framework involving training HCP on person-centred communication, holistic assessment of PLWHIV needs in the domains of physical, psychological, social and spiritual wellbeing, with collaborative care planning and delivery.

Conclusion: These results revealed aspects of care that matter to PLWHIV, barriers to accessing standard care services and the need to train and mentor HCP to be person-centred. We developed a framework of person-centred care based on the results to improve care quality and wellbeing for PLWHIV.

Abstract number: P02-375

Abstract type: Poster 
Where Do I Go Now? A Mixed-method Systematic Review into the Availability, Effectiveness, Economics, Experience, and Policy Context of Respite Care for Young Adults with Complex Healthcare Needs Knighting K. ${ }^{1}$, Pilkington G. ${ }^{1}$, Bray L. ${ }^{1}$, Downing J. ${ }^{2}$, Jack B.A. ${ }^{1}$, Maden M. ${ }^{3}$, Mateus C. ${ }^{4}$, Noyes J. ${ }^{5}, O^{\prime}$ Brien M. R. $^{1}$, Roe B. ${ }^{1}$, Spencer ${ }^{1}{ }^{1}$ ${ }^{1}$ Faculty of Health and Social Care, Edge Hill University, Ormskirk, United Kingdom, 'International Children's Palliative Care Network, Assagay, South Africa, ${ }^{3}$ Liverpool Reviews and Implementation Group, University of Liverpool, Liverpool, United Kingdom, ${ }^{4}$ Faculty of Health and Medicine, Lancaster University, Lancaster, United Kingdom, ${ }^{5}$ School of Social Sciences, Bangor University, Bangor, United Kingdom

Background: The number of children with complex healthcare needs ( $\mathrm{CHCN})$ due to a life-limiting condition or complex physical disability surviving into adulthood is growing internationally; estimated to be $>55,000$ and 100,000 respectively in the UK. Respite care and short breaks are an essential component of care for children with $\mathrm{CHCN}$ but following transition to adult services provision is often inadequate and inappropriate for young adults, and described as 'like falling off a cliff'. Poor continuity of care significantly impacts on the health and wellbeing of families, and can shorten lives.

Aim: To identify, characterise and evaluate respite care provision for young adults with $\mathrm{CHCN}$ (18-40 years); develop a knowledge map of service types and a conceptual framework.

Methods: A 2-stage mixed-methods systematic review to identify and select evidence: electronic databases and grey literature searched from 2002-current; evidence selected based on a priori inclusion criteria by two reviewers; data extraction into bespoke forms; and data synthesised according to service type and evidence stream (effectiveness, economics, experience and attitudes, policy and guidelines) using appropriate methods. An overarching synthesis will inform a conceptual framework describing the circumstances and contexts which affect how service types work. Advisory groups of young adults and family members, alongside professionals with service commissioning and delivery expertise have guided the project.

Results: To date we have identified nine service types across five categories: planned residential, day care, home-based care, emergency care, and holidays. We will present the full knowledge map, with a summary of the identified evidence including gaps for each evidence stream and service type.

Conclusions: The review will add to shared learning of conducting complex evidence synthesis, and findings will inform future research agendas and optimal service development for this growing population.

Abstract number: P02-376

Abstract type: Poster

Optimising the Management of Patients with Cancer Pain: Development and Evaluation of the Rapid Access Multidisciplinary Palliative Assessment and RadioTherapy (RAMPART) Clinic

Jenks A., Fenton P., Brooks C., Clode L., Driver I., Carol D.

University Hospital Southampton NHS Foundation Trust, Southampton, United Kingdom

Background: Patients with cancer-induced bone pain often wait weeks to receive palliative radiotherapy treatment and to be assessed by specialist palliative care and allied health services. While waiting, they experience debilitating physical problems and psychological distress. This paper reports on the development and evaluation of RAMPART, a onestop multidisciplinary clinic at University Hospital Southampton.

Methods: The clinic model involves a single visit and combines assessment by palliative medicine, clinical oncology and allied health professionals, with the planning and delivery of palliative radiotherapy. The intervention also involves signposting, onward referrals and supported self-management of physical, psychological and social concerns. A patient satisfaction questionnaire and Macmillan's Holistic Needs Assessment are performed on the clinic day and repeated one month later. Open response questions are asked on the day and at 1 month. Results: Overall, $87 \%$ of patients were very satisfied and $13 \%$ were satisfied. Patients' global concern score decreased by 1.9 points, mean score 7.1 (range 4-10) on clinic day to 5.2 (range 2-8) at 1 month. There was a reduction in pain score by 2 points, mean score 6.8 (range 3-10) on clinic day to 4.8 (range 0-8) at 1 month. The RAMPART clinic model successfully reduced the median time from referral to radiotherapy from 22 days in the comparator cohort to 8 days in the RAMPART cohort. Qualitative data findings are that patients felt supported, enlightened, informed and valued by the comprehensive nature of the assessment. Patients felt their symptoms and quality of life had improved.

Conclusions: Implementing a multidisciplinary palliative radiotherapy clinic is feasible, valued by patients and effective in reducing pain, other patient concerns and time from referral to treatment. This model helps to bridge the gap between hospital and community services and may be transferrable to other areas.

Abstract number: P02-377

Abstract type: Poster

High Technology, High Personality: Establishment of a Palliative Care Consult Service in an University Hospital Setting

Engel P.T. ${ }^{1}$, Görlich D. ${ }^{2}$, Lenz P. ${ }^{1}$

${ }^{1}$ Department of Palliative Care, University Medicine Muenster, Muenster, Germany, ${ }^{2}$ Institute of Biostatistics and Clinical Research, University of Muenster, Muenster, Germany

Background and study aims: Acute health services still lack specialized palliative care. We conducted this study to provide guidance for the establishment of a palliative care consult service (PCCS).

Patients and methods: The study included patients at the University Hospital of Muenster with advanced life-limiting diseases identified to be in need of palliative care. It is conceived as a retrospective singlecenter study analyzing the first years of building up a PCCS from 05/01/2015 to $05 / 31 / 2018$.

Results: In this period, all departments of Muenster University Hospital requested the PCCS. We found increasing referral rates from 20 to 80 patients per month leading to a total of 2362 patients at the end of the study period. Among the departments internal medicine $(27.6 \%)$, radiotherapy $(17,6 \%)$ and gynecology $(15.7 \%)$ showed the highest numbers of referrals. In the first year patients most frequently reported fatigue, reduced mood, loss of appetite, constipation, pain and insomnia. The symptom pain was most frequently localized in the stomach $(20.4 \%)$, at the back $(17.1 \%)$ or in the head and neck area (14.9\%).

Despite the recommendation to introduce or modify painkillers for 142 inpatients (28.9\% of all inpatients), only in $57.0 \%$ of these cases it was directly taken into effect by the respective department. Similarities could be observed with other reported symptoms, where rates of around $50 \%$ of PCCS' recommendations had been realized.

Conclusion: The establishment of a PCCS includes building up a team, which should be able to deal with the most frequent symptoms in terminally ill patients this study reported about. In order to facilitate the interaction of PCCS with other departments, it is advisable to emphasize the role and possibilities of this service both in regular ward rounds and individual staff contacts. As well, the implementation of PCCS' recommendations could be monitored.

Abstract number: P02-378

Abstract type: Poster

Scattergun to Strategy - Our Approach to Dementia

Brady J. 
North London Hospice, London, United Kingdom Presenting author email address: jbrady@northlondonhospice.co.uk

The number of people living and dying with dementia is rising. Our local population has a high prevalence of dementia due to demographics and high number of care homes. Initially we recognised dementia as a priority area for improvement and our enthusiasm led to a scattergun approach of ideas and actions. However, we had not taken stock of local needs and considered our role as specialists. We therefore decided to formalise our aims and develop a hospice strategy.

We approached this by mapping ourselves against the Hospice UK Dementia Enabled Hospice Care Checklist to ascertain what we had achieved and where there were gaps.

Our strategy was firstly, to develop, educate and support staff and volunteers in dementia care - achieved through a 3 level training programme from dementia friends mandatory for all staff and volunteers to internal and external training for generalist and specialist staff. We introduced a pain assessment tool and 1 page patient profile to support individualised care. Secondly, to scope and recommend models of care for the organisation - achieved by reviewing and refining our data collection to ensure accurate information of need. Then collating information about models of care so we are now ready to apply for grants to support implementing best practice models. We conducted audits of our buildings to establish then ensure they were dementia friendly.

Finally, to build collaborative relationships within our community by participating in Dementia Action Alliances, meeting and understanding our local dementia services and attending the Hospice UK Community of Practice to facilitate networking and benchmark with progress throughout the UK.

By understanding local need and existing services we have identified gaps. We considered our strengths in palliative care and what we could contribute through direct care but also via collaboration and partnership models of care. We provided leadership locally and nationally via NICE to drive change.

\section{Abstract number: P02-379 \\ Abstract type: Poster}

The 'Palliative Care Ambulance': Patient and Carer Perspectives of a Paramedic Service

Collier A. ${ }^{1,2}$, Jeffs $C^{3}{ }^{3}$, Houthuysen P. $^{3}$, Avery A. ${ }^{3}$, Dadich A. ${ }^{4}$, Noble A. ${ }^{5}$, Crawford $G^{3}$

${ }^{1}$ School of Nursing, University of Auckland, Auckland, New Zealand, ${ }^{2}$ School of Health Sciences, University of Tasmania, Hobart, Australia, ${ }^{3}$ North Adelaide Palliative Care Service, North Adelaide Local Health Network, Adelaide, Australia, ${ }^{4}$ School of Business, Western Sydney University, Sydney, Australia, ${ }^{5}$ South Australia Ambulance Service, Government of South Australia, Adelaide, Australia

Background: The need for home-based palliative care is growing at the same time as health systems face increased complexity and funding constraints. As such, ambulance services are increasingly tasked with providing 24-hour care, and potentially averting unnecessary hospital admissions. However, there is a dearth of research focused on their role in palliative care. Most studies to date have focused on issues at the very end of life and/or withholding resuscitation. The wider role of paramedics is yet to be examined to determine their value, particularly for patients and carers.

Aim: Explore patient and carer experiences of home-based palliative care delivered by trained paramedics.

Methods: From January to August 2018, 17 patients and carers participated in semi-structured interviews to explore their experiences with, and perspectives of the palliative care delivered by paramedics. Interviews were recorded and transcribed verbatim. Data were analysed within a social constructionist framework using Braun and Clarke's thematic analysis.
Results: Participants valued the palliative care delivered by trained paramedics. Notably, they appreciated their promptness, their compassion, their expertise in symptom management, and the ways they coordinated their efforts with palliative care specialists and other team members. Furthermore, carers reported feeling more confident in their capacity to support the patient, cognisant that timely assistance was always available.

Conclusion: Despite the importance of home-based palliative care, 24-hour care is not always available given resource constraints. This study suggests that ambulance services represent a helpful complement to specialist palliative care services. They enabled patients and carers to receive what they require, when they require it. Further research is thus required to determine best practice models of ambulance home care that can accommodate the changing needs and preferences of patients and their carers.

Russell B. ${ }^{1,2,3}$, Sundararajan V..$^{3,4,5}$, Vogrin S. ${ }^{4}$, Collins A. ${ }^{1}$, Le B..$^{6,7}$, Brand C. ${ }^{8,9}$, Hudson P. ${ }^{3,10,11}$, Philip J.1,7

${ }^{1}$ University of Melbourne and St Vincent's Hospital Melbourne, VCCC Palliative Medicine Research Group, Department of Medicine, Fitzroy, Australia, ${ }^{2}$ Department of Palliative Care, St Vincent's Hospital Melbourne, Melbourne, Australia, ${ }^{3}$ St Vincent's Hospital Melbourne, Centre for Palliative Care, Melbourne, Australia, ${ }^{4}$ Department of Medicine, University of Melbourne and St Vincent's Hospital Melbourne, Melbourne, Australia, ${ }^{5}$ Department of Public Health, La Trobe University, Melbourne, Australia, ${ }^{6}$ Department of Palliative Care, Royal Melbourne Hospital, Melbourne, Australia, ${ }^{7}$ Victorian Comprehensive Cancer Centre, Melbourne, Australia, ${ }^{8}$ University of Melbourne and Melbourne Health, Melbourne Epicentre, Melbourne, Australia, ${ }^{9}$ Monash University, Department of Preventive Medicine, Melbourne, Australia, ${ }^{10}$ Vrije University, Brussels, Belgium, ${ }^{11}$ University of Melbourne, School of Health Sciences, Melbourne, Australia

Aims: Palliative care services are challenged with a growing diverse workload as patients are referred with a range of goals and needs at varied points along their disease trajectory. In the context of finite resources, decisions must be made daily about whose needs are more urgent.

To address the need for an evidence-based, transparent and equitable method by which to manage waiting lists, the Responding to Urgency of Need in Palliative Care (RUN-PC) Triage Tool was developed through a pioneering body of research. RUN-PC is a 7-item tool covering physical, psychosocial and caregiver domains, scored accordingly. This study aimed to investigate the feasibility of implementing the RUN-PC Triage Tool in clinical practice.

Design: A prospective multi-site stepped-wedge mixed methods pilot was conducted in Victoria, Australia. Baseline practice audits were conducted and triangulated with qualitative data collection to characterise pre-existing triage practices and workflow. The RUN-PC triage tool and accompanying user manual was then implemented via a site initiation workshop at each service followed by a one-month period of intensive support and education, with a further audit and qualitative data collection at the conclusion of this period and again after a two-month sustainability phase.

Results: Ten palliative care services participated across metropolitan Melbourne including community, inpatient and hospital liaison teams. User confidence and satisfaction was acceptable, and instances of noncompliance or tool deviation were informative. Site initiation processes and education materials were adjusted iteratively to reflect learnings. Impact on relationships with referrers appeared positive likely due to increased transparency.

Conclusion: The results of this study have demonstrated the feasibility of the RUN-PC triage tool implementation model to ensure sustainable uptake of the tool into clinical practice.

Funded by the Department of Health Victoria. 
Abstract number: P02-382

Abstract type: Poster

Team Work between the Intensive Care Unit, The Hospice Care Unit and the Local Organ Procurement Unit of the Livorno Hospital

D'Imporzano E. ${ }^{1}$, Lopane P. ${ }^{2}$, Meini L. ${ }^{2}$, Roncucci P. ${ }^{3}$, Quartarone M. ${ }^{3}$, Cardone L. ${ }^{1}$, Tofani R. ${ }^{3}$, La Rocca F. ${ }^{1}$, Galli C. ${ }^{1}$

${ }^{1}$ Palliative Care, Livorno Hospital, Livorno, Italy, ${ }^{2}$ Coordinamento Locale Donazioni, Livorno Hospital, Livorno, Italy, ${ }^{3}$ Intensive Therapy, Livorno Hospital, Livorno, Italy

Introduction: The anesthesiologist usually asses very different welfare setting in the hospital environment. Facing a chronic patient who is terminal or a patient who irreversibly deteriorates, the anesthesiologist has to choose appropriately between an intensive care therapy or a merely palliative therapy. Howewer whereby the patient is a suitable candidate organ donor after death, the ICU admission is appropriate even without the expectation of the therapeutic benefit.

Materials and methods: The Italian Law 219/17 dealing with DAT (anticipate treatment dispositions) and PAC (anticipate cure planning) guarantees the shared caregiving with the patient and the relatives, the patient self-determination and the suspension of the excessive treatments; the end of life path also can't exclude the legal right of everyone to become an organ donor. The combined evaluation of medical case between the anesthesiologist and the hospice clinician allows the decision to admit in the hospice ward the patients who are unable of an adequate recovery so to increase the palliative treatments.

Results: Between January and October 2018, 10 patients were transferred from the ICU to the Hospice ward; we provided to register on the medical record the result of the evaluation about the impossibility of any benefit of the therapies, the lack of eligibility at the donation and the difficulty of a brain death development. The admission at the hospice was always the result of a shared decision between the clinicians involved and the family.

Conclusions: The end of the intensive care treatment that can only lead to an end of life process is a legal and deontological obligation. The Hospice is the adequate setting because allows the right privacy for the patient and the family, the planning of palliative sedation, the pain therapy and the psychological and spiritual support for the patient and the family as well. The NBHD tissue donation and the postmortem family support complete the end of life program.

Abstract number: $\mathrm{P} 02-383$

Abstract type: Poster

Spotlight: Hospital Palliative Care Team at University Hospital Southampton (UHS) - Progressive and Proactive

Banting M., Cawley M., Haynes K., Davis C.

University Hospital Southampton NHS Foundation Trust, Southampton, United Kingdom

UHS: 1300 beds, provides local services and specialist services to Southern England. It is a centre of excellence for major trauma, cancer including teenagers and young adults (TYA), cardiology, intestinal failure and respiratory medicine including cystic fibrosis (CF).

The UHS Hospital Palliative Care Team has four consultants, two junior doctors, nine clinical nurse specialists (CNSs) and an academic advanced nurse practitioner (ANP).

Statistics for 12 months to April '18: 2358 referrals, 33\% non-cancer, 7,980 face-to-face contacts, $30 \%$ died as inpatients.

In the last 12 months our team's remit has grown. 2 new consultants spend a third of their time working with the heart failure team or the ED/ acute medical unit (AMU) doing proactive ward rounds. A specialist palliative care (SPC) heart failure clinic started with very positive patient feedback. The numbers of referrals have increased three fold for heart failure and doubled for AMU.

The current consultants consolidated their time with other specialties. One with oncology including TYA, rapid MDT radiotherapy clinic and supportive care clinic. The other does intensive care (ICU) ward rounds (number of referrals rising: 107 in 2017-18). We are conducting a funded study to develop an ICU Palliative Assessment Tool. She also provides proactive input to the CF unit (29 referrals in 2017-18; 2 patients died); CF team, patients and families provide very positive feedback. Our ANP is a leading international voice for renal disease and dialysis and runs a busy SPC renal clinic.

The CNSs have developed new proactive roles (hepatology, dementia, MND, pancreatic disease, CF and interstitial lung disease) with input to MDTs, teaching and proactive patient contact. One CNS leads education for SPC and end of life care (EOLC). We run an annual EOLC Conference. We are proud of the way our team and remit has evolved and believe it provides the blueprint for Acute Hospital Palliative Care in the future.

Abstract number: P02-384

Abstract type: Poster

Characteristics of Out-of-Office Hours Hospital Consultations by a Regional Palliative Care Team

van der Werff G.F.M. ${ }^{1}$, Ruivenkamp J. ${ }^{2}$, Costongs L.G.P. ${ }^{3}$, Geerling J.I. ${ }^{4}$, van Lune P.F. ${ }^{5}$, Thoma M. ${ }^{6}$, de Jong J.W. ${ }^{2}$, van der Velden A.W.G. ${ }^{1}$, Reyners A.K.L.'

${ }^{1}$ Palliative Care, Martini Hospital, Groningen, Netherlands, 2Palliative Care, Ommelander Hospital, Scheemda, Netherlands, ${ }^{3}$ Palliative Care, Zonnehuisgroep Noord, Zuidhorn, Netherlands, ${ }^{4}$ Palliative Care, University of Groningen, University Medical Center, Groningen, Netherlands, ${ }^{5}$ General Practitioner, Lopperum, Netherlands, ${ }^{6}$ TSN Home Care, Zwolle, Netherlands, ?University of Groningen, University Medical Center, Groningen, Netherlands

Aim: In 2013, a multidisciplinary, transmural provincial palliative consultation team (PPTG) was set up in Groningen. All hospitals have a palliative care team during office hours. However, during out-of-office hours, the $24 / 7$ available PPTG is approached by primary care professionals as well as hospital staff for consultations. This study describes the characteristics of those hospital consultations.

Design: All hospital based PPTG consultations between July 2016 and July 2018 were identified. Of those, consultation records and hospital records were studied. In the consultation records the characteristics of the questioner, the question and whether the provided advice was in accordance with the national guidelines was assessed. In the hospital records, whether the provided advice was followed was assessed. Moreover, we determined if the consultation could have been postponed to office hours. Analyses were performed by experienced PPTG consultants, discussing one another when in doubt.

Results: Of the 242 performed consultations, 56 were hospital based. Only 41 consultations were included (15 insufficient data).

Physicians consulted the PPTG 29 times, 16 of them being residents. Nurses called 12 times. Most consultations originated from cardiology (13), internal medicine (6) and ENT surgery (6). Mean age of patients was 74 (range 21-92) years, 19M. Underlying diseases were cancer (22), vascular disease (8), infections (3) and multiple organ failure (3). In 19 cases, life expectancy was estimated $<3$ days, in 11 cases $4-14$ days.

67 questions were addressed, concerning symptom management (34, most frequently dyspnoea (10), pain (9) and agitation (8)), end-of-life decisions (13) and organization of care (11).

All advices were given according to national guidelines and all advices were followed. We considered that two consultations could have waited until office hours.

Conclusion: Hospital based out-of-office hour consultations consider acute problems in symptom management. 
Abstract number: P02-385

Abstract type: Poster

Adaptation of a Palliative Care Approach in the Context of Intensive Care: A Systematic Literature Review Hamdan H., Olausson S., Wolf A., Öhlén J.

Institute of Health and Care Sciences, Sahlgrenska Academy, University of Gothenburg, Gothenburg, Sweden

Background: Palliative care is assumed to be an integral part of care in Intensive Care Units (ICU), yet, research still indicates lack knowledge about how to adapt, integrate and adopt palliative care in curative focused contexts such as in intensive care.

Aim: To identify factors influencing the integration of a palliative care approach in the context of ICU.

Method: A systematic review based on quantitative and qualitative studies using a thematic synthesis of the articles was performed. The following search term were used: Implementation, palliative care and ICU. In total 1843 articles were screened, and 25 articles were eligible for this review.

Result: The findings show that the influencing factors for integrating a palliative care approach are multifaceted. Themes were identified; facilitators and barriers for implementation and adaptation on organizational level (facilitating policies and environmental support, care pathways, prognostication, multidisciplinary team, training and education) and interpersonal level (communication conflict, emotional and spiritual support, person-centred care and patient and family involvement).

Conclusion: To adopt a palliative care approach into the ICU setting, the integration process requires adaption of organizational support and personal beliefs and attitudes within the entire multi-professional team including the manager as well as patient and family.

Abstract number: P02-386

Abstract type: Poster

From No Palliative Care to a National Sustainable Palliative Care Program: Experiences from Togo with the Partnership with Africa Palliative Care Association and MdM Suisse Alinon K.N.

Ministry of Health and Social Protection, Non Communicable Disease, Atlanta, Togo

Togo has participated to the First African Ministers of Health Session in 2013, the Second Session of the African Ministers of Health on Palliative Care in Kampala 2016 and took the engagement for the implementation of the 2014 WHA Resolution on Palliative Care. Therefore, the country had been involved in developing and implementing palliative care programs during the last year.

Objective: Our objective is to describe the currently situation of palliative care services and some Indicators for measuring the integration of Palliative Care in Togo's Health System.

Method: A systematic review of the currently situation of palliative care has been done.

Result: Some Indicators for measuring the integration of Palliative Care in the Health System were identified. Indeed, Togo has already integrated Palliative Care in his Health Sector Strategic Plan (HSSP) and in the National Development Plan for Non Communicable Diseases (NCD). There's a functional multidisciplinary and multi-sectoral Palliative Care task force and a full-time staff for Palliative Care within the National NCD program. Some national, regional and private hospitals' management teams got a sensitization on Palliative Care. 25 Health Care workers in $\mathrm{CHU}$ SO have received 5 day of training on Palliative Care. 25 Policy makers were also sensitized; 60 Health Care workers have been also oriented to Pediatrics Palliative Care through a 2 day conference and one day training. 8 Health Care workers got an international training on Palliative Care through 6 weeks course in Hospice Uganda. Another 8 Heath Care workers have been trained through a 2 weeks course by a team from Korea.

Conclusion: There's many challenges to deal with in term of low funding for Palliative Care; the Physicians' fear of morphine products prescription and the weakness of the Health System. But, Togo still have a very strong political will for the integration of Palliative Care in the Health System.

Abstract number: $\mathrm{P} 02-387$

Abstract type: Poster

Implementation of Best Practice Recommendations for Palliative Care in German Comprehensive Cancer Centers Gahr S. ${ }^{1}$, Loedel S. ${ }^{1}$, Berendt J. ${ }^{2}$, Thomas M. ${ }^{3}$, Ostgathe C. ${ }^{1}$ ${ }^{1}$ Department of Palliative Medicine, University Hospital Erlangen, Friedrich-Alexander University Erlangen-Nürnberg (FAU), Erlangen, Germany, ${ }^{2}$ Section Health System Analysis, Health Regions plus and Health Services Research, Bavarian Health and Food Safety Authority, Nuremberg, Germany, ${ }^{3}$ Department of Thoracic Oncology, University of Heidelberg, Heidelberg, Germany

Background: The Comprehensive Cancer Centers' (CCCs) working group on palliative medicine developed best practice recommendations for the integration of general and specialized palliative medicine (SPM) in CCCs, funded by the German Cancer Aid, in Germany. They were published in December 2017 containing 29 recommendations in different thematic areas such as inpatient service, day-care and outpatients, regional networking, research or education and teachings.

Aim: Rating the degree of current implementation of these recommendations in the CCCs.

Methods: An online survey was conducted between 12/2017 and $2 / 2018$ to assess the degree of implementation of these recommendations in the 15 CCCs. The CCC directors of each site were eligible participant. The implementation status of each recommendation in every CCC was evaluated using a verbal scale with stages of change according to the transtheoretical model (Prochaska, 1997).

Results: Participation rate of all requested sites was $100 \%(n=15)$. In all CCCs according to the consented best practice model a palliative care unit is available. The recommendation providing a palliative care hospital support service was followed by 11 CCCs. Twelve CCCs quoted a possibility for an interdisciplinary outpatient consultation with expertise in SPM or its integration into the research structures. Regional networking and the provision of a palliative ward were approved by all CCCs. Only one CCC followed the recommendation that the employees of the emergency department had a basic palliative care qualification.

Conclusions: The majority of German CCCs already fulfil essential organizational and structural requirements. More efforts are needed in education and teaching of palliative care to foster general palliative care in the CCCs. The results of this study will be used as a basis to establish strategic tandem partnerships between the CCCs to promote further local development through mutual assistance in implementation.

Abstract number: P02-388

Abstract type: Poster

Shaping Integrated Palliative Care: Impact of A 6 Year Strategy in the Makerere and Mulago Palliative Care Unit National Hospital and Academic Setting in Uganda

Leng M. ${ }^{1,2,3}$, Grant L. ${ }^{3}$, Downing J. ${ }^{1,4}$, Murray S. ${ }^{5}$, Turyahikyio J. ${ }^{1}$, Bagasha P. ${ }^{1}$, Namukwaya E. ${ }^{1}$

${ }^{1}$ Internal Medicine, Makerere and Mulago Palliative Care Unit, Kampala, Uganda, ${ }^{2}$ Cairdeas International Palliative Care Trust, Aberdeen, United Kingdom, ${ }^{3}$ Global Health Academy, Edinburgh 
University, Edinburgh, United Kingdom, 'International Children's Palliative Care Network, Assagay, South Africa, ${ }^{5}$ Primary Palliative Care Research Group, Edinburgh University, Edinburgh, United Kingdom

Background: National hospital and academic settings with integrated palliative care $(P C)$ can offer leadership for shaping the future of PC through: improved access to quality, evidence-based PC for patients and families as well as clinical modelling; training \& capacity-building; developing leaders \& national \& international partnerships.

Aim: To evaluate \& assess outputs \& outcome indicators in the strategic plan 2011-2016.

Methods: A review of indicators using outcomes integrated within the research agenda including: capacity building; training numbers; staff development \& collation of impact assessments.

Results: 3,189 patients received care from the specialist PC team with an additional 6,669 identified for \& received generalist PC by link nurses (731 referred for specialist support). 4,630 individuals were trained including undergraduates (medicine, nursing, pharmacy), postgraduate (Diploma, Degree and 4 Med programmes), and in-service short courses. 1,223 of those trained were in 8 additional countries. Staff gained 5 BSc, 2 Masters, 1 PhD. 192 abstracts at national \& international conferences \& 53 publications were achieved. Impact assessments include evaluation of integrated models including link nurses, nurse prescribing, outcome of PC interventions, educational impact, research capacity building, morphine use, experience of patients \& staff \& service evaluations. Conclusions: As an essential component of Universal Health Coverage PC can be integrated with in a tertiary hospital in Sub-Saharan Africa. A robust strategic plan, clear research agenda, strong partnerships \& focus on leadership, research \& training alongside clinical care can make a significant impact. PC Specialists can empower hospital staff to integrate PC in their routine practice and refer only when necessary. This integrated model is also applicable in District Hospitals \& Health Centers to render $\mathrm{PC}$ in the community and wider scale up has implications for shaping the future of global PC.

\section{Abstract number: P02-389 \\ Abstract type: Poster}

Perspectives of Physicians Working in Thoracic Oncology on Interprofessional Collaboration to Facilitate Early Palliative Care: Results of a Qualitative Study Villalobos M. ${ }^{1}$, Handtke V. ${ }^{1}$, Hagelskamp L. ${ }^{1}$, Siegle A. ${ }^{1}$, Jung $C^{1}{ }^{1}$, Krug $K_{.}{ }^{2}$, Bossert J. ${ }^{2}$, Deis N. ${ }^{3}$, Jünger J. ${ }^{3}$, Wensing $M{ }^{2}$, Thomas $M .^{1}$ ${ }^{1}$ Thoracic Oncology, University of Heidelberg, Heidelberg, Germany, ${ }^{2}$ General Practice and Health Services Research, University of Heidelberg, Heidelberg, Germany, ${ }^{3}$ Institut für medizinische und pharmazeutische Prüfungsfragen, Mainz, Germany

Background: Oncological guidelines recommend early integration of palliative care into lung cancer care. However, there is still a perceived threshold for physicians working in oncology to offer palliative care early in the course of disease. Interprofessional interventions have shown to significantly support in overcoming this barrier. We developed a tandem approach to patient consultations: physicians and nurse navigators with experience in oncology and palliative care hold joint consultations at pivotal moments in the disease trajectory starting from diagnosis with the aim of facilitating illness understanding, coping, advance care planning and early integration of palliative care. This approach is new in Germany. Aims: Exploration of attitudes of physicians working in oncology towards interprofessional collaboration before implementing the new nursing role and the tandem approach.

Methods: Qualitative thematic analysis of 2 focus groups (in total 10 physicians) using a semi-structured interview guide.

Results: We found controverting views on the new nurses' role: on the one hand the expected role is described as rather passive (listening) and reduced to topics related to nursing; on the other hand, the nurse is seen as a gate-keeper and advocate for patients' issues and with psycho-social skills, that are expected to be helpful in a crisis intervention which the physician is unable to cope with. Participants emphasize the lack of time in consultations and fear excessive workload. In general, strengthening of continuity in care, improved information flow and a more holistic care are expected.

Conclusion: As interprofessional interventions lead to improved patient outcomes in regard to early integration of palliative care, institutional efforts should not neglect barriers to collaboration. Clarification of roles and interprofessional training are important means to strengthen this collaboration.

Funding: National Center for Tumor diseases (NCT 3.0, G835), Germany.

Abstract number: P02-390

Abstract type: Poster

Dare to Care: Patients with Advanced Cancer and Their Informal Caregivers Want Personal Well-organized Care van Roij J.1,2, de Zeeuw B. ${ }^{1}$, Zijlstra M. ${ }^{1,3}$, Claessens N. ${ }^{4}$, Raijmakers N. ${ }^{1}$, van de Poll-Franse L. ${ }^{1,2,5}$, Brom L. ${ }^{1}$, Boddaert M. ${ }^{1}$

${ }^{1}$ Netherlands Comprehensive Cancer Organisation, Utrecht, Netherlands, ${ }^{2}$ Tilburg University, Tilburg, Netherlands, ${ }^{3}$ Maxima Medical Centre, Eindhoven, Netherlands, ${ }^{4}$ Rijnstate, Nijmegen, Netherlands, ${ }^{5}$ Netherlands Cancer Institute, Amsterdam, Netherlands

Background: Palliative care is focussed on improving quality of life of patients with a life-threatening illness and their informal caregivers. It remains unclear what good quality of care entails for patients with advanced cancer and their informal caregivers. Therefore, the aim of this study is to explore the essential aspects of palliative care according to patients with advanced cancer and their informal caregivers.

Methods: Seven focus groups and seven in-depth semi-structured interviews with 18 patients suffering from advanced cancer and 15 informal caregivers were conducted in the Netherlands. All interviews were audiotaped, transcribed verbatim, and open coded using a thematic analysis approach.

Results: Two essential aspects of quality of care from the patients' and informal caregivers' perspective arose: 'communication and relation' and 'organisation of care'. Regarding communication and relation patients and informal caregivers found it essential that health care professionals were personally engaged and provided support and compassion. Regarding organization of care, patients and informal caregivers expressed the importance of well-organized logistics tailored to needs of the receiver. Patients and informal caregivers also expressed the importance of supportive care being proactively offered multiple times during the disease trajectory.

Discussion: This study shows that personally engaged health care professionals are essential in high quality palliative care according to patients and their informal caregivers. Health care professionals are dared to really engage with their patients to gain a deeper understanding of their needs. Therefore, it is of utmost importance that health care professionals have good communication skills and take the time to explore the patients' and informal caregivers' unique experiences, needs, preferences, and expectations.

Abstract number: P02-391

Abstract type: Poster

Changes in Consultation and Palliative Care Awareness: Outcomes after Six Years of Specialized In-hospital Palliative Care Consultation

van der Stap L. ${ }^{1}$, de Nijs E. ${ }^{1}$, Oomes M. ${ }^{1}$, Juffermans C. ${ }^{2}$, Ravensbergen $W^{2}{ }^{2}$, Luelmo S. ${ }^{3}$, Horeweg $N^{4}{ }^{4}$, van der Linden $Y^{5}$

${ }^{1}$ Center of Expertise Palliative Care, Leiden University Medical Center, Leiden, Netherlands, '2Department of Public Health and Primary Care, Leiden University Medical Center, Leiden, Netherlands, ${ }^{3}$ Department 
of Medical Oncology, Leiden University Medical Center, Leiden, Netherlands, ${ }^{4}$ Department of Radiation Oncology, Leiden University Medical Center, Leiden, Netherlands, ${ }^{5}$ Center of Expertise Palliative Care and Department of Radiation Oncology, Leiden University Medical Center, Leiden, Netherlands

Background: All Dutch health care providers (HCPs) provide generalist palliative care (PC). If necessary, $P C$ specialists are consulted. About $4 \%$ of hospitalized patients have specialist PC needs. In our academic hospital a PC consultation team (PCCT) was introduced in 2012.

Aim: To evaluate consultation characteristics and effect of PCCT presence on PC awareness of generalist HCPs.

Methods: Survival, patient and consultation characteristics (2012-2017) were reviewed. Referring HCPs estimated survival, categorized in $>3$ months, $>14$ days to $\leqslant 3$ months and $\leqslant 14$ days. Estimations were compared to observed overall survival (OS) using weighted Kappa. Outcomes of two e-questionnaires (2012 and 2016) among hospital HCPs were compared using descriptive statistics and chi-square tests.

Results: In total,1404 consultations were registered with a mean increase of $28 \%$ per year. Medical oncology consulted most frequently and increasingly for patients with observed OS > 3 months (9 in 2012; 40 in 2017). Of all consultations, $86 \%$ were in oncology. Non-oncology increased from 5 in 2012 to 65 in 2017. Median survival was 0.9 months (range $0-68$ ). Physicians estimated in $31 \%$ a survival of $>3$ months, in $52 \%>14$ days to $\leqslant 3$ months and in $17 \% \leqslant 14$ days ( $49 \%$ correct estimation, $44 \%$ overestimation (kappa $0.34,95 \% \mathrm{Cl}: 0.28-0.40)$ ). The e-questionnaires had 291 (2012) and 195 (2016) respondents from 25 departments. PCCT familiarity increased from $56 \%$ to $85 \%(p<0.001)$. Among physicians, PC guideline use increased from $37 \%$ to $87 \%$ ( $p<$ 0.001 ). In $2016,86 \%$ of physicians and $89 \%$ of nurses reported good or excellent experiences with the PCCT.

Discussion: Although PCCT presence increased hospital palliative care awareness, survival was overestimated in nearly half of the patients and, over time, only medical oncology consulted earlier in the disease trajectory. To integrate PC into standard care, PCCT policy should focus on early referral and improved PC access, especially for non-oncology patients.

Abstract number: P02-392

Abstract type: Poster

Is it Really Early Our Early Intervention? Activity of a Hospitalbased Palliative Care Consultation Team at the Clínica Universidad de Navarra

Noguera A., Urdiroz J., Muro E., Garcia-Consuegra A., Centeno C. Clínica Universidad de Navarra, Pamplona, Spain

Background: The Palliative Care Consultation Team started its activity at the Clínica Universidad de Navarra 15 years ago. Since the beginning we tried to establish a close relationship with the Oncology Department receiving from them early referrals of advanced patients for evaluation and follow-up. After the 2010 Temel's study, with confirmed results in over 19 randomized trials, we ask ourselves how early is our intervention in the oncological setting.

Aim: To describe the activity and integration of the PCCTs at the patient level considering early integration parameters.

Method: Cohort study with newly referred consecutive patients from the department of Oncology, monitoring these patients until death. We recorded the time from diagnosis of advanced disease to the referral, ECOG, main problems in first evaluation, and proportion of patients participating in clinical trials and time of survival. As the criteria for early integration, we have adapted the Hui et al (2016) international consensus. Results: 52 new patients were included in the three months recruitment period. The follow-up time of the cohort was six months. Twenty-one patients (40\%) were within 3 months of diagnosis of advanced disease and initial ECOG was $1-2$ in 30 patients (57\%). The main problem in the initial evaluation was pain for the whole cohort though there is also a highly reported level of emotional distress at the first evaluation. Nine out of 52 patients were in a clinical trial regime (17\%). When writing the abstract, 22 patients (42\%) were alive with a median survival time of 3,5 months.

Conclusion: The preliminary analysis of the cohort suggests that nearly one in two new patients referred to Palliative Care has criteria of early intervention. Patients under clinical trials represent today a significant proportion of the activity of the palliative care consultation team in a university hospital.

\section{Abstract number: $\mathrm{P} 02-393$ \\ Abstract type: Poster}

\section{Changing Minds in a Small Hospital with a Big Proportion of} Elderly People

Nakaema K.E. ${ }^{1}$, Furuya S.Y. ${ }^{1}$, Toroshima T. ${ }^{1}$, Matsumoto L.K. ${ }^{1}$, Chiba T. ${ }^{2}$

${ }^{1}$ Palliative Care Group, Hospital SBC, São Paulo, Brazil, 2Palliative Care, Instituto do Câncer do Estado de São Paulo, São Paulo, Brazil

This year, in January, we started a service of Palliative care at a small hospital in Sao Paulo. This hospital is characterized by a prevalence of Japanese descendants because it was created by a cooperative of farmers in the last century. Nowadays, there are over 5.400 people assisted by this hospital with 30 beds and more than $54 \%$ are over 60 years old. Despite the number of elderly people, discussions about death and preferences in the end of life were very infrequently and many patients die in suffering or at intensive care unit.

There are many people with 80 years old or more with a good functionality but it fall abruptly when a hospital admission is necessary.

Our Palliative care team is composed of 2 nurses, 1 psychologist and 1 doctor and we started with a consultation service at the nursery. We have made family conference with all patients' families.

The Project counts on financial help from a Japanese governamental development organ and on tecnical colaboration from a Japanese hospital with Palliative care team.

Since January until October, we followed 18 patients in more then 32 hospitalizations. There was only one who refused the Program - a patient of 56 years old with cerebellar ataxia.

With a median age of 82 years, $44 \%$ patients had neoplasia, $50 \%$ had neurodegenerative diseases (Alzheimer's Dementia or Vascular dementia) and 1 patient had fragility syndrome, $83 \%$ of the patients have already died.

We have been doing Death Conference with the care team, when we can discuss about care of patients who died. And we can perceive that the team is more comfortable with end of life care of patients followed by the palliative care team.

The number of consultations are increasing between these months. In October we also started Palliative care multidisciplinary outpatient consultation.

Palliative care mind is slowly spreading between medical body, nurses, administrative body and the community of this hospital.

Change minds to Palliative care minds is possible.

Abstract number: P02-394

Abstract type: Poster

Reducing Hospital Deaths in People with Dementia, a New Approach Led by a Hospice Based Specialist Dementia Team Santos Lopes N., Priestley S., Mclvor K. Royal Trinity Hospice, London, United Kingdom

Background: Dementia is the leading cause of death in England and Wales. There is growing pressure on the National Health Service to provide cost effective, evidence-based care and to avoid unnecessary hospital admissions at the end-of-life. The number of people with Dementia is 
increasing yearly and recent data in England shows that $32 \%$ of these people die in Hospital. Audit was the method chosen to monitor the activity of the team in regards to Preferred Place of Death (PPD) and Place of Death (PD).

Aims: Develop the Hospice Dementia Team and understand its impact with regards to the PPD and PD in patients with Dementia, compared with the PD in the general population.

Methods: Facing the increasing demand for support for people with Dementia reaching end-of-life the Dementia Team was restructured. An operational policy was designed, to standardise referral criteria enabling patients to self-refer, an additional dementia nurse specialist was employed and advanced care planning was prioritized. PD and PPD data was collected using Hospice electronic records for patients with Dementia who died under the care of the specialist dementia team between 1st June 2017 and 31st May 2018. PD was categorized as Home, Hospice, Hospital and Care Home.

Results: Within this period $69 \%$ of the people with Dementia died in their PPD. From the 39 deaths, $49 \%$ happened at home, $26 \%$ in a Care Home, $18 \%$ in Hospital and $8 \%$ at the Hospice.

Discussion/ conclusion: Hospice dementia team intervention was associated with a higher home death rate of $49 \%$, compared with the $8 \%$ in England and a lower rate of hospital deaths - 18\% compared with $32 \%$ nationally. The majority of patients achieved their PPD. These findings support the benefits of a specialist dementia team in supporting patients to die at their PPD, as well as reducing the economic impact of unnecessary hospital admissions. There is a need for further studies into this field.

Abstract number: P02-395

Abstract type: Poster

The Importance of Early Identifications of Palliative Care Needs in Emergency Wards

Grillfors Mård M., Gullberg Sundin C., Dreilich M.

Palliative Care Unit, Uppsala University Hospital, Uppsala, Sweden

Aim: The authors consider it important with early identifications of palliative care needs in patients cared for in the emergency care unit. A structured and regular meeting at the ward can help to identify palliative care needs in the early trajectory of the illness and ensure that the patient receives a good, safe and equal palliative care.

Design, methods and approach taken: We planned on weekly meetings with the emergency ward team and developed a screening tool for palliative care needs as well as advanced care planning tool. Our aim was to learn the team to identify palliative care needs using the screening tool and from our weekly meetings where palliative issues and dilemmas is discussed.

The structured questionnaire has been developed to meet the demands for advanced care planning including items concerning diagnosis, medical plan, symptoms, activity of daily life and the patient's own concerns and wishes.

Results: Introducing palliative care into emergency wards that traditionally worked on investigating and treating curable patients takes time. Since 2011, the palliative care unit has been working to bring palliative care in emergency wards. With structured weekly palliative meetings, a greater awareness of the care of patients with palliative care needs has been achieved. Regular meetings once a week and the screening of palliative care needs has become a successful combination. Patients concerns and advanced care plannig where the patient wants to be cared for the last time in life, social networking and the need for support the patient and his / her relatives need.

Conslusion: There is a need to make palliative care integrated in emergency care in order to early identification and offer patients good ad safe care in the end of life.

Abstract number: P02-396

Abstract type: Poster
Building Clinical Trial Capability in Palliative Care: Strategies and Lessons Learned

Philip J. ${ }^{1,2,3}$, Le B. ${ }^{4}$, Pasanen L. ${ }^{5}$, Boughey M. ${ }^{5}$, Weil J. ${ }^{5}$, Moran J. ${ }^{6}$,

Coperchini M. ${ }^{7}$, Rosens E. ${ }^{3}$, Woollett $A^{3}{ }^{3}$

${ }^{1}$ Department of Medicine, University of Melbourne, Fitzroy, Australia,

2Palliative Care Service, St Vincent's Hospital Melbourne, Fitzroy,

Australia, ${ }^{3}$ Victorian Comprehensive Cancer Centre, Parkville, Australia,

${ }^{4}$ Palliative Care Service, Melbourne Health, Parkville, Australia,

${ }^{5}$ Palliative Care Service, St Vincent's Hospital Melbourne, Fitzroy,

Australia, ${ }^{6}$ Palliative Care Service, Austin Health, Melbourne, Australia,

${ }^{7}$ Palliative Care Service, Western Hospital, Sunshine, Australia

Patients enrolled in clinical trials, and cared for by trial active health services have better outcomes. A key strategic objective of a multisite cancer centre alliance was to increase clinical trial expertise in targeted groups, with a focus on palliative care. The alliance palliative care services presented substantial trial development opportunities with large patient numbers, established relationships and few trial-active centres.

Aim: To increase clinical trial expertise and patient access to clinical trials in palliative care.

Method: Areas targeted for building capability included:

- Infrastructure: establishing trial nurses and fellow positions

- Mentorship and training: cross pollination of trial experienced nurses; overarching clinical trials coordinator; establishment of Scientific Committee to assess/ guide site investigators; early career researchers included in scientific committee.

- $\quad$ Setting studies priorities: Grading study complexity to match site capability; mix of drug and non-drug studies; Consumer priorities included.

- Sustainability: mixed trial portfolio development (sponsored and investigator initiated); review of trial funding models; development of higher research degree positions.

- Measures of success: reflecting quantitative and qualitative outcomes.

Early results:

- $\quad$ Contractual negotiations take time

- Strong relationships and governance essential to facilitate contracts.

- $\quad$ Baseline data critical

- Already identified key personnel for targeted professional development

Early "wins" :

- $\quad$ Prompted an international paediatric palliative care phase 4 studies program;

- Leveraging infrastructure to: roll out competitively funded studies, source additional fellow support;

- $\quad$ Trials registrar training position created, \&

- $\quad$ Trials fellows identified for key early career positions in national collaboratives.

The program is supported by state government funding.

Abstract number: P02-397

Abstract type: Poster

Palliative Care Needs, a Qualitative Proxy Considering Patient and Professional Local Perspectives

De Allende-Salazar M.I. ${ }^{1}$, Tupper L. ${ }^{2}$

${ }^{1}$ Chile ID, Santiago, Chile, ${ }^{2}$ Palliative Care, Hospital Sótero del Río, Santiago, Chile

Background: Among Latin American (LA) countries, Chile has one of the highest levels of development in palliative care (PC) provision. Facing 
demographic and epidemiological changes, palliative care needs (PCN) are expected to rise in next decades. This report is part of a broader national PCN assessment: we estimated that nearly 50.000 new patients would require PC annually. Very little data are published on PCN from the patient and professional perspective in LA, although cultural differences are relevant to design adequate in $\mathrm{PC}$ local programs.

Aim: To asses type of PC services and which attributes are valuable for patients and professionals.

Methods: An exploratory mixed-methods study was conducted including relatives and professionals, exploring their perceptions of the essential attributes of PC services. Four focus groups were conducted including 19 adult bereaved caregivers as a proxy of patient perspective and 9 PC professionals completed semi structured interviews. Data were analyzed using thematic analysis techniques.

Results: The study results indicated that attributes could be classified into 6 categories: general aspects of care, physical and psychological symptoms, social and spiritual support and organizational issues. Most relevant themes were related to patient-centered care focused on quality of life, a warm relationship with professionals, excellent communication skills and training of healthcare practitioners. Symptom control, psychosocial and spiritual support were identified as key by both groups. After-hours services, continuity and coordination of care, as well as hospice bed availability and short waiting times were highly valued.

Conclusion: Pain relief and excellent communication and warm personal contact were the most relevant topics. Valued palliative care attributes were identified for Chilean context, providing detailed information to guide the improvement of current and design of future palliative care services across the country.

Abstract number: P02-398

Abstract type: Poster

An Evaluation of the Contribution of the Specialist Palliative Care Nursing Service to Patient Care in an Acute Hospital Setting Connolly M. ${ }^{1}$, Ryder M. ${ }^{2}$, Furlong E. ${ }^{3}$, Carruthers E. ${ }^{4}$, McGuigan E. ${ }^{4}$, Larkin P. ${ }^{5}$, Project Steering Group

${ }^{1}$ School of Nursing, Midwifery and Health Systems, University College Dublin, Dublin, Ireland, ${ }^{2}$ University College Dublin, Dublin, Ireland, ${ }^{3}$ School of Nursing, Midwifery \& Health Systems, University College Dublin, Dublin, Ireland, ${ }^{4}$ Specialist Palliative Care Services - HSE North East, Drogheda, Ireland, 5 Université de Lausanne, Lausanne, Switzerland

Background: In Ireland Specialist Palliative Care (SPC) is a consultant led service provided in a range of settings with Clinical nurse specialists (CNS) providing specialist care particularly in the hospital setting. Nurses often work independently, and are a reference point for clinical advice, care and discharge planning and family support. In Ireland five core competences have been identified for the role of CNS: clinical, advocacy, consultation, education, audit and research.

Aim: The aim of the study was to evaluate the contribution of the SPCCNS service to patient care in an acute hospital.

Method: A validated questionnaire survey was adopted for use, to assess current understanding and contribution of the SPCCNS service. 200 questionnaires were circulated to medical, nursing and allied health care professional staff working in an acute hospital. 121 completed questionnaires were returned representing a response rate of $60.5 \%$. Responses were received from a range of health care professionals.

Findings: Findings indicate that the majority (99.2\%) of respondents were aware that a SPCCNS service is provided at the hospital. To date $54 \%(n=65)$ of respondents indicated that they had referred patients to the SPCCNS service. The majority of referrals sought advice on symptom management and discharge planning. Findings indicate that respondents identify clinical, advocacy and consultancy as the most important core competences. Audit and research were measured as least important aspects of the SPCCNS role, particularly by non-nursing staff who responded. It is also worth noting that the education role of the SPCCNS was viewed as important by nurses, but was not seen as an important part of the SPCCNS service by other health care professionals.

Conclusions: The study highlights gaps in understanding of the role of the SPCCNS service in a hospital setting. Further education of staff regarding the role and the core competences inherent in the role is needed.

Abstract number: P02-399

Abstract type: Poster

Healthcare Providers' Views and Experiences of Non-specialist

Palliative Care (NSPC) in the Acute Care Hospital Setting: A

Systematic Review

Nevin M., Smith V., Hynes G.

School of Nursing and Midwifery, Trinity College Dublin, Dublin, Ireland

Aim of review: Healthcare providers working in acute care hospitals have significant exposure to patients with palliative care needs, and for the majority of patients these reflect non-specialist rather than specialist palliative care needs. However, unlike a palliative philosophy of care in environments such as the hospice services, it is often the medical model of care that is dominant in acute hospital-based care. This represents a specific challenge as to how we may embed Non-Specialist Palliative Care (NSPC) within the organisation of services in hospitals. Understanding the viewpoints of those providing NSPC in hospitals is an important aspect of this.

Search and review methodology: Six databases were searched (The Cochrane Central Register of Controlled Trials (CENTRAL), PubMed, EMBASE, Medline, CINAHL, Web of Science) from inception to March 2018. Grey literature and reference list/citation searches of included papers were undertaken. The review considered studies that focused on qualitative data, and a quality assessment tool was used to assess the quality of included studies. The method of qualitative evidence synthesis used in this review was thematic synthesis.

Results: A total of thirty-nine papers (reporting on thirty-seven studies) were identified and selected for inclusion. Four themes emerged; knowledge and competency in NSPC; communication; differing perspectives of nurses and physicians; and the acute hospital care environment. Healthcare providers expressed fears around communicating bad news with patients and families. Lack of private rooms, time constraints, staff shortages, lack of clarity of role within NSPC provision and an emphasis on acute care goals were significant barriers to delivering good NSPC in the hospital setting.

Conclusion: Based on the review findings, a multi-level and multi-disciplinary approach will be needed to address the organisation of services in hospitals in order to meet the challenges of providing good NSPC.

Abstract number: P02-400

Abstract type: Poster

Palliative and End of Life Care Chain: From Plan to Perfection Mikkonen $\mathrm{H}$.

Helsinki University Hospital, Palliative Care Center, Department of Oncology, Helsinki HUS, Finland Presenting author email address: heli.mikkonen@hus.fi

Background: When delivering palliative and end of life care in multiorganizational model, there is a risk for inadequacy of care. In Finland palliative care and end of life care has been determined to be a part of primary health care, assisted by specialized palliative and hospice organizations in a need based three-level model. Organization of care are formulated in to care chains.

Aims: To evaluate the Helsinki metropolitan area (HMA) palliative and end of life care chain and to improve practical implementation of the care chain in co-operation with professionals working in the care chain. 
Methods: The study was conducted as an action research. In the first cycle, the palliative and end of life care chain was described. In the second cycle the actors' views on the care chain principles and functionalities were evaluated (N33). Based on the second cycle's results, in the third cycle the participants (N27) determined the focus for development. The collected data and results were produced by communicative group work and analyzed by qualitative content analysis.

Results: The HMA care chain is not equally known, does not have unified and determined values but actors' have a coherent value frame. The practice has improved but there are more challenges than strengths. The strengths are continuity, co-operation, homecare and increased knowledge. The challenges arise from timing, co-operation, features of the service structure and deficiencies in care delivery. The determined focuses for development are delivery of information, enhancing of knowledge and delivery and organization of care. The focuses were formulated into the development frame sheet

Conclusion: Development of the palliative and end of life care chain requires networking, communication, organization and education. The results can be utilized in various care environments and areas by administrators, care organizations and individual professionals in promoting and delivering palliative and end of life care.

Abstract number: P02-401

Abstract type: Poster

\section{Characteristics of Urgent versus Routine Referrals to an}

Ambulatory Palliative Care Clinic

Alam S., Pope A., Zimmermann C., Hannon B.

Princess Margaret Cancer Centre, University Health Network, Supportive Care, Toronto, ON, Canada

Background: Ambulatory palliative care clinics are increasingly recommended as the optimal venue for early palliative care engagement. Patients continue to be referred late to palliative care services, however. Little is known about the characteristics of patients referred urgently to these services, and how, if at all, they differ from patients referred for routine assessments.

Aims: To characterise patients referred urgently to the ambulatory palliative care clinic at a tertiary cancer centre, and compare with those referred for routine assessments.

Methods: We retrospectively reviewed all urgent referrals to the ambulatory palliative care clinic between January 2016 and December 2017. We will compare these to a random selection of patients referred for routine assessment to the clinic in a 1:2 ratio. Patient and referring tumour site group demographics were collected, along with reason for referral, symptom scores (using the Edmonton Symptom Assessment System), performance status (using the Palliative Performance Scale), as well as immediate and longer-term clinical outcomes.

Results: Data collection and analyses are ongoing. 113 patients were referred urgently to the palliative care clinic. Mean age was 64 years (SD15); 60\% were female. $79.6 \%$ were referred for management of urgent physical symptoms, with medical oncology accounting for $58.4 \%$ of referrals, of whom the most common tumour sites were lung (24.8\%) and gynecology (16.8\%). 20.4\% required urgent advance care planning discussions. $16 \%$ were admitted to hospital directly following their visit; while $60.2 \%$ continued to be followed in the ambulatory clinic longitudinally.

Conclusion / Discussion: Patients continue to be referred late to ambulatory palliative care services. Further exploration of the potential facilitators and barriers to timely referral is needed. Ambulatory palliative care services need to consider how to incorporate urgent referrals into their scheduling as part of service development.
Development of Palliative Care (PC) in Albania through Setting up and Development of New PC Units in the Public Healthcare Institutions

Koleci G., Laska I.

Palliative Care, 'Mary Potter' Palliative Care Center, Korce, Albania

Background: PC in Albania started in 1993 with creation of three PC teams.The increased number of patients in need and the enactment of PC law brought the necessity of establishment of PC units in other regions of Albania.

Methodology: In 2007 the staff of "Mary Potter" PC Center (MPPCC) began to train the healthcare personnel (HCP) all over Albania.These training included based,intermediate and advanced level of information for PC.After that there were some of the trained doctors and nurses who created volunteers PC groups to provide care for the terminally ill in their areas.Later on,this service was institutionalized with creation of PC units within the public healthcare institutions.In 2014 the first PC units were opened in the three regional hospitals.The HCP of these units were part of residential courses in MPPCC with theoretical and practical information and on-job trainings.They were also part of national, international $\mathrm{PC}$ trainings and began to exchange the experiences between the new PC units.Based on the positive experience of these PC units, some other units were opened during 2016.The staff of these unites followed the same way.The new units were helped with drugs and special equipment for needed patients.

Results: During the period 2014-2017 five new PC units were established in the regional hospitals in Albania which have appropriate rooms for offering PC with quality and standards.The staff of these units already has increased theoretical and practical capacities.These units are equipped with necessary drugs and special equipment for terminally ill. Now there is the strategic plan the national training manual and the treatment protocol,designed by national PC working group.

Conclusions: PC in Albania is really a developing service; it is available in all the regions of Albania.The trained healthcare personnel of all the PC units offer a qualitative service for needed patients, based in the $P C$ standards and according to treatment protocols in PC.

Abstract number: P02-403

Abstract type: Poster

Innovative Models of Palliative Care Delivery: Integrated Palliative Care in Internal Medicine Hospital Department Keller N. ${ }^{1}$, Lopez M. ${ }^{1}$, Brumana E. ${ }^{2}$, Ottolini L. ${ }^{1}$, Fusi-Schmidhauser T. ${ }^{1}$, Vanini G. ${ }^{2}$, Gamondi C. ${ }^{1}$

1Palliative and Supportive Care Clinic, Institute of Oncology of Southern Switzerland and Ente Ospedaliero Cantonale, Bellinzona, Switzerland, 2Internal Medicine Department, Ospedale Regionale di Lugano, Sede Italiano, Ente Ospedaliero Cantonale, Lugano, Switzerland

Introduction: Internal medicine departments (IMD) are recognized to be caring for a high number of palliative care (PC) patients, including those in need of specialized PC. In Southern Switzerland, 1200 patients are seen each year by the PC mobile consultation team in the seven public hospitals of the Canton and more of half of those hospitalized in the IMD are expected to present complex PC needs. Therefore, a new model of specialized PC delivery within an internal medicine ward was developed and tested. The model has been based on a collaborative partnership between IMD and PC team with two principal aims: to offer specialized PC for patients in need of it and to foster PC competencies in the IMD professionals through shared clinical practice and bedside teaching. Setting: The internal medicine ward of the Ospedale Italiano (Lugano) was identified to test the model. The model is constituted by:

A. Early identification of patients in need of specialized PC with an appropriate tool 
B. Interdisciplinary specialized PC delivered with a collaborative model of shared clinical responsibility

c. Protected home discharge or appropriate relocation

Results: The pilot project started in September 2017 and more than 250 patients have been included until September 2018. A database has been created to monitor the project outcomes and to collect clinical and epidemiological data.

Conclusions: The integrated model resulted feasible and well accepted by both teams. As a result of this project, other wards are interested in implementing this innovative model.

\section{Abstract number: $\mathrm{P} 02-404$}

Abstract type: Poster

Identification and Assessment of Integrated Palliative Care Initiatives in the Netherlands

Pereira C.F.R. ${ }^{1,2}$, de Wit A. ${ }^{1,3}$, Middelburg M. ${ }^{1,2}$, Boddaert M. ${ }^{1,2}$ Raijmakers N.J.H. ${ }^{1,2}$

1PZNL- Association for Palliative Care in the Netherlands, Utrecht, Netherlands, ${ }^{2}$ Netherlands Comprehensive Cancer Organisation (IKNL), Utrecht, Netherlands, ${ }^{3}$ Fibula, Platform of Regional Palliative Care Networks, Utrecht, Netherlands

Background: The Netherlands has a national program for the improvement of quality of palliative care (PC). Hence, the Ministry of Health supports healthcare professionals and affiliated organizations to provide generalist PC, supported by PC specialists if needed: a cumulative stepped process of engagement. Effective integration of generalist PC and PC specialists that transcends the borders of different care settings is essential for high quality PC.

Aim: To gain insight into integrated palliative care initiatives (IPCI) in the Netherlands and to identify potential best practices.

Method: To make an inventory of existing IPCI, all 65 PC networks in the Netherlands were requested to share documentation on IPCI in their region. The quality of the identified initiatives was determined by document analysis based on essential elements derived from the recently launched Dutch national quality framework for PC. Two assessors evaluated all documents and consensus was reached in discussion with an independent researcher.

Results: In total, documentation of 39 IPCI was evaluated. Fourteen initiatives were identified as potential best practices. 13 (93\%) Had a high score on advance care planning, 11 (79\%) on identification, 11 (79\%) on shared-decision making, 10 (71\%) on expertise, $8(57 \%)$ on individual care plan, $6(43 \%)$ on coordination \& continuity, 4 (29\%) on effective communication and $3(21 \%)$ on care for healthcare professionals. Three organizational models for PC became apparent in the fourteen best practices: integrated PC case management, integrated PC pathway and integrated PC team.

Conclusion: Several best practices for integrated PC are present in the Netherlands and they generally make use of three organizational models. These models can potentially be used for nationwide organization of integrated PC. Further research is needed into the quality of care and cost-effectiveness of these models and suitable reimbursement methods will need to be developed.

Abstract number: $\mathrm{P} 02-405$

Abstract type: Poster

Trends of Dying at our National Cancer Center

Ebert Moltara M., Benedik J., Ivanetič Pantar M., Bernot M., Zavratnik B.

Institute of Oncology Ljubljana, Ljubljana, Slovenia

Introduction: Our hospital represents main cancer centre in the region with 287 beds and over 14.000 hospitalizations per year. Many cancer patients die in our hospital. There are three clinical departments, where patients are treated: Department for medical oncology (DMO), Department for Radiotherapy (DRT) and Department for Oncological Surgery (DOS). Since 2007 our institution established also a specialised Department for acute palliative care (DAPC) with 6 beds. One of main task of DAPC is providing palliative care support/consult to all of the departments of the cancer centre.

Method: Aim of the analysis was to assess the trends of dying in our cancer centre from 2000-2017 and to distinguish the differences among departments needs according to specialised palliative care (PC) support. Results: In our analysis we are observing a rising trends of annual number of deaths in a hospitals, from 438 hospital deaths in 2000, 500 in 2010 to 592 in 2016. In 2017 number of deaths declined to 519 cases, since new cancer centre in a close by region has been opened. Among departments there is raising trends of deaths in DMO (2000: 136, 2010: 147, 2016: 250), DAPC (2010: 48, 2013: 51, 2016: 79) and intensive care unit (2000:7, 2010: 8, 2016: 14), falling trends in DRT (2000: 232, 2010: 257, 2016: 209), stable in DOS (2000: 63, 2010: 40, 2016: 40). $65 \%$ of patients dying at our cancer centre are from central region of the country, where also hospital is based, and there is no trend seen between 2000-2017.

Conclusion: Raising numbers of deaths in our hospitals can be explained by raising cancer incidence and at the same time, insufficient palliative care support in dying at home. In our hospital there is a huge demand in an additional PC support in DMO and DAPC. In order to lower the number of dying in a hospital and to provide $P C$ at home further PC education is needed as well as early PC implementation, in all regions of the country.

Abstract number: P02-406

Abstract type: Poster

Dying Worlds in Austria - The Perspectives of Professional Carers on 'Good Dying'

Heimerl K. ${ }^{1}$, Egger B. ${ }^{1}$, Schuchter P. ${ }^{2}$, Lang A. ${ }^{3}$

${ }^{1}$ Department of Nursing Sciences, University of Vienna, Vienna, Austria, 2Palliative Care and Organizational Ethics, University of Graz, Graz, Austria, ${ }^{3}$ Technoscience and Societal Transformation, Instute for Advanced Studies, Vienna, Austria

Background: The project "Dying worlds in Austria" primarily investigated the perspectives on 'good dying' of those affected. We conducted 30 qualitative interviews with people that had close experiences with death and dying. For our interview partners their caring networks were of utmost importance. Although for some of our interview partners dying is never beautiful, many of them told us about beautiful moments at the end of life.

Methods: We conducted three group discussions with professional carers. We asked the carers about their images of 'good death' and generated their feedback on selected quotes of the interviews with people affected.

Findings: Although the professional carers were occupied with death and dying on a daily basis, they were deeply moved by the quotes of the affected persons. As much as in the interviews with those affected, beautiful and elevating aspects were in the center of the statements of the professional carers, even if they alluded to 'bad situations'. In contrary to those affected, the professionals emphasized the difficult organizational conditions and caring networks were of minor importance.

Abstract number: $\mathrm{P} 02-407$

Abstract type: Poster

A Review of the Regional use of Palliative Care Support Beds Mulqueen L.

Our Lady of Lourdes Hospital, Palliative, Drogheda, Ireland Presenting author email address: laramul101@hotmail.com 
Background: The National Advisory Committee on Palliative Care report introduced the idea of palliative care support beds (PCSBs) as a way to provide an intermediate level of in-patient palliative care for patients in a local environment.

The National Clinical Programme for Palliative Care Working Group conducted a review of the organisation and function of PCSBs in Ireland in 2011, and found great regional variability.

In the region reviewed the criteria for admission is referral by the Palliative care team; predicted length of stay of 2-8 weeks; admission for end of life care, symptom management, respite or rehab.

Aims and objectives: To review the use of PCSBs and compare to regional guidelines and available national data.

Methods: Patient data from the 6 PCSBs in this region was collected and analysed using excel. Data from a 36 week period in 2017 was included.

Findings: The average age of admissions was 76.4 years.

6 patients $(11.7 \%)$ were under the age of 65.

$47 \%$ were female, $53 \%$ male.

$86 \%$ of patients had a diagnosis of malignancy.

Median length of stay was 14 days. Average length of stay excluding outliers was 19.7 days.

$47 \%$ of patients were admitted from home, $53 \%$ were referred from a hospital setting.

$54.9 \%$ of patients died in a PCSB, $33 \%$ were discharged home, $11.7 \%$ were discharged to another location.

The occupancy rate of PCSBs over this period was $89.6 \%$.

Conclusions: It is clear from the above data that referral to PCSBs in this region is not limited by age or diagnosis. It can be extrapolated that referrals are largely appropriate as evidenced by the low number of patients (2) who required emergency transfer to acute setting, and the average length of stay. This would imply that the current medical model is safe and effective for patient care.

Abstract number: P02-408

Abstract type: Poster

Rapid 24/7 Access to Hospice Inpatient Beds and End of Life Care Preferences

Das A. ${ }^{1,2}$, Lewis $K_{.}{ }^{1,2}$, Stark Toller C. ${ }^{1,2}$

${ }^{1}$ Palliative Medicine, University Hospitals Southampton, Southampton, United Kingdom, ${ }^{2}$ Palliative Medicine, Countess Mountbatten Hospice, Southampton, United Kingdom

Background: Place of death is a key quality indicator for end of life care. There is an increasing focus on developing services to enable people to die in their preferred place of death (PPD). Extensive literature suggests that most people would prefer to die at home; however, there is limited literature on people's preference to die in a hospice.

Aim: To investigate if a rapid $24 / 7$ access to a hospice inpatient bed enables people to die in their PPD.

Method: Rapid 24/7 access to this hospice's inpatient beds is defined as a category

A admission. End of life preferences were reviewed for all patients who died following a category A admission. The data for this study was collected for a six month period from April-October 2018.

Result: 100 patients were admitted as category A during this period. 41 patients ( $41 \%)$ died during the admission. Of these, 17 patients $(41.4 \%)$ expressed hospice as PPD. 9 patients (21\%) expressed no preference between hospice or home initially and confirmed their PPD as hospice at the time of admission. 7 patients (17\%) expressed home as PPD.

Some of the reasons for choosing the hospice as PPD included uncontrolled symptoms, young family at home, family or care giver not coping with death at home, or rapid deterioration.

Conclusion: Most patients who died during the admission expressed hospice as their PPD or confirmed their PPD as hospice at the time of admission. This suggests that for some people, death at home is not as important as other factors such as rapid access to symptom control and relieving care giver burden from the family.
This information on patients choosing the hospice as PPD and changing PPD to hospice has to be taken in to account while developing hospice services. Rapid 24/7 access to hospice inpatient beds will help meet end of life preferences for this group of patients.

Abstract number: P02-409

Abstract type: Poster

Analysis of Weekend Out of Hours Support as Part of Home Hospice Care Service

Katz G.J., Moss K.R., Sabar R.

Sabar Health, Even Yehuda, Israel

Background: Out of hours support is an essential part of the continuum of care provided to home hospice patients and caregivers. Addressing this need is a challenge, particularly for a large home hospice care service that treats $>550$ patients nation-wide at any time. A unique model of out of hours support by primary team during weekdays, and a central team over weekends, has been established.

Aim: The goal was to determine which parameters are associated with on-call team contacts during weekends, and try to further customize the ongoing support to better address patient and caregiver needs.

Methods: Over a six month period of Oct 2017 - Feb 2018, a de-identified call log was reviewed, including various parameters such as: caller role; reason for call; baseline disease; phase of illness; and number of days in home hospice.

Results: 922 calls were made over the period, accounting for 383 unique patients. $42 \%$ of patients treated during this period required weekend support. The average number of calls per patient was similar across indications (oncology/non-oncology). The majority of the calls were made within the patient's two weeks (median time in care 26 days). Unstable phase of illness accounted for nearly $50 \%$ of all calls during the initial weeks, and later decreased. Deteriorating remained stable, and Dying accounted for few calls. This finding led to the introduction of a mandatory call by teams to all Unstable patients prior to the weekend during the first 2 weeks. The call ensured adequate PRN treatment plan in place, as well as availability of medications and other tools to anticipate patient needs over the weekend. The impact of this call on need for weekend support is being evaluated.

Conclusion: Unstable phase of illness within the first weeks in home hospice is a combination of two significant risk factors for requiring weekend support. Awareness of this has allowed for better customization of support, and improved the overall patient care.

Abstract number: P02-410

Abstract type: Poster

\section{Outlook on Community Based Approach in Palliative Care at} Home in France

Genin F. ${ }^{1}$, Vie J.-F. ${ }^{1}$, Croixmarie F. ${ }^{1,2}$

${ }^{1}$ Visitatio, Boulogne Billancourt, France, ${ }^{2}$ Maison Médicale Jeanne Garnier, Paris, France

Problem: In France, 10,000 caregivers and 6,000 volunteers are dedicated to palliative care. Unfortunately the needs coverage remains insufficient, estimates varying from 20 to $44 \%$ coverage. In connection with the ambulatory shift and the axis 3 of the Palliative Care Plan 2015-2018, the solution to go further lies in the development of new approaches at home.

Method: Inspired by international experiences NNPC \& Saiatu, the model is based on 3 axes:

(i) palliative care at home is a global / human issue and a community / non-caring leadership helps to give every player its role (family, GP, nurses...) thus increasing eachother's engagement, 
(ii) to get volunteers involvement in palliative care at home, they need to belong to a local community,

(iii) to consolidate the involvement of everyone (ie local caregivers and as well as volunteers), the physical intervention of a local \& specialized medical team is requiered.

Results: To demonstrate the French ability to implement this model, an association was created in Jan 2017. In 15 months of operations (July 2017-Oct 2018), the association took in charge 42 people, $71 \%$ at home and $29 \%$ in hospices. 24 people died and 18 are still accompanied. $100 \%$ of those living in hospices died in the hospices -French average is $75 \%$-, the $25 \%$ remaining die at the hospital. For those previously living at home, $56 \%$ died at home, $25 \%$ in a palliative care unit and $19 \%$ at the hospital, where French average is $29 \%$ at home, $8 \%$ in a palliative care unit and $63 \%$ at the hospital. Social impact measuring through a specialized firm is under investigation. We already know that satisfaction scoring by all stakeholders is tremendously high and potential economical savings high.

Outlook: French authorities set a special regulation to have organizational innovations and reimbursment scheme innovations raising. Association is currently applying to start an official evaluation over a 2-3 years period.

The association is $100 \%$ funded by charity

Abstract number: P02-412

Abstract type: Poster

Optimising Patient Choice through Multi-professional Collaboration to Develop an Effective Referral Pathway in Enhanced Supportive Care

Franks A. ${ }^{1}$, Oliver K. ${ }^{1}$, Hollyoak S. ${ }^{1}$, McKelvie J. ${ }^{1}$, Malaczkoski A. ${ }^{1}$, Kelly B. ${ }^{1}$, Acquah D. ${ }^{2}$

${ }^{1}$ Arden Cancer Centre, University Hospitals Coventry \& Warwickshire NHS Trust, Coventry, United Kingdom, ${ }^{2}$ Arden Cancer Centre, UHCW NHS Trust, Coventry, United Kingdom

Aim: To ensure equity of choice for all patients attending our chemotherapy suite (CTS)for systemic anti-cancer treatment given with palliative intent to be offered referral to the Enhanced Supportive Care (ESC) team. Design, Method and Approach: Our centre is part of NHS England's pilot project looking at incorporating palliative care/medicine principles, attitudes and skills earlier in the disease trajectory, for patients with incurable cancers which are still potentially amenable to systemic anti-cancer treatments. Our biggest challenges have included convincing colleagues of the added value this service offers (to patients \& staff) and devising an efficient way to identify appropriate patients \& ensure that they are offered access to the ESC team.

Initially, the lead nurse personally trawled the daily CTS lists to identify potential patients \& spoke to each of them, but this was inefficient \& too dependent on one person. Secondly we attended the CTS morning board round, 'tagged' potential patients \& asked the CTS nurses to offer ESC leaflets to the patients, but this only worked very haphazardly, so failed on equity of offer. Our third method was developed following review of the existing pre-chemo. checks protocol with a senior CTS nurse \& subsequent discussion with our in house technology (IT) team.

This multi-professional approach has led to the development of a new, simple \& efficient referral management pathway being added to our comprehensive oncology therapy system, triggered by the CTS nurses as part of their comprehensive chemo. pre-administration checks.

Results: Referrals to the ESC team have now significantly increased. For those seen by the team, scores using the International Patient Outcome Scores (i-POS) in the majority of domains are improved by $5-72 \%$. Those not improved were nausea \& lack of energy.

Conclusion and Lessons learned: When introducing a new service multiprofessional collaboration, thinking laterally \& exploiting IT can be invaluable.
Abstract number: $\mathrm{P} 02-413$

Abstract type: Poster

How Many Outpatients Are Receiving Early Palliative Care in a

Cancer Palliative Care Clinic in a University Hospital?

Noguera A., Sanchez-Bayona R., Larumbe A., López-Fidalgo J., Muro E., Centeno $C$.

University of Navarra, Pamplona, Spain

Background: Outpatient clinics facilitates an ideal setting where to provide early palliative care. On $1^{\text {st }}$ January 2018 , we established a permanent palliative care consultant team (PCCT) at the outpatient cancer clinic of the Clínica Universidad de Navarra (Spain).

Aim: To describe the patients assisted by our team according to the major criteria of early intervention of an international consensus panel (Hui et al., 2018).

Method: Retrospective analysis on consecutive patients attended in the palliative care outpatient clinic needs-based and time-based criteria. We also describe reason for referral and problems found in the first evaluation.

Results: We include 50 patients in the analysis, males $34(68 \%)$ and age 61 years, $60 \%$ gastrointestinal cancer, $20 \%$ lung cancer. 38 patients $(68 \%)$ met both needs-based and time-based criteria (sample test of proportion $p<0.001)$. The main reasons for referral was severe physical symptoms (96\%), assistance with decision making $(16 \%)$, and severe emotional symptoms (14\%). The PCCT during the first evaluation identified severe emotional symptoms in 35 cases $(70 \%)$ and provide support in the decision making in 16 cases (32\%).

Conclusion: In our palliative care clinic, $68 \%$ of the patients referred are receiving early palliative care including the support in the decision-making process and detecting more easily emotional problems.

Abstract number: P02-414

Abstract type: Poster

Experiences from 10 Years of a Perinatal Palliative Care Program

Doherty M. ${ }^{1}$, Power L. ${ }^{2}$, Williams R. ${ }^{1}$, Stoppels N. ${ }^{1}$, Grandmaison Dumond L. ${ }^{1}$

${ }^{1}$ Pediatrics, Children's Hospital of Eastern Ontario, Ottawa, Canada,

${ }^{2}$ Roger Neilson House, Ottawa, Canada

Background: Perinatal palliative care is a relatively new component of paediatric palliative care supporting families who are expecting the birth of a child with a life-limiting condition. This study seeks to understand the characteristics of the babies and families referred for perinatal palliative care and the context for referrals in terms of diagnoses, referral characteristics, interventions, and outcomes.

Methods: A 10-year retrospective chart review of a perinatal palliative care program from 2007 to 2017 at a paediatric palliative care hospice.

Results: A total of 85 referrals were made for perinatal palliative care in the 10 -year period. Stillbirths were common, occurring in $27 \%$ cases. Of live-births, $57 \%$ of infants survived for 2 days, and $76 \%$ died within 30 days. Average gestational age at diagnosis was 23 weeks, and the mean gap between diagnosis and referral was 7 weeks. The most commonly referred types of conditions were trisomy 13 and $18(25 \%)$, severe CNS malformations (20\%), and cardiac disease (16\%). The majority of families (65\%) had at least one other child, identifying the need for specific supports for siblings in perinatal palliative care programs.

Discussion: Our study adds to the growing body of research concerning perinatal palliative care programming, confirming previously observed characteristics of diagnosis, referrals, and outcomes, while offering a more detailed account of outcomes for particular diagnoses. Comparison with existing literature is complicated by a wide variety of social contexts in which perinatal palliative programs are situated. Future investigation of the societal context concerning termination of pregnancy in a given setting and its influence on the dynamics of screening and referral processes, 
and decision-making by families and physicians in the presence of lifelimiting illness is needed. This research should take a collaborative approach amongst perinatal palliative care programs in various cultural settings.

\section{Abstract number: $\mathrm{P} 02-415$}

Abstract type: Poster

\section{Challenges of Integrated Palliative Care in the Health Care System \\ Lukacs M. ${ }^{1}$, Pakai A. ${ }^{2}$}

${ }^{1}$ Hospice-Palliative, University of Pecs Medical School, Pécs, Hungary, ${ }^{2}$ Faculty of Health Sciences, University of Pecs, Szombathely, Hungary

Objectives: The aim of our study was to estimate the relationship of the chances of dying in hospital or at home, based on demographic data, social support, healthcare categories and additional service needed in palliative care provided by multidisciplinar teams.

Methods: The study is a retrospective, quantitative analysis. Through a purposive sampling we analyzed records of tumor- and terminal stage patients who were involved in the service of the Pécs-Baranya County Hospice Foundation and met enroll criteria. ( $N=1389)$. Descriptive (absolute- and reltive frequency, variance,mean, modus, and mathematical statistics (correlation, chi-square test, varianceanalysis (ANOVA), T-test) were done on the sample $(p<0,05)$ using MS Excel and SPSS 22.0.

Results: Men are more likely to die in hospice care unit (OR $=1.369)$ compared with those who die at home. The county town living $(69.10 \%$, $\mathrm{OR}=0.625$ ), however, are less likely to die in hospice care. The number of days spent in care of $30.5 \%$ of a maximum of six days, and average length of stay in home care was 23.84 days (SD $=29.98$ ) and $46.1 \%$ of patients die in home care. We found significant positive correlation between the role of the healthcare scene and social support $(p=0.024)$, while the tumor disease type, deterioration of general status of the patient is significantly rise the chance of the integration into the intramural than home care $(p=0.002)$. The team providing complementary homecare tasks increase the outcome of optimal hospice-palliative care, chance of dying at the patient's home and the length of survival time $(p=0.027)$.

Conclusions: Providing an optimal hospice-palliative care needs a complex, holistic, integrated model of care to be implemented.

\section{Abstract number: P02-416}

Abstract type: Poster

\author{
Initiating the Development of Palliative Care Service in \\ Transnistria \\ Carafizi N. ${ }^{1}$, Isac V. ${ }^{2}$ \\ ${ }^{1}$ Hospice 'Angelus', Charity Foundation for Public Health 'Angelus \\ Moldova', Chisinau, Moldova, Republic of, ${ }^{2}$ National Association of \\ Palliative Treatment of Moldova, Chisinau, Moldova, Republic of
}

Introduction: Palliative care in Moldova was initiated in 2000 by some non-governmental organizations. It was recognized at the state level in 2008 and became a component part of the National Healthcare system. In Transnistria this kind of services is a new concept for both local medical society and general public of the region.

Objectives: To assess initiation of the palliative care development in Transnistria in regards of the developed activities.

Methods: Review of the reports on educational activities of the Charity Foundation for Public Health "Angelus-Moldova".

Results: During the year 2018, 15 medical specialists, including one clinical psychologist from Transnistria took part in 2-week intensive educational course. National clinical protocols and a Clinical Guideline were translated from Romanian into Russian. There was drafted further needs' assessment on palliative care in the region and development of the home-based and in-patients palliative care services.

Abstract number: $\mathrm{P} 02-417$

Abstract type: Poster

Palliative Care Matters: Progress Toward an Integrated Palliative Care Strategy for Canada

Fassbender K. ${ }^{1,2}$, Brenneis $\mathrm{C}^{2}$, Xiao J. ${ }^{2}$, Anderson S. ${ }^{2}$, Macmillan K. ${ }^{2}$

${ }^{1}$ University of Alberta, Department of Oncology, Edmonton, $A B$, Canada, ${ }^{2}$ Covenant Health, Palliative Institute, Edmonton, AB, Canada

Presenting author email address: konrad.fassbender@ualberta.ca

Palliative Care Matters (PCM) is a collaboration intended to develop a consensus toward optimal palliative care for all Canadians. An inaugural steering committee of 14 national stakeholders included government representatives, healthcare providers, non-governmental organizations, and patient-family advocates. To date, over 100,000 Canadians were engaged by PCM. A national survey of 1,540 Canadians generated evidence supporting urgent need for a framework. Experts in palliative care systematically reviewed the evidence. A consensus development conference was anchored by a distinguished lay panel and resulted in a report of 20 recommendations. The Conference Board of Canada provided context, insights and feasibility of those recommendations and potential next steps. The collective impact framework (CIF) was employed to identify 30 additional stakeholders from across Canada to develop an action plan for an integrated palliative care strategy (IPCS). The CIF represents a structured approach to help inform an integrated palliative care action plan. Fifty-five individuals met February 18, 2018 in Ottawa, Canada to identify five core elements of an action plan:

1) Essential elements of an IPCS;

2) Education, training and standards for health professionals;

3) Caregiver supports;

4) National secretariat and national center of research; and

5) Public awareness campaign.

Many organizations declared themselves as core for each of these five domains: 9, 10, 7, 7 and 4, respectively (note some organizations chose more than one domain as core). A high level of commitment from a large number of organizations necessitates a formal stakeholder analysis. A shared understanding of core stakeholders, their activities and strategic plans will help PCM with the following:

1) An agreement on the scope and nature of an IPCS;

2) Identification of the high-level components of an action plan for an IPCS; and

3) Development of the initial structure and process for the priorities of an IPCS.

Abstract number: P02-418

Abstract type: Poster

How Do Patients Followed by a Palliative Care Team Use the Emergency Department?

Tavares T. ${ }^{1}$, Rema J. ${ }^{2}$, Tavares M. ${ }^{3}$, Gonçalves E. ${ }^{2}$

${ }^{1}$ Serviço de Medicina Interna, Unidade Local de Saúde de Matosinhos, Leça da Palmeira, Portugal, ${ }^{2}$ Serviço de Cuidados Paliativos, Centro Hospitalar São João, Porto, Portugal, ${ }^{3}$ Instituto Português de Oncologia do Porto Francisco Gentil, Porto, Portugal

Background: Patients with palliative needs often use the Emergency Department (ED), which is usually assumed as a poor quality indicator. It 
is important to describe this reality and identify modifiable factors that can improve the care dynamics of this population.

Aim: To characterize the use of ED by patients accompanied by a Palliative Care team (PCT).

Methods: A retrospective observational study was performed to analyse the ED episodes of patients accompanied by a PCT, in their last year of life (LYL). They were included if they had 2 or more ED episodes after being accompanied by PCT. This PCT includes in-hospital support team, clinic and home team, and runs from $8 \mathrm{am}$ to $6 \mathrm{pm}$ on weekdays and on Saturday mornings. Data was collected from hospital computer systems and PCT database and statistically analysed with Excel 2011.

Results: Of the 680 patients followed by PCT, 39 (5.7\%) used the ED 2 times or more, totalizing 118 episodes. Median age 69 years, 59\% male, 90\% oncological and Palliative Performance Scale median 50\%. Each patient used the ED on average 4 times during the LYL. After starting PCT accompaniment, patients visited the ED 1.3 times per month. The most frequent complaints were dyspnea (31.4\%) and pain (23.7\%). In 19 episodes there was therapeutic adjustment, in 46 hospitalization and in 2 the patients died in the ED. Only in 4 of the episodes the patient was being followed by the PCT at home. Over $50 \%$ of the episodes happened outside of the team schedule. In the LYL, each patient had about 3 consultations with their general practitioner (GP).

Conclusion/ discussion: In the LYL, only a small percentage of patients (5.7\%) accompanied by a PCT (without 24-hour care) continued to rely on the ED, most of them outside of the team schedule. The reported data point out possible measures of improvement in the approach of these patients, such as greater support from GP and home teams. Future research on the patient's and family's perspectives is important.

Abstract number: $\mathrm{P} 02-420$

Abstract type: Poster

Hospice-Palliative Care in Hungary. Data, Standards and Integration Model

Lukacs M.1,2, Munk K. ${ }^{2}$, Hegedus K.2,3

${ }^{1}$ Hospice-Palliative, University of Pecs Medical School, Pécs, Hungary, ${ }^{2}$ Hungarian Hospice-Palliative Association, Budapest, Hungary, ${ }^{3}$ Faculty of Medicine Institute of Behavioural Sciences, Semmelweis University, Budapest, Hungary

Objectives: Modern palliative-hospice care has gained space in Europe for more than 50 years. The palliative medicine has gained its place in evidence-based medicine in more and more countries. Hungarian palliative-hospice care has developed significantly over the past 15 years. The review mainly demonstrates the hospice care data of 2017.

Methods: In order to review the development of the nationwide palliative care the Hungarian Hospice- Palliative Association sends a detailed online questionnaire to each hospice services every year. At the beginning of 2018 we contacted 84 care providers. In the questionnaire we asked them - among other things - about the form of hospice care, the number of patients, the period of care, the mortality data and about the service providing team and their qualifications. We paid special attention to the available resources and the main difficulties of the care providers.

Results: According to the survey, 93 health care providers performed hospice activities in Hungary. In 2017 Hungary's population is 9,781 million. The number of deaths per year is 103,600 of which deaths from cancer are annually 32,000 . Of those who died of cancer, 9091 people taken care of palliative care. The number of nursing days was 232,636 days (average: 25.6 days), 24.8 days for institutional care, 29.1 days for home care. Bed occupancy was $74.8 \%$. Of the patients in hospice care, $5798(63.8 \%)$ died; $76.8 \%$ of the patients in the institutions died, while $61.4 \%$ of those in the home care sector died. The proportion of cancer patients was $90.5 \%$

Conclusions: The integration of palliative care into standard oncology is an international requirement, which also appears in the form of professional guidelines. Hungary has also played a role in the development of the European model of integrated palliative care of which Hungarian implementation, the Pecs model, is discussed in detail in our paper.

Keywords: Palliative-hospice care; integrated

Abstract number: P02-421

Abstract type: Poster

The Palliative Care Consultation in a University Hospital

Cerullo G. ${ }^{1}$, Abreu K. ${ }^{2}$, Carrancha M. ${ }^{3}$, Rodrigues M. ${ }^{3}$, Silveira A. ${ }^{3}$, Lima

$D .^{3}$

${ }^{1}$ Internal Medicine, University Hospital of Algarve, Faro, Portugal,

${ }^{2}$ Family Health Unit - Farol, Faro, Portugal, ${ }^{3}$ University Hospital of

Algarve, Faro, Portugal

Background: Palliative Care (PC) aims is to improve the quality of life (QOL) of people with a serious and / or incurable disease with a limited prognosis, preventing and alleviating suffering, identifying and treating: physically, physical, psychological, social and psychological problems, in a holistic approach.

Aims: Describe the activity in outpatient consultation in Palliative Care of a University Hospital.

Methods: Retrospective descriptive study, consulted the processes of the patients observed in Palliative Care consultation between 01.01.2017 to 31.12.2017.

Results: A total of 663 consultations were recorded, corresponding to 215 (32.4\%) for first consultations and a total of 272 patients: 156 males (57.4\%), mean age of 74 years ( 37 to 95 years).

Regarding the origin of the request for consultation: $19.7 \%$ of the patients were referred by the Medical Oncology, followed by Pneumology with $14.9 \%$. The three most frequent diagnoses in the first consultations were: colorectal cancer in 40 patients (18.6\%), followed by lung cancer in $32(14.9 \%)$ and stomach in $23(10.7 \%)$. Of the patients seen in the consultation, $144(70 \%)$ died and $16(7.4 \%)$ were transferred to the Home Support Team in Palliative Care. The mean follow-up time in the outpatient clinic was 235 days ( 1 to 1095 days).

Discussion: Palliative Care consultations allow for symptomatic control and improvement of patients' quality of life during their stay at home. The importance of carrying out the evaluation of the casuistry of the external consultation is necessary in the face of the need to characterize the users in order to optimize the care delivery. The data obtained show a relatively short follow-up time, since the population that we follow because of its advanced age and its comorbidities consequently has a shorter survival. This fact can also be boosted by a late referral of the patients, being essential the training of health professionals in the early recognition of palliative cases.

Abstract number: $\mathrm{P} 02-422$

Abstract type: Poster

Patients Admitted to a Palliative Care Unit in a University

Hospital

Cerullo G. ${ }^{1}$, Abreu K. ${ }^{2}$, Carrancha M. ${ }^{3}$, Rodrigues M. ${ }^{3}$, Silveira A. ${ }^{3}$, Lima

$D .^{3}$

${ }^{1}$ Internal Medicine, University Hospital of Algarve, Faro, Portugal,

${ }^{2}$ Family Health Unit - Farol, Faro, Portugal, 3University Hospital of

Algarve, Faro, Portugal

Background: Palliative Care (PC) are all actions aimed at improving the quality of life (QOL) ofpeople suffering from a serious and / or incurable disease with limited prognosis, preventing and alleviating suffering: early identification and treatment of physical, psychological, social and spiritual symptom, with holistic approach. These care are more effective if organized in defined services.

Aim: This study intends to analyse the hospitalizations in the PC Service of a University Hospital. 
Methods: Retrospective descriptive study, the hospitalization processes were consulted between 01.01.2017 and 31.12.2017, and analysing (Excel ${ }^{\circledR}$ software)

Results: Control complex symptoms(CS) led to the hospitalization of 249 patients (258 hospitalizations), average delay of 10.9 days. CS: pain, hemorrhage, intestinal occlusion, delirium and agony. 53.8\% males, mean age of 70.4 years (37-95 years), Karnofsky's performance status less than $60 \% .45 .4 \%$ came from other wards, $37.7 \%$ from the Emergency Department and $16.8 \%$ from the PC consultation. The three most frequent diagnoses: colorectal neoplasia (22.9\%), lung neoplasm (12.4\%) and stomach neoplasia (8.4\%). All patients were treated with opioids. Administration: transdermal in $92 \%$, oral $71.2 \%$, intravenous in $41 \%$, subcutaneous in $26 \%$ and rectal in $2 \% .188$ deaths. In addition to discharge due to death, patients were referred to the home and PC consultation, to the Home Support Team in PC or other units.

Discussion: Internment in palliative care is recommended for the management of complex symptomatology. The number of deaths observed during hospitalization reflects not only the fact that patients are suffering from complex and consequently more severe symptoms, as well as the inadequacy of the resources available in the community to the needs of these patients. We continue to observe a late referral of these patients, and it is important to continue to focus on the training of health professionals.

\section{Abstract number: $\mathrm{P} 02-423$ \\ Abstract type: Poster}

Last Aid International - Implementation of an International Working Group on Last Aid Courses for the Public Bollig G. $^{1,2,3}$

${ }^{1}$ University of Southern Denmark / Institute of Regional Health Research, Palliative Care Research Group, Sønderborg, Denmark, 2South Jutland Hospital, Palliative Care Team, Medical Department, Sønderborg, Denmark, 'ast Aid International, Schleswig, Germany Presenting author email address: georg.bollig@rsyd.dk

Aims: Although most people want to die at home many people die in institutions. Last Aid Courses can serve as basic palliative care education for the public and compassionate communities and may enhance the possibility of dying at the preferred place. Pilot courses in Norway, Denmark and Germany have shown the feasibility and acceptance of the course. The main goals of the current project were to establish an international Last Aid working group and to reach consensus on the curriculum for an international Last Aid course.

Methods: Associations engaged in palliative care in European countries were invited to participate in an international working group. A variety of national organisations such as palliative care organisations, healthservices, the church or other national organisations were invited. Participating countries send two delegates as participants to the meetings of the working group. All working group members reviewed the existing Last Aid Course curriculum.

Results: National organisations from seven countries participate in the international Last Aid working group. Consensus on an international Last Aid Course curriculum was established during a two-day meeting of the working group consisting of experts from different professions working within palliative care. The adaptation of the international presentation is finished. Work with translation into different languages and education of Last Aid course instructors is ongoing.

Conclusions: The first experiences with Last Aid courses are very promising. The formation of an international Last Aid working group and consensus on a curriculum has been achieved in 2018. Research on the implementation and effect of Last Aid courses and a wider distribution and inclusion of other countries is ongoing. Meetings of the working group in order to evaluate and adapt the curriculum will be held with regular intervals of 1-2 years.
Abstract number: P02-424

Abstract type: Poster

Soul Injury; How Stressful Experiences from the Past and Posttraumatic Stress Disorder (PTSD) Can Become Exacerbated at the End of Life and Complicating Peaceful Dying Ulrich $S^{1,2}$

${ }^{1}$ University of Berne, Switzerland, CAS: Theological, Phil. Hum., Faculty of Medicine, Berne, Switzerland, ${ }^{2}$ RFM Royal Foundation Management, Palliative Counseling, Zug, Switzerland Presenting author email address: info@palliative-counseling.com

Background: Soul Injury, originally emerged in a VA-Hospice in the USA, is not only used to explain emotional suffering but stands for a healthcare concept also used in crisis intervention and in End of Life Care.

Aim: Spiritual Care providers in Europe also, are confronted with patients diagnosed with PTSD or patients that may had stressful or traumatic experiences, such as for example refugees, victims of physical and mental violence, terror etc. Professionals should become sensitized why such stressful experiences may complicate peaceful dying. Soul Injury, stoicism and PTSD are a few issues that need to be considered.

Method: The study contained a CAS paper and educational trainings that informed about the unique care needs of patients struggling to overcome events from the past and application method used within the concept of Soul Injury. At the trainings, participants learnt why stressful experiences in the past or PTSD may exacerbate and influence End of Life Care. An overlooked wound, identified as Soul Injury, is explained in the context of Spiritual Care. Surveys were answered by the participants to determine the applicability of Soul Injury in Europe. The trainings took place at the University in Berne and in other cities in Switzerland.

Results: Participants understood why unmourned loss, unforgiven guilt and shame can burden for a lifetime. Interestingly, the evaluation showed that $87 \%$ of the participants thought that they may know someone with a Soul Injury and are more prepared to support them with the understanding gained.

Conclusion: The outcome demonstrated, that the tools and knowledge about the concept of Soul Injury were valuable in the attendees' professional and private lives. Thus, the understanding of its tools, was confirmed as professional, yet simple in its application. Moreover, it also suggested that Spiritual Care and generally End of Life Care providers in Europe should benefit from the lessons of Soul Injury.

Abstract number: $\mathrm{P} 02-425$

Abstract type: Poster

"People Want to Talk about Death and Dying" - Experiences from the Implementation of Last Aid Courses in Germany Bollig G. ${ }^{1,2,3}$, Schmidt M. ${ }^{3}$, Hornke I. ${ }^{3}$, Knopf B. ${ }^{3}$

${ }^{1}$ Palliative Care Team, Medical Department, South Jutland Hospital, Sønderborg, Denmark, 2Palliative Care Research Group, University of Southern Denmark / Institute of Regional Health Research, Sønderborg, Denmark, ${ }^{3}$ Letzte Hilfe Deutschland, Schleswig, Germany

Aims: The concept of Last Aid courses for the public was first described by Bollig in 2008. Last Aid courses aim at introducing the public to palliative care and to empower citizens to participate in the provision of endof-life care at home. The feasibility and acceptance of such courses have been shown after starting pilot courses in Norway, Germany and Denmark in the years 2014 and 2015. The main goal of the current project was to implement Last Aid courses in different German regions and to educate instructors.

Methods: People with practical experience from the field of palliative care and different backgrounds were educated as Last Aid course instructors during a one day instructor course. Potential instructors had to show 
their ability to teach the public during the course by giving a trial lecture.

Results: Within the period from 2015 to September 2018750 instructors have been educated and more than 6000 people have participated in Last Aid courses in Germany. Most participants appreciate the course, its content and discussion death and dying with others. In 2015 the concept was awarded by the German Association for Palliative Medicine. A scientific evaluation of the questionnaires of more than 5000 participants is ongoing. A first German Last Aid symposium was held in 2017 and the second held in October 2018.

Conclusions: Within three years the Last Aid courses in Germany have been transformed from being a pilot project to a well-known educational concept for palliative care and end-of-life care for the public. In order to met the great demand for Last Aid instructor courses an own $n$ on-governmental organization has been established under the name of "Letzte Hilfe Deutschland". More info from www.letztehilfe.info.

Abstract number: $\mathrm{P} 02-426$

Abstract type: Poster

What We See Is What You Get (We Hope): Progress for Competency Based Medical Education in Postgraduate Hospice and Palliative Medicine Education in the USA

Radwany S. ${ }^{1}$, Gustin J. ${ }^{1}$, Landzaat L. ${ }^{2}$, Morrison L. ${ }^{3}$, Barnett M. ${ }^{4}$, Buckholz G. ${ }^{5}$, Hwang J. ${ }^{6}$, Levine S. ${ }^{7}$, Okon T. ${ }^{8}$, Yang H. ${ }^{9}$, Christensen A. ${ }^{10}$, Johnston C.B. ${ }^{11}$, Edgar L. ${ }^{12}$

${ }^{1}$ Palliative Medicine, Ohio State University, Columbus, United States, 2Internal Medicine, University of Kansas Medical Center, Kansas City, United States, ${ }^{3}$ Yale Palliative Care Program, Internal Medicine, Yale School of Medicine, New Haven, United States, ${ }^{4}$ University of Alabama at Birmingham, Birmingham, United States, ${ }^{5} \mathrm{H}$ ospice and Palliative Medicine, University of California San Diego / Scripps Health, San Diego, United States, ${ }^{6}$ Children's Hospital Philadelphia, Perelman School of Medicine, University of Pennsylvania, Philadelphia, United States, ${ }^{7}$ University of Chicago, Chicago, United States, ${ }^{8}$ Marshfield Clinic, Marshfield, United States, ${ }^{9}$ University of California San Diego/ Scripps Health, San Diego, United States, ${ }^{10}$ Palliative Medicine, Mayo Clinic, Rochester, United States, ${ }^{11}$ Geriatric and Palliative Medicine, University of Arizona, Tucson, United States, ${ }^{12}$ Vice President for Milestone Development, Accreditation Council for Graduate Medical Education, Chicago, United States

Background: The Accreditation Council for Graduate Medical Education (ACGME) in the USA provided a framework of competency-based medical education (CBME) for residents and fellows called the Next Accreditation System (NAS) in 2012. Within the NAS, ACGME emphasizes "measurement and reporting of outcomes through the educational milestones" including: Entrustable Professional Activities (EPAs), Curricular Milestones (CMs) and Reporting Milestones (RMs).

Aims: Describe the process and results of the development of EPAs, CMs and RMs for Hospice and Palliative Medicine (HPM) fellowship training in the United States.

Methods: From 2014 to 2018 HPM EPAs and then CMs were developed through an iterative consensus process involving an expert workgroup, vetting at a national meeting with HPM educators, and an electronic survey of practicing HPM physicians and physician educators respectively. The HPM RM's were developed in 2017 and 2018 with the ACGME convening broadly based interdisciplinary expert panels of adult and pediatric providers, educators, trainees and community members.

Results: In a national survey, 362 HPM specialists responded (10\%), including 58 of 126 fellowship program directors (46\%) indicating that the set of 17 EPAs well represented the core activities of HPM physician practice (mean 4.72 on a five-point Likert scale) and considered all EPAs to either be "essential" or "important". In a national survey of 203 HPM Fellowship Directors and physician educators regarding the $22 \mathrm{CMs}$ there was a $57 \%$ response rate. All CMs met the minimum agreement level of $75 \%$ or greater with most over $85 \%$.
Conclusion / Discussion: Efforts at developing these tools for improving postgraduate medical education in HPM were thorough and rigorous. Next steps will include the creation of evaluation tools based on the EPAs and $\mathrm{CMs}$, tracking their use in curricular development and analyzing results of RM reporting in their first years of use.

\section{Abstract number: $\mathrm{P} 02-427$ \\ Abstract type: Poster}

Palliative Care Education in the Medical Curriculum: Views from Master Students

Wens J., Van den Broek K.

Primary and Interdisciplinary Care, University of Antwerp, Wilrijk, Belgium

Recently, the medical education in Belgium scaled down from 7 year training to 6 year, involving considerable curriculum changes. One of these is the reduced visibility of some courses as palliative care, despite the recommendations from the Belgian Royal Academy of Medicine, the Belgian Healthcare Knowledge Centre and the EAPC.

During a palliative care teaching class we inquired all last year medical students $(n=88)$ about their views on obtained knowledge on palliative care during their past training using an adapted version of the PEAT (Palliative Education Assessment Tool).

In global, according to most students, their knowledge about pain treatment and symptom relief was sufficient, unless for complementary treatments. Also their broader communication skills were assessed as sufficient, but with the exception of specific topics as "assigning treatment targets with patients or relatives", "reporting the death to relatives", "discussing advanced care planning", "assigning abstinence or quitting further treatment". More problematic were psychosocial and spiritual topics of palliative care in which less than half of all students felt sufficiently trained. Finally, and most dramatically, far most of all students felt knowledge shortage on ethical and legal topics about DNR dilemmas, death certification, assessment of autonomy of decision, proportionality in treatment, and the concept of inappropriate care at the end of life. Most obvious shortcomings according to these students were gaps in the education about legislation and clinical performance of palliative sedation and euthanasia. Half of all students (44/88) missed a clinical internship in palliative care during their educational pathway. Training in palliative care seen by students proved serious gaps in education that was evaluated overall as on hand but rather fragmented. Based on this survey, guided initiatives will be taken to improve the presence of palliative care content in the newly developed curriculum.

Abstract number: P02-428

Abstract type: Poster

Teaching to Teach: Palliative Care Specialist Nurse Perceptions of the Impact of an Education and Skills Training Programme Groves K. ${ }^{1}$, Jack B. ${ }^{2}, O^{\prime}$ Brien M..$^{2}$, Kinloch K. ${ }^{2}$

${ }^{1}$ Palliative Medicine, Supportive \& Specialist Palliative Care Services, Southport, Merseyside, United Kingdom, 2Evidence Based Practice Research Centre, Edge Hill University, Ormskirk, United Kingdom

Background: Central to national and international policies is the need for generalist healthcare staff to have education in end-of-life care. Much end-of-life care education is provided by specialist nurses, who often have no specific educator development, to prepare them to teach. To address this identified gap, an Educator Development Programme (EDP) was developed and delivered to specialist nurses. We report on the independent evaluation of the programme.

Methods: A mixed methods evaluation was adopted, with purposive sampling of 20 participants who completed the EDP and agreed to participate in the evaluation. Each participant provided a pre and post test score on a Likert scale for willingness and confidence to teach junior staff, staff at the same grade and medical staff. Data were analysed using 
descriptive statistics. Focus groups were undertaken post-course at 3 education centres in Northwest England. Data were digitally recorded and subjected to thematic analysis. Ethical approval was obtained.

Results: An improvement was found in all participants' reported willingness and confidence to teach all grades of staff, particularly medical staff. From the focus groups two main themes were identified; learning to teach and building skills to change practice. Participants reported greater confidence and preparedness for their teaching roles. Their growth in confidence and the practical skills they have gained have equipped the participants to be able to teach a diverse workforce.

Conclusions: It cannot be assumed that specialist staff, with teaching as part of their role, have the skills to facilitate learning. This programme offers a potential method of improving facilitation skills. Specialist staff with teaching responsibilities should be provided with education and training to develop their teaching and facilitation skills

Funding: Cheshire \& Merseyside Palliative and End of Life Network Education Strategy Group

Abstract number: P02-429

Abstract type: Poster

Growing in Confidence: Impact of New One-day Advance Care Planning Course on Frontline Clinical Staff Groves K. ${ }^{1}$, O'Brien M. ${ }^{2}$, Jack B. ${ }^{2}$, Kinloch K. $^{2}$

${ }^{1}$ Palliative Medicine, Supportive \& Specialist Palliative Care Services, Southport, Merseyside, United Kingdom, ${ }^{2}$ Evidence Based Practice Research Centre, Edge Hill University, Ormskirk, United Kingdom

Background: Advance care planning (ACP) is a central part of UK and international end of life care policies. Yet studies have shown barriers exist with staff reporting concerns about undertaking ACP conversations.

A one day ACP course was developed for frontline health and care staff and an ACP "train the trainer" course was developed to train facilitators who subsequently delivered it. We report an independent evaluation of this training.

Aim: To explore frontline staff views of the impact of a one-day Advance Care Planning course on their confidence and knowledge of ACP

Methods: All frontline staff in the first cohort (August 2017 - June 2018) were invited to complete a confidential 5-item survey online or in hard copy after the end of their training. Quantitative data was analysed using descriptive statistics. Qualitative responses to the free text question, on what one thing from the course would be taken back to practice, were analysed using content analysis.

Results: 248 staff responded. The quantitative data indicated $94 \%$ of respondents agreed their knowledge of ACP had increased, 95\% agreed that they were more confident about undertaking ACP in their role. Over 95\% responded positively about the course structure and how it was easy to follow. Four themes were identified in the open text responses including; the importance of $\mathrm{ACP}$, confident communication, recording and documenting ACP and being an ACP advocate. The most frequently identified theme was confident communication.

Conclusions: Staff who undertake training in ACP are more confident incorporating ACP into their everyday practice. The key aspect frontline staff perceive as important to their role is confidence in communicating with patients and families.

Funded by Health Education England via the Cheshire and Merseyside Palliative \& End of Life Care Clinical Network \& Greater Manchester \& Cheshire Strategic Clinical Network.

Abstract number: P02-430

Abstract type: Poster

Danish Nurses' Perceived Confidence within Palliative Care of Patients with Heart Disease

Holmegaard B. ${ }^{1}$, Ellekrog Ingwersen G. ${ }^{2}$, Sig - Palliation
${ }^{1}$ Medicine, Holbaek Hospital, Holbaek, Denmark, ${ }^{2}$ Cardiology, Zealand University Hospital, Roskilde, Denmark

Background: Palliative Care within cardiology are evolving in Denmark. European and Danish Society of Cardiology have published position papers on palliative care. However, studies indicate that cardiovascular nurses perceive lack of confidence on palliative care.

Purpose: This study is to explore Danish nurses' perceived confidence within palliative care of patients with heart disease.

Method: An electronic survey were distributed September to November 2017 to nurses engaged in palliative care from hospitals, hospices and municipalities. Using validated measures of The Self-Efficacy in Palliative Care (SEPC) scoring confidence (0) versus anxiety (100) in three domains; management, communication and multidisciplinary activities, and Approach to Death and Dying Patients Attitude Scale (ADDPAS) containing two domains rated on a Likert Scale. Descriptive statistics present results.

Results: Out of 131 nurses, $86(66 \%)$ responded. Nurses do not perceive themselves as very confident. Mean scores of the three domains; communication 38.2( $\mathrm{Cl} 32-53)$ management $42.6(\mathrm{Cl} 32-53)$ and multidisciplinary activities $43.3(\mathrm{Cl} 37-50)$ Nurses perceive less confidence in discussions with relatives about death to occur 51.4, consultations about suffering 52.6, identifying the patient with a remaining life within 1 year 53.0 and remediating medication 50.9 . Nurses perceive most confidence in discussing issues of dying 31.7 and $96 \%$ perceive confidence in communication within end-of life care. Most confident are nurses in managing dyspnea (89\%), edema (89\%) and pain relief (88\%). Related to multidisciplinary activities $39 \%$ perceive lack of knowledge of social support.

Conclusion: Danish nurses' perceive neither very confident nor very anxious within palliative care, but nurses perceive confidence within end-oflife care. Nurses lack confidence in communicating and multidisciplinary activities in supportive palliative care. This reveals a need for educational activities.

\section{Abstract number: P02-431}

Abstract type: Poster

Education Needs of Nurses and Healthcare Assistants Providing Level II Palliative Care

Agbana S. ${ }^{1,2}$, White L. ${ }^{3}$, Connolly M. ${ }^{1}$, Guerin S. ${ }^{3}$

${ }^{1}$ School of Nursing, Midwifery \& Health Systems, University College Dublin, Dublin, Ireland, ${ }^{2}$ Our Lady's Hospice and Care Services, Blackrock, Dublin, Ireland, ${ }^{3}$ School of Psychology, University College Dublin, Dublin, Ireland

Palliative care is an approach of care that aims to improving quality for patients with life limiting conditions and their families through the prevention and relief of suffering, through early identification, assessment, and treatment of pain and other distressing symptoms: could be physical, psychological and spiritual. Palliative care is delivered at three levels; Levels 1 (General Palliative care), Level 2 (Supportive Palliative care) and Level 3 (Specialist Palliative Care).

This study sought to explore the experiences of nurses and healthcare assistants providing Level 2 Palliative Care with particular focus on their education needs. The research was carried out in a resident care setting which provides level 2 palliative care. Ethical approval for the study was obtained from a University Research Ethics Committee.

A qualitative methodological approach was employed. A purposive sampling of nurses and health care assistants working in the residential care setting were invited to take part in focus group interviews. Interviews were recorded electronically, transcribed verbatim and analysed using van-Manen's method of analysis.

Findings demonstrated the importance of continuing education for the delivery of palliative care regardless of the years of experience of the 
individual. The findings also highlighted the education needs of the Nurses and Healthcare Assistants working in Level 2 Palliative Care. Addressing these education needs will ensure that the individual healthcare professional and the organisation needs are met while delivering effective care to patients diagnosed with life limiting illnesses and their families.

Abstract number: $\mathrm{P} 02-432$

Abstract type: Poster

Increased Competence Can Prevent Complications and Unnecessary Hospital Admissions of the Frail Old Dale G.H., Eikemo S.B.

The Dignity Center, Bergen, Norway

Background: Research has shown a lack of competence among Norwegian nurses and nurse assistants in elderly care regarding recognition of early signs of severe and acute disease, and how to respond in these situations in order to avoid further complications and hospital admission.

Aim: To develop and evaluate an interdisciplinary education program for employees in the elderly care, intended to increase competence regarding acute and severe illness in the frail old.

Methods: An education program spanning six days was developed. The program consisted of a combination of lectures and training in practical procedures. The themes concerned mainly acute and severe conditions such as respiratory and circulatory failure, in addition to advance care planning and palliative care. Ethical reflections regarding treatment vs over-treatment were central.

All 122 participants in 2017 were invited to fill out an electronic evaluation form three months after completing the education. The purpose was to find out if the education program had been useful in the participants' everyday work. Ninety-eight (80\%) replied.

Results: Most of the respondents were nurses with/without specialization $(78 \%)$ or nurse assistants with/without specialization (19\%). The evaluation showed for instance that $93 \%$ felt more confident in their everyday work, both theoretically and during practical procedures. Many gave examples of situations where they had actively used the achieved knowledge and for instance been able to avoid hospitalization of patients. An unexpected, but positive consequence, was that many had afterwards taught their colleagues.

Conclusion: An interdisciplinary education program developed for employees in the elderly care, can increase the confidence in caring for the frail old when acute and severe illness occurs. Increased competence can help prevent unnecessary hospital admissions, so the frail old instead can be cared for in familiar surroundings and by staff they know.

Abstract number: $\mathrm{P} 02-433$

Abstract type: Poster

Innovative Approaches in Education: Lightbulb Moments in the Asia Pacific Region

Aggarwal G. ${ }^{1,2}$

${ }^{1}$ Concord Centre for Palliative Care, Concord West, Australia, ${ }^{2}$ The University of Sydney, Sydney, Australia

Background: The scope of education is a paramount responsibility of all clinicians in the development of palliative care. It is not enough to practice without a focus on the dissemination of palliative care concepts to generalists, administrators and the community at large. Education abilities for clinicians take time and structure to become effective and useful, requiring a dynamic process of learning and refining over time.

Aim: To describe strategies utilised in the development of a palliative care education program in the Asia Pacific Region.

Methods: As a Palliative Care Physician in a university teaching hospital in Sydney, Australia, it was a learning experience to refine and improve teaching and facilitation skills to adequately meet the challenges of culture, language and limited resources when teaching in the Asia Pacific Region over 15 years.

Results: Education methods needed to be tweaked to meet different needs of the target audiences: to influence government organisations to commence development of palliative care and make opioids available; to educate the general public to improve death literacy; to teach senior specialist consultants who are well versed in their specialty but have not considered palliative care, quality of life, symptom control as priorities in their practice; to train young doctors and nurses who are considering future specialisation in this new field of practice. How does one effectively gear the education experience to this wide diverse group with varying outcome needs!

Conclusion: This presentation explores the challenges and dynamic changes required through the education process to target various learning outcomes, dependent on the audience and their needs within resource limitations of the country. An experiential perspective gained showing outcomes both achieved and errors made over a decade will be demonstrated.

Abstract number: $\mathrm{P} 02-434$

Abstract type: Poster

Integrated Palliative Care Champions

Parr P., Campbell M., Victoria Ali.

Specialist Palliative Care, Aintree University Hospital, Liverpool, United Kingdom

Background: Specialist Palliative Care services in the locality are integrated; yet no integrated 'link nurse' programme of education had been delivered to Nurses and Allied Health Professionals (AHPs) from across clinical settings. Areas had historically held their own version of a programme aimed at Nurses within their clinical setting with no element of integration.

Aim: To implement an integrated Palliative Care Champion programme of education aimed at Nurses and AHPs from across locality settings in order to enhance knowledge, skills and confidence of the Champions when providing palliative and end of life care and assist them in the delivery of cascade training in their own area.

Method: In partnership with community colleagues we developed a programme that would incorporate all aspects of palliative and end of life care.

The Champions programme has Nurses and AHPs from the acute hospital, district nursing teams, specialist neurological centre, prison and local hospice.

The programme is delivered with a full launch day to bring the group together and outline the purpose and aim of the group and programme. Champions are invited to make a 'pledge' to change or improve an area of practice in their clinical area with the support of the programme. Subsequent sessions are then held monthly for two hours with a different subject each month and an evaluation session at the end of the programme.

Results: The programme has brought together Professionals from across settings, encouraging shared learning and a recognition of challenges faced when providing palliative and end of life care in any setting. Innovations in practice have occurred, knowledge and confidence in care provision has grown. The programme has been well evaluated by participants and continues to expand.

Conclusion.The programme is in its third year in order to ensure no gaps develop in areas as staff move role. Champions are now taught skills needed to train and develop others to expand the impact of the programme.

Abstract number: $\mathrm{P} 02-435$

Abstract type: Poster 
Learning Field Palliative Care in the Region of the Palliative Care Network Zuid Gelderland in the Netherlands

Nogarede R. ${ }^{1}$, Verschuur E.M.L. ${ }^{2}$

${ }^{1}$ Palliative Care Network Zuid Gelderland, Nijmegen, Netherlands, ${ }^{2} \mathrm{HAN}$ VDO, HAN University of Applied Sciences, Nijmegen, Netherlands

Background: One of the goals of the Palliative Care Network Zuid Gelderland is to improve the offer of education and training programs for healthcare professionals. For that reason we initiated the project "Working together on professional development". The aim is to develop a high standard and future proof regional Learning Field Palliative Care for healthcare professionals, especially nurses and nurse assistants.

Methods: During 8 meetings with educators and nurses, several domains were explored: Care \& Well-being, Development, Knowledge, Management, Social and Role \& Function domain. Didactic or educational models and methods that could serve as an instrument for educational purposes were thoroughly discussed.

Results: The outcomes of the meetings resulted in developing objectives and criteria regarding competences of nurses and nurse assistants in palliative care. These criteria are described in so-called Rubrics, ordered according to the CanMeds roles. Rubric means "a scoring guide used to evaluate the quality of students' constructed responses". Each Rubric is focused on measuring behavior and contains specific performance characteristics in levels indicating the degree to which the standard has been met. The Rubrics we developed can be used for different purposes: as a self-assessment tool, a feedback tool for trainees, for the development of education programs and as a Human Research tool.

Conclusions: The co-creation of the Rubrics has led to commitment of all participants in our Palliative Care Network. It is a first step towards the development of a Learning Field Palliative Care, with the aim improving the quality of palliative caregiving in our network.

Abstract number: P02-436

Abstract type: Poster

Palliative Medicine Core Competencies for the Geriatrician in Brazil: A Proposal of a Curricular Matrix

Moraes Dias L. ${ }^{1}$, Junqueira dos Santos A.F. ${ }^{2}$, Rebello Bezerra M. ${ }^{3}$, dos

Santos G. ${ }^{4}$, Mendes Barreto Arantes A. ${ }^{5}$

${ }^{1}$ Sociedade Brasileira de Geriatria e Gerontologia, Belem, Brazil, IInstituto de Oncologia de Ribeirão Preto, Ribeirão Preto, Brazil, ${ }^{3}$ Insituto de Medicina Integral Professor Armando Figueira, Recife, Brazil, ${ }^{4}$ Sociedade Brasileira de Geriatria e Gerontologia, Curitiba, Brazil, ${ }^{5}$ Hospital de Apoio de Brasília, Brasília, Brazil

Background and aims: Despite the great interface between palliative care (PC) and geriatrics, there are still no proposals for a syllabus content based on palliative medicine competencies for the geriatrician in Brazil. On this basis, the objective was to develop a curricular matrix of palliative care skills for the geriatrician, especially for the one in post graduating training.

Methodology: In first stage the curricular pilot was established by a committee of specialists in geriatrics and palliative medicine. In second stage a group of geriatricians with certification in palliative medicine from all regions of the Brazil gave opinions about each thematic area of PC on the pilot matrix, in two different moments (3rd and 4rd stages). The modified Delphi methodology was used to obtain the consensus in theses stages (agreement level greater than $50 \%$ and $80 \%$ ). Finally, the public consultation phase ( $5 \mathrm{rd}$ stage) was carried out through the Brazilian Academy of Palliative Care website and in the Brazilian Congress of Geriatrics and Gerontology.

Results: Nineteen palliative medicine and geriatrics experts collaborated with the curricular matrix. There was an agreement level of all thematic areas greater than the determined, except for the palliative sedation with $20 \%$ of disagreement in the fourth stage. The matrix curricular finalized with 13 thematic areas with 105 competencies: 11 Prerequisites, 52 Essential, 24 desirable and 18 advanced.

Conclusion: After a review of international consensus and research with specialists from all regions of Brazil was defined a palliative care skills matrix with 105 competencies, that can be integrated into medical education. It is proposed that all these programs in Brazil offer at least the 'essential' skills training and ideally the 'desirable' and advanced skills in the 13 thematic areas proposed in the matrix, strengthening palliative care education in a homogeneous way throughout the country.

\section{Abstract number: $\mathrm{P} 02-437$}

Abstract type: Poster

Therapies by Movies, Theater and Drama Activities in the End of Life Care. Review of the Literature

Krakowiak P., Romianowska E., Romianowska J.

Educational Sciences, Nicolaus Copernicus University, Torun, Poland

Purpose: This literature review has examined the use of specific part of art therapy - therapies by movies, theatre and drama activities - in the EOL care. Their use among patients and caregivers, and effects on the negative symptoms often associated with end of life care has been studied and will be presented. The contribution of professionals and volunteers in creating theatre arts beneficially affecting psycho-emotional sphere of patients and participation in art therapy of patients and their relatives allowing enduring the process of serious illness will also be presented.

Data sources: An electronic database search was used to collect articles for this literature review in the following databases: Academic Search Complete, MEDLINE, CINAHL Complete, Caresearch, PubMed, CINAHL Plus with full text and psychINFO, EBSCO, SpringerLink, Google Schoolar, FBC- Federacja Bibliotek Polskich, LIBRA IBOOK.

Implications: The universal appeal of movies, theatre and drama makes it possible for most individuals to relate to and participate in these forms of art therapy in meaningful ways. It is important that all practitioners, as well as family caregivers understand this treatment modality so they may offer it, if appropriate. Various forms of art therapy could make an excellent tool for all providers, from the beginning of PC care, through all stages of care. They can be used in grief and bereavement care as well as useful care tool for caregivers.

Conclusion: Analysis of the studies have found that therapies by movies, theatre and drama activities are often used in the end of life care. They can be effective for meeting needs of patients and their families, inspiring life review, and discover meaning in difficult moments of life. They can serve as support tools for caregivers and for those in bereavement process. These forms of therapies could be an encouragement for volunteers to engage in caring process by their involvement in these forms of art therapy.

\section{Abstract number: $\mathrm{P} 02-438$}

Abstract type: Poster

Attitudes towards Care of the Dying: A Cross Sectional Study on Italian Undergraduate Nursing Students

Mastroianni C. ${ }^{1}$, Magnani C. ${ }^{1}$, Stefanelli M.C. ${ }^{1}$, Carbonara L. ${ }^{1}$, Surdo

L. ${ }^{1}$, Giannarelli D. ${ }^{2}$, Ramon Codina M. ${ }^{3}$, D'Angelo D. ${ }^{4}$, De Marinis M.G. ${ }^{5}$, Monaco R. ${ }^{6}$, Piscitelli F. ${ }^{6}$, Delli Poggi A. ${ }^{6}$, Zaino L. ${ }^{6}$, Alvaro R. ${ }^{6}$, Latina $R^{6}{ }^{6}$, Chavarapuzha S. ${ }^{6}$, Bialek J. ${ }^{1}$, Casale G. ${ }^{1}$

${ }^{1}$ Antea Palliative Care Centre, Roma, Italy, ${ }^{2}$ Regina Elena National Intstitute for Cancer Treatment and Research, Roma, Italy, ${ }^{3}$ Universidad Nacional de Educacion a Distancia (UNED), Roma, Italy, ${ }^{4}$ Centre for Clinical Excellence and Quality of Care, Istituto Superiore di Sanità, Roma, Italy, ${ }^{5}$ Campus Bio Medico University, Roma, Italy, ${ }^{6}$ Antea Palliative Care Center, Roma, Italy 
Background: International literature reports that the development of professionals competences in Palliative Care (PC) are influenced by attitudes towards death and care of dying. Attitudes constantly affect the nursing care and represent the nurses personal and professional value system. Nurses' attitudes come from the interaction between PC knowledge, emotional experience and professional behavior. Despite the growing attention in PC undergraduate education and competences, there is a lack of Italian data on this issue.

Aim: To assess the attitudes of undergraduate nursing students towards care for the dying and to evaluate the influence of demographic, religious, experiential and educational factors.

Methods: A cross sectional study was performed with a convenience sample of undergraduate nursing students of the four universities of Rome. The students of first, second and third year of education, completed the Italian version of Frommelt Attitudes Towards Care of the Dying Scale (FATCOD B-I), between September 2017 and March 2018.

Results: A sample of 1193 students completed the FATCOD B-I. The mean score of attitudes were 97,37 $\pm 7,37$ (range score $30-150$ ). PC education ( $p<0,0001)$, experience with terminally ill patients $(p<0,0001)$ and students academic level $(p<0,0001)$ resulted as primary variables affecting the development of positive attitudes toward care of dying. Also Age $(p<0,02)$ resulted as a factor influencing students attitudes. Religious beliefs and gender are significantly associate only with a few factors of FATCOD B-I scale.

Discussion: Findings revealed that student nurses' attitudes was similar to nursing students from other countries. PC Education and experience are predictor of positive nursing attitudes. Attitudes can change and improve gradually, based on new experiences and greater knowledge. This study suggests that PC courses and training are essential and should be extensively integrated to the undergraduate nursing curriculum.

Abstract number: P02-439

Abstract type: Poster

\section{Development of a Check-list to Evaluate Physicians' Education in Palliative Care \\ Hedman C.}

Karolinska Institutet, Department of Molecular Medicine and Surgery, Stockholm, Sweden Presenting author email address: christel. hedman@stockholmssjukhem.se

Aim: In the Swedish education plan for all resident physicians, palliative care is obligatory. Thus, many resident physicians work at a specialised palliative care unit for a couple of months. In order to provide a high quality education and to ensure the goals of education to be fulfilled, an educational plan is crucial. To ensure the educational quality, the aim was to develop check-list containing the most important knowledge and skills resident physicians need to learn during the education.

Methods: To develop the check-list, a review of existing educational plans for resident physicians in all clinical specialties was performed. In addition, information from the national palliative care guidelines were included. Based on the information, a preliminary check-list including common symptoms in palliative care and organizational aspects were included. The preliminary check-list was discussed with all physicians at the palliative care unit at Stockholms Sjukhem, a large palliative care unit in Stockholm. The content was revised four times according to suggestions. Initially the check-list was to evaluate the knowledge and skills at the end of the education. During development it was divided into three parts to enable evaluation of the progress of knowledge and skills.

Results: The final version of the check-list contains 22 goals of education, including symptoms, communication, organisational issues, consultation with other specialties and the dying process. The check-list is to be filled in at three different time points, at the beginning, at half-time and at the end of the internship. Thus, progression of knowledge and skills can be evaluated. The check-list has been implemented into clinical use and is very appreciated of both resident physicians and their supervisors.
Conclusions: A check-list to enable follow up of the progression of knowledge and skills of resident physicians in palliative care is both appreciated and ensures good quality of education.

\section{Abstract number: P02-440}

Abstract type: Poster

Integrating Palliative Medicine Within the Medical School Curriculum: Improving the Care Given by the Next Generation Mackey R. ${ }^{1}$, Eick M. ${ }^{1}$, Dev R. ${ }^{2}$, Strand J. ${ }^{1}$

${ }^{1}$ Palliative Medicine, Mayo Clinic, Rochester, United States, ${ }^{2}$ Palliative Medicine, MD Anderson Cancer Center, Houston, United States

Background: In the US and most developed countries there is a consensus that teaching Palliative Medicine competencies to students is essential. Medical students must be educated and trained in the specialty of Palliative Medicine to improve quality of life in chronically ill patients and provide compassionate end-of-life care.

Goal: Teach Palliative Medicine competencies at Medical School, start Year 1 and continuing until graduation. This course provides a general level of Palliative Medicine knowledge, incorporates the Palliative Care training rotation into the core clinical clerkship and provides communication skills training such as delivering bad news and discussing prognostication.

Design and Methods: This case report series, starts year 1 at a leading U.S. Medical School, and follows the same class until graduation. A series of meetings will teach communication skills and provide guidance and support as the students progress with their education while interacting with patients. Students will have access to rotations with a Palliative Care team, with mentorship, and participate in rounds, family meetings, and be able to interview patients and their families.

Results: Preliminary results from Year 1: from 53 Med Students, 35 (66\%) participated in the first meeting.

Conclusion: Medical education is changing continuously in response to the increasing need to acquire scientific knowledge and adapt to new technology. Medical education emphasizes scientific knowledge over other critical skills such as clinical judgment, communication with patients, working as a part of an interdisciplinary team, and the development of cardinal virtues such as compassion, empathy, and integrity. The purpose of Palliative care is to improve quality-of-life for patients and their families facing life-threatening illness, through the prevention and relief of unnecessary suffering. Incorporating the Palliative Care core principals would enrich the medical school curriculum.

Abstract number: $\mathrm{P} 02-441$

Abstract type: Poster

Problems that Hygienists Have on Performing Multidisciplinary Approach on Palliative Care

Nakajima $N$.

University of the Ryukyus, Division of Community-based Medicine and Primary Care, Okinawa, Japan Presenting author email address: nakajy@med.u-ryukyu.ac.jp

Aims: Palliative care is constructed by various kinds of specialists including physicians, nurses, pharmacists, dieticians, hygienists, etc. Multidisciplinary approach is important in palliative care, but the degree of maturity of the cooperation with other specialists varies according to the type of specialists. The purpose of this study is to clarify the problems that hygienists have on performing multidisciplinary approach on palliative care.

Methods:

1) We performed a questionnaire about "oral care for terminal cancer patients" for all 1,290 hygienists who belonged to the Japanese Society of Oral Care. 
2) We performed a questionaire about the education of palliative care for the school of oral health sciences in one university in Japan.

Results:

1) We got answers from 515 hygienists (response rate= 40\%). They had the experience of oral care for terminal cancer patients (73\%), but no experience to take the education on palliative care $(92 \%)$, and felt the lack of knowledge on palliative care $(94 \%)$.

2) We got answers from all of 75 students. They took lecture on palliative care (55\%) and more than $90 \%$ of them replied "It's a rewarding experience". But, $88 \%$ had anxiety, and $78 \%$ felt the shortage of the classes on palliative care.

Conclusions: This is, to our best knowledge, first nationwide survay about the problems on palliative care which arise on the hygienists. Many of the hygienists performed oral care for terminal cancer patients, but they felt that they lacked the opportunity for education and insufficient knowledge on palliative care. University students were highly interested in palliative care, but insufficient chance for the education on palliative care might lead to their anxiety. Improvement of 'pre- and post-graduate education system' on palliative care is necessary for hygienists in order to play important role positively as a member of multidisciplinary team. Resolving these problems will be able to lead the multidisciplinary approach better.

Abstract number: P02-442

Abstract type: Poster

Improving Clinical Examination Skills of Hospice Community Nurses to Enhance Patient Care - An Educational Intervention Taylor A.G.S., Ritchie A., White C.

Northern Ireland Hospice, Belfast, United Kingdom

Background: Hospice community nurses (HCN) undertake Health Assessment Skills modules to develop competency in history taking and clinical examination. However, ongoing support and education in this area can be lacking.

Aims: To improve the confidence and competence of $\mathrm{HCN}$ in clinical examination, improving patient care and inter-professional discussions. Methods: Five tutorials were organised for $15 \mathrm{HCN}$ who had previously completed Health Assessment Skills training. Facilitated by 2 junior doctors, sessions involved a presentation, practical demonstration and small group practice. General, respiratory, gastrointestinal and neurological systems were taught. Participants maintained practice logs. Skills development was supported with a 5 day placement in the hospice inpatient unit. An anonymous feedback survey after the programme assessed clinical impact.

Results: $10 \mathrm{HCN}$ completed the survey. All strongly agreed that the sessions were useful and improved their clinical practice. Nurses described the practical demonstrations and supervised practice as most beneficial. The informal environment and tutor availability were highlighted as important.

Prior to this, $60 \%$ last received clinical teaching over 2 years previously. $30 \%$ reported examining patients at least daily, $40 \%$ weekly and others less frequently. After this course, all $\mathrm{HCN}$ were examining patients at least weekly, with $70 \%$ examining patients at least daily.

$90 \%$ declared greater confidence in discussing patients with other healthcare professionals, including general practitioners. $70 \%$ stated that this course had reduced the need for another healthcare professional to review a patient at least weekly. $20 \%$ reported this monthly. Conclusion: A focused clinical skills teaching programme, facilitated by junior doctors, improves $\mathrm{HCN}$ confidence in examination skills and interprofessional discussions, increases the frequency that $\mathrm{HCN}$ undertake examinations and reduces requirements for reviews by other healthcare professionals.

Abstract number: $\mathrm{P} 02-443$

Abstract type: Poster

Growing our Own: Developing a Training Programme for Clinical Nurse Specialists New to Palliative Care

Gillon U.

Imperial College London NHS Trust, Palliative Care, London, United Kingdom Presenting author email address: ulrike.gillon@nhs.net

Background: The recruitment of experienced Clinical Nurse Specialists ( CNSs) in Palliative Care is very difficult. Few applicants had worked in Palliative Care. They were enthusiastic but inexperienced. We realised we needed to grow our own.

Aim: We could not find a programme elsewhere that suited our needs. So to support our new CNSs and to enable them to become confident, safe and independent practitioners we developed an in-house Palliative Care Clinical Nurse Specialist in-training programme.

Design: Based on the UK Speciality Training Curriculum for Palliative Medicine with added nursing specific knowledge, skills and abilities we built a comprehensive teaching and training programme for CNSs new to Palliative Care.

Approach taken: Collaborating closely in our team of CNSs and Consultants in Palliative Medicine we formulated a training programme that included theoretical and practical aspects deemed vital in securing our aim. We developed a detailed induction booklet that included competencies to achieve on completion of the programme and a workbook that included work and assessment sheets, reflections sheets and holistic assessment proformas. Our new CNSs in-training then started a six month teaching schedule that followed the curriculum and competencies to be achieved alongside the daily practical work with patients.

Results: The curriculum, induction booklet and competencies gave a good overall structure to the daily work and on-the-job teaching. Meetings to discuss and review progress, and to present case studies complemented the learning and assessment structure.Ongoing feedback helped address issues particularly with regard to the practical element of the programme.

Conclusions: While the theoretical aspects of the programme were clear and worked well, some practical elements and the assessment of some of the competencies proved challenging for the mentors. Our new CNSs are growing and flourishing, and we are working on improving our programme further.

\section{Abstract number: P02-444}

Abstract type: Poster

Skills to Take Anywhere after Graduation: Updating Palliative and End-of-Life Care Competencies for Undergraduate Medical Learners in Canada

Bush S.H. ${ }^{1,2,3}$, Chary S. ${ }^{4,5}$, Roze des Ordons A. ${ }^{4,6}$, Boyle A. $^{7}$

${ }^{1}$ Department of Medicine, Division of Palliative Care, University of Ottawa, Ottawa, Canada, ${ }^{2}$ Ottawa Hospital Research Institute, Ottawa, Canada, ${ }^{3}$ Bruyère Research Institute, Ottawa, Canada, ${ }^{4}$ Division of Palliative Medicine, Department of Oncology, Cumming School of Medicine, University of Calgary, Calgary, Canada, ${ }^{5}$ Pallium Canada, Ottawa, Canada, ${ }^{6}$ Department of Critical Care Medicine, Cumming School of Medicine, University of Calgary, Calgary, Canada, ${ }^{7}$ Division of Palliative Care, Department of Family Medicine, McMaster University, Hamilton, Canada

Background/ aim: The original Educating Future Physicians in Palliative and End-of-Life Care (EFPPEC) project released national medical undergraduate competencies for palliative and end-of-life care (PEOLC) in 2008. Although approved by the Deans of all Canadian medical schools, 
uptake was variable. Recent practice environment and legislative changes in Canada have led to a crucial need for an update.

Methods: From February 2017 - April 2018, a core project team for the Canadian Society of Palliative Care Physicians (CSPCP) Undergraduate Education Committee updated the EFPPEC competencies. They also aligned them with the revised 2015 CanMEDs framework. Support was provided by the project partners: Association of the Faculties of Medicine of Canada and Canadian Hospice Palliative Care Association. A multi-stage validation process for the bilingual document was conducted with stakeholders from across Canada, including representatives of all 17 medical schools, the Medical Council of Canada, palliative medicine practitioners, residents, and medical students.

Results: To reflect current discourse, we shifted from solely 'PEOLC' with the addition of 'an early, integrated, collaborative palliative approach to care' starting earlier in the course of both life-threatening malignant and non-malignant illness. Topic areas for new competencies and specific objectives in the 2018 EFPPEC update included opioid prescribing in the context of palliative care and the opioid crisis, role of cannabinoids, legalisation of Medical Assistance in Dying in Canada, and paediatric palliative care.

Conclusion: The final EFPPEC document has been circulated to all Canadian medical schools, with early integration and implementation occurring at some sites. The 2018 EFPPEC update will provide a foundation for undergraduate medical education programs transitioning towards competency-based models of education.

(Funded by CSPCP and grant from College of Family Physicians of Canada)

Abstract number: P02-445

Abstract type: Poster

\section{Pediatric Palliative Care Training in a Referral Hospital in Bhutan}

Vesel T. ${ }^{1}$, O'Connor M. $^{2}$, Beveridge C. ${ }^{3}$, Vesel L. ${ }^{2}$, Nishizawa Y.4, Mize C. ${ }^{5}$, Jullien $S^{6}$

${ }^{1}$ Tufts Medical Center, Boston, United States, ${ }^{2}$ Harvard T.H. Chan School of Public Health, Boston, United States, ${ }^{3}$ University of Texas Southwestern Medical Center, Dallas, United States, ${ }^{4}$ KhesarGyalpo University of Medical Sciences of Bhutan, Thimphu, Bhutan, ${ }^{5}$ Children's Hospital of Philadelphia, Philadelphia, United States, ${ }^{6}$ Jigme Dorji Wangchuck National Referral Hospital, Thimphu, Bhutan

Background: Pediatric palliative care and effective communication is much needed but often overlooked, particularly in low- and middleincome settings. Lack of resources and specialized training are major barriers to care provision. A number of curricula exist that address these educational needs in the United States. Our objective was to adapt our interactive curriculum to the Bhutanese context and evaluate the effect of its implementation on provider knowledge, skills and attitudes related to pediatric palliative care.

Methods: The workshop was conducted in January 2017 at Jigme Dorji Wangchuck National Referral Hospital in Bhutan. Mixed methods were used to collect and analyze responses of interdisciplinary pediatric providers to a pre-workshop, immediately post-workshop and 6-month post-workshop survey.

Results: A total of 41 providers participated in the workshop and preworkshop survey; 38 completed the post-workshop survey; and 27 completed in the 6-month post-workshop survey. Respondents' comfort levels managing pediatric palliative care patients peaked immediately following the workshop, and remained higher 6-months later. Providers understood the importance of their role in communicating with patients and families experiencing life-threatening illnesses and reported feeling more capable initiating and guiding such difficult conversations. Respondents reported feeling better equipped to identify, assess, and manage pain in pediatric patients. Finally, respondents reported utilizing self-care techniques and spiritual beliefs in their own coping and ability to connect with children and their families.
Conclusion: The results of this first palliative care training in Bhutan suggests that even a short intervention, adapted to the local context, can improve providers' knowledge, skills, and abilities to provide high-quality pediatric palliative care and address gaps in capacity.

Funding source: The workshop and study were self-funded.

Abstract number: P02-446

Abstract type: Poster

Palliative Care Nursing Education Across the EU: Results from an International Survey Study

Martins Pereira S. ${ }^{1,2,3}$, Hernández-Marrero P. ${ }^{1,2,3}$, Capelas M.L. 4,5,6, Pasman H.R. ${ }^{7}$, Larkin P. 8,9 , Francke A. ${ }^{10,11}$, EAPC Task Force on

Preparation for Practice in Palliative Care Nursing across the EU

${ }^{1}$ Instituto de Bioética, Universidade Católica Portuguesa, Porto, Portugal, ${ }^{2}$ UNESCO Chair in Bioethics, Institute of Bioethics, Universidade Católica Portuguesa, Porto, Portugal, ${ }^{3}$ CEGE: Centro de Estudos em Gestão e Economia, Porto Católica Business School, Universidade Católica Portuguesa, Porto, Portugal, ${ }^{4}$ Institute of Health Sciences, Universidade Católica Portuguesa, Lisboa, Portugal, ${ }^{5}$ Centre for Interdisciplinary Research in Health, Universidade Católica Portuguesa, Lisboa, Portugal, ${ }^{6}$ Portuguese Observatory for Palliative Care, Universidade Católica Portuguesa, Lisboa, Portugal, ${ }^{7}$ Amsterdam Public Health Research Institute, Department of Public and Occupational Health, Expertice Center for Palliative Care, Amsterdam UMC, Vrije Universiteit Amsterdam, Amsterdam, Netherlands, ${ }^{8}$ UNIL | Université de Lausanne, CHUV | Centre Hospitalier Universitaire Vaudois, Lausanne, Switzerland, ${ }^{9}$ Faculté de Biologie et de Médecine - FBM, Institut Universitaire de Formation et de Recherche en Soins - IUFRS, Lausanne, Switzerland, ${ }^{10}$ Nivel: Netherlands Institute for Health Services Research, Utrecht, Netherlands, ${ }^{11}$ Department of Public and Occupational Health, Amsterdam Public Health Research Institute, Amsterdam UMC, Vrije Universiteit Amsterdam, Amsterdam, Netherlands

Background: In 2004, an EAPC taskforce on palliative nurse education presented guidelines for the preparation of nurses for practice in palliative care (PC). There was an indication that the document was useful, but no systematic evidence of its impact through the EU was undertaken. Since its publication, there have been considerable national and international developments in relation to PC education.

Aims: To determine education and competencies of nurses working in PC in Europe; to assess the impact and uptake of the EAPC 2004 document for the development of PC nursing practice.

Methods: International online survey study with expert nurses (ie, nurses with relevant experience in nurse education and/or role in PC nursing) recruited via the EAPC collective associations and members of the current EAPC Nursing Taskforce Steering and Advisory Groups. The survey was built de novo for this study. Data collection was performed throughout 2016. All data were pseudo-anonymized. Descriptive analyses were conducted; country reports were built based on country-level descriptive analyses.

Results: A total of 135 expert nurses from 25 countries completed the online survey. In the majority $(n=14 ; 56 \%)$ of the countries represented in our sample, PC was not identified as a mandatory subject within undergraduate nursing education. Post-graduate educational programmes for PC nursing were offered in 19 (76\%) of these countries, and were mostly interdisciplinary. In the majority of the participant countries $(n=13 ; 52 \%)$ there was no specialization in $P C$ nursing with formal recognition and certification. A vast majority $(n=20 ; 80 \%)$ of the participants was aware of the EAPC 2004 document. Large variations were found across and within country responses.

Conclusions: Palliative care nursing education varies largely in Europe. This taskforce will contribute to the work of the education stream of the EAPC and help to inform future initiatives for member associations and EAPC itself. 
Abstract number: $\mathrm{P} 02-447$

Abstract type: Poster

A Descriptive Study about Self Assessment on Competencies for Caring of Dying Patients among Nursing Students after Experiencing a Patient Death

Bobnar A.

University of Ljubljana Faculty of Health Sciences, Nursing, Ljubljana, Slovenia

Background: Caring for dying patients is one of the most stressful and traumatic experiences reported by the nursing students. Preregistration nursing curricula have traditionally had a limited emphasis on dying and death and nurses report feeling unprepared to care for dying patients.

Aims: The study was assessing nursing students' competencies for caring of dying and death patients after experiencing a patient death during clinical practice.

Methods: A descriptive qualitative method was employed from second study-year nursing students. Data were collected in 2014/2015. The nursing students' written descriptions ( $n=38,32 \%)$ of their experiences and competencies were analyzed using Colaizzi's seven step procedure.

Results: Four themes emerged from this analysis. While caring for dying patients, nursing students experienced with many negative emotions such as fear, frustration, anger, sadness, disappointment and anxiety. Contemplation of the patient's dignity and death occurred as the students cared for their patients. In addition they feel unprepared, as well as helpless for providing physical, emotional, social and spiritual support for dying patients, an integral part of nursing students' care. The mentors should offer the nursing students their constant, understanding support and counselling, drawn from their own life experiences.

Conclusion / discussion: Nursing students reported that they are not professionally competent for caring of dying patients. Findings demonstrate the importance of understanding such first experiences, and the results are beneficial to school and clinical mentors and nursing personnel. Several recommendations have been made: teaching about understanding of death, openness to examining dying and death, and reduced negative emotional state in the period of dying, moment of patient death and post mortality. In clinical training, the care for dying patients should be introduced, supported and monitored by the school and clinical mentors.

\section{Abstract number: P02-448 \\ Abstract type: Poster}

\section{Learning Outcomes of an Inter-professional Training Palliative Care Program in Greece \\ Patiraki E. ${ }^{1,2}$, Katsaragakis S. ${ }^{1}$, Los $C^{2}$, Protogiros D. ${ }^{1}$, Avgetidou H. ${ }^{2}$, Deskou F. ${ }^{1}{ }^{2}$, Dionysi M. ${ }^{2}$, Mpiskemi V. ${ }^{2}$, Paliourgiotou G. ${ }^{2}$, Tsotakos $S^{2}{ }^{2}$, Xenikou M. ${ }^{2}$, Tserkezoglou A. ${ }^{2}$ \\ ${ }^{1}$ Nursing, National and Kapodistrian Univesrity of Athens, Athens, Greece, ${ }^{2}$ Palliative Care Unit Galilee, Spata, Greece}

Background: An inter professional educational program in palliative care, has been organized for many years in collaboration of Galilee palliative care unit and department of Nursing, National and Kapodistrian University of Athens.

Aims: Learning outcomes evaluation of an inter-professional palliative care training program (IPTP).

Methods: A pre- and post-test study design was used. Interactive training on fundamentals aspects of palliative care was offered in 40hours/5days (April 2018) to 34 professionals. Only 24 (response rate 64.7\%) (11 nurses, 4 doctors, 2 social workers, 1 psychologist, 1 physiotherapist, 1 pharmacist, 1 priest, and 1 administrator) consented to participate. The evaluation of the IPTP included a 48 true/false items self-administered knowledge tool (Cronbach's a $=.067)$, at baseline $\left(\mathrm{T}_{0}\right)$, IPTP completion $\left(T_{1}\right)$, and one month later $\left(T_{2}\right)$. The statistical significance level: 0.05 .

Results: The participants were female (86.4\%), with a mean age 42.1 years, and a mean work duration 17 years and gave a high percentage of correct answers for the majority of items. The prevalence of the 10 most frequent correct answers were $\mathrm{T}_{0} 81.8 \%, \mathrm{~T}_{1} 90.9 \%$ and $\mathrm{T}_{2} 90.9 \%$. The mean total number of correct answers was statistically significant different over the 3 evaluations $\left(x^{2}(2)=19.3, p=<.001\right)\left(T_{0} 29.0, T_{1} 33.4, T_{2}\right.$ 33.7). The total number of correct answers at baseline were statistically significant improved after the IPTP $(z=-3.4, p=.001)$ and one month later $(z=-3.5, p<.0001)$. Knowledge was statistically significant improved after IPTP, at 5 items related with opioids addiction, dying interventions and bereavement $(\mathrm{p}<.0001$ to .016$)$, and a month later, at 2 items regarding meaning of suffering, and use of placebo as analgesic $(p<$ .0001 to $p=.005$ ).

Conclusion/ discussion: Attendance at IPTP had a measurable effect on the learning outcomes. Future planning should focus on testing the inter-disciplinary utility of this program and ensuring the maintenance its gains.

Abstract number: P02-449

Abstract type: Poster

Expanding Palliative Care Teaching at a Graduate Entry Medical School

Prosser A. ${ }^{1}$, Baker I. ${ }^{1,2}$, Walrond H. ${ }^{3}$

${ }^{1}$ Palliative Medicine, Abertawe Bro Morgannwg University Health Board, Swansea, United Kingdom, ${ }^{2}$ Palliative Medicine, Swansea University, Swansea, United Kingdom, ${ }^{3}$ Swansea University, Swansea, United Kingdom

Background: It is recognised internationally that basic training in palliative care should be a part of all undergraduate medical curricula. According to the UK Association of Palliative Medicine, doctors in their F1 year will care for 40 patients who die, and another 120 in the last few months of life. $48 \%$ of UK deaths occur in hospital; highlighting the need to develop general palliative care skills in the whole medical workforce. Aims: At this medical school, minimal palliative care teaching was previously scattered throughout the curriculum. We were given an exciting opportunity to develop a new combined pain and palliative care learning week for second year students; aiming to design a structured and balanced week, with well chosen content to build on the knowledge and skills required.

Methods: We took a student-led approach by surveying previous students to attain their thoughts on existing and future learning opportunities; with responses influential in the development of the week. To reflect the holistic and multi-disciplinary team approach within palliative care, a multi-disciplinary teaching faculty and wide variety of teaching methods were employed. To ensure our teaching met the students' learning needs, surveys were also sent to those who had attended the week.

Results: There was a significant increase in those who felt they had received sufficient teaching in palliative care (62.5\%), as compared to those surveyed in the development phase of this project (11\%). There was also a decrease in those who felt 'not confident' about their knowledge of palliative care ( $8 \%$ v $39 \%)$.

Conclusion: One week is insufficient to teach the entirety of the palliative care curriculum. However, this exciting opportunity to expand the palliative care teaching at our university is a step in the right direction towards building a workforce better equipped to deliver general palliative care, as well as nurturing and inspiring the next generation of experts to join our expanding specialty.

Abstract number: P02-450

Abstract type: Poster 
"Let's See a Human Being in a Medical Student". What are the Areas for Empowering Future Doctors in Facing Death and Dying?

Wyszadko A. ${ }^{1}$, Lichodziejewska-Niemierko M. ${ }^{1}$, Nowicka-Sauer K. ${ }^{2}$ ${ }^{1}$ Palliative Medicine Department, Medical University of Gdansk, Gdańsk, Poland, ${ }^{2}$ Family Medicine Department, Medical University of Gdansk, Gdańsk, Poland

Background: Some studies show that providing help to others may cause an emotional burden in carers. On the other hand, experience facilitates learning and personal development.

Aims: We aimed to assess personal perspectives of medical students (MS) from accompaniment dying people (AD) in order to recognise areas for improving training in palliative care.

Methods: MS (176) of different years of education, who experienced $A D$ answered open questions about experienced difficulty and what they have learned from such situation. Interpretative phenomenological analysis was conducted to identify repeated themes. With added 282 other students' results (not having AD experience), the analysis of impact of the type of difficulties/lessons learned on opinions about euthanasia and persistent treatment was done (regression analysis).

Results: Perceived difficulties in AD were: helplessness (25\%), own feelings ( $21 \%, 23 \%$ when relative died, $16 \%$ in case of patient's death), witnessing suffering (18\%), $17 \%$ existential issues, $14 \%$ own behaviour, $8 \%$ others' behaviour, $3 \%$ "nothing". Lessons learned from AD included: existential self-discoveries $(27 \%)$, what to do $(26 \%$, more often when assisting patient), $27 \%$ did not give answer, $3 \%$ described a difficulty, $10 \%$ gave a description of observation (instead of a lesson learned). $4 \%$ MS made destructive conclusions. Some significant factors allowing prediction of support for euthanasia were found (experiencing of existential difficulty or no difficulty, seeing difficulty instead of a lesson learned).

Conclusion: $1 / 3$ of MS did not learn from AD. Support for MS in integrating experiences from $A D$ is necessary. Coping with (own and others) emotions, learning how to deal with different challenges and open communication with MS about issues of dying are the areas to be consciously included in education. Implementation of these subjects is necessary from the beginning of studies, as MS face death and dying not only within palliative medicine course.

Abstract number: P02-451

Abstract type: Poster

"To Cooperate or Not Cooperate? That lis the Question"Medical Students Experiences, Opinions and Plans for Interprofessional Cooperation

Wyszadko A., Pawłowski L., Mróz P., Lichodziejewska-Niemierko M. Palliative Medicine Department, Medical University of Gdansk, Gdańsk, Poland

Background: Interprofessional cooperation (IC) is essential for good patient care and especially important in the end-of-life. This subject in medical education (ME) has not been researched much in Poland.

Aims: We aimed to research students' experiences and plans of IC with other specialists.

Methods: 178 students of final year of medical studies filled in diagnostic survey: $71 \%$ women (W), 28\% men (M), $1 \%$ missed answer. Descriptive statistics, association and logistic regression analysis were used.

Results: In the scope of commissioning, accepting orders, consulting, providing advice and discussing cases students claimed that at least $75 \%$ plan to use this forms of IC and they observed them within ME. $44 \%$ feel prepared to commissioning, $48 \%$ accepting orders, $84 \%$ discussing cases. Consulting was perceived as the most difficult: $47 \%$, but $91 \%$ want to use it in future. Students observed IC with other physicians and nurses, also psychologists and physiotherapists: $84,82,68,66 \%$ respectively. $51 \%$ of the students observed cooperation with patients' relatives. The percent of those who practiced IC with these groups was smaller: $82,56,34,31 \%$ respectively. IC with other professions was less represented. The mean percent of good cooperation observed within training was $59 \%, \mathrm{sd}=19 \%$, bad examples: $39 \%, s d=17 \%$. The main reasons chosen to explain lack of IC: lack of time (84\%), others not willing to cooperate $(69 \%)$, no procedures (59\%), no good models of IC (51\%). The last reason increased (4\%) students' conviction that discussing cases of patients with other specialists is difficult. $\mathrm{W}$ and $\mathrm{M}$ differed in opinions about benefits of IC: women paid more attention to quality of life and patient satisfaction with medical care.

Conclusion: Most students appreciate IC, but need more education, especially in seeking advice from other specialists. Results inspire for involving more practical training in IC, as palliative approach may be necessary in many settings.

Abstract number: $\mathrm{P} 02-452$

Abstract type: Poster

Don't You Forget About Me: Assessing the Palliative and End of Life Care Learning Needs of Physician Associates in a University Teaching Hospital in England

Jena M., Tomas J.

Queen Elizabeth Hospital Birmingham, Birmingham, United Kingdom

Background: The number of persons needing Palliative/End-of-Life Care $(\mathrm{P} / \mathrm{EOLC})$ is increasing and large numbers of hospital inpatients have entered the last year of their lives. Physician Associates (PAs), a new NHS practitioner working across a range of specialities, are increasingly being asked to attend to patients with P/EoLC needs. Little is known, however, about their P/EoLC learning needs and relevant training is highly variable.

Objectives:

1. To assess PA involvement in P/EoLC and their confidence in symptom management.

2. To identify PA educational needs and preferences to facilitate the design of a P/EoLC teaching programme.

Methods: 20 -item bespoke and 16-item e-ELCA assessment tools were distributed to all PAs working at a University Teaching Hospital in England. Participation was voluntary and collected data anonymised. Data were analysed using descriptive statistics via Microsoft Excel.

Results: $100 \%$ of questionnaires were returned (16/16 PAs). $69 \%$ of PAs are directly involved in providing $\mathrm{P} /$ EoLC for patients at least monthly; $38 \%$ at least weekly. $63 \%$ agreed that delivering P/EoLC is extremely rewarding; $69 \%$ reported never having had any formal P/EoLC education. $13 \%$ reported any degree of confidence in managing symptoms in noncancer patients at the end of life.

$69-75 \%$ of respondents were 'not so confident' or 'not at all confident' in managing dyspnoea, communicating with patients/carers about dying, assessing palliative care emergencies, and managing agitation and confusion.

The most requested education topics were communication skills, P/EoLC pathways and symptom control for non-cancer patients; with lectures with other PAs, study days and case discussions the preferred methods of instruction.

Conclusion: To our knowledge this study represents the first formal assessment of P/EoLC learning needs amongst PAs. PAs at our hospital are regularly involved in P/EoLC, but most lack confidence in key skills. There is a need for a relevant PA teaching programme.

Abstract number: $\mathrm{P} 02-453$

Abstract type: Poster 
Cultural Adaptation and Psychometric Properties of the End-ofLife Professional Caregiver Survey - Brazilian Version Garcia A.C.M. ${ }^{1}$, Spineli V.M.C.D. ${ }^{2}$, Eduardo A.H.A. ${ }^{3}$, Lazenby M. ${ }^{4}$, Meireles E.C.d.A. ${ }^{5}$, Barros G.A.M. ${ }^{6}$, Calixto Damasceno Spinelli V.M. ${ }^{1}$ ${ }^{1}$ Federal University of Alfenas, School of Nursing, Alfenas, Brazil, ${ }^{2}$ University of São Paulo, School of Nursing, São Paulo, Brazil, ${ }^{3}$ Federal University of São Carlos, Nursing Departament, São Carlos, Brazil, ${ }^{4}$ Yale Universit, School of Nursing, New Haven, CT, United States, ${ }^{5}$ Federal University of Recôncavo da Bahia, Health Sciences Center, Cruz das Almas, Brazil, ${ }^{6}$ São Paulo State University (Unesp), Medical School, Botucatu, Brazil

Background: The lack of training of health professionals in Palliative Care is one of the great barriers to improving the population's access to care. The End-of-Life Professional Caregiver Survey (EPCS) identifies the educational needs of health professionals in Palliative Care.

Aims: To describe the Brazilian cultural adaptation and preliminary psychometric properties (validity and reliability) of the End-of-life Professional Caregiver Survey (BR-EPCS), a self-report questionnaire to assess the educational needs of multidisciplinary health professionals in palliative care.

Methods: Methodological study conducted in 2018 with 300 health professionals working in palliative care (pilot-testing, $n=15$; cross-sectional analysis of the initial psychometric properties, $n=285$ ). The EPCS, established in the USA, consists of 28 items divided into 3 factors: "Patient and family centered communication" (12 items), "Cultural and ethical values" ( 8 items) and "Effective care delivery" ( 8 items). Higher values reflect greater knowledge and, therefore, lower levels of educational needs [Total possible score $=112$ ]. The cultural adaptation process went through the steps of European Organisation for Research and Treatment of Cancer - Quality of Life Group Translation Procedure (EORTC-QLGTP). For the analysis of dimensionality of the BR-EPCS, parallel analyzes were performed. We used Cronbach's alpha coefficient to analyze reliability.

Results: The EORTC - QLGTP resulted in an adapted instrument semantically equivalent to the established English EPCS. The BR-EPCS has two factors. Cronbach's alpha was 0.94 (Factor 1) and 0.89 (Factor 2).

Conclusions/ discussion: The BR-EPCS has strong psychometric properties at the item and factor level and can be used to measure the educational needs of health professionals working in palliative care in Brazil.

\section{Abstract number: $\mathrm{P} 02-454$}

Abstract type: Poster

\section{The Effect of Facing Dying Patients on Medical Students - An Outcome Evaluation \\ Thyson T., Schallenburger M., Scherg A., Schwartz J., Neukirchen M. Interdisciplinary Centre for Palliative Medicine, University Hospital Dusseldorf, Dusseldorf, Germany}

Background: Within our compulsory optional subject "Communication in borderline situations", which is divided into three phases (eLearning, encounter, reflection) advanced medical students talk to patients during their last weeks of life. Expanding their knowledge about communication, practicing their communication skills and professionalizing handling of their own emotions are the main goals.

Aim: Palliative Care teaching wants to reach students on a level of selfreflection. A specific outcome-evaluation should represent an enhancement of knowledge and communication skills. Particularly a change of attitudes in meaning of social- and self-competences is expected.

Method: Beside global evaluation of learning outcomes, specific outcome of learning objectives were assessed. Those outcomes were examined by comparative self-assessment (CSA) of 14 items, presenting specific learning objectives of the subject. In a pre-post (e. g. prospective) and post-then (e. g. retrospective) procedure the learning progress is measured on a six-point Likert-scale.
Results: The first cohort (between april and july 2018) included 15 medical students. Post-then comparisons showed above 50 percent increase in learning abilities by ten out of 14 items (range $37 \%$ up to $77.66 \%$ ). Highest CSA-gain was identified by definition of the NURSE communication model (post-then $77.66 \%$ ) and approach to own emotions while facing a terminally ill patient (post-then $73.88 \%$ ). The settlement with one's own finiteness revealed less learning increase (post-then 37\%). There are only negligible differences between pre-post and post-then comparison.

Discussion: The compulsory optional subject supports medical students not only in terms of gaining knowledge and skills, but also in developing attitude and emotional engagement on death and dying. Notably students are touched on a level of self-reflection. As a longitudinal outcome, patient-physician relationship could be enhanced by higher communicative competences.

Abstract number: P02-455

Abstract type: Poster

Assessment of Knowledge of Ethical and Palliative Care Issues in Professionals who Are Working in Palliative Care Villavicencio-Chávez C.1,2, Esquirol-Caussa J.3, Garzón-Rodríguez C. 1,4, Vaquero-Cruzado J. ${ }^{1,5}$, Loncan-Vidal P. ${ }^{6}$, Guerrero-Torrelles M. ${ }^{7}$, SanchezAldeguer J. ${ }^{3}$, García-Navarro Y. ${ }^{8}$, García-Abejas A. ${ }^{1,9}$, Ortega-Carrillo A. ${ }^{1}$ ${ }^{1}$ Palliative Home Care Service, Caredoctors, Barcelona, Spain, ${ }^{2}$ Medicina Humana, Universitat Internacional de Catalunya, Sant Cugat, Spain, ${ }^{3}$ Fisioteràpia, Fundació Privada Escolas Universitarias Gimbernat, Sant Cugat, Spain, ${ }^{4}$ Geriatría, Centres Blau Clinic Isabel Roig, Barcelona, Spain, ${ }^{5}$ Equip de Gestió de la Cronicitat, Institut Català de la Salut, Baix Llobregat Centre, Spain, ${ }^{6}$ Home Care Team Service, Fundació Santa Susanna, Caldes de Montbui, Spain, ${ }^{7}$ Catedra We Care, Universitat Internacional de Catalunya, Sant Cugat, Spain, ${ }^{8}$ EAP Sant Antoni, Institut Català de la Salut, Barcelona, Spain, ${ }^{9}$ Geriatría, José de Mello Residências e Serviços, Lisboa, Portugal

Aim: To assess the knowledge of the ethical(ET) and palliative care(PC) aspects in professionals(Ps) that are working with patients(Px) with advanced chronic disease(ACD)

Method: From June to August 2018 we design a questionnaire using Delphi methodology:12 questions(6 ethical and 6 PC concepts).It was published online in September and disseminated through social networks. Socio-demographics were recorded including age, profession and workplace.

Results: 128 answers from physicians (50.8\%), nurses (32\%) and other health professions(17.2\%). The average age was $44.4 \pm 10.8$. The sample working environment was hospital $(28.9 \%)$, in primary care $(21.1 \%)$, intermediate care (18\%) and in nursing home (13.3\%).

PC aspects: $77.3 \%$ consider that the assessment in PC includes physical, emotional, social and spiritual dimensions. $98.4 \%$ contemplated withdrawing chemotherapy from decision making at end of life issues (EOL). 46.4\% considered Px with ACD as Px with PC need. 81.9\% pondered palliative sedation to reduce consciousness to relieve suffering. $97.6 \%$ considered that decision making in ACD was integrative (patient, family and the healthcare team).83.6\% believed family members should be informed that the patient in agony does not perceive suffering.

ET aspects: $86.7 \%$ consider as a wrong that, in the EOL period prevails the opinion of the doctor over the patient and family opinions.77.8\% that, the ethical criterion to decide the treatments in a non-autonomous patient, should be the duty of care of the Px as part of the community. $58.6 \%$ recognized advance directives document is not the same as the advance care planning. $81.9 \%$ that, the euthanasia is direct, active and voluntary. $99.2 \%$ endorsed constant monitoring of symptom control and emotional support to the family of Px in EOL.

Conclusions: Our overall scores support that Ps are aware of many ET and PC issues of patients of ACD. Yet they also raise the possibility that a considerable number of Ps could need further training in ET and PC. 
Abstract number: P02-456

Abstract type: Poster

Evaluating the Experiences of Health Workers on Initiators' Course and Their Impact to Palliative Care Services in Africa Basemera B. ${ }^{1}$, Merriman A. ${ }^{1}$, Low M. ${ }^{2}$, Mwebesa E. ${ }^{1}$, Dive S. ${ }^{1}$, Basirika D. ${ }^{1}$, Tan You G. ${ }^{2}$

${ }^{1}$ Hospice Africa Uganda, Kampala, Uganda, ${ }^{2}$ Columbia University, New York, United States

Background: After 10 years of training health care professionals in Africa and beyond in supporting palliative care integration, an evaluation was conducted to analyse whether the five week Palliative Care Initiators' Course has impacted palliative care provision in Africa.

Aim or goal of the work: To evaluate the impact of the initiators' course on health workers in own countries through experiences.

Design, methods and approach taken: A cross sectional survey was carried out using self-designed questionnaires which were sent to respondents via email. The questionnaire comprised of mostly open ended questions to allow for in depth responses to phenomena under investigation.

Results: Out of 150 alumni contacted, responses from 50 participants were received. Out of the 50 respondents, 37 were from online survey and 13 were from WhatsApp calls.

The outcomes of initiators course include; Increased number of trained health care professionals providing palliative care services in own countries. Most rated useful part of the course was the clinical placement where $89 \%$ of the respondents mentioned it in response to this question. The responses are an indication that palliative care integration and development is taking place in African countries.

Few responses were received because of different reasons ranging from change in personal emails shared during the course.

Conclusion / lessons learned: Most participants have found the Initiators' Course extremely helpful in advancing palliative care in their country. Most $(79 \%)$ have achieved their desired outcomes though they still face difficulties owing to lack of recognition from the authorities and colleagues, and ignorance and lack of knowledge.

Abstract number: P02-457

Abstract type: Poster

Improving theTherapeutic Choices in the Palliative Care: The Healthcare Professionals' Point of View on the End-of-Life Patients' Dignity

Bovero A., Botto R., Tosi C., Pidinchedda A., Torta R.

Clinical and Oncological Psychology Unit, Department of Neuroscience, University of Turin, Turin, Turin, Italy

Background: Patients' dignity preservation in the end-of-life represents one of the core goal of the palliative care.

Aims: To compare the perceptions of the patients' dignity of four different healthcare professionals' groups, involved in hospital end-of-life care, such as the nurse assistants, the nurses, the physicians and the psychologists, to observe differences and similarities among their perspectives. Methods: A semi-structured interview was administered to 306 healthcare professionals involved in the hospital palliative care. The Patient Dignity Inventory (PDI), adapted by the researches to fit the professionals, a demographic form and a brief qualitative survey were administered. Statistical analyses was performed using SPSS software.

Results: The sample's average age and working years were, respectively, $43.7 \pm 10.7$ and $16.4 \pm 11.3$. Most of the physicians (33.7\%), nurses (36.9\%) and nurse assistants (47.7\%) believed that the psychological dimension was the most problematic for the patients' dignity maintenance; most of the psychologists attributed more relevance to the physical one (46.9\%). Healthcare workers' age, working years and having attended to specific training on dignity significantly correlated with the
PDI total scores $(p<.01)$. The nurse assistants and the nurses obtained significant higher scores on all the PDI subscales than the physicians; psychologists scored significantly higher than the physicians on the "Existential Distress" subscale $(p<.01)$. These results highlighted that the work experience and an adequate training might be associated to a major sensitivity towards the various aspect of dignity.

Conclusion: The integration of different healthcare professionals' perspectives appeared relevant for the therapeutic processes to consider the dignity related patients' needs. Favoring interdisciplinary palliative interventions, oriented to the maintenance of patients' dignity at the end of life, means giving value to the whole person.

Abstract number: P02-458

Abstract type: Poster

Effects of an Interprofessional Collaborative Training for Healthcare Teams on the Level of Care Determination Process with Patients and Their Relatives

Fortin G., Dumont $S$.

School of Social Work and Criminology, Université Laval, Quebec, Canada

Background: The level of care determination [LOCD] is a shared decision making that involves physicians and patients in identifying the appropriate care for their health condition which respects their life plan. LOCD is often complex, since it depends on the uncertainty of the prognosis, the patients beliefs, understanding and wishes for the end of life. In order to improve this LOCD in these situations, interprofessional collaboration [IPC] focused on patients and their relatives is essential.

Aims: This research project aims to evaluate the short-terms outcomes (efficiency and relevance) of an IPC training for health care providers developed to enhance the LOCD process.

Methods: The study was conducted in 2018 using a mixed method research design. Two groups of healthcare providers from various disciplines received and IPC training in winter $(N=26)$. Semi-directive interviews were conducted three months later to identify successes and failures of the training to change IPC practices in context of LOCD. The sense of self-competency was measured with a self-reported questionnaire at 3 different times during the study.

Results: The participants deem the training relevant when it increased their awareness on the importance of the LOCD process for their patients' end-of-life planning, and on the acquisition of useful knowledge for their practice. Useful knowledge includes: the tools available to support communication about LOCD; the concept of life project; the concept of collaborative leadership. When the training is perceived as relevant, participants noted a greater sense of competence in: discussing levels of care with patients and their relatives; their role of teaching, listening and guiding patients in LOCD situations; their roles and those of their colleagues participating in the LOCD.

Conclusion: This study shows that IPC training can be relevant and beneficial to improve the communication with patients and their relatives in context of end-of-life decisions.

\section{Abstract number: P02-459}

Abstract type: Poster

\section{Project ECHO: Filling the Gap in Pediatric Palliative Care Training} in India

Palat G. ${ }^{1}$, Rayala S. ${ }^{2}$, Doherty M. ${ }^{3}$

1Palliative Medicine, Consultant, MNJ Institute of Oncology and Director, Palliative Care, Asia, Two World Cancer Collaboration, Canada, Hyderabad, India, ${ }^{2}$ Palliative Medicine, Consultant, MNJ Institute of Oncology and Two World Cancer Collaboration, Canada, Hyderabad, India, ${ }^{3}$ Pediatric Palliative Care, Two Worlds Cancer Collaboration, Canada, Ontario, Canada 
Background: More than $98 \%$ of children who need palliative care live in low- or middle-income countries where access to palliative care is extremely limited. A lack of education about palliative care has been identified as a significant barrier to palliative care. Project ECHO uses web-based videoconference sessions to train healthcare professionals. We developed Project ECHO Pediatric Palliative Care, which focuses on training health professionals in South Asia to provide palliative care for children. Summary of Innovation: We developed the content and structure of our ECHO program by reviewing the literature, a web-based survey of stakeholders, and consultation with experts. Project ECHO PPC consists of a 90 min session occurring regularly every 2 weeks. On average, there are 34 participants, who join from 15 different health centers in India and Bangladesh. A link to the video recording and the key learning points are sent participants after each session. The sessions format is consisted of Welcome and introductions (10 mins), Didactic teaching (30 mins), Questions (10mins), Case presentation (10 mins), Case discussion (25 mins), Summary (5 mins). The goal is to create a virtual learning community where participants can reflect and engage to deepen their knowledge of PPC. The use of question and discussions is intended to encourage deeper engagement with the material, and collaboration with colleagues. We have conducted baseline evaluations of the program, with plans for future evaluation.

Conclusion: Project ECHO PPC is an innovative educational format for delivering pediatric palliative care education in resource-limited sessions, which can bring together content experts and practitioners in a collaborative online community.

\section{Abstract number: P02-460 \\ Abstract type: Poster}

Labour Pains: Beyond the Spectrum to Pre-Fatal Care -

Strengthening the Labour Needs of Dying

Lewis $S$.

Memorial University of Newfoundland, Social Work, Great Village, NS, Canada

Background: Nova Scotia is striving to identify labour challenges of integrating services to support end of life care throughout communities. A rapidly growing demographic of aging adults, as well as challenges of a rural provincial workforce have created mounting demands of caregiving labour teams. Attention to evidence based research, population shifts and social justice for the care of the dying challenges proactive, visionary engagement for all involved. Death, dying and grief care will require collaborative engagement of communities, healthcare and social sectors to strengthen a more holistic framework of care. Beyond labour force issues lies the challenge of creating discourse and opportunity to evolve in conversations with clients and families as we improve diverse support in dying and grief.

Aims: Community engagement models that support stronger discourse and death literacy are proving to stimulate changes in society. Building upon existing concepts and theories, this paper describes a new curriculum that has been developed that supports staff in critically assessing what a palliative approach to care means today. By actualizing the concepts of a person (family) integrated palliative approach to care, the End of Life Communications course supports provincial and national front line workers with diverse roles in stimulating discourse and demonstrating an evolving continuum of care.

Methods: The overall goal of the End of Life Communications course was to actively shift paradigms beyond traditional, medical approaches to care of the dying. With the support of Nova Scotia's Department of Labour and Advanced Education-Workplace Education and other hosting agencies, 1000 frontline workers in health and social care sectors took part in the course.

Conclusion: This paper will describe how this innovative, collaborative approach is supporting the labour force through active engagement with people dying and grieving in the province.
Abstract number: P02-461

Abstract type: Poster

Knowledge and Understanding of Healthcare Ethics: A Survey of Palliative care Teams at the Prince and Princess of Wales Hospice and the Queen Elizabeth University Hospital in Glasgow Devanney M. ${ }^{1}$, Datta C. ${ }^{2}$

${ }^{1}$ University of Glasgow, Glasgow, United Kingdom, 2 Prince and Princess of Wales Hospice, Glasgow, United Kingdom

Introduction: Ethics is integral to palliative care, making staff knowledge of healthcare ethics critical. A survey was distributed to palliative care teams at a hospice and hospital to assess staff self-reported knowledge of ethics and ethical issues encountered.

Aims:

- To assess staff knowledge of ethics and confidence in applying knowledge

- To determine the incidence of ethical issues in palliative care

- To gain insight into the types of ethical issues staff encounter in their work and how they currently deal with these

- To assess the desire for further staff training in ethics.

Methods: The survey was distributed to healthcare assistants, nurses, doctors, allied healthcare professionals and family support services at a hospice and hospital. Data was analysed using Microsoft Excel. Results:

- 35 out of 75 surveys were completed; $51 \%$ of respondents were nurses, 23\% healthcare assistants, $17 \%$ doctors, 3\% allied healthcare professionals, and 6\% family support services

- $69 \%$ of participants had training in healthcare ethics

- Participants demonstrated a high knowledge of the four main principles of ethics

- There was ambiguity as to what constitutes an ethical issue

- $86 \%$ had encountered at least one ethical issue at work

- Most commonly reported issue was a patient who stopped eating, cited by $44 \%$ of hospice participants

- $50 \%$ of hospital staff raised concerns about medical interventions at end of life

- Majority of respondents felt able to deal with these ethical issues

- $\quad$ Participants often turned to colleagues for support, benefiting from discussing ethical issues

- $\quad 77 \%$ desired ethical training; a course was the most popular method.

Conclusion:

- Staff knowledge of ethics appears solid

- Self-reported staff confidence measures on applying ethical knowledge was high

- $\quad$ There is a high incidence of ethical issues in palliative care

- Common ethical issues reported were patients stopping eating and issues surrounding medical interventions

- The majority of staff were desirous of additional ethical training.

Abstract number: P02-462

Abstract type: Poster

Development and Psychometric Tests of the "Cuidar" Instrument - Short Version for the Assessment of the Competences of Students for Care Palliative

Carrillo G M., Gómez O.J.,

Grupo de Cuidado Facultad de Enfermería Universidad Nacional de Colombia

Universidad Nacional de Colombia, Bogota, Colombia 
Background: Measurement scales for the competencies needed for palliative care are required, and need to be developed by undergraduate students.

Aim: develop and conduct psychometric analysis of the instrument CUIDAR designed to assess the competencies required for palliative care in undergraduate students.

Method: Cross-sectional study to validate and instrument designed from original scale CUIDAR and adapted for undergraduate students. Contents and face validity tests were conducted with an experts team, as well as construct validity by running a Cronbach's Alpha test to assess internal consistency of the scale.

Results: The CUIDAR-PC instrument assess the competencies for palliative care that undergraduate students are required to develop. Composed by 20 items, the scale includes 6 dimensions: knowledge, unicity or personal conditions to be a formal caregiver, instrumental or procedural, enjoyment or well-being, anticipation or the capacity to prevent and identify alarm situations and social interactions and relationships with social support networks. Its stratification has three levels, low, moderate and high. The experts group validation evidenced clarity, coherence and sufficiency of the scale. A factorial analysis with varimax rotation confirmed the six components by showing an aggrupation of the items into de original categories. Cronbach's Alpha level was 0.85 . Conclusions: the instrument CUIDAR-CP assess the competencies for palliative care required by undergraduate students integrally, demonstrated content and construct validity as well as a high internal consistency, suggesting its pertinence to be used in the Colombian context.

\section{Abstract number: P02-463 \\ Abstract type: Poster}

\section{“My Patient is Going to Die!” Medical Students' High Resilience Correlates with Higher Self-efficacy in Palliative Care \\ Gryschek G. ${ }^{1}$, Cecilio-Fernandes D. ${ }^{2}$, Carvalho-Filho M.A. ${ }^{1,2}$, Mason S. ${ }^{3}$ ${ }^{1}$ UNICAMP - School of Medical Sciences, Campinas, Brazil, ${ }^{2}$ Center for Education Development and Research in Health Professions (CEDAR), University of Medical Center Groningen, Groningen, Netherlands, ${ }^{3}$ Palliative Care Institute Liverpool Academic Palliative \& End of Life Care Centre, University of Liverpool, Liverpool, United Kingdom}

Background: As the world population is ages, patients with chronic illness need help to deal with worsening symptoms and eventually their own mortality. Unfortunately, medical curricula often disregard palliative care training, undervaluing the importance of providing medical students with the necessary skills to provide care. To design and implement pedagogical strategies in palliative medicine, it's essential to understand student's psychological characteristics. We hypothesized that students who engage better with palliative care patients, possess high resilience; the ability to adjust to difficult situations. Thus, our study investigated the relation of medical students' resilience with thanatophobia and selfefficacy in palliative care (SEPC).

Methods: A cross-sectional study, which enrolled 107 third-year medical students, with Ethics Committee's approval. Resilience was measured using the Resilience Scale, and performance in Palliative Care were measured using the SEPC and Thanatophobia Scales, which were translated and validated to Brazilian Portuguese during the study.

Results: We have found evidence of weak Pearson's correlation coefficient between resilience and SEPC $(\rho=0.2 ; p=0.02)$ and no correlation between resilience and thanatophobia $(\rho=-0.18 ; p=0.06)$. However, comparing the students' resilience quartiles, we found higher self-efficacy $(\mathrm{t}=2,5 ; \mathrm{p}<0.05)$ and lower thanatophobia $(\mathrm{t}=2.3 ; \mathrm{p}<0.05)$ in those with higher resilience levels.

Discussion: Our data suggest that students with higher resilience may perform better in palliative care. So, resilience may be an essential psychological characteristic for medical students in palliative care training, which should include opportunities for discussion, self-reflection, and emotional awareness, with constant feedback. This pedagogical approach should acknowledge the different psychological readiness of medical students and promote the development of resilience to deal with death and the dying process.

Abstract number: P02-464

Abstract type: Poster

Multidisciplinary Development of Nursing and Medical

Education of Palliative Care in Finland

Hökkä M. ${ }^{1}$, Sulosaari V. ${ }^{2}$, Saarto $T^{3,4}$, Lehto J.5,6

${ }^{1}$ School of Health, Kajaani University of Applied Sciences, Kajaani,

Finland, ${ }^{2}$ Turku University of Applied Sciences, Turku, Finland,

${ }^{3}$ Department of Palliative Care and Faculty of Medicine, University of

Helsinki, Helsinki, Finland, ${ }^{4}$ Comprehensive Cancer Center, Helsinki

University Hospital, Helsinki, Finland, ${ }^{5}$ Faculty of Medicine and Life

Sciences, University of Tampere, Tampere, Finland, ${ }^{6}$ Department of

Oncology, Palliative Care Unit, Tampere University Hospital, Tampere, Finland

In Finland, the undergraduate education of palliative care varies widely in nursing education and a curriculum of palliative medicine exists in only two of the five faculties of medicine. Postgraduate education in palliative care for nurses and physicians are not available at the universities. The goal of EduPal-project (2018-2020), granted by the Finnish Ministry of Education and Culture, is to develop national recommendations for undergraduate curriculum and postgraduate education in palliative care for both nurses and physicians. The project is multidisciplinary and performed in collaboration with working life.

The first phase of the project is national surveys. Twenty multidisciplinary workshops are made in different parts of the country. A national survey is ongoing among nurses working in palliative care and physicians with special competency in palliative medicine. Evaluation on nursing and medical undergraduate curriculums is completed. In addition, a nationwide questionnaire to nursing and medical students will be conducted in autumn 2018.

The project has four phases. In the first phase, surveys and evaluation are performed and analyzed. In the second phase, competence description will be made for palliative nursing and medicine (basic and specialist levels) in multidisciplinary cooperation based on the results of the first phase. In the third phase, a national recommendation for undergraduate and postgraduate education in palliative nursing and medicine will be made. In the fourth phase, new teaching methods are developed, and future research \& development activities are established.

The strength of the project is multidisciplinarity and collaboration with professionals and stakeholders. A network of palliative educators and professionals are working together to provide interprofessional palliative care education. In the conference, a description of the overall design of the project, planned outcomes and preliminary results of the phase one will be provided.

\section{Abstract number: P02-465}

Abstract type: Poster

Ivy Street Views: Evaluating Perceptions of a Novel Use of a Virtual Learning Environment

Clabburn O. ${ }^{1}$, Groves $K^{2}{ }^{2}$

${ }^{1}$ Faculty of Health \& Social Care, Edge Hill University, Ormskirk, Lancashire, United Kingdom, ${ }^{2}$ Queenscourt Hospice, Southport, United Kingdom

Study Aim: The aim of the study was to evaluate student and facilitator perceptions regarding the novel use of a Virtual Learning Environment (VLE) through the development and implementation of 'Ivy Street', a web and app based virtual community and its associated newsfeed.

Sample and Methods: All postgraduate clinicians $(n=6)$ and healthcare professional participants enrolled in the palliative care module were 
invited to participate in the study $(n=24)$. Two online surveys were developed which consisted of five open-ended questions to gain both student perceptions and facilitator perceptions of Ivy Street.

Findings: 16 (67\%) participants and 4 (67\%) facilitators responded. Data was analysed thematically to create two overarching themes of 'Positive Perceptions of Ivy Street', and 'Critical Feedback of Ivy Street'. Respondents perceived the use of Ivy Street to enable and promote peer discussion, whilst also having a high impact on student engagement. Additional benefits in using vignettes enabled facilitators to encourage students to view material learnt in classroom sessions through the lens of a known Ivy Street character or household. Respondents also commented on how Ivy Street reduced concerns regarding confidentiality when discussing patient cases through utilisation of standardised Ivy Street characters. Suggested improvements were made regarding the quantity, frequency and management of Ivy Street updates to the VLE to improve student experience for future cohorts.

Conclusion: The novel use of a virtual community within a VLE, through developing characters, a story, and vignettes, is considered to be an effective and engaging method of learning for clinicians and healthcare professionals enrolled on a palliative and end-of-life care module.

Abstract number: P02-466

Abstract type: Poster

The Training Program for Nursing Care Staff at Palliative Care Unit was Effective in Easing Anxiety about Death and EOL Care Kosugi T., Kubo M., Uike N., Yumiba T., Inoue E., Ishikawa A., Hiura A., Mori N., Fujimori S., Onisaki M., Miyazaki M.

Department of Palliative Care, Saga-Ken Medical Centre Koseikan, Saga, Japan

Background: In Japan, around $80 \%$ of people die in hospital. Many older adults live in a nursing care facility, but many such facilities cannot provide end-of-life (EOL) care for various reasons. One is that the staff lacks confidence and knowledge in dealing with death and EOL care, having little experience in either

Aims: The study aimed to examine whether a training program is effective for improving nursing care staff's confidence and attitude regarding mortality, equipping them with knowledge and skills in EOL care, and thereby increasing the quality of EOL care.

Methods: The participants underwent three to five days of practical training at palliative care units and completed pre- and post-intervention surveys. The surveys included items on the participants' anxiety about death and EOL care, knowledge of EOL care, and experience in caring for someone as they die. Each item was scored on a five-point scale, with 1 indicating strong anxiety or negative emotion and 5 , minimal anxiety or positive emotion. The responses were analyzed using a Wilcoxon signed-rank test. Results: The training was undertaken by 24 participants in nine facilities. Nine question items on anxiety/attitude regarding death and EOL care received significantly better scores in the post-intervention survey $(p<$ 0.05 ) compared with the pre-intervention survey. The difference in preand post-intervention scores was not related to differences in experience of attending palliative care course or experience of EOL care prior to the training. After the training, four of the facilities, which had been unable to provide EOL care, provided it to seven patients.

Conclusion: The training program was effective in easing anxiety about death and EOL care.

Abstract number: P02-467

Abstract type: Poster

Are Young Nurses Well Equipped in Dealing with Paediatric Pallitive Care?

Mwari Rithara S. ${ }^{1,2}$, Gitonga S. ${ }^{3}$, Gakii Kimani G. ${ }^{4}$, Muriithi N. ${ }^{5}$, Education
${ }^{1}$ Nursing, Kenya Medical Training College, Nairobi, Kenya, ${ }^{2}$ Nursing, Ongata Ngong Palliative Community Care, Nairobi, Kenya, ${ }^{3}$ Nursing, Kenyatta National Hospital, Nairobi, Kenya, ${ }^{4}$ Research, Nairobi University, Nairobi, Kenya, ${ }^{5}$ Chaplain, Kenya Prisons, Nairobi, Kenya

Background: Young nurses avoids death and dying discussion in children during training. Neverthless, it is important for students to understand death and dying early in training, hence cope with difficult situation in clinical placements.

Aims: To explore knowledge on paediatric Palliative Care among young nurses.

Methods: 62 nursing students were selected to take role play of nurses, parent, friend, sick child and complete questions on chronic illness, breaking bad news, loss, bereavements and coping strategies knowledge.

Results: Majority didnt know the defination of peadiatric palliative care and important of breaking news. $68 \%$ had some informations about PC and the $32 \%$ of the students knew the definition of PPC. $72 \%$ didn't know the different between palliative care nursing and general nursing; $55 \%$ didnt like idea of working in PCU: $45 \%$ avoided loss questions. Most of the students $76 \%$ requested more accessability to the information. $48 \%$ had relatives/friends who with chronic illness or died with, $92 \%$ interested in attending bearevements session: $32 \%$ declined to discuss own death and dying: $38 \%$ wished to attend advanced training, $62 \%$ preferred other courses, $62 \%$ were not kin in roleplaying as a sick child. $68 \%$ PPC is difficuilt to understand that general nursing, $88 \%$ need of supportive counselling centre.

Conclusions: There is need to introduce paediatric palliative care early in training for young nurses benefits. Most of palliative care assignment need be roleplay for nurses to understand and have, confident caring for children. Results indicated lot of work needed to be done by palliative providers.

\section{Abstract number: P02-468 \\ Abstract type: Poster}

Simulation-based Medical Education in Palliative Care: A Systematic Review of the Literature

Kozhevnikov D., Morrison L., Ellman M.

Yale School of Medicine, New Haven, United States

Background: The growing need for Palliative Care (PC) is outstripped by the short supply of PC specialists. This mismatch calls for competency of all clinicians in primary PC, including relationship-centered communication, management of pain and other symptoms, and interprofessional teamwork. Simulation-based medical education (SBME) has emerged as a promising modality to teach core skills and help close the educational gap.

Aims: To describe the current state of SBME in PC skills training and to identify gaps and opportunities.

Methods: We conducted a systematic review of the literature on simulation experiences addressing PC skills for learners in medicine and nursing. We collected data on learner characteristics, the method and content of the simulation, and outcome assessments.

Results: In a total of 78 studies, $77 \%$ involved learners from medicine and $38 \%$ from nursing, while social work $(6 \%)$ and spiritual care $(3 \%)$ learners were less frequently involved. Only $16 \%$ of studies involved collaboration between participants at different training levels. The standardized patient encounter was the most popular simulation method, accounting for $68 \%$ of all studies. Eliciting treatment preferences (50\%), delivering bad news $(41 \%)$, and providing empathic communication (40\%) were the most commonly addressed skills, while symptom management was only addressed in $13 \%$ of studies. The most common method of simulation evaluation was subjective participant feedback (62\%). Only $4 \%$ of studies examined patient outcomes. 
Conclusions/ discussion: We describe the current state of SBME in PC education, highlighting advances over recent decades and identifying gaps and opportunities for future directions. We recommend designing SBME for a broader range of learners and for interprofessional skill building. We advocate for expansion of skill content, especially symptom management. Finally, evaluation of SBME in PC training should be more rigorous with a shift to include more patient outcomes.

Abstract number: P02-469

Abstract type: Poster

Implementing and Evaluating a Four-year Integrated Palliative Care Curriculum for Medical Students

Ellman M., Fortin A., Putnam A., Bia M.

Yale School of Medicine, New Haven, United States

Background: Meeting the needs of patients with life-limiting illnesses requires that all physicians acquire competency in primary palliative care (PPC). Graduating medical students do not consistently feel prepared in PCC.

Aims: Develop, implement, and evaluate a longitudinal, integrated and developmental curriculum to teach PPC to medical students.

Methods: We created and implemented a curriculum with diverse educational strategies, emphasizing skill-building activities, self-reflection, and interprofessional learning. A mixed method evaluation included content analysis of written reflections, graduating student surveys, and demonstration of PCC competency in a newly created observed structured clinical examination (OSCE).

Results: Graduating students' who participated in the curriculum had significantly $(p<0.5)$ higher self-reported preparedness scores on a 13-item palliative care questionnaire (median response score $=3.8$ on a 5 -point Likert scale) compared with historical controls (median response $=3.5$ ). Analysis of the AAMC survey of our graduates from 2005-2013 (over the course of the curriculum implementation) demonstrated significant time trend improvements in perceptions of adequacy of training in End-of-life care $(p=0.0003)$ and in Palliative care $(p=0.002)$. Further, $4^{\text {th }}$ year students demonstrated competency in PPC skills in an OSCE, scoring an average of $74 \%$ ( $S D=13 \%$ ) on a 14-item palliative care history checklist.

Conclusion / Discussion: A 4-year longitudinal, integrated curriculum enhanced students' skills and preparedness in PPC. Blended learning strategies (online modules with live workshops) promoted interprofessional learning, flexibility and adaptability. As curricular structures, faculty and clinical resources vary across institutions, proven and adaptable strategies will be beneficial to improve PCC education. The teaching strategies and curricular components can be adapted for inclusion in other medical education programs.

\section{Abstract number: $\mathrm{P} 02-470$}

Abstract type: Poster

In Case of Life-limiting Illness: What is Most Important for the Public in Norway?

Karlsen L. ${ }^{1}$, Dale K.Y. ${ }^{2}$, Winsjansen B. ${ }^{3,4}$, Lindseth G. ${ }^{5}$, Driller B. ${ }^{6}$, Care and Treatment of Critically ill and Dying Patients

${ }^{1}$ Centre for Development of Institutional and Home Care Services, Kristiansund Department, Ålesund, Norway, ${ }^{2}$ Molde University College, Faculty of Health Sciences and Social Care, Molde, Norway, ${ }^{3}$ Fræna Municipalities, Cancer Coordination, Elnesvågen, Norway, ${ }^{4}$ Eide Municipalities, Cancer Coordination, Eide, Norway, ${ }^{5}$ Kristiansund Municipality, Cancer Coordination, Kristiansund, Norway, ${ }^{6} \mathrm{M} \varnothing \mathrm{re}$ og Romsdal Hospital Trust, Cancer Department, Ålesund, Norway

Background: Research suggests that there is lack of knowledge and mostly negative perceptions towards palliative care in the public. An aging population and it's associated increase in the number of palliative patients urges us to explore the public's understanding of the concept of palliative care. This is important to inform policymakers and to target educational strategies. Resulting public education needs to facilitate regional and cultural characteristics as well as challenges that may be associated with demographic details.

Aims: To explore approaches of the general public towards a palliative disease and check if the respondents' priorities are associated with gender, age and level of education.

Methods: A paper survey with closed questions was sent to a sample of the general public by random distribution among employees in the eight municipalities in Romsdal, Norway $(n=530)$. A question about what is most important in case of serious illness, like cancer, with limited life expectancy included four main topics; view of life, symptom management, practical support and relation to family and friends. Descriptive statistical analysis was used for demographic data and preferences, Chisquare test was applied for association analyses.

Results: The response rate was $57 \%(n=300)$. Most important points were to get relief of pain and suffering (54\%), positive view of life (24\%), secure that family and friends are not anxious or distressed $(15 \%)$ and getting practical issues solved (7\%). Gender and education did not affect the results. The priority of symptom management was significantly higher in people from 59 to 80 years $(p=0.05)$.

Conclusion: A sample of people in Norway, especially in older age, value symptom management as the most important point for them in case of serious life-limiting illness. Identifying educational strategies reflecting the public's current perception is a key factor to empower individuals and to involve communities in future palliative care.

Abstract number: $\mathrm{P} 02-471$

Abstract type: Poster

Post-graduate Palliative Care Education for All Healthcare Providers in Europe: Results from an EAPC Survey Paal P. ${ }^{1}$, Brandstötter C. ${ }^{1}$, Lorenzl S. ${ }^{1,2}$, Larkin P. ${ }^{1,3}$, Elsner F. ${ }^{4}$

${ }_{1}$ Paracelsus Medical University, Salzburg, Austria, ${ }^{2}$ Ludwig-MaximilianUniversity, Munich, Germany, ${ }^{3}$ Lausanne University Hospital, Lausanne, Switzerland, ${ }^{4}$ RWTH Aachen University, Aachen, Germany

Palliative care training at basic, intermediate and specialist levels, recommended by the WHO is challenging to access in resource-poor countries and regions. Providing support in this regard would seem a moral imperative for all countries with established palliative care education systems and a strong resource base. In collaboration with WHO European Office and EAPC this paper looks into the educational requirements in palliative care at post-graduate level within Europe.

A survey was specifically designed to gather opinions and comments on elements of palliative care education from European experts. Participants were invited to assess the EAPC core competencies on a 5-item scale and to define essential learning goals. Survey data was statistically analysed using IBM SPSS Statistics Software. Qualitative data was thematically analysed.

195 data sets were recorded, 82 were completed fully. The statistical analysis revealed a high agreement regarding the key elements of palliative care education. The thematic analysis indicated that at post-graduate level all health care providers need to

(1) comprehend the palliative care philosophy,

(2) be able to demonstrate the complex symptom assessment and management competencies,

(3) be able to design care plans based on patients and families wishes integrating multi-professional and interdisciplinary approaches, and

(4) be able to listen and self-reflect.

Inadequate skills and capacities of healthcare workers are one of the four barriers hindering the access to palliative care. This paper contains 
a new and comprehensive list of learning goals essential for multidisciplinary post-graduate palliative care education. Besides highlighting the relevant competencies, the article provides best-practice toolboxes with teaching and assessment methods. The article comments on the WHO's palliative care definition and underpins the importance of the role of the education in knowledge development and skills acquisition.

Abstract number: P02-472

Abstract type: Poster

\author{
A Cost Effective, Home Study Palliative Care Course to Improve \\ and Sustain Healthcare Professionals Confidence to Deliver \\ Palliative Care \\ Sadler C. ${ }^{1}$, Reed L. ${ }^{1}$, Todd J. ${ }^{2}$ \\ ${ }^{1}$ Education and Research, Princess Alice Hospice, Esher, United \\ Kingdom, ${ }^{2}$ Medicine, Princess Alice Hospice, Esher, United Kingdom
}

Background: There is a need to develop the knowledge and skills of specialist and generalist practitioners in palliative and end of life care in for them to better care for their patients. The European Certificate in Essential Palliative Care (ECEPC) is an eight week home-study based programme for healthcare professionals (HCP). Since its inception in 2001, over 5000 people have successfully completed the course, now run in 10 centres throughout Europe. It is biannual with an average of 150 HCP's in each cohort.

Aim: To provide and evaluate the impact of a home study educational intervention that is cost effective and accessible to a diverse range of HCP's (doctors, nurses and allied HCP's) in different healthcare settings.

Method: One hospice acts as the hub with 10 hospices as the spokes with collaboration and discussion between the local and coordinating sites. The collaboration acts as a governance mechanism, and ensures development and sustainability of the programme. The home study model combines a handbook; virtual learning environment to make it cost effective and accessible to a diverse range of healthcare professionals. The course assessment is coordinated by the hub, but run simultaneously at each site, and seeks to examine knowledge, skills and attitudes. This is comprised of a written portfolio and an assessment day. A multicentre longitudinal mixed methods study was undertaken to explore the impact of the ECEPC.

Results: The research study demonstrated that the ECEPC is consistently well evaluated and increases confidence in symptom control, holistic care, communication and multi-professional working which is sustained over time.

Conclusion: The ECEPC is a cost effective and accessible way to educate a wide range of HCP's as a multi-site hub and spoke home study educational intervention. Despite the current reduced opportunities for both study funding and time for healthcare professionals generally, the ECEPC is oversubscribed.

\section{Abstract number: $\mathrm{P} 02-473$ \\ Abstract type: Poster}

\section{Palliative Care Learning in an Electronic Era}

Xu Y., Morrison L., Ellman M.

Internal Medicine, Yale University School of Medicine, New Haven, United States

Background: All healthcare providers draw on palliative care (PC) principles and practices to care for patients with serious illnesses. However, variability and gaps exist in the quality of structured PC education. With increasing reliance on smartphones and computers, the demand grows for electronic resources to supplement textbooks and classroom teaching. These electronic resources are scattered across different platforms, therefore posing challenges for learners to identify and access appropriate resources for their educational and clinical needs.
Aims: To identify and categorize electronic PC educational resources suitable for learners and practicing graduates.

Methods: We performed a search of PC educational electronic resources and categorized these according to consensus competencies across five domains: Communication; Pain and Symptom Management; Palliative Principles and Practice; Psychosocial, Spiritual, and Cultural Aspects of Care; and Terminal Care and Bereavement. We also characterized these resources by

1) amount of time needed to complete the material,

2) ease of access, and

3) media format (text, video, mixed).

We chose these categories to determine a resource's suitability as a point of care guide to improve patient care.

Results: 39 electronic resources were identified. Most (87\%) required at least an hour to complete and were not suitable for point of care use. Only $40 \%$ of the resources addressed psychosocial, spiritual, and cultural aspects of care and only approximately $30 \%$ allowed for interactive learning.

Discussion / Conclusions: Our analysis revealed gaps in electronic PC educational tools, especially those suitable for easy bedside use and those targeting different learner styles. There is a need for easily accessible tools that offer focused guidance to improve their PC at the bedside. Similarly, resources designed for various learning styles will allow all healthcare learners and professionals more options to enhance their PC learning.

\section{Abstract number: $\mathrm{P} 02-474$}

Abstract type: Poster

\section{Compassionate End of Life - CEOL Networks}

Steele K., Lynch $M$.

Healthcare Department, Irish Hospice Foundation, Dublin, Ireland

Background: Each year in Ireland over 7,000 people die in residential settings yet there is no formal framework to sustain quality delivery of end of life care (EOLC) in these settings. CEOL, a quality improvement (QI) programme assists RCCs to identify, reflect and implement changes to enable delivery of compassionate end-of-life. Key to the success and sustainability of the programme is the CEOL Regional Network structure.

Aims and objectives: The aims are to

1. establish Regional CEOL Networks,

2. to provide staff with on-going.support and educations,

3. to provide staff with a peer support/ networking structure,

4. to assess the impact and usefulness of the networks to attendees.

Methods: 2 professionally accredited CEOL networks were established. Each network held three meetings per year. To date 10 network meeting have been held from 2017-2018. 272 staff members from both private and public care centres have attended. Learning inputs from community services such as specialist palliative care, gerontology, acute hospital are facilitated along with review of the CEOL programme, how it is working in each RCC incorporating shared learning, peer support and networking opportunities.

Findings/ results: Attendees complete an evaluation after each network meeting with a $70 \%$ response rate (191 evaluations received). The main themes that emerged from analysis of the evaluation forms were

1. importance of providing staff with a structured forum to meet,

2. networking opportunities,

3. increasing knowledge and skills,

4. a peer support structure. 
Conclusions: The CEOL Networks develops staff skills and confidence in communicating on end-of-life care. The network structure operates a community learning practice which supports the sustainability of the CEOL programme. It also provides opportunity for links with specialist services and other relevant end of life initiatives. Further regional networks are planned to support a national foundation for the CEOL programme.

Abstract number: P02-475

Abstract type: Poster

\section{Global Palliative Care Education}

Fink R., Arora K., Bailey F.A., Scott M., Robertson N., Lawanto K. University of Colorado Anschutz Medical Campus, Aurora, United States

Introduction and Aim of the project: Palliative care (PC) is an interdisciplinary approach to care for the seriously ill. PC is rapidly establishing services within hospital systems yet current estimates reveal that $1 / 3$ of the world has no access to PC (Reville \& Foxwell,2014). In 2014, the World Health Assembly called growing PC capacity in the community an ethical imperative. Major obstacles to providing PC in the community include lack of available and affordable educational programs for professional and family caregivers. To address this problem, we developed 5 Massive Open Online Courses (MOOCs) in PC. Courses launched in October 2018, bringing the expertise of interdisciplinary PC educators to an online global educational network of 150 partners, 35 million registered learners and 29 countries, making it possible to reach almost anyone desiring primary PC education.

Methods: Learners take five courses over 25 weeks: What is Palliative Care, Pain Management, Easing Physical Symptoms, Psychosocial and Spiritual Aspects of Palliative Care, and a cumulative course where learners apply what they learned in the first four courses. Upon completion of each course, learners earn a Coursera Course Certificate. If desired, learners can submit one or more certificates for continuing education credits. After completion of all 5 courses, learners earn a Specialization Certificate in Palliative Care.

Conclusions: Serious illness and the suffering it causes is a human experience crossing all cultural, economic, and geographic boundaries. PC is a proven approach to decrease suffering, increase quality of life, and support seriously-ill persons and their caregivers. A high-quality, affordable, online PC education program helps take primary PC skills across the globe.

Funding: The authors acknowledge minimal funding given by the online educational platform for faculty support of this project.

Abstract number: P02-476

Abstract type: Poster

Healthcare students' Knowledge on Palliative Care and End-ofLife Care from Two Portuguese Universities Sickmüller Nunes F. ${ }^{1}$, Rego G. ${ }^{1}$, Laranjeira A. ${ }^{2}$

${ }^{1}$ Faculty of Medicine of Porto University, Porto, Portugal, ${ }^{2}$ School of Health Sciences, University of Aveiro, Aveiro, Portugal

Background: In today's complex health care delivery system, the needs of patients with serious illness and their families demand that healthcare professionals be educated in palliative care(PC) and end-of-life care(ELC).

Although there are evidence supporting the value of these types of care and the importance of healthcare professional's education on these fields, the number of healthcare professionals enabled to provide it is inadequate to meet the needs of patients and families across the lifespan and the illness trajectory.

Aim: This study aimed to assess and characterize the knowledge on PC and ELC of healthcare students from two Portuguese Universities.
Methods: An explorative quantitative design was developed to collect data between January and Mars of 2018 among students from the School of Health Sciences of the University of Aveiro and the Faculty of Medicine of the University of Porto. After the data was collected, it was analyzed using the version 22.0 from IBM SPSS.

Results: Study respondents ( $n=143$ ) were mostly woman $(91 \%)$, who had a curricular unit on PC (57\%) and reported having "medium" knowledge on the subjects covered (61\%).

CONCLUSION / Discussion: Although the knowledge on PC and ELC of the questioned healthcare students was good, the data obtained showed that there are still misconceptions about these themes among them, namely to the introduction of PC as a care and the concept of Dysthanasia and Advanced Directives.

Since these misconceptions can lead to delays in meeting patients' needs, a late transition to PC and discussing end-of-life issues with patients/families, it's important to introduce specific training and curricular units on ELC and PC since undergraduate courses.

Healthcare educators should initiate changes in undergraduate, graduate, and continuing education to ensure that future and practicing healthcare professionals have relevant attitudes, knowledge, and skills to care well for severally ill and dying patients.

Abstract number: P02-477

Abstract type: Poster

Delivery of Ward Based Education in a Regional Cancer Centre Griffiths A.

The Clatterbridge Cancer Centre, CReST, Wirral, United Kingdom Presenting author email address: ann.griffiths2@nhs.net

Background: Following the discontinuation of the Liverpool Care Pathway (LCP) A Regional Cancer Centre designed and implemented The End of Life Care and Communication Record. This was introduced in 2015.

It is recognised that all healthcare staff caring for dying patients must ensure they are aware of and compliant with evidence-based practice and guidance.

Aim: Design, implement and evaluate a bite size education programme for end of life care for clinical staff focusing on Symptom Control.

Methods: In July 2018 bite size sessions began on a single ward and then rotated to another ward the following week. The aim was to focus on one symptom and to deliver 4 sessions per month to each ward also inviting staff from other wards to join in on the training.

We used a bite sized blended learning approach to deliver education on 4 of the 5 main symptoms at end of life.

Written qualitative feedback was received from all participants to aid evaluation.

Results: 91 members of staff have had training in symptom control at the end of life covering Pain/agitation/Respiratory tract secretions/nausea and vomiting. We are yet to cover Shortness of Breath. We receive feedback in the form of one thing you have learned from the session however a final evaluation will be sent out at the end of all sessions.

The process evaluation has highlighted the need to provide on-going education and training for all members of staff caring for patients in the last hours/days of life. It has also been noted that staff used the sessions for debrief and reflection.

Conclusions: Although we have not completed the training it has already become apparent the need for ongoing education and training across all wards. We have also provided information regarding access and content of E-elca for ongoing professional development.

Abstract number: P02-479

Abstract type: Poster

How Can Nurses Be Prepared for End-of-Life Care? Griffith S. 
Farleigh Hospice, Education, Chelmsford, United Kingdom Presenting author email address: sue.griffith@farleighhospice.org

Background: Little research currently exists informing how nurses have become prepared for end-of-life care. Available evidence indicates that lack of preparation can lead to avoidance of dying patients.

Aims: The study's aim was to establish how hospice nurses have become prepared, to offer insight into future educational strategies.

Methods: A classic Grounded Theory approach was used to investigate the experiences of 22 hospice nurses in one UK hospice. Theoretically sampled nurses participated in individual and focus group interviews. Data was analysed using the constant comparative approach, throughout data collection, until data saturation was reached. Findings were discussed with participants and palliative care experts to establish validity. Results: Findings were synthesised into five categories: the shared ideal, feeling good at the job, making a difference, experience/exposure to hospice work, and the importance of role models. The shared ideal formed the core category, which explained how hospice nurses feel a sense of 'fit' with their organisation.

Discussion: Data, previous research and a concept analysis of prepared for end-of-life care were analysed. From this a substantive theory was developed which incorporated the conceptual framework of the Person/ Environment Fit theory. Nurses need to have a willing attitude toward care of the dying and understand that cure is not always possible, which requires more emphasis in the curriculum. All nurses need to have experience of caring for dying patients, and being with people as they die, with opportunities for support and de-briefing from experienced and knowledgeable colleagues. In an area where there is currently little research to inform practice, the findings can be used to guide nurse education strategies.

Conclusion: Although based on a small study of one site, the substantive theory concurs with the small amount of international literature currently available and may have salience for nurse education.

Abstract number: P02-480

Abstract type: Poster

Two Years after Implementing Medical Assistance in Dying in Canada: Educational Needs of Health Care Professionals and the General Public Wilson D.M. ${ }^{1}$, Triscott J. ${ }^{2}$

${ }^{1}$ Faculty of Nursing, University of Alberta, Edmonton, Canada, ${ }^{2}$ Faculty of Medicine, University of Alberta, Edmonton, Canada

Background: Medical Assistance in Dying (MAID) was implemented across Canada in June of 2016. Every province and territory, however, has a different process for citizens to apply for and have MAID approved. Two years after this publicly-funded healthcare service was initiated, it was time to assess baseline knowledge and determine educational needs, if any.

Aims: To explore healthcare professional and general public educational needs regarding MAID.

Methods: An online 2017-18 survey of Alberta physicians, nurses, and members of the general public was conducted. Following ethics approval, a brief devised survey was posted online and advertised in a wide variety of community and healthcare professional sites.

Results: 282 voluntary participants $(50 \%$ healthcare professionals and $50 \%$ members of the general public) completed the questionnaire. Only $30.5 \%$ identified the correct approximate number of MAID deaths to date in Alberta, 33.0\% correctly identified when MAID can be done, and $48.9 \%$ correctly identified the locations or places where MAID can be practiced. Nearly one half of the respondents $(49.3 \%)$ correctly identified who can stop MAID once approved and slightly more correctly identified how MAID is performed to end life (52.8\%). Higher percentages were obtained in relation to identifying where reliable information about MAID can be found (63.1\%), and in identifying which people can qualify for MAID (67.7\%). Surprisingly, few differences in the knowledge level of healthcare professionals and members of the general public were found in relation to the research questions.

Conclusion: As MAID is a new policy and practice, and relatively rare, it is not surprising that educational needs exist for both healthcare professionals and members of the general public. Given that it is a service that it is legally provided, it is imperative that healthcare professionals all gain knowledge about MAID.

Abstract number: $\mathrm{P} 02-481$

Abstract type: Poster

Educational Intervention of Palliative and End-of-Life Care for Staff Working in Long-term Care Facilities: An Integrative Review lida K., Mcllfatrick S., Ryan A., Hasson F. Institute of Nursing and Health Research, Ulster University, Newtownabbey, United Kingdom

Background: Globally, the need for palliative and end-of-life (EOL) care in long-term care facilities (LTCFs) is increasing due to ageing populations. Staff education is a vital aspect of improving care quality; however, many LTCFs face limitations in the provision of such education due to resource shortage. This review is part of a study on the development of a palliative and EOL care educational programme for Japanese LTCFs.

Aim: To examine recent literature for palliative and EOL care educational intervention for staff working at LTCFs.

Methods: An integrative review framework (Whittemore \& Knafl 2005) was used. Seven electronic databases were searched and five key journals were hand-searched with limitations on language (English and Japanese) and dates (2007-2018). Search terms related to palliative care, LTCF and education were combined to increase search sensitivity. Descriptive and thematic analysis were conducted.

Results: Forty-six papers were included in synthesis. Locations of studies were UK ( $n=17)$, North America $(n=13)$, Japan $(n=8)$, EU $(n=4)$, Oceania $(n=3)$ and China $(n=1)$ with no cross-country studies. Various study methods were used: surveys (20\%), interviews (32\%) and mixed/multimethods (48\%). Most employed an uncontrolled before-and-after study design (61\%), whereas two employed a cluster-RCT. There was variation in education duration (one-day to over 12 months) and content (generalist palliative/EOL care, dementia, ACP, cultural competence and symptom management). Barriers (e.g. staff shortages, culture and values in learning) and facilitators (e.g. on-site intensive facilitation) for implementation were reported.

Conclusion: Variation in educational development and research activities were highlighted among countries. Most Japanese studies remained questionable due to the programme's credibility, implementation and outcome evaluations. A methodologically robust and culturally appropriate educational intervention is required for Japanese LTCFs.

Abstract number: $\mathrm{P} 02-482$

Abstract type: Poster

Phenomenological Texts as Pedagogic Instruments: A Pilot Study to Determine the Value of Reading what an Authentic Nursepatient Relationship is for Educating Nursing Students to Be Better Able to Provide Expert Person-centered Care for People Nearing the End of Life

Errasti-Ibarrondo B..$^{1,2}$, Wilson D.M. ${ }^{3,4}$

${ }^{1}$ Adult Person Nursing Department, Faculty of Nursing, University of Navarra, Pamplona, Spain, ${ }^{2}$ IdiSNA, Instituto de Investigación Sanitaria de Navarra, Pamplona, Spain, ${ }^{3}$ University of Alberta, Edmonton, Canada, ${ }^{4}$ University of Limerick, Limerick, Ireland

Background: When people become aware they have a limited time left to live, they usually need highly personalized supportive care. Nurses are very important for dying people, and the nurse-patient 
relationship is critical for person-centered care to occur in hospitals and other end-of-life care settings. Teaching nursing students to be able to establish authentic interpersonal relationships with dying patients is a real challenge, as it is not something that can be easily learned or taught. Phenomenological Texts -the result of hermeneutic-phenomenological research studies- are a new and promising form of "teaching material." However, there is little evidence of the benefit of using short phenomenologically-derived reports about what an ideal nurse-patient relationship for student nurses to learn how to provide person-centered care.

Aims: Explore the self-identified impact of reading phenomenological texts and reveal the teaching usefulness of brief phenomenological texts for enhancing nursing student ability to provide person-centered end-oflife care.

Methods: Qualitative descriptive pilot study; 30 nursing students in Alberta read a short passage, wrote reflections on what they read and completed an evaluation form about how useful it was for them to read the text and write a reflection.

Results: $26 / 30$ participants reported this was a useful activity. The reflections showed the text helped them gain a more insightful and more broad-based view of the importance of an authentic relationship for patients. Moreover, reading the text helped students discover additional nuances and traits of the role they will have as practicing nurses. Conclusions: Phenomenological texts are a promising teaching tool for nurses and perhaps others to gain a better understanding of intangible aspects of the care needed to foster authentic relationships for personcentered care at the end-of-life.

Abstract number: P02-483

Abstract type: Poster

\section{Educational Needs of Primary Health Care Nurses on Palliative \\ Care \\ Spineli V.M.C.D., Minosso J.S.M., Costa G.D., Oliveira M.A.C.}

School of Nursing, University of São Paulo, Sao Paulo, Brazil

Introduction: The education of Primary Health Care (PHC) nurses in Palliative Care (PC) is made necessary to provide comfort and alleviate the suffering of patients and their relatives with life-limiting chronic diseases.

Objective:To verify the needs of education in PC of nurses working in PHC.

Method: This is a descriptive and exploratory study with a qualitative approach. For data collection, 19 focal groups were performed, with the participation of 183 nurses working in Basic Health Facilities and/or the Family Health Strategy of 19 municipalities in the South and Southwest of Minas Gerais, Brazil, with a mean of 9.6 nurses per group. The ethical and legal aspects for research with human beings in Brazil were preserved. The corpus of analysis of the study was formed by the data obtained in the focal groups and analyzed according to thematic analysis, performed in three stages: pre-analysis, content exploration, outcome treatment and interpretation; in light of Katharine Kolcaba's theory of comfort.

Results: The data treatment and interpretation has allowed the categorization of these outcomes into specific themes. Such themes represent the needs of education in PC, according to the participating nurses. Among themes which were highlighted themselves: The process of communication and care in the end of life phase as fundamental aspects to the professional's education to provide relationship of trust, support in situations of fragility and suffering, and to avoid the distancing between the professional, the patient and the family.

Discussion: The lack of preparation to communicate and face situations of physical, emotional, social and spiritual suffering, especially in the end of life phase, corroborates that nurses have difficulties to provide comfort to the patient and his/her family in all these dimensions.
Conclusion: The identification of the needs of education in Palliative Care of nurses from PHC contributes to the elaboration of proposals of education in PC.

\section{Abstract number: P02-484 \\ Abstract type: Poster}

Professional Education in Spiritual Care in Palliative Care: An EAPC White Paper

Best M. ${ }^{1,2}$, Leget $C^{3}{ }^{3}$ Goodhead A. ${ }^{4}$, Paal P. ${ }^{5}$

1Psycho-Oncology Co-operative Research Group (PoCoG), The University of Sydney, Sydney, Australia, ${ }^{2}$ Institute for Ethics and Society, The University of Notre Dame Australia, Sydney, Australia, ${ }^{3}$ University of Humanistic Studies, Utrecht, Netherlands, ${ }^{4} \mathrm{St}$ Christopher's Hospice, London, United Kingdom, ${ }^{5}$ Paracelsus Medical University, Salzburg, Austria

The EAPC White Paper addresses the issue of spiritual care education for all palliative care professionals. It is to guide health care professionals involved in teaching or training palliative care and spiritual care; stakeholders, leaders and decision makers responsible for training and education; as well as national and local curricula development groups.

Early in 2018, preliminary draft paper was written by members of the EAPC spiritual care taskforce inviting comment on the four core elements of spiritual care as outlined by Gamondi et al.(2013) in their paper on palliative care core competencies. The preliminary draft paper was circulated to experts from the EAPC spiritual care reference group for feedback. At the second stage (July-August) feedback was incorporated into a second draft paper and experts and representatives of national palliative care organizations were invited to provide feedback and suggest revisions. The final version incorporated the subsequent criticism and as a result, the Gamondi framework was explored and critically revised leading to updated suggestions for spiritual care education in palliative care.

The EAPC white paper points out the importance of spiritual care as an integral part of palliative care and suggest incorporating it accordingly into educational activities and training models in palliative care. The revised spiritual care competencies for all palliative care providers are accompanied by the best practice models and research evidence at the same time being sensitive towards different development stages of the palliative care services across the European region.

Better education can help the healthcare practitioner to avoid being distracted by their own fears, prejudices, and restraints and attend to the patient and his/her family. The EAPC white paper encourages and facilities high quality, multi-disciplinary, academically and financially accessible spiritual care education to all palliative care staff.

\section{Abstract number: P02-485}

Abstract type: Poster

\section{Home Made 5 Minute Videos as a Teaching Tool in Paediatric} Palliative Medicine

Craig F., Kerr-Elliott T., Bayliss J.

The Louis Dundas Centre for Palliative Care, Great Ormond Street Hospital for Children NHS Foundation Trust, London, United Kingdom

Aim: Specific topics in palliative care need to be taught to a wide group of professionals, so similar teaching sessions are delivered repeatedly. Our aim was to design an effective method of delivering recurrent teaching on a single theme.

Design, method and approach: We chose video as our teaching tool as we wanted something that would engage the audience and would work as a stand-alone short teaching session for those accessing on-line learning and could also be used as an interactive tool during formal teaching sessions. We chose 2 topics that were taught regularly and where we felt 
the key message could be delivered in 5 minutes. None of the production team had any previous experience of filming or editing.

The first film 'The A,B,C and D of Dignity Care' was developed on story boards and scripted. We felt the key messages would be most effective if delivered by children (none of whom were patients). Volunteer children used a combination of the script and improvisation. The second film 'What school age children understand about death' was made with the same group of children. This was unscripted and the children spoke from their own experiences and understanding. Written consent for participation was obtained from the children and their parents.

The videos were filmed using an iphone and ipad and edited using standard computer software. Each took around 8 hours to film and 8-10 hours to edit. Where a storyboard had not been used, editing required key points to be pulled from the dialogue and organized into themes.

Results: Both films delivered the key messages intended and have received positive feedback from participants and those delivering teaching. Conclusions: Short home made films can help deliver palliative care teaching. They are easy but time-consuming to make and are perhaps best used where a specific teaching session has to be delivered recurrently.

Abstract number: P02-486

Abstract type: Poster

\section{Designing an Educational Program of Person Centred End-of-Life} Care for Registered Nurses who Work in Non-Palliative Care Specialist Settings. First Steps

Carvajal A. ${ }^{1}$, Haraldsdottir E. ${ }^{2}$, Kroll T. ${ }^{3}$, McCormack B. ${ }^{4}$, Errasti B. ${ }^{1}$, Larkin $P^{5}$

${ }^{1}$ Universidad de Navarra, Faculty of Nursing, Pamplona, Spain, ${ }^{2}$ Queen Margareth University, Edimburgh, United Kingdom, ${ }^{3}$ University College Dublin, Dublin, Ireland, ${ }^{4}$ Margaret Queen University, Edimburgh, United Kingdom, ${ }^{5}$ Université de Lausanne, Lausanne, Sweden

Background: The demand for providing palliative care outside specialist palliative care setting is increasing. In this context, registered nurses working in the generalist health care setting need to be prepared to provide end of life care. Thus, experts in Palliative care and Person Centred Care are developing an education program that aims to equip registered nurses with the knowledge and skills needed to provide endof-life person centred care. This study is the first step of the design of the program.

Aim: To identify the essential elements nurses, who care for people in the general health care settings, need to provide person centred end-oflife care.

Method: A scoping review. Articles written in English and Spanish from 2000 to 2018 were reviewed in Medline, Cinahl, PsycINFO, Cochrane, Web of Science and Embase databases. The main keywords used in the search were 'nurse', 'person centred care' and 'end of life' with their respective synonyms.

Results: The review yielded twenty-seven articles from thirteen countries. It was found that nurses need knowledge of the whole person (patient and family) to provide personal comfort through symptom management and support, as well as the education of the whole family. Nurses also need to develop an interpersonal relationship with people they care for and with other healthcare professionals and this is vital for effective palliative care. Moreover, nurses need to have professional and personal self-knowledge to provide person-centred care. Support from the organization and managers appears to be of high importance.

Conclusions: These results can be used as the basis of a framework for an educational program focused on the philosophy of person centred end-of-life care for nurses working in the contexts of general health care system. This will enable the professional and personal development of nurses needed to improve the wellbeing of dying patients and their families.
Abstract number: P02-487

Abstract type: Poster

Lessons Learned from Introducing "Last-Aid" Courses at a

University Hospital in Germany

Mueller E. ${ }^{1}$, Bollig G. ${ }^{2,3,4}$, Becker G. ${ }^{1}$, Boehlke C. ${ }^{1}$

${ }^{1}$ Clinic for Palliative Medicine, University Hospital, Freiburg, Germany,

2Palliative Care Team, Medical Department, University of Southern

Denmark, South Jutland Hospital, Sønderborg, Denmark, ${ }^{3}$ Palliative

Care Research Group, Institute of Regional Health Research,

Sønderborg, Denmark, ${ }^{4}$ Letzte Hilfe Deutschland, Schleswig, Germany

Background: The "Last-Aid" course curriculum has been created by an international working group to educate citizens about the care of the dying. Over the last three years "Last-Aid" courses were successfully established in the community setting in several European countries. We hypothesized that education needs concerning care of the dying are especially high in hospital staff not directly involved in the treatment of patients, because care of the dying is not included in their vocational training. However, they are often indirectly involved in the processes of dying patients.

Aims: To introduce "Last-Aid" courses at a university hospital and evaluate their acceptance.

Methods: The courses are comprised of four sessions of 45-minutes each: care at the end of life, advance care planning and decisions making, symptom management, cultural aspects of death and bereavement. The courses were announced on the hospital's intranet platform and evaluated by an anonymous survey in the last session including Likertscale and open response questions.

Results: All offered courses were fully booked. 55 of 56 participants took part in the evaluation survey. They included the full range of professions reaching from hairdresser, laboratory staff, administration, but also nurses and physicians. 25 (45.5\%) participants had only rare or no contact to patients. On a four-level Likert-scale $100 \%$ of the participants ( $n=42, n=13$ no answer) rated the course as (very) good and 52 participants ( $94.5 \%, n=53, n=2$ no answer) would recommend the course to others. Reasons to participate included the wish to prepare for emotional aspects of care of the dying, to be informed about care givers specialized in palliative care, to reduce own fears of dying, and to contribute to society's discourse of dying.

Conclusion: "Last-Aid" courses were well accepted and helped to reduce information deficits on care of the dying especially in staff without direct contact to patients.

Funding: None

Abstract number: P02-488

Abstract type: Poster

Education of Palliative Representatives in Nursing Homes

Simmross I., Witalis K., Ousi Z., Sandlund F., Ekeström M.-L., Strang P.

Palliativt Kunskapscentrum i Stockholms Län, Stockholm, Sweden

Aim and goal of work: Health professionals that care for older people need to learn more about a palliative care approach, to improve quality of care in end of life. Therefore, an education model was designed to improve knowledge of palliative care.

The goal of the training is that the Palliative Representative will learn how to:

- Explain what palliative care approach means

- Identify the patient's nursing needs in early and late palliative phase

- $\quad$ Relate and discuss palliative care approach with coworkers

Method: Managers in the municipality appointed persons to be educated and become palliative representatives. The education took place 
on three occasions (three whole days) with identified learning goals based on National Guidelines. After finishing the education, the participant has a personal assignment to disseminate knowledge about palliative care and palliative care approach within their work settings.

The education was held by registered nurses certified in palliative care. A written evaluation took place in connection with the last education cession.

Results: 419 health professionals have completed the education to become a palliative representative during 2016 - 2018.

According to the evaluation, participants assessed that they acquired knowledge and a deeper understanding of palliative care- and approach and that they will benefit from the education. According to the evaluation 406/419 stated that they to a large extent will have good use of the education at their workplace. The evaluation also shows that 388/419 of the participants felt, to a large extent, that they had received enough knowledge after the education to educate their coworkers about the palliative approach.

Conclusion: With support from management and with personal involvement, Palliative representatives are prepared with tools for education of coworkers about a palliative care approach.

Abstract number: P02-489

Abstract type: Poster

\section{"It's Like They're Showing that They Car",: A Qualitative Study of} the Second Conversation Model

Weil A. ${ }^{1}$, Bristowe $K .^{1}$, Wakefield D. ${ }^{1,2}$, Mathew R. ${ }^{3}$, St John $K .^{2}$, Carey.$^{2}{ }^{2}$, Sleeman K.E. ${ }^{1}$

${ }^{1}$ Cicely Saunders Institute, King's College London, London, United Kingdom, ' 'Guys and St Thomas' NHS Foundation Trust, London, United Kingdom, ${ }^{3}$ The Royal College of Physicians, London, United Kingdom

Background: The Second Conversation Model is an on-the-job end of life care communication training intervention for junior doctors. The training involves 4 components: observation of a senior-led first conversation with patient and/or caregivers; subsequent preparatory discussion; a junior-led 'second conversation' to address questions or concerns; finally, a structured debrief to consolidate learning. A pilot study found benefits of the Second Conversation Model for clinicians but did not explore patient or caregiver views or potential harms.

Aim: To explore the benefits and risks of the Second Conversation Model from the perspectives of junior doctors, senior doctors, and patients and/or their caregivers.

Methods: Qualitative study using semi-structured interviews, analysed using Framework Analysis. Participants were purposively sampled from 3 London hospitals and aimed to capture triads consisting of a caregiver, senior doctor and junior doctor from a single Second Conversation.

Findings: 17 interviews were conducted with 4 caregivers, 5 senior and 9 junior doctors, capturing 4 dyads and 2 triads across SurgicalMedicine, Oncology and Elderly Care specialties. Junior doctors felt the Second Conversation Model prepared them to lead end of life care conversations and they valued the opportunity to receive feedback, a theme also echoed by senior doctors. Caregivers felt supported by proactive follow-up discussions, whilst juniors appreciated providing good care. The theme 'Trust and Mistrust' was prominent, influenced by a presence or absence of factors including empathy, compassionate care, clinician knowledge and confidence, and communication skills. No harms were identified from use of the Second Conversation Model.

Conclusion: The Second Conversation Model has potential to improve end of life care communication through enhancing caregiver trust in clinicians, building confidence in junior doctors and providing a framework to support assessment of this key skill.

Abstract number: P02-490

Abstract type: Poster

\section{ACP: Lets Talk About It}

Worlock P. ${ }^{1}$, Gleeson A. ${ }^{1,2}$, Fretwell C. ${ }^{1}$

${ }^{1}$ Palliative Care, Aneurin Bevan University Health Board, Gwent, United Kingdom, ${ }^{2}$ Cardiff University School of Medicine, Cardiff, United Kingdom

Background: Advance care planning (ACP) has become increasingly popular in recent years. The importance of public engagement in promoting ACP discussions and incorporation of ACP into culture cannot be under estimated

Aim: To address the need for public engagement, this health board included Engagement as one of the 3 pillars in the ACP Triple E model (Engagement, Empowerment, and Education).

Methods: Engagement focused on members of the general public, health and social care professionals, and stakeholder. This included engagement activities across diverse clinical and public settings. The activities were adapted to complement the specific event and audience and included: display stands, public information leaflets, 1 to 1 conversations, formal presentations, interactive question and answer sessions, round table discussions, and a novel approach - an interactive LOTTO game! In this game individuals were asked to list their top 5 ACP wishes and preferences

Results (to date): There have been 4000 individual contacts, including 15001 to 1 conversations. Basic demographic data, examples of personal opinions, anecdotes and experiences will be presented. The outcomes of the LOTTO Game will also be presented. The results of the engagement process have been used to shape ACP developments within the health board to ensure they meet the needs of the diverse population.

Conclusion: ACP engagement has provided a wealth of information and opportunity to explore the rich qualitative narrative feedback volunteered from the public. Changing culture is a slow process but our results demonstrate public support for engagement and indeed for ACP.

Abstract number: P02-491

Abstract type: Poster

\section{Basic Model für Palliativ Nursing in Germany}

Hach M. ${ }^{1}$, Klimsch C. ${ }^{1}$, Müller-Mundt G. ${ }^{1}$, Becker D. ${ }^{1}$, Doll A. ${ }^{2}$, Goudinoudis K. ${ }^{1}$

${ }^{1}$ Section Nursing, German Association for Palliative Care, Berlin, Germany, ${ }^{2}$ Sektion Nursing, German Association of Palliativ Medicine, Berlin, Germany

Background: Palliative nursing (pn) in Germany is faced with the question of how to make the manifold implicit knowledge explicit and how to reach an evidence based nursing practice. The basis is the description of the core and specifics of professional pn. As a first step for this the section "nursing" in the DGP has developed through a consensual process, the care guideline for pn and a guideline-process of central nursing phenomenons in palliative care. However, these are not sufficient enough for the theoretical and empirical foundation of palliative care. In order to tackle pressing challenges in German palliative care in a knowledgebased and research-oriented manner, an independent professional profile and a uniform technical language is needed.

Methods: Preliminary literature analysis of nursing theories and concept development in palliative care. Two workshops were held with experts from the members of the section $(n=24)$ : Card inquiry and thematic clustering, world café and plenary discussion, final consensus finding

Previous results: Based on previous analysis, five concepts were identified as central and put up for discussion in the expert workshops. With slight shifts in emphasis, four concepts were found essential in the discourse: care ethics, corporeality, interpretative diagnosis and evidence based nursing, which are rudimentary also reflected in the Hospice and Palliative Act, in the Nursing Profession Act and Social Code XI underlying concept of care. The elaborated central phenomena and meta 
paradigms show a high degree of congruence with the interdisciplinary EAPC core competences.

Discussion: By consensus, the expert group developed key dimensions for the foundation of a theoretical conceptualization of pn, whose empirical foundation is to be strived based on studies on pn practice. What is also needed is a broader discourse on practice, pedagogy, research and science in pn.

Abstract number: P02-492

Abstract type: Poster

Nursing Competences across Different Levels of Palliative Care Education and Provision: A Systematically Constructed Review with Narrative Synthesis

Hökkä M. ${ }^{1}$, Martins Pereira S. ${ }^{2,3,4}$, Kyngäs H. ${ }^{5}$, Pölkki T. ${ }^{6}$, HernándezMarrero P. ${ }^{2,3,4}$, EduPal-Developing Palliative Nursing and Medical Education through Multidisciplinary Cooperation and Working Life Collaboration

${ }^{1}$ Kajaani University of Applied Sciences, Kajaani, Finland, ${ }^{2}$ Instituto de Bioética, Universidade Católica Portuguesa, Porto, Portugal, ${ }^{3}$ UNESCO Chair in Bioethics, Institute of Bioethics, Universidade Católica Portuguesa, Porto, Portugal, ${ }^{4} \mathrm{CEGE}$, Centro de Estudos em Gestão e Economia, Porto Católica Business School, Universidade Católica Portuguesa, Porto, Portugal, ${ }^{5}$ Research Unit of Nursing Science and Health Management, University of Oulu, Oulu, Finland, ${ }^{6}$ Oulu University Hospital, Oulu, Finland

Background: Palliative care (PC) is provided across healthcare settings and nurses play an active role in its provision. Three levels of PC provision and education have been recognized: basic ( $A$ ), and specialist ( $B$ and C) levels. Nurses need competences to provide appropriate PC in all levels.

Aims: To systematically assess and synthesize the evidence of empirical studies with respect to the identification of nursing competences in PC and the assessment of whether these differ across levels of PC.

Methods: Systematically constructed review with narrative synthesis, following the Centre for Reviews and Dissemination (2008) and PRISMA guidelines. Data sources: CINAHL, MEDLINE, PubMed, Academic Search Premier, Scopus. Inclusion criteria: empirical studies on nursing competences in PC in different contexts, in English, Swedish, Finnish, Spanish, Portuguese or German language, with full text available. Main search terms: 'palliative care or hospice care or end-of-life care' and 'competence or professional competence' and 'nursing'. Articles were independently screened and reviewed by two researchers. Quality appraisal was conducted.

Results: 2922 articles retrieved; 16 included for analysis. The levels of PC $(A, B / C)$ were rarely defined in these studies. Most of the studies focused on different contexts of PC provision (e.g., hospice, home care, intensive care unit and inpatient units). A wide range of competences were identified as needed in different contexts.

Conclusions: This study shows that nurses need a wide range of competences in PC depending on the setting where they develop their practice. There are barely no studies focusing on competences aligned with the different levels of PC provision and education. A better systematization of nursing competences in PC considering the different levels of PC provision is needed to enhance PC development, education and provision. This calls for more research.

Acknowledgment: Finnish Ministry of Education and Culture.

Abstract number: P02-493

Abstract type: Poster

What Is Difficult about Talking to Friends and Relatives about Dying, and Do Clinical Communication Training Methods Help? Analysis of the Omega Course Communication Training Day
Briffa C. ${ }^{1}$, Meystre C. ${ }^{1,2}$

${ }^{1}$ The Omega Course, Kenilworth, United Kingdom, ${ }^{2}$ Haematology

Oncology, University Hospitals Birmingham, Solihull, United Kingdom

Background: Whilst healthcare use escalates exponentially in the last year of life, with commensurate increase in clinician contact, people still spend $90 \%$ of their time outside of hospital with their family and friends. Much is known about the professional's difficulties initiating discussion about death and dying, but very little about the confidence or facility with which friends and family members approach the topic.

Aims: The Omega Course was designed to give lay people the opportunity to talk freely about death and dying in a safe environment, to develop understanding and improve skills. A key part of the 14 hour course is a communication skills training day using role play modelled on communications skills training for clinicians. Course members contribute scenarios where they have had difficulties, the group vote on the most pertinent and the top 3 are role-played using participant volunteers.

Methods: Thematic analysis was applied to the 58 scenarios recorded on a flip chart by 49 participants on 5 training days. Course feedback sheets were also analysed.

Results: 8 themes emerge from the analysis: Crossing Cultural Chasms; Starting the Conversation; Confidence in my Competence; Taking Responsibility; How Will Others Respond to my Bad News?; Resilience for the Hearers of Bad News; Bereavement Issues; Dealing with Distress on the Phone. On feedback forms the participants rated this day the most valuable part of the course because they were able to observe actual scenarios being addressed in a controlled simulated way.

Conclusion: These themes demonstrate the difficulties experienced by the 'helpful friend'; and that traditional clinical communication skills training is effective in a lay setting. The Omega Course thus contributes to Ambition 6 "People are ready, willing and confident to have conversations about living and dying well, and to support each other in emotional and practical ways".

Abstract number: P02-494

Abstract type: Poster

Why Death and Dying Education for Firefighters Matters to

Palliative Care Professionals?

Leiva Santos J.P. 1,2

${ }_{1}^{1}$ Hospital de Manacor, Palliative Care, Mallorca, Spain, ${ }^{2}$ Asociación FIT for CARE, Madrid, Spain Presenting author email address: eliopabav@ gmail.com

Background: A study dealing with the issue of Death \& Dying (D\&D) in the Spanish Fire Services explicitly highlighted that end-of-life issues are not the sole province of hospice workers. Many other professionals whose job is not explicitly "end-of-life care" also work with D\&D daily.

Methodology: The ongoing integration of the new program into the existing plan began in February 2017 for firefighters at a regional level in Spain. The pedagogy was based on Kolb's learning styles. It consisted of five phases:

1) Introduction workshop (2h);

2) Teaching the full program (16h) for junior firefighters.

3) Teaching the full program (16h) for senior firefighters;

4) Teaching an extended program (64h) for firefighters applying to become corporals and sergeants.

Every phase had a mixed method assessment: ad-hoc surveys, semistructured interviews, and video-recording testimonials.

Results: 192 firefighters were enrolled. Their mean age was 42 y.o. ranging from $28-58$ y.o. Two participants were female. The top five most valued topics were: 
1) The opportunity for an open talk about $D \& D$ among colleagues, from professional and personal perspectives;

2) Concern for developing leadership skills to improve problemsolving abilities when facing $D \& D$ issues and its impact on victims.

3) Learning skills to balance how their professional experiences regarding $D \& D$ might negatively impact their families;

4) Awareness for improving: counselling, fitness, resilience, communication skills, and their understanding of PTSD.

The general satisfaction was $100 \%$ positive leading the Fire Service to extend the program to 64 hours and offering three new editions for phase four. Firefighters also suggested the D\&D education for police officers, A\&E, and rescue teams.

Conclusions: This educational program about $D \& D$ issues for firefighters shows that it is a legitimate need, and, through this, society as a whole is benefited. Palliative care professionals need to continue exploring this field.

Abstract number: P02-495

Abstract type: Poster

Building Capacity, Knowledge and Skills. Developing a

Registered Nurse Training Position within the Consultative Palliaitve Care Team

Magann L., Offner K., Morris S., Davis J.M.

Palliative Care, St George Hospital, Sydney, Australia

Background: Patients with complex palliative care needs can present in any area within the acute hospital setting. The Palliative Care Team at St George Hospital in Sydney, Australia undertakes a Registered Nurse Training Program to develop core competencies in caring for palliative care patients with complex needs. A selection process exists to ensure equity across the organisation. The training position runs for 6 months at 0.5 FTE.

Aim: To develop specialist palliative care skills, knowledge and competence so that the Registered Nurse can return to their substantive position within the organisation as a palliative care champion to improve patient outcomes.

Method: The perceptions and experiences of the trainee are measured along with the experience of the mentors within the Palliative Care Team. Identifying organisational factors which support and encourage this advanced learning process are explored.

Identification of learning goals, provision of learning packages, close mentorship and education opportunities are all part of the training process.

Implications for practice: Improved knowledge, skill and capacity of the individual RN, fostering palliative care champions within the organisation, strengthening the organisations commitment to patient care and, better outcomes for palliative care patients throughout the organisation. While initially the $\mathrm{RN}$ is closely mentored the goal is by the end of the training position that they are independent and displaying advanced palliative care skills, improved communication and an awareness of evidence based practice.

Conclusion: A need was identified to develop and improve training for RNs caring for palliative care patients within the acute hospital setting. Building capacity and competence to improve outcomes for patients and their loved ones is vital in ensuring quality of life and best practice palliative care.

Abstract number: P02-496

Abstract type: Poster

Success Story: Developing Palliative Care as Specialty for Nurses in Romania
Mitrea N.1,2, Mosoiu D.2,3

${ }^{1}$ Faculty of Medicine, Nursing Division, University of Transylvania, Brasov, Romania, ${ }^{2}$ Education and Development, Hospice Casa Sperantei, Brasov, Romania, ${ }^{3}$ Faculty of Medicine, University of Transylvania, Brasov, Romania

Background: Since the beginning of Palliative Care (PC) in Romania in 1992, education of the interdisciplinary team has been a priority. In 1999, PC was recognized as a sub-specialty for physicians and after sustained advocacy efforts in December 2017, through the health ministry order 942/2017, PC specialty for nurses in Romania was approved.

Aim: To ensure quality training in PC for nurses in Romania, through the design of the specialized program in PC nursing based on the EAPC's standards.

Method: The development of the PC nursing specialty program started with competencies development by an international expert panel, and their testing among nurses in PC services. Further steps: literature search, translation and adaptation to internal context of relevant resources; weekly working meetings of nursing PC trainers for one year; experts agreement on topics, objectives and format of the curriculum; training materials development; rolling out the $1^{\text {st }} \mathrm{PC}$ specialty program between October 2018- June 2019.

Results: based on the EAPC nursing curricula for specialized level the Romanian curriculum was developed. The PC specialty training is delivered over a 9 months period, with a total number of 750 hours, divided in : 150 hours $(20 \%)$ of theory (5 residential courses, 480 hours (65\%) of mentored clinical practice and 120 hours $(15 \%)$ of practical work. A toolkit for trainers and trainees was produced including ppt presentations, teaching exercises, cases studies, a practice log book, formative assessment tools, sumative assessment questionnaire, reflective writing. 10 centers are accredited nationally to run the course. Training for the 1st cohort is taking place in 4 locations and has 77 nurses enrolled.

Conclusions: The success story of PC as specialty for nurses took time and sustained collective effort to develop the program in order to have national unitary education program. Funding for the program development was provided by BMS foundation.

Abstract number: P02-497

Abstract type: Poster

Measuring the Impact of the Introductory and Advanced

Modules on Palliative Nursing Education on the Hospital Nurses'

Clinical Practice

Mitrea N. ${ }^{1,2}$, Ancuta C. ${ }^{2}$

${ }^{1}$ Faculty of Medicine, Nursing Division, University of Transylvania, Brasov, Romania, ${ }^{2}$ Education and Development, Hospice Casa Sperantei, Brasov, Romania

Background: Evaluation of impact of training on clinical practice is needed. In Romania, standardized Palliative Care (PC) educational programs for nurses, at levels of education, recommended by EAPC:

A - introductory (18 hours),

B - advanced (18 hours) and

C - specialized (750 hours) were developed, implemented and evaluated.

A 36 hours credited program in Palliative Nursing Education (PNE) including A plus B level is mandatory for nurses in order to comply with minimum quality standard for education of hospital nurses.

Aim: To evaluate if hospital nurses attending PNE implement in their clinical practice the $\mathrm{PC}$ competencies acquired in the training.

Method: The PNE program was evaluated using a pre-test/ post-test administered with a self-evaluation quiz on the competencies implemented in clinical practice. The competencies were structured on 7 
domains: Patient, Patient and Family, Team, Ethics, PC in the Health System, Education, Research.

Results: 375 nurses from 20 different specialties in 21 counties of Romania attended the 36 hours PNE in 2018. Pre and post tests evaluation showed an average of 3 points (out of 10) increase in knowledge. The self-assessed quiz with 20 competencies in PC showed that $44.2 \%$ of nurses implement all competencies and $55.8 \%$ partially. Ethics, nursing interventions and communication competencies were applied in a higher percent. The 3 main reasons for not implementing PC competencies: interventions involve major routine change (22\%), interventions not important in practice (10\%) topic insufficiently explained during classes (7\%); other barriers: physician's recommendation is mandatory $(8 \%)$, lack of time (2.6\%), family driven culture $(2 \%)$, lack of resources human, material (1.6\%).

Conclusions: Hospital nurses benefited from attending the 36 hours PNE program. A change in the delivery of some sessions is required. Further research is necessary in order to determine solutions for minimizing or exclusion of barriers.

\section{Abstract number: $\mathrm{P} 02-498$ \\ Abstract type: Poster}

My Learning: Online Educational Insights

Rawlings D., Tieman J., Moores C.

Flinders University, Bedford Park, Australia

Background: Online learning is increasingly being used to deliver ongoing education and CPD to health professionals with well-understood benefits including accessibility, efficacy, cost effectiveness, learner flexibility and interactivity. This study reports on the contribution of a set of online palliative care modules, their uptake, and their contribution to the learners' palliative care practice.

Methods: Two sets of questions were embedded within a set of "using evidence in practice" modules to capture data to describe the reach (pre-module) and implied effectiveness (post-module). Both online survey forms were optional to complete, simple to collect, and brief. Discussion with the Chair of the Social and Behavioural Ethics Committee, Flinders University a priori determined ethics approval was not required as the research fits the exempt category involving the collection and use of non-identifiable data.

Results: Over a 9-month study period from 1 April 2016 to 4 Jan 2017, 1,852 learners visited one of the eight modules. Of those, 1,137 opted in to the evaluation, with $92 \%$ completing pre-module evaluation $(n=1043)$ and $32 \%$ completing post-module evaluation $(n=366)$. The majority of respondents were middle-aged (i.e. ( $\geqslant 41 \mathrm{y}$ ), Australian, female nurses. Post-module evaluation indicated that learners found the modules were easy to use (99\%). Many reported that they would undertake another module (96\%). Ninety two percent indicated they would use the information presented in their practice.

Conclusions: This study showed that a set of online modules is useful, and has the potential to support awareness and use of evidence by health professionals. The increasing friendliness of learning platforms means that health professional educators can easily create relevant online content for particular target groups that can be readily updated.

Abstract number: P02-499

Abstract type: Poster

An Education Model that Empowers Individuals to Engage in the Advance Care Planning (ACP) Conversation

Fretwell C., Worlock P., Gleeson A.

Aneurin Bevan University Health Board, Newport, United Kingdom

Advance care planning (ACP) is recognised as an integral part of achieving excellence at end-of-life but for a variety of reasons ACP conversations are avoided or not offered appropriately to those we support. This often leads to negative consequences for individuals particularly in relation to their end-of-life care.

This ACP triple E education model aims to empower individuals through education with the knowledge, skills and confidence to engage with the ACP discussion with appropriately identified individuals. The model has been developed collaboratively with all organisations within a large university health board covering a population of over 600,000 in both urban and rural areas directly in line with strategic and policy guidelines related to end-of-life care. This includes primary, secondary, care home and voluntary sectors

The model aims to:

- use a newly developed bespoke blended e-learning model to educate all health and social care professionals with the appropriate knowledge and skills, to engage with ACP discussions within the limits of their roles and document and share these discussions with other significant people.

- empower ACP champions in all clinical areas using a structured education programme including the e-learning combined with interactive workshops to enable cascade training and enhance sustainability.

- increase awareness of the public, health and social care professionals and stakeholders of the benefits of ACP by working with national initiatives and facilitating an array of interactive public events.

The model has been evaluated. Quantitative pre and post training data as well as a qualitative focus group study have been analysed.

Results clearly demonstrate the positive impact this model is having on changing practice and integrating ACP in a positive and proactive way. The data also supports the model's transferability to multidisciplinary areas of practice and the potential vision to be adopted nationally and internationally.

\section{Abstract number: P02-500 \\ Abstract type: Poster}

Meaningful Learning in Palliative Care - Like a "Red Thread" due to the Pedagogy Method

Berggren E., Strang P.P.

Karolinska Institutet, Stockholm, Sweden

Background: Within the Centre for Palliative Knowledge (PKC) we have chosen to work for a strengthened learning based on a theory and on the principles of the constructive alignment [1]. Through intended learning outcomes for each education, both education, learning activities and assessment tasks work together, according to a "red thread".

Aim: To achieve meaningful learning by means of defined ILO:s (intended learning outcomes.

Method: Intended learning outcomes, ILO:s, become the hub for the content of the education, the educational activity and for what to assess and evaluate. For each ILO, verbs are used for the appropriate level of learning. The verbs are supported by the SOLO taxonomy, the Structure of Observed Learning Outcome $e^{[1,2]}$. Then, ILO:s are assessed with a webbased evaluation with answers according to the ranking: fully agree; mainly agree; partly agree; do not agree at all.

Examples of intended learning outcomes:

- Identify... what... among whom

- Describe and in collaboration discuss... any problems ... among whom

- Relate one's own role and responsibility regarding care to whom and in which context in relation to roles and responsibility among other professions and caregivers

- Reflect and connect new knowledge with own experience. 
Results: 2017 When evaluating whether the participants had gained knowledge according to the ILO:S, our assessment showed that $84 \%$ "fully agreed - mainly agreed" and that $86 \%$ considered that they would benefit from the continuing education in their work.

Conclusion: Continuing education including ILO:S proves to lead to knowledge useful in the palliative care context, and thus provide the conditions for a conscious palliative approach

References:

Biggs J, Tang C (Eds.): Teaching for Quality Learning at University: The McGraw-Hill Companies; 2011.

Designing a Course for Meaningful Learning, Medical Education Guide NO. 1. Edited by Weurlander M: Karolinska Institutet; 2006.

Abstract number: P02-501

Abstract type: Poster

Cross Border Knowledge Brokering: Driving Knowledge Transfer and Exchange within Palliative Care Research on the Island of Ireland

Rabbitte M. ${ }^{1}$, McKeon M. ${ }^{1}$, Leahy A. ${ }^{1}$, Boylan J. ${ }^{1}$, Charnley $K .^{2}$

${ }^{1}$ Research Programme, All Ireland Institute of Hospice \& Palliative

Care, Dublin, Ireland, ${ }^{2}$ All Ireland Institute of Hospice \& Palliative Care, Dublin, Ireland

The island of Ireland consists of two jurisdictions, Republic of Ireland and Northern Ireland; each has a public healthcare provider, health research funding body and advocacy groups. The All Ireland Institute of Hospice and Palliative Care (AIIHPC) as a knowledge broker for palliative care research on the island of Ireland aims to: facilitate transfer of research evidence into practice and policy, support collaborative interdisciplinary research, and coordinate funding opportunities to address the top 10 all Ireland palliative care research priorities [1]. In 2012 AllHPC formed the all-Ireland Palliative Care Research Network (PCRN) to support researchers to undertake internationally recognised research that enhances knowledge and expertise. The PCRN connects researchers with each other and to people with life-limiting conditions, carers, service providers, policy makers and research funders. In 2016 The Palliative Hub Professional website aimed at health and social care professionals, academics, researchers and educators was launched. The research zone on the hub and social media presence (\#PCRN) provides a platform to exchange knowledge using an interactive blog, research project webpages and promotion of publications. In 2017 two additional project managers were appointed to support the PCRN, build capacity in Knowledge Transfer and Exchange (KTE) and offer expert advice in health research, education, policy and practice, public engagement and traditional- and digital- marketing. In 2018 All Ireland events to support interdisciplinary research, knowledge sharing and funding opportunities included Palliative Care, Disabilities and Legislation Seminar, Research Impact Workshops and 7th Annual PCRN Symposium. To be a cross border knowledge broker requires a diverse team to promote inclusive ongoing activity that meets the needs of all key stakeholders.

[1] AllHPC (2015): http://aiihpc.org/wp-content/uploads/2015/06/ PeolcPSP-AIIHPC-report.-FINAL.-april.15.pdf

Abstract number: P02-502

Abstract type: Poster

Tomorrow's Nurses: Availability and Appreciation of Palliative Care Learning Experiences in Portugal

Minosso J.S.M. ${ }^{1}$, Spineli V.M.C.D. ${ }^{1}$, Martins M.M.F.P.S. ${ }^{2}$, Oliveira M.A.C. ${ }^{1}$

${ }^{1}$ School of Nursing, University of São Paulo, São Paulo, Brazil, ${ }^{2}$ Escola

Superior de Enfermagem do Porto, Porto, Portugal

Background: To increase support in society for palliative care (PC), the students' educational experiences during nursing undergraduate courses must be able to change paradigms, going beyond of the development of technic skills. It is necessary to demonstrate the importance of PC and stimulate the student to perceive the potential development in their communities through PC.

Objective: This study aimed to evaluate PC learning experiences availability during an undergraduate nursing degree of a Portuguese public institution and the importance attributed by the students to the theme.

Methods: A cross-sectional survey was conducted with 403 students distributed among the different years of the course.

Results: $92 \%$ of the students declared that they were not receiving or received adequate training on $\mathrm{CP}$ during the course and $88 \%$ considered themselves incapable to provide them. Only $46 \%$ of final year's students reported having the desire to work in palliative care units, in contrast to $58 \%$ of the first year. Nevertheless, almost all the students (98\%) considered important to incorporate content on palliative care in the undergraduate degree.

Conclusion: The results endorse the need for rapid adaptation of the curricula of the nursing programs to the growing demand that has been established in society in order to form competent professionals with a comprehensive vision for the care of the patients with life-threatening diseases.

Abstract number: P02-503

Abstract type: Poster

The Pedagogical Transposition Process of an Integrated Competency-based Approach in Palliative Medicine

Mathe A. ${ }^{1}$, Lafaye D. ${ }^{1}$, Lejeune A. ${ }^{1}$, Melin M. ${ }^{1}$, Pouget J. ${ }^{2}$, Roumiguiere

C. ${ }^{1}$, Saussac $C .{ }^{1}$, Burucoa $B .{ }^{1}$

1 University Hospital and University Bordeaux, Bordeaux, France,

${ }^{2}$ Bagatelle Hospital, Bordeaux, France

Background: The Integrated Competency-Based Approach (ICBA) is an original and innovative teaching approach, allowing reflexive practitioner training by the association of contextualized and analytical approaches.

In this context, an integrated skills reference guide to palliative medicine (ISRG) was drafted by a multi-professional working group.

A first ICBA's pedagogical transposition was conducted in post-graduate diploma of palliative care in 2016-17. At the request of the students, the transposition was renewed in 2017-18.

Aims: Describe and criticize the second ICBA's pedagogical transposition during the first year of this diploma in 2017 - 2018.

Methods: The study was conducted as an action research, analyzing two populations: the teachers (leaders) and the students (learners). The data collected were the learners' productions, the leaders' notes and the leaders' meeting reports.

Instructions were given for learners: to identify " problem-situation ", to confronte themselves to the "problem-situation" in practice using skills and macro skills, to analyze their practice using feedback and auto-analysis.

Results: 43 of the 53 learners have attended to all 4 sessions offered, supervised by the 8 leaders. 53 "problem-situations" were validated, the majority exploring the "care and accompaniment" function. The dimensions of accompaniment and relationship were acclaimed by the learners in the choice of skills. A closer guidance was proposed by using a pedagogical platform (half of the students registered). 44/46 learners wished for further transposition in 2018-2019.

Conclusion: The ICBA is an innovative but complex teaching approach, valuing reflective activities in professional practice. This transposition has been renewed this year in the first year and in the second year of this diploma. Other transpositions are planned for the coming years (pregraduate curriculum for medical students...). 
Abstract number: P02-504

Abstract type: Poster

Does the Omega Course Change Perceptions and Behaviour to Support Community Action towards Better Dying? Davies I. ${ }^{1}$, Hiskett I. ${ }^{1}$, Meystre C. ${ }^{2,3}$

${ }^{1}$ Medical School, University of Warwick, Coventry, United Kingdom, ${ }^{2}$ The Omega Course, Kenilworth, United Kingdom, ${ }^{3}$ University Hospitals Birmingham, Birmingham, United Kingdom

Background: Death can be difficult to address personally, discuss and to plan for. Since 2016 The Omega Course (OC) has educated local people about death and dying; broaching these issues. It aspires to produce practical outcomes with positive implications for end of life planning and future care.

Aim: To assess if OC changed participant's attitudes to dying and if this led to practical action.

Method: Anonymous questionnaires, distributed by Qualtrics or by post if preferred, were sent to 62 participants of OC aged 22-94. Thematic analysis and inferential statistics were used.

Results: 23 replies (37\%) scoring changes across four areas; barriers to discussion, ease discussing death, fears about death and levels of preparedness all showed a significant beneficial change with a Paired Sample T-test $(\mathrm{P}<0.01)$.

Respondents noted common fears of death and dying; regrets from life, suffering, powerlessness and overzealous medical care: "the determination of the medical profession to keep me alive at all costs is not attractive".

The information and skills training delivered empowered participants; feeling "more aware of what I can do to influence the process" and "accepting that life ends for us all". This translated into intentions to plan e.g. Lasting Power of Attorney or Living Will, but less reliably into actioned change e.g. "I haven't made any changes at this moment in time but understand it will be important to put some things in place". Some participants found the concepts difficult to engage with at their stage in life, "I'm still unlikely to make a will any time soon. I have nothing of value!". Others highlighted areas not covered, such as the religious aspects of death.

Conclusion: The Omega Course changed participant's attitude towards their death, promoting autonomy and encouraging planning. The effect on lives and care outcomes remains to be seen and is the crucial link, yet to be evidenced between community public health education and health benefit.

\section{Abstract number: P02-505 \\ Abstract type: Poster}

Simulating the Palliative Patient: A High-fidelity In-

situ Simulation for the Multi-disciplinary Team, and an Undergraduate Simulation Programme

Wells H. ${ }^{1}$, Mclldowie B. ${ }^{1}$, Benbow A. ${ }^{1}$, Jones $T .{ }^{1}$, Wollaston A. ${ }^{2}$, Simonds L. ${ }^{3}$, Tulloch L. ${ }^{3}$, Connell A. ${ }^{3}$, Simpson S.-A. ${ }^{3}$ ${ }^{1}$ University of Birmingham, Birmingham, United Kingdom, ${ }^{2}$ Sandwell and West Birmingham Hospitals NHS Trust, Birmingham, United Kingdom, ${ }^{3}$ Worcestershire Acute Hospitals NHS Trust, Worcester, United Kingdom

Background: Medical education through high-fidelity simulation has largely been confined to the simulation of the acutely unwell patient with a reversible pathology. We proposed simulation could be a promising new framework for providing education surrounding a patient with palliative care needs.

We developed a collection of simulation scenarios with the common theme of the palliative patient. We delivered these scenarios to both undergraduate medical students in a simulation suite and in-situ to the multi-disciplinary team on general medical wards.
Aims: We aimed to improve both participant confidence and knowledge in dealing with a patient with palliative care needs. We also aimed to address communication issues that may arise, and to improve teamwork within ward team. We hoped to encourage a more holistic approach to the palliative patient.

Methods: Final year medical students on their acute medicine rotation completed the palliative care scenarios in a simulation suite. Healthcare professionals participated in-situ on two medical wards.

Results: A total of 30 medical students participated in the undergraduate simulations (feedback was collected from 12), and 10 healthcare professionals in the in-situ simulations.

For the in-situ simulation, all participants agreed with the statement "I feel it has improved my skills in dealing with a palliative patient". 75\% agreed that participating in a simulation on a ward environment made the scenario more realistic, and all participants agreed that the simulation had improved their teamworking. All participants agreed that the simulation improved their confidence and reduced their anxiety in dealing with a palliative patient.

Conclusions: Both the undergraduate simulation programme and the insitu simulation programme have proved excellent alternatives to traditional teaching techniques.

We propose simulation as a promising new method for education surrounding the palliative patient.

\section{Abstract number: P02-506 \\ Abstract type: Poster}

\section{Medicinal Cannabis: What Do Patients and Clinicians Want to} Know?

Panozzo S. 1,2,3, Philip J.3,4, Collins A..$^{3,4}$, Whyte J. ${ }^{5}$, Barton M.5, Weil J.6, Coperchini M. ${ }^{7}$, Rametta M. ${ }^{8}$, Ong K. ${ }^{9}$, Bayliss J. ${ }^{8}$, Le B. ${ }^{1}$

${ }_{1}^{1}$ Peter MacCallum Cancer Centre, Parkville Integrated Palliative Care Service, Melbourne, Australia, ${ }^{2}$ St Vincent's Hospital Melbourne, VCCC Palliative Medicine Research Group, Fitzroy, Melbourne, Australia, ${ }^{3}$ Victorian Comprehensive Cancer Centre, VCcC Palliative Medicine Research Group, Melbourne, Australia, ${ }^{4}$ University of Melbourne, Department of Medicine, St Vincent's Hospital, Melbourne, Australia, ${ }^{5}$ Western and Central Melbourne Integrated Cancer Service, Melbourne, Australia, ${ }^{6}$ St Vincent's Hospital Melbourne, Centre for Palliative Care, Melbourne, Australia, ${ }^{7}$ Western Health, Melbourne, Australia, ${ }^{8}$ Peter MacCallum Cancer Centre, Melbourne, Australia, ${ }^{9}$ Department of Health and Human Services, Office of Medicinal Cannabis, Melbourne, Australia

Background: While there is increasing interest in the use of medicinal cannabis for patients with cancer, the evidence underpinning its use is not yet well established. Recent Australian legislation changes allow for prescribing of medicinal cannabis by registered clinicians, creating an imperative for new information resources for use in clinical practice. Aims:

1. To identify the understanding and information needs of patients, carers and clinicians concerning the role of medicinal cannabis in cancer.

2. To develop information resources that respond to identified needs.

Methods: Co-design methodology was used to actively engage and involve patients, clinicians and researchers throughout the project design, development and evaluation. Focus groups and interviews with cancer patients and survivors (consumers) and clinicians were conducted until saturation. Key themes were used to inform the development of patient and clinician information resources. Resources were then evaluated by cancer consumers and clinicians.

Results: 14 consumers and 10 palliative care and oncology clinicians identified key information needs to be addressed. Key themes around 
identified needs included: products, treatment, administration and dosage, side effects, monitoring, precautions, access and prescribing, expense, and evidence of efficacy. Most consumers indicated their preference for clinicians to raise medicinal cannabis as a possible symptom treatment option, while most clinicians indicated they would wait for patients to raise. Evaluation confirmed information needs were adequately addressed by resources and considered suitable for implementation across four tertiary cancer services.

Conclusion: This study demonstrated the value of co-design methodology in the development of resources to support both patients and clinicians understand areas of practice with emerging evidence and national legislative change.

Source of funding: Western and Central Melbourne Integrated Cancer Service

Abstract number: P02-507

Abstract type: Poster

Reflective Essays as an Assessment Tool in Palliative Care Evaluation of Penetration Depth

Kriesen U., Diwoky S., Schmidt S., Große-Thie C., Murua Escobar H., Junghanss $C$.

Clinic III, Hematology, Oncology, Palliative Care, Universitätsmedizin

Rostock, Rostock, Germany

Introduction: In palliative care (PC) training, students are taught facts and attitudes to ensure patient centered care. While written multiple choice (MC) exams have been the common examination for PC knowledge, we report on a pilot study evaluating reflection essays as exam form.

Material and methods: Students in the $10^{\text {th }}$ semester of human medicine were asked to write a reflection essay on a free chosen topic as an examination performance at the end of their PC training. All essays were graded by at least one member of faculty, $20 \%$ were evaluated by two faculty members independently for standardization of grading. The penetration depth into PC was differentiated into 5 stages (low to very deep), according to pre-established evaluation anchors. Depth of PC penetration was differentiated in three categories:

i) personal contribution,

ii) scientific reasoning level and

iii) relation to palliative medicine. Penetration levels of $M C$ questions were compared to the level achieved by reflective writing.

Results: Out of the 224 students writing reflective assays, 184 students gave their informed consent for scientific evaluation. None of the students fulfilled all three categories with a high degree of penetration. A high degree of penetration could be rated for a maximum of two of the three aspects for each single essay, either in personal involvement and relationship to palliative care or in terms of scientific reasoning and relationship to palliative care. The MC questions each concerned only one aspect, either in terms of clinical reasoning or simply querying knowledge in low to deep penetration.

Conclusions: Reflective essays are a very useful tool to allow students confront themselves with important topics concerning end-of-life-care. The penetration depth of reflective writing is very high but addresses only narrow parts of the "whole". In contrast, MC questions aim for clinical reasoning and knowledge, but not for attitudes.

Abstract number: P02-508

Abstract type: Poster

Palliative Care: Because Every Moment Matters - A Public Education Campaign with Personal Stories Challenging Public Perceptions of Palliative Care
O'Hara B. ${ }^{1}$, Charnley K. ${ }^{2}$

${ }^{1}$ Policy and Practice Programme, All Ireland Institute of Hospice and Palliative Care, Dublin, Ireland, 2Director, All Ireland Institute of Hospice and Palliative Care, Dublin, Ireland

Aim/goal: To deliver the Palliative Care Week 2018 awareness-raising campaign from 9 to 15 September 2018 on the island of Ireland aimed at the public and health and social care professionals.

Design, methods and approach: The campaign theme was: Palliative Care, Because Every Moment Matters.

We set out to deliver a campaign informed by service user stories/narratives and by an understanding of palliative care at a population level.

An independent survey (1500 people) to measure public understanding of palliative care was carried out in June 2018.

Eight videos involving people with palliative care needs were produced. Campaign messaging combined key statistical information from the survey with patient narratives.

Stories, photographs and personal experiences of illness and palliative care featured in promotional materials, on social media, including videos, and media engagement for the campaign.

Results: Important campaign messages were personalised, for example, by one video participant featuring in a poster stating: "I have had nothing but good experience since receiving palliative care ... it will give you a better quality of life".

More than 50 media interactions included seven TV interviews, two national and nine regional radio interviews. One on-line media story placed for Palliative Care Week had almost 26,000 viewers.

More than 13,500 leaflets and posters were distributed for events.

Conclusion / lessons learned: The personal experiences of palliative care enabled the public to connect with the campaign.

Most promotional and media activity featured those involved in the eight videos. Participants in five videos took part in media interviews. The high level of media interest was due to their involvement.

By enabling the public to connect with the campaign, the personal stories helped people to learn more about the quality of life benefits of palliative care.

Abstract number: P02-509

Abstract type: Poster

Development of Educational Program for Mixed Groups of Citizens and Healthcare Providers to Facilitate Readiness toward Advance Care Planning

Nagae H. ${ }^{1}$, Takahashi Z. ${ }^{2}$, Sakai S. ${ }^{1}$, Harasawa N. ${ }^{1}$, Iwasaki T. ${ }^{1}$, Kawahara M. ${ }^{1}$, Ikeda M. ${ }^{3}$, Tanigaki S. ${ }^{4}$, Katayama Y. ${ }^{5}$, Watanabe ${ }^{6} .^{6}$, Takenouchi S. ${ }^{7}$, Tamura $K^{8}{ }^{8}$

1End-of-Life Care/ Gerontological Nursing, Tokyo Women's Medical University, Tokyo, Japan, ${ }^{2}$ Graduate School of NursingInterprofessional, Education Research Center, Chiba University, Chiba, Japan, ${ }^{3}$ School of Nursing, Nursing Administration, Tokyo Women's Medical University, Tokyo, Japan, ${ }^{4}$ Community Health Nursing, Okayama University, Okayama, Japan, ${ }^{5}$ Home Health Care Nursing, Department of Nursing, Kagawa Prefectural University of Health Sciences, Kagawa, Japan, 6 Jichi Medical University, Tochigi, Japan, ${ }^{7}$ Kyoto University Hospital, Kyoto, Japan, ${ }^{8}$ kyoto Univeisity, kyoto, Japan

Background: Advance Care Planning(ACP) has been considered to promote communication which could help people express their values or preferences on end of life. However, competences of readiness for expressing those issues and what kind of support is necessary are not clear.

Aim: The aim of the study is to conduct educational program for mixed groups of citizens and healthcare providers to cultivate the readiness to express their values and preferences, and to explore the influence on participants' competences of readiness. 
Methods: Research design is an mixed method. The content of the educational program is to learn the basic knowledge of ACP, discuss participants' values, lifestyle and preferences about their way of living or good death. We conducted a three-hour session twice in a mixed group of citizens and healthcare providers during a month. Citizen participants were recruited on the website of the city.We measured readiness for expressing values or preferences before and after intervention by using ACP Readiness Scale, Attitude toward Death Scale and Perspective Scale. Also, we asked questions about changes in value with a questionnaire. We merged the result of scale and questionnaire with mixed method. Results: 21 participants: women $61.9 \%$, men $38.1 \%$; average age was $59.1 \pm 13.7$. Wilcox's signed rank test of the three scales found significant difference only in "Perspective Scale" $(P=0.003)$.The scores of subscale 'confusion and dilemma' were significantly decreased. Results of questionnaire showed that conversation with citizens was fruitful for providers, but citizens answered they wanted to think about more realistic examples and problems.

Conclusion: It was shown that the feelings of conflict and confusion were reduced by the conversation experiences, and that participants were able to be consciously aware of their values and preferences on end-of-life.

This work was supported by JSPS KAKENHI Grant Number JP15H02586.

Abstract number: P02-510

Abstract type: Poster

Development of a Joint Undergraduate Course for Medical and Law Student to Enhance Using Advance Directives in Practice Csikos A. ${ }^{1,2}$, Pozsgai E. ${ }^{1}$, Lukacs M..$^{1,2}$, Zeller J. ${ }^{3}$, Busa $C^{1,{ }^{1,2}}$

${ }^{1}$ University of Pecs Medical School, Pecs, Hungary, ${ }^{2}$ Pecs-Baranya Hospice Program, Pecs, Hungary, ${ }^{3}$ Faculty of Law, University of Pecs, Pecs, Hungary

Aims: Advance Directive (AD) is still not put into practice within the Hungarian health care system, although the legal background has made $A D$ possible for the past 20 years. Education regarding this topic has been missing from the training of medical and law students. The University of Pecs (UP) is the first university in Hungary to have begun the integration of an $A D$ course into the undergraduate curriculum.

Methods: A joint project between the UP Medical School and Faculty of Law was initiated in 2015 in collaboration with the Pecs-Baranya Hospice Program. The curriculum was developed following the review of the international theoretical and practical background of AD. An AD template was also drafted based on current Hungarian laws and regulations.

Results: An undergraduate course available to both medical and law students, titled "Who should decide at the end of life?" was launched in 2016 as a 2-credit optional subject. The course has three components.

(1) The classroom component focuses on the legal background and the current practices of end-of-life decisions.

(2) During the classroom communication training, students learn interviewing techniques and counselling skills.

(3) The third component is practical, where issues concerning AD can be discussed between students and palliative care patients under supervision of the tutor.

Feedbacks about the course were positive from students and patients alike. A number of advance directives were completed in accordance with Hungarian regulations.

Conclusions: During the practical part of the course additional issues came up. Further discussions between professions should be initiated and education about $A D$ needs to be integrated into postgraduate trainings for the wider application of $A D$ in practice.

The project was founded by the Open Society Institute.
Abstract number: P02-511

Abstract type: Poster

Engaging and Supporting Staff in Residential Care Settings by Sharing Palliative Care Expertise

Payne C. ${ }^{1}$, Flanagan M. ${ }^{2,3}$

${ }^{1}$ All Ireland Institute of Hospice \& Palliative Care, Dublin, Ireland, ${ }^{2} \mathrm{Our}$ Ladys Hospice \& Care Services, Dublin, Ireland, ${ }^{3}$ University College

Dublin, Dublin, Ireland

Background: Our Lady's Hospice \& Care services provides specialist palliative care to over 12,000 people in their homes including nursing homes. Nursing homes are providing increasingly complex care and support to people with life limiting conditions to live as well as they can during the last phase of their life and to have their personal wishes considered regarding disease management and place of death. Project ECHO (Extension for Community Healthcare Outcomes) is an internationally recognised collaborative model of health education and care management that empowers health professionals everywhere to provide better care to more people, right where they live. The ECHO mode ${ }^{\mathrm{TM}}$ provides front-line services with the knowledge and support they need to better manage patients with complex conditions through online education seminars.

Aims: To use ECHO so support the management of chronic life limiting conditions, through support, mentoring and development of Nursing Home staff.

Method: The palliative care multidisciplinary team from Our Lady's Hospice \& Care Services supported by All Ireland Institute of Hospice and Palliative Care (AllHPC) invited subject experts (the hub) together with nursing home staff in the South Dublin area (the spokes) in a community of learning.

Results: Nursing home staff significantly increased their confidence in supporting residents with palliative care needs. Involvement in ECHO also enhanced the confidence of palliative care specialists in supporting the learning needs of nursing home staff with a range of experience and professional backgrounds

Conclusion: A spirit of peer-learning and reflection through the development of a community of practice was created through Project ECHO which should enhance future care and treatment for nursing home residents and their families requiring a palliative care approach.

Abstract number: P02-512

Abstract type: Poster

Translating International Recommendations into an Undergraduate Palliative Care Curriculum: The EDUPALL Project Mosoiu D. ${ }^{1}$, Charnley K. ${ }^{2}$, Payne C. ${ }^{3}$, Elsner F. ${ }^{4}$, Predoiu O. ${ }^{5}$, Stanciulescu L. ${ }^{5}$, Albu S. ${ }^{6}$, Boeriu E. ${ }^{7}$, Poroch V.8,9, Noguera A. ${ }^{10}$, Paal P. ${ }^{11}$, Ling J..$^{12}$, Pascu A. ${ }^{1}$, Rogozea L. ${ }^{1}$, Kovacs J. ${ }^{6}$, Urtila P. ${ }^{13}$, Tarniceru C.C. ${ }^{8}$, Vranceanu A.R. ${ }^{8,9}$, Spiru L. ${ }^{14}$, Mason S. ${ }^{12}$

${ }^{1}$ Transilvania University, Brasov, Romania, ${ }^{2}$ All Ireland Institute of Hospice and Palliative Care, Dublin, Ireland, ${ }^{3}$ All Ireland Institute of Hospice \& Palliative Care, Dublin, Ireland, ${ }^{4}$ RWTH Aachen University, Aach, Germany, ${ }^{5}$ Hospice Casa Sperantei, Brasov, Romania,

${ }^{6}$ University of Medicine and Pharmacy, Targu Mures, Romania, 7University of Medicine and Pharmacy "Victor Babes, Timisoara, Romania, ${ }^{8}$ University of Medicine and Pharmacy "Grigore T. Popa, lasi, Romania, ${ }^{9}$ Regional Institute of Oncology, lasi, Romania,

${ }^{10}$ Atlantes Research Program, Institute for Culture and Society of the University of Navarra, Navara, Spain, ${ }^{11}$ Paracelsus Medical University of Salzburg, Institute for Nursing Science and Practice, Salzburg, Austria, ${ }^{12}$ European Association for Palliative Care, Vilvoorde, Belgium, ${ }^{13}$ University of Medicine and Pharmacy "Victor Babes, Timisoara, Romania, ${ }^{14}$ University of Medicine and Pharmacy "Carol Davila, Bucharest, Romania 
An aging population and changing disease patterns across the globe has prompted the World Health Organisation to recommend "palliative care (PC) should be integrated as a routine element of all Undergraduate medical education." However, training is limited: only 6/43 European countries have compulsory programmes in PC.To address this, clinicians/ educators/researchers from Romania, Germany, Ireland, Austria and the European Association of Palliative Care (EAPC) have formed the EDUPALL collaborative (supported by ERASMUS +) to develop, implement and evaluate a practical undergraduate curriculum in PC.

Aim: To develop a complete undergraduate PC curriculum for adoption in medical schools across Europe.

Method:

Phase1-Development: EDUPALL employs the "EAPC recommendations for the development of undergraduate curricula" to drive the development of the curriculum.

Phase2-Training: Faculty will be empowered in specific teaching methods: experiential clinical learning; self-reflection; problem-based learning; mentoring skills; technology enhanced learning; and assessment methods.

Phase3-Implementation: The curriculum will be piloted in six Medical schools (Romania and Ireland) where students will be provided with training focused on the key domains of practice.

Phase 4-Evaluation: Examination of the effect of the curriculum upon training for future practice.

Results: Still in its first (of three) years, EDUPALL has: systematically developed a core curriculum and specific learning objectives; engaged review by an expert group engaging 17 European Countries; systematically developed universal lesson plans employing didactic, small groupwork, experiential and online learning methods.

EDUPALL addresses a problem shared by many countries. The development of a universally adaptable and applicable curriculum, freely available in multiple languages, will provide a platform for the greater integration of PC within undergraduate medical training programmes across Europe.

\section{Abstract number: P02-513 \\ Abstract type: Poster}

Difficult Decisions about End of Life Care: What Medical and Nurses Residents Think about it in a Private Hospital Located in Rio de Janeiro, Brazil

Fonseca A., Vidal T.

Adventis Silvestre Hospital, Rio de Janeiro, Brazil

Background: The suffering of patients demand in their care, especially when in the end of life stage, a special attitude of health professionals, which can influence a just graduated profissional on how they think their own end of life. Knowing the Palliative Care (PC) might be a differencial for their perfomance.

Aims: This study has the objective of knowing end of life preferences among resident physicians and nurses, based on their previous knowledge of PC.

Methodology: Qualitative study based on semi-structured interviews about end of life care preferences of resident physicians and nurses, performed in the last 4 years.

Results: Total of 38 among physicians and nurses residents were interviewed. $26,31 \%$, mostly nurses, said they did not have any knowledge about PC before residency; $52,63 \%$, most part of them nurses and internal medicine residents, affirmed difficult in end of life patients care; $71,05 \%$, mostly nurses, affirmed they would accept invasive procedures to be performed for diagnosis and treatment, although, they did not wish to artificially prolong their lives. Uncertainty in the end of life care was common among resident physicians, mostly related to the decision of what palliative care strategy should be performed.
Discussion: Previous knowledge of PC was important to residents involved in the end of life patients care; uncertainty of nurses is, probably, related to their unfamiliarity after they graduate. Otherwise, uncertainties among physicians are, probably, related that to the fact that they are expected to make decisions despite their technical inexperience, even when under supervision, which implies a responsibility they are not yet mature to assume.

Conclusion: The presence of PC since graduation was vital for residents in the end of life patients care and a previous knowledge about this subject was important for young professionals to assume technical responsibilities as soon as possible and to reduce uncertainties in making decisions.

Abstract number: P02-514

Abstract type: Poster

Evidence-based Professional Education Programs for Hospice and Palliative Care in Taiwan

Wang Y.-W. ${ }^{1}$, Chen R. ${ }^{1}$, Wu C.-Y. ${ }^{2}$, Hsiao S.-C. ${ }^{2}$, Huang C.-W. ${ }^{2}$, Cheng

K.-S. ${ }^{2}$, Chen Y.-H. ${ }^{2}$

${ }^{1}$ Health Promotion Administration, Ministry of Health and Welfare, Taipei, Taiwan, Republic of China, ${ }^{2}$ Cancer Prevention and Control Division, Health Promotion Administration, Ministry of Health and Welfare, Taipei, Taiwan, Republic of China

Aim of the work: We launched a 3-year project which established evidence-based professional education programs for hospice and palliative care (HPC) to enhance the capability of healthcare providers( physicians, nurses, social workers, psychologists, pastoral care workers, long-term care workers), caregivers, volunteers and NGOs in Taiwan.

Design, methods and approach taken: The project adopted bio-psychosocial-spiritual (BPSS) model to develop HPC guidelines and training courses. It included 5 subprojects:

1) pediatric and geriatric care,

2) long-term care,

3) psycho-oncology care,

4) social care, and

5) spiritual care.

Foursocieties and one university got the government grants to set up theprofessional education programs. For the first year (2017), they conducted focus groups, consensus meetings, workshops and specialist meetings and proposed the first year HPC guideline and training curriculum drafts. Each draft should be modified and tested by on three trial sites at hospitals or long-term care settings.The finalized guideline and training curriculum (flipped classroom model) will be expected to spread to 100 cancer hospitals in thenext year (2019). For the spiritual care subproject, 20 NGOs havechosen to create compassionate communities in their own communities.

Results: We have 5 focus groups and hold 18 specialist meetings, 10 consensus meetings, and 36 workshops. We will launch evidence-based professional education programs with BPSS model for health workers and volunteers to provide better services to meet the patients' needs in Taiwan.

Conclusion: HPC in Taiwan is no longer reserved for cancer patients, but also for patients with non-cancer. We will face a growing number of patients dying from non-cancer diseases. Apart from the professionals' efforts in improving skills, we recognize capacity building is vital to holistic palliative care. We also recognize the feasibility of cooperation between different professionals.

Abstract number: P02-515

Abstract type: Poster 
Applying the EAPC Recommendations in the Development of a Standardized Undergraduate Curriculum in Palliative Medicine: The EDUPALL Project

Mason S. ${ }^{1,2}$, Payne C. ${ }^{3}$, Stanciulescu L. ${ }^{4}$, Albu S. ${ }^{5}$, Paal P. ${ }^{6}$, Mosoiu D. ${ }^{7}$ 1Palliative Care Institute Liverpool, University of Liverpool, Liverpool, United Kingdom, ${ }^{2}$ European Association for Palliative Care, Vilvoorde, Belgium, ${ }^{3} \mathrm{All}$ Ireland Institute of Hospice \& Palliative Care, Dublin, Ireland, ${ }^{4}$ Hospice Casa Sperantei, Bucharest, Romania, ${ }^{5}$ University of Medicine and Pharmacy, Targu Mures, Romania, ${ }^{6}$ Paracelsus Medical University of Salzburg, Institute for Nursing Science and Practice, Salzburg, Austria, ${ }^{7}$ Transilvania University, Brasov, Romania

Provision of training for medical undergraduates is variable; only $30 \%$ of 43 European countries having mandatory training in Palliative Medicine. EDUPALL is an ERASMUS + funded international collaborative project to develop and pilot an undergraduate programme for training in Palliative Medicine: Standardised Curriculum; Faculty Training; Implementation; and Evaluation. The first step of this project was to systematically review the current EAPC Recommendations for the Development of Undergraduate Curricula in Palliative Medicine, translating these into an updated curriculum document.

Method: Clinicians, Academics and Researchers from Romania, Ireland, Germany, Austria, Spain and the UK (the core working group of the EDUPALL Project), systematically reviewed the EAPC recommendations for relevance, purpose and potential omissions; using a variant of consensus methodology. From the updated document, four working groups translated each recommendation into a specific Learning Objective, and developed associated learning outcomes, stratified by domain: Attitude; Cognition; Skills. The Outcomes and Objectives were organised into discrete teaching units and transferred into a Curriculum Template, identifying notional hours, teaching and assessment strategies. To ensure Quality Control, the draft template was circulated to experts from 17 European Countries, together with a brief survey instrument, for peer review purposes.

Results: All 17 reviewers returned overwhelmingly positive comments on the Curriculum Template draft. There was large agreement that: the teaching units were logically organized; Learning Outcomes covered core training needs; Learning Objectives provided guidance for teaching sessions; learning modalities were appropriately aligned; assessment strategies were fit for purpose.

Conclusion: An updated and standardized curriculum has been developed and provides a platform for the sequential development of the next phases of the EDUPALL project.

Abstract number: P02-516

Abstract type: Poster

Evaluation of Palliative Care Postgraduate Course Held by the National Association of Palliative and Hospice Care

Golob N. ${ }^{1}$, Bernot M. ${ }^{2}$, Ebert Moltara M. ${ }^{2}$

${ }^{1}$ Division of Internal Medicine, University Medical Center Ljubljana, Ljubljana, Slovenia, ${ }^{2}$ Acute Palliative Care Department, Institute of Oncology Ljubljana, Ljubljana, Slovenia

Background: The aim of the National Association of Palliative and Hospice Care is the promotion and professional development of palliative care. Among their activities is a 60 -hour postgraduate intensive course on basic knowledge in palliative care. Since its beginning in spring 2012, 425 medical professionals attended the course.

Aim: The aim of the study was to reveal how participants assess the course, how much of the knowledge they acquired they use in practice, which topics would they like to deepen and how much time are they prepared to dedicate to that.

Methods (design, data collection, analysis): Participants were asked to join a web survey composed of 12 structured questions that referred to 5 main topics of interest.
Results: 215 (63\%) surveys were completely answered and used for the analysis. Participants were medical doctors $(40 \%)$ and nurses (46\%) with 20 or more years of practice $(46 \%)$ who work in the central Slovenian region (39\%). 147 (68\%) of participants rated the course as excellent and $55(26 \%)$ as very good. $69(32 \%)$ of the participants apply the knowledge acquired in every day practice a lot. The topics in which participants feel most comfortable are: treatment of nephrology patients, law issues, treatment of neuropsychiatric symptoms and treatment of neurology patients. They feel less competent and wish to improve these topics: communication, treatment of the dying patient, ethical and moral issues and pain control. 101 (47\%) of the interviewed would attend a refresh course in the capital city and dedicate several days to that.

Conclusion / Discussion: Palliative care is insufficiently covered in the curriculum of medical professionals and many medical professionals attend a postgraduate course in palliative care. Although the majority evaluate the training with high grades, they also express the need to cover several topics in greater details. Up-grading and refreshing courses would be welcome and should be organise.

\section{Abstract number: P02-517 \\ Abstract type: Poster}

Spanish Strategy for the Pediatric Palliative Care Education Navarro Mingorance A. ${ }^{1}$, Ortiz-San Roman L. ${ }^{2}$, Martino-Alba R. ${ }^{1}$, Navarro Vilarubi S. ${ }^{3}$, Grupo de Formación de PEDPAL

${ }_{1}^{1}$ Pediatria-Cuidados Paliativos Pediátricos, Hospital Infantil Universitario Niño Jesús, Madrid, Spain, ${ }^{2}$ Cuidados Paliativos Pediátricos, Hospital Unversitario de Alicante, Alicante, Spain, ${ }^{3}$ Cuidados Paliativos Pediátricos, Hospital Sant Joan de Déu, Barcelona, Spain

Background: On the WHPCA categorization of palliative care development Spain is considered as a level 4A (preliminary integration). At the moment there are few resources on Pediatric Palliative Care (PPC) and not all of them meets the European Standards. The Spanish Association of PPC (PEDPAL) was created in 2016 to develop children's palliative care in Spain. Improving education in PPC among health professionals is one of the main goals of this association.

Aim: To describe the strategy for PPC education.

Results: The PEDPAL strategy on Health professional education is organized in three levels (Basic, Intermediate and advanced PPC training).

Basic PPC education is performed through an Online Course addressed to all general pediatric trainees and pediatric nurses. On it first edition running at the moment 455 pupils have been enrolled ( $47 \%$ pediatric trainees). A new edition of this course will take place every year.

Intermediate training has been assured with courses and conferences participation. The courses are addressed to professionals from adult palliative care departments and health-area-reference teams (pediatrician and nurses). PEDPAL organizes plenaries and workshops in all the pediatric specific areas conferences (ie. neonatology, pediatric neurology, intensive care and pediatric oncology).

Advanced training in PPC is provided by an official master's degree. It is a problem based learning program with 60 ECTS ( 1500 hours) and internship. To the date 193 pupils have finished the master's (60\% pediatricians and $40 \%$ nurses).

Conclusions: With this strategy PEDPAL is improving de knowledge on PPC: training new health professionals who are founding new advanced PPC resources, improving intermediate PPC in pediatric specialist and allowing all the children who may need, access to a basic palliative care approach trough general pediatrics on primary care.

Abstract number: P02-518

Abstract type: Poster 
Medicine Resident and Death Disclosure to Relatives: Their Experiences, from 14 Guided Interviews. Bordeaux in 2018 Grenier M., Burucoa B.

Service d'Accompagnement et de Soins Palliatifs, Université de Bordeaux, Bordeaux, France

Aim: The death disclosure to patient's relatives is an important moment generating strong feelings for the one receiving the information. The medical resident, with his special status halfway between doctor and student, grows around a theoretical knowledge, poor concerning breaking news and death, and a practical knowledge learning by doing. The objective of this study was to describe their's experiences in general practice during death disclosure to relatives.

Method: This is a cross-sectional qualitative observational study by 14 semi-directed interviews, conducted between July and August 2018, and among general practice residents. Axial and open coding of the verbatim was performed (Nvivo software) according to the Anchored Theorization method.

Results: Five components were identified.

1- Their feelings, full of emotions as positive as negative, strongly reported in number or intensity

" confronted with so many... cope ".

2- The expression of a relationship, materialized by communication tools and attitudes, little mastered " the contact... just be close ".

3- Several difficulties arising from the first two points, whether related to the patient, to the relatives, to the death, or to the resident himself " it's complicated... you have to answer ".

4- The absence of initial training but an " on-the-job » learning with the emergence of an experience, positive or negative " helpless... on-the-job ».

5- Finally some form of taboo on this subject within the profession " we do not speak... big omerta ».

Conclusion: The death disclosure requires to develop an emotional intelligence and to free speech on emotions. Students find themselves struggling with in the meeting, to communicate and adopt their attitudes. Finally, collegiality, companionship, co-construction and the return to experience through debriefing appear very useful in learning. Keywords: death disclosure, medical student, experiences, palliative care

\section{Abstract number: P02-519 \\ Abstract type: Poster}

Developing and Evaluating an Educational Programme for Health and Social Care Professionals Providing Palliative and End of Life Care for Gender and Sexual Minorities Chidiac C. ${ }^{1,2}$

${ }^{1}$ Saint Francis Hospice Hospice, Romford, United Kingdom, ${ }^{2}$ London South Bank University, Adult Nursing, London, United Kingdom Presenting author email address: claudechidiac@sfh.org.uk

Background: Lesbian, gay, bisexual and transgender (LGBT) individuals represent a significant underrepresented and underprivileged minority group, with distinct healthcare needs. A significant literature indicates that LGBT people face challenges when accessing palliative and end of life care services. This is mainly associated with lack or biased understanding of LGBT distinct needs, overpowering heteronormative behaviours, discrimination, and homophobia within the health services.

Aim: To develop and evaluate an education programme for health and social care professionals providing palliative and end of life care for LGBT people.

Method: An interdisciplinary Project Steering Group was formed to oversee the project, provide leadership, and influence strategic decisions. An education programme was developed based on systematic review of national and international evidence, national reports, and consultation with external experts and members of the LGBT community, using Kotter's 8 Step Change Model. A 90 minutes interactive education programme was delivered to health and social care professionals $(n=140)$ across 4 hospices in England. The programme was evaluated using pretest and post-test self-report questionnaires. Data was analysed using SPSS-21.

Results: There was a significant increase in the overall knowledge of general LGBT issues, knowledge of LGBT issues and needs in palliative and end of life care, confidence in providing palliative and end of life care for LGBT people, and comfort with using LGBT terms in the post-tests. The majority of participants indicated that this programme is beneficial to their practice, would recommend it to their colleagues, and would like further education in this area.

Conclusion: The education programme is now embedded in undergraduate and postgraduate palliative care curricula. The organisations that participated in this programme have initiated changes to support the delivery of LGBT-affirmative care.

Abstract number: P02-520

Abstract type: Poster

Delivering Pediatric Palliative Care Education through ECHO (Extension for Community Healthcare Outcomes) - Are the Key Learning Theories Applicable in a Resource Limited Setting? Doherty M. ${ }^{1}$, Rayala S. ${ }^{2}$, Evans E. ${ }^{3}$, Palat G. ${ }^{2}$

${ }^{1}$ Pediatrics, Children's Hospital of Eastern Ontario, Ottawa, Canada, ${ }^{2}$ Hyderabad Centre for Palliative Care, Hyderabad, India, ${ }^{3}$ Faculty of Medicine, University of Ottawa, Ottawa, Canada

Background: ECHO (Extension for Community Healthcare Outcomes) uses web-based videoconferencing to train health care professionals in remote areas, creates a virtual community of learners through discussion and sharing clinical experiences to empower and support participants. The structure of ECHO, which includes didactic teaching and case-based learning, was developed from principles of Social Cognitive Theory, Situated Learning Theory and Community of Practice Theory. The role of these learning theories in an $\mathrm{ECHO}$ program in a resourcelimited setting has not been explored.

Methods: We implemented a 1-year pilot ECHO program based in Hyderabad, India, targeting physicians, nurses and allied health professionals in India, Nepal, Sri Lanka and Bangladesh. We used analysis of session video recordings and participant surveys to explore the experiences and characteristics of this ECHO program related to key educational theories and to find ways to improve incorporation these principles into the ECHO program.

Results: There were 17 international faculty members who delivered didactic teaching. The average duration of discussion after the didactic session was 10 mins, with an average of 4 (range 0-9) questions. Participants from 20 health centers presented cases for discussion, followed by an average of 22 minutes of discussion and 6 (range 0-12) questions. Participants spoke for $30 \%$ of the discussion and content experts for the remaining $70 \%$. Results of participant surveys highlighted benefits from Social Cognitive Theory, Situated Learning Theory and Community of Practice Theory.

Discussion: Our results indicate that delivery of ECHO in a resource limited setting continues to reflect the original educational principles of Social Cognitive Theory, Situated Learning Theory and Community of Practice Theory. Our findings also suggest several modifications which may improve the benefits which participants derive from an ECHO program in a resource limited setting.

Abstract number: P02-521

Abstract type: Poster 
Impact of Theoretical Palliative Care Training for Oncology Residents in Romania

Mosoiu D. ${ }^{1,2}$, Strasser $F^{3}{ }^{3}$ Hinshaw D. ${ }^{4}$, losub L. ${ }^{5}$, Predoiu $0 .^{5}$

${ }^{1}$ Transilvania University, Brasov, Romania, ${ }^{2}$ Educatie, Hospice Casa Sperantei, Brasov, Romania, ${ }^{3}$ Cantonal Hospital, Saint Gallen, Switzerland, ${ }^{4}$ Michigan University, Michigan, United States, ${ }^{5}$ Hospice Casa Sperantei, Brasov, Romania

Introduction: Although palliative care (PC) services have started in Romania since 1992 at present just a limited number of cancer patients receive specialized PC. Training oncologist to deliver basic PC is a must. $A$ theoretical and practical PC training program for oncology residents based on ESMO/ASCO recommendation was developed and piloted.

Aim: To assess the impact of the palliative care theoretical training of oncology residents.

Method: PC curriculum for medical oncology was developed and piloted in all the 5 training centers for oncology residents in Romania. From 270 oncology residents in Romania, 144 participated in PC theoretical courses. The course had 42 hours and was delivered using a common training material and teaching method. Participants received a pre-test before and a post-test after the theoretical training for testing knowledge related to PC. Means for each topic from pre and post-test were compared using paired Sample T Test.

Results: Main content items covered in the multiple-choice examination included pain, non-pain symptom assessment and management, communication (with patient and family), psychosocial and spiritual care, knowledge of/referral to hospice, end-of-life care. From 144 participants to theoretical training, 86 had both pre and post-test completed. Comparing pre and post test scores there was a $70 \%$ increase of knowledge related to PC. For all the topics except of three- breaking bad news, fatigue and management of neuropsychiatric symptoms- there was a statistically significant increase $(p \leqslant 0.001)$ in knowledge after the theoretical training.

Conclusion: Including a PC module in oncology residents training program will increase their knowledge regarding PC and hopefully will improve quality of care to oncology patients.

Abstract number: P02-522

Abstract type: Poster

Community Views: Analysis of 'Before I Die' Statements

Rawlings D., Miller-Lewis L., Tieman J.

Flinders University, Bedford Park, Australia

Background: 'Before I Die' is a participatory public art project created by Candy Chang where people could start a conversation, writing their wishes and aspirations on a public chalkboard. It has since grown into a global project with more than 4,000 'Before I Die' walls presented in over 75 countries and in 36 languages (https://beforeidieproject.com/)

Aim: This study describes the responses made to a virtual 'Before I Die' wall within an online learning platform.

Methods: The 'Before I Die' activity was undertaken as part of an online massive open online course, or MOOC, Dying2Learn, conducted i2016 and 2017. Responses from both courses were extracted and de-identified. Generation of an initial coding system occurred through independent coding of 50 responses by the three investigators. A trial of the coding system using a further 100 responses was undertaken and discrepancies in coding quantified and analysed. Two research assistant then independently coded the entire response set of 633 statements. Ethical approval was granted by Flinders University (SBREC 7247).

Results: Respondents were predominantly female (93.3\%), with a mean age of 49.8 years. Twelve themes were identified from the responses. From the coding and analysis, three higher order themes emerged suggesting that wishes and aspirations related to the self (eg do some activity), were other focused (eg make the world a better place) or encapsulated the family (eg doing things with the family, wanting to see grandchildren grow up).

Conclusions: The data shows how 'well' community members view what is important while considering their death. While this study is limited by a sample of convenience, it is one of the first to systematically and empirically code and analyse the way that people respond to the question 'Before I Die, I want to...' This activity encourages people to articulate values in their life more consciously, within the context of understanding that life is finite.

\section{Abstract number: P02-523}

Abstract type: Poster

Impact of Advance Directives on the Decision-making in Intensive Care Unit

Smirdec M. ${ }^{1}$, Pereira B. ${ }^{1,2}$, Van Lander A. ${ }^{1,2}$, Lautrette A..$^{2,3}$

${ }^{1}$ University Hospital of Clermont-Ferrand, Clermont-Ferrand, France, ${ }^{2}$ University of Medicine, Clermont-Ferrand, France, ${ }^{3}$ Centre Jean-Perrin, Clermont-Ferrand, France

Introduction: Physicians do not know how to follow the patients' wishes when they are unable to express themselves. We assessed the impact of Advances Directives (AD) and how they have been written on the physician's decisions.

Methods: A multicentre, prospective, interventional, simulation study was carried out. 8 patients were recruited and wrote $A D$ after receiving clear and complete information by video and interview with one Intensive Care Unit (ICU) physician. 2 simulation scenarios including 10 questions about ICU admission and situations of withholding/withdrawing therapies using the patients' characteristics were submitted to ICU physicians from 28 French ICU, in 3 rounds (R): simulation without knowledge of the patient's AD (R1), with these $A D(R 2)$ and with these $A D$ and the knowledge of how they were carried out (R3).

Results: The qualitative analysis of these 8 AD highlights a form of living will or end-of-life will and not formal guidelines on medical care expected. The results were performed on complete data of 102 physicians. The variability between physicians themselves was high: among the 80 questions of R1, there were 37, 26 and 17 questions with an agreement $>80 \%, 80-60 \%$ and $<60 \%$ respectively. The AD significantly decreased the agreement between the physicians $(p=0.02)$. There was no difference between the rounds 2 and $3(p=0.84)$.The intra-individual variability between R1 and R2 was very high and the AD were significantly associated with this variability after adjustment on characteristics of the physicians and the patients $(p<0,001)$. It is relevant to notice that the knowledge of how AD were carried out has a very low impact on the physicians' decisions.

Conclusion: The AD have a major impact on the physicians' decisions for admission, withholding and withdrawing therapies decisions and increase the inter-individual variability but not the knowledge of how these $A D$ have been written. It might be a limit of the autonomous model.

Abstract number: P02-524

Abstract type: Poster

Support for Physicians Confronted with Euthanasia Requests from People with Dementia: A Nominal Group Expert Meeting Schuurmans J. ${ }^{1}$, Vos $S .{ }^{2}$, Tilburgs $B .{ }^{3}$, Engels $Y^{2}$

${ }^{1}$ General Practice Ottenhoff, Groesbeek, Netherlands, ${ }^{2}$ Radboud University Medical Center, Anesthesiology, Pain, Palliative Medicine, Nijmegen, Netherlands, ${ }^{3}$ Radboud University Medical Center, IQ-Healthcare, Nijmegen, Netherlands Antilles Presenting author email address: yvonne.engels@radboudumc.nl

Background: Under strict conditions, euthanasia is regulated by law in the Netherlands. The decision-making process following a euthanasia request can have a major impact on physicians. Recently, an interview 
study showed that, when confronted with a person with dementia (PWD)'s euthanasia request, physicians experience problems with communication, pressure from relatives, workload, interpretation of the law and ethical considerations concerning euthanasia in general. Moreover, physicians and patients may disagree about the right moment for euthanasia. Therefore, we aimed to identify types of support for physicians confronted with a request for euthanasia from a PWD.

Methods: In 2018, we organized two expert nominal group meetings. Experts were a lawyer, an ethicist, a chaplain, elderly care physicians, general practitioners, a palliative care consultant and a psychologist. Both meetings were audio-recorded, transcribed verbatim, and analysed using a combination of conventional and directed content analysis.

Results and discussion: Fifteen experts participated in the meeting, and five themes emerged:

1. Support provided by healthcare professionals (by individual professionals with different expertise, by multidisciplinary meetings and by awareness of and easy accessibility to these sources),

2. Public opinion (euthanasia is no right, impact on the physician, alternatives for euthanasia),

3. Training and tools (communication skills, observational tools for assessing (unbearable) suffering),

4. Legislation (a clear and understandable explanation of the law including advance directives),

5. Time and work pressure (time for reflection, administrative assistant, etc.) The urgent need for support that directly concerns the physician's decision-making process will be further explored during a survey among general practitioners. A concise and perspicuous explanation of the law is currently in process.

Abstract number: P02-525

Abstract type: Poster

Comparison of Ethical Issues Experienced during Palliative Care Provision in Nursing Homes in the UK and Canada: Findings from a Cross-sectional Survey

Muldrew D. ${ }^{1}$, Kaasalainen S. ${ }^{2}$, McLaughlin D. ${ }^{3}$, Brazil K. ${ }^{3}$

${ }^{1}$ Institute of Nursing and Health Research, Ulster University, Newtownabbey, United Kingdom, ${ }^{2}$ School of Nursing, McMaster University, Hamilton, Canada, ${ }^{3}$ School of Nursing and Midwifery, Queen's University Belfast, Belfast, United Kingdom

Background: Increased dependency on nursing homes to provide palliative care to an ageing population with complex care needs may lead to an increase in ethical issues, which may in turn lead to burnout and moral distress for staff.

Aim: To identify the type of ethical issues and level of associated distress experienced by nurses providing palliative care in nursing homes in the UK and Canada.

Methods: A cross-sectional survey design was utilised. On the day of data collection, all nurses working in 21 nursing homes across the UK and Canada received the "Ethical issues in Palliative Care for Nursing Homes" survey instrument. The EPiCNH instrument consists of 26 items, scored on two subscales; Frequency and Distress. One hundred and twenty three nurses completed the instrument across two days of data collection. Descriptive and inferential statistics were used to compare the frequency and level of distress caused by ethical issues in the UK and Canada.

Results: Frequent ethical issues include upholding resident autonomy, managing family distress, lack of staff communication, and lack of time in both countries. Higher levels of distress resulted from poor communication, insufficient training, lack of time, and family disagreements. Nurses in Canada experienced a greater frequency of ethical issues $(p=.022)$, however, there was no statistical difference in reported distress levels $(p=.53)$.

Conclusions: Nurses' reported comparable experiences of providing palliative care in UK and Canadian nursing homes. The majority of items overlap between the two countries suggesting pervasiveness of these ethical issues. These findings have implications on the practise of care in nursing homes, including how care is organized as well as capacity of staff to care for residents at the end of life. Training staff to take account of patient and family values during decision-making may address many ethical issues, in line with global policy recommendations.

Funder: Queen's University Belfast

\section{Abstract number: P02-526}

Abstract type: Poster

Deep Continuous Sedation until Death Requested by the Patient in Palliative Care a Multicentric Study

Filbet M., Serey A., Phan Hoang N., Perceau-Chambard E., Tricou C. Palliative Medecine, Centre Hospitalier Universitaire de Lyon Hospices Civils de Lyon, Lyon, France

Goal: In 2016, a new law was adopted in France granting patients the right, under specific conditions, to continuous deep sedation (CDS) until death. The goal of this study was to measure the frequency of requests for CDS from patients in palliative care.

Design: The data collected from the medical records of patients in palliative care units (PCU) or followed by palliative care support teams (PCST) in the Rhone-Alpes area, who died after CDS, focused on the patient's characteristics, the drugs used,and compliance with regulatory processes.

Results: All 12 PCU and 12 of the 24 PCST were included. Among the 8,500 patients followed, $42(0.5 \%)$ requested CDS until death. The patients were: $65.7(\mathrm{SD}=13.7)$ years old, highly educated $(69 \%)$, had cancer $(81 \%)$, refractory symptoms (98\%), mostly psycho-existential distress (69\%). The request was rejected for 2 (5\%) patients and delayed for 31 (74\%) patients. After a delay of a mean 8 days, 13 (31\%) patients were granted CDS. The drug used was midazolam at $115 \mathrm{mg} / 24 \mathrm{~h}(15-480)$, during a mean of 3 days. PCUs used lower dosages than PCSTs ( 83 vs 147), with significantly lower initial doses (39 mg vs $132 \mathrm{mg} \mathrm{p}=0.01$ ). A life-threatening condition was recorded in 13 cases (31\%) and a collegial decision was taken in 25 cases (60\%).

Conclusion: This study highlights the low rate of request and the even lower rate of CDS in specialized palliative care. However, the sedation for psycho-existential distress and the lack of procedure records raises ethical questions.

Abstract number: P02-527

Abstract type: Poster

End-of-Life Decision-making and Factors Affecting it: Has There been a Change?

Piili R. ${ }^{1,2}$, Lehto J. ${ }^{1,2}$, Metsänoja R. $^{3}$, Hinkka H. ${ }^{4}$, Kellokumpu-Lehtinen

P.-L. ${ }^{1,2}$

${ }^{1}$ Faculty of Medicine and Life Sciences, University of Tampere, Tampere, Finland, ${ }^{2}$ Department of Oncology, Tampere University Hospital, Tampere, Finland, ${ }^{3}$ Faculty of Social Sciences, University of Tampere, Tampere, Finland, ${ }^{4}$ Rehabilitation Center Apila, Kangasala, Finland

Background: Today most of the physicians and patients prefer shared decision-making and patient-centered care, especially in end-of-life. Aims: The aim of this study was to explore how physicians' decisions concerning end-of-life care have changed during the past 16 years.

Methods: A questionnaire including hypothetical patient scenarios was sent to 1182 and 1258 Finnish physicians in 1999 and 2015, respectively. Two patient scenarios were presented; 
1) a comatose prostate cancer patient with alternative situations for whom physicians were asked to choose either palliative, active or intensive care approach and

2) a breast cancer patient asking for a voucher for hospice. Physicians' attitudes, values and background factors were also queried. The response rate was $56 \%$.

Results: Physicians in 2015 chose the palliative approach more often than in 1999 in the following situations of the prostate cancer patient: probable iatrogenic Gl-bleeding due to ketoprophen (53\% vs 59\%, $p=0.014$ ), when his son was coming to see him ( $46 \%$ vs $60 \%, p<0.001$ ) and when he had spiritual anxiety ( 53 vs $71 \% \mathrm{P}<0.001$ ). Patients benefit (96\% vs 99\%, $p=0.001)$, ethical values ( $83 \%$ vs $93 \%, p<0.001$ ) and patient's (68 vs $86 \%, p<0.001$ ) or physician's ( $44 \%$ vs $63 \%, p<0.001$ ) legal protection were considered more influential to the decisions today than 16 years ago, whereas the influence of family's benefit decreased ( $37 \%$ vs $25 \%, p<0.001$ ). Physicians were more willing to give a voucher for the breast cancer patient for hospice in 2015 (34\% vs 58\%, $p<0.001$ ). Post-graduate end-of-life training was one of the most constant factors explaining these decisions.

Discussion: Our findings can be seen as a reflection of the changes in the surrounding society and medical atmosphere towards individualism and patient-centered care. The results also highlights the importance of education in end-of-life care, which should be incorporated to post-graduate training in all specialties taking care of the dying patients.

Abstract number: P02-528

Abstract type: Poster

Ethics of Using Prognostic Tools to Screen General Practice Populations to Identify People Approaching the End of Life - A Comparison with the WHO Wilson and Jungner 1968 Criteria for Screening Programmes

Verne J.

Public Health England, Bristol, United Kingdom

Background: In England, there is policy interest to identify patients in their last year of life to offer Advance Care Planning (ACP). Primary care doctors are encouraged to find their $1 \%$ of patients who may die within a year and discuss ACP ( $1 \%$ Campaign). Methods to identify patients range from the frailty index, to the 'surprise question' - would the doctor be surprised if the patient died within the next year. Population Screening Programmes are judged against different ethical criteria to normal doctor-patient interactions.

Aim: To test the concept of primary care screening to identify patients in the last year of life against the 10 Wilson and Jungner Criteria (WJC10) for assessing whether a screening programme can be ethically implemented. Method: Assessment of the 1\% Campaign against the WJC10 using empiric evidence from literature review.

Results:

1) Important public health problem - yes, in England 0.5 million die each year.

2) There is a treatment - not a cure but yes palliation.

3) Disease trajectory understood - yes for cancer more problematic in organ failure and frail elderly.

4) Identifiable earlier stage which can be where intervention is more effective - good evidence for this.

5) A good screening test (high sensitivity, specificity and predictive value) - current methods do not fulfil this criteria.

6) Acceptable test - poor evidence on patient and doctor acceptability.

7) Facilities for treatment available for the cases identified - good evidence of significant geographical variation in quantity and quality of care in England.
8) Agreement who to treat - evidence for elderly poor.

9) Favourable economic analysis - some evidence to support.

10) Screening should be a repeated process - it would be for it to work.

Conclusions: The 1\% Campaign does not fulfil the WJC10 for a population screening programme. Care should be exercised in implementation and such programmes usually require evidence from randomised controlled trials before implementation.

Abstract number: P02-529

Abstract type: Poster

"Confound their Language that They May Not Understand One Another's Speech": An Examination of the Ethical Arguments against and in Defence of the Liverpool Care Pathway (LCP) Keeley $P .^{1,2}$

${ }^{1}$ Glasgow Royal Infirmary, Glasgow, United Kingdom, ${ }^{2}$ University of Glasgow, Centre for the History of Medicine, Glasgow, United Kingdom

Aim: Intimately connected to the press coverage of the Liverpool Care Pathway was the ethical debate surrounding the issues that emerged around the end of life. The aim of this study is an examination of the ethical arguments against and in defence of the LCP

Methods: A literature search was undertaken using the broad search terms 'Liverpool Care Pathway' and 'ethics'. Papers were also sought via a wider search of literature and personal communications obtained from participants in a research project on the LCP were used, including submissions to the Neuberger Review of the LCP.

Results: Three principle ethical standpoints were developed in the ethical furore surrounding the LCP. The main critics of the LCP used an ethical standpoint of traditional Aristotelean-Thomistic ethics to argue from principles of virtue and intent that the LCP was unethical. Such an outlook is sometimes labelled 'vitalist'. A more nuanced and less critical stance was taken from a similar ethical standpoint was advanced by other Catholic ethicists. Finally, an ethical stance entirely divorced from traditional ethics was advanced, but ultimately failed to address, the ethical arguments of the so-called 'vitalists'.

Conclusion: Contemporary medical ethics, drawing on secular exponents, will maintain the pillars of beneficence, non-maleficence, autonomy and justice, while failing to address the persisting issues of virtue and ontology as expounded by Aristotle and Aquinas (among others). The fact that the debate was conducted in essentially different languages, where the metaphysics of one is ignored by the other, means there was unikely to be ground for agreement or consensus.

Abstract number: P02-530

Abstract type: Poster

Moral Distress in End-of-Life Care - Medical Students' Perspectives

Thurn T., Anneser J.

Psychosomatic Medicine and Psychotherapy, Technical University of Munich, Munich, Germany

Background: Moral distress (MD) is a frequent phenomenon in end-oflife care. It occurs when one knows the morally correct response to an ethically challenging situation, but cannot act because of institutional or hierarchical constraints. Medical students - having a perceived low level in a hierarchy - may be particularly vulnerable for MD.

Aim: To assess the frequency and intensity of medical students' MD occurring in end-of-life care.

Methods: Based on the moral distress scale -revised (MDS-R), we developed a self-administered questionnaire describing ten potentially morally distressing scenarios in end-of-life care. The questionnaire was 
distributed to all $4^{\text {th }}$ year medical students of the Technical University of Munich/Germany. We asked students

1) if they had ever witnessed the described scenarios

2) to rate the extent (NRS 1-5) they felt disturbed (experienced $\mathrm{MD}$ ) or they would feel, if it happened to them (hypothetical $\mathrm{MD)}$.

In addition, we asked for thoughts about dropping out of medical school or choosing a non-clinical specialty because of these experiences.

Results: Out of 340 students, 217 completed the survey (64\%). On average, students had experienced 2.51 morally distressing situations (SD \pm 2.23). The majority (75\%) had experienced at least one situation. "Providing futile care with the basic intention to earn money" was the item with the highest levels of experienced $(2.88 \pm 1.05)$ and hypothetical (3.34 \pm 0.95$)$ distress, witnessed by $25 \%$ of participants. 25 students (12\%) reported that they had thought about dropping out of medical school or choosing to a non-clinical specialty due to the MD experienced in the described situations.

Conclusions: Medical students experience MD on a regular basis and most frequently in scenarios of "futile care". MD in end-of life care may be an underestimated factor for medical school attrition. Interventions should address the sources of MD and help the students to overcome their perceived powerlessness.

Abstract number: P02-531

Abstract type: Poster

\author{
End-of-Life Decision-making in a Deaf and Mute Patient: A Case \\ Report \\ Provido M., Neo H.Y.
}

Palliative Medicine, Tan Tock Seng Hospital, Singapore, Singapore

Case Vignette: Mr. M was a 74 year-old Chinese gentleman who was born deaf and mute. He never had any formal education and communicated through rudimentary hand signals. His sister described him to be slow-witted and easily deceived. He has no legal surrogate.

He was newly diagnosed of HIV/AIDS. Radiologic scans showed enhancing intracranial lesions and a lung mass. Prognosis varied between short months should the cerebral lesions be metastatic versus long years should they be curable opportunistic infection from AIDS.

The diagnosis of HIV was not disclosed owing to challenges in conveying the complexity of his treatment due to profound hearing and speech impairment. The physician also could not obtain informed consent for biopsy, which is crucial to diagnosis, prognosis and treatment.

\section{Ethical Questions:}

1. Does the patient possess capacity for therapeutic decisionmaking?

2. Are external translators ethically allowed even if there would be a breach of confidentiality to his retroviral diagnosis?

3. Should the patient be judged competent but healthcare providers are unable to convey sufficient information or establish his level of appreciation of the issues, who should hold principal authority over clinical management?

Conclusion: Two translators, one of whom was a deaf-mute were engaged to facilitate communication. A joint session involving psychiatrist, ethicist, physicians and the patient was held. Patient was assessed to possess capacity for only simple tasks. As he was unable to understand, appreciate and weigh the complex clinical issues, he could not give informed consent or refusal of recommended treatment. However, he should be engaged in discussions as far as possible. Decisions on clinical management and aggressiveness of resuscitation would be best discussed amongst doctors and through departmental consensus. Such decisions should be reviewed regularly, as factors such as response to empirical treatment, will influence aggressiveness of further management.

Abstract number: P02-532

Abstract type: Poster

How Do Patients Talk about Wishes to Hasten Death? A

Qualitative Study

Chassagne A., Mathieu-Nicot F., Cretin E.

University Hospital of Besançon, Besancon, France

In France, euthanasia and physician-assisted suicide are illegal. Yet, some terminally ill patients express wishes to hasten death (WTHD). The literature suggests that WTHD disappear when patients are provided with effective care. We hypothesized that WTHD expression remains but evolves over time and may not be recognized by healthcare professionals.

We aimed to describe the initial WTHD and analyze their evolution over one week.

We performed a qualitative study on 31 patients (in 11 French palliative care units) whose explicit euthanasia or physician-assisted suicide request was written in their medical records, and referred to us by a healthcare professional. Of the patients, 18 were interviewed 24 hours after the initial request, and 9 one week later. We performed lexical and thematic analyses of the requests to hasten death found in the medical records and the interviews.

Five main themes emerged from the initial requests transcribed from medical records and the first interview:

1/The will of the person ("I want");

2/The capacity or incapacity ("to can"; "could");

3/Involvement of a third party ("you"; "help me");

4/Temporalities of the act ("right now", "quickly");

5/The use of metonymies ("injection", "drug").

During the second interview, we observed a semantic shift, the involvement of the patient himself appeared ("give me"; "I need material") and the word suicide.

In conclusion, initial request tended to be euthanasia requests. The WTHD remained after 1 week but their expression changed: requests tended to be physician-assisted suicide requests. Paying attention to the phrasing used by patients to express their WTHD may be important to fully and precisely understand their intentions and to adapt care delivery.

Abstract number: P02-533

Abstract type: Poster

How to Optimize Informed Consent in Patients with Dementia? A Qualitative Study on Palliative Care Professionals' Views Hernández-Marrero P..$^{1,2,3}$, Carvalho A.S. ${ }^{1,2,3}$, Araújo J. ${ }^{1,2,4}$, Martins Pereira S. ${ }^{1,2,3}$, Project ENSURE, Enhancing the Informed Consent Process. Supported Decision-Making and Capacity Assessment in Clinical Dementia Research

1 Instituto de Bioética, Universidade Católica Portuguesa, Porto, Portugal, ${ }^{2}$ UNESCO Chair in Bioethics, Institute of Bioethics, Universidade Católica Portuguesa, Porto, Portugal, ${ }^{3} \mathrm{CEGE}$, Centro de Estudos em Gestão e Economia, Porto Católica Business School, Universidade Católica Portuguesa, Porto, Portugal, ${ }^{4}$ CEGE: Centro de Estudos em Gestão e Economia, Porto Católica Business School, Universidade Católica Portuguesa, Porto, Portugal

Background: Cognitive impairment is frequently present among patients with dementia. This challenges the process of informed consent (IC) both in clinical practice and palliative care (PC) research. International 
research suggests the use of diverse measures and tools to improve the IC process in such cases. Very little is known on the application of such tools in PC and research.

Aims: To understand PC professionals' views about the actual practice and measures to optimize IC in clinical practice and research.

Methods: Qualitative study, using in-depth interviews with professionals working in PC in Portugal. Purposive sampling combined with snowball sampling was conducted until reaching theoretical saturation. Thematic content analysis was performed to the transcript of interviews. QSR-NVIVO11 was used for data analysis. A total of 24 professionals from diverse institutions and cities were interviewed (10 nurses, 9 physicians, 2 psychologists, 2 social care worker, and 1 physiotherapist).

Results: Four main themes emerged in our analysis: impossibility to apply the IC process in face of cognitive impairment; efforts to involve the patient in the decision-making process; proxy consent, via family carer, as the preferred way to meet patients' wishes; innovative measures (e.g., visual aids) perceived as potentially useful, but not used in clinical practice or research. For all the participants, proxy and supported decision-making were the most common strategies used to obtain IC. Innovative measures were perceived as potentially useful, but none of the participants ever applied them in practice.

Conclusions: This study highlights the need to further research the use of strategies to improve the informed consent process in palliative care and research. It shows the potential of innovative measures, which need to be designed in a tailored manner and require more research on their applicability and usefulness.

Acknowledgment: ERA-NET NEURON II ELSA and FCT Portugal.

Abstract number: P02-534

Abstract type: Poster

\section{Compassionate Use Programs and Palliative Care: Ethical} Guidelines

De Panfilis L. ${ }^{1}$, Satolli R. ${ }^{2}$, Costantini M. ${ }^{1}$

${ }^{1}$ Azienda USL-IRCCS di Reggio Emilia, Reggio Emilia, Italy, ${ }^{2}$ Ethics Committe AVEN, Modena, Italy

Aim: This study proposes a retrospective ethical analysis of a compassionate use (CU). It is the result of a discussion within an italian Provincial Ethics Committee (EC) following the finding of a high number of requests for CU program.

The primary aim of the study is to illustrate the specific ethical and clinical profiles that emerge from the compassionate use program (CUP) issue. The secondary goals are:

a) to promote a moral reflection among physicians who require approval for the CUP and

b) provide the basis for recommendations on how to request CUP.

Methods: The instruments for carrying out the analysis of the case study and the discussion are as follows:

- Analysis of the audio-recording of the EC meeting regarding the selected Case study.

In-depth discussion of topics that emerged during the meeting by means of administration of 5 semi-structured interviews with 2 doctors involved in the case (proposing physician and palliative physician) and with 3 components of the EC who

played a major role in the EC internal discussion.

Results: In an exploration of emerging clinical and ethical issues, four primary themes arise:

1. efficacy, safety of the treatment and patient's quality of life;

2. clear, realistic, adequate communication;

3. right to hope;

4. simultaneous Palliative Care approach
Conclusions: The provincial EC of Reggio Emilia chose to steer the request for drugs

for compassionate use through recommendations for good clinical and ethical practice based on the following assumptions:

1) the "simultaneous care" approach must be preferred. In fact, it helps to provide a more complete and realistic picture of the disease, the prognosis and the possibilities of curing and/or the quality of remaining life being higher than that proposed only by the oncologist's point of view; secondly,

2) the EC's assessment must be part of the decision-making process that the care team conducts before proposing compassionate use to the patient.

Abstract number: P02-535

Abstract type: Poster

How Can We Support Advance Care Planning? Seeking Ways for Nurses to Assist Patients to Have Conversations about Death and Dying in Japan

Takenouchi S. ${ }^{1}$, Okada H. ${ }^{2}$

${ }^{1}$ Department of Ethics Support, Kyoto University Hospital, Kyoto, Japan, ${ }^{2}$ Department of Health Communication, Tokyo University, Tokyo, Japan

Background: Nurses often struggle to have explicit conversations about death and dying with patients. Although many nurses in Japan are aware of its benefits, they still fear Advance Care Planning (ACP) may impose more burdens than benefits on patients due to lack of knowledge about how to support ACP and understand patients' attitudes toward it.

Aims: To investigate experiences with and attitudes toward ACP among advanced lung cancer patients who undergo ambulatory chemotherapy and explore ways in which nurses can adequately support ACP.

Methods: This mixed method study comprised two sequential phases. A convenience sample of patients was recruited after screening.

Phase 1: Semi-structured interviews were conducted to understand participants' perceptions of ACP. Content analysis was applied to the qualitative data.

Phase 2: Participants' experiences, readiness, and perceptions of ACP were assessed using questionnaires, including an ACP engagement survey.

Results: A total of 10 patients (mean age $69.7 \pm 5.7$ years, $50 \%$ women) participated in the interview and 83 patients (mean age $67.3 \pm 10.5$ years, $43.4 \%$ women) in the qualitative research. Although $58.1 \%$ had some ideas concerning their end-of-life treatment preferences and $57.9 \%$ had talked with their family and friends, only $11.7 \%$ had conversations with their physicians about ACP. Only $6 \%$ of the participants expected nurses to discuss ACP, but $63.9 \%$ were willing to have conversations with nurses with ACP expertise.

Conclusion: This study explored patients' experiences with and attitudes toward ACP in Japan. We identified barriers and challenges specific to the Japanese culture that prevent patients from talking to their physicians about their preferences. Our findings could provide an important foundation for developing a model facilitating ACP conversations between Japanese nurses and seriously ill patients.

This work was supported by Japan Society for the Promotion of Science Grant Number JP16K20751.

Abstract number: P02-536

Abstract type: Poster

Putting Clinical Ethics into Guideline Format: When and how to Limit Therapeutic Interventions in Palliative Care as a Part of the German Evidenced-Based Guideline 'Palliative Care for Patients with Incurable Cancer' 
Alt-Epping B. ${ }^{1}$, Bausewein C. ${ }^{2}$, Voltz R. ${ }^{3}$, Simon S.T. ${ }^{3}$, Simon A. ${ }^{4}$, on behalf of the German Guideline Group 'Palliative Care'

${ }^{1}$ Dept. of Palliative Medicine, University Medical Center Goettingen, Goettingen, Germany, ${ }^{2}$ Dept. of Palliative Medicine, University Hospital Munich, Munich, Germany, ${ }^{3}$ Dept. of Palliative Medicine, University Hospital Cologne, Cologne, Germany, ${ }^{4}$ Academy for Ethics in Medicine (AEM), Goettingen, Germany

Background: The German Association for Palliative Medicine (DGP) has launched an evidence-based guideline in 2012; seven chapters were published in 2015; eight further topics are currently being consented by the participating 61 scientific associations.

To determine when and how therapeutic interventions should be limited constitutes not only an objective, but a normative process. This question remains one of the most controversial topics in the care of incurably affected patients and may lead to ethical conflicts between medical disciplines, professions, within teams and individuals. We therefore addressed the criteria for clinical decision making, focusing on limiting interventions, as an explicit part of the current guideline.

Methods: Study evidence, the legal framework and ethical positions were reviewed. Recommendations were made based on their clinical and normative relevance, and approved by the formal consensus process. Further legal explanations and ethical discourse was placed in the background text. Results: Patient participation, the role of relatives, anticipation of problems, the role of ethics consultation, determining the therapeutic aim, indication and consent, role of proxies in decision making, prognostically uncertain situations, cultural differences in therapeutic decision making, and formal aspects like validity for specific patient cohorts were identified as relevant aspects. Of 20 recommendations, 19 remained on an expert consensus level, one was consented as evidence based.

Discussion: Illustrating ethical principles and criteria of therapeutic decision making by means of an evidence-based guideline is feasible. Further research will have to demonstrate whether confidence, certainty and reproducibility of normative decision processes may increase and ethical conflicts in clinical routine may be reduced by implementation of this guideline in palliative care and beyond.

Abstract number: P02-537

Abstract type: Poster

"Tailoring a Special Therapy by Pertinence": A Grounded Theory (GT) Model Describing the Proposal of a Compassionate Use (CU) Therapy for Cancer Patients

Bertocchi E. ${ }^{1}$, Giovanardi F. ${ }^{2}$, Sireci F. ${ }^{3}$, Nunziante F. ${ }^{4}$, Capodanno 1. ${ }^{4}$, Sacchi S. ${ }^{1}$, Di Leo S. ${ }^{5}$, Ghirotto L. ${ }^{6}$

${ }^{1}$ Palliative Care Unit, Azienda USL - IRCCS di Reggio Emilia, Reggio Emilia, Italy, ${ }^{2}$ Oncology, Azienda USL - IRCCS di Reggio Emilia, Reggio Emilia, Italy, ${ }^{3}$ Neurology, Azienda USL - IRCCS di Reggio Emilia, Reggio Emilia, Italy, ${ }^{4}$ Haematology, Azienda USL - IRCCS di Reggio Emilia, Reggio Emilia, Italy, ${ }^{5}$ Psycho-oncology Unit, Azienda USL - IRCCS di Reggio Emilia, Reggio Emilia, Italy, ${ }^{6}$ Scientific Directorate, Azienda USL IRCCS di Reggio Emilia, Reggio Emilia, Italy

Background/aims: CU programs are pathways that allow cancer patients without medical treatment options to benefit from not yet approved therapy. Many studies highlight a complex interplay among medical needs, ethics, medical law and commercial interests. Up to now no qualitative studies about the perspective of patients and professionals exist. We aimed to model a qualitative explanation about the process of $\mathrm{CU}$ proposal to cancer patients.

Methods: We carried out a GT through semi-structured interviews, using Charmaz's constructivist approach. We involved 21 participants (professionals, patients and other stakeholders) from a Clinical Cancer Centre. We did data collection and data analysis concurrently; theoretical sampling allowed the definition of an explicative model of the process.
Results: From analysis we identified three main phases: "Giving a new opportunity", "A special therapy", "Ensuring pertinence". First, the sense of uncertainty and clinical instability bring patients and physicians to build together a new therapeutic chance. Doctors are in doubt about the efficacy but they overbalance toward "trying" and therapy becomes "special" (phase II): it strengthens care relationship and generates "little hopes". Finally, the therapeutic proposal passes from hand to hand through different professionals who verify its pertinence. A lot of resources are employed: different institutional filters go on while time is running out. Clinical and ethical debate among professionals is enhancing through responsibility and emotional load. We named the overall process: "Tailoring a special therapy by pertinence".

Conclusions: Our model describes the process of CU proposal to cancer patients, showing important areas for ethical interdisciplinary debate and target improvements. Results reveal how important is the role of Palliative Care in advocating the centrality of patient and connecting patients' preferences and values with clinical pertinence.

Abstract number: $\mathrm{P} 02-538$

Abstract type: Poster

Insights into Control in Advanced Cancer Patients: A

Phenomenological Study

Rodríguez-Prat A. ${ }^{1}$, Crespo I. ${ }^{2}$, Balaguer A. ${ }^{3}$, Monforte-Royo C. ${ }^{4}$, We

Care, End-of-life Care

${ }^{1}$ Faculty of Humanities, Universitat Internacional de Catalunya,

Barcelona, Spain, ${ }^{2}$ School of Medicine and Health Sciences, Universitat Internacional de Catalunya, Barcelona, Spain, ${ }^{3}$ Universitat Internacional de Catalunya, Barcelona, Spain, ${ }^{4}$ Nursing Department, Uniersitat Internacional de Catalunya, Sant Cugat del Vallès. Barcelona, Spain

Background: It has been proposed that control is a key aspect at the end of life in terms of the identity, quality of life and wellbeing of patients. Patients who expressed a wish to hasten death referred to the loss of control as one of the main causes of their suffering. As such, having a degree of control over the process of dying has been seen as a way of overcoming the lack of control that a life-threatening condition entails. It is also a way of maintaining personal independence. However, little is known about patients' experiences of control in this context.

Aims: To explore the construct of control in the experiences of advanced cancer patients.

Methods: We conducted semi-structured qualitative interviews using an interpretive phenomenological analysis approach. Eight advanced cancer patients from an oncology unit from Barcelona (Spain) were interviewed. A purposive sample was used. The interviews were recorded and analysed using Atlas.ti v.8.

Results: We identified 240 codes, which we grouped into 2 themes. The first theme "the experience of illness as a form of lack of control" included the subthemes
a) loss of functionality,
b) identity,
c) social relationships and
d) healthcare.

The second theme "domains of control" was described by the following subthemes

a) "what I can control",

b) strategies and mechanisms of control,

c) social control and d) interior liberty.

Conclusion / Discussion: This study allowed us to identify key strategies to promote a sense of control in patients with advanced cancer. This illness, according to the participants, was experienced as series of losses. 
However, attention was often focussed on areas where they continued to have control. Furthermore, the results highlight the fact that both family members and healthcare professionals can help to empower patients.

Abstract number: P02-539

Abstract type: Poster

How Do Patients with Advanced Disease Feel about their Participation in Palliative Care Research?

Vlčková K., Tučková A., Poláková K., Houska A., Loučka M. Center for Palliative Care, Prague, Czech Republic

Background: Research in palliative care is limited by the vulnerability of this patient population. There is a controversy about ethical justification for involvement of such fragile patients in research studies. The aim of this study was to explore how patients themselves feel about their participation in palliative care research.

Methods: We conducted a multicenter cross-sectional study in patients with advanced illness and limited prognosis (assessed by 12-month surprise question). Patients ( $n=170)$ completed questionnaires about their preferences with life-threatening illness and as a final part of questionnaire we asked them using 5 points Likert scale to evaluate participation in this palliative care research.

Results: Only $7 \%$ of patients found it unpleasant or very unpleasant and the rest did not evaluate it as bad experience. We found that more than half of patients (53\%) did not mind participate in this study and for $41 \%$ it was even interesting or very interesting experience. There were no differences in the evaluation of participation according to age, religion, sex, education or belief that their disease is curable/incurable.

Discussion: We found that most of patients included in this study did not mind participate in research and they even found it as interesting experience which can encourage scientist to do research in palliative care. The evaluation of participation in research did not differ according to demographic characteristics which is not completely consistent with previous results. This could be explained by the role of culture on evaluation of participation in research, therefore it is important to conduct national research on this topic.

\section{Abstract number: P02-540}

Abstract type: Poster

Advance Directives: Expectation vs Reality

González Jaramillo V. ${ }^{1}$, Maessen $M .^{1}$, Pautex $S .^{2}$, Gudat $H^{3}{ }^{3}$, Eychmüller S. 4

${ }^{1}$ ISPM, University of Bern, Bern, Switzerland, ${ }^{2}$ Hôpitaux Universitaires de Genève, Genève, Switzerland, ${ }^{3}$ Hospiz im Park, Arlesheim, Switzerland, ${ }^{4}$ University Centre for Palliative Care, University of Bern, Bern, Switzerland

Background: Advance directive (AD) is the form by which patients express their autonomy and empowerment with regard to medical decision-making. Each time, more emphasis is placed on the importance of patient's autonomy. Despite this, AD is underutilized in some settings. This makes important to understand the pattern of use of $A D$ in patients receiving palliative care $(\mathrm{PC})$.

Aim: We aimed to assess the prevalence of $A D$ among patients in $P C$ units in Switzerland and to explore which characteristics may be related with its completion.

Methods: A pilot national registry for PC was initiated in 2014. Data from six cantons was collected between December of 2014 and April of 2016. We compared different characteristics between patients according to the presence of $A D$.

Results: Data from 419 patients was available, $52 \%$ of whom were women. The mean age was 71 . Thirty-one percent reported to have AD at either their admission or discharge from the PC unit. The prevalence of $A D$ did not change regardless patients' cognitive status or psychiatric comorbidities. There was no difference between patients with or without $A D$ in terms of sex, age or modified Karnofsky. Although not statistically significant, there was a trend regarding religion. Only $8 \%$ of Islamic patients had $A D$, compared to Christians, other religions, or agnostics (30\%, 43\%, and 40\% respectively). Even in patients with more severe conditions, or patients who died during the hospitalization, the prevalence of AD was less than 35\%, although a higher proportion would be expected in this subpopulation.

Conclusion: In this setting of patients, the use of AD was unexpectedly low. These results should encourage the multidisciplinary team to promote the expression of autonomy in patients in order to prevent controversy about treatments, which often arise between decisions made by surrogates and patient's wishes. For this, new strategies must be designed according to the patient's owns values, culture and religion.

Abstract number: P02-541

Abstract type: Poster

\section{Palliative Care for the Elderly - Balancing Inevitable}

\section{Contradictions}

Schuchter $P .^{1}$, Heimerl $K^{2}{ }^{2}$

${ }^{1}$ Palliative Care and Organizational Ethics, University of Graz, Graz, Austria, ${ }^{2}$ IFF-Palliative Care and Organizational Ethics, University of Vienna, Vienna, Austria

Background: Palliative Care for the elderly has become an important issue that is recognized also internationally. As early as 2004 the WHO has begun to publish a series of brochures dedicated to palliative care for the elderly. Likewise the issue of implementing palliative care in nursing homes has increasingly gained attention. At the same time projects to implement palliative care for the eldery face considerable challenges.

Methods: We carried out a series five group discussions with managers of projects that aimed at implementing Palliative Care for elderly people. Overall, 54 project managers and 30 projects participated in the discussions. Two members of the research team facilitated, documented and analysed the discussions. In addition, we collected relevant documents about the projects. We used thematic analysis to analyse the data. Findings: All discussions were provoked by and/or revealed by contradictions that characterize the projects for implementation of palliative care for the elderly. The most important contradictions that the project managers discussed are:

(1) General ethics of care vs. specialized services,

(2) Inclusion and understanding vs. exclusion,

(3) Quality: Measurements vs. narratives,

(4) Cooperation vs. Competition,

(5) Research vs. practice,

(6) Clinical ethics vs. ethics consultation for elderly care.

Conclusion: The project managers discussed contradictions, which are inevitable. They cannot easily be solved or disappear completely. The importance of dealing with such somehow inevitable contradictions (and necessary conflicts) has been highlighted by Krainer/Heintel (2010, 2015) in their model of "process-ethics" in the tradition of dialectical philosophy. The discussions highlight the inequality between autonomy and dependency in palliative care for the elderly. We therefore consider palliative care for the elderly to be an expression of ethics of care.

Abstract number: P02-542

Abstract type: Poster

Self-neglect and End-of-Life Care - A Conceptualization in View of the Idea of Stigma 


\section{Dunger C., Schnell M.W.}

Lehrstuhl für Sozialphilosophie und Ethik, Witten/Herdecke University, Witten, Germany

Neglect is often described as a syndrome with different manifestations and causes. The term is also controversial, as it mainly describes an individual deviation from the socially desired normality (cf. Goffman). In this effect, emphasis is regularly put on chaotic/ neglected home environments or housing fulfillment. The exact number of people affected is not known. Nevertheless, many social and health care professionals from different settings describe an increase in those affected. They also feel that dealing with these people is particularly stressful and challenging As we claim to facilitate palliative care (PC) to all persons with PC needs at the end of life (EOL), we also need to deal with neglect as a relevant subject. Therefore, we examine the importance of the self-neglect (SN) concept for the care of people at the EOL. At the same time, we question, which contribution the special perspective of $\mathrm{PC}$ can make to care for those affected at the EOL.

Bases on a literature analysis, we

(1) conceptualized SN, including the stigma term of Goffman, such as legal provisions and

(2) discuss it in terms of the understanding and values of PC.

SN includes physical, mental and social neglect caused by physical as well as psycho-social life-situations and illnesses. It appears in different intensity and consciousness. Some affected people decided to life e.g. in a chaotic home environment, but many are not aware of their situation. Therefore, professional care needs to be individual and to embower them to make their own decisions and to react to the stigma.

The stigma term and legal provisions help to focus on the connection between the normative perspective of the socially desired normality and the individual deviation. Possible reactions to the stigma do not necessarily require an adaption of affected persons. Thus, PC as individual and empowering discipline, with a focus of self-determined quality of live, can contribute to the EOL-care for affected people.

Abstract number: P02-543

Abstract type: Poster

Are there Benefits for Palliative Care Patients in Participating in Research? Systematic Literature Review and Narrative Synthesis Červ B., Lunder U., Kodba-Čeh H.

University Clinic Golnik, Golnik, Slovenia

Background: Fear of burdening patients is one of the prevailing reasons for gatekeeping in palliative care research. A thorough insight into patients' experiences is needed.

Aim: To establish palliative care patients' beneficial experiences from their participation in research.

Methods: A systematic literature review was undertaken by searching PubMed and CINAHL databases for the literature published from 2008 until September 2018, involving adult palliative care patients' reports, written in English language. References and citation searching of the included articles and literature review articles was performed. Patients' reports were analysed using inductive thematic analysis.

Results: In the 11 included articles, participants were mainly patients with advanced cancer. Reported were experiences of clinical/psychosocial trials (10) and qualitative research participation (1) in hospitals, patients' homes and nursing homes. Analysis showed that the majority of palliative care patients find research participation beneficial. Five themes describing benefits of participation were identified: empowerment through contribution (helping others, giving back to society), helpful insights (more information about their situation/symptoms), feeling secure (being visible, receiving more attention and care), therapeutic value of interaction (sharing thoughts, vent frustrations, forgetting troubles and symptoms for a while) and self-reflection (deeper thinking, contemplation, accepting situation, establishing new hopes). Majority of patients did not experience burden associated with participation in research.

Conclusion: Despite often expressed fear of burdening palliative care patients with inclusion in research, our review shows that majority of even very sick patients experience many benefits. The intention to protect palliative care patients might bereave them from empowering, comforting and even therapeutic benefits of research participation. Financed: EU $7^{\text {th }}$ framework

Abstract number: P02-544

Abstract type: Poster

CURA - Developing Ethics Support for Professionals in Palliative

Care

Metselaar S. ${ }^{1}$, Van Schaik M. ${ }^{1}$, Kröger $C .{ }^{1}$, Van Bennekom D. ${ }^{1}$, Pasman

R. $^{2}$

${ }^{1}$ Medical Humanities, Amsterdam UMC, Location VU University, Amsterdam, Netherlands, ${ }^{2}$ Public and Occupational Health, Amsterdam UMC, Location VU University, Amsterdam, Netherlands

Background: Professionals in palliative care are frequently confronted with moral dilemmas. For instance, whether to follow a patient's wishes whereas this is not in line with the best care one could provide. This can cause 'moral distress', i.e. distress that arises either when being unable to do what one thinks is right, or when in doubt about the right thing to do. Ultimately, if moral conflicts remain unresolved, and professionals are not equipped or supported to deal with them, the quality of patient care may suffer. Our research has shown that existing ethics support does not suffice, and that there is a need for accessible ethics support that is to be used in daily care practice and does not require extensive training or supervision.

Aims: Our study aims to develop, implement, and evaluate a new instrument for ethics support in palliative care. It focuses on nurses, but may be used by other professionals too.

Methods: We developed an ethics support instrument for palliative care on the basis of a participatory design, closely involving a diverse group of stakeholders in a process of iterative, responsive co-creation. The moral dynamics in palliative care was studied on the basis of analyzing data of ethics sessions and interviews with professionals, family and patients. The instrument is evaluated on the basis of focus groups and questionnaires. Conclusion: The ethics support instrument that we developed is called CURA, and consists of a four-step method for reflection. CURA is to provide an accessible way for professionals to reflect on moral dilemmas in palliative care practice. It is complemented with an overview of moral dilemmas in palliative care and values that are found to be important in providing good palliative care (in the Netherlands). CURA is to promote sensitivity to moral dilemmas, 'moral competences' and 'moral resilience'. CURA is piloted in both practice and education. This study is funded by the Dutch government (ZonMW/Palliantie)

Abstract number: P02-545

Abstract type: Poster

Private Health Insurance in Palliative Care: An Overview of the Portuguese Reality

Pinto S. ${ }^{1,2}$, Conceição A.P. ${ }^{1}$, Machado D. ${ }^{1}$, Simões C. ${ }^{1,3}$

${ }^{1}$ Escola Superior de Saúde de Santa Maria, Porto, Portugal, ${ }^{2}$ NursID, CINTESIS - Center for Health Technology and Services Research, Oporto, Portugal, ${ }^{3}$ Hospital Luz Arrábida, Oporto, Portugal

Background: In many countries Palliative Care (PC) is not completely integrated within the main-stream public health services. Yet, with the life expectancy increasement and the overcrowding of National Health 
Systems (NHS), new health financing options should be explored, particularly within the private sector.

Aims: To analyze private insurance health care plans regarding the practice of PC in Portugal.

Methods: Comprehensive search strategy of data published until September $30^{\text {th }} 2018$, on the website of the Portuguese Supervision Authority of Insurance and Pension Funds (P-ASF), with the search terms seguro de saúde AND cuidados paliativos. Eligibility criteria included the identification of insurance companies, health insurance plans and specific plans for PC. Motor insurance, road accidents and housing insurance were excluded. Data were systematically collected through an Excel grid, and independently analysed by 3 researchers.

Results: 73 insurance companies were registered in the P-ASF website, and 20 health insurance plans were found (life insurance premium mean=307.02€). Information regarding PC insurance plans is unclear, and often not included in key discourse. Preliminary analysis suggests that private insurance plans do not provide coverage for PC. Very few plans include some benefits for cancer patients. When included, only the basic health services are provided and are exclusive for home care, which means hospital costs are not included (eg: chemotherapy is not included).

Conclusion: Though the Portuguese Foundation Health Law considers health insurance an important activity complementary to the provision of public health care, private health insurance plans, as well policies to enhance different financial approaches in PC, are practically non-existent, which raises important ethical and moral issues. The market for private health insurance must follow the changes in the course of diseases, along with the reforms of the NHS, to ensure optimal PC.

Abstract number: P02-547

Abstract type: Poster

Are we Providing the Services Valued by Patients and their Carers: Preferences for the Breathlessness Support Service in England

Yi D. ${ }^{1}$, Jonston A. ${ }^{1}$, Burssens E. ${ }^{1,2}$, Teixeira M.J.C. ${ }^{1,2}$, Gao W. ${ }^{1}$, Reilly $C .{ }^{3}$, Marion D. ${ }^{1}$, Higginson I. $^{1}$

${ }^{1}$ Cicely Saunders Institute of Palliative Care, Policy \& Rehabilitation, King's College London, London, United Kingdom, ${ }^{2}$ King's College Hospital NHS Foundation Trust, London, United Kingdom, ${ }^{3}$ Department of Physiotherapy, King's College Hospital NHS Foundation Trust, London, United Kingdom

Background: Chronic breathlessness is a common symptom, impairs quality of life and often results in A\&E visits or hospital admissions. Breathlessness Support Service (BSS) was developed as a holistic intervention combining palliative and respiratory medicine to help patients and families at an earlier stage than usual.

Aims: To identify attributes of BSS valued by older patients ( $65>=)$ and families; and suggest models of BSS to be prioritised.

Methods: Face to face interviews among patients with COPD, lung cancer or ILD, and carers. Attributes and levels were determined from literature, expert consultation and interviews. 6 attributes (consultation; treatment review; additional support; expectation for mobility; expectation for health service use; time to get registered) were identified. 3 blocks of 6 questions, developed by the discrete choice experiment, were used at random. Regression was used to analyse the data.

Results: 190 patients and 68 carers were recruited from 9 sites in England. was All configurations of BSS were preferred to no service. BSS with a consultant was preferred but BSS with a GP or nurse at a GP clinic was not. BSS with additional support from a social worker was only preferred if combined with a therapist. Expectation for mobility or health service use were not important but review of medicinal treatments and waiting time were. Subgroup analysis of patients and carers found that a preference for BSS with home visits by a GP or nurse, with combined support from a therapist and social worker was stronger in carers. Patient's preference was stronger for the BSS with a consultant in an outpatient clinic.

Conclusion: Older patients and their carers want services provided by a consultant, reviewing medicinal treatments, with home visits by a therapist and support from a social worker. They are willing to wait longer for their preferred service. BSS based on the preferences of service users will increase the acceptance rate and improve outcomes.

Abstract number: P02-548

Abstract type: Poster

\section{Project "Opioid Price Watch (OPW)" - Trends and Differences in} Two Cohorts

Pastrana T. ${ }^{1,2}$, Wenk R. ${ }^{1,3}$, Radbruch L. ${ }^{1,4}$, De Lima L. ${ }^{1}$

${ }^{1}$ International Association for Hospice and Palliative Care, Houston, United States, ${ }^{2}$ Department of Palliative Medicine, University Hospital RWTH Aachen, Aachen, Germany, ${ }^{3}$ Federación Médica de la Provincia de Buenos Aires - FEMEBA, Buenos Aires, Argentina, ${ }^{4}$ Malteser Hospital Bonn/Rhein-Sieg, Bonn, Germany

Background: Strong opioids are a cornerstone of pain treatment. However, in many countries limited or no access still is a significant problem due to a range of barriers including restrictive laws and regulations on controlled essential medicines, lack of education, and high prices.

Aim: To provide information on access to pain treatment, as measured by the availability and accessibility of five strong opioids in 13 formulations, and the affordability of oral immediate-release (IR) morphine.

Method: A prospective study was implemented in countries in all income groups in 2013 and 2016 asking for information on the availability and price of the smallest selling unit and strength for 13 opioid formulations in the pharmacy located closest to a facility that provides diagnosis/treatment for life-threatening conditions. Countries represented in both cohorts were included in the study. An analysis was carried to determine trends and differences between both years.

Results: 12 countries were included (Bangladesh, Brazil, Guatemala, India, Kenya, Nepal, New Zealand, Nigeria, Norway, Poland, Uganda, and United Kingdom). The average availability of opioids and formulations increased slightly ( 1.9 to $2.3 ; p=0.218$ and 3.5 to $4 ; p=0.409$ respectively). The median price of morphine oral IR increased from 29.7 to 32.8 USD for 30 -Day treatment (Defined Daily Dose $=100 \mathrm{mg}$ oral morphine) $(p=0.878)$. The average affordability for 30 -Days treatment for minimum workers wage improved from 20.8 to 15.4 days $(p=0.578)$. In High Income Countries (HIC) substances as well as different formulations of strong opioids are more available compared to Low-Middle Income Countries (LMIC) $(p \leqslant 0.000 ; p \leqslant 0.000)$ and usually are available free of cost for patients.

Conclusions: Patients in LMIC have less access to opioid medications than patients in HIC. Efforts should be made by governments, health care administrators and pharmacists to ensure that opioids are available and affordable in local pharmacies.

Abstract number: P02-549

Abstract type: Poster

Disentangling Hope from Beliefs about Prognosis among Advanced Cancer Patients in Singapore

Finkelstein E. ${ }^{1,2}$, Malhotra C. ${ }^{1,2}$, Wong G.C. ${ }^{3}$, Volpp K. ${ }^{4}$, Lee L.H. ${ }^{3}$, Schweitzer M. ${ }^{4}$, Kanesvaran R. ${ }^{3}$, Cheung Y.B. ${ }^{5}$

${ }^{1}$ Health Services and Systems Research Program, Duke-NUS Medical School, Singapore, Singapore, ${ }^{2}$ Lien Centre for Palliative Care, Duke-NUS Medical School, Singapore, Singapore, ${ }^{3}$ Singapore General Hospital, Singapore, Singapore, ${ }^{4}$ University of Pennsylvania, Philadelphia, United States, ${ }^{5}$ Duke-NUS Medical School, Singapore, Singapore 
Background: Many international studies report that advanced cancer patients are overly optimistic about their prognosis. This is likely because many patients report not what they believe, but what they would like to believe (i.e., what they hope).

Aim: To use an incentive-compatible randomized trial design with 200 participants to discern patient's beliefs about their prognosis independent of hope in efforts to identify whether patients are truly overly optimistic about their prognosis. The incentive-compatible approach has been shown to be effective in increasing accuracy of responses in a wide range of domains, including mortality risk predictions. We hypothesise that although many participants will report an overly optimistic assessment of their prognosis, those responding to the incentive-compatible version will provide more accurate estimates.

Methods: Patients diagnosed with solid cancer, leukaemia or lymphoma and an expected prognosis of 1 year (as determined by their treating physicians) are recruited from outpatient clinics in Singapore and randomized to receive one of two survey versions. Both versions ask the participant whether they believe their illness is curable and their expected survival in years. However, in the incentive-compatible version participants are offered a financial incentive for answering 'correctly' (determined by their treating physician).

Results: Blinded review of interim data on $n=100$ shows that participants responding to one survey version answer more accurately than others when asked about whether their illness is curable $(p<0.10)$. We also find statistically significant differences in expected survival across the two arms $(p<0.10)$.

Conclusions: Due to blinding in this ongoing trial, we are unable to disclose which group reports a more accurate estimate of their prognosis until recruitment ends (April 2019) but the incentive compatible version appears to have an effect on expected survival and self-reported curability.

Abstract number: P02-550

Abstract type: Poster

Evaluating out of Pocket Health Expenditure: Collaboration with Patients and Policy Makers to Report on Palliative Care and Poverty in Low and Middle-income Countries

Bates M.J. ${ }^{1,2}$, Manthalu G. ${ }^{3}$, Masamba L. ${ }^{4,5}$, Tomeny E. ${ }^{6}$, Grant L. ${ }^{7}$, Squire $\overline{S . B .}{ }^{6}$, Niessen L. ${ }^{2}$

${ }^{1}$ School of Public Health and Family Medicine, College of Medicine, Blantyre, Malawi, ${ }^{2}$ Clinical Sciences, Liverpool School of Tropical Medicine, Liverpool, United Kingdom, ${ }^{3}$ Planning and Budgeting, Ministry of Health, Lilongwe, Malawi, ${ }^{4}$ Department of Medicine, College of Medicine, Blantyre, Malawi, ${ }^{5}$ Department of Medicine, Ministry of Health, Blantyre, Malawi, ${ }^{6}$ Collaboration for Applied Health Research and Delivery, Liverpool School of Tropical Medicine, Liverpool, United Kingdom, ${ }^{7}$ Department of Global Health, University of Edinburgh, Edinburgh, United Kingdom

Goal: Early evidence suggests that access to palliative care can reduce out of pocket expenditure (OOPE) though research tools and approaches are still under development in this area, particularly in low and middleincome countries (LMIC). The Patient and Carer Cancer Cost (PaCCCt) survey is being developed in Malawi for the collection of health-related expenditure and wellbeing data for households affected by advanced cancer.

Design: Palliative care users' priorities for household expenditure were extracted from data within a locally conducted Photovoice study exploring wellbeing for patients affected by advanced cancer. A literature search was conducted to identify studies reporting household expenditure in similar populations. The WHO TB patient cost survey, assessing catastrophic costs in LMICs was identified for adaptation. Content validity was tested through the involvement of Malawian palliative, oncology and home-based care nurses and reviewed at a national meeting of country stakeholders and experts.
Results: Questionnaires previously used were examined. Suitable metric components (income generation and the ability to keep dependents in school) have been added based on the priorities of palliative care users. A section on funeral-related expenditure is included. The EQ5D-3L, APCA African POS and VAS score will report wellbeing. An electronic version of the PaCcCt survey is to be piloted before use in a prospective cohort study in Blantyre, Malawi.

Lessons learned: Questionnaires which report on a comprehensive range of contextually relevant societal costs are required to explore associations between palliative care, wellbeing and poverty at household level in LMIC. The PaCCCt survey incorporates priorities of palliative care users. Early involvement of national stakeholders in survey development will facilitate evidence to policy pathways in Malawi, promoting access to palliative care as part of Universal Health Coverage.

Abstract number: P02-551

Abstract type: Poster

The Establishment of a Three-dimensional Service System for Hospice \& Palliative Care: A Stakeholder Theory Perspective Li Y.-G. ${ }^{1}$, Peng X.-F. ${ }^{1}$, Zhang Z. ${ }^{2,3}$

${ }^{1}$ Graduate School, Chinese Academy of Social Sciences, Beijing, China, ${ }^{2}$ Hospice Palliative Care Unit, Beijing Haidian Hospital, Beijing, China, ${ }^{3}$ Hospice Palliative Care Alliance of China Foundation, Eagleville, United States

Aim: The great demand caused by problems such as the aging and the high incidence of malignant diseases has formed a great contrast with the lagging hospice palliative care in China. From the perspective of the national pilot programs and studies the authors participated in, it is both imperative and urgent to accelerate the development of hospice palliative care and service system.

Methods: This article analyzes the current situation and problems of the development of the hospice palliative care in China, summarizes the research progress of the hospice system, and comprehensively sorts out the stakeholders of hospice palliative care for the first time, classifies them into eight categories and uses Mitchell scoring method identifies core stakeholders.

Results: On this basis, a three-dimensional service system for hospice palliative care was constructed: First, the framework of the three-dimensional service system for hospice palliative care was designed, and the horizontal and vertical relationships among various stakeholders were straightened out; Second, propose countermeasures to build a threedimensional service system for hospice palliative care, including propaganda and education, policies and regulations, quality management, talent supply, and information systems, etc.

Discussion: According to the combing and induction of the literature, this article believes that the current academic community lacks systematic research and in-depth analysis of the construction of hospice palliative care stakeholders and service system with inter-departmental mechanisms. There are few studies focusing on strategic level and micro-practice, and they are relatively scattered. There is a lack of deep research on the value consensus, concept, and universal model of collaboration, and lack of representative theory; especially at strategic heights to examine China. There are also few studies on the government inter-departmental mechanism construction under the big governments.

Abstract number: P02-552

Abstract type: Poster

Handover Information: A Complex Intervention Underpinned by a Process which Offers Care and Savings Beyond Information Tranferal

Garcia-Baquero Merino M.T. ${ }^{1,2,3}$, Abarshi E. ${ }^{3}$, Cooper H. ${ }^{2}$, Williams E. ${ }^{2}$, Bartlam K. ${ }^{2}$ 
${ }^{1}$ Facultad de Medicina, Universidad Catolica San Antonio de Murcia, Guadalupe. Murcia., Spain, ${ }^{2}$ Isabel Hospice, Welwyn Garden City, United Kingdom, ${ }^{3}$ Wisdom Hospice., Medway Community Healthcare, Rochester, United Kingdom

Background: Continuity of care is crucial for palliative care populations and an ever rising issue owing to working patterns. The impact on excellence of care and financial outcome can be significant. The concept of real time clinical information available to all professionals working from different work areas looking after same population on a rotational basis is a currently developing model, unsustainable when rigorous handover mechanism are not in place.

Aims: To identify what minimum information needs to be addressed when handing over care for a given period of time.

To establish possible advantage of on-line, real time recorded plan versus fixed one - off, printed or email data sheet

Methods: Retrospective analysis of one thousand ( $24 \%$ of all available) real time plans were compared with 1000 (85\% of those available) printed/ email, non-real-time handover documents from over 100 European centres. Outcomes were analysed with the help of a simple, comprehensive tool designed by a palliative care financial adviser.

Results: 7 variables were analysed including: number of healthcare professionals needed per time period, (up to $95 \%$ less inappropriate patient transfer (76\% per month per centre), medication rotation (up to $60 \%$ less), IPOS results (maintained or improver in 60\%), team engagement $(87 \%$ increase new entries in clinical records, complaints (down per centre per year up to $90 \%$ ) and gratitude showed significant better results when real-time, online information was accessible by all those involved.

They included cost savings between 15 and $74 \%$ per patient per year, even when IT development costs were included.

Conclusions: The application of IT advances to palliative care practice revolutionizes care making it available to larger populations because of the large cost savings to out of hours costs when fewer clinicians can care for larger populations.

Abstract number: P02-553

Abstract type: Poster

A Systematic Review of the Economic Costs of Gastrointestinal Consequences of Treatment for Cancer Patients and Healthcare Providers

Longo M. ${ }^{1}$, Weightman A. ${ }^{2}$, Muls A. ${ }^{3}$, Nelson A. ${ }^{1}$, Andreyev J. ${ }^{3}$, Staffurth J. ${ }^{4}$, Smith L. ${ }^{5}$, Mann M. ${ }^{1,2}$

${ }^{1}$ Cardiff University School of Medicine, Marie Curie Palliative Care Research Centre, Cardiff, United Kingdom, ${ }^{2}$ Cardiff University, Specialist Unit for Review Evidence (SURE), Cardiff, United Kingdom, ${ }^{3}$ The Royal Marsden NHS Foundation Trust, London, United Kingdom, ${ }^{4}$ School of Medicine, Cardiff University, Cardiff, United Kingdom, ${ }^{5}$ Macmillan Cancer Support, London, United Kingdom

Background: One in two patients treated with cancer is expected to survive 10 years after diagnosis. Research so far has focused on toxicities directly interfering with the delivery of treatment. Gastrointestinal (GI) toxicity can seriously impact functioning and quality of life: nearly 1 in 2 patients has chronic side effects (eg diarrhoea, faecal incontinence, abdominal pain) after pelvic radiotherapy. These reflect also an economic burden to patients, healthcare providers and society as a whole. We carried out a systematic review to evaluate the economic costs of GI consequences of treatment for cancer patients and healthcare providers.

Methodology: Databases were searched from 2006. Published and unpublished studies were included. The data abstraction followed the CRD guidelines. Quality assessment was carried out independently by two members of the team. Health economic data retrieved from the main paper and additional references if necessary (e.g. model-based study). Costs not expressed in Sterling Pounds were converted using the Purchasing Power Currency (PPP) exchange rate. All costs were inflated using the Bank of England calculator.

Results: 18 relevant studies were identified. The majority of studies were decision models and based in the USA. The study sample size ranged from 204 to 68,462 and the follow-up period varied from 6 weeks to 10 years following the completion of cancer treatment. Cost per episode of $\mathrm{GI}$ toxicity event ranged from $£ 345$ to $f 11,548$ whereas cost per patient varied from $£ 1,606$ to $£ 120,440$ (following non-fatal intestinal perforation)

Conclusions: Evidence is limited, nonetheless it offers a good sense of the burden on the NHS/third party payer perspective. Hospitalization was the cost driver, however, the costs falling on the primary care sector on the patients and to the society were not included in any of the studies. Policy should aim to improve patients' awareness of cancer treatment long term adverse events.

Abstract number: P02-554

Abstract type: Poster

What Are the National Development Contexts in which

Palliative Care Services Have (and Have Not) Developed? A

Worldwide Ecological Study

Clark J. ${ }^{1}$, Barnes A. ${ }^{2}$, Campbell M. ${ }^{2}$, Gardiner C. ${ }^{3}$

${ }^{1}$ Wolfson Palliative Care Research Centre, Hull York Medical School, Hull, United Kingdom, ${ }^{2}$ School of Health and Related Research, University of Sheffield, Sheffield, United Kingdom, ${ }^{3}$ The School of Nursing \& Midwifery, University of Sheffield, Sheffield, United Kingdom

Background: Advocates for Palliative Care (PC) argue that service implementation is feasible in all settings. Yet, services have developed patchily in low and middle-income settings. Beyond Human Development Index indicators, little thought has been given to the broader development challenges facing nations tasked with implementing PC.

Aim: To describe how indicators of national development relate to levels of PC services in nations around the world.

Methods: An ecological study to identify relationships between potential predictor variables and countries' level of PC development. 28 predictor variables from 6 domains were selected using hypothesised relationships with levels of PC development: disease demographics, socioeconomics, health systems, politics, demographics and economics. The outcome variable was level of national PC development on a six-point scale. Spearman's correlation test was used, significance level $<0.05$.

Results: $26 / 28$ variables were significantly associated with levels of PC development in 207 countries. PC is more developed in countries with high: percentage of deaths from non-communicable disease, population proportion aged $65+$, gross national income and tourism. Development is lower in countries with high levels of: political corruption, infant mortality, deaths by infectious disease and weak democracy. Prevalence of undernourishment and levels of private health expenditure were not associated with PC development.

Discussion: PC development is highly consistent with broader national development indicators. Development is less in countries where acute deaths are more likely and any benefits from PC provision, short-term. In such countries, resources may be prioritised towards life prolonging therapies and key aspects of PC need only, prior to fully integrated palliative services. Calls for accelerated development of PC services must be informed by in-depth understanding of national development contexts, local needs and opportunity costs.

Abstract number: P02-555

Abstract type: Poster 
Addressing the Needs of Terminally-ill Patients in BosniaHerzogovina: Patients' Perceptions and Expectations Pautex S. ${ }^{1}$, Aebischer Perone S. ${ }^{2}$, Lab B. ${ }^{2}$, Lachat S. ${ }^{2}$, Vogel T. ${ }^{2}$, Matis $C .{ }^{1}$, Dropic E. ${ }^{3}$, Lazic R. ${ }^{4}$, Radoslav N. ${ }^{4}$, Chappuis F. ${ }^{2}$

${ }^{1}$ Rehabilitation and Geriatrics, Division of Palliative Medicine, CollongeBellerive, Switzerland, '2Division of Tropical and Humanitarian Medicine, Geneva, Switzerland, ${ }^{3}$ Fondacija Fami, Doboj, Bosnia and Herzegovina, ${ }^{4}$ Domzdravlja, Doboj, Bosnia and Herzegovina

Background: Many terminally ill patients in Bosnia-Herzegovina (BiH) fail to receive needed medical attention and social support. To address the needs of terminally ill patients living at home and in order to design some acceptable, affordable and sustainable solutions, we involved patients and their families in exploring needs, existing barriers and available resources.

Methods: Semi-structured interviews with 62 purposively selected patients living at home in $\mathrm{BiH}$. Both qualitative and quantitative analyses were conducted, using an inductive thematic approach.

Results: While patients were aware that their illnesses were incurable, they were poorly informed about medical and social support resources available to them. Family members appeared to be patients' main source of support, and often suffered exhaustion and financial strain. Patients expressed feelings of helplessness and a lack of control - both for them and for their caregivers - over their health, and wanted health professionals to help more with pain control and other symptoms management as well as with anxiety and depression. Patients who were bedridden or with reduced mobility expressed strong feelings of loneliness, social exclusion, and stigma from community members and - occasionally - from health workers.

Conclusions: Our findings suggest a wide gap between patients' end-oflife care needs and existing services. In order to address the medical, psychological and social needs of terminally ill patients, a multi-pronged approach is called for, including not only better symptom management through training of health professionals and improved access to medication and equipment, but also a coordinated inter-professional, inter-institutional and multi-stakeholder effort aimed at offering comprehensive psycho-social, educational and spiritual support.

Abstract number: P02-556

Abstract type: Poster

The Changing Landscape of Palliative Care Research in Ireland from 2002 to 2017: A Comparison of Two Systematic Reviews within a Global Context

Mcllfatrick S. ${ }^{1}$, Muldrew D. ${ }^{1}$, Hasson $F^{1}{ }^{1}$, Payne S. ${ }^{2}$

${ }^{1}$ Institute of Nursing and Health Research, Ulster University, Newtownabbey, United Kingdom, ${ }^{2}$ International Observatory on End of Life Care, Lancaster University, Lancaster, United Kingdom

Background: Whilst there have been significant developments in palliative care in recent years, a lack of global information pertaining to palliative care research capacity has led to individual countries seeking to assess their own state of play.

Aim: To examine how palliative care research in Ireland has changed within an international context.

Methods: The findings from two systematic reviews conducted in Ireland in 2012 and 2017 were compared to see what impact additional investment in palliative care research has made to the state of play. A further comparison was undertaken against systematic reviews from Scotland, Sweden, and south Asia to map palliative care research capacity in Ireland globally.

Results: Whilst research outputs almost doubled in Ireland from 2012 ( $n=151$ over 10 years) to 2017 ( $n=151$ over five years), many were still experiential and needs based, in line with Scotland, Sweden, and south Asia. Only one trial was noted in Ireland per review, compared to 11 in
Scotland, ten in Sweden, and four in south Asia. Despite a majority of relatively small-scale studies, systematic reviews increased in popularity from $3 \%(2012)$ to $17 \%$ (2017). Collaborative working is evident $(66 \%$ showed national or international collaboration in Ireland) despite a large percentage of unfunded research across all reviews. Research gaps include out of hours' care and psychological symptom control, with over reliance on healthcare professionals' perspectives.

Conclusions: Whilst overall palliative care research has increased, these findings, in accordance with the international reviews, indicates that further progress is required. Key areas for more research are continuity of care and non-physical symptom control. Research should include patients and family perspectives, and use trial methodology. Additional funding for large scale, international collaborative projects would substantially advance the palliative care research field.

This work received no funding.

Abstract number: P02-557

Abstract type: Poster

Realising the Vision: Striving to Achieve the Best Care for a Dying Person through International Collaboration in Research, Education, Training, and Quality Assurance Processes Supporting an Individual's Plan for Care

Boughey M. ${ }^{1}$, Ellershaw J. ${ }^{2}$, Wilkinson S. ${ }^{2}$

${ }^{1}$ St Vincent's Hospital Melbourne Palliative Care Services, Centre for Palliative Care, Fitzroy, Australia, ${ }^{2}$ Palliative Care Institute, University of Liverpool, Liverpool, United Kingdom

Can you envision a world 'where all people experience a good death, as an integral part of their individual life, supported by the very best personalised care'? The International Collaborative for Best Care for the Dying Person (the Collaborative) does.

Established in 2014, and currently globally representing 21 countries, the Collaborative strives to drive forward the vision of best care for all dying patients. Through the work of its leading practitioners, researchers, educators and facilitators to achieve this goal, the Collaborative has established a framework to articulate the values, strategic aims, obligations and quality indicators across the international clinical community in caring for a dying person; developed an international core 'clinical care for the dying person guidance document', with materials for implementation and auditing within a 5 year Quality Assurance Cycle; created an agenda to undertake an international 'care for the dying' research; created a facilitated program of international education and training collaborations; and developed and participated in the societal debate about death and care for the dying and in society.

In realising the vision, twenty project working groups have been created, foundation courses have been provided for organization developing a Care for the Dying program and funding has been secured from two significant research calls from the European Union Horizon 2020 call to inform the development of sustainable mechanisms for continuous improvement in care for the dying and the European Research Area (ERA-NET) Latin American and Caribbean call to conduct a multi centred trial to evaluate the International Care of the Dying Evaluation (CODE) questionnaire.

Sharing the key steps and building blocks, of this foundational work, will demonstrate the ability of international collaborators, across a variety of jurisdictions, to achieve embedded, sustainable and quality care for the dying person.

Abstract number: P02-558

Abstract type: Poster

Cross-cultural Evaluation of the Quality of Dying and Death Questionnaire with Caregivers of Advanced Cancer Patients in Kenya 
Chalklin L. ${ }^{1}$, Mah K. ${ }^{1}$, Powell R. ${ }^{2}$, Malfitano C. ${ }^{1}$, Gikaara N. ${ }^{2}$, Hales S. ${ }^{3}$, Rydall A. ${ }^{1}$, Zimmermann C. ${ }^{1}$, Mwangi-Powell F. ${ }^{2}$, Rodin G. ${ }^{1}$

${ }^{1}$ Supportive Care, Princess Margaret Cancer Centre, Toronto, Canada, ${ }^{2}$ MWAPO Health Development Group, Nairobi, Kenya, ${ }^{3}$ Princess Margaret Cancer Centre, Toronto, Canada

Background: Access to palliative care (PC) is of growing global concern, but PC services reach less than $5 \%$ of those in need in Africa. Locally validated patient-centered outcome measures are needed to assess and improve PC services in Africa.

Aim: The 31-item Quality of Dying and Death (QODD) questionnaire, the most widely employed measure of experiences of concerns in the quality of dying and death, was evaluated with bereaved caregivers of patients with advanced cancer who received care at Kenyan hospices.

Methods: 127 bereaved caregivers completed translated versions of the QODD and FAMCARE measure of family satisfaction with care 2-12 months after patient death. We compared item ratings to 602 Ontario caregivers and examined the relationships between item ratings and overall quality of dying and death ratings and the FAMCARE. Alpha was set at 01 .

Results: Kenyan caregivers showed worse experiences than Ontario caregivers on 14 QODD items, including pain control/functioning, treatment preference, and moment of death items, $p^{\prime} s \leqslant .001$; better experiences on 5 interpersonal, religious, and personal items, $p$ 's $\leqslant .005$; and no differences on 12 interpersonal, preparatory, and symptom items, $p^{\prime} s>.01$. Quality of dying and family satisfaction were better with enhanced experiences with symptom control/functioning and interpersonal concerns. Quality of dying was worse with better experiences with visits from a religious advisor, $p^{\prime} s<.01$. Better quality of death was related to better experiences with location of death and state of consciousness before death, $p^{\prime} s<.01$. Substantial missing data were evident across mostly treatment preference, preparatory, and moment of death items.

Conclusion: The QODD detected cultural differences in quality of dying and death but requires further evaluation of the cultural relevance of some items to African concepts of "a good death." With revisions and further validation, the QODD may beneficially inform quality improvement of PC in Africa.

\section{Abstract number: P02-559 \\ Abstract type: Poster}

\section{General Public Awareness, Knowledge and Beliefs toward Palliative Care in a Saudi Population \\ Alkhudairi $\mathrm{H}$. \\ King Saud Univerity, Oncology Center, Riyadh, Saudi Arabia}

Objectives: Public awareness of palliative care (PC) is satisfactory in only twenty countries in 2013, which does not include Saudi Arabia in spite of the global efforts done to enhance the public awareness of PC. This study was conducted to evaluate the awareness, knowledge, and beliefs of the Saudi adult population about PC.

Methods: A cross-sectional design study was conducted in December 2017 using a self-administered questionnaire survey that was sent through Google Docs to assess the Saudi general public on the awareness of PC.

Results: There were 1987 (out of 3164, 62.8\% response rate) Saudi adults who responded to the survey, in which $60.3 \%$ were males (mean age: 39.50 years, median: 39.0 years, and range: $15-77$ years). There were $321(16.2 \%)$ respondents who reported that they know PC and 454 $(22.8 \%)$ answered that they have heard or were aware of PC. A total of 755 (38.2\%) believe that PC improves the quality of life of sick people and $684(34.4 \%)$ believe that PC can reduce the physical suffering of patients. Knowledge of PC was not statistically significantly affected by gender, place of residence, and marital status. On the other hand, employment and having higher levels of education led to better knowledge and more awareness of PC.

Conclusion: The current study demonstrated that the knowledge and awareness of PC is still low among surveyed Saudis. There is a need for the government to advocate institutions to functionally devote programs and initiatives and promote literacy of PC in the general population. Nongovernmental sectors should also participate in programs to improve the knowledge and awareness of PC.

Abstract number: P02-560

Abstract type: Poster

Initiation of Perinatal Palliative (PeriPal) Service in a Tertiary Women's and Children's Hospital in Singapore

Tewani K.1,2

${ }^{1}$ KK Women's and Children's Hospital, Palliative Medicine Dept of Gynaecological Oncology, Singapore, Singapore, ²Duke-Nus, OBGYN ACP, Singapre, Singapore Presenting author email address: komal. tewani@singhealth.com.sg

Background: Perinatal Palliative Care is comprehensive and holistic care for expectant parents who receive a diagnosis of life-limiting fetal condition (LLFC). Kandang Karbau Hospital (KKH), Singapore, sees more than 10,000 deliveries in a year and is a regional referral center for high risk and complex obstetrics cases. In view of the relatively large number of complex cases seen, a multidisciplinary team lead by a palliative specialist to provide perinatal palliative care was initiated in Jan 2017.

Aims: Primary aim was to provide, in addition to standard medical care, individually tailored holistic care during pregnancy, labor, birth, postnatal and bereavement period. Secondary aim was to have Advance Care Plans (ACP) including birth plans made before delivery and communicated clearly to various teams involved.

Method: In the aftermath of the Neonatal Palliative Symposium held in Singapore, a Perinatal Palliative (PeriPal) team lead by palliative specialist and comprising of high risk obstetricians, neonatologist, nurses, medical social worker was set up. A list of 'lethal' fetal conditions that would qualify for referral to PeriPal team was put together and during Birth Defect Clinic (BDC) these cases were highlighted to the PeriPal team. A meeting of parents with the team is fixed subsequently with the aim of introducing the team and discussing parents understanding, decision making process, answering questions and initiating discussion about care plans. In the next consult the care plans are finalized and documented. After delivery, neonatal palliative care is delivered.

Conclusion: Clear workflow in Birth defect clinic results in adequate pickup and appropriate referral. ACP is ready and communicated for most PeriPal cases before delivery making neonatal care in accordance to pre determined goals of care. The workflow still needs to be tweaked to ensure appropriate care for emergency cases Eg Abruptio placenta and resultant severe fetal injury.

Abstract number: P02-561

Abstract type: Poster

Evaluation of Nurse Prescribing in Palliative Care in Uganda: Shaping the Future for Global Palliative Care Downing J. 1,2,3, Acuda W. ${ }^{4}$, Adong D. ${ }^{4}$, Luyirika E. ${ }^{5}$, Namisango E. ${ }^{5}$, Kiyange F. ${ }^{5}$, Kiwanuka R. ${ }^{6}$, Amandua J.7, Grant L. ${ }^{2}$, Leng M.2,8,9,

Evaluation Study Team

${ }^{1}$ Makerere and Mulago Palliative Care Unit, Makerere University, Kampala, Uganda, ${ }^{2}$ Global Health Academy, University of Edinburgh, Edinburgh, United Kingdom, 3International Children's Palliative Care Network (ICPCN), Durban, South Africa, ${ }^{4}$ Hospice Africa Uganda, Kampala, Uganda, ${ }^{5}$ African Palliative Care Association, Kampala, Uganda, ${ }^{6}$ Palliative Care Association of Uganda, Kampala, Uganda, ${ }^{7}$ Ministry of Health, Kampala, Uganda, ${ }^{8}$ Makerere and Mulago Palliative 
Care Unit, Kampala, Uganda, ${ }^{9}$ Cairdeas International Palliative Care Trust, Edinburgh, United Kingdom

Background: The WHA Resolution calls for the integration of palliative care $(P C)$ into the health system, thus ensuring access to pain \& symptom management for all. Uganda has led the way through PC trained nurses prescribing medications including oral morphine. However, no evaluation had been undertaken since its inception in 2004.

Aims: To determine the effectiveness of nurse prescribing in PC in Uganda.

Methods: A 3-phase mixed-methods evaluation examining:

1) training for nurse prescribing;

2) analysis of nurse competency to assess \& manage pain, and appropriately prescribe;

3) appraisal of the health-care system in 10 districts to understand the context within which nurses are working.

Initially 13 nurses, 4 trainers \& $1 \mathrm{MoH}$ colleague were interviewed, core competencies for prescribing agreed \& curriculum review undertaken. 22 nurses managed 20 patients each, assessing \& managing their pain \& other symptoms, using the APCA POS on 3 sequential visits. Ethical approval was gained from HAUREC/UNCST.

Results: Results show nurses can assess \& manage pain, including prescribing oral morphine.

1) Themes impacting nurse preparation included: training, supervision \& mentorship, competency, boundaries, beliefs \& system issues. The current curriculum, with minor adaptions, is 'fit-for-purpose'.

2) Nurses can assess \& manage pain $(p<0.001)$ utilising appropriate medications $\&$ requesting support as patients required.

3) Nurses work in a system where: access to medications varies; there is limited understanding of PC \& myths persist about the use of morphine. However they are resilient \& able to overcome challenges.

Conclusion: Training PC nurses to prescribe medications in order to increase access to PC is an important transferable model. Whilstchallenges exist \& recommendations are made to improve the system, this evaluation demonstrates the benefit \& safety of nurse prescribing for PC in Uganda. This has important implications for shaping the future of global PC.

Abstract number: P02-562

Abstract type: Poster

The International Primary Palliative Care Network - Promoting Palliative Care in Primary Health Care for Universal Health Coverage

Barnard A. ${ }^{1}$, Moine S. ${ }^{2}$, Murray S. ${ }^{2}$, Mitchell G. ${ }^{3}$

${ }^{1}$ University of Cape Town, Cape Town, South Africa, ${ }^{2}$ University of Edinburgh, Edinburgh, United Kingdom, ${ }^{3}$ University of Queensland, Brisbane, Australia

This international network was established in 2005 to support and develop palliative care in all primary care contexts across the world. It advocates early integrated palliative care, for all diseases, in all locations and in all parts of the world.

The vision of the IPPCN is to contribute to comprehensive universal health coverage by empowering individuals working in different health systems to deliver primary palliative care, having identified those who need it early in the trajectory of the illness and ensuring optimal pain and symptom control and comprehensive planned care.

The group has members on all five continents and helps to develop collaborative clinical, advocacy and educational activities. Collaborative research in the field of primary palliative care conducted by members of the network has contributed to the evidence base of primary palliative care internationally.

The IPPCN has developed relationships with WONCA (The World Organisation of Family Doctors) and members helped form the EAPC Task Force and Reference Group in Primary Care. It has contributed in recent WHO publications about integrated and primary palliative care. It successfully advocated for palliative care to be a core component of primary health care, and this vision and commitment is included in the Astana Declaration.

This poster sketches the history of the IPPCN and illustrates its research and education output with examples from the collaborative work of members. The networking function is illustrated by accounts of conference presentations and academic visits clinicians and researchers. The group has shown through collaborative advocacy and teaching that palliative care can be integrated with curative care in hospital, care home and primary care settings for patients with all life-threatening illnesses, early in the course of the illness.

Abstract number: P02-563

Abstract type: Poster

Development of the Evidence Base for Palliative Care in Low and Middle Income Countries: Bibliometric Study (1999-2018)

Sleeman K.E., Leniz J., De Brito M., Nkhoma K., Guo P., Namisango E., Harding $R$.

Cicely Saunders Institute of Palliative Care, Polity and Rehabilitation, King's College London, London, United Kingdom

Introduction: Reducing end of life suffering in Low and Middle Income Countries (LMICs) relies on effective policies, and these require evidence that is both high quality and setting-appropriate. Our aim was to measure development of the evidence base for palliative care in LMICs.

Methods: Observational bibliometric study. We used Web of Science to identify palliative care literature using key terms (hospice* or "palliative care" or "terminally ill" or "terminal care" or "end-of-life care"), from 1999 to 2018. LMIC publications were identified by searching author affiliations, using World Bank Income Classifications (2018). We extracted information on publication year, countries and organizations to which authors were affiliated. For a random subsample of 500 publications that involved LMIC authors, we extracted information on research setting, methods, collaborations and funding.

Results: Of 27,752 palliative care publications, 2,183 (7.9\%) included at least one author affiliated to a LMIC. There were eight times as many publications with a LMIC author during the decade 2009-2018 compared to $1999-2008$, and LMIC-author publications accounted for $1.5 \%$ of all publications in 1998, rising to $11.5 \%$ in 2017. Of a random sample of 500 LMIC publications, 284 (56.8\%) presented primary data, $76(15.2 \%)$ were opinion pieces, 32 (6.4\%) were systematic reviews and 108 (21.6\%) were 'other'. 247 papers (49.4\%) presented data collected in a LMIC. $89.4 \%$ of papers had a LMIC author as either first or last author.

Discussion: It is sobering that while $80 \%$ of people dying with Serious Health Related Suffering live in LMICs, just 7.9\% of palliative care articles include a LMIC-affiliated author, and of these fewer than half $(49.4 \%)$ present primary data collected in LMIC settings. The WHA resolution on palliative care calls for high quality locally-relevant evidence to support public health strategies. This is urgently needed for equitable and efficiently delivered health services.

Abstract number: P02-564

Abstract type: Poster

French National Platform for End-of-Life Research: A First

Overview of Research Forces in France

Cretin E. ${ }^{1,2}$, Baudry P. ${ }^{3}$, Aupet S. ${ }^{1}$, Aubry R. ${ }^{2}$ 
1 University Bourgogne Franche-Comté, Besançon, France, 2University Hospital of Besançon - Clinical Investigation Center, Insem 1431, Besançon, France, ${ }^{3}$ University Bordeaux Montaigne, Bordeaux, France

In October 2018, the French national Platform for end-of-life (EOL) research was launched at the French Ministry of Research, in Paris. As part of the national plan for development of palliative care, the Platform aims to foster collaborations between different academic disciplines, support and promote research on EOL issues in France.

So far, the Platform has identified and connects 66 research teams - from empirical biomedical research to theoretical research in philosophy who declared their interest in the actions of the Platform. These actions will focus on 3 mains key levers:

- Know: identify and index research teams, researchers and projects. Create a national directory and therefore a research network to facilitate interdisciplinary interfaces.

- Support: provide resources, training, and scientific events to improve the quality of research, support international collaborations and encourage emerging projects.

- Promote: initiate strategic actions to foster the implementation of specific calls for end-of-life research in France and disseminate results among both the scientific community and the general public.

A national survey will be conducted from January to April 2019, based on already identified teams and following a snowball sampling scheme. The survey will analyze the teams academic affiliations, research areas, types of methods used, types of funding, geographic locations, and existing international collaborations.

The results of this survey will be presented: participants will be able to visualize how many French teams are involved in EOL research in France, their research areas and methods. This map will provide a first scope of French research forces, as well as research areas that still need to be investigated or strengthened.

Funding: French Ministry of Higher Education, Research, and Innovation, French Ministry of Health, and Fondation de France.

Abstract number: P02-565

Abstract type: Poster

Providing Palliative Care in Ecuador: Development, Current Status and Areas of Improvement from the Perspective of Five Ecuadorian Palliative Care Pioneers

Suarez D., Orellana-Rios C.L., Becker G.

Clinic for Palliative Care, Medical Center, University of Freiburg, Faculty of Medicine, University of Freiburg, Freiburg, Germany

Background/Aim: The development of palliative care in Ecuador remains in early stages. There is a lack of studies exploring its areas of improvement and future perspectives. Furthermore an analysis of the cultural and social characteristics of Ecuador is necessary to adapt existing guidelines for palliative care to the reality of Latin American countries. This study aimed at closing this gap by exploring the perspectives and experiences of key Ecuadorian professionals involved in this field.

Methods: A qualitative research approach involving semi-structured interviews was carried out with five palliative care pioneers from different care settings in Ecuador. In order to obtain a systematic classification of the topics mentioned, the verbatim-transcribed interviews were analyzed through qualitative content analysis.
Results: Two main themes were identified: palliative care development in Ecuador and characteristics of the Ecuadorian society regarding palliative care. Within the first theme, results showed that the beginning of palliative care in Ecuador was only possible thanks to non-governmental actors. The main deficiencies of local palliative care structures are the lack of knowledge and the precarious health system. Experts emphasized the need for an implementation of palliative care within the health care system and therefore the urgency of local training options. The second theme refers to the social and cultural aspects to be considered in order to plan and to develop palliative care structures in Ecuador. Hereby, the importance of family and community networks as well as the attachment to religion stood out. Conclusions: This study provides important knowledge, not only about the development and current status of palliative care in Ecuador, but also about aspects to be developed in the future such as training options or the awareness of society. These results represent a starting point for future in-depth investigations regarding these topics.

Abstract number: P02-566

Abstract type: Poster

What Are the Best Indicators to Assess National-level Development of Palliative Care in Europe? Results of an International Delphi Consensus Process

Arias N. ${ }^{1}$, Garralda E. ${ }^{2}$, López-Fidalgo J. ${ }^{3}$, Rhee J.Y. ${ }^{4}$, de Lima L. ${ }^{5}$, Centeno C. ${ }^{2}$

${ }^{1}$ Universidad de Navarra, Instituto Cultura y Sociedad, Pamplona, Spain, ${ }^{2}$ Institute for Culture and Society, Universidad de Navarra, Instituto Cultura y Sociedad, Pamplona, Spain, ${ }^{3}$ Institute for Culture and Society, Úniversidad de Navarra, Pamplona, Spain, ${ }^{4}$ Icahn School at Mount Sinai, New York, United States, ${ }^{5}$ International Association for Hospice and Palliative Care, Houston, United States

Background: Specific indicators to accurately assess the national-level development of PC in Europe are needed.

Aim: To identify the indicators that better assess national-level PC development in Europe.

Methods: Indicators retrieved from a detailed systematic review on PC development macro-indicators used for cross-national comparison in global studies were rated (1-9) by relevance, measurability and feasibility in a two-round RAND/UCLA Delphi with international experts in PC development. These parameters were used to calculate a Global Score. Indicators scoring over $7 / 9$ proceeded to next round. In a second round, experts rated indicators by Global Score (1-9). Indicators scoring Median $\geqslant 7 / 9$, a lower limit $95 \%$ IC $\geqslant 7$, Content Validity Index $\geqslant 0.30$ and Disagreement Index $\leqslant 0.30$ were selected as the best indicators. Results: 24 experts rated 45 indicators. 33/45 scoring over 7/9 Global Score proceeded to a second round. Lastly, 25 indicators fulfilled the inclusion criteria and were selected as the best indicators. Policy indicators $(n=8)$ included existence of national law, policies, standards and norms; inclusion of PC in the list of service of the national health systems and designated human resource in the department of health for PC. Education indicators $(n=4)$ focused on processes of official specialisation for physicians, inclusion of mandatory teaching at the undergraduate level and PC professorship. Medicines indicators $(n=4)$ consisted in opioids consumption, availability and prescription requirements. Services indicators $(n=6)$ included number and type of services for adults and children. Additional indicators $(n=3)$ for professional activity were identified. Conclusion: A Delphi consensus process has identified 25 indicators as the most suitable for the assessment of national-level PC development, representing all domains of the WHO Public Health Strategy. 


\section{Author Index}

Aabom B.

Aass N.

Abarshi E.

Abas M.

Abboah-Offei M.

Abbott U.

Abdelhafeez A.

Abdelrazeq $\mathrm{H}$.

Abel Aal $\mathrm{H}$.

Abraham C.

Abreu K.

Abu-Saad Huijer $\mathrm{H}$.

Achette D.

Achterberg W.

Acquah D.

Acuda W.

Adamidis $\mathrm{F}$.

Adams N.

Adamson $\mathrm{G}$.

Adang E.M.M.

Addo Opare-Lokko E.

Adlitzer $\mathrm{H}$.

Adong D.

Adra M.

Adsersen M.

Aebischer Perone S.

Aertsen M.

Affolter B.

Afrin $\mathrm{S}$.

Agapinaki E.

Agar M.

Agbana S.

Aggarwal G.

Ahlborn M.

Ahmad Ainuddin $\mathrm{H}$.

Ahn H.-Y.

Ahrenholz M.

Akande $\mathrm{S}$.

Akers $\mathrm{E}$.

Akhter $\mathrm{F}$.

Akhter R.

Akita K.

Akyea R.

Al Tabba' A.

Alam S.

Al-Awamer A.

Albarracin D.

Albu S.

Aldawagreh A.

Alex Pellegrin M.

Ali $\mathrm{H}$.

Ali M.

Ali N.

Alinon K.N.

Alkharabsheh A.

Alkhudairi $\mathrm{H}$.

Allão $C$.

Allcroft P.

Allende-Pérez $S$.

Allgar V.

Allongham $\mathrm{S}$.

Alluin $\mathrm{R}$.

Almack K.

Almansa Gimeno M.

Almaraz M.J.

Alme T.

Almeida R.

Alnes R.E.

Alonso Ruiz M.T.
Alonso-Babarro A.

Alsirafy $\mathrm{S}$.

Al-Tarawneh M.

P02-037 Alt-Epping B.

FC 16 Althaus B.

P02-552 Altini M.

P02-374 Alvarez C.

P02-374 Álvarez M.

P02-209 Alvariza A

P02-118, P02-172

PS 17 Alvaro R.

P02-172 Amanda F.

P02-294 Amandua J.

P01-495, P02-421, P02-422 Amar S.

P02-179 Amoakwa E.

P01-019 Amorim C.

FC 10, P01-537, P02-217, P02-199 Anagnostou D.

P02-412 Ancuta C.

P02-561 Andersag M.

P02-289 Andersen M.F.

P02-046 Anderson A.

P02-048 Anderson A.-K.

FC 23 Anderson $\mathrm{R}$.

P02-313 Anderson S.

P01-454 Andreyev J.

P02-561 Andriishyn L.

P02-179 Anestis E.

FC 4, FC 12 Anjos C.

P02-555 Anllo H.

P01-435 Anne P.

P01-568 Annemans L.

P01-241 Anneser J.

P02-314 Anota A.

PS 8, FC 2, FC 4, FC 9, FC 22, ME 1 ,

P01-242, P01-243, P01-483, P02-216

P01-252, P02-431

P02-262, P02-433

FC 16

FC 3

P01-368

P01-402

P02-089

P01-044

P01-241

P01-241

P01-462, P02-154

P02-131

PS 17

P01-481, P02-401

P01-207

P02-006

P02-512, P02-515

P01-161

FC 18

P02-234

P02-346

P01-458, P02-267

P02-386

P01-161

P02-559

P01-451

P01-465

P01-408

FC 8

FC 2

P02-268

P01-260, P01-343

P02-014

FC 8

P01-304

P02-330

P01-304

P02-328, P02-329
Ansa G.

Antiñolo F.

Antunes B.

Anyane $\mathrm{G}$.

Aoun S.

Aoyama M.

Aparecida Caliente E.

Aparicio A.I.

Aparicio M.

Apondo E.

Appleton L.

Aradilla-Herrero A.

Aragão $\mathrm{E}$.

Arantzamendi M.

Araújo J.

Araujo Hernández M.

Archer S.

Årestedt K.

Arias $\mathrm{M}$

Arias N.

Ariyoshi K.

Arkin S.

Armstrong $\mathrm{M}$.

Armstrong Wilson J.

Arnaud J.

Arora K.

Arquez Pianetta M.

Arraiza B.

Arraras-Torrelles $\mathrm{N}$.

Arriaga I.

Arrizabalaga M.J.

Arrue B.

Arshinoff R.

Aruga $\mathrm{E}$.

Arumugam $J$.

Ashby J.

Ashton G.

Ashton W.

Asiimwe Buhiire M.

Atallah C.
$\mathrm{P} 02-220$

P02-118, P02-172

PS 17

FC 17, FC 16, P02-536

P01-414

P01-233

$\mathrm{P} 02-344$

P01-157, P01-400

FC 13, FC 25, P01-148, P01-191, P01-244, P01-467, P02-053, P02-189, P02-194

P02-438

FC 8

P02-561

P01-397

FC 8, P02-366

P01-494

P01-114, P02-224, P02-252

P02-227, P02-497

P02-200

P01-299

P01-105, P02-283

P01-034, P02-204

P01-098, P02-264

$\mathrm{P} 02-417$

P02-553

P01-115

P01-563

P01-532

FC 3

P01-090

FC 2, P01-335

P02-530

FC 16, P02-051

P02-374

P01-468

FC 21, P01-039

$\mathrm{P} 02-367$

FC 11, P02-056

FC 11

P02-121

P02-102

P02-169

P01-089

P01-429, P01-430

P02-235

P01-538

FC 4, P01-121, P02-007, P02-246 P02-533

P01-268, P01-549, P01-550 P01-189

FC 25, P01-148, P01-467, P02-194

FC 7, P01-079, P02-176

P01-339, P02-566

P02-096

P01-153

P02-126

P02-322, P02-323, P02-324

P01-442

P02-475

$\mathrm{P} 02-094$

$\mathrm{P} 02-310$

P02-084, P02-087, P02-088

P01-444

P01-444

P01-286

FC 19, P01-133, P01-457

P02-072, P02-073

P02-311

FC 21

P01-009

P01-388

P01-150

P02-172 
Ateş $\mathrm{G}$

Atout M.

Atsuda K.

Attala M.

Aubry R.

August $\mathrm{H}$.

Aujoulat I.

Aulmann C.

Aupet S.

Auschra C.

Autelitano C.

Avery A.

Avgetidou $\mathrm{H}$.

Axelsson L.

Aydinli B.

Ayre D.

Ayre R.

Ayres B.

Azab R.

Azenha S.

Bętkowska I.

B. Mikkelsen T.

Baastrup Jørgensen M.

Badim S.

Baer K.

Bagasha P.

Bagchus C.

Bahloul S.

Bailey C.

Bailey F.A.

Bailey K.

Bailey S.

Bajwah S.

Baker I.

Baker-Schuster L.

Baks N.

Balaguer A.

Balan A.

Balboni C.

Balfe J.

Ballentine J.

Balmer D.

Banerjee S.

Banse C.

Banting M.

Bar M.

Barallat-Gimeno E.

Barata J.

Barbaret C.

Barbato M.

Barbero E.

Barbette C.

Barbour L.

Barclay D.

Barclay G.

Barclay S.

Bardenheuer $\mathrm{H}$.

Bargfeldt $\mathrm{E}$.

Barker F.

Barnard A.

Barnes A.

Barnes D.E.

Barnes $\mathrm{H}$.

Barnett $\mathrm{M}$.

Barone G.

Barra F.

Barrios P.

Barrioso P.D.C.

Barros G.A.M.

Barros N.

Barry C.
P01-231, P01-234, P01-474

PPC 4, P01-142, P01-161

P02-129

P01-004

ME 4, FC 16, P01-273, P01-320, P01-399, P02-051, P02-564

P01-373

P01-022

P01-231

P02-564

P02-031

P02-185

P02-379

P02-252, P02-448

P02-194

P02-361

P02-125

P02-025

P02-118

FC 21

P01-187

P01-293

P01-372

$\mathrm{P} 02-316$

P01-186

FC 12, P01-437, P01-551, P02-388

P01-537, P02-217

P01-402

P01-198

FC 18, P02-475

PS 2

FC 19

PS 5

P02-449

FC 20

P01-530

FC 17, P02-538

P02-228

P02-368

P01-008, P01-020, P01-064, P01-065

P01-539

P02-156

FC 12, P01-424, P01-547

P02-159, P02-383

$\mathrm{P} 01-460$

P02-084, P02-087, P02-088

$\mathrm{P} 02-024$

P02-268

FC 9

P02-191, P02-206

P02-038

P01-023

P02-275

FC 9

FC 7, FC 13, P01-217, P01-221, P01-222

FC 16, P01-224

P01-372

P01-044

P02-562

P02-554

PS 12

P01-149, P01-421, P02-356

P02-426

P01-048

P01-002

P01-532

FC 8

$\mathrm{P} 02-453$

FC 22, P01-220, P01-453

P01-189
Barth M.

Bartlam K.

Barton M.

Bártová P.

Basemera B.

Basirika D.

Bates M.J.

Bates U.

Batista S.

Baudry P.

Bauer A.

Bausewein C.

Baxter S.

Bayliss J.

Bazzan C.

Beas E.

Bechara F.

Beck E.

Beck E.-R.

Beck I.

P01-025

$\mathrm{P} 02-552$

P02-506

P01-460

P02-456

P02-456

P02-345

P01-235

P02-564

P01-196

PS 2, FC 2, FC 6, FC 10, P01-090, P01-107, P01-236, P01-489, P02-029, P02-155, P02-173, P02-212, P02-536

$\mathrm{P} 02-175, \mathrm{P} 02-251$

PPC 4, P01-044, P01-048, P01-054, P02-485, P02-506

P01-544, P01-545

P02-191, P02-206

P02-156

P01-137

P01-508

P02-189, P02-208, P02-214 $\mathrm{P} 02-218, \mathrm{P} 02-491$

Becker G. FC 6, FC 16, P01-118, P01-224, P01-232, P02-112, P02-339, P02-487,

Becker S.

$\mathrm{P} 02-565$

P01-066

-Commissaris A

P02-028

Beckmann $\mathrm{M}$.

Becque $Y$.

$\mathrm{P} 02-044$

Beecham E.

FC 25, P01-169

PPC 4

Beermann $\mathrm{M}$.

$\mathrm{P} 02-311$

Beernaert K. FC 3, PPC 2, PPC 2, P01-049, P01-052, P01-055, P01-097, P01-228,

Behlendorf T.

P01-334

P02-155

Behzadi A.

Beighton J.E.

Belar A.

Bell J.

$\mathrm{P} 02-292$

FC 4, P02-246

P02-135

P02-094

P01-158

P01-325

P01-435

P01-443

P02-505

P02-405

P01-001

P02-013

P02-344

Benito E.

Benkel I.

Bennett M.

Bennetts J.

Benoit C.

Benson S.

Benze G.

Berall A.

Berendt J.

Beresford B.

Bergbrant I.-M

Bergelt C.

Bergenholtz $\mathrm{H}$.

Berger S.

Berggren $\mathrm{E}$.

Bergh S.

Berghaus D.

Bergianti M.L.

Bergmann D.

Bergmann L.

Bergqvist J.

Bernal J.

Bernard L.

Bernard M.

Bernot M.

Berrocal Higuero M.A.
PS $3, \mathrm{P} 01-148, \mathrm{P} 01-365, \mathrm{P} 02-071$

FC 8, P02-185

$\mathrm{P} 02-311$

P01-009

P02-274, P02-336

P02-155

P02-229

P02-387

P01-024

P02-260

FC 17, P01-168

P01-093, P02-349

P01-092

P02-500

P02-023

P02-180

P01-383

P01-245

P01-230

P02-120

P01-088

P02-021

P01-414, P02-226

P02-067, P02-405, P02-516

P01-485 
Berthold D.

Bertino G.

Bertocchi E.

Best A.

Best M.

Best $\mathrm{S}$.

Beveridge $\mathrm{C}$.

Beyer $\mathrm{F}$.

Beyer S.

Bhagwandien R.E.

Bharadwaj P.

Bharmal A.

Bhatnagar S.

Bhattarai P.

Bia M.

Bialek J.

Biddle J.

Bidegain-Sabas A.

Bidstrup P.E.

Bieber A.

Bieberstein $\mathrm{H}$.

Bijnsdorp F.M.

Billett $\mathrm{H}$.

Bingley A.

Bioy A.

Birgisdottir D.

Birkholz C.B.

Birmingham L.

Bithi M.J.

Bitschnau K.

Björkhem Bergman L.

Björkman-Randström K.

Bjuresäter K.

Black C.

Black S.

Blackburn K.

Blackburn P.

Blenkinsopp J.

Bleznak A.

Bloch S.

Bloomer M.

Blot $\mathrm{F}$.

Bluebond-Langner $\mathrm{M}$.

Blum D.

Boateng A.

Bobnar A.

Bocahu Y.

Bockenkamp B.

Boddaert M.

Boden A.

Boehlke C.

Boelsbjerg H.B.

Boeriu E.

Boesner S.

Bokan D.

Bokemeyer C.

Boland J.W.

Bollig G.

Bolt S.

Bolzani A.

Boman J.

Bonares M.

Bond R.

Bondesson $\mathrm{E}$.

Bone A.

Bonini L.

Bonino Timmerman F.

Bonniaud P.

Boot C.R.L.

Bora S.

Borasio G.D.

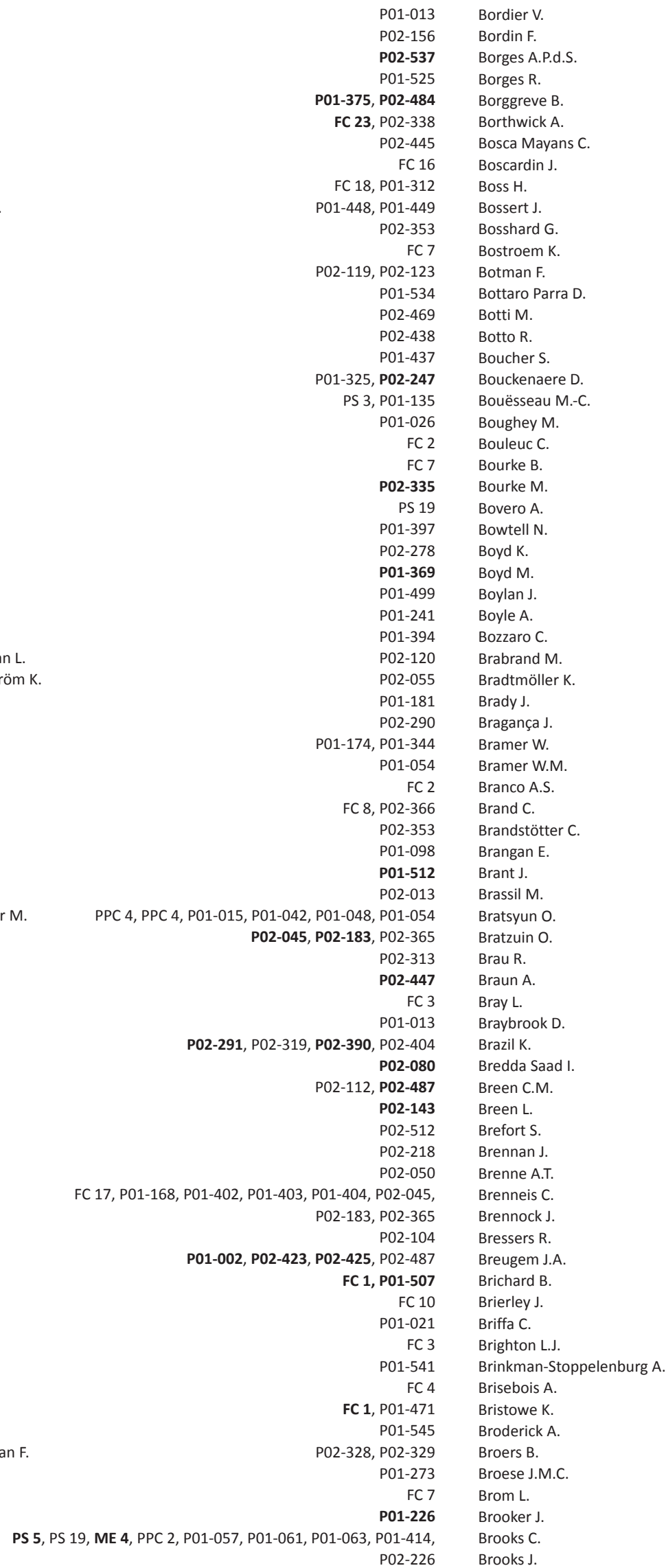

\section{Bratsyun 0 .}

Bratzuin O.

Brau R.

Braun A.

Bray L.

Braybrook D.

Brazil K.

Bredda Saad I.

Breen C.M.

Breen L.

Brefort S.

Brennan J.

Brenne A.T.

Brenneis C.

Brennock J.

Bressers R.

Breugem J.A.

Brichard B.

Brierley J.

Briffa C.

Brighton L.J.

Brinkman-Stoppelenburg A

Brisebois A.

Bristowe K.

Broderick A.

Broers B.

Broese J.M.C.

Brom L.

Brooker J.

Brooks C.

Brooks J.

P02-021, P02-107 P01-019

P01-532

P02-308

P02-030, P02-085

PS 12

P02-192

P01-092, P02-389

P01-520

P01-081

P01-257

P02-094

P01-512

P02-092, P02-457

P01-035, P01-038, P02-210

P01-022

$\mathrm{P} 02-233$

P02-396, P02-557

FC 16, P01-399, P02-051, P02-276

$\mathrm{P} 02-132$

P01-113

P02-092, P02-457

FC 12, P01-322

ME 16, P01-212

FC 19, P01-539

P01-562, P02-501

P02-444

P01-236

P01-514

P01-112, P01-342

P01-478, P02-378

P02-169

P01-359

P01-117

P02-330

FC 25

P02-471

FC 17

P01-315

FC 4

P02-223

P01-115

P01-385

P01-520

P02-375

P01-343

FC 10, P01-473, P02-525

FC 22

P02-197

P02-056

FC 3

P02-283

P01-096

P02-417

P01-219, P02-293

P01-256

P02-342

P01-022

P01-044

P02-493

FC 19

P01-199, P01-227, P01-448, P01-449

P01-214

PS 2, P01-343, P02-374, P02-489

$\mathrm{P} 02-132$$$
\text { FC } 1
$$

P01-438

P02-390

P01-376

P02-376

P01-452 
Brown L.

Brown M.J.

Brown P.

Bruera E.

Brumana E.

Brunder P.

Bruun Wyller T.

Bryan T.

Brząkała J.

Buchhold B.

Buckholz G.

Budo R.

Buentzel J.

Buisan M.

Buiting $\mathrm{H}$.

Bulilete $\mathrm{O}$.

Bulli F.

Bullock R.

Bülow H.-H.

Bunn F.

Burgos Sánchez J.

Burke K.

Burkhardt S.

Burlacu G.

Burley L.

Burnod A.

Burns B.

Burns E.

Burnside G

Burssens $\mathrm{E}$.

Burtonwood J.

Burucoa B.

Busa C.

Buscham H.

Bush S.

Busink V.

Busquets E.

Butler A.E.

Butow P.

Buurman B.M.

Buyinza N.

Buzea L.

Bužgová R.

Byamukama $\mathrm{P}$.

Bye A.

Byrne A.

Byrne P.

Cabañero Martínez M.J.

Caceres-Becerra M.

Cáceres-Muñoz A

Cadwallader $\mathrm{C}$.

Cahill F.

Caldas J.M.

Caldeira S.

Calixto Damasceno Spinelli V.M.

Callenbach M.

Callewaert C.

Calman L.

Calvieri A.

Calvo Espinos C.

Cameron M.G.

Campana L.

Campbell M.

Campbell R.

Campo M.A.

Campos F.

Campos L.

Campos M.

Cañado Y.

Canal-Sotelo J.

Cancelinha C.
FC 4 Candrian C.

P01-197 Candy B.

P01-257 Cano Garzón G.M.

EE1, P01-130, P02-188 Cano-del Olmo K.

P02-403 Cantegreil-Kallen I.

P01-013 Capel M.

P02-023 Capelas M.L.

FC 24, P01-542 Caplan G.

P02-114 Capodannol.

P02-354 Carafizi N.

P02-426 Carbonara L.

P02-129 Cardenas Mori J.

P02-173 Cardenas Turanzas M.

P02-206 Cardone L.

P01-257, P01-274, P01-275 Cardoso P.

P02-344 Carduff E.

P01-415 Carey E.

P02-343 Carey I.

P01-518 Carinci P.

P01-243, P01-464, P01-472 Carling L.

P01-535 Carlsson N.

P02-204 Carmo A.

P01-522 Carneiro A.

P02-228 Carneiro R.

P02-314 Carol D.

P02-098 Carrancha M.

P02-074, P02-097 Carrasco Gimeno J.M.

P02-307 Carratalá A.

P01-525 Carreño $S$

FC 21, P01-540, P02-011

P01-180, P01-325, P02-113, P02-247, P02-503, P02-518

$\mathrm{P} 02-510$

P02-368

FC 4, ME 1, P02-279, P02-444

P01-275

P01-413

P01-015, P01-042

P01-375

P01-140, P01-528, P01-530

P01-546

P02-227

P01-460

P01-552

P02-022

P01-114, P02-060

P01-482

P02-239, P02-288

$\mathrm{P} 02-153$

P02-243

$\mathrm{P} 02-336$

P02-337

P01-379, P01-380

P02-453

P01-075

P01-303

P01-429, P01-430

$\mathrm{P} 02-081, \mathrm{P} 02-141$

P02-310

P02-095

P02-156

P02-434, P02-554

P01-242

$\mathrm{P} 01-444$

P01-046

FC 22, P01-220, P01-453

P01-532

P02-030, P02-085

P02-084, P02-087, P02-088, P02-110, P02-153

P01-046, P01-324, P01-338
Carrillo G.M.

Carruthers E.

Carson-Stevens A.

Carter G.

Carter J.

Carvajal A.

Carvalho A.S.

Carvalho R.T.

Carvalho-Filho M.A.

Casale G.

Casellas-Grau A.

Casey M.

Cass K.

Casseb G.

Cassidy M.

Casson K.

Castro L.

Caswell G.

Cauldwell K.

P01-125, P01-432

P01-132, P02-126

P01-536

P02-153

FC 1

$\mathrm{P} 02-253, \mathrm{P} 02-346$

P01-235, P01-314, P02-446

FC 4

P02-537

P02-416

$\mathrm{P} 02-438$

P01-108, P01-111, P02-309

P01-286

P02-382

P01-494

P01-212, P01-282, P01-475, P02-137, P02-138

P02-077

P02-489

P02-141

P02-260

P02-194

P01-502

FC 24

FC 24

P02-376

P02-421, P02-422

P02-233

P02-085

FC 7, P01-079, P02-176

P02-462

FC 20

P02-398

P01-237

P01-151

P02-311

P02-007, P02-486

P02-362, P02-533

P01-461, P02-340

$\mathrm{P} 02-463$

P01-297, P01-347, P02-081, P02-141, P02-438

P01-400

P02-269, P02-270

P01-084

P01-502

P01-064, P01-065

P01-340

P01-175

P01-260, P01-415

P01-120

P02-267, P02-311

$\mathrm{P} 02-080$

P02-383

$\mathrm{P} 02-215$

Cecilio-Fernandes D. P02-463
Centeno C. FC 4, P01-121, P01-339, P02-246, P02-392, P02-413, P02-566

P01-503

P01-495, P02-421, P02-422

PL 3, P02-543

Červ B.

P01-555

Chaila M.J.

Chalklin L.

Chambaere K.

P02-558

Chamberlain C.

PL 4, FC 3, FC 10, PPC 2, P01-049, P01-052, P01-055,

$\mathrm{P} 01-228, \mathrm{P} 01-420$

$\mathrm{P} 01-223$

Chambers L.

P01-425

Chan F.

Chand A.

P01-459

Chang H.-T.

P01-533

P01-192

P01-433

Chang W.-P.

P02-005

Chao C.-T.

Chaplin D.
P01-433

FC 15 
Chapman E.

Chapman L.

Chapman R.

Chappuis F.

Charles H.

Charlieux B.

Charnley K.

Charnock L.

Chary S.

Chassagne A.

Chaturvedi S.K.

Chauhan A.

Chaumier $\mathrm{F}$.

Chavannes N.H.

Chavarapuzha S.

Chaves A.

Chaves P.

Cheah S.L.

Chen C.-K.

Chen H.-F.

Chen P.-J.

Chen R.

Chen T.-J.

Chen Y.-H.

Chen Y.-J.

Cheng K.-S.

Cheng S.-Y.

Cheong S.K.

Cheung Y.B.

Chiang Y.-T.

Chiba T.

Chidiac C.

Chikhladze N.

Chin J.J.

Chiou Y.-G.

Chitani A

Cho M.

Chochinov $\mathrm{H}$.

Choi K.C.

Choi M.Y.

Chojnicki M.

Choo A.

Choudhary N.

Chow J.

Christensen A.

Christensen C.A.

Christensen P.L.

Christiaens T.

Christiansen C.F.

Christodoulides K.

Chua Z.R.

Chuang E.

Chuang H.J.

Chukwusa E.

Chye R.

Cirac M.

Ciurlionis M.

Clabburn $\mathrm{O}$.

Cladellas-Pros R.

Claessens N.

Clara M.D.J.

Clark D.

Clark J.

Clark K.

Clarke M.

Classon C.

Clayton J.

Clement Carbonell V.

Clep C.

Clifford C.

Clode L.

Clover J.

Coackley A.

$\begin{aligned} \text { FC } 19 & \text { Coad J. } \\ \text { P02-136, P02-358 } & \text { Coast J. } \\ \text { P01-541 } & \text { Cobbaut J.-P. } \\ \text { P02-555 } & \text { Cochran C. } \\ \text { P02-311 } & \text { Coelho M. } \\ \text { FC 1 } & \text { Coelho S.P. }\end{aligned}$

P01-564

P02-137

P02-003

P01-001

P01-502

PL 4, PS 20, FC 2, FC 3, PPC 2, P01-049, P01-052, P01-097, P01-182,

P01-310, P02-501, P02-508, P02-512

P02-282

$02-444$

PS 10, P02-532

$\mathrm{P} 01-412$

P02-299

P01-442

$\mathrm{P} 01-438$

P02-438

P01-532

P01-039

FC 4

P01-192

P01-192

P01-215, P02-373

$\mathrm{P} 02-514$

P01-192

P02-514

P01-192

P02-514

P01-215

FC 4

P02-549

P01-192

P02-025, P02-393

P02-519

P01-377

P01-458

P02-301

P01-265

FC 15

P01-039

P02-111

P01-187

FC 4

P02-119

P01-504

P02-426

P01-415

P02-037

P01-486, P01-487

PPC 5

P01-558

P01-141

P01-111, P02-309

P02-047

FC 19

FC 4

P02-315

P02-357

P02-465

P01-157, P01-400

$\mathrm{P} 02-390$

P02-102

FC 14

FC 8, P02-366, P02-554

FC 9, P01-242, P01-482

FC 7, P02-137, P02-138

P02-120

FC 5, P01-375

P01-171, P02-244

P02-196, P02-258, P02-259

$\mathrm{P} 02-324$

P02-376

$\mathrm{P} 02-156$

P02-269, P02-270, P02-336
Cohen $k$. P01-183, P01-334, P01-335, P01-420, P01-487, P02-232, P02-237

Cohen S.R.

Colleran M.

Collidge T.

Collier A.

Collingridge Moore D.

Collins A.

Collins $\mathrm{M}$.

Collis E.

Colman R.

Colombet I.

Colotto M.

Comas-Carbonell E.

Conceição A.P.

Condesa M.

Conneely A.

Connell A

Connell T.

Connolly D.

Connolly J.

Connolly M.

Connor K.

Connor S.

Conrad R.

Consigli S.

Consortium N.

Consortium N.I.C.U.

Contel E.

Conti F.

Cooke M.

Cooke R.

Cookson T.

Cools F.

Coombes L.

Cooper C.

Cooper H.

Cooper M.

Coperchini M.

Coppé H.

Corbett L.

Corcoy de Febrer B.

Corfias E.

Cornes $\mathrm{M}$.

Cornet C.

Cornette L.

Corona G.V.

Corquaye $\mathrm{O}$.

Corredor M.T.

Cortés Coll P.

Cory S.

Cosgrove S.

Costa F.F.S.

Costa G.D.

Costa I.

Costantini M.

Costello M.

Coster J.

Costongs L.G.P.

Cosulich E.

Cottrell L.

Courtens A.

Couto E.

Cox S.

Cox T.F.
02-222

P02-194

C 3, P01-446

02-379

PS 7

P01-240, P02-132, P02-506

P02-282

P02-311

EE 2

P02-276

P01-347

P01-413

P02-545

P02-316

ME 2

P02-505

P02-345

P02-337

FC 2

P01-252, P02-116, P02-133, P02-398, P02-431

P01-361

ME 13

P02-108

P02-246

P01-049

P01-052

P02-085

P02-141

$\mathrm{P} 02-274$

P01-044

P02-125

PPC 2, P01-049, P01-052

$\mathrm{P} 02-204$

FC 22, SPS6, P01-223

PS 3

P02-552

P02-125, P02-336

$\mathrm{P} 02-396, \mathrm{P} 02-506$

P01-303

P02-343

P02-115

FC 9

FC 21

FC 16, P01-399, P02-051

P01-052

P02-141

P02-313

P02-344

P02-014

P02-175, P02-251

FC 20

P02-340

$\mathrm{P} 02-483$

P02-093

P02-185, P02-534

FC 3

P01-443

P02-384

P01-545

P02-374

P01-110

FC 22

P02-267

P02-135 
Coyle S.

Coym A.

Craig A.

Craig $\mathrm{F}$.

Cramm J.

Crawford C.

Crawford G.

Crawley S.

Creemers J.

Crespo I.

Cretin E.

Crispim D.H.

Cristino Agudo B.

Croft R.

Croixmarie $\mathrm{F}$.

Croke S.

Crosby V.

Cross S.

Crumpler C.

Cruz R.P.

Cruz Quintana F.

Cruz-Sequeiros C.

Csikos A.

Cuervo Pinna M.Á.

Cuesta Vargas A.I.

Cuhls $\mathrm{H}$.

Cullati S.

Cullen A.

Culver J.

Cumming A.

Cunha T.

Cunningham J.

Curatolo P.

Cureton S.

Curfs L.

Currow D.

Curtis P.

Cuthbertson L.

Czudnochowski D.

Dadich A.

Dahl M.

Daisley-Devoy T.

Dakessian Sailian S.

Dale G.H.

Dale K.Y.

Damani A.

Damarell R.

Dance C.

D’Angelo $D$.

Daniela D.N.

Daniels A.

Danneberg M.

Dardeli A.

Darlington A.-S

Das A.

Datsiuk N.

Datta C.

Dauchy S

Davenport A

Daveson B.A.

David A.

David C.

Davidson Lund A.

Davies A.

Davies I.

Davies J.M.

Davies L.

Davies N.

Davila Quintana C.D.

Davis C.

Davis J.M.

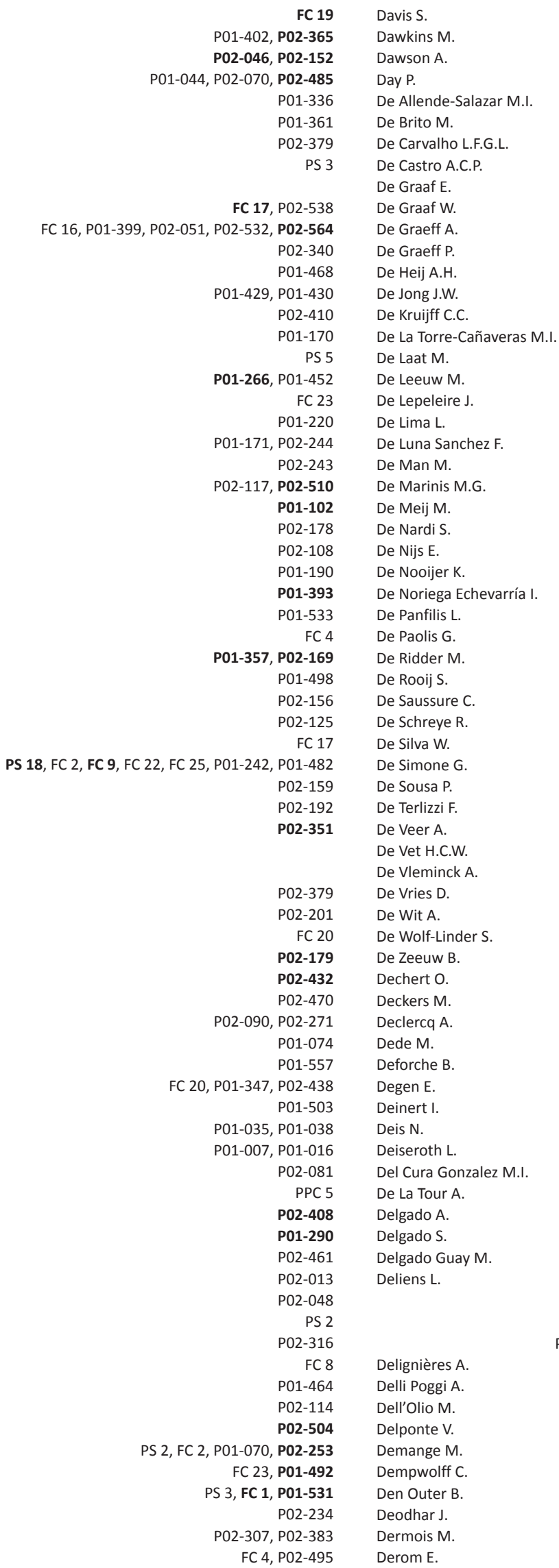

FC 19

P01-402, P02-365

P02-046, P02-152

$\mathrm{P} 01-044, \mathrm{P} 02-070, \mathrm{P} 02-485$

P01-336

P01-361

P02-379

PS 3

FC 17, P02-538

FC 16, P01-399, P02-051, P02-532, P02-564

P02-340

P01-468

P01-429, P01-430

P02-410

P01-170

PS 5

P01-266, P01-452

FC 23

P01-220

P01-171, P02-244

$\mathrm{P} 02-243$

P02-117, P02-510

P01-102

$\mathrm{P} 02-178$

P02-108

P01-190

P01-393

P01-533

FC 4

P01-357, P02-169

P01-498

P02-156

$\mathrm{P} 02-125$

FC 17

PS 18, FC 2, FC 9, FC 22, FC 25, P01-242, P01-482

P02-159

P02-192

P02-351

P02-379

P02-201

FC 20

P02-179

P02-432

$\mathrm{P} 02-470$

P02-090, P02-271

$\mathrm{P} 01-074$

P01-557

FC 20, P01-347, P02-438

P01-503

P01-035, P01-038

P01-007, P01-016

P02-081

PPC 5

P02-408

P01-290

P02-461

$\mathrm{P} 02-013$

P02-048

PS 2

P02-316

FC 8

P01-464

P02-114

P02-504

PS 2, FC 2, P01-070, P02-253

FC 23, P01-492

PS 3, FC 1, P01-531

$\mathrm{P} 02-234$

P02-307, P02-383

FC 4, P02-495

Davis S.

Dawkins M.

Dawson A.

Day P.

De Allende-Salazar M.I

De Brito M.

De Carvalho L.F.G.L.

De Castro A.C.P.

De Graaf E.

De Graaf W.

De Graeff A.

De Graeff P.

De Heij A.H.

De Jong J.W.

De Kruijff C.C.

De La Torre-Cañaveras M.I.

De Laat M.

De Leeuw M.

De Lepeleire J.

De Lima L.

De Luna Sanchez F.

De Man M.

De Marinis M.G.

De Meij M.

De Nardi S.

De Nijs E.

De Nooijer K.

De Noriega Echevarría I.

De Panfilis L.

De Paolis G.

De Ridder M.

De Rooij S

De Saussure C.

De Schreye R.

De Silva W.

De Simone G.

De Sousa P.

De Terlizzi F.

De Veer A.

De Vet H.C.W.

De Vleminck A

De Vries D.

De Wit A.

De Wolf-Linder $\mathrm{S}$

De Zeeuw B.

Dechert $\mathrm{O}$.

Deckers M.

Declercq A.

Dede $M$

Deforche B.

Degen E.

Deinert I.

Deis N.

Deiseroth L.

Del Cura Gonzalez M.I.

De La Tour A.

Delgado A.

Delgado $\mathrm{S}$.

Delgado Guay M.

Deliens L.

Delignières $A$.

Delli Poggi A.

Dell'Olio M.

Delponte V.

Demange $\mathrm{M}$.

Dempwolff $C$.

Den Outer B.

Deodhar J.

Dermois M.

Derom E.

P01-132

P01-480

P01-237, P02-221

P02-299

P02-397

FC 23, P02-170, P02-563

P01-019

P01-019

$\mathrm{P} 01-256, \mathrm{P} 01-386, \mathrm{P} 01-561, \mathrm{P} 02-167$

P01-294

P01-435, P02-308

P01-443

P01-438

P02-384

P01-067

P02-220

EE 2

P02-249

P01-144

FC 9, P01-339, P02-548, P02-566

P02-030

EE 2

P01-347, P02-438

P01-528

P01-122, P02-391

P01-336, P01-513

P01-036

P01-383, P02-534

$\mathrm{P} 01-347$

P02-237

P01-443

FC 1

FC 2, P01-335, P01-487, P02-237

P01-376

P02-205

P01-316

P02-156

FC 13, P01-110, P02-286

FC 10

PS 12, P01-182, P01-183, P02-027

P01-274

P02-319, P02-404

FC 19, P01-520

P02-390

P01-007, P01-016, P01-053

P01-025

P02-232

P01-416

P01-097

P02-371

P01-060

P01-089, P01-092, P02-389

P01-424

P01-485

P01-397

FC 8

P02-235

P01-130

PL 3, PL 4, PS 12, PS 12, FC 2, FC 3, FC 10, FC 10 FC 14, FC 23, EE 2, PPC 2, P01-049, P01-052, P01-055, P01-082, P01-097, P01-182, P01-183, P01-334, P01-335, P01-420, P01-486, P01-487, P02-027, P02-237

FC 3

$\mathrm{P} 02-438$

P02-104

P01-019

FC 1

FC 6

P01-031

P02-090, P02-271

P02-308

FC 3 
Deskou F.

Deskur-Śmielecka E.

Desmonds C.

Detsyk O.

Dev $\mathrm{R}$

Devalois B.

Devanney M.

Devery K.

Devins M.

Devlin L.

Dewes $\mathrm{O}$.

Dewulf C.

Dey $P$.

Dhaini S.

Dhollander N.

Di Leo $\mathrm{S}$

Diaz Sierra G.

Dickie C.

Dickman A

Diehl E.

Dieperink K.

Dieperink K.B.

Dierckx S.

Dieterich M.

Dietz A.

Dieudonné Rahm N.

Diez-Porres L.

Diffin J.

Digiovanni Y.

Dijxhoorn A.F.Q

Dilles T.

Dimitri A.

D'Imporzano E.

D'Incà M.

Dinges $S$.

Dinkelbach L.

Dinsdale A.

Dionysi M.

Distelmaier F.

Ditscheid B.

Dittborn M

Dive S.

Diwoky S.

D'Mello S.

Dodd S.

Doherty D.

Doherty M.

Dojan T.

Doll A.

Dombrecht L.

Domingues T.D.

Donker G.A.

Doran $\mathrm{T}$.

Dorange C.

Dorantes Romandía R

Dos Santos C.E.

Dos Santos G.

Doughty $\mathrm{H}$.

Douglas-Jones A.

Douma J.

Doveson S.

Downham E.

Downie J.

Downing J.

Draeger D.L.

Drake R.

Draper K.

Dreher K.

Dreier L.A.

Dreilich M.

Drennan J.

Drenth D.J.

Dressel G.
$\mathrm{P} 02-252, \mathrm{PO2}-448$

P01-187

P01-385

P01-229

P02-440

P02-247

P02-461

P02-303

PPC 4, PPC 5, P01-006, P01-008, P02-132

P02-125

P01-172

P01-385

P01-555

P02-179

P02-027

$\mathrm{P} 02-537$

P01-485

$\mathrm{P} 01-041$

PS 11, P02-269, P02-270

P01-250, P01-323

P01-556

$\mathrm{P} 02-348$

PS 12, P01-055, P01-228

FC 16

FC 16

P01-522

$\mathrm{P} 02-220$

PS 16

P02-092

P02-291

P01-486, P01-487

P02-225

P02-382

P01-383

P01-289

P01-007

P01-048

P02-448

P01-053

FC 6, FC 6, P01-309

FC 17

$\mathrm{P} 02-456$

P02-507

P01-021

P01-426

PPC 4, P02-149

PS 13, P01-004, P02-414, P02-459, P02-520

P01-081

P02-155, P02-173, P02-491

PPC 2, P01-049, P01-052

$\mathrm{P} 01-379, \mathrm{P} 01-380$

P01-082

P01-029

P01-385

P02-009

P01-220

P02-436

P01-555

P02-221

P02-291

$\mathrm{P} 02-016$

P02-265

P01-044, P01-048, P01-054, P01-490

FC 12, P01-035, P01-551, P02-210, P02-375, P02-388, P02-561

$\mathrm{P} 02-043$

FC 2

P01-072

P01-028

$\mathrm{P} 02-395$

PS 7, P01-529

P01-443

P01-329
Driller B.

Driver I.

Droney J.

Dropic E.

Drost J.

Du Pré $P$.

Duart-Clemente $C$.

Duarte A.

Dubech M.

Ducharlet K.

Ducoin M.-H.

Ducreux M.

Dumble K.

Dumbović N.

Dumitrescu M.

Dumont $\mathrm{S}$.

Dunger $\mathrm{C}$.

Dunleavy L.

Dunne M.

Dupont C.

Duran A.

Durley K.E.

Durmała J.

Duschinsky R.

Dwyer J.

Dybvik T.K.

Dykes N.M.

Dymond E.

Dzaka D.

Dzaka N.

Eagar K.

Earp M.

Eason R.

Ebert M.

Echteld M.A.

Economos G.

Edgar L.

Edmunds L.

Edmunds P.

Edra N.

Eduardo A.H.A.

Edwards G.

Edwards J.

Edwards L.

Edwards S.

Edwin A.

Eecloo K.

Eeden-van Dam E.

Effendy C.

Egger B.

Egido A.

Eibel M.

Eich H.T.

Eick $M$.

Eikemo S.B.

Eilegård Wallin $A$.

Eisenmann $\mathrm{Y}$.

Ekeström M.-L.

Ekholm O.

Eklund R.

Ekström M.

El Aamraoui N.

Ela $\mathrm{S}$.

Ellekrog Ingwersen $\mathrm{G}$.

Ellershaw J.

Ellis-Smith C.

Ellman $\mathrm{M}$.

Elme A.

Elseviers M.

El-Sherif W.

Elsner $\mathrm{F}$.
P01-096, P01-304, P02-470

$\mathrm{P} 02-376$

FC 24, P02-334, P02-363

$\mathrm{P} 02-555$

P01-499

P01-044

P02-196, P02-258, P02-259

P02-278

P01-162

P01-246, P01-247

$\mathrm{P} 02-113$

P02-013

$\mathrm{P} 02-311$

$\mathrm{P} 02-302$

FC 20, P01-056

$\mathrm{P} 02-458$

P02-542

P01-348, P01-354, P01-525, P02-182

, PPC 5

$\mathrm{P} 02-237$

P02-115

FC 11

P01-524

FC 7

FC 15

P01-304

FC 24

P02-186

P02-367

FC 18

FC 2

P01-213

P02-266

P01-306, P02-067, P02-405, P02-516

FC 21, P01-566

$\mathrm{P} 02-021, \mathrm{P} 02-107$

P02-426

P01-452, P02-125

P01-506

P02-316

P02-453

P02-323

FC 2

FC 4

P01-279

P02-313

EE 2

P01-257

P01-163, P01-164, P02-202

P01-289, P02-406

$\mathrm{P} 02-085$

$\mathrm{P} 01-312$

P02-297

P02-440

P02-432

P01-011

P02-036

$\mathrm{P} 02-488$

P01-126

FC 13

FC 9

P01-080

P02-191, P02-206, P02-235

P02-430

FC 19, P02-135, P02-557

$P 01-480, P 02-212$

FC 20, P02-468, P02-469, P02-473

$\mathrm{P} 02-164$

P01-486, P01-487

$\mathrm{P} 02-172$

P02-471, P02-512 


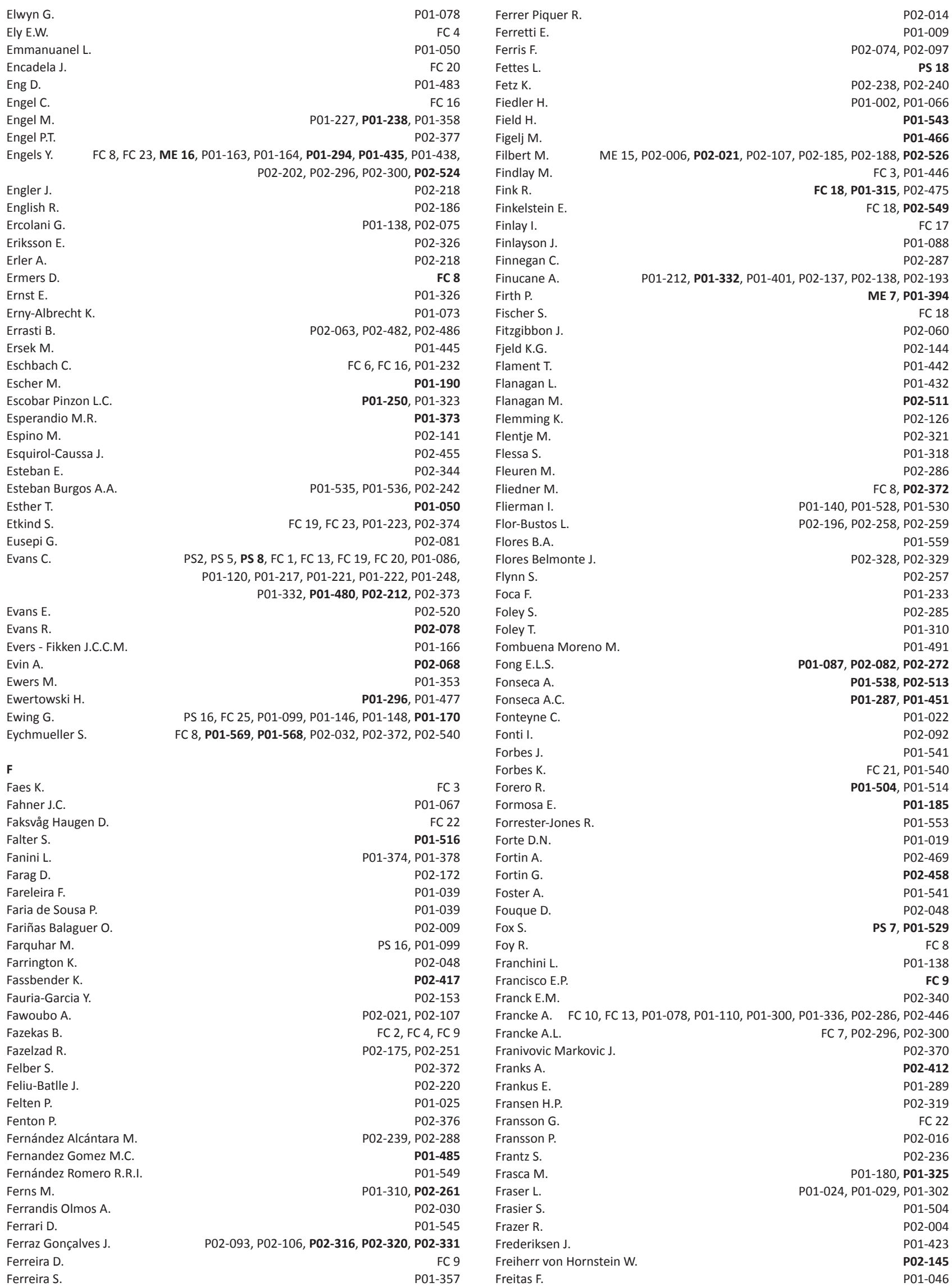




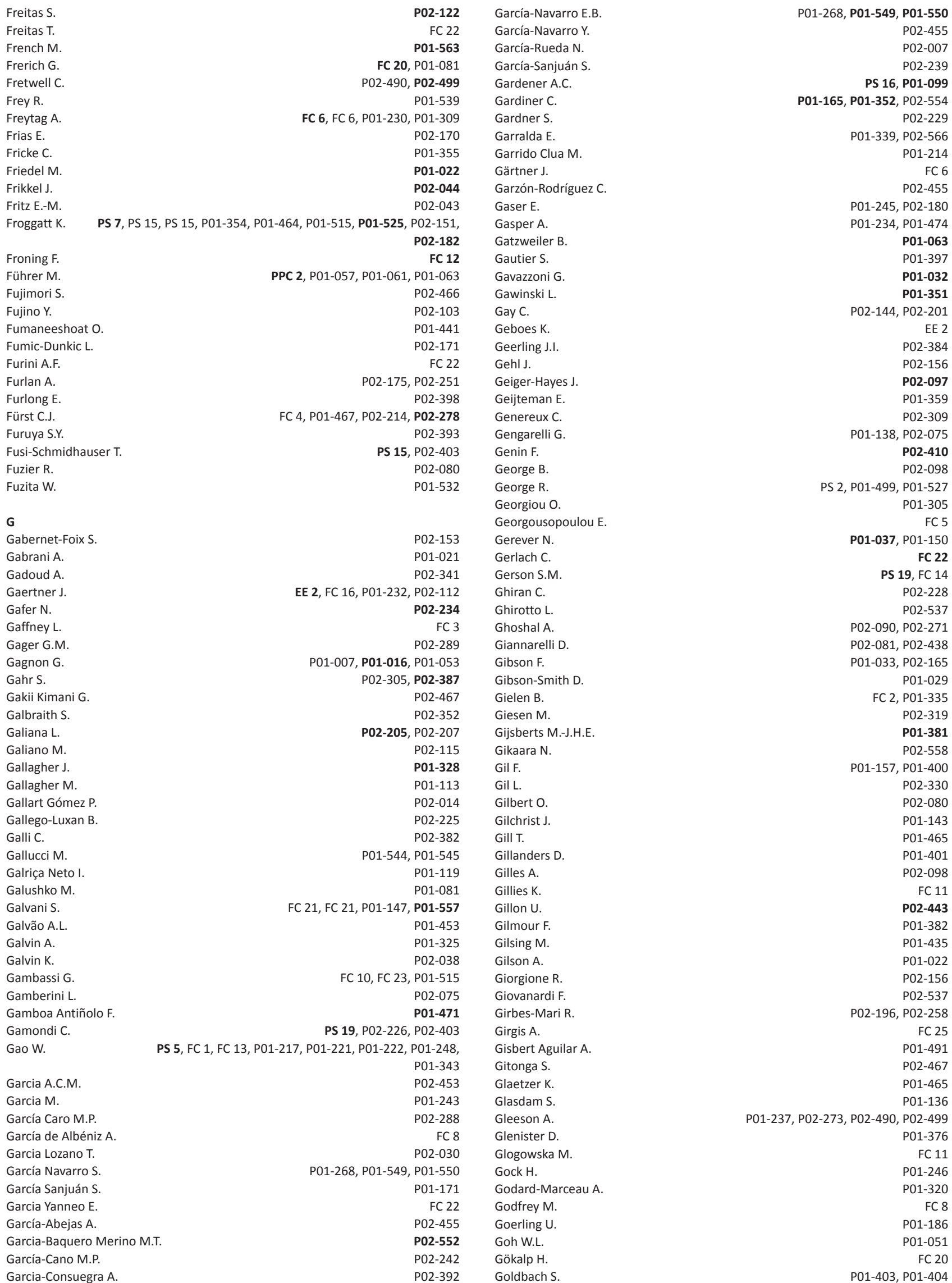


Goldhammer E.

Goldraij G.

Goletti M.

Goliath I.

Golob N.

Gomes A.A.

Gomes B.

Gomes Esperandio M.R.

Gómez O.J.

Gómez Bravo R.

Gómez García R.

Gomez-Batiste X

Gómez-Romero M.J.

Gonçalves C.

Gonçalves E.

Gonçalves F.

Gonçalves L.

Gonzalez N.

Gonzalez R.

González Campos L.G.C.

Gonzalez de Etxabarri S.

González Jaramillo V.

González-Barboteo J.

Gonzalez-Rubio R.

Goodhead A.

Goodman C.

Goossens L.

Goossensen A.

Gootjes J.

Gootjes J.R.G.

Gorbal A

Gorlen T.

Görlich D.

Goryaeva M.

Gorzewska A.

Gosliga F.

Gott M.

Gottschling S.

Goudinoudis K.

Gough N.

Gould T.

Gourlay D.

Govers A.C.

Graaf E.

Grabenhorst U.

Graça J.

Graff S.

Grah C.

Graham-Wisener L.

Grance G.

Grande G.

Grandmaison Dumond L.

Grant J.

Grant L.

Grant M.

Grassau P.A.

Gratz M.

Graven V.

Gray A.

Green A.

Green P.

Gregan P.

Gregori Guillem L.

Gregory A.

Grenier M.

Grief C.

Griffith $S$

Griffiths A.

Griffiths J.

Grillfors Mård M.

Grimes C.

Grindrod A.

Grinyer A.

\begin{tabular}{|c|c|c|}
\hline P02-155 & Grischke E.-M. & P02-156 \\
\hline FC 22 & Grodard G. & FC 16, P01-399, P02-051 \\
\hline P01-297 & Groen-van de Ven L. & P01-101 \\
\hline P01-244, P01-331 & Groenvold M. & PS 12 , FC 4, P02-130, P02-139 \\
\hline P02-516 & Grønberg B.H. & P02-023 \\
\hline P01-019 & Grönholm L. & P02-017 \\
\hline FC 23, P01-248, P01-332, P02-170 & Groninger $\mathrm{H}$. & FC 11, P01-091 \\
\hline P01-374, P01-378 & Gronvold M. & FC 12, P01-183 \\
\hline P02-462 & Gronwald B. & PPC 4 \\
\hline FC 16 & Groselj A. & P02-156 \\
\hline P02-178 & Große-Thie C. & P02-230, P02-507 \\
\hline P02-191, P02-206, P02-235 & Grossman D. & P02-229 \\
\hline P01-157, P01-400 & Grov E.K. & P01-181 \\
\hline P01-039 & Groves K. & FC 5, P02-282, P02-284, P02-287, P02-428, P02-429, P02-465 \\
\hline P02-170, P02-418 & Grozic Zivolic S. & P02-370 \\
\hline P02-122 & Gruber D. & P02-218 \\
\hline P02-170 & Grüneberg $\mathrm{E}$. & P01-040 \\
\hline P01-504 & Gruvebäck A. & P02-208 \\
\hline P01-188 & Gryschek G. & P02-463 \\
\hline P01-468 & Gu C. & P02-360 \\
\hline P01-444 & Guarda M. & FC 20 \\
\hline P02-540 & Guardia Mancilla P. & P01-171, P02-110 \\
\hline P02-191, P02-206 & Guastella V. & P01-397, P02-109, P02-177 \\
\hline P02-084, P02-087, P02-088 & Gudat $\mathrm{H}$. & P02-540 \\
\hline P02-484 & Guelfi K. & FC 9 \\
\hline P01-243, P01-464, P01-472 & Guell Perez E. & P02-009 \\
\hline PPC 2, P01-049, P01-052 & Guenther M. & FC 4 \\
\hline P01-427 & Guerin S. & PS 7, ME 3, P01-197, P01-252, P01-529, P01-562, P02-431 \\
\hline P02-167 & Guerrero-Torrelles M. & FC 17, P02-455 \\
\hline P01-166, P01-389, P02-277 & Guillén M. & P01-292 \\
\hline P02-223 & Guinan E. & P02-337 \\
\hline P01-299 & Guirimand F. & P02-247 \\
\hline P02-377 & Guldin M. & PS 3 \\
\hline P02-267 & Guldin M.-B. & PS 3, P01-135, P02-071 \\
\hline P02-140 & Gullberg Sundin C. & P02-395 \\
\hline P01-031 & Guma S. & P01-037, P01-150 \\
\hline PL 3, P01-165, P01-174, P01-286, P01-344 & Gumà Padrò J. & P02-094 \\
\hline PPC 4 & Gunnink N. & P01-199 \\
\hline P02-491 & Guo J. & P01-295 \\
\hline P02-314, P02-332 & Guo P. & PS 2, FC 23, P02-563 \\
\hline P02-325 & Gupta N. & P02-119 \\
\hline ME 10 & Gurung S. & P02-325 \\
\hline P01-106 & Gustin J. & P02-426 \\
\hline P01-362, P02-249, P02-250 & Guthrie C. & P02-262 \\
\hline P01-253 & Guthrie D. & P02-232 \\
\hline P02-024 & Gutiérrez Rivero S. & P02-110 \\
\hline P01-465 & Gutiérrez Sánchez D. & P02-178 \\
\hline P01-249, P01-251 & Guyot M. & P02-044 \\
\hline FC 7, P02-137, P02-138 & Gwyther L. & PS 17, P01-202, P01-203, P01-204, P01-205, P01-206 \\
\hline \multicolumn{3}{|l|}{ P02-205 } \\
\hline FC 25, P01-146, P01-148, P01-170, P01-429, P01-430 & $\mathbf{H}$ & \\
\hline P02-414 & H Rasmussen B. & P02-278 \\
\hline P01-014 & Haan M. & P01-134 \\
\hline FC 12, P01-551, P02-263, P02-388, P02-561 & Haas N. & P01-076 \\
\hline FC 14 & Haasenritter J. & P02-218 \\
\hline P02-279 & Haberland B. & P02-029 \\
\hline P01-366 & Habermann M. & FC 24 \\
\hline P01-264, P02-369 & Hach M. & P01-230, P01-309, P02-218, P02-491 \\
\hline FC 23 & Hack T. & P02-192 \\
\hline FC 4 & Hackett J. & FC 8, P01-024 \\
\hline P01-445 & Hagelskamp L. & P01-092, P02-389 \\
\hline P01-310 & Hagemann M. & P02-372 \\
\hline P02-014 & Hagemann V. & PS 11, P01-489 \\
\hline P01-216 & Hagenguth $\mathrm{A}$. & P01-013 \\
\hline P02-518 & Hahn L. & P01-025 \\
\hline P02-229 & Hainsch-Müller I. & P01-231 \\
\hline P02-479 & Håkanson C. & P01-148, P01-191, P01-467 \\
\hline P02-269, P02-270, P02-336, P02-477 & Hakata S. & P02-103 \\
\hline P01-558 & Hakenberg O.W. & P02-043 \\
\hline P02-395 & Hales S. & P02-558 \\
\hline FC 11, FC 23, P02-152 & Hall A. & P01-146 \\
\hline FC 14 & Halley A. & P02-257, P02-266, P02-298, P02-363 \\
\hline PS 19, FC 19, P01-133, P01-457 & Haltia O. & P02-017 \\
\hline
\end{tabular}

Grischke E.-M

2-051

02-139

02-017

1, P01-091

P02-507

02-229

2-465

P02-218

2-463

-110

02-540

FC 9

2-009

01-292

02-247

PS 3

02-395

P02-094

P01-199

$02-426$

02-110

2-178

2-278

2-218

02-029

02-192

02-389

P01-489

01-013

01-231

P02-103

P02-043

1-146

P02-017 


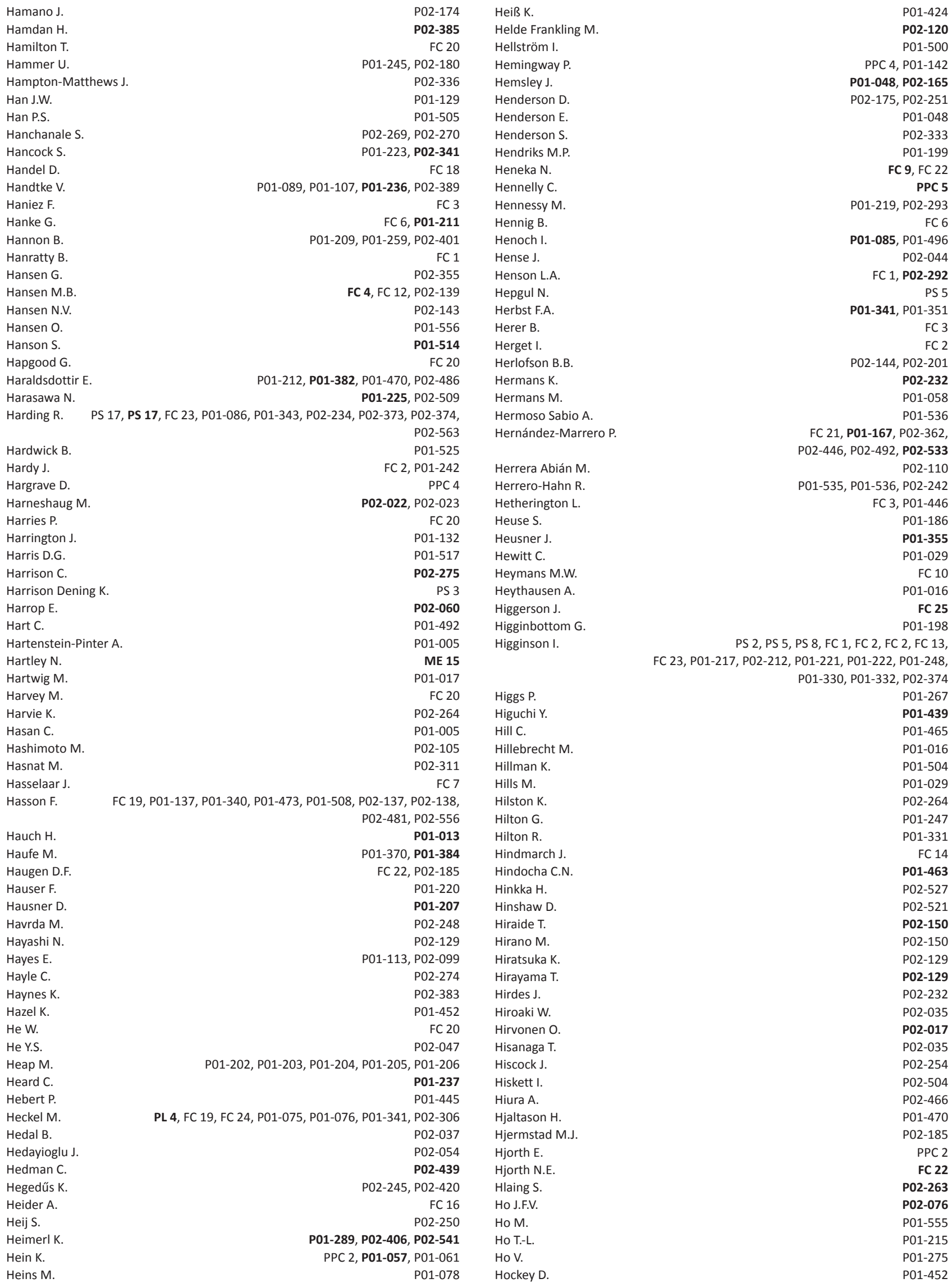


Hockley J.

Hodiamont $\mathrm{F}$.

Hodock K.

Hoell J.

Hofmeister U.

Högberg C.

Hoic L.

Hökkä M.

Holland N.

Hollburg W.

Holliday N.

Hollins S.

Hollyoak S.

Holm M.

Holmberg B.

Holmegaard B.

Holmes $\mathrm{H}$.

Holtom N.

Holyoke P.

Homma M.

Hong J.

Hong Y.S.

Honjo K.

Honohan C.

Hoondert M.

Hooper D.

Horeica R.

Horeweg N.

Hori K.

Horne K.

Hornke I.

Horsfall D.

Horstmann M.

Hosie A.

Hou M.-F

Houben $\mathrm{C}$.

Houska A.

Houthuysen P.

Hov R.

Hove L.H.

Hrda K.

Hrdličková L.

Hsiao S.-C.

Hsieh W.-T.

Hsu S.-H.

$\mathrm{Hu} \mathrm{M}$

Huang B.W.-Y

Huang C.C.

Huang C.-W.

Huang Y.-J.

Hubbard C.

Hubik D.

Hudelson $\mathrm{P}$.

Hudson B.

Hudson B.F.

Hudson P.

Hueso-Montoro C.

Hufthammer K.O.

Hughes D.

Hughes R.

Hughes S.

Hui D.

Huijgens P.C.

Huisman T.F.

Huizinga $C$.

Hulbert-Williams $\mathrm{N}$.

Hum A.

Hum A.Y.M

Hum Y.M.A.

Hummers E.

Humphreys R.

Hung K.-Y.

Hunt L.
Hupcey J.

Hurley I.

Husbands E.

Hussey $\mathrm{S}$.

$\begin{array}{rr}\text { FC } 4 & \text { Hutchinson A. } \\ \text { P02-189 Hutchinson C. }\end{array}$

P02-171 Hutt E.

P02-464, P02-492 Huvane B.

P01-310 Huxtable R

P01-403, P01-404 Hvidt N.C.

P01-564 Hwang J.

FC 17 Hwang S.-J.

P02-412 Hwang S.W.

FC 25, P01-148, P02-016 Hyland C.

P01-500 Hynes G.

P02-430

PS 4

P02-263 Ibrahim M

P02-359 Ichihara $\mathrm{K}$

P02-129 Igarashi N.

P01-051 lida K.

P02-111 lijima T.

P01-439 Ikander T.

P01-218 Ikeda M.

FC 5 Ikegaki J.

FC 2 Imai K.

P02-215 Imamura $Y$.

P01-122, P02-391 Ingravallo F.

P02-150 Ingviya T.

P02-186 Inoue A.

P02-425 Inoue $E$.

FC 14, P01-337 Inoue S

FC 5 losub L.

FC 4 Iranzo Tatay A.

P02-373 Irvine

Isa R.

FC 17, P02-248, P02-539 Isac V.

P02-379 Isherwood R.

P01-181 Ishida S.

P02-144 Ishiguro $\mathrm{H}$.

P01-286 Ishii R.

P01-068 Ishikawa A.

P02-514 Ishiki $\mathrm{H}$.

P01-280 Ivancic A.

P01-086 Ivanetič Pantar M

Ivanetič Pant

202-083 Iwase S.

P02-057 Izquierdo-Gómez J.

Izquierdo-Trigo $\mathrm{P}$.

P01-375 Izumi H.

P01-555

FC 15

P01-190

P01-543, P02-338

FC 21, P01-361

FC 25

$\mathrm{P} 02-242$

FC 22

FC 19

P01-533, P02-304

P01-149, P01-421, P02-151, P02-356

P01-209, P01-368

P02-291

P01-166

P01-030, P01-031

P01-401

P01-458, P02-086, P02-347

FC 9

P02-181

FC 6

P01-479

P01-433

P02-353

Jaatinen A.

Jabbarian L.

Jack B.

Jackson C.

Jacob J.

Jacobs-de Leur J.

Jacobsen M.H.

Jagannath J.

Jaggi P.

Jaillet $\mathrm{H}$.

Jakda A

Jales S.M.C.P.

Jamali A.

James B.M.

Janowicz A.

Jansen J.

Jansen-Segers $M$.

Janssen A.

Janssen D.J.A.
Jääskeläinen A.-S. $\quad$ P02-164

P01-143, P01-155

$\mathrm{P} 01-045$

P02-131

$\mathrm{P} 02-132$

$\mathrm{P} 01-512, \mathrm{P} 02-038$

P02-303

P01-050

P02-309

FC 17

FC 5

P02-426

P01-192

P01-178

P02-125

P02-399

P02-313

FC 5

FC 11

P02-481

P01-462, P02-154

P01-556

P01-225, P02-509

P02-100

P02-035, P02-203

P01-450

P01-183

P01-441

P02-096

P02-466

P01-450

P02-521

P02-030

P02-193

FC 3

P02-416

P02-147, P02-148

P02-162

P02-154

P01-071, P01-488, P02-105

$\mathrm{P} 02-466$

FC 15, P01-069, P02-174

$\mathrm{P} 02-370$

$\mathrm{P} 02-405$

P02-509

$\mathrm{P} 02-174$

P02-243

P02-115

P02-174

P02-364

PL 3

FC 5, P01-555, P02-375, P02-428, P02-429

P01-525

P01-207

P01-058

P01-423

P01-308

P02-192

FC 3

$\mathrm{P} 02-232$

P02-340

$\mathrm{P} 02-267$

P02-361

FC 20, P01-152

$\mathrm{P} 02-240$

P02-319

Jansky M. FC 6, FC 6, FC 6, FC 12, P01-196, P01-230, P01-309, P01-424, P01-547
P01-294

P01-438 
Janßen G.

Janssen $\mathrm{G}$.

Jarlbæk L.

Jarvis $S$.

Jaspers B.

Jassal $\mathrm{S}$.

Jaufeerally $F$.

Jeffs $C$.

Jena $\mathrm{M}$.

Jenions $\mathrm{L}$.

Jenks A.

Jennifer $P$.

Jennings $\mathrm{V}$.

Jensen H.I.

Jensen L.A.

Jensen L.H.

Jensen M.-B.

Jensen W.

Jensen Hjermstad M.

Jentschke $\mathrm{E}$.

Jeppesen J.

Jernelöv S

Jerwood J.

Jespersen B.A.

Jess $M$

Jimeno-Uclés $\mathrm{R}$.

Johns $\mathrm{H}$.

Johnsen S.P.

Johnson $\mathrm{H}$.

Johnson K.

Johnson M.

Johnson S.

Johnston B.

Johnston B.M.

Johnston C.B.

Johnstone R.

Joist T.M.

Joling $\mathrm{K}$.

Joly C.

Jones $\mathrm{H}$.

Jones I.R.

Jones $\mathrm{K}$.

Jones L.

Jones $\mathrm{T}$.

Jonsdottir G.

Jonsdottir $\mathrm{H}$.

Jonston A.

Joos $\mathrm{G}$.

Jordan S.

Jordhøy M.

Jørgensen B.

Joshi R.

Jox R.

Judge $C$.

Juelich A.

Juenger J.

Juffermans $C$.

Juliana A

Julião $M$.

Jülich $A$.

Jullien S.

Jung $C$.

Jünger J.

Junghanß C.

Junghanss $C$.

Junqueira dos Santos A.F.

Juodaite Rackauskiene A.

Kılıc Ozturk Y.

Kaasa S.

Kaasalainen S.

Kabir M

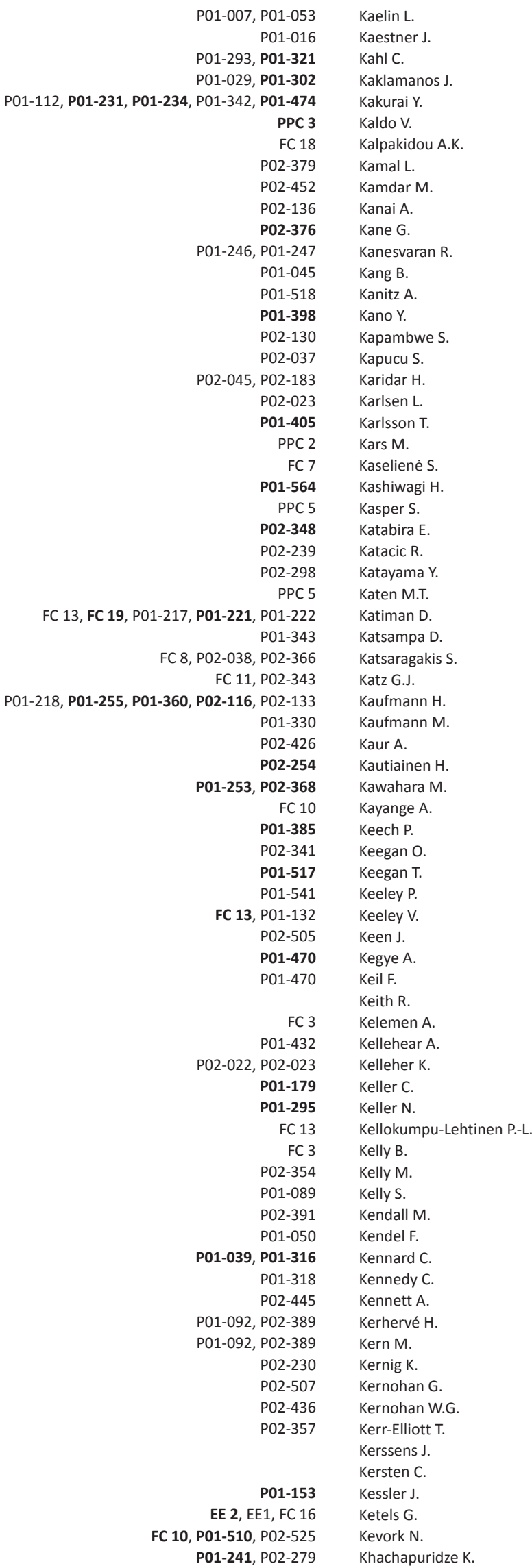

Kaelin L.

Kaestner J.

Kahl C.

Kaklamanos J.

Kakurai Y.

Kaldo V.

Kalpakidou A.K.

Kamal L.

Kamdar M.

Kanai A

Kane G.

Kanesvaran $\mathrm{R}$.

Kang B.

Kanitz A.

Kano Y.

Kapambwe S.

Kapucu S.

Katiman D.

Katsampa D.

Katsaragakis S.

Katz G.J.

Kaufmann $\mathrm{H}$.

Kaufmann M.

Kaur A.

Kautiainen $\mathrm{H}$.

Kawahara M.

Kayange A.

Keech P.

Keegan $\mathrm{O}$.

Keegan T.

Keeley P.

Keeley V.

Keen J.

Kegye A.

Keil $\mathrm{F}$.

Keith R.

Kelleher K.

Keller C.

Keller N.

Kellokumpu-Lehtinen P.-L.

Kelly B.

Kelly M.

Kelly S.

Kendall M.

Kendel $\mathrm{F}$.

Kennard C.

Kennedy $\mathrm{C}$.

Kennett A.

Kerhervé $\mathrm{H}$.

Kern M.

Kernig K.

Kernohan G.

Kernohan W.G.

Kerr-Elliott T.

Kerssens J.

Kersten C.

Kessler J.

Ketels G.

Kevork N.

Khachapuridze K.

P01-289

P01-013

FC 16, P02-155

P02-195

$\mathrm{P} 02-073$

FC 7

P01-558

FC 24

P01-456, P02-077

P02-129

P02-298

P02-549

P01-368

P02-365

FC 24

P02-002

P01-136

P02-470

P02-037

ME 6, P01-067, P01-183, P01-415

P01-364

P01-450

$\mathrm{P} 02-044$

P01-437

P02-370

P02-509

PS 12

FC 3

P01-558

P02-195, P02-224, P02-252, P02-448 P02-409

P01-239, P02-161, P02-164 P02-509

FC 5, P01-059, P01-062 P01-504

PS 3, FC 11, P02-071 P01-563

FC 7, P01-345, P02-529

P01-558

$\mathrm{P} 01-212$

P02-245

P02-015

P02-264

FC 11, P01-091

ME 12, P02-056

P02-049

PPC 4

P02-403

$\mathrm{P} 02-527$

P02-412

FC 14

P01-020

P01-212, P01-469

P01-186

P02-311

FC 20, P01-181

FC 24

FC 1

P01-234, P01-474 $\mathrm{P} 02-043$

PS 7, ME 3, P01-529 P01-197

P01-033, P01-044, P02-485 P01-212 P02-095

FC 6, P01-232

P02-045, P02-183

P02-175, P02-251 P01-377 
Khair Allah M.

Khalid D.S.

Khan F.

Khan S.A.

Khemlani M.

Khodabukus A.

Kiely F.

Kilbane L.

Kim S.

Kim Y.J.

Kimmig A.

King A.

King $M$.

King-Shier K.

Kinley J.

Kinloch K.

Kinoshita F.

Kioroglo P.

Kirkhus L.

Kirkwood J.

Kirsch D.

Kirschner P.

Kis E.

Kissane D.

Kitko L.

Kitta A

Kiuchi D.

Kiwanga F.

Kiwanuka R.

Kiyange $F$.

Kizawa Y.

Kjær T.

Klapwijk M.S.

Klein C.

Klepstad P.

Klimsch C.

Kline D.

Klint Å.

Klug D.

Kneen K.

Knight E.

Knighting K.

Knochel K.

Knopf B.

Knorrenschild J.R.

Knudsen A.K.

Koçer S.

Koch M.

Koch Jamal S.

Kochovska S.

Kodba Čeh H.

Kodsi A.

Koekoek B.I.

Koffman J. FC 13, FC 19, FC 20, P01-086, P01-217, P01-221, P01-222, P01-223,

P01-469, P02-040, P02-373, P02-374

$\mathrm{P} 02-315$

Kögl K.

Koh M.

Koh S.-J.

Koh T.

Koh Y.H.M.

Kojima R.

Kokkonen K.

Kokot M.

Koksvik G.

Koleci G.

Kolen M.

Kölpin A.

Komesaroff $P$.

König P.

König S.

Koorn R.

Koppitz A.L.

Kopplin-Förtsch K.

01-198

PS 13

P02-292

FC 18

P02-136

P02-049

P01-498

P01-368, P02-111

P01-368

P01-013, P01-025

P01-189

PS 3, FC 2, P01-132, P01-343

FC 12

P01-464

FC 5, P02-428, P02-429

FC 5

P02-022, P02-023

FC 7

P01-403, P01-404

P02-031

P02-156

FC 5, P01-376

P01-143, P01-155

P02-289

FC 15, P01-069

FC 5, P01-059, P01-062

P02-561

P02-561

P01-409, P02-035, P02-096, P02-100, P02-174, P02-203

P02-369

P02-212, P02-199

FC 2, P01-076, P02-306

FC 16

P02-491

FC 18

FC 4

FC 20, P01-220

$\mathrm{FC} 2$

$\mathrm{P} 02-304$

$\mathrm{P} 02-375$

PPC 2, P01-057, P01-061, P01-063

$\mathrm{P} 02-425$

P01-109

P01-096

P01-109

$\mathrm{P} 02-093$

FC 4, P01-243

$\mathrm{P} 01-286, \mathrm{P} 01-415, \mathrm{P} 02-543$

P02-225

P02-225

P01-458, P02-347, P02-360

P02-111

P02-360

$\mathrm{P} 02-181$

FC 15, P01-069

P01-239, P02-164

P01-524

FC 14

P02-402

P01-566

FC 19

FC 14

P02-351

FC 6

P01-362, P01-561

P01-520

P01-403, P01-404
Kordes U.

Kordzaia D.

Korfage I.

Kosminsky L.

Kosugi T.

Kotoda M.

Kovacevic T.

Kovacovic B.

Kovacs J.

Koyama A.

Kozáková R.

Kozhevnikov D.

Kraft S.

Krakauer E.

Krakowiak P.

Krause M.

Krause $\mathrm{O}$.

Krauss S.

Krauss S.H.

Kravchenko D.

Kreft J.

Kreicbergs U.

Kremeike $\mathrm{K}$.

Kremling A.

Kreye G.

Kreyer C.

Kriesen U.

Krikorian A.

Kristanti M.S.

Kristiansen M.

Kröger C.

Kroik L.

Kroll T.

Krooupa A.-M.

Kröz M.

Krueger J.

Krueger W.

Krug K.

Kruithof J.W.F.

Krull E.

Kruschel I.

Kruse R.L.

Krzyzanowska M.

Kubin T.

Kubo M.

Kuhlberg J.

Kuhlen M

Kulasegaram $\mathrm{P}$.

Kulla A.

Kumakura Y.

Kumar V.

Kumari P.

Kunte C.

Kuo L.C.

Kuon J.

Kupeli N.

Kurita D.

Kurita G.

Kurita G.P.

Kurkowski S.

Kurrle S.

Kuss K.

Kustura A.

Kwaitana D.P.

Kwok A.O.L.

Kwon J.H.

Kylanen M.

Kyngäs $\mathrm{H}$.

L

L'hoest H.

La Rocca F.

Lab B.
P01-017

P01-377

PL 3, PS 12, FC 21, P01-116, P01-117, P01-336

P01-111

P02-466

P01-462, P02-154

P02-050

P02-370

P02-512

P02-096

P01-460

P02-468

P01-026

PS 9, ME 5

FC 20, P01-152, P02-059, P02-064, P02-066, P02-437

FC 6, FC 6, P01-309

P01-477

P01-309

P01-196

P02-108

P01-401

FC 7, FC 13, PPC 2, P01-011, P02-053, P02-058

P01-081, P01-090

P01-236, P01-281

EE1

PS 16, FC 25

$\mathrm{P} 02-230, \mathrm{P} 02-507$

$\mathrm{P} 01-175$

P01-117, P01-163, P01-164, P02-202

FC 12

$\mathrm{P} 02-544$

P01-263

P02-486

FC 19

P01-251

FC 4

$\mathrm{P} 02-354$

P01-089, P01-092, P02-389

P01-389

P02-173

P01-245

FC 10

P01-259

FC 16

P02-466

P02-351

P01-007, P01-107, P01-053

P01-510

P01-076

P01-462, P02-154

P02-119

P01-308

P02-156

P01-554

FC 16

P01-132, P01-327, P01-479, P02-126

P02-150

P02-143

PL 4

P02-306

FC 4

P02-218

P02-171

P02-280

P01-368

FC 10, FC 23, P01-515

P02-492

FC 6

P02-382

P02-555 
Lacasta M.

Lacasta-Reverte M.

Lacerda A.

Lachat S.

Lafaye D.

Lafaye $F$.

Lafuente-Sanchis $\mathrm{P}$.

Lagan N.

Lahaye M.

Lai T.T.K.

Lai Y.-L.

Laidlaw S.

Laier G.

Lam C.

Lam E.

Lam J.C.Y.

Lam L.

Lam L.P.L.

Lam M.

Lama M.

Landzaat L.

Lane J.

Lang A

Langlois A.

Langner R.

Lapeire L.

Lapkin S.

Laranjeira A.

Larkin M.

Larkin P.

Larsen A.M.

Larsson M.

Larue $F$.

Larumbe A.

Laska I.

Lasker-Hertz S.

Lasmarías $\mathrm{C}$.

Lasserson D.

Latina R.

Latino N.

Lau J.

Laurent C.

Laurent S.

Lautrette A.

Lautwein F.

Lauwerier $\mathrm{E}$.

Law B.M.H.

Law P.T.W.

Lawanto K.

Lawlor P.

Lazenby M.

Lazic $\mathrm{R}$.

Le $B$.

Le L.

Le Berre R.

Le Boutillier C.

Leahy $A$

Leahy $\mathrm{H}$.

Leavey $\mathrm{G}$

Lee $A$.

Lee C.S.

Lee E.S.

Lee $\mathrm{G}$

Lee J.

Lee K.H.

Lee K.T.

Lee L.H.

Lee L.T.

Lee S.H.

Lee S.N.

Leemans $\mathrm{K}$.

Lefranc A.

$\begin{aligned} & \text { P02-071 } \text { Leget C. } \\ & \text { P02-220 } \text { Legoupil C. } \\ & \text { P01-324, P01-338 } \text { Legowska E. } \\ & \text { P02-555 } \text { Legrumber M. } \\ & \text { P02-503 } \text { Lehmann N. } \\ & \text { P02-113 } \text { Lehmann T. } \\ & \text { Lehto J. } \\ & \text { P01-020 } \text { Lehto J.T. } \\ & \text { P01-022 } \text { Leiva A. } \\ & \text { Leiva Santos J.P. } \\ & \text { P02-083 } \text { Lejeune A. } \\ & \text { P01-212, P01-475 } \text { Lemaire A. } \\ & \text { P02-037 } \text { Lemonidou C. } \\ & \text { P01-088 } \text { Lemos Dekker N. } \\ & \text { P01-201 } \text { Lemus P. } \\ & \text { Leners J.-C. } \\ & \text { FC 4 } \text { Leng M. } \\ & \text { Leniz J. } \\ & \text { P01-201 } \text { Lenz P. } \\ & \text { FC 4 } \text { Leo O. } \\ & \text { P02-426 } \text { Leon Loza G.A. }\end{aligned}$

P01-370, P01-386, P01-384, P02-484

P02-013

P01-152, P02-059, P02-064, P02-066

P01-538

FC 8

FC 6, FC 6, P01-230, P01-309

$\mathrm{P} 02-164, \mathrm{P} 02-464, \mathrm{P} 02-527$

P02-017, P02-161

P01-292, P02-344

P02-494

P02-503

P02-098

P02-195

P01-537, P02-199, P02-217

P01-079

P02-010

PS 13, FC 12, P01-437, P01-551, P02-388, P02-561

FC 2, P02-563

FC 4, P02-219, P02-297, P02-377

P02-049

$\mathrm{P} 02-310$

$\begin{array}{lll}\text { P01-237 Leonard R. FC 14, P01-337 } & \end{array}$

P01-289, P02-406 Leong I. P01-458

FC 22, P02-114

PL 2, P01-109

PPC 4

P01-183 Lerdal A.

FC 9 Leskelä R.-L.

P02-476 Lester J.

P01-113, P02-099 Letcher Lazo C.J.

Lethborg C.

Letsch A.

Letzel S.

Levine $S$.

Lewis D.A.

Lewis K.

Lewis $\mathrm{S}$.

P01-432 Lewis V.

P02-235 Lex K.

FC 11 Li L.

P02-438 LiY.

EE1 Li Y.-G.

P02-175, P02-251 Li Y.H.

P02-013 Liakopoulos G

P02-013 Liakopoulou P.

P02-523 Lian W.Q.S.

FC 15 Lichodziejewska-Niemierko M.

P01-144 Lieber K.

Liebig B.

Liefbroer A.I.

P02-475 Liew D.

FC 4, P02-279 Liew S.H.

P02-453 Lima D.

P02-555 Limonero J.T.

FC 4, FC 25, P01-240, P02-396, P02-506

P01-259 Lin C.C.

P02-003 Lin C.L.

P01-189 Lin C.-P.

P02-501 Lin H.-J.

P02-049 Lin M.-H.

P01-132 Lin W.-Y.

P01-458 Lin Y.-C.

P02-181 Lind $S$.

P01-368 Lindner $R$.

P01-051 Lindqvist $O$.

P02-262 Lindseth G.

P02-111 Lindwedel-Reime U.

P02-111 Ling G.C.C.

P02-549 Ling J.

FC 4 Linge-Dahl L.

P01-181 Linhart M.

P02-111 Linzitto J.P.

P02-237 Lisa W.

P01-162 Lisboa M.
P02-017

P01-114

P01-342

$\mathrm{P} 01-542$

FC 18, P01-186, P02-031

$\mathrm{P} 01-250, \mathrm{P} 01-323$

P02-426

PS 19

P02-408

P02-460

P01-073

P01-476, P01-519

P02-360

P02-353

P01-288, P02-551

P01-315

P01-305, P01-307

P01-416

P01-087, P02-082, P02-272

P01-283, P02-166, P02-450, P02-451

$\mathrm{P} 01-013$

P02-371

P01-381

FC 25

P02-156

P01-495, P02-421, P02-422

PS 3, P01-157, P01-176, P01-400, P02-071, P02-191, P02-206

P01-554, P02-005

P02-047

P01-086, P02-373

$\mathrm{P} 01-192$

P01-192

P01-215

P01-280

P02-214

P01-090

P01-263

P02-470

P02-351

P01-006, P02-512

P01-406

FC 8

P02-205

P01-174

P02-320, P02-331
P02-144, P02-201 
Listina I.

Liva E.

Llagostera M.

Llewellyn $\mathrm{H}$.

Llisterri Sánchez P.

Llobera J.

Llobera-Solanas M.

Llorens-Torromé $\mathrm{S}$.

Lloyd Williams M.

Lo $\mathrm{C}$.

Lo M.L.

Lo T.J.

Loedel S.

Loge J.H.

Lokker M.E.

Loncan-Vidal P.

London L.

Longo $\mathrm{M}$

Lookabaugh B.

Lopane P.

Lopes A.

Lopes S.

Lopes Ferreira P.

Lopez M.

López Bermudo C.

Lopez García M.

López Navarro A.

López-Fidalgo J.

Lopez-Ribes J.

López-Saca M.

Loquai C.

Lordick F.

Lorenzl S

Lormans A.H.M.

Lormans T.

Los C.

Lotz J.

Loučka M.

Louw S.

Lovell $\mathrm{M}$.

Lövgren $\mathrm{M}$.

Low G.S.H.

Low J.

Low M.

Low Z.J.C.

Lowney A.C.

Lucas $C$.

Lucas $\mathrm{H}$.

Luckett T.

Lueg A.

Luelmo S.

Luís $\mathrm{M}$.

Lukacs M.

Lum $\mathrm{H}$.

Lundeby T.

Lunder U.

Lundorff $\mathrm{L}$.

Lundström S

Lundy $\mathrm{E}$.

Luo Z.-W.

Luque-Blanco $\mathrm{A}$.

Luyirika $\mathrm{E}$.

Lykke C.

Lynch J.

Lynch M.

Lynch S.

M

Macareavy D.

Macarow K.

MacArtney J.

Mach V.
P01-445

Machado D.

Machado E.

P01-305, P01-307 Machado J.

P01-292, P02-344 Maclnnis C.

P01-267 MacKenzie C.

P02-014 Mackey R.

P01-292, P02-344 Macková M.

P02-153 Mackuline A.

P02-115 MacLeod R.

P01-429, P01-430 MacLeod U.

FC 16 MacMahon $\mathrm{H}$

P02-057 Macmillan K.

P01-286 Macpherson S.

P02-305, P02-387 Maddela S.

EE 2 Maden $\mathrm{M}$.

P02-160 Madero-Jarabo R.

P02-455 Madigan M.

P01-202, P01-203, P01-204, P01-205, P01-206 P01-114, P02-060, P02-553

P02-097

P02-382

P01-495

P01-324, P01-338

$\mathrm{P} 02-170$

$\mathrm{P} 02-403$

$\mathrm{P} 02-110$

P02-328, P02-329

$\mathrm{P} 01-471$

FC 4, P01-339, P02-246, P02-413, P02-566 $\mathrm{P} 02-084, \mathrm{P} 02-087, \mathrm{P} 02-088$ $\mathrm{P} 02-246$

FC 16

FC 16

P01-476, P01-519, P02-471

P01-386

$\mathrm{P} 02-249, \mathrm{P} 02-250$

$\mathrm{P} 02-448$

PPC 2

FC 13, FC 17, P02-184, P02-248, P02-539

FC 9

FC 4

FC 13, PPC 2, P01-011, P01-100

P02-076

FC 18, P01-098

$\mathrm{P} 02-456$

P02-181

FC 11

P01-435

P01-417, P02-330

FC 22, P01-243

P01-253

P02-391

P02-320, P02-331

P02-117, P02-415, P02-420, P02-510

FC 18, P01-432

EE 2

PS 12, PS 15, P01-183, P01-415, P01-466, P02-543

FC 22

FC 22

P02-255

P01-288

P02-243

P02-561

P01-126

P01-464, P01-472

P01-310, P02-295, P02-474

P01-376, P02-145

P01-555

P01-331

P02-137
Madsen L.D.

Maeda I.

Maessen M.

Maetens A.

Mafra A.B.B.

Magalhães S.

Magann L.

Magee C.

Magnani C.

Magri-Mari R.

Mah K.

Mahboub A.

Mahdavi $\mathrm{H}$.

Mahieu A.

Maia M.

Maia R.

Maier B.O.

Maillard B.

Maincent $\mathrm{C}$.

Maindet C.

Mainzer $\mathrm{K}$

Malaczkoski A

Malcolm C.

Malerba M.B.

Malessa A.

Malfitano $\mathrm{C}$.

Malheiro M.

Malhotra C.

Malinverni E.

Mallett J.

Mallon A.

Maltoni M.

Manalo M.F.

Manase $\mathrm{F}$.

Mancheño Alvaro A.

Mandal N.

Mander T.

Mann M.

Mann T.

Manna A.

Mannion E.

Mannion R.

Mansfield L.

Mansour A.

Manthalu G.

Mantziou V.

Manus W.C.

Marđetko R.

Maradey P.

Marco D.

Marheineke A.

Mariam L.

Marini J.

Marinova M

Marion D.

Marlow C.
P02-545

P01-220

P02-320, P02-331

P02-192

P01-411

P02-077, P02-440

P01-395

P01-050

FC 8

P01-310

P02-417

P01-212

FC 6, FC 6

P02-375

P02-220

P02-261

FC 5

P01-071, P02-035

P01-569, P02-540

P01-334

P01-019

P01-127

P02-495

FC 22

P01-297, P01-347, P02-081, P02-141, P02-438

P02-259

FC 3, FC 16, P02-558

P01-022

P01-349

P01-097

P02-157, P02-158

P02-102

P02-155

P02-068

P01-273

P02-098

P01-002

P02-412

P01-014

P01-138

FC 19, P01-075

FC 16, P02-558

$\mathrm{P} 02-024$

FC 18, P02-549

FC 20

$\mathrm{P} 02-048$

P01-340

P01-233, P02-211

P02-026

FC 5, P01-059, P01-062

P02-014, P02-030, P02-085

P01-077

FC 20

P02-553

P02-311

P01-276, P01-418

P02-281

P02-132

P01-189

PS 17

P02-195

P01-511

P02-302

$\mathrm{P} 02-110$

FC 24, P01-240

FC 10

P02-020

P01-544

P02-108

SPS6 
Marmar C

Marques G.

Marques L.

Marques-Aguilar E.

Marsden M.

Marsden P.

Marsden S

Marshall S.

Marston J.

Martín S.

Martín Fuentes de la Rosa M.A.

Martín Jiménez L.

Martín Roselló M.

Martin Utrilla S.

Martina D.

Martina W.

Martinez M.

Martínez Gomá R.

Martínez Granero A.

Martin-Marco A.

Martín-Martín J.

Martino Alba R.

Martin-Roselló M.L.

Martins A.

Martins E.

Martins $\mathrm{H}$.

Martins J.C.

Martins M.M.F.P.S.

Martins T.

Martins Cunha O.

Martins Pereira S.

Martins-Branco D.

Martinsson L.

Martin-Utrilla S.

Marx G.

März L.

Masamba L.

Mascherini M.

Masel E.K.

Mason B.

Mason S.

\section{FC 19}

Massaiochi Tanimoto $\mathrm{H}$.

Massam J.

Masson J.

Mastroianni C.

Maté J.

Maté-Méndez J.

Mateo-Ortega D.

Mateus C.

Mathe A.

Mathew R.

Mathieu-Nicot F.

Mathis G.

Mathoulin-Pellissier S

Matis C.

Matsuda Y.

Matsukawa T.

Matsumoto L.K.

Matsumoto $\mathrm{Y}$.

Matsuoka $\mathrm{H}$.

Matsushima E.

Matteucci P.

Matthes B.

Matthes $\mathrm{H}$.

Matthews A.

Matthews F.

Matthews R.

Maxwell P.

May P.

Mayer-Steinacker R.

Mayland C.

Mayoral V.
FC 16

P01-407

P02-316

P02-258

P01-310

PS 15

P02-054

ME 7, P01-041, P01-343

PS 13

P01-491

P02-328, P02-329

P01-036

P02-178

P02-030, P02-085

P01-117

FC 5

FC 4, P02-246

P01-491

P02-014

P02-196, P02-258, P02-259

P01-139

P01-036, P02-517

P02-344

P02-024

$\mathrm{P} 02-102$

P01-379, P01-380

P01-521

P02-502

P01-407

P02-106

FC 21, P01-167, P02-362, P02-446, P02-492, P02-533

P02-330

FC 22

P02-014

FC 17, P01-168, P01-424, P01-547

P01-254

P02-156

P02-289

P01-212

02-512,

P02-515

P02-121

P02-282

P01-273

FC 20, P01-297, P01-347, P02-081, P02-141, P02-438

P01-157, P01-400

P02-191, P02-206

P01-157, P01-400, P01-413, P02-191, P02-206

P02-375

P02-503

P02-489

FC 16, P01-399, P02-051, P02-532

P01-313

P01-325

P02-555

P01-071, P01-488, P02-035, P02-103, P02-105, P02-168

$\mathrm{P} 01-462, \mathrm{P} 02-154$

$\mathrm{P} 02-393$

P01-409

P02-035, P02-096, P02-174

P02-203

P02-156

P01-249, P01-251

P01-249, P01-251

P02-325

FC

P02-343

$\mathrm{P} 02-048$

$\mathrm{P} 01-218, \mathrm{P} 01-330$

P01-224

FC 22, P01-555, P02-135

P02-115
Mazzotta P.

Mboulla M.

Mbozi P.

Mc Carthy S.

Mc Donald D.

McArdle M.

McAuley J.

McCaffrey N.

McCann M.-A.

McCaul J.

McCauley R.

McClement S.

McCloud P.

McConnell T.

McCormack B.

McCorry N.

McCoubrie R.

McCulloch $\mathrm{R}$.

McCullough J.

McDonald C.

McDonald R.

McDougall A.

McEnerney $\mathrm{N}$.

McGannon M

McGhie J.

McGinn G.

McGirr D.

McGlinchey T.

McGreevy B.

McGuigan E.

McGuigan $\mathrm{H}$.

Mclldowie B.

Mcllfatrick S.

Mclver J.

Mclvor K.

McKeaveney C.

McKeever S.

McKelvie J.

McKenna G.

McKeon M.

McLaughlin D.

McLintock S

McMahon E.

McNulty M.

McPherson M.L.

McQuillan R.

McVeigh J.

Mead G.

Meade R.

Meads D.

Medgyasszay F.B.

Meertens D.

Meesters S.

Mehnert-Theuerkauf A

Mehr D.R.

Meijers J.M.M.

Meijers M

Meini L.

Meireles E.C.d.A.

Meißner W.

Meissner W.

Mejdal S.

Mejon-Casas O.

Mele F.

Meléndez A.

Melia A.

Melin M.

Melin-Johansson C.

Mellon A.

Melnyk R

Mendes F.

Mendes Barreto Arantes A.

P02-175, P02-251

P01-006

P01-020

P01-375

P02-197

FC 2, FC 9

P02-197

P02-156

P01-218

P02-192

FC 9

FC 7

P02-486

P02-137, P02-138

P02-011

PPC 3, P01-048, P01-490

P02-046

FC 9

P02-269, P02-270

$\mathrm{P} 02-135, \mathrm{P} 02-274$

P01-064

P01-551

P02-078, P02-186

$\mathrm{P} 02-186$

P01-014

$\mathrm{P} 01-346, \mathrm{P} 02-136$

P02-039, P02-046

P02-398

P02-147, P02-148

P02-505

FC 19, P01-137, P01-340, P01-508, P02-137, P02-138,

P02-481, P02-556

$$
\text { FC } 3
$$

P02-394

P02-048

FC 19

$\mathrm{P} 02-412$

$\mathrm{P} 02-361$

ME 3, P02-501

P01-473, P02-525

P02-269, P02-270

P02-149

P02-363

PS 4, ME 10

P01-218, P02-185

P02-049

P01-469

P01-282, P01-332, P01-541

FC 8

P02-354

P02-319

FC 10, P01-107

FC 16

FC 10

FC 1, P01-507

P01-078

P02-382

P02-453

FC 6, FC 6, P01-230, P01-245, P01-309

P02-180

FC 14

P02-153

P01-503

FC 8

P01-376

P01-180, P02-503

FC 22, P01-100

P02-046

P01-229

P01-494 
Mendez A.

Merkle A.

Merriman A.

Merriman R.

Mertens F.

Messerer J.

Messum S.-J.

Metsänoja R.

Metselaar S.

Metsemakers J.

Metzenmacher $\mathrm{M}$.

Metzner S.

Meyburg J.

Meyer I.

Meystre C.

Miccinesi G.

Michael N.

Micheletti S.

Michler F.

Middelburg M

Middeljans L.

Mieras A.

Miguel S.

Mikkonen $\mathrm{H}$.

Mikołajczyk M.

Milerski S.

Milewski V.

Miller-Lewis L.

Millington S.

Mills M.

Mina M.

Minatel M.D.I.A.

Minello $C$.

Minosso J.S.M.

Minton $\mathrm{O}$.

Miranda R.

Mishra S.

Missala I.

Missel M.

Mitchell A.

Mitchell G.

Mitchell L.

Mitrea N.

Mitsimponas $\mathrm{N}$.

Miyamoto $\mathrm{S}$.

Miyashita M.

Miyazaki M.

Mize C.

Mizushima A.

Mjörnberg M.

Mochizuki M

Mockler D.

Modi M.

Modlińska A.

Modlinska A.

Moeda Adesla L.

Moeke-Maxwell T.

Moeschler S.

Mohammed K.

Moine $S$.

Moir G.

Molander U.

Molloy E.

Molloy U.

Momber S.

Monaco R.

Monaghan $\mathrm{C}$.

Monforte-Royo C.

Monnery D.

Monreal-Carrillo E.

Monroe B.

Monsen R.

Montag T.

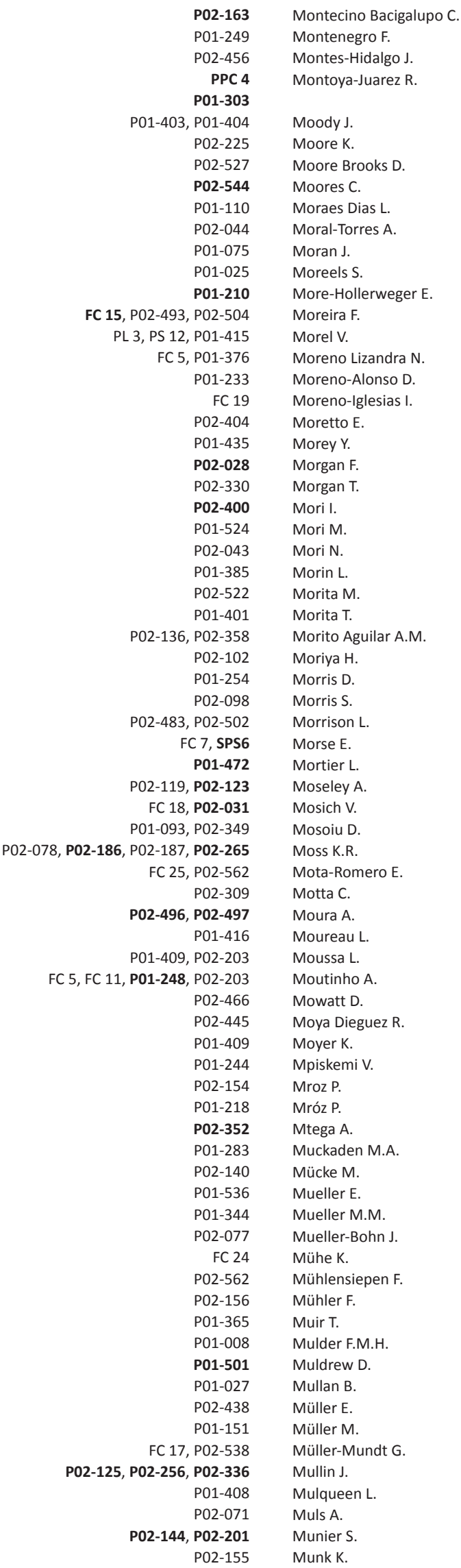

P02-163

P01-249

P02-456

PPC 4

P01-303

P01-403, P01-404

$\mathrm{P} 02-225$

P02-527

P02-544

P01-110

P02-044

P01-075

P01-025

P01-210

FC 15, P02-493, P02-504

PL 3, PS 12, P01-415

FC 5, P01-376

P01-233

FC 19

P02-404

P01-435

P02-028

P02-330

P02-400

P01-524

P02-043

P01-385

P02-522

P01-401

P02-136, P02-358

P02-102

P01-254

P02-098

P02-483, P02-502

FC 7, SPS6

P01-472

P02-119, P02-123

FC 18, P02-031

P01-093, P02-349

P02-078, P02-186, P02-187, P02-265

FC 25, P02-562

$\mathrm{P02}-309$

P02-496, P02-497

P01-416

P01-409, P02-203

FC 5, FC 11, P01-248, P02-203

$\mathrm{P} 02-466$

P02-445

P01-409

P01-244

P02-154

P01-218

P02-352

P01-283

P02-140

P01-536

P01-344

P02-077

FC 24

P02-562

P02-156

P01-365

P01-008

P01-501

P01-027

P02-438

P01-151

FC 17, P02-538

P02-125, P02-256, P02-336

P01-408

P02-071

P02-144, P02-201

P02-155

Montecino Bacigalupo C.

Montenegro $\mathrm{F}$.

Montes-Hidalgo J.

Montoya-Juarez $\mathrm{R}$.

Moody J.

Moore K.

Moore Brooks D.

Moores C.

Moraes Dias L.

Moral-Torres A.

Moran J.

Moreels S.

More-Hollerweger E.

Moreira F.

Morel V.

Moreno Lizandra N.

Moreno-Alonso D.

Moreno-Iglesias I.

Moretto E.

Morey Y.

Morgan F.

Morgan T.

Mori I.

Mori M.

Mori N.

Morin L.

Morita M.

Morita T.

Morito Aguilar A.M.

Moriya $\mathrm{H}$.

Morris D.

Morris S.

Morrison L.

Morse E.

Mortier L.

Moseley A.

Mosich V.

Mosoiu D.

Moss K.R.

Mota-Romero E.

Motta C.

Moura A.

Moureau L.

Moussa L.

Moutinho A.

Mowatt D.

Moya Dieguez R.

Moyer K.

Mpiskemi V.

Mroz P.

Mróz P.

Mtega A.

Muckaden M.A.

Mücke $M$.

Mueller E.

Mueller M.M.

Mueller-Bohn J.

Mühe K.

Mühlensiepen $\mathrm{F}$.

Mühler F.

Muir T.

Mulder F.M.H.

Muldrew D.

Mullan B.

Müller E.

Müller M.

Müller-Mundt G.

Mullin J.

Mulqueen L.

Muls A.

Munier S.

Munk K.

P01-188

P01-451

P01-157, P01-400

P01-171, P01-535, P01-536, P02-239, P02-242, P02-244,

P02-288

$$
\text { FC } 2
$$

PS 3, P01-132, P01-463

P01-044

P02-498

P02-436

$\mathrm{P} 02-243$

FC 25, P02-396

P01-082

P01-312

P01-502

P01-397

P02-094

P02-115

P02-153

P01-261, P01-422

P02-060

FC 7

P01-071

P01-117, P01-409

P02-466

PS 20

P01-439

FC 5, FC 11, P01-409, P02-203

FC 16

P01-225

P02-353

$P 02-495$

FC 3, FC 20, P02-426, P02-468, P02-473

P02-091

P02-003

P01-064

P02-200

FC 20, P02-496, P02-512, P02-515, P02-521

P02-409

P01-535, P01-536, P02-239

$\mathrm{P} 01-502$

P01-495

P01-410

P01-212

P02-093

P02-156

P01-535

FC 20

P02-448

P02-140

P01-283, P02-451

$\mathrm{P} 02-364$

P02-090, P02-271

P02-108

P02-487

$\mathrm{P} 02-043$

P01-075

FC 17

P01-195

P01-230

P02-156

P01-140

FC 19, P02-137, P02-138, P02-525, P02-556

P02-048

P01-568

FC 2, P01-403, P01-404

P01-477, P01-516, P02-491

P01-469

FC 18, P02-407

$\mathrm{P} 02-553$ 
Muramatsu M.

Murdock C.

Muriithi N.

Muro E.

Murphy A.

Murphy I.

Murphy M.

Murray A

Murray N.

Murray S

Mursi M.

Murtagh F.

Murua Escobar $\mathrm{H}$.

Muscheites W.

Musimenta R.

Mutabbir A.

Muwanguzi D.

Mwangi-Powell $F$

Mwari Rithara S.

Mwebesa E.

Myllärniemi M.

Mystakidou K.

Nadarajan G.D.

Naert E.

Nafae W.

Nagae $\mathrm{H}$.

Nagaoka H.

Nagashima R.

Nagelschmidt K.

Nakaema K.E.

Nakagawa S.

Nakajima E.

Nakajima K.

Nakajima N.

Nakamura M.

Nakanishi M.

Nakayama T.

Nalin Passarini J.

Nalutaaya F.

Namisango $E$.

Namora P.

Namukwaya E.

Nancarrow C.

Nandi S.

Nanni O.

Näppä U.

Nash S.

Nasridinov R.

Nauck F.

Naughton J.P.

Naulaers G.

Navarro Mingorance A.

Navarro Verdú P.

Navarro Vilarubi S.

Navarro-Sánchez A

Nazareth I.

Neergaard M.A.

Nekolaichuk C.

Nelson A

Nendaz M.

Neo H.Y.

Neo S.H.P.

Nethagani J.

Neto $\mathrm{R}$

Neukirchen M.

Neumann $\mathrm{N}$.

Neves $\mathrm{E}$.

Nevin J.

Nevin M.

Newman S.

$\mathrm{Ng} \mathrm{W}$.

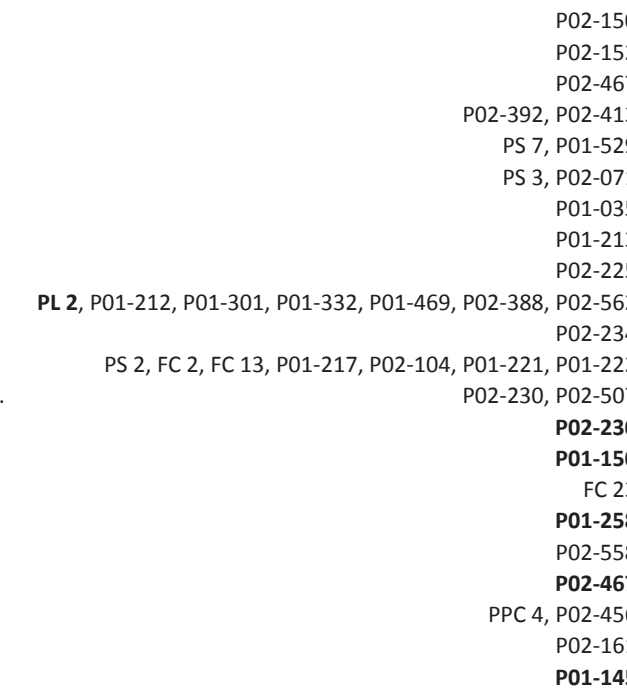

P01-087, P02-082, P02-272

EE 2

P02-101

P01-225, P02-509

$\mathrm{P} 02-174$

P01-367

PL 2, P01-109, P01-124

P02-393

P02-162

P01-462, P02-154

$P 02-154$

P02-198, P02-441

P01-439

P01-286

P01-462, P02-154

FC 22

P01-551

P02-561, P02-563

P01-046

FC 12, P02-388

P01-261, P01-422

P01-241

P01-233, P02-211

P02-055

P02-325

P02-223

PS 13, FC 6, FC 12, FC 17, P01-230, P01-424, P01-547

P02-273

PPC 2, P01-049, P01-052

P01-036, P02-517

P01-171, P02-288

$\mathrm{P} 02-517$

P02-239

P01-132

FC 12, PPC 5, P01-135, P01-299

P02-188

P01-114, P02-060, P02-553

P01-190

FC 4, P02-531

P01-051

P01-308

$\mathrm{P} 02-025$

FC 15, P02-454

FC 19

$\mathrm{P} 02-316$

FC 24

P02-399

P01-541

P01-459
Ng Y.H.

Ni Laoire A.

Ní Laoire Á.

Ní Nualláin A.

Nicholson C.

Nicholson E.

Nicolas-Casem M.E.

Nicolas-Robin A.

Nielsen D.

Nielsen M.K

Niemi-murola L.

Nienhaus A.

Niessen L.

Niezink D.M.

Nikander P.

Niki $\mathrm{K}$.

Nikoloudi M.

NiLaoire A.

Nilsson $\mathrm{F}$.

Nishi T.

Nishiyama N.

Nishizawa Y.

Nissim R.

Nizard J.

Nkhoma K.

Nkosi B.

Noakes S.

Noble A.

Noble B.

Noble $\mathrm{H}$.

Noble $\mathrm{S}$.

Noeske L.

Nogarede R.

Noguera A.

Nohavicka L.

Nolte-Buchholtz S.

Nomura K.

Noonan K.

Noor $\mathrm{N}$.

Noordam C.

Noorlandt H.W.

Norberg A.

Nordström M.

Norinder M.

Normand C.

Norris J.

Norwood S.

Notenboom M.

Nottelmann L.

Novis Y.A.

Nowicka-Sauer K.

Noyes J.

Nozato J.

Ntizimira C.

Numata $\mathrm{C}$.

Nunes B.

Nunes $T$.

Núñez García B.

Núñez Viejo M.

Nunziante F.

Nwosu A.C.

Nyblom S.

O' Mahony S.

O Reilly L.

$O$ Reilly M.

Obaid M.

Obenaus L.

O’Brien M.

$\mathrm{O}^{\prime}$ Callaghan $\mathrm{C}$.

O'Caoimh R.

O'Connor A.
P01-051

P01-484

PPC 4

P01-484

PS 2, P01-527

FC 19

P01-559

P02-268

P01-514, P01-548

PS 3, P01-135

FC 24

P01-250, P01-323

P01-528

P01-239

P01-071, P01-488

P01-145

P01-045

P01-331

P01-409

P02-168

$\mathrm{P} 02-445$

FC 16

P02-068

FC 23, P02-563

P01-035, P01-038

$\mathrm{P} 02-322$

P02-379

FC 4, P02-338

$\mathrm{P} 02-048$

P01-114, P01-223, P01-237, P02-221

P02-241

$\mathrm{P} 02-435$

P02-392, P02-413, P02-512

P01-012, P01-060

P01-069

FC 14, P01-337

P01-459

P01-058

FC 21

P01-500

P02-120

P01-148

P01-330

SPS6, SPS6, P01-070

P01-401

P01-431

P02-130

P01-019

P02-450

P02-375

P01-409

PL 5

P01-488

P01-316

P01-453

FC 16

P02-110

P02-537

P01-346, P02-135, P02-136, P02-358

P01-365

P02-049

P01-113

PPC 4, P01-484

P01-021

P02-015

FC 5, P02-375, P02-428, P02-429

FC 5, FC 15, P01-376

FC 3

P01-492 


\begin{tabular}{|c|c|c|c|}
\hline O’Connor L. & P02-116, P02-133 & Osborne T. & P01-120 \\
\hline O'Connor M. & P02-445 & O’Shea B. & P01-310 \\
\hline Oderbein S. & FC 2 & Osowicka M. & P02-140 \\
\hline Odili J. & P02-156 & Österlind J. & P01-085, P01-500 \\
\hline O’Donoghue D. & P02-048 & Ostgathe $\mathrm{C}$. & EC 19, FC 24, P01-075, P01-076, P01-341, P02-305, P02-306, \\
\hline O’Donoghue E. & P01-045 & & P02-387 \\
\hline \multirow[t]{2}{*}{ Oechsle K. } & C 17, FC 16, P01-168, P01-402, P01-403, P01-404, P02-045, & Osua S. & PS 12 \\
\hline & P02-183, P02-365 & O’Sullivan A. & P01-191 \\
\hline Oei S.L. & P01-251 & Otani H. & P02-203 \\
\hline Oertel M. & P02-297 & Ottolini L. & P02-403 \\
\hline Offner K. & P02-112, P02-495 & Ouchi S. & FC 5 \\
\hline Ogden M. & FC 19 & Oude Veldhuis S. & P01-184 \\
\hline O'Gorman A. & P01-219, P02-293 & Oulton K. & P01-033 \\
\hline O'Gorman B. & P02-070 & Ousi Z. & P02-488 \\
\hline Oh H.-S. & P02-111 & Oustalet A. & P01-083 \\
\hline Oh S. & P01-129, P02-061 & Ouvrard C. & P01-180 \\
\hline O’Hara B. & P02-508 & Owen M. & P02-186 \\
\hline O'Hare A. & P01-445 & Owens C. & P02-125 \\
\hline Öhlen J. & FC 25, P01-148, P01-191 & Owusu Appiah M. & P02-367 \\
\hline Öhlén J. & PS 16, P01-467, P02-385 & Owusuaa C. & P01-123 \\
\hline Ohmori T. & P01-450 & Owusu-Ansah M. & P02-367 \\
\hline Ohta T. & P02-129 & Owusu-Boakye S. & FC 12, P01-547 \\
\hline Ohvanainen A. & FC 24 & Oyamada S. & P02-096, P02-174 \\
\hline Oishi A. & P02-203 & Ozanne A. & P01-467, P01-496 \\
\hline Okada H. & P02-535 & Ozbič P. & P02-067 \\
\hline Okamoto Y. & P01-071, P01-488, P02-105, P02-162 & & \\
\hline Okamura H. & P02-168 & $\mathbf{P}$ & \\
\hline O'Kane R. & P02-290 & Paal P. & $\mathrm{P} 02-471, \mathrm{P} 02-484, \mathrm{P} 02-512, \mathrm{P} 02-515$ \\
\hline Okayama S. & FC 5, P02-105 & Pace V. & P01-527 \\
\hline Okon T. & P02-426 & Paczkowska A. & P02-059, P02-066 \\
\hline Olano-Lizarraga M. & P01-139 & Padmanabhan S. & P02-294 \\
\hline Olausson S. & P02-385 & Pairona G. & FC 20 \\
\hline Oldenmenger $\mathrm{W}$. & P01-336, P01-359 & Pais D. & P02-316 \\
\hline Oliete Ramírez E. & P01-491 & Pakai A. & P02-415 \\
\hline Olij B.F. & P01-199 & Palacio C. & P01-176 \\
\hline Oliveira G. & P01-324, P01-338 & Palat G. & P01-308, P02-459, P02-520 \\
\hline Oliveira M.A.C. & P02-483, P02-502 & Palfrey C. & P01-452 \\
\hline Oliver A. & P02-205, P02-207 & Palzer J. & P02-236 \\
\hline Oliver D. & P01-553, P02-054 & Panagiotou I. & P01-305, P01-307, P02-224 \\
\hline Oliver K. & P02-412 & Pancu A. & P02-215 \\
\hline Oliver P. & PS 19 & Pannuti F. & P01-138, P02-075 \\
\hline Olivier V. & P01-385 & Pannuti R. & P01-138, P02-075 \\
\hline Oloko R. & FC 24 & Panozzo S. & P01-542, P02-506 \\
\hline Olsen L.A. & P01-304 & Papadatou D. & PS 13, PPC 5 \\
\hline Olsen M. & PPC 5, P01-126 & Papageorgiou N. & P01-145 \\
\hline Olsman E. & P01-047, P01-381 & Paque K. & P01-486, P01-487 \\
\hline Olsson C. & P01-181, P02-326 & Pardo Sere J. & P02-030 \\
\hline Olsson Möller U. & P02-208 & Pardon K. & PS 12, EE 2, P01-486, P01-487, P02-027 \\
\hline Olthuis G. & P01-134 & Parhizkari S. & P01-230 \\
\hline Oluyase A. & FC 19 & Parinyajittha S. & P01-526 \\
\hline Omar R.Z. & P01-558 & Park J. & P01-368 \\
\hline Ong E.H.M. & P01-087, P02-082, P02-272 & Park K. & P01-368 \\
\hline Ong K. & P02-506 & Park S.M. & P01-178 \\
\hline Ong W. & P02-347, P02-360 & Park Y. & P01-368 \\
\hline Onisaki M. & P02-466 & Parker D. & PS 7, FC 10, FC 25, P01-243 \\
\hline \multirow[t]{2}{*}{ Onwuteaka-Philipsen B. } & PS 20, FC 23, P01-082, P01-515, P01-537, P02-028, & Parkinson A. & P02-131 \\
\hline & P02-217 & Parmar K. & P02-333 \\
\hline Oomes M. & P01-122, P02-391 & Parpa E. & P01-145 \\
\hline Oostendorp L. & FC 20 & Parr P. & P02-434 \\
\hline Opia V. & FC 12, P01-551 & Parra S. & P01-175 \\
\hline Opoku R. & P02-367 & Parsons C. & P02-290 \\
\hline Opsomer S. & P01-144 & Parsons H.A. & P02-279 \\
\hline Oravec M. & P01-499 & Partain D. & P01-456, P02-077 \\
\hline O’Reilly M. & PPC 5, P01-006, P01-008 & Pasanen L. & P02-396 \\
\hline Orellana-Rios C.L. & FC 6, P01-118, P01-232, P02-339, P02-565 & Pascu A. & P02-512 \\
\hline O’Riordan J. & P02-281 & Pascual A. & P01-292 \\
\hline Orlando A. & P02-156 & Pascual Lopez A. & P02-009 \\
\hline Ortega San Martin A.M. & P01-485 & Pascual Pla F.J. & P02-014, P02-030, P02-085 \\
\hline Ortega-Carrillo A. & P02-455 & Pask S. & PS 2, P02-104 \\
\hline Ortiz-San Roman L. & P02-517 & Pasman H.R. & FC 7, FC 10, P01-537, P02-028, P02-217, P02-446, P02-544 \\
\hline Ortmann $\mathrm{O}$. & FC 16 & Pastrana T. & FC 9, FC 12, P01-040, P01-254, P02-548 \\
\hline Orzechowski R. & P01-453 & Patel B. & PPC 4, P01-054, P01-490 \\
\hline Osaka I. & P02-035 & Patel M. & 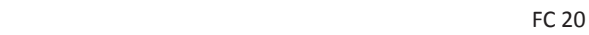 \\
\hline
\end{tabular}


Patel S.

Patiraki E.

Patterson A.

Paula S.

Paulsen $\varnothing$.

Pautex S.

Pawłowski L.

Payne C.

Payne S.

Paz E.

Pazart L.

Peacock M.

Peddinti P.

Pedruco A.

Peeters C.

Peinado Cañaveras M.C.

Peixoto Caldas J.M.

Pektas M

Pelgrum-Keurhorst M.

Pelin De Nardi S.

Pellini G.

Pelttari L.

Peña M.

Peña-Nieves A

Penders Y.W.H.

Peng X.-F.

Penttinen $\mathrm{H}$.

Perceau E.

Perceau -Chambard E.

Perdamaian T.

Pereira B.

Pereira C

Perez Algorta G.

Pérez Espina R.

Pérez Martín M.Á

Pérez-Diez del Corral M

Perez-Manrique T.

Perkins M

Perkins P.

Perneger T.

Perpiñá Galváñ J.

Perry M.

Perry R.

Peter S.

Peters E.A.

Peters J.

Petersen M.A.

Petitti T.

Petrova M.

Petruckevitch A.

Pettifer A.

Pettus K.

Petursdottir A.B.

Peuckmann-Post V.

Peuten S.

Pfaff $\mathrm{H}$

Pham T.-N.

Phan Hoang $\mathrm{N}$.

Phelan A

Philip J.

Phillips C

Phillips J.

Phillips M.

Phimister D.

Pichler B.

Pickett $\mathrm{S}$.

Pickles A.

Pidinchedda A.

Pieri M.

Pierre S.

Pieterse A.

Piette V.
01-197, P02-511, P02-512, P02-515

P01-354, P01-464, P01-525, P02-182

P02-195, P02-224, P02-252, P02-448

P01-201

P01-357

FC 16

FC 1, P01-515, P01-522, P02-540, P02-555

P01-283, P02-166, P02-451

7. PS 19, FC 10, FC 19, FC 23, P01-183,

P01-426, P01-515, P02-556

P02-222

FC 16, P01-320, P01-399, P02-051$$
\text { FC } 21
$$

P01-555

P01-046

P01-047

P01-485

01-101

P01-383

FC 18, P01-312, P01-427

P02-085

P01-408

P01-082, P01-513

P02-551

P01-239

P02-021, P02-107

P02-006, P02-188, P02-526

P01-301, P01-511

P02-523

P01-235, P02-316, P02-404

P01-429, P01-430, P01-525, P02-182

P01-268, P01-549, P01-550

P01-036

P01-139

P02-220

FC 24

P02-131, P02-343

P01-190

P01-171, P02-244

FC 8, P01-294

P01-558

P01-193, P01-194, P01-200

P01-199

FC 24

FC 4, FC 12, P02-130, P02-139

P01-347

P01-437

P02-204

P02-151

FC 9

FC 11

P01-072, P02-108

P01-231

P01-193, P01-194, P01-200

P01-295

P02-006, P02-526

P01-501

FC 14, FC 25, P01-240, P01-542, P02-396, P02-506

FC 4, FC 9, FC 22, P01-242, P01-243

P01-027, P01-043

P01-564

P01-329

P02-060

FC 13, P01-217, P01-221, P01-222

P02-457

$\mathrm{P} 01-233$

P02-080

P01-122

P01-052
Pietz J.

Piili R.

Pilastri P.

Pilkington G.

Pinheiro $P$.

Pino $M$.

Pinto $\mathrm{C}$.

Pinto $S$.

Pinto S.M.

Pinto Machado J.

Piolot A.

Piriz G.

Piscitelli F.

Pissarek A.

Pitman A.

Pivodic L.

Placido A.

Pleno P.

Pleschberger S.

Pluchino $\mathrm{P}$.

Podlesnik K.

Pohlkamp L.

Poi C.H.

Poláková K.

Polinder S.

Pölkki T.

Pollock K.

Pollok F.

Poolman M.

Poon Y.L.E.

Pop Csog B.

Popa C.

Popat S.

Pope A.

Popowicz N.

Poroch V.

Porta-Sales J.

Porter S

Portnoy S.

Porz J.

Poth A.

Pothmann R.

Potma M.C.

Potter J.

Pottle J.

Pouget J.

Pouymayou J.

Powell P.

Powell R.

Power E.

Power J.

Power L.

Pöyhiä R.

Pozsgai E.

Pralong A.

Prandi C.

Predoiu O.

Preisler M.

Prentice J.

Prentice W.

Preston N.

Prevezanou M.

Priestley S.

Pring A.

Privett-Cook $\mathrm{H}$.

Probert C.

Prod'homme C.

Prosser A.

Protogiros D.

Protz K.

Provencio Pulla M.
P01-066

P02-527

P01-545

P02-375

P02-145

FC 1

PS 2

P01-127, P01-371, P01-407, P01-521, P02-158, P02-545

P02-157

P02-093

P02-276

P02-163

P02-438

P01-427

P01-343

FC 10, P01-286, P01-513

P02-024

P01-046

PS 16, FC 25

P02-075

P02-067

FC 7, P02-058

$\mathrm{P} 02-347, \mathrm{P} 02-360$

FC 13, FC 17, P02-539

P01-199

P02-492

PS $12, \mathrm{P} 01-415$

P02-332

P01-541, P02-254

P01-051

P02-328, P02-329

P01-391

FC 24

P01-209, P02-401

FC 9

P02-512

FC 17, P02-243

FC 7, P02-048

P02-070

P01-253

P02-097

P01-002

P01-370, P01-384

FC 9

P02-254

P02-503

$\mathrm{P} 02-080$

P02-256

P02-558

P01-361

P01-218

P02-414

FC 24, P02-364

P02-510

FC 6, P02-173

FC 20

P02-512, P02-521

FC 18, P01-186, P02-031

FC 3, P01-446

P01-041

PS 15, PS 15, PS 19, FC 19, ME 6, P01-133,

P01-348, P01-354, P01-415, P01-426, P01-457, P01-464, P01-525, P01-563, P02-182, P02-341

P01-416

P02-394

FC 12, P01-322

$\mathrm{P} 02-269, \mathrm{P} 02-270$

FC 19

P02-003

P02-449

P02-448

$\mathrm{P} 02-173$

FC 16 
Provido M.

Psychogyiou A.

Puente-Fernàndez D.

Puertas Martín V.

Pugh C.W.

Puls N.

Pungchompoo S.

Pungchompoo W.

Purewal G.

Putnam A.

Pype P.

Pytlak C.

Pyykkönen T.

Oin Y.

Quaglino P.

Quah S.C.D.

Quartarone M.

Queiroz J.C.

Quenot J.P.

Quiles B.

Quinn C.

Rabbitte M.

Raccichini M.

Radbruch L.

Radlanski K.

Radley A.

Radoslav N.

Radwany S.

Rahim S.

Rahko E.

Rahmah R.

Raijmakers N.

Raijmakers N.J.H.

Rainsford S.

Rait G.

Raja Casillas I.

Rajagopal M.R.

Rajala K.

Rajapakse D.

Rakbek J.

Ramakrishnan K.

Ramaswamy A.

Ramelet A.-S.

Rametta M.

Ramon Codina M.

Ramos Gonzalez M.

Ramos-Guerrero J.

Ramsay J.

Ramsenthaler C.

Rao S.

Raoux N.

Rasmussen B.

Rasmussen B.H.

Rauh S.P.

Raunkiær M.

Ravensbergen W.

Ravindrarasan $\mathrm{S}$.

Rawlings D.

Rawlinson F.

Rayala S.

Rayens M.K.

Reaper Reynolds S.

Rebello Bezerra M.

Redondo P.

Redondo Moralo M.J.

Reed K.

Reed L.

Reedy F.
$\mathrm{P} 01-474, \mathrm{P} 02-108, \mathrm{P} 02-548$

P02-531

$\mathrm{P} 02-195$

P01-535, P02-239, P02-242, P02-244

P01-036

FC 11

P02-365

P01-526

P01-526

FC $12, \mathrm{P} 01-551$

P02-469

PS 2, PS 12, FC 3, FC 10, P01-144, P01-303, P01-513

FC 3
-239

P01-239

P01-288

P02-156

P01-051

P02-382

P01-502

P01-273

$\mathrm{P} 02-085$

, P01-006

P01-218, P01-562, P02-501

P01-017

P02-265

P02-555

P01-499, P02-426

P01-459

P02-017

P01-363

FC 13, P02-390

$\mathrm{P} 02-404$

FC 1, P01-531

P02-110

PL 1, PS 14

P02-161

P01-044

PPC 2

P02-294

P01-505

P02-226

$\mathrm{P} 02-506$

FC 20, P01-347, P02-438

$\mathrm{P} 02-328, \mathrm{P} 02-329$

P01-040

P02-298

FC 2, P02-029, P02-040

P02-271

P01-180

P02-208

FC 4

FC 10

P01-293, P01-423, P01-548, P01-556

P02-391

P02-099, P02-281

P02-498, P02-522

P01-466

P02-459, P02-520

$$
\text { FC } 11
$$

P02-285

$\mathrm{P} 02-436$

P02-320, P02-331

P02-328, P02-329

$\mathrm{P} 02-312$

P01-216, P02-472

$\mathrm{P} 02-366$
Rees S.

Reeves E.

Regan J.

Regel Y.U.

Rego G.

Reichmann J.

Reid J.

Reigada C.

Reilly C.

Reilly L.

Reimann D.

Reinke L.

Reis Carreira N.

Reitinger $\mathrm{E}$.

Relf M.

Rema J.

Remi C.

Remke S.

Renard C.

Rente J.

Renz M.

Reschke F.

Ressner P.

Restuccia M.R.

Reuther M.

Reuvers E.

Revol A.

Reyes E.

Rezgiene L.R.

Rhee J.Y.

Rhee Y.

Rho H.

Ribbers S.

Ricciardi F.

Riccobono R.

Rice B.

Richard B.

Richard M.

Richards C.

Richards N.

Richardson $\mathrm{H}$.

Richardson L.

Ricou B.

Rief W.

Rieger S.

Riemer $\mathrm{M}$.

Riera-Tornés G.

Rietjens J.

Rietz C.

Rigaud A.-S.

Riglietti A.

Riklikienè $\mathrm{O}$.

Riley J.

Ristau P.

Ristimäki K.

Ritchie A.

Ritchie L.

Rizza K.

Roa Mompó J.

Robbie F.

Robbins S.

Roberts A.

Roberts C.

Roberts D.

Roberts J.

Robertson N.

Robijn L.

Robins A.

Robinson C.A.

Robinson J.

Robinson S.

Roch C.
P02-253, P02-346

P02-371

FC 7

FC 15

P02-476

P01-253

FC 7, P01-473, P02-048

FC 8, P01-121

P02-099, P02-149, P02-281

PPC 2, P01-057, P01-061

P01-445

P01-119

P01-329

PS 3, PS 3, ME 8

$\mathrm{P} 02-418$

PS 11, P01-489

P01-392

ME 14

P02-170

PL 2

P01-012

P01-460

P02-081

P01-010

P01-009

FC 1

P02-207

P02-318

P01-339, P02-566

P01-286

P02-175, P02-251

P01-010

FC 20

P01-382

FC 21

P01-306

P01-385

P01-445

FC 14

P01-527

P02-358

P01-190

PL 2, P01-109, P01-124

P01-250, P01-323

P01-186

$\mathrm{P} 02-243$

PL 3, PS 12, FC 10, FC 25, P01-116, P01-169, P01-183, P01-336,

P01-359, P01-415, P01-448, P01-449, P02-160

FC 6, P01-211

FC 1

PS 15

P01-364

P02-334

P02-001

P01-239

$\mathrm{P} 02-442$

$\mathrm{P} 01-212$

P01-313

P02-014

FC 8

P01-223

FC 11

P01-541

P01-429, P01-430

P02-274

P02-475

FC 10

P01-529

P02-007

P01-165, P01-174

FC 3

P02-236, P02-241, P02-315, P02-321 
Rocha J.A.

Rocha T.

Rocha V.

Rochmawati E.

Rodin G.

Rodin R.

Rodrigues A.

Rodrigues A.P.

Rodrigues M.

Rodriguez Mesa D.

Rodríguez Pérez $\mathrm{B}$.

Rodríguez-Mayoral O.

Rodríguez-Morera A.

Rodríguez-Nuñez A.

Rodríguez-Ortíz B.

Rodriguez-Prat A

Rodríguez-Prat $A$.

Roe B.

Roelens K.

Roets E.

Roganti D.

Rogers S.

Roggeveen B.

Rognsvåg $\mathrm{M}$.

Rogozea L.

Rohde M.

Rohrmoser A

Roikjaer S.G.

Rojas-Concha L.

Rokhanskiy A

Rola Morilla G.L.

Rolke R.

Romeo A.

Romero Gabino V.

Romianowska E.

Romianowska J.

Rommel A.

Romotzky V.

Roncucci P.

Roper J.

Rosenberg J.

Rosens E.

Roser T.

Ross $\mathrm{C}$

Ross $\mathrm{H}$.

Ross L.

Rossi R.

Rostoft S

Rotger A

Rother $\mathrm{M}$.

Roughneen S.

Roulston G.

Roumeliotou A.

Roumiguiere $\mathrm{C}$

Rowe $\mathrm{B}$.

Rowett D.

Rowland C.

Royce $\mathrm{C}$.

Royer-Garabige V.

Roze des Ordons A

Ruano Raviña A

Rubio L.

Rubli Truchard E.

Rudilla D.

Rudolph A.

Ruellan A.

Ruer $\mathrm{M}$.

Ruivenkamp J.

Ruiz P.

Ruiz de Gaona Lana E.

Rullán M.

Rumbold B.

Runa D.

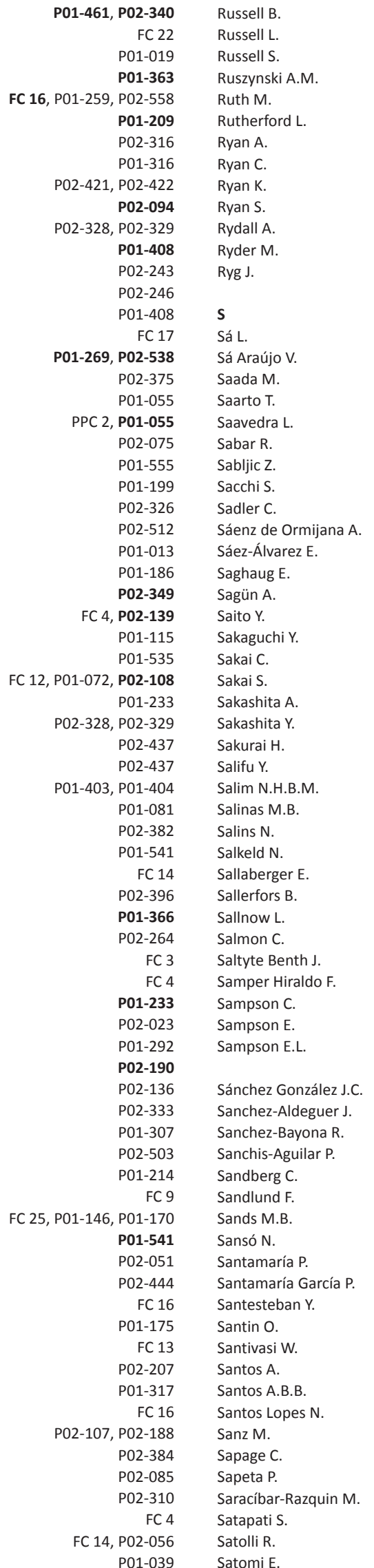

002-192

P01-360

P02-339

P02-116

P01-473

P02-481

P01-546

P01-330, P02-116, P02-133

P02-175

FC 16, P02-558

P02-398

P01-514

P01-314

P02-093

FC 3

P01-239, P02-017, P02-161, P02-164, P02-464

P02-409

P02-171

$\mathrm{P} 01-383, \mathrm{P} 02-537$

$\mathrm{P} 02-472$

FC 8

$\mathrm{P} 02-014$

P02-144, P02-201

P02-072, P02-073

FC 11

P01-439

P01-225, P02-509

P02-174

P01-367

P02-203

P01-260

P01-141

P01-444

P02-271

P01-317

P02-015

P02-278

PS 14, ME 12, P01-327

P02-149

$\mathrm{P} 02-023$

$\mathrm{P} 02-030$

P01-114

P01-243, P01-479

PS 3, FC 1, P01-132, P01-267, P01-327, P01-463,

P01-531

FC 16

P02-455

$\mathrm{P} 02-413$

P02-259

P02-120

P02-488

P02-216, P02-225

P02-205, P02-207, P02-344

P02-085

P02-030

FC 4, P02-246

P01-151

P02-077

P01-039

P02-340

P02-394

P01-444

P01-371

P01-235

P01-139

P02-123

P02-534

FC 15, P01-069 
Saunders Z.

Saurman E.

Saussac C.

Savage E.

Sawatzky R.

Sawyer J.M.

Scandone S.

Scarpi E.

Schablon A.

Schablon A.S.

Schad F.

Schade F.

Schaekel U.

Schaepe C.

Schallenburger M.

Schallner J.

Schäper S.

Schaperdoth E.

Schaufel M.A.

Scheer M.

Scheerens C.

Schefold J.

Schelin M.E.

Schell M.

Schellenberger S.

Schenell R.

Schenker Y

Scherer F.

Scherg A.

Scherrens A.-L.

Schers $\mathrm{H}$.

Schildmann E.

Schildmann J.

Schillinger D.

Schimmer A

Schindler C.

Schippel N.

Schlesinger A

Schlieper D.

Schlösser K.

Schmidt C.

Schmidt M.

Schmidt P.

Schmidt R.

Schmidt S.

Schmiegelow $\mathrm{K}$.

Schmitt T.

Schmitz A.

Schneider N

Schneider W.

Schnell M.W.

Schoensteiner S.

Schofield G.

Schofield S.

Schols J.M.G.A

Scholten $\mathrm{N}$.

Scholz C.

Schønau M.N.

Schoof-van der Ham S.

Schoonheim-Lunenborg $\mathrm{H}$

Schouten-van Meeteren A.Y.N.

Schrikker T.

Schroeder $\mathrm{H}$.

Schroer B.

Schubert I.

Schuchter P.

Schuetz C.

Schuiling-Otten $\mathrm{M}$.

Schuler M.

Schultz T.

Schulz $\mathrm{H}$.

Schulz S.

Schulz-Kindermann F.

$\begin{aligned} \text { P01-356 } & \text { Schulz-Quach C. } \\ \text { FC 2 } & \text { Schütz A. } \\ \text { P02-113, P02-503 } & \text { Schuurmans J. } \\ \text { P02-314 } & \text { Schwabe S. } \\ \text { P01-467, P02-194 } & \text { Schwartz J. } \\ \text { P01-327 } & \text { Schweighoffer R. } \\ \text { P01-503 } & \text { Schweitzer M. } \\ \text { P02-211 } & \text { Scott A. } \\ \text { P01-323 } & \text { Scott D. } \\ \text { P01-250 } & \text { Scott H. } \\ \text { P01-249, P01-251 } & \text { Scott M. } \\ \text { FC 12, P01-547 } & \text { Scott R. } \\ \text { FC 6, FC 16, P01-232 } & \text { Seabra S. }\end{aligned}$

P01-353 Seah D.

FC 15, P02-454 Secundo I.

P01-012 Seddon K.

P01-565 Seibel K.

P02-173 Seifart C

FC 22 Seiger Cronfalk B.

P01-013 Seipp H.

FC 3 Sejersen T.

P01-568 Selbæk G.

FC 4 Selman L.

P01-162 Semlali I.

FC 19 Senderovich H.

P01-496 Seppälä S.S.

P02-304 Serafim J.A.

P01-190 Seres D.S.

FC 15, P02-454 Serey A.

P01-097 Serrano A.

FC 8 Serrano $C$

PS 2, FC 10, P01-107, P01-236 Serrano Pons J.

P01-236, P01-281 Serrano-Bermúdez G.

PS 12 Sersa G.

FC 16 Sewtz C.

P01-318 Seymour J.

FC 6, P01-211 Shabnam J.

P02-368 Shadat R.

FC 15 Shamieh O.

P02-036 Shanagher D.

P02-354 Shanahan E.M

P02-031, P02-425 Shapoval-Deinega K.

P01-005 Sharma M.P.

P02-297 Shaw T.

P01-118, P02-507 Sheldrick M

P01-126 Sheu S.-J.

P02-321 Shi K.

P01-411, P02-238 Shi Y.

P01-296, P01-341, P01-351, P01-477, P01-516 Shibata K.

P01-196, P01-230, P01-231 Shida H.

P02-542 Shields J.

FC 6, FC 16, P01-232 Shima Y.

FC 17, P01-223 Shimizu M.

P02-125, P02-256 Shin S.-H.

FC 1 Shulman C.

P01-193, P01-194, P01-200 Shyu C.-H.

P01-060 Sichi V.

P02-349 Sickmüller Nunes F.

P01-122 Siddig S.

P02-319 Sidgwick P.

P01-067 Siegle A

P02-275 Siemens W.

PPC 5 Sigilipoe M.A.

P01-565 Sigurdardottir K.

P01-210 Sigurdardottir V.

P01-289, P02-406, P02-541 Silbermann M.

P02-045, P02-183 Sills E.

P01-030, P01-031, P01-058 Silove L.

P02-044 Silva C.

P01-454 Silva N.

P01-403, P01-404 Silva Moreira Macari K.

Silva Soares D.

Silveira A

$\mathrm{P} 01-403, \mathrm{P} 01-404$
P01-411, P02-238, P02-240

$\mathrm{P} 02-180$

P02-524

P01-112, P01-342

FC 15, P02-454

P02-371

P02-549

P02-125

P01-464

P02-060

$\mathrm{P} 02-475$

FC 14, ME 8, P01-425, P01-427

P01-046

FC 5

FC 3

$\mathrm{P} 02-060$

FC 6, P01-224

P01-109

P01-080

P02-218

PPC 2

$\mathrm{P} 02-023$

FC 17

P01-094

P01-440

P02-161

P02-048

P02-526

$\mathrm{P} 02-115$

P01-292, P02-344

$\mathrm{P} 02-233$

P02-115

P02-156

P02-230

PPC 4, P01-142, P01-183

P01-548

P02-311

PS 17

P01-310, P02-285

P01-465

P01-115, P01-290, P02-223

P01-412

$$
\text { FC } 9
$$

P02-209

P01-086

FC 19

PS 12

P02-150

P02-150

P02-048

FC 11

FC 15, P01-069, P01-069, P02-154

P02-111

FC 21, ME 2, P01-543

P01-192

P02-075

P02-476

P02-234

P01-044

P01-089, P01-092, P02-389

FC 6, FC 16, P01-232, P02-112

P01-511

FC 22, P02-185, P02-188

FC 11, P01-470

P01-315

P01-216

ME 14

P01-521

P02-316

P02-121

P01-039 
$\operatorname{Sim}$ A.

Sim D.K.L.

Sim L.K.

Simanek R.

Simion A.

Simmross I.

Simões $C$.

Simon A.

Simon S.

Simon S.T.

Simonds L.

Simpson S.-A

Sinclair $\mathrm{S}$.

Singh $P$

Singh R.

Singy $P$.

Sinnarajah A.

Sireci F.

Sistermanns J.

Sithole L.

Sithole Z.

Sivell S.

Sjøgren P.

Skellett $\mathrm{S}$.

Skevington S.

Skinner E.

Skjelvan L.

Skliros S.

Skov Zellweger B.

Škutová M.

Slaaen J.

Slater P.

Slee A.

Sleeman K.E.

Sliwa T.

Smaling $\mathrm{H}$.

Small C.

Smets T.

Smirdec M

Smith A

Smith C.

Smith $\mathrm{E}$.

Smith J.

Smith L.

Smith P.

Smith V.

Smits $C$.

Sneesby L.

Snook A.

Snow V.

So W.K.W.

Sobanski P.

Sobral M.A.

Soler D.

Somerville L.

Sondaal S.F.V.

Song E.-K.

Sono $\mathrm{S}$.

Sorensen A.

Soriano Soto M

Sotoudeh S.

Soutar D.

Spangenberg $\mathrm{K}$.

Spencer K.

Spencer S.

Spichiger $F$.

Spickermann $M$

Spiegel A.

Spiller J.

Spineli V.M.C.D.

Spirgiene L.

Spiru L.

Spronk I.

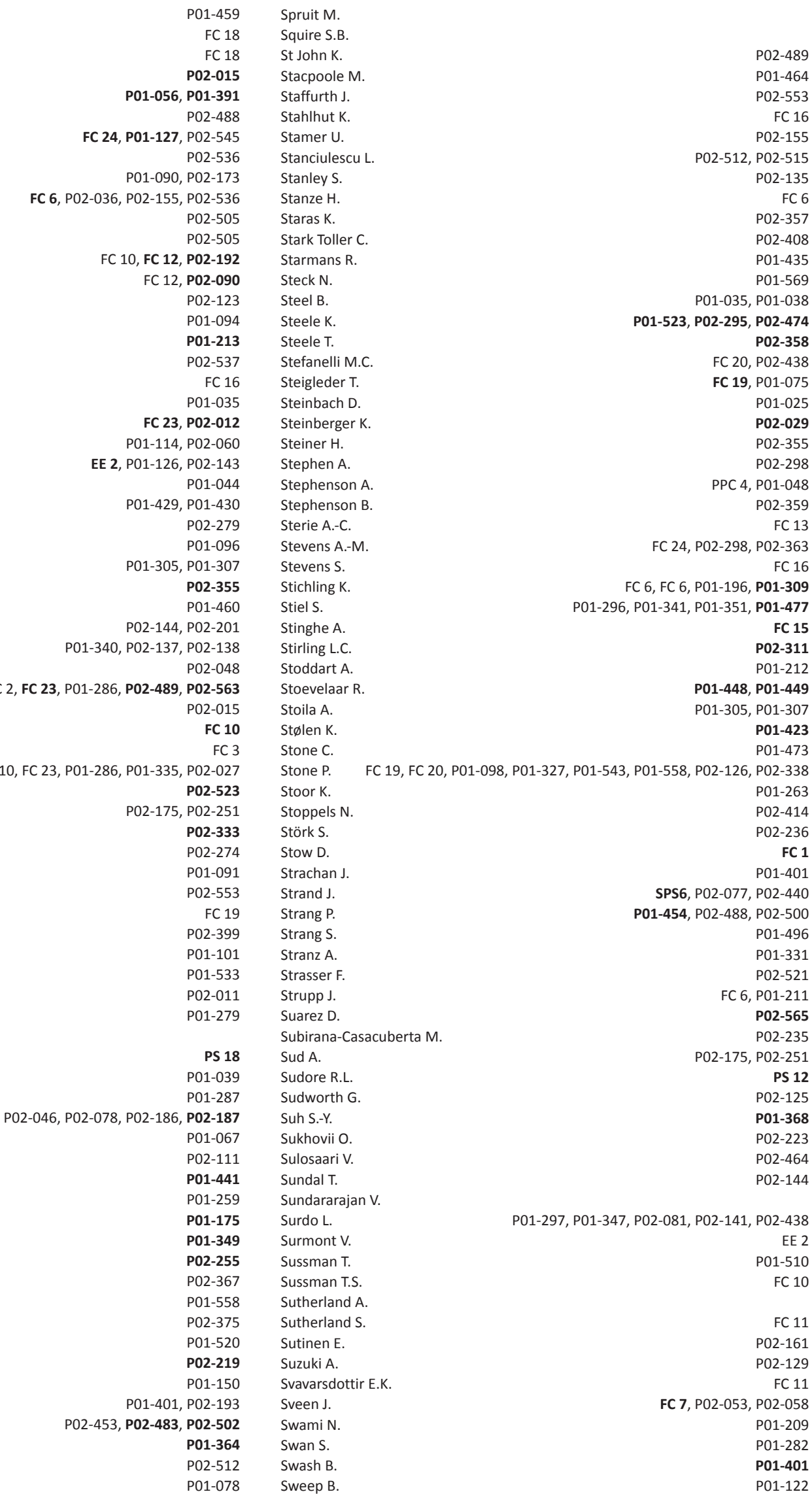


Sym S.J.

Symons X.

Szczerbińska K.

Tabak R.

Taberner-Bonastre M.T

Tagami K.

Taggart L.

Taglini A.

Tahmasebi M.

Takács S.

Takada $\mathrm{H}$.

Takagi T.

Takahashi H.

Takahashi Z.

Takenouchi S.

Taltavull J.M.

Tamerus R.

Tamura K.

Tan A.

Tan C.

Tan H.L.

Tan L.

Tan L.L.

Tan S.B.

Tan Y.P.

Tan Y.Y

Tan You G.

Tanaka A.

Tanaka I.

Tanaka T.

Tang S.T.

Tanigaki S.

Tapp A.

Tarniceru C.C.

Tarot A.

Tarraso-Gomez L.

Tarraso-Gomez M.L.

Tasmuth T.

Taubert M.

PL 4, FC 7, FC 20, SPS6, SPS6, P01-070, P01-084, P01-279, P01-317,

P01-360, P02-004

Tavares F.

Tavares M.

Tavares $\mathrm{T}$.

Tay R.Y.

Taylor A.G.S.

Taylor B.

Taylor J.

Taylor L.

Taylor T.

Tebidze N.

Teike Lüthi F.

Teixeira C.M.

Teixeira M.J.C.

Templeton L.

Templier C.

Ten Koppel M.

Ter Horst - van Oord M.E.

Terheggen $\mathrm{F}$.

Terzi K.

Tetzlaff F.

Teufel M.

Teunissen S.

Teunissen S.C.C.M.

Tewani K.

Tewes M.

Thakrar S.

Thalgi M.

Theochari M.

Theoneste $\mathrm{N}$.

Theuns D.A.M.J.

Thimm A.

P02-111

P01-376

FC 10, FC 23, P01-515

P01-435

P02-196, P02-258, P02-259

P01-450, P02-096

P01-088

P01-503

P01-349

P02-245

FC 15, P01-069

P01-071

P02-072, P02-073

P02-509

P02-509, P02-535

$\mathrm{P} 02-344$

P01-227, P01-238, P01-358

FC 5, P02-509

$\mathrm{P} 01-141$

P02-086

$\mathrm{P} 02-360$

FC 18, P01-141

P01-459

P02-076

P01-051

P01-051

P02-456

P01-439

P01-488, P02-105

P01-439

P02-057, P02-301

P02-509

P01-261, P01-422

P02-512

P02-109, P02-177

P02-196, P02-259

$\mathrm{P} 02-258$

FC 13

P02-418

P02-418

FC 9, P01-458

P02-197, P02-442

P01-165

P01-029

P01-445

P02-358

$\mathrm{P} 01-377$

P02-226

P02-362

P01-147

P02-003

P01-286

P01-166, P02-277

P01-199

P02-002

P01-516

$\mathrm{P} 02-044$

P01-256, P01-362, P01-561, P02-167, P02-249, P02-250

$\mathrm{P} 01-370, \mathrm{P} 01-386, \mathrm{P} 01-384$

P02-560

$\mathrm{P} 02-044$

FC 23

P01-161

P01-168

P02-079

P01-448, P01-449

$\mathrm{P} 01-565$
Thit Johnsen A

Tho L.M.

Thoma M.

Thomas K.

Thomas M.

Thomas S.

Thompson C.

Thompson G.

Thompson G.T.

Thomsen I.H.

Thomson G.

Thorsnes S.L.

Thronicke A.

Thurn T.

Thygesen L.C.

Thyson T.

Tielen L.

Tieman J.

Tienken M.

Tierney S.

Tilburgs B.

Tilburt J.

Tilma M.

Tils $\mathrm{M}$.

Timm H.

Timm H.U.

Timmermann M.

Timmesfeld N.

Timmins L.

Timmons S.

Tio $\mathrm{N}$.

Tipping G.

Tishelman C.

Tiwari A.K.

Tkachenko O.

Toccafondi A.

Todd A.

Todd C.

Todd J.

Todd S.

Todd-Fordham F.

Tofani R.

Toft S.

Tojar Grisolia T.

Tokoro A.

Tollisen A.

Tomas J.

Tomás J.M.

Tomás-Sábado J.

Tomeny E.

Tomkevičiūtè J.

Tomlinson C.

Tomlinson S.

Tonoki Y.

Tontini L.

Topp J.

Törnquist $\mathrm{K}$.

Toroshima T.

Torres Cavazos J.L.

Torres Royo L.

Torrijos-Pastor A.

Torta R.

Tosi C.

Tosi M.C.

Touzet L.

Tracey G.

Tremellat F.

Tretiakowa I.

Treutlein M.

Trevisani D.

Tricou C.

Trimaille $\mathrm{H}$.

Tripodoro V.
PL 3

P02-076

P02-384

FC 25, P02-322, P02-323, P02-324

FC 16, P01-089, P01-092, P01-224, P02-387, P02-389

P01-218, P01-237

P01-315

P01-510

FC 10

FC 14

P02-264

P01-304

P01-249, P01-251

P02-530

FC 12

P02-454

P01-566

PS 7, P01-073, P01-074, P01-534, P02-303, P02-498, P02-522

FC 16

$\mathrm{P} 02-279$

P02-524

$\mathrm{P} 02-077$

$\mathrm{P} 02-308$

$\mathrm{P} 02-108$

P01-093, P01-264, P01-321, P01-548, P02-348, P02-369

P02-349

FC 5

P01-109

P01-343

PS 7, P01-529

P01-047

P01-219

P01-263, P01-331

P02-090, P02-271

P01-290

P01-415

PS 4

P01-558

P01-216, P02-472

PS 6, P01-088

P01-558

P02-382

P02-285

FC 16

P02-174

P02-144

P02-452

P02-207

P01-157, P01-400

P01-364

FC 20

P02-343

P01-439

P01-233

P02-351

P02-208

$\mathrm{P} 02-393$

P02-306

P02-094

P02-196, P02-258, P02-259

$\mathrm{P} 02-092, \mathrm{P} 02-457$

P02-457

P01-502

P02-003

P02-345

P02-268

P01-013

FC 17

P02-121

P02-006, P02-021, P02-107, P02-185, P02-188, P02-526

P01-320

FC 22 
Triscott J.

Trocan L.

Trujillano-Cabello J.

Tryphonos A.

Tsai H.-B.

Tserkezoglou A.

Tsilika E.

Tsotakos S.

Tsuji K.

Tsuneto S.

Tsunoda N.

Tučková A.

Tuca Rodríguez A.

Tucker S.

Tuen Hansen M.I.

Tuesen L.D.

Tuffin $P$.

Tuffrey-Wijne I.

Tuil W.

Tulloch L.

Tunnard I.

Tupper L.

Turgut Ö.

Turner M.

Turyahikyio J.

Tweya $\mathrm{H}$.

Twiddy M.

Twomey M.

Tybor D.

Tymoshevska V.

Tyynelä-Korhonen K.

Udo C.

Uebach B.

Uehara Y.

Uejima E.

Uemori M.

Uike $\mathrm{N}$.

Uitdehaag $\mathrm{M}$.

Ullrich A.

Ulrich $S$.

Unseld M.

Urch C.

Urdiroz J.

Urtila P.

Urzay V.

Usborne C.

Utarini A.

Utriainen $\mathrm{M}$.

V

Vadeboncoeur C.

Valencia-Gil S.

Valle A

Vallianatos S.

Van Audenhove C.

Van Belle S.

Van Bennekom D.

Van Bruchem-Visser R.L.

Van Buiren M.

Van Bussel K.

Van Cauwenberg J.

Van de Geer J.

Van de Graaf G.

Van de Poll-Franse L.

Van de Water C.R.T.

Van Delden $\mathrm{H}$.

Van Delden J.J.M.

Van den Berg B.

Van den Block L.

Van den Boogaart V.
$\mathrm{P} 02-480$
$\mathrm{P} 01-007, \mathrm{P} 01-016, \mathrm{P} 01-053$

P02-084, P02-087, P02-088

P01-158

P01-433

P02-224, P02-252, P02-448

P01-145

P02-448

P02-162

P02-035, P02-072, P02-073

P02-162

FC 13, FC 17, P02-248, P02-539

$\mathrm{P} 02-110$

P01-212

FC 22

P01-518

FC 9, P01-483

PS 6, FC 17, P01-088

P01-058

P02-505

$\mathrm{P} 01-480$

P02-397

PS 10, P01-149, P01-421, P02-311, P02-356

P02-388

$\mathrm{P} 02-313$

P02-038

, PPC 4, PPC 5, P01-006, P01-008, P01-484

P01-326

P01-115, P01-290, P02-223

P02-017

P01-011, P01-100

P02-173

P01-409

P01-071

P02-072

P02-466

P01-101, P01-184

FC 17, EE1, P01-168, P01-402, P01-403, P01-404, P02-365

P02-424

P02-289

P01-189

$\mathrm{P} 02-392$

P02-512

P01-444

P02-254

P01-163, P01-164, P02-202

P01-239

P01-004, P01-009

P01-139

P02-141

P01-030, P01-058

P01-182

EE 2, P02-027

$\mathrm{P} 02-544$

P01-448, P01-449

P01-025

FC 8

ME 16

P01-566

P02-390

P01-106

PL 3

PS 12

P01-199

PS 7, PS 8, FC 10, FC 23, P01-082, P01-286, P01-334, $\mathrm{P} 01-472, \mathrm{P} 01-513, \mathrm{P} 01-515, \mathrm{P} 02-237$
Van den Brink J.

P01-389

Van den Broek K.

Van Den Noortgate N.

Van der Ark A.

Van der Baan F.

Van der Baan F.H.

Van der Kleij R.M.J.J.

Van der Lent L.G.G.

Van der Linden $Y$.

Van der Maaden T.

Van der Padt-Pruijsten A.

Van der Rijt C.C.D.

Van der Sande R.

Van der Smissen D.

Van der Stap L.

Van der Steen J.T.

Van der Velden A.W.G.

Van der Vorst M.J.

Van der Weegen $\mathrm{K}$.

Van der Werff G.F.M.

Van Dongen $\mathrm{S}$.

Van Driel A.G.

Van Dulmen S.

Van Eijk M.

Van Erp J.

Van Esch H.J.

Van Esch T.E.M.

Van Gemert-Pijnen J.E.W.C.

Van Gurp J.

Van Hooft S.M.

Van Klinken $\mathrm{M}$.

Van Lander A.

Van Leen $\mathrm{M}$.

Van Lindert A.

Van Lune P.F.

Van Marion P.

Van Noord-Stijlen J.

Van Nus J.L.

Van Nus-Stad J.L.

Van Oorschot B.

Van Rijn M.

Van Roij J.

Van Schaik M.

P02-427

P01-513

P01-227, P01-238, P01-358

P01-256, P01-362, P01-561, P02-167, P02-249, P02-250

P01-386

P01-438

P01-123

P01-122, P01-199, P02-391

FC 10

P01-199

P01-117, P01-123, P02-160, P02-291

P01-300

P01-116

P02-391

FC 1, FC 10, P01-243, P01-473, P01-507, P01-537, P02-212, P02-217, P02-199

P02-296, P02-300, P01-106, P02-384 P01-199

FC 5

P01-106, P02-384

P01-336

FC 25, P01-169

P01-078, P01-116

P01-274

P01-435

P02-160

P02-296

P01-116

FC 7, P01-134

FC 7

P01-256, P01-362, P01-561

P01-397, P02-109, P02-177, P02-523

P01-435

P01-078

P02-384

P02-319

P01-122

P01-389

P01-166, P02-277

FC 16, P02-236, P02-241, P02-315, P02-321

P01-140, P01-528, P01-530

P02-390

$\mathrm{P} 02-544$

Van Staa A.

Van Tol D.

Van Veldhuisen D.J.

Van Vliet L.

Van Wesemael Y.

Van Wijngaarden J.

Van Zanten S.

Van Zuylen L.

Vanbutsele G.

Vandenbogaerde I.

Vander Stichele R.

Vanderlinden $\mathrm{K}$.

Vanderpuye - Donton N.A.

Vanderstichelen S.

Vanini G.

Vaquero-Cruzado J.

Varani S.

Vardy J.

Varela-Cerdeira M.

FC 7
$01-275$

P01-443

PS 2, FC 13, P01-078

PL 4, P01-420

FC 7

P01-009

P02-160

EE 2

P01-182, P01-183

P01-486, P01-487

P02-226

$\mathrm{P} 02-374$

PL 4, P01-420

P02-403

P02-455

P01-138, P02-075

$\mathrm{P} 02-216$

P02-220

ME 13

Vasconcelos de Matos L.

P02-135

P02-024

$\mathrm{P} 02-173$

P01-188

P01-082

Vega Alonso A.T.

Vehmanen L.

Vejlgaard T.B.

Veldhoven C.

Velijanashvili M. 
Veltman P.G.

Vemuri S.

Venkateswaran C.

Vennat B.

Verbeek L.M.H.

Verberne L.M.

Verbree P.P.

Verdet M.

Verga S.

Vergara López S.

Vergroesen $D$.

Verhaert R.

Verhage $\mathrm{S}$.

Verhagen $\mathrm{E}$.

Verhoef M.-J.

Verkissen M.N.

Vermorgen $\mathrm{M}$.

Verne J.

Vernooij-Dassen M.

Vernooij-Dassen M.J.F.J.

Verschuur E.M.L.

Vesel L.

Vesel T.

Viant L.

Vickerstaff V.

Victor J.

Vidal C.

Vidal T.

Vidal Rodriguez A.

Vie J.-F.

Viegas $\mathrm{S}$.

Viehrig M.

Viftrup D.T.

Vikas N.N.

Vilches-Aguirre $\mathrm{Y}$.

Vilfaillot A.

Villalobos $\mathrm{M}$.

Villavicencio-Chávez C.

Vinant $P$.

Vincent $C$.

Vincent $\mathrm{K}$.

Vineela R.

Vinke B.

Vinkeroye $\mathrm{C}$.

Virdun C.

Vissers K.

Vissers K.C.P.

Vivat B.

Vlčková K.

Vogel A.

Vogel T.

Vogrin S.

Vogt J.

Voisin-Saltiel S.

Völkel M.

Volken T.

Volkert A.

Vollmar H.C.

Volpp K.

Voltz R.

Von Blanckenbur Gunten C.

Von Hayek J.

Von Trott P.

Vos S.

Voss $\mathrm{H}$.

Vranceanu A.R.

Vriens J.

W

Wachterman M.

Wagatsuma Y.
P01-048

FC 12

P02-177

P01-507

P01-067

P02-277

P01-385

P02-353

P01-468

P01-122

P01-228

P01-199

PPC 5, P01-030

P01-122

P01-082, P01-183

P01-182

FC 12, P01-261, P01-322, P01-422, P02-528 P01-163, P01-164, P02-202

FC 23

P01-300, P02-435

P02-445

P01-326, P02-445

P02-013

PS 3, P01-558, P02-126

P02-070

P01-320

P02-513

P02-094

P02-410

$\mathrm{P} 02-330$

FC 6, FC 16, P01-224, P01-232

FC 5

FC 18

P02-220

$\mathrm{P} 02-276$

P01-089, P01-092, P02-389

P02-455

P02-276

P02-299

P01-015, P01-042

P01-308

P01-329

P01-486

FC 22

P01-294

FC 23, P02-291

FC 19

FC 13, FC 17, P02-184, P02-539

P01-110

P02-555

FC 16

P02-276

P01-329

P01-520

P01-193, P01-194, P01-200

FC 6, FC 6, P01-309

P02-549

5, FC 6, FC 6, FC 20, P01-081, P01-090, P01-211, P02-155,

P02-173, P02-345, P02-536

PL 2, P01-109, P01-124

P02-074, P02-097

P01-196, P01-231

P02-040

$\mathrm{P} 02-524$

P01-110, P02-286

P02-512

P01-216

P01-445

P02-035
Wagemans A.

Wager J.

Waghorn $\mathrm{M}$.

Wagland R.

Wagner S.

Wakefield D.

Waldron D.

Waldron P.D.

Wallin L.

Walls E.

Walrond $\mathrm{H}$.

Walshe C.

Wälter R.

Walters L.

Wampfler J.

Wang Y.-W.

Wanklyn S.

Wannke L.

Ward G.

Ward L.

Warren S.

Warren T.

Warriner K.

Warter C.

Watanabe K.

Waters L.

Watson A.

Watson M.

Watson R.

Watts $\mathrm{G}$.

Watzke $\mathrm{H}$.

Watzke H.H.

Weafer J.

Webber L.

Webber N.

Weber M.

Weber $\mathrm{O}$.

Webley J.

Weck C.

Weckbecker K.

Wedding U.

Wee I.

Wegleitner K.

Wei T.-Y.

Weightman A.

Weil A.

Weil J.

Wells $\mathrm{H}$.

Welsch K.

Wenk R.

Wennman Larsen A.

P01-110

P01-005, P01-010, P01-028

P01-216

P02-280

P01-253, P02-368

P02-489

P01-113, P02-099

P02-149

P02-214

P01-310

P02-449

PS 15, P01-149, P01-348, P01-354, P01-421, P01-426, P01-429,

P01-430, P01-464, P01-525, P02-182, P02-356

P01-329

P01-048

FC 1

P02-514

P02-292, P02-332

P02-045, P02-183

P01-564

P01-044

P01-261, P01-422

P01-332

P02-136

P01-076

P01-225, P02-509

P02-125

P02-336

P02-137

P01-223

P01-482

P02-200

P02-289

FC 11

FC 20

P02-257

P02-053

P01-094

P01-506

P01-476

P01-253

FC 6, FC 6, P01-230, P01-245, P01-309, P02-180

P02-216

P01-289

P02-301

P02-553

FC 2, P02-489

FC 24, P01-247, P02-396, P02-506

P02-505

PPC 4

P02-548

P02-016

P02-427

P01-089, P01-092, P02-389

FC 3, P01-259

PPC 2

P01-026

P02-277

Werner $\mathrm{P}$.

Wesseling $\mathrm{E}$.

Wesseling $\mathrm{G}$.

Westerholm B.

P01-331

P02-135

P01-041

FC 24

P02-442

White $\mathrm{C}$.

P02-056

P01-252, P02-431

White L.

White N.

Whitla L.

Whitman M.

FC 20

P01-008

P02-048

P02-506

Wichmann A.B.

FC 23

Wicki M.T. 
Widagdo T.M.M.

Wieles G.A.M.

Wieles-Griffioen G.A.M.

Wikert J.

Wiksuarini E.

Wilcock A.

Wilde Larsson B.

Wiles J.

Wilkinson A.

Wilkinson E.

Wilkinson S.

Wille-Friederichs A.

Willemen I.

Willems D.L.

Williams E.

Williams $\mathrm{H}$.

Williams $\mathrm{J}$

Williams K.

Williams L.

Williams R.

Wilson D.

Wilson D.M.

Wilson M.P.

Wilson R.

Winsjansen B.

Wirz S.

Wiseman T.

Witalis $\mathrm{K}$.

Witham G.

Witkamp E.

Witkamp F.E.

Woellert K.

Wojcik T.

Wojtacki J.

Wolf A

Wolff S.L.

Wollaston A.

Wolszczak K.

Wong C.H.

Wong G.C.

Wong J.

Wong K.Y.Y.

Wong L.H.

Wong S.L.

Wong V.

Wong Y.-L.

Wood A.

Wood J.

Woollett A.

Workentin K.

Worlock P.

Worthley K.

Wouters E.

Wright E.

Wright $\mathrm{H}$.

Wright S.

Wright-Hughes A

Wu C.-Y.

Wu H.Y

Wu J.

Wu K.

Wyszadko A.
P01-511 $X$

P01-389 Xenikou M.

P02-252, P02-448

P02-417

$\mathrm{P} 02-473$

XuY.

P01-363

PL 3, P01-183 Y

P01-181 Yaakup $\mathrm{H}$ P02-076

P01-174, P01-344 Yamada Y. $\quad$ P02-150

P01-278 Yamaguchi T. P02-035

P02-333 Yamazaki M. P02-129

P02-126, P02-557 Yang C. P01-192

P02-368 Yang C.-H. P02-373

P01-199 Yang $\mathrm{H}$. P02-426

P01-140, P01-528, P01-530 Yang W.-L. P01-192

P02-552 YardleyS. FC 20

P02-273 Yarwood G. P01-147

$\begin{array}{ll}\text { P01-130 Yates P. PS 7, FC 20 } & \end{array}$

P01-172 Ybarra-Huesa C

$\begin{array}{lll}\text { P01-344 Yee C.M. P02-181 } & \text { P. }\end{array}$

P01-504, P02-414 Yeo W.

Yi D.

Yi D.H.

P02-145 Yik P.

P02-470 Yoon S.J.

P02-155 Yorganci E.

Yoshida M.

P02-488 Yoshikawa Y.

FC 21 Yoshinobu M

FC 25, P01-336 Yow F.

P01-169, P02-296 Yu P.

P01-168 Yumiba T.

P01-022

P01-283, P02-166 Z

P02-385 Zaimovic V.

PPC 5 Zainal Abidin H.A.

P02-505 Zainal Abidin M.Z.

Zaino L.

P01-505 Zana Á.

P02-086 Zaric B.

P02-181 Zavratnik B. P02-405

P01-505 Zehm A. P01-456

P02-076 Zeller J. P02-510

P01-051 Zeni E. P02-025

P02-360 Zernikow B. PPC 3, P01-005, P01-010, P01-028

P01-048 Zhang Z. P01-288, P02-551

FC 24 Ziegler L. FC

P02-396 Zigan N. P01-520

P01-295 Zijlstra M. P02-390

P02-490, P02-499 Zimmermann C. FC 3, FC 16, P01-209, P01-259, P02-175, P02-251,

$\begin{array}{ll}\mathrm{P} 02-353 & \mathrm{P} 02-401, \mathrm{P} 02-558\end{array}$

Zoboli E.L.C.P.

P02-137 Zolotarova Z
P02-209

FC 21, P01-147, P01-557 Zwahlen M.

FC 8 Zwahlen S.

P01-215, P02-514 Zwakhalen S.M.G.

Zwakman M.

$\begin{array}{ll}\text { P01-213 } & \text { Zybell L. } \\ \text { P02-077 } & \text { Zygogianni A }\end{array}$

$\mathrm{P} 01-283, \mathrm{P} 02-450, \mathrm{P} 02-451$
P01-229

P01-569

P02-032

FC 1

P01-415

P01-329

P01-145 